\title{
SANITARY LANDFILL 1991 ANNUAL REPORT - GROUNDWATER MONITORING REPORT (U)
}

RECEIVED

FEB - 41993

ก c.

by C.Y. Thompson

Westinghouse Savannah River Company

Savannah River Site

Aiken, South Carolina 29808

Other Authors:

\section{G. T. Norrell}

(WSAC)

C. B. Bennett

(WSRC)

This paper was prepared in connection with work done under Contract No. DE-AC09-89SR18035 with the U. S. Department of Energy. By acceptance of this paper, the publisher and/or recipient acknowledges the U. S. Government's right to retain a nonexclusive, royalty-free license in and to any copyright covering this paper, along with the right to reproduce and to authorize others to reproduce all or part of the copyrighted paper.

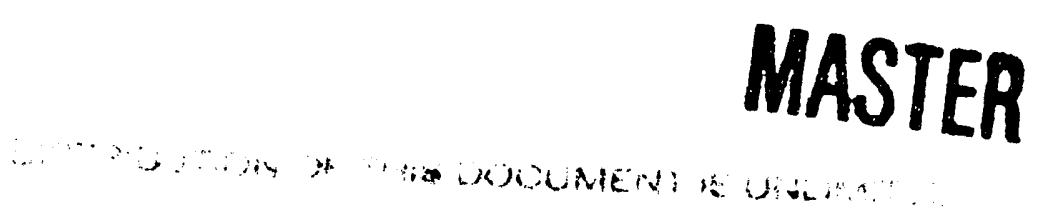


WSRC-TR-92-111

\section{SANITARY LANDFILL 1991 ANNUAL GROUNDWATER MONITORING REPORT (U)}

Publication Date: February 1992

Mestinghouse Savannah River Company

Savannah River Site

Niken. SC 298018

1.1'1

$\therefore$ rapson

qP

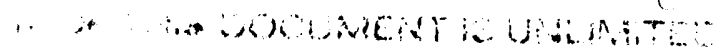

Christine y. Thumpson 


\section{DISCLAIMER}

This report was prepared as an account of work sponsored by an agency of the United States Government. Neither the United States Government nor any agency thereof, nor any of their employees, makes any warranty, express or implied, or assumes any legal liability or responsibility for the accuracy, completeness, or usefulness of any information, apparatus, product, or process disclosed, or represents that its use would not infringe privately owned rights. Reference herein to any specific commercial product, process, or service by trade name, trademark, manufacturer, or otherwise does not necessarily constitute or imply its endorsement, recommendation, or favoring by the United States Government or any agency thereof. The views and opinions of authors expressed herein do not necessarily state or reflect those of the United States Government or any agency thereof.

This report has been reproduced directly from the best available copy.

Available to DOE and DOE contracters from the Office of Scientific and Technical Information, P. O. Box 62, Oak Ridge, TN 37831; prices available from (615) 576-8401.

Available to the public from the National Technical Information Service, U. S. Department of Commerce, 5285 Port Royal Rd., Springfield, VA 22161. 


\section{SANITARY LANDFILL 1991 ANNUAL GROUNDWATER MONITORING REPORT (U)}

Gregory T. Norrell

Christoprei B. Bénnett

Cr istine "mpson

Unclassified

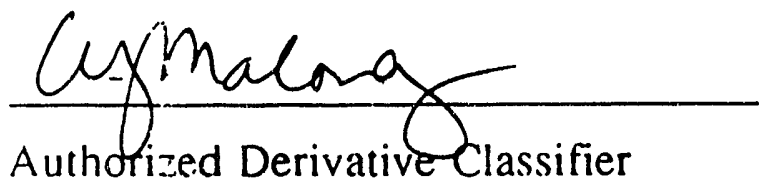




\section{ABSTRACT}

Concentrations of at least one of the following constituents: tritium, vinyl chloride, total radium, cadmium, 1,1,1-trichloroethane, 1,2 dichloroethane, 1,4 dichlorobenzene, trichloroethylene (TCE), tetrachloroethylene, and 1,1 dichloroethylene in excess of the primary drinking water standards were observed in at least one well monitoring the Sanitary Landfill during the third quarter of 1991. All of these constituents, except radium, were observed in the lower half of the original thirty-two acre site or the southern expansion site.

Trichloroethylene and vinyl chloride are the primary organic contaminants in groundwater beneath the Sanitary Landfill. Vinyl chloride has become the primary contaminant during 1991. Elevated levels of benzene were consistently detected in LFW 7 in the past, but were not present in any LFW wells during the third quarter of 1991. A minor tritium plume is present in the central part of original thirty-two acre landfill. Elevated levels of tritium above the PDWS were consistently present in LFW 10A through 1991. This well has exhibited elevated tritium activities since the second quarter of 1989 . 


\section{EXECUTIVE SUMMARY}

Results from the first through thirc quarter 1991 groundwater monitoring data continue to show evidence of elevated levels of several hazardous constituents beneath the Sanitary Landfill. These data also indicate that the southern expansion, as well as the original thirty-two acre site, may be contributing to the contamination. Estimated horizontal flow rates for the Water Table hydrologic unit support the supposition that some impact is from the southern expansion, since it is unlikely that contamination from the original site has had sufficient time to travel to points downgradient of the southern expansion. Point sources for contamination appear to be present in both the original landfill and the southern expansion.

Groundwater samples from the Sanitary Landfill exceeded the Primary Drinking Water Standards (PDWS) for ten constituents (eight hazardous and two radioactive) for the third quarter of 1991 . Tritium, trichloroethylene (TCE), and vinyl chloride are the primary contaminants present in the groundwater. Only one well (LFW 10A) had a tritium activity $(63 \mathrm{pCi} / \mathrm{ml})$ exceeding the PDWS during the third quarter of 1991. Thirteen wells (LFW 10A, 16, 21, 22, 37, 38, 39, 40, 58D, 59C, 59D, 61C, and 61D) had third quarter TCE concentrations in excess of the PDWS. The highest TCE concentration for the quarter was observed at LFW $10 \mathrm{~A}(28 \mathrm{ug} / \mathrm{l})$. Most of the elevated levels of contamination can be observed in the lower half of the original thirty-two acre site or the southern expansion. Groundwater flow in the Water Table hydrologic unit below the Sanitary Landfill is southerly (SRS coordinates) toward Upper Three Runs (UTR).

\section{INTRODUCTION}

The Savannah River Site (SRS) Sanitary Landfill is an approximately seventy acre site located just south of SRS Road C between the Savannah River Site's B-Area and Upper Three Runs Creek (Figure 1). Figure 2 depicts the topographic setting of the Sanitary Landfill. 


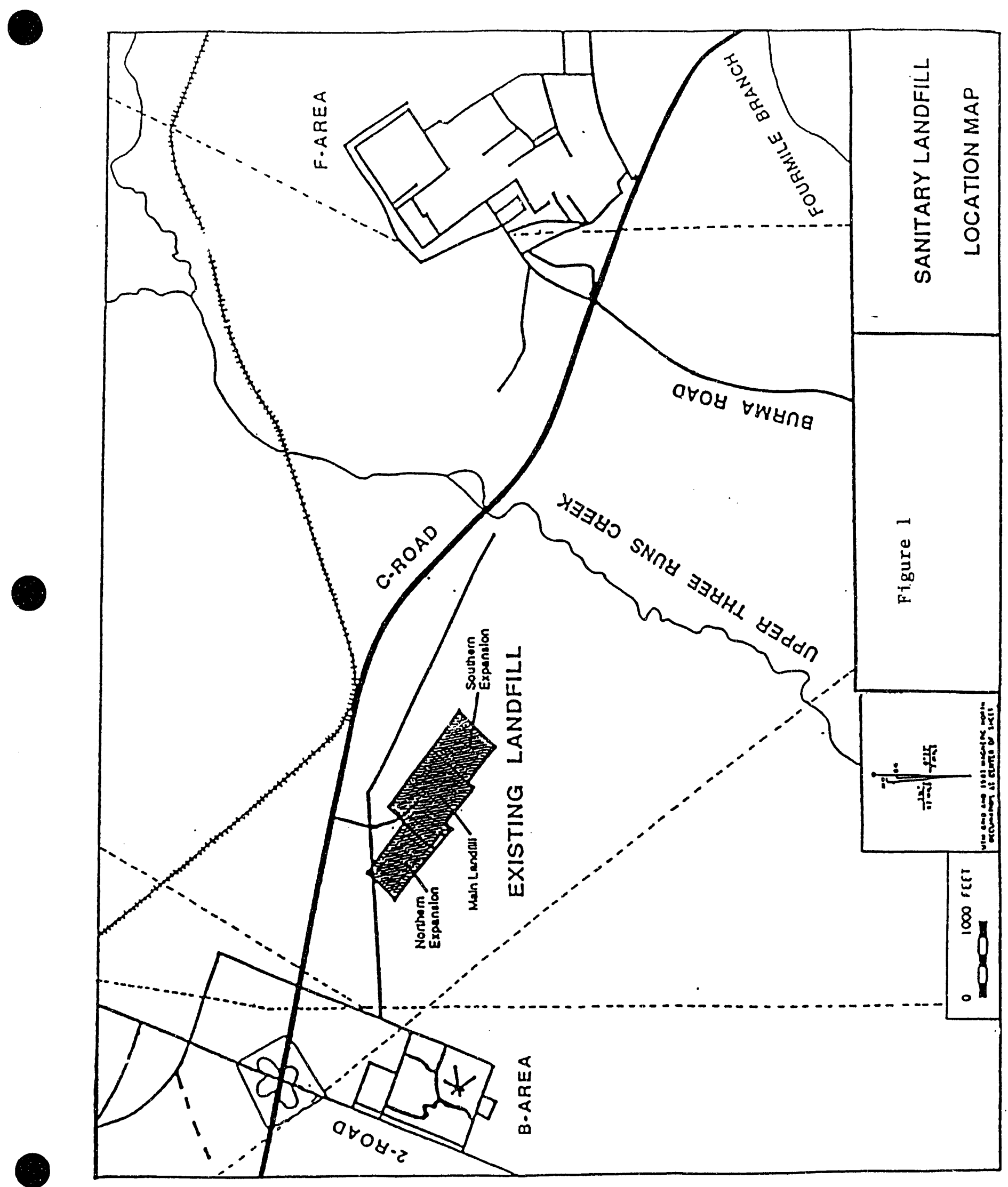




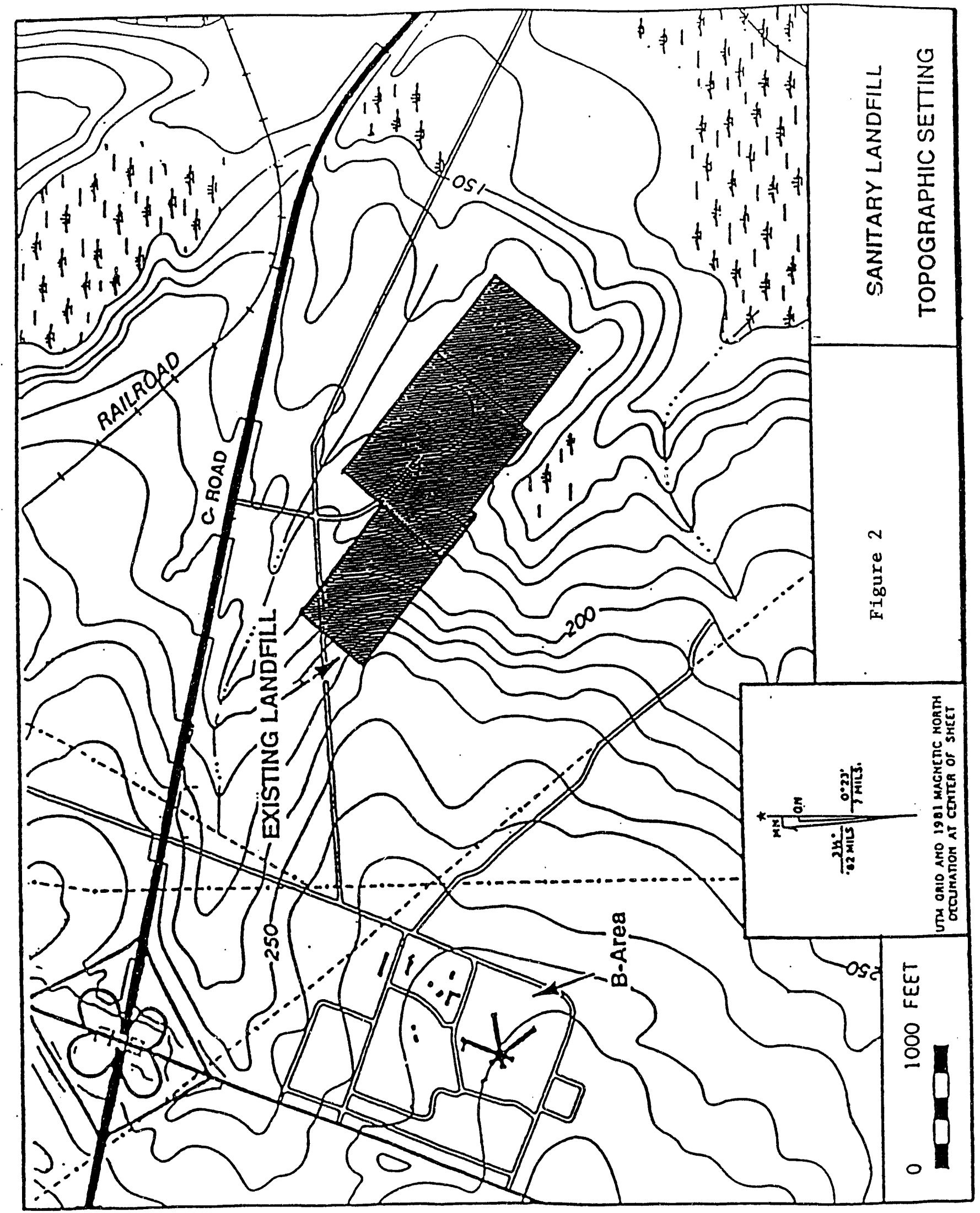


The Sanitary Landfill has been receiving waste since 1974, and is currently operating under Domestic Waste Permit Modification \#087-A which was renewed on August 24,1991. The new reporting schedule, specified in the renewed permit, is effective for 1992 Groundwater Reports. This Groundwater Quality Assessment Report reviews field and analytical data through the third quarter of 1991. A previously submitted report was based on data from the first and second quarters of 1991. Future annual reports will include data for the entire previous year, but data interpretation will be based primarily on data from quarters four through three. Fourth quarter data will be included in an appendix, since it is not possible to incorporate that data in the text and figures under the permitted reporting schedule.

The original landfill is a thirty-two acre square shaped area that was filled to capacity in early 1987 . In 1987 , two expansion areas were permitted to the north and south of the original landfill. The northern expansion is a sixteen acre tract and the southern expansion is a twenty-two acre tract. The southern expansion is approximately three-fourths full at this time. The Domestic Waste Permit Modification \#087-A has been revised and implemented allowing the utilization of the northern expansion for waste disposal and requires groundwater monitoring and reporting. The northern expansion has not yet been used. Figure 3 is a map depicting the boundaries of the original tract and the northern and southern expansion.

Fifty-seven weils currently monitor groundwater quality at the Sanitary Landfill (Figure 4). Most of the wells are screened in the Water Table hydrologic unit. Fourteen of the wells are in or around the boundaries of the original thirty two acre landfill and were installed from 1981 to 1984 . These wells include: LFW 6, 7, 8 , $10 \mathrm{~A}, 16,17,18,19,20,21,22,23,24$, and 25 . Seventeen additional wells were installed in late 1986, around the southern and northern expansions. These wells include: LFW 26, 27, 28, 29, 30, 31, 32, 33, 34, 35, 36, 37, 38, 39, 40, 41, and 42. Groundwater monitoring data from all of the LFW wells for the third and fourth quarters of 1991 are included in Appendices B and C, respectively.

Eight wells (LFW 6, 7, 8, 16, 17, 18, 19, and 20) were installed in 1981. LFW 6,7 , and 8 are constructed of 4 " galvanized steel casings with PVC screens. LFW 16-20 are constructed with PVC casings and screens. LFW 10A, 21, 22, 23, 24, 
SANITARY LANDFILL BOUNDARY

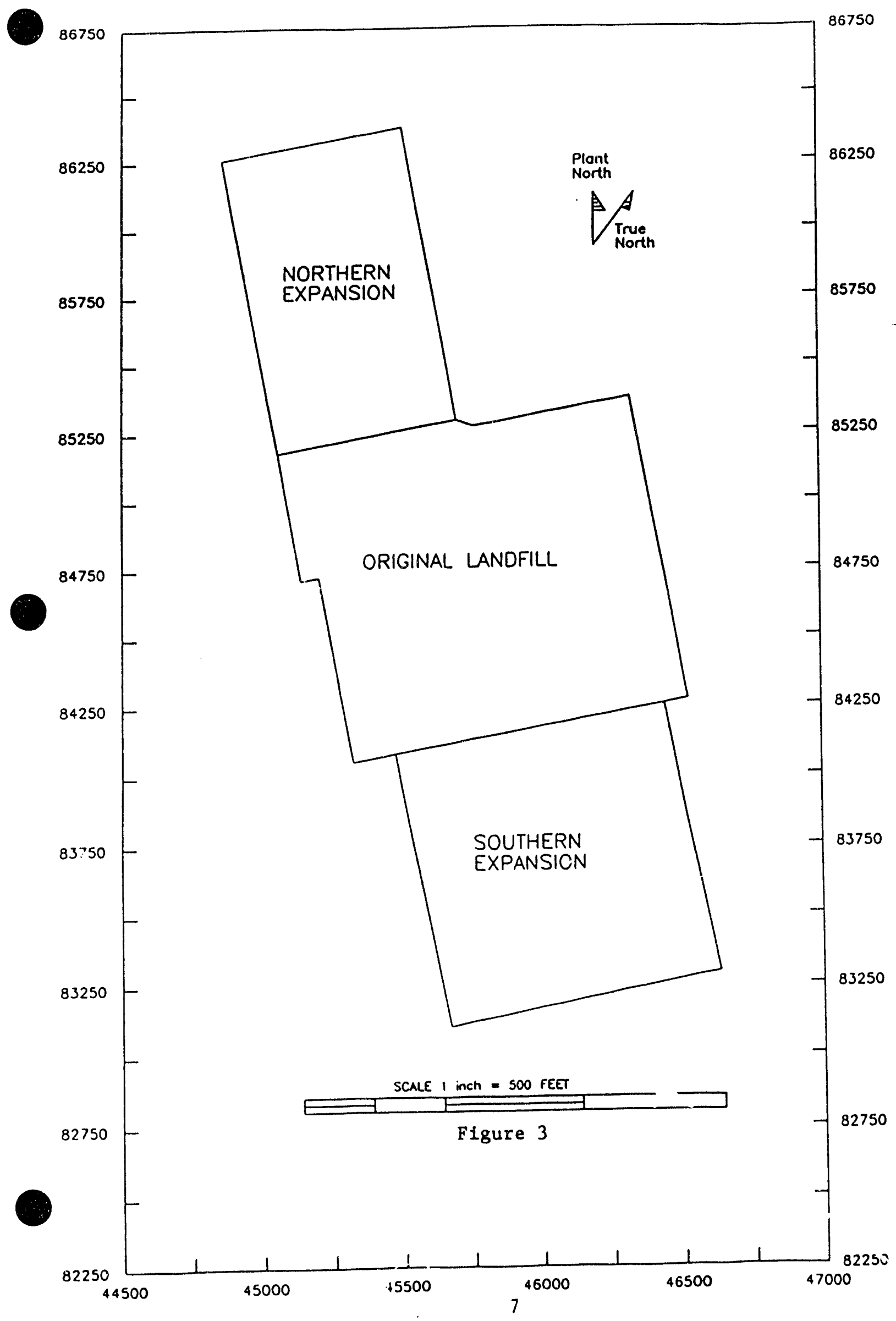


SANITARY LANDFILL WELL LOCATIONS

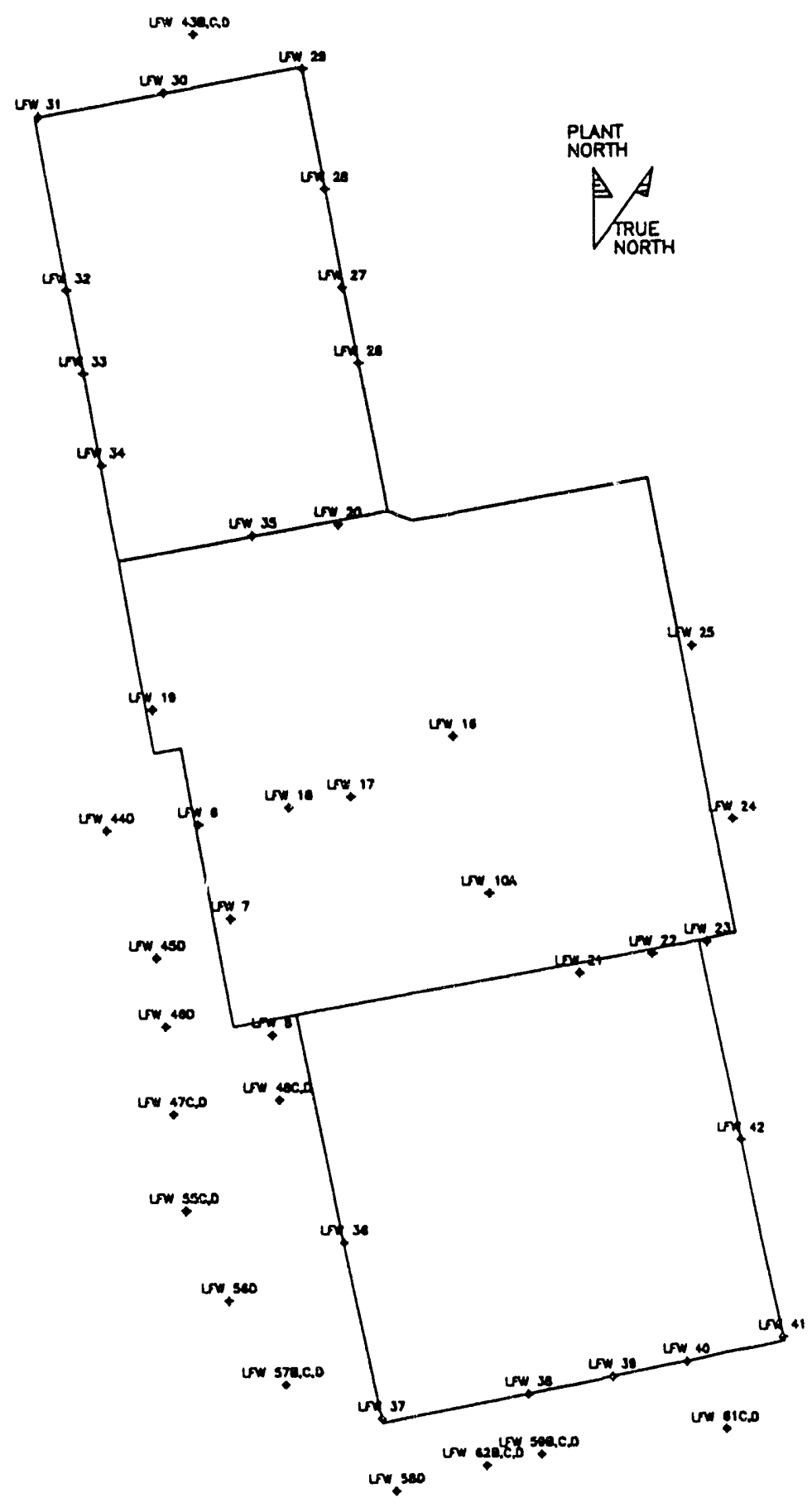

SC $E$ I inch $=500$ FEET

Eigure 4

$$
\infty n+\infty
$$


and 25 were installed in 1984, and are constructed with PVC casings and screens. LFW 26 through 42 were installed in late 1986 around the southern and northern expansions of the landfill and are constructed with PVC casings and screens. LFW43B through 62D were installed in mid 1991, and are constructed with PVC casings and screens.

A Groundwater Quality Assessment Plan was approved by SCDHEC to install additional monitoring wells. Twenty-six wells (43B,C,D; 44D; 45D; 46D; 47C,D; 48C,D; 55C,D; 56D; 57B,C,D; 58D; 59B,C,D; 60D; 61 C,D; 62B,C,D) were installed in accordance with this plan during 1991. These wells are located outside the boundaries of the sanitary landfill (Figure 4). LFW 43B, C, D are upgradient wells and well clusters LFW 58, 59,60,61, and 62 are downgradient wells. Well clusters LFW 44, 45, 46, 47, 48, 55, 56, and 57 are located west of the original landfill and southern expansion. Due to health and safety risks from high methane gas concentrations within the sanitary landfill, nine of the originally proposed wells were not installed (approved by SCDHEC). The remaining wells were installed in accordance with the approved ( ondwater Quality Assessment Plan.

\section{MONITORING DATA}

The Sanitary Landfill groundwater quality data include field and lab analyses from monitoring wells sampled during the third quarter of 1991. Most of the wells monitor the Water Table unit. A map indicating the locations of the wells in and around the landfill is included as Figure 4.

Concentrations of at least one of the following constituents: tritium, vinyl chloride, 1,2 dichloroethylene, 1,4 dichlorobenzene, 1,1 dichloroethylene, total radium, tetrachloroethylene, trichloroethylene, 1,1,1-trichloroethane, and cadmium were obs ed in at least one well in excess of the primary drinking water standards monitoring the Sanitary Landfill for the third quarter of 1991. The following wells exceeded the primary drinking water standards for the indicated constituents as shown below: 
THIRD QUARTER 1991

\begin{tabular}{|c|c|}
\hline Cintaminiant and Walls & Lecalion \\
\hline NCADNIUNA: $1.1 \cdot$ W.22 & SE \\
\hline 13) 1.1 DKCIII.OROLEIIIYIIINI: I.IWW 17 & o \\
\hline (CTRIILAN: II:W ION & o \\
\hline D) IOINI. KAIDIUM: I.LW 7. 16, 25, 27.42,46D & O.NE.SE \\
\hline 1:) $\mathrm{TCl}:=1.1 \mathrm{~W} 10 \mathrm{~N}, 16,21,22,37,38,39,40,58 \mathrm{D}, 59 \mathrm{C}, 59 \mathrm{D}, 61 \mathrm{C}, 61 \mathrm{D}$ & O.SE \\
\hline 1.) VINYI. CIII.ORIDE: LFW 7,8,47C,48D & 0 \\
\hline (i) TETR $\wedge$ CIII.OROETHYYLENE: 10A, 16, 21, 39. & O,SE \\
\hline (i) 1.4 DICIII.OROBENZENE: LFW 7 & o \\
\hline 1) 1.2 DICIII.OKOT:THANE: I.FW 17 & 0 \\
\hline
\end{tabular}

$$
\begin{aligned}
& O)=\text { Original Sitc } \\
& S 1:=\text { Southern Expansion } \\
& N E=\text { Northern Expansion }
\end{aligned}
$$

Most of the wells are downgradient of areas that have been used to landfill waste. Groundwater wells monitoring the northern expansion did not exhibit elevated levels of contaminants above the PDWS. In general, wells which produced samples with elevated radioactive parameters are located in the original landfill and wells which produced samples with elevated organic constituents are located mostly in the southern expansion. Figures 5 through 7 are maps depicting tritium, total radium, trichloroethylene, and vinyl chloride, (the most consistently detected constituents) for all of the LFW wells from the third quarter of 1991. Figure 7A represents the vinyl chloride concentrations measured in the fourth quarter of 1991.

As can be seen above, virtually all of the contaminants are present in wells in the original thirty-two acre site or the southern expansion. In past quarters, wells which produced samples with elevated radioactive parameters were typically located in the original landfill and wells which produced samples with elevated organic constituents were located mostly in the southern expansion.

Appendix A contains time-series plots for potential groundwater contaminants at the SRS Sanitary Landfill. 
SANITARY LANDFILL 3091 WATER TABLE: TRITIUM $(p \mathrm{Ci} / \mathrm{mL})$

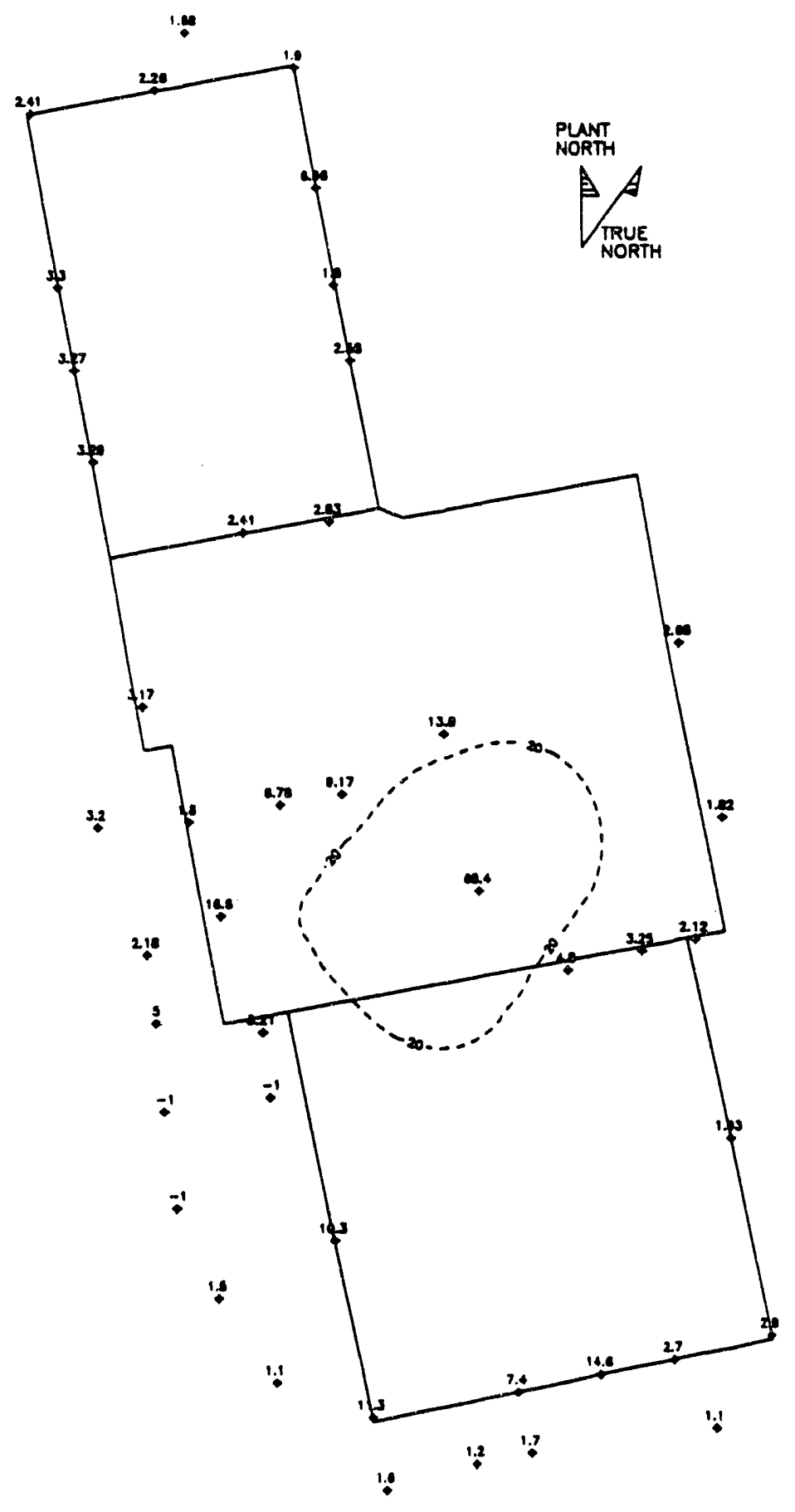

SCNE 1 inch - 500 FEET

Figure 5

(Lans sew conotes uss nuw octection unm) 
SANITARY LANDFILL 3091 WATER TABLE: TOTAL RADIUM ( $D C i / L$ )

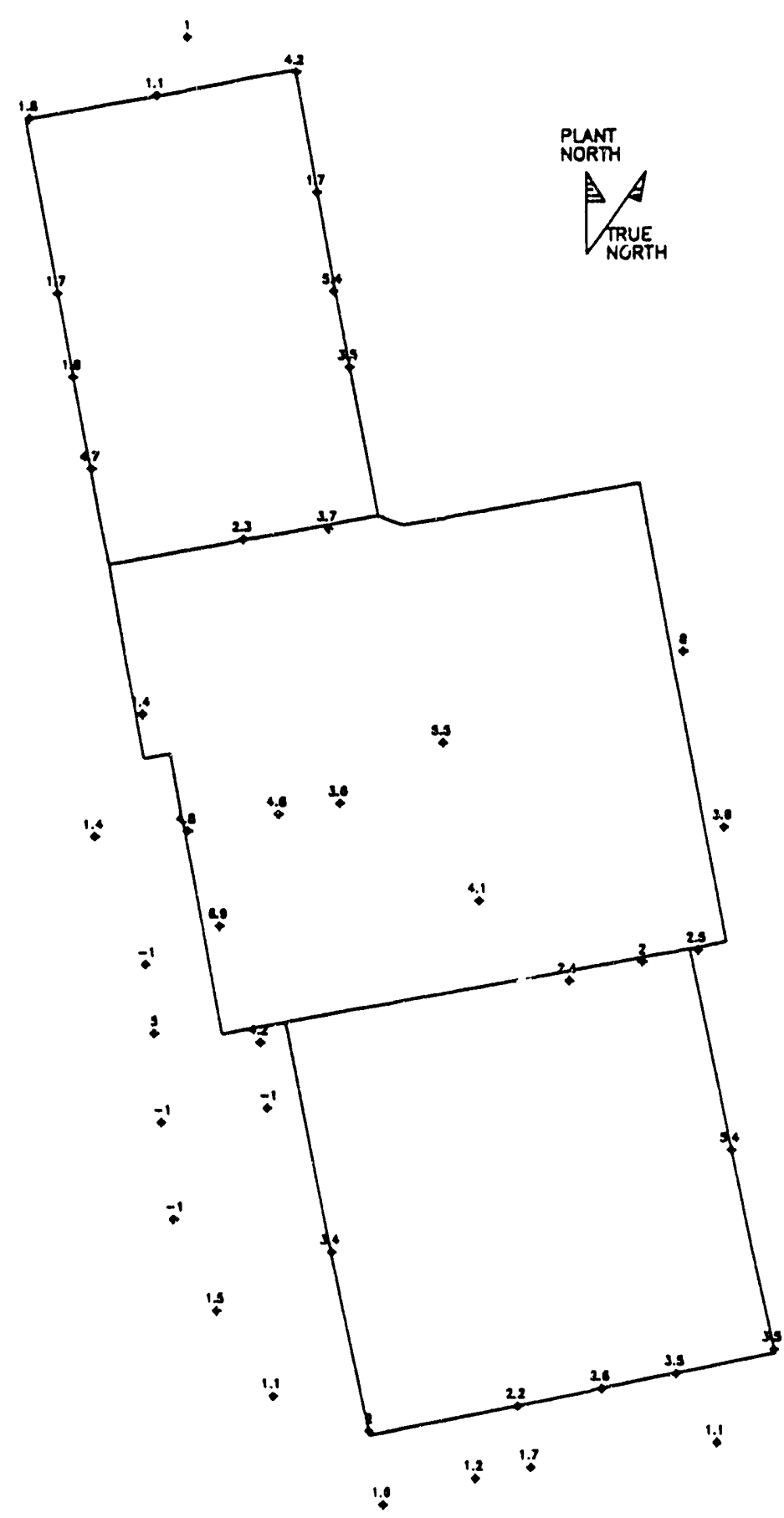

F1gure 5A

SCALE. 1 inch $=500$ FEET

(MNAS SICN DENOTES LESS RWN OETECTION UMT)

$\because$

$11 \mathrm{~A}$ 
SANITARY LANDFILL 3091 WATER TABLE: TRICHLOROETHYLENE (ug/l)

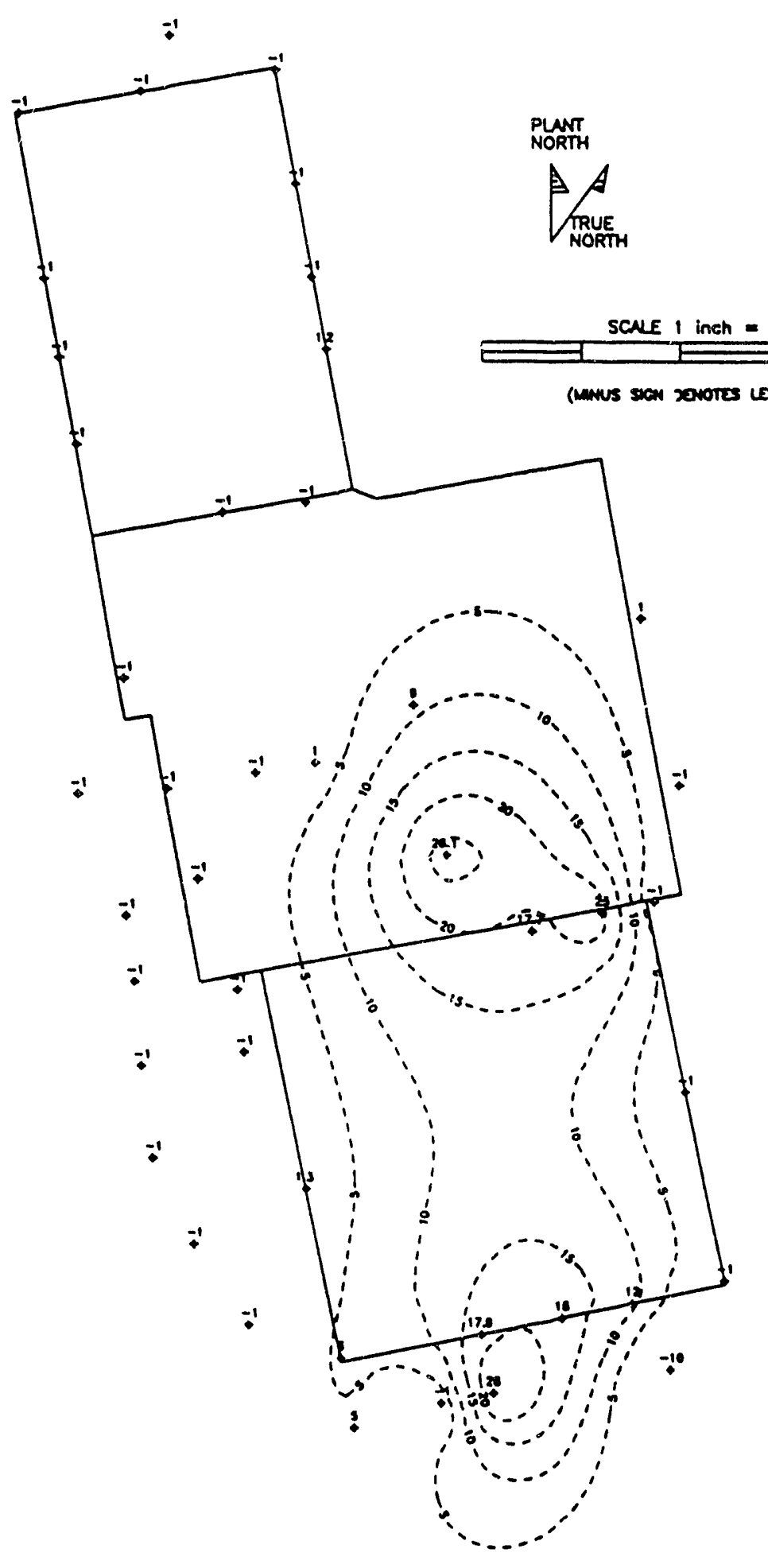


SANITARY LANDFILL 3091 WATER TABLE: VINYL CHLORIDE (ug/L)

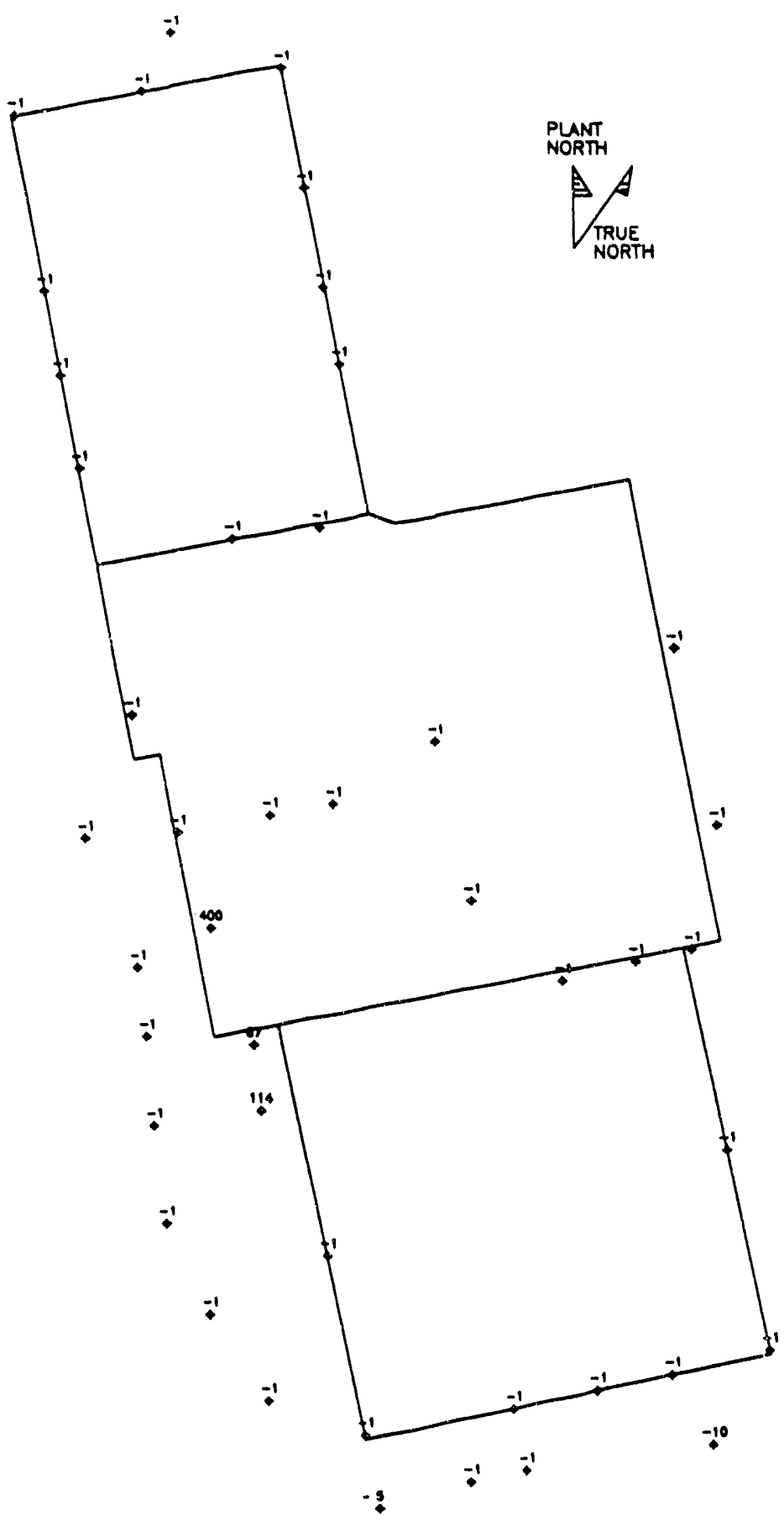

Figure 7

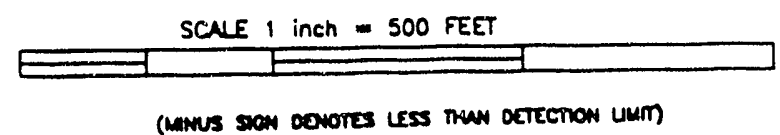

$-1$ 
SANITARY LANDFILL $4 Q 91$ WATER TABLE: VINYL CHLORIDE (ug/L)

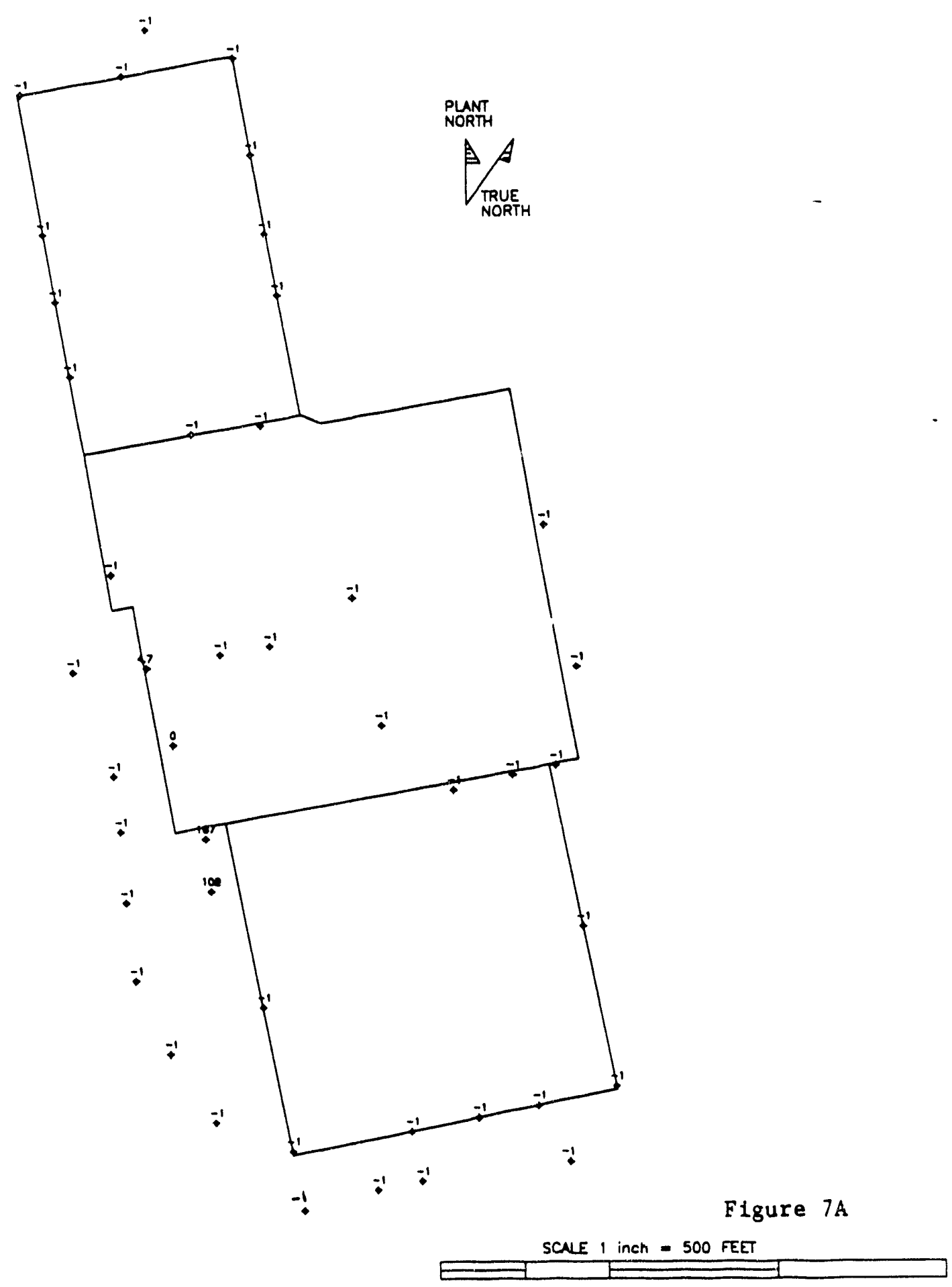

(MINUS SIEN DENOTES LESS THW DETECTION UMIT)

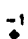

o - nolcates well Not SMMPLED

$13 \mathrm{~A}$ 


\section{Radionuclides}

The only recorded tritium concentration above the PDWS in the third quarter of 1991 was observed in LFW 10A. Most of the LFW monitoring wells exhibited slightly elevated tritium activities typical of tritium in the shallow aquifer from percolation of weakly tritiated rainfall (Figure 5). In the lower half of the original thirty-two acre site and the western portion of the southern expansion, a plume of tritiated groundwater exists indicative of a point source release. This point source most likely occurred in the south-central portion of the original landfill.

Figure 5 depicts tritium concentrations during the third quarter of 1991 , throughout the Sanitary Landfill. The elevated levels of tritium are centered around the lower portion of the original site and the northern end of the southern expansion. LFW 10A continues to exhibit the highest concentrations of tritium above the PDWS. During the third quarter of 1989, LFW 10A exhibited a sharp upward trend in tritium concentrations. Since the increase, this well has consistently exhibited elevated tritium activities, although values have fluctuated from quarter to quarter.

\section{Tritium in the LFW-Series Monitoring Wells}

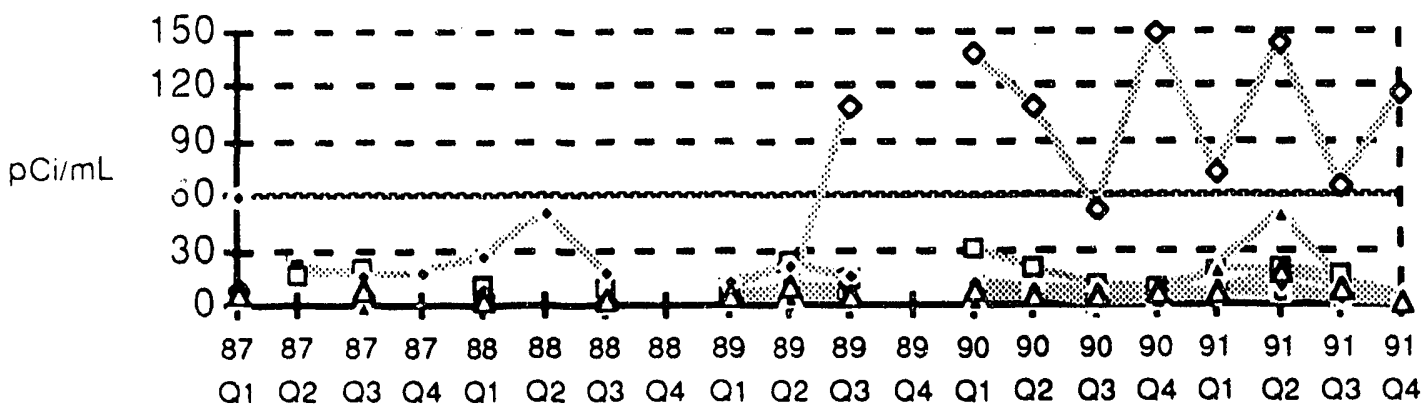

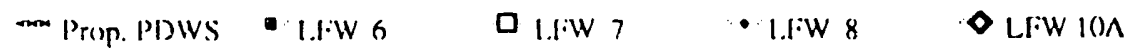

$$
\begin{aligned}
& \text { - I.1:W la } \quad \Delta \text { I.1.W } 17
\end{aligned}
$$

Six wells (LFW 7, 16, 25, 27, 42, 46D) were observed to have elevated total radium activities above the PDWS during the third quarter of 1991 . In each case, the activity is very close to the PDWS of $5 \mathrm{pCi} / \mathrm{L}$. Examination of Figure $5 \mathrm{~A}$ 
that the slightly elevated radium activites occur in all portions of the landfill, without regard for flow directions. The natural $\mathrm{pH}$ of groundwater beneath the sanitary landfill is acidic, and is potentially responsible for the dissoluticn of natuial radium from the sedimentary matrix in sufficient concentrations to generate the observed activity levels. Below are time-series plots for total radium at several LFW wells. Appendix A contains the time-series data for all the LFW wells.

\section{Total Radium in the LFW-Series Monitoring Wells}

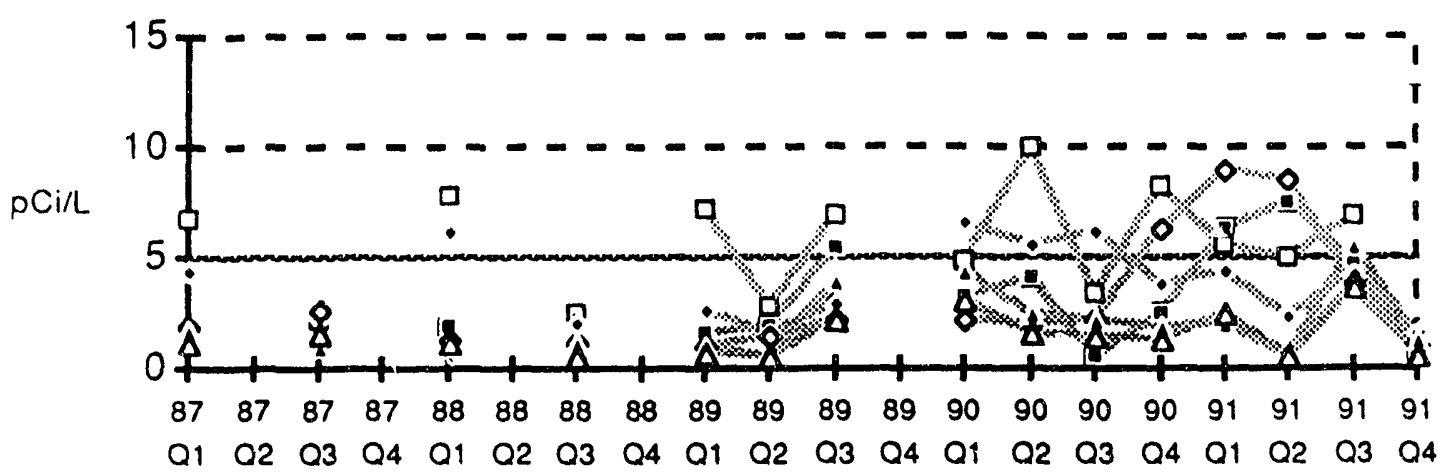

$$
\begin{array}{lllll}
\cdots \text { PDWS } & \cdots \text { LFW } 6 & \text { QLFW } 7 & \cdots \text { LFW } 8 & \diamond \text { LFW 10A } \\
\cdots \text { LFW } 16 & \triangle \text { LFW } 17
\end{array}
$$

Total Radium in the LFW-Series Monitoring Wells

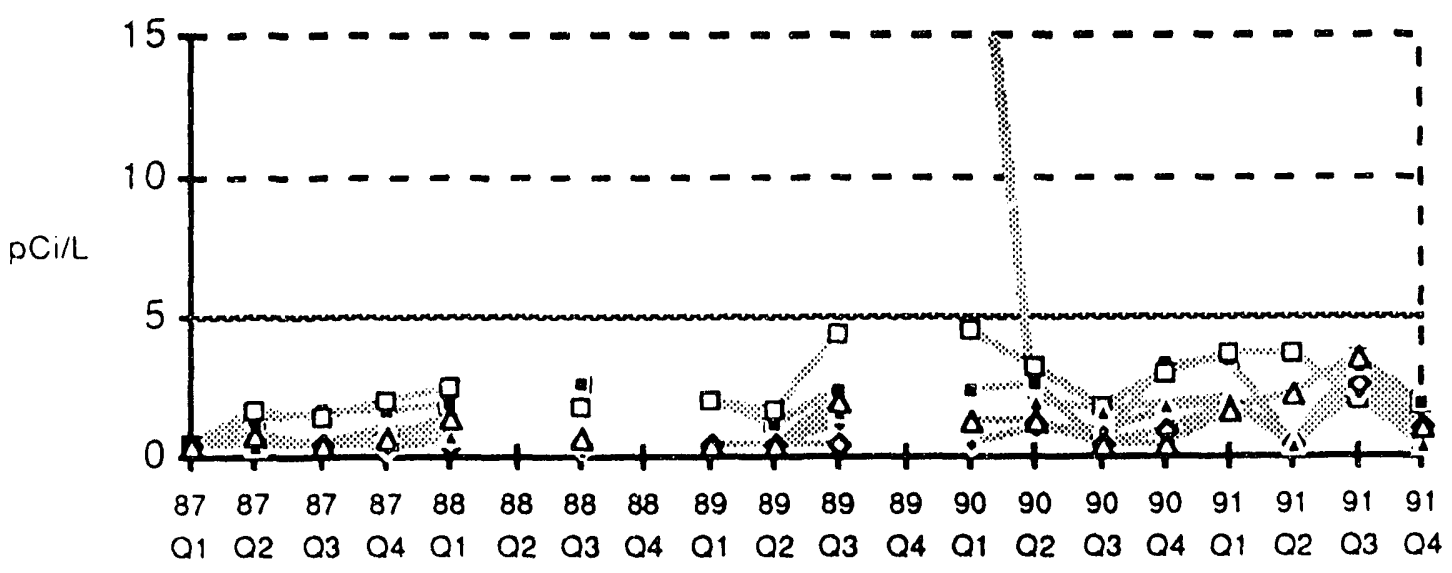

m POWS $\cdots$ LFW $36 \quad \square$ LFW $37 \cdots$ LFW $38 \quad \bullet$ LFW $39 \quad \cdots$ LFW $40 \quad \cdots$ LFW 41 


\section{Organics}

TCE was observed in concentrations above the PDWS at thirteen groundwater monitoring wells during the third quarter (Table 1). The highest level of TCE was observed in LFW 10A (28 ug/l). LFW 10A has historically been the most contaminated well in the Sanitary Landfill well network. It currently possesses elevated levels of TCE, tritium, and tetrachloroethylene above the PDWS's. Figure 6 illustrates the contoured TCE data for the LFW wells. Two point sources for this contaminant appear to be present, one in the south-central portion of the original landfill (identical to the tritium point source) and one in the lower portion of the southern expansion.

Figure 6 clepicts TCE concentrations during the third quarter of 1991. LFW 1)A was observed to have the highest TCE concentration $(28 \mathrm{ug} / \mathrm{l})$. Until second quarter 1991, LFW 10A had been experiencing an upward trend in TCE concentrations; at that time, however, there was a slight decrease, and that decrease has continued through the third quarter. LlFW 21 has exhibited the highest TCE concentrations in the past, however, this well is also experiencing a downward trend of TC.E.

In late 1987, groundwater monitoring wells LFW 37 and 38 exhibited levels of TCE above $30 \mathrm{ug} / \mathrm{l}$. Since then, these wells have experienced a downward trend of TCE concentrations. As depicted on Figure 4, these wells are located in the southwest corner of the southern expansion site. The source of the elevated TCE may be attributable to wastes deposited in the southern section of the southern expansion in late 1987.

TCE and tetrachloroethylene have been the primary, consistently detected organic contaminants in the Sanitary Landfill groundwater monitoring network. I.FW I()A. 21, 22, 37, 38, and 39 have consistently exhibited elevated levels of TCE above detection during 1991. Vinyl chloride, however, has become a primary contaminant during 1991, as shown in the below time series plots and in Appendix $A$. Figures 7 and $7 \mathrm{~A}$ represent the concentrations of vinyl chloride measured in the monitoring wells during the third and fourth quarters of 1991 , respectively. 
Vinyl Chloride in the LFW-Series Monitoring Wells

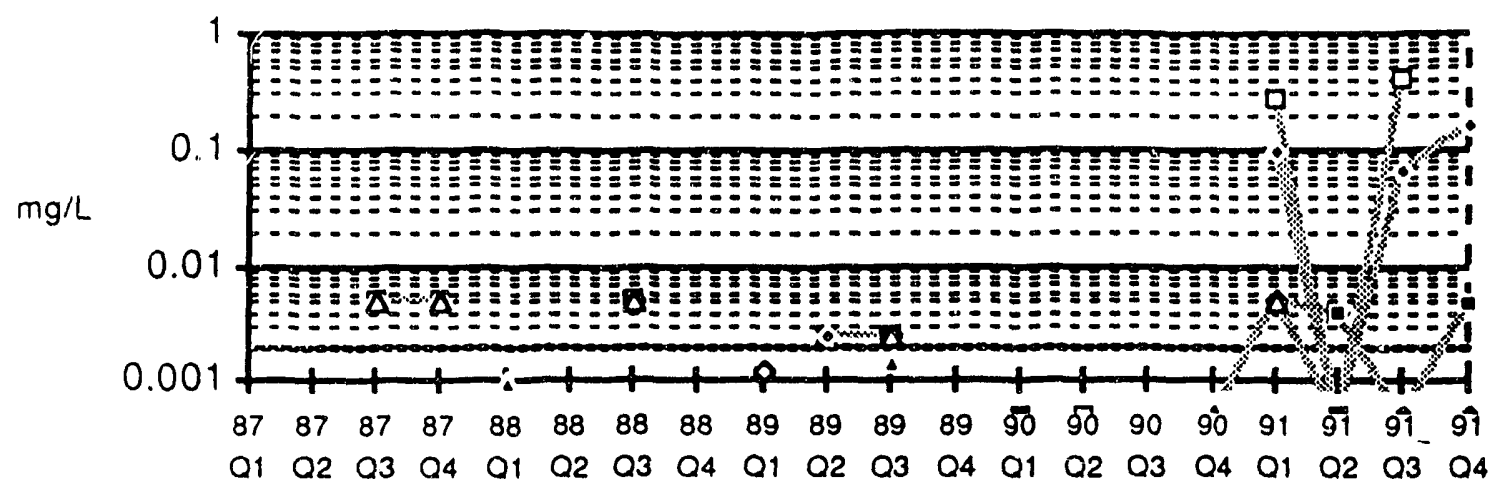

\begin{tabular}{|c|c|c|c|c|}
\hline$\sim$ PDWS & $\cdots$ LFW 6 & : LFW 7 & $\cdots$ LFW 8 & $\sigma$ LFW $10 A$ \\
\hline$\cdots$ " LFW 16 & $\triangle$ LFW 17 & & & \\
\hline
\end{tabular}

Vinyl Chloride in the LFW-Series Monitoring Wells

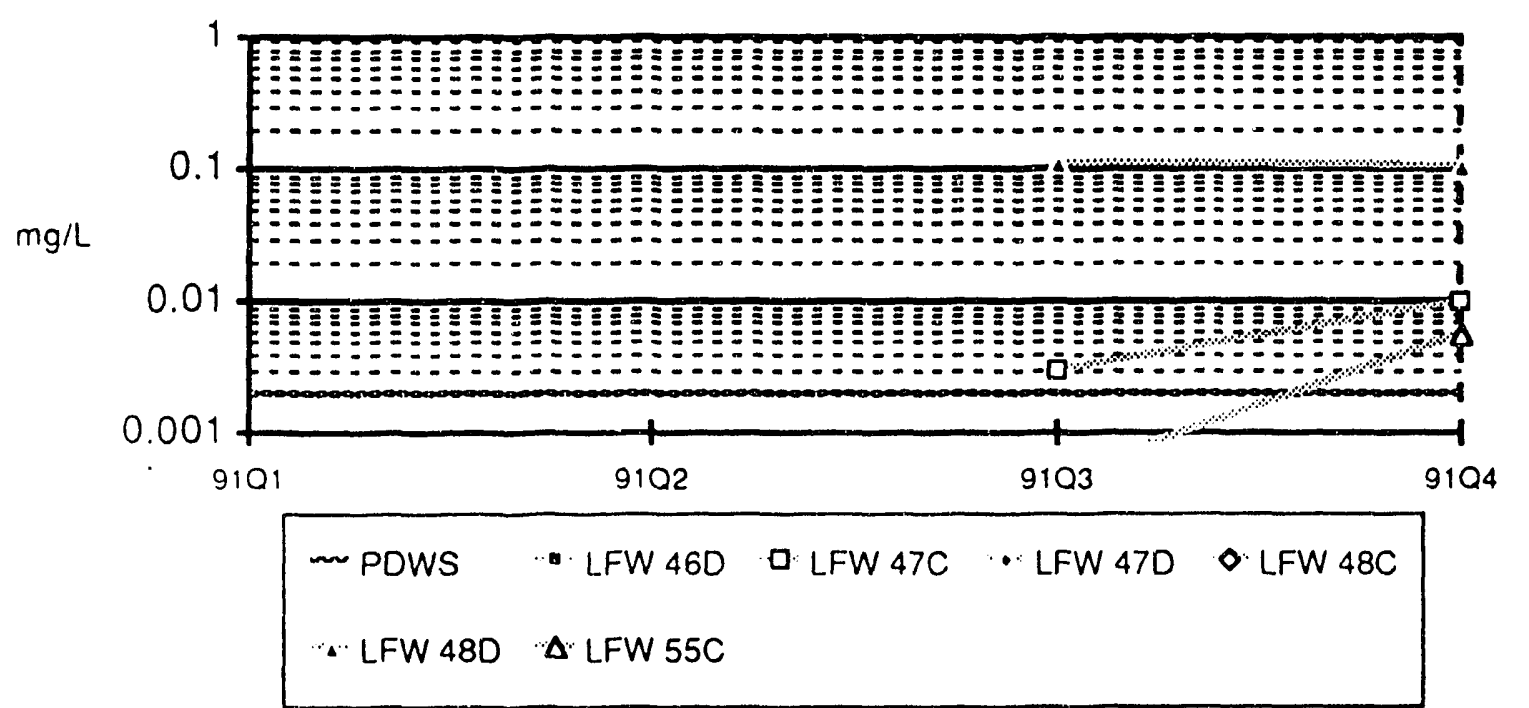

There are several possible origins for the vinyl chloride to be considered. First, vinyl chloricle $\left(\mathrm{C}_{2} \mathrm{H}_{3} \mathrm{Cl}\right)$ and 1,2-dichloroethylene $\left(\mathrm{C}_{2} \mathrm{H}_{2} \mathrm{Cl}_{2}\right)$ can be produced by microbial dehalogenation of TCE $\left(\mathrm{C}_{2} \mathrm{HCl}_{3}\right)$ under methanogenic conditions. Since methanogenic conditions are favored at the landfill, this biodegradation origin for the vinyl chloride (VC) is plausible. One potential problem with this hypothesis 
stems from the relative proportions of TCE and VC in the landfill. Specifically, the recently observed VC concentrations are substantially higher than the maximum observed TCE concentrations in the landfill. It is not likely that TCE dissolved in groundwater at the 50-100 ppb range could be dehalogenated to produce VC in the $100-400 \mathrm{ppb}$ range downgradient of the source. If however, due to the low solubility of TCE, the biodegradation occurred at presumed solvent rag source areas of TCE in concentrations substantially greater than that dispersed in groundwater, VC could be generated and then subsequently disperse into the surrounding groundwater. Since VC is a more polar substance than TCE, the theoretical solubility should be greater, and higher concentrations of VC than TCE might result in the groundwater.

Another possible source for the VC is the potential reaction of plastics in the landfill with other chlorinated solvents. VC is used in copolymers, adhesives for plastics, and in other organic synthetic processes. Since plasticizers used to manufacture plastics typically contain phthalates, the deterioration of plastics could release both vinyl chloride (from the copolymers) and phthalates (from the plasticizer) to the environment. Bis(2-ethylhexyl)phthalate is a commonly used plasticizing agent. A review of the historical database reveals that this compound has been present in high concentrations in the past.

\section{Bis(2-ethylhexyl) Phthalate in the LFW-Series Monitoring Wells}
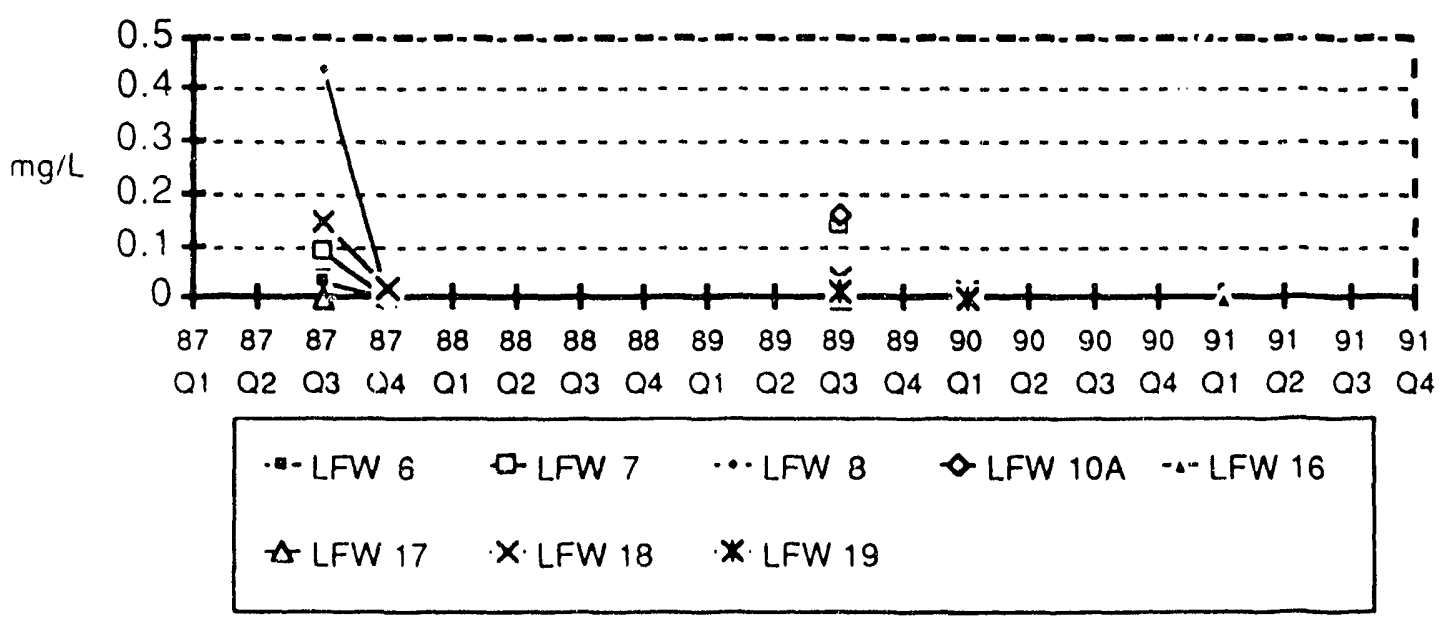


\section{Bis(2-ethylhexyl) Pilthalate in the LFW-Series Monitoring Wells}

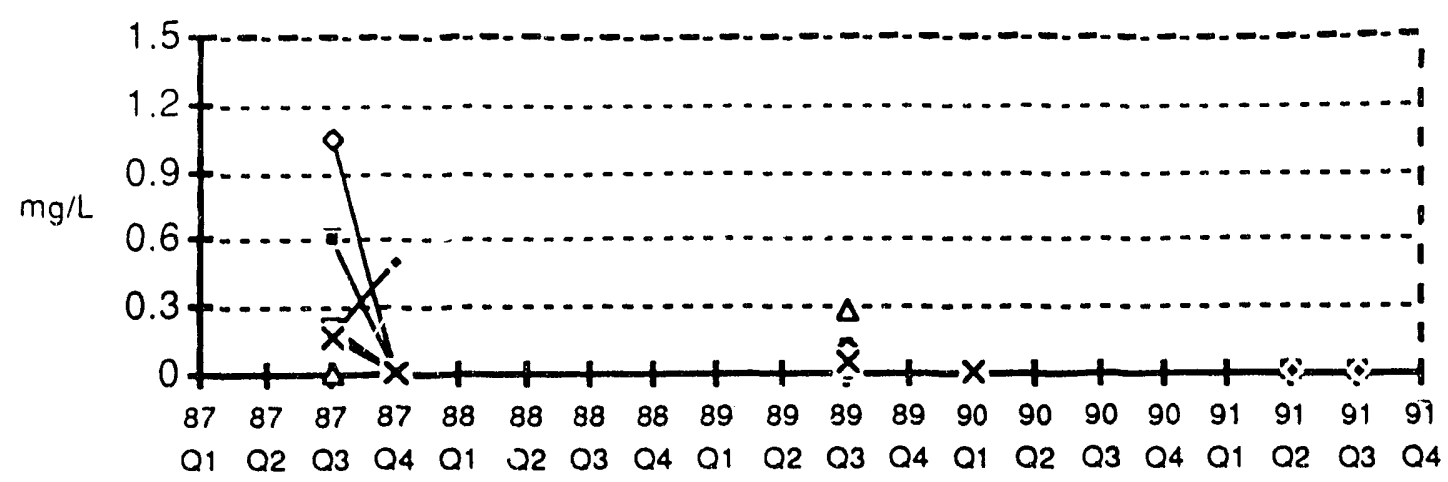

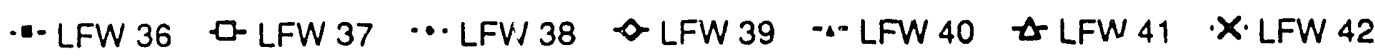

\section{Bis(2-ethylhexyl) Phthalate in the LFW-Series Monitoring Wells}

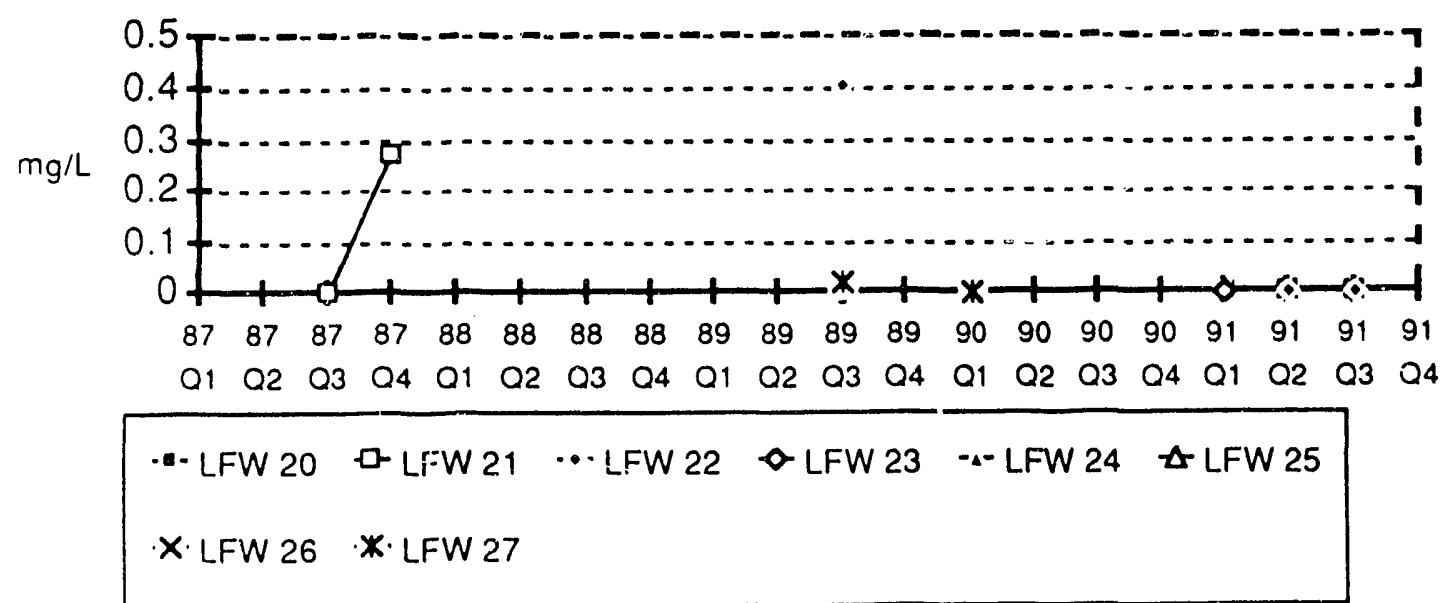

Bcnzene has been above drinking water standards at multiple wells in past quarters, but was not elevated above the PDWS at any well during the third quarter of 1991. Other organic contaminants are sporadically elevated in the Sanitary Landfill groundwater monitoring network. Sporadic hits of other organics in low concentrations may be related to biological degradation or trichoroethylene and tetrachloroethylene. 


\section{WATER LEVELS}

A comparison of water level data from the second quarter of 1991 to the third quarter of 1991 reveals that water levels increased an average of .0145 feet over that time period. Appendix E displays time-trend plots for water elevations in the Water Table.

\section{GROUNDWATER FLOW RATES AND DIRECTIONS}

The flow direction in the Water Table unit underlying the Sanitary Landfill is southerly (site coordinates) toward Upper Three Runs Creek, as determined from a potentiometric map of the Water Table aquifer.. The average change in head is approximately twenty feet from the extreme northern boundary of the landfill to the extreme southern boundary (Figure 8).

To derive flow directions at specific areas within the landfill, we can calculate flow triplets from the historical water levels at three or more given monitoring wells. All possible triangular planes are determined for the set of wells, and the flow potentiais, which represents the maximum dip direction of a given calculated plane, is graphically illustrated. Positive values are degrees clockwise from site north. Negative values represent degrees counter-clockwise from site north.

The following time-series plots (Figures A-F) illustrate derived flow potentials for specific well triplets through the Sanitary Landfill. Figure 8 shows the well locations and the locations of the flow triplets used in the calculations. 
SANITARY LANOFILL WELL LOCATIONS

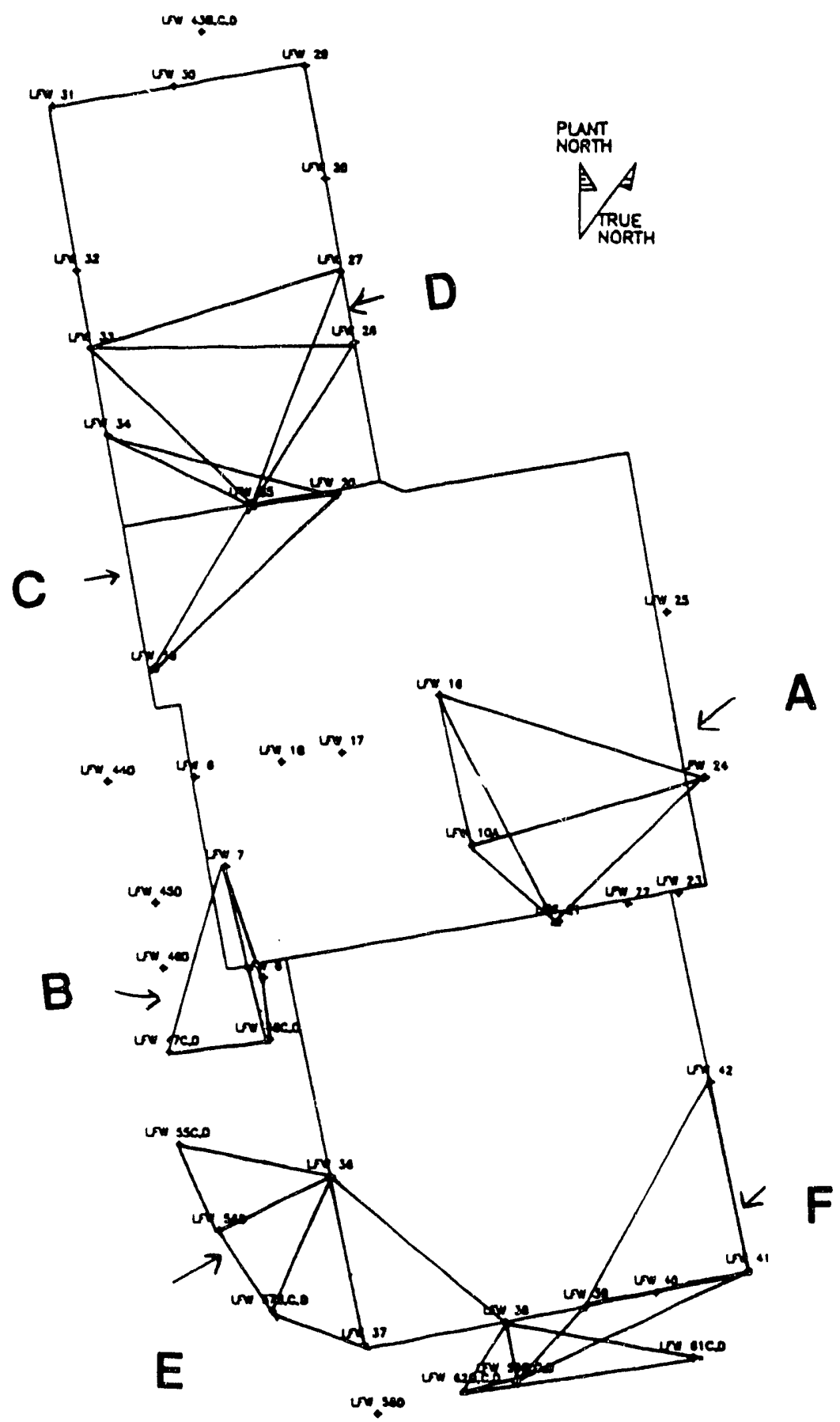

Figure 8

SCNE 1 ineh - 500 FEET 
SAINTARY LANDFILL 3091 WATER TABLE FLOW RATE ANO DIRECTIONS

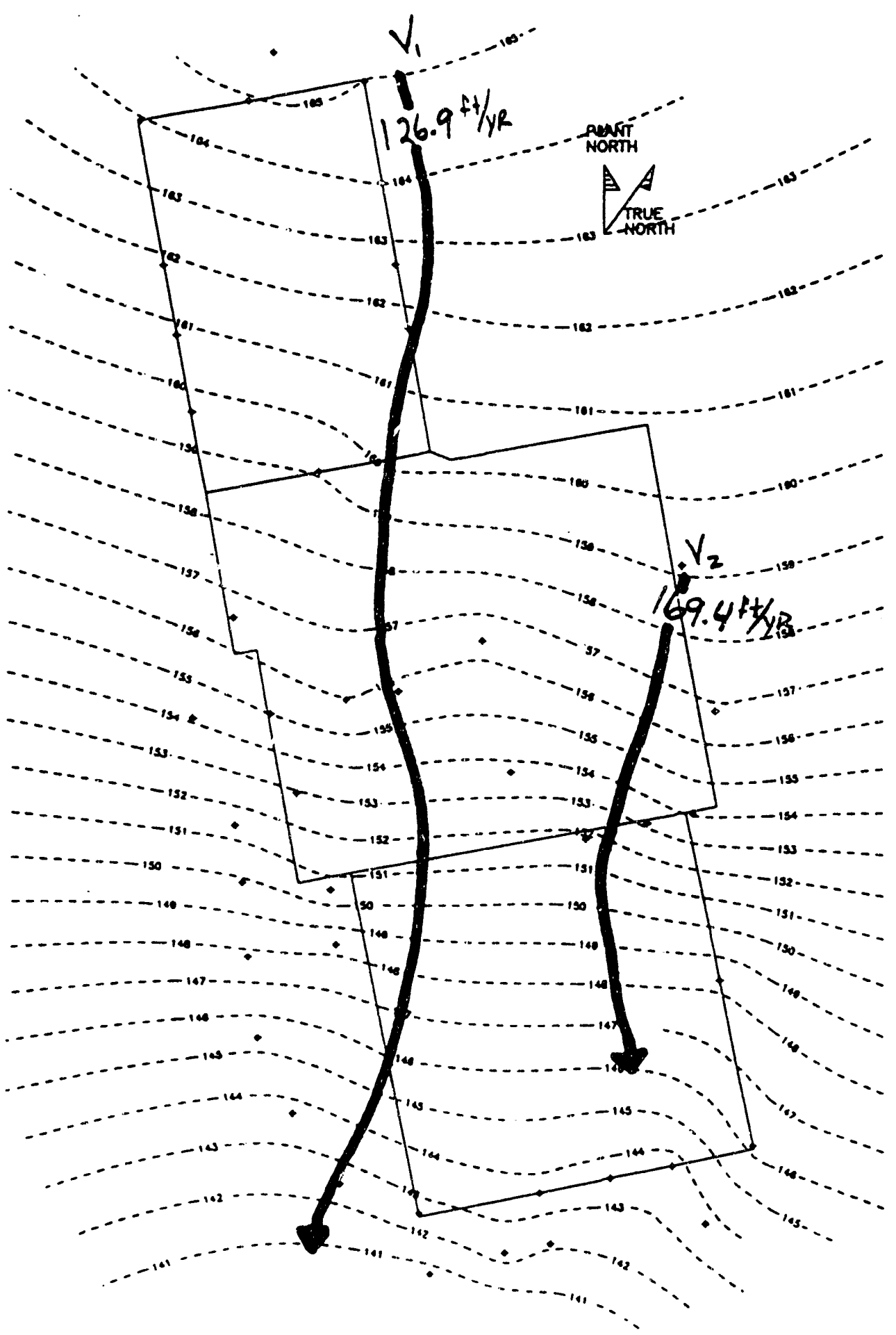

SCALE 1 inCh = SO0 FEET 
A: Groundwater Flow Directions at Triplets of LFW-Series Wells

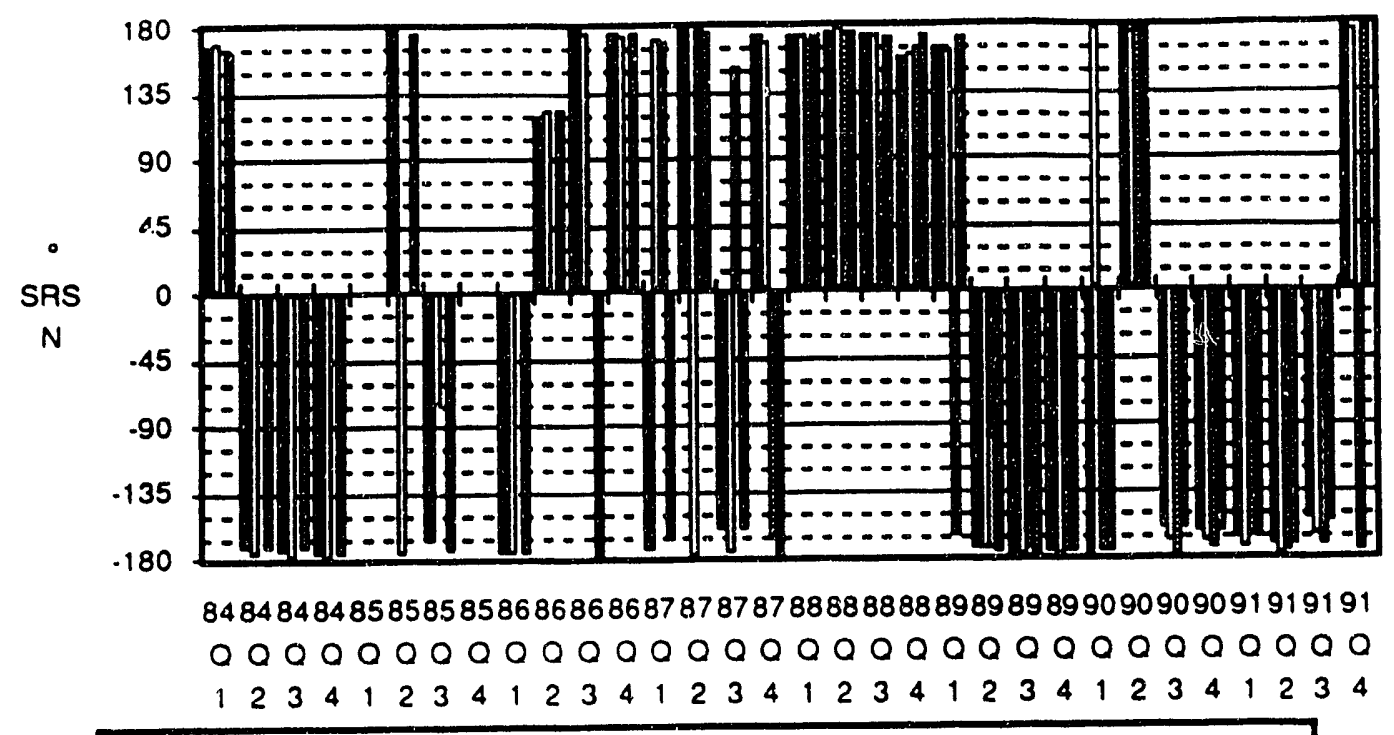

LFW $\{10 A, 16,24\} \square \operatorname{LFW}\{10 A, 21,24\}$ LFW $\{16,20,35\}$ LFW $\{16,21,24\}$

Examination of Figure $A$ reveals that the groundwater flow potential in the Water Table aquifer in the southeastern portion of the original landfill is 180 degrees from site north, or due south. This direction has been relatively consistent since at least 1984.

Figure B. below, graphically presents the flow potentials over time in the southwestern part of the original landfill, where the vinyl chloride plume has developed. 
B: Groundwater Flow Directions at Triplets of LFW-Series Wells

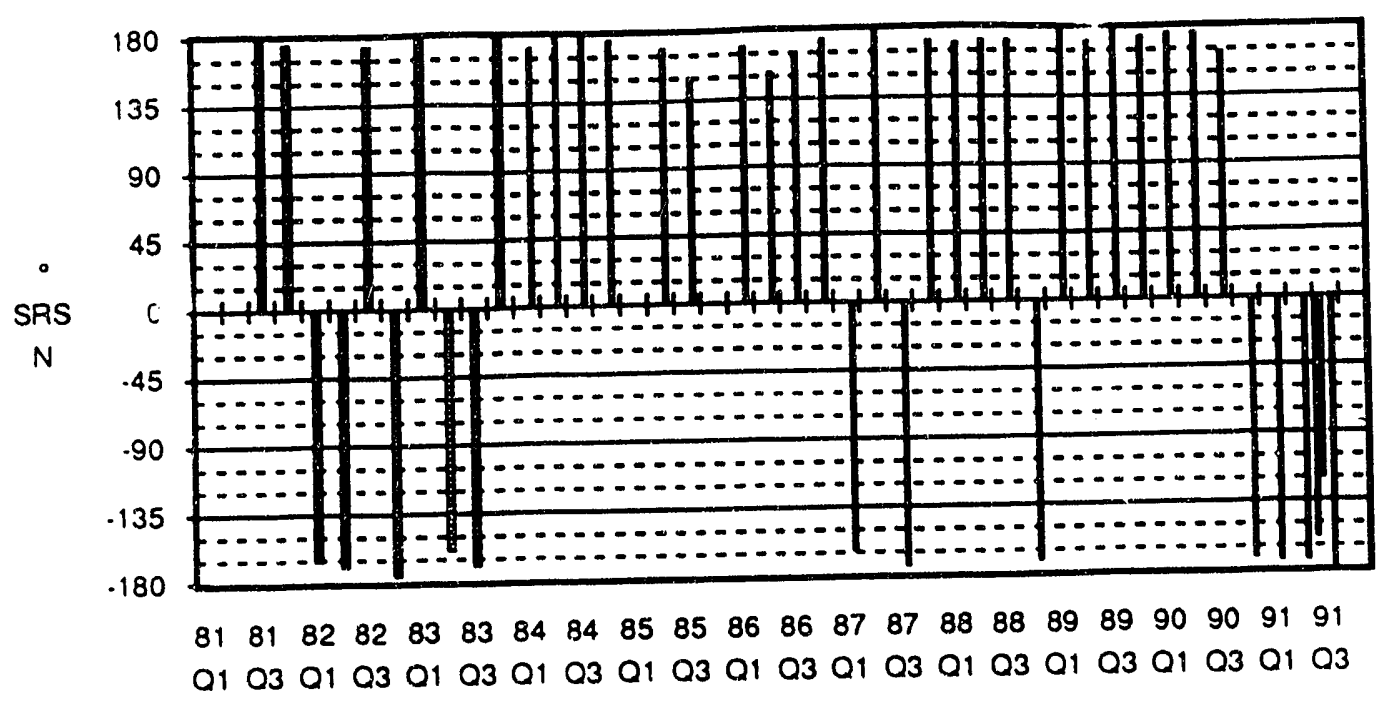

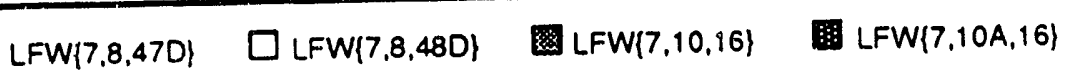

Flow directions for this area through time are slightly to the east of site south. During 1991, the potentials have been slightly to the west of site south.

Figures C and D illustrate flow potentials in the Water Table aquifer for the northern expansion of the landfill. 
C: Groundwater Flow Directions at Triplets of LFW-Series Wells

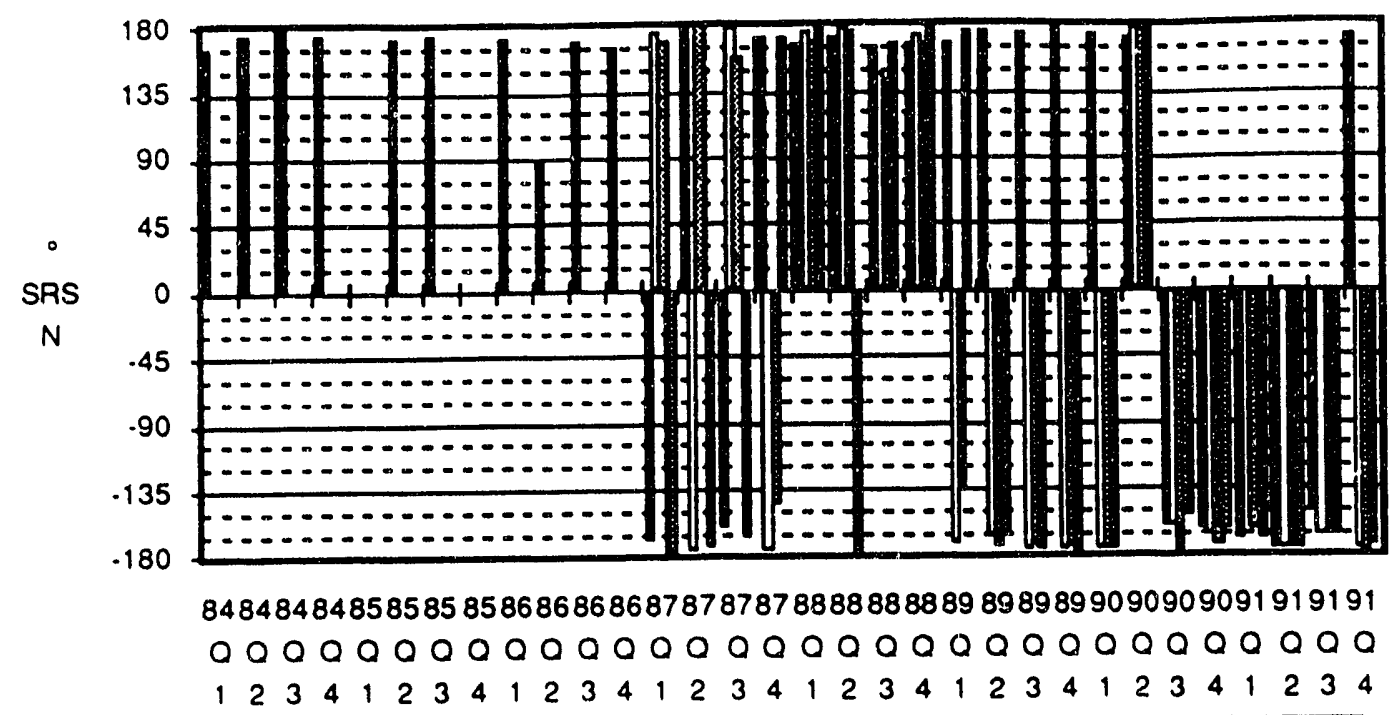

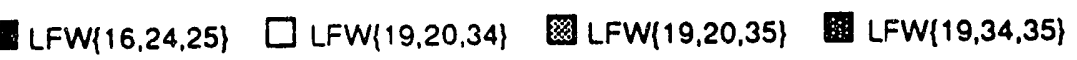

D: Groundwater Flow Directions at Triplets of LFW-Series Wells

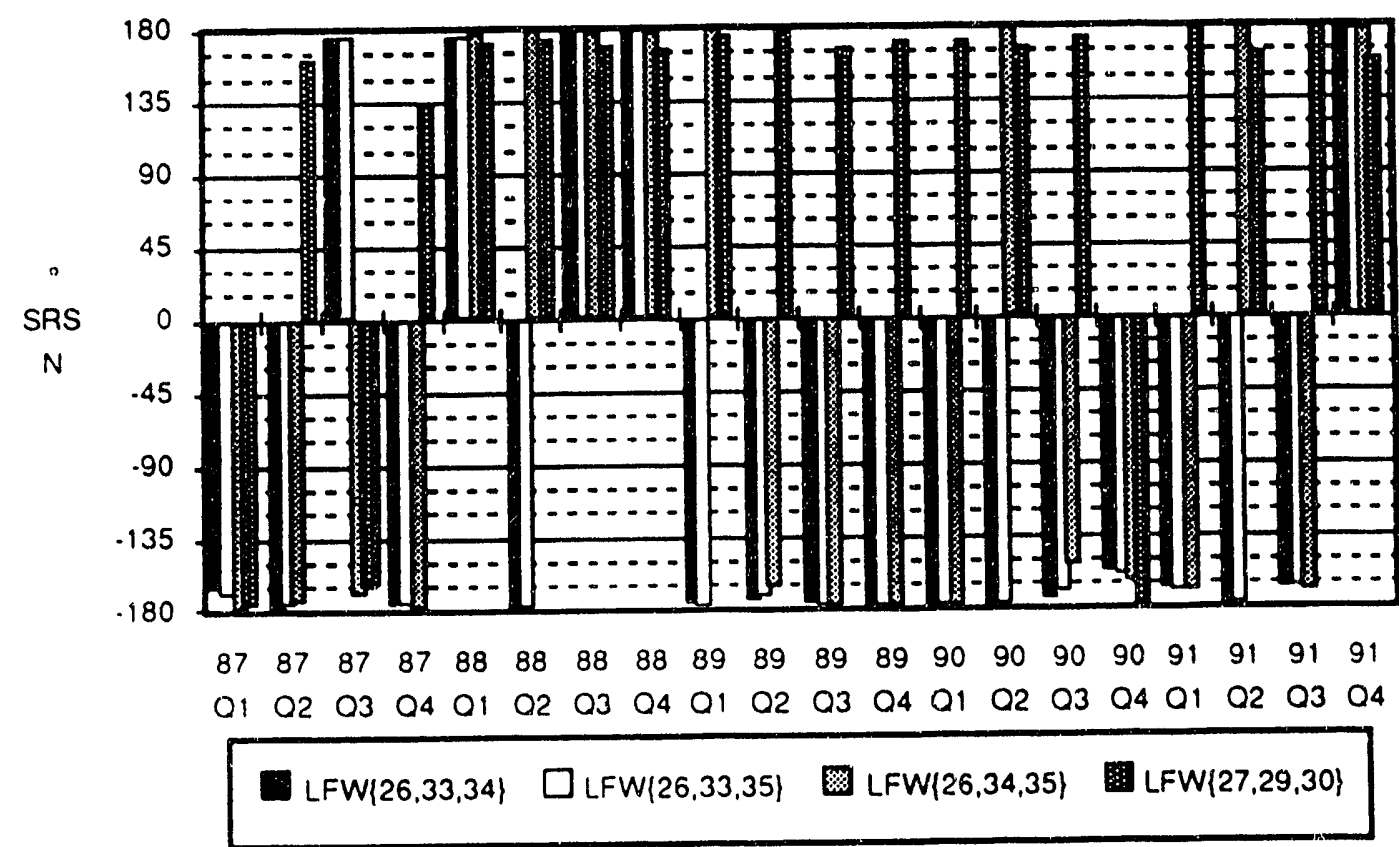


As shown, flow potentials for the northern expansion are close to site south, similar to those observed from the original landfill area.

Figures $E$ and $F$ illustrate the flow potentials, respectively, for the southwestern part and the southeastern part of the landfill's southern expansion. The data available for this area are more limited, but the same general relationship holds, with primarily southerly flow potentials.

\section{E: Groundwater Flow Directions at Triplets of LFW-Series Wells}

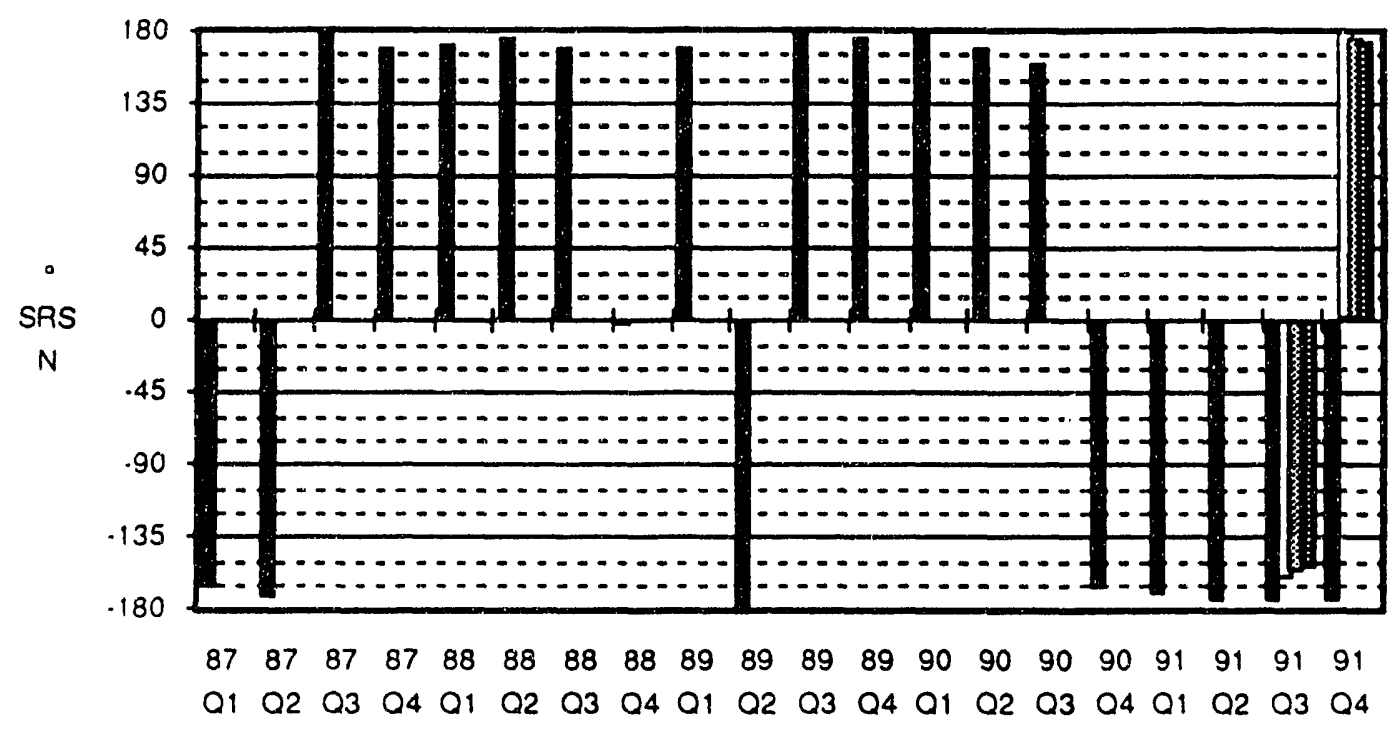

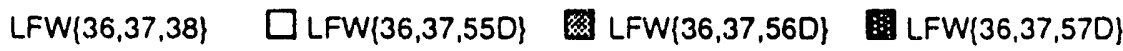




\section{F: Groundwater Flow Directions at Triplets of LFW-Series Wells}

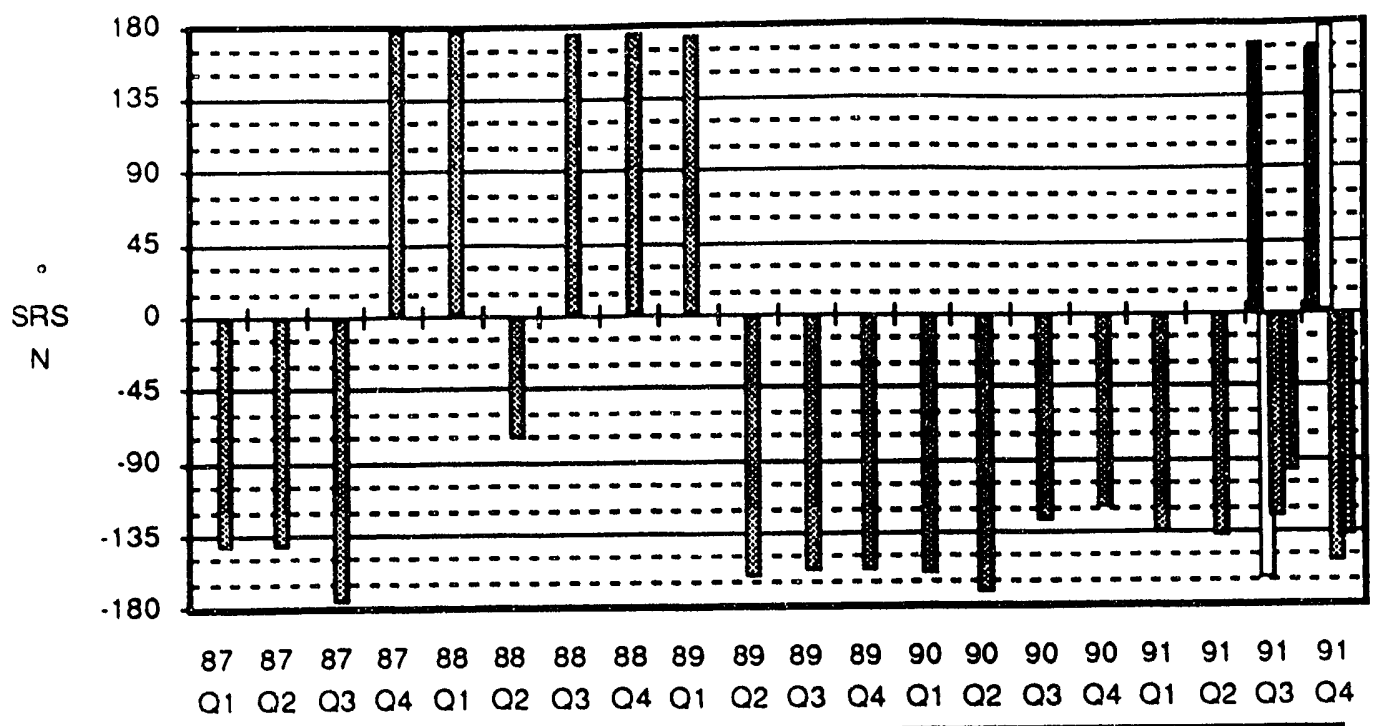

$\operatorname{LFW}\{38,590,62 \mathrm{D}\} \square \operatorname{LFW}\{38,610,62 \mathrm{D}\} \mathrm{LFW}\{39,41,42\}$

To estimate an average flow rate in the Water Table unit under the Sanitary Landfill, one can take the gradient just described, an estimated hydraulic conductivity $(\mathrm{Kh})$ for the Water Table hydrologic unit at SRS (10.0 ft/day), a conservatively low estimate of effective porosity (n) of $20 \%$, and use the following one-dimensional horizontal flow equation:

$$
\begin{aligned}
& \mathrm{Kh}^{\mathrm{h}} \text { dh } \\
& \mathrm{V}=\quad-\mathrm{x}-\mathrm{K} \quad \text { where } \mathrm{K}^{\mathrm{h}}=\text { hydraulic conductivity } \\
& \text { n dl } \\
& \mathrm{n}=\text { porosity } \\
& \mathrm{dh}=\text { change in head } \\
& \mathrm{dl}=\text { length of the flow path }
\end{aligned}
$$

Figure 9 depicts the average groundwater flow direction underneath the landfill for the third quarter of 1991. The change in head (dh) and length of the run (dl) were established by using Figure 9. By utilization of the base equation to derive an average horizontal groundwater velocity underneath the landfill during the third quarter of 1991, we obtain: 


$$
\begin{aligned}
& 10 \quad 24 \\
& \mathrm{~V}_{1}=\begin{array}{ccc}
-- & \mathrm{x} & -- \\
0.20 & 3450
\end{array} \quad=0.347 \mathrm{ft} / \text { day } \mathrm{x} 365 \text { days } \\
& =127 \mathrm{ft} / \mathrm{yr} \\
& \mathrm{V}_{2}=\begin{array}{ccc}
10 & 13 \\
-- & \mathrm{x} & -- \\
0.20 & 1400
\end{array} \quad=0.464 \mathrm{ft} / \text { day } \times 365 \text { days } \\
& =170 \mathrm{ft} / \mathrm{yr}
\end{aligned}
$$

$V_{1}$ and $V_{2}$ represent averaged horizontal flow rates along two separate flow paths throughout the landfill, as illustrated in Figure 9. V1 was determined by using the hydraulic gradient $(\mathrm{dh} / \mathrm{dl})$ from the northern end of the facility to the southern end to estimate total groundwater flow throughout the entire facility. $V_{2}$ was determined by using the hydraulic gradient $(\mathrm{dh} / \mathrm{dl})$ from the potentiometric surface line 158. This was an arbitrary point picked to determine flow rate from another location within the facility..

Values for the dips of the triplet planes can be used as hydraulic gradients in the one-dimensional horizontal flow rate equation. Figures $A^{\prime}$ through $F^{\prime}$ graphically present the angles of dip of the calculated triplet planes through time. Again, A' and $B^{\prime}$ are from the original landfill area. $C^{\prime}$ and $D^{\prime}$ are from the northern expansion, and $E^{\prime}$ and $F^{\prime}$ are from the southern expansion 
A': Groundwater Flow Gradients at Triplets of LFW-Series Wells

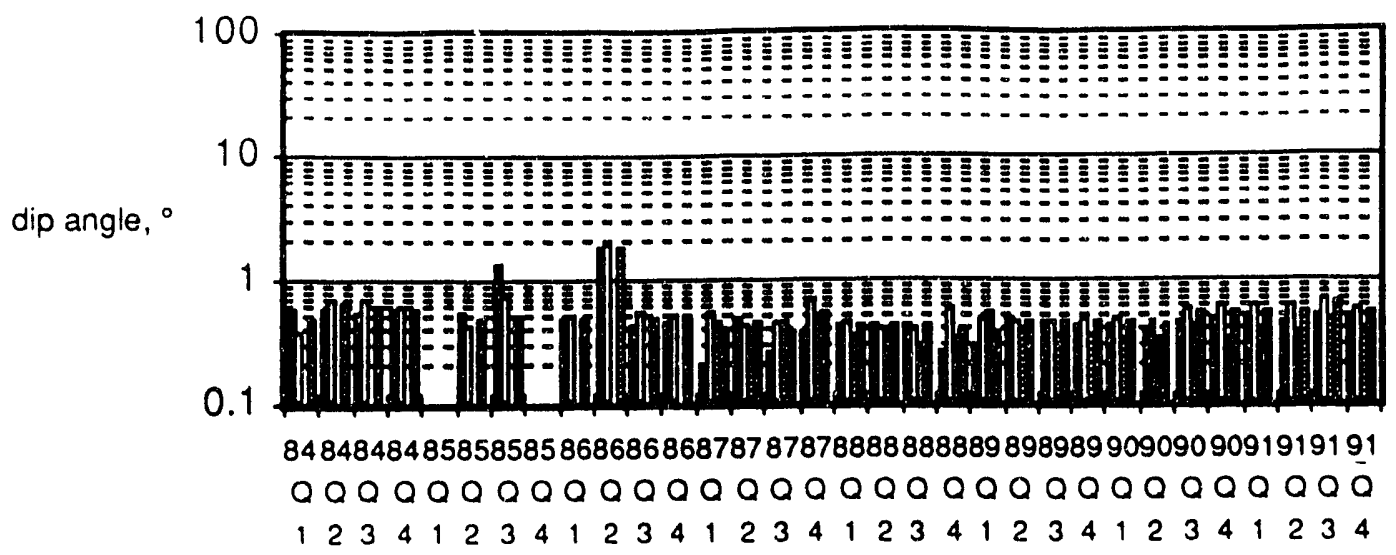

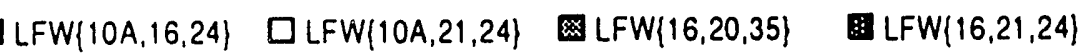

B': Groundwater Flow Gradients at Triplets of LFW-Series Wells

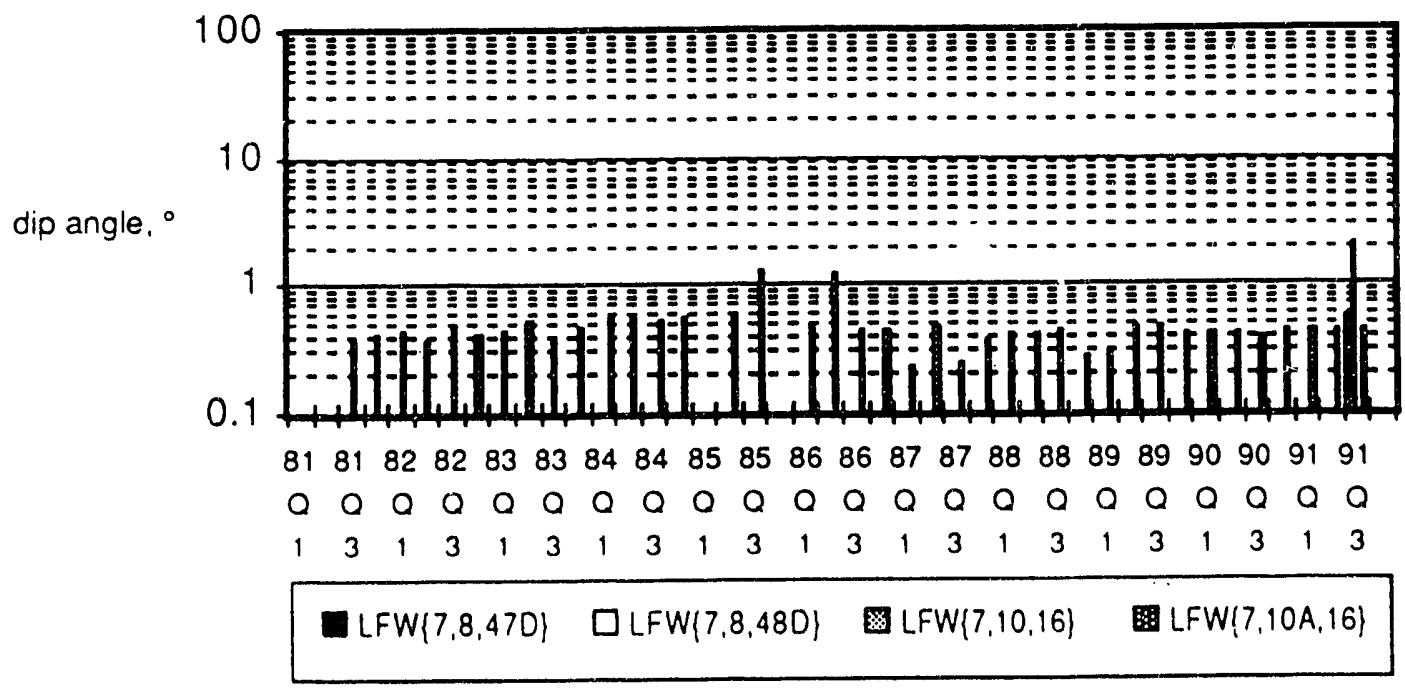


C': Groundwater Flow Gradients at Triplets of LFW-Series Wells

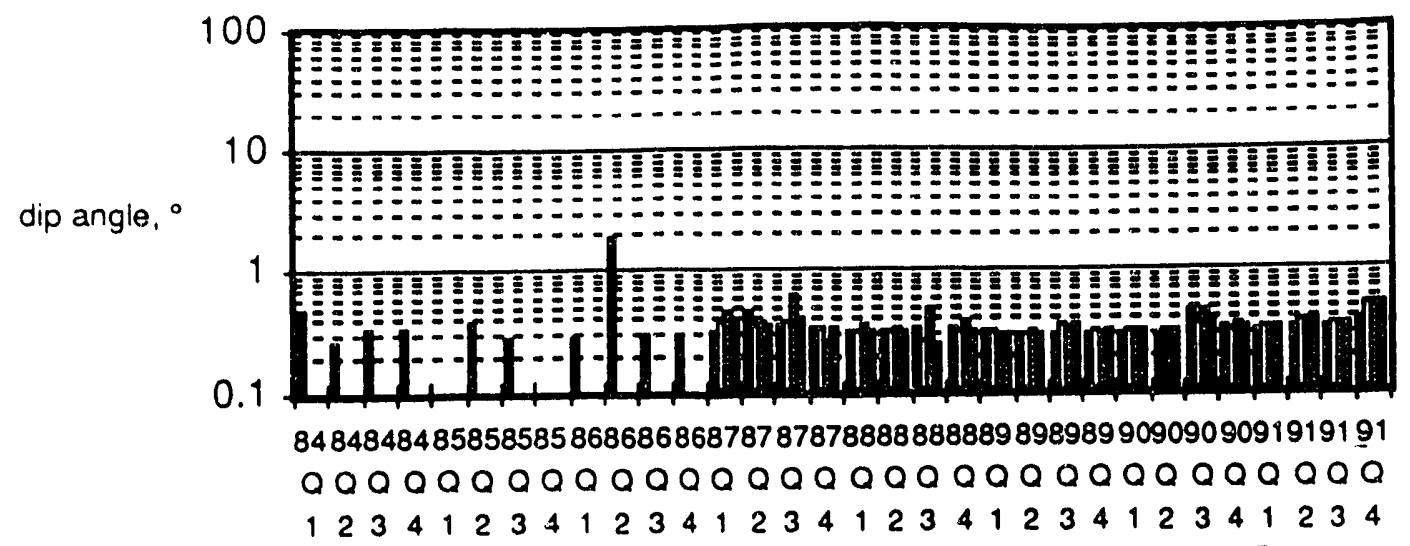

$\operatorname{LFW}\{16,24,25\} \square \operatorname{LFW}\{19,20,34\} \operatorname{LFW}\{19,20,35\} \quad \operatorname{LFW}\{19,34,35\}$

D': Groundwater Flow Gradients at Triplets of LFW-Series Wells

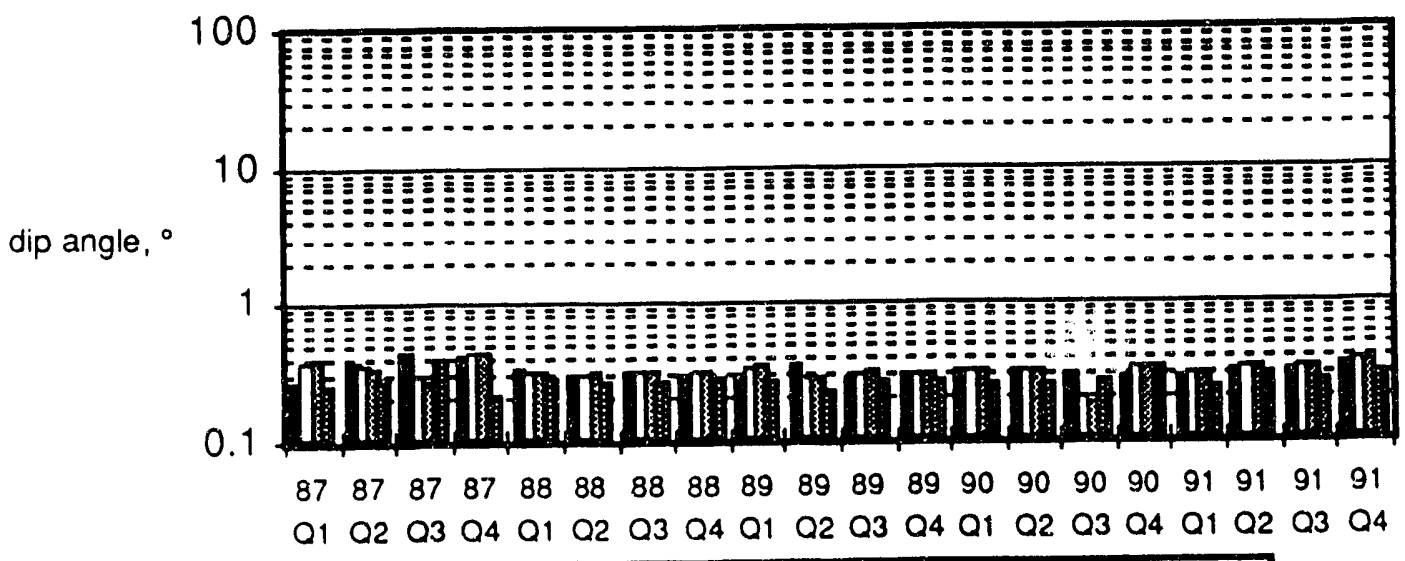

$\operatorname{LFW}\{26,33,34\} \square \operatorname{LFW}\{26,33,35\}$ 낪 $\operatorname{LF}\{26,34,35\}$ 田FW $\{27,29,30\}$ 


\section{E': Groundwater Flow Gradients at Triplets of LFW-Series Wells}

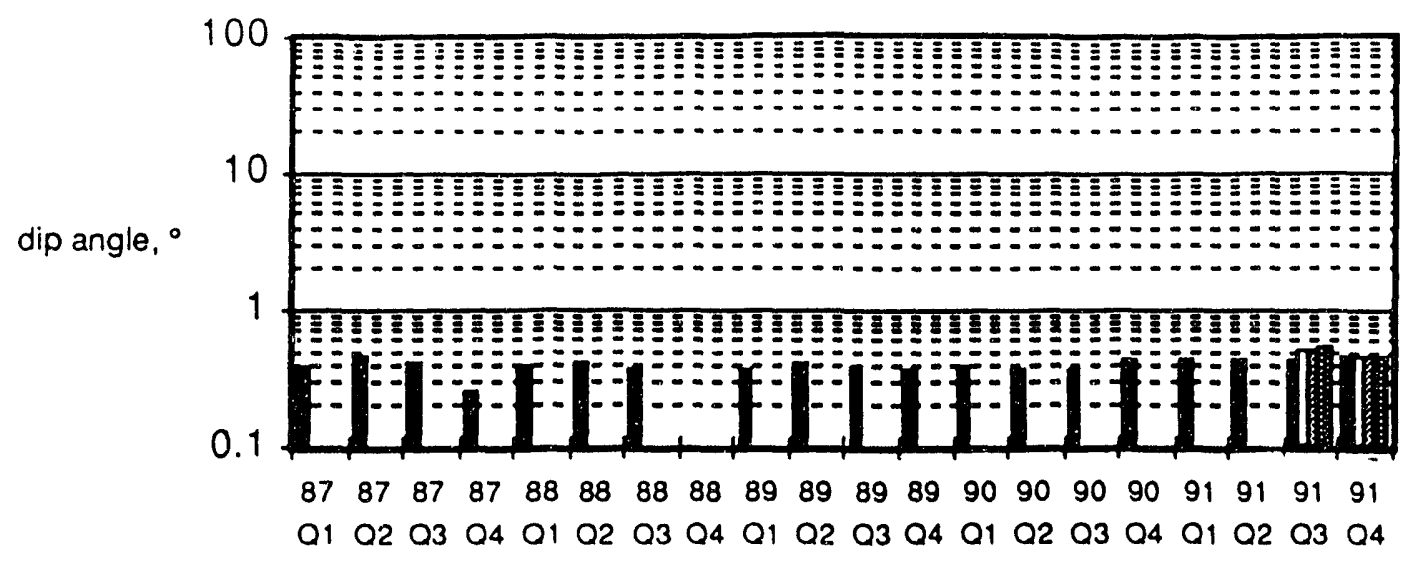

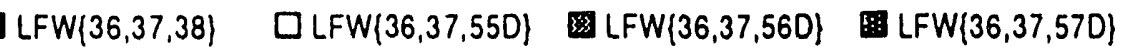

F: Groundwater Flow Gradients at Triplets of LFW-Series Wells

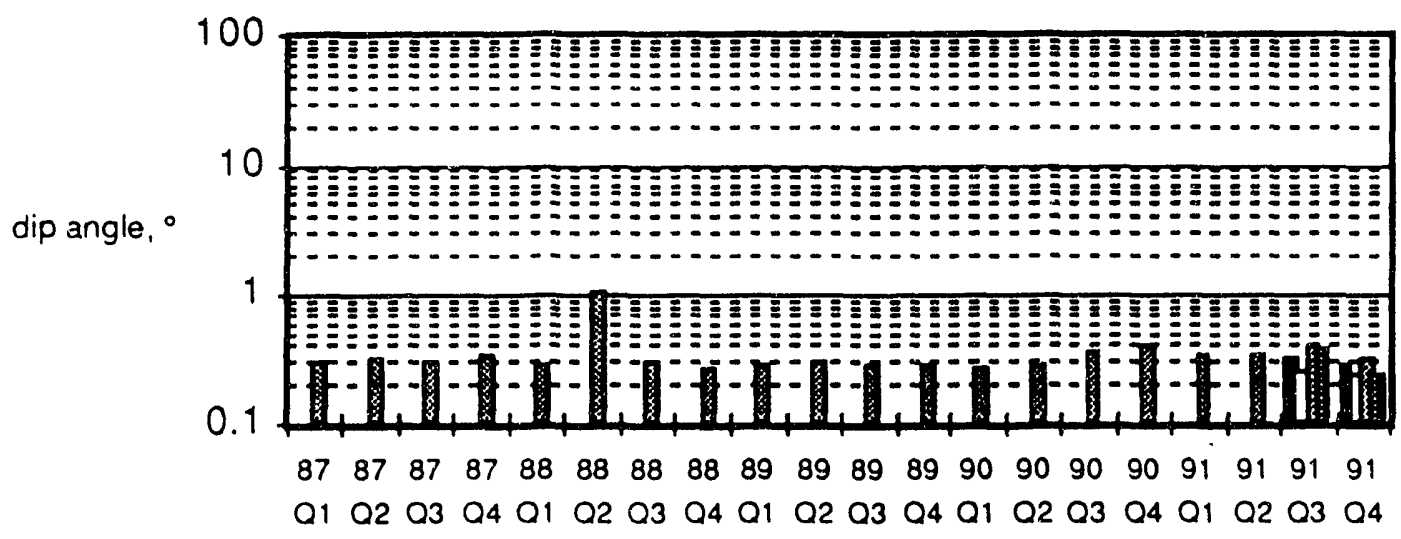

LFW $\{38,590,620\} \quad \square L F W\{38,610,620\}$ LFW $\{39,41,42\}$ 用LFW $\{39,41,590\}$

The averaged hydraulic gradients along the two flow paths used for calculating $V_{1}$ and $V_{2}$ can be converted to dip angles with the following equation:

$$
\alpha=\arctan (\mathrm{dh} / \mathrm{dl})
$$

The resulting calculated dips are $\alpha_{1}=0.40$ and $\alpha_{2}=0.53$, which are consistent with the dip angles determined from the calculated triplets (Figures $A^{\prime}$ through $E^{\prime}$ ). Growndwater flow rates during the third quarter of 1991 in the Water Table average approximately $1.50 \mathrm{ft} / \mathrm{yr}$. 


\section{CONCLUSIONS}

Groundwater beneath the SRS Sanitary Landfill has been contaminated with tritium (low levels) and organics. Elevated levels of tritium are consistently being detected in LFW 10A. Cadmium has been observed in low concentrations. Total radium has also been observed at levels near the PDWS, but does not appear to be related to a point source of contamination. Elevated radium is likely due to naturally low $\mathrm{pH}$ groundwater leaching radium of matrix sediments. Elevated levels of total radium, 1,2 dichloroethane, 1,1 dichloroethylene, and tetrachloroethylene continue to be detected at levels above the primary drinking water standards in different wells from quarter to quarter that monitor the Sanitary Landfill. TCE has been the most consistently elevated organic contaminant in the landfill groundwater wells. Other organic contaminants periodically are observed in a number of wells throughout the facility. The appearance of the periodic organic plumes could be from an isolated plume migrating south/southeast with the groundwater and being discharged into Upper Three Runs Creek. Also, these periodic observances of elevated levels organic contaminants (other then TCE), could be from buried waste decomposing in a particular area within the Sanitary Landfill that is independent of the larger organic plume. Vinyl chloride has recently become a primary contaminant in groundwater beneath the sanitary landfill. The recent occurrence could be from microbial dehalogenation of TCE, or from the decomposition of plastics in the landfill. Current data best support the latter hypothesis. Further analysis will be directed toward clearly elucidating the origin of the vinyl chloride.

Several point sources appear to be present for contamination in the Sanitary Landfill. A single source of tritium appears to be present in the south-central part of the original 32 acre landfill. This area also appears to be a point source for TCE contamination. A second TCE point source appears to be present in the lower part of the southern extension of the landfill. Vinyl chloride has become a primary contaminant during 1991, and appears to originate near the southwestern corner of the original 32 acre landfill. 
FEDERAL PRIMARY DRINKING WATER STANDARDS FOR RADIOACTIVE AND NONRADIOACTIVE CONSTITUENTS

CONSTITUENT

ARSENIC (As)

BARIUM (Ba)

BENZENE

CADMIUM (Cd)

CARBON TETRACHLORIDE

CHROMIUM (Cr)

CHLOROFORM

COLIFORM BACTERIA

1,1-DICHLOROETHYLENE

1,2 DICHLOROETHANE

2,4-D

ENDRIN

FLUORIDE (F) limit is temp. dependent

GROSS ALPHA

GROSS BETA

$\operatorname{LEAD}(\mathrm{Pb})$

LINDANE

MERCURY (Hg)

METHOXYCHLOR

NITRATE AS NITROGEN $\left(\mathrm{NO}_{3}\right)$

PARA-DICHLOROBENZENE

RADIUM ( $\mathrm{Ra}^{226}$ plus $\mathrm{Ra}^{228}$ )

SELENIUM (Se)

SILVER (Ag)

2,4,5-TP (SILVEX)

STRONTIUM-90

TETRACHLOROETHYLENE

TOXAPHENE

TRICHLOROETHYLENE (TCE)

1,1,1-TRICHLOROETHANE (1,1,1-TCA)

TRIHALOMETHANES (CHX 3 ;

$\mathrm{X}$ is any grouping of $\mathrm{F}, \mathrm{Cl}, \mathrm{Br}, \mathrm{OR} \mathrm{I}$ )

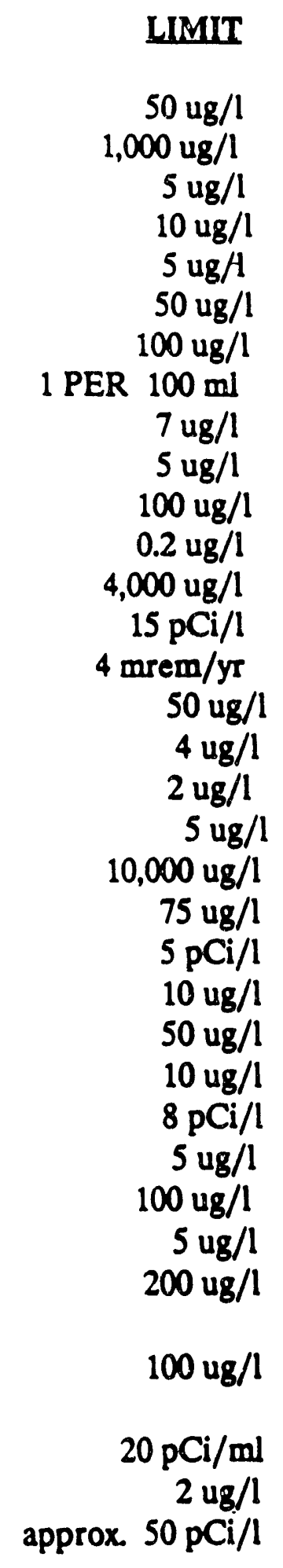

LIMIT

$50 \mathrm{ug} / \mathrm{l}$

$1,000 \mathrm{ug} / 1$

$5 \mathrm{ug} / \mathrm{l}$

$10 \mathrm{ug} / \mathrm{l}$

5 ug $A$

$50 \mathrm{ug} / 1$

$100 \mathrm{ug} / 1$

1 PER $100 \mathrm{ml}$

$7 \mathrm{ug} / 1$

$5 \mathrm{ug} / \mathrm{l}$

$100 \mathrm{ug} / \mathrm{l}$

$0.2 \mathrm{ug} / 1$

$4,000 \mathrm{ug} / 1$

$15 \mathrm{pCi} / \mathrm{l}$

$4 \mathrm{mrem} / \mathrm{yr}$

$50 \mathrm{ug} / 1$

4 ug/l

$2 \mathrm{ug} / \mathrm{l}$

$5 \mathrm{ug} / \mathrm{l}$

$10,000 \mathrm{ug} / 1$

75 ug/l

$5 \mathrm{pCi} / 1$

10 ug/l

$50 \mathrm{ug} / \mathrm{l}$

$10 \mathrm{ug} / 1$

$8 \mathrm{pCi} / \mathrm{l}$

5 ug/l

$100 \mathrm{ug} / 1$

$5 \mathrm{ug} / 1$

$200 \mathrm{ug} / \mathrm{l}$
$100 \mathrm{ug} / 1$
$20 \mathrm{pCi} / \mathrm{ml}$ 2 ug/l
approx. $50 \mathrm{pCi} / \mathrm{l}$

TRITIUM $\left(\mathrm{H}^{3}\right)$

VINYL CHLORIDE

NONVOLATILE BETA 


\section{APPENDIX A}

\section{TIME-SERIES PLOTS OF ANALYTICAL DATA}


Tetrachloroethylene in the LFW-Series Monitoring Wells

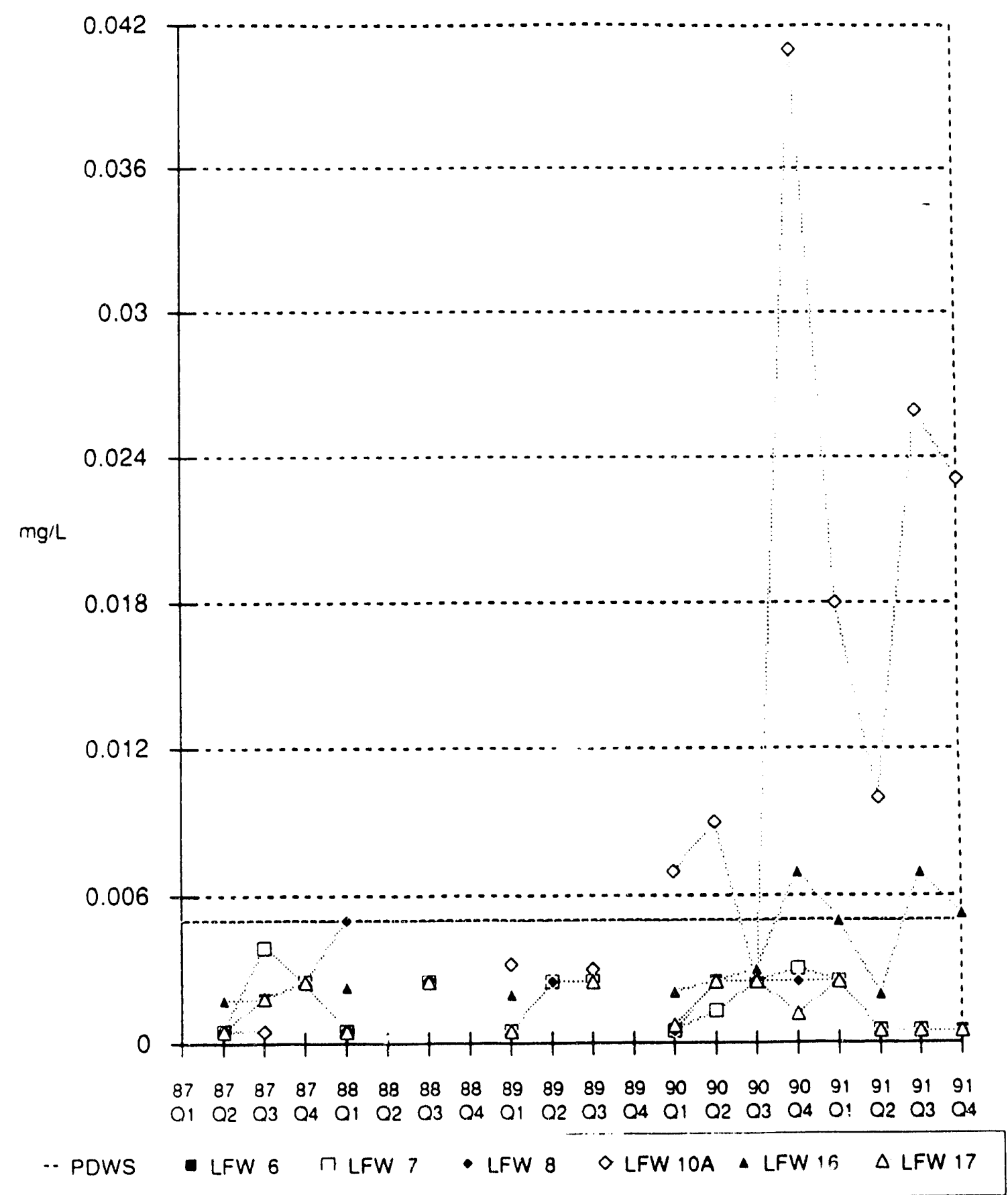




\section{Benzene in the LFW-Series Monitoring Wells}

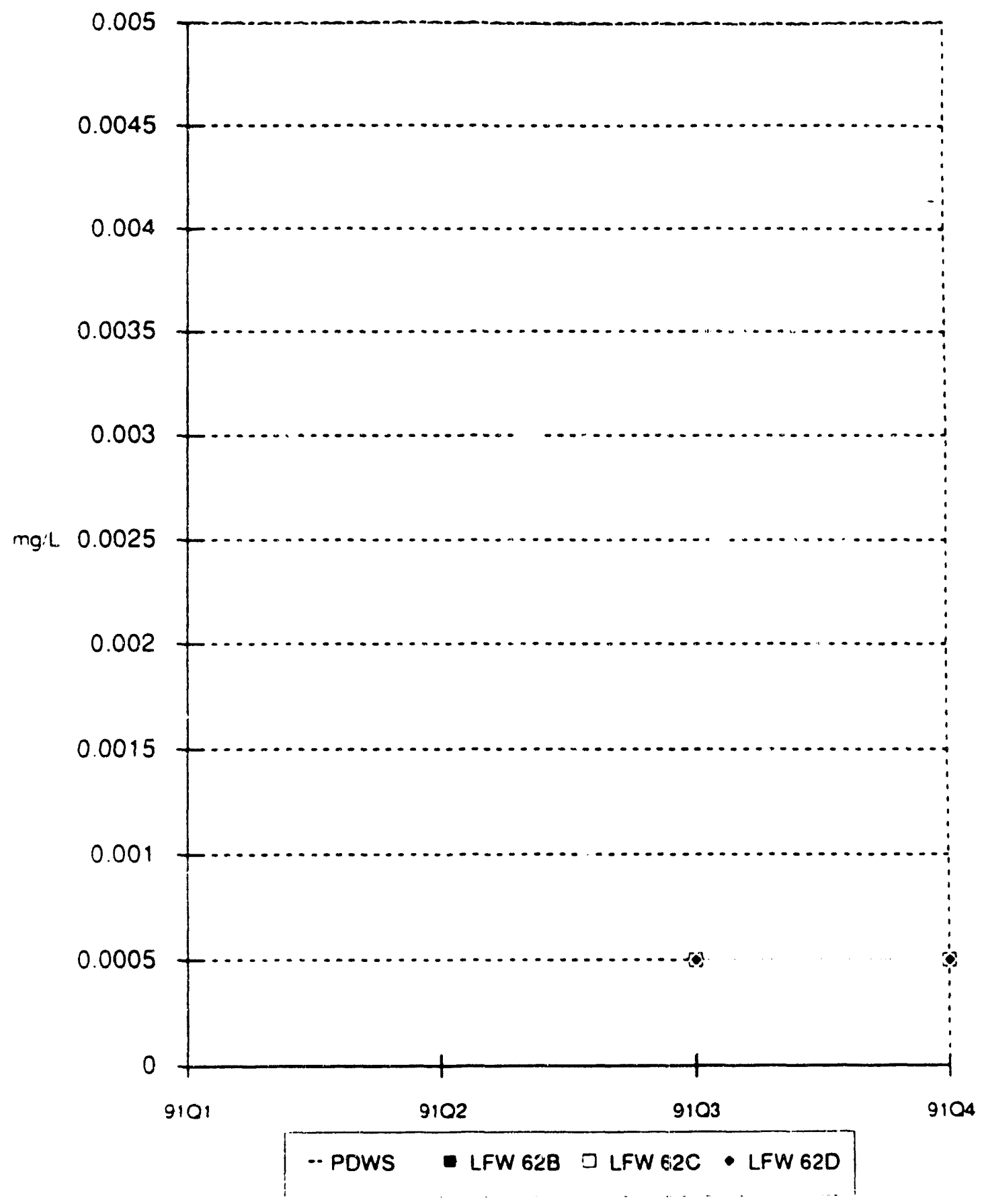


Benzene in the LFW-Series Monitoring Wells

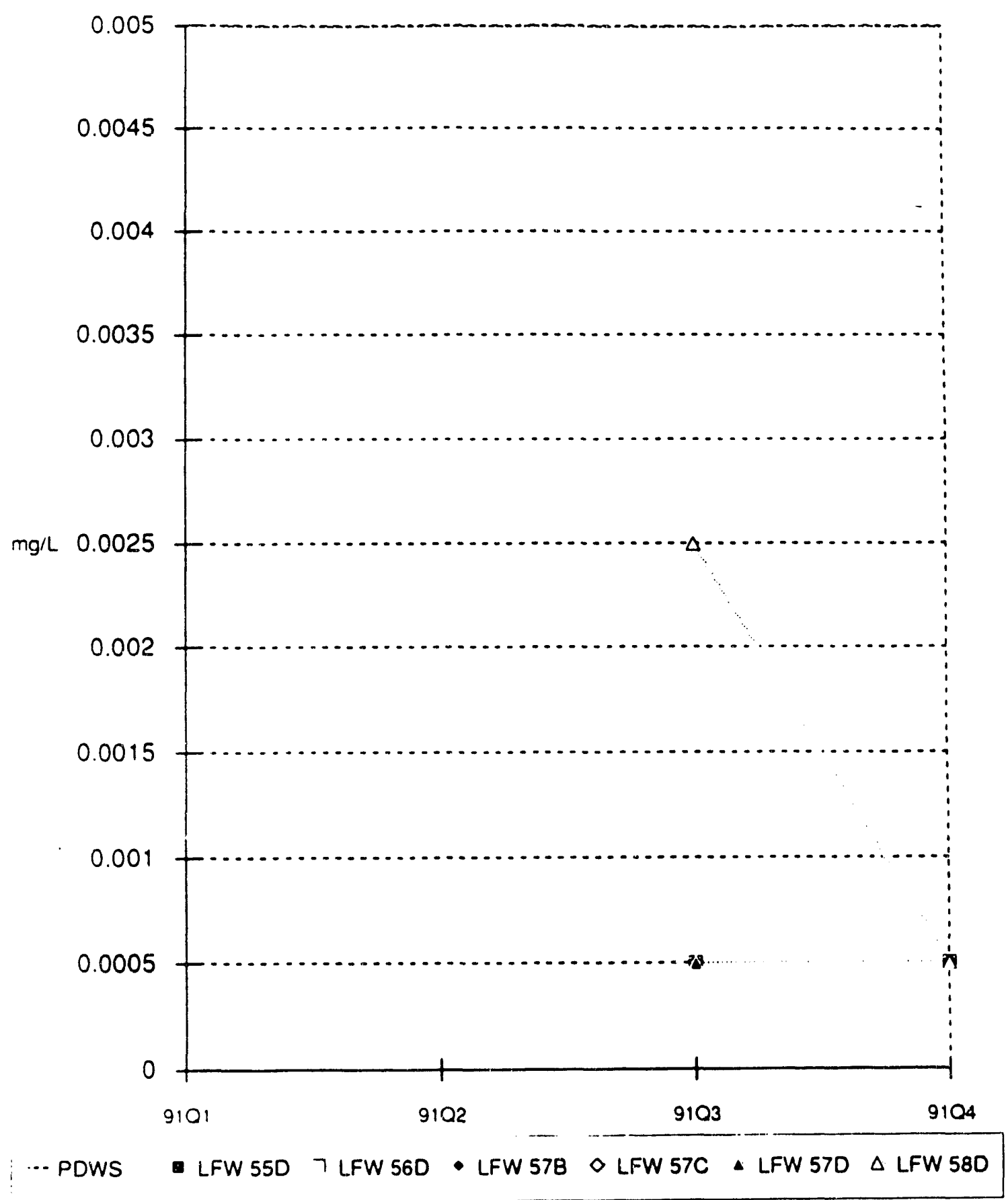


Benzene in the LFW-Series Monitoring Wells

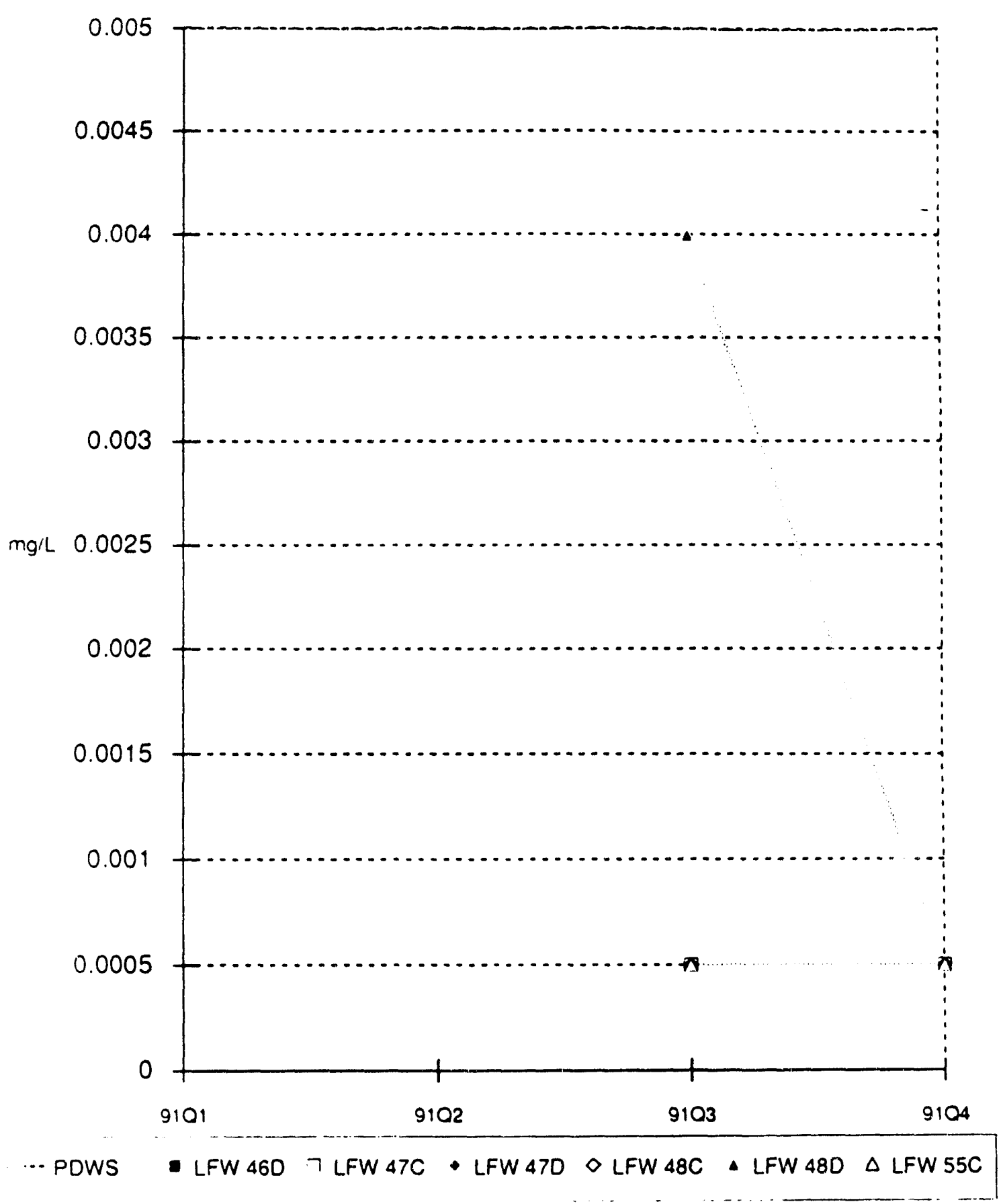


Benzene in the LFW-Series Monitoring Wells

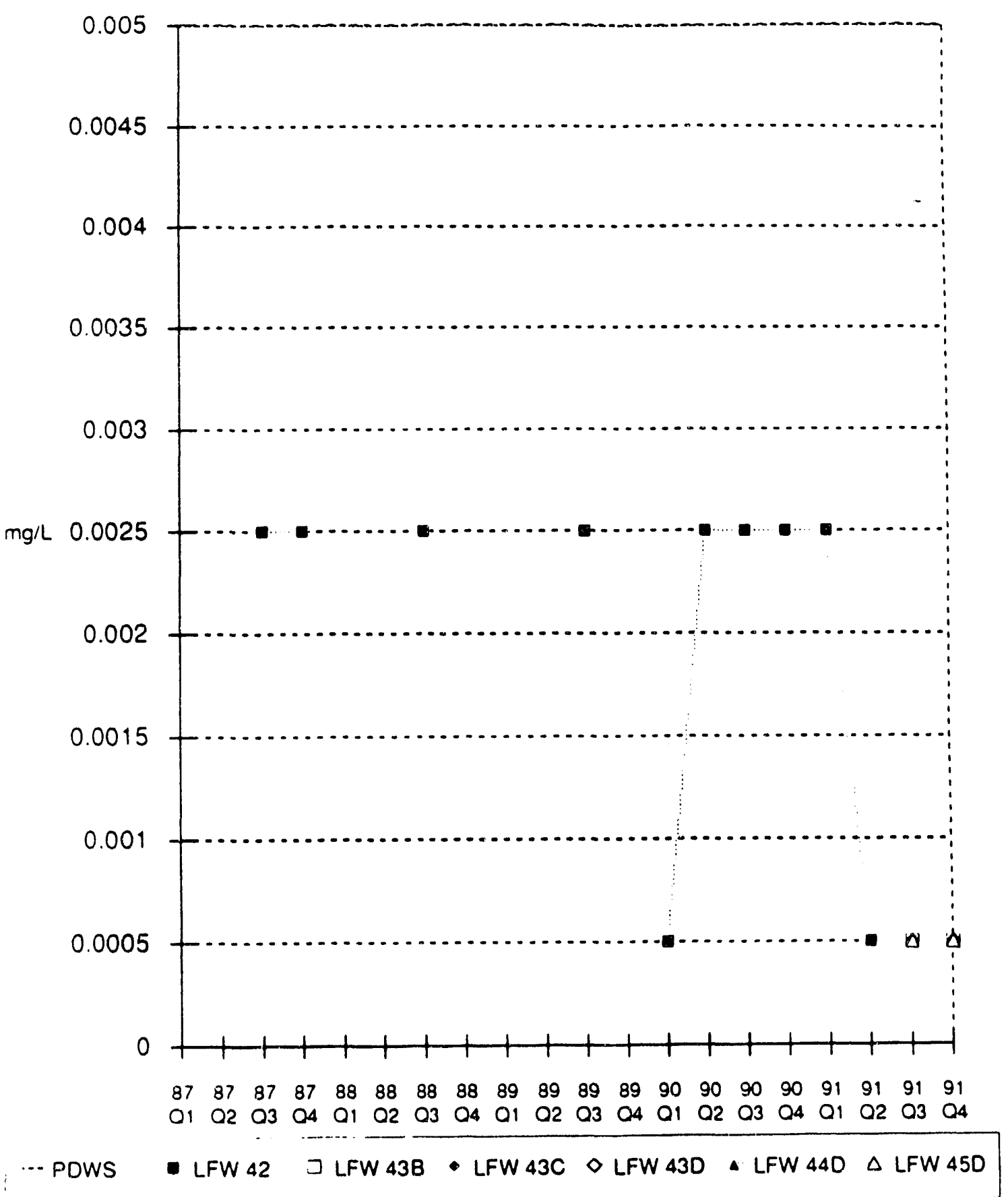




\section{Benzene in the LFW-Series Monitoring Wells}

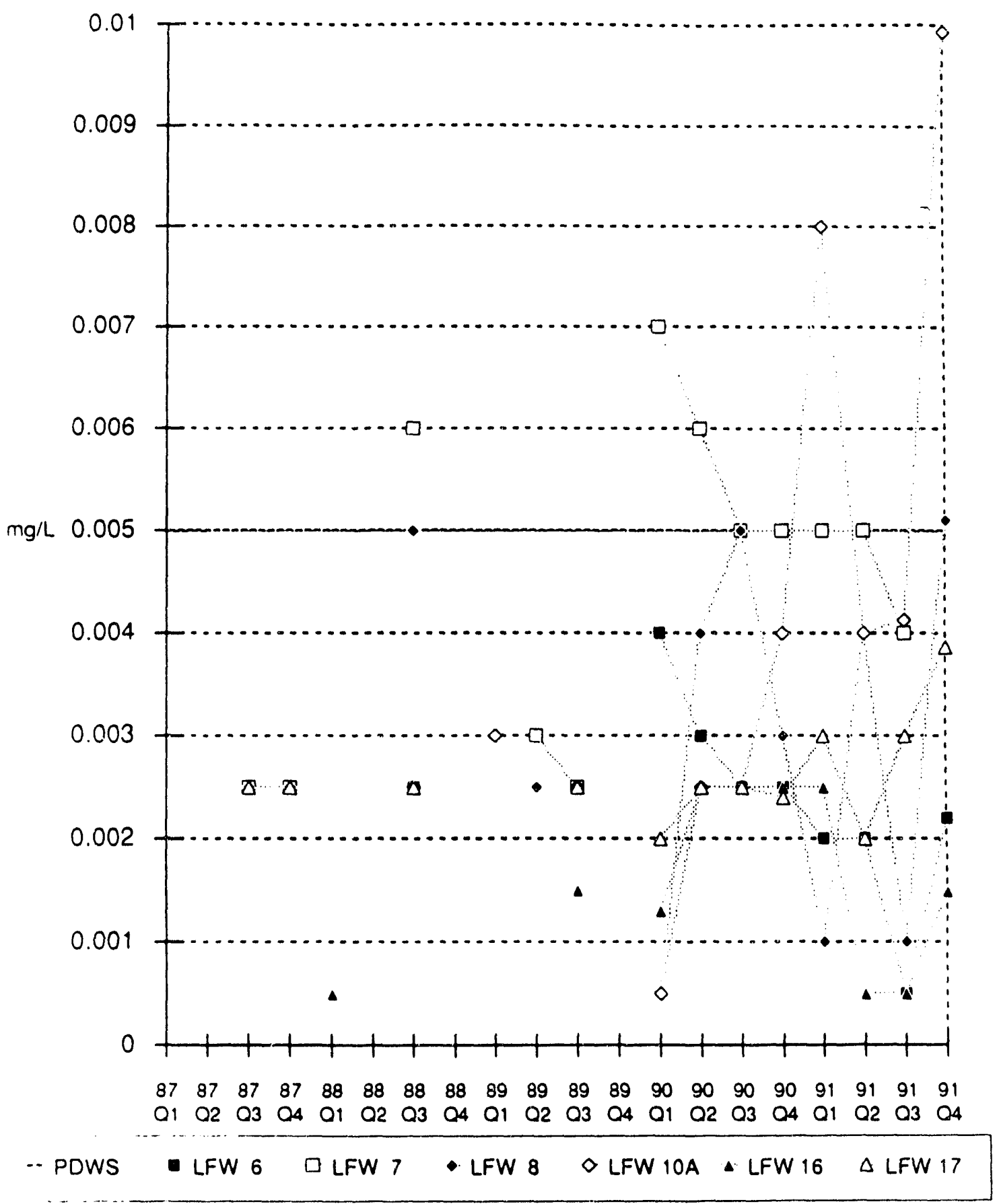


Benzene in the LFW-Series Monitoring Wells

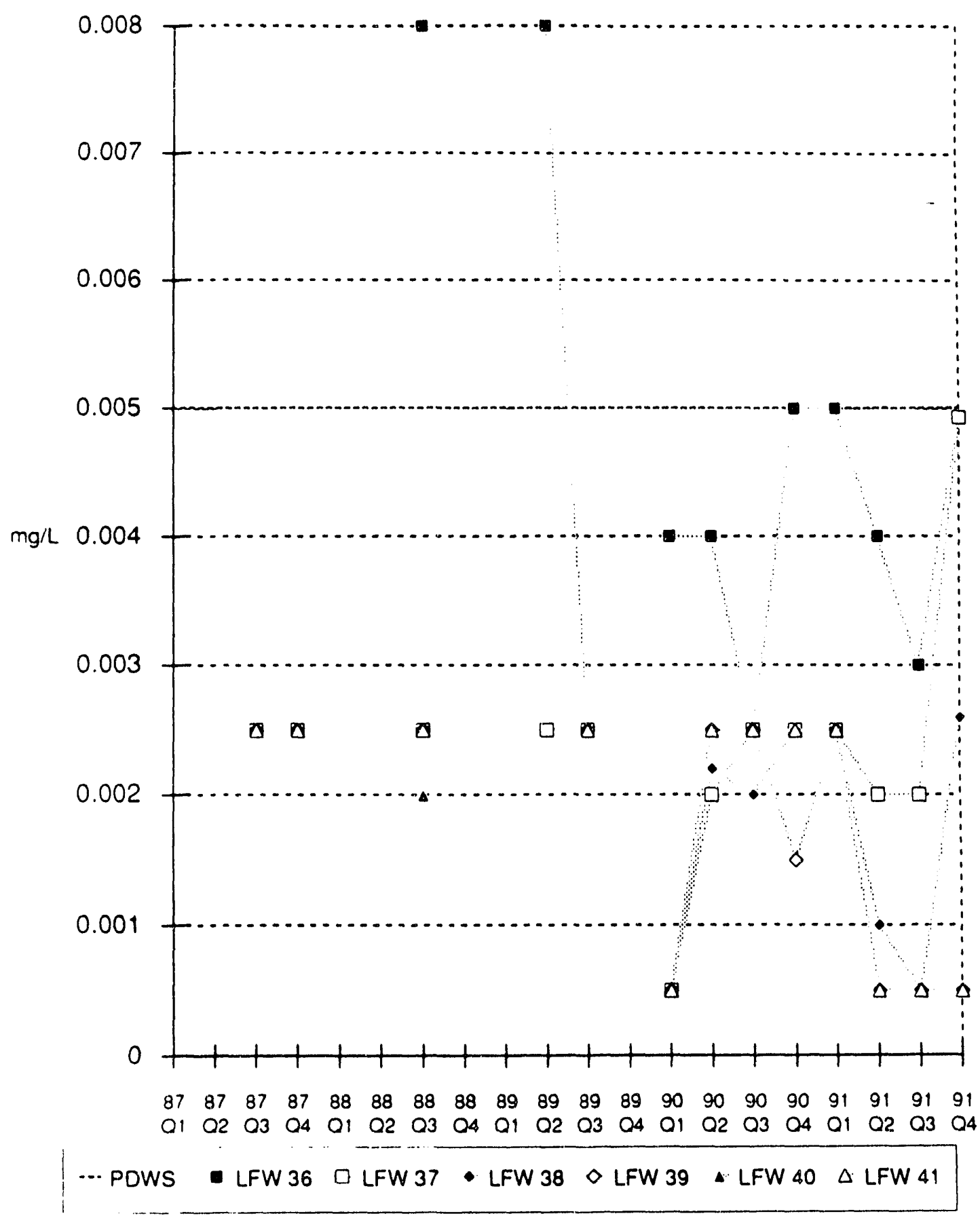


Benzene in the LFW-Series Monitoring Wells

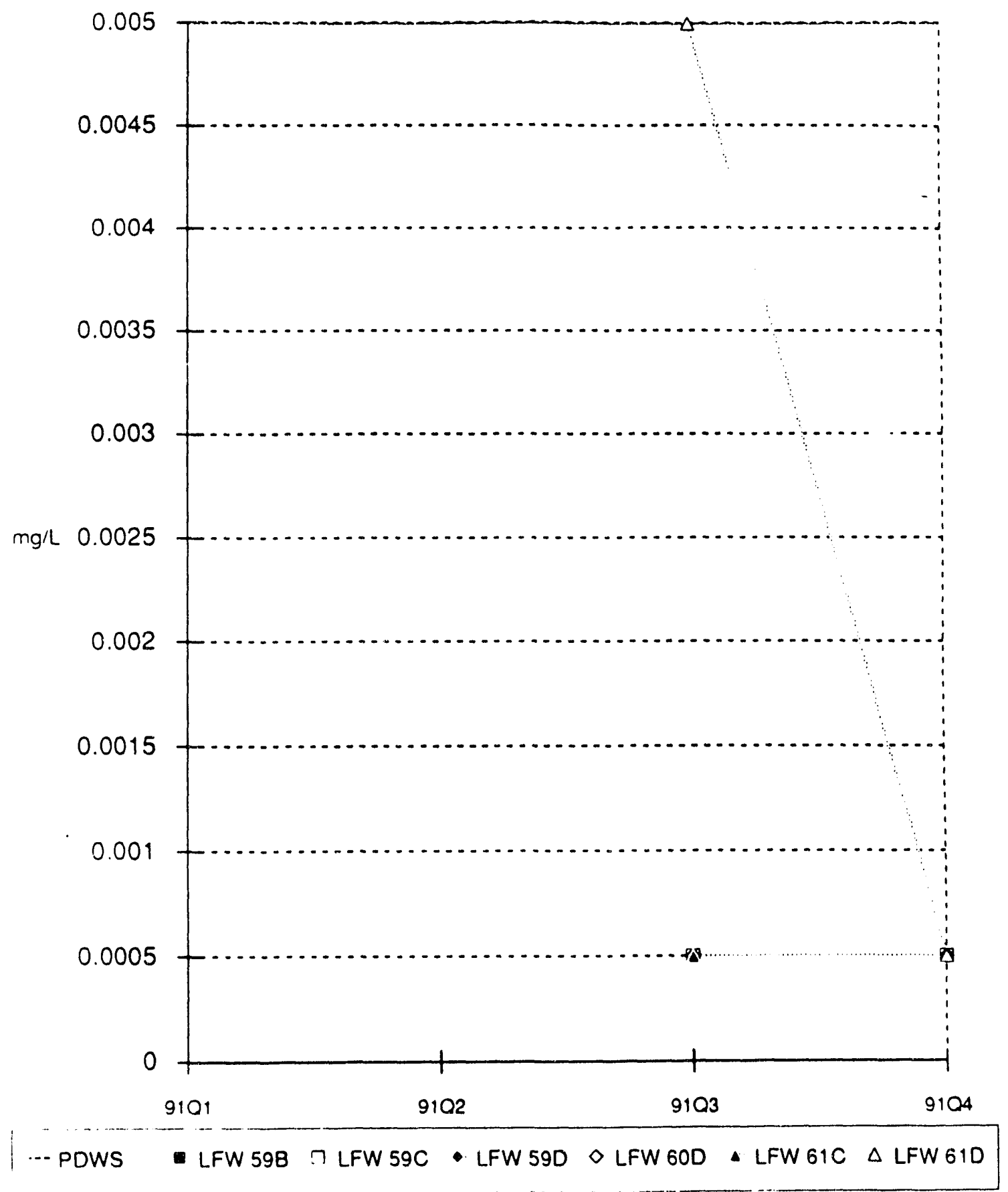


Benzene in the LFW-Series Monitoring Wells

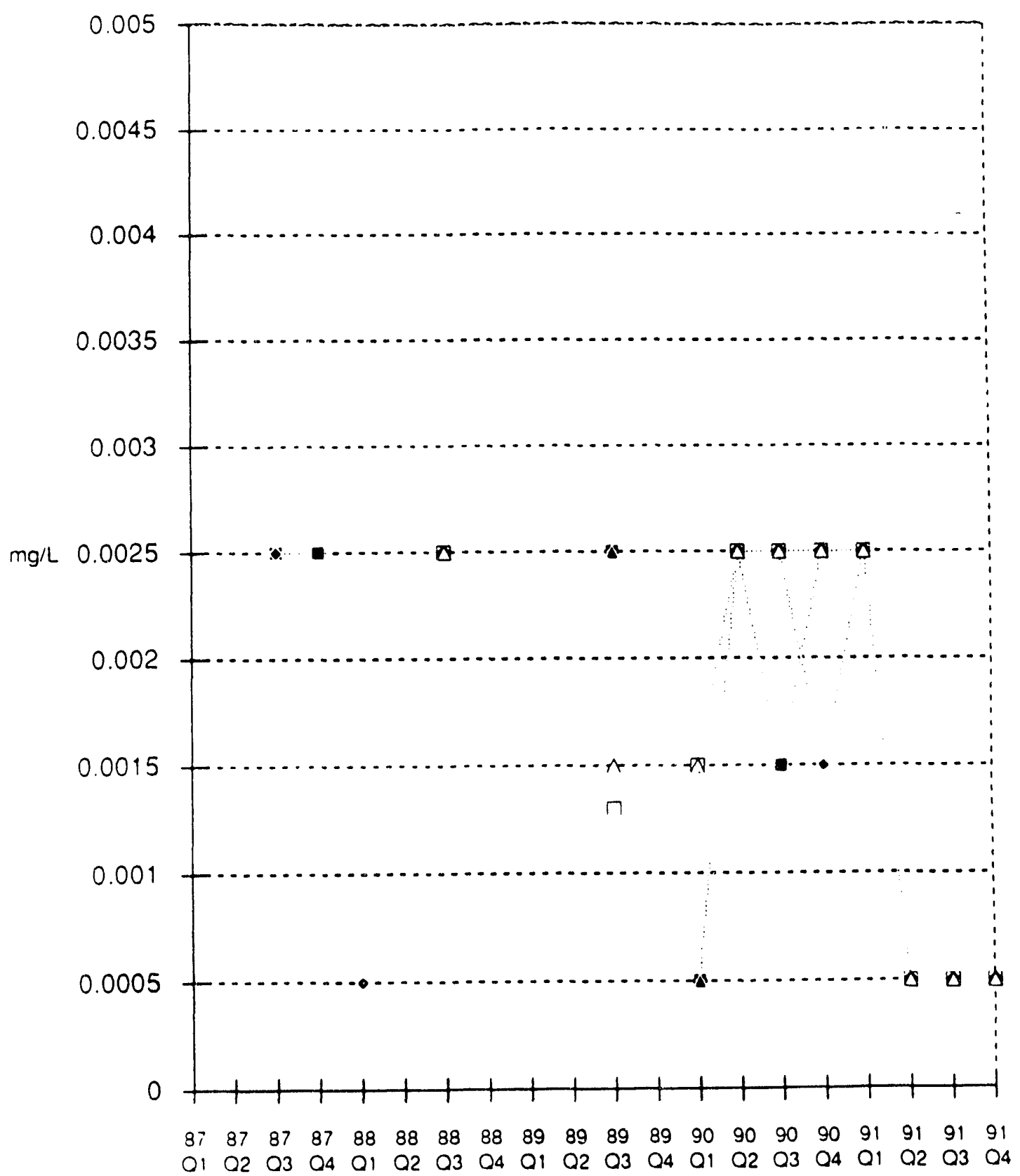

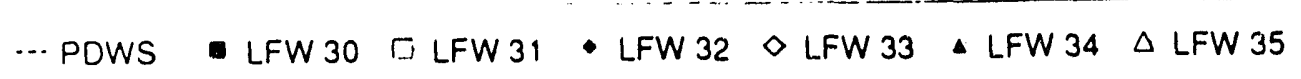


Benzene in the LFW-Series Monitoring Wells

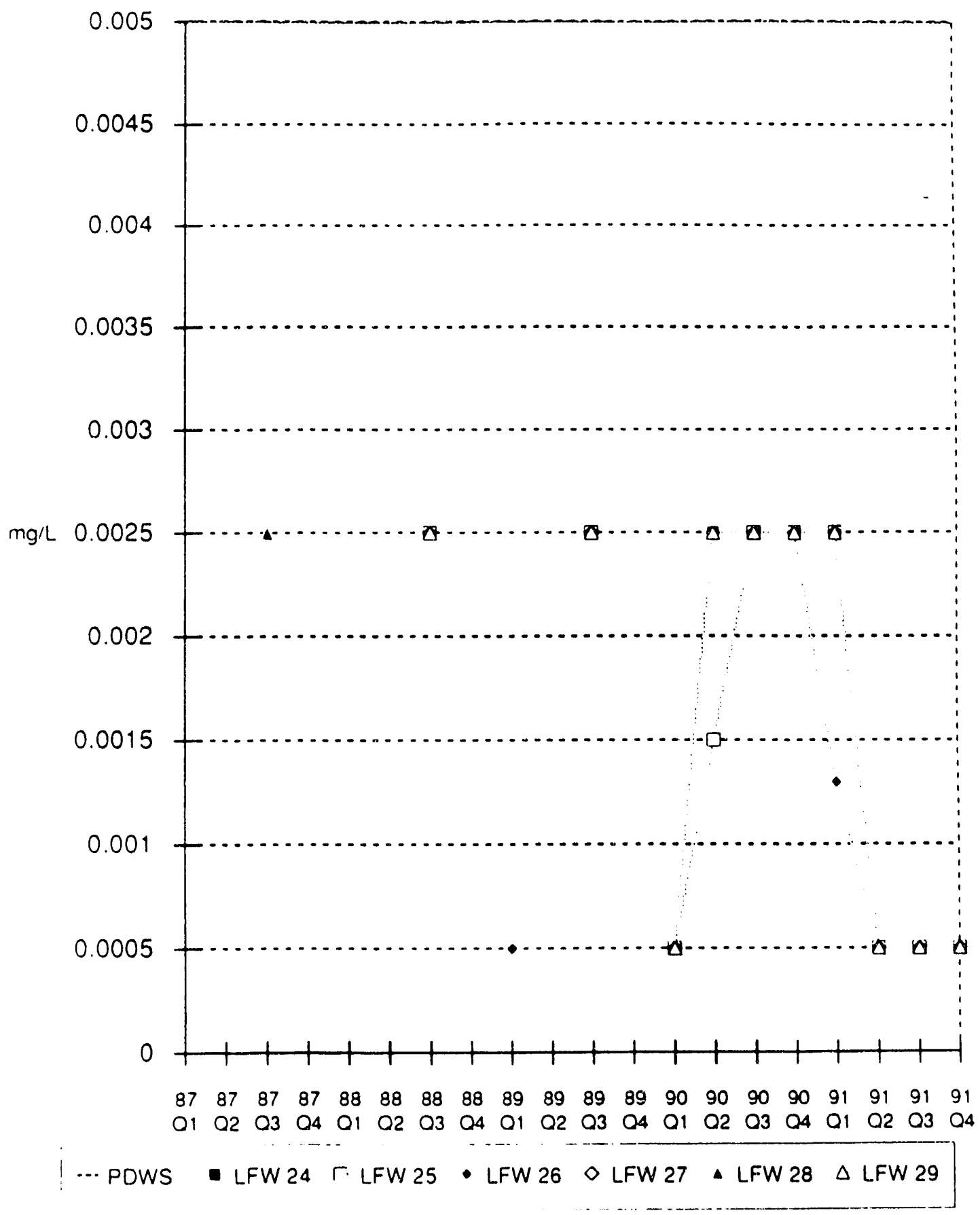


Benzene in the LFW-Series Monitoring Wells

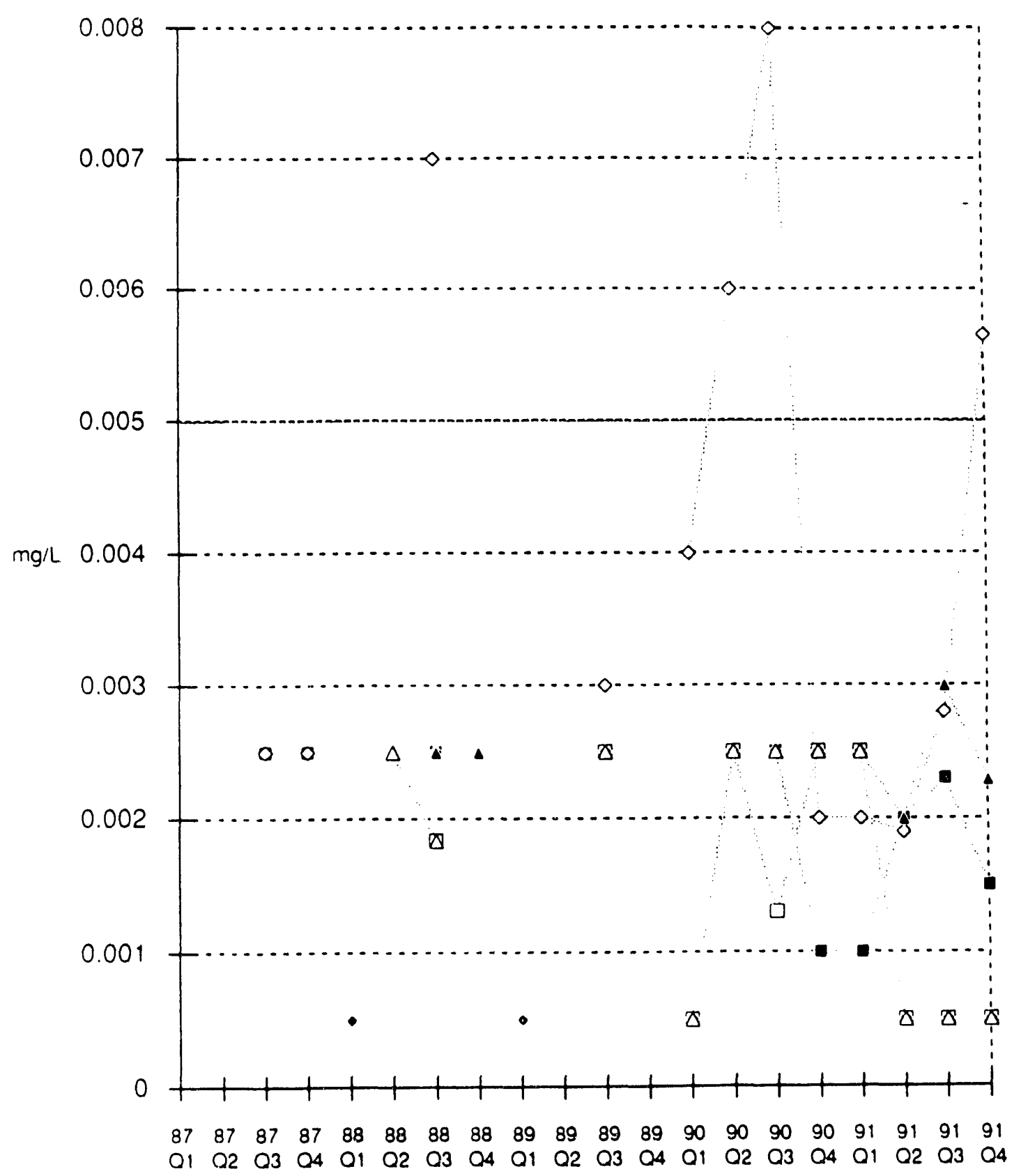

... POWS - LFW 18 LFW 19 • LFW 20 ○ LFW 21 • LFW $22 \triangle$ LFW 23 
Total Organic Halogens in the LFW-Series Monitoring Wells

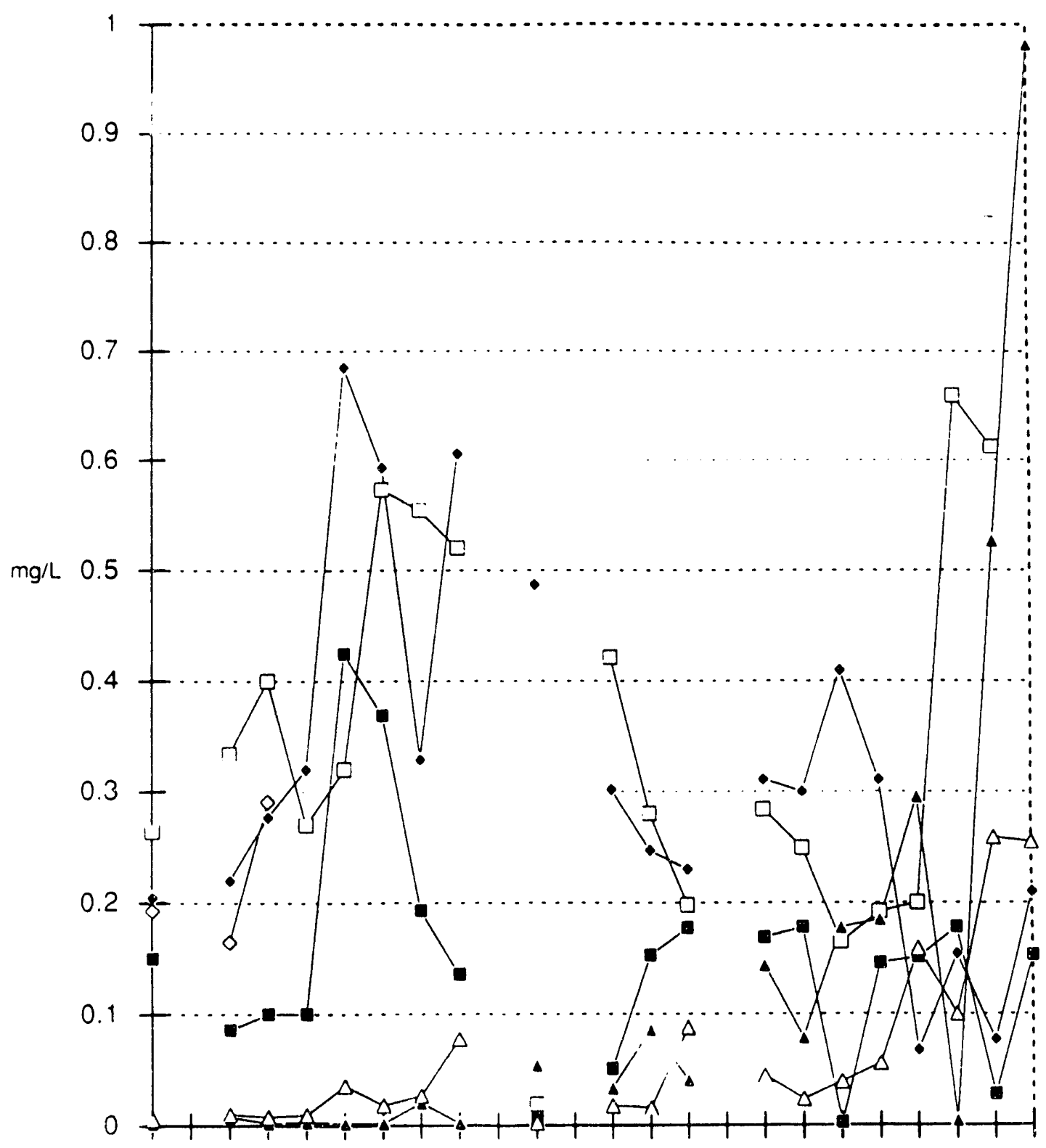

$\begin{array}{llllllllllllllllllllllll}86 & 86 & 86 & 86 & 87 & 87 & 87 & 87 & 88 & 88 & 88 & 88 & 89 & 89 & 89 & 89 & 90 & 90 & 90 & 90 & 91 & 91 & 91 & 91\end{array}$ Q1 $\mathrm{Q}_{2} \mathrm{Q}_{3} \mathrm{Q}_{4} \mathrm{Q}_{1} \mathrm{Q}_{2} \mathrm{Q}_{3} \mathrm{Q}_{4} \mathrm{Q}_{1} \mathrm{Q}_{2} \mathrm{Q}_{3} \mathrm{Q}_{4} \mathrm{Q}_{1} \mathrm{Q}_{2} \mathrm{Q}_{3} \mathrm{Q}_{4} \mathrm{Q}_{1} \mathrm{Q}_{2} \mathrm{Q}_{3} \mathrm{Q}_{4} \mathrm{Q}_{1} \mathrm{Q}_{2} \mathrm{Q}_{3} \mathrm{Q}_{4}$

- LFW $6 \quad \square$ LFW 7 - LFW 8 - LFW 9 - LFW 10A $\triangle$ LFW 16 
Total Organic Halogens in the LFW-Series Monitoring Wells

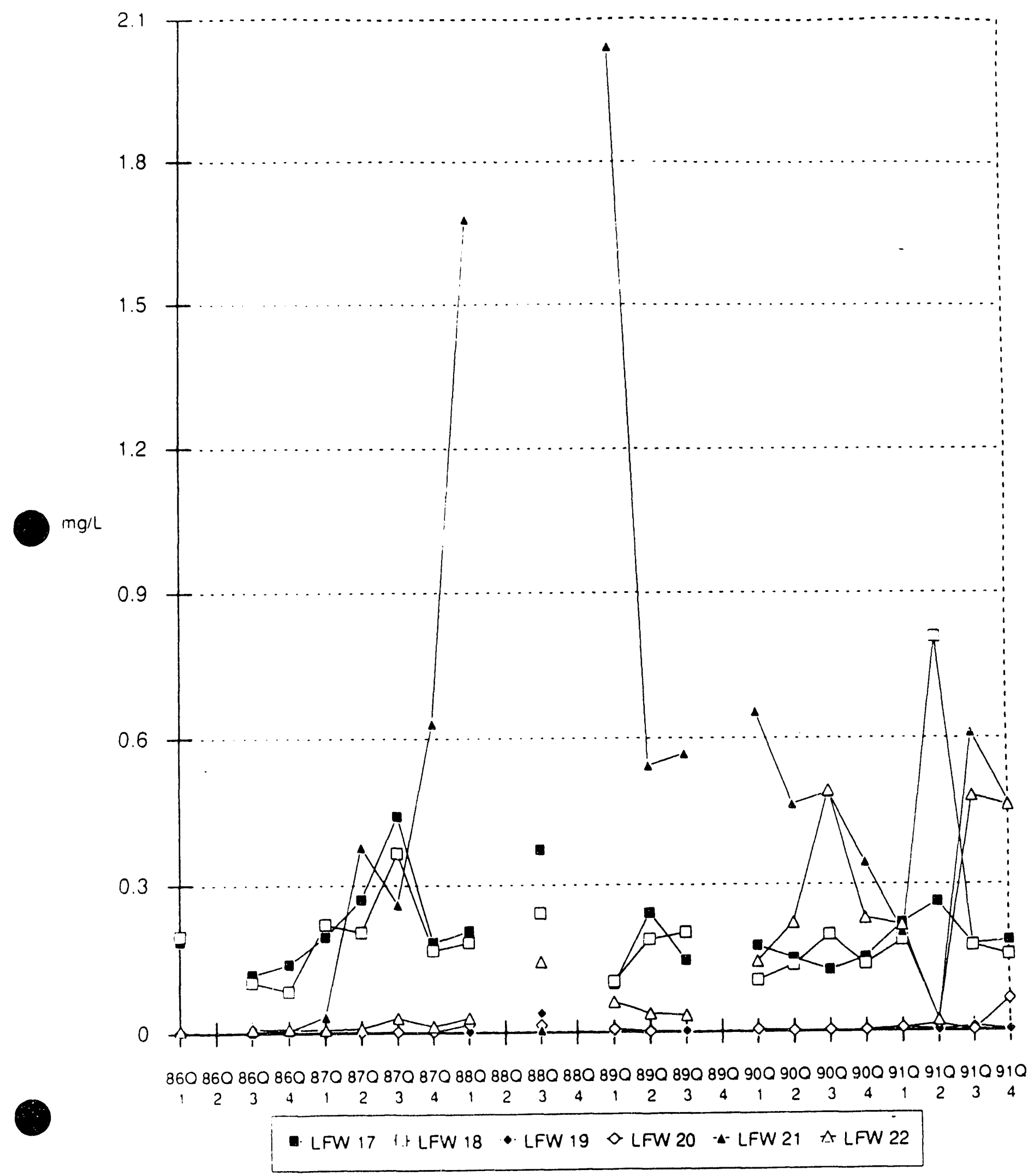


Total Organic Halogens in the LFW-Series Monitoring Wells

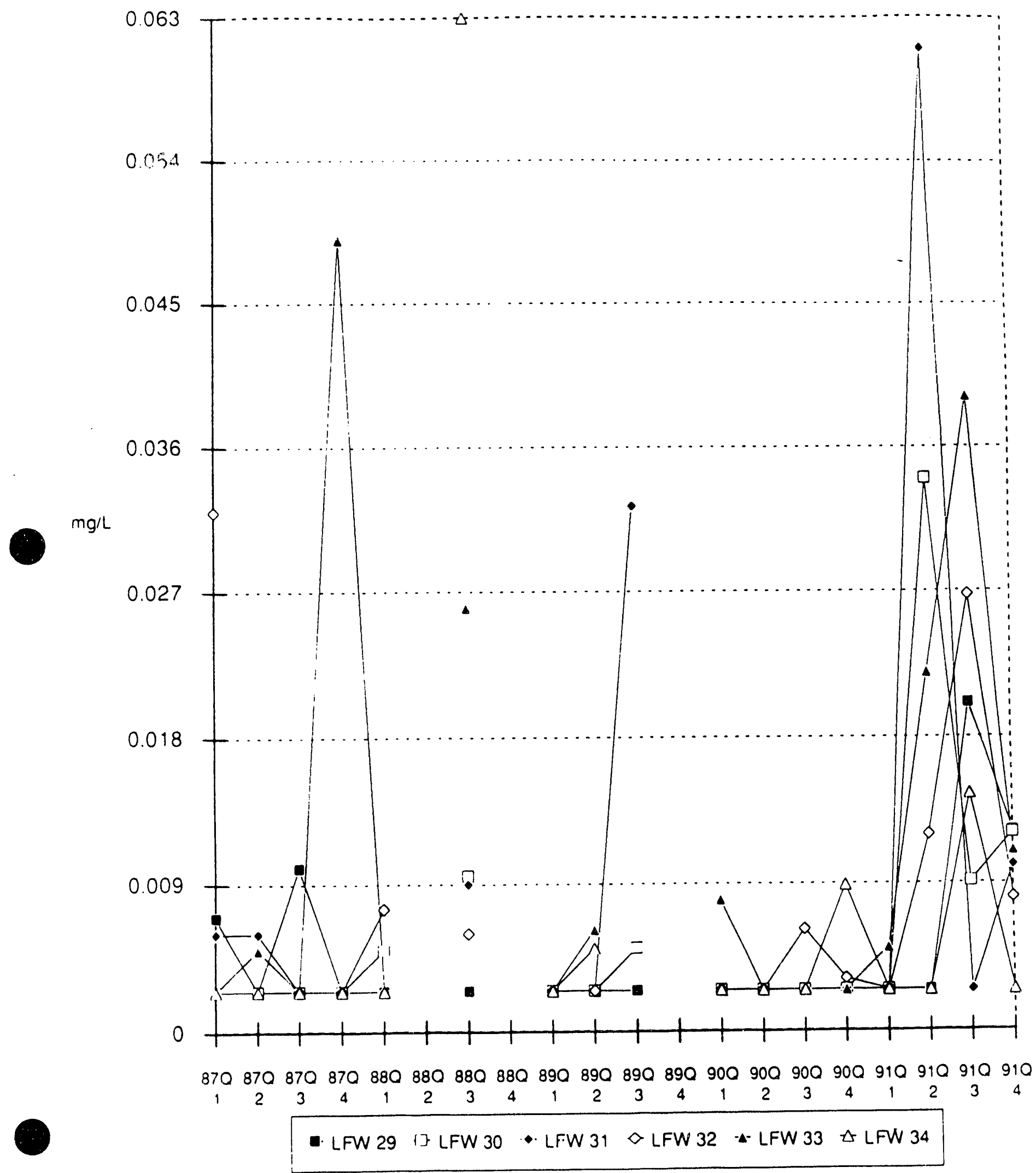


Total Organic Halogens in the LFW-Series Monitoring Wells

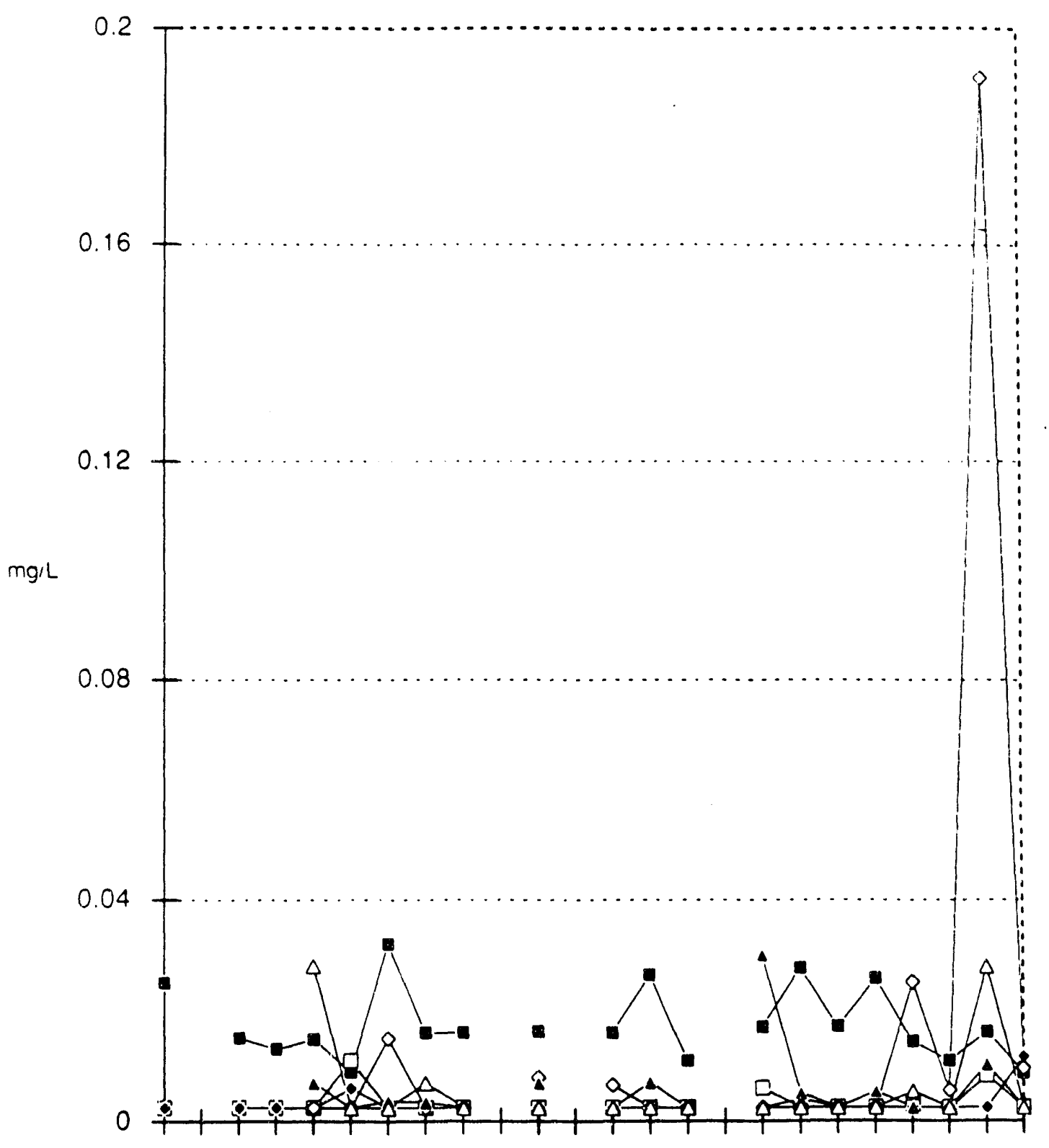

$\begin{array}{llllllllllllllllllllllll}86 & 86 & 86 & 86 & 87 & 87 & 87 & 87 & 88 & 88 & 88 & 88 & 89 & 89 & 89 & 89 & 90 & 90 & 90 & 90 & 91 & 91 & 91 & 91\end{array}$

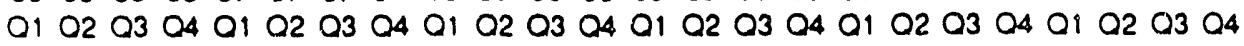

- LFW 23 ก LFW 24 • LFW $25 \diamond$ LFW 26 -LFW $27 \Delta$ LFW 28 
Total Organic Halogens iti the LFW-Series Monitoring Wells

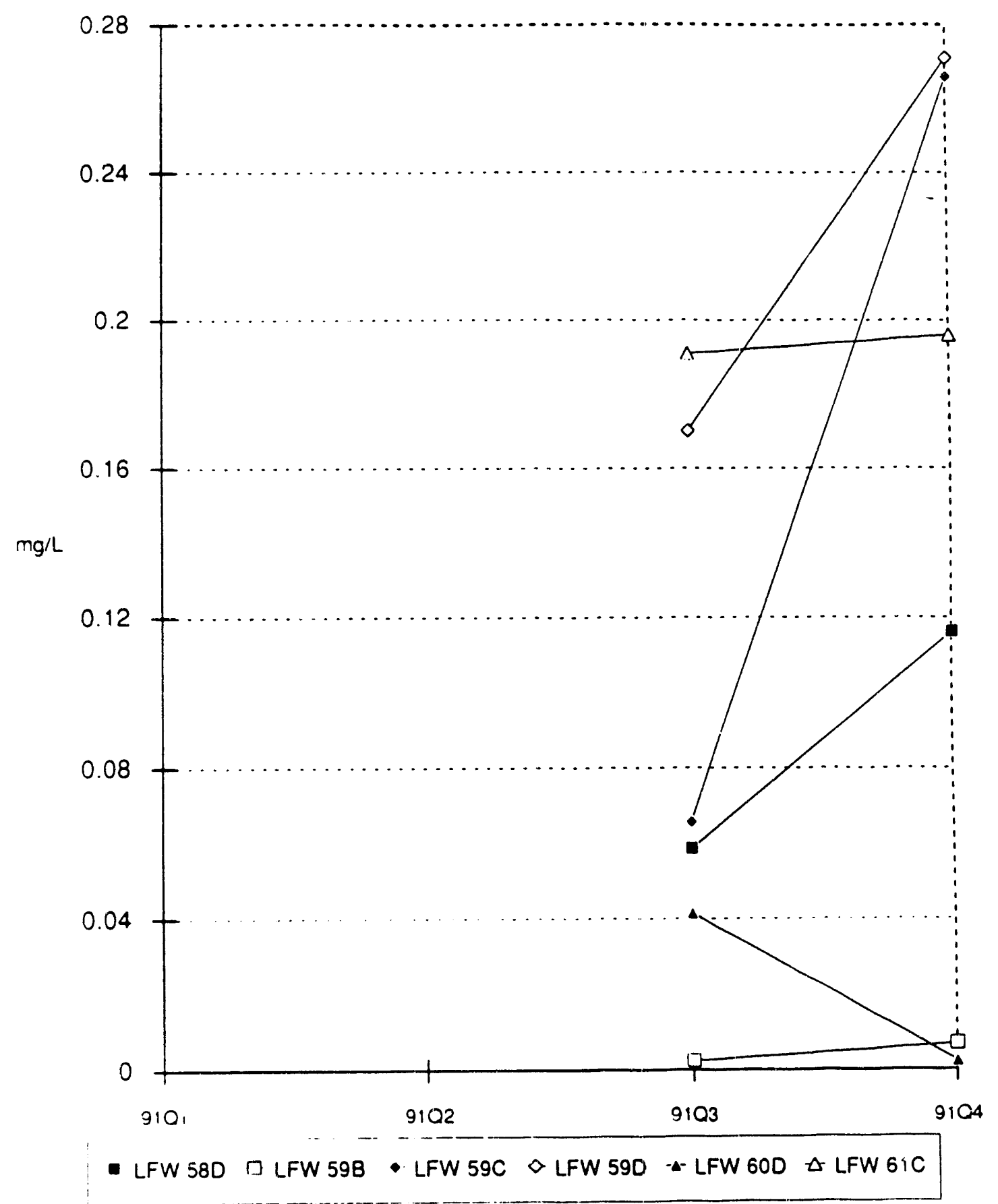


Total Organic Halogens in the LFW-Series Monitoring Wells

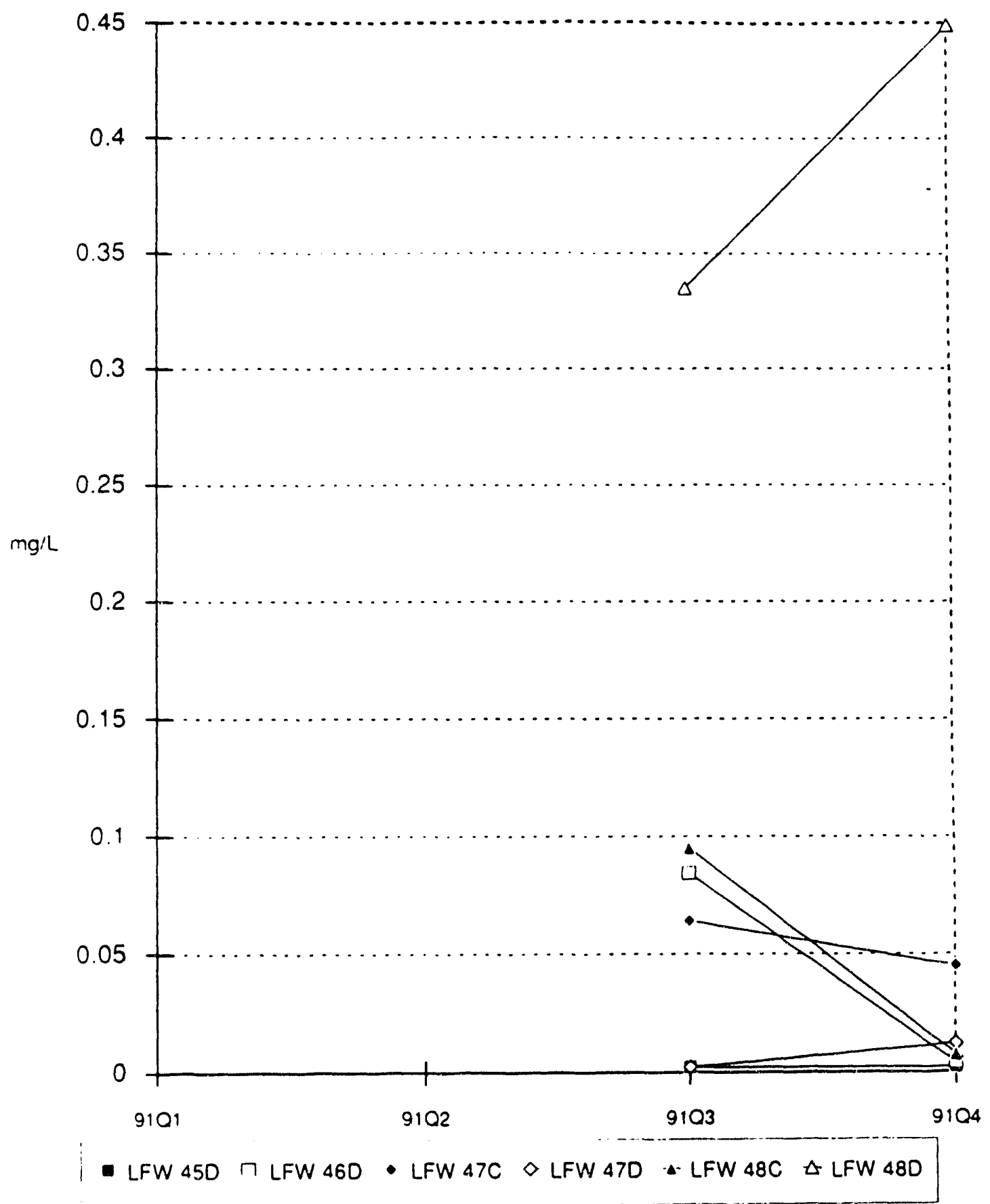


Total Organic Halogens in the LFW-Series Monitoring Wells

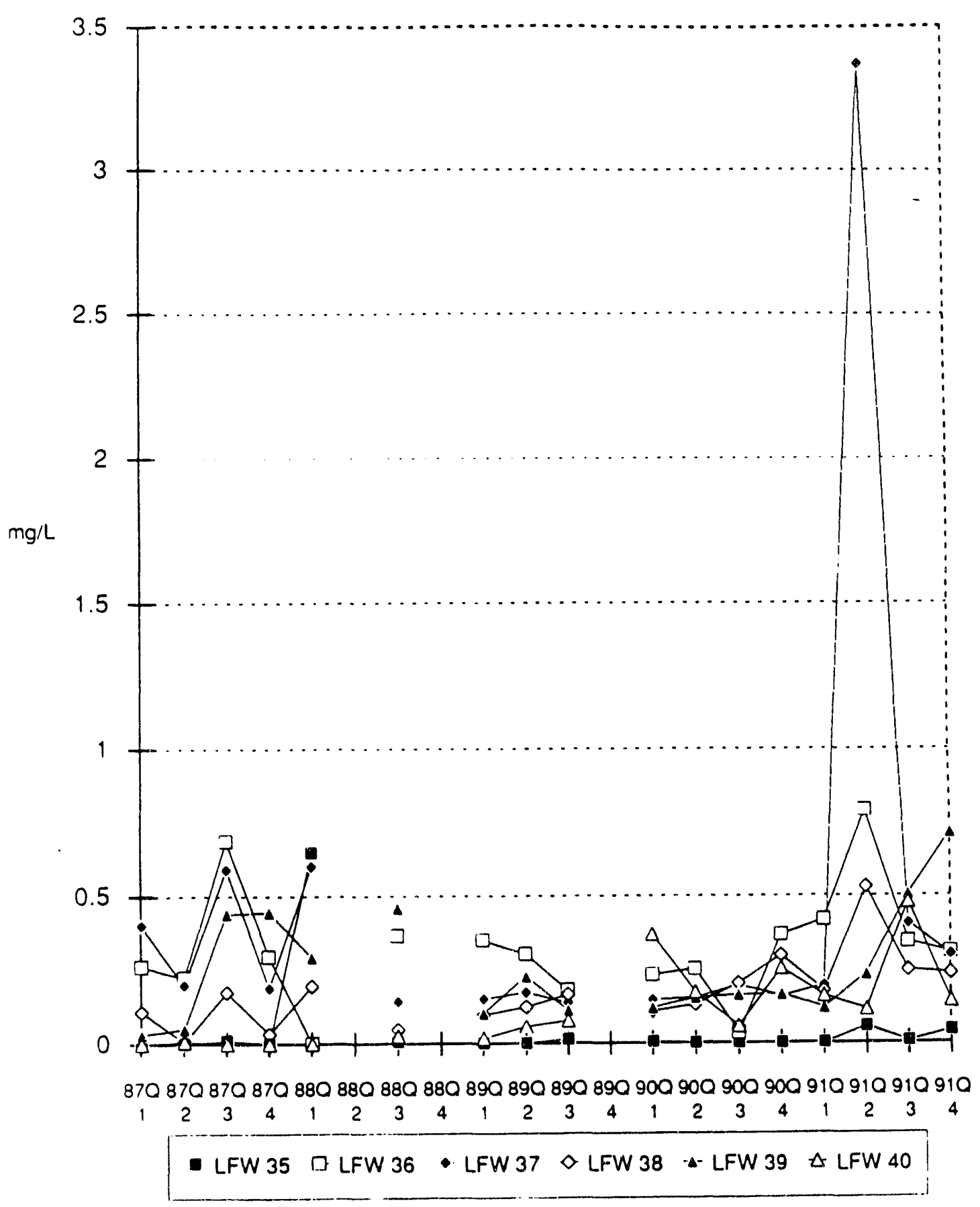


Total Organic Halogens in the LFW-Series Monitoring Wells

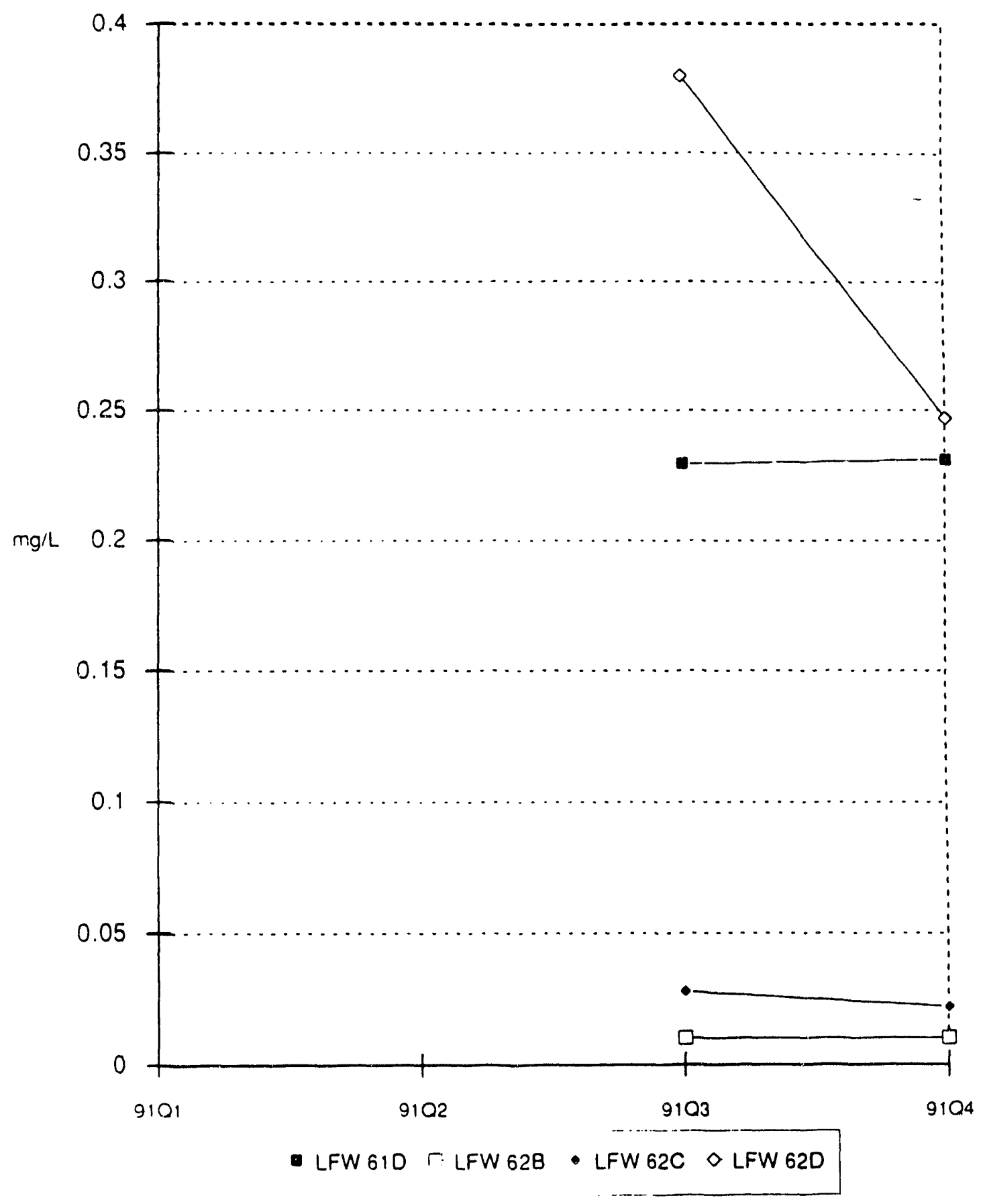


Total Organic Halogens in the LFW-Series Monitoring Wells

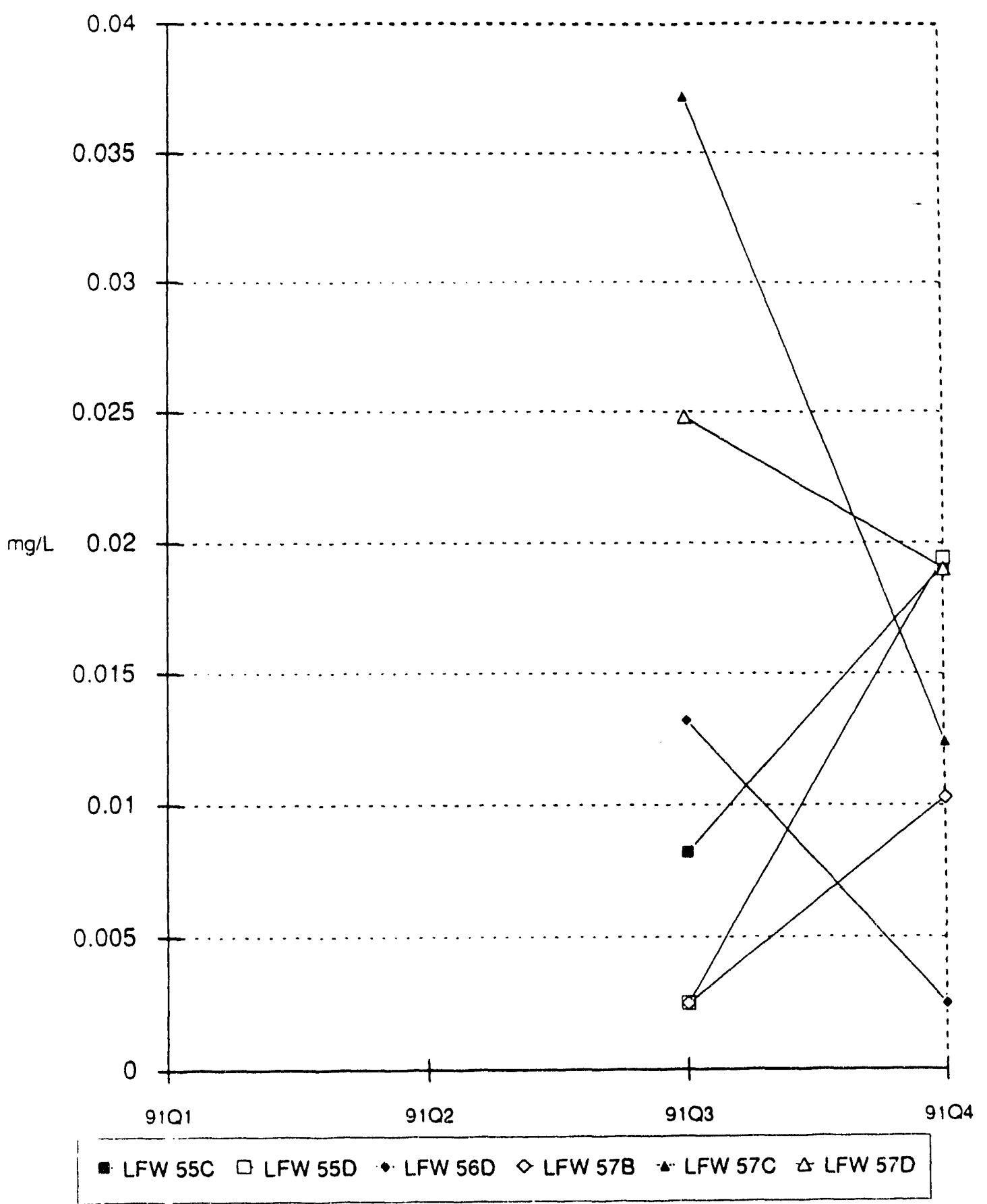


Total Organic Halogens in the LFW-Series Monitoring Wells

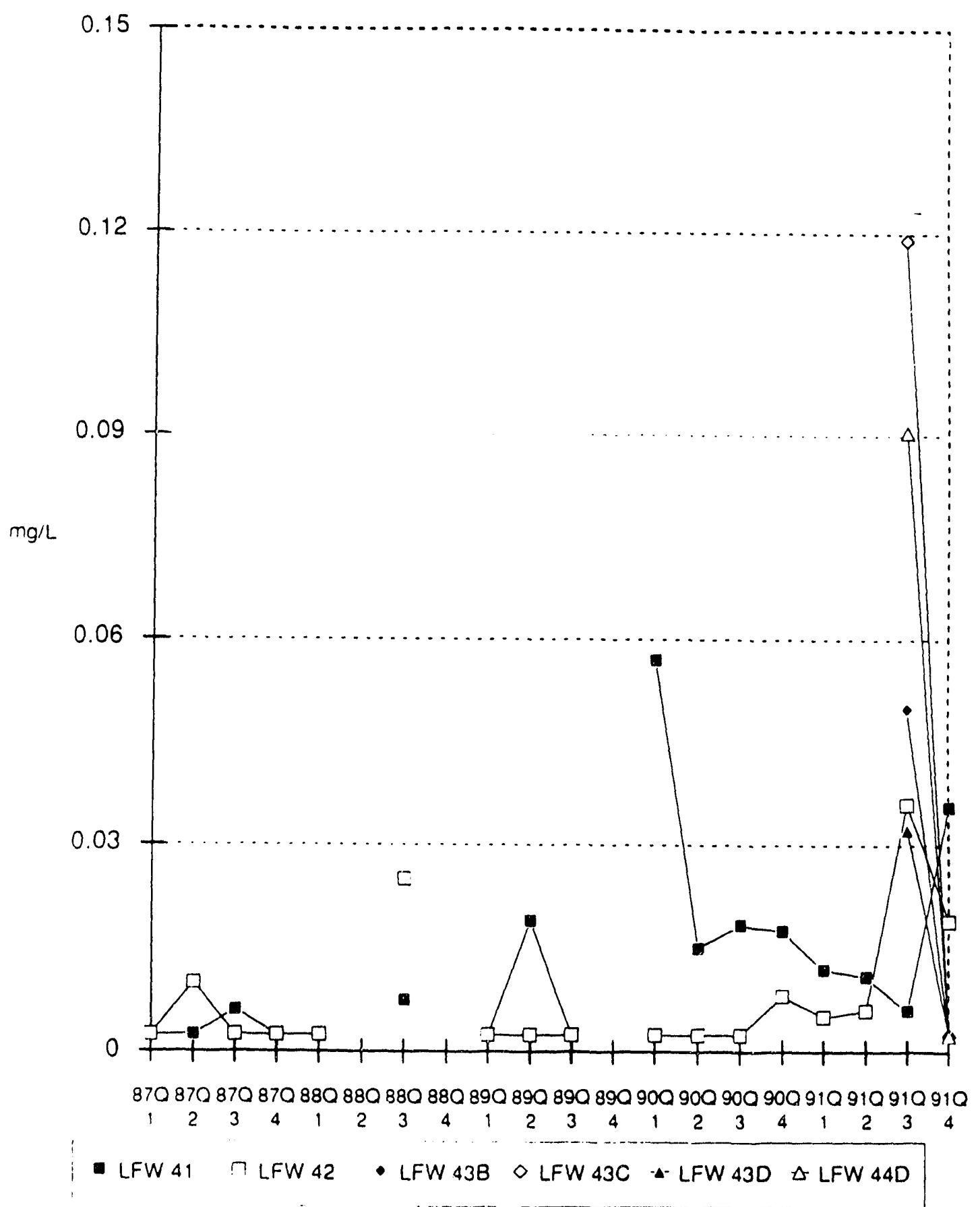


1,1,1-Trichloroethane in the LFW-Series Monitoring Wells

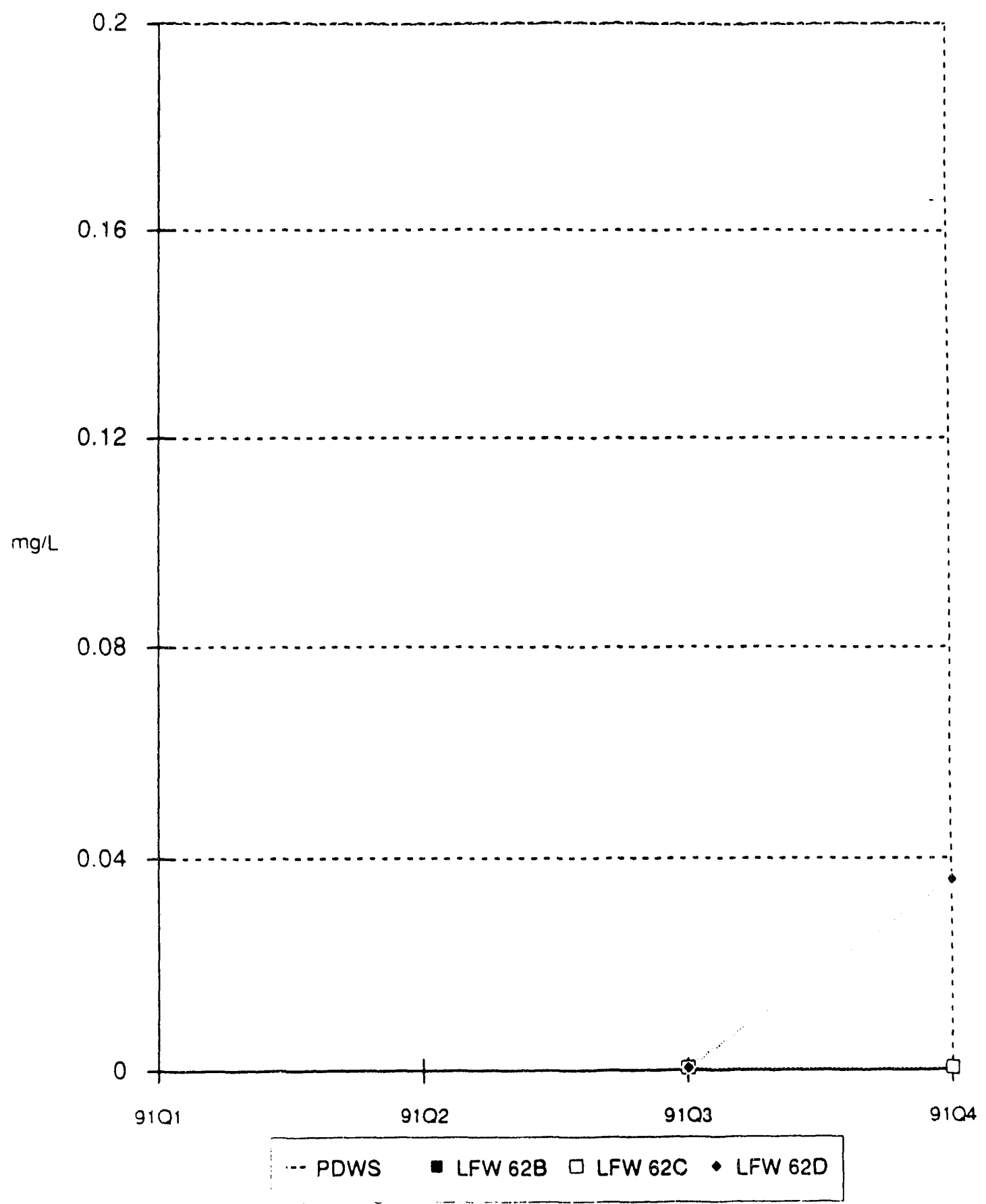


$1,1,1$-Trichloroethane in the LFW-Series Monitoring Wells

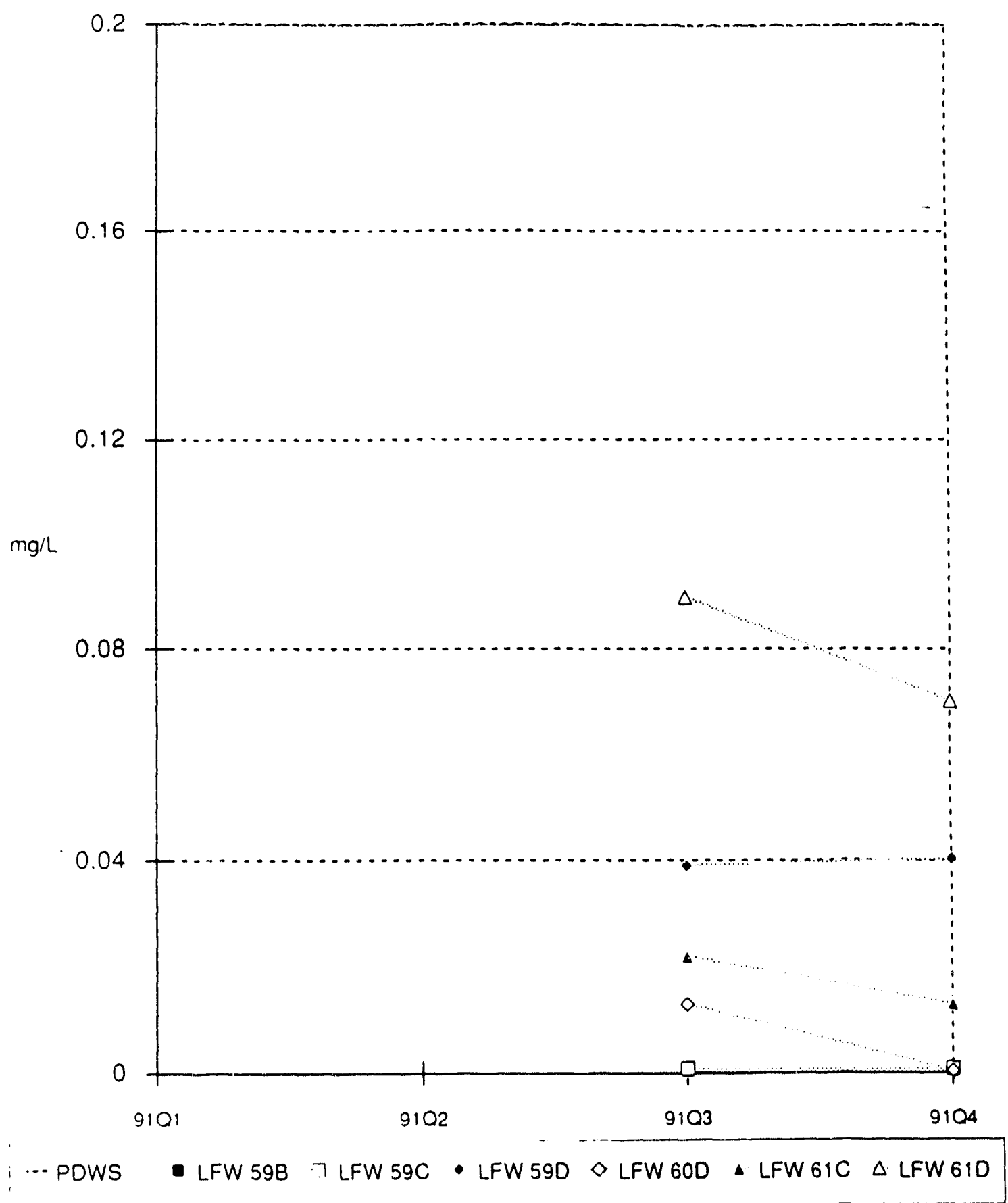


$1,1,1 \cdot$ Trichloroethane in the LFW-Series Monitoring Wells

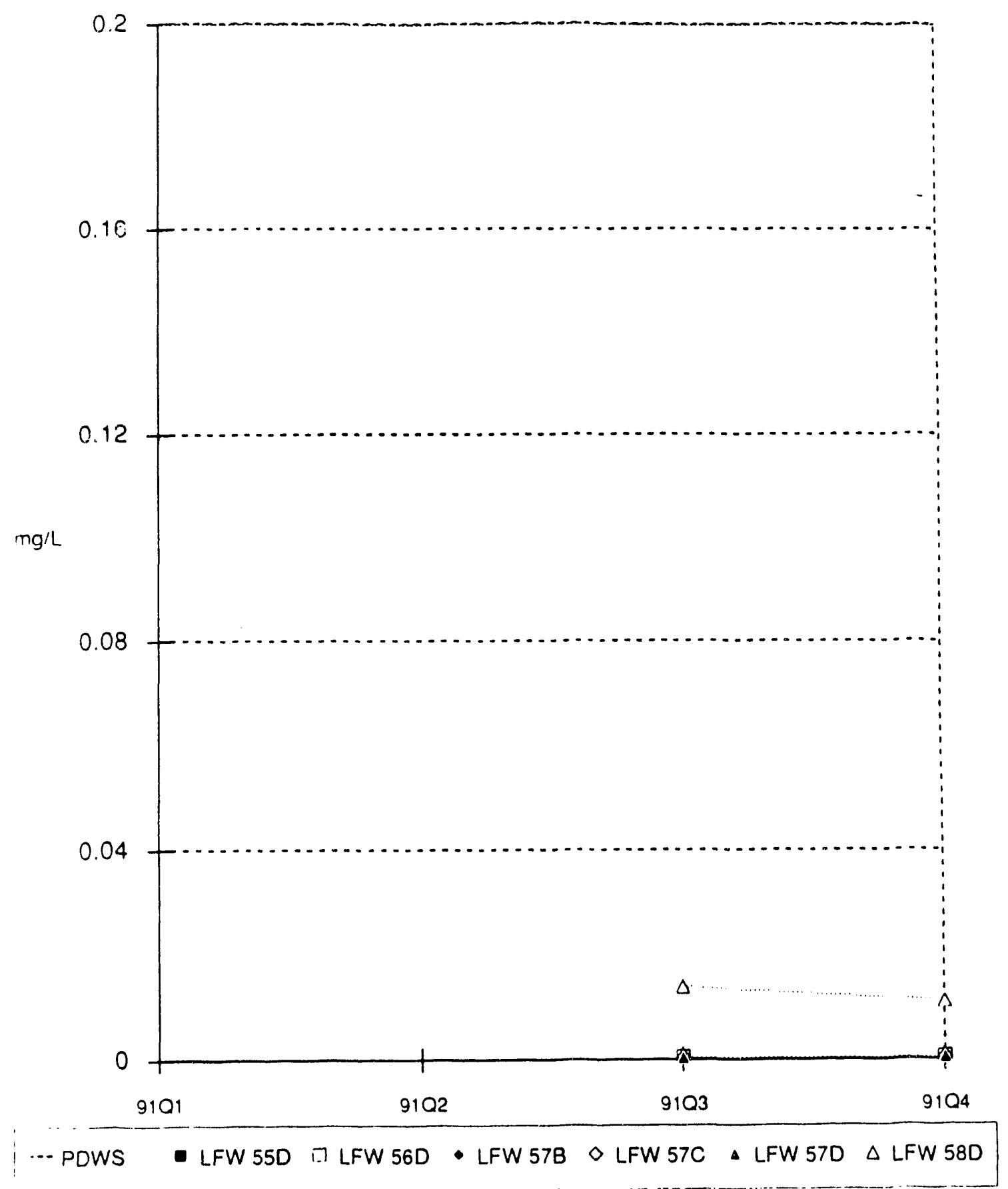


1,1,1-Trichloroethane in the LFW-Series Monitoring Wells

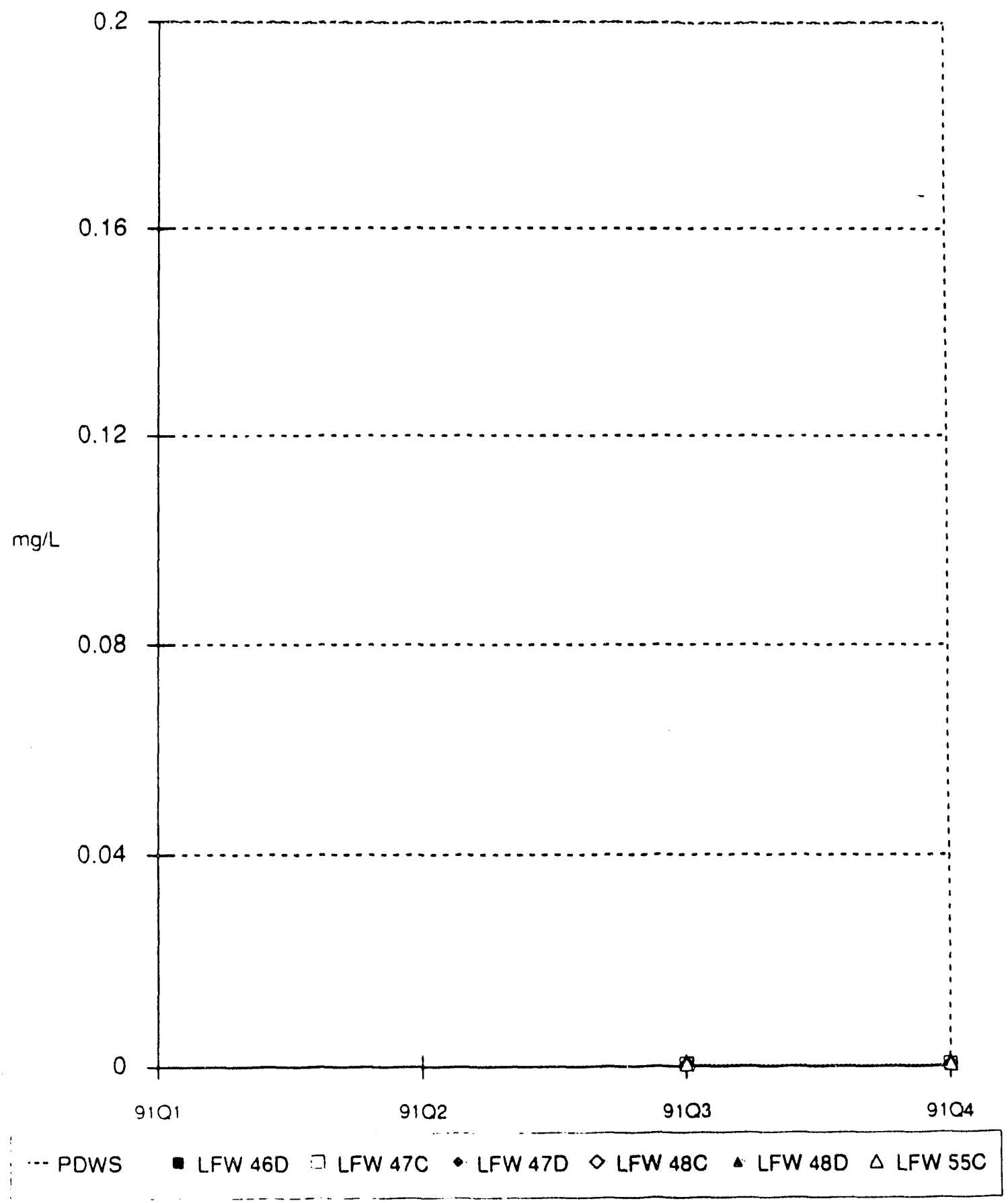




\section{1,1,1-Trichloroethane in the LFW-Series Monitoring Wells}

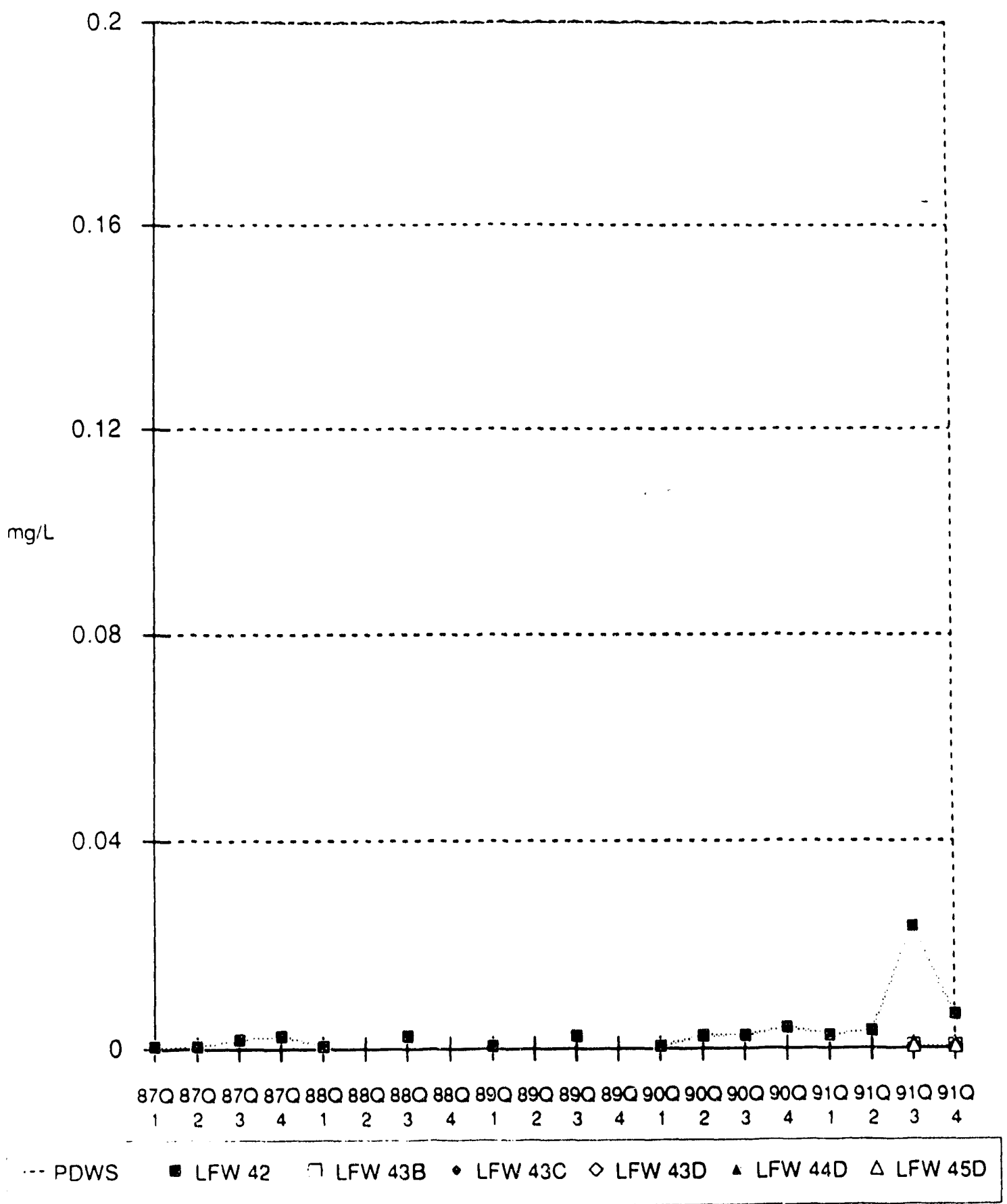


1,1,1-Trichloroethane in the LFW-Series Monitoring Wells

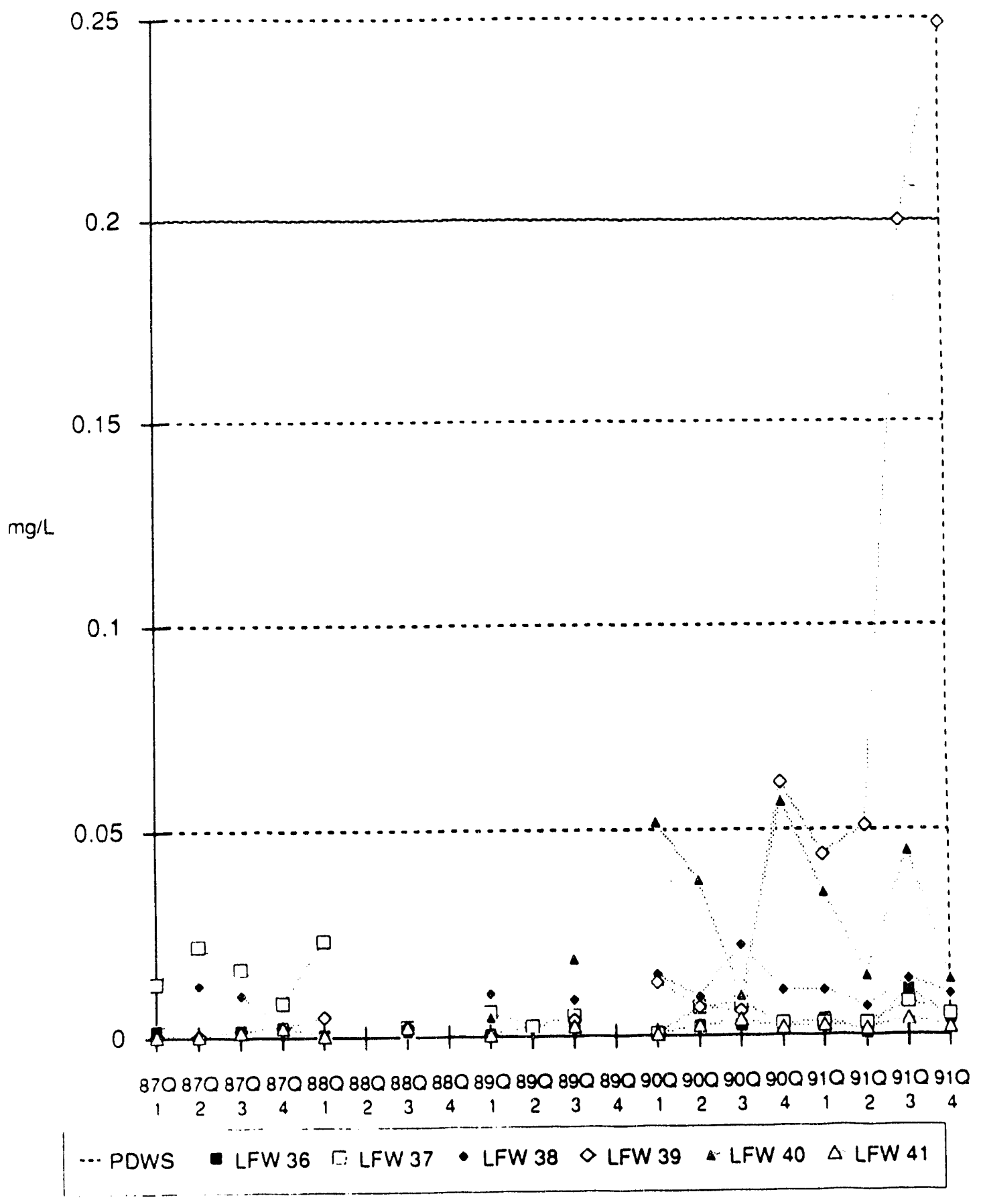


1,1,1-Trichloroethane in the LFW-Series Monitoring Wells

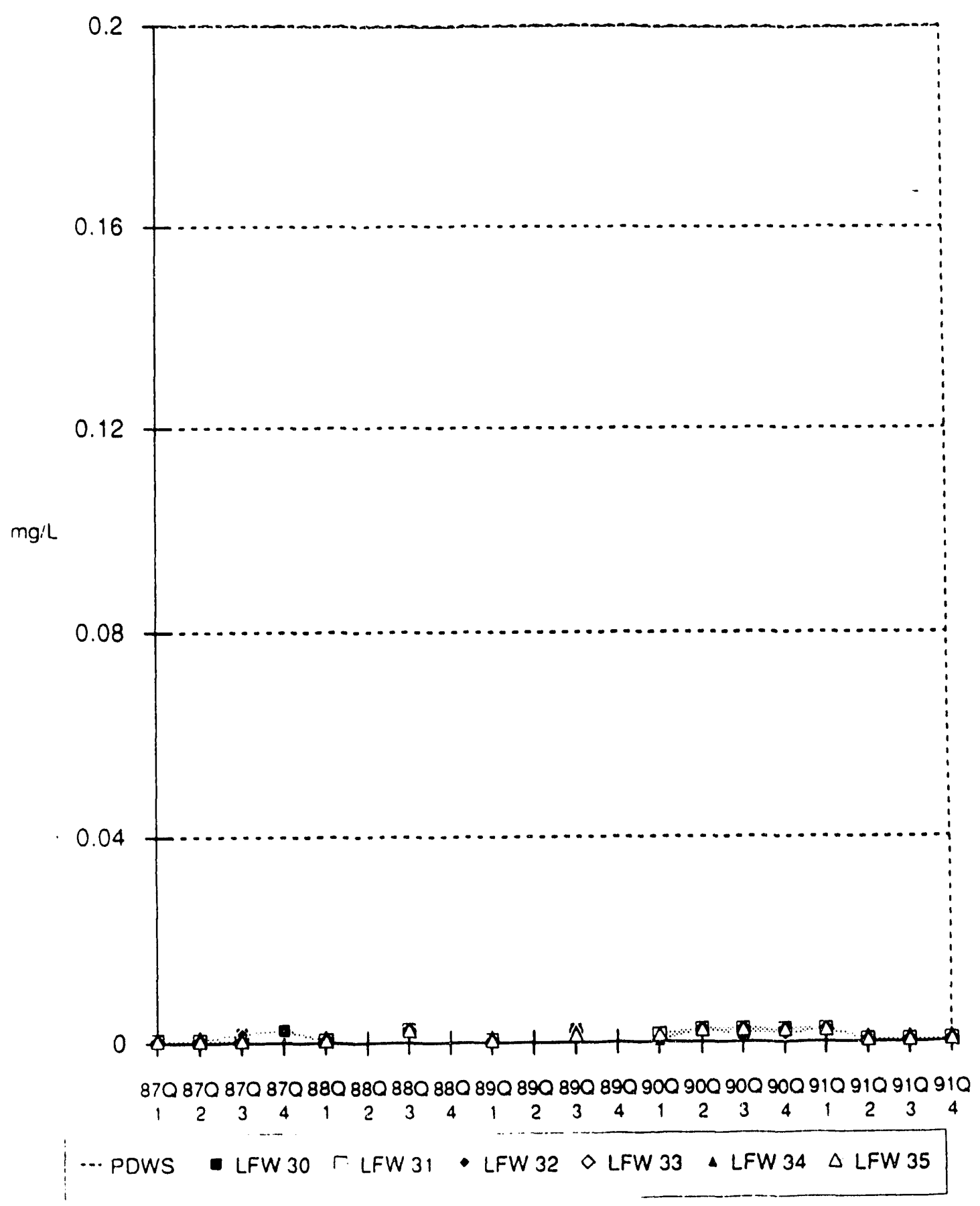


1,1,1-Trichloroethane in the LFW-Series Monitoring Wells

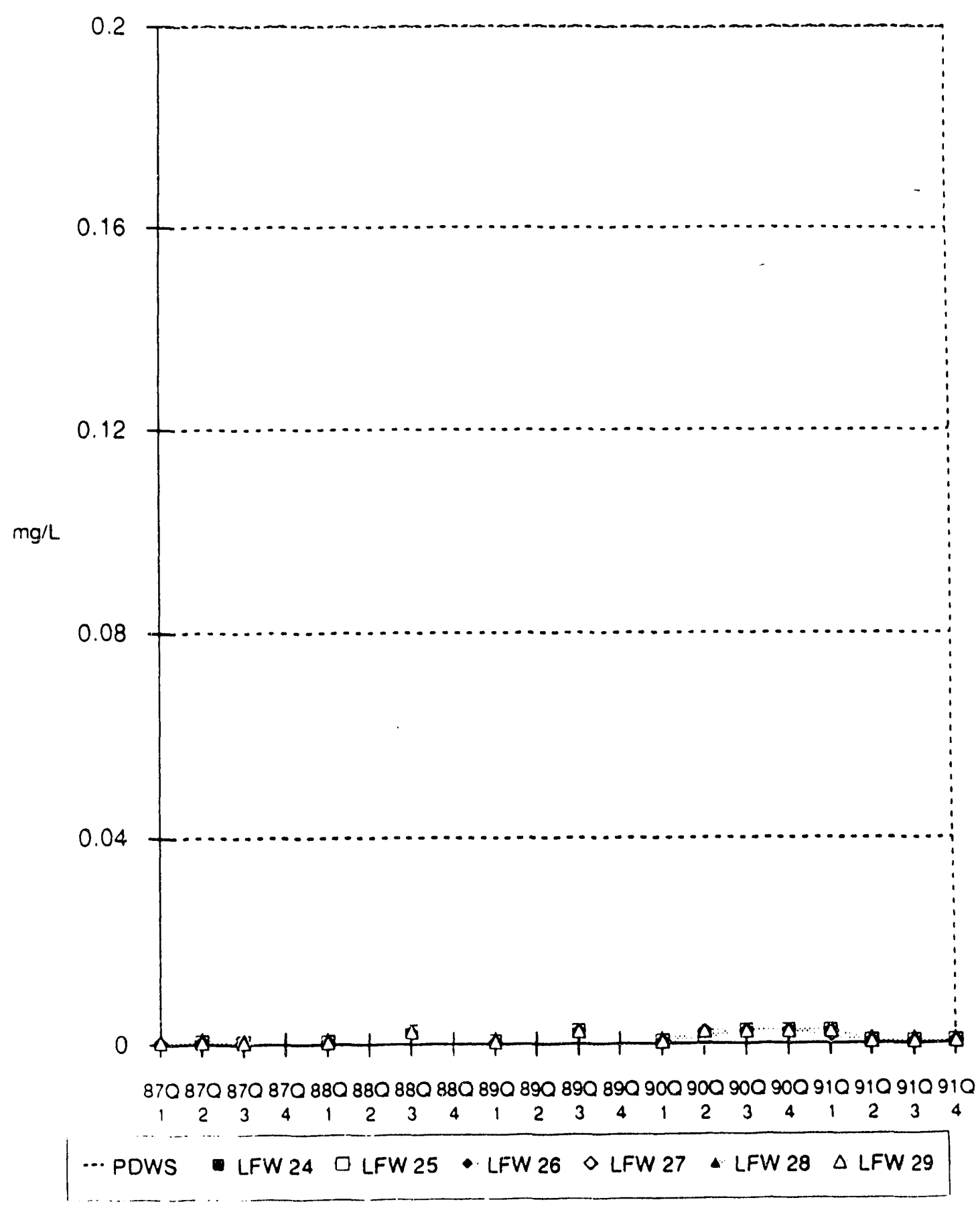


1,1,1-Trichloroethane in the LFW-Series Monitoring Wells

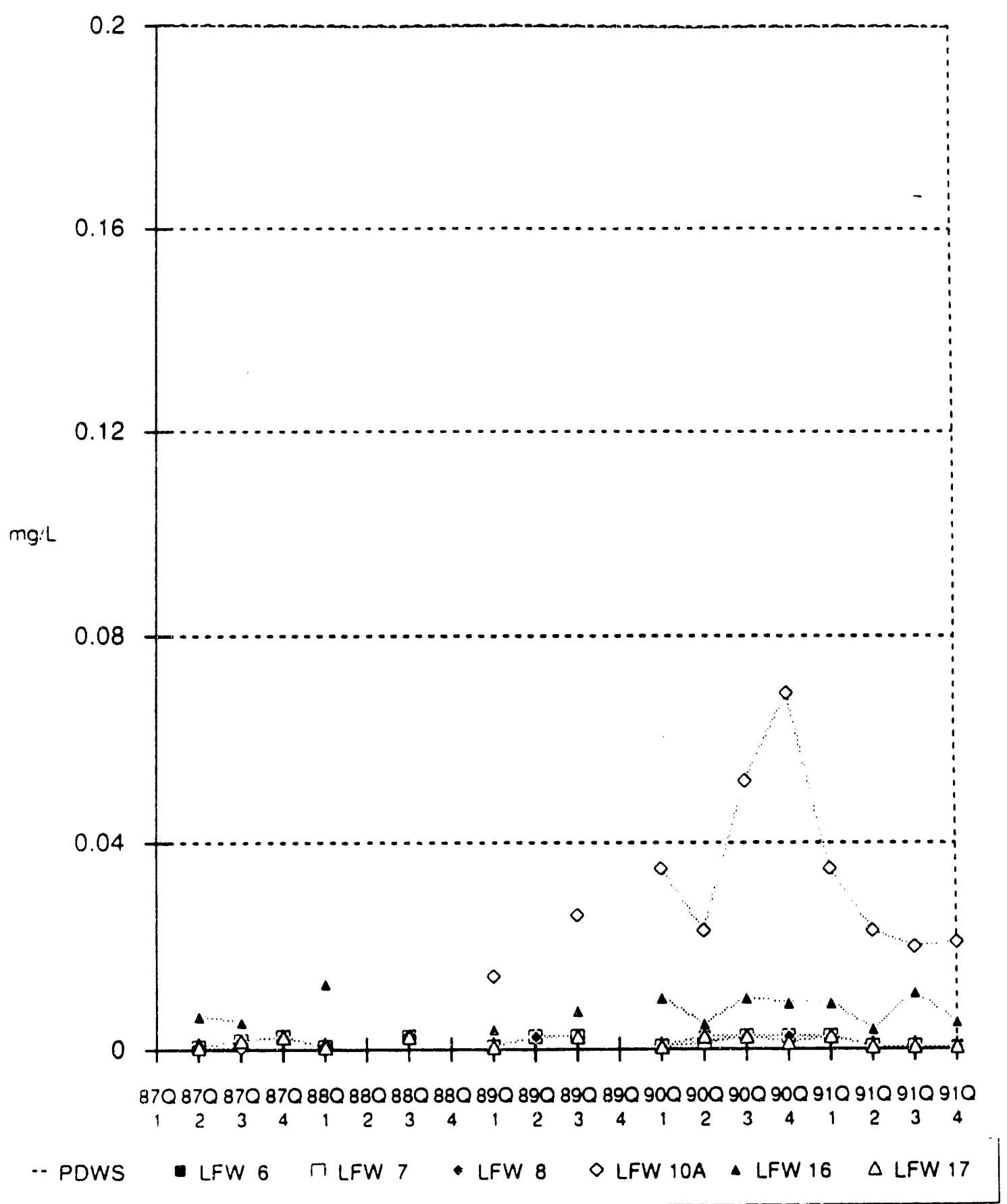


1,1,1-Trichloroethane in the LFW-Series Monitoring Wells

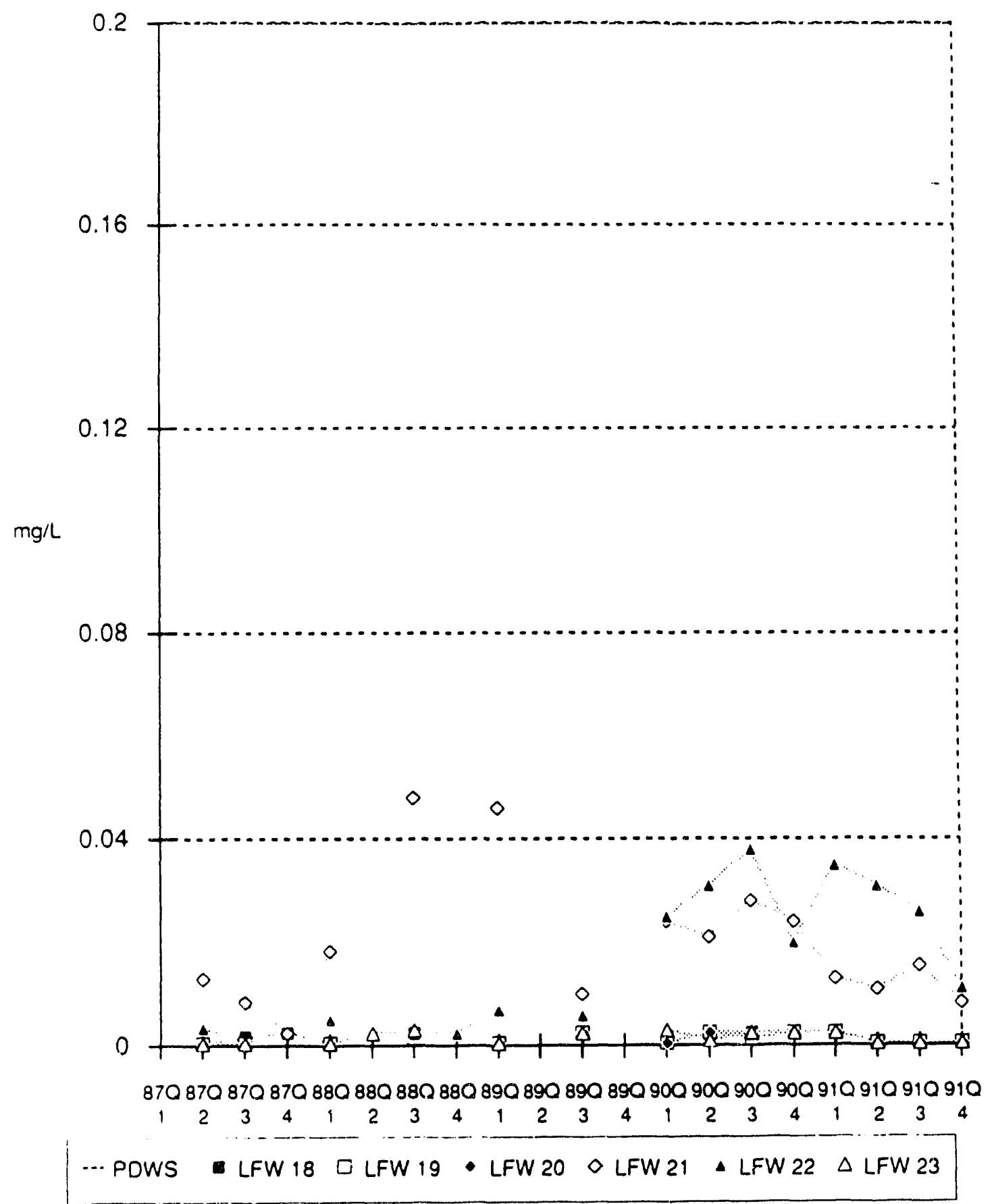


$1,1,1$-Trichloroethane in the LFW-Series Monitoring Wells

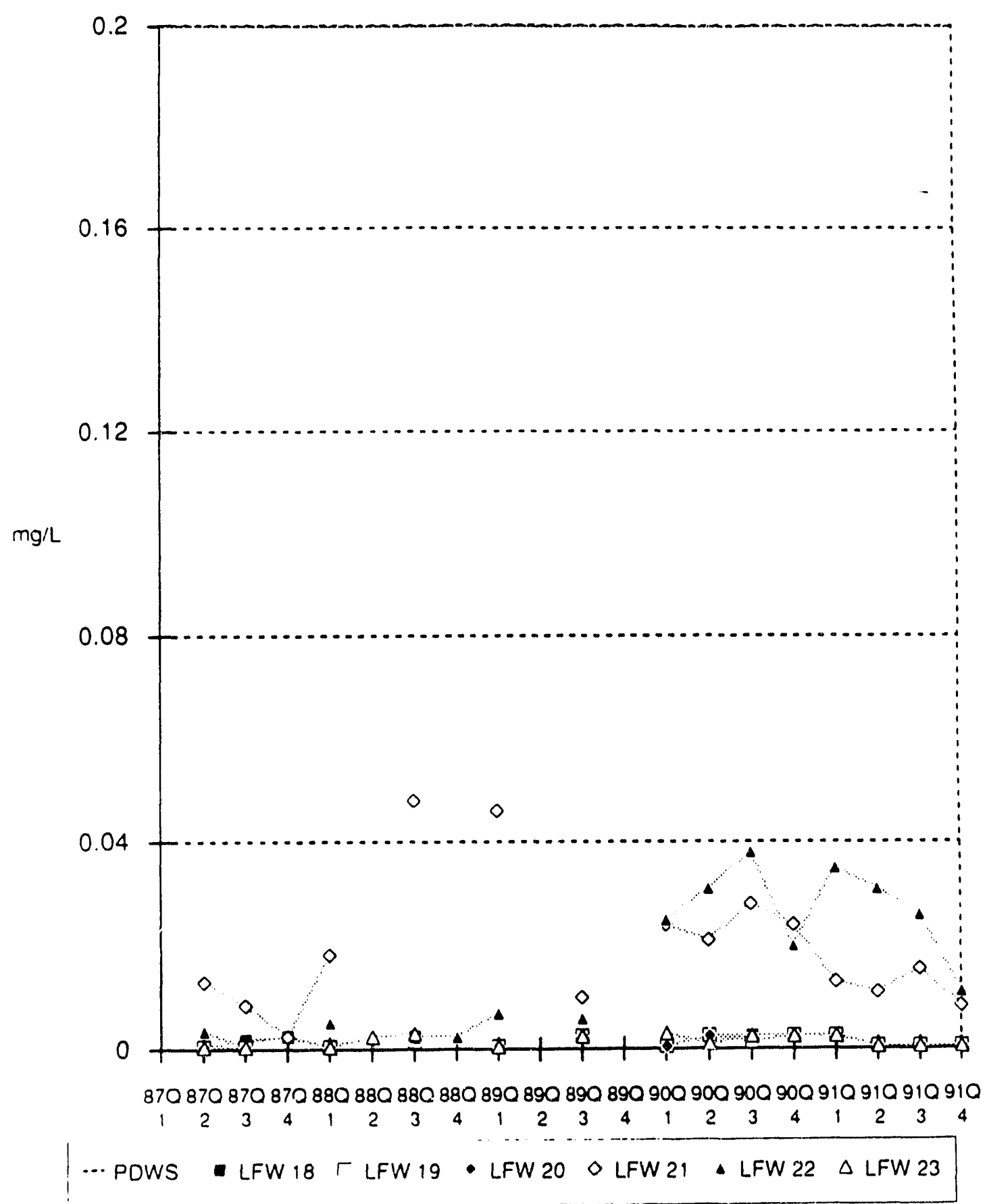




\section{1,1,2-Trichloroethane in the LFW-Series Monitoring Wells}

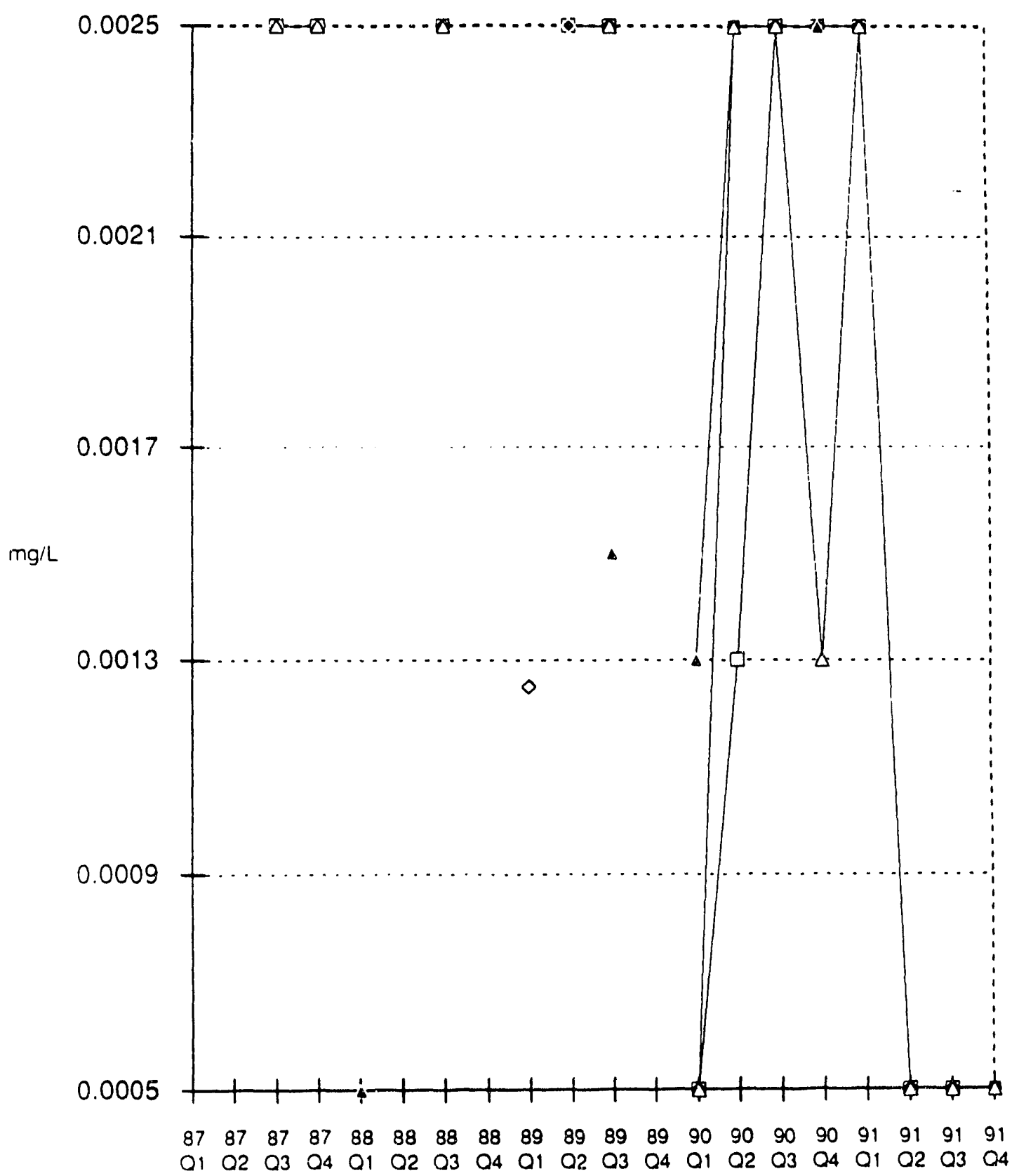

- LFW $6 \quad \Pi \operatorname{LFW} 7$ - LFW 8 \&LF 10A A LFW $16 \Delta$ LFW 17 
1,1,2-Trichloroethane in the LFW-Series Monitoring Wells

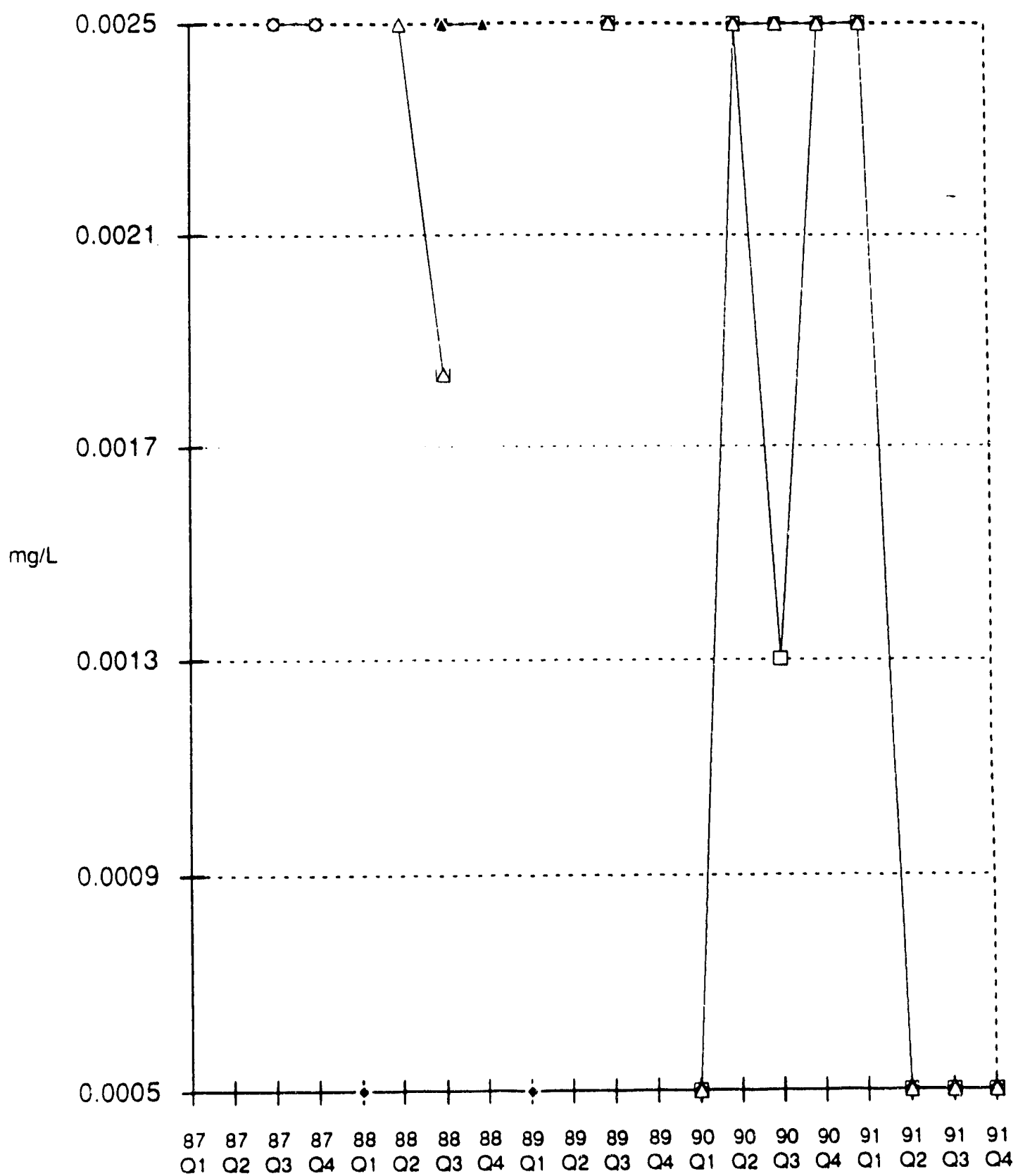

- LFW 18 口 LFW 19 • LFW $20 \diamond$ LFW 21 - LFW $22 \Delta$ LFW 23 
1,1,2-Trichloroethane in the LFW-Series Monitoring Wells

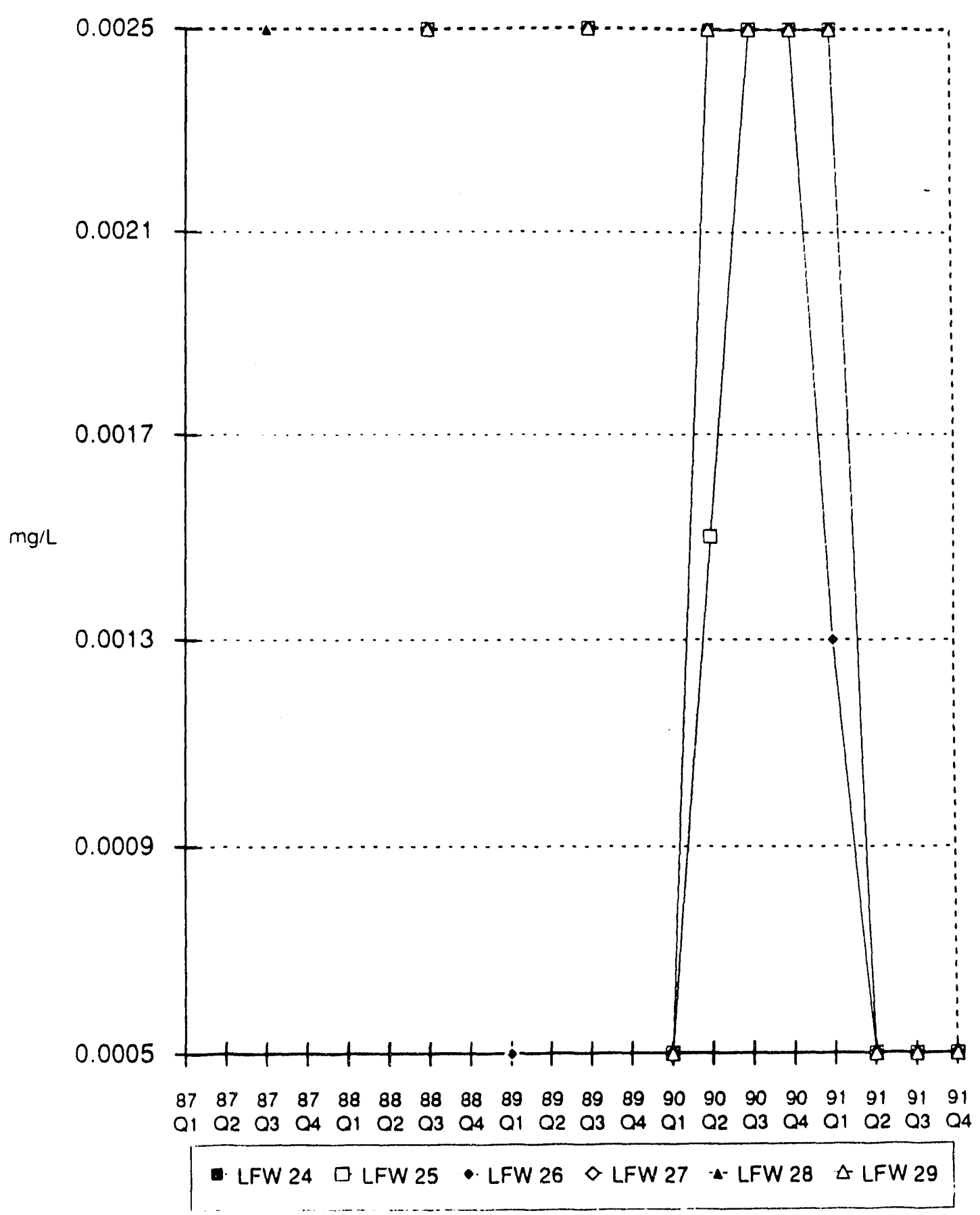




\section{1,1,2-Trichloroethane in the LFW-Series Monitoring Wells}

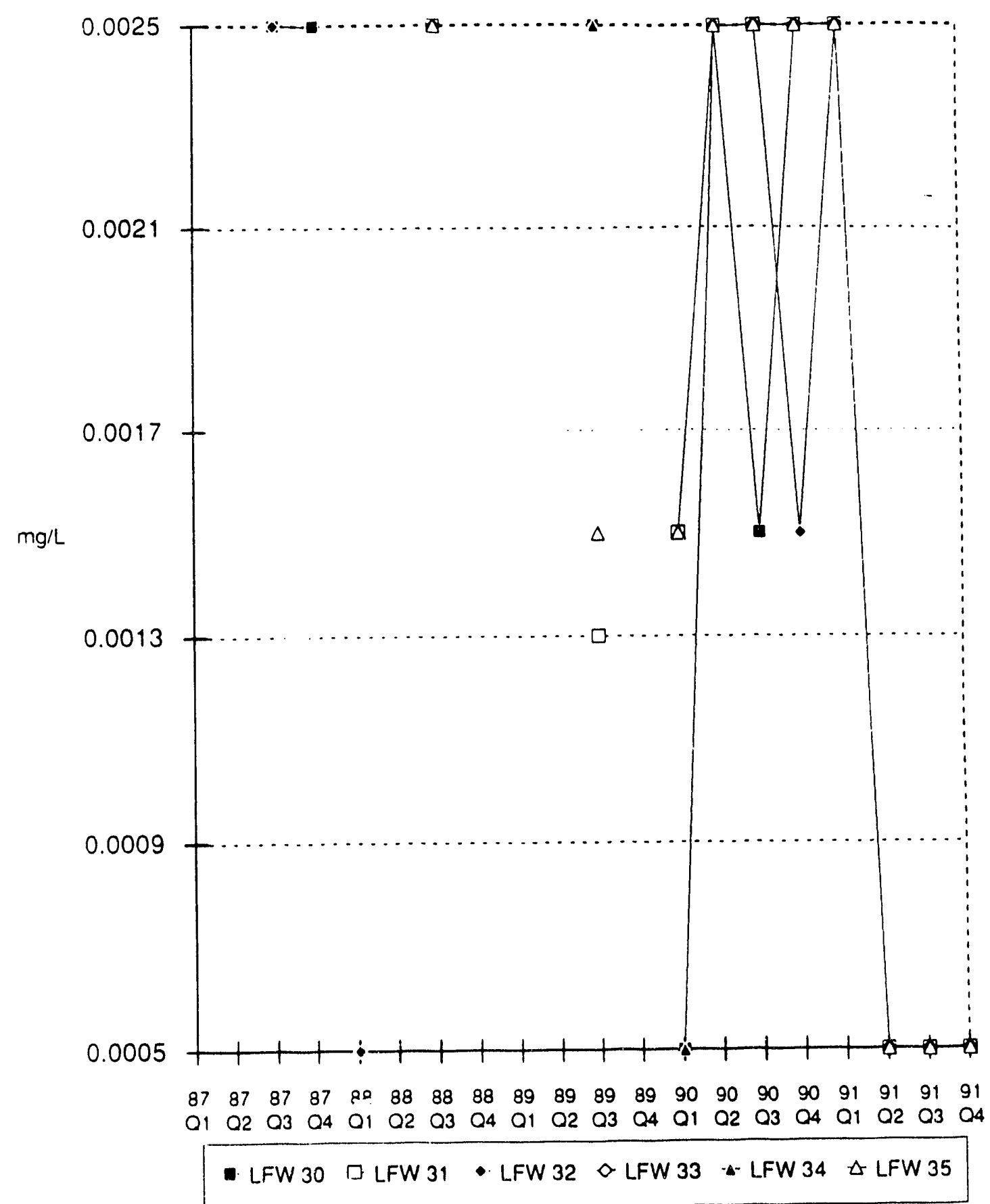




\section{1,1,2-Trichloroethane in the LFW-Series Monitoring Wells}

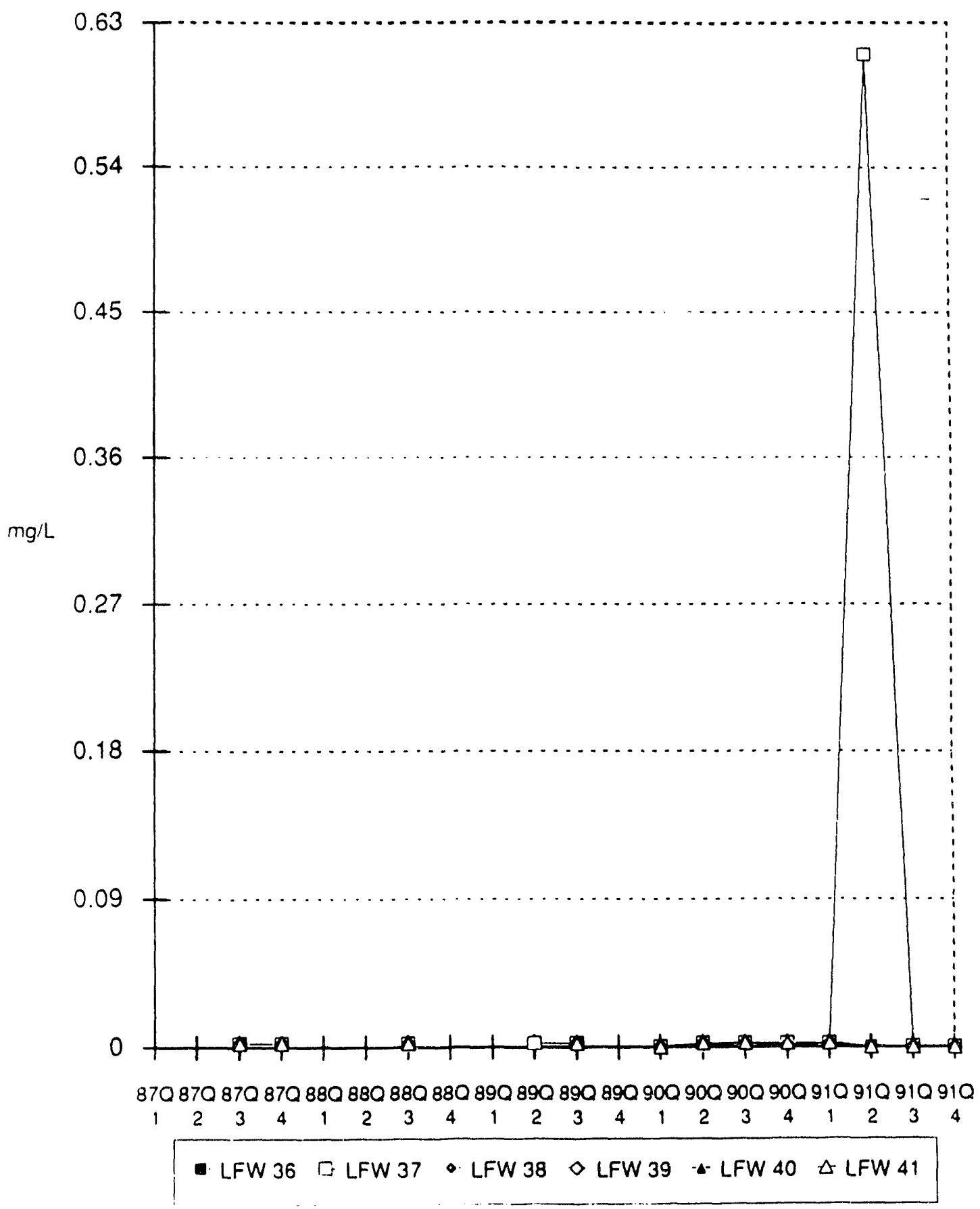


1,1,2-Trichloroethane in the LFW-Series Monitoring Wells

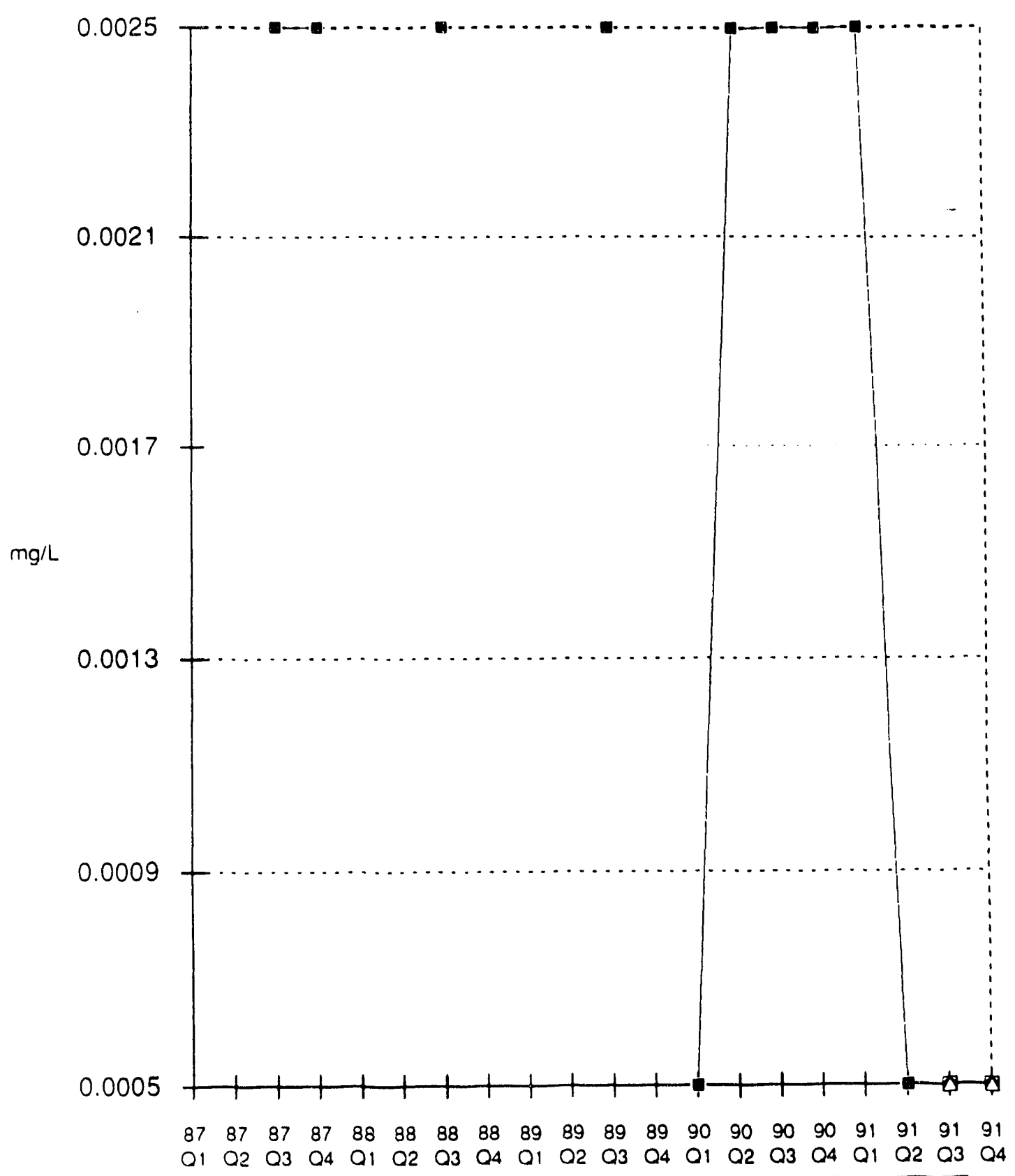

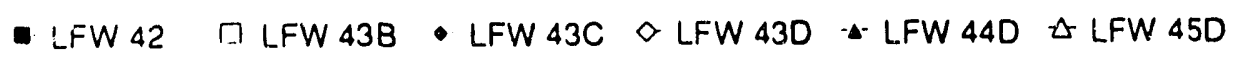


$1,1,2$-Trichloroethane in the LFW-Series Monitoring Wells

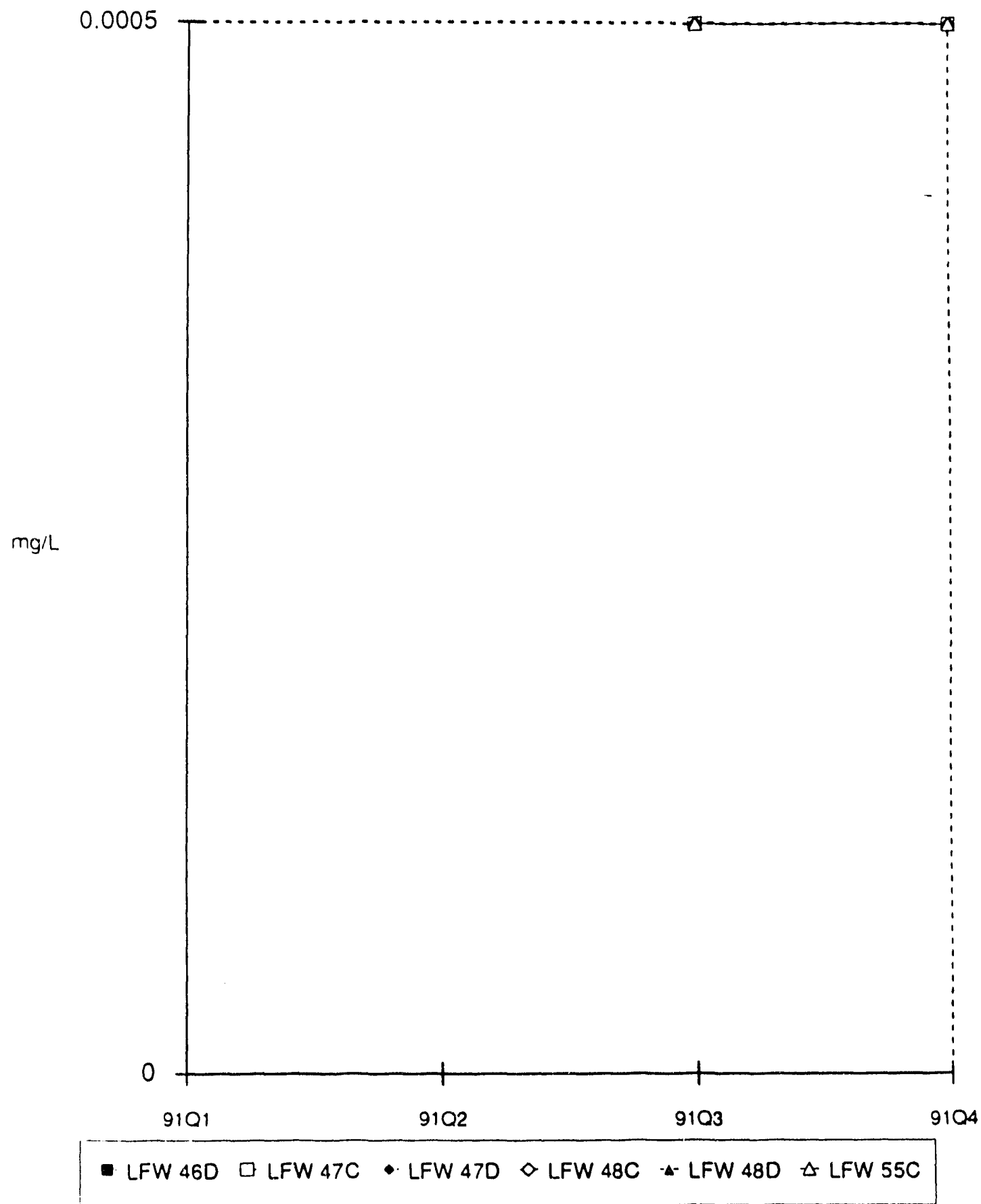




\section{1,1,2-Trichloroethane in the LFW-Series Monitoring Wells}

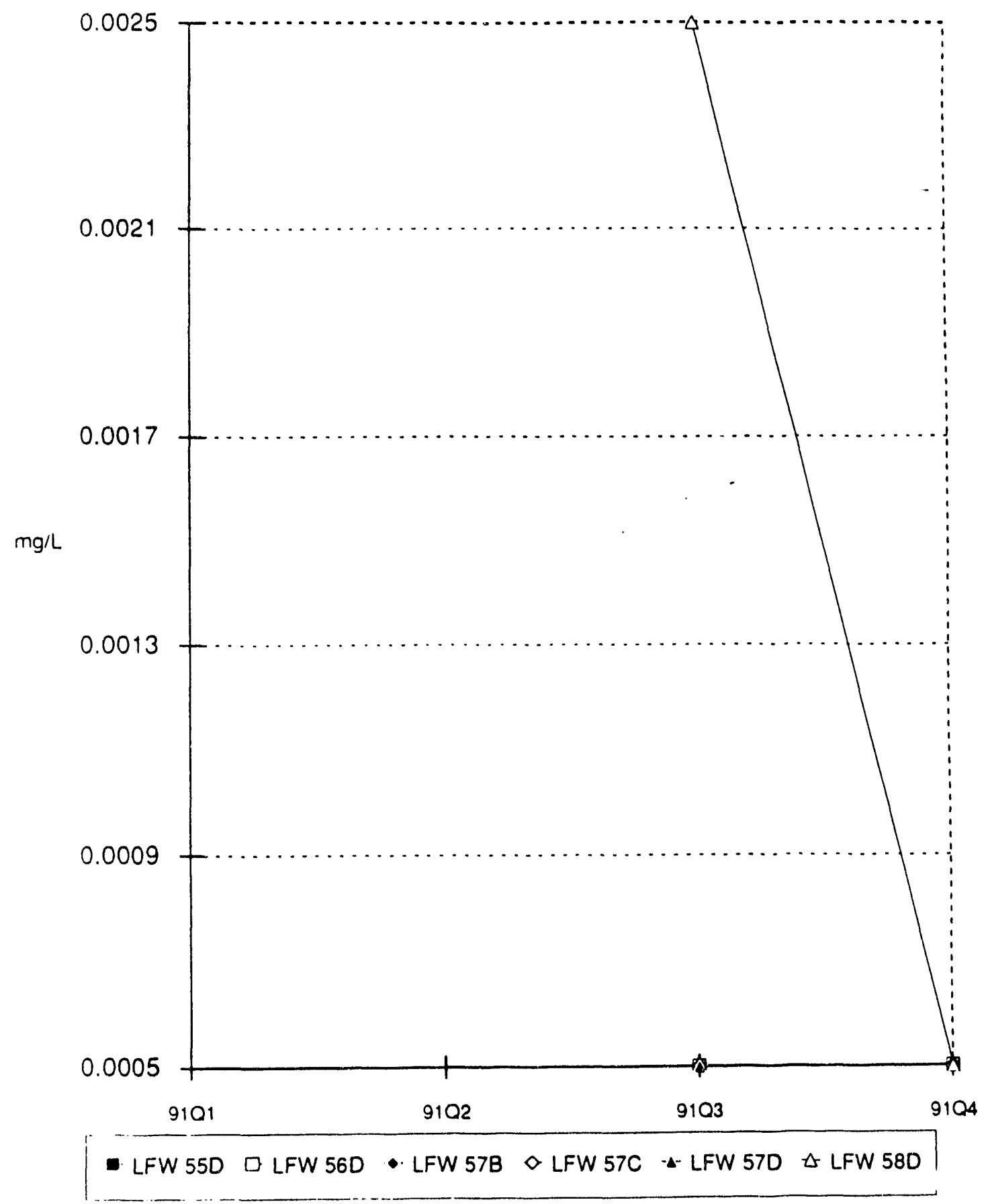




\section{1,1,2-Trichloroethane in the LFW-Serles Monitoring Wells}

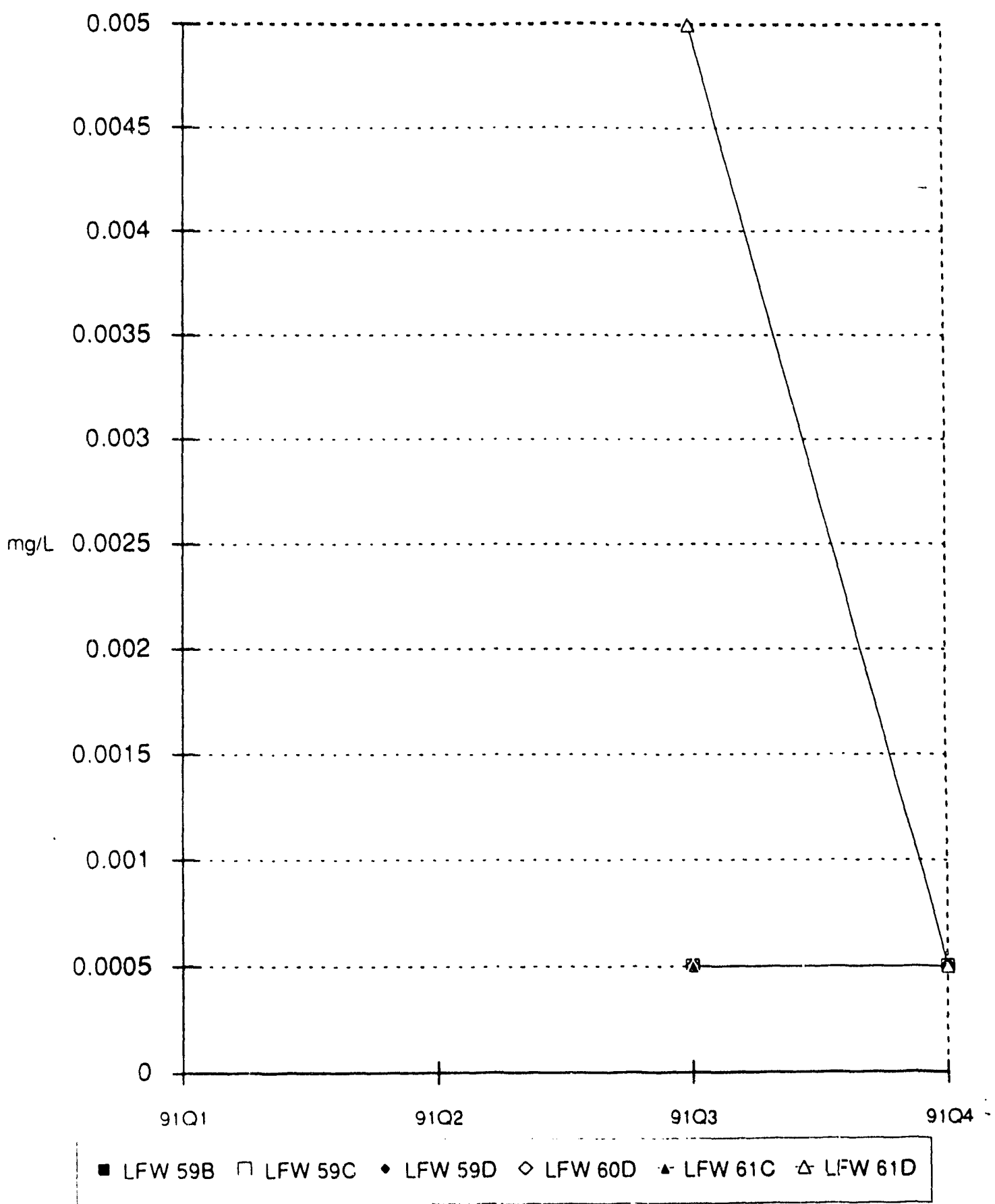


1,1,2-Trichloroethane in the LFW-Series Monitoring Wells

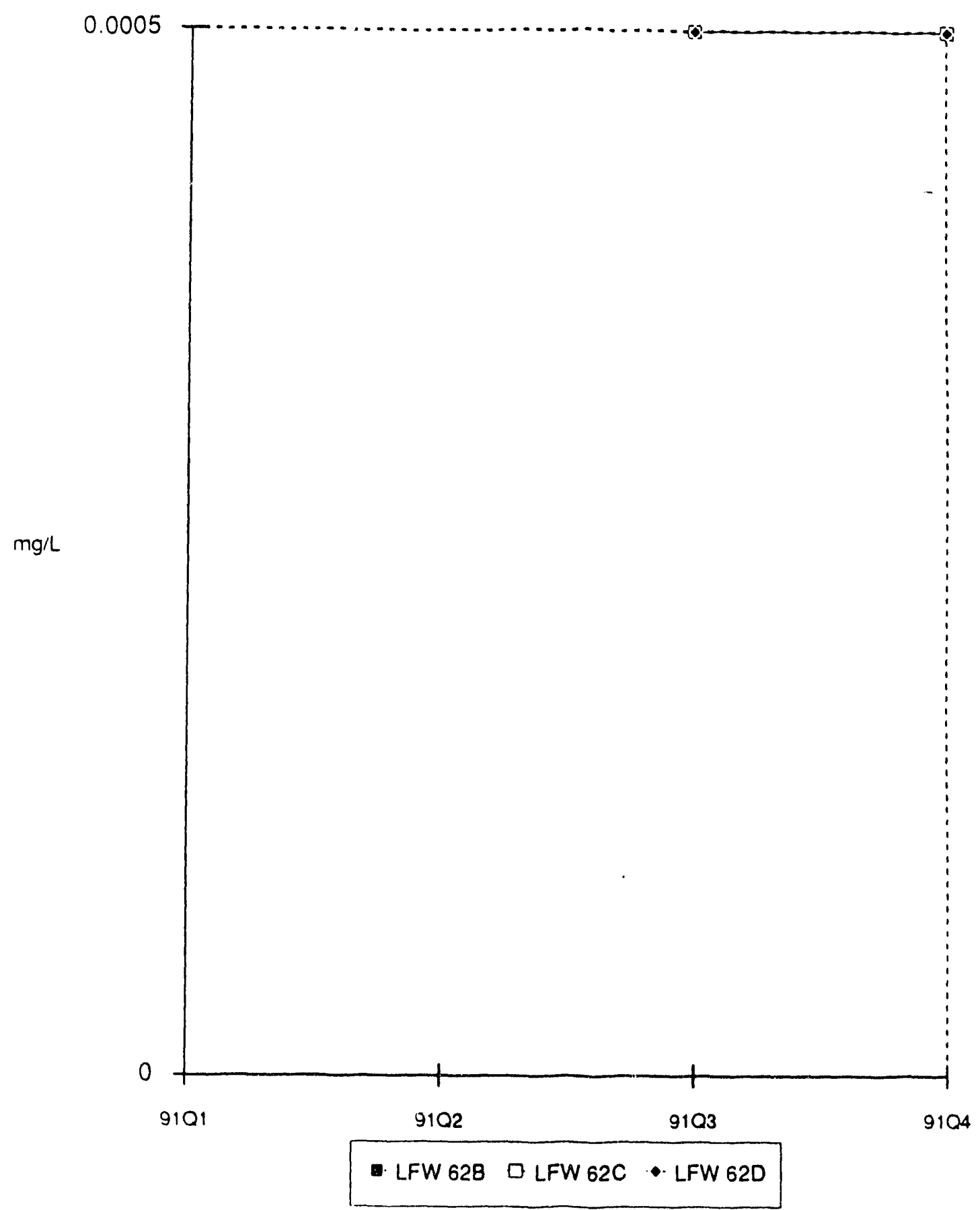




\section{1,1-Dichloroethylene in the LFW-Series Monitoring Wells}

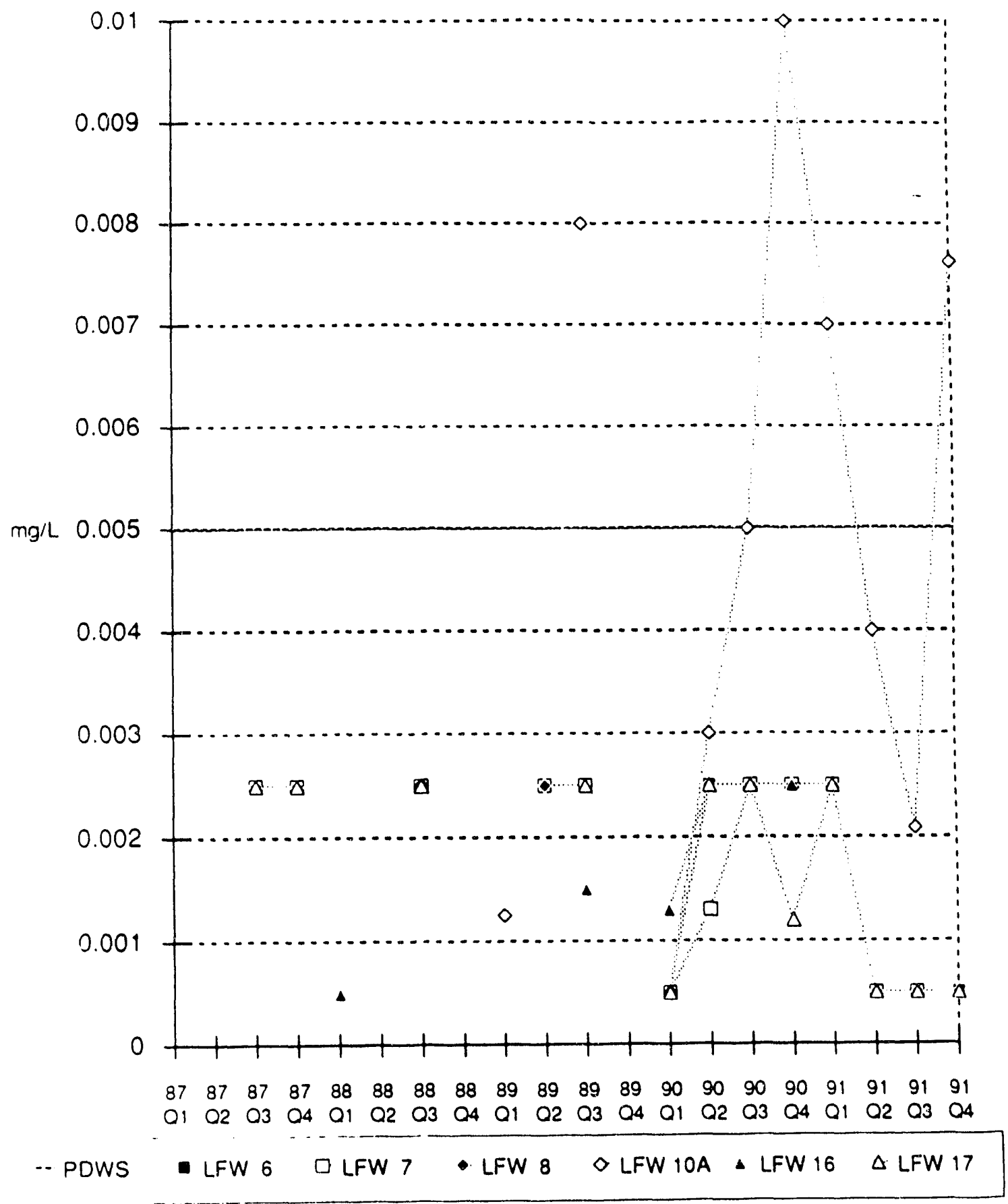


1,1-Dichlorc-thylene in the LFW-Series Monitoring Wells

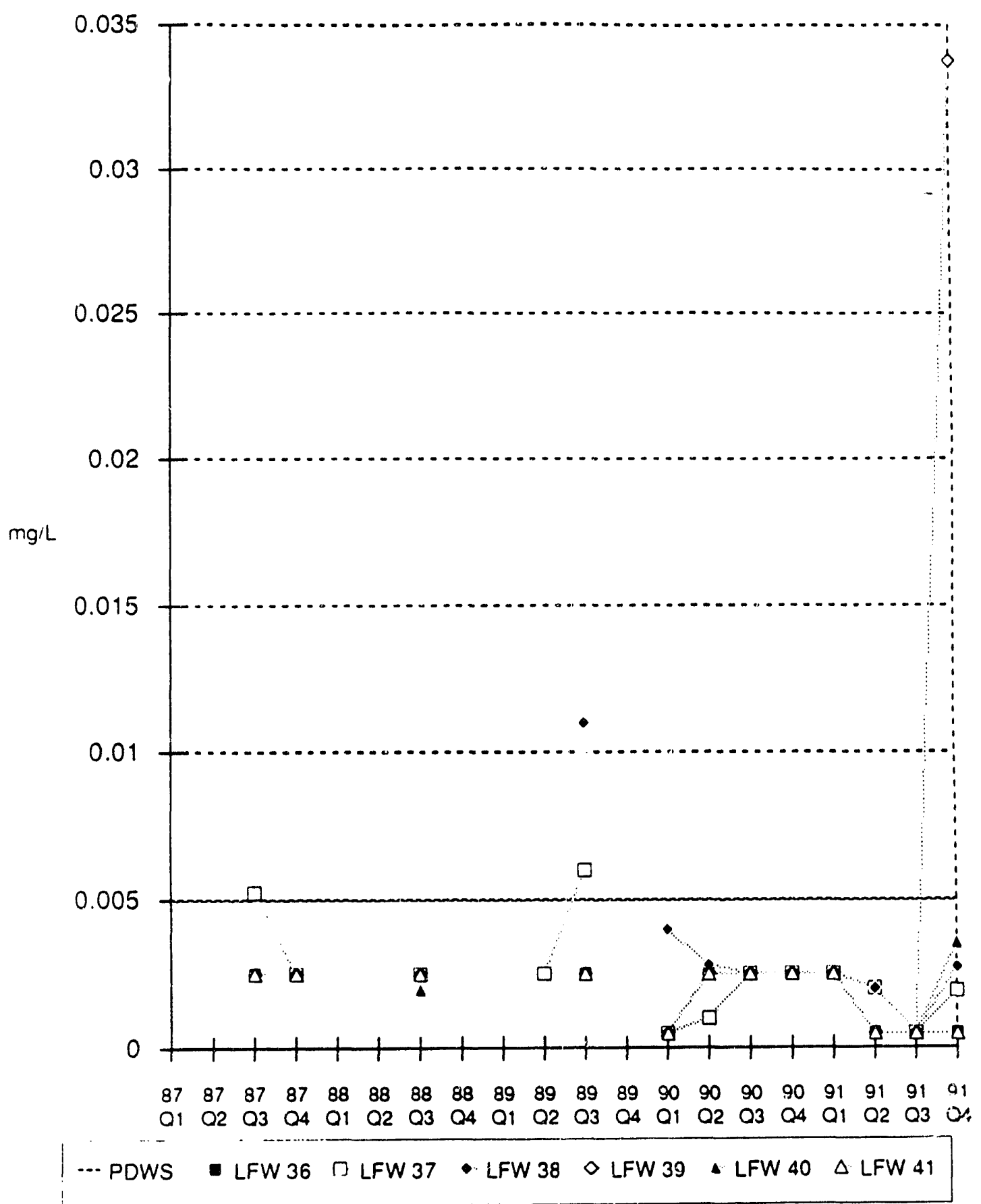


1,1-Dichloroethylene in the LFW-Series Monitoring Wells

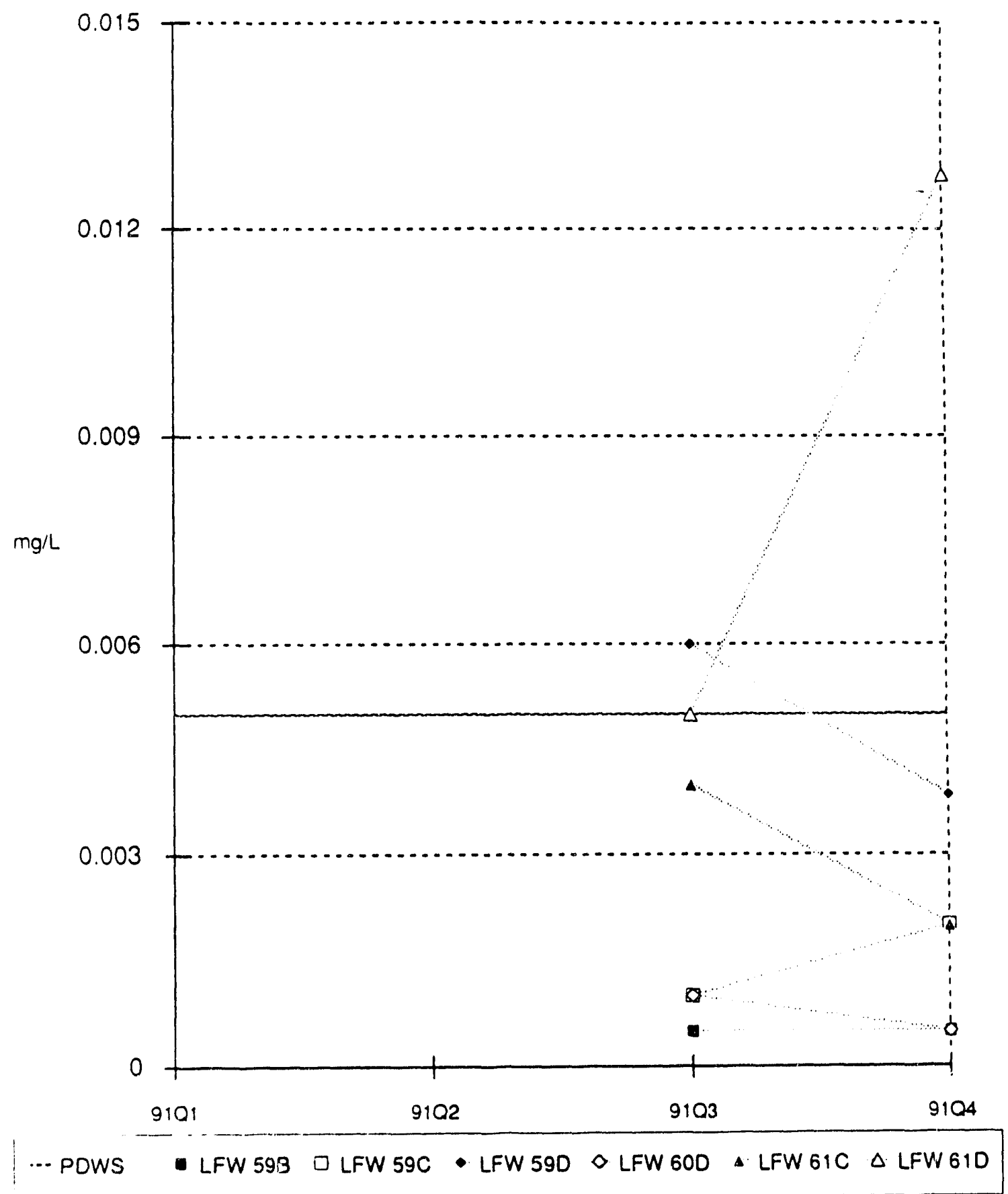


1,1-Dichloroethylene in the LFW-Series Monitoring Wells

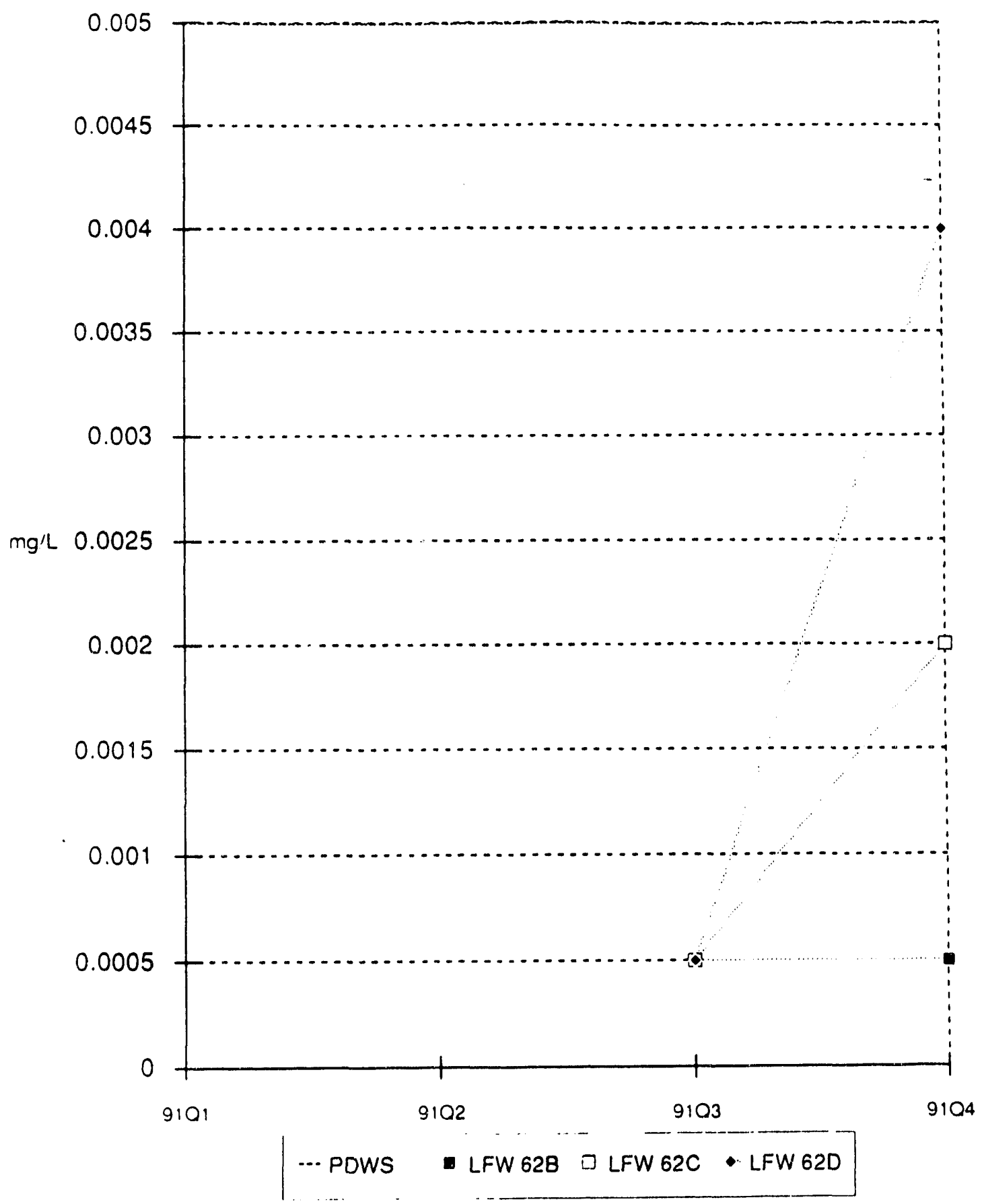


1,1-Dichloroethylene in the LFW-Series Monitoring Wells

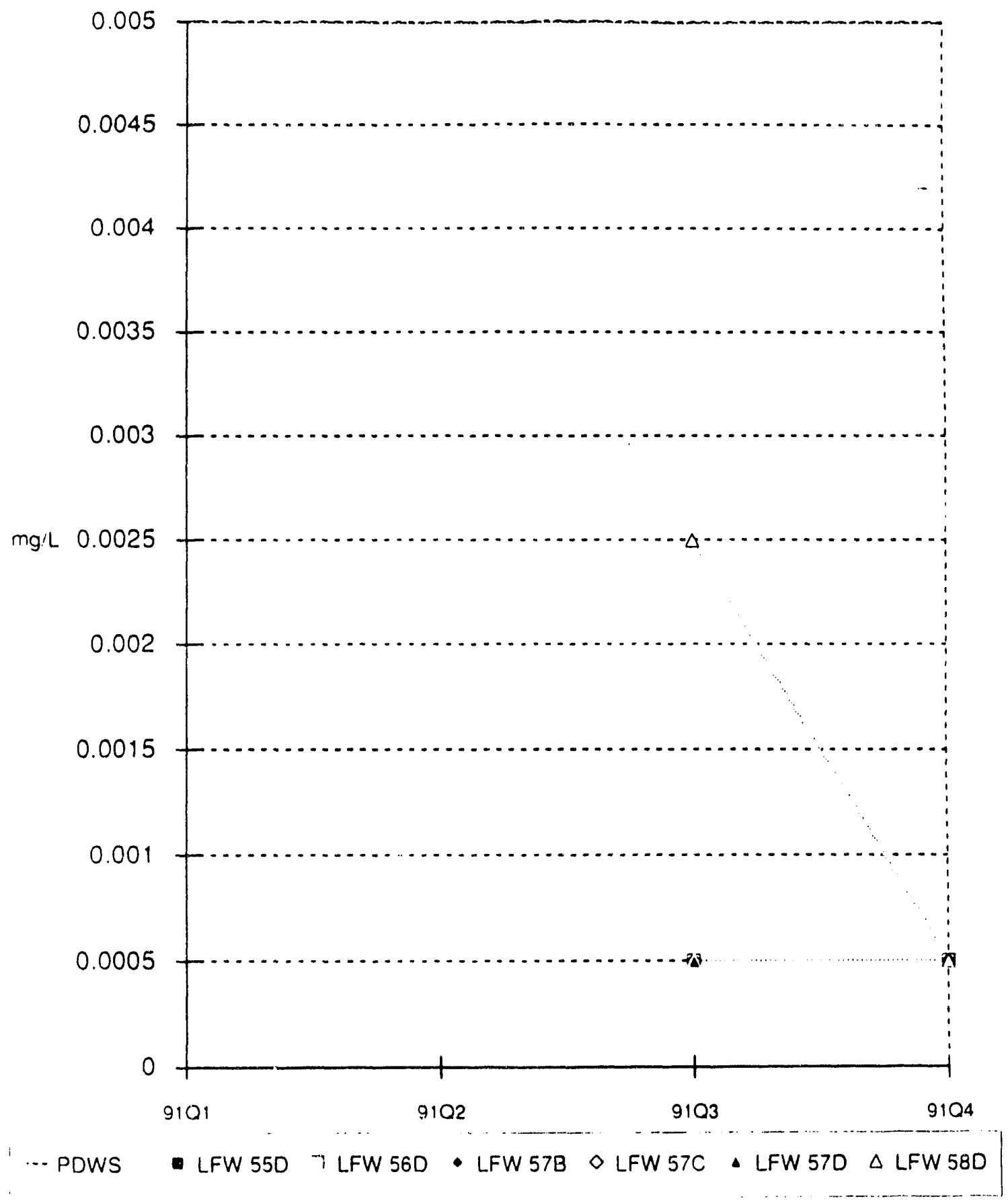


1,1-Dlchloroethylene in the LFW-Series Monltoring Wells

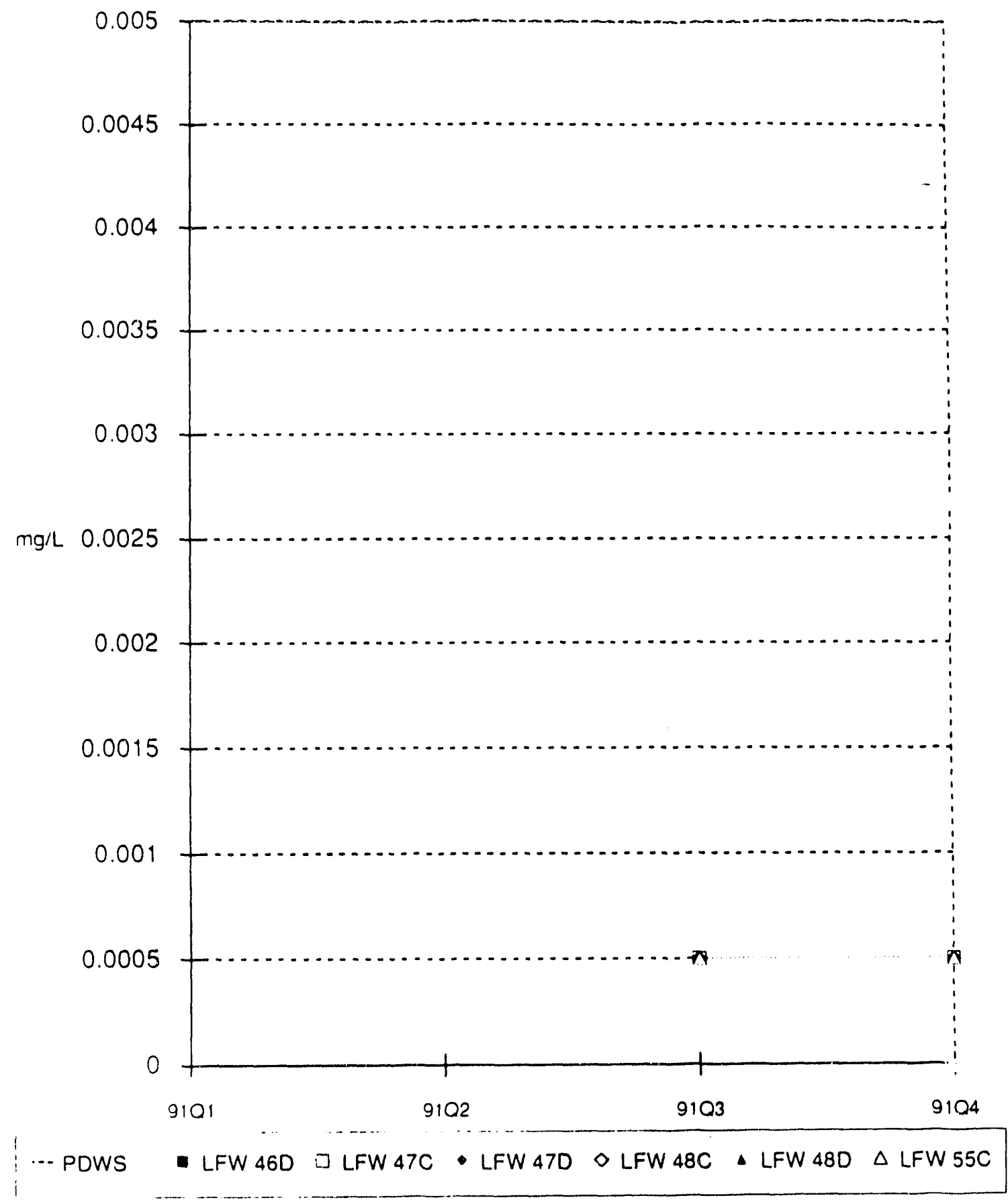


1,1-Dichloroethylene in the LFW-Series Monitoring Wells

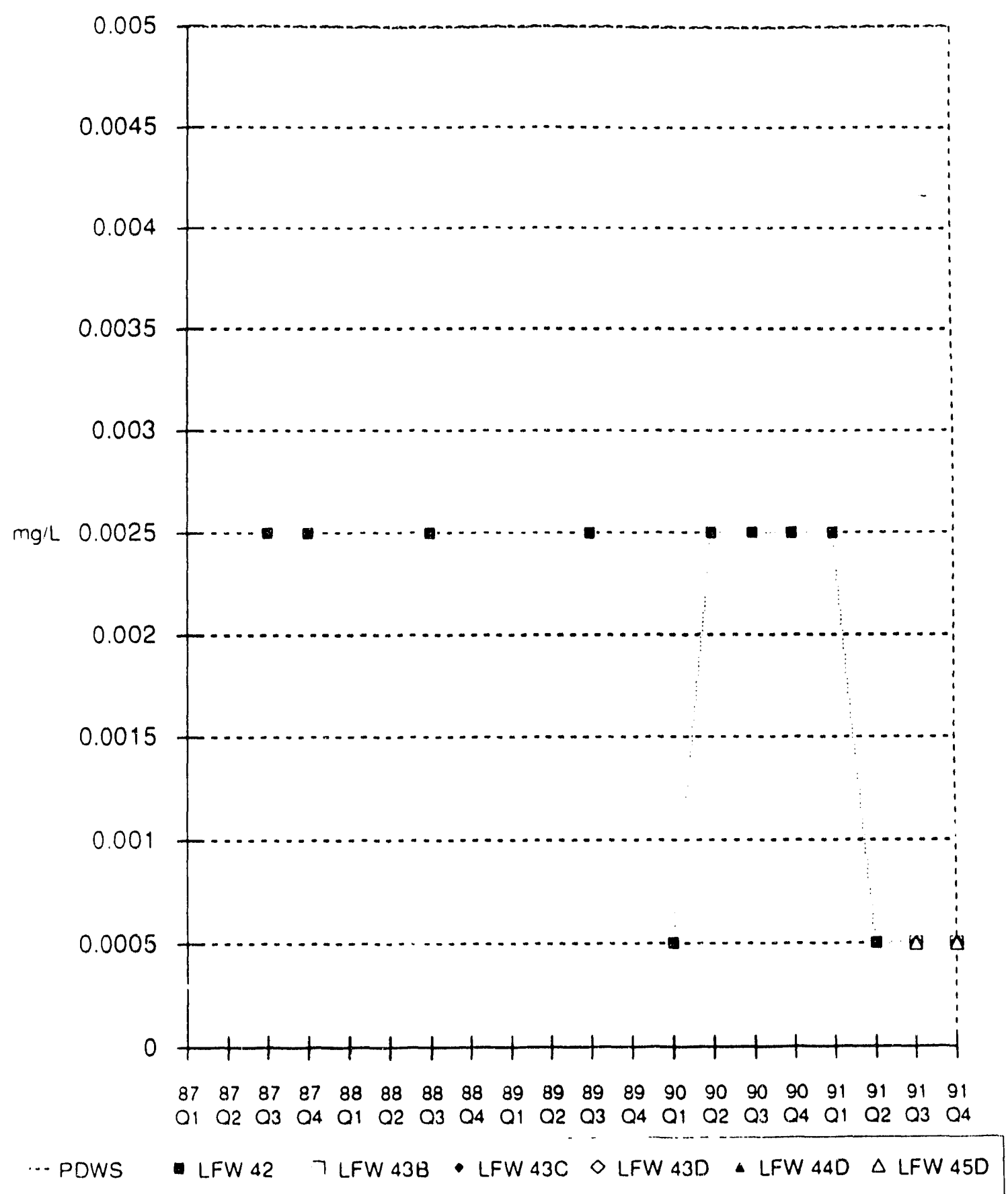


1,1-Dichloroethylene in the LFW-Series Monitoring Wells

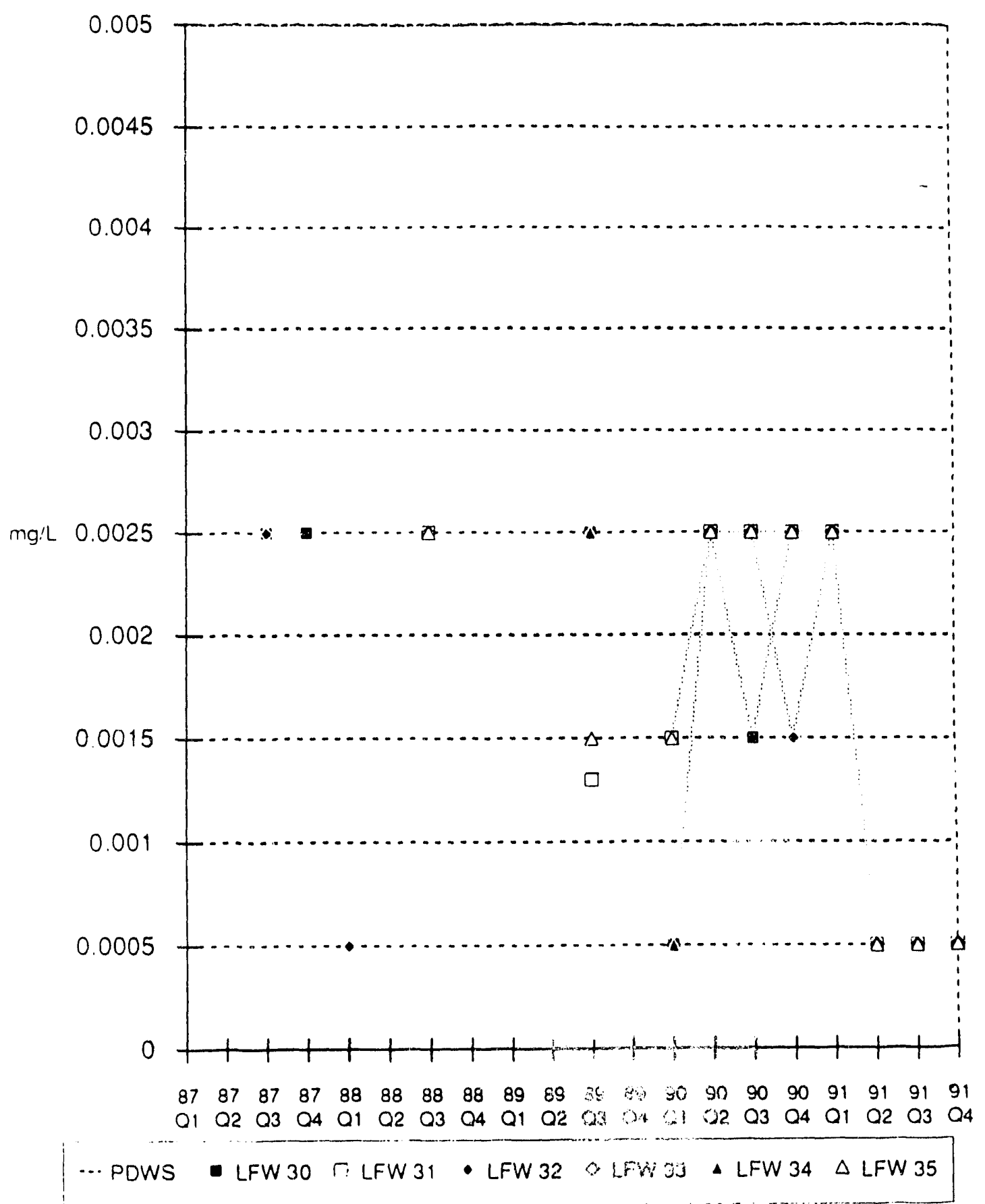




\section{1,1-Dichloroethylene in the LFW-Series Monitoring Wells}

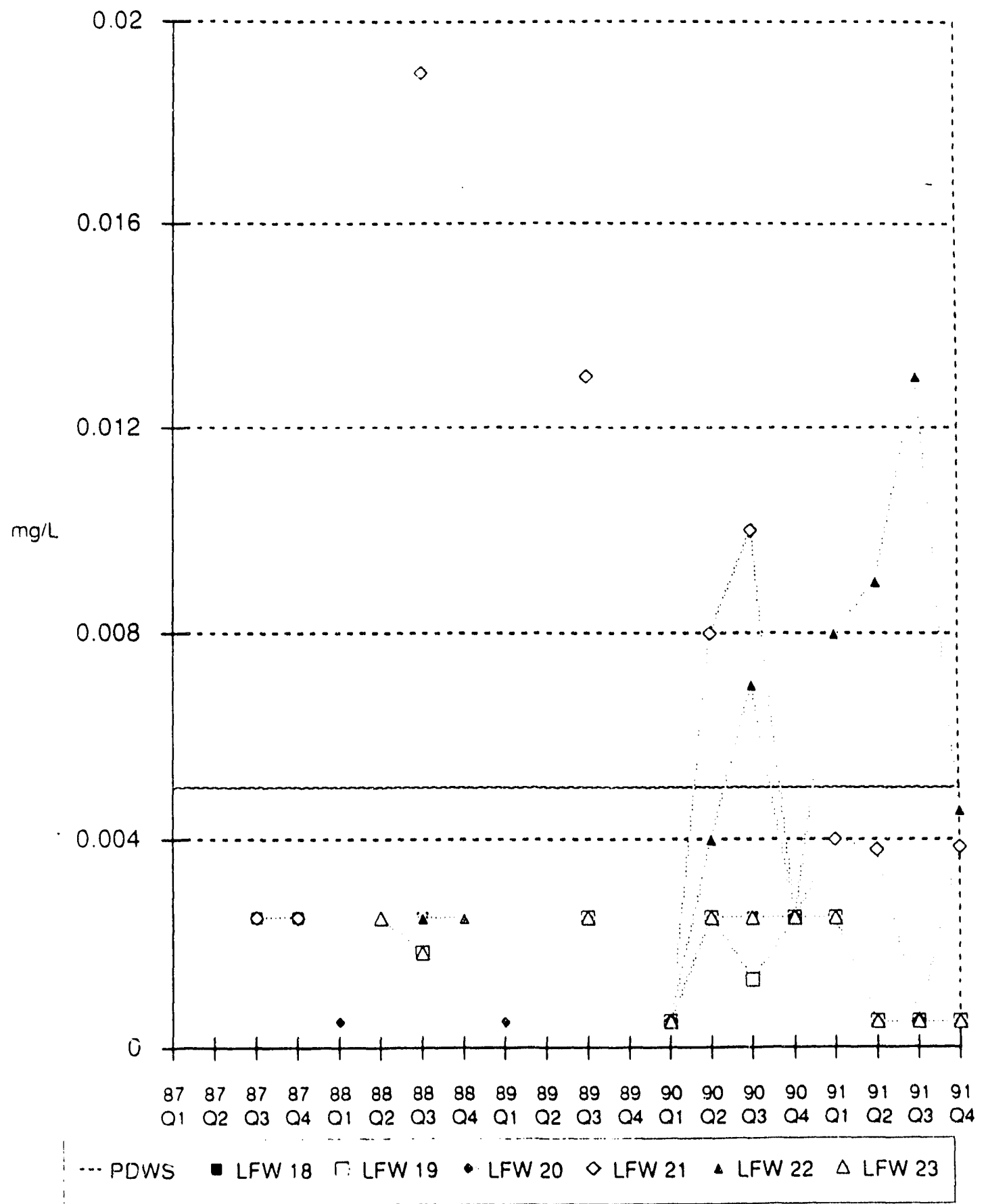


1,1-Dichloroethylene in the LFW-Series Monitoring Wells

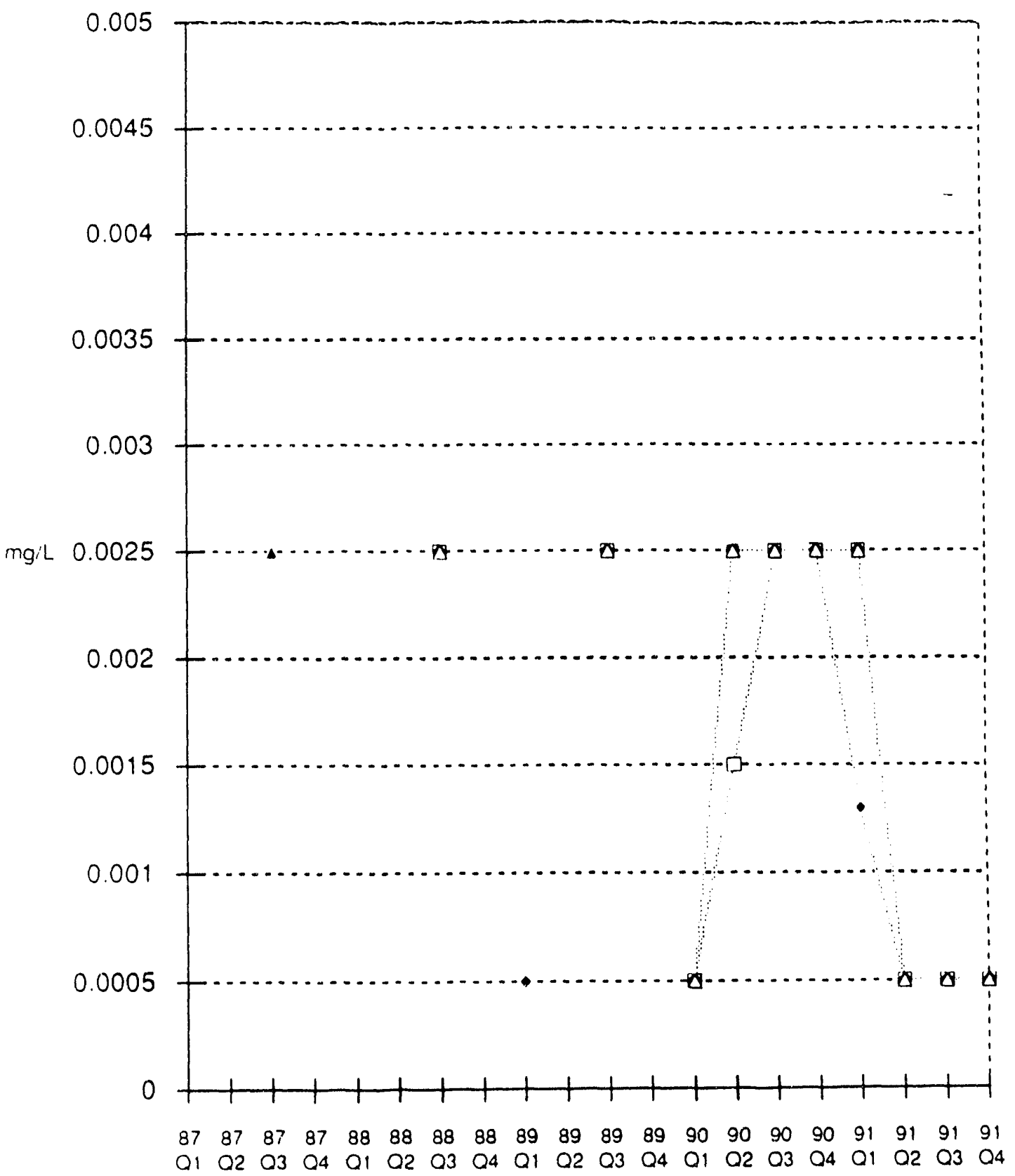

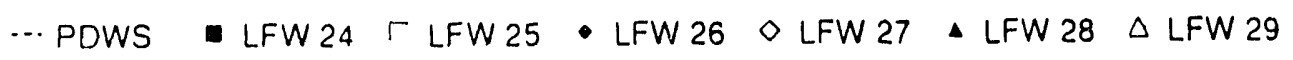


1,2-Dichloroethane in the LFW-Series Monitoring Wells

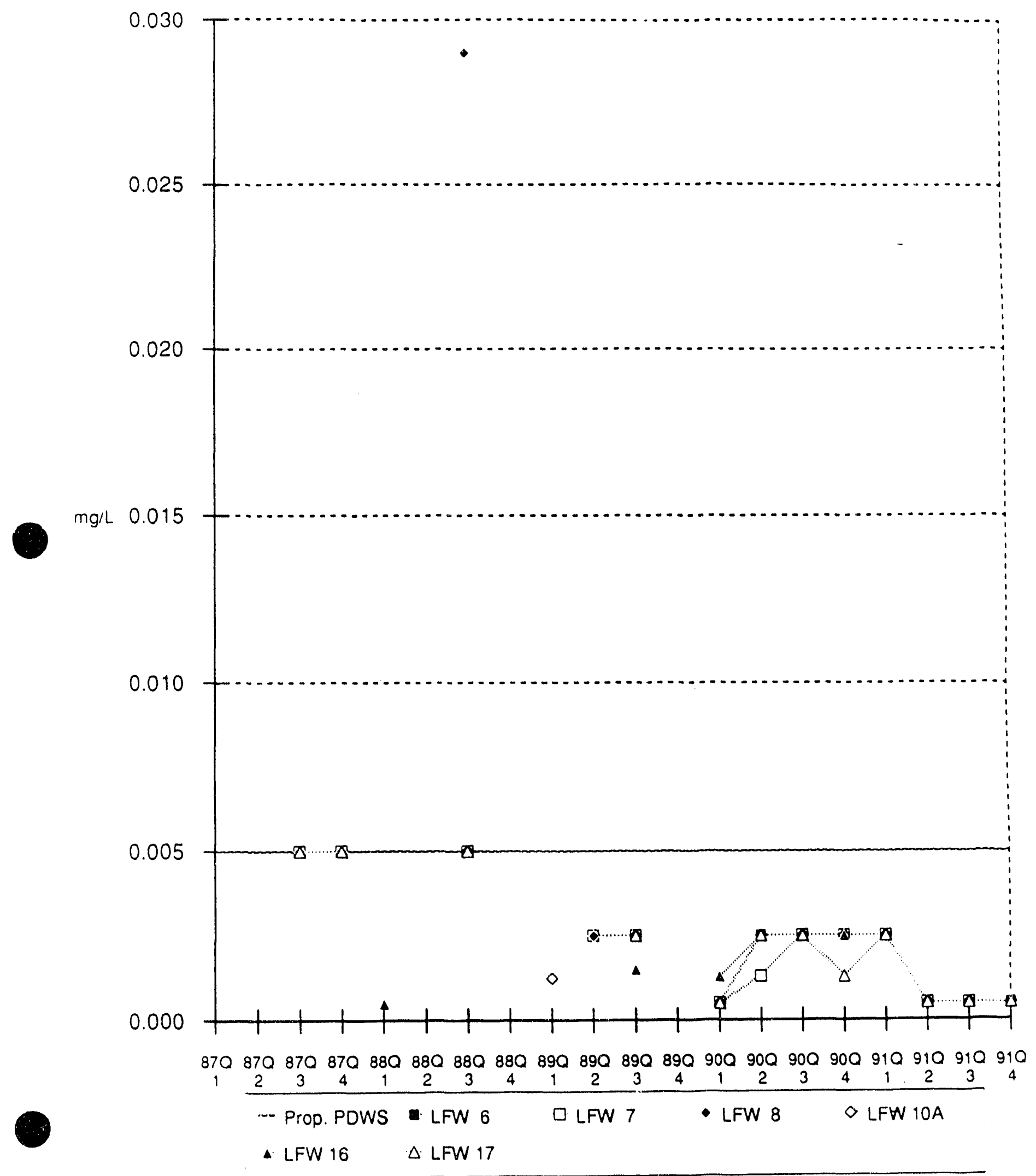


1,2-Dichloroethane in the LFW-Series Monitoring Wells

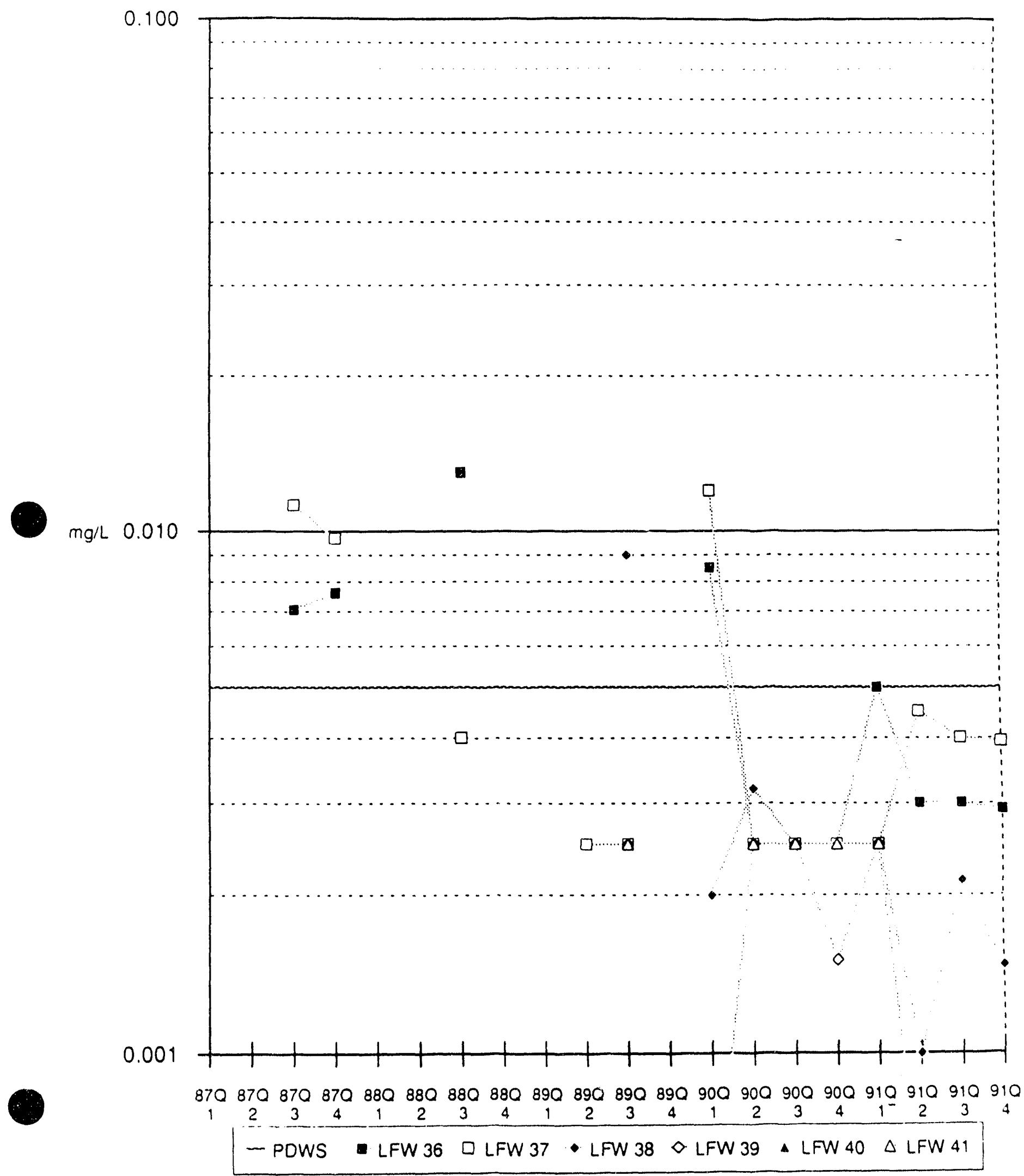


1,2-Dichloropropane in the LFW-Series Monitoring Wells

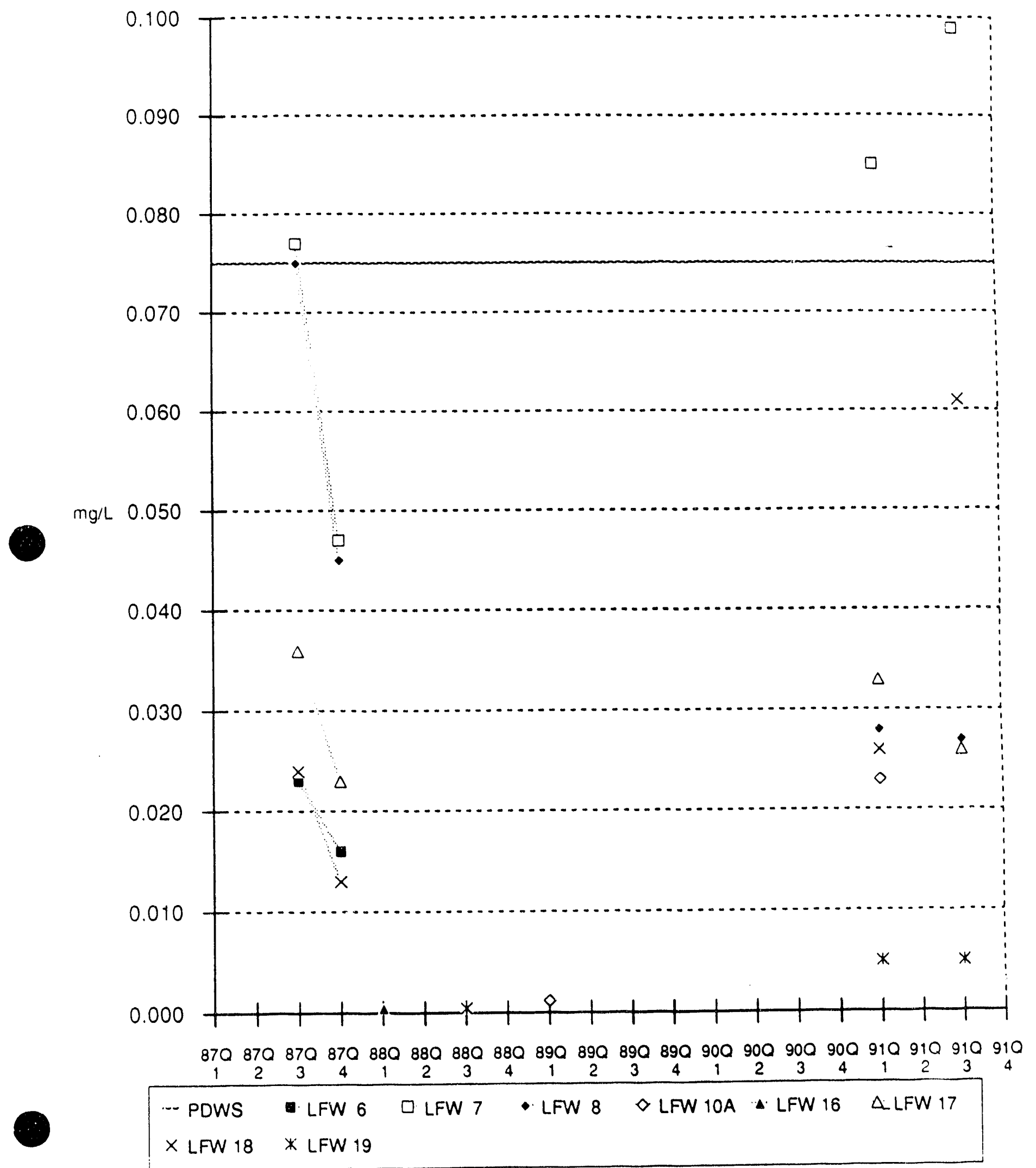


1,2-Dichloropropane in the LFW-Series Monitoring Wells

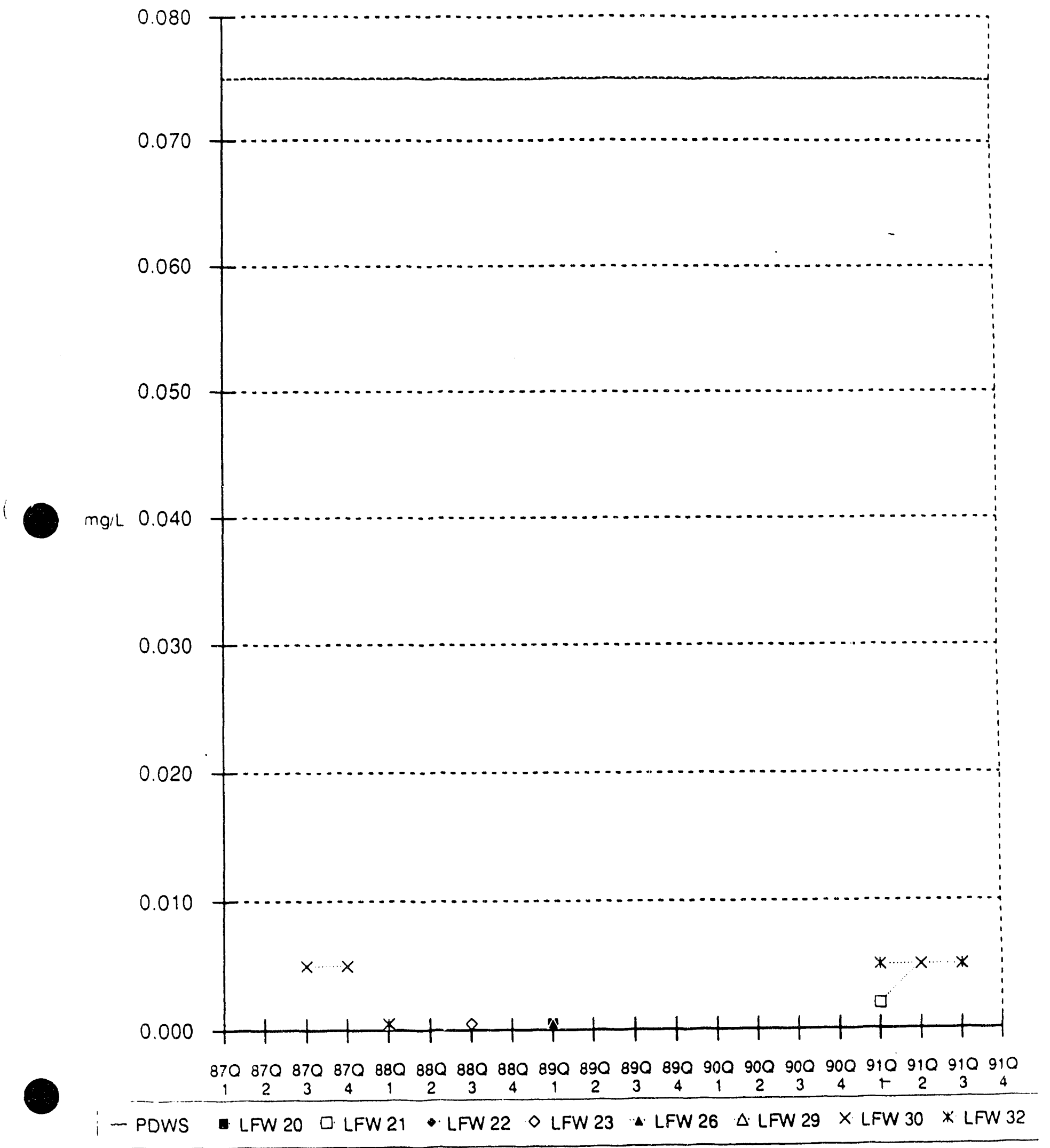


1,2-Dichloropropane in the LFW-Series Monitoring Wells

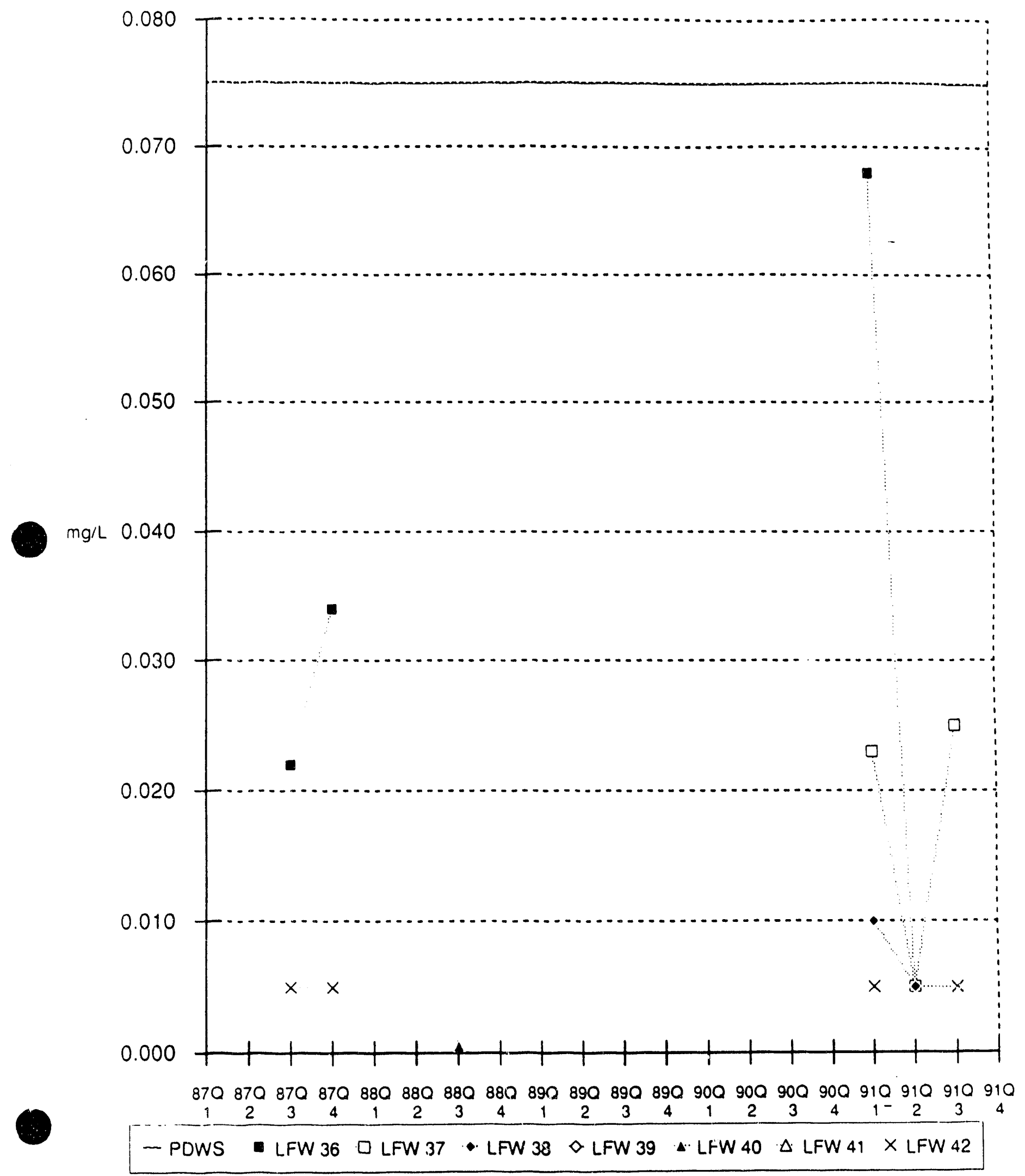




\section{1,4-Dichlorobenzene in the LFW-Series Monitoring Wells}

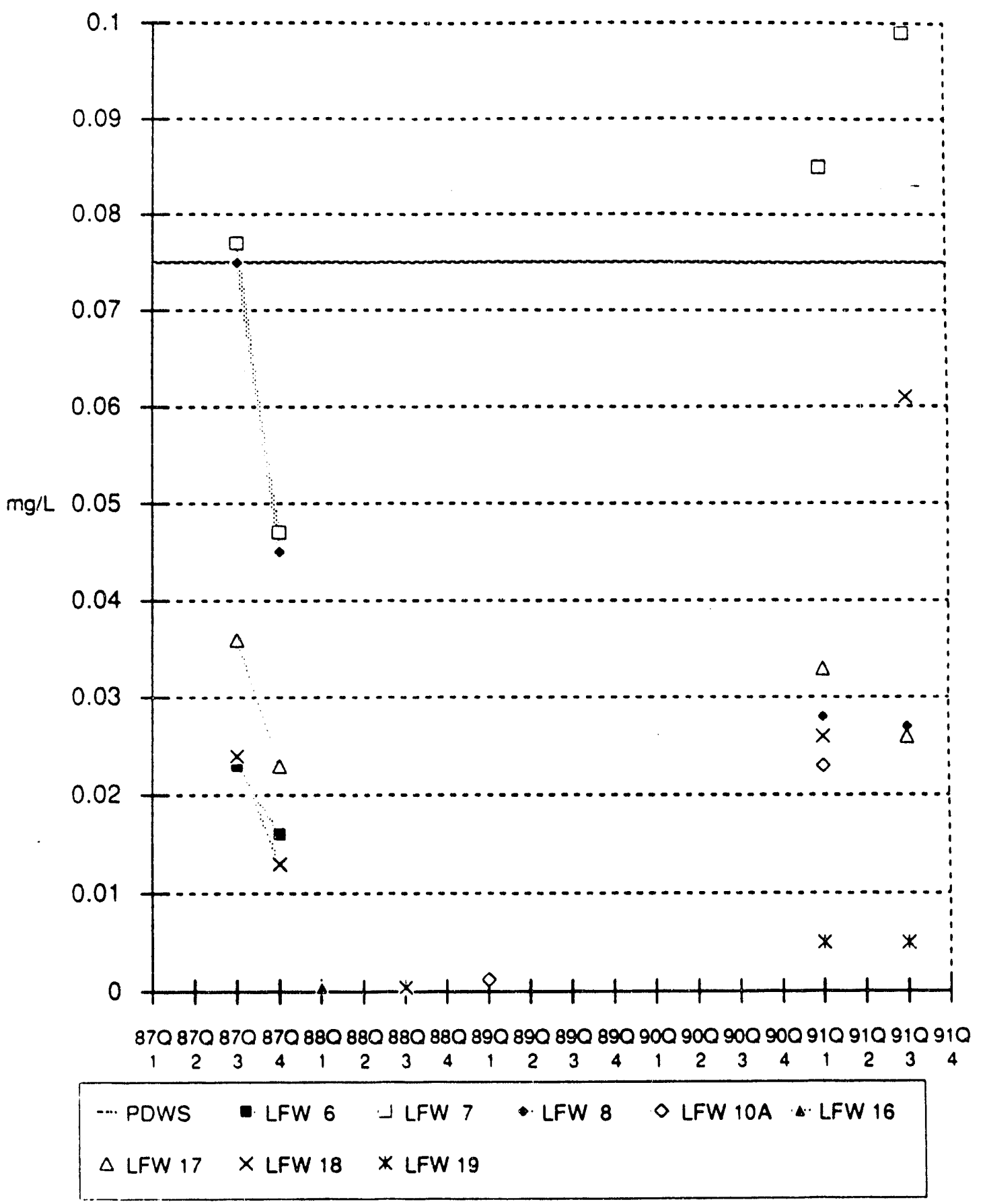


1,4-Dichlorobenzene in the LFW-Series Monitoring Wells

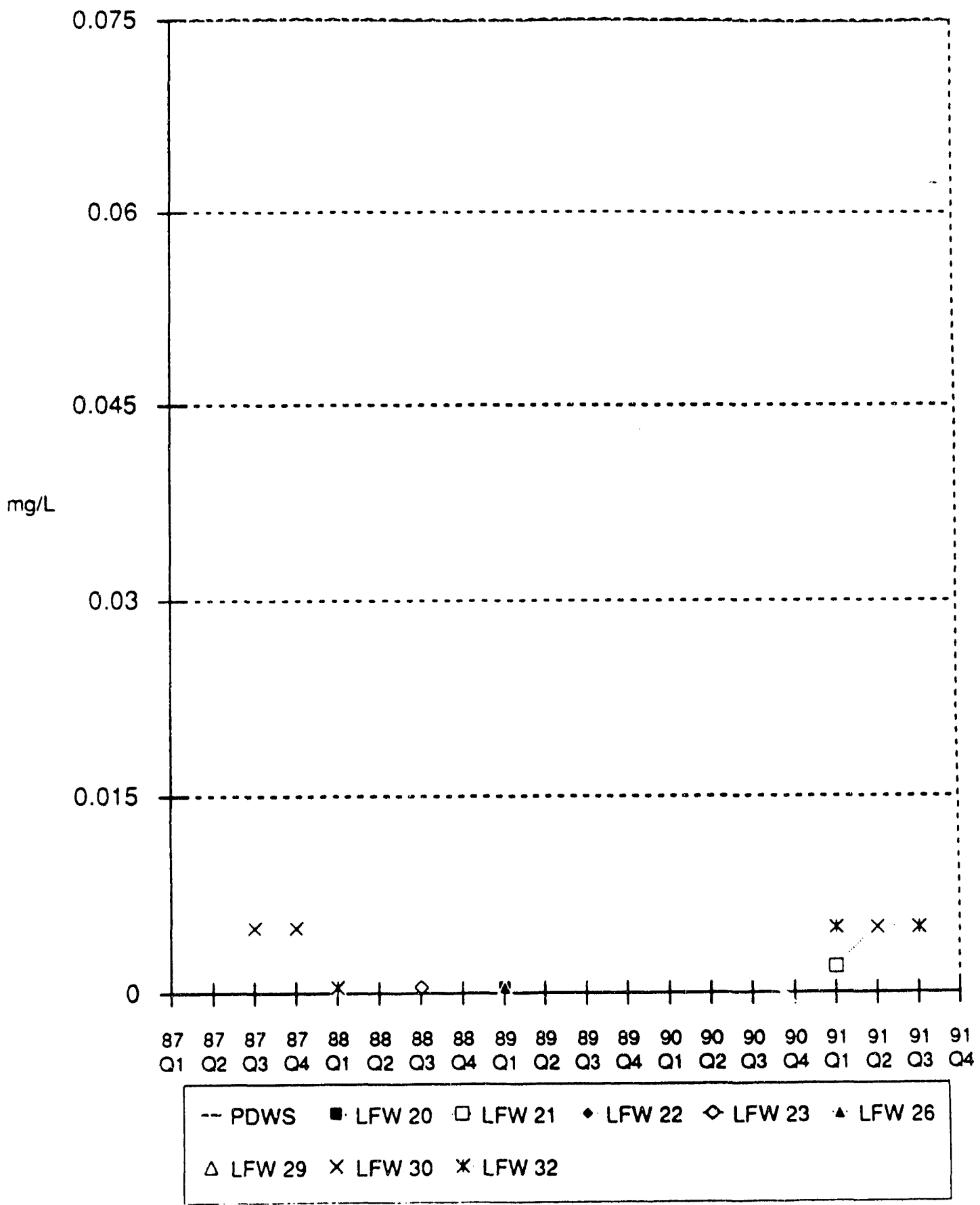


1,4-Dichlorobenzene in the LFW-Serles Monitoring Wells

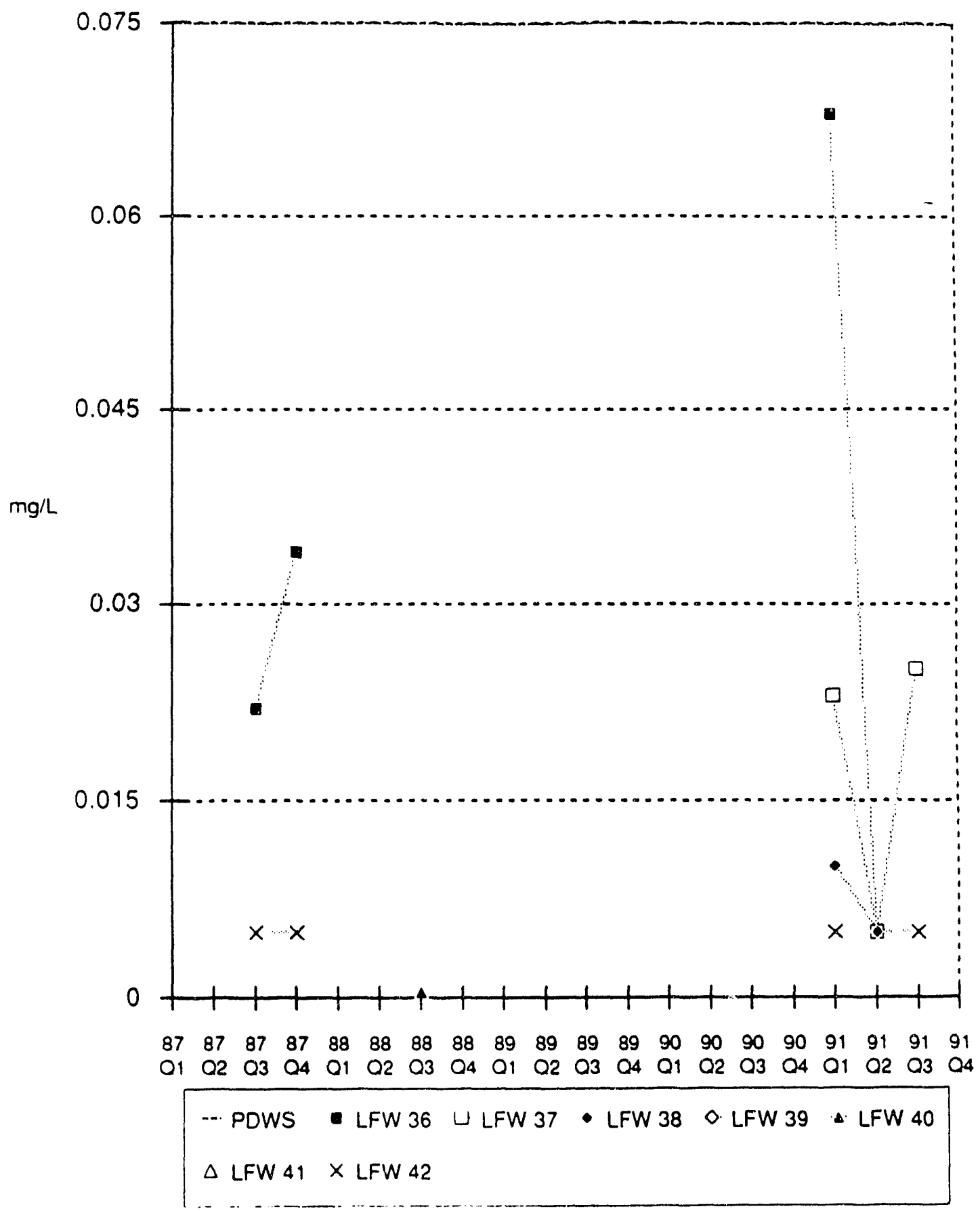


Tritium in the LFW-Series Monitoring Wells

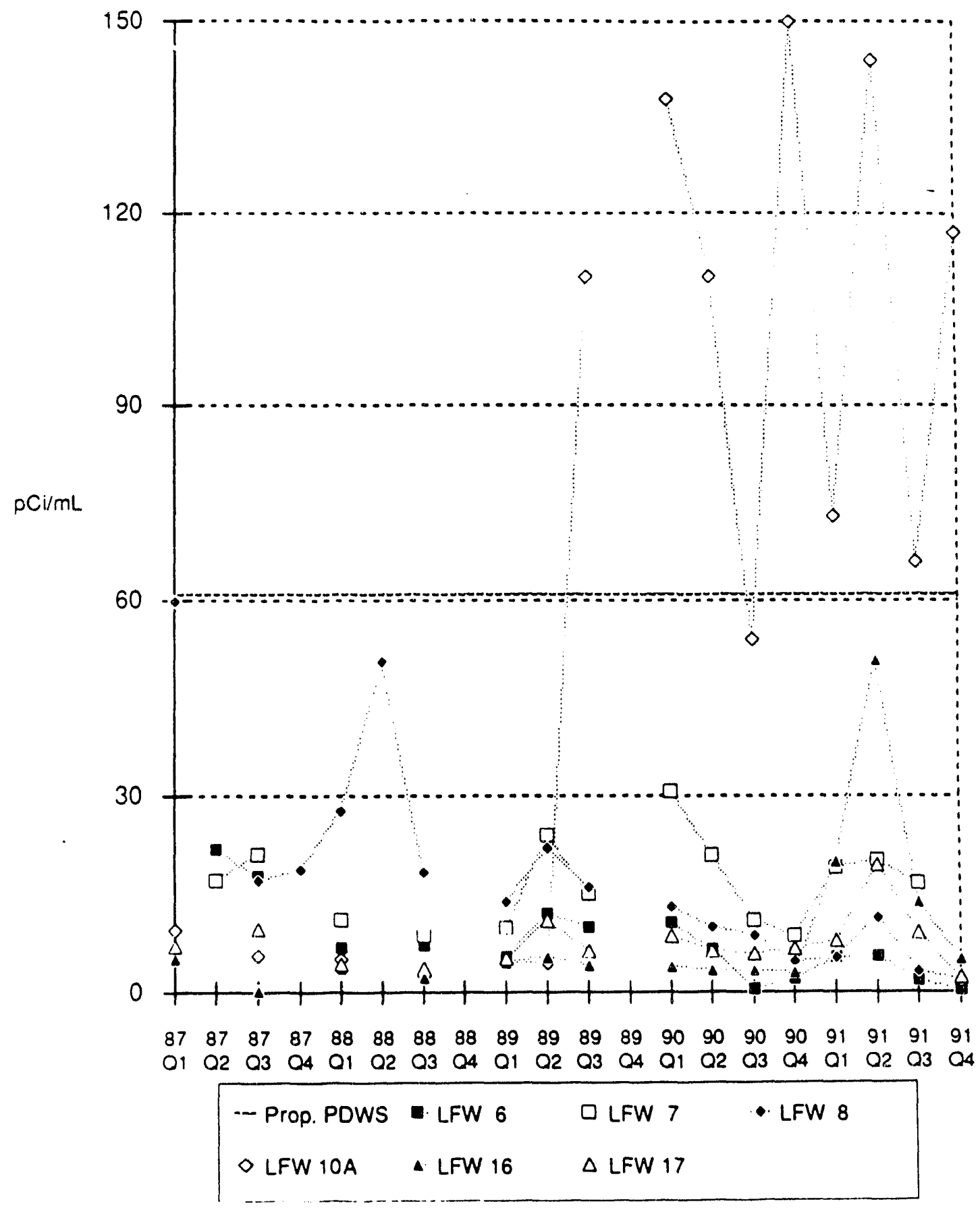


Tritium in the LFW-Series Monitoring Wells

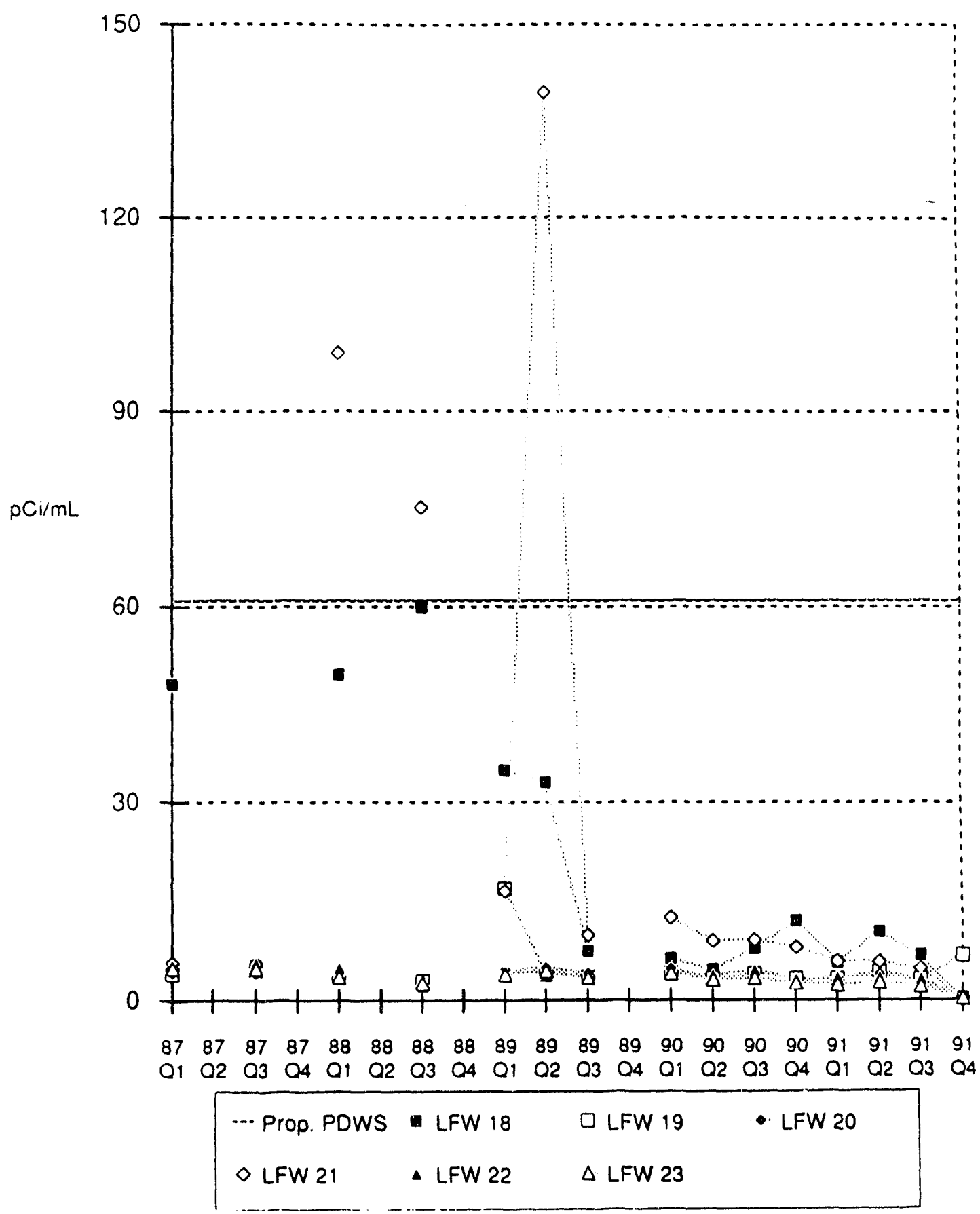


Tritium in the LFW-Serles Monitoring Wells

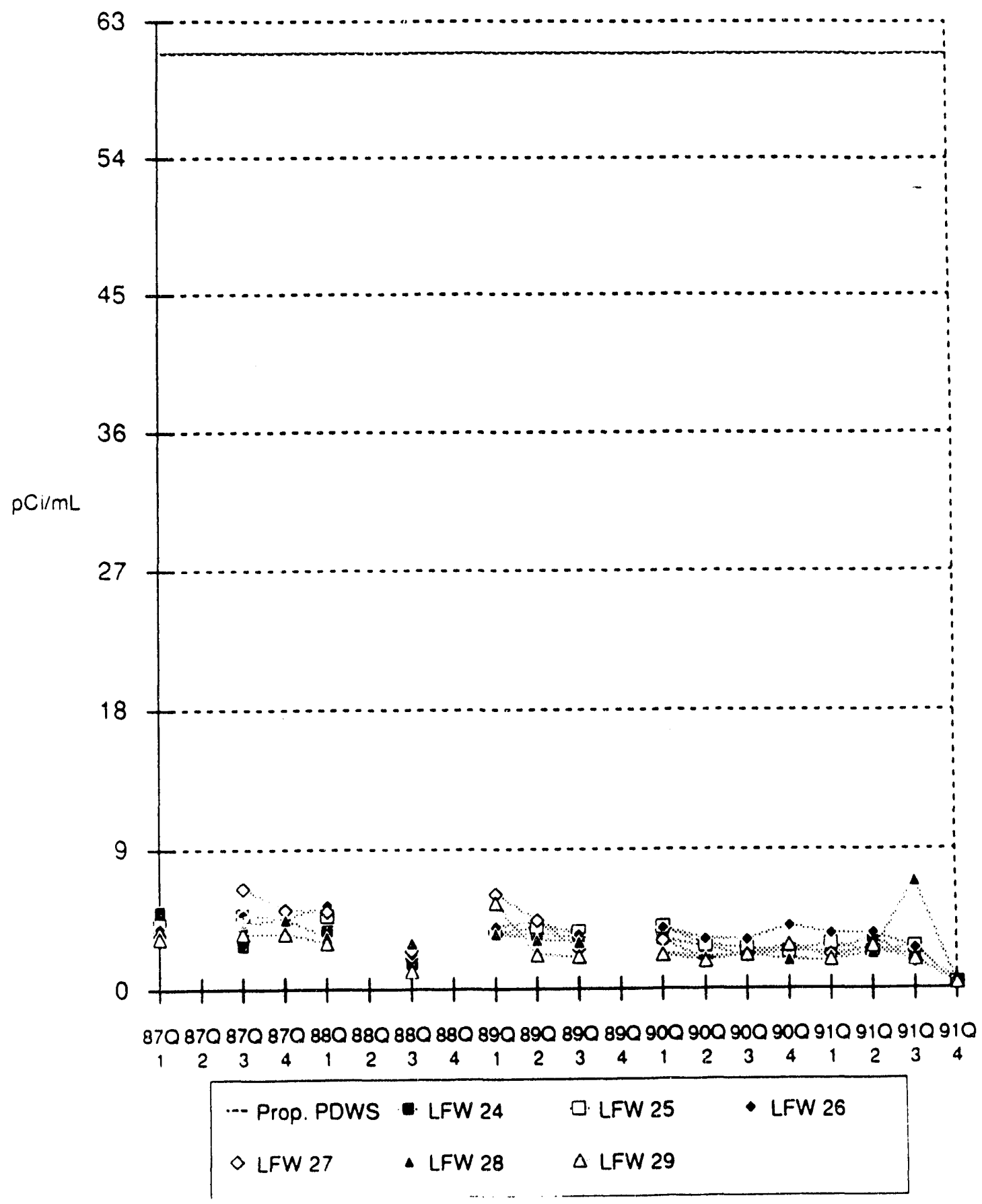


Tritium in the LFW-Series Monitoring Wells

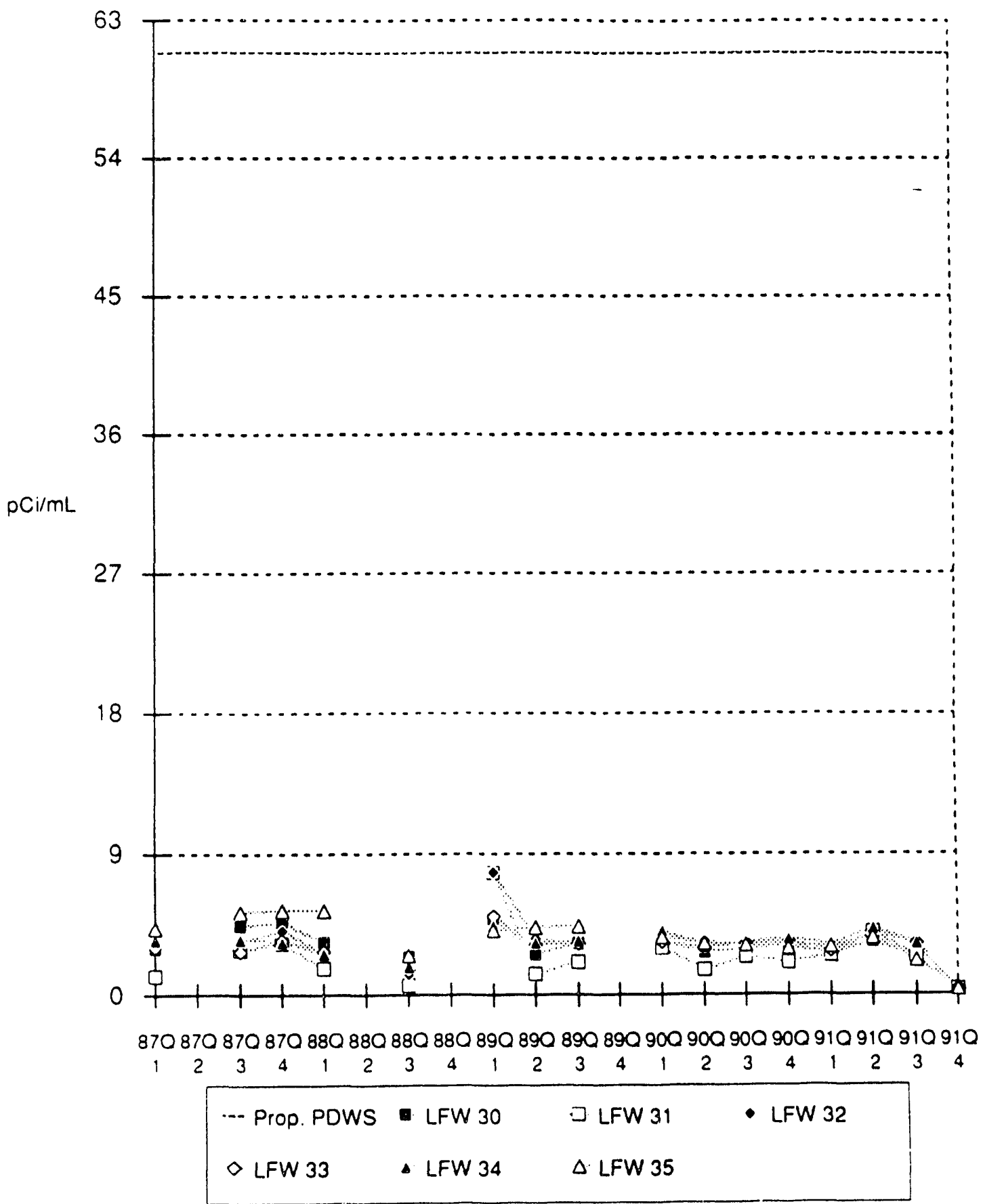


Tritium in the LFW-Series Monitoring Wells

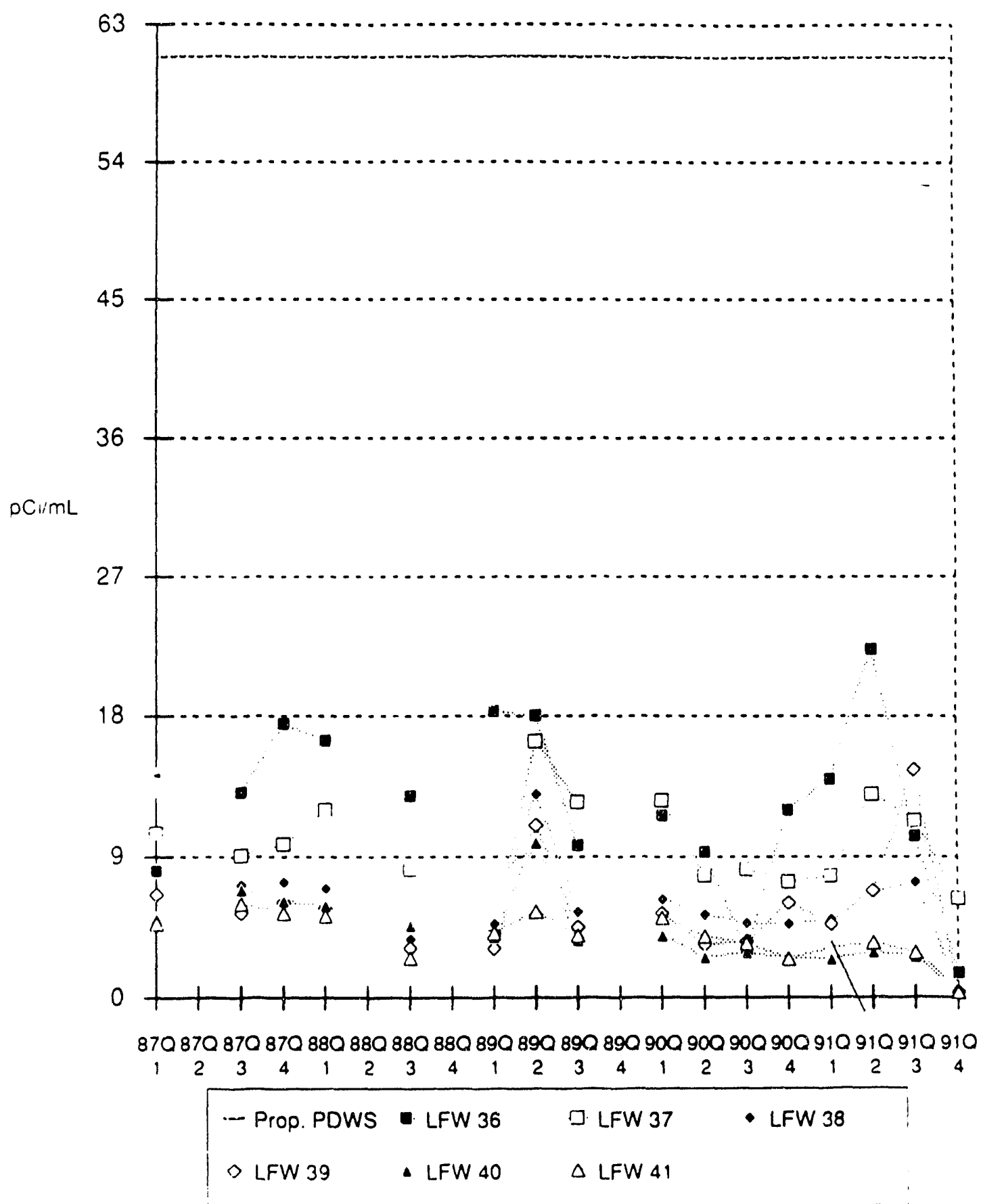


Tritium in the LFW-Series Monitoring Wells

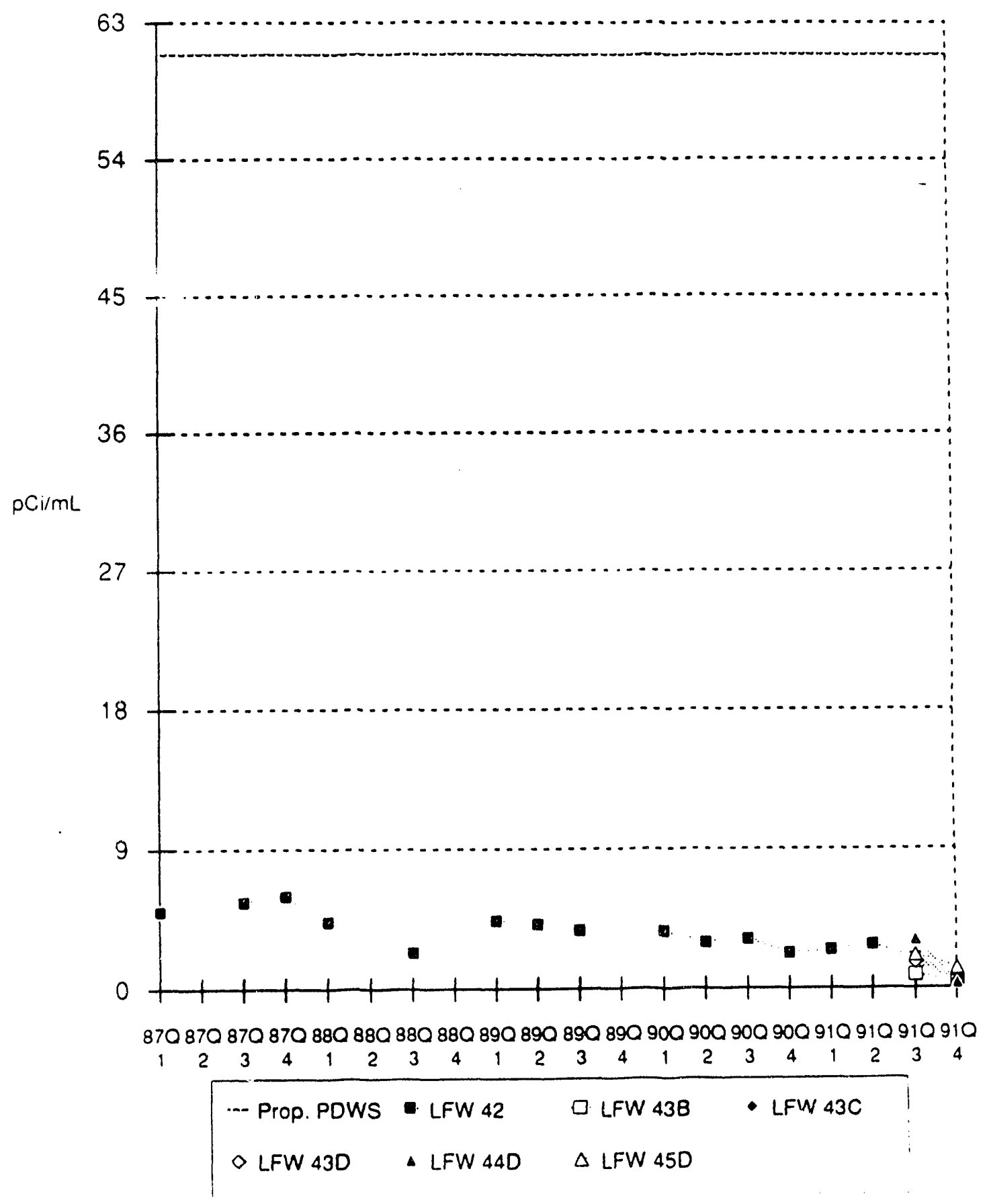


Tritium in the LFW-Series Monitoring Wells

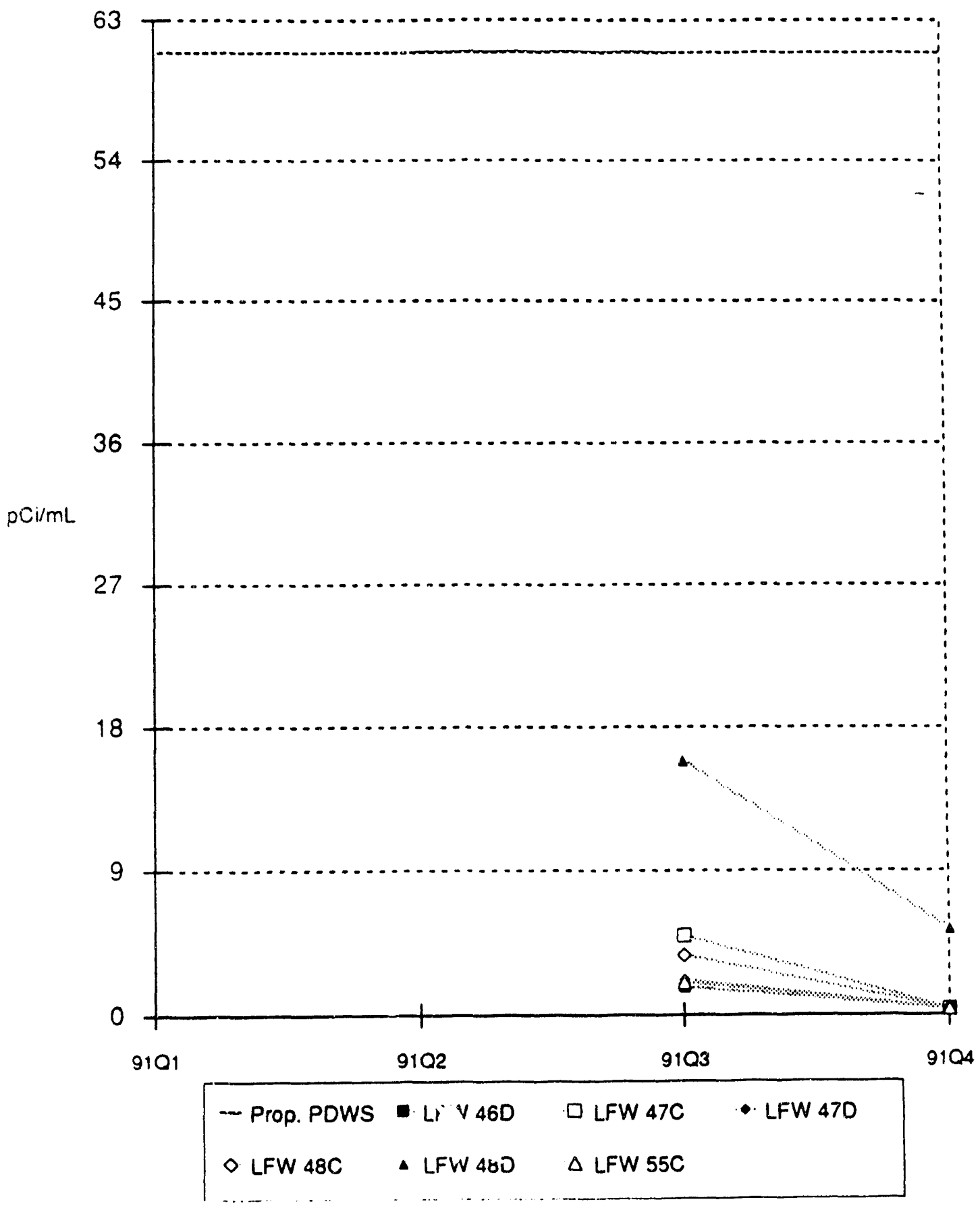


Tritium in the LFW-Series Monitoring Wells

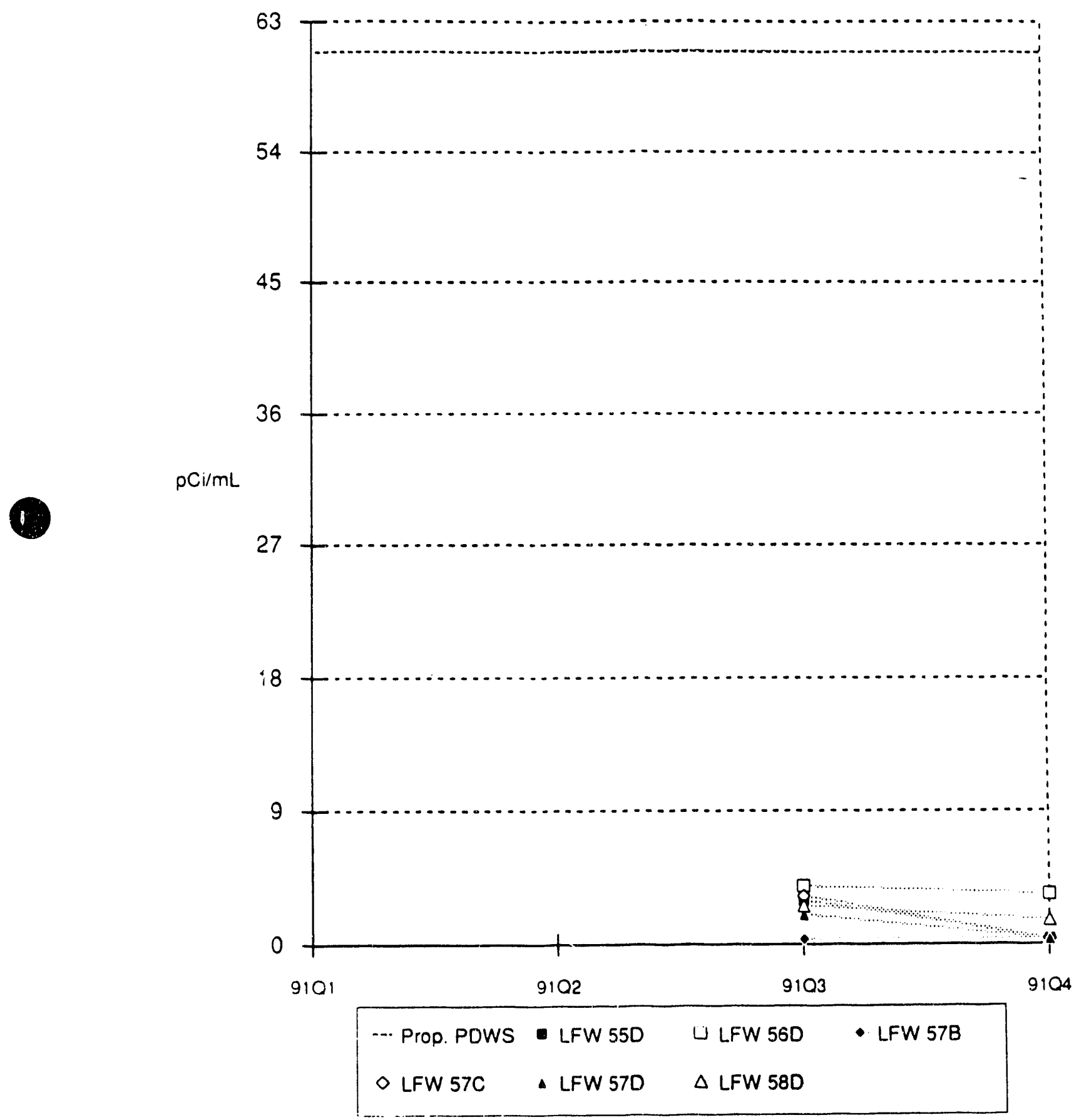


Tritlum In the LFW-Series Monitoring Wells

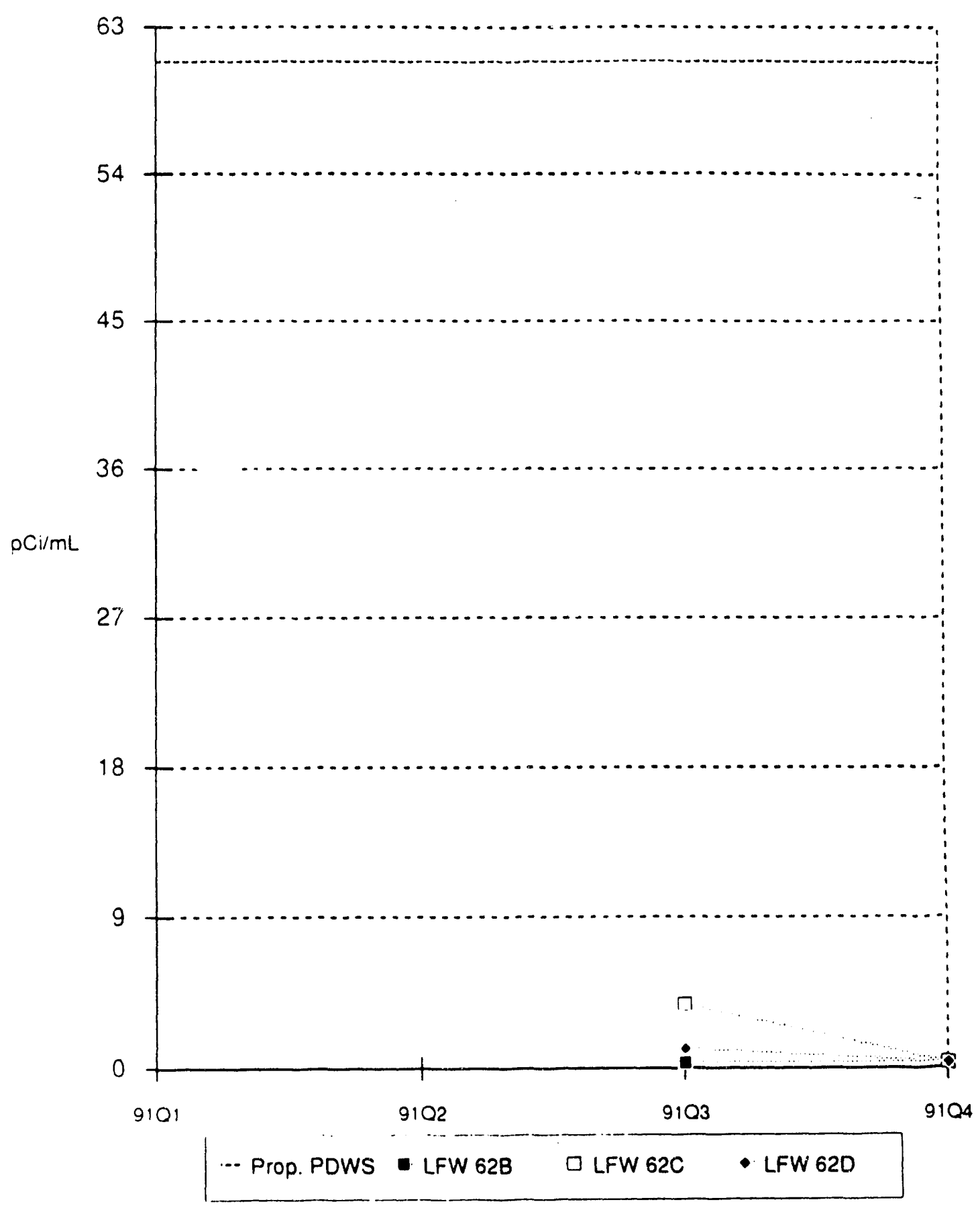


Total Radium in the LFW-Series Monitoring Wells

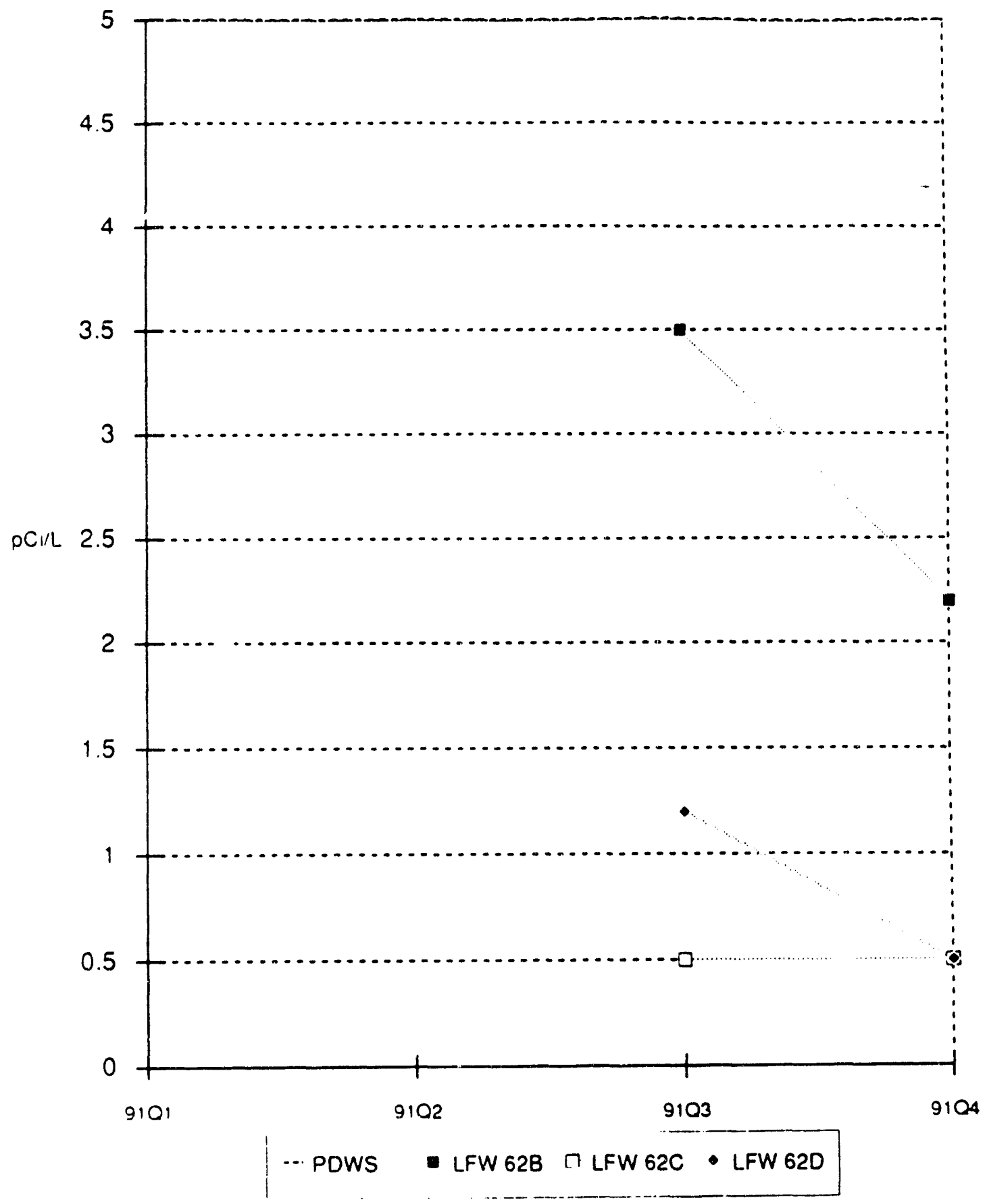




\section{Total Radium in the LFW-Series Monitoring Wells}

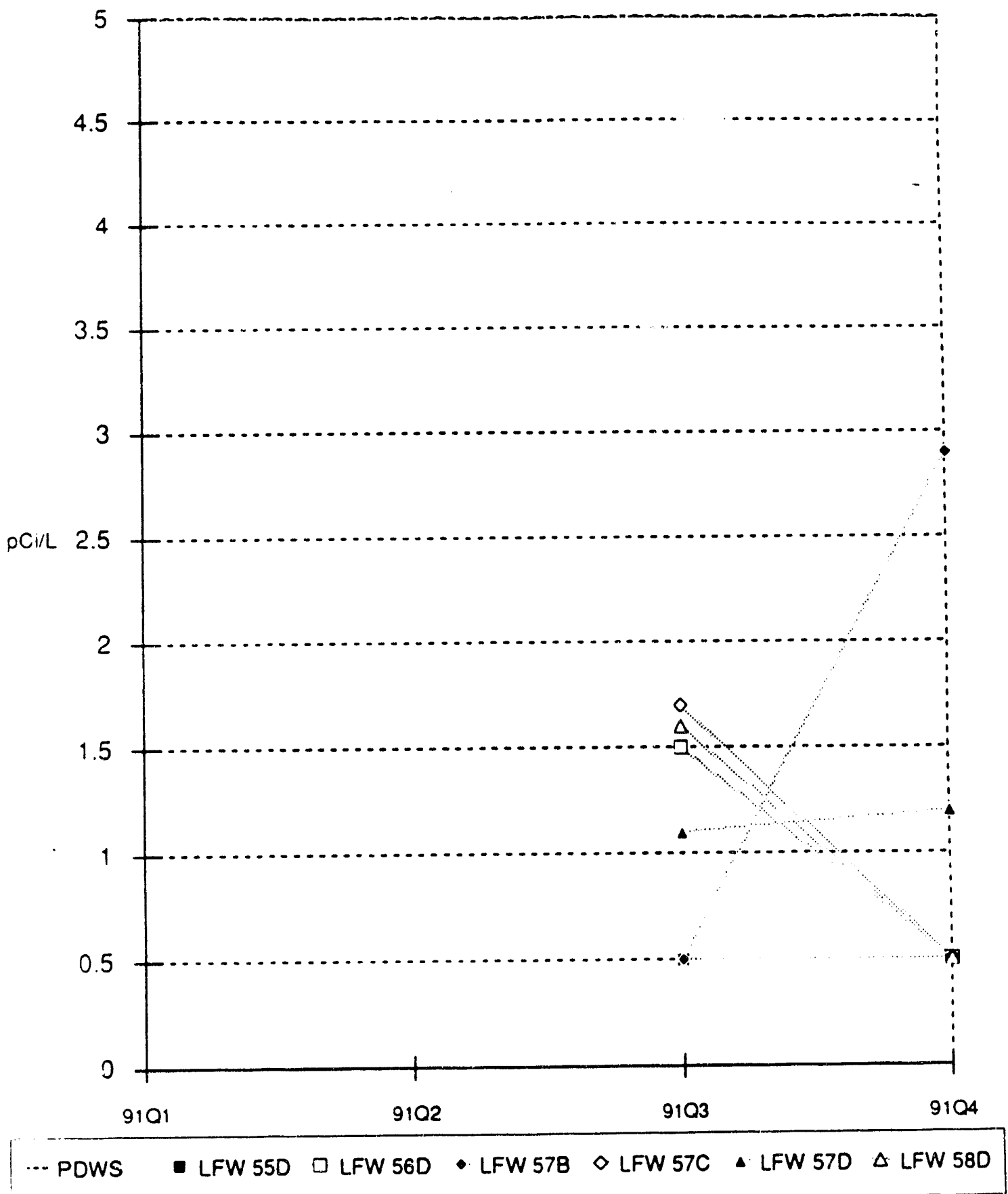


Total Radium in the LFW-Series Monitoring Wells

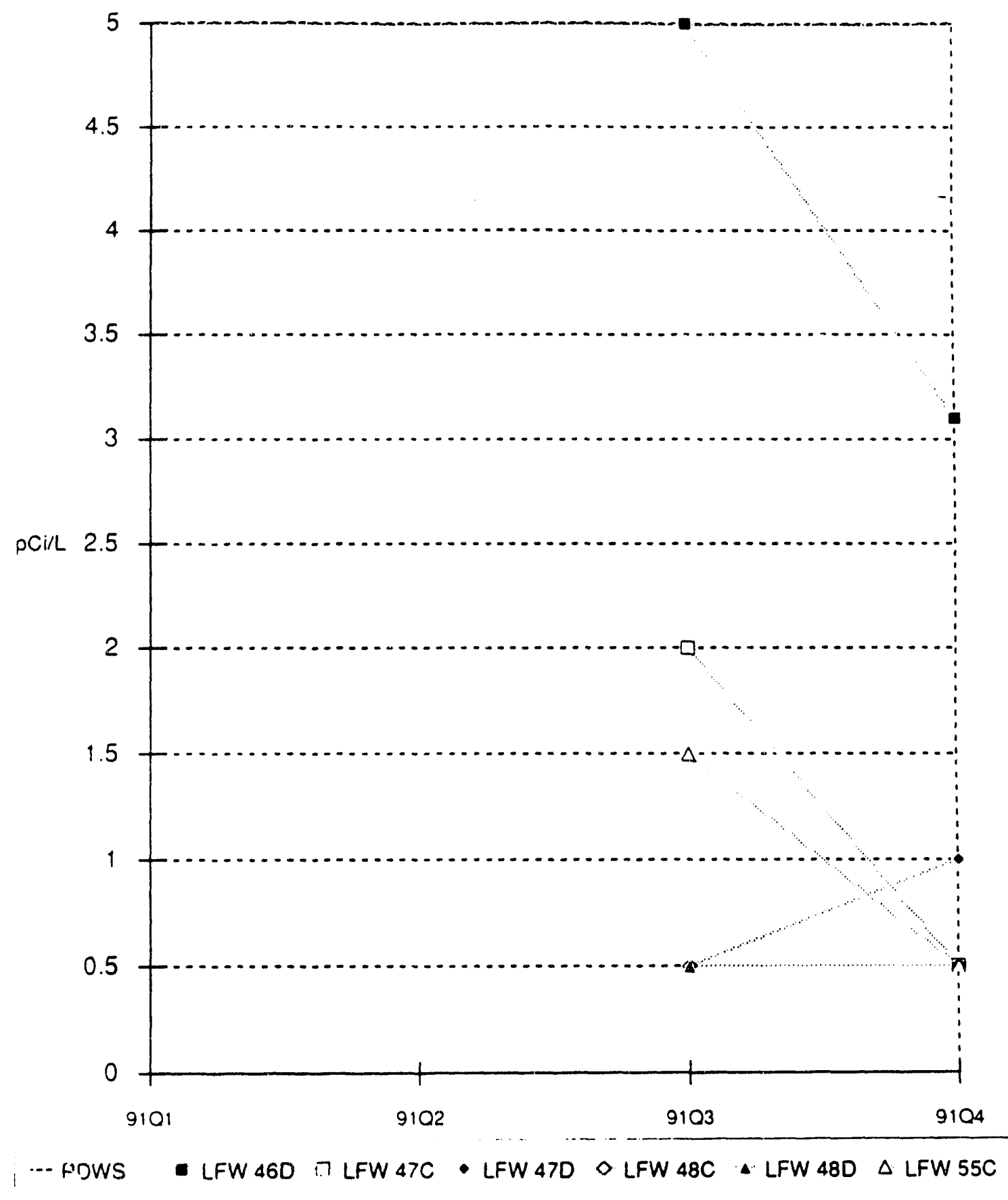


Total Radium in the LFW-Series Monitoring Wells

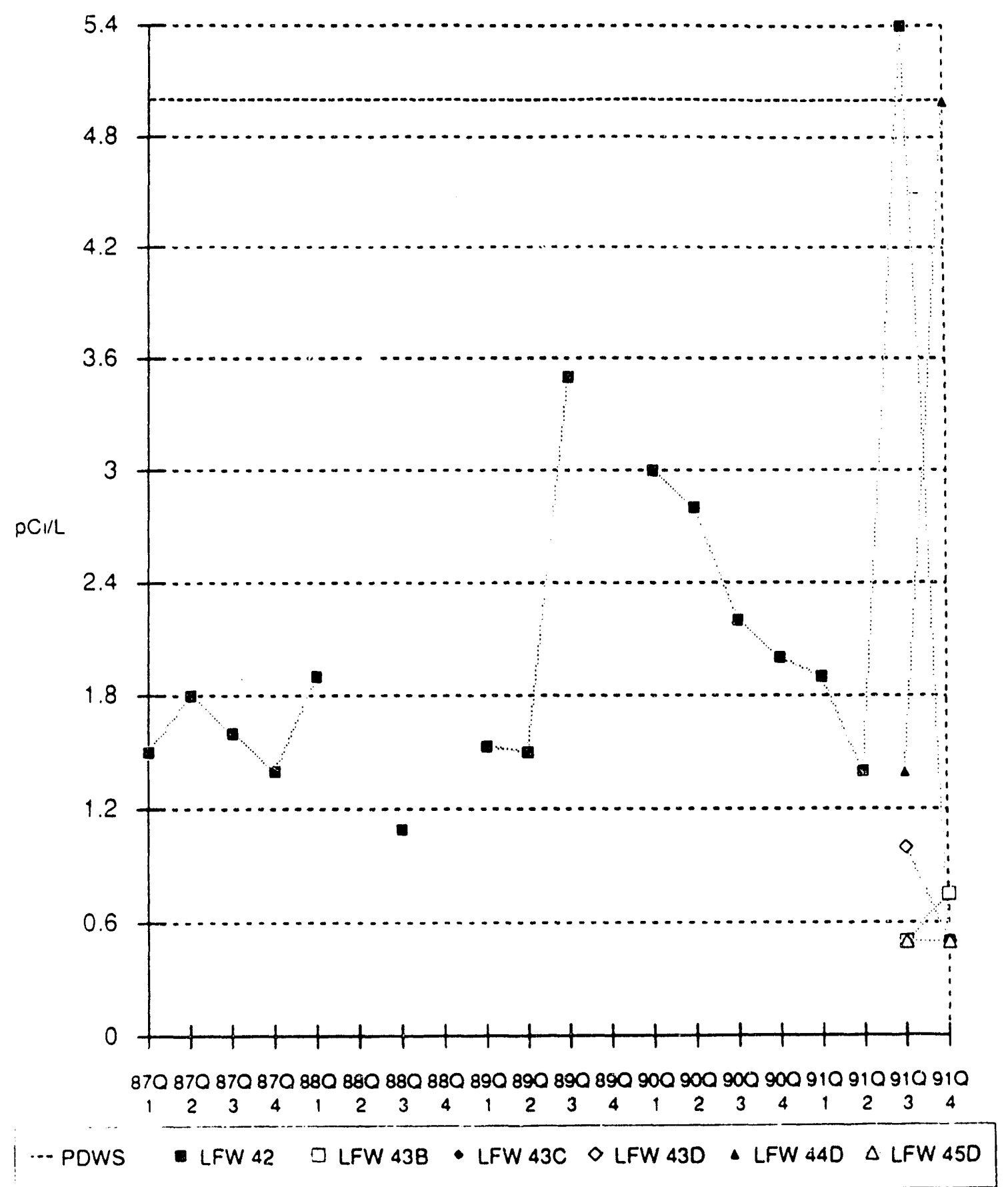


Total Radium in the LFW-Series Monitoring Wells

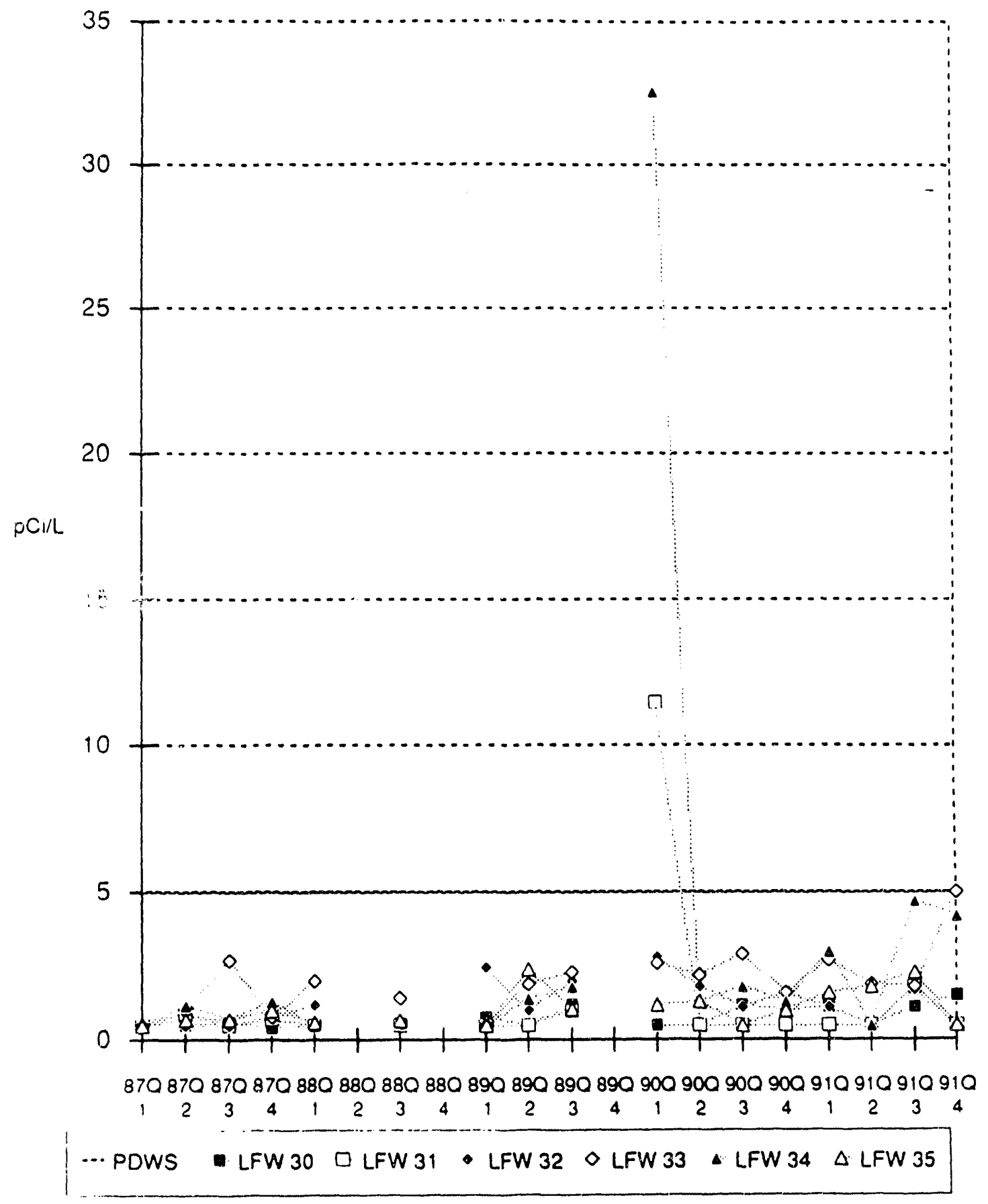


Total Radium in the LFW-Series Monitoring Wells

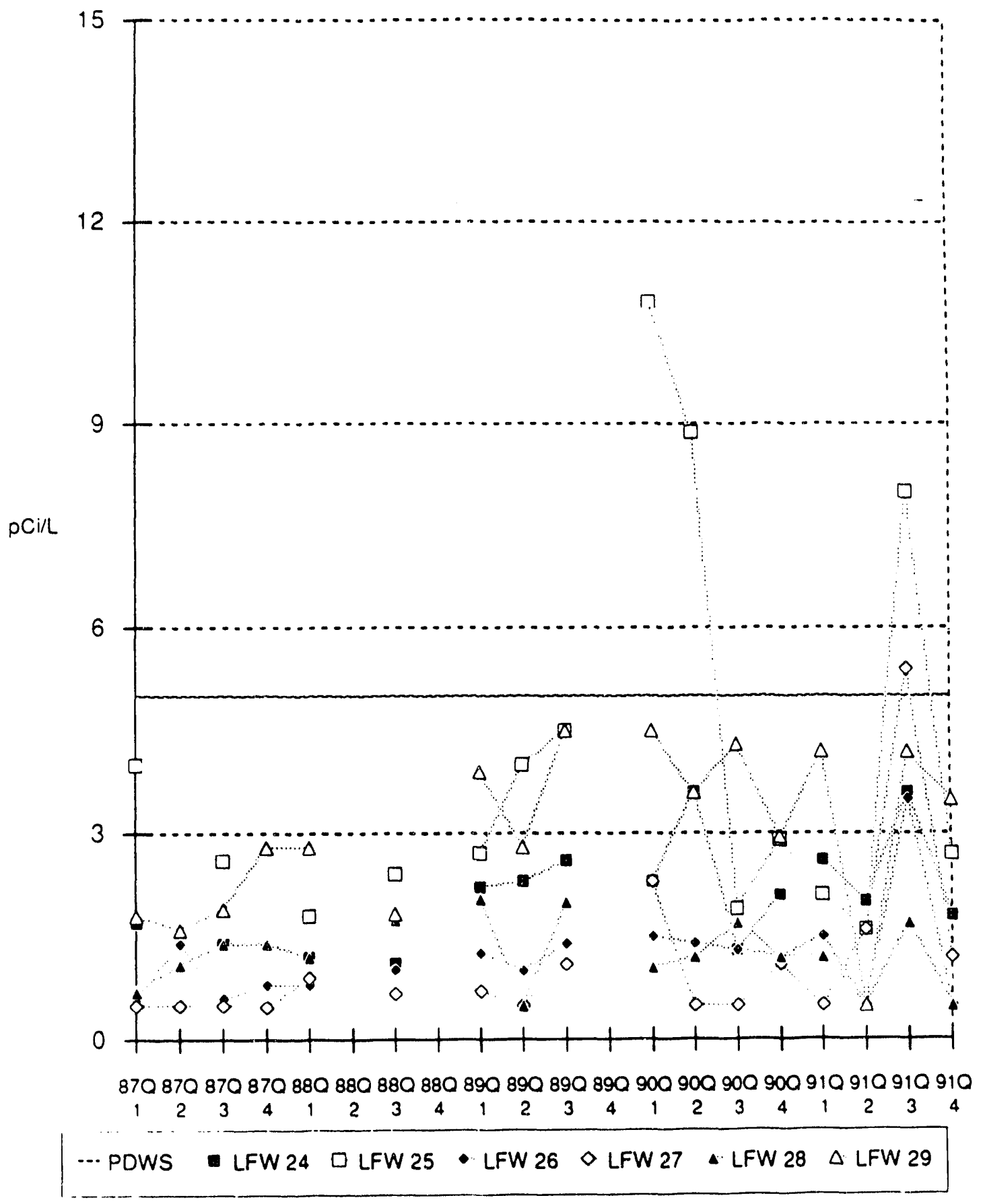


Total Radium in the LFW-Series Monitoring Wells

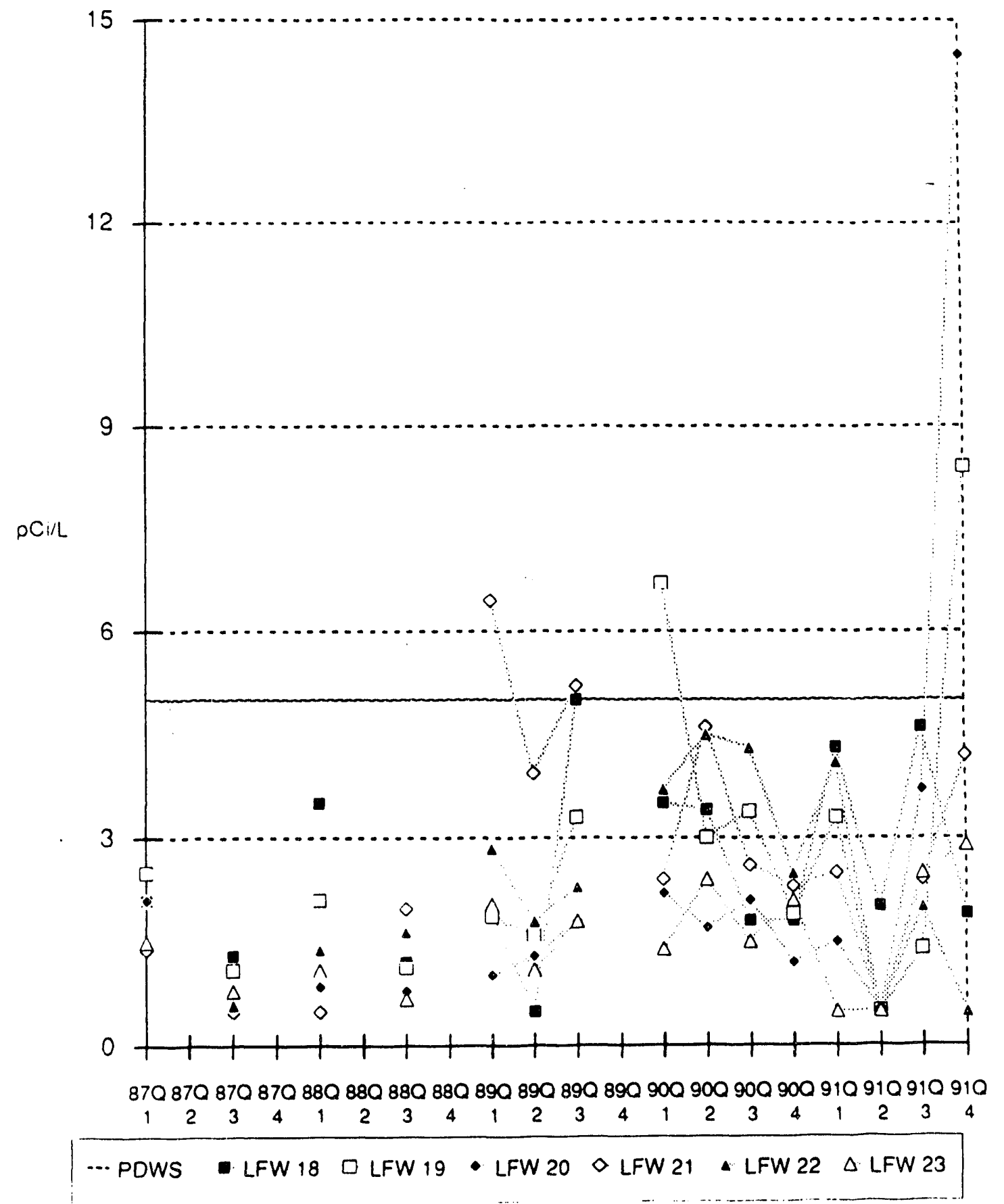


Gross Alpha in the LFW-Series Monitoring Wells

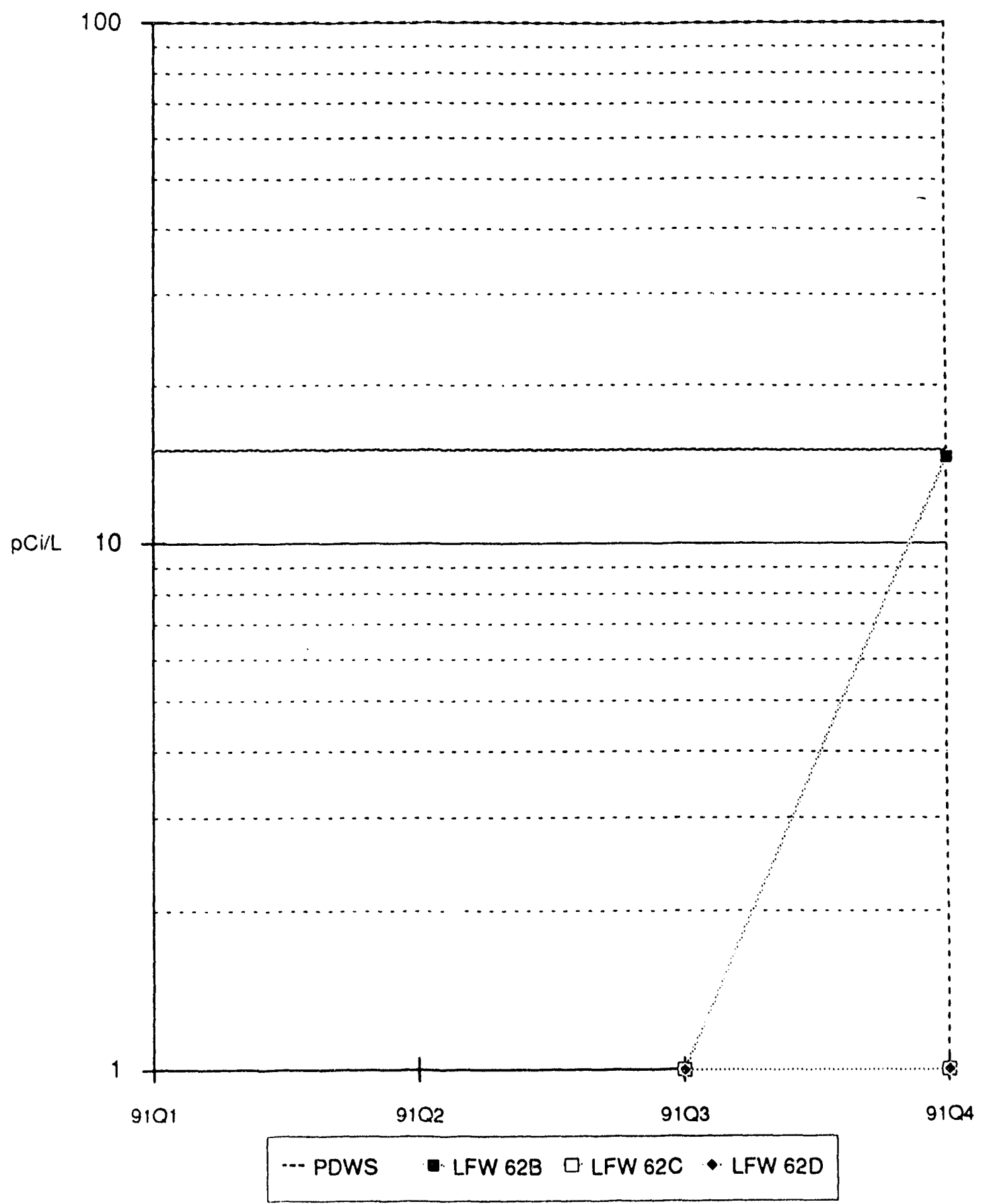


Gross Alpha in the LFW-Series Monitoring Wells

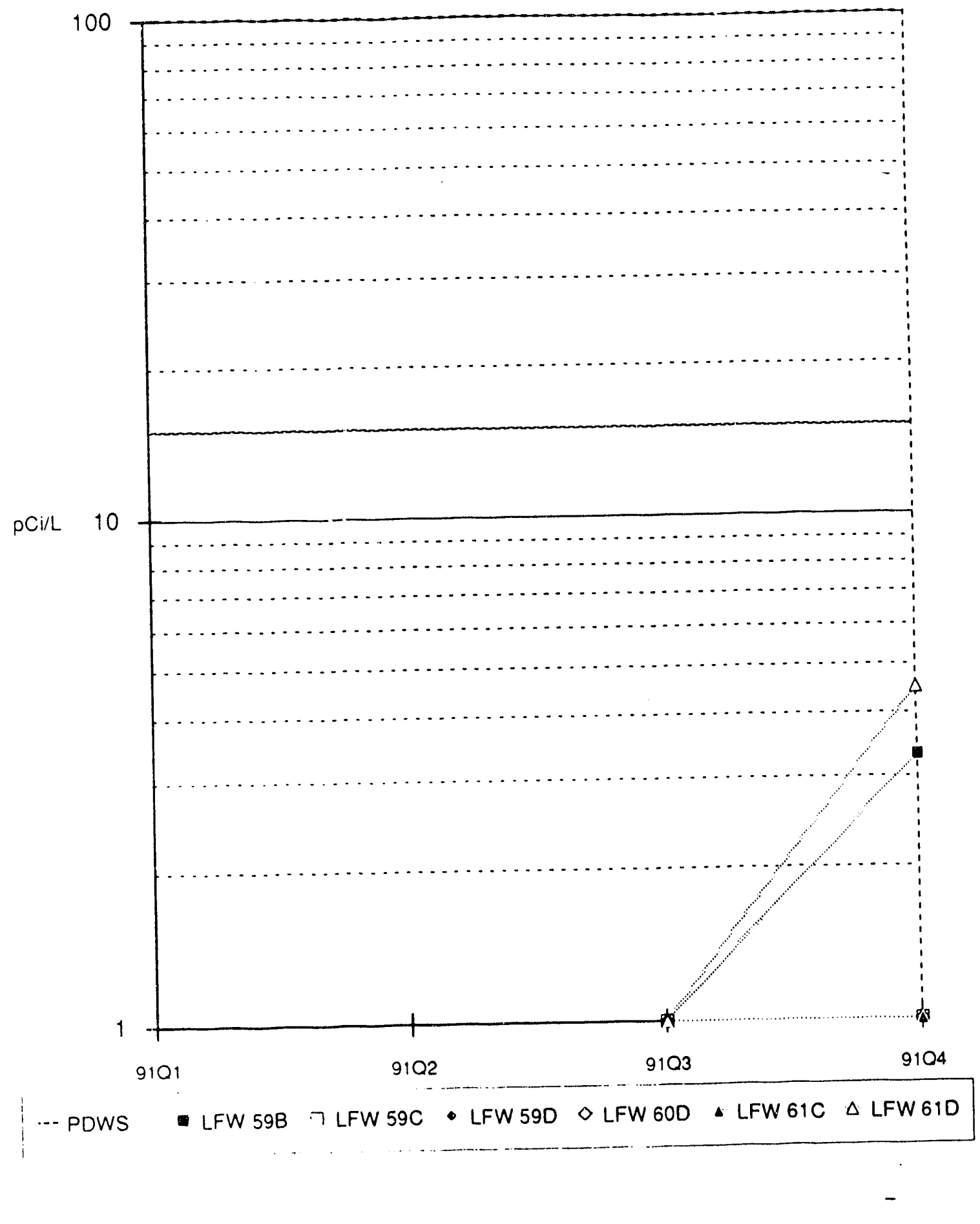


Gross Alpha in the LFW-Series Monitoring Wells

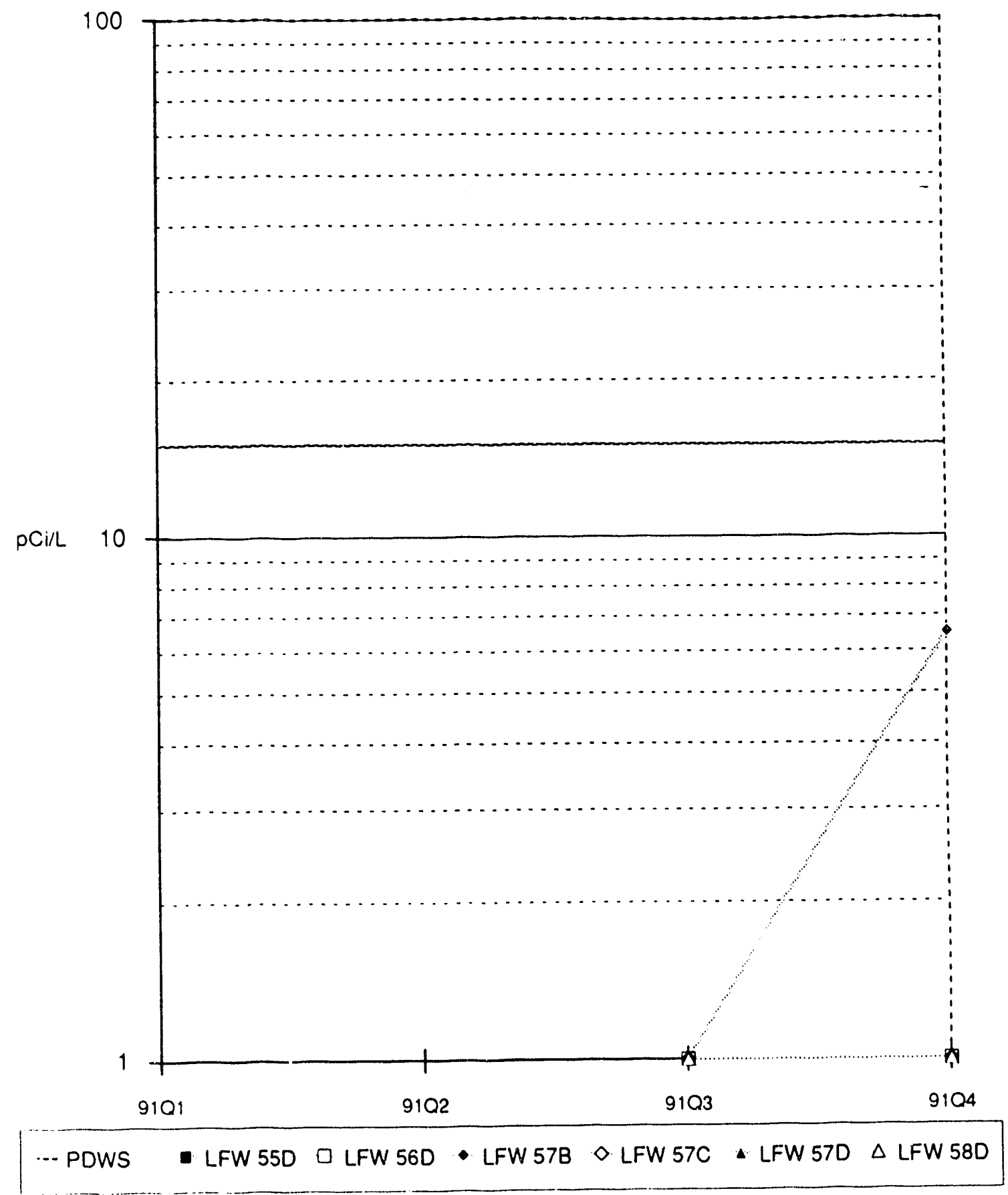




\section{Gross Alpha in the LFW-Series Monitoring Wells}

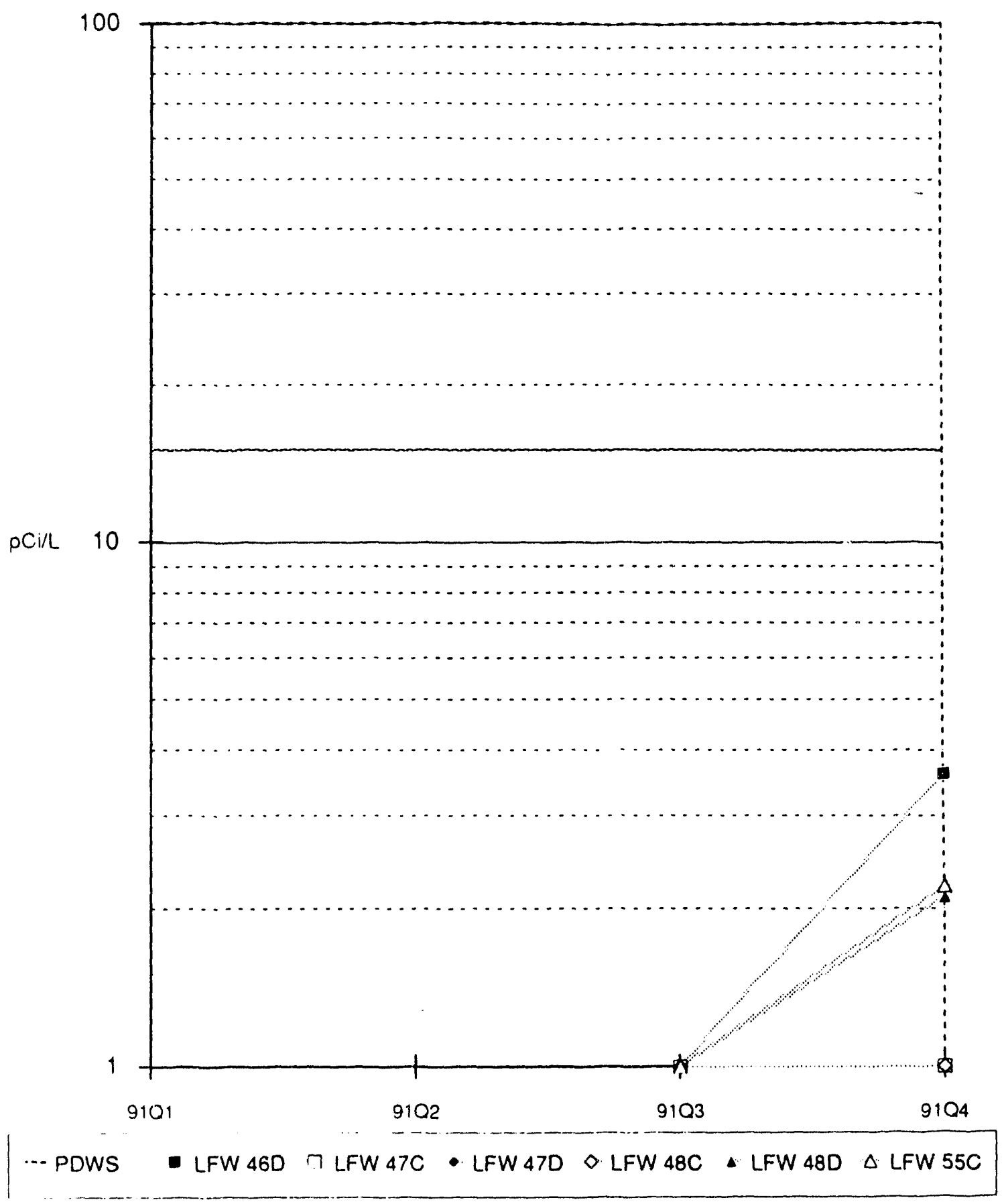


Gross Alpha in the LFW-Series Monitoring Wells

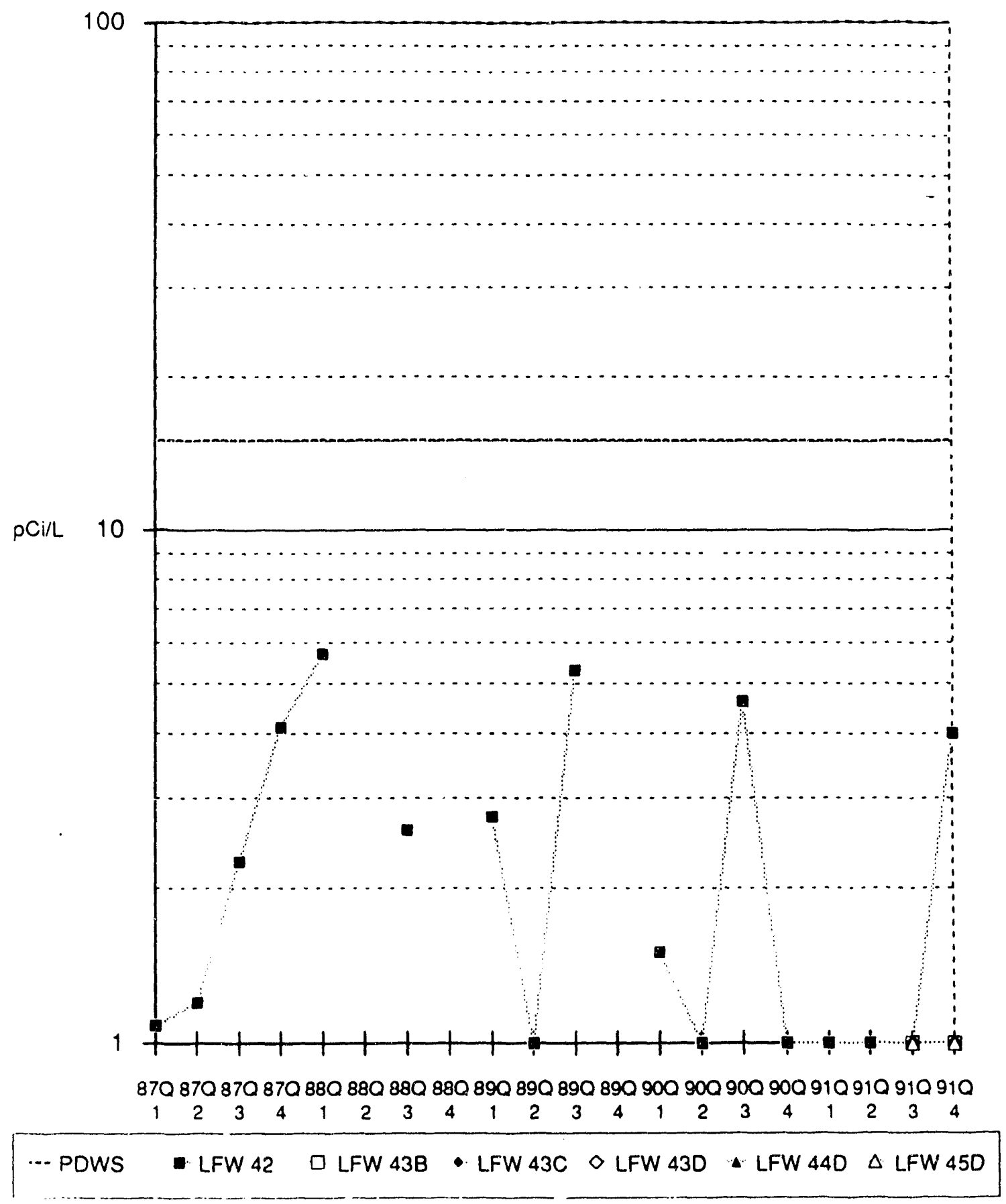


Gross Alpha in the LFW-Series Monitoring Wells

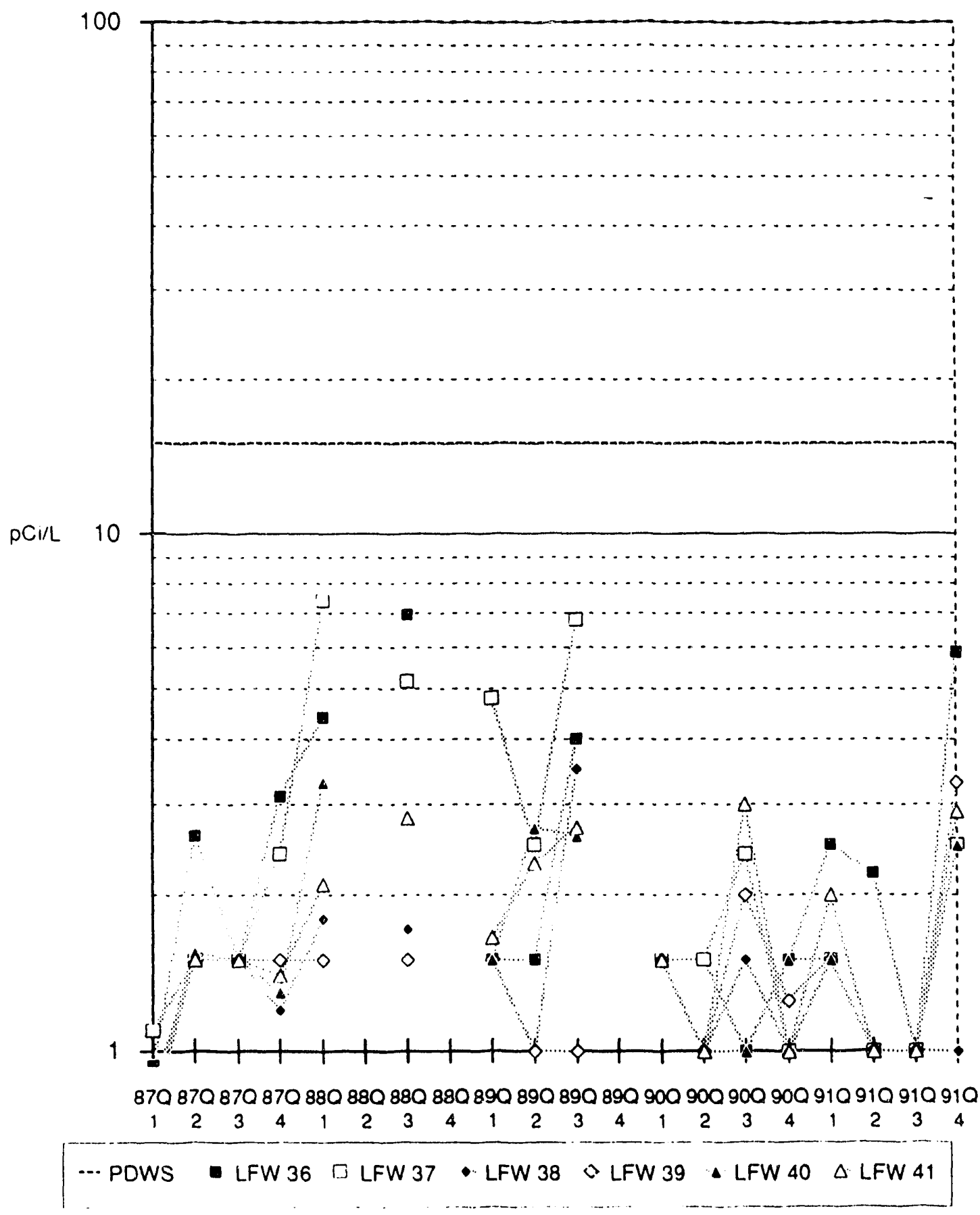


Gross Alpha in the LFW-Series Monitoring Wells

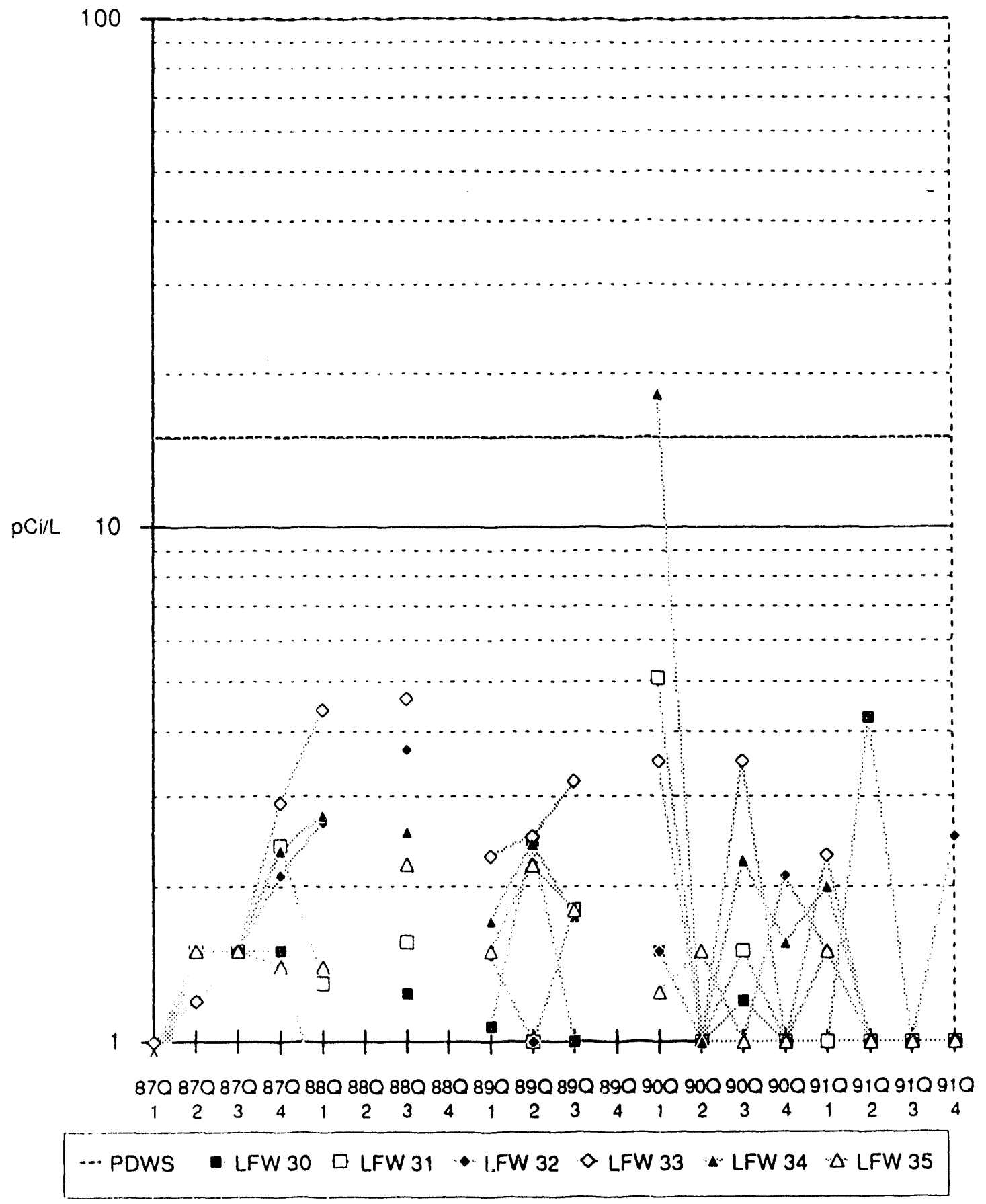


Gross Alpha in the LFW-Series Monitoring Wells

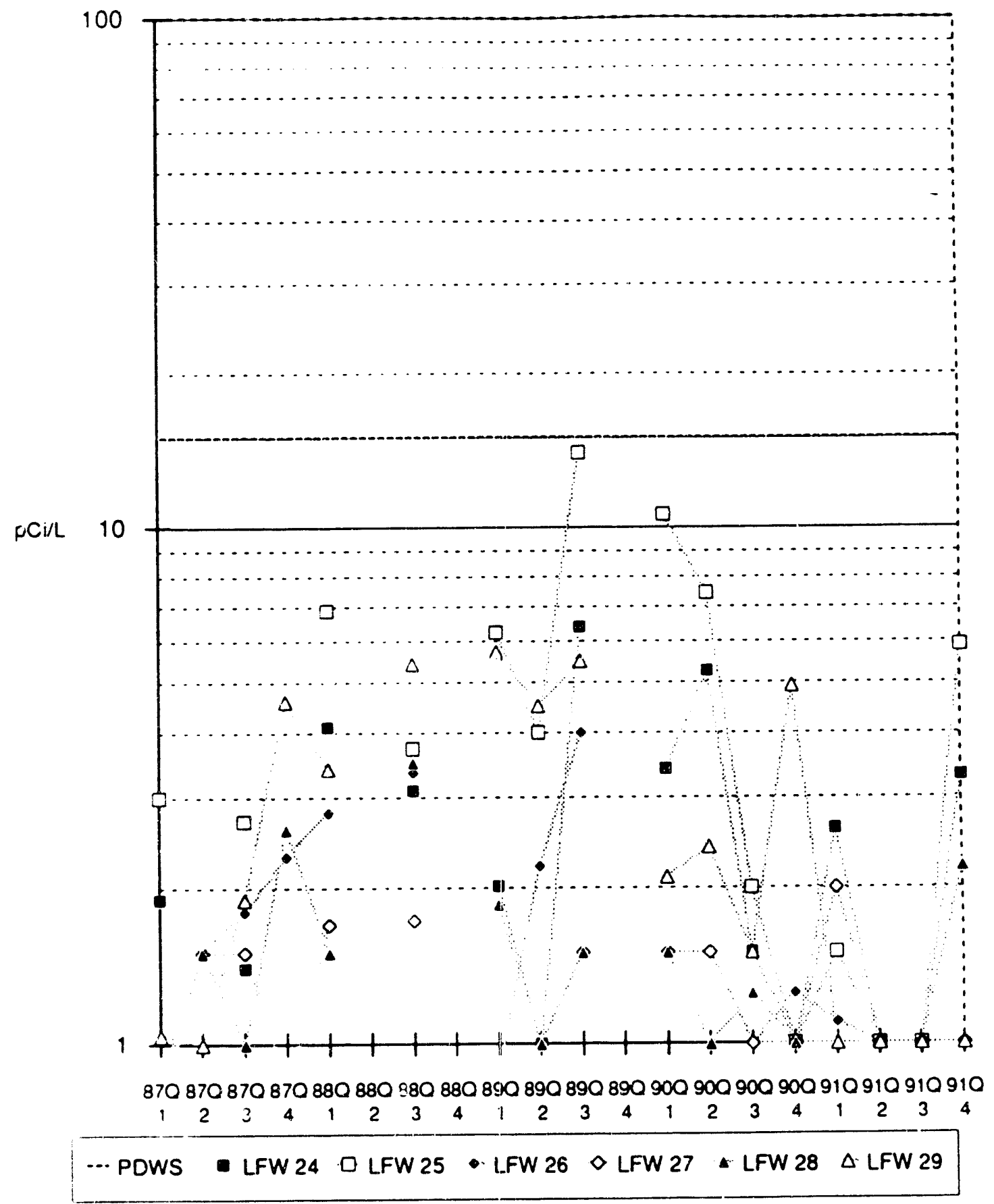


Gross Alpha in the LFW-Series Monitoring Wells

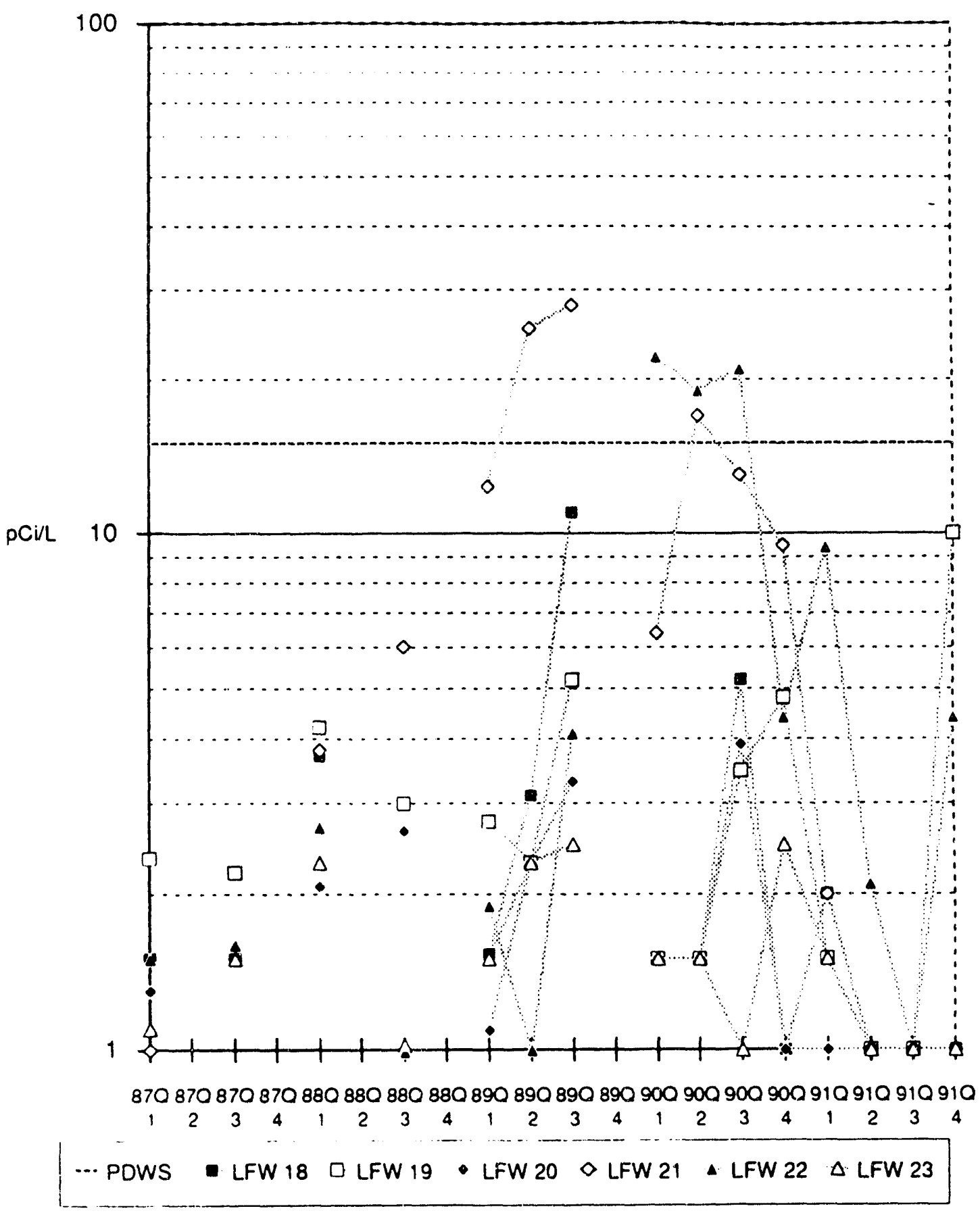




\section{Gross Alpha in the LFW-Series Monitoring Wells}

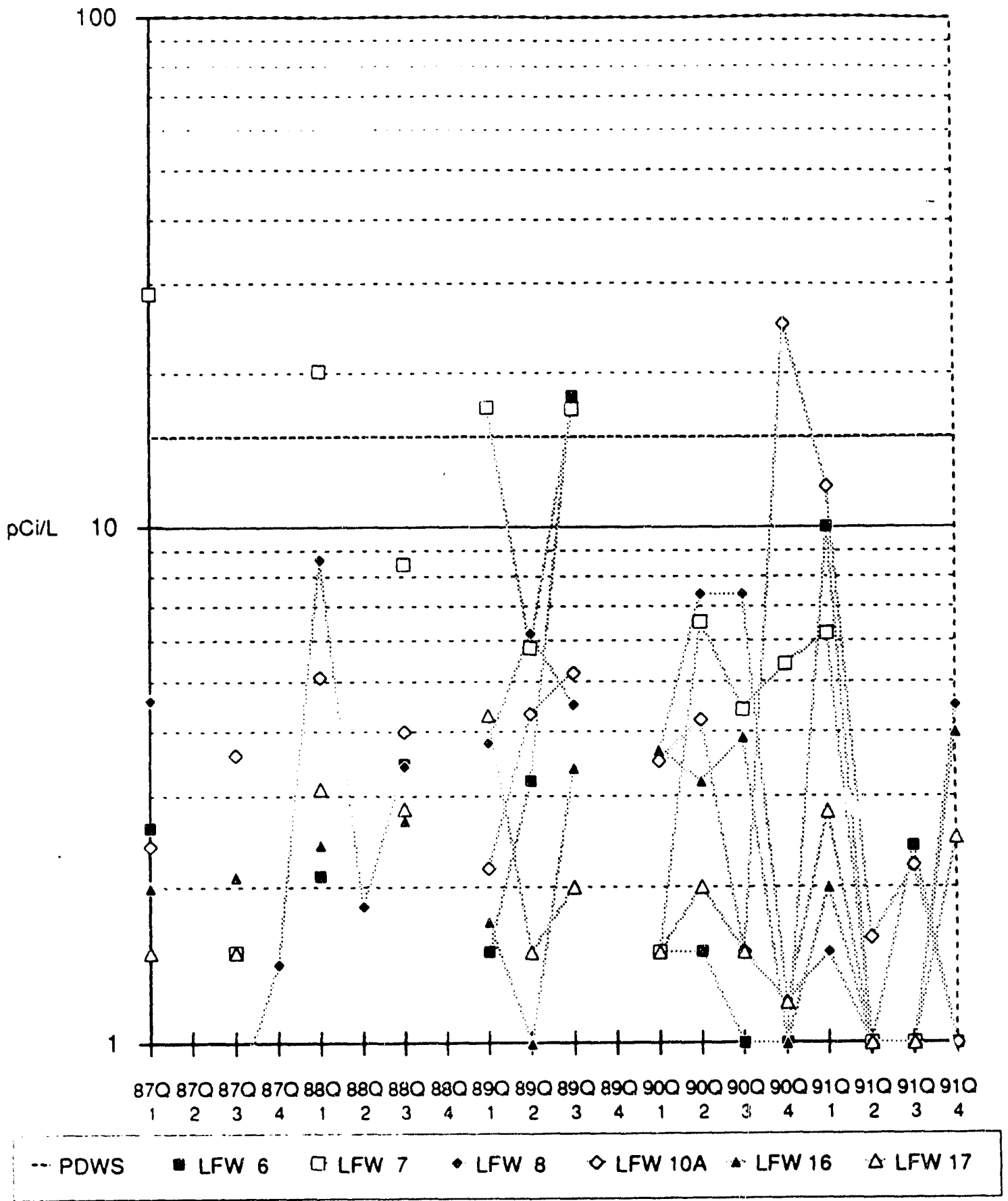


Cadmium in the LFW-Series Monitoring Wells

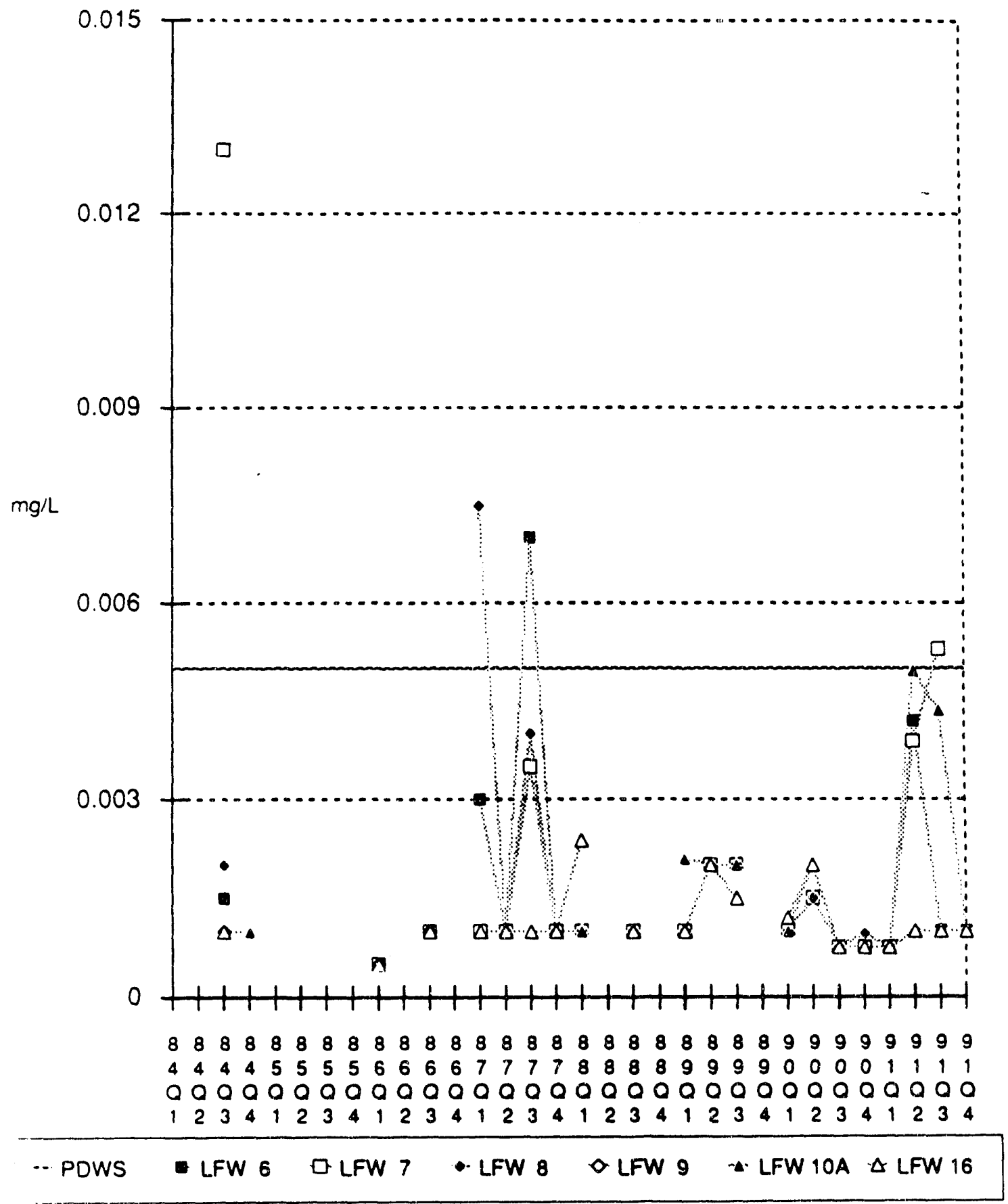




\section{Cadmium in the LFW-Series Monitoring Wells}

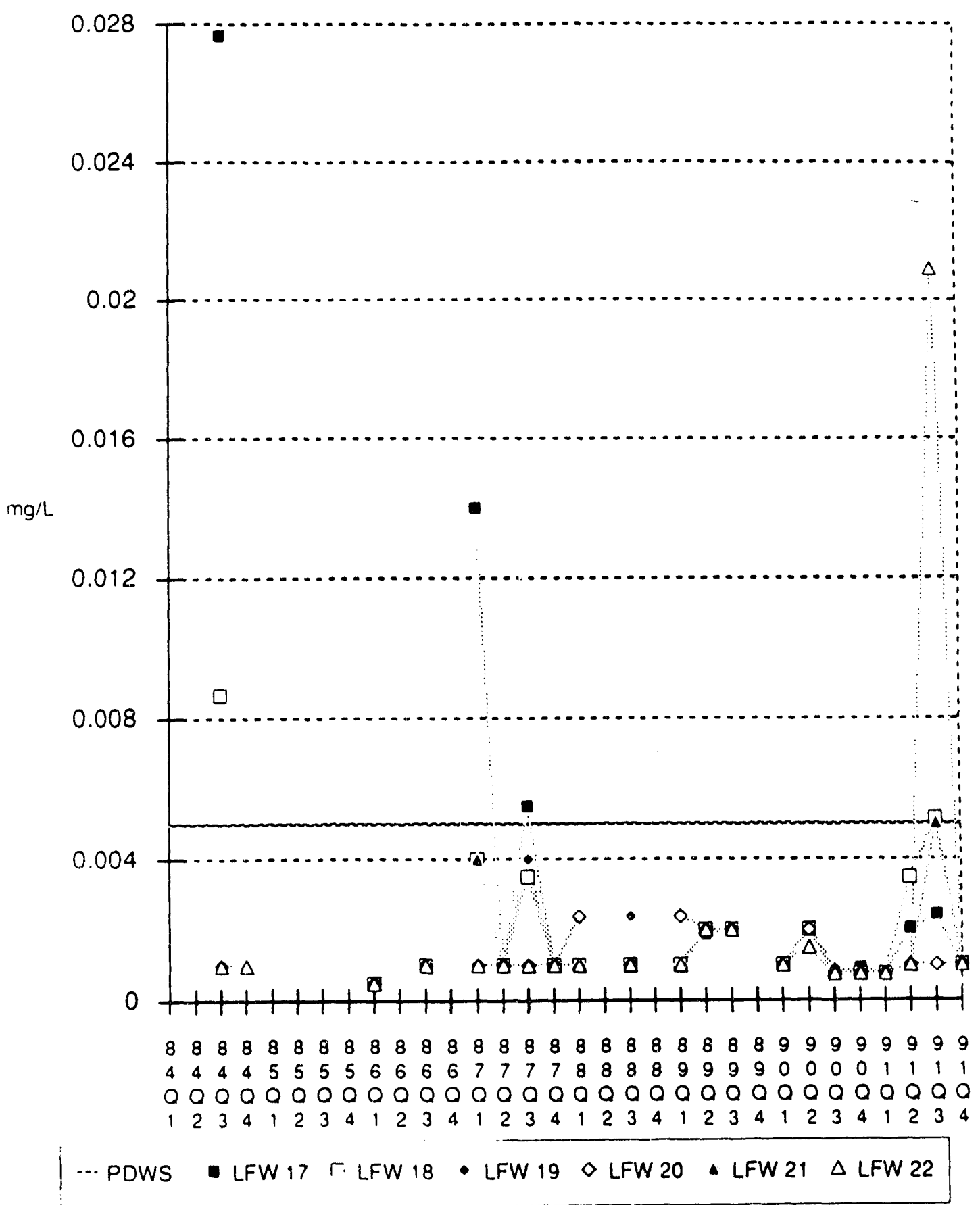




\section{Cadmium in the LFW-Series Monitoring Wells}

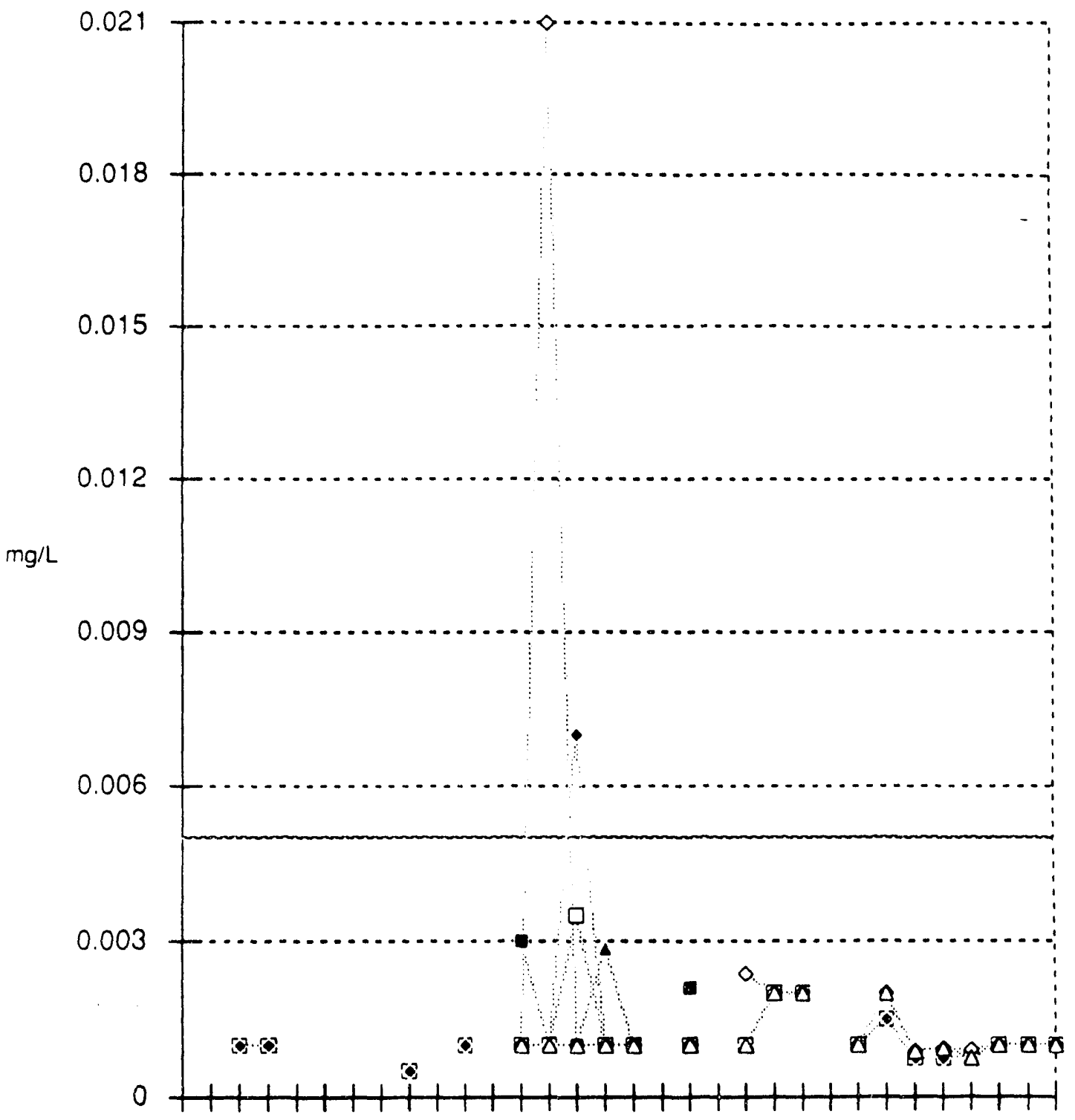

$\begin{array}{llllllllllllllllllllllllllllllll}8 & 8 & 8 & 8 & 8 & 8 & 8 & 8 & 8 & 8 & 8 & 8 & 8 & 8 & 8 & 8 & 8 & 8 & 8 & 8 & 8 & 8 & 8 & 8 & 9 & 9 & 9 & 9 & 9 & 9 & 9 & 9\end{array}$ $\begin{array}{lllllllllllllllllllllllllllllllll}4 & 4 & 4 & 4 & 5 & 5 & 5 & 5 & 6 & 6 & 6 & 6 & 7 & 7 & 7 & 7 & 8 & 8 & 8 & 8 & 9 & 9 & 9 & 9 & 0 & 0 & 0 & 0 & 1 & 1 & 1 & 1\end{array}$

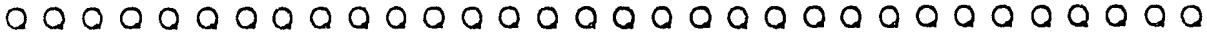
$\begin{array}{llllllllllllllllllllllllllllllll}1 & 2 & 3 & 4 & 1 & 2 & 3 & 4 & 1 & 2 & 3 & 4 & 1 & 2 & 3 & 4 & 1 & 2 & 3 & 4 & 1 & 2 & 3 & 4 & 1 & 2 & 3 & 4 & 1 & 2 & 3 & 4\end{array}$

-.. POWS LFW 23 ! LFW 24 - LFW $25 \diamond$ LFW 26 \& LFW $27 \triangle$ LFW 28 


\section{Cadmium in the LFW-Series Monitoring Wells}

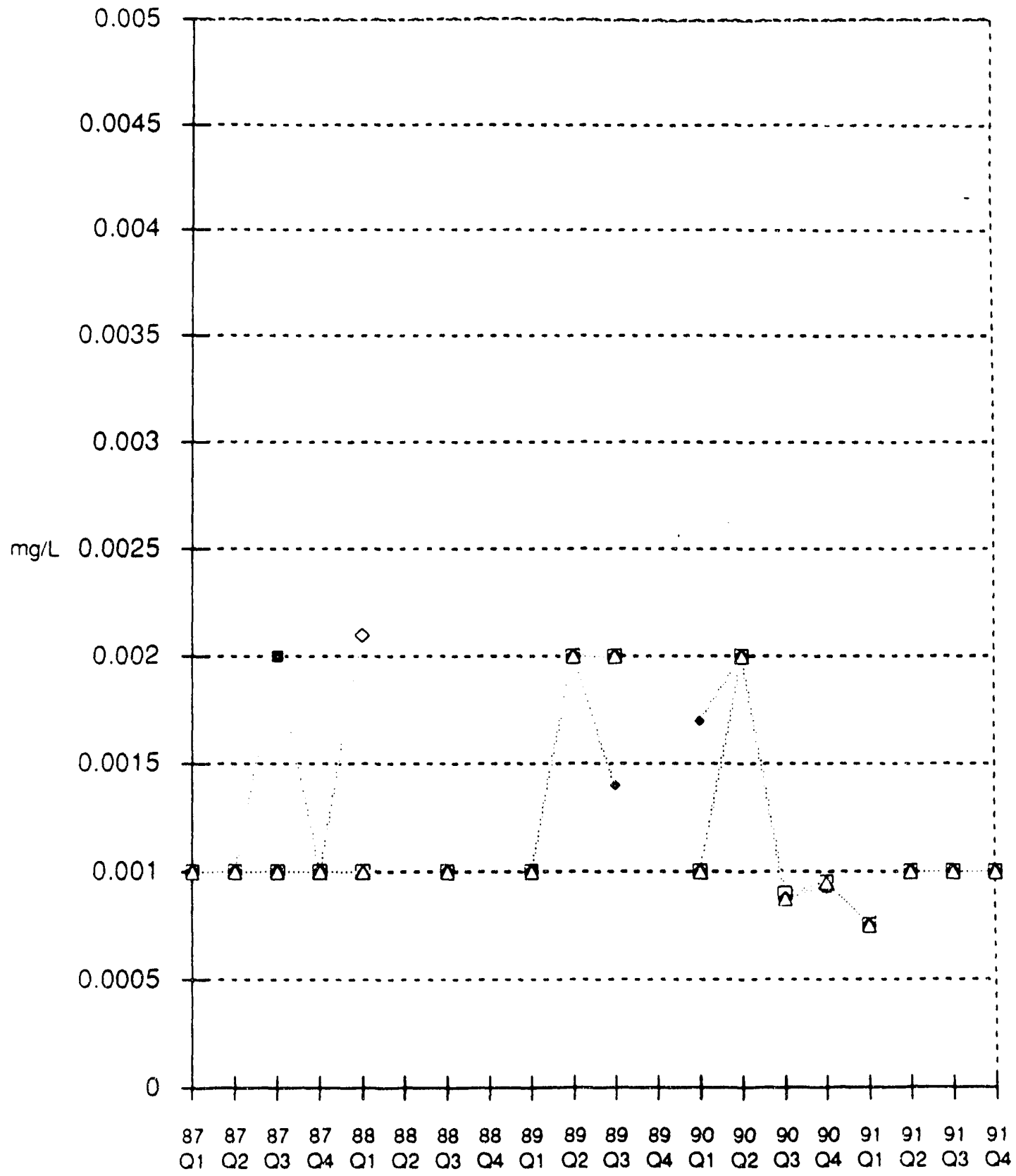

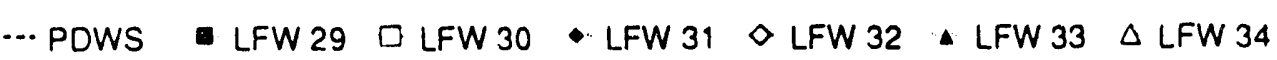




\section{Cadmium in the LFW-Series Monitoring Wells}

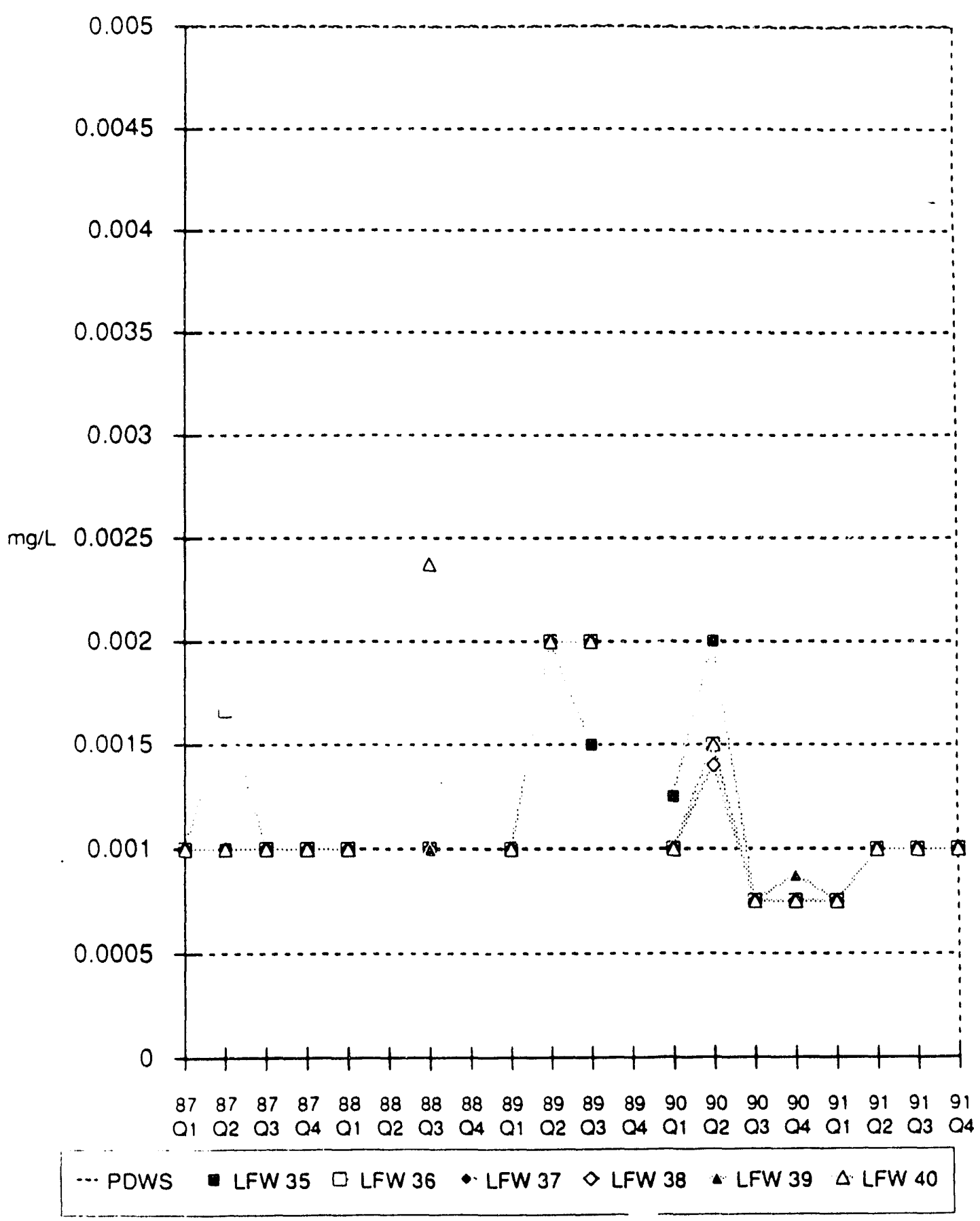


Cadmium in the LFW-Series Monitoring Wells

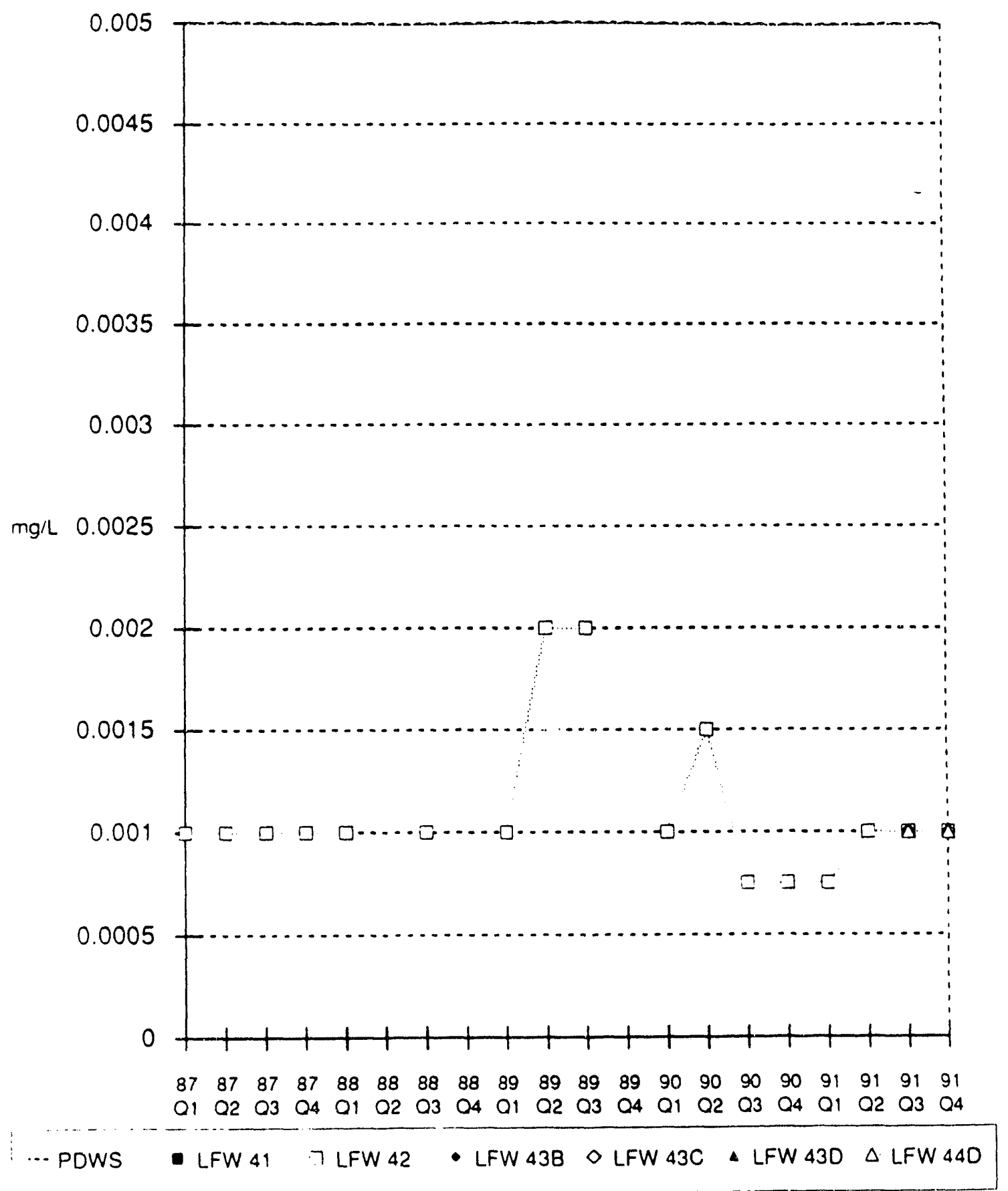


Cadmium in the LFW-Series Monitoring Wells

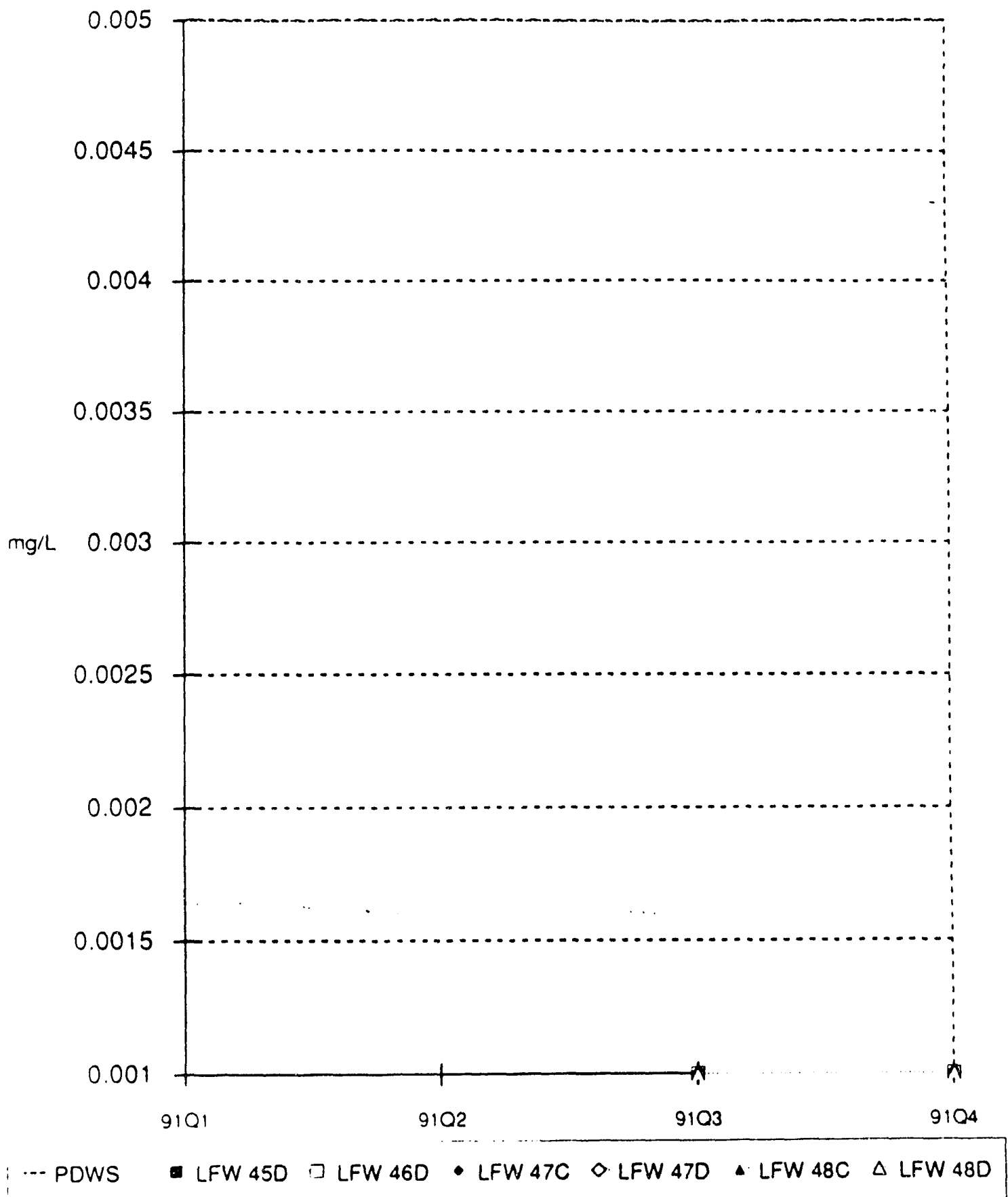


Cadmium in the LFW-Series Monitoring Wells

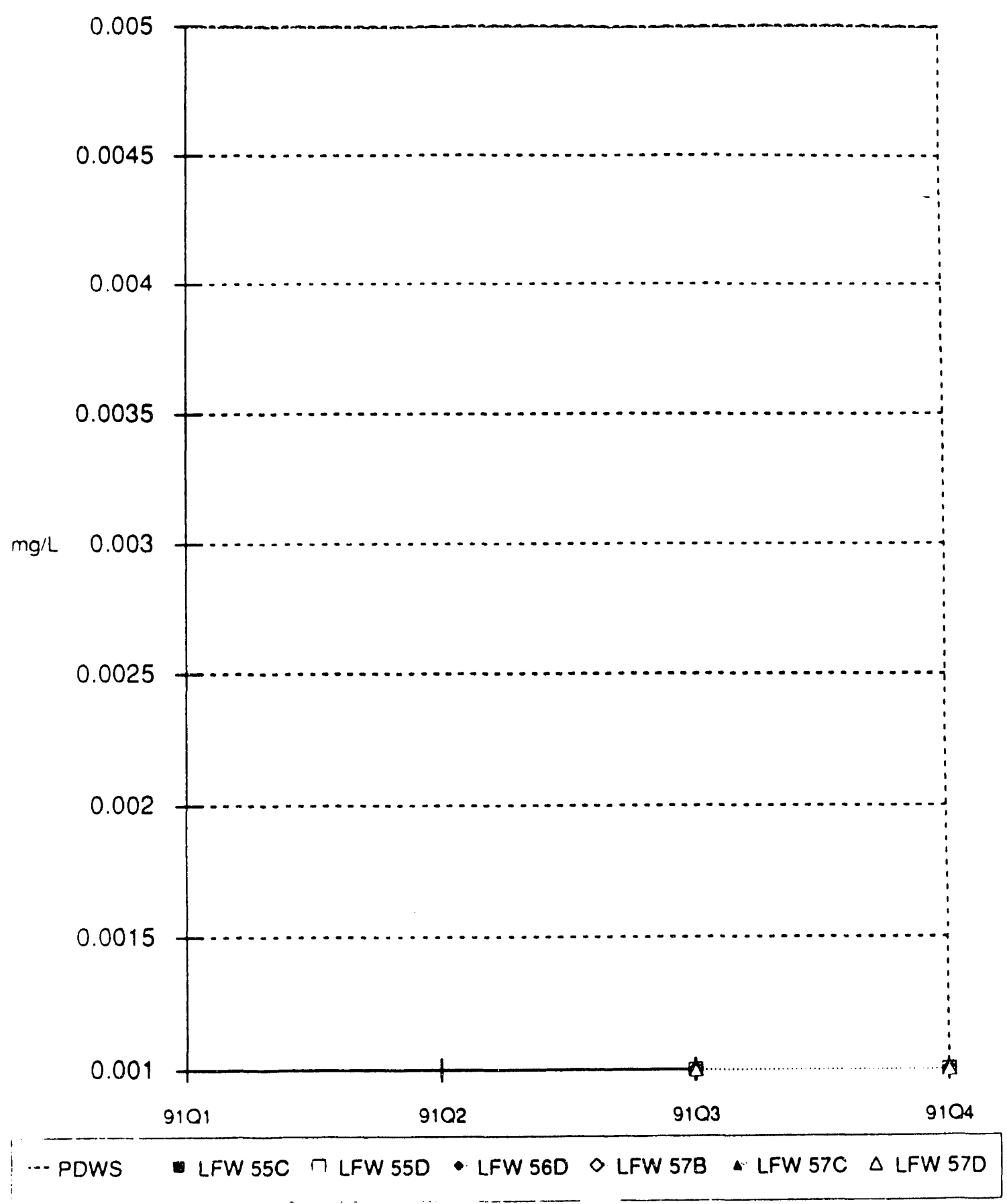


Cadmium in the LFW-Series Monitoring Wells

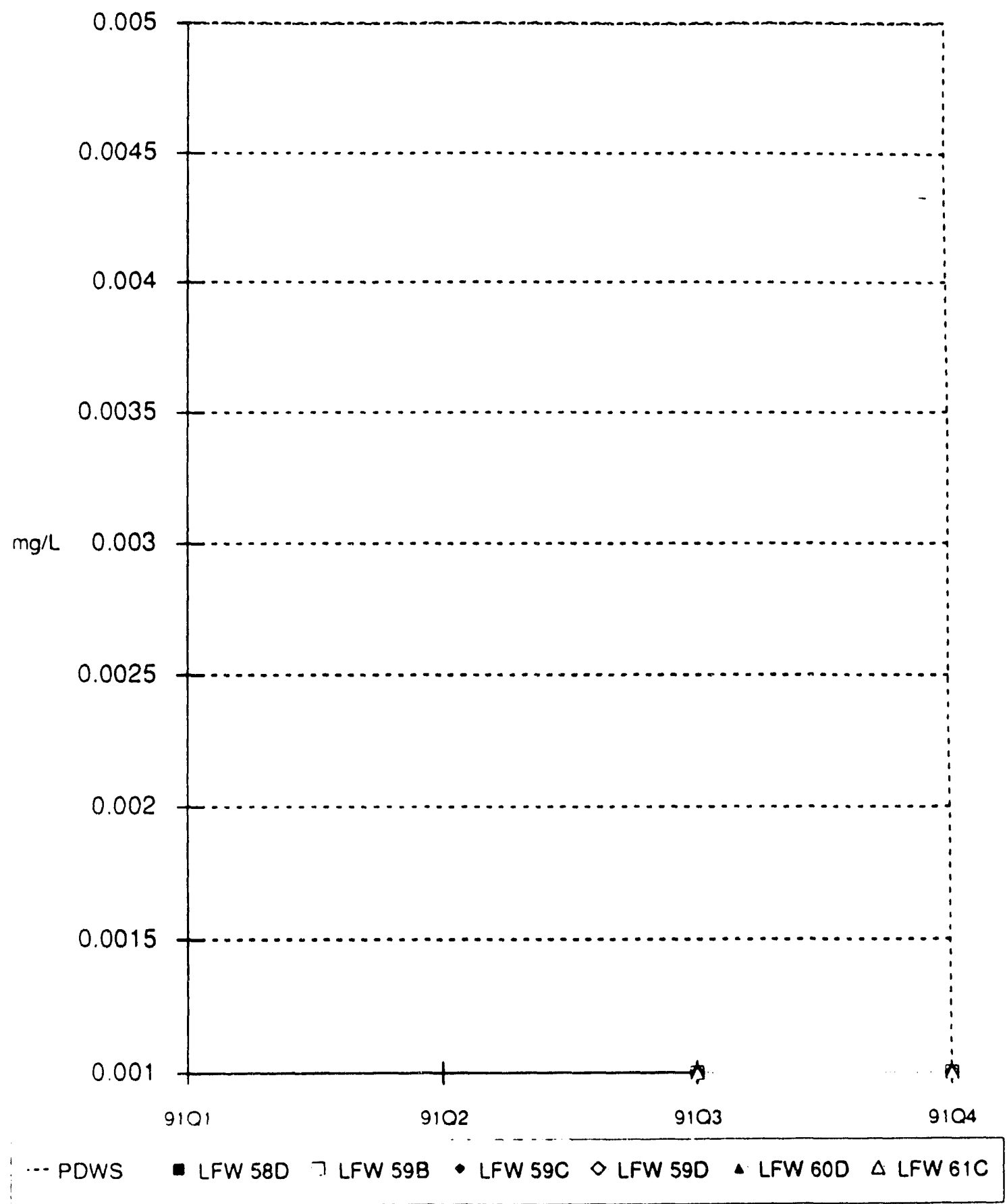


Cadmium in the LFW-Series Monitoring Wells

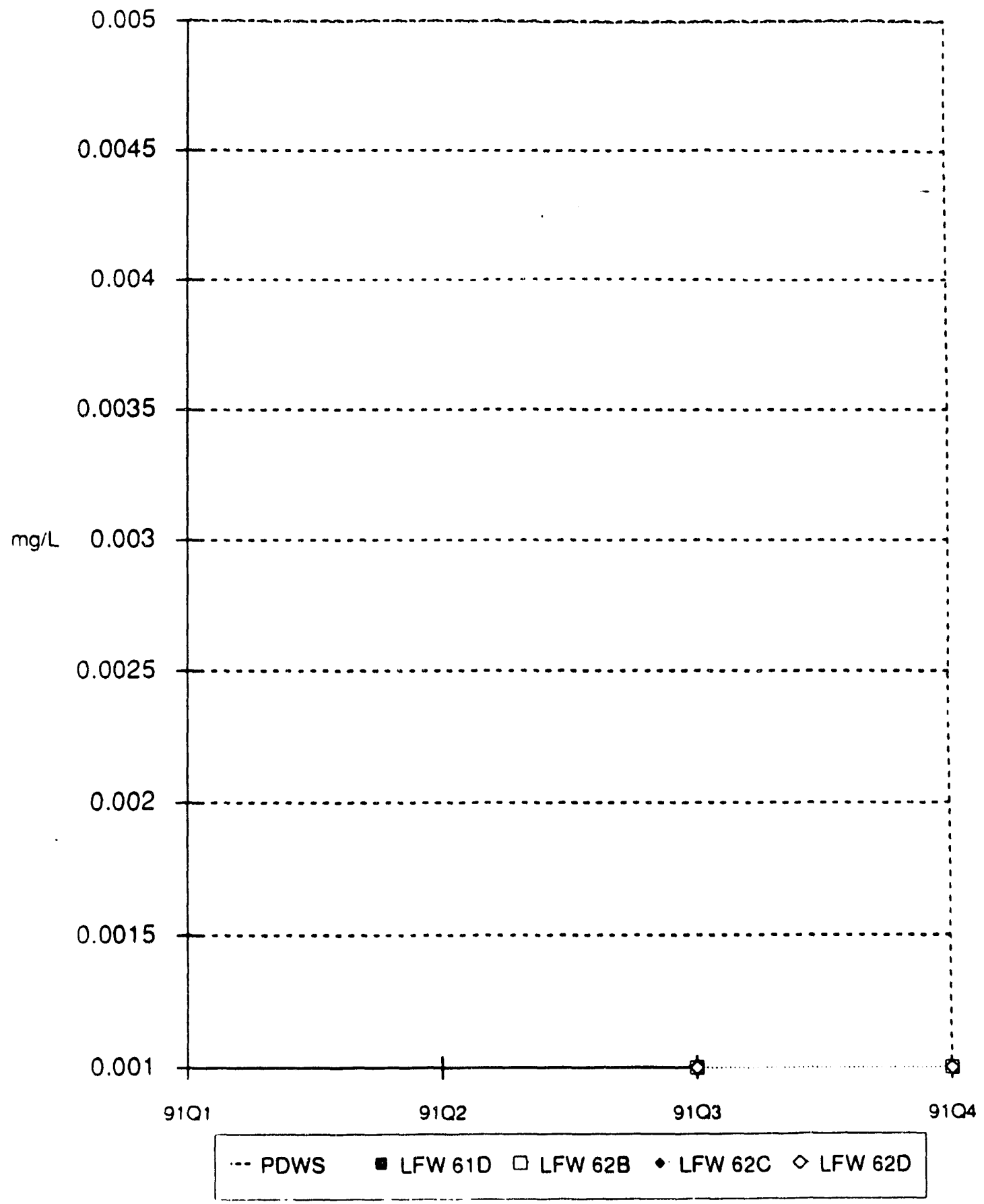


Trichloroethylene in the LFW-Series Monitoring Wells

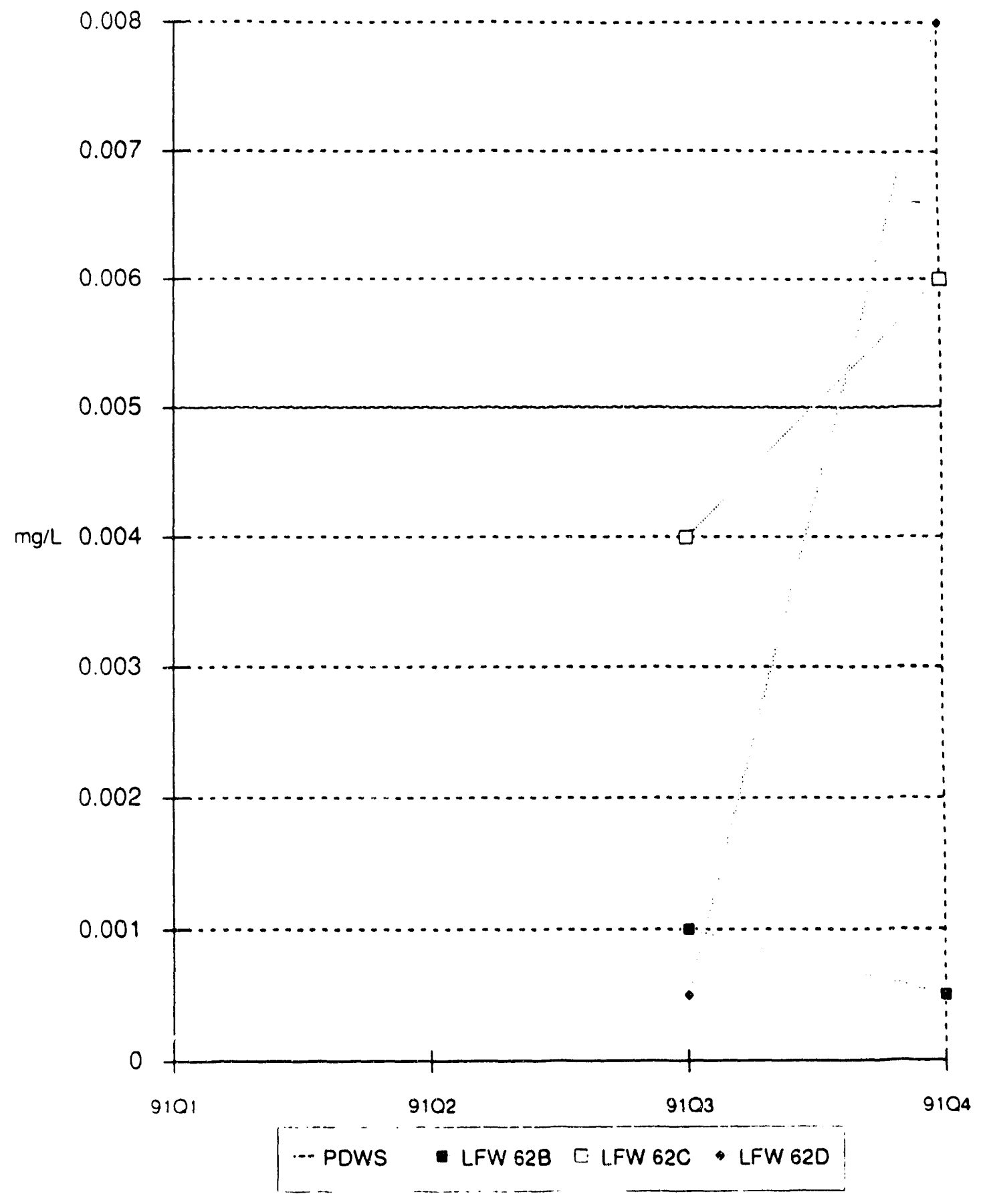


Trichloroethylene in the LFW-Series Monitoring Wells

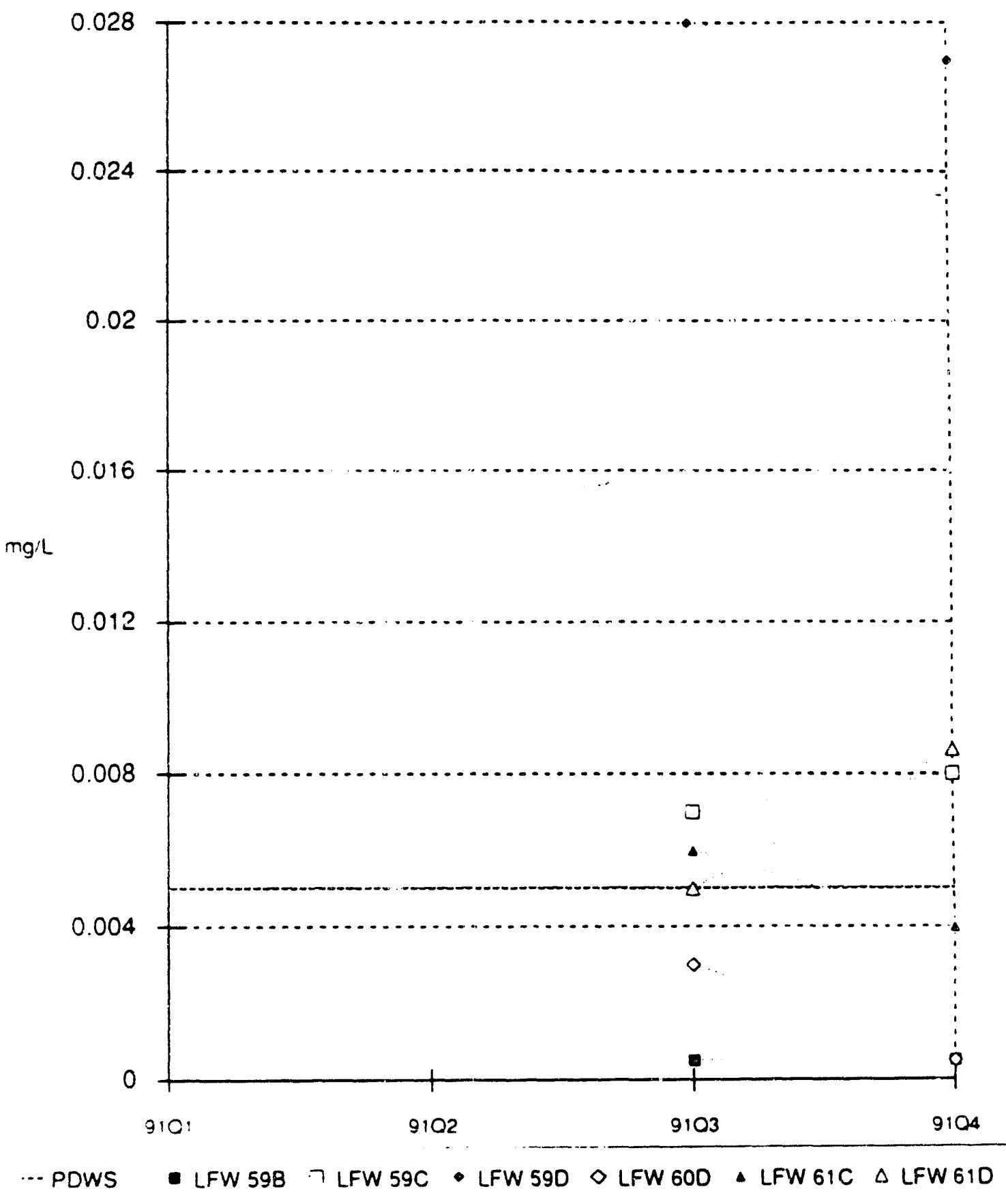


Trichloroethylene in the LFW-Series Monitoring Wolls

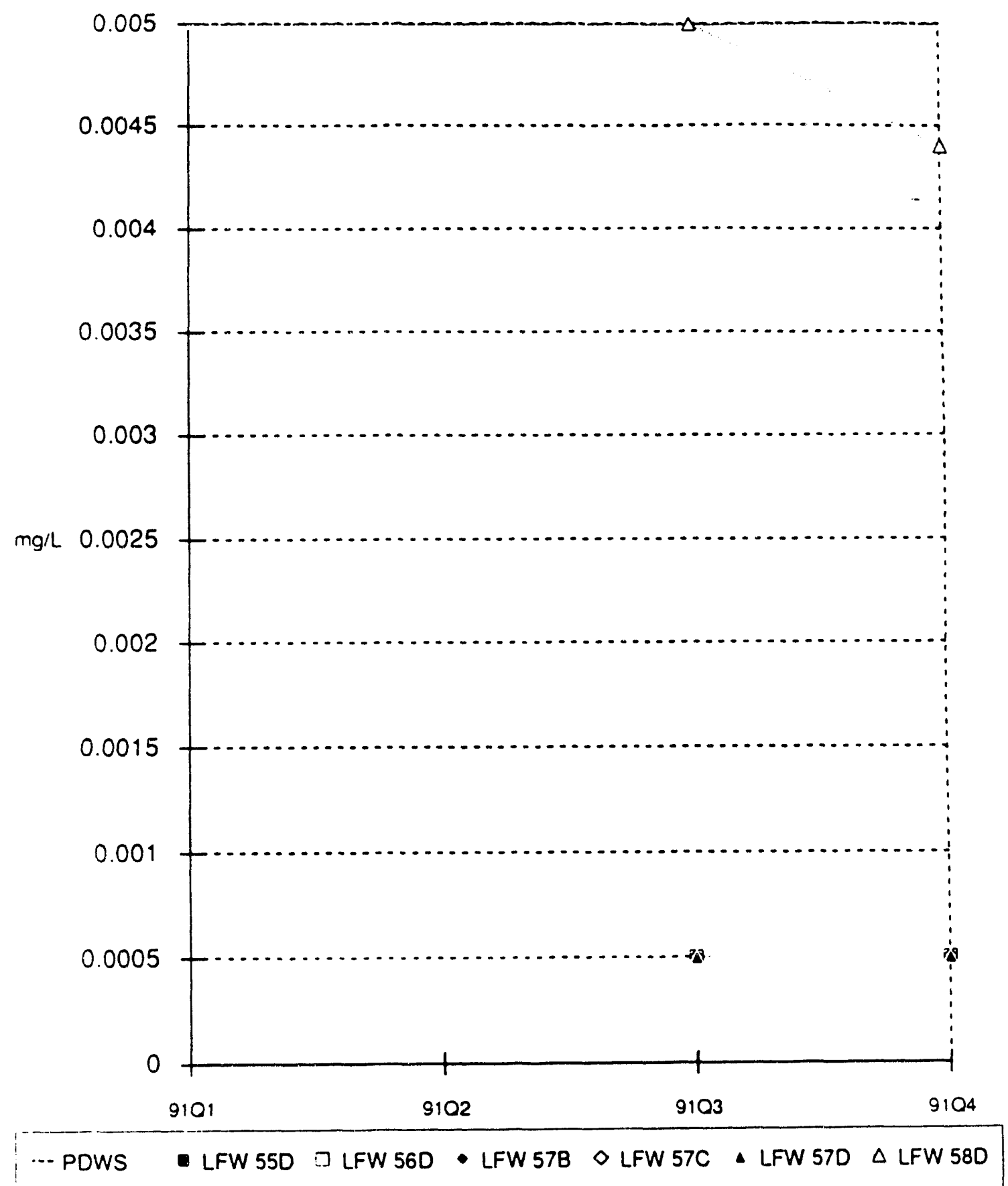


Trichloroethylene in the LFW-Series Monitoring Wells

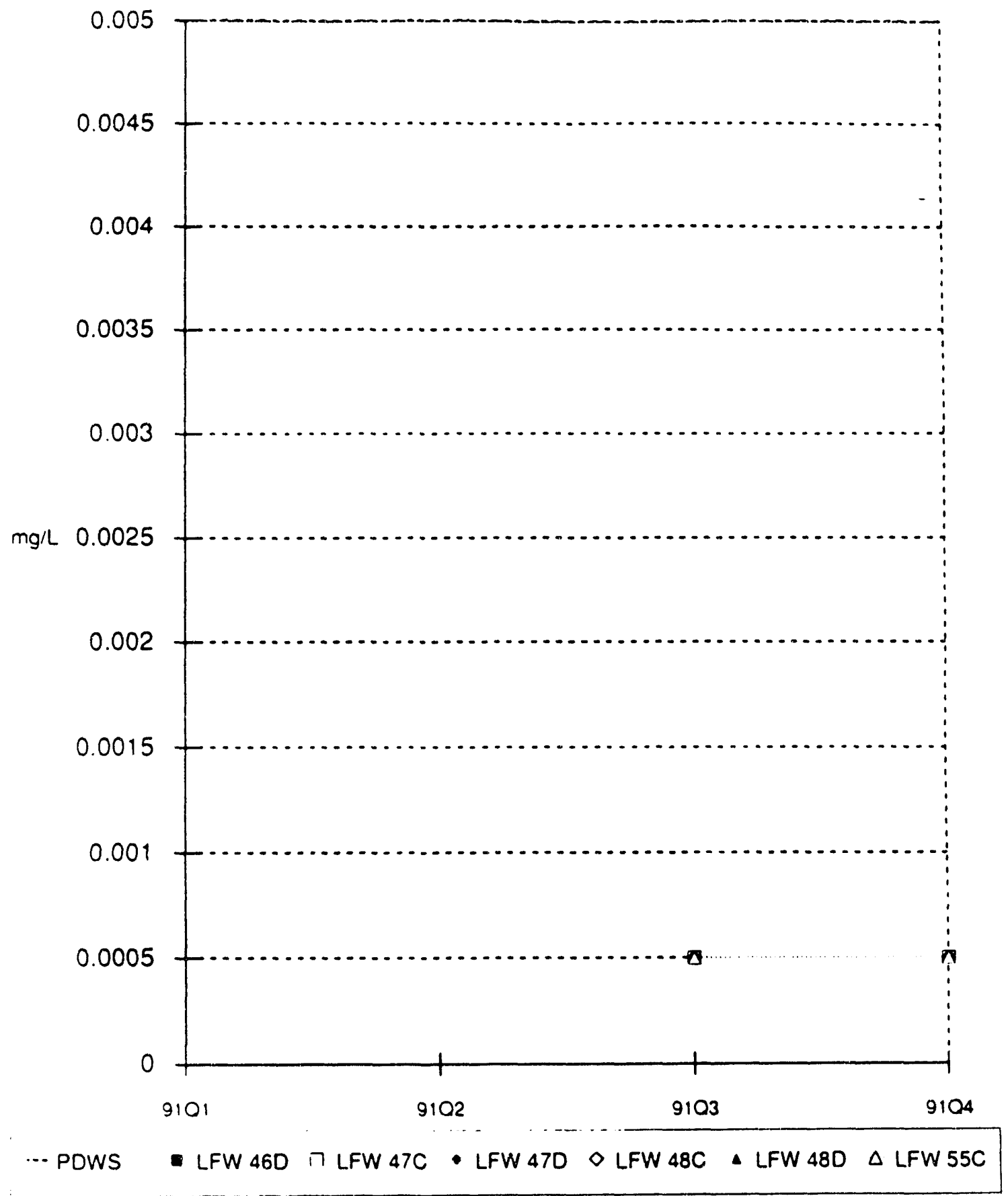


Trichloroethylene in the LFW-Series Monitoring Wells

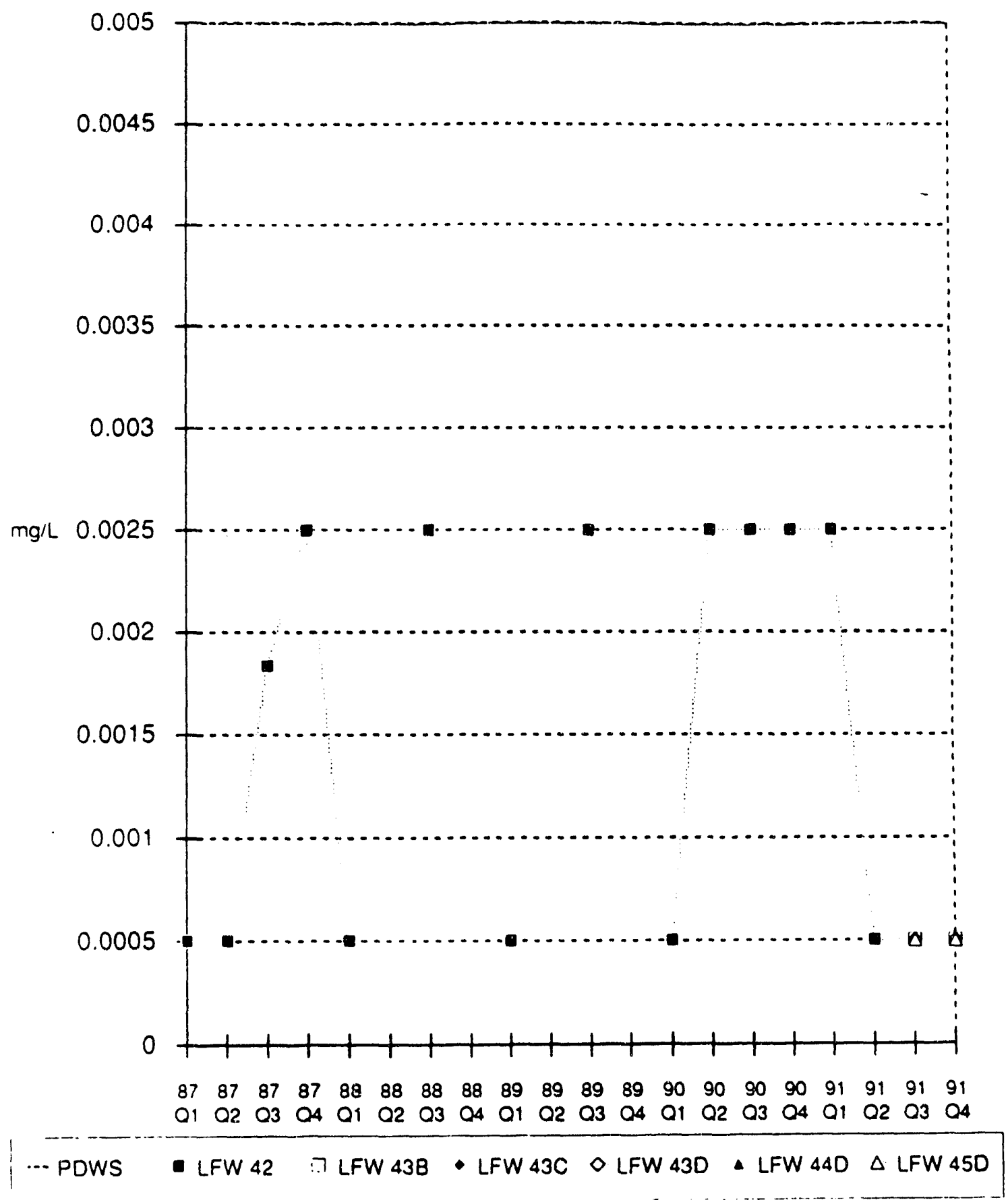


Trichloroethylene in the LFW-Series Monitoring Wells

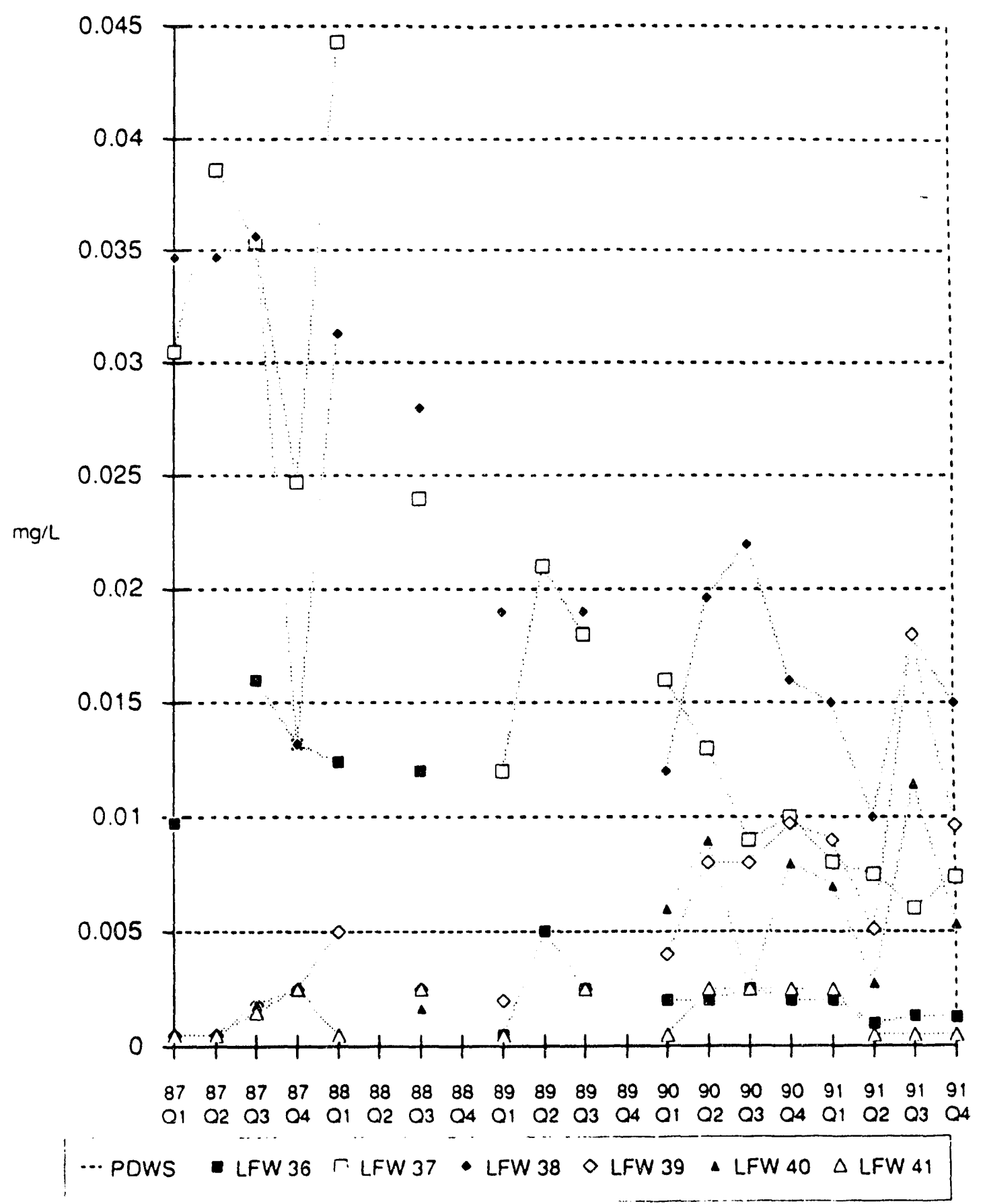


Trichloroethylene in the LFW-Series Monitoring Wells

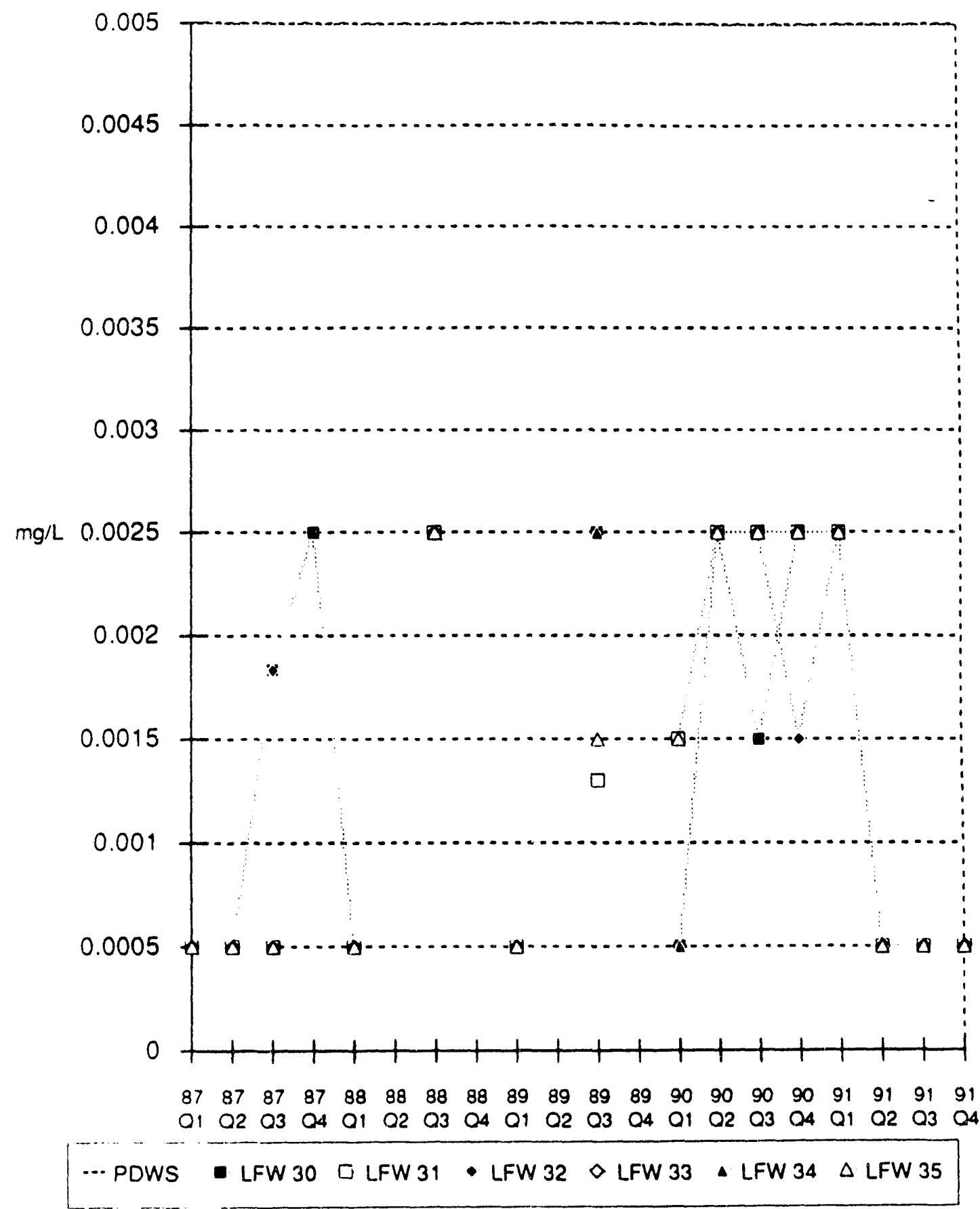


Trichloroethylene in the LFW-Series Monitoring Wells

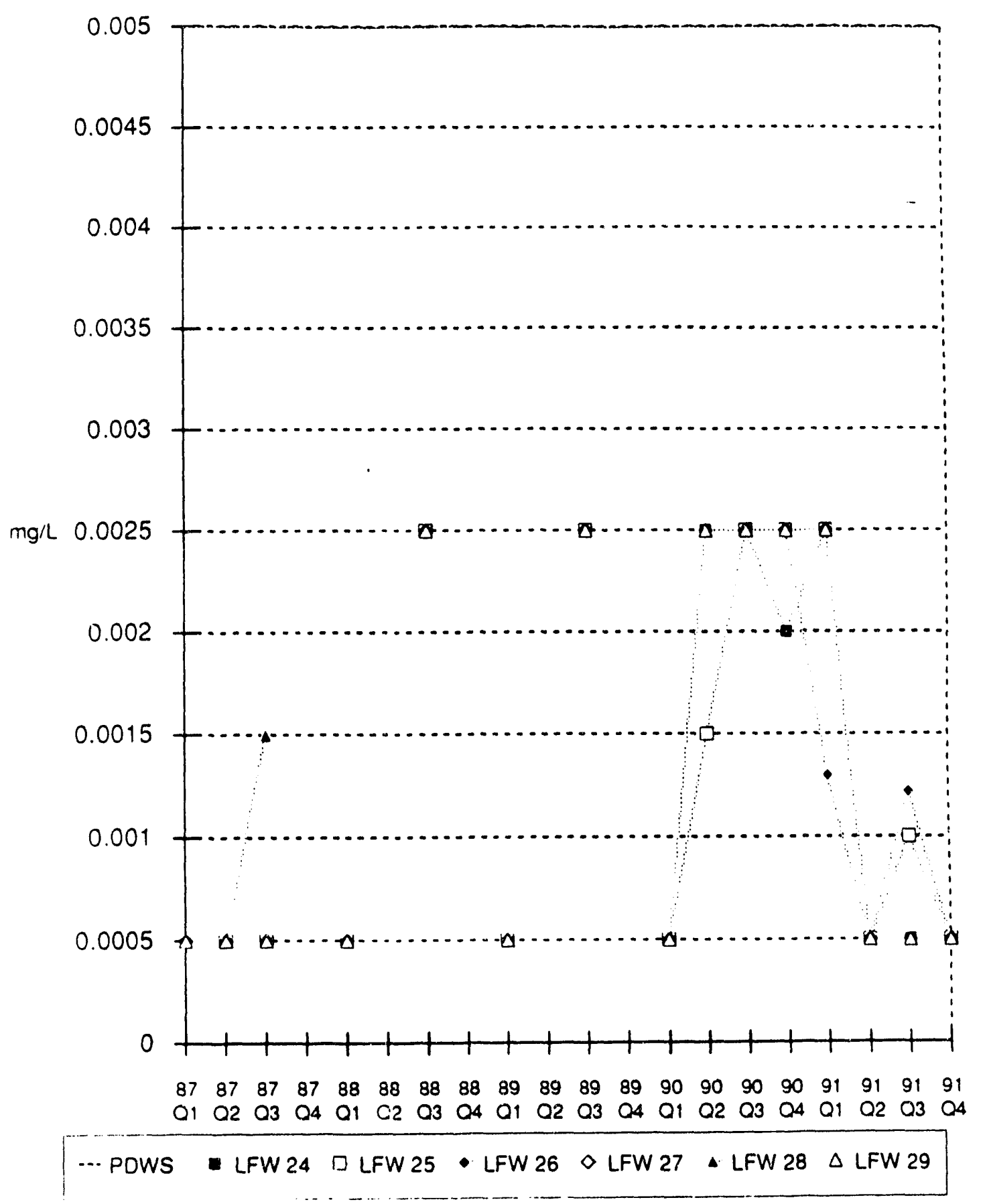


Trichloroethylene in the LFW-Series Monitoring Wells

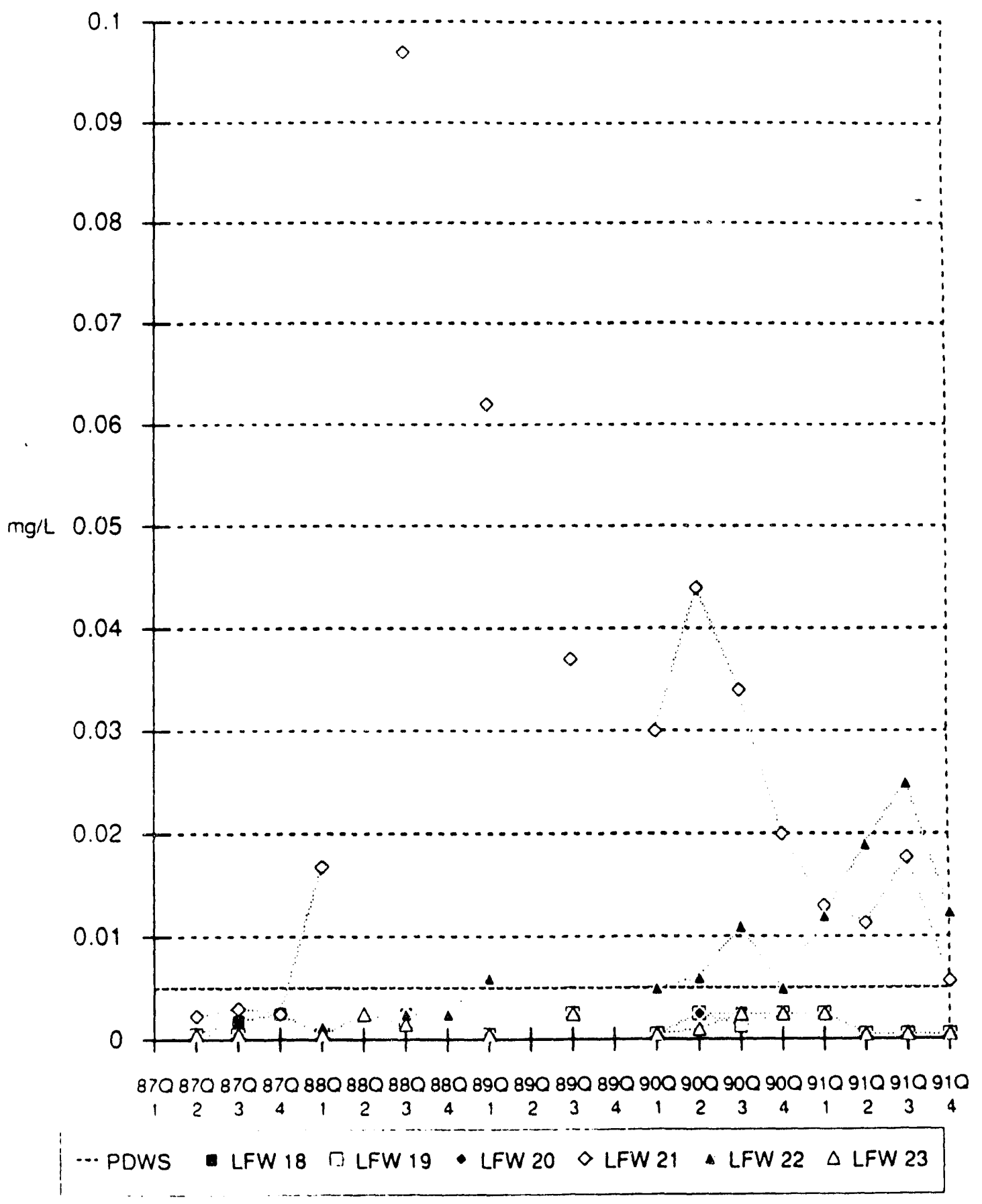


Trichloroethylene in the LFW-Series Monitoring Wells

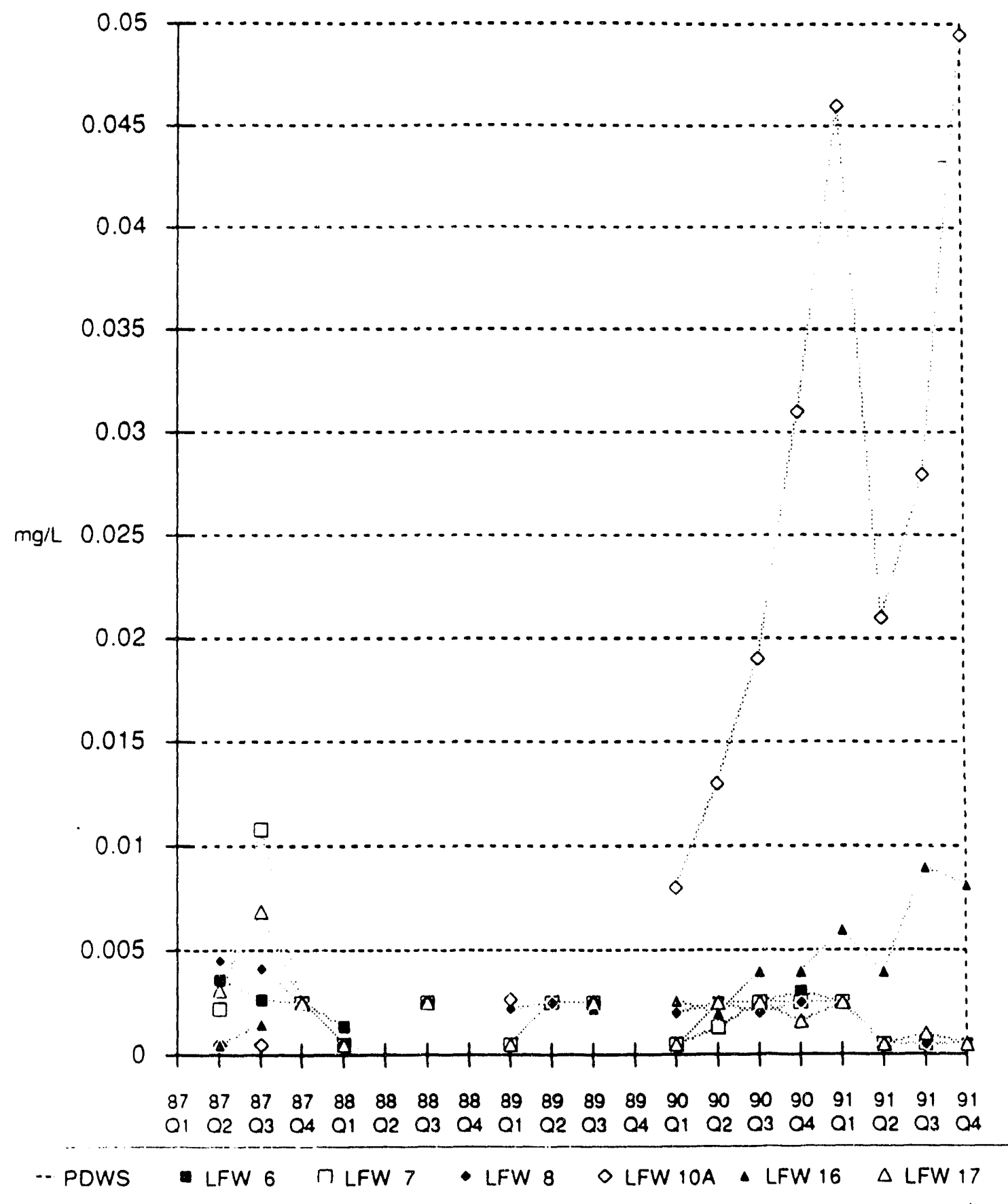




\section{Vinyl Chloride in the LFW-Series Monitoring Wells}

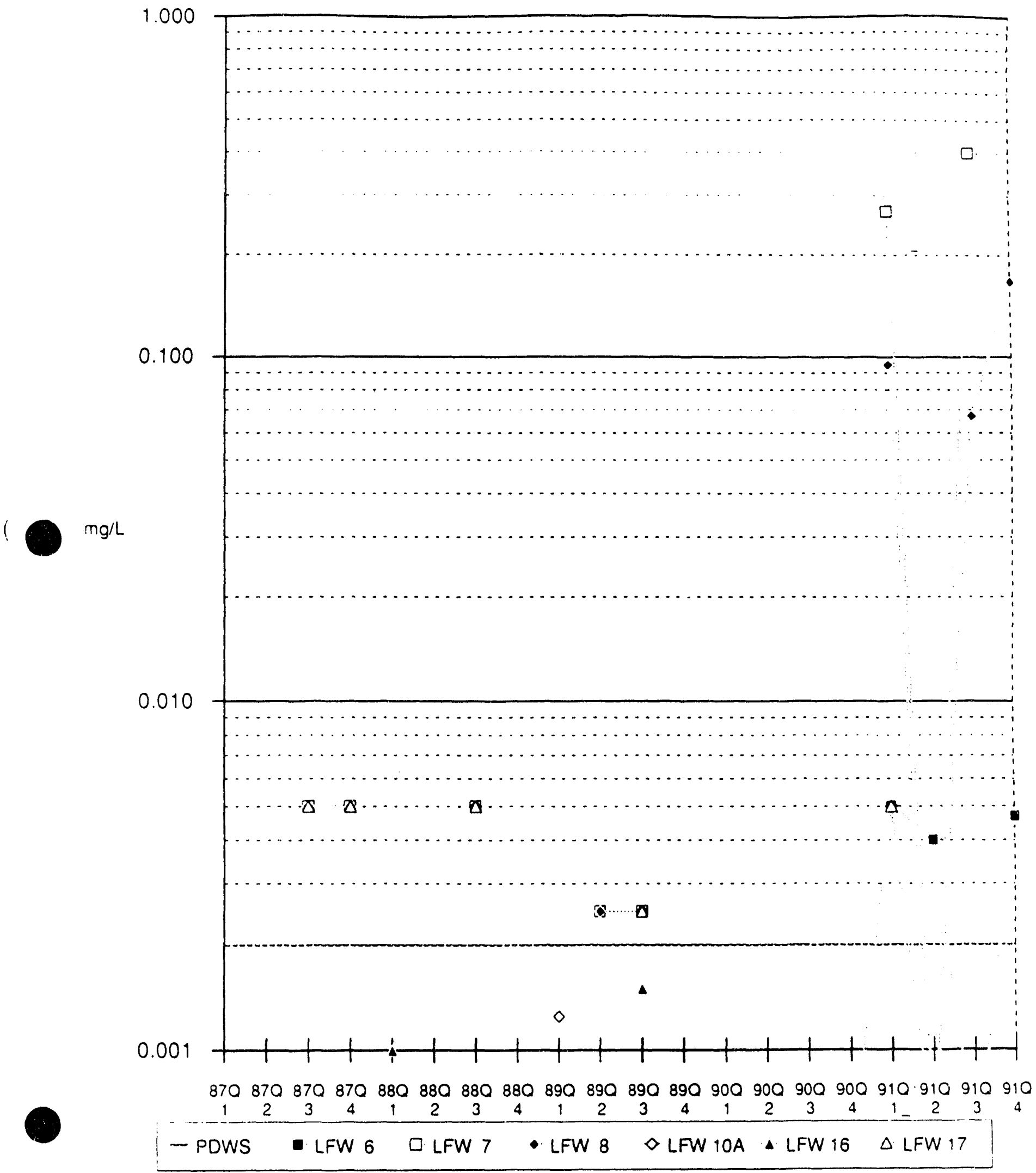


Vinyl Chloride in the LFW-Series Monitoring Wells

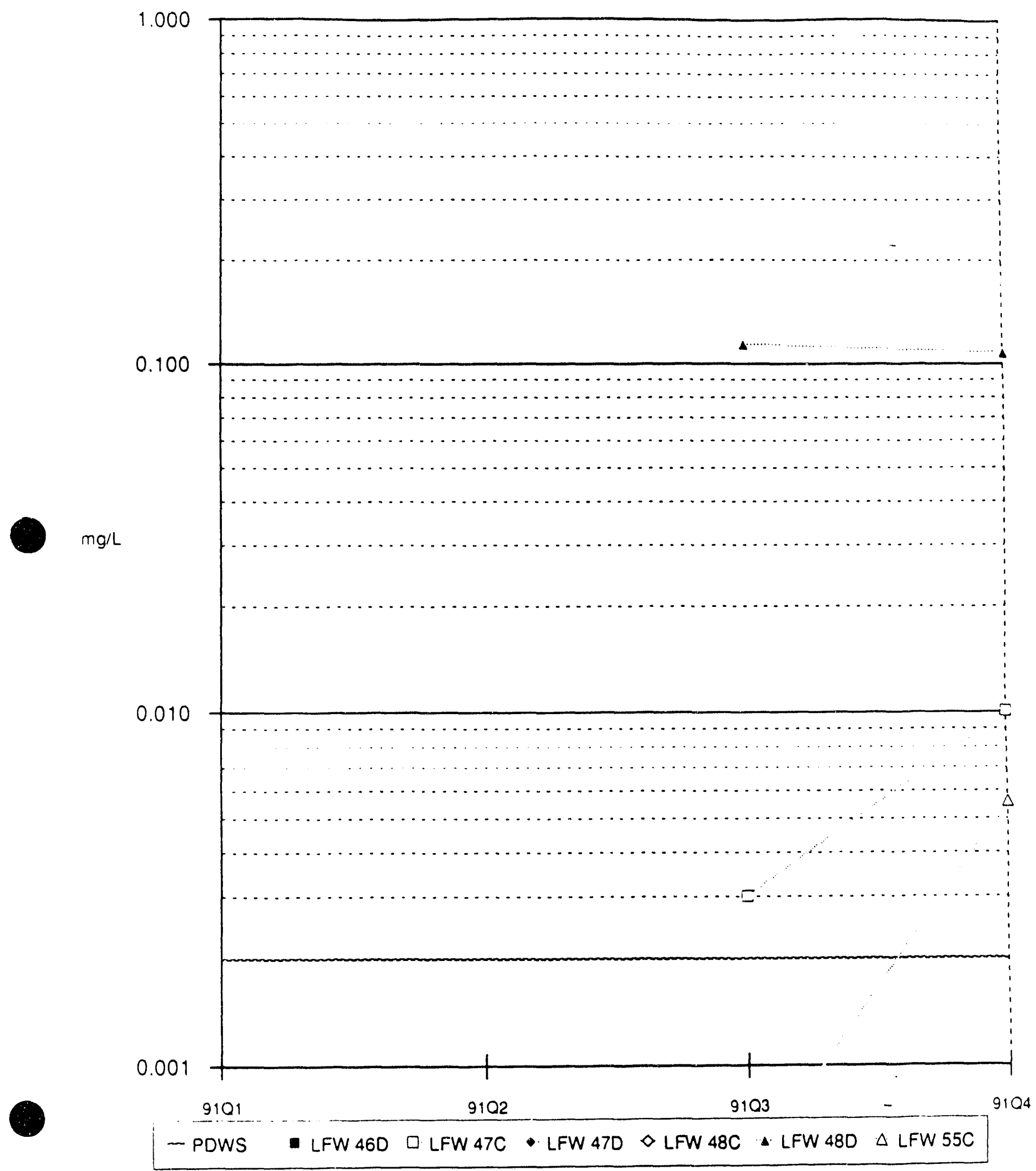


Vinyl Chloride in the LFW-Series Monitoring Wells

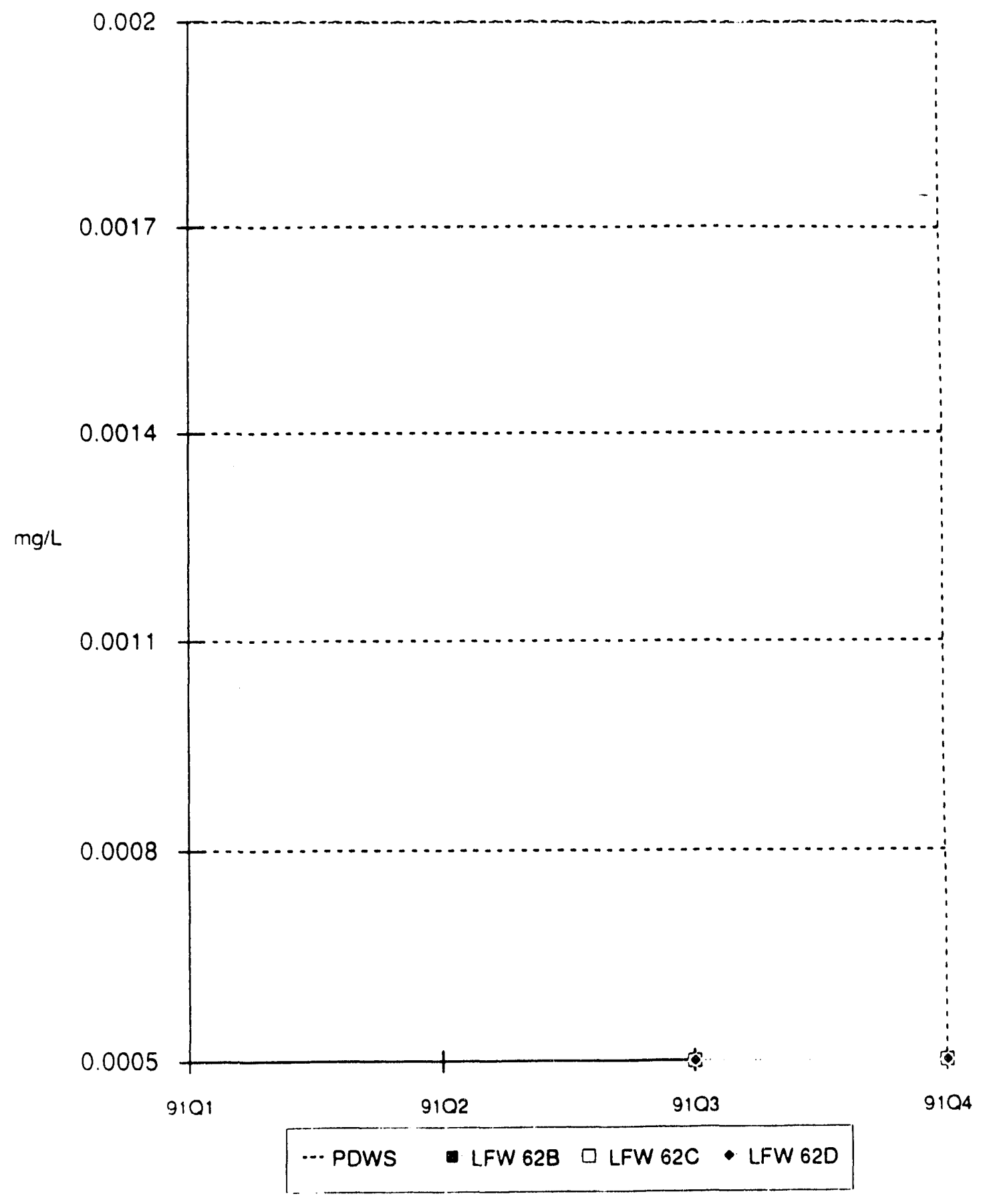


Vinyl Chloride in the LFW-Series Monitoring Wells

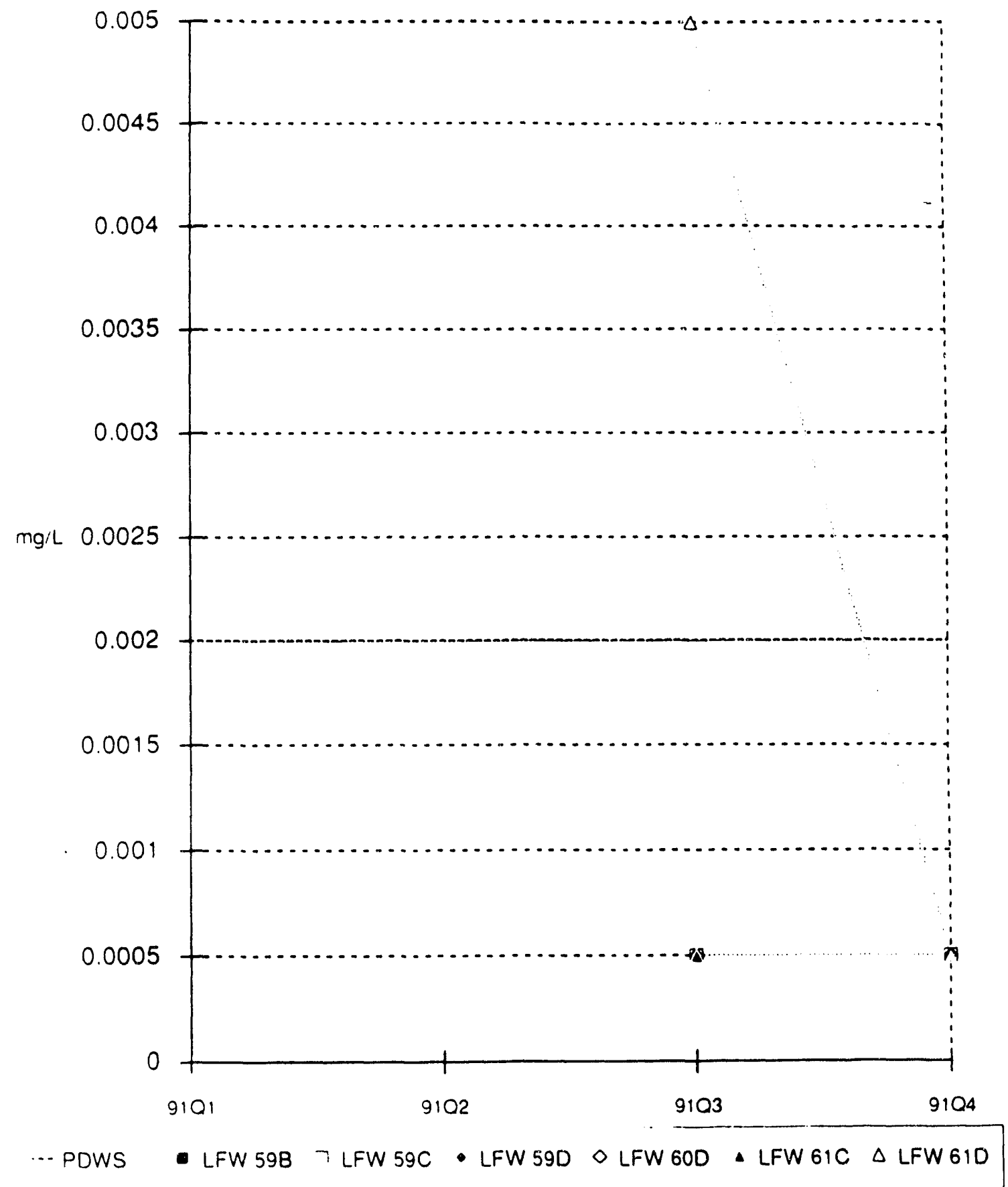


Vinyl Chloride in the LFW-Series Monitoring Wells

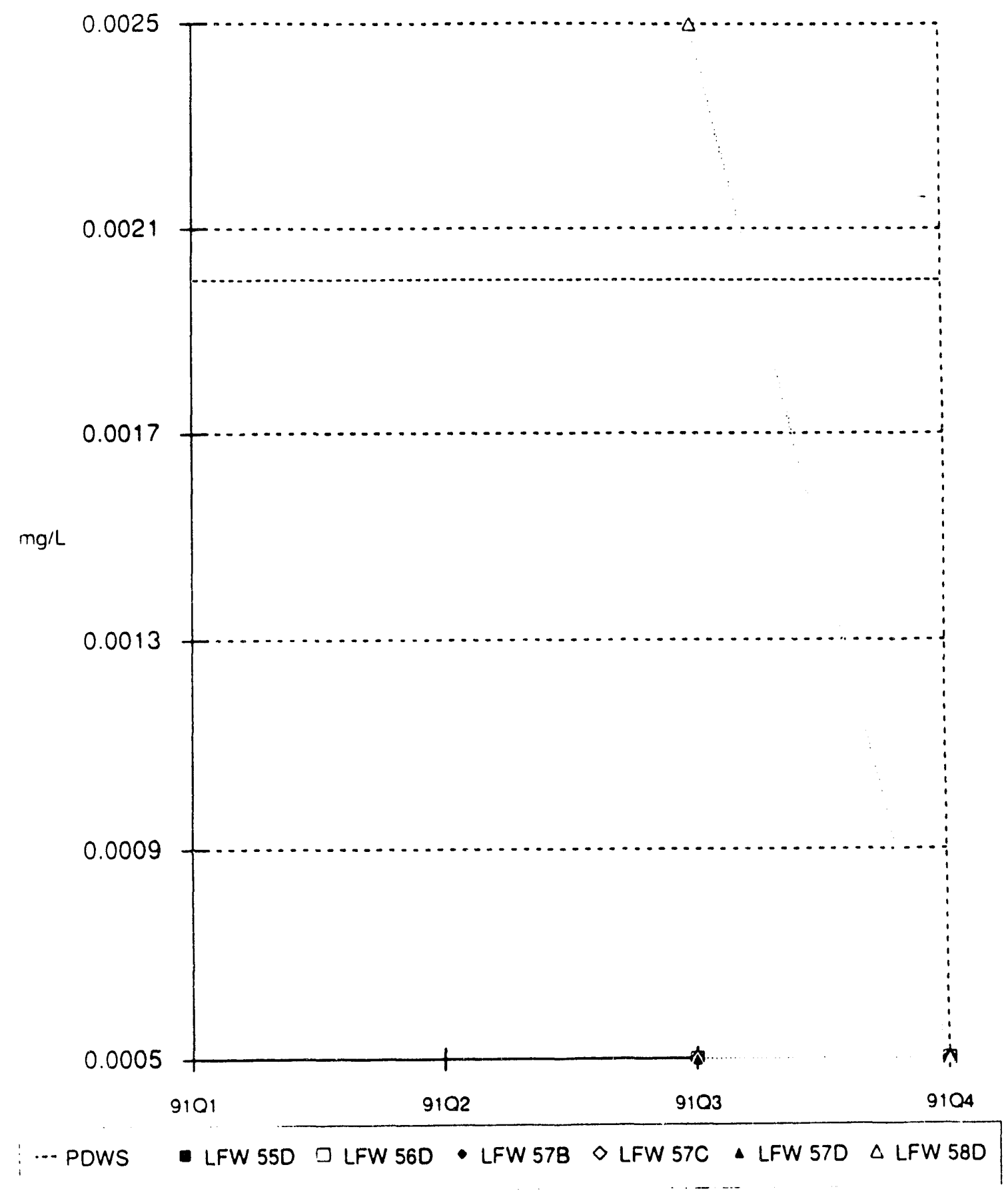


Vinyl Chloride in the LFW-Series Monitoring Wells

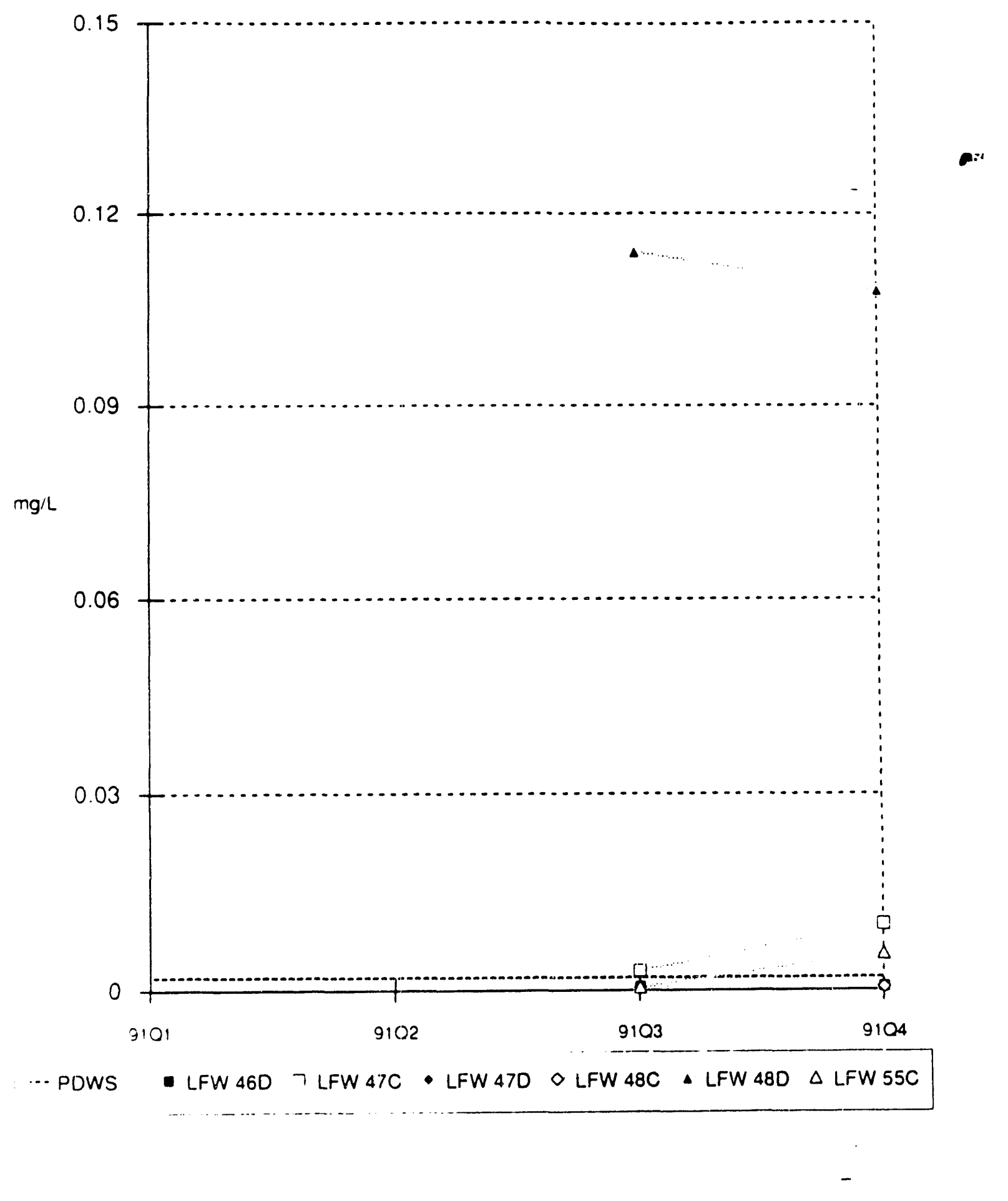


Vinyl Chloride in the LFW-Series Monitoring Wells

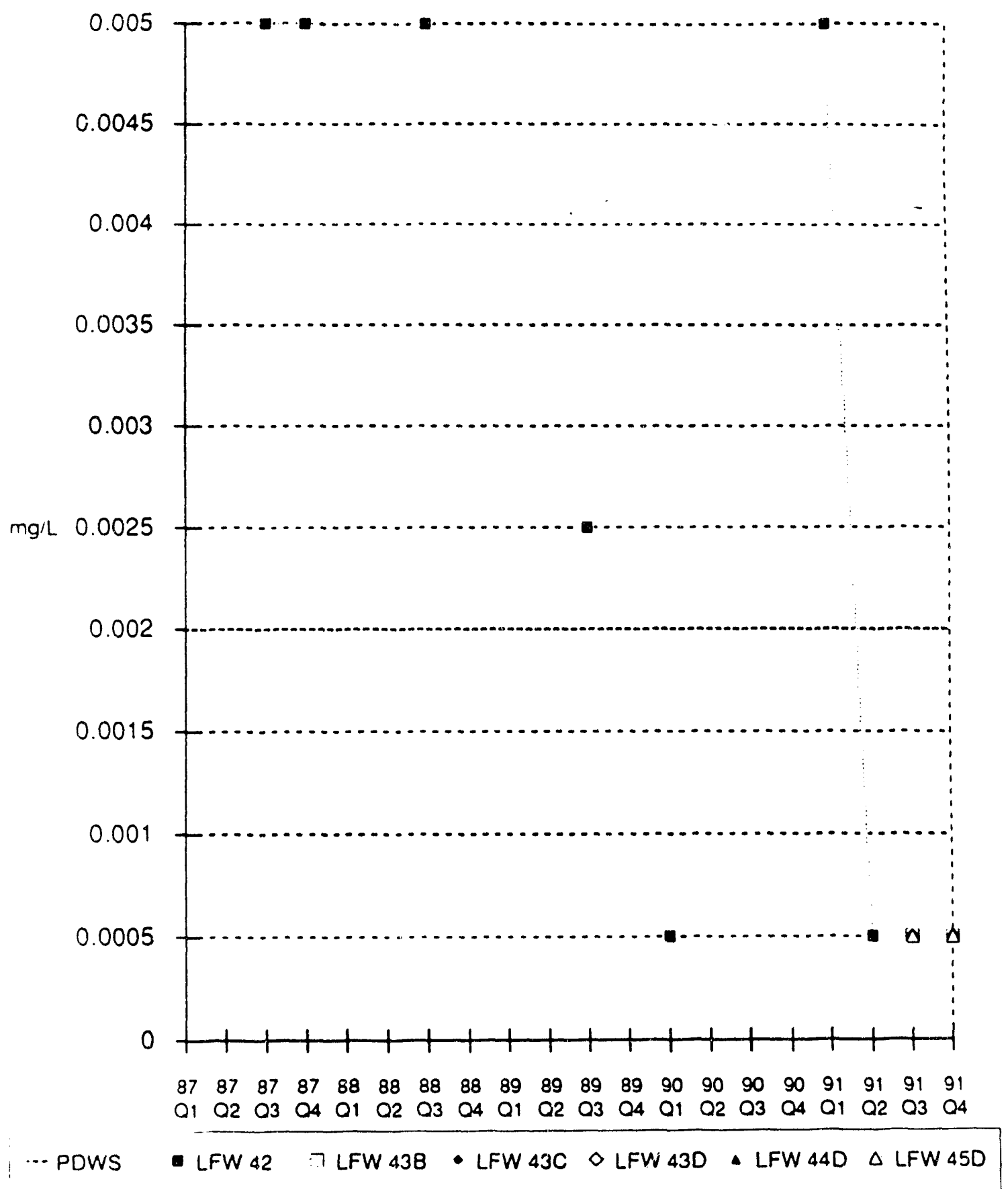




\section{Vinyl Chloride in the LFW-Series Monitoring Wells}

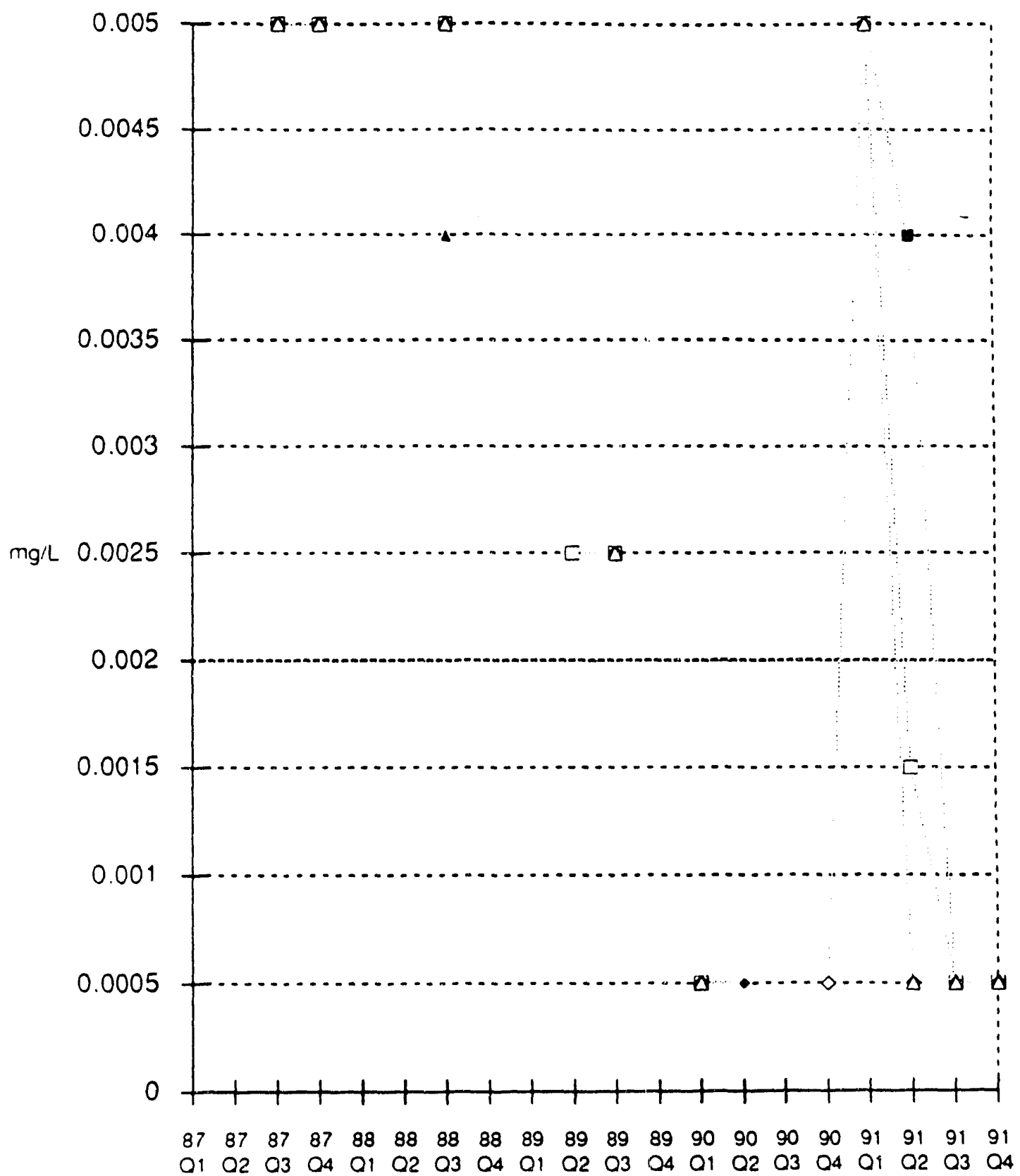

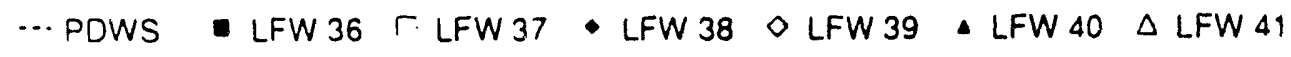




\section{Vinyl Chloride in the LFW-Series Monitoring Wells}

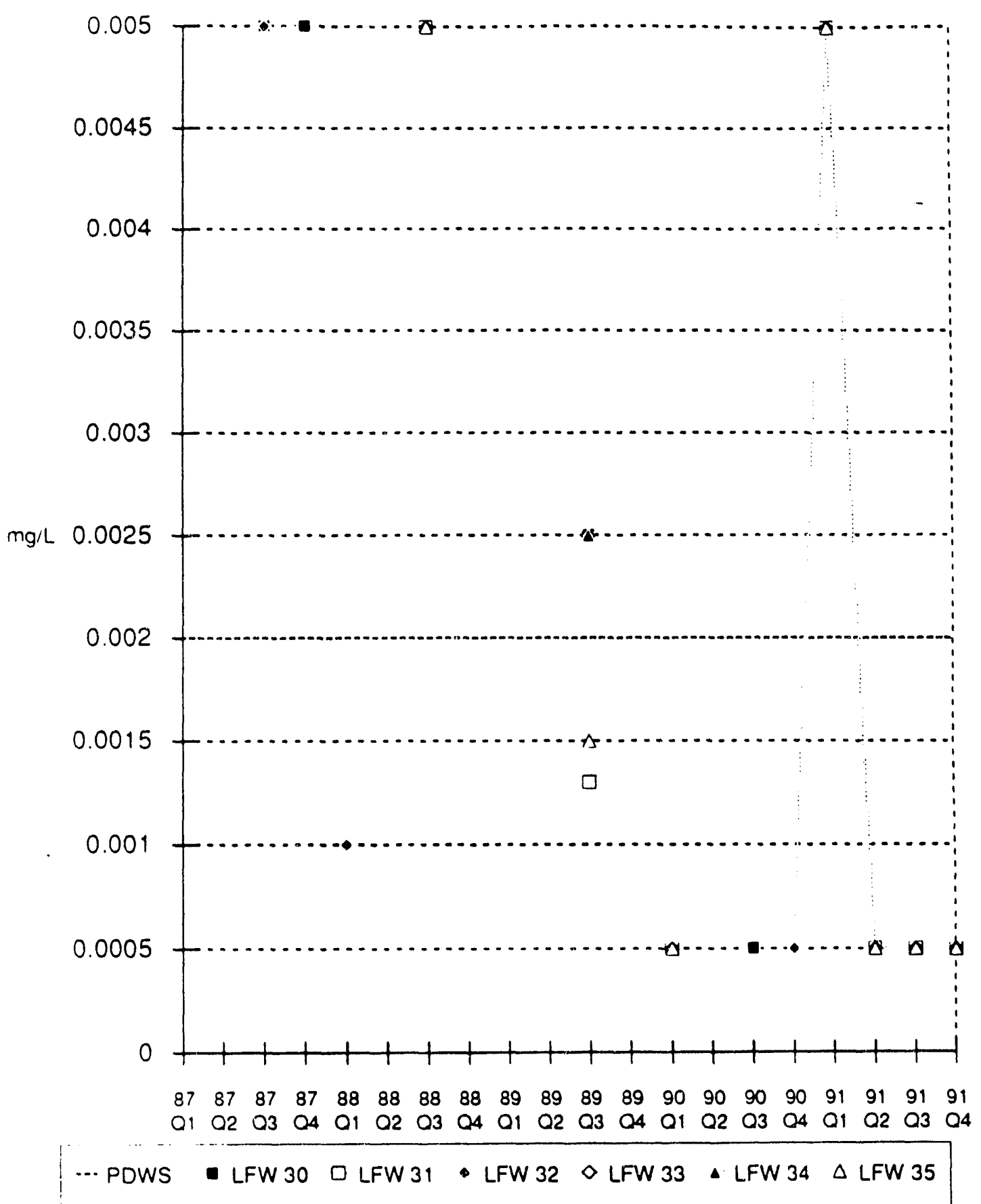




\section{Vinyl Chloride in the LFW-Series Monitoring Wells}

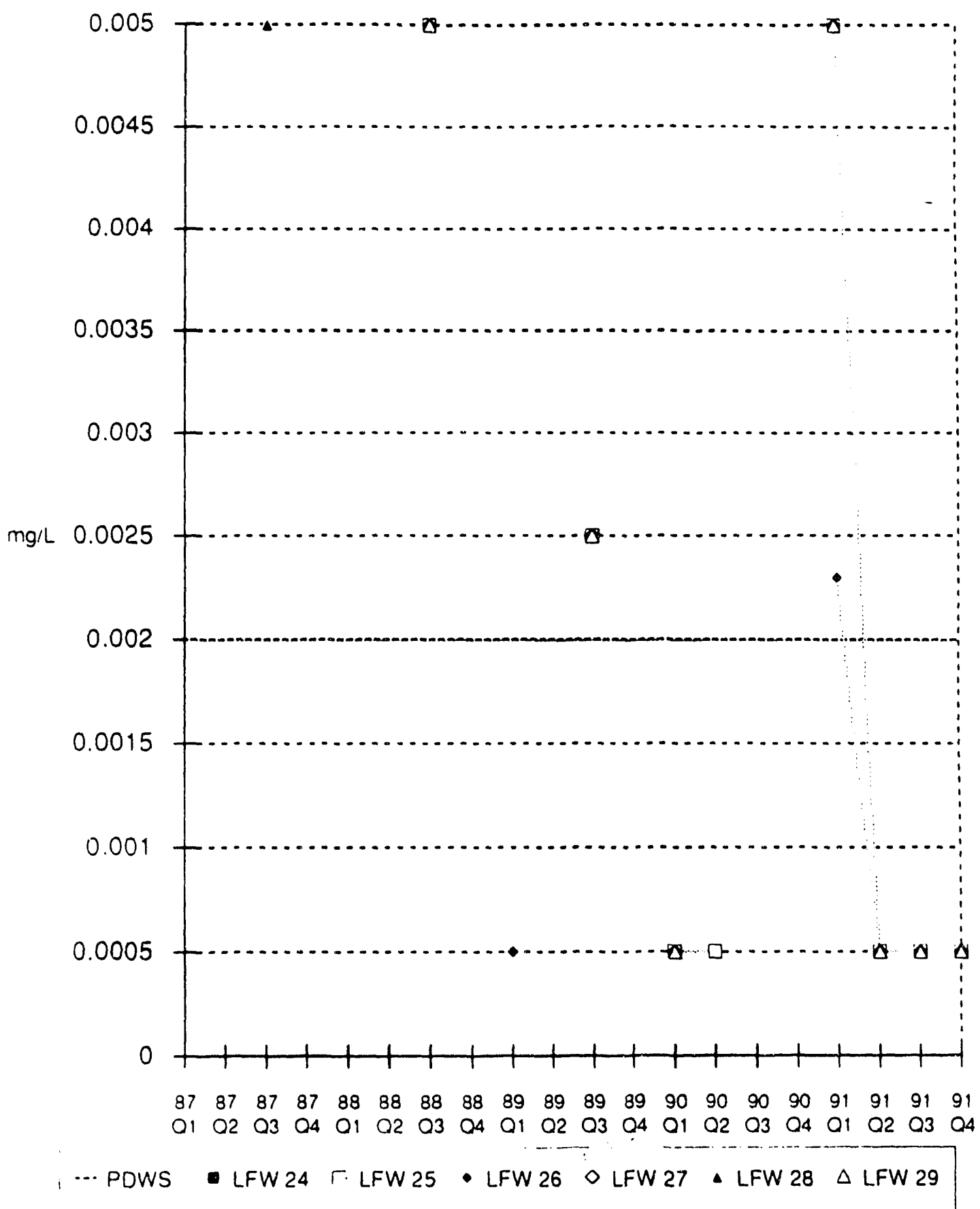




\section{Vinyl Chloride in the LFW-Series Monitoring Wells}

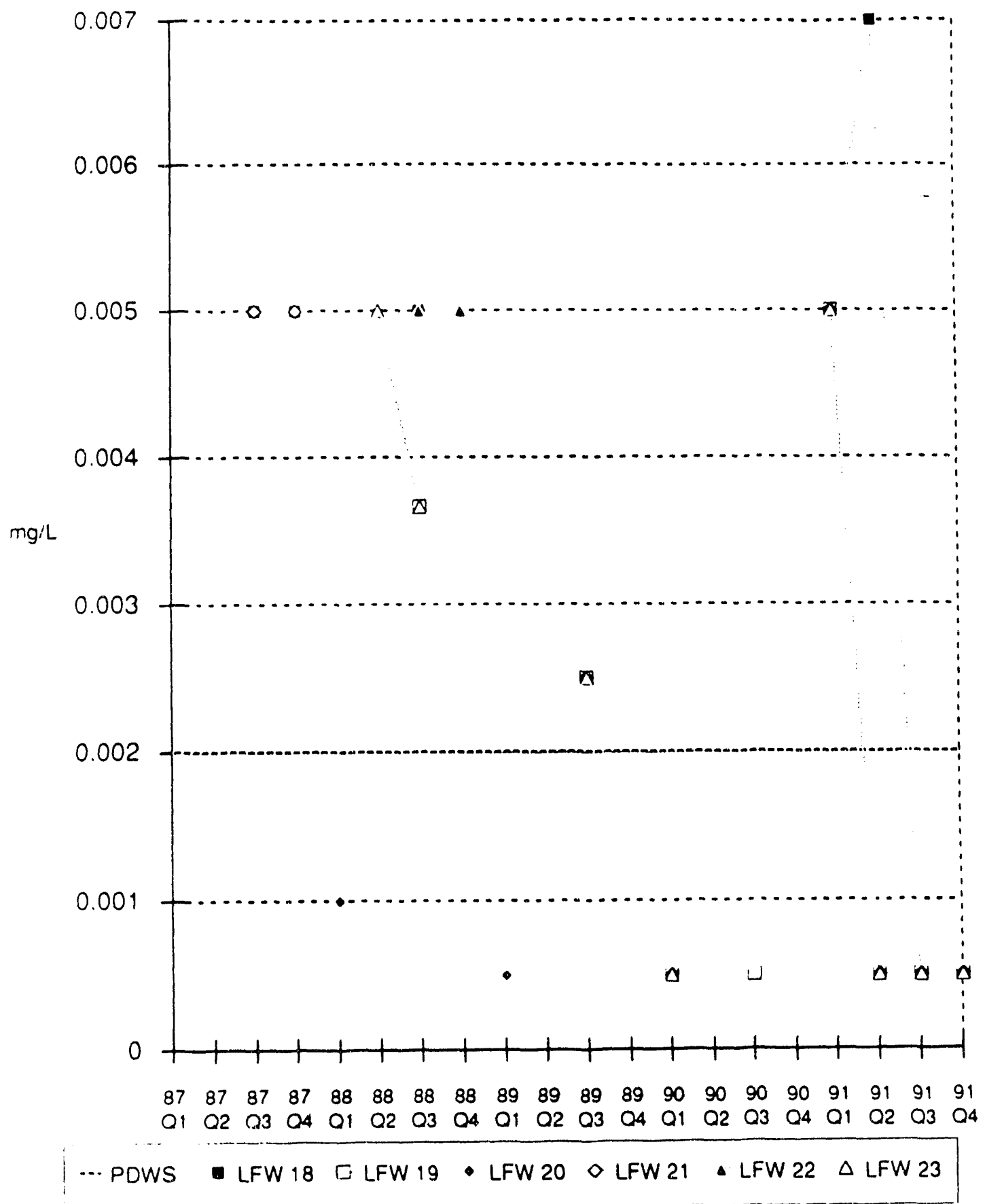


Vinyl Chloride in the LFW-Series Monitoring Wells

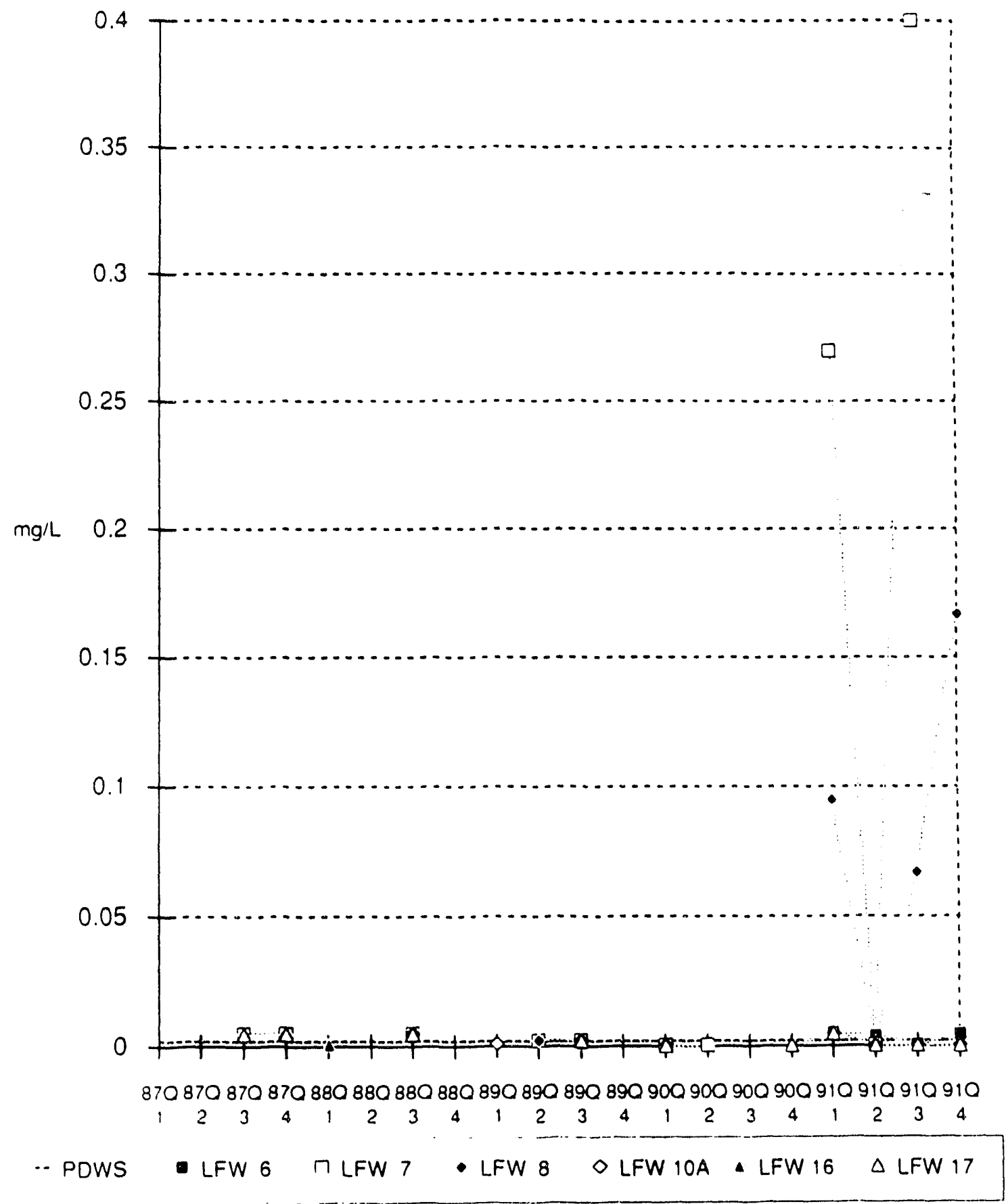


Tetrachloroethylene In the LFW-Series Monitoring Wells

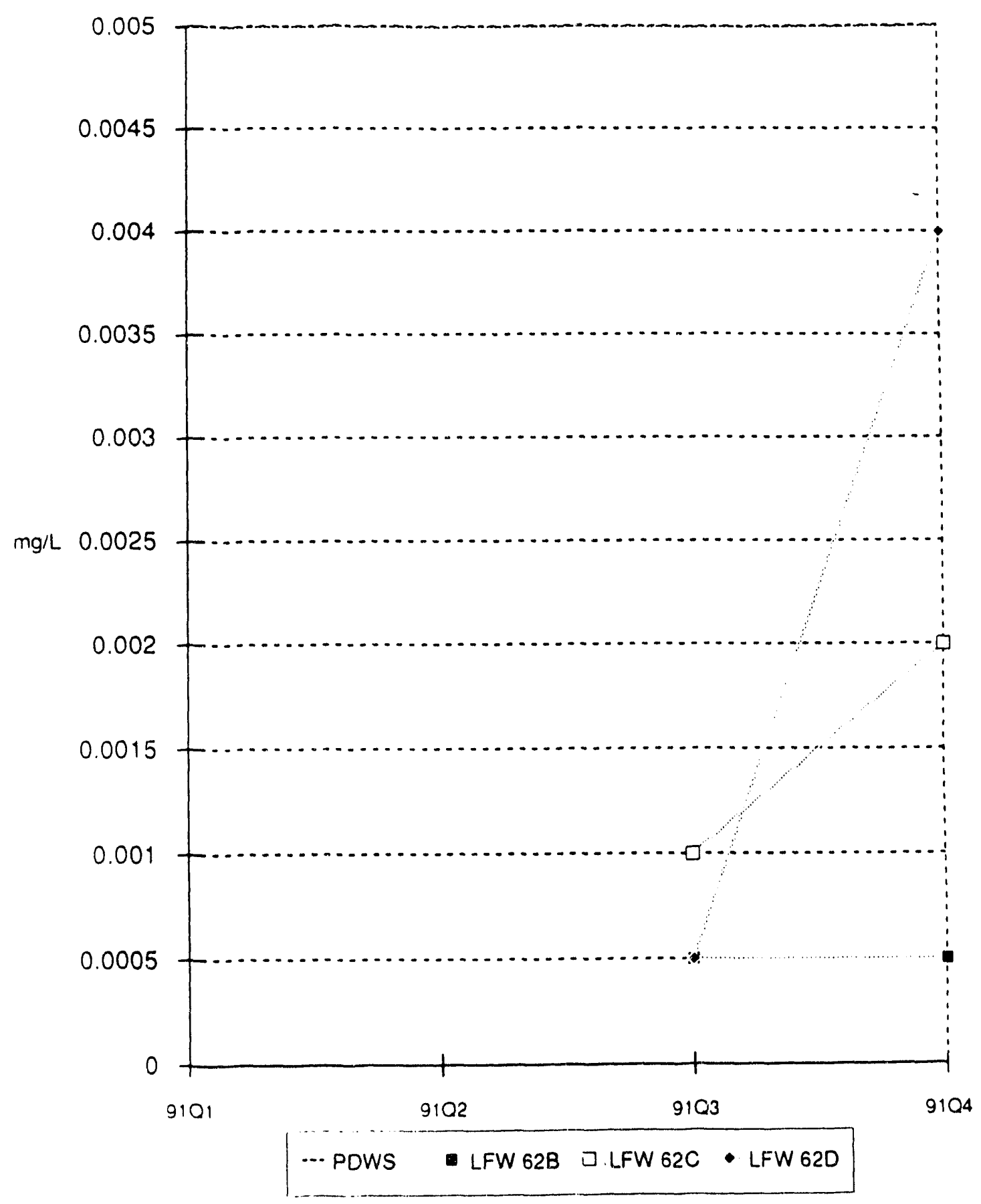


Tetrachloroethylene in the LFW-Series Monitoring Wells

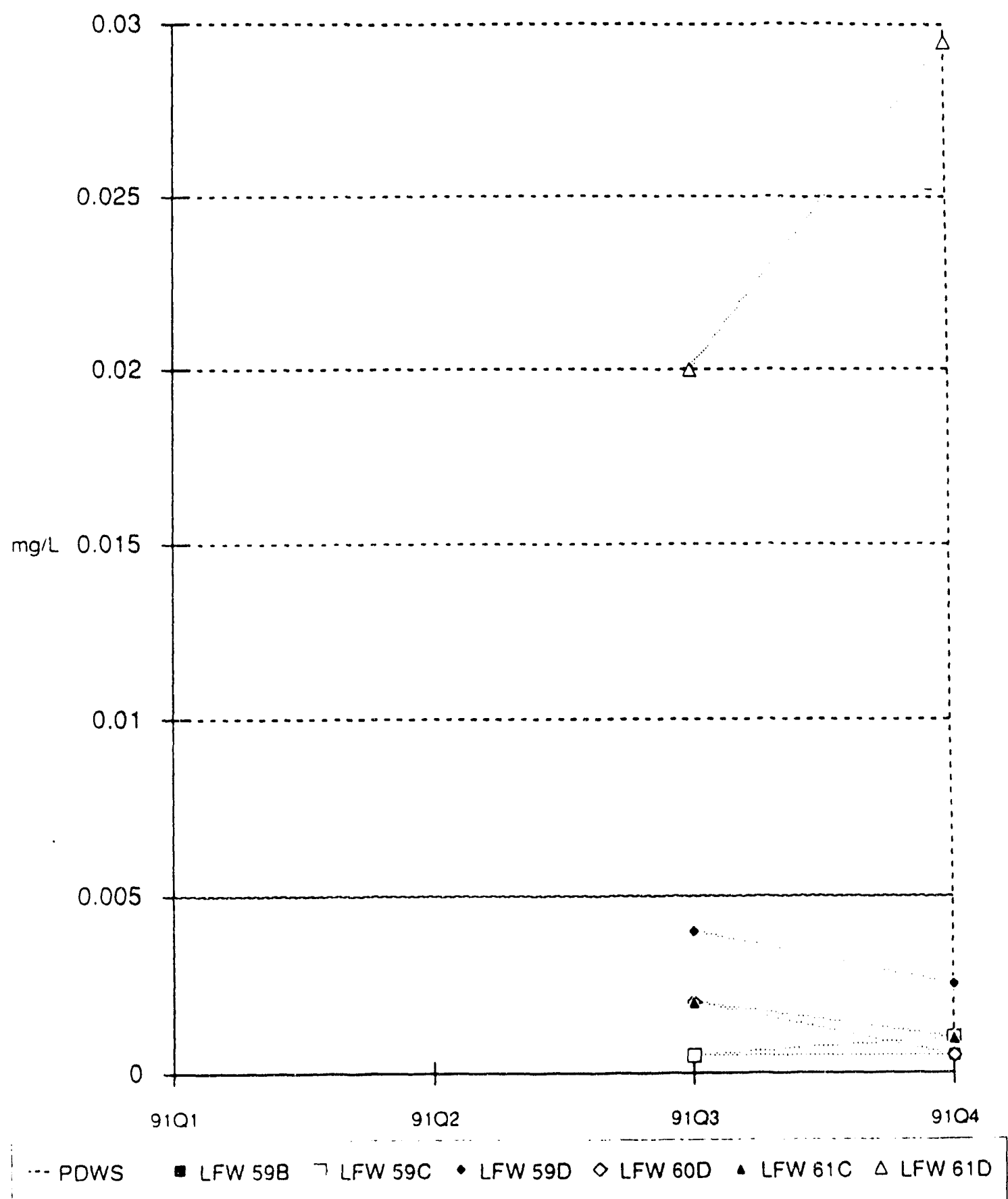


Tetrachloroethylene in the LFW-Series Monitoring Wells

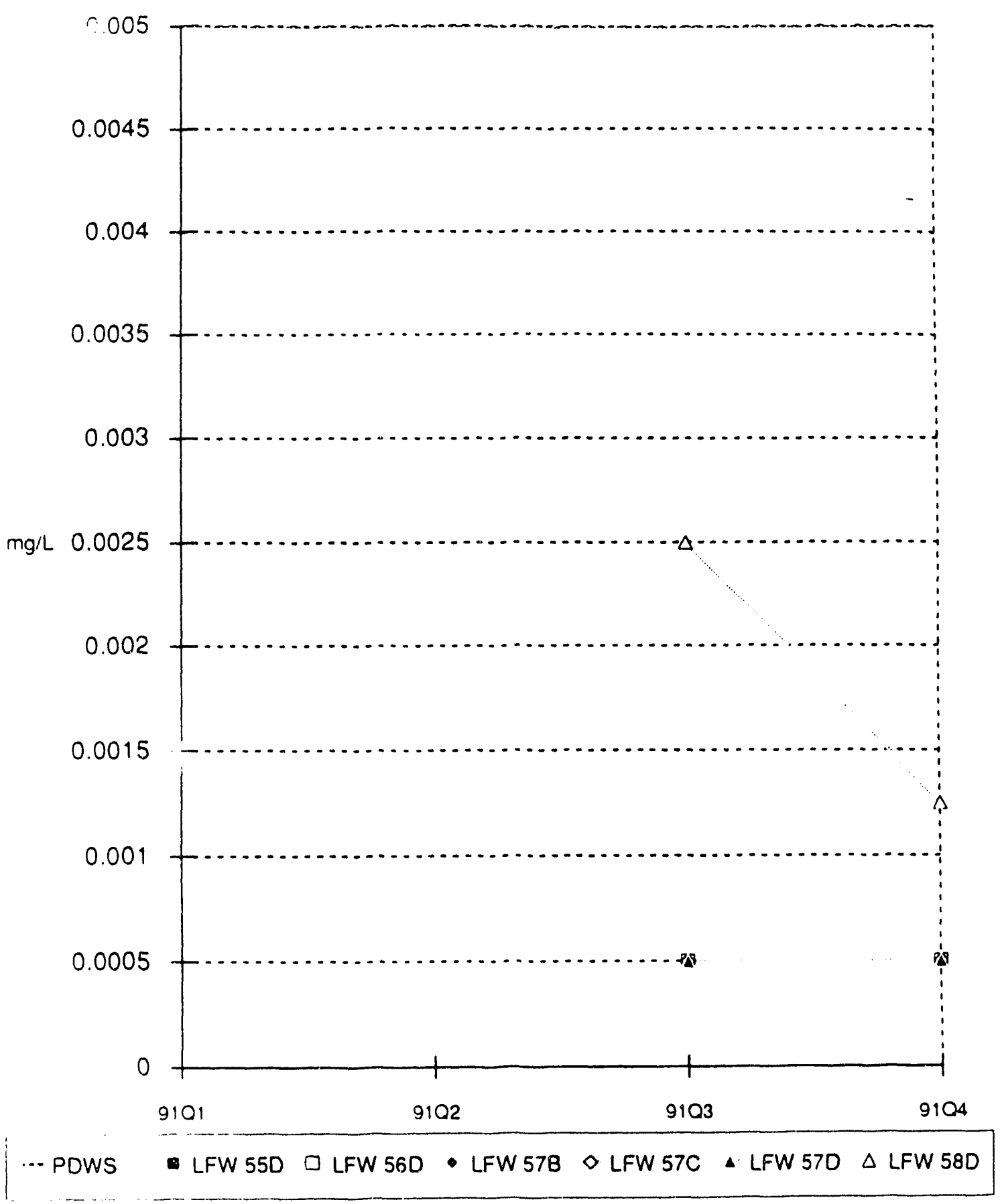


Tetrachloroethylene in the LFW-Series Monitoring Wells

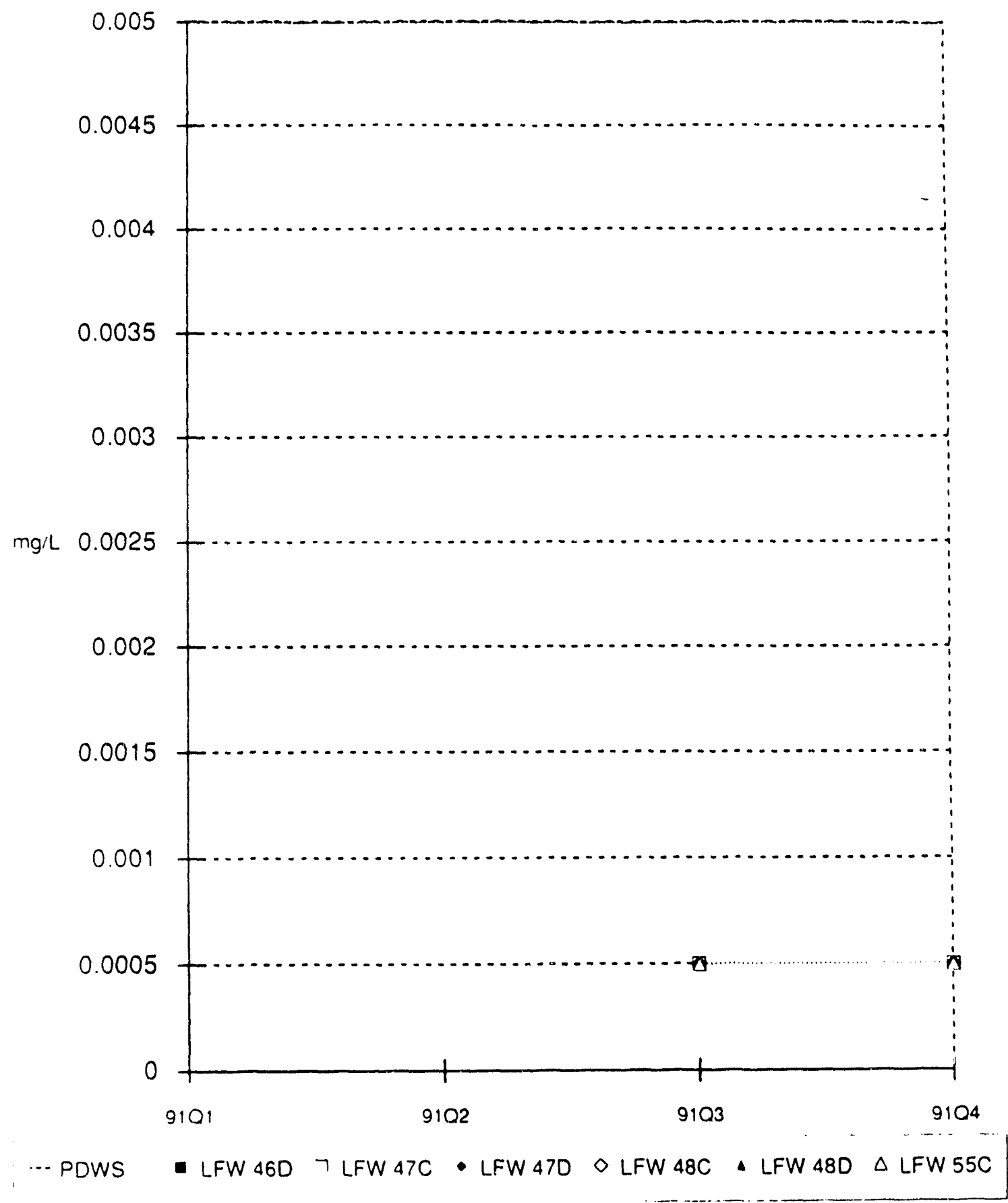


Tetrachloroethylene in the LFW-Series Monitoring Wells

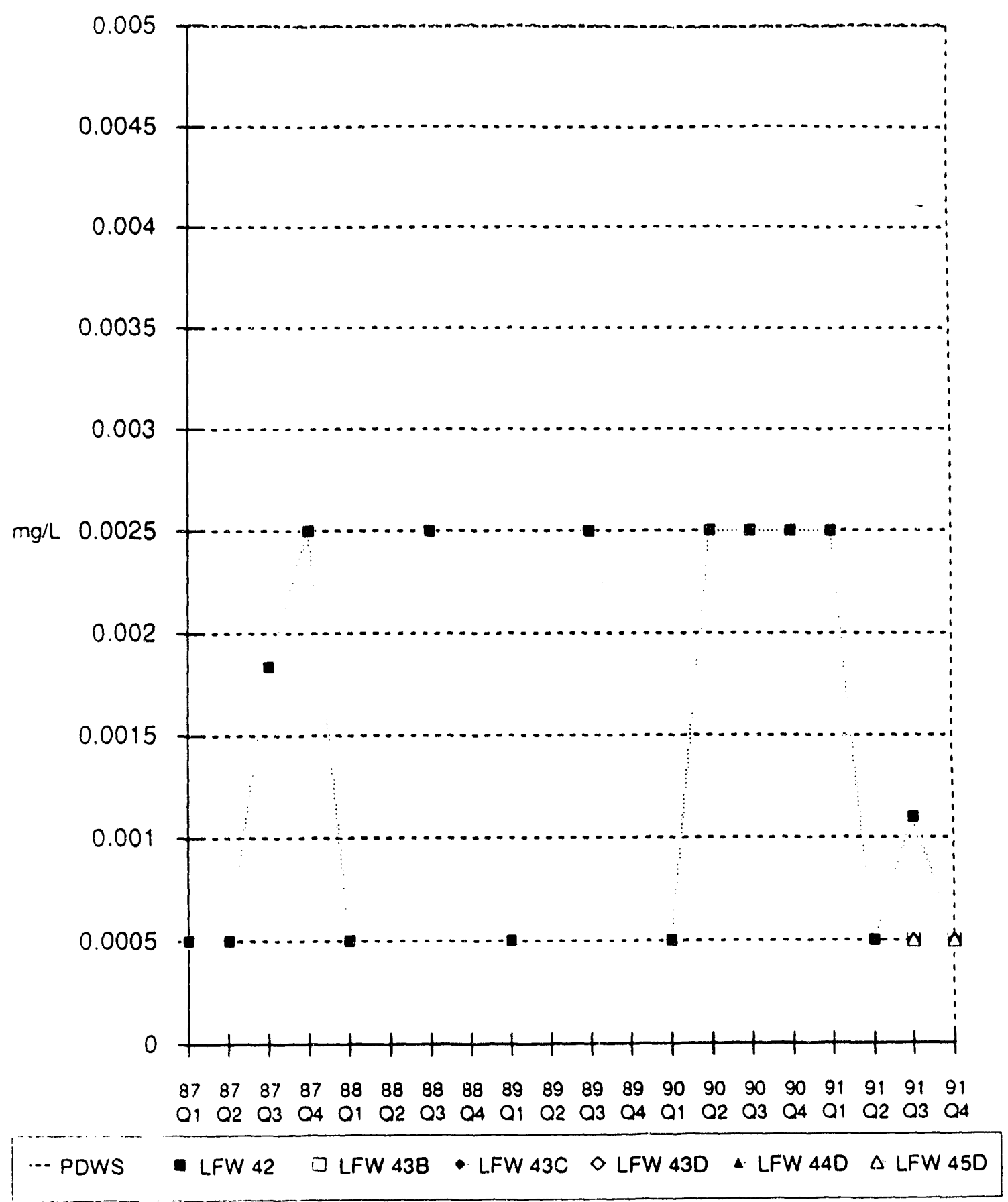


Tetrachloroethylene in the LFW-Series Monitoring Wells

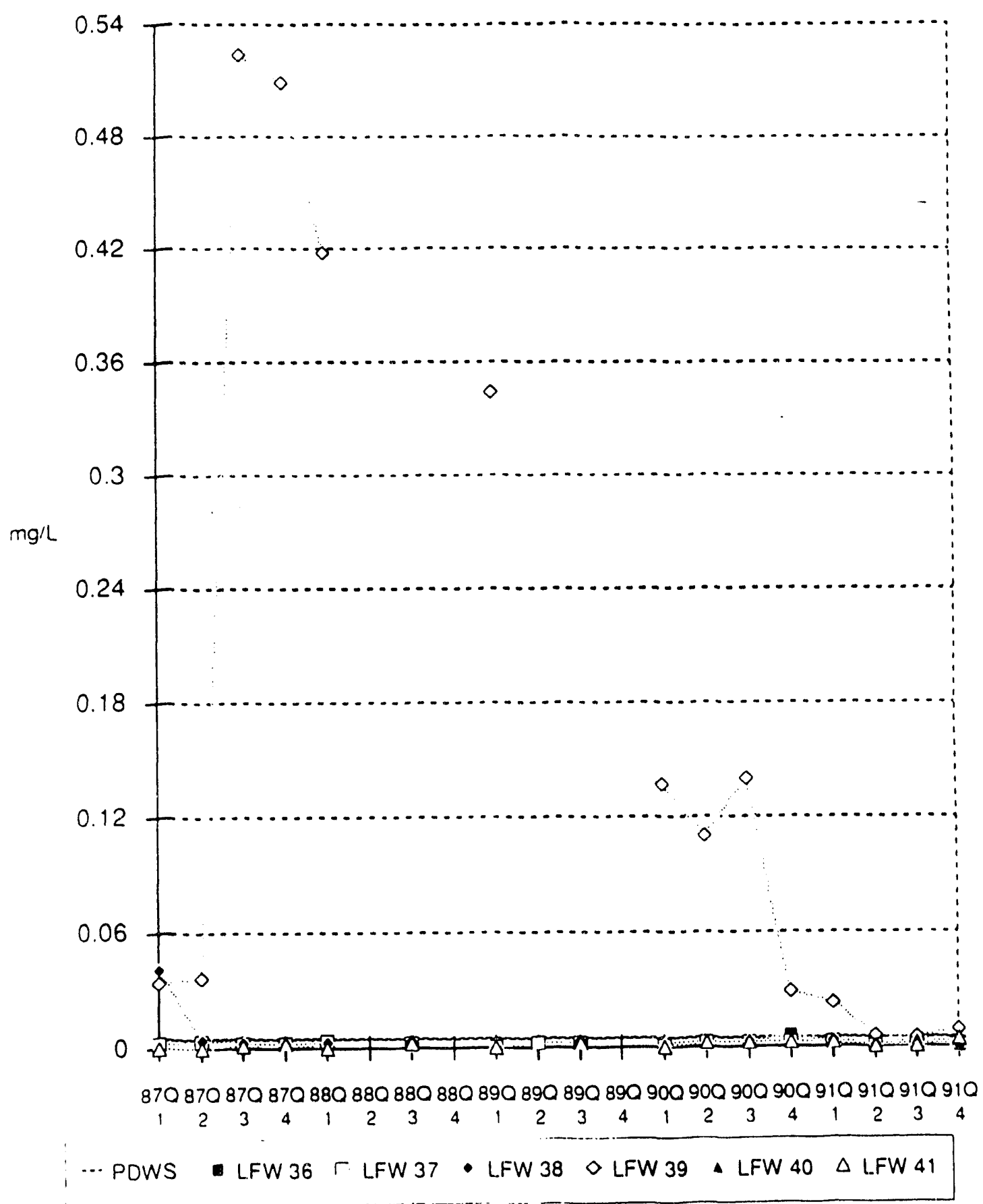


Tetrachloroethylene in the LFW-Series Monitoring Wells

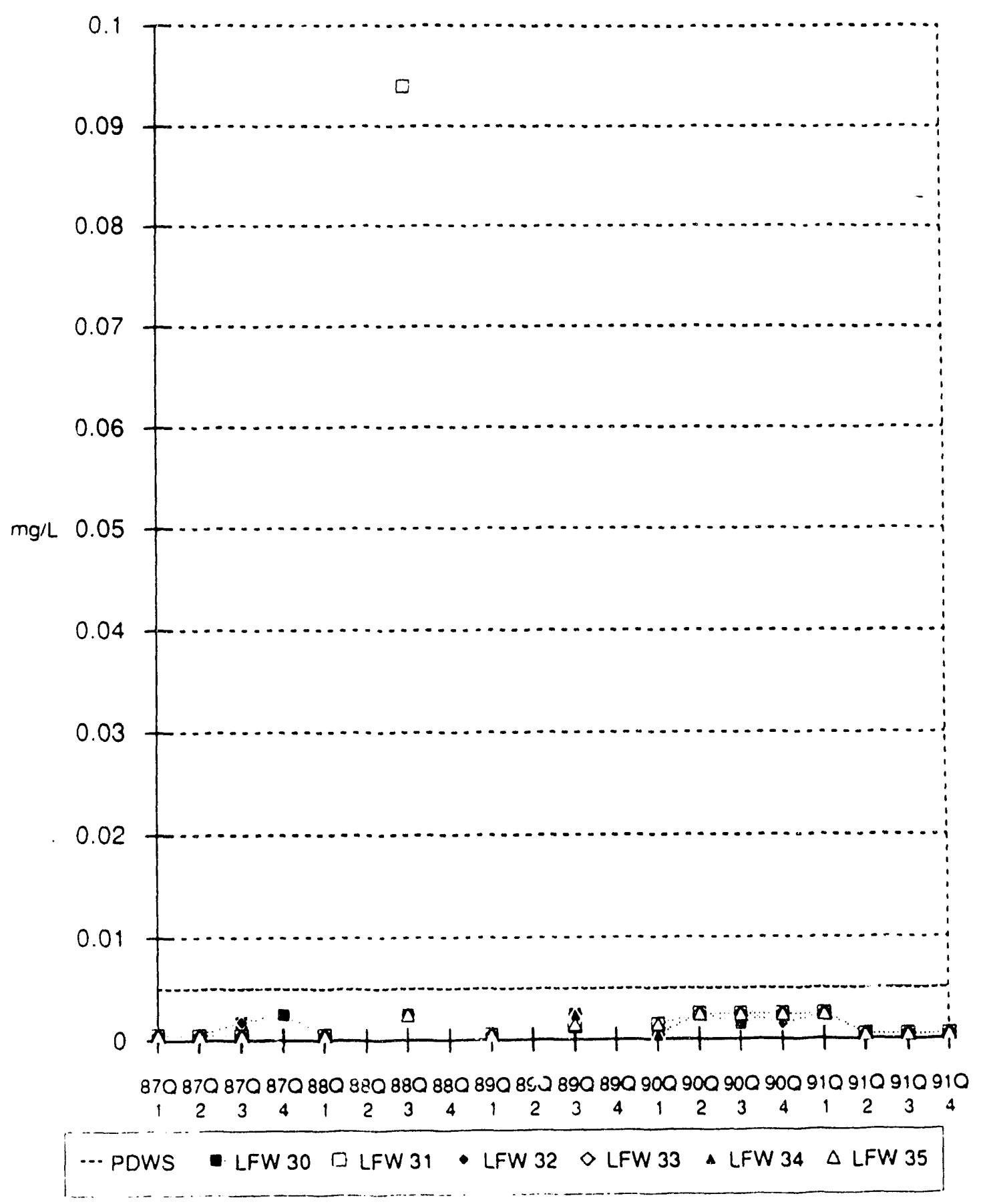


Tetrachloroethylene in the LFW-Series Monitoring Wells

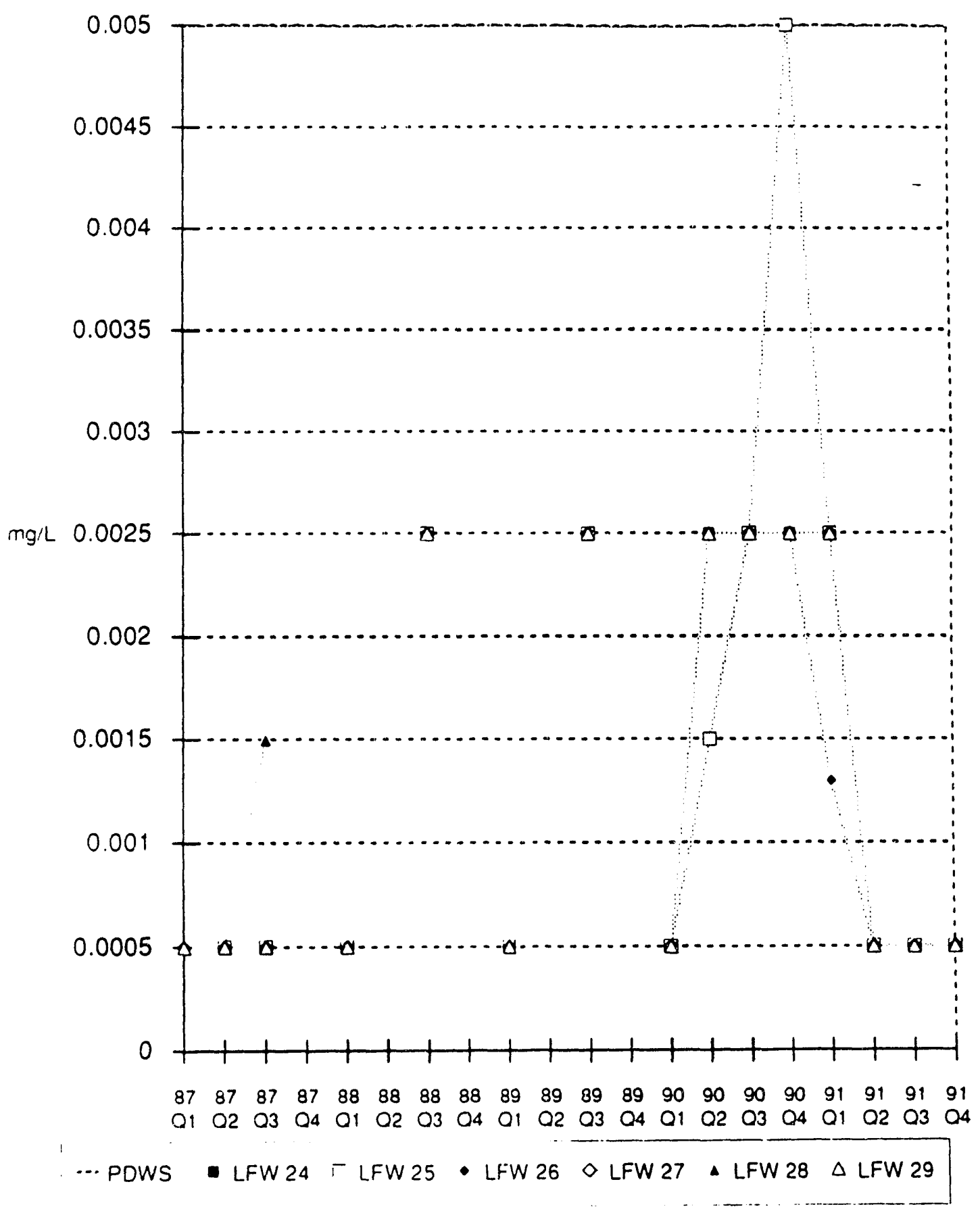


Tetrachloroethylene in the LFW-Series Monitoring Wells

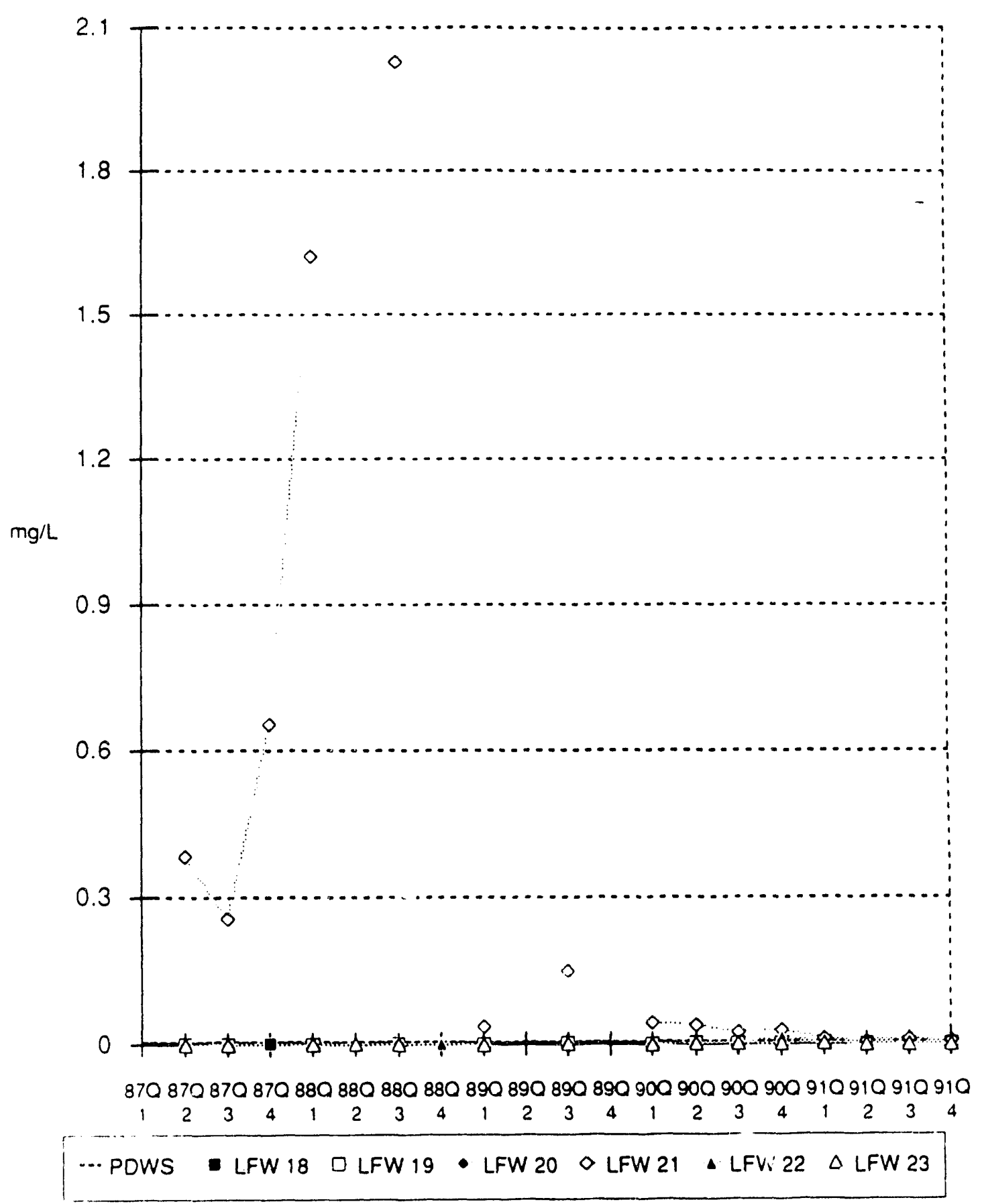




\section{APPENDIX B}

\section{THIRD QUARTER 1991 ANALYTICAL DATA}


WELL LFW 6

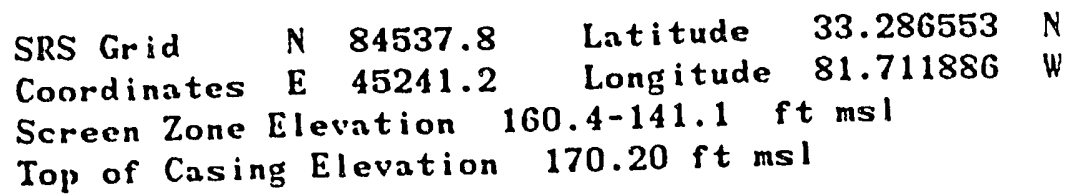

MEASUREMENTS CONDUCTED IN THE FIELD

Sample date 08/13/91 Time 1205

Depth to water = $15.17 \mathrm{ft}(4.62 \mathrm{~m})$ below the TOC

Water elevation - $155.03 \mathrm{ft}(47.25 \mathrm{~m}) \mathrm{msl}$

$\mathrm{pH}-5.3$ Alkalinity $=19 \mathrm{mg} / \mathrm{L}$

Specific conductance - $84 \mathrm{umhos} / \mathrm{cm}$

Water temperature - 20.9 degrees Celsius

Wnter evacunted from the well prior to sampling - $43 \mathrm{gal}$

\section{LABORATORY ANALYSES}

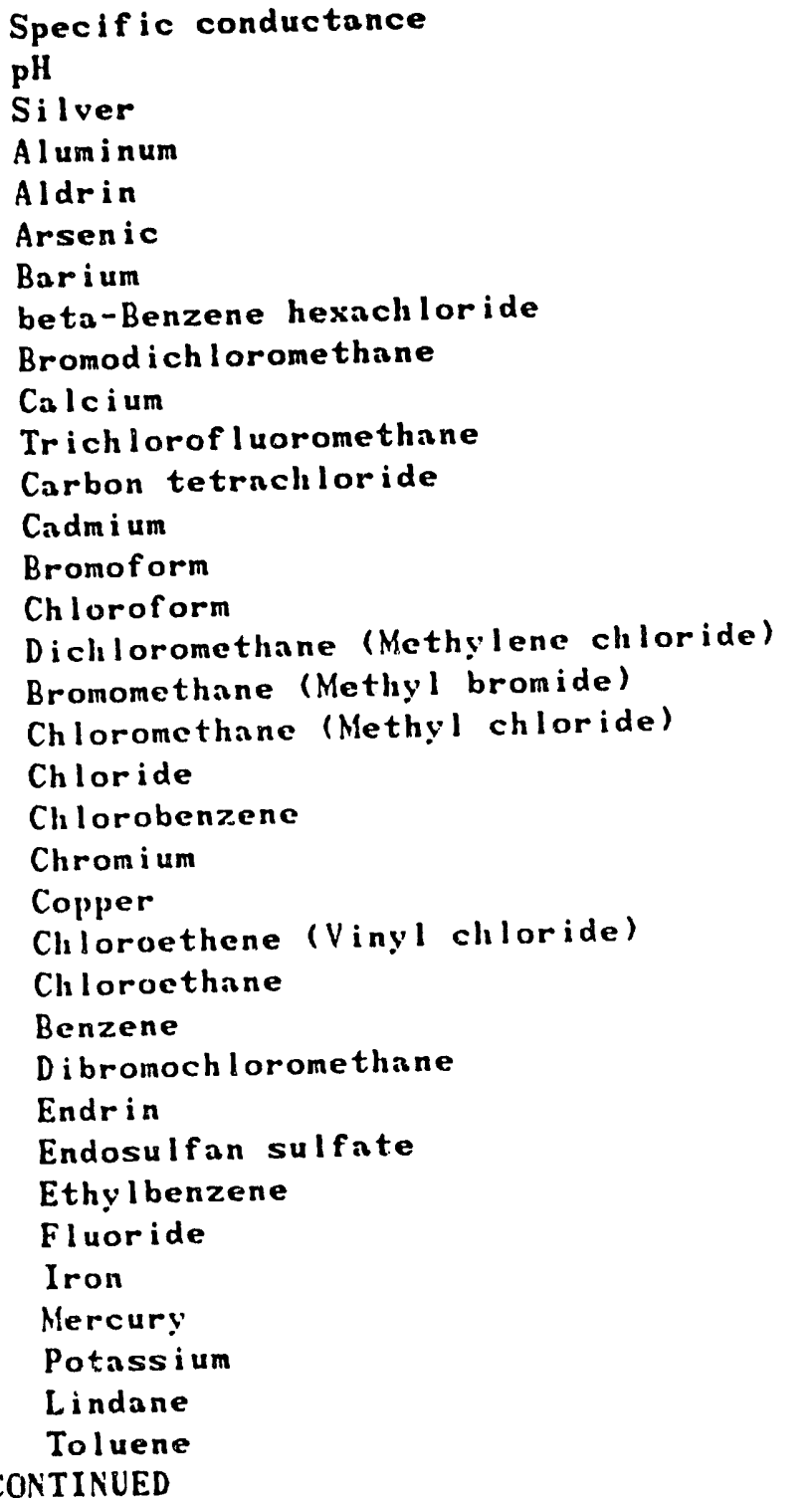

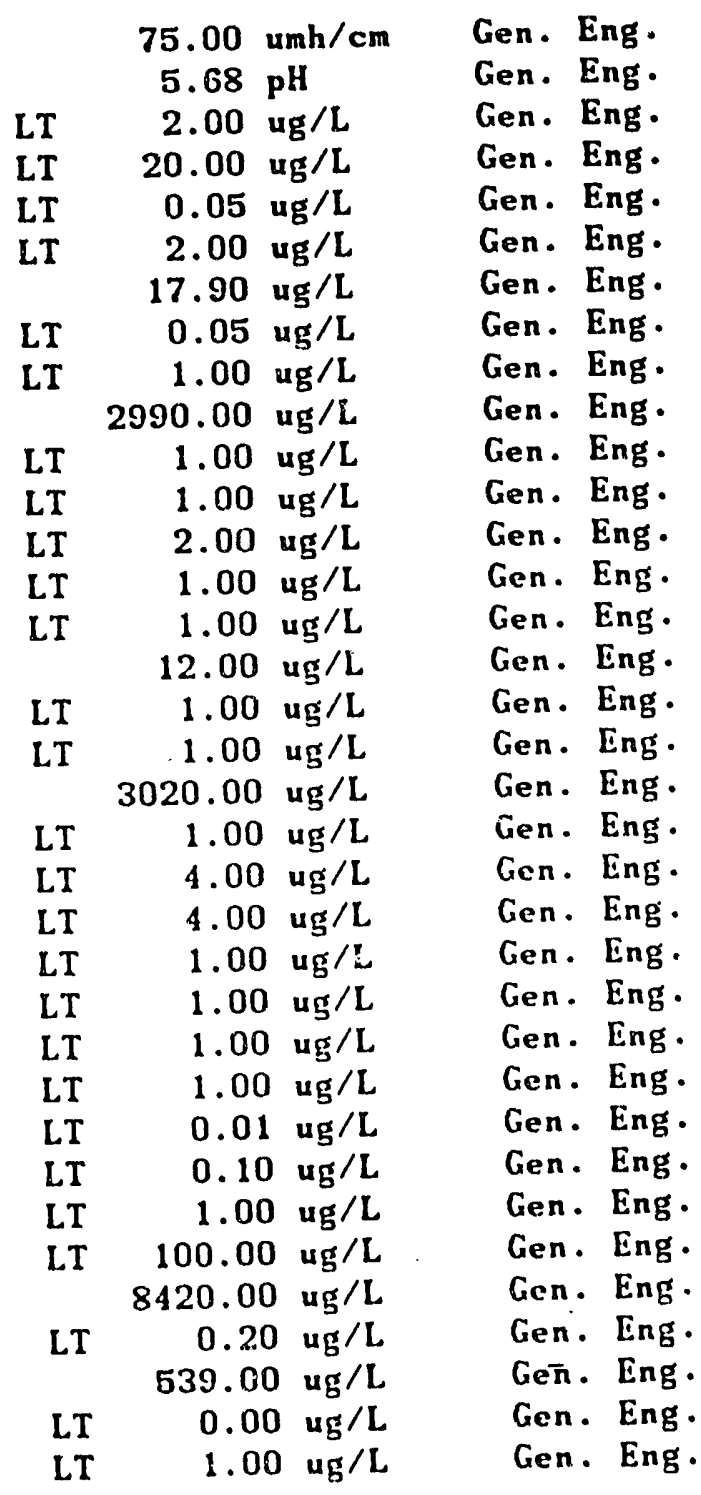


WELL LFW 6 COLLECTED ON OS/13/91 LABORATORY ANAI.YSES CONTINUED

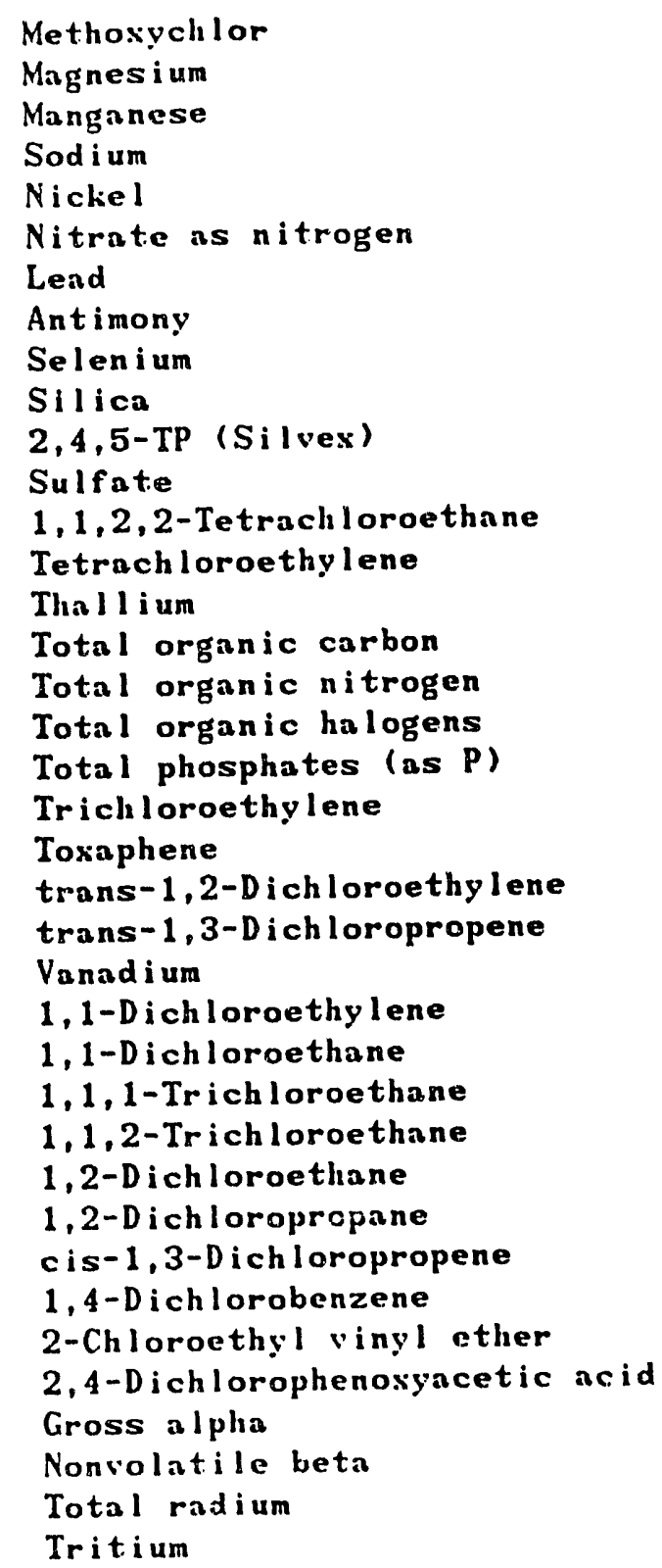

\begin{tabular}{|c|c|c|c|c|}
\hline LT & 0.50 & ug $/ \mathrm{L}$ & Gen. & Eng. \\
\hline & 610.00 & ug/L & Gen. & Eng. \\
\hline & 18.40 & ug $/ \mathrm{L}$ & Gen. & Eng. \\
\hline & 670.00 & $u g / L$ & Gen. & Eng. \\
\hline LT & 4.00 & ug $/ \mathrm{L}$ & Gen. & Eng. \\
\hline LT & 50.00 & $\mathrm{ug} / \mathrm{L}$ & Gen. & Eng. \\
\hline LT & 3.00 & ug $/ \mathrm{L}$ & Gen. & Eng. \\
\hline LT & 2.00 & ug $/ L$ & Gen. & Eng. \\
\hline LT & 2.00 & $u g / L$ & Gen. & Eng. \\
\hline & 710.00 & $\mathrm{ug} / \mathrm{L}$ & Gen. & Eng. \\
\hline LT & 0.09 & $u g / L$ & Gen. & Eng. \\
\hline & 710.00 & $u g / L$ & Gen. & Eng. \\
\hline LT & 1.00 & ug $/ \mathrm{L}$ & Gen.- & Eng. \\
\hline LT & 1.00 & ug/L & Gen. & Eng. \\
\hline LT & 2.00 & $\mathrm{ug} / \mathrm{L}$ & Gen. & Eng. \\
\hline & 000.00 & $u_{g} / \mathrm{L}$. & Gen. & Eng. \\
\hline LT & 100.00 & ug/L & Gen. & Eng. \\
\hline & 28.40 & ug/L & Gen. & Eng. \\
\hline LT & 100.00 & $u_{g} / L$ & Gen. & Eng. \\
\hline LT & 1.00 & ug/L & Gen. & Eng. \\
\hline LT & 0.24 & ug/L & Gen. & Eng. \\
\hline LT & 1.00 & ug $/ \mathrm{L}$ & Gen. & Eng. \\
\hline LT & 1.00 & ug/L & Gen. & Eng. \\
\hline LT & 10.00 & ug/L & Gen. & Eng. \\
\hline LI & 1.00 & $\mathrm{ug} / \mathrm{L}$ & Gen. & Eng. \\
\hline & 3.00 & $u g / L$ & Gen. & Eng. \\
\hline LT & 1.00 & ug /L & Gen. & Eng. \\
\hline LT & 1.00 & ug/L & Gen. & Eng. \\
\hline LT & 1.00 & ug/L & Gen. & Eng. \\
\hline LT & 1.00 & ug $/ \mathrm{L}$ & Gen. & Eng. \\
\hline LT & 1.00 & $\mathrm{ug} / \mathrm{L}$ & Gen. & Eng. \\
\hline LT & 10.00 & $\mathrm{ug} / \mathrm{L}$ & Gen. & Eng. \\
\hline LT & 1.00 & ug $/ \mathrm{L}$ & Gen. & Eng. \\
\hline LT & 0.30 & $\operatorname{ug} / \mathrm{L}$ & Gen. & Eng. \\
\hline $2.40+-$ & 1.60 & $\mathrm{pCi} / \mathrm{L}$ & Gen. & Eng. \\
\hline LT & 2.00 & $\mathrm{pCi} / \mathrm{L}$ & Gen. & - Eng. \\
\hline $4.80+-$ & 3.50 & $\mathrm{pCi} / \mathrm{L}$ & Gen. & - Eng. \\
\hline $1.82+-$ & 0.20 & $\mathrm{pC} \cdot \mathrm{i} / \mathrm{mL}$ & Gen. & - En \\
\hline
\end{tabular}


WELL LFW 7

$\begin{array}{llllll}\text { SRS Grid } & N & 84310.3 & \text { Latitude } & 33.286177 & N \\ \text { Coordinates } & E & 45318.9 & \text { Longitude } & \mathbf{8 1 . 7 1 1 2 3 9} & W\end{array}$

Screen Zone Elevation 159.8-140.5 ft ms

Top of Casing Elevation $169.60 \mathrm{ft} \mathrm{msl}$

MEASUREMENTS CONDUCTED IN THE FIELD

Sample date 08/13/91 Time 1130

Depth to water - $16.70 \mathrm{ft}(5.09 \mathrm{~m})$ below the TOC

Water elevation - $152.90 \mathrm{ft}(46.60 \mathrm{~m}) \mathrm{msl}$

$\mathrm{pH}=6.4$ Alkalinity $=121 \mathrm{mg} / \mathrm{L}$

Specific conductance - 491 umhos/cm

Water temperature - 19.5 degrees Celsius

Water evacuated from the well prior to sampling - $40 \mathrm{gal}$

\section{LABORATORY ANALYSES}

Specific conductance

$\mathrm{pH}$

Silver

Al um inum

Aldrin

Arsenic

Barium

beta-Benzene hexachloride

Bromodich loromethane

Calcium

Trichlorof luoromethane

Carbon tetrachloride

Cadmi um

Bromoform

Chloroform

Dichloromethane (Methylene chloride)

Bromomethane (Methyl bromide)

Chloromethane (Methyl chloride)

Chloride

Chlorobenzene

Chromium

Copper

* Chloroethene (Vingl chloride)

Chloroethane

Berizene

Dibromochloromethane

Endrin

Endosulfan sulfate

Ethylbenzene

Fluoride

Iron

Mercury

Potassium

Lindane

Toluene

Methosychlor

Magnesium

CONTINUED

$\begin{array}{lrl} & 325.00 \mathrm{umh} / \mathrm{cm} \\ & 6.42 \mathrm{uH} \\ \mathrm{LT} & 2.00 \mathrm{ug} / \mathrm{L} \\ \mathrm{LT} & 20.00 \mathrm{ug} / \mathrm{L} \\ \mathrm{LT} & 0.05 \mathrm{ug} / \mathrm{L} \\ & 34.10 \mathrm{ug} / \mathrm{L} \\ & 26.90 \mathrm{ug} / \mathrm{L} \\ \mathrm{LT} & 0.05 \mathrm{ug} / \mathrm{L} \\ \mathrm{LT} & 1.00 \mathrm{ug} / \mathrm{L} \\ & 11000.00 \mathrm{ug} / \mathrm{L} \\ \mathrm{LT} & 1.00 \mathrm{ug} / \mathrm{L} \\ \mathrm{LT} & 1.00 \mathrm{ug} / \mathrm{L} \\ & 5.29 \mathrm{ug} / \mathrm{L} \\ \mathrm{LT} & 1.00 \mathrm{ug} / \mathrm{L} \\ \mathrm{LT} & 1.00 \mathrm{ug} / \mathrm{L} \\ & 23.00 \mathrm{ug} / \mathrm{L} \\ \mathrm{LT} & 1.00 \mathrm{ug} / \mathrm{L} \\ \mathrm{LT} & 1.00 \mathrm{ug} / \mathrm{L} \\ & 26600.00 \mathrm{ug} / \mathrm{L} \\ & 52.00 \mathrm{ug} / \mathrm{L} \\ \mathrm{LT} & 4.00 \mathrm{ug} / \mathrm{L} \\ \mathrm{LT} & 4.00 \mathrm{ug} / \mathrm{L} \\ & 400.00 \mathrm{ug} / \mathrm{L} \\ \mathrm{LT} & 1.00 \mathrm{ug} / \mathrm{L} \\ & 4.00 \mathrm{ug} / \mathrm{L} \\ \mathrm{LT} & 1.00 \mathrm{ug} / \mathrm{L} \\ \mathrm{LT} & 0.01 \mathrm{ug} / \mathrm{L} \\ \mathrm{LT} & 0.10 \mathrm{ug} / \mathrm{L} \\ & 18.00 \mathrm{ug} / \mathrm{L} \\ & 116.00 \mathrm{ug} / \mathrm{L} \\ & 58400.00 \mathrm{ug} / \mathrm{L} \\ \mathrm{LT} & 0.20 \mathrm{ug} / \mathrm{L} \\ & 3810.00 \mathrm{ug} / \mathrm{L} \\ \mathrm{LT} & 0.00 \mathrm{ug} / \mathrm{L} \\ & 17.00 \mathrm{ug} / \mathrm{L} \\ \mathrm{LT} & 0.50 \mathrm{ug} / \mathrm{L} \\ & 18300.00 \mathrm{ug} / \mathrm{L} \\ & & \end{array}$

Gen, Eng -

Gen. Eng.

Gen. Eng.

Gen. Eng.

Gen. Eng.

Gen. Eng.

Gen. Eng -

Gen. Eng.

Gen. Eng .

Gen. Eng.

Gen. Eng.

Gen. Eng.

Gen. Eng.

Gen. Eng.

Gen. Eng.

Gen. Eng.

Gen. Eng.

Gen. Eng.

Gen. Eng.

Gen. Eng.

Gen. Eng.

Gen. Eng.

Gen. Eng.

Gen. Eng .

Gen. Eng.

Gen. Eng.

Gen. Eng -

Gen. Eng.

Gen. Eng.

Gen. Eng .

Gen. Eng .

Gen. Eng.

Gen: Eng.

Gerr. Eng .

Gen. Eng .

Gen. Eng.

Gen. Eng. 
WELL LFW 7 COLLECTED ON 08/13/91 LABORATORY ANALYSES CONTINUED

\author{
Manganese \\ Sod i um \\ Nickel \\ Nitrate as nitrogen \\ Lead \\ Antimony \\ Selenium \\ Silica \\ 2,4,5-TP (Silvex) \\ Sulfate \\ 1,1,2,2-Tetrachloroethane \\ Tetrachloroethylene \\ Thallium \\ Total organic carbon \\ Total organic nitrogen \\ Total organic halogens \\ Total phosphates (as P) \\ Trichloroethylene \\ Toxaphene \\ trans-1,2-Dichloroethylene \\ trans-1,3-Dichloropropene \\ Vanadium \\ 1,1-Dichloroethylene \\ 1,1-Dichloroethane \\ 1,1,1-Trichloroethane \\ 1,1,2-Trichloroethane \\ 1,2-Dichloroethane \\ 1,2-Dichloropropane \\ cis-1,3-D ich loropropene \\ * 1,4-Dichlorobenzene \\ 2-Chloroethyl vinyl ether \\ 2,4-Dichlorophenoxyacetic acid \\ Gross a lpha \\ Nonvolatile beta \\ * Total radium \\ Tritium
}

$\begin{array}{rr} & 3.70 \mathrm{ug} / \mathrm{L} \\ & 32100.00 \mathrm{ug} / \mathrm{L} \\ \mathrm{LT} & 4.00 \mathrm{ug} / \mathrm{L} \\ \mathrm{LT} & 50.00 \mathrm{ug} / \mathrm{L} \\ \mathrm{LT} & 3.00 \mathrm{ug} / \mathrm{L} \\ \mathrm{LT} & 2.00 \mathrm{ug} / \mathrm{L} \\ \mathrm{LT} & 2.00 \mathrm{ug} / \mathrm{L} \\ & 6750.00 \mathrm{ug} / \mathrm{L} \\ \mathrm{LT} & 0.09 \mathrm{ug} / \mathrm{L} \\ \mathrm{LT} & 1000.00 \mathrm{ug} / \mathrm{L} \\ \mathrm{LT} & 1.00 \mathrm{ug} / \mathrm{L} \\ \mathrm{LT} & 1.00 \mathrm{ug} / \mathrm{L} \\ \mathrm{LT} & 2.00 \mathrm{ug} / \mathrm{L} \\ & 10000.00 \mathrm{ug} / \mathrm{L} \\ \mathrm{LT} & 100.00 \mathrm{ug} / \mathrm{L} \\ & 612.00 \mathrm{ug} / \mathrm{L} \\ \mathrm{LT} & 100.00 \mathrm{ug} / \mathrm{L} \\ \mathrm{LT} & 1.00 \mathrm{ug} / \mathrm{L} \\ \mathrm{LT} & 0.24 \mathrm{ug} / \mathrm{L} \\ \mathrm{LT} & 1.00 \mathrm{ug} / \mathrm{L} \\ \mathrm{LT} & 1.00 \mathrm{ug} / \mathrm{L} \\ \mathrm{LT} & 10.00 \mathrm{ug} / \mathrm{L} \\ \mathrm{LT} & 1.00 \mathrm{ug} / \mathrm{L} \\ & 28.00 \mathrm{ug} / \mathrm{L} \\ \mathrm{LT} & 1.00 \mathrm{ug} / \mathrm{L} \\ \mathrm{LT} & 1.00 \mathrm{ug} / \mathrm{L} \\ \mathrm{LT} & 1.00 \mathrm{ug} / \mathrm{L} \\ \mathrm{LT} & 1.00 \mathrm{ug} / \mathrm{L} \\ \mathrm{LT} & 1.00 \mathrm{ug} / \mathrm{L} \\ & 99.00 \mathrm{ug} / \mathrm{L} \\ \mathrm{LT} & 1.00 \mathrm{ug} / \mathrm{L} \\ \mathrm{LT} & 0.30 \mathrm{ug} / \mathrm{L} \\ \mathrm{LT} & 2.00 \mathrm{pC} \mathrm{i} / \mathrm{L} \\ 24.80+- & 16.00 \mathrm{\mu Ci} / \mathrm{L} \\ 6.90+- & 4.00 \mathrm{pC} / \mathrm{i} / \mathrm{L} \\ 16.60+- & .0 .60 \mathrm{pC} \mathrm{i} / \mathrm{mL}\end{array}$

Gen. Eng.

Gen. Eng.

Gen. Eng.

Gen. Eng.

Gen. Eng.

Gen. Eng.

Gen. Eng.

Gen. Eng.

Gen. Eng.

Gen. Eng.

Gen. Eng.

Gen. Eng .

Gen.- Eng.

Gen. Eng.

Gen. Eng.

Gen. Eng.

Gen. Eng.

Gen. Eng.

Gen. Eng -

Gen. Eng .

Gen. Eng.

Gen. Eng.

Gen. Eng.

Gen. Eng.

Gen. Eng .

Gen. Eng.

Gen. Eng .

Gen. Eng.

Gen. Eng .

Gen. Eng .

Gen. Eng.

Gen. Eng.

Gen. Eng.

Gen. Eng.

Gen. Eng.

Gen. Eng. 
HELL LFH' 8

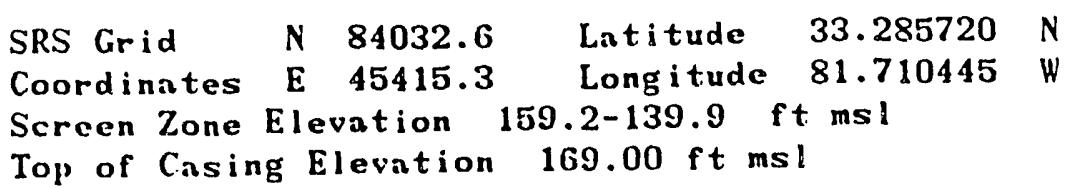

MEASUREMENTS CONDUCTED IN TIE FIELD

Sample date 08/14/91 Time 1340

Depth to water $=18.24 \mathrm{ft}(5.56 \mathrm{~m})$ below the TOC

Water elevation - $150.76 \mathrm{ft}(45.95 \mathrm{~m}) \mathrm{msl}$

ph -5.8 Alkalinity $=15 \mathrm{mg} / \mathrm{L}$

Specific conductance $=86 \mathrm{umhos} / \mathrm{cm}$

Water temperature $-\mathbf{2 0 . 5}$ degrees Celsius

Water evacuated from the well prior to sampling - $44 \mathrm{gal}$

\section{LABORATORY ANALYSES}

Specific conductance
pH
Silver
Aluminum
Aldrin
Arsenic
Barium
beta-Benzene hexachloride
Bromodichloromethane
Calcium
Trichlorofluoromethane
Carbon tetrachloride
Cadmium
Bromoform
Chloroform
Dichloromethane (Methylene chloride)
Bromomethane (Methyl bromide)
Chloromethane (Methyl chloride)
Chloride
Chlorobenzene
Chromium
Copper
Chloroethene (Vinyl chloride)
Chloroethane
Benzene
Dibromochloromethane
Endrin
Endosulfan sulfate
Ethylbenzene
Fluoride
Iron
Mercury
Potassium
Lindane
Toluene
Methosychlor
Magnesium
ONTiNuED
Ch

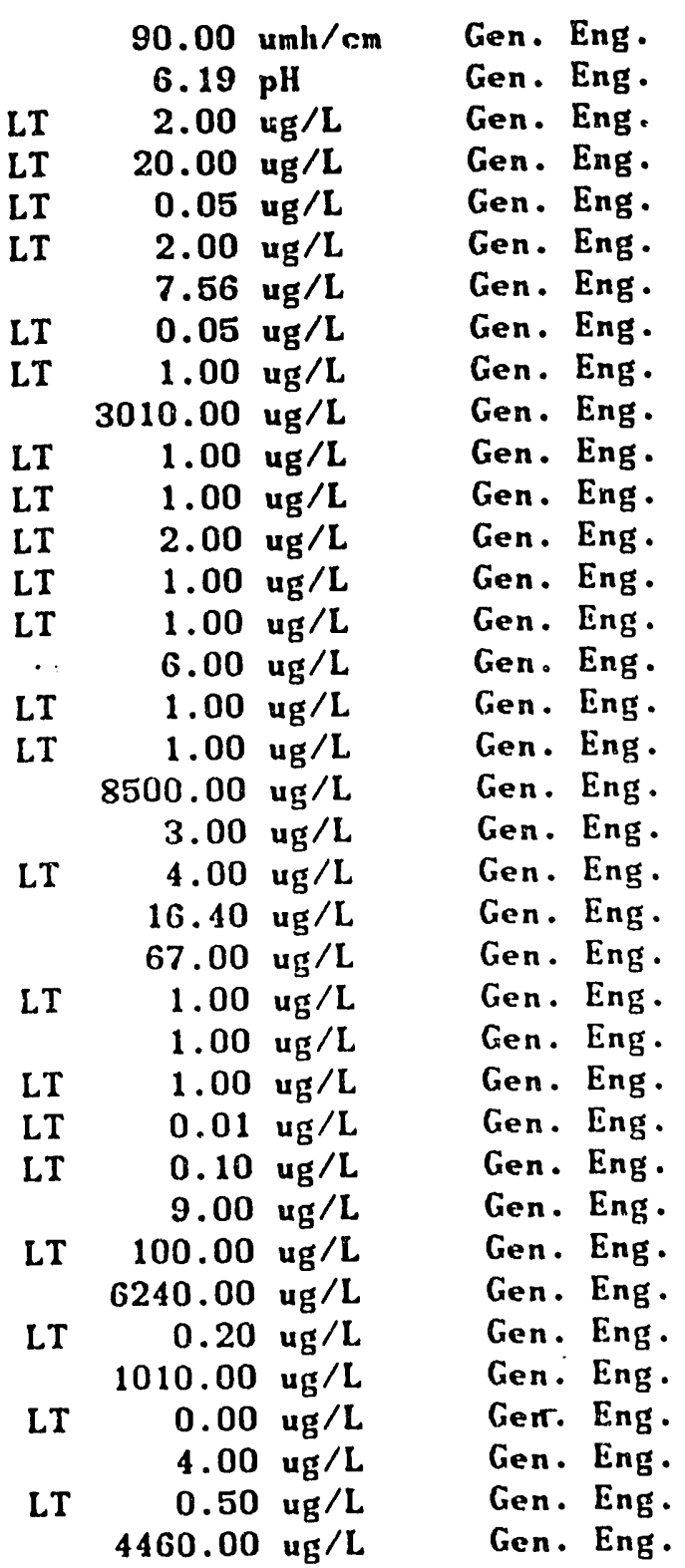


WELL LFW 8 COLLECTED ON 08/14/91 LABORATORY ANALYSES C.ONTINUED

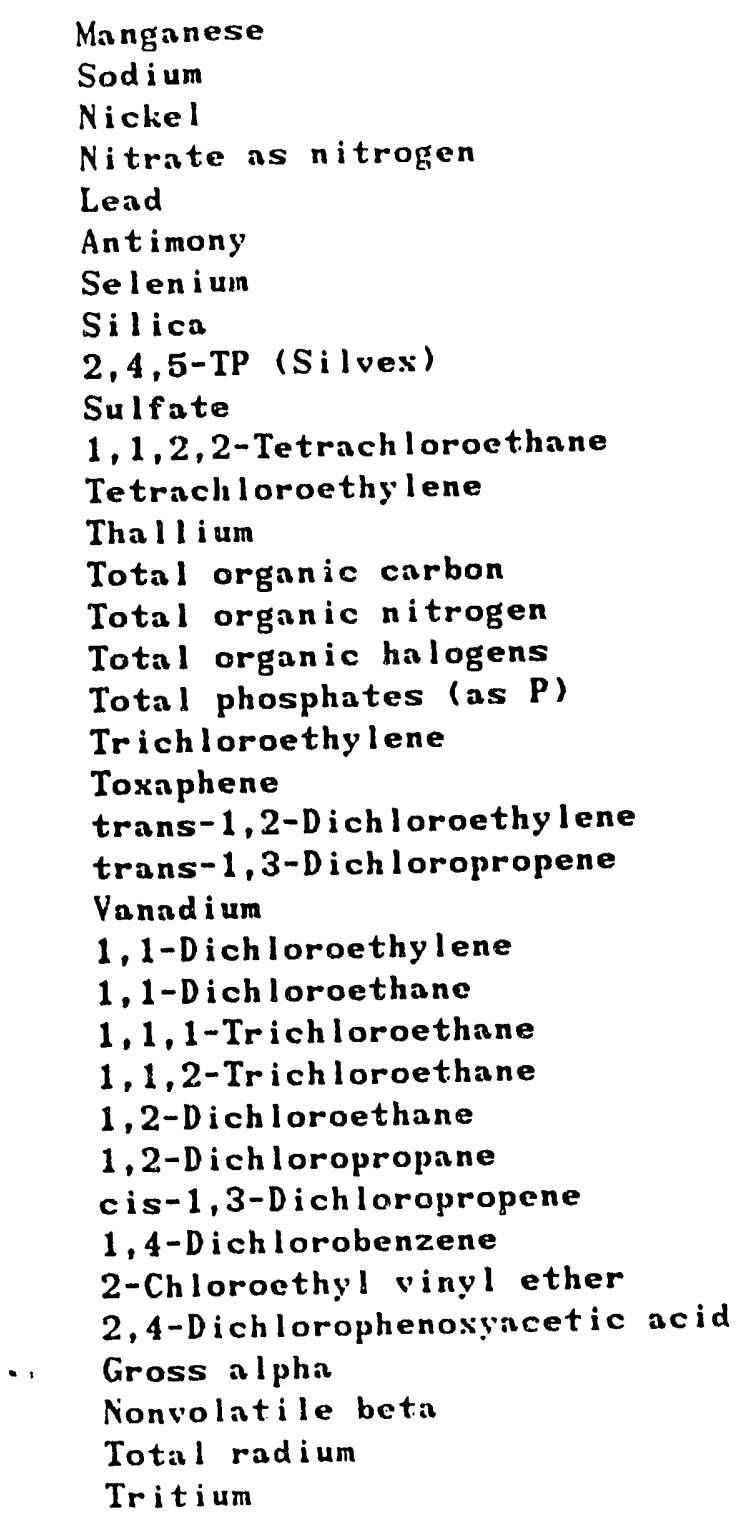

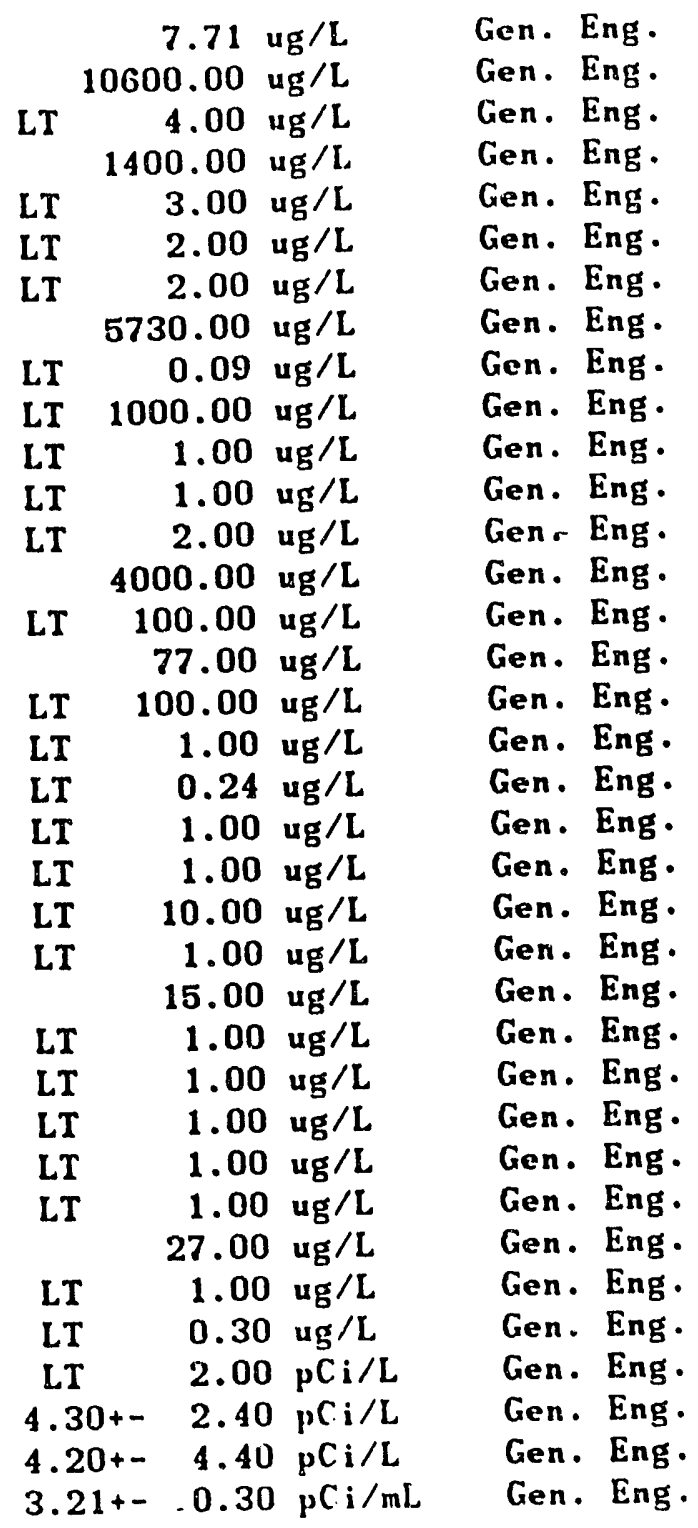


WELL LFH $10 \mathrm{~A}$

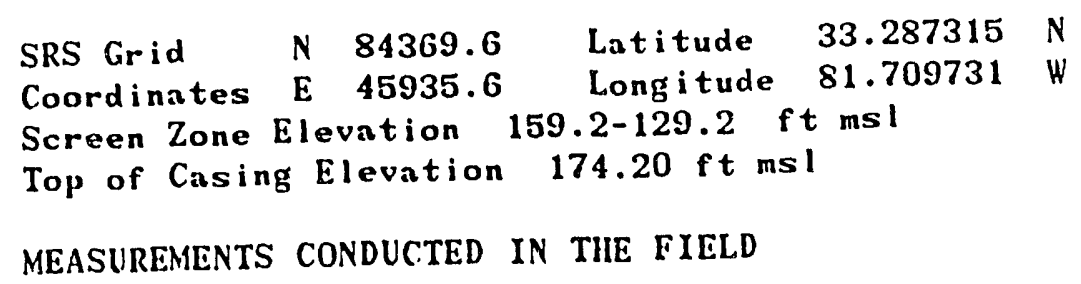

\section{LABORATORY ANALYSES}

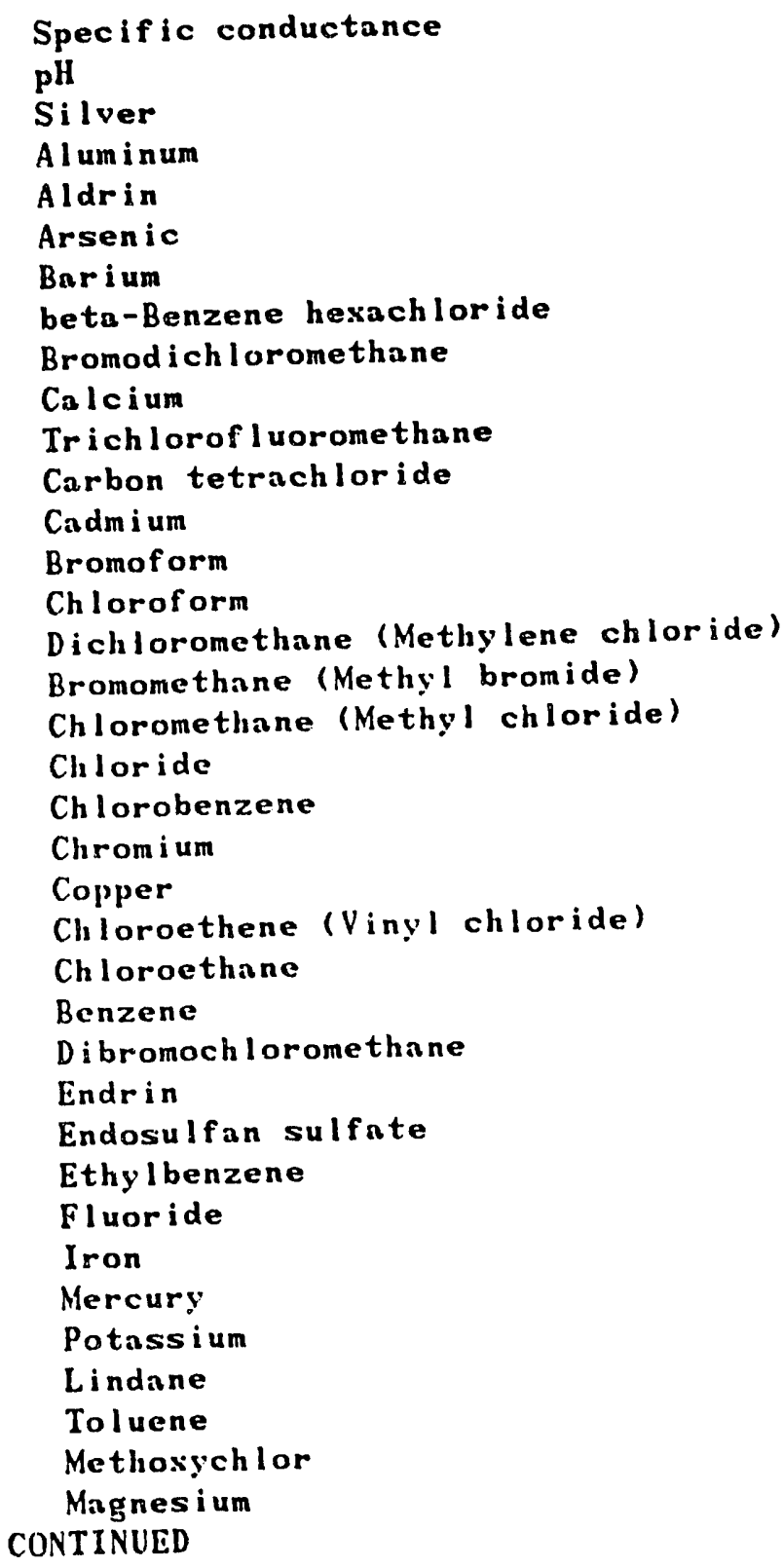

\begin{tabular}{|c|c|c|c|c|}
\hline & $\begin{array}{r}285.00 \\
6.11 \\
2.00\end{array}$ & $\begin{array}{l}\mathrm{umh} / \mathrm{cm} \\
\mathrm{pH} \\
\mathrm{ug} / \mathrm{L}\end{array}$ & $\begin{array}{l}\text { Gen. } \\
\text { Gen. } \\
\text { Gen. }\end{array}$ & $\begin{array}{l}\text { Eng. } \\
\text { Eng. } \\
\text { Eng. }\end{array}$ \\
\hline LI & 20.00 & ug $/ L$ & Gen. & Eng. \\
\hline LT & 0.05 & $u g / L$ & Gen. & Eng. \\
\hline & 2.61 & $\mathrm{ug} / \mathrm{L}$ & Gen. & Eng. \\
\hline & 12.20 & $u g / L$ & Gen. & Eng. \\
\hline$T$ & 0.05 & $u g / L$ & Gen. & Eng. \\
\hline 1 & $\begin{array}{r}1.00 \\
11600.00\end{array}$ & $\begin{array}{l}u g / L \\
u g / L\end{array}$ & $\begin{array}{l}\text { Gen. } \\
\text { Gen. }\end{array}$ & $\begin{array}{l}\text { Eng. } \\
\text { Eng. }\end{array}$ \\
\hline & 43.00 & $\mathrm{ug} / \mathrm{L}$ & Gen. & Eng. \\
\hline & 1.77 & $u g / L$ & Gen. & Eng. \\
\hline & 4.26 & $\mathbf{u g} / \mathrm{L}$ & Gen. & Eng. \\
\hline LT & 1.00 & $\mathbf{u g} / \mathrm{L}$ & Gen. & Eng. \\
\hline LT & 1.00 & $\mathrm{ug} / \mathrm{L}$ & Gen. & Eng. \\
\hline & 215.00 & ug/L & Gen. & Eng. \\
\hline LT & 1.00 & $u g / L$ & Gen. & Eng. \\
\hline LT & 1.00 & $u g / L$ & Gen. & Eng. \\
\hline & 24000.00 & $u g / L$ & Gen. & - Eng. \\
\hline LT & 1.00 & $\mathbf{u g} / \mathrm{L}$ & Gen. & - Eng. \\
\hline LT & 4.00 & $u g / L$ & Gen. & - Eng. \\
\hline LT & 4.00 & ug /L & Gen. & - Eng. \\
\hline LT & 1.00 & $u g / L$ & Gen. & - Eng. \\
\hline LT & 1.00 & ug / L & Gen. & . Eng. \\
\hline & 4.22 & ug/L & Gen. & . Eng. \\
\hline L.T & 1.00 & $\mathrm{ug} / \mathrm{L}$ & Gen. & . Eng. \\
\hline LT & 0.01 & $u g / L$ & Gen. & . Eng. \\
\hline LT & 0.10 & ug/L & Gen. & Eng \\
\hline LT & 1.00 & $u g / L$ & Gen. & - Er \\
\hline & 101.00 & ug/L & en. & . Eng \\
\hline & 56100.00 & $u g / L$ & Gen. & 1. Eng \\
\hline LT & 0.20 & $\mathrm{ug} / \mathrm{L}$ & $\begin{array}{l}\text { Gen. } \\
\text { Gen }\end{array}$ & : \\
\hline LT & $\begin{array}{r}2390.00 \\
0.00\end{array}$ & $u_{g} / L$ & Gen & \\
\hline LT & 1.00 & ug $/ \mathrm{L}$ & Gen & \\
\hline LT & 0.50 & ug $/ \mathrm{L}$ & $\mathbf{n}$ & . \\
\hline & 8160.00 & ug/L & n & \\
\hline
\end{tabular}


WELL LFW 10A COLLECTED ON 08/15/91 LABORATORY ANALYSES CONTINUED

Manganese

Sod i um

Nickel

Nitrate as nitrogen

Lead

Antimony

Selenium

Silica

$2,4,5-T P$ ( $S$ i lvex)

Sulfate

$1,1,2,2-T e t r a c h l o r o e t h a n e$

Tetrachloroethylene

Tha 11 i um

Total organic carbon

Total organic nitrogen

Total organic halogens

Total phosphates (as P)

* Trichloroethylene

Toxaphene

trans-1,2-Dichloroethylene

trans-1,3-Dichloropropene

Vanad i um

1,1-Dichloroethylene

1,1-Dichloroethane

1,1,1-Trich loroethane

1,1,2-Trichloroethane

1,2-Dichloroethane

1,2-Dichloropropane

cis-1, 3-D ich loropropene

1,4-Dichlorobenzene

2-Chloroethyl vinyl ether

2,4-Dichlorophenosyncetic acid

Gross alpha

Nonvolatile beta

Total radium

* Tritium

$\begin{array}{lrll} & 30.30 \mathrm{ug} / \mathrm{L} & \text { Gen. Eng. } \\ & 18400.00 \mathrm{ug} / \mathrm{L} & \text { Gen. Eng. } \\ \mathrm{LT} & 4.00 \mathrm{ug} / \mathrm{L} & \text { Gen. Eng. } \\ & 220.00 \mathrm{ug} / \mathrm{L} & \text { Gen. Eng. } \\ \mathrm{LT} & 3.00 \mathrm{ug} / \mathrm{L} & \text { Gen. Eng. } \\ \mathrm{LT} & 2.00 \mathrm{ug} / \mathrm{L} & \text { Gen. Eng. } \\ \mathrm{LT} & 2.00 \mathrm{ug} / \mathrm{L} & \text { Gen. Eng. } \\ & 10100.00 \mathrm{ug} / \mathrm{L} & \text { Gen. Eng. } \\ \mathrm{LT} & 0.09 \mathrm{ug} / \mathrm{L} & \text { Gen. Eng. } \\ & 4790.00 \mathrm{ug} / \mathrm{L} & \text { Gen. Eng. } \\ \mathrm{LT} & 1.00 \mathrm{ug} / \mathrm{L} & \text { Gen. Eng. } \\ \mathrm{LT} & 1.00 \mathrm{ug} / \mathrm{L} & \text { Gen. Eng. } \\ \mathrm{LT} & 2.00 \mathrm{ug} / \mathrm{L} & \text { Gen. Eng. } \\ & 38000.00 \mathrm{ug} / \mathrm{L} & \text { Gen. Eng. } \\ & 170.00 \mathrm{ug} / \mathrm{L} & \text { Gen. Eng. } \\ & 561.00 \mathrm{ug} / \mathrm{L} & \text { Gen. Eng. } \\ \mathrm{LT} & 100.00 \mathrm{ug} / \mathrm{L} & \text { Gen. Eng. } \\ & 28.10 \mathrm{ug} / \mathrm{L} & \text { Gen. Eng. } \\ \mathrm{LT} & 0.24 \mathrm{ug} / \mathrm{L} & \text { Gen. Eng. } \\ \mathrm{LT} & 1.00 \mathrm{ug} / \mathrm{L} & \text { Gen. Eng. } \\ \mathrm{LT} & 1.00 \mathrm{ug} / \mathrm{L} & \text { Gen. Eng. } \\ \mathrm{LT} & 10.00 \mathrm{ug} / \mathrm{L} & \text { Gen. Eng. } \\ \mathrm{LT} & 1.00 \mathrm{ug} / \mathrm{L} & \text { Gen. Eng. } \\ & 70.00 \mathrm{ug} / \mathrm{L} & \text { Gen. Eng. } \\ & 15.10 \mathrm{ug} / \mathrm{L} & \text { Gen. Eng. } \\ \mathrm{LT} & 1.00 \mathrm{ug} / \mathrm{L} & \text { Gen. Eng. } \\ \mathrm{LT} & 1.00 \mathrm{ug} / \mathrm{L} & \text { Gen. Eng. } \\ \mathrm{LT} & 1.00 \mathrm{ug} / \mathrm{L} & \text { Gen. Eng. } \\ \mathrm{LT} & 1.00 \mathrm{ug} / \mathrm{L} & \text { Gen. Eng. } \\ \mathrm{LT} & 10.00 \mathrm{ug} / \mathrm{L} & \text { Gen. Eng. } \\ \mathrm{LT} & 1.00 \mathrm{ug} / \mathrm{L} & \text { Gen. Eng. } \\ \mathrm{LT} & 0.30 \mathrm{ug} / \mathrm{L} & \text { Gen. Eng. } \\ 2.30+- & 1.80 \mathrm{pC} \mathrm{i} / \mathrm{L} & \text { Gen. Eng. } \\ 4.80+- & 2.40 \mathrm{pC} \mathrm{i} / \mathrm{L} & \text { Gen. Eng. } \\ 3.50+- & 3.40 \mathrm{pC} / \mathrm{L} & \text { Gen. Eng. } \\ \mathrm{G3} .30+- & 1.40 \mathrm{pC} \mathrm{i} / \mathrm{mL} & \text { Gen. Eng. } \\ & & & \end{array}$


WELL LFW 10 A

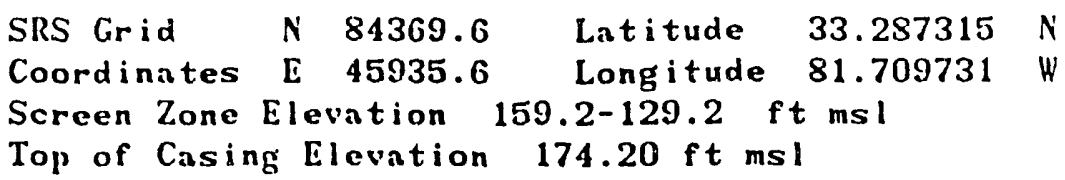

MEASUREMENTS CONDUCTED IN THE FIELD

Sample date 08/15/91 Time 920

Depth to water - $20.47 \mathrm{ft}(6.24 \mathrm{~m})$ below the TOC

Water elevation $-153.73 \mathrm{ft}(46.86 \mathrm{~m}) \mathrm{msl}$

$\mathrm{pH}=6.0 \quad$ Alkalinity $=79 \mathrm{mg} / \mathrm{L}$

Specific conductance - 265 umhos/cm

Water temperature -20.9 degrees Celsius

Water evacunted from the well prior to sampling $=88 \mathrm{gal}$

\section{LABORATORY ANALYSES}

Specific conductance

$\mathrm{pH}$

Silver

Aluminum

Aldrin

Arsenic

Barium

beta-Benzene hexachloride

Bromodichloromethane

Calcium

Trichlorof luoromethane

Carbon tetrachloride

Cadmi um

Bromoform

Chloroform

Dichloromethane (Methylene chloride)

Bromomethane (Methyl bromide)

Chloromethane (Methyl chloride)

Chloride

Chlorobenzene

Chromium

Copper

Chloroethene (Vingl chloride)

Chloroethane

Benzene

Dibromochloromethane

Endrin

Endosulfan sulfate

Ethylbenzene

Fluoride

Iron

Mercury

Potassium

Lindane

Toluene

Methoxychlor

Magnesium

CONTINUED

$\begin{array}{lrll} & 290.00 \mathrm{umh} / \mathrm{cm} & \text { Gen. Eng. } \\ & 6.06 \mathrm{uH} & \text { Gen. Eng. } \\ \text { LT } & 2.00 \mathrm{ug} / \mathrm{L} & \text { Gen. Eng. } \\ \text { LT } & 20.00 \mathrm{ug} / \mathrm{L} & \text { Gen. Eng. } \\ \text { LT } & 0.05 \mathrm{ug} / \mathrm{L} & \text { Gen. Eng. } \\ & 2.60 \mathrm{ug} / \mathrm{L} & \text { Gen. Eng. } \\ & 12.20 \mathrm{ug} / \mathrm{L} & \text { Gen. Eng. } \\ \text { LT } & 0.05 \mathrm{ug} / \mathrm{L} & \text { Gen. Eng. } \\ \text { LT } & 1.00 \mathrm{ug} / \mathrm{L} & \text { Gen. Eng. } \\ & 11600.00 \mathrm{ug} / \mathrm{L} & \text { Gen. Eng. } \\ & 63.60 \mathrm{ug} / \mathrm{L} & \text { Gen. Eng. } \\ & 2.90 \mathrm{ug} / \mathrm{L} & \text { Gen. Eng. } \\ & 4.46 \mathrm{ug} / \mathrm{L} & \text { Gen. Eng. } \\ \text { LT } & 1.00 \mathrm{ug} / \mathrm{L} & \text { Gen. Eng. } \\ \text { LT } & 1.00 \mathrm{ug} / \mathrm{L} & \text { Gen. Eng. } \\ & 219.00 \mathrm{ug} / \mathrm{L} & \text { Gen. Eng. } \\ \text { LT } & 1.00 \mathrm{ug} / \mathrm{L} & \text { Gen. Eng. } \\ \text { LT } & 1.00 \mathrm{ug} / \mathrm{L} & \text { Gen. Eng. } \\ & 23400.00 \mathrm{ug} / \mathrm{L} & \text { Gen. Eng. } \\ \text { LT } & 1.00 \mathrm{ug} / \mathrm{L} & \text { Gen. Eng. } \\ \text { LT } & 4.00 \mathrm{ug} / \mathrm{L} & \text { Gen. Eng. } \\ \text { LT } & 4.00 \mathrm{ug} / \mathrm{L} & \text { Gen. Eng. } \\ \text { LT } & 1.00 \mathrm{ug} / \mathrm{L} & \text { Gen. Eng. } \\ \text { LT } & 1.00 \mathrm{ug} / \mathrm{L} & \text { Gen. Eng. } \\ & 4.04 \mathrm{ug} / \mathrm{L} & \text { Gen. Eng. } \\ \text { LT } & 1.00 \mathrm{ug} / \mathrm{L} & \text { Gen. Eng. } \\ \text { LT } & 0.01 \mathrm{ug} / \mathrm{L} & \text { Gen. Eng. } \\ \text { LT } & 0.10 \mathrm{ug} / \mathrm{L} & \text { Gen. Eng. } \\ & 21.20 \mathrm{ug} / \mathrm{L} & \text { Gen. Eng. } \\ & 105.00 \mathrm{ug} / \mathrm{L} & \text { Gen. Eng. } \\ & 56000.00 \mathrm{ug} / \mathrm{L} & \text { Gen. Eng. } \\ \text { LT } & 0.20 \mathrm{ug} / \mathrm{L} & \text { Gen. Eng. } \\ & 2350.00 \mathrm{ug} / \mathrm{L} & \text { Gen. Eng. } \\ \text { LT } & 0.00 \mathrm{ug} / \mathrm{L} & \text { Gen. Eng. } \\ & 53.50 \mathrm{ug} / \mathrm{L} & \text { Gen. Eng. } \\ \text { LT } & 0.50 \mathrm{ug} / \mathrm{L} & \text { Gen. Eng. } \\ & 8140.00 \mathrm{ug} / \mathrm{L} & \text { Gen. Eng. } \\ & & & \end{array}$


WELL LFW 10 A COLLECTED ON 08/15/91 LABORATORY ANALYSES CONTINUED

Manganese

Sod i um

Nickel

Nitrate as nitrogen

Lead

Antimony

Selenium

Silica

$2,4,5-T P$ (Silvex)

Sulfate

1,1,2,2-Tetrach loroethane

* Tetrachloroethylene

Thallium

Total organic carbon

Total organic carbon

Total organic nitrogen

Total organic halogens

Total phosphates (as P)

* Trichloroethylene

Toxaphene

trans-1,2-Dichloroethylene

trans-1,3-Dichloropropene

Vannd i um

1,1-Dichloroethylene

1,1-Dichloroethane

1,1,1-Trichloroethane

1,1,2-Trichloroethane

1,2-Dichloroethane

1,2-Dich loropropane

cis-1,3-Dichloropropene

1,4-D ichlorobenzene

2-Chloroethyl vinyl ether

2,4-Dichlorophenoxyacetic acid

Gross a lpha

Nonvolatile beta

Total radium

* Tritium

\begin{tabular}{|c|c|c|c|c|}
\hline & 29.60 & ug $/ \mathrm{L}$ & Gen. & Eng. \\
\hline & 8500.00 & ug/L & Gen. & Eng. \\
\hline LT & 4.00 & ug/L & Gen. & Eng. \\
\hline & 200.00 & ug/L & Gen. & Eng. \\
\hline LT & 3.00 & ug/L & Gen. & Eng. \\
\hline LT & 2.00 & ug/L & Gen. & Eng. \\
\hline LT & 2.00 & ug/L & Gen. & Eng. \\
\hline & 0100.00 & ug $/ \mathrm{L}$ & Gen. & Eng. \\
\hline LT & 0.09 & ug/L & Gen. & Eng. \\
\hline & 4540.00 & ug/L & Gen. & Eng. \\
\hline LT & 1.00 & ug/L & Gen. & Eng. \\
\hline & 15.60 & ug/L & Gen. & Eng. \\
\hline LT & 2.00 & $u g / L$ & Gen .- & Eng. \\
\hline & 1000.00 & ug/L & Gen. & Eng. \\
\hline & 1000.00 & $\mathrm{ug} / \mathrm{L}$ & Gen. & Eng. \\
\hline & 250.00 & ug/L & Gen. & Eng. \\
\hline & 493.00 & ug/L & Gen. & Eng. \\
\hline & 120.00 & ug/L & Gen. & Eng \\
\hline & 27.80 & ug/L & Gen. & $\mathrm{ng}$ \\
\hline LT & 0.24 & ug $/ \mathrm{L}$ & Gen. & Eng. \\
\hline LT & 1.00 & ug $/ \mathrm{L}$ & en. & Eng. \\
\hline LT & 1.00 & $\operatorname{ug} / \mathrm{L}$ & Gen. & Eng \\
\hline LT & 10.00 & ug/L & Gen. & Eng \\
\hline & 3.68 & ug /L & Gen. & Ex \\
\hline & 74.40 & ug $/ \mathrm{L}$ & Gen. & \\
\hline & 24.70 & ug $/ L$ & Gen. & Eng \\
\hline LT & 1.00 & ug/L & Gen. & Eng \\
\hline LT & 1.00 & ug $/ \mathrm{L}$ & Gen. & Eng \\
\hline LT & 1.00 & ug $/ \mathrm{L}$ & Gen. & Eng \\
\hline LT & 1.00 & ug $/ L$ & Gen. & Eng \\
\hline LT & 10.00 & ug $/ \mathrm{L}$ & Gen. & Eng \\
\hline LT & 1.00 & $\operatorname{ug} / \mathrm{L}$ & Gen. & Eng \\
\hline LT & 0.30 & ug $/ \mathrm{L}$ & Gen. & $\mathbf{E n}_{\mathrm{g}}$ \\
\hline $2.10+-$ & 2.30 & $p C i / L$ & Gen. & \\
\hline $5.30+-$ & 4.10 & $p C i / L$ & Gen. & \\
\hline $4.10+-$ & $-\quad .3 .60$ & $\mathrm{Ci} / \mathrm{L}$ & Gen. & \\
\hline 38.40 & 1.40 & $\mathrm{~mL}$ & Gen. & \\
\hline
\end{tabular}


WELL LFW 16

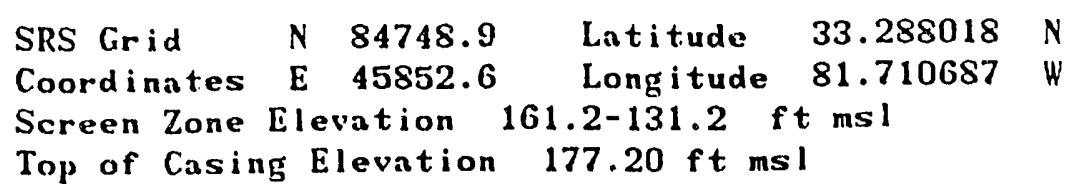

\section{MEASUREMENTS CONDUCTED IN THE FIELD}

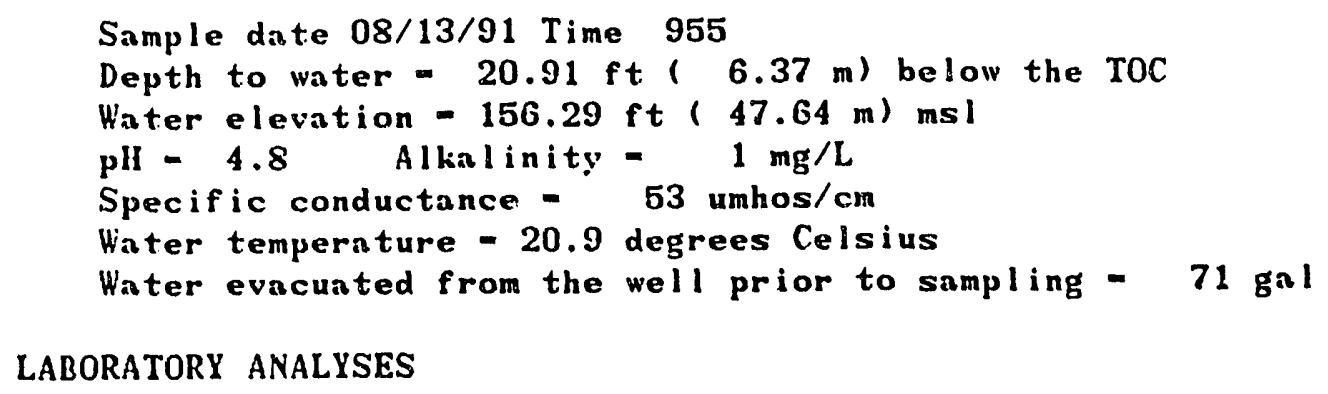

\section{LABORATORY ANALYSES}

Specific conductance
pH
Silver
Aluminum
Aldrin
Arsenic
Barium
beta-Benzene hexachloride
Bromodichloromethane
Calcium
Trichlorofluoromethane
Carbon tetrachloride
Cadmium
Bromoform
Chloroform
Dichloromethane (Methylene chloride)
Bromomethane (Methyl bromide)
Chloromethane (Methyl chloride)
Chloride
Chlorobenzene
Chromium
Copper
Chloroethene (Vinyl chloride)
Chlorocthane
Benzene
Dibromochloromethane
Endrin
Endosulfan sulfate
Ethylbenzene
Fluoride
Iron
Mercury
Potassium
Lindane
Toluene
Methosychlor
Magnesium
NTINuED
Mid

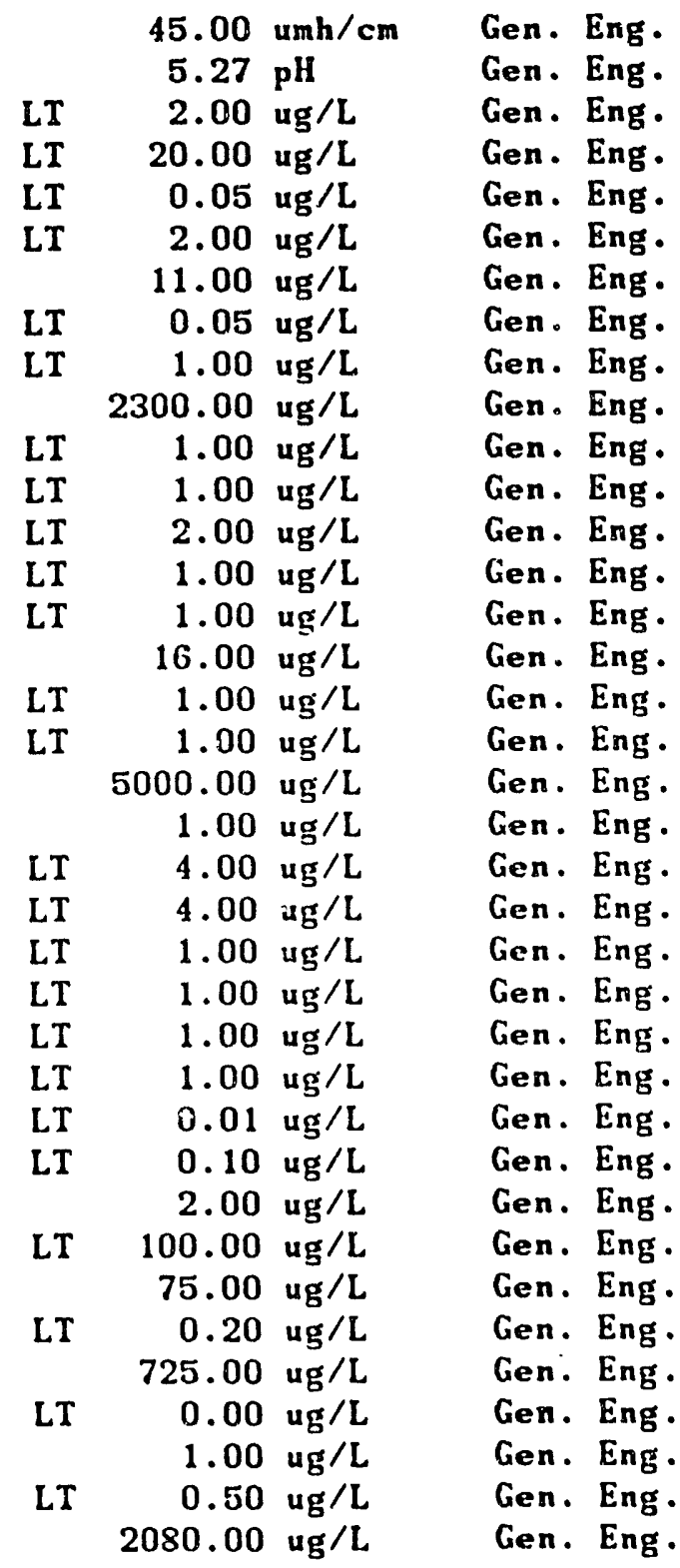


WELL LFW 16 COLLECTED ON 08/13/91 LABORATORY ANALYSES CONTINUED

Manganese

Sod i um

Nickel

Nitrate as ritrogen

Lead

Antimony

Selen ium

Silica

$2,4,5-T P$ (Silvex)

Sulfate

1,1,2,2-Tetrach loroethane

* Tetrachloroethylene

Tha I I i um

Total organic carbon

Total organic nitrogen

Total organic halogens

Total phosphates (as P)

* Trichloroethylene

Toxaphene

trans-1,2-D ich loroethylent

trans-1,3-Dich loropropene

Vanadium

1,1-Dichloroethylene

1,1-Dichloroethane

1,1,1-Trichloroethane

$1,1,2-T r i c h l o r o e t h a n e$

1,2-Dichloroethane

1,2-Dich loropropane

c is-1,3-D ichloropropene

1,4-Dich lorobenzene

2-Chloroethyl vinyl ether

2,4-D chlorophenoxyacetic acid

Gross a Ipha

Nonvolatile beta

* Total radium

Tritium
LI

$2700.00 \mathrm{ug} / \mathrm{L}$

$9.84 \mathrm{ug} / \mathrm{L}$

$1140.00 \mathrm{ug} / \mathrm{L}$

$3.00 \mathrm{ug} / \mathrm{L}$

LT

LT

$2.00 \mathrm{ug} / \mathrm{L}$

LT

$2.00 \mathrm{ug} / \mathrm{L}$

$11700.00 \mathrm{ug} / \mathrm{L}$

LT $\quad 0.09 \mathrm{ug} / \mathrm{L}$

$1350.00 \mathrm{ug} / \mathrm{L}$

LT

$1.00 \mathrm{ug} / \mathrm{L}$

$7.00 \mathrm{ug} / \mathrm{L}$

L.T

$2.00 \mathrm{ug} / \mathrm{L}$ $1000.00 \mathrm{ug} / \mathrm{L}$

LT $100.00 \mathrm{ug} / \mathrm{L}$ $259.00 \mathrm{ug} / \mathrm{L}$

LT

$100.00 \mathrm{ug} / \mathrm{L}$

$9.00 \mathrm{ug} / \mathrm{L}$

LT $\quad 0.24 \mathrm{ug} / \mathrm{L}$

LT $\quad 1.00 \mathrm{ug} / \mathrm{L}$

LT $\quad 1.00 \mathrm{ug} / \mathrm{L}$

LT $10.00 \mathrm{ug} / \mathrm{L}$

LT

$1.00 \mathrm{ug} / \mathrm{L}$

$34.00 \mathrm{ug} / \mathrm{L}$

$11.00 \mathrm{ug} / \mathrm{L}$

LT $\quad 1.00 \mathrm{ug} / \mathrm{L}$

LT $\quad 1.00 \mathrm{ug} / \mathrm{L}$

LT $\quad 1.00 \mathrm{ug} / \mathrm{L}$

LT $\quad 1.00 \mathrm{ug} / \mathrm{L}$

LT $10.00 \mathrm{ug} / \mathrm{L}$

LT

LT

LT

$1.00 \mathrm{ug} / \mathrm{L}$

$0.30 \mathrm{ug} / \mathrm{L}$

$2.00 \mathrm{pCi} / \mathrm{L}$

LT

$2.00 \mathrm{pCi} / \mathrm{L}$

$5.50+-3.70 \mathrm{pCi} / \mathrm{L}$

$13.80+-.0 .50 \mathrm{pCi} / \mathrm{mL}$
Gen. Eng.

Gen. Eng.

Gen. Eng.

Gen. Eng.

Gen. Eng.

Gen. Eng

Gen. Eng.

Gen. Eng.

Gen. Eng.

Gen. Eng.

Gen. Eng.

Gen. Eng.

Gen - Eng.

Gen. Eng.

Gen. Eng.

Gen. Eng.

Gen. Eng.

Gen. Eng.

Gen. Eng.

Gen. Eng.

Gen. Eng.

Gen. Eng.

Gen. Eng.

Gen. Eng.

Gen. Eng.

Gen. Eng.

Gen. Eng.

Gen. Eng.

Gen. Eng.

Gen. Eng.

Gen. Eng.

Gen. Eng.

Gen. Eng.

Gen. Eng.

Gen. Eng.

Gen. Eng. 
WELL LF' 17

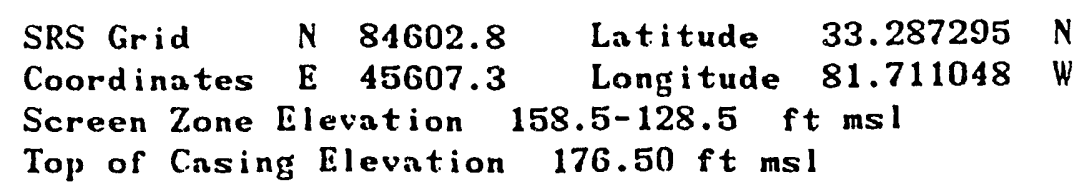

\section{MEASUREMENTS CONDUCTED IN THE FIELD}

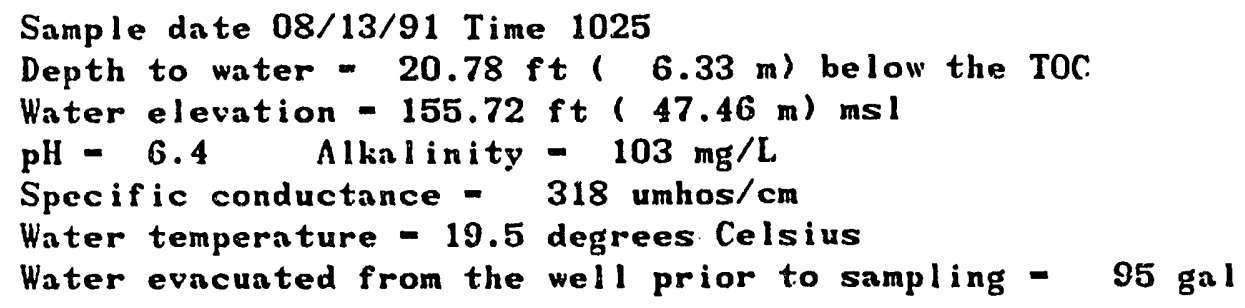

\section{LABORATORY ANALYSES}

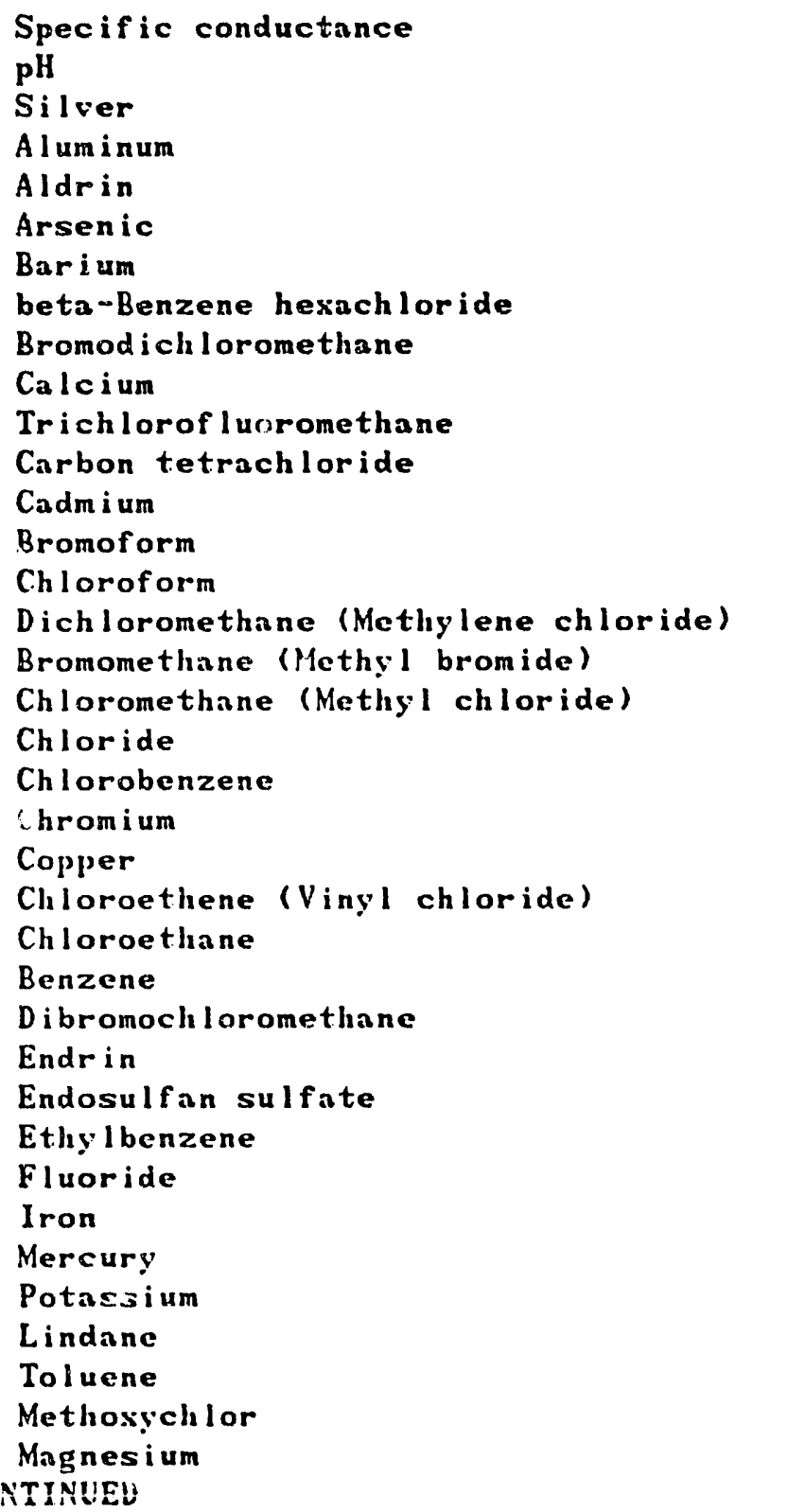

\begin{tabular}{|c|c|c|c|c|}
\hline & $\begin{array}{r}200.00 \\
6.38\end{array}$ & $\begin{array}{l}\mathrm{umh} / \mathrm{cm} \\
\mathrm{pH}\end{array}$ & Gen. & $\begin{array}{l}\text { Eng. } \\
\text { Eng. }\end{array}$ \\
\hline T & 2.00 & ug / L & Gen. & Eng \\
\hline LT & 20.00 & ug/L & Gen. & Eng \\
\hline $\mathrm{T}$ & $\begin{array}{r}0.05 \\
21.70\end{array}$ & $\begin{array}{l}u g / L \\
u g / L\end{array}$ & $\begin{array}{l}\text { Gen. } \\
\text { Gen. }\end{array}$ & $\begin{array}{l}\text { Eng } \\
\text { Eng }\end{array}$ \\
\hline & 5.05 & ug $/ \mathrm{L}$ & Gen. & Eng \\
\hline $\mathbf{T}$ & 0.05 & ug/L & Gen. & Eng \\
\hline$T$ & 1.00 & $\mathrm{ug} / \mathrm{L}$ & Gen. & Eng \\
\hline & 5340.00 & ug $/ \mathrm{L}$ & Gen. & Eng \\
\hline LT & 1.00 & ug/L & Gen. & Eng \\
\hline LT & 1.00 & ug $/ \mathrm{L}$ & Gen. & \\
\hline$T$ & 1.00 & ug $/ \mathrm{L}$ & Gen. & Eng \\
\hline LT & 1.00 & $\mathrm{ug} / \mathrm{L}$ & en. & ng \\
\hline & 8.00 & $u g / L$ & . & Eng. \\
\hline LT & 1.00 & ug /L & n. & Ling. \\
\hline$L T$ & 1.00 & ug/L & n. & ng \\
\hline & 24800.00 & $\mathbf{u g} / \mathrm{L}$ & Gen. & Eng \\
\hline & 55.00 & ug $/ L$ & ien. & En! \\
\hline LT & 4.00 & ug/L & ien. & En \\
\hline LT & 4.00 & ug $/ L$ & ien. & En \\
\hline LT & 1.00 & $\mathrm{ug} / \mathrm{L}$ & ien. & En \\
\hline LT & 1.00 & ug $/ \mathrm{L}$ & n. & \\
\hline & 3.00 & ug $/ \mathrm{L}$ & Gen. & \\
\hline LT & 1.00 & ug $/ \mathrm{L}$ & en. & \\
\hline LT & 0.01 & $\mathbf{u g} / \mathrm{L}$ & en. & \\
\hline LT & 0.10 & ug $/ L$ & & \\
\hline & 22.00 & ug $/ \mathrm{L}$ & & \\
\hline LT & 100.00 & $\mathbf{u g} / \mathrm{L}$ & $n$. & $\mathbf{E}$ \\
\hline & 22700.00 & $u_{g} / L$ & Gen. & \\
\hline LT & 0.20 & ug $/ \mathrm{L}$ & üen. & \\
\hline & 758.00 & $u g / L$ & & \\
\hline LT & 0.00 & $u g / L$ & & \\
\hline LT & $\begin{array}{l}6.00 \\
0.50\end{array}$ & ug $/ \mathrm{L}$ & & \\
\hline & 8910.00 & $\mathrm{ug} / \mathrm{L}$ & & \\
\hline
\end{tabular}


Manganese

Sod i um

Nickel

Nitrate as nitrogen

Lead

Antimony

Selen ium

Silica

$2,4,5-\mathrm{TP}$ (Si lvex)

Sulfate

$1,1,2,2$-Tetrach loroethane

Tetrachloroethylene

Thall ium

Total organic carbon

Total organic carbon

Total organic nitrogen

Total organic halogens

Total phosphates (as P)

Trichloroethylene

Toxaphene

trans-1,2-D ichloroethylene

trans-1, 3-D ichloropropene

Vanadium

1,1-Dichloroethylene

1,1-Dichloroethane

1,1,1-Trichloroethane

$1,1,2-$ Trichloroethane

* 1,2-Dichloroethane

1,2-Dichloropropane

c is-1,3-Dirhloropropene

1,4-D ich lorobenzene

2-Chloroethyl vinyl ether

2,4-Dichlorophenosyacetic acid

Gross a lpha

Nonvolatile beta

Total radium

Tritium

Tritium

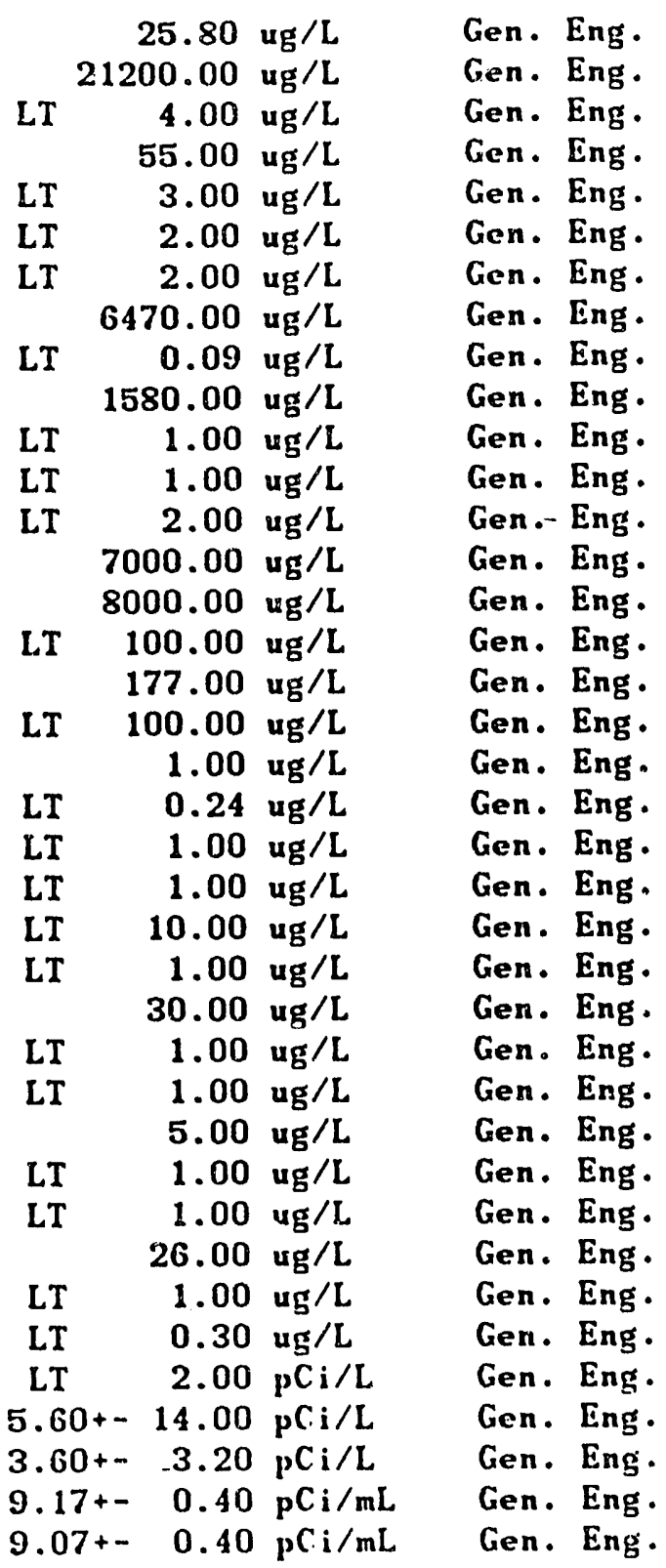


WELL EFW 18

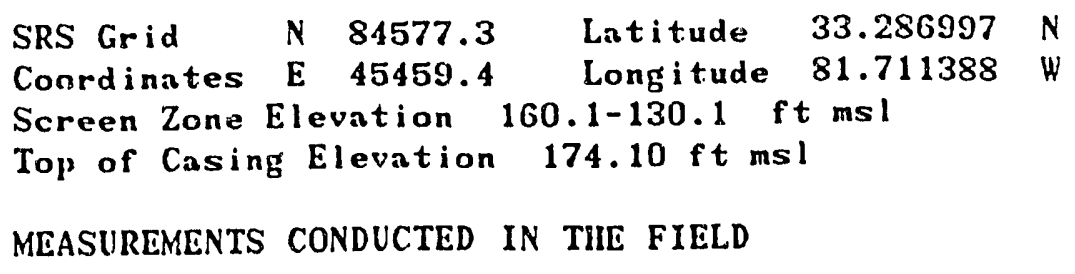

Sample date 08/13/91 Time 1055

Depth to water - $17.98 \mathrm{ft}(5.48 \mathrm{~m})$ below the TOC

Water elevation $-156.12 \mathrm{ft}(47.59 \mathrm{~m}) \mathrm{msl}$

$\mathrm{pH}=6.4$ Alkalinity $=145 \mathrm{mg} / \mathrm{L}$

Specific conductance - 352 umhos/cm

Water temperature - 20.1 degrees Celsius

Water evacuated from the well prior to sampling - $73 \mathrm{gal}$

\section{LABORATORY ANALYSES}

Specific conductance

$\mathrm{pH}$

Silver

Aluminum

Aldrin

Arsenic

Bari um

beta-Benzene hexachloride

Bromodichloromethane

Calcium

Trichlor of luoromethane

Carbon tetrachloride

Cadm i um

Bromoform

Chloroform

Dichloromethane (Methylene chloride)

Bromomethane (Methyl bromide)

Chloromethane (Methyl chloride)

Chloride

Chlorobenzene

Chromium

Copper

Chloroethene (Vinyl chloride)

Chloroethane

Benzene

Dibromochloromethane

Endrin

Endosulfan sulfate

Ethylbenzene

Fluoride

Iron

Mercury

Potassium

Lindane

Toluene

Methoxychlor

Magnesi um

CONTINUED
$200.00 \mathrm{umh} / \mathrm{cm}$

$6.26 \mathrm{pH}$

LT

LT

$2.00 \mathrm{ug} / \mathrm{L}$

$20.00 \mathrm{ug} / \mathrm{L}$

LT

$0.05 \mathrm{ug} / \mathrm{L}$

$25.40 \mathrm{ug} / \mathrm{L}$

$7.25 \mathrm{ug} / \mathrm{L}$

LT $\quad 0.05 \mathrm{ug} / \mathrm{L}$

LT $\quad 1.00 \mathrm{ug} / \mathrm{L}$

$5320.00 \mathrm{ug} / \mathrm{L}$

LT $\quad 1.00 \mathrm{ug} / \mathrm{L}$

LT $\quad 1.00 \mathrm{ug} / \mathrm{L}$

$5.15 \mathrm{ug} / \mathrm{L}$

LT $\quad 1.00 \mathrm{ug} / \mathrm{L}$

LT $\quad 1.00 \mathrm{ug} / \mathrm{L}$

$4.42 \mathrm{ug} / \mathrm{L}$

LT $\quad 1.00 \mathrm{ug} / \mathrm{L}$

LT $\quad 1.00 \mathrm{ug} / \mathrm{L}$

$17400.00 \mathrm{ug} / \mathrm{L}$

$2.00 \mathrm{ug} / \mathrm{L}$

LT $\quad 4.00 \mathrm{ug} / \mathrm{L}$

LT $4.00 \mathrm{ug} / \mathrm{L}$

LT $\quad 1.00 \mathrm{ug} / \mathrm{L}$

$18.50 \mathrm{ug} / \mathrm{L}$

$2.30 \mathrm{ug} / \mathrm{L}$

LT $\quad 1.00 \mathrm{ug} / \mathrm{L}$

LT $0.01 \mathrm{ug} / \mathrm{L}$

LT $0.10 \mathrm{ug} / \mathrm{L}$

$18.60 \mathrm{ug} / \mathrm{L}$

$117.00 \mathrm{ug} / \mathrm{L}$

$55300.00 \mathrm{ug} / \mathrm{L}$

LT $\quad 0.20 \mathrm{ug} / \mathrm{L}$

$1880.00 \mathrm{ug} / \mathrm{L}$

L.T

$0.00 \mathrm{ug} / \mathrm{L}$

$7.91 \mathrm{ug} / \mathrm{L}$

LT

$0.50 \mathrm{ug} / \mathrm{L}$

$10500.00 \mathrm{ug} / \mathrm{L}$
Gen. Eng -

Gen. Eng.

Gen. Eng.

Gen. Eng.

Gen. Eng .

Gen. Eng.

Gen. Eng.

Gen. Eng.

Gen. Eng.

Gen. Eng.

Gen. Eng.

Gen. Eng.

Gen. Eng.

Gen. Eng.

Gen. Eng.

Gen. Eng.

Gen. Eng.

Gen. Eng.

Gen. Eng.

Gen. Eng.

Gen. Eng.

Gen. Eng.

Gen. Eng.

Gen. Eng.

Gen. Eng.

Gen. Eng.

Gen. Eng.

Gen. Eng.

Gen. Eng.

Gen. Eng .

Gen. Eng.

Gen. Eng.

Gen. Eng.

Geñ. Eng.

Gen. Eng.

Gen. Eng.

Gen. Eng. 
WELL LFW 18 COLLECTED ON 08/13/91 LABORATORY ANALYSES CONTINUED

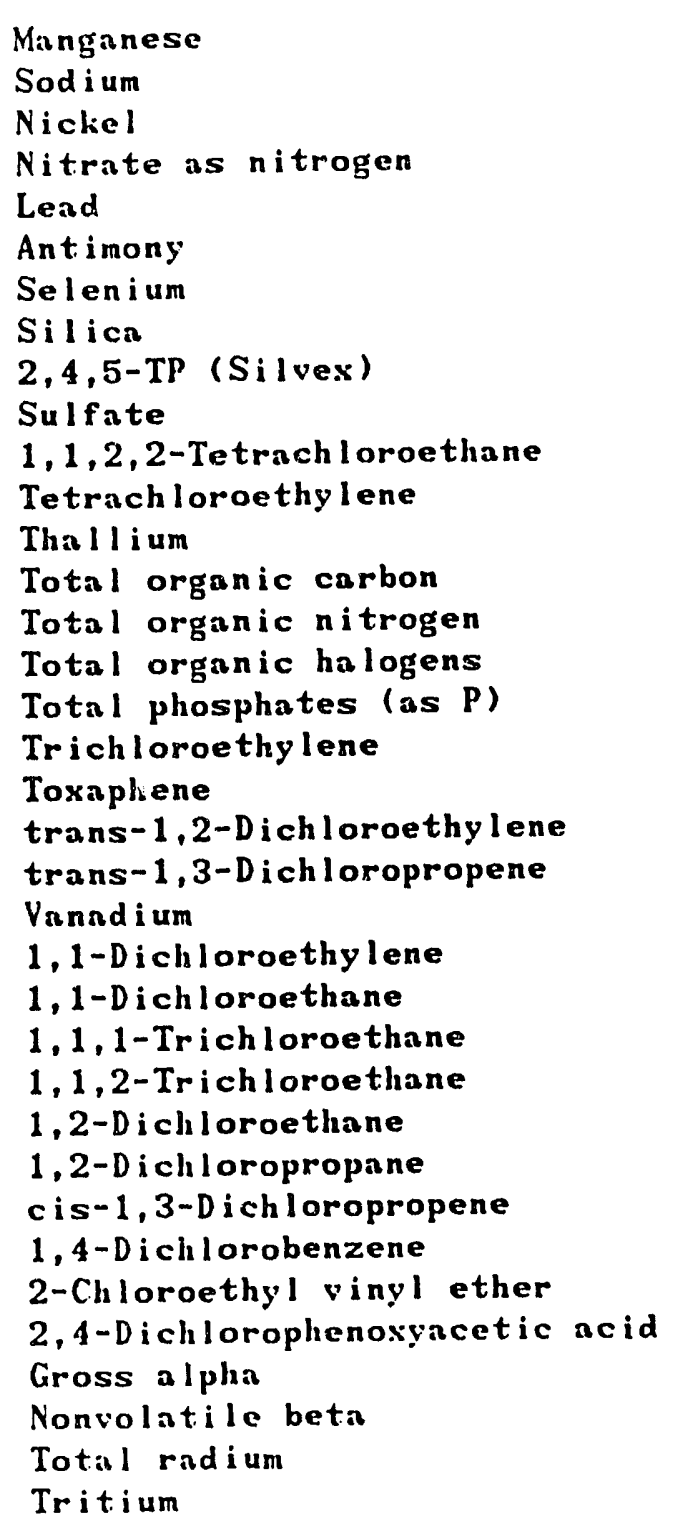

Manganese

Nitrate as nitrogen

Antimony

Selenium

Silica

$2,4,5-T P$ (Silvex)

$1,1,2,2$-Tetrachloroethane

Tetrachloroethylene

Thalli um

Total organic carbon

Total organic nitrogen

Total organic halogens

Total phosphates (as P)

Toxaphene

trans-1,2-Dichloroethy lene

trans-1,3-Dichloropropene

Vannd i um

1,1-Dichloroethylene

1,1-Dichloroethane

1,1,1-Trichloroethan

1,2-Dichloroethane

1,2-Dichloropropane

c is-1,3-D ichloropropene

1, 4-D ich lorobenzene

2-Chloroethyl vinyl ether

Gross a lpha

Total radium

Tritium

\begin{tabular}{|c|c|c|c|c|}
\hline & 15.80 & ug $/ \mathrm{L}$ & Gen & Eng. \\
\hline & 3600.00 & ug $/ \mathrm{L}$ & Gen. & Eng. \\
\hline LT & 4.00 & ug $/ \mathrm{L}$ & Gen. & Eng. \\
\hline & 79.00 & ug $/ \mathrm{L}$ & Gen. & Eng. \\
\hline LT & 3.00 & ug $/ \mathrm{L}$ & Gen. & Eng. \\
\hline LT & 2.00 & ug $/ \mathrm{L}$ & Gen. & ing \\
\hline LT & 2.00 & ug $/ \mathrm{L}$ & Gen. & ing \\
\hline & 3390.00 & ug $/ \mathrm{L}$ & Gen. & ing \\
\hline LT & 0.09 & ug $/ \mathrm{L}$ & Gen. & ng \\
\hline LT & 1000.00 & $u_{g} / L$ & Gen. & ng \\
\hline LT & 1.00 & ug $/ \mathrm{L}$ & en. & ng \\
\hline$L T$ & 1.00 & $\mathrm{ug} / \mathrm{L}$ & Gen. & $\mathrm{ng}$ \\
\hline LT & 2.00 & ug $/ \mathrm{L}$ & en .- & ng \\
\hline & 5000.00 & ug/L & en. & ng \\
\hline LT & 100.00 & ug/L & en. & ng \\
\hline & 174.00 & ug/L & en. & ng \\
\hline LT & 100.00 & ug $/ \mathrm{L}$ & Gen. & Eng \\
\hline LT & 1.00 & ug /L & Gen. & n \\
\hline LT & 0.24 & ug/L & Gen. & \\
\hline L.T & 1.00 & ug/L & en. & \\
\hline LT & 1.00 & $u g / L$ & ien. & $n_{E}$ \\
\hline LT & 10.00 & ug/L & Gen. & me \\
\hline LT & 1.00 & ug/L & Gen. & En \\
\hline & 15.90 & $\mathbf{u g} / \mathrm{L}$ & Gen. & En \\
\hline LT & 1.00 & ug/L & Gen. & En \\
\hline LT & 1.00 & ug $/ \mathrm{L}$ & Gen. & 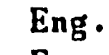 \\
\hline & 4.57 & ug $/ \mathrm{L}$ & ien. & 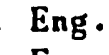 \\
\hline LT & 1.00 & $\mathrm{ug} / \mathrm{L}$ & Gen. & - En \\
\hline LT & 1.00 & $u g / L$ & Gen. & \\
\hline & 61.00 & $u g / L$ & Gen. & \\
\hline LT & 1.00 & $\mathrm{ug} / \mathrm{L}$ & Gen. & \\
\hline LT & 0.30 & $u g / L$ & Gen. & \\
\hline LT & 2.00 & $\mathrm{pCi} / \mathrm{L}$ & Gen. & • \\
\hline $.20+-$ & 2.40 & $\mathrm{pCi} / \mathrm{L}$ & Gen. & \\
\hline $4.60+-$ & 3.40 & $\mathrm{pCi} / \mathrm{L}$ & Gen. & - \\
\hline $6.78+-$ & $-\quad .0 .40$ & $\mathrm{pCi} / \mathrm{mL}$ & Gen. & \\
\hline
\end{tabular}


HELL. LFW 19

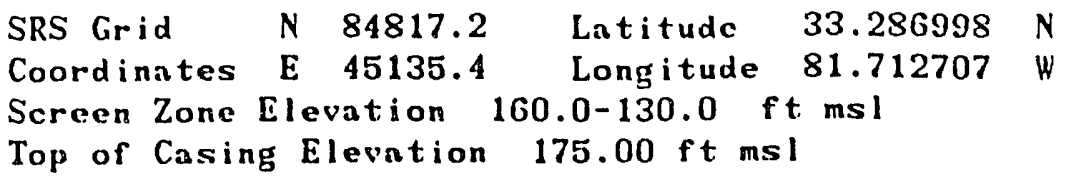

MEASUREMENTS CONDUCTED IN THE FIELD

Sample date $08 / 13 / 91$ Time 1230

Depth to water - $18.40 \mathrm{ft}(5.61 \mathrm{~m})$ below the TOC

Water elevation $-156.60 \mathrm{ft}(47.73 \mathrm{~m}) \mathrm{ms}$ l

$\mathrm{pH}=4.8$ Alkalinity - $1 \mathrm{mg} / \mathrm{L}$

Specific conductance - 17 umhos/cm

Water temperature - 19.1 degrees Celsius

Water evacuated from the well prior to sampling - $75 \mathrm{gal}$

\section{LABORATORY ANALYSES}

Specific conductance

pII

Silver

Al um inum

Aldrin

Arsenic

Barium

beta-Benzene hexachloride

Bromodich loromethane

Calcium

Trich lor of luoromethane

Carbon tetrachloride

Cadmi um

Bromof orm

Chlor of orm

Dichloromethane (Mcthylene chloride)

Bromomethane (Methyl bromide)

Chloromethane (Methyl chloride)

Chloride

Chlorobenzene

Chromium

Copper

Chlorocthene (Vingl chloride)

Chlorocthane

Benzene

Dibromoch loromethane

Endrin

Endosulfan sulfate

Ethylbenzene

Fluoride

Iron

Mercury

Potassium

Lindane

Toluene

Methoxychlor

Magnesium

CONTINUED
$15.00 \mathrm{umh} / \mathrm{cm}$

$5.35 \mathrm{pH}$

LT

LT

LT

LT

LT

LT

LT

LT

LT

LT

LT

LT

LT

LI

\section{LT}

LT

LT

LT

LT

LT

LT

LT

LT

LT

LT

LT

LT

LT

LT

LT
$2.00 \mathrm{ug} / \mathrm{L}$

$20.00 \mathrm{ug} / \mathrm{L}$

$0.05 \mathrm{ug} / \mathrm{L}$

$2.00 \mathrm{ug} / \mathrm{L}$

$3.00 \mathrm{ug} / \mathrm{L}$

$0.05 \mathrm{ug} / \mathrm{L}$

$1.00 \mathrm{ug} / \mathrm{L}$

$353.00 \mathrm{ug} / \mathrm{L}$

$1.00 \mathrm{ug} / \mathrm{L}$

$1.00 \mathrm{ug} / \mathrm{L}$

$2.00 \mathrm{ug} / \mathrm{L}$

$1.00 \mathrm{ug} / \mathrm{L}$

$1.00 \mathrm{ug} / \mathrm{L}$

$1.62 \mathrm{ug} / \mathrm{L}$

$1.00 \mathrm{ug} / \mathrm{L}$

$1.00 \mathrm{ug} / \mathrm{L}$

$2320.00 \mathrm{ug} / \mathrm{L}$

$1.00 \mathrm{ug} / \mathrm{L}$

$4.00 \mathrm{ug} / \mathrm{L}$

$4.00 \mathrm{ug} / \mathrm{L}$

$1.00 \mathrm{ug} / \mathrm{L}$

$1.00 \mathrm{ug} / \mathrm{L}$

$1.00 \mathrm{ug} / \mathrm{L}$

$1.00 \mathrm{ug} / \mathrm{L}$

$0.01 \mathrm{ug} / \mathrm{L}$.

$0.10 \mathrm{ug} / \mathrm{L}$

$1.00 \mathrm{ug} / \mathrm{L}$

$100.00 \mathrm{ug} / \mathrm{L}$

$54.20 \mathrm{ug} / \mathrm{L}$.

$0.20 \mathrm{ug} / \mathrm{L}$

$500.00 \mathrm{ug} / \mathrm{L}$

$0.00 \mathrm{ug} / \mathrm{L}$

$1.00 \mathrm{ug} / \mathrm{L}$

$0.50 \mathrm{ug} / \mathrm{L}$

$421.00 \mathrm{ug} / \mathrm{L}$
Gen. Eng.

Gen. Eng.

Gen. Eng.

Gen. Eng.

Gen. Eng.

Gen. Eng.

Gen. Eng.

Gen. Eng.

Gen. Eng.

Gen. Eng.

Gen. Eng.

Gen. Eng.

Gen. Eng.

Gen. Eng.

Gen. Eng.

Gen. Eng.

Gen. Eng.

Gen. Eng.

Gen. Eng.

Gen. Eng.

Gen. Eng.

Gen. Eng.

Gen. Eng.

Gen. Eng.

Gen. Eng.

Gen. Eng.

Gen. Eng.

Gen. Eng.

Gen. Eng.

Gen. Eng.

Gen. Eng.

Gen. Eng.

Gen. Eng.

Geñ. Eng.

Gen. Eng.

Gen. Eng.

Gen. Eng. 
Manganese

Sod i um

Nicliel

Nitrate ns nitrogen

Lead

Antimony

Selenium

Silica

$2,4,5-T P$ (Silvex)

Sulfate

1,1,2,2-Tetrach loroethane

Tetrachloroethylene

Tha 11 ium

Total organic carbon

Total organic nitrogen

Total organic halogens

Total phosphates (as P)

Trich loroethylene

Toxaphene

trans-1,2-Dich loroethylene

trans-1,3-D ich loropropene

Vanadium

1,1-Dichloroethylene

1,1-Dichloroethane

1,1,1-Trichloroethane

1,1,2-Trichloroethane

1,2-Dichloroethane

1,2-Dichloropropane

c is-1,3-Dich lopopropene

1,4-Dichlorobenzene

2-Chloroethyl vinyl ether

2,4-Dichlorophenosyacetic iscid

Gross a lpha

Nonvolatile beta

Total radium

Tritium
LT

$$
\begin{array}{r}
2.00 \mathrm{ug} / \mathrm{L} \\
1370.00 \mathrm{ug} / \mathrm{L} \\
4.60 \mathrm{ug} / \mathrm{L}
\end{array}
$$

$170.00 \mathrm{ug} / \mathrm{L}$

$4.05 \mathrm{ug} / \mathrm{L}$

LT

$2.00 \mathrm{ug} / \mathrm{L}$

LT

$2.00 \mathrm{ug} / \mathrm{L}$

$6810.00 \mathrm{ug} / \mathrm{L}$

$0.09 \mathrm{ug} / \mathrm{L}$

LT

LT

$1000.00 \mathrm{ug} / \mathrm{L}$

$1.00 \mathrm{ug} / \mathrm{L}$

$1.00 \mathrm{ug} / \mathrm{L}$

$2.00 \mathrm{ug} / \mathrm{L}$

$1000.00 \mathrm{ug} / \mathrm{L}$

$100.00 \mathrm{ug} / \mathrm{L}$

$9.90 \mathrm{ug} / \mathrm{L}$

$100.00 \mathrm{ug} / \mathrm{L}$

$1.00 \mathrm{ug} / \mathrm{L}$

$0.24 \mathrm{ug} / \mathrm{L}$

$1.00 \mathrm{ug} / \mathrm{L}$

$1.00 \mathrm{ug} / \mathrm{L}$

$10.00 \mathrm{ug} / \mathrm{L}$

$1.00 \mathrm{ug} / \mathrm{L}$

$1.00 \mathrm{ug} / \mathrm{L}$

$1.00 \mathrm{ug} / \mathrm{L}$

$1.00 \mathrm{ug} / \mathrm{L}$

$1.00 \mathrm{ug} / \mathrm{L}$

$1.00 \mathrm{ug} / \mathrm{L}$

$1.00 \mathrm{ug} / \mathrm{L}$

$10.00 \mathrm{ug} / \mathrm{L}$

$1.00 \mathrm{ug} / \mathrm{L}$

$0.30 \mathrm{ug} / \mathrm{L}$

$2.00 \mathrm{pC} i / L$

LT

3.90+-

$2.10 \mathrm{pCi} / \mathrm{L}$

1.

$2.40 \mathrm{pCi} / \mathrm{L}$

3. $14 \cdots$

$0.30 \mathrm{pCi} / \mathrm{mL}$
Gen. Eng .

Gen. Eng.

Gen. Eng.

Gen. Eng.

Gen. Eng.

Gen. Eng.

Gen. Eng.

Gen. Eng.

Gen. Eng.

Gen. Eng.

Gen. Eng.

Gen. Eng.

Gen.- Eng.

Gen. Eng.

Gen. Eng.

Gen. Eng.

Gen. Eng.

Gen. Eng.

Gen. Eng.

Gen. Eng.

Gen. Eng.

Gen. Eng.

Gen. Eng.

Gen. Eng.

Gen. Eng.

Gen. Eng.

Gen. Eng.

Gen. Eng.

Gen. Eng.

Gen. Eng.

Gen. Eng.

Gen. Eng.

Gen. Eng.

Gen. Eng.

Gen. Eng.

Gen. Eng. 
HELL LFW 20

SRS Grid N 85262.6 Latitude $33.288714 \mathrm{~N}$

Coordinates E 45582.9 Longitude $81.712395 \mathrm{~W}$

Screen Zone Elevation 165.0-135.0 ft ms

Top of Casing Elevation $179.00 \mathrm{ft} \mathrm{msl}$

MEASUREMENTS CONDUCTED IN THE FIELD

Sample date 08/13/91 Time 925

Depth to water - $18.78 \mathrm{ft}(5.72 \mathrm{~m})$ below the TOC

Water elevation $-160.22 \mathrm{ft}(48.84 \mathrm{~m}) \mathrm{msl}$

pll $=4.8 \quad$ Alkalinity $=0 \mathrm{mg} / \mathrm{L}$

Specific conductance = 17 umhos $/ \mathrm{cm}$

Water temperature -20.0 degrees Celsius

Water evacuated from the well prior to sampling - 72 gal

\section{LABORATORY ANALYSES}

Specific conductance

$\mathrm{pH}$

Silver

A l um i num

Aldrin

Arsenic

Bari um

beta-Benzene hexachloride

Bromodichloromethane

Calcium

Trichlorof luoromethane

Carbon tetrachloride

Cadm i um

Bromof orm

Chloroform

Dichloromethane (Methylene chloride)

Bromomethane (Methyl bromide)

Chloromethane (Methyl chloride)

Chloride

Chlorobenzene

Chromium

Copper

Chloroethene (Vinyl chloride)

Chlorocthane

Benzene

Dibromochloromethane

Endrin

Endosulfan sulfate

Ethylbenzene

Fluoride

Fluoride

Iron

Mercury

Potassium

Lindane

Toluene

Methosychlor

CONTINUED

$$
15.00 \mathrm{umh} / \mathrm{cm}
$$

$5.24 \mathrm{pH}$

LT

LT

LT

LT

LT

LT

LT

LT

L.T

LT

LT

LT

LT

LT

LT

LT

LT

LT

LT

LT

LT

LT

LT

LT

$2.00 \mathrm{ug} / \mathrm{L}$

$20.00 \mathrm{ug} / \mathrm{L}$

0.05 ug/L

$2.00 \mathrm{ug} / \mathrm{L}$

$4.13 \mathrm{ug} / \mathrm{L}$

$0.05 \mathrm{ug} / \mathrm{L}$

$1.00 \mathrm{ug} / \mathrm{L}$

$187.00 \mathrm{ug} / \mathrm{L}$

$1.00 \mathrm{ug} / \mathrm{L}$

$1.00 \mathrm{ug} / \mathrm{L}$

$2.00 \mathrm{ug} / \mathrm{L}$

$1.00 \mathrm{ug} / \mathrm{L}$

$1.00 \mathrm{ug} / \mathrm{L}$

$2.22 \mathrm{ug} / \mathrm{L}$

$1.00 \mathrm{ug} / \mathrm{L}$

$1.00 \mathrm{ug} / \mathrm{L}$

$2530.00 \mathrm{ug} / \mathrm{L}$

$1.00 \mathrm{ug} / \mathrm{L}$

$4.00 \mathrm{ug} / \mathrm{L}$

$7.55 \mathrm{ug} / \mathrm{L}$

$1.00 \mathrm{ug} / \mathrm{L}$

$1.00 \mathrm{ug} / \mathrm{L}$

$1.00 \mathrm{ug} / \mathrm{L}$

$1.00 \mathrm{ug} / \mathrm{L}$

$0.01 \mathrm{ug} / \mathrm{L}$

$0.10 \mathrm{ug} / \mathrm{L}$

$1.00 \mathrm{ug} / \mathrm{L}$

$100.00 \mathrm{ug} / \mathrm{L}$

$100.00 \mathrm{ug} / \mathrm{L}$

$55.40 \mathrm{ug} / \mathrm{L}$

LT

LT

LT

LT

$0.20 \mathrm{ug} / \mathrm{L}$

$500.00 \mathrm{ug} / \mathrm{L}$

$0.00 \mathrm{ug} / \mathrm{L}$

LT
$1.00 \mathrm{ug} / \mathrm{L}$

$0.50 \mathrm{ug} / \mathrm{L}$
Gen. Eng.

Gen. Eng.

Gen. Eng.

Gen. Eng.

Gen. Eng.

Gen. Eng .

Gen. Eng.

Gen. Eng.

Gen. Eng.

Gen. Eng .

Gen. Eng.

Gen. Eng -

Gen. Eng.

Gen. Eng.

Gen. Eng.

Gen. Eng.

Gen. Eng.

Gen. Eng.

Gen. Eng.

Gen. Eng.

Gen. Eng.

Gen. Eng.

Gen. Eng.

Gen. Eng.

Gen. Eng.

Gen. Eng.

Gen. Eng.

Gen. Eng.

Gen. Eng.

Gen. Eng.

Gen. Eng.

Gen. Eng.

Gen. Eng.

Geñ. Eng.

Gen. Eng.

Gen. Eng.

Gen. Eng. 
WELL LFW 20 COLLECTED ON 08/13/91 LABORATORY ANALYSES CONTINUED

\author{
Magnesium \\ Manganese \\ Sod i um \\ Nickel \\ Nitrate as nitrogen \\ Lead \\ Antimony \\ Selenium \\ Silica \\ $2,4,5-T P$ (Silvex) \\ Sulf ate \\ 1,1,2,2-Tetrachloroethane \\ Tetrachloroethylene \\ Thalli um \\ Total organic carbon \\ Total organic nitrogen \\ Total organic halogens \\ Total phosphates (as P) \\ Trichloroethylene \\ Toxaphene \\ trans-1,2-Dichloroethylene \\ trans-1,3-Dichloropropene \\ Vanadium \\ 1,1-Dichloroethylene \\ 1,1-Dichloroethane \\ 1,1,1-Trichloroethane \\ 1,1,2-Trichloroethane \\ 1,2-Dichloroethane \\ 1,2-Dichloropropane \\ c is-1,3-Dichloropropene \\ 1,4-D ichlorobenzene \\ 2-Chloroethyl vinyl ether \\ 2,4-Dichlorophenosyacetic acid \\ Gross a lpha \\ Nonvolatile beta \\ Total radium \\ Tritium
}

$$
\begin{array}{r}
309.00 \mathrm{ug} / \mathrm{L} \\
2.20 \mathrm{ug} / \mathrm{L} \\
1330.00 \mathrm{ug} / \mathrm{L} \\
5.66 \mathrm{ug} / \mathrm{L} \\
170.00 \mathrm{ug} / \mathrm{L} \\
4.83 \mathrm{ug} / \mathrm{L} \\
2.00 \mathrm{ug} / \mathrm{L} \\
2.00 \mathrm{ug} / \mathrm{L} \\
6180.00 \mathrm{ug} / \mathrm{L}
\end{array}
$$

LT

LT

$0.09 \mathrm{ug} / \mathrm{L}$

LT $1000.00 \mathrm{ug} / \mathrm{L}$

LT

LT

LT

LT

LT

LT

LT

L.T

LT

LT

LT

LT

LT

LT

LT

LT

LT

LT

LT

LT

LT

LT

LT

$1.00 \mathrm{ug} / \mathrm{L}$

$1.00 \mathrm{ug} / \mathrm{L}$

$2.00 \mathrm{ug} / \mathrm{L}$

$1000.00 \mathrm{ug} / \mathrm{L}$

$100.00 \mathrm{ug} / \mathrm{L}$

$5.00 \mathrm{ug} / \mathrm{L}$

$100.00 \mathrm{ug} / \mathrm{L}$

$1.00 \mathrm{ug} / \mathrm{L}$

$0.24 \mathrm{ug} / \mathrm{L}$

$1.00 \mathrm{ug} / \mathrm{L}$

$1.00 \mathrm{ug} / \mathrm{L}$

$10.00 \mathrm{ug} / \mathrm{L}$

$1.00 \mathrm{ug} / \mathrm{L}$

$1.00 \mathrm{ug} / \mathrm{L}$

$1.00 \mathrm{ug} / \mathrm{L}$

$1.00 \mathrm{ug} / \mathrm{L}$

$1.00 \mathrm{ug} / \mathrm{L}$

$1.00 \mathrm{ug} / \mathrm{L}$

$1.00 \mathrm{ug} / \mathrm{L}$

$10.00 \mathrm{ug} / \mathrm{L}$

$1.00 \mathrm{ug} / \mathrm{L}$

$0.30 \mathrm{ug} / \mathrm{L}$

$2.20+-$

$2.20+-1.90 \mathrm{pCi} / \mathrm{L}$
$3.70+-\quad 3.90 \mathrm{pCi} / \mathrm{L}$

$2.83+-$
$2.00 \mathrm{pCi} / \mathrm{L}$

$0.30 \mathrm{pCi} / \mathrm{mL}$
Gen. Eng.

Gen. Eng.

Gen. Eng.

Gen. Eng.

Gen. Eng.

Gen. Eng.

Gen. Eng.

Gen. Eng.

Gen. Eng.

Gen. Eng.

Gen. Eng.

Gen. Eng.

Gen - Eng.

Gen. Eng.

Gen. Eng.

Gen. Eng.

Gen. Eng.

Gen. Eng.

Gen. Eng.

Gen. Eng.

Gen. Eng.

Gen. Eng .

Gen. Eng.

Gen. Eng.

Gen. Eng.

Gen. Eng.

Gen. Eng.

Gen. Eng.

Gen. Eng.

Gen. Eng.

Gen. Eng.

Gen. Eng.

Gen. Eng.

Gen. Eng.

Gen. Eng.

Gen. Eng.

Gen. Eng. 
WELL LFW 21

$\begin{array}{llllll}\text { SRS Grid } & N & 84178.3 & \text { Latitude } & 33.287241 & N \\ \text { Coordinates } & E & 46149.4 & \text { Longitude } 81.708796 & W\end{array}$

Screen Zone Elevation 158.9-128.9 ft msl

Top of Casing Elevntion $173.90 \mathrm{ft} \mathrm{msl}$

\section{MEASUREMENTS CONDUCTED IN THE FIELD}

Sample date 08/14/91 Time 1415

Depth to water - $22.23 \mathrm{ft}(6.78 \mathrm{~m})$ below the TOC

Water elevation $=151.67 \mathrm{ft}(46.23 \mathrm{~m}) \mathrm{msl}$

pH = 6.0 Alkalinity - $106 \mathrm{mg} / \mathrm{L}$

Specific conductance - $301 \mathrm{umhos} / \mathrm{cm}$

Water temporature - 20.6 degrees Celsius

Water evacuated from the well prior to sampling - 64 gal

\section{LABORATORY ANALYSES}

Specific conductance

Carbon 12-labeled 2,3,7,8-TC.DD

Carbon 12-labeled TCDF

Heptachlorodibenzo-p-dioxin isomers

Heptachlorodibenzo-p-furan isomers

Hexachlorodibenzo-p-dioxin isomers

Hexachlorodibenzo-p-furan isomers

Octachlorodibenzo-p-dioxin isomers

Octachlorodibenzo-p-furan isomers

Pentachlorodibenzo-p-dioxin isomers

Pentachlorodibenzo-p-furan isomers

pll

Tetrachlorodibenzo-p-dioxin isomers

Tetrachlorodibenzo-p-furan isomers

$1,2,3,4,6,7,8-\mathrm{HPCDD}$

$1,2,3,4,6,7,8-$ IIHPCDF

$1,2,3,4,7,8$-Hesachlorodibenzo-p-dioxin LT

$1,2,3,4,7,8$-Hexachlorodibenzo-p-furan LT

$1,2,3,7,8$-Pentachlorodibenzo-p-dioxin LT

$1,2,3,7,8$-Pentachlorodibenzo-p-furan

a, a-Dimethylphenethylamine

alplua-Benzene hexachloride

Acetone

Acetophenone

Acrolein

Acrylonitrile

Silver

Aluminum

Aldrin

Acenaphthene

Acenaphthylene

Aniline

Anthracene

Aramite

Arsenic

Allyi chloride

Bar i um

CONTINUED
$250.00 \mathrm{umh} / \mathrm{cm}$

$0.45 \mathrm{ng} / \mathrm{L}$

LT $0.40 \mathrm{ng} / \mathrm{L}$

LT $\quad 0.65 \mathrm{ng} / \mathrm{L}$

LT $0.45 \mathrm{ng} / \mathrm{L}$

LI

LT

LT

LT

LT

I.T

LT

LT

LT

LT

\section{TT}

LT

LT

LT

LT

LT

LT

LT

LT

LI

LT

LT

LT

LT

LT

LT

LT

LT
$0.45 \mathrm{ng} / \mathrm{L}$

$0.40 \mathrm{ng} / \mathrm{L}$

$1.00 \mathrm{ng} / \mathrm{L}$

$1.00 \mathrm{ng} / \mathrm{L}$

$0.55 \mathrm{ng} / \mathrm{L}$

$0.55 \mathrm{ng} / \mathrm{L}$

$6.32 \mathrm{pll}$

$0.45 \mathrm{ng} / \mathrm{L}$

$0.40 \mathrm{ng} / \mathrm{L}$

$0.65 \mathrm{ng} / \mathrm{L}$

$0.45 \mathrm{ng} / \mathrm{L}$

$0.45 \mathrm{ng} / \mathrm{L}$

$0.40 \mathrm{ng} / \mathrm{L}$

$.0 .55 \mathrm{ng} / \mathrm{L}$

$0.55 \mathrm{ng} / \mathrm{L}$

$10.00 \mathrm{ug} / \mathrm{L}$

$0.05 \mathrm{ug} / \mathrm{L}$

$1.00 \mathrm{ug} / \mathrm{L}$

$10.00 \mathrm{ug} / \mathrm{L}$

$20.00 \mathrm{ug} / \mathrm{L}$

$20.00 \mathrm{ug} / \mathrm{L}$

$2.00 \mathrm{ug} / \mathrm{L}$

$20.00 \mathrm{ug} / \mathrm{L}$

$0.05 \mathrm{ug} / \mathrm{L}$

$10.00 \mathrm{ug} / \mathrm{L}$

$10.00 \mathrm{ug} / \mathrm{L}$

$10.00 \mathrm{ug} / \mathrm{L}$

$10.00 \mathrm{ug} / \mathrm{L}$

$10.00 \mathrm{ug} / \mathrm{L}$

$2.00 \mathrm{ug} / \mathrm{L}$

$50.00 \mathrm{ug} / \mathrm{L}$

$6.53 \mathrm{ug} / \mathrm{L}$
Gen. Eng.

Gen. Eng.

Gen. Eng.

Gen. Eng.

Gen. Eng.

Gen. Eng.

Gen. Eng.

Gen. Eng.

Gen. Eng.

Gen. Eng.

Gen. Eng.

Gen. Eng.

Gen. Eng.

Gen. Eng.

Gen. Eng.

Gen. Eng.

Gen. Eng.

Gen. Engु.

Gen. Eng.

Gen. Eng.

Gen. Eng.

Gen. Eng.

Gen. Eng.

Gen. Eng.

Gen. Eng.

Gen. Eng.

Gen. Eng.

Gen. Eng.

Gen. Eng.

Gen. Eng.

Gen. Eng.

Gen. Eng.

Gen. Eng.

Gert. Eng.

Gen. Eng .

Gen. Eng.

Gen. Eng. 


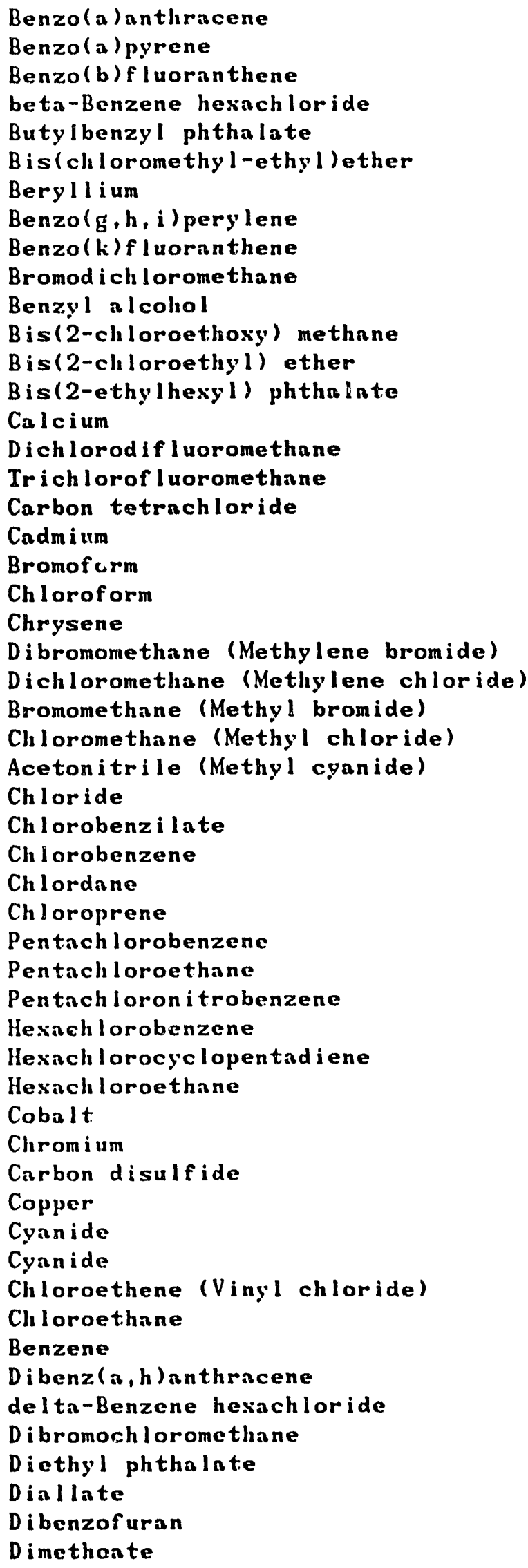

\begin{tabular}{|c|c|c|c|c|}
\hline LT & 10.00 & ug/L & Gen. & Eng \\
\hline LT & 10.00 & $u g / L$ & Gen. & Eng \\
\hline LT & 10.00 & $u g / L$ & Gen. & Eng \\
\hline LT & 0.05 & ug/L & Gen. & Eng \\
\hline LT & 10.00 & $u g / L$ & Gen. & Eng \\
\hline LT & 10.00 & ug /L & Gen. & Eng \\
\hline LT & 5.00 & $u_{g} / L$ & Gen. & Eng \\
\hline LT & 10.00 & ug/L & Gen. & Eng \\
\hline LT & 10.00 & $u g / L$ & Gen. & Eng \\
\hline LT & 1.00 & ug/L & Gen. & Engr. \\
\hline LT & 10.00 & ug/L & Gen. & Eng. \\
\hline LT & 10.00 & $u g / L$ & Gen. & Eng. \\
\hline LT & 10.00 & $u g / L$ & Gen. & Eng. \\
\hline LT & 10.00 & ug/L & Gen. & Eng \\
\hline & 6530.00 & ug/L & Gen. & Eng. \\
\hline LI & 1.00 & $u g / L$ & Gen. & Eng. \\
\hline LT & 1.00 & ug/L & Gen. & Eng. \\
\hline $\mathbf{L T}$ & 1.00 & $u g / L$ & Gen. & Eng. \\
\hline & 5.03 & $\mathrm{ug} / \mathrm{L}$ & Gen. & Eng. \\
\hline LT & 1.00 & ug/L & Gen. & Eng. \\
\hline LT & 1.00 & ug $/ L$ & Gen. & Eng. \\
\hline $\mathrm{LT}$ & 10.00 & $u g / L$ & Gen. & Eng. \\
\hline $\mathrm{LT}$ & 1.00 & $u g / L$ & Gen. & Eng. \\
\hline & 275.00 & $u_{g} / L$ & Gen. & Eng. \\
\hline LT & 1.00 & $u g / L$ & Gen. & Eng. \\
\hline LT & 1.00 & ug/L & Gen. & Eng. \\
\hline LT & 1.00 & ug/L & Gen. & Eng \\
\hline & 11400.00 & ug /L & Gen. & Eng. \\
\hline LT & 10.00 & ug/L & Gen. & Eng \\
\hline & 1.00 & $\mathrm{ug} / \mathrm{L}$ & Gen. & Eng. \\
\hline LT & 0.50 & $u g / L$ & Gen. & Eng. \\
\hline LT & 200.00 & $u g / L$ & Gen. & Eng \\
\hline LT & $10 ! 00$ & $\mathrm{ug} / \mathrm{L}$ & Gen. & Eng. \\
\hline LT & 10.00 & ug /L & Gen. & Eng. \\
\hline L.T & 10.00 & $\mathbf{u g} / \mathrm{L}$ & Gen. & Eng \\
\hline LT & 10.00 & ug /L & Gen. & Eng \\
\hline LT & 10.00 & ug/L & Gen. & Eng. \\
\hline $\mathrm{LI}$ & 10.00 & $u g / L$ & Gen. & Eng. \\
\hline LT & 4.00 & $u g / L$ & Gen. & Eng \\
\hline LT & 4.00 & $\mathbf{u g} / \mathrm{L}$ & Gen. & Enğ \\
\hline LT & 1.00 & $u g / L$ & Gen. & Eng \\
\hline LT & 4.00 & $u g / L$ & Gen. & Eng \\
\hline LT & 5.00 & $w g / L$ & Gen. & Eng \\
\hline LT & 5.00 & $u_{g} / L$ & Gen. & Eng \\
\hline LT & 1.00 & $\mathrm{ug} / \mathrm{L}$ & Gen. & Eng \\
\hline LT & 1.00 & $u g / L$ & Gen. & Eng \\
\hline & 2.80 & $u g / L$ & Gen. & Eng \\
\hline LT & 10.00 & ug $/ L$ & Gen. & Eng \\
\hline LT & 0.05 & $u g / L$ & & Eng \\
\hline LT & 1.00 & $u g / L$ & Gen. & Eng \\
\hline LT & 10.00 & ug $/ L$ & Gert. & Eng \\
\hline LT & 10.00 & $\mathrm{ug} / \mathrm{L}$ & Gen. & Eng \\
\hline LT & 10.00 & ug $/ \mathrm{L}$ & Gen. & Eng \\
\hline LT & 10.00 & $\mathrm{ug} / \mathrm{L}$ & Gen. & En \\
\hline
\end{tabular}


WELL LFW 21 COLLECTED ON 08/14/91 LABORATORY ANALYSES CONTINUED

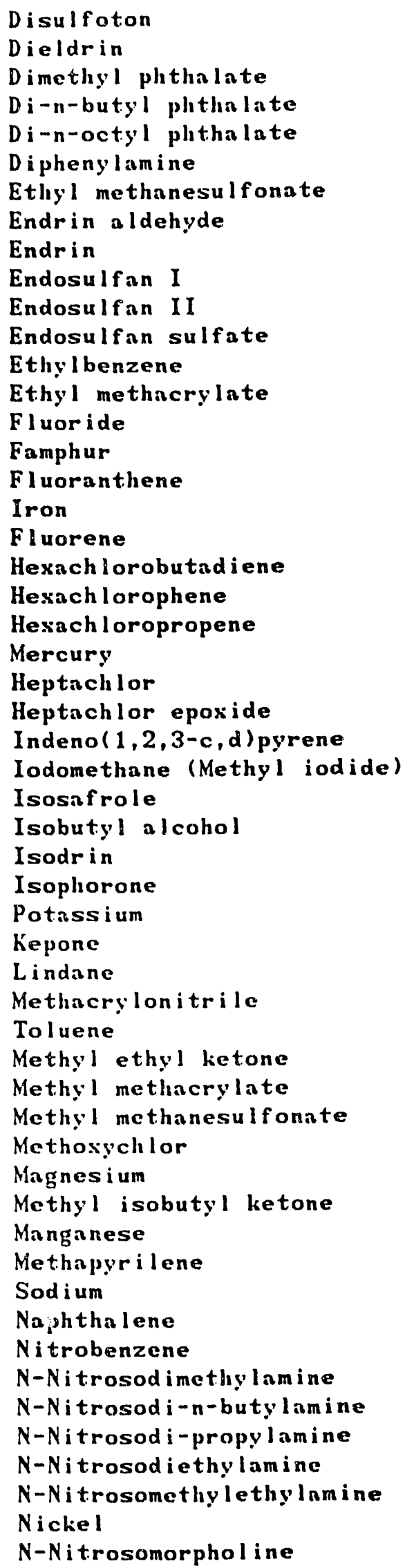

\begin{tabular}{|c|c|c|c|c|}
\hline $\mathrm{LT}$ & 10.00 & $u g / L$ & Gen. & Eng. \\
\hline $\mathrm{LT}$ & 0.50 & ug $/ \mathrm{L}$ & Gen. & Eng. \\
\hline LT & 10.00 & $u g / L$ & Gen. & Eng. \\
\hline LT & 10.00 & ug $/ \mathrm{L}$ & Gen. & Eng \\
\hline LT & 10.00 & $u g / L$ & Gen. & Eng. \\
\hline LT & 10.00 & ug $/ L$ & Gen. & Eng. \\
\hline LT & 10.00 & $u g / L$ & Gen. & Eng. \\
\hline $\mathrm{LT}$ & 0.10 & ug $/ L$ & Gen. & Eng. \\
\hline LT & 0.01 & $\mathbf{u g} / \mathrm{L}$ & Gen. & Eng. \\
\hline LT & 0.10 & ug $/ L$ & Gen. & Eng. \\
\hline LT & 0.10 & $u g / L$ & Gen. & Eng. \\
\hline LT & 0.10 & $u_{g} / L$ & Gen. & Eng. \\
\hline & 18.10 & ug/L & Gen... & Eng. \\
\hline$L T$ & 10.00 & ug $/ L$ & Gen. & Eng . \\
\hline & 137.00 & $u_{g} / L$ & Gen. & Eng. \\
\hline LT & 10.00 & ug/L & Gen. & Eng. \\
\hline $\mathbf{L T}$ & 10.00 & $\mathbf{u g}_{\mathrm{g}} / \mathrm{L}$ & Gen. & Eng. \\
\hline & 63500.00 & ug $/ L$ & Gen. & Eng. \\
\hline LT & 10.00 & ug/L & Gen. & Eng. \\
\hline LT & 10.00 & ug/L & Gen. & Eng. \\
\hline LT & 10.00 & ug/L & Gen. & Eng. \\
\hline LT & 10.00 & $u g / L$ & Gen. & Eng. \\
\hline LT & 0.20 & $u g / L$ & Gen. & Eng. \\
\hline LT & 0.05 & $u_{g} / L$ & Gen. & Eng. \\
\hline LT & 0.05 & $u g / L$ & Gen. & Eng. \\
\hline LT & 10.00 & $u_{g} / L$ & Gen. & Eng. \\
\hline L.T & 15.00 & ug $/ \mathrm{L}$ & & Eng \\
\hline LT & 10.00 & $u g / L$ & Gen. & Eng. \\
\hline LT & 100.00 & ug $/ L$ & Gen. & Eng. \\
\hline LT & 10.00 & ug /L & & En \\
\hline LT & 10.00 & $\mathrm{ug} / \mathrm{L}$ & Gen. & Eng. \\
\hline LT & 500.00 & ug $/ L$ & & Eng. \\
\hline LT & 10.00 & ugु /L & Gen. & Eng. \\
\hline LT & 0.00 & ug/L & Gen. & Eng. \\
\hline LT & 50.00 & $\mathbf{u} \mathfrak{G} / \mathrm{L}$ & & Eng \\
\hline & 19.10 & ug/L & Gen. & Eng \\
\hline & 39.40 & ug/L & Gen. & Eng \\
\hline LT & 10.00 & $\mathbf{u g} / \mathrm{L}$ & & \\
\hline LT & 10.00 & ug/L & Gen. & Eng \\
\hline $\mathrm{LT}$ & 0.50 & ug /L & & En \\
\hline & 6660.00 & $u_{g} / L$ & Gen. & Eng \\
\hline & 74.00 & ug $/ \mathrm{L}$ & Gen. & Eng \\
\hline & 120.00 & $\mathbf{u g} / \mathrm{L}$ & Gen. & \\
\hline LT & 10.00 & ug/L & Gen. & Eng \\
\hline & 18500.00 & $\mathbf{u g} / \mathrm{L}$ & Gen. & Eng \\
\hline LT & 10.00 & ug/L & Gen. & $E_{r}$ \\
\hline LT & 10.00 & ug/L & Gen. & Eng \\
\hline LT & 10.00 & $u g / L$ & Gen. & Eng \\
\hline LT & 10.00 & $u g / L$ & Gen. & $\Gamma$ \\
\hline LT & 10.00 & $u g / L$ & Gen: & Er \\
\hline LT & 10.00 & $u g / L$ & Gerr. & Es \\
\hline LT & 10.00 & $u g / L$ & & $\pi$ \\
\hline LT & 4.00 & $u g / L$ & Gen. & 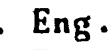 \\
\hline $\mathrm{LT}$ & 10.00 & $\mathbf{u g} / \mathrm{L}$ & Gen. & E \\
\hline
\end{tabular}


$N-N i$ trosodiphenylamine

$\mathrm{N}-\mathrm{Ni}$ trosopiperidine

$N-N i$ trosopyrolidine

Nitrate as nitrogen

Nitrate as nitrogen

4-Nitroquinoline-1-oxide

5-Nitro-o-toluidine

o-Toluidine

Lead

PCB 1016

PCB 1221

PC.B 1232

PCB 1242

PCB 1248

PCB 1254

PCB 1260

Pentachlorophenol

p-Phenylenediamine

p-Dimethy laminonzobenzene

Phenacetin

Phenanthrene

Phenol

Phorate

$\mathbf{p}, \mathbf{p}^{\prime}-\mathrm{DDD}$

$p, p^{\prime}-D D E$

$p, p^{\prime}-D D T$

Pronamid

Propionitrile

Parathion

Parathion methyl

Pyrene

Pyridine

Safrole

Antimony

2-sec-Butyl-4,6-d in itrophenol

Selen ium

Silica

$2,4,5-T P$ (Silvex)

Tin

Sulfate

Sulfate

Sulf otepp

Styrene

Sulf ide

1,2,4,5-Tetrach lorobenzene

$1,1,2,2$-Tetrachloroethane

$1,1,1,2$-Tetrachloroethane

* Tetrachloroethylene

$0,0,0$-Triethyl phosphorothionte

Thionazin

Thall i um

Total organic carbon

Total organic carbon

Total organic nitrogen CONTINUED

\begin{tabular}{|c|c|c|}
\hline $\mathrm{T}$ & 10.00 & ug $/$ \\
\hline LT & 10.00 & ug/ \\
\hline \multirow[t]{3}{*}{$\mathrm{LT}$} & 10.00 & ug/ \\
\hline & 150.00 & 1 \\
\hline & 170.00 & $g / 1$ \\
\hline & 10.00 & 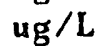 \\
\hline & 10. & $\mathbf{u g} / 1$ \\
\hline & 10 & $g / 1$ \\
\hline & & 5 \\
\hline & & $g /$ \\
\hline & 0 . & $\mathrm{g} /$ \\
\hline & 0 & $\mathbf{u g g}_{\mathbf{g}} /$ \\
\hline & & ug/ \\
\hline & & 5 \\
\hline & 1.00 & $g / 1$ \\
\hline & & $\mathrm{g} /$ \\
\hline & 10. & $\mathbf{u g} / 1$ \\
\hline & 10 & ug/ \\
\hline & 10 & 0 \\
\hline & 10. & ug $/ 1$ \\
\hline & 10 & ug/l \\
\hline & 10 & \\
\hline & 0 & ug $/ 1$ \\
\hline & & $\mathrm{ug}$ \\
\hline.$T$ & 0.10 & $\mathbf{u g} / 1$ \\
\hline & 0.10 & ug $/ 1$ \\
\hline & 10 & \\
\hline & 200 . & ug $/ 1$ \\
\hline & 0 . & $\mathrm{ug} / 1$ \\
\hline & & \\
\hline & 10 & ug $/ 1$ \\
\hline & & \\
\hline$T$ & 10. & ug .1 \\
\hline & 2 & $\mathbf{u g g} / \mathrm{I}$ \\
\hline & 10 & \\
\hline & 2 . & ugg/I \\
\hline & 11300 & ug $/ 1$ \\
\hline & & \\
\hline & 2 & ug $/ 1$ \\
\hline & 4240 & ug $/ 1$ \\
\hline & 4300 & ugg/l \\
\hline & 10.00 & $u_{g} / 1$ \\
\hline & & \\
\hline & 1000.00 & ug $/$ \\
\hline$T$ & 10.00 & ug $/ 1$ \\
\hline $\mathrm{LT}$ & & \\
\hline & 1.00 & $\mathrm{ug} / 1$ \\
\hline & 9.80 & ug $/ 1$ \\
\hline & 10.00 & ug $/$ \\
\hline & 10.00 & ug $/ 1$ \\
\hline$\pi$ & & \\
\hline & 11000.00 & ug / \\
\hline & 12000 . & \\
\hline & 100.00 & \\
\hline
\end{tabular}

Gen. Eng.

Gen. Eng.

Gen. Eng.

Gen. Eng.

Gen. Eng.

Gen. Eng.

Gen. Eng.

Gen. Eng.

Gen. Eng.

Gen. Eng.

Gen. Erz.

Gen. Eng.

Gen.- Eng .

Gen. Eng.

Gen. Eng .

Gen. Eng.

Gen. Eng.

Gen. Eng.

Gen. Eng.

Gen. Eng.

Gen. Eng.

Gen. Eng .

Gen. Eng.

Ge: . Eng.

Geil. Eng.

Gen. Eng.

Gen. Eng.

Gen. Eng.

Gen. Eng.

Gen. Eng.

Gen. Eng.

Gen. Eng.

Gen. Eng.

Gen. Eng.

Gen. Eng.

Gen. Eng.

Gen. Eng.

Gen. Eng.

Gen. Eng.

Gen. Eng .

Gen. Eng.

Gen. Eng.

Gen. Eng.

Gen. Eng.

Gen. Eng.

Gen. Eng.

Gen. Eng.

Gen. Eng.

Gen. Erig.

Gen. Eng.

Ger. Eng.

Gen. Eng.

Gen. Eng.

Gen. Eng. 
Total organic halogens

Total phosphates (as P)

* Trichloroethylene

Tosaphene

trans-1,2-Dichloroethylene

trans-1,3-Dichloropropene

trans-1,4-Dichloro-2-butene

Vanadi um

Vinyl acetate

Xylenes

1-Naphthylamine

1,1-Dichloroethylene

1,1-Dichloroethane

1,1,1-Trichloroethane

1,1,2-Trichloroethane

1,2-Dibromoethane

1,2-Dibromo-3-chloropropane

1,2-Dichlorobenzene

1,2-Dichloroethane

1,2-Dichloropropane

1,2,3-Trich loropropane

1,2,4-Trich lorobenzene

1,3-Dich lorobenzene

cis-1,3-D ichloropropene

1,3-Dinitrobenzene

1,3,5-Trinitrobenzene

1,4-Dichlorobenzene

1,4-Dioxane

1,4 -Naphthoquinone

2-Acetylaminof luorene

2-Chloroethyl vinyl ether

2-Chlorophenol

2-Chloronaphthalene

2-Hexanonc

2-Methylnaphthalene

o-Cresol (2-Metlyylphenol)

2-Nitroaniline

2-Naphthylamine

2-Nitrophenol

2-Picoline

2,3,4,6-Tetrach lorophenol

2,4-Diclulorophenosyacetic acid

2,4-Dichlorophenol

2,4-Dimethyl phenol

2,4-Dinitrophenol

2,4-Dinitrotoluene

2,4,5-Trichlorophenoxyacetic acid

2,4,5-Trichlorophenol

2, 4,6-Trichlorophenol

2, 6-Dichlorophenol

2,6-Dinitrotoluene

3-Methylcholanthrene

m-Cresol (3-Methylphenol)

3-Nitroaniline

CONTINUED
$612.00 \mathrm{ug} / \mathrm{L}$

LT

LT

LT

LT

LT

LT

LT

LT

LT

LT

LiT

LT

LT

LT

LT

LT

LT

LT

LT

LT

LT

LT

LT

LT

LT

LT

LT

LT

LT

LT

LT

LT

LT

LT

LT

LT

LT

LT

LT

LT

LT

LT

LT

LT

LT

LT

LT

LT
$100.00 \mathrm{ug} / \mathrm{L}$

$17.70 \mathrm{ug} / \mathrm{L}$

0.24 ug $/ \mathrm{L}$

$1.00 \mathrm{ug} / \mathrm{L}$

$1.00 \mathrm{ug} / \mathrm{L}$

$30.00 \mathrm{ug} / \mathrm{L}$

$10.00 \mathrm{ug} / \mathrm{L}$

$1.00 \mathrm{ug} / \mathrm{L}$.

$70.00 \mathrm{ug} / \mathrm{L}$

$10.00 \mathrm{ug} / \mathrm{L}$

$1.00 \mathrm{ug} / \mathrm{L}$

$74.20 \mathrm{ug} / \mathrm{L}$

$15.50 \mathrm{ug} / \mathrm{L}$

$1.00 \mathrm{ug} / \mathrm{L}$

$20.00 \mathrm{ug} / \mathrm{L}$

$1.00 \mathrm{ug} / \mathrm{L}$

$10.00 \mathrm{ug} / \mathrm{L}$

$1.00 \mathrm{ug} / \mathrm{L}$

$1.20 \mathrm{ug} / \mathrm{L}$

$20.00 \mathrm{ug} / \mathrm{L}$

$10.00 \mathrm{ug} / \mathrm{L}$

$10.00 \mathrm{ug} / \mathrm{L}$

$1.00 \mathrm{ug} / \mathrm{L}$

$10.00 \mathrm{ug} / \mathrm{L}$

$10.00 \mathrm{ug} / \mathrm{L}$

$10.00 \mathrm{ug} / \mathrm{L}$

$10.00 \mathrm{ug} / \mathrm{L}$

$10.00 \mathrm{ug} / \mathrm{L}$

$10.00 \mathrm{ug} / \mathrm{L}$

$10.00 \mathrm{ug} / \mathrm{L}$

$10.00 \mathrm{ug} / \mathrm{L}$

$10.00 \mathrm{ug} / \mathrm{L}$

$1.00 \mathrm{ug} / \mathrm{L}$

$10.00 \mathrm{ug} / \mathrm{L}$.

$10.00 \mathrm{ug} / \mathrm{L}$

$10.00 \mathrm{ug} / \mathrm{L}$

$10.00 \mathrm{ug} / \mathrm{L}$

$10.00 \mathrm{ug} / \mathrm{L}$

$10.00 \mathrm{ug} / \mathrm{L}$

$10.00 \mathrm{ug} / \mathrm{L}$

$0.30 \mathrm{ug} / \mathrm{L}$

$10.00 \mathrm{ug} / \mathrm{L}$

$10.00 \mathrm{ug} / \mathrm{L}$

$45.00 \mathrm{ug} / \mathrm{L}$

10.00 ug $/ \mathrm{L}$

$0.09 \mathrm{ug} / \mathrm{L}$

$10.00 \mathrm{ug} / \mathrm{L}$

$10.00 \mathrm{ug} / \mathrm{L}$

$10.00 \mathrm{ug} / \mathrm{L}$

$10.00 \mathrm{ug} / \mathrm{L}$

$10.00 \mathrm{ug} / \mathrm{L}$

$10.00 \mathrm{ug} / \mathrm{L}$

10.00 ug $/ \mathrm{L}$
Gen . Eng .

Gen. Eng .

Gen. Eng.

Gen. Eng.

Gen. Eng.

Gen. Eng.

Gen. Eng.

Gen. Eng.

Gen. Eng.

Gen. Eng.

Gen. Eng.

Gen. Eng.

Gen . Eng .

Gen. Eng.

Gen. Eng.

Gen. Eng.

Gen. Eng.

Gen. Eng.

Gen. Eng .

Gen. Eng.

Gen. Eng .

Gen. Eng.

Gen. Eng.

Gen. Eng.

Gen. Eng.

Gen. Eng.

Gen. Eng.

Gen. Eng.

Gen. Eng.

Gen. Eng.

Gen. Eng.

Gen. Eng.

Gen. Eng.

Gen. Eng.

Gen. Eng.

Gen. Eng.

Gen. Eng.

Gen. Eng.

Gen. Eng.

Gen. Eng.

Gen. Eng.

Gen. Eng .

Gen. Eng .

Gen. Eng.

Gen. Eng.

Gen. Eng.

Gen. Eng.

Gen. Eng.

Gen. Eng.

Gen: Eng.

Ger. Eng.

Gen. Eng.

Gen. Eng.

Gen. Eng. 
WELL LFW 21 COLLECTED ON 08/14/91 LABORATORY ANALYSES CONTINUED

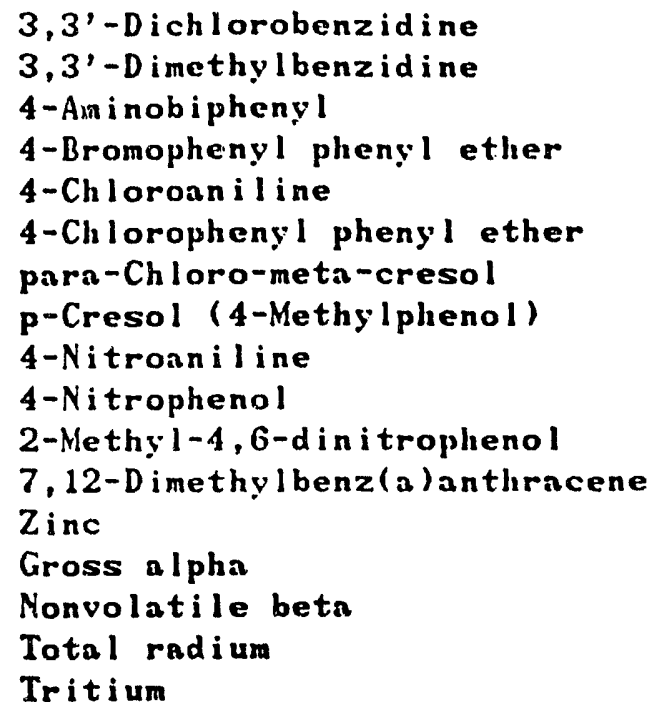

$\begin{array}{lrl}\mathrm{LT} & 20.00 \mathrm{ug} / \mathrm{L} \\ \mathrm{LT} & 10.00 \mathrm{ug} / \mathrm{L} \\ \mathrm{LT} & 10.00 \mathrm{ug} / \mathrm{L} \\ \mathrm{LT} & 10.00 \mathrm{ug} / \mathrm{L} \\ \mathrm{LT} & 10.00 \mathrm{ug} / \mathrm{L} \\ \mathrm{LT} & 10.00 \mathrm{ug} / \mathrm{L} \\ \mathrm{LT} & 10.00 \mathrm{ug} / \mathrm{L} \\ \mathrm{LT} & 10.00 \mathrm{ug} / \mathrm{L} \\ \mathrm{LT} & 10.00 \mathrm{ug} / \mathrm{L} \\ \mathrm{LT} & 10.00 \mathrm{ug} / \mathrm{L} \\ \mathrm{LT} & 50.00 \mathrm{ug} / \mathrm{L} \\ \mathrm{LT} & 10.00 \mathrm{ug} / \mathrm{L} \\ & 13.40 \mathrm{ug} / \mathrm{L} \\ \mathrm{LT} & 2.00 \mathrm{pCi} / \mathrm{L} \\ 2.70+- & 2.20 \mathrm{pCi} / \mathrm{L} \\ 2.40+- & 3.00 \mathrm{pCi} / \mathrm{L} \\ 4.80+- & 0.40 \mathrm{pCi} / \mathrm{mL}\end{array}$

Gen. Eng.

Gen. Eng.

Gen. Eng.

Gen. Eng.

Gen. Eng.

Gen. Eng.

Gen. Eng.

Gen. Eng.

Gen. Eng.

Gen. Eng.

Gen. Eng.

Gen. Eng.

Gen -- Eng -

Gen. Eng.

Gen. Eng.

Gen. Eng.

Gen. Eng.

\section{WELL LFW 22}

\section{SRS Grid N 84223.6 Latitude 33.287628 N \\ Coordinates E 46325.2 Longitude 81.708421 W \\ Screen Zone Elevation 152.4-122.4 ft ms I \\ Top of Casing Elevation $172.40 \mathrm{ft} \mathrm{msl}$}

\section{MEASUREMENTS CONDUCTED IN THE FIELD}

Sample date 08/15/91 Time 1450

Depth to water - $19.22 \mathrm{ft}(5.86 \mathrm{~m})$ below the TOC

Water elevation $-153.18 \mathrm{ft}(46.69 \mathrm{~m}) \mathrm{msl}$

$\mathrm{pH}=6.3$ Alkalinity $=202 \mathrm{mg} / \mathrm{L}$

Specif ic conductance $=597 \mathrm{um} / \mathrm{hos} / \mathrm{cm}$

Water temperature - 20.8 degrees Celsius

Hater evacuated from the well prior to sampling - $86 \mathrm{gal}$

\section{LABORATORY ANALYSES}

Specific conductance

Carbon 12-labeled 2,3,7,8-TCDD

Carbon 12-labeled TCDF

Heptachlorodibenzo-p-diosin isomers

Heptachlorodibenzo-p-furan isomers

Hesnchlorodibenzo-p-diosin isomers

Hexachlorodibenzo-p-furan isomers

Octachlorodibenzo-p-diosin isomers

Octachlorodibenzo-p-furan isomers

Pentachlorodibenzo-p-dioxin isomers

Pentachlorodibenzo-p-furan isomers pH

Tetrachlorodibenzo-p-dioxin isomers Tetrachlorodibenzo-p-furan isomers $1,2,3,4,6,7,8-H P C D D$

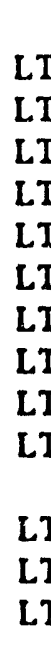

LT

L.T

LT

LT

LT

LT

LT

LT

LT

LT

LT

LT

LT
$430.00 \mathrm{umh} / \mathrm{cm}$

$0.45 \mathrm{ng} / \mathrm{L}$

$0.40 \mathrm{ng} / \mathrm{L}$

$0.65 \mathrm{ng} / \mathrm{L}$

$0.45 \mathrm{ng} / \mathrm{L}$

$0.45 \mathrm{ng} / \mathrm{L}$

$0.40 \mathrm{ng} / \mathrm{L}$

$1.00 \mathrm{ng} / \mathrm{L}$

$1.00 \mathrm{ng} / \mathrm{L}$

$0.55 \mathrm{ng} / \mathrm{L}$

$0.55 \mathrm{ng} / \mathrm{L}$

6.08 pll

$0.45 \mathrm{ng} / \mathrm{L}$

$0.40 \mathrm{ng} / \mathrm{L}$

$0.65 \mathrm{ng} / \mathrm{L}$
Gen. Eng.

Gen. Eng.

Gen. Eng.

Gen. Eng.

Gen. Eng.

Gen. Eng.

Gen. Eng.

Gen. Eng.

Gen. Eng.

Gen. Eng.

Gen. Eng.

Gen. Eng.

Gerr. Eng.

Gen. Eng.

Gen. Eng. CONTINUED 
$1,2,3,4,6,7,8$-HIIPCDF

$1,2,3,4,7,8$-Hexachlorodibenzo-p-dioxin $L T$

$1,2,3,4,7,8$-Hexachlorodibenzo-p-furan LT

$1,2,3,7,8$-Pentachlorodibenzo-p-dioxin LT

$1,2,3,7,8$-Pentachlorodibenzo-p-furnn LT

a, a-Dimethylphenethy lamine

alpha-Benzene hesachloride

Acetone

Acetophenone

Acrolein

Acrylonitrile

Silver

Aluminum

Aldrin

Acenaphthene

Acenaphthylene

Aniline

Anthracene

Aramite

Arsenic

Allyl chloride

Barium

Benzo(a) anthracene

Benzo( $a$ ) pyrene

Benzo ( $b$ ) f luoranthene

beta-Benzene hexachloride

Butylbenzyl phthalate

Bis(chloromethyl-ethyl)ether

Beryllium

Benzo( $g, h, i)$ perylene

Benzo( $k$ ) f luoranthene

Bromodichloromethane

Benzyl alcohol

Bis(2-chloroethosy) methane

Bis(2-chloroethyl) ether

Bis(2-ethylhesyl) phthalate

Calcium

Dichlorod if luoromethane

Trichlorof luorome thane

Carbon tetrachloride

* Cadmi um

Bromoform

Chlorrform

Chrysene

Dibromomethane (Methylene bromide)

Dichloromethane (Methylene chloride)

Bromomethane (Methyl bromide)

Chloromethane (Methyl chloride)

Acetonitrile (Methyl cyanide)

Chloride

Chlorobenzi inte

Chlorobenzene

Chlordane

Ch loroprene

CONTINUED
T
LT

LT

LT

LT

LT

LT

LT

LT

LT

LT

LT

LT

LT

LI

LI

LT

LT

LT

LT

LT

LT

LT

LT

LT

LT

LT

LT

LT

LT

LT

LT

LT

LT

LT

LT

LT

LT

LT

LT

LT

$T$

$T$

$T$

LT

$0.45 \mathrm{ng} / \mathrm{L}$

$0.45 \mathrm{ng} / \mathrm{L}$

$0.40 \mathrm{ng} / \mathrm{L}$

$0.55 \mathrm{ng} / \mathrm{L}$

$0.55 \mathrm{ng} / \mathrm{L}$

$10.00 \mathrm{ug} / \mathrm{L}$

$0.05 \mathrm{ug} / \mathrm{L}$

$24.80 \mathrm{ug} / \mathrm{L}$

$10.00 \mathrm{ug} / \mathrm{L}$

$20.00 \mathrm{ug} / \mathrm{L}$

$20.00 \mathrm{ug} / \mathrm{L}$

$2.00 \mathrm{ug} / \mathrm{L}$

$20.00 \mathrm{ug} / \mathrm{L}$

C. 05 ug/L

$10.00 \mathrm{ug} / \mathrm{L}$

$10.00 \mathrm{ug} / \mathrm{L}$

$10.00 \mathrm{ug} / \mathrm{L}$

$10.00 \mathrm{ug} / \mathrm{L}$

$10.00 \mathrm{ug} / \mathrm{L}$

$5.96 \mathrm{ug} / \mathrm{L}$

$50.00 \mathrm{ug} / \mathrm{L}$

$13.80 \mathrm{ug} / \mathrm{L}$

$10.00 \mathrm{ug} / \mathrm{L}$

$10.00 \mathrm{ug} / \mathrm{L}$

$10.00 \mathrm{ug} / \mathrm{L}$

$0.05 \mathrm{ug} / \mathrm{L}$

$10.00 \mathrm{ug} / \mathrm{L}$

$10.00 \mathrm{ug} / \mathrm{L}$

$5.00 \mathrm{ug} / \mathrm{L}$

$10.00 \mathrm{ug} / \mathrm{L}$

$10.00 \mathrm{ug} / \mathrm{L}$

$1.00 \mathrm{ug} / \mathrm{L}$

$10.00 \mathrm{ug} / \mathrm{L}$

$10.00 \mathrm{ug} / \mathrm{L}$

$10.00 \mathrm{ug} / \mathrm{L}$

$10.00 \mathrm{ug} / \mathrm{L}$

$4710.00 \mathrm{ug} / \mathrm{L}$

$1.00 \mathrm{ug} / \mathrm{L}$

$625.00 \mathrm{ug} / \mathrm{L}$

$1.00 \mathrm{ug} / \mathrm{L}$

$20.90 \mathrm{ug} / \mathrm{L}$

$1.00 \mathrm{ug} / \mathrm{L}$

$1.00 \mathrm{ug} / \mathrm{L}$

$10.00 \mathrm{ug} / \mathrm{L}$

$1.00 \mathrm{ug} / \mathrm{L}$

$1.00 \mathrm{ug} / \mathrm{L}$

$1.00 \mathrm{ug} / \mathrm{L}$

$1.00 \mathrm{ug} / \mathrm{L}$

$1.00 \mathrm{ug} / \mathrm{L}$

$25200.00 \mathrm{ug} / \mathrm{L}$

$10.00 \mathrm{ug} / \mathrm{L}$

$1.00 \mathrm{ug} / \mathrm{L}$

$0.50 \mathrm{ug} / \mathrm{L}$

$200.00 \mathrm{ug} / \mathrm{L}$
Gen. Eng.

Gen. Eng.

Gen. Eng.

Gen. Eng.

Gen. Eng .

Gen. Eng.

Gen. Eng.

Gen. Eng.

Gen. Eng.

Gen. Eng.

Gen. Eng .

Gen. Eng.

Gen - Eng.

Gen. Eng.

Gen. Eng.

Gen. Eng.

Gen. Eng.

Gen. Eng.

Gen. Eng.

Gen. Eng.

Gen. Eng.

Gen. Eng.

Gen. Eng.

Gen. Eng -

Gen. Eng .

Gen. Eng .

Gen. Eng .

Gen. Eng.

Gen. Eng.

Gen. Eng.

Gen. Eng.

Gen. Eng.

Gen. Eng.

Gen. Eng.

Gen. Eng.

Gen. Eng.

Gen. Eng.

Gen. Eng.

Gen. Eng.

Gen. Eng.

Gen. Eng.

Gen. Eng.

Gen. Eng.

Gen. Eng.

Gen. Eng.

Gen. Eng.

Gen. Eng .

Gen. Eng.

Gen. Eng.

Gen: Eng.

Gen. Erg.

Gen. Eng.

Gen. Eng.

Gen. Eng. 
WELL LFH' 22 COLLECTED ON 08/15/91 LABORATORY ANALYSES CONTINUED

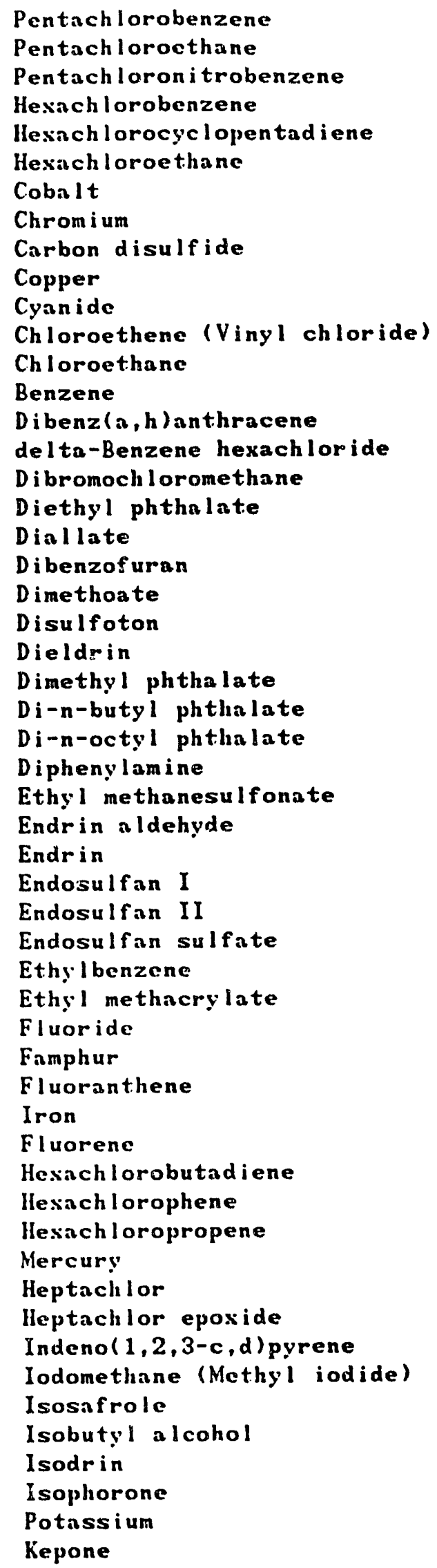

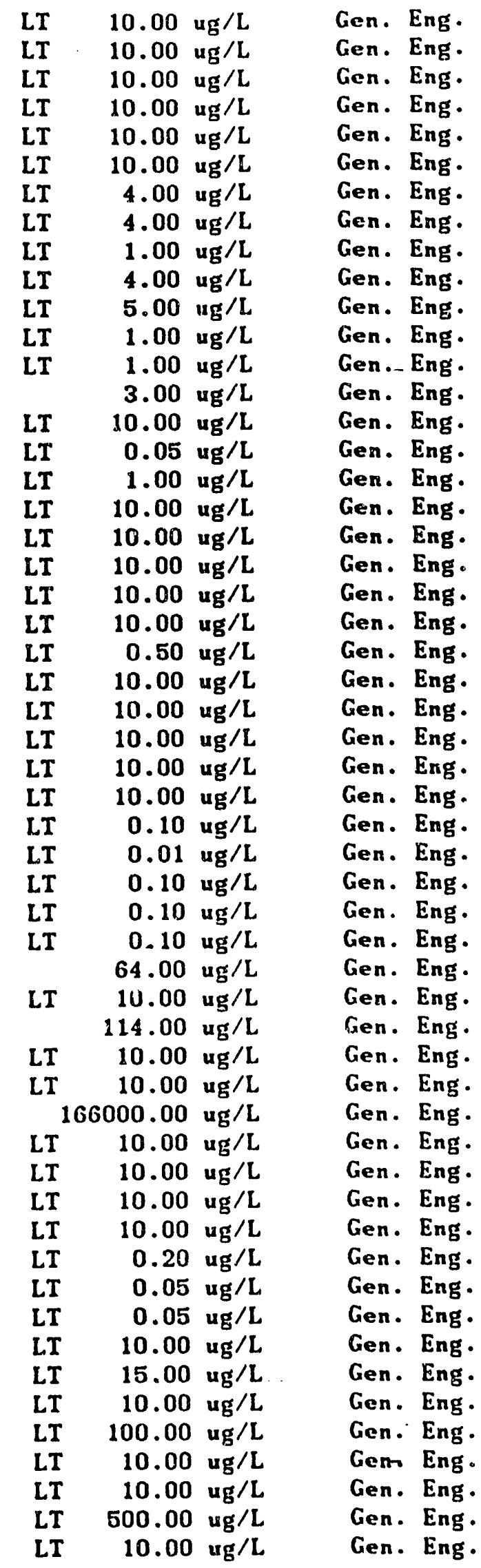


WELL LFW 22. WLECTED ON 08/15/91 LABORATORY ANALYSES CONTINUED

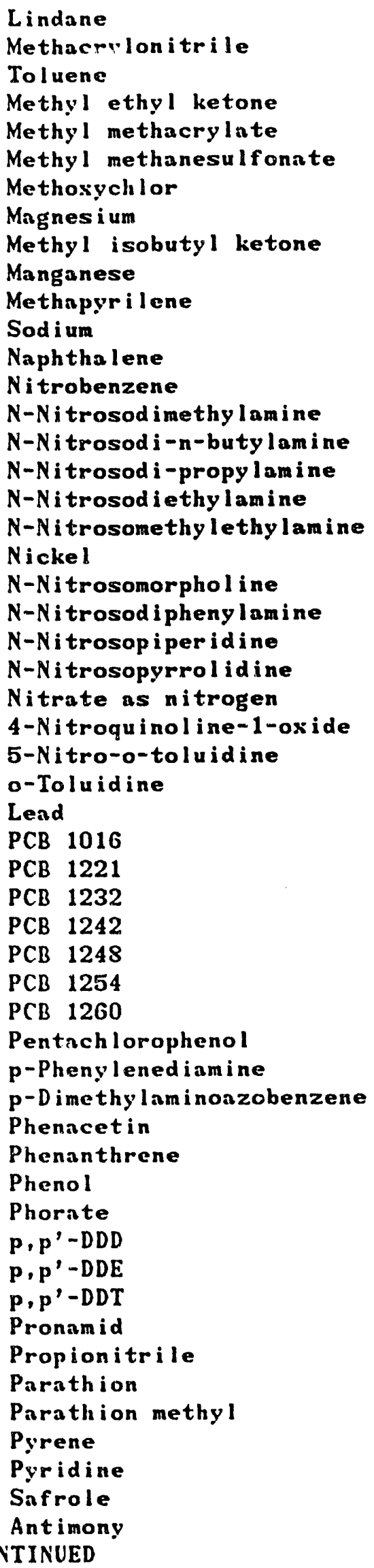

Lindane

Methacrvlonitrile

Toluene

Methyl ethyl ketone

Methyl methacrylnte

Methosychlor

Magnes i um

Methyl isobutyl ketone

Manganese

Methapyrilene

Naphthalene

Nitrobenzene

$\mathrm{N}-\mathrm{Ni}$ trosodimethylamine

$\mathrm{N}-\mathrm{Nitrosodi}-\mathrm{n}$-butylamine

$\mathrm{N}-\mathrm{Ni}$ trosodi-propy lamine

$\mathrm{N}$-Nitrosomethylethylamine

Nickel

$\mathrm{N}-\mathrm{N}$ itrosomorphol ine

$\mathrm{N}-\mathrm{N}$ itrosodi phenylamine

$\mathrm{N}$-Nitrosopiperidine

$\mathrm{N}$-Nitrosopyrrolidine

Nitrate as nitrogen

4-Nitroquinoline-1-oxide

o-Toluidine

Lead

PCB 1016

PCB 1248

PCB 1260

Pentachlorophenol

p-Phenylenediamine

$p-D$ ime thylaminoazobenzene

Phenacet in

Phenanthrene

$p, p^{\prime}-D D$

$p, p^{\prime}-D D E$

$p, p^{\prime}-D D T$

Pronamid

Propionitrile

Parathion

Parathion methy

LT

LT

$00 \mathrm{ug} / \mathrm{L}$

$50.00 \mathrm{ug} / \mathrm{L}$

$170.00 \mathrm{ug} / \mathrm{L}$

$638.00 \mathrm{ug} / \mathrm{L}$

LT

LT

LT

$10.00 \mathrm{ug} / \mathrm{L}$

$10.00 \mathrm{ug} / \mathrm{L}$

$0.50 \mathrm{ug} / \mathrm{L}$

$8380.00 \mathrm{ug} / \mathrm{L}$

$1680.00 \mathrm{ug} / \mathrm{L}$

$21.60 \mathrm{ug} / \mathrm{L}$

LT $\quad 10.00 \mathrm{ug} / \mathrm{L}$

$21400.00 \mathrm{ug} / \mathrm{L}$

LT $\quad 10.00 \mathrm{ug} / \mathrm{L}$

LT $10.00 \mathrm{ug} / \mathrm{L}$

LT $10.00 \mathrm{ug} / \mathrm{L}$

LT $10.00 \mathrm{ug} / \mathrm{L}$

LT $10.00 \mathrm{ug} / \mathrm{L}$

LT $\quad 10.00 \mathrm{ug} / \mathrm{L}$

LT $10.00 \mathrm{ug} / \mathrm{L}$

LT $4.00 \mathrm{ug} / \mathrm{L}$

LT $10.00 \mathrm{ug} / \mathrm{L}$

LT $\quad 10.00 \mathrm{ug} / \mathrm{L}$

LT $10.00 \mathrm{ug} / \mathrm{L}$

LT $10.00 \mathrm{ug} / \mathrm{L}$

$310.00 \mathrm{ug} / \mathrm{L}$

LT

LT

LT

LT

LT

LT

LT

LT

LT

LT

LT

LT

LT

LT

LT

LT

LT

LT

LT

LT

LT

LT

LT

LT

LT

LT

LT

LT

LT
$10.00 \mathrm{ug} / \mathrm{L}$

$10.00 \mathrm{ug} / \mathrm{L}$

$10.00 \mathrm{ug} / \mathrm{L}$

$3.00 \mathrm{ug} / \mathrm{L}$

$0.50 \mathrm{ug} / \mathrm{L}$

$0.50 \mathrm{ug} / \mathrm{L}$

$0.50 \mathrm{ug} / \mathrm{L}$

$0.50 \mathrm{ug} / \mathrm{L}$

$0.50 \mathrm{ug} / \mathrm{L}$

$1.00 \mathrm{ug} / \mathrm{L}$

$1.00 \mathrm{ug} / \mathrm{L}$

$10.00 \mathrm{ug} / \mathrm{L}$

$10.00 \mathrm{ug} / \mathrm{L}$

$10.00 \mathrm{ug} / \mathrm{L}$

$10.00 \mathrm{ug} / \mathrm{L}$

$10.00 \mathrm{ug} / \mathrm{L}$

$10.00 \mathrm{ug} / \mathrm{L}$

$0.10 \mathrm{ug} / \mathrm{L}$

$0.10 \mathrm{ug} / \mathrm{L}$

$0.10 \mathrm{ug} / \mathrm{L}$

$0.10 \mathrm{ug} / \mathrm{L}$

$10.00 \mathrm{ug} / \mathrm{L}$

$200.00 \mathrm{ug} / \mathrm{L}$

$0.05 \mathrm{ug} / \mathrm{L}$

$0.05 \mathrm{ug} / \mathrm{L}$

$10.00 \mathrm{ug} / \mathrm{L}$

$10.00 \mathrm{ug} / \mathrm{L}$

$10.00 \mathrm{ug} / \mathrm{L}$

$2.00 \mathrm{ug} / \mathrm{L}$
Gen. Eng .

Gen. Eng.

Gen. Eng.

Gen. Eng.

Gen. Eng.

Gen. Eng.

Gen. Eng.

Gen. Eng.

Gen. Eng.

Gen. Eng .

Gen. Eng.

Gen. Eng .

Gen.- Eng.

Gen. Eng.

Gen. Eng.

Gen. Eng .

Gen. Eng .

Gen. Eng .

Gen. Eng.

Gen. Eng.

Gen. Eng.

Gen. Eng.

Gen. Eng.

Gen. Eng.

Gen. Eng.

Gen. Eng.

Gen. Eng.

Gen. Eng.

Gen. Eng.

Gen. Eng.

Gen. Eng.

Gen. Eng.

Gen. Eng.

Gen. Eng.

Gen. Eng.

Gen. Eng.

Gen. Eng.

Gen. Eng.

Gen. Eng.

Gen. Eng.

Gen. Eng.

Gen. Eng.

Gen. Eng.

Gen. Eng.

Gen. Eng.

Gen. Eng.

Gen. Eng.

Gen. Eng.

Gen. Eng.

Gen. Eng.

Geñ. Eng.

Gen. Eng.

Gen. Eng.

Gen. Eng. 
WELL LFW 22 COLLECTED ON 08/15/91 LABORATORY ANALYSES CONTINUED

2-sec-Butyl-4,6-dinitrophenol

Selen i um

Silica

$2,4,5-T P$ (Silvex)

Tin

Sulfate

Sulfotepp

Styrene -

Sulf ide

$1,2,4,5$-Tetrach lorobenzene

$1,1,2,2$-Tetrach loroethane

1,1,1,2-Tetrach loroethane

Tetrachloroethylene

0,0,0-Triethyl phosphorothionte

Thionazin

Thallium

Total organic carbon

Total organic nitrogen

Total organic halogens

Total phosphates (as P)

* Trichloroethylene

Toxaphene

trans-1,2-Dichloroethylene

trans-1,3-D ich loropropene

trans-1,4-Dich loro-2-butene

Vanadi um

Vinyl acetate

Xylenes

1-Naphthylamine

* 1,1-Dichloroetlyylene

1,1-Dichloroethane

1,1,1-Trich loroethane

$1,1,2-\mathrm{Tr}$ ichloroethane

1.2-D ibromoethane

1, 2-D i hromo-3-ch loropropane

1,2-D i ch lorobenzene

1,2-Dichloroethane

1,2-D ich loropropane

1,2,3-Trich loropropane

1,2,4-Trich lorobenzenc

1,3-Dichlorobenzene

c is-1,3-D ich loropropene

1,3-D in itrobenzene

$1,3,5-T_{r}$ in itrobenzene

1,4-Dichlorobenzene

1,4-Dioxane

1,4-Naph thoqu inone

2-Acety Iaminof I uorene

2-Chloroethyl vingl ether

2-Chlorophenol

2-Chloronaph thalene

2-Hexanone

2-Methylnaphthalene

o-Cresol (2-Methylphenol)

CONTINUED

\begin{tabular}{|c|c|c|c|c|}
\hline $\begin{array}{l}\text { LT } \\
\text { LT }\end{array}$ & $\begin{array}{r}10.00 \\
2.00\end{array}$ & $\begin{array}{l}u g / L \\
u g / L\end{array}$ & $\begin{array}{l}\text { Gen. } \\
\text { Gen. }\end{array}$ & $\begin{array}{l}\text { Eng. } \\
\text { Eng. }\end{array}$ \\
\hline & 13900.00 & ug $/ \mathrm{L}$ & Gen. & Eng. \\
\hline LT & 0.09 & ug/L & Gen. & Eng. \\
\hline L.T & 2.00 & ug /L & Gen. & Eng. \\
\hline & 2200.00 & ug / L & Gen. & $\begin{array}{l}\text { Eng. } \\
\text { Ens. }\end{array}$ \\
\hline $\begin{array}{l}\text { LT } \\
\text { LT }\end{array}$ & $\begin{array}{r}10.00 \\
1.00\end{array}$ & ug / L & $\begin{array}{l}\text { Gen. } \\
\text { Gen. }\end{array}$ & $\begin{array}{l}\text { Eng. } \\
\text { Eng. }\end{array}$ \\
\hline LT & $\begin{array}{r}1.00 \\
1000.00\end{array}$ & $\begin{array}{l}u g / L \\
u g / L\end{array}$ & $\begin{array}{l}\text { Gen. } \\
\text { Gen. }\end{array}$ & Eng. \\
\hline LT & 10.00 & $u_{g} / L$ & Gen. & Eng. \\
\hline LT & 1.00 & ug/L & Gen. & Eng. \\
\hline LT & $\begin{array}{l}1.00 \\
3.00\end{array}$ & $\begin{array}{l}u g / L \\
u g / L\end{array}$ & $\begin{array}{l}\text { Gen. } \\
\text { Gen :- }\end{array}$ & $\begin{array}{l}\text { Eng. } \\
\text { Eng. }\end{array}$ \\
\hline LT & 10.00 & $u g / L$ & Gen. & Eng. \\
\hline LT & 10.00 & ug/L & Gen. & Eng. \\
\hline LT & 2.00 & ug/L & Gen. & Eng. \\
\hline & 83000.00 & ug/L & Gen. & Eng. \\
\hline & 460.00 & ug /L & Gen. & Eng. \\
\hline & 481.00 & ug/L & Gen. & Eng. \\
\hline$L T$ & $\begin{array}{r}100.00 \\
25.00\end{array}$ & $\begin{array}{l}\mathrm{ug} / \mathrm{L} \\
\text { ug/L }\end{array}$ & $\begin{array}{l}\text { Gen. } \\
\text { Gen. }\end{array}$ & $\begin{array}{l}\text { Eng. } \\
\text { Eng. }\end{array}$ \\
\hline LT & 0.24 & ug $/ L$ & Gies. & Eng. \\
\hline LT & 1.00 & ug $/ \mathrm{L}$ & Gen. & Eng. \\
\hline LT & 1.00 & ug/L & Gen. & k. ig. \\
\hline LT & 30.00 & ug/L & Gen. & Eng. \\
\hline LT & 10.00 & ug/L & Gen. & Eng. \\
\hline LT & 1.00 & ug $/ L$ & Gen. & Eng. \\
\hline & 140.00 & ug $/ \mathrm{L}$ & Gen. & Eng. \\
\hline LT & 10.00 & ug/L & en. & ng. \\
\hline & 13.00 & ug/L & Gen. & . \\
\hline & 100.00 & ug/L & Gen. & ng. \\
\hline & 26.00 & ug/L & Gen. & - Eng. \\
\hline LT & 1.00 & ug $/ \mathrm{L}$ & Gen. & - Eng. \\
\hline LT & 20.00 & ug/L & Gen. & - Eng. \\
\hline LT & 1.00 & ug $/ \mathrm{L}$ & Gen. & - Eng. \\
\hline LT & 10.00 & $\mathrm{ug} / \mathrm{L}$ & Gen. & - Eng. \\
\hline LT & 1.00 & ug $/ \mathrm{L}$ & Gen. & - Eng. \\
\hline & 2.00 & $\mathbf{u g} / \mathrm{L}$ & . & ng. \\
\hline LT & 20.00 & $\mathrm{ug} / \mathrm{L}$ & Gen. & - Eng. \\
\hline LT & 10.00 & $u_{g} / L$ & Gen. & - Eng. \\
\hline LT & 10.00 & $\mathbf{u g} / \mathrm{L}$ & Gen. & . Eng \\
\hline LT & 1.00 & $u_{g} / L$ & Gen. & . Eng \\
\hline LT & 10.00 & ug $/ \mathrm{L}$ & Gen. & - Eng \\
\hline LT & 10.00 & ug $/ \mathrm{L}$ & Gen. & - Eng \\
\hline LT & 10.00 & $u g / L$ & Gen. & . Eng \\
\hline LT & 10.00 & ug/L & Gen. & - En \\
\hline LT & 10.00 & ug/L & Gen. & - Eng \\
\hline LT & 10.00 & $u g / L$ & Gen. & - Eng \\
\hline LI & 10.00 & ug $/ \mathrm{L}$ & Gen: & : Eng \\
\hline LT & 10.00 & ug $/ \mathrm{L}$ & Gen. & . En \\
\hline LI & 10.00 & ug/L & Gē̄. & . Eng \\
\hline LT & $\begin{array}{r}4.00 \\
\end{array}$ & ug/L & $\begin{array}{l}\text { Gen. } \\
\text { Gen. }\end{array}$ & . \\
\hline LT & $\begin{array}{l}10.00 \\
10.00\end{array}$ & ug/L & Gen. & . Eng \\
\hline
\end{tabular}




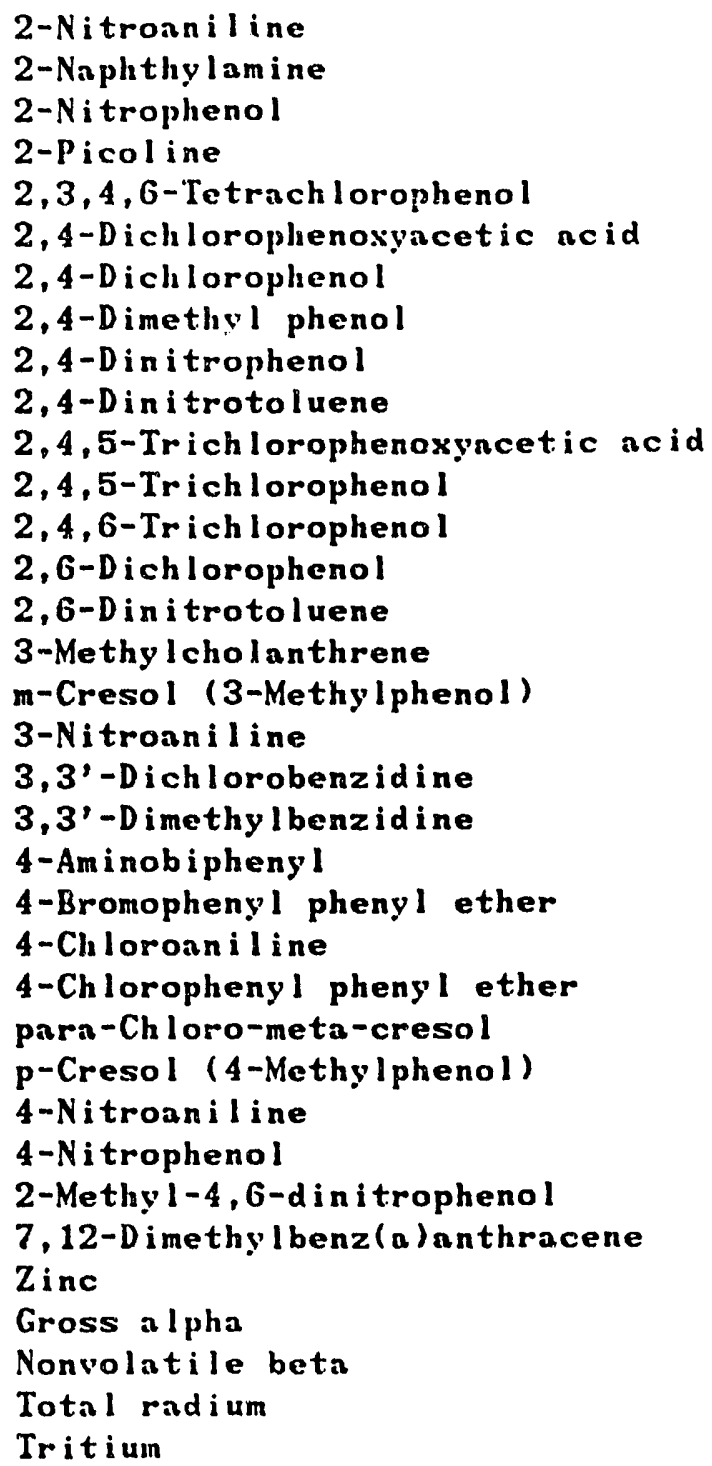

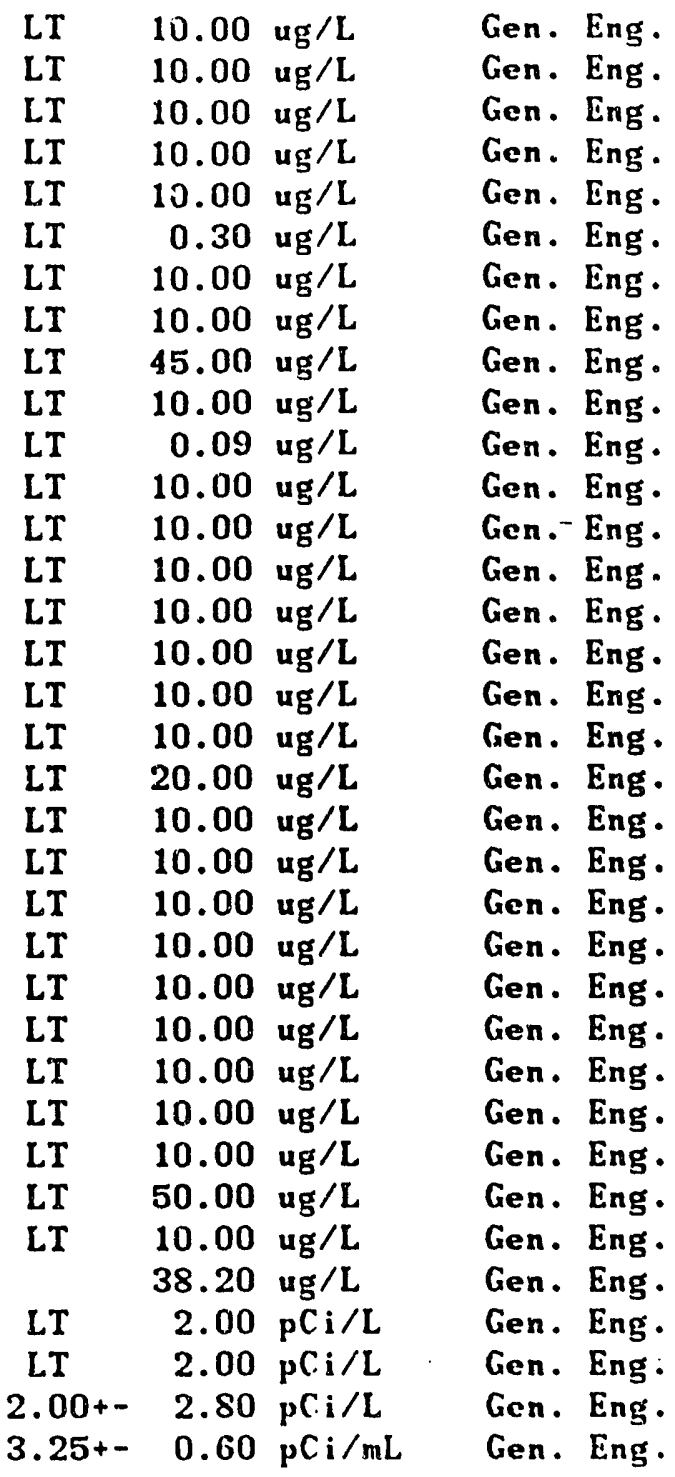


WELL LFW 23

SRS Grid N 84251.3 Latitude $33.287903 \quad N$

Coordinates E 46456.1 Longitude 81.708131 W

Screen Zone Elevation 155.1-125.1 ft ms I

Top of Casing Elevation 170.10 ft msl

\section{MEASUREMENTS CONDUCTED IN THE FIELD}

Sample date 08/14/91 Time 1500

Depth to water - $15.92 \mathrm{ft}(4.85 \mathrm{~m})$ below the TOC.

Water elevation $-154.18 \mathrm{ft}(46.99 \mathrm{~m}) \mathrm{msl}$

$\mathrm{pH}-4.6 \quad$ Alkalinity $-0 \mathrm{mg} / \mathrm{L}$

Specific conductance - 27 umhos/cm

Water temperature - 20.1 degrees Celsius

Water evacuated from the well prior to sampling - $82 \mathrm{gal}$

\section{LABORATORY ANALYSES}

Specific conductance

pH

Silver

Aluminum

Aldrin

Arsenic

Bari um

beta-Benzene hexachloride

Bromodichloromethane

Calcium

Trichlorof luoromethane

Carbor: tetrachloride

Cadmi um

Bromoform

Chloroform

Dichloromethane (Methylene chloride)

Bromomethane (Methyl bromide)

Chloromethane (Methyl chloride)

Chloride

Chlorobenzene

Chromium

Copper

Chloroethene (Vinyl chloride)

Chloroethane

Benzene

Dibromoch lorometlane

Endrin

Endosulfan sulfate

Ethylbenzene

Fluoride

Iron

Mercury

Potassium

Lindane

Toluene

Methoxychlor

Magnesium

C.ONTINUED
$25.00 \mathrm{umh} / \mathrm{cm}$

$5.10 \mathrm{pH}$

LT

LT

LT

LT

LT

LT

LT

LT

LT

LT

LT

LT

LT

LT

$2.00 \mathrm{ug} / \mathrm{L}$

$20.00 \mathrm{ug} / \mathrm{L}$

$0.05 \mathrm{ug} / \mathrm{L}$

$2.00 \mathrm{ug} / \mathrm{L}$

$4.90 \mathrm{ug} / \mathrm{L}$

0.05 ug $/ \mathrm{L}$

$1.00 \mathrm{ug} / \mathrm{L}$

$341.00 \mathrm{ug} / \mathrm{L}$

$1.00 \mathrm{ug} / \mathrm{L}$

$1.00 \mathrm{ug} / \mathrm{L}$

$2.00 \mathrm{ug} / \mathrm{L}$

$1.00 \mathrm{ug} / \mathrm{L}$

$1.00 \mathrm{ug} / \mathrm{L}$

$9.00 \mathrm{ug} / \mathrm{L}$

$1.00 \mathrm{ug} / \mathrm{L}$

$1.00 \mathrm{ug} / \mathrm{L}$

$2270.00 \mathrm{ug} / \mathrm{L}$

$1.00 \mathrm{ug} / \mathrm{L}$

LT

LT

LT

LT

LT

LT

LT

LT

$4.00 \mathrm{ug} / \mathrm{L}$

$4.25 \mathrm{ug} / \mathrm{L}$

$1.00 \mathrm{ug} / \mathrm{L}$

$1.00 \mathrm{ug} / \mathrm{L}$

$1.00 \mathrm{ug} / \mathrm{L}$

$1.00 \mathrm{ug} / \mathrm{L}$

$0.01 \mathrm{ug} / \mathrm{L}$

$0.10 \mathrm{ug} / \mathrm{L}$

$1.00 \mathrm{ug} / \mathrm{L}$

$100.00 \mathrm{ug} / \mathrm{L}$

$51.50 \mathrm{ug} / \mathrm{L}$

LT $\quad 0.20 \mathrm{ug} / \mathrm{L}$

LT $500.00 \mathrm{ug} / \mathrm{L}$

LT

LT

LT
$0.00 \mathrm{ug} / \mathrm{L}$

$1.00 \mathrm{ug} / \mathrm{L}$

$0.50 \mathrm{ug} / \mathrm{L}$ $528.00 \mathrm{ug} / \mathrm{L}$
Gen. Eng.

Gen. Eng.

Gen. Eng.

Gen. Eng.

Gen. Eng.

Gen. Eng .

Gen. Eng.

Gen. Eng .

Gen. Eng.

Gen. Eng.

Gen. Eng.

Gen. Eng.

Gen. Eng .

Gen. Eng .

Gen. Eng.

Gen. Eng.

Gen. Eng.

Gen. Eng.

Gen. Eng.

Gen. Eng.

Gen. Eng.

Gen. Eng.

Gen. Eng.

Gen. Eng.

Gen. Eng.

Gen. Eng.

Gen. Eng.

Gen. Eng.

Gen. Eng .

Gen. Eng.

Gen. Eng.

Gen. Eng.

Ger. Eng.

Geñ. Eng.

Gen. Eng.

Gen. Eng.

Gen. Eng. 
Manganese

Sod $\mathrm{i}$ um

Nickel

Nitrate as nitrogen

Lead

Antimony

Selenium

Silica

$2,4,5-T P$ (Silvex)

Sulfate

$1,1,2,2$-Tetrachloroethane

Tetrachloroethylene

Tha 11 ium

Total organic carbon

Total organic nitrogen

Total organic halogens

Total phosphates (as P)

Trichloroethylene

Toxaphene

trans-1,2-Dich loroethy lene trans-1, 3-D ich loropropene

Vanadi um

1,1-D ich loroethylene

1,1-Dichloroethane

1,1,1-Trichloroethane

1,1,2-Trichloroethane

1,2-D ichloroethane

1.2-D ich loropropane

cis-1,3-Dichloropropene

1,4-D ichlorobenzene

2-Chloroethyl vinyl ether

2,4-Dichlorophenosyacetic acid

Gross alpha

Nonvolatile beta

Total radium

Tritium
LT

$$
\begin{array}{r}
2580.00 \mathrm{ug} / \mathrm{L} \\
4.17 \mathrm{ug} / \mathrm{L}
\end{array}
$$

$1100.00 \mathrm{ug} / \mathrm{L}$

$5.45 \mathrm{ug} / \mathrm{L}$

LT

$2.00 \mathrm{ug} / \mathrm{L}$

LT

$2.00 \mathrm{ug} / \mathrm{L}$

$8540.00 \mathrm{ug} / \mathrm{L}$

$0.09 \mathrm{ug} / \mathrm{L}$

LT

LT

LT

LT

LT

LT

LI

$1000.00 \mathrm{ug} / \mathrm{L}$

$1.00 \mathrm{ug} / \mathrm{L}$

$1.00 \mathrm{ug} / \mathrm{L}$

$2.00 \mathrm{ug} / \mathrm{L}$

$1000.00 \mathrm{ug} / \mathrm{L}$

$100.00 \mathrm{ug} / \mathrm{L}$

$16.10 \mathrm{ug} / \mathrm{L}$

LT

LT

LI

$00.00 \mathrm{ug} / \mathrm{L}$

$1.00 \mathrm{ug} / \mathrm{L}$

$0.24 \mathrm{ug} / \mathrm{L}$

$1.00 \mathrm{ug} / \mathrm{L}$

$1.00 \mathrm{ug} / \mathrm{L}$

$10.00 \mathrm{ug} / \mathrm{L}$

$1.00 \mathrm{ug} / \mathrm{L}$

$1.00 \mathrm{ug} / \mathrm{L}$

$1.00 \mathrm{ug} / \mathrm{L}$

$1.00 \mathrm{ug} / \mathrm{L}$

$1.00 \mathrm{ug} / \mathrm{L}$

$1.00 \mathrm{ug} / \mathrm{L}$

$1.00 \mathrm{ug} / \mathrm{L}$

$10.00 \mathrm{ug} / \mathrm{L}$

$1.00 \mathrm{ug} / \mathrm{L}$

$0.30 \mathrm{ug} / \mathrm{L}$

$2.00 \mathrm{pCi} / \mathrm{L}$

$2.50+-3.00 \mathrm{pCi} / \mathrm{L}$

$2.12+-\quad 0.20 \mathrm{pCi} / \mathrm{mL}$
Gen. Eng.

Gen. Eng.

Gen. Eng.

Gen. Eng.

Gen. Eng.

Gen. Eng.

Gen. Eng.

Gen. Eng -

Gen. Eng.

Gen. Eng.

Gen. Eng.

Gen. Eng.

Gen - Eng .

Gen. Eng -

Gen. Eng.

Gen. Eng.

Gen. Eng.

Gen. Eng.

Gen. Eng.

Gen. Eng.

Gen. Eng.

Gen. Eng.

Gen. Eng.

Gen. Eng.

Gen. Eng.

Gen. Eng.

Gen. Eng.

Gen. Eng.

Gen. Eng.

Gen. Eng.

Gen. Eng.

Gen. Eng.

Gen. Eng.

Gen. Eng .

Gen. Eng .

Gen. Eng. 
WELL LFW 24

SRS Grid N 84544.2 Latitude 33.288656 N

Coordinates E 46520.8 Longitude $81.708530 \mathrm{~W}$

Screen Zone Elevation 154.5-124.5 ft msl

Top of Casing Elevation $169.50 \mathrm{ft}$ msl

MEASUREMENTS CONDUCTED IN TIE FIELD

Sample date 08/15/91 Time 1420

Depth to water - $12.61 \mathrm{ft}(3.84 \mathrm{~m})$ below the TOC

Water elevation $=156.89 \mathrm{ft}(47.82 \mathrm{~m}) \mathrm{msl}$

$\mathrm{pH}=5.1$ Alkalinity $-0 \mathrm{mg} / \mathrm{L}$

Specific conductance - 19 umhos/cm

Water temperature $=19.5$ degrees Celsius

Water evacuated from the well prior to sampling - $91 \mathrm{gal}$

\section{LABORATORY ANALYSES}

Specific conductance

pH

Silver

Al um inum

Aldrin

Arsenic

Bari um

beta-Benzene hexachloride

Bromodichloromethane

Calcium

Trichlorof luoromethane

Carbon tetrachloride

Cadmi um

Bromoform

Chloroform

Dichloromethane (Methylene chloride)

Bromomethane (Methyl bromide)

Chloromethane (Methyl chloride)

Chloride

Chlorobenzene

Chronium

Copper

Chloroethene (Vinyl chloride)

Chloroethane

Benzene

Dibromochloromethane

Endrin

Endosulfan sulfate

Ethylbenzene

Fluoride

Iron

Mercury

Potassium

Lindane

Toluene

Methosychlor

Magnesi um

CONTINUED
$20.00 \mathrm{umh} / \mathrm{cm}$

$5.27 \mathrm{pH}$

LT

LT

LT

LT

LT

LT

LT

LT

LT

LT

LT

LT

LT

LT

LT

LT

LT

LT

LT

LT

LT

LT

LT

LT

LT

LT

LT

LT

$2.00 \mathrm{ug} / \mathrm{L}$

$20.00 \mathrm{ug} / \mathrm{L}$

$0.05 \mathrm{ug} / \mathrm{L}$

$2.00 \mathrm{ug} / \mathrm{L}$

$4.46 \mathrm{ug} / \mathrm{L}$

$0.05 \mathrm{ug} / \mathrm{L}$

$1.00 \mathrm{ug} / \mathrm{L}$

$480.00 \mathrm{ug} / \mathrm{L}$

$1.00 \mathrm{ug} / \mathrm{L}$

$1.00 \mathrm{ug} / \mathrm{L}$

$2.00 \mathrm{ug} / \mathrm{L}$

$1.00 \mathrm{ug} / \mathrm{L}$

$1.00 \mathrm{ug} / \mathrm{L}$

$12.90 \mathrm{ug} / \mathrm{L}$

$1.00 \mathrm{ug} / \mathrm{L}$

$1.00 \mathrm{ug} / \mathrm{L}$

$2080.00 \mathrm{ug} / \mathrm{L}$

$1.00 \mathrm{v} / \mathrm{s} / \mathrm{L}$

$4.00 \mathrm{ug}^{\prime} \mathrm{L}$

$4.81 \mathrm{ug} / \mathrm{L}$

$1.00 \mathrm{u} / / \mathrm{L}$

$1.00 \mathrm{ug} / \mathrm{L}$

$1.00 \mathrm{ug} / \mathrm{L}$

$1.00 \mathrm{ug} / \mathrm{L}$

$0.01 \mathrm{ug} / \mathrm{L}$

$0.10 \mathrm{ug} / \mathrm{L}$

$1.00 \mathrm{ug} / \mathrm{L}$

$100.00 \mathrm{ug} / \mathrm{L}$

$18.10 \mathrm{ug} / \mathrm{L}$

$0.20 \mathrm{ug} / \mathrm{L}$

$500.00 \mathrm{ug} / \mathrm{L}$

$0.00 \mathrm{ug} / \mathrm{L}$

$1.00 \mathrm{ug} / \mathrm{L}$

$0.50 \mathrm{ug} / \mathrm{L}$

$477.00 \mathrm{ug} / \mathrm{L}$
Gen. Eng -

Gen. Eng.

Gen. Eng .

Gen. Eng -

Gen. Eng .

Gen. Eng .

Gen. Eng.

Gen. Eng.

Gen. Eng .

Gen. Eng .

Gen. Eng.

Gen. Eng.

Gen. Eng .

Gen. Eng.

Gen. Eng.

Gen. Eng.

Gen. Eng.

Gen. Eng.

Gen. Eng.

Gen. Eng.

Gen. Eng .

Gen. Eng.

Gen. Eng.

Gen. Eng.

Gen. Eng.

Gen. Eng.

Gen. Eng.

Gen. Eng.

Gen. Eng.

Gen. Eng.

Gen. Eng.

Gen. Eng.

Gen. Eng.

Gen-: Eng.

Gen. Eng.

Gen. Eng.

Gen. Eng. 
WELL LFW 24 COLLECTED ON 08/15/91 LABORATORY ANALYSES CONTINUED

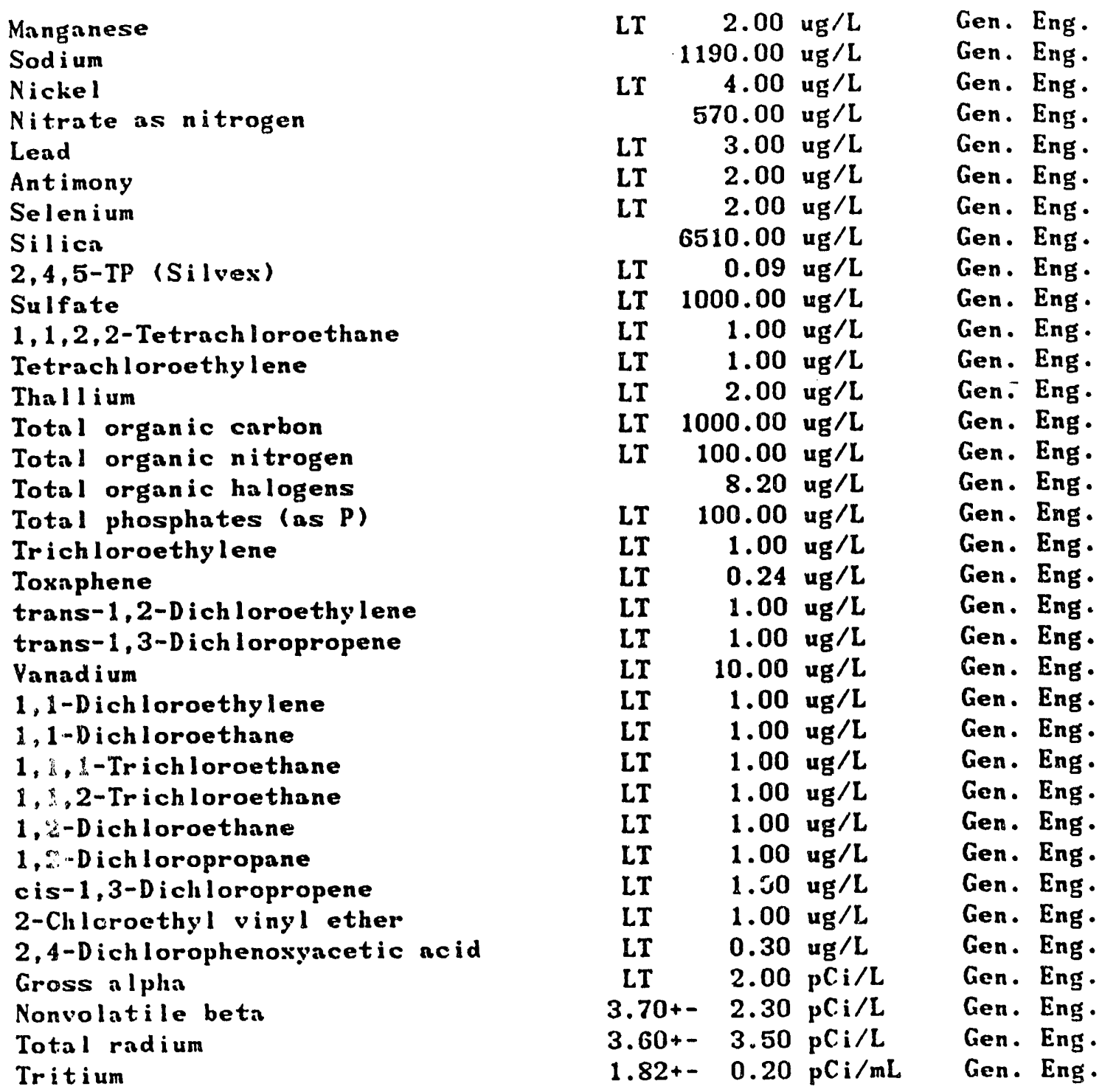


WELL LFW 25

$\begin{array}{llllll}\text { SRS Grid } & \text { N } 84967.2 & \text { Latitude } & 33.289436 & \text { N }\end{array}$

Coordinates E 46425.7 Longitude 81.709602 W

Screen Zone Elevation 153.2-123.2 ft ms l

Top of Casing Elevation $173.20 \mathrm{ft} \mathrm{msl}$

MEASUREMENTS CONDUCTED IN THE FIELD

Sample date 08/12/91 Time 840

Depth to water - $14.00 \mathrm{ft}(4.27 \mathrm{~m})$ below the TOC

Water elevation $=159.20 \mathrm{ft}(48.52 \mathrm{~m}) \mathrm{msl}$

$\mathrm{pH}=4.9$ Alkalinity $=0 \mathrm{mg} / \mathrm{L}$

Specific conductance - 16 umhos/cm

Water temperature - 19.0 degrees Celsius

Water euncuated from the well prior to sampling - $99 \mathrm{gal}$

\section{LABORATORY ANALYSES}

Specific conductance

PHI

Silver

Al umi inum

Aldrin

Arsenic

Barium

beta-Benzene hexachloride

Bromodichloromethane

Calcium

Trichlorof luoromethane

Carbon tetrachloride

Cadmi um

Bromoform

Chlor of or'n

Dichloroyethane (Methylene chloride)

Bromomethane (Methyl bromide)

Chloromethane (Methyl chloride)

Chloride

Chloride

Chlorobenzene

Chromium

Copper

Chloroethene (Vinyl chloride)

Chloroethane

Benzene

Dibromochloromethane

Endrin

Endosulfan sulfate

Ethylbenzene

Fluoride

Iron

Mercury

Potassium

Lindane

Toluene

Methosycinlor

CONTINUED
$15.00 \mathrm{umh} / \mathrm{cm}$

$5.05 \mathrm{pH}$

LT

LT

LT

LT

LT

LT

LT

LT

LT

LT

LT

LT

LT

$2.00 \mathrm{ug} / \mathrm{L}$

$20.00 \mathrm{ug} / \mathrm{L}$

0.05 ug $/ \mathrm{L}$

$2.00 \mathrm{ug} / \mathrm{L}$

$5.00 \mathrm{ug} / \mathrm{L}$

$0.05 \mathrm{ug} / \mathrm{L}$

$1.00 \mathrm{ug} / \mathrm{L}$

$239.00 \mathrm{ug} / \mathrm{L}$

$1.00 \mathrm{ug} / \mathrm{L}$

$1.00 \mathrm{ug} / \mathrm{L}$

$2.00 \mathrm{ug} / \mathrm{L}$

$1.00 \mathrm{ug} / \mathrm{L}$

$1.00 \mathrm{ug} / \mathrm{L}$

$7.00 \mathrm{ug} / \mathrm{L}$

$1.00 \mathrm{ug} / \mathrm{L}$

$1.00 \mathrm{ug} / \mathrm{L}$

$1640.00 \mathrm{ug} / \mathrm{L}$

$1680.00 \mathrm{ug} / \mathrm{L}$

$1.00 \mathrm{ug} / \mathrm{L}$

$4.00 \mathrm{ug} / \mathrm{L}$

$4.00 \mathrm{ug} / \mathrm{L}$

$1.00 \mathrm{ug} / \mathrm{L}$

$1.00 \mathrm{ug} / \mathrm{L}$

$1.00 \mathrm{ug} / \mathrm{L}$

$1.00 \mathrm{ug} / \mathrm{L}$

$0.01 \mathrm{ug} / \mathrm{L}$

$0.10 \mathrm{ug} / \mathrm{L}$

$1.00 \mathrm{ug} / \mathrm{L}$

$100.00 \mathrm{ug} / \mathrm{L}$

$13.70 \mathrm{ug} / \mathrm{L}$

LT

ST

LT

LT

LT
$0.20 \mathrm{ug} / \mathrm{L}$ $500.00 \mathrm{ug} / \mathrm{L}$

$0.00 \mathrm{ug} / \mathrm{L}$

$1.00 \mathrm{ug} / \mathrm{L}$

$0.50 \mathrm{ug} / \mathrm{L}$
Gen. Eng .

Gen. Eng .

Gen. Eng.

Gen. Eng.

Gen. Eng.

Gen. Eng.

Gen. Eng.

Gen. Eng.

Gen. Eng.

Gen. Eng.

Gen. Eng.

Gen. Eng.

Gen. Eng.

Gen. Eng.

Gen. Eng.

Gen. Eng.

Gen. Eng.

Gen. Eng.

Gen. Eng.

Gen. Eng.

Gen. Eng.

Gen. Eng.

Gen. Eng.

Gen. Eng.

Gen. Eng.

Gen. Eng.

Gen. Eng.

Gen. Eng.

Gen. Eng.

Gen. Eng.

Gen. Eng.

Gen. Eng.

Gen. Eng.

Geñ. Eng.

Gen. Eng.

Gen. Eng.

Gen. Eng. 
WELL LFW 25 COLLECTED ON 08/12/91 LABORATORY ANALYSES CONTINUED

Magnesium

Manganese

Sod i um

Nickel

Nitrate as nitrogen

Nitrate as nitrogen

Lead

Antimony

Selen ium

Silica

2,4,5-TP (Silvex)

Sulfate

Sulfate

1,1,2,2-Tetrach loroethane

Tetrachloroethylene

Tha 11 i um

Total organic carbon

Total organic nitrogen

Total organic halogens

Total phosphates (as P)

Trichloroethylene

Toxaphene

trans-1,2-Dichloro.

trans-1,3-D ich loropropene

Vanadi um

1,1-Dichloroethylene

1,1-Dichloroethane

$1,1,1-$ Trichloroethane

1, 1,2-Trichloroethane

$1,2-D$ ichloroethane

1,2-Dichloropropane

c is-1,3-D ichloropropene

2-Chloroethyl vinyl ether

2,4-Dichlorophenoxyacetic acid

Gross alpha

Nonvolatile beta

* Total radium

Tritium

$\begin{array}{lrl} & 430.00 \mathrm{ug} / \mathrm{L} \\ \mathrm{LT} & 2.00 \mathrm{ug} / \mathrm{L} \\ & 957.00 \mathrm{ug} / \mathrm{L} \\ \mathrm{LT} & 4.00 \mathrm{ug} / \mathrm{L} \\ & 550.00 \mathrm{ug} / \mathrm{L} \\ & 540.00 \mathrm{ug} / \mathrm{L} \\ \mathrm{LT} & 3.00 \mathrm{ug} / \mathrm{L} \\ \mathrm{LT} & 2.00 \mathrm{ug} / \mathrm{L} \\ \mathrm{LT} & 2.00 \mathrm{ug} / \mathrm{L} \\ & 6640.00 \mathrm{ug} / \mathrm{L} \\ \mathrm{IT} & 0.09 \mathrm{ug} / \mathrm{L} \\ \mathrm{LT} & 1000.00 \mathrm{ug} / \mathrm{L} \\ \mathrm{LT} & 1000.00 \mathrm{ug} / \mathrm{L} \\ \mathrm{LT} & 1.00 \mathrm{ug} / \mathrm{L} \\ \mathrm{LT} & 1.00 \mathrm{ug} / \mathrm{L} \\ \mathrm{LT} & 2.00 \mathrm{ug} / \mathrm{L} \\ & 6000.00 \mathrm{ug} / \mathrm{L} \\ & 360.00 \mathrm{ug} / \mathrm{L} \\ \mathrm{LT} & 5.00 \mathrm{ug} / \mathrm{L} \\ \mathrm{LT} & 100.00 \mathrm{ug} / \mathrm{L} \\ \mathrm{LT} & 2.00 \mathrm{uC} \mathbf{i} / \mathrm{L} \\ \mathrm{LT} & 1.00 \mathrm{ug} / \mathrm{L} \\ \mathrm{LT} & 0.24 \mathrm{ug} / \mathrm{L} \\ \mathrm{LT} & 1.00 \mathrm{ug} / \mathrm{L} \\ \mathrm{LT} & 1.00 \mathrm{ug} / \mathrm{L} \\ \mathrm{LT} & 10.00 \mathrm{ug} / \mathrm{L} \\ \mathrm{LT} & 1.00 \mathrm{ug} / \mathrm{L} \\ \mathrm{LT} & 1.00 \mathrm{ug} / \mathrm{L} \\ \mathrm{LT} & 1.00 \mathrm{ug} / \mathrm{L} \\ \mathrm{LT} & 1.00 \mathrm{ug} / \mathrm{L} \\ \mathrm{LT} & 1.00 \mathrm{ug} / \mathrm{L} \\ \mathrm{LT} & 1.00 \mathrm{ug} / \mathrm{L} \\ \mathrm{LT} & 1.00 \mathrm{ug} / \mathrm{L} \\ \mathrm{LT} & 1.00 \mathrm{ug} / \mathrm{L} \\ \mathrm{LT} & 0.30 \mathrm{ug} / \mathrm{L} \\ \mathrm{LT} & 0.30 \mathrm{uC} \mathrm{i} / \mathrm{mL}\end{array}$

Gen. Eng.

Gen. Eng.

Gen. Eng.

Gen. Eng.

Gen. Eng.

Gen. Eng.

Gen. Eng.

Gen. Eng.

Gen. Eng.

Gen. Eng.

Gen. Eng.

Gen. Eng.

Gen.- Eng.

Gen. Eng.

Gen. Eng.

Gen. Eng.

Gen. Eng .

Gen. Eng .

Gen. Eng.

Gen. Eng.

Gen. Eng.

Gen. Eng.

Gen. Eng.

Gen. Eng.

Gen. Eng.

Gen. Eng.

Gen. Eng.

Gen. Eng.

Gen. Engt.

Gen. Eng.

Gen. Eng.

Gen. Eng.

Gen. Eng.

Gen. Eng.

Gen. Eng.

Gen. Eng.

Gen. Eng.

Gen. Eng. 
WTLLL LFW 26

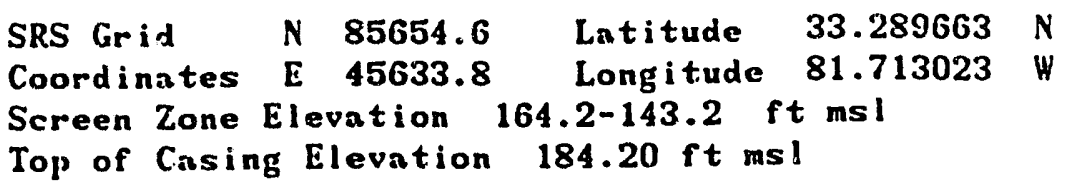

MEASUREMENTS CONDUCTED IN TIIE FIELD

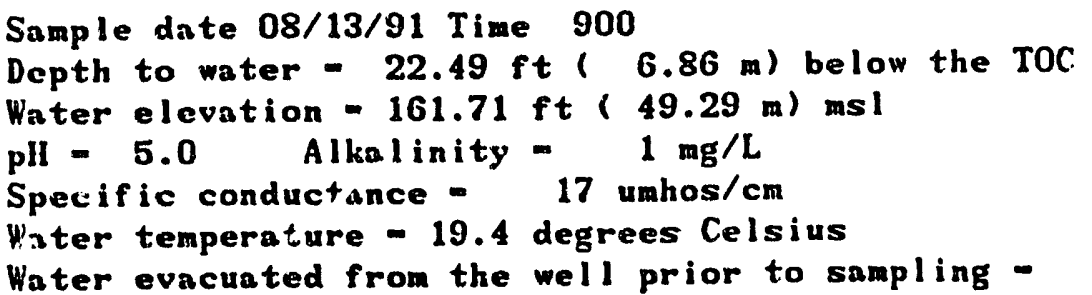

\section{LABORATORY ANALYSES}

Specific conductance
H
Silver
Aluminum
Aldrin
Arsenic
Barium
beta-Benzene hexachloride
Bromodichloromethane
Calcium
Trichlorofluoromethane
Carbon tetrachloride
Cadmium
Bromoform
Chloroform
Dichloromethane (Methylene chloride)
Bromomethane (Methyl bromide)
Chloromethane (Methyl chloride)
Chloride
Chlorobenzene
Chromium
Copper
Chloroethene (Vingl chloride)
Chloroethane
Benzene
Dibromochloromethane
Endrin
Endosulfan sulfate
Ethylbenzene
Fluoride
Iron
Mercury
Potassium
Lindane
Toluene
Methoxychlor
Magnesium
TiNuED
Ch

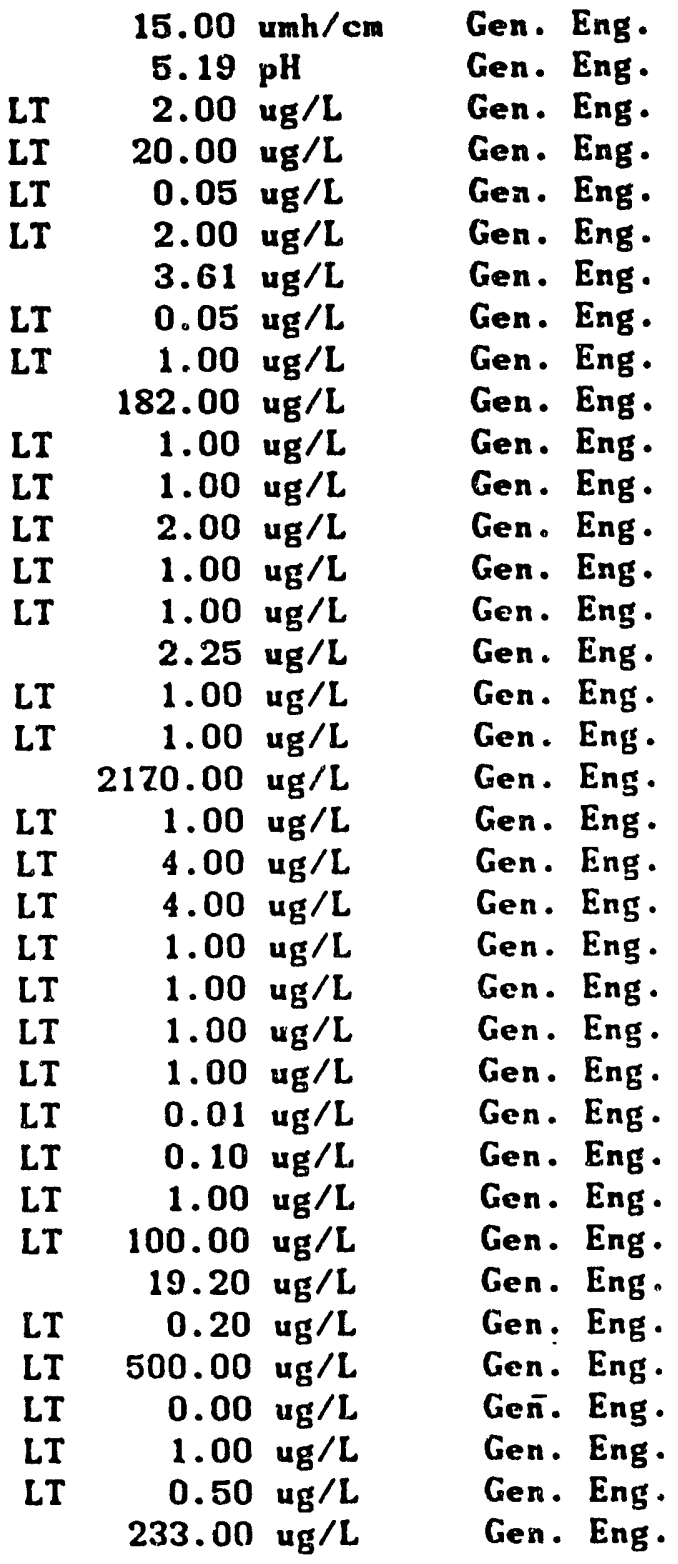


Manganese

Sodi um

Nickel

Nitrate as nitrogen

Lead

Antimony

Selen i um

Silica

2,4,5-TP (Silvex)

Sulfate

Sulfate

1,1,2,2-Tetrach loroethane

Tetrachloroethylene

Thallium

Total organic carbon

Total organic nitrogen

Total organic halogens.

Total phosphates (as P)

Trichloroe thylene

Toxaphene

trans-1,2-Dichloroethylene

trans-1, 3-Dich loropropene

Vanadium

1,1-Dichloroethylene

1,1-Dichloroethane

1,1,1-Trich loroethane

1,1,2-Trichloroethane

1,2-Dichloroethane

1,2-Dichloropropane

c is-1, 3-D ich loropropene

1,4-Dich lorobenzene

2-Chlorocthyl vingl ether

- 2,4-Dichlorophenoxyacetic ncid

Gross alpha

Nonvolatile beta

Total radium

Tritium
LT

LT

LT

LT

LT

LT

LT

LT

LI

LI

LT

LT

LI

LT

LT

I.T

L:

LI

LI

LT

LT

LI

LT

L.T

LT

LT

LT

LT

LT

LT

$3.50+$

$2.58+-$
$2.00 \mathrm{ug} / \mathrm{L}$ $1220.00 \mathrm{ug} / \mathrm{L}$

$4.00 \mathrm{ug} / \mathrm{L}$ $100.00 \mathrm{ug} / \mathrm{L}$

$3.00 \mathrm{ug} / \mathrm{L}$

$2.00 \mathrm{ug} / \mathrm{L}$

$2.00 \mathrm{ug} / \mathrm{L}$ $5750.00 \mathrm{ug} / \mathrm{L}$

$0.09 \mathrm{ug} / \mathrm{T}$.

1000.00 uE

$1000.00 \mathrm{ug} / \mathrm{L}$

$1.00 \mathrm{ug} / \mathrm{L}$

$1.00 \mathrm{ug} / \mathrm{L}$

$2.00 \mathrm{ug} / \mathrm{L}$

$1000.00 \mathrm{ug} / \mathrm{L}$

$100.0 \% \mathrm{~g} / \mathrm{L}$

$191.0 \mathrm{ag} / \mathrm{L}$

$100.00 \mathrm{ug} / \mathrm{L}$

$1.22 \mathrm{ug} / \mathrm{L}$

$0.24 \mathrm{ug} / \mathrm{L}$

$1.00 \mathrm{ug} / \mathrm{L}$

$1.00 \mathrm{ug} / \mathrm{L}$

$10.00 \mathrm{ug}^{\prime} / \mathrm{L}$

$1.00 \mathrm{ug} / \mathrm{L}$

$1.00 \mathrm{ug} / \mathrm{L}$

$1.00: \xi / L$

$1.00 \mathrm{ug} / \mathrm{L}$

$1.00 \mathrm{ug} / \mathrm{L}$

$1.00 \mathrm{ug} / \mathrm{L}$

$1.00 \mathrm{ug} / \mathrm{L}$

$10.00 \mathrm{ug} / \mathrm{L}$

$1.00 \mathrm{ug} / \mathrm{L}$

$0.30 \mathrm{ug} / \mathrm{L}$

$2.00 \mathrm{pC} . \mathrm{i} / \mathrm{L}$

$2.00 \mathrm{pCi} / \mathrm{L}$

$.3 .30 \mathrm{pC} . \mathrm{i} / \mathrm{L}$

$0.30 \mathrm{pCi} / \mathrm{mL}$
Gen. Eng.

Gen. Eng.

Gen. Eng.

Gen. Eng.

Gen. Eng.

Gen. Eng.

Gen. Eng.

Gen. Eng.

Gen. Eng.

Gen. Eng.

Gen. Eng.

Gen. Eng.

Gen: Eng.

Gen. Eng.

Gen. Eng.

Gen. Eng.

Gen. Eng.

Gen. Eng.

Gen. Eng .

Gen. Eng.

Gen. Eng.

Gen. Eng.

Gen. Eng.

Gen. Eng.

Gen. Eng.

Gen. Eng .

Gen. Eng.

Gen. Eng.

Gen. Eng.

Gen. Eng.

Gen. Eng.

Gen. Eng.

Gen. Eng.

Gen. Eng.

Gen. Eng.

Gen. Eng.

Gen. Eng. 
WELL LFW 27

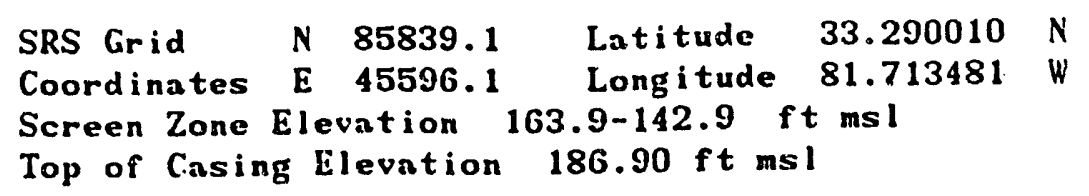

\section{MEASUREMENTS CONDUCTED IN THE FIELD}

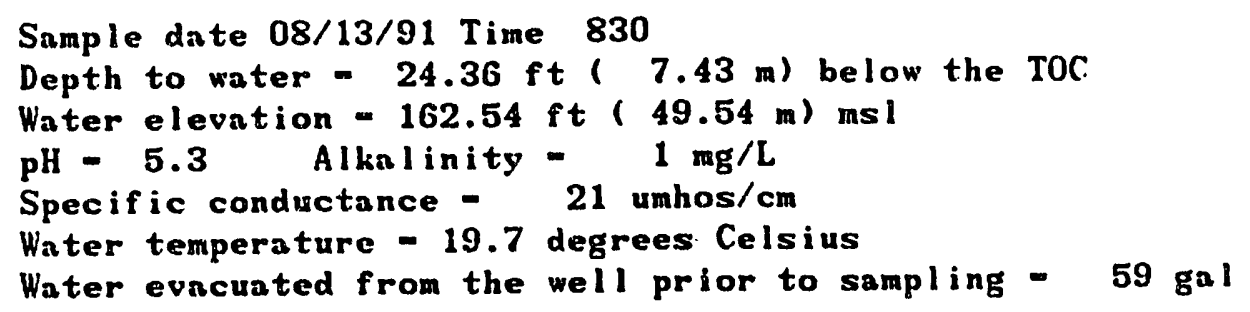

Specific conductance

pH.

Silver

Aluminum

Aldrin

Arsenic

Barium

beta-Benzene hexachloride

Bromodichloromethane

Calcium

Trichlorof luoromethane

Carbon tetrachloride

Cadmi um

Bromoform

Chloroform

Dichloromethane (Methylene chloride)

Bromomethane (Methyl bromicie)

Chloromethane (Methyl chloride)

Chloride

Ch lorobenzene

Chromium

Copper

Chloroethene (Vinyl chloride)

Chloroethane

Benzene

Dibromochloromethane

Endrin

Endosulfan sulfate

Ethylbenzene

Fluoride

Iron

Mercury

Potassium

Lindane

Tol uene

Methoxychlor

Magnesi um

CONTINUED

$$
\begin{aligned}
& 15.00 \mathrm{umh} / \mathrm{cm} \\
& 5.38 \mathrm{pH}
\end{aligned}
$$

LI

LT

LT

LT

LI

LI

LT

LT

LT

LT

LT

LT

LT

LI

LI

LT

LT

LT

LT

LT

LI

LT

LT

LT

$2.00 \mathrm{ug} / \mathrm{L}$ $20.00 \mathrm{ug} / \mathrm{L}$

$0.05 \mathrm{ug} / \mathrm{L}$

$2.00 \mathrm{ug} / \mathrm{L}$

$4.06 \mathrm{ug} / \mathrm{L}$

$0.05 \mathrm{ug} / \mathrm{L}$

$1.00 \mathrm{ug} / \mathrm{L}$ $356.00 \mathrm{ug} / \mathrm{L}$.

$1.00 \mathrm{ug} / \mathrm{L}$

$1.00 \mathrm{ug} / \mathrm{L}$

$2.00 \mathrm{vg} / \mathrm{L}$

$1.00 \mathrm{ug} / \mathrm{L}$

$1.00 \mathrm{ug} / \mathrm{L}$

$12.00 \mathrm{ug} / \mathrm{L}$

$1.00 \mathrm{ug} / \mathrm{L}$

$1.00 \mathrm{ug} / \mathrm{L}$

$1880.00 \mathrm{ug} / \mathrm{L}$

$1.00 \mathrm{ug} / \mathrm{L}$

$4.00 \mathrm{ug} / \mathrm{L}$

$4.00 \mathrm{ug} / \mathrm{L}$

$1.00 \mathrm{ug} / \mathrm{L}$

$1.00 \mathrm{ug} / \mathrm{L}$

$1.00 \mathrm{ug} / \mathrm{L}$

$1.00 \mathrm{ug} / \mathrm{L}$

$0.01 \mathrm{ug} / \mathrm{L}$

$0.10 \mathrm{ug} / \mathrm{L}$

$1.00 \mathrm{ug} / \mathrm{L}$

$100.00 \mathrm{ug} / \mathrm{L}$

$24.50 \mathrm{ug} / \mathrm{L}$

LT

$0.20 \mathrm{ug} / \mathrm{L}$

LT $500.00 \mathrm{ug} / \mathrm{L}$

LT

$0.00 \mathrm{ug} / \mathrm{L}$

$1.00 \mathrm{ug} / \mathrm{L}$

LI
$0.50 \mathrm{ug} / \mathrm{L}$ $240.00 \mathrm{ug} / \mathrm{L}$
Gen. Eng.

Gen. Eng.

Gen. Eng.

Gen. Eng.

Gen. Eng.

Gen. Eng.

Gen. Eng.

Gen. Eng .

Gen. Eng .

Gen. Eng.

Gen. Eng.

Gen. Eng.

Gen. Eng.

Gen. Eng.

Gen. Eng.

Gen. Eng.

Gen. Eng.

Gen. Eng.

Gen. Eng.

Gen. Eng.

Gen. Eng.

Gen. Eng.

Gen. Eng.

Gen. Eng.

Gen. Eng.

Gen. Eng.

Gen. Eng.

Gen. Eng.

Gen. Eng.

Gen. Eng.

Gen. Eng.

Gen. Eng.

Gen. Eng.

Geri. Eng.

Gen. Eng.

Gen. Eng.

Gen. Eng. 
Manganese

Sod i um

Nicliel

Nitrate as nitrogen

Lead

Ant imony

Selen ium

Silica

$2,4,5-T P$ (Silvex)

Sulfate

1,1,2,2-Tetrach loroethane

Tetrach loroethylene

Tha 11 i um

Total organic carbon

Total organic nitrogen

Total organic halogens

Total phosphates (as P)

Total phosphates (as P)

Trichloroethylene

Coxaphene

trans-1,2-Dich loroethylene

trans-1,3-Dichloropropene

Vanadi um

1,1-Dichloroethylene

1,1-Dichloroethane

1,1,1-Trichloroethane

1,1,2-Trichloroethane

$1,2-D$ ichloroethane

1,2-Dich loropropane

c is-1,3-D ichloropropene

2-Chloroethyl vinyl ether

2,4-Dichlorophenosyacetic acid

Gross alpha

Nonvolatile beta

* Total radium

Tritium
$2.28 \mathrm{ug} / \mathrm{L}$

$2160.00 \mathrm{ug} / \mathrm{L}$

$4.00 \mathrm{ug} / \mathrm{L}$

$96.00 \mathrm{ug} / \mathrm{L}$

LI

LT

LT

LT

$3.00 \mathrm{ug} / \mathrm{L}$

$2.00 \mathrm{ug} / \mathrm{L}$

$2.00 \mathrm{ug} / \mathrm{L}$

$6380.00 \mathrm{ug} / \mathrm{L}$

$0.09 \mathrm{ug} / \mathrm{L}$

$1390.00 \mathrm{ug} / \mathrm{L}$

$\begin{array}{ll}\text { LT } & 1.00 \mathrm{ug} / \mathrm{L} \\ \mathrm{LT} & 1.00 \mathrm{ug} / \mathrm{L}\end{array}$

LT $2.00 \mathrm{ug} / \mathrm{L}$

LT $1000.00 \mathrm{ug} / \mathrm{L}$

LT

$100.00 \mathrm{ug} / \mathrm{L}$

$10.30 \mathrm{ug} / \mathrm{L}$

LI

LT

LT

$100.00 \mathrm{ug} / \mathrm{L}$

$1.00 \mathrm{ug} / \mathrm{L}$

$0.24 \mathrm{ug} / \mathrm{L}$

$1.00 \mathrm{ug} / \mathrm{L}$

$1.00 \mathrm{ug} / \mathrm{L}$

$10.00 \mathrm{ug} / \mathrm{L}$

$1.00 \mathrm{ug} / \mathrm{L}$

$1.00 \mathrm{ug} / \mathrm{L}$

$1.00 \mathrm{ug} / \mathrm{L}$

$1.00 \mathrm{ug} / \mathrm{L}$

$1.00 \mathrm{ug} / \mathrm{L}$

$1.00 \mathrm{ug} / \mathrm{L}$

$1.00 \mathrm{ug} / \mathrm{L}$

$1.00 \mathrm{ug} / \mathrm{L}$

$0.30 \mathrm{ug} / \mathrm{L}$

$2.00 \mathrm{pCi} / \mathrm{L}$

$\begin{array}{cl}\mathrm{LT} & 2.00 \mathrm{pCi} / \mathrm{L} \\ 5.40+- & 3.60 \mathrm{pCi} / \mathrm{L}\end{array}$

$1.80+-\quad 0.20 \mathrm{pCi} / \mathrm{mL}$
Gen. Eng.

Gen. Eng.

Gen. Eng.

Gen. Eng.

Gen. Eng.

Gen. Eng.

Gen. Eng.

Gen. Eng.

Gen. Eng.

Gen. Eng.

Ge:. Eng.

Gen. Eng.

Gen:- Eng.

Gen. Eng.

Gen. Eng.

Gen. Eng.

Gen. Eng.

Gen. Eng.

Gen. Eng.

Gen. Eng .

Gen. Eng.

Gen. Eng.

Gen. Eng.

Gen. Eng.

Gen. Eng.

Gen. Eng.

Gen. Eng.

Gen. Eng.

Gen. Eng.

Gen. Eng.

Gen. Eng.

Gen. Eng.

Gen. Eng.

Gen. Eng.

Gen. Eng.

Gen. Eng. 
WELL ".FW 28

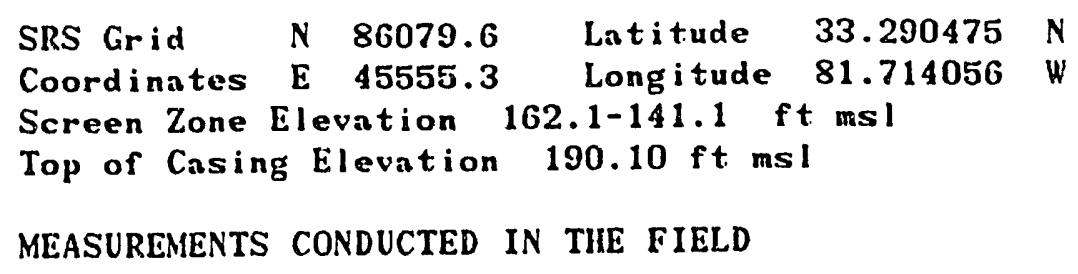

\section{MEASUREMENTS CONDUCTED IN THE FIELD}

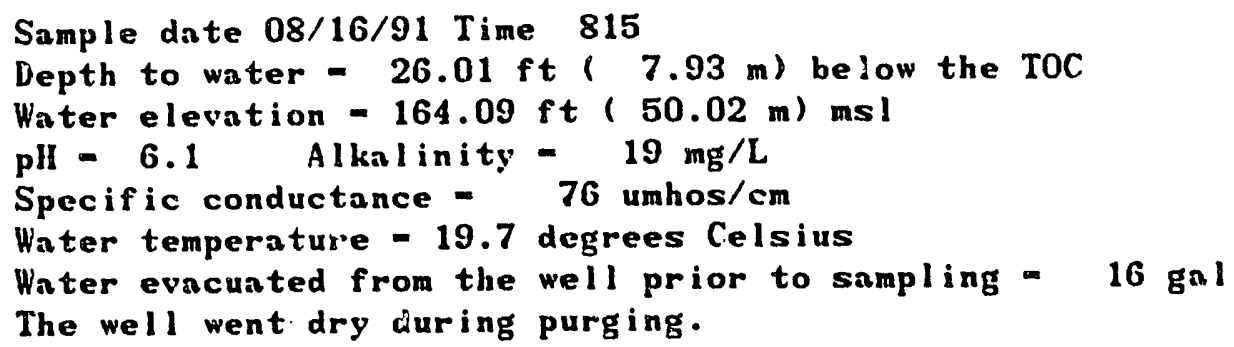

\section{LABORATORY ANALYSES}

Specific conductance
pll
Silver
Aluminum
Aldirin
Arsenic
Barium
beta-Benzene nexachloride
Bromodichl
Calcium
Trichlorofluoromethane
Carbon tetrachloride
Cadmium
Bromoform
Chloroform
Dichloromethane (iethylene chloride)
Bromomethane (Methyl bromide)
Chloromethane (Methyl chloride)
Chloride
Chloride
Chlorobenzene
Chromium
Copper
Chlorocthene (Vingl chloride)
Chloroethane
Benzene
Dibromochloromethane
Endrin
Endosulfan sulfate
Ethylbenzene
Fluoride
Iron
Mercury
Potassium
Lindane
Toluene
Methosychlor
CTINED

$\begin{array}{lrll} & 75.00 \mathrm{umh} / \mathrm{cm} & \text { Gen. Eng. } \\ & 6.35 \mathrm{ull} & \text { Gen. Eng. } \\ \text { LT } & 2.00 \mathrm{ug} / \mathrm{L} & \text { Gen. Eng. } \\ \text { LT } & 20.00 \mathrm{ug} / \mathrm{L} & \text { Gen. Eng. } \\ \text { LT } & 0.05 \mathrm{ug} / \mathrm{L} & \text { Gen. Eng. } \\ \text { LT } & 2.00 \mathrm{ug} / \mathrm{L} & \text { Gen. Eng. } \\ & 14.80 \mathrm{ug} / \mathrm{L} & \text { Gen. Eng. } \\ \text { LT } & 0.05 \mathrm{ug} / \mathrm{L} & \text { Gen. Eng. } \\ \text { LT } & 1.00 \mathrm{ug} / \mathrm{L} & \text { Gen. Eng. } \\ & 4760.00 \mathrm{ug} / \mathrm{L} & \text { Gen. Eng. } \\ \text { LT } & 1.00 \mathrm{ug} / \mathrm{L} & \text { Gen. Eng. } \\ \text { LT } & 1.00 \mathrm{ug} / \mathrm{L} & \text { Gen. Eng. } \\ \text { LT } & 2.00 \mathrm{ug} / \mathrm{L} & \text { Gen. Eng. } \\ \text { LT } & 1.00 \mathrm{ug} / \mathrm{L} & \text { Gen. Eng. } \\ \text { LT } & 1.00 \mathrm{ug} / \mathrm{L} & \text { Gen. Eng. } \\ & 12.10 \mathrm{ug} / \mathrm{L} & \text { Gen. Eng. } \\ \text { LT } & 1.00 \mathrm{ug} / \mathrm{L} & \text { Gen. Eng. } \\ \text { LT } & 1.00 \mathrm{ug} / \mathrm{L} & \text { Gen. Eng. } \\ & 1460.00 \mathrm{ug} / \mathrm{L} & \text { Gen. Eng. } \\ & 1460.00 \mathrm{ug} / \mathrm{L} & \text { Gen. Eng. } \\ \text { LT } & 1.00 \mathrm{ug} / \mathrm{L} & \text { Gen. Eng. } \\ \text { LT } & 4.00 \mathrm{ug} / \mathrm{L} & \text { Gen. Eng. } \\ \text { LT } & 4.00 \mathrm{ug} / \mathrm{L} & \text { Gen. Eng. } \\ \text { LT } & 1.00 \mathrm{ug} / \mathrm{L} & \text { Gen. Eng. } \\ \text { LT } & 1.00 \mathrm{ug} / \mathrm{L} & \text { Gen. Eng. } \\ \text { LT } & 1.00 \mathrm{ug} / \mathrm{L} & \text { Gen. Eng. } \\ \text { LT } & 1.00 \mathrm{ug} / \mathrm{L} & \text { Gen. Eng. } \\ \text { LT } & 0.01 \mathrm{ug} / \mathrm{L} & \text { Gen. Eng. } \\ \text { LT } & 0.10 \mathrm{ug} / \mathrm{L} & \text { Gen. Eng. } \\ \text { LT } & 1.00 \mathrm{ug} / \mathrm{L} & \text { Gen. Eng. } \\ \text { LT } & 100.00 \mathrm{ug} / \mathrm{L} & \text { Gen. Eng. } \\ & 21.00 \mathrm{ug} / \mathrm{L} & \text { Gen. Eng. } \\ \text { LT } & 0.20 \mathrm{ug} / \mathrm{L} & \text { Gen. Eng. } \\ & 7150.00 \mathrm{ug} / \mathrm{L} & \text { Gen. Eng. } \\ \text { LT } & 0.00 \mathrm{ug} / \mathrm{L} & \text { Gen. Eng. } \\ \text { LT } & 1.00 \mathrm{ug} / \mathrm{L} & \text { Gen. Eng. } \\ \text { LT } & 0.50 \mathrm{ug} / \mathrm{L} & \text { Gen. Eng. } \\ & & & \end{array}$


WELL LFW 28 COLLECTED ON 08/16/91 LABORATORY ANALYSES CONTINUED

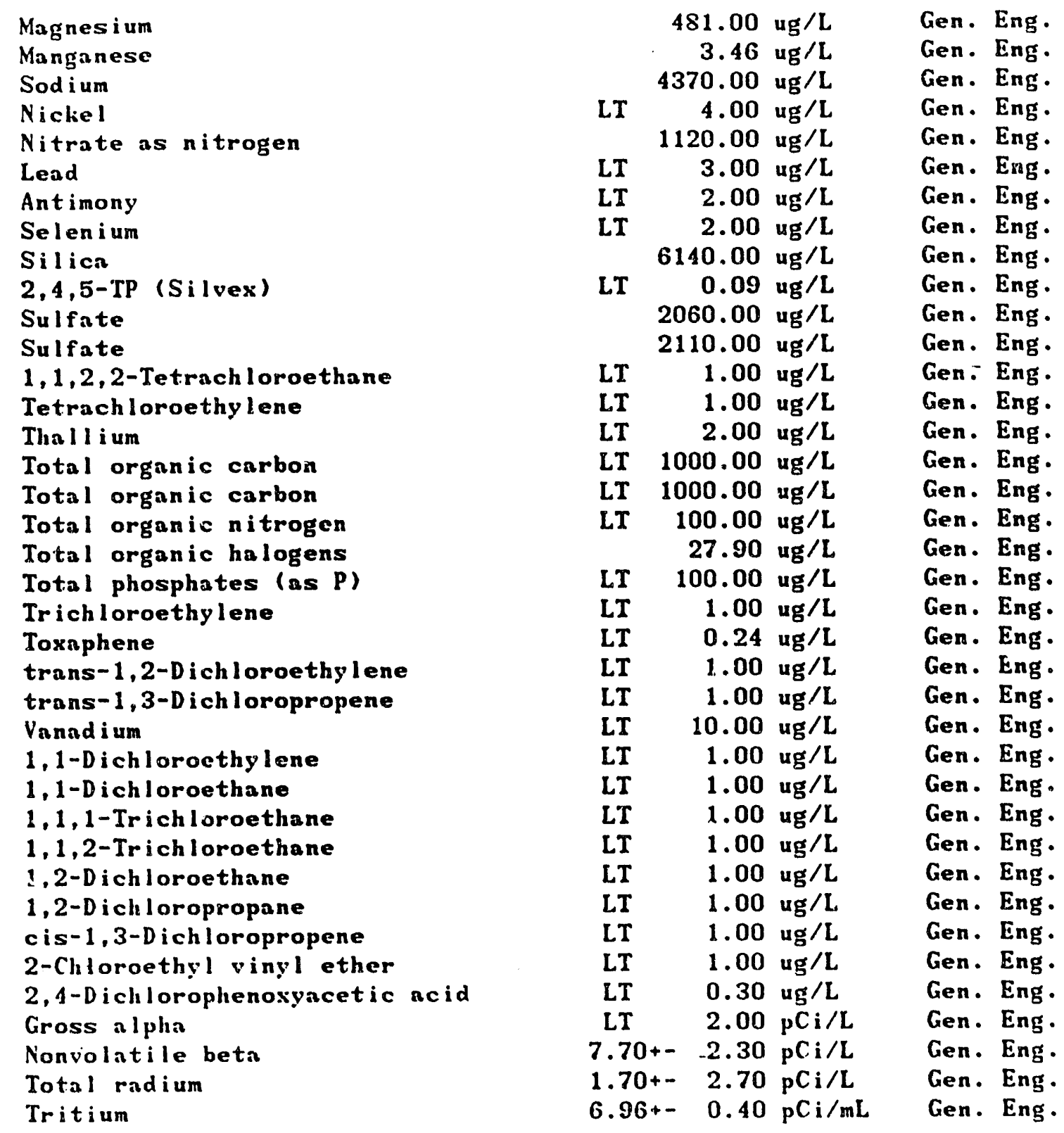


WELL LFW 29

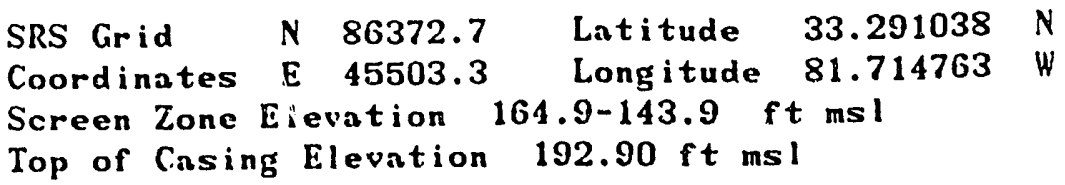

\section{MEASUREMENTS CONDUCTED IN THE FIELD}

Sample date 08/15/91 Time 1250

Depth to water $=27.94 \mathrm{ft}(8.52 \mathrm{~m})$ below the TOC

Water elevation - $164.96 \mathrm{ft}(50.28 \mathrm{~m}) \mathrm{msl}$

$\mathrm{pH}=4.5 \quad$ Alkalinity - $0 \mathrm{mg} / \mathrm{L}$

Specific conductance - 25 umhos/cm

Water temperature -18.5 degrees Celsius

Water evacuated from the well prior to sampling - $63 \mathrm{gal}$

\section{LABORATORY ANALYSES}

Specific conductance

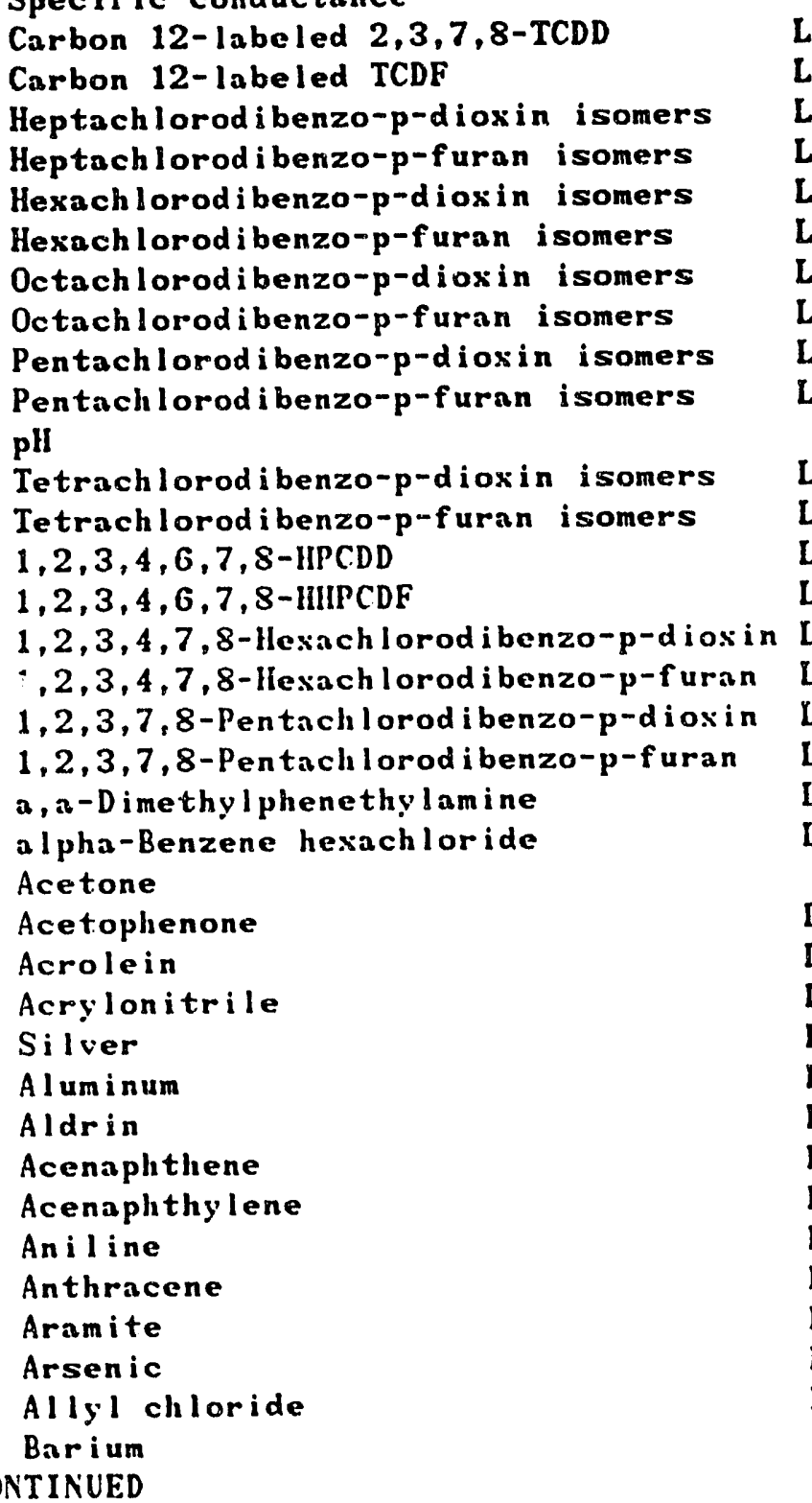

$20.00 \mathrm{umh} / \mathrm{em}$

$0.45 \mathrm{ng} / \mathrm{L}$

$0.40 \mathrm{ng} / \mathrm{L}$

$0.65 \mathrm{ng} / \mathrm{L}$

$0.45 \mathrm{ng} / \mathrm{L}$

$0.45 \mathrm{ng} / \mathrm{L}$

$0.40 \mathrm{ng} / \mathrm{L}$

$1.00 \mathrm{ng} / \mathrm{L}$

$1.00 \mathrm{ng} / \mathrm{L}$

$0.55 \mathrm{ng} / \mathrm{L}$

$0.55 \mathrm{ng} / \mathrm{L}$

$5.12 \mathrm{pH}$

$0.45 \mathrm{ng} / \mathrm{L}$

$0.40 \mathrm{ng} / \mathrm{L}$

$0.65 \mathrm{ng} / \mathrm{L}$

$0.45 \mathrm{ng} / \mathrm{L}$

$0.45 \mathrm{ng} / \mathrm{L}$

$0.40 \mathrm{ng} / \mathrm{L}$

$-0.55 \mathrm{ng} / \mathrm{L}$

$0.55 \mathrm{ng} / \mathrm{L}$

$10.00 \mathrm{ug} / \mathrm{L}$

$0.05 \mathrm{ug} / \mathrm{L}$

$11.00 \mathrm{ug} / \mathrm{L}$

$10.00 \mathrm{ug} / \mathrm{L}$

$20.00 \mathrm{ug} / \mathrm{L}$

$20.00 \mathrm{ug} / \mathrm{L}$

$2.00 \mathrm{ug} / \mathrm{L}$

$20.00 \mathrm{ug} / \mathrm{L}$

$0.05 \mathrm{ug} / \mathrm{L}$

$10.00 \mathrm{ug} / \mathrm{L}$

$10.00 \mathrm{ug} / \mathrm{L}$

$10.00 \mathrm{ug} / \mathrm{L}$

$10.00 \mathrm{ug} / \mathrm{L}$

$10.00 \mathrm{ug} / \mathrm{L}$

$2.00 \mathrm{ug} / \mathrm{L}$

$50.00 \mathrm{ug} / \mathrm{L}$

$7.67 \mathrm{ug} / \mathrm{L}$
Gen. Eng.

Gen. Eng.

Gen. Eng.

Gen. Eng.

Gen. Eng.

Ger. Eng.

Gen. Eng.

Gen. Eng.

Gen. Eng.

Gen. Eng.

Gen. Eng.

Gen. Eng.

Gen, Eng.

Gen. Eng.

Gen. Eng.

Gen. Eng.

Gen. Eng.

Gen. Eng.

Gen. Eng.

Gen. Eng.

Gen. Eng.

Gen. Eng.

Gen. Eng.

Gen. Eng.

Gen. Eng.

Gen. Eng.

Gen. Eng.

Gen. Eng.

Gen. Eng.

Gen. Eng.

Gen. Eng.

Gen. Eng.

Gen. Eng.

Geñ. Eng.

Gen. Eng.

Gen. Eng.

Gen. Eng. 
Benzola)anthracene

Benzo(a)pyrene

Benzo(b)f I uoranthene

beta-Benzene hesachloride

Butylbenzyl phthalate

Bis(chloromethyl-ethyl)ether

Berylli um

Benzo( $g, h, i)$ perylene

Benzo(k)f luoranthene

Bromodichloromethane

Benzy: alcohol

Bis(2-chloroethoxy) methane

Bis(2-chloroethyl) ether

Bis(2-ethylhexyl) phthalate

Calcium

Dich lorad if I uoromethane

Trichlorof I uoromethane

Carbon tetrachloride

Cadmi um

Bromof or'm

Chloroform

Chrysene

Dibromomethane (Methylene bromide)

Dichloromethane (Methylene chloride)

Bromomethane (Methyl bromide)

Chloromethane (Methyl chloride)

Acetonitrile (Methyl cyanide)

Chloride

Chlorobenzilate

Chlorobenzene

Chlordane

Chloroprene

Pentachlorobenzene

Pentachloroethane

Pentach loron i trobenzene

Hexach lorobenzene

Hexach lorocyclopentadiene

Hexachloroe thane

Coba $1 t$

Chromi um

Carbon disulfide

Copper

Cyanide

Chloroethene ( $V$ inyl chloride)

Chloroethane

Benzene

Dibenz $(a, h) a n t h r a c e n e$

delta-Benzene hesachloride

Dibromochloromethane

Diethyl phthalate

Diallate

Dibenzof uran

Dimethonte

Disulfoton

CONTINUED
LT

LT

LI

LT

LT

LT

LI

LT

LI

LT

LT

LT

LI

LT

LI

LT

LI

LI

LT

LI

LI

LI

LT

LT

LT

L.T

LT

LT

LT

LT

LT

LT

IT

LT

LT

LT

LT

LT

LT

LT

LT

LT

LT

LT

LT

LT

LT

LT

LT

LT
$10.00 \mathrm{ug} / \mathrm{L}$

$10.00 \mathrm{ug} / \mathrm{L}$

$10.00 \mathrm{ug} / \mathrm{L}$

$0.05 \mathrm{ug} / \mathrm{L}$

$10.00 \mathrm{ug} / \mathrm{L}$

$10.00 \mathrm{ug} / \mathrm{L}$

$5.00 \mathrm{ug} / \mathrm{L}$

$10.00 \mathrm{ug} / \mathrm{L}$

$10.00 \mathrm{ug} / \mathrm{L}$

$1.00 \mathrm{ug} / \mathrm{L}$

$10.00 \mathrm{ug} / \mathrm{L}$

$10.00 \mathrm{ug} / \mathrm{L}$

$10.00 \mathrm{ug} / \mathrm{L}$

$10.00 \mathrm{ug} / \mathrm{L}$

$502.00 \mathrm{ug} / \mathrm{L}$

$1.00 \mathrm{ug} / \mathrm{L}$

$6.00 \mathrm{ug} / \mathrm{L}$

$1.00 \mathrm{ug} / \mathrm{L}$

$2.00 \mathrm{ug} / \mathrm{L}$

$1.00 \mathrm{ug} / \mathrm{L}$

$1.00 \mathrm{ug} / \mathrm{L}$

$10.00 \mathrm{ug} / \mathrm{L}$

$1.00 \mathrm{ug} / \mathrm{L}$

$1.00 \mathrm{ug} / \mathrm{L}$

$1.00 \mathrm{ug} / \mathrm{L}$

$1.00 \mathrm{ug} / \mathrm{L}$

$1.00 \mathrm{ug} / \mathrm{L}$

$2140.00 \mathrm{ug} / \mathrm{L}$

$10.00 \mathrm{ug} / \mathrm{L}$

$1.00 \mathrm{ug} / \mathrm{L}$

$0.50 \mathrm{ug} / \mathrm{L}$

$200.00 \mathrm{ug} / \mathrm{L}$

$10.00 \mathrm{ug} / \mathrm{L}$

$10.00 \mathrm{ug} / \mathrm{L}$

$10.00 \mathrm{ug} / \mathrm{L}$

$10.00 \mathrm{ug} / \mathrm{L}$

$10.00 \mathrm{ug} / \mathrm{L}$

$10.00 \mathrm{ug} / \mathrm{L}$

$4.00 \mathrm{ug} / \mathrm{L}$

$4.00 \mathrm{ug} / \mathrm{L}$

$1.00 \mathrm{ug} / \mathrm{L}$

$4.00 \mathrm{ug} / \mathrm{L}$

$5.00 \mathrm{ug} / \mathrm{L}$

$1.00 \mathrm{ug} / \mathrm{L}$

$1.00 \mathrm{ug} / \mathrm{L}$

$1.00 \mathrm{ug} / \mathrm{L}$

$10.00 \mathrm{ug} / \mathrm{L}$

$0.05 \mathrm{ug} / \mathrm{L}$

$1.00 \mathrm{ug} / \mathrm{L}$

$10.00 \mathrm{ug} / \mathrm{L}$

$10.00 \mathrm{ug} / \mathrm{L}$

$10.00 \mathrm{ug} / \mathrm{L}$

$10.00 \mathrm{ug} / \mathrm{L}$

$10.00 \mathrm{ug} / \mathrm{L}$
Gen. Eng •

Gen. Eng.

Gen. Eng.

Gen. Eng.

Gen. Eng.

Gen. Eng.

Gen. Eng.

Gen. Eng.

Gen. Eng.

Gen. Eng.

Gen. Eng.

Gen. Eng.

Gen.- Eng.

Gen. Eng .

Gen. Eng.

Gen. Eng.

Gen. Eng.

Gen. Eng.

Gen. Enz?.

Gen. Eng.

Gen. Eng.

Gen. Eng.

Gen. Eng.

Gen. Eng.

Gen. Eng.

Gen. Eng.

Gen. Eng.

Gen. Eng.

Gen. Eng.

Gen. Eng.

Gen. Eng.

Gen. Eng.

Gen. Eng.

Gen. Eng.

Gen. Eng.

Gen. Eng.

Gen. Eng.

Gen. Eng.

Gen. Eng.

Gen. Eng .

Gen. Eng.

Gen. Eng.

Gen. Eng.

Gen. Eng.

Gen. Eng.

Gen. Eng.

Gen. Eng .

Gen. Eng.

Gen. Eng.

Gen. Eng.

Geñ. Eng.

Gen. Eng.

Gen. Eng.

Gen. Eng. 
WELL LFW 29 COLLECTED ON 08/15/91 LABORATORY ANALYSES CONTINUED

\author{
Dieldrin \\ Dimethyl phthalate \\ Di-n-butyl phthalate \\ Di-n-octyl phthalate \\ Dipheny lamine \\ Ethyl methanesulfonate \\ Endr in aldehyde \\ Endrin \\ Endosulfan I \\ Endosulfan II \\ Endosulfan sulfate \\ Ethylbenzene \\ Ethyl methacrylate \\ Fluoride \\ Firmphur \\ Fluoranthene \\ Iron \\ iluorene \\ Hexach lorobutadiene \\ Hexachlorophene \\ Hexachloropropene \\ Mercury \\ Heptach lor \\ Heptachlor epoxide \\ Indeno $(1,2,3-c, d)$ pyrene \\ Iodomethane (Methyl iodide) \\ Isosafrole \\ Isobutyl alcohol \\ Isodrin \\ Isophorone \\ Potassium \\ Kepone \\ Lindane \\ Methacrylonitrile \\ Toluene \\ Methyl ethyl ketone \\ Methyl methacrylate \\ Methyl methanesulfonate \\ Methoxychlor \\ Magnes ium \\ Metliyl isobutyl ketone \\ Manganese \\ Metliapyri lene \\ Sod i um \\ Naphthalene \\ Nitrobenzene \\ $N-N i$ trosodimethylamine \\ $N-N i$ trosod $i-n-b u t y$ lamine \\ $N-N i$ trosodi-propylamine \\ $N-N i t r o s o d i c t h y l a m i n e$ \\ $\mathrm{N}$-Nitrosomethylethylnmine \\ Nickel \\ $\mathrm{N}-\mathrm{Ni}$ trosomorpholine \\ $\mathrm{N}-\mathrm{N}$ i trosodipheny lamine
}

CONTINUED

\begin{tabular}{|c|c|c|c|c|}
\hline LT & 0.50 & ug $/ L$ & Gen. & Eng \\
\hline LT & 10.00 & $u g / L$ & Gen. & Eng \\
\hline LT & 10.00 & $u g / L$ & Gen. & Eng \\
\hline LT & 10.00 & ug/L & Gen. & Eng \\
\hline LT & 10.00 & ug $/ \mathrm{L}$ & Gen. & ing \\
\hline LT & 10.00 & ug $/ \mathrm{L}$ & Gen. & ng \\
\hline LT & 0.10 & ug/L & Gen. & ing \\
\hline LT & 0.01 & $\mathrm{ug} / \mathrm{L}$ & Gen. & Eng \\
\hline$T$ & 0.10 & ug $/ \mathrm{L}$ & Gen. & Eng \\
\hline $\mathrm{T}$ & 0.10 & ug/L & Gen. & ng \\
\hline LT & 0.10 & ug/L & Gen. & b \\
\hline LT & 1.00 & ug/L & en. & 1 \\
\hline LT & 10.00 & ug/L & Gen. & $\mathbf{E}$ \\
\hline LT & 100.00 & ug $/ L$ & Gen. & Eng \\
\hline LT & 10.00 & $u g / L$ & Gen. & Eng \\
\hline $\mathrm{LT}$ & 10.00 & ug $/ \mathrm{L}$ & Gen. & Eng \\
\hline LT & $\begin{array}{l}13.80 \\
10.00\end{array}$ & $u_{\mathrm{g}} / \mathrm{L}$ & Gen. & E \\
\hline LT & $\begin{array}{l}10.00 \\
10.00\end{array}$ & $\begin{array}{l}\text { ug } / L \\
\text { ug } / L\end{array}$ & Gen. & \\
\hline LT & 10.00 & ug $/ \mathrm{L}$ & Gen. & Eng \\
\hline LT & 10.00 & ug $/ L$ & Gen. & En \\
\hline LI & 0.20 & $\mathrm{ug} / \mathrm{L}$ & Gen. & EI \\
\hline LT & 0.05 & ug/L & Gen. & $\mathbf{E}$ \\
\hline LT & 0.05 & $u g / L$ & Gen. & $\mathbf{E}$ \\
\hline LT & 10.00 & $\mathrm{ug} / \mathrm{L}$ & Gen. & EI \\
\hline LT & 15.00 & $\mathrm{ug} / \mathrm{L}$ & Gen. & Er \\
\hline LT & 10.00 & ug/L & Gen. & $\mathbf{E}$ \\
\hline LT & 100.00 & $\mathbf{u g} / \mathrm{L}$ & Gen. & $\mathbf{E}$ \\
\hline LT & 10.00 & $u g / L$ & Gen. & \\
\hline LT & 10.00 & ug/L & Gen. & Eng̨ \\
\hline LT & 500.00 & ug / L & Gen. & $\mathbf{E}$ \\
\hline LT & 10.00 & $\mathrm{ug} / \mathrm{L}$ & Gen. & En \\
\hline LT & 0.00 & ug $/ \mathrm{L}$ & en. & $\mathbf{E}$ \\
\hline LT & 50.00 & ug/L & Gen. & \\
\hline LT & 1.00 & $\mathbf{u g} / \mathrm{L}$ & n. & \\
\hline $\mathrm{LT}$ & -1.00 & ug/L & Gen. & \\
\hline $\mathrm{LT}$ & 10.00 & ug/L & Gen. & \\
\hline LT & 10.00 & $\mathrm{ug} / \mathrm{L}$ & en. & Eng \\
\hline LT & 0.50 & ug/L & en. & \\
\hline & 535.00 & ug/L & $n$. & \\
\hline & 2.00 & ug $/ \mathrm{L}$ & en. & \\
\hline & 3.63 & $\mathrm{ug} / \mathrm{L}$ & Gen & \\
\hline LT & 10.00 & $\mathbf{u g} / \mathrm{L}$ & Gen & \\
\hline & 1290.00 & $u g / L$ & Gon & \\
\hline LT & 10.00 & $u g / L$ & Gen. & \\
\hline LT & 10.00 & ug/L & Gen & \\
\hline LT & 10.00 & $\mathrm{ug} / \mathrm{L}$ & Gen. & \\
\hline LT & 10.00 & $u g / L$ & en & \\
\hline LT & 10.00 & $\mathbf{u g} / \mathrm{L}$ & G. & \\
\hline LT & 10.00 & $\mathrm{gg} / \mathrm{L}$ & Gen & \\
\hline LT & 10.00 & ug/h & Ger & \\
\hline LT & 4.00 & $\mathbf{u g} / \mathrm{L}$ & Ge & \\
\hline LT & 10.00 & "g/L & Ge & \\
\hline LT & 10.00 & $u_{g} / L$ & Ge & \\
\hline
\end{tabular}


$N-N$ itrosopiperidine

$N-N i$ trosopyrrolidine

Nitrate as nitrogen

4-Nitroquinoline. 1 -oxide

5-Nitro-o-toluidine

o-Toluidine

Lead

PCB 1016

PCB 1221

PCB 1232

PCB 1242

PCB 1248

PCB 1254

PCB 1260

Pentachlorophenol

$p$-Phenylenediamine

$P$-D imethylaminoazobenzene

Phenacetin

Phenanthrene

Phenol

Phorate

$\mathbf{p}, \mathbf{p}^{\prime}-\mathrm{DDD}$

$P, P^{\prime}-D D E$

$p, p^{\prime}-D D T$

Pronamid

Propionitrile

Parathion

Parathion methyl

Pyrene

Pyridine

Safrole

Antimony

2-sec-Butyl-4,6-dinitrophenol

Selen i um

Silica

$2,4,5-T P$ ( $S$ i l vex)

Tin

Sulf ate

Sulf otepp

Styrene

Sulf ide

Sulf ide

$1,2,4,5$-Tetrachlorobenzene

$1,1,2,2$-Tetrachloroethane

$1,1,1,2$-Tetrachloroethane

Tetrachloroethylene

0,0,0-Triethyl phosphorothioate

Thionazin

Tha I I i um

Total organic carbon

Total organic nitrogen

Total organic halogens

Total phosphates (ns P)

Trichlorocthylene

CONTINUED
LT

LT

LT

LT

LT

L. T

LT

LT

LI

LT

LT

LT

LI

LI

LI

LT

LT

LT

LT

LT

LT

LI

LT

LT

LT

LT

LT

LT

LT

LT

LT

LT

LT

LT

LT

LT

LT

LT

LT

LT

LT

LT

LT

LI

LT

LT

LT

LT

LT

LT
$10.00 \mathrm{ug} / \mathrm{L}$ $10.00 \mathrm{ug} / \mathrm{L}$ $410.00 \mathrm{ug} / \mathrm{L}$ $10.00 \mathrm{ug} / \mathrm{L}$ $10.00 \mathrm{ug} / \mathrm{L}$ $10.00 \mathrm{ug} / \mathrm{L}$ $3.00 \mathrm{ug} / \mathrm{L}$ $0.50 \mathrm{ug} / \mathrm{L}$ $0.50 \mathrm{ug} / \mathrm{L}$ $0.50 \mathrm{ug} / \mathrm{L}$ $0.50 \mathrm{ug} / \mathrm{I}$. $0.50 \mathrm{ug} / \mathrm{L}$ $1.00 \mathrm{ug} / \mathrm{L}$ $1.00 \mathrm{ug} / \mathrm{L}$ $10.00 \mathrm{ug} / \mathrm{L}$ $10.00 \mathrm{ug} / \mathrm{L}$ $10.00 \mathrm{ug} / \mathrm{L}$ $10.00 \mathrm{ug} / \mathrm{L}$ $10.00 \mathrm{ug} / \mathrm{L}$ $10.00 \mathrm{ug} / \mathrm{L}$ $0.10 \mathrm{ug} / \mathrm{L}$ $0.10 \mathrm{ug} / \mathrm{L}$ $0.10 \mathrm{ug} / \mathrm{L}$ $0.10 \mathrm{ug} / \mathrm{L}$ $10.00 \mathrm{ug} / \mathrm{L}$ $200.00 \mathrm{ug} / \mathrm{L}$

$0.05 \mathrm{ug} / \mathrm{L}$

$0.05 \mathrm{ug} / \mathrm{L}$ $10.00 \mathrm{ug} / \mathrm{L}$ $10.00 \mathrm{ug} / \mathrm{L}$ $10.00 \mathrm{ug} / \mathrm{L}$

$2.00 \mathrm{ug} / \mathrm{L}$ $10.00 \mathrm{ug} / \mathrm{L}$

$2.00 \mathrm{ug} / \mathrm{L}$ $6320.00 \mathrm{ug} / \mathrm{L}$

$0.09 \mathrm{ug} / \mathrm{L}$

$2.00 \mathrm{ug} / \mathrm{L}$ $1420.00 \mathrm{ug} / \mathrm{L}$. $10.00 \mathrm{ug} / \mathrm{L}$ $1.00 \mathrm{ug} / \mathrm{L}$ $1000.00 \mathrm{ug} / \mathrm{L}$ $1000.00 \mathrm{ug} / \mathrm{L}$ $10.00 \mathrm{ug} / \mathrm{L}$ $1.00 \mathrm{ug} / \mathrm{L}$ $1.00 \mathrm{ug} / \mathrm{L}$ $1.00 \mathrm{ug} / \mathrm{L}$ $10.00 \mathrm{ug} / \mathrm{L}$ $10.00 \mathrm{ug} / \mathrm{L}$ $2.00 \mathrm{ug} / \mathrm{L}$ $1000.00 \mathrm{ug} / \mathrm{L}$ $100.00 \mathrm{ug} / \mathrm{L}$ $20.10 \mathrm{ug} / \mathrm{L}$ $100.00 \mathrm{ug} / \mathrm{L}$ $1.00 \mathrm{ug} / \mathrm{L}$
Gen. Eng.

Gen. Eng.

Gen. Eng -

Gen. Eng.

Gen. Eng.

Gen. Eng.

Gen. Eng.

Gen. Eng.

Gen. Eng.

Gen. Eng.

Gen. Eng .

Gen. Eng.

Gen.- Eng.

Gen. Eng.

Gen. Eng.

Gen. Eng.

Gen. Eng .

Gen. Eng.

Gen. Eng.

Gen. Eng.

Gen. Eng .

Gen. Eng.

Gen. Eng.

Gen. Eng.

Gen. Eng.

Gen. Eng.

Gen. Eng.

Gen. Eng.

Gen. Eng.

Gen. Eng.

Gen. Eng.

Gen. Eng.

Gen. Eng.

Gen. Eng.

Gen. Eng.

Gen. Eng.

Gen. Eng.

Gen. Eng .

Gen. Eng.

Gen. Eng.

Gen. Eng.

Gen. Eng.

Gen. Eng.

Gen. Eng.

Gen. Eng.

Gen. Eng.

Gen. Eng -

Gen. Eng.

Gen. Eng.

Gen. Eng.

Geñ. Eng.

Gen. Eng.

Gen. Eng.

Gen. Eng. 
Toxaphene

trans-1,2-Dich loroethylene

trans-1, 3-D i ch loropropene

trans-1,4-Dichloro-2-butene

Vanadium

Vinyl acetate

X'ylenes

1-Naphthylamine

1,1-Dichloroethylene

1,1-Dichloroethane

1,1,1-Trichloroethane

$1,1,2-$ Trich loroethane

1,2-D ibromoiethane

1,2-D ibromo-3-chlisropropane

1,2-Dichlorobenzene

1,2-Dichloroethane

1,2-Dichloropropane

1,2,3-Tr i ch loropropane

$1,2,4$-Trich lorobenzene

1,3-D ich lorobenzene

c is-1,3-Dichloropropene

1,3-Din itrobenzene

1,3,5-Trinitrobenzene

1,4-Dichlorobenzene

1,4-Dioxane

1,4-Naphthoquinone

2-Acety laminof luorene

2-Chloroethyl vinyl ether

2-Chlorophenol

2-Chloronaphthalene

2-Hexanone

2-Methyl naphthalene

o-Cresol (2-Methylphenol)

2-Nitroaniline

2-Naphthylamine

2-Nitrophenol

2-Picoline

$2,3,4,6$-Tetrachlorophenol

2,4-Dichlorophenoxyacetic acid

2,4-Dichlorophenol

2,4-Dimethyl phenol

2,4-Din itrophenol

2,4-Dinitrotoluene

2,4,5-Trichlorophenosyacetic ncid

$2,4,5-\mathrm{Tr}$ ichlorophenol

2,4,6-Trichlorophenol

2,6-Dichlorophenol

2,6-Dinitrotoluene

3-Methylcholanthrene

m-Cresol (3-Methylphenol)

3-Nitronniline

3, 3'-D ichlorobenzidine

$3,3^{\prime}$-Dimethylbenzidine

4-Aminobipheny!

CONTINUED
LT

LT

LT

LT

LT

LT

LT

LT

LT

LT

LT

LT

LT

LT

LT

I.T

LT

LT

LT

LT

LT

LT

LT

LT

LT

LT

LT

LT

LT

LT

LT

LT

LT

LT

LT

LT

LT

LT

LT

LT

LT

LT

LT

LT

LT

LT

LT

LT

LT

LT

LT

LT

LT

LT

\section{$0.24 \mathrm{ug} / \mathrm{L}$}

$1.00 \mathrm{ug} / \mathrm{L}$

$1.00 \mathrm{ug} / \mathrm{L}$

$30.00 \mathrm{ug} / \mathrm{L}$

$10.00 \mathrm{ug} / \mathrm{L}$

$1.00 \mathrm{ug} / \mathrm{L}$

$1.00 \mathrm{ug} / \mathrm{L}$

$10.00 \mathrm{ug} / \mathrm{L}$

$1.00 \mathrm{ug} / \mathrm{L}$

$1.00 \mathrm{ug} / \mathrm{L}$

$1.00 \mathrm{ug} / \mathrm{L}$

$1.00 \mathrm{ug} / \mathrm{L}$

$20.00 \mathrm{ug} / \mathrm{L}$

$1.00 \mathrm{ug} / \mathrm{L}$

$10.00 \mathrm{ug} / \mathrm{T}$.

$1.00 \mathrm{ug} / \mathrm{L}$

$1.00 \mathrm{ug} / \mathrm{L}$

$20.00 \mathrm{ug} / \mathrm{L}$

$10.00 \mathrm{ug} / \mathrm{L}$

$10.00 \mathrm{ug} / \mathrm{L}$

$1.00 \mathrm{ug} / \mathrm{L}$

$10.00 \mathrm{ug} / \mathrm{L}$

$10.00 \mathrm{ug} / \mathrm{L}$

$10.00 \mathrm{ug} / \mathrm{L}$

$10.00 \mathrm{ug} / \mathrm{L}$

$10.00 \mathrm{ug} / \mathrm{L}$

$10.00 \mathrm{ug} / \mathrm{L}$

$10.00 \mathrm{ug} / \mathrm{L}$

$10.00 \mathrm{ug} / \mathrm{L}$

$10.00 \mathrm{wg} / \mathrm{L}$

$1.1,0 \mathrm{ug} / \mathrm{L}$

$10.00 \mathrm{ug} / \mathrm{L}$

$10.00 \mathrm{ug} / \mathrm{L}$

$1 \mathrm{~J} .00 \mathrm{ug} / \mathrm{L}$

$10.00 \mathrm{ug} / \mathrm{L}$

$10.00 \mathrm{ug} / \mathrm{L}$

$10.00 \mathrm{ug} / \mathrm{L}$

$10.00 \mathrm{ug} / \mathrm{L}$

$0.30 \mathrm{ug} / \mathrm{L}$

$10.00 \mathrm{ug} / \mathrm{L}$

$10.00 \mathrm{ug} / \mathrm{L}$

$45.00 \mathrm{ug} / \mathrm{L}$

$10.00 \mathrm{ug} / \mathrm{L}$

0.09 ug $/ \mathrm{L}$

$10.00 \mathrm{ug} / \mathrm{L}$

$10.00 \mathrm{ug} / \mathrm{L}$

$10.00 \mathrm{ug} / \mathrm{L}$

$10.00 \mathrm{ug} / \mathrm{L}$

$10.00 \mathrm{ug} / \mathrm{L}$

$10.00 \mathrm{ug} / \mathrm{L}$

$10.00 \mathrm{ug} / \mathrm{L}$

$20.00 \mathrm{ug} / \mathrm{L}$

$10.00 \mathrm{ug} / \mathrm{L}$

$10.00 \mathrm{ug} / \mathrm{L}$
Gen. Eng.

Gen. Eng.

Gen. Eng.

Gen. Eng.

Gen. Eng .

Gen. Eng.

Gen. Eng.

Gen. Eng.

Gen. Eng.

Gen. Eng.

Gen. Eng .

Gen. Eng.

Gen.- Eng.

Gen. Eng.

Gen. Eng.

Gen. Eng.

Gen. Eng.

Gen. Eng.

Gen. Eng.

Gen. Eng.

Gen. Eng.

Gen. Eng.

Gen. Eng.

Gen. Eng.

Gen, Eng.

Gen. Eng.

Gen. Eng.

Gen. Eng.

Gen. Eng.

Gen. Eng.

Gen. Eng.

Gen. Eng.

Gen. Eng.

Gen. Eng.

Gen. Eng.

Gen. Eng.

Gen. Eng.

Gen. Eng.

Gen. Eng.

Gen. Eng.

Gen. Eng.

Gen. Eng.

Gen. Eng.

Gen. Eng.

Gen. Eng.

Gen. Eng.

Gen. Eng.

Gen. Eng.

Gen. Eng.

Gen. Eng.

Geñ. Eng.

Gen. Eng.

Gen. Eng.

Gen. Eng. 
WELL LFW 29 COLLECTED ON 08/15/91 LABORATORX. ANALYSES CONTINUED

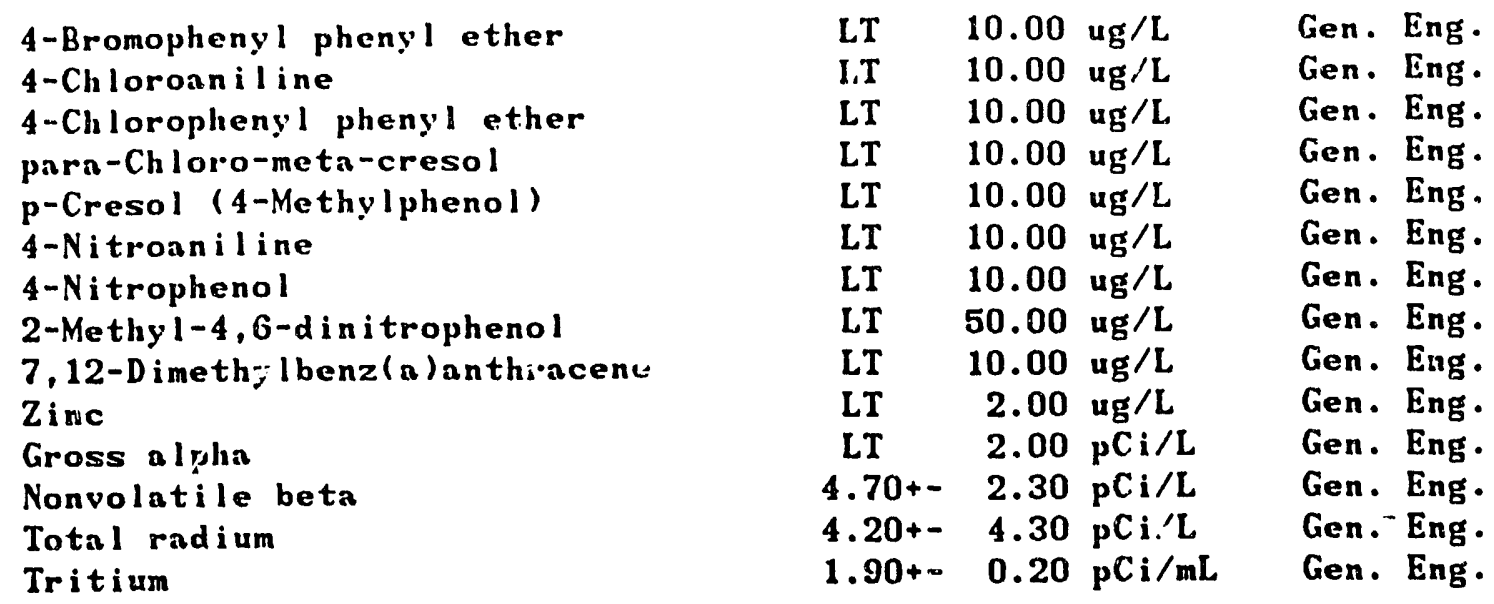

WELL LFW 30

SRS Grid N 86318.4 Latitude $33.290375 \mathrm{~N}$

Coordinates I! 45170.9 Longitude $81.715532 W$

Screen Zone Elevation 162.7-141.7 ft msl

Top of Casing Elevation $207.70 \mathrm{ft} \mathrm{msl}$

\section{MEASUREMENTS CONDUCTED IN TIIE FIELD}

Sample date $08 / 15 / 91$ Time 1210

Depth to water - $42.87 \mathrm{ft}(13.07 \mathrm{~m})$ below the TOC

Water elevation - $164.83 \mathrm{ft}(50.24 \mathrm{~m}) \mathrm{msl}$

pll $=5.1 \quad$ Alkalinity $-1 \mathrm{mg} / \mathrm{L}$

Specific conductance - $18 \mathrm{umhos} / \mathrm{cm}$

Water temperature - 18.5 degrees Celsius

Hater evacuated from the well prior to sampling - 61 gal

\section{LABORATORY ANALYSES}

Specific conductance

Carbon 12-labeled 2,3,7,8-TCDD

Carbon 12-labeled TCDF

Heptachlorodibenza-p-dioxin isomers

Heptachlorodibenzo-p-furan isomers

Hexachlorodibenzo-p-dioxin isomers

Hexachlorodibenzo-p-furan isomers

Octachlorodibenzo-p-dioxin isomers

Octachlorodibenzo-p-furan isomers

Pentachlorodibenzo-p-dioxin isomers

Pentachlorodibenzo-p-furan isomers

plI

Tetrachlorodibenzo-p-dioxin isomers

Tetrachlorodibenzo-p-furan isomers

$1,2,3,4,6,7,8-I I P C D D$

$1,2,3,4,6,7,8$-HIIPC.DF

$1,2,3,4,7,8$-Hexachlorodibenzo-p-dioxin LT

$1,2,3,4,7,8$-llexachlorodibenzo-p-furan IT

CONTINUED
$20.00 \mathrm{umh} / \mathrm{cm}$

$0.45 \mathrm{ng} / \mathrm{L}$

$0.40 \mathrm{ng} / \mathrm{L}$

$0.65 \mathrm{ng} / \mathrm{L}$

$0.45 \mathrm{ng} / \mathrm{L}$

$0.45 \mathrm{ng} / \mathrm{L}$

$0.40 \mathrm{ng} / \mathrm{L}$

$1.00 \mathrm{ng} / \mathrm{L}$

$1.00 \mathrm{ng} / \mathrm{L}$

$0.55 \mathrm{ng} / \mathrm{L}$

$0.55 \mathrm{ng} / \mathrm{L}$

$5.30 \mathrm{pll}$

$0.45 \mathrm{ng} / \mathrm{L}$

$0.40 \mathrm{ng} / \mathrm{L}$

$0.65 \mathrm{ng} / \mathrm{L}$

$0.45 \mathrm{ng} / \mathrm{L}$

$0.45 \mathrm{ng} / \mathrm{L}$

$0.40 \mathrm{ng} / \mathrm{L}$
Gen. Eng.

Gen. Eng.

Gen. Eng.

Gen. Eng.

Gen. Eng.

Gen. Eng.

Gen. Eng.

Gen. Eng.

Gen. Eng.

Gen. Eng.

Gen. Eng.

Gen. Eng.

Gen. Eng.

Gen. Eng.

Gen. Eng.

Geñ. Eng.

Gen. Eng.

Gen. Eng. 


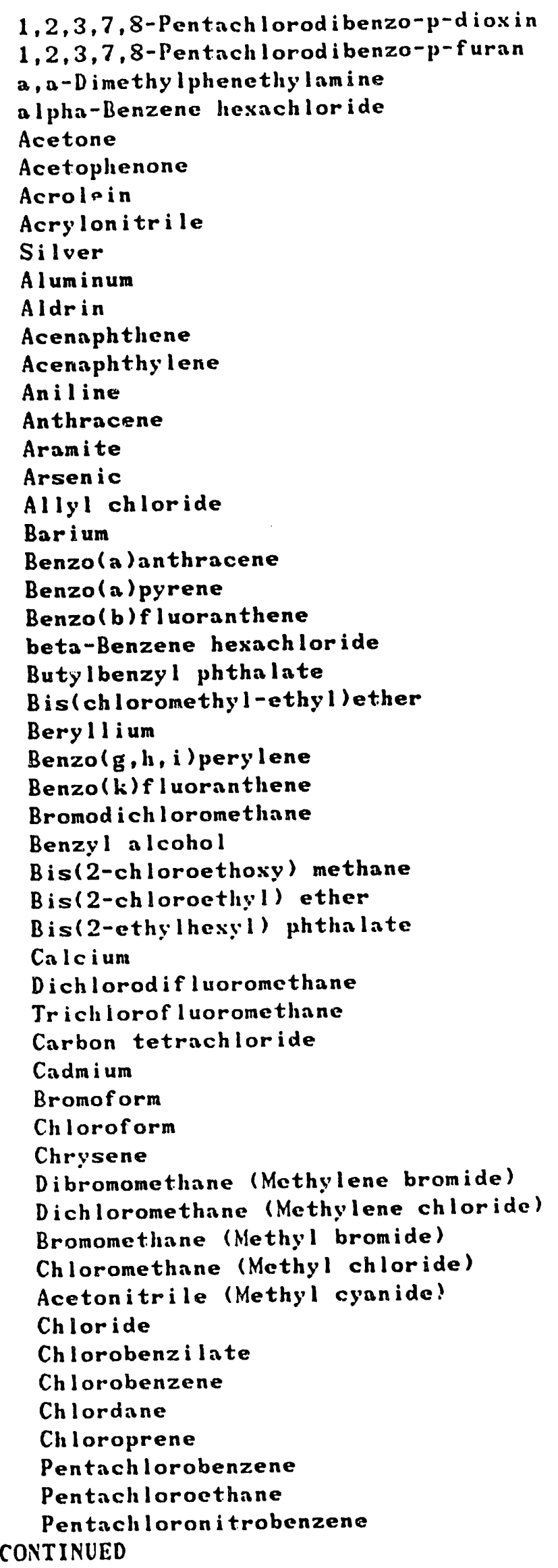

LT

LT

LI

LI

LT

LT

LT

LT

LI

L.T

LT

LI

LT

LT

LT

LT

LT

LT

LI

LT

LI

LI

LT

LI

LI

LT

LT

LT

LI

LT

LT

LT

LI

LT

LI

LI

LT

LT

LT

LT

\section{LT}

LT

LT

LT

LT

LT

LT

LT

LT

LT
$0.55 \mathrm{ng} / \mathrm{L}$

$0.55 \mathrm{ng} / \mathrm{L}$

$10.00 \mathrm{ug} / \mathrm{L}$

$0.05 \mathrm{ug} / \mathrm{L}$

$1.00 \mathrm{ug} / \mathrm{L}$

$10.00 \mathrm{ug} / \mathrm{L}$.

$20.00 \mathrm{ug} / \mathrm{L}$

$20.00 \mathrm{ug} / \mathrm{L}$

$2.00 \mathrm{ug} / \mathrm{L}$

$20.00 \mathrm{ug} / \mathrm{L}$

$0.05 \mathrm{ug} / \mathrm{L}$

$10.00 \mathrm{ug} / \mathrm{L}$

$10.00 \mathrm{ug} / \mathrm{L}$

$10.00 \mathrm{ug} / \mathrm{L}$

$10.00 \mathrm{ug} / \mathrm{L}$

$10.00 \mathrm{ug} / \mathrm{L}$

$2.00 \mathrm{ug} / \mathrm{L}$

$50.00 \mathrm{ug} / \mathrm{L}$

$5.57 \mathrm{ug} / \mathrm{L}$

$10.00 \mathrm{ug} / \mathrm{L}$

$10.00 \mathrm{ug} / \mathrm{L}$

$10.00 \mathrm{ug} / \mathrm{L}$

$0.05 \mathrm{ug} / \mathrm{L}$

$10.00 \mathrm{ug} / \mathrm{L}$

$10.00 \mathrm{ug} / \mathrm{L}$

$5.00 \mathrm{ug} / \mathrm{L}$

$10.00 \mathrm{ug} / \mathrm{L}$

$10.00 \mathrm{ug} / \mathrm{L}$

$1.00 \mathrm{ug} / \mathrm{L}$

$10.00 \mathrm{ug} / \mathrm{L}$

$10.00 \mathrm{ug} / \mathrm{L}$

$10.00 \mathrm{ug} / \mathrm{L}$

$10.00 \mathrm{ug} / \mathrm{L}$

$878.00 \mathrm{ug} / \mathrm{L}$

$1.00 \mathrm{ug} / \mathrm{L}$

$1.00 \mathrm{ug} / \mathrm{L}$

$1.00 \mathrm{ug} / \mathrm{L}$

$2.00 \mathrm{ug} / \mathrm{L}$

$1.00 \mathrm{ug} / \mathrm{L}$

$1.00 \mathrm{ug} / \mathrm{L}$

$10.00 \mathrm{ug} / \mathrm{L}$

$1.00 \mathrm{ug} / \mathrm{L}$

$1.70 \mathrm{ug} / \mathrm{L}$

$1.00 \mathrm{ug} / \mathrm{L}$

$1.00 \mathrm{ug} / \mathrm{L}$

$1.00 \mathrm{ug} / \mathrm{L}$

$1620.00 \mathrm{ug} / \mathrm{L}$

$10.00 \mathrm{ug} / \mathrm{L}$

$1.00 \mathrm{ug} / \mathrm{L}$

$0.50 \mathrm{ug} / \mathrm{L}$

$200.00 \mathrm{ug} / \mathrm{L}$

$10.00 \mathrm{ug} / \mathrm{L}$

$10.00 \mathrm{ug} / \mathrm{L}$

$10.00 \mathrm{ug} / \mathrm{L}$
Gen. Eng.

Gen. Eng.

Gen. Eng.

Gen. Eng.

Gen. Eng.

Gen. Eng.

Gen. Eng.

Gen. Eng.

Gen. Eng.

Gen. Eng.

Gen. Eng.

Gen. Eng.

Gen.- Eng.

Gen. Eng.

Gen. Eng.

Gen. Eng.

Gen. Eng.

Gen. Eng.

Gen. Eng.

Gen. Eng .

Gen. Eng.

Gen. Eng.

Gen. Eng.

Gen. Eng.

Gen. Eng .

Gen. Eng.

Gen. Eng -

Gen. Eng.

Gen. Eng.

Gen. Eng.

Gen. Eng -

Gen. Eng.

Gen. Eng.

Gen. Eng.

Gen. Eng.

Gen. Eng.

Gen. Eng.

Gen. Eng.

Gen. Eng.

Gen. Eng.

Gen. Eng.

Gen. Eng.

Gen. Eng .

Gen. Eng.

Gen. Eng.

Gen. Eng.

Gen. Eng.

Gen. Eng.

Gen. Eng.

Gen. Eng.

Geñ. Eng.

Gen. Eng.

Gen. Eng.

Gen. Eng. 
Hesachlorobenzene

Hexachlorocyclopentadiene

Hesach loroethane

Cobalt

Chromium

Carbon disulfide

Copper

Cyanide

Chioroethene (Vinyl chloride)

Chloroethane

Benzene

Dibenz $(a, h)$ anthracene

delta-Benzene hexachloride

Dibromoch loromethane

Diethyl phthalate

Diallate

Dibenzof uran

Dimethoate

Disulfoton

Dieldrin

Dimethyl phthalate

Di-n-butyl phthalate

Di-n-octyl phthalate

Diphenylamine

Ethyl methanesulfonate

Endrin aldehyde

Endrin

Endosulfan I

Endosulfan II

Endosulfan sulfate

Ethyl benzene

Ethyl methacrylate

Fluor ide

Famphur

Fluoranthene

Iron

Fl uorene

Hexachlorobutadiene

Hexachlorophene

Hexach loropropene

Mercury

Heptachlor

Heptachlor epoxide

Indeno $(1,2,3-c, d)$ pyrene

Iodomethane (Methyl iodide)

Isosafrole

Isobutyl alcohol

Isodrin

Isophorone

Potassium

Kepone

Lindane

Methacrylonitrile

Toluene

CONTINUED

\begin{tabular}{|c|c|c|c|c|}
\hline LT & $\begin{array}{l}10.00 \\
10.00\end{array}$ & $\begin{array}{l}u g / L \\
u g / L\end{array}$ & $\begin{array}{l}\text { Gen. } \\
\text { Gen. }\end{array}$ & Eng. \\
\hline $\begin{array}{l}\text { LT } \\
\text { LT }\end{array}$ & $\begin{array}{l}10.00 \\
10.00\end{array}$ & $\begin{array}{l}\text { ug } / L \\
\text { ug } / L\end{array}$ & $\begin{array}{l}\text { Gen. } \\
\text { Gen. }\end{array}$ & $\begin{array}{l}\text { Eng. } \\
\text { Eng. }\end{array}$ \\
\hline $\begin{array}{l}\mathrm{LT} \\
\mathrm{LT}\end{array}$ & 4.00 & $\mathrm{ug} / \mathrm{L}$ & Gen. & Eng. \\
\hline LT & 4.00 & ug $/ \mathrm{L}$ & Gen. & Eng. \\
\hline LT & 1.00 & $u g / L$ & Gen. & Eng. \\
\hline LT & 4.00 & ug $/ \mathrm{L}$ & Gen. & Eng. \\
\hline LT & 5.00 & ug $/ \mathrm{L}$ & Gen. & Eng. \\
\hline LT & 1.00 & ug/L & Gen. & Eng. \\
\hline LT & 1.00 & ug $/ \mathrm{L}$ & Gen. & Eng. \\
\hline LT & 1.00 & ug $/ \mathrm{L}$ & Gen. & Eng. \\
\hline LT & 10.00 & ug $/ L$ & Gen. & Eng. \\
\hline LT & 0.05 & ug $/ L$ & Gen.- & Eng. \\
\hline LT & 1.00 & ug/L & Gen. & Eng. \\
\hline LT & 10.00 & ug/L & Gen. & Eng. \\
\hline LT & 10.00 & ug/L & Gen. & Eng. \\
\hline LT & 10.00 & ug/L & Gen. & $\mathbf{E}$ \\
\hline LT & 10.00 & ug/L & Gen. & Eng. \\
\hline LI & 10.00 & ug/L & Gen. & Eng. \\
\hline LT & 0.50 & ug/L & Gen. & Eng. \\
\hline LT & 10.00 & ug/L & Gen. & Eng. \\
\hline LT & 10.00 & ug/L & Gen. & Eng • \\
\hline LT & 10.00 & ug/L & Gen. & Eng. \\
\hline LT & 10.00 & $u_{g} / L$ & Gen. & Eng - \\
\hline LT & 10.00 & ug/L & Gen. & Eng. \\
\hline LT & 0.10 & ug/L & Gen. & Eng. \\
\hline LT & 0.01 & ug/L & Gen. & Eng. \\
\hline LT & 0.10 & ug/L & Gen. & Eng. \\
\hline LT & 0.10 & ug/L & Gen. & Eng. \\
\hline LI & 0.10 & $u_{g} / L$ & Gen. & Eng. \\
\hline LT & 1.00 & ug/L & Gen. & Eng. \\
\hline LT & 10.00 & ug/L & Gen. & Eng. \\
\hline LT & 100.00 & $\mathbf{u g} / \mathrm{L}$ & Gen. & Eng \\
\hline LT & 10.00 & ug/L & Gen. & Eng. \\
\hline LT & 10.00 & ug/L & Gen. & Eng. \\
\hline & -8.41 & $\mathrm{ug} / \mathrm{L}$ & & Eng. \\
\hline 21 & 10.00 & ug/L & Gen. & \\
\hline LT & 10.00 & ug $/ L$ & Gen & \\
\hline LT & 10.00 & $4 \mathrm{~g} / \mathrm{L}$ & Gen. & Er \\
\hline LT & 10.00 & ug/L & Gen. & Eng. \\
\hline LT & 0.20 & ug $/ \mathrm{L}$ & Gen. & Eng. \\
\hline LT & 0.05 & ug/L & Gen. & En \\
\hline LT & 0.05 & ug $/ \mathrm{L}$ & Gen. & En \\
\hline LT & 10.00 & ug /L & Gen & $\mathbf{E}$ \\
\hline LT & 15.00 & ug/L & $\mathrm{Ge}$ & $\mathbf{E}_{1}$ \\
\hline LT & 10.00 & $u g / L$ & Ge & $E_{1}$ \\
\hline LT & 100.00 & ug /L & Gen & En \\
\hline LT & 10.00 & $\mathbf{u g}_{\mathrm{g} / \mathrm{L}}$ & Gen. & En \\
\hline LT & 10.00 & $\mathbf{u g} / \mathrm{L}$ & Gen. & En \\
\hline LT & 500.00 & ug/L & Gen. & En \\
\hline LT & 10.00 & Ig/L & Ge & Ex \\
\hline LT & 0.00 & $\mathrm{gg} / \mathrm{L}$ & n. & EI \\
\hline$L T$ & 50.00 & $\mu g / 2$ & & \\
\hline LT & 1.00 & $\operatorname{gg} / \mathrm{L}$ & & 20 \\
\hline
\end{tabular}


WELL LFW 30 COLLECTED ON 08/15/91 LABORATORY ANALYSES CONTINUED

\begin{tabular}{|c|c|c|c|c|}
\hline 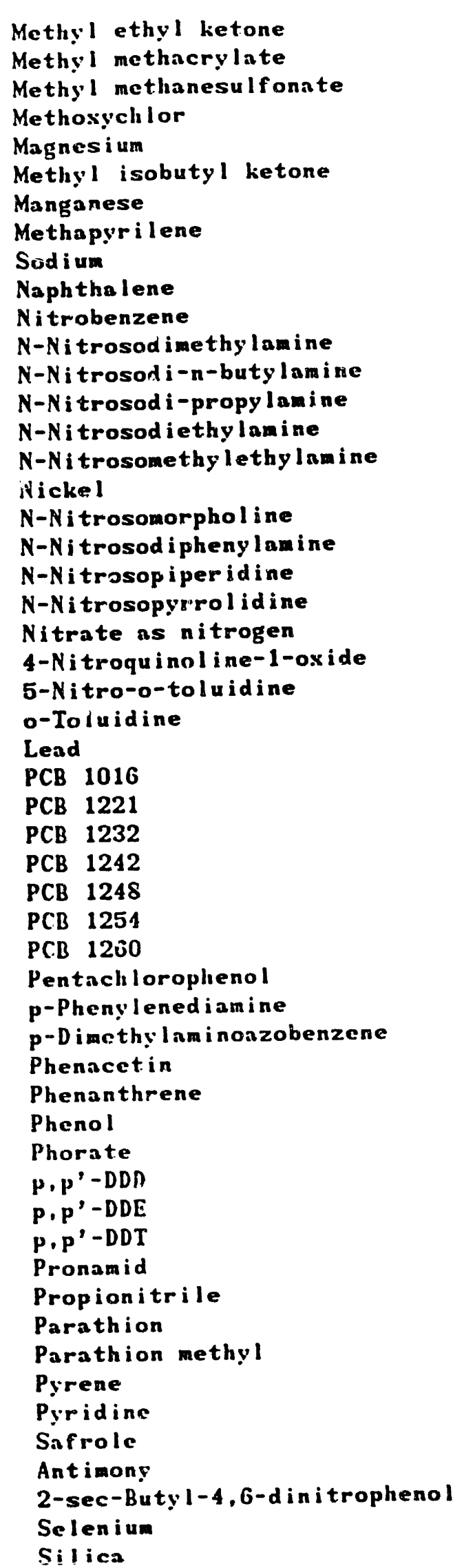 & $\begin{array}{l}\text { LT } \\
\text { LT } \\
\text { LT } \\
\text { LT } \\
\text { LI } \\
\text { LI } \\
\text { LT } \\
\text { LT } \\
\text { LT } \\
\text { LT } \\
\text { LT } \\
\text { LT } \\
\text { LT } \\
\\
\text { LT } \\
\text { LT } \\
\text { LT } \\
\text { LT } \\
\text { LT } \\
\text { LT } \\
\text { LT } \\
\text { LT } \\
\text { LT } \\
\text { LT } \\
\text { LT } \\
\text { LT } \\
\text { LT } \\
\text { LT } \\
\text { LT } \\
\text { LT } \\
\text { LT } \\
\text { LT } \\
\text { LT } \\
\text { LT } \\
\text { LT } \\
\text { LT } \\
\text { LT } \\
\text { LT } \\
\text { LT } \\
\text { LT } \\
\text { LT } \\
\text { LT } \\
\text { LT } \\
\text { LT } \\
\text { LT }\end{array}$ & $\begin{array}{r}1.00 \\
10.00 \\
10.00 \\
0.50 \\
471.00 \\
1.00 \\
3.79 \\
10.00 \\
888.00 \\
10.00 \\
10.00 \\
10.00 \\
10.00 \\
10.00 \\
10.00 \\
10.00 \\
4.00 \\
10.00 \\
10.00 \\
10.00 \\
10.00 \\
470.00 \\
10.00 \\
10.00 \\
10.00 \\
3.00 \\
0.50 \\
0.50 \\
0.50 \\
0.50 \\
0.50 \\
1.00 \\
1.00 \\
10.00 \\
10.00 \\
10.00 \\
10.00 \\
10.00 \\
10.00 \\
0.100 .00 \\
2.00 \\
0.10 \\
0.10 \\
0.10 \\
10.00 \\
200.00 \\
0.05 \\
0.05 \\
10.00 \\
10.00 \\
10.00 \\
2.00 \\
100\end{array}$ & $\begin{array}{l}\mathrm{ug} / \mathrm{L} \\
\mathrm{ug} / \mathrm{L} \\
\mathrm{ug} / \mathrm{L} \\
\mathrm{ug} / \mathrm{L} \\
\mathrm{ug} / \mathrm{L} \\
\mathrm{ug} / \mathrm{L} \\
\mathrm{ug} / \mathrm{L} \\
\mathrm{ug} / \mathrm{L} \\
\mathrm{ug} / \mathrm{L} \\
\mathrm{ug} / \mathrm{L} \\
\mathrm{ug} / \mathrm{L} \\
\mathrm{ug} / \mathrm{L} \\
\mathrm{ug} / \mathrm{L} \\
\mathrm{ug} / \mathrm{L} \\
\mathrm{ug} / \mathrm{L} \\
\mathrm{ug} / \mathrm{L} \\
\mathrm{ug} / \mathrm{L} \\
0 \mathrm{ug} / \mathrm{L} \\
0 \mathrm{ug} / \mathrm{L} \\
0 \mathrm{ug} / \mathrm{L} \\
0 \\
\mathrm{ug} / \mathrm{L} \\
0 \\
\mathrm{ug} / \mathrm{L}\end{array}$ & $\begin{array}{l}\text { Gen. } \\
\text { Gen. } \\
\text { Gen. } \\
\text { Gen. } \\
\text { Gen. } \\
\text { Gen. } \\
\text { Gen. } \\
\text { Gen. } \\
\text { Gen. } \\
\text { Gen. } \\
\text { Gen. } \\
\text { Gen. } \\
\text { Gen. } \\
\text { Gen. } \\
\text { Gen. } \\
\text { Gen. } \\
\text { Gen. } \\
\text { Gen. } \\
\text { Gen. } \\
\text { Gen. } \\
\text { Gen. } \\
\text { Gen. } \\
\text { Gen. } \\
\text { Gen. } \\
\text { Gen. } \\
\text { Gen. } \\
\text { Gen. } \\
\text { Gen. } \\
\text { Gen. } \\
\text { Gen. } \\
\text { Gen. } \\
\text { Gen. } \\
\text { Gen. } \\
\text { Gen. } \\
\text { Gen. } \\
\text { Gen. } \\
\text { Gen. } \\
\text { Gen. } \\
\text { Gen. } \\
\text { Gen. } \\
\text { Gen. } \\
\text { Gen. } \\
\text { Gen. } \\
\text { Gen. } \\
\text { Gen. } \\
\text { Gen. } \\
\text { Gen. } \\
\text { Gen. } \\
\text { Gen. } \\
\text { Gen. }\end{array}$ \\
\hline
\end{tabular}


WELL LFW 30 COLLECTED ON 08/15/91 LABORATORY ANALYSES CONTINUED

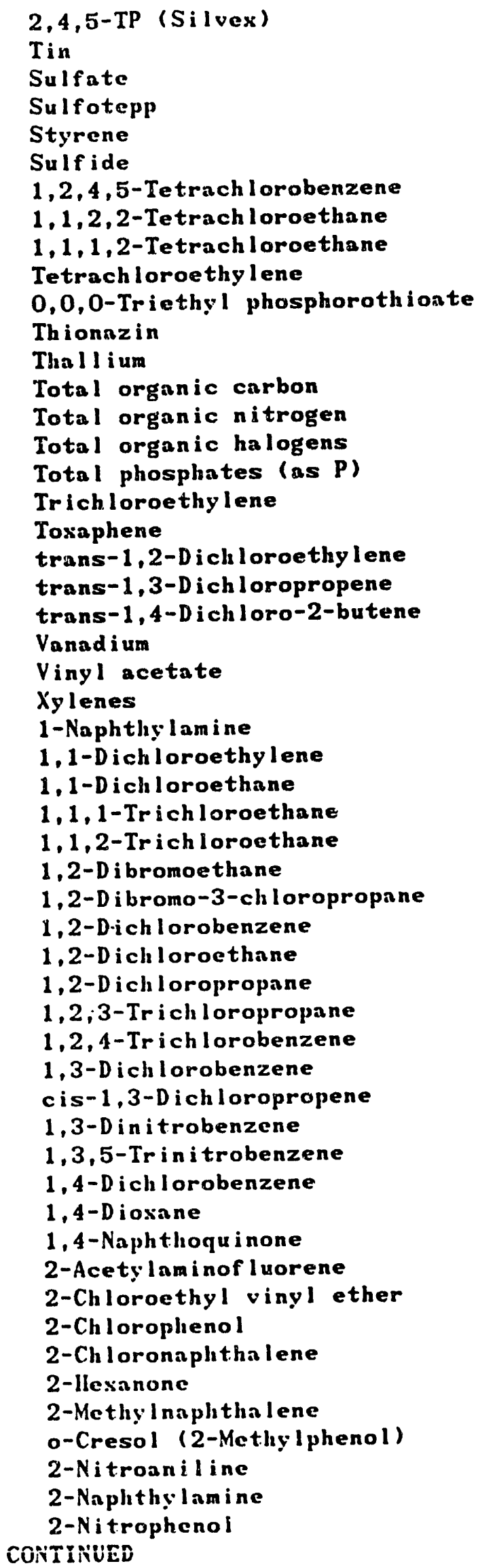

LI

LT

LT

LT

LT

LT

LT

LT

LT

LT

LT

LT

LT

LT

LT

LT

LT

LT

LT

LT

LI

LT

\section{LT}

LT

LT

LT

LI

LT

LT

LI

LT

LT

LT

LI

LT

LT

LT

LT

LT

LT

LT

LI

LI

LT

LT

LT

LT

LT

LT

LT

LT

LT
$0.09 \mathrm{ug} / \mathrm{L}$

$2.00 \mathrm{ug} / \mathrm{L}$

$1000.00 \mathrm{ug} / \mathrm{L}$

$10.00 \mathrm{ug} / \mathrm{L}$

$1.00 \mathrm{ug} / \mathrm{L}$

$1000.00 \mathrm{ug} / \mathrm{L}$

$10.00 \mathrm{ug} / \mathrm{L}$

$1.00 \mathrm{ug} / \mathrm{L}$

$1.00 \mathrm{ug} / \mathrm{L}$

$1.00 \mathrm{ug} / \mathrm{L}$

$10.00 \mathrm{ug} / \mathrm{L}$

$10.00 \mathrm{ug} / \mathrm{L}$

$2.00 \mathrm{ug} / \mathrm{L}$

$2000.00 \mathrm{ug} / \mathrm{L}$

$100.00 \mathrm{ug} / \mathrm{L}$

$9.10 \mathrm{ug} / \mathrm{L}$

$100.00 \mathrm{ug} / \mathrm{L}$

$1.00 \mathrm{ug} / \mathrm{L}$

$0.24 \mathrm{ug} / \mathrm{L}$

$1.00 \mathrm{ug} / \mathrm{L}$

$1.00 \mathrm{mg} / \mathrm{L}$

$30.00 \mathrm{ug} / \mathrm{L}$

$10.00 \mathrm{ug} / \mathrm{L}$

$1.00 \mathrm{ug} / \mathrm{L}$

$1.00 \mathrm{ug} / \mathrm{L}$

$10.00 \mathrm{ug} / \mathrm{L}$

$1.00 \mathrm{ug} / \mathrm{L}$

$1.00 \mathrm{ug} / \mathrm{L}$

$1.00 \mathrm{ug} / \mathrm{L}$

$1.00 \mathrm{ug} / \mathrm{L}$

$20.00 \mathrm{ug} / \mathrm{L}$

$1.00 \mathrm{ug} / \mathrm{L}$

$10.00 \mathrm{ug} / \mathrm{L}$

$1.00 \mathrm{ug} / \mathrm{L}$

$1.00 \mathrm{ug} / \mathrm{L}$

$20.00 \mathrm{ug} / \mathrm{L}$

$10.00 \mathrm{ug} / \mathrm{L}$

$10.00 \mathrm{ug} / \mathrm{L}$

$1.00 \mathrm{ug} / \mathrm{L}$

$10.00 \mathrm{ug} / \mathrm{L}$

$10.00 \mathrm{ug} / \mathrm{L}$

$10.00 \mathrm{ug} / \mathrm{L}$

$10.00 \mathrm{ug} / \mathrm{L}$

$10.00 \mathrm{ug} / \mathrm{L}$

$10.00 \mathrm{ug} / \mathrm{L}$

$10.00 \mathrm{ug} / \mathrm{L}$

$10.00 \mathrm{ug} / \mathrm{L}$

$10.00 \mathrm{ug} / \mathrm{L}$

$1.00 \mathrm{ug} / \mathrm{L}$

$10.00 \mathrm{ug} / \mathrm{L}$

$10.00 \mathrm{ug} / \mathrm{L}$

$10.00 \mathrm{ug} / \mathrm{L}$

$10.00 \mathrm{ug} / \mathrm{L}$

$10.00 \mathrm{ug} / \mathrm{L}$
Gen. Eng -

Gen. Eng.

Gen. Eng.

Gen. Eng.

Gen. Eng.

Gen. Eng -

Gen. Eng .

Gen. Eng .

Gen. Eng.

Gen. Eng.

Gen. Eng.

Gen. Eng .

Gen:- Eng.

Gen. Eng.

Gen. Eng.

Gen. Eng.

Gen. Eng .

Gen. Eng.

Gen. Eng .

Gen. Eng.

Gen. Eng -

Gen. Eng.

Gen. Eng.

Gen. Eng.

Gen. Eng.

Gen. Eng.

Gen. Eng.

Gen. Eng.

Gen. Eng.

Gen. Eng.

Gen. Eng .

Gen. Eng.

Gen. Eng.

Gen. Eng.

Gen. Eng.

Gen. Eng.

Gen. Eng.

Gen. Eng.

Gen. Eng.

Gen. Eng.

Gen. Eng.

Gen. Eng .

Gen. Eng.

Gen. Eng.

Gen. Eng.

Gen. Eng .

Gen. Eng.

Gen. Eng.

Gen. Eng.

Gen. Eng.

Geñ. Eng.

Gen. Eng.

Gen. Eng.

Gen. Eng. 
WELL LFW 30 COLLECTED ON 08/15/91 LABORATORY ANALYSES CONTINUED

2-Picoline

$2,3,4,6$-Tetrach lorophenol

2,4-Dichlorophenoxyacetic acid

2,4-D ichlorophenol

2,4-Dimethyl phenol

2,4-D in itrophenol

2,4-D in itrotoluene

$2,4,5-$ Ir ichlorophenoxyacetic acid

2,4,5-Trich lorophenol

2,4,6-Trich lorophenol

2,6-Dichlorophenol

2,6-Dinitrotoluene

3-Methylcholanthrere

m-Cresol (3-Methylphenol)

3-Nitroanil ine

3, $3^{\prime}-D$ ich lorobenzid ine

3, 3'-Dimethyl benzidine

4-Ami nobiphenyl

4-Bromophenyl phenyl ether

4-Chloroani I ine

4-Chlorophenyl phenyl ether

para-Chloro-meta-cresol

p-Cresol (4-Methylphenol)

4-Nitroaniline

4-Nitrophenol

2-Methyl-4,6-dinitrophenol

7, 12-Dimethylbenz(a) anthracene

$\mathrm{Zinc}$

Gross alpha

Nonvolatile beta

Total radium

Tritium

$\begin{array}{lrl}\mathrm{LT} & 10.00 \mathrm{ug} / \mathrm{L} \\ \mathrm{LT} & 10.00 \mathrm{ug} / \mathrm{L} \\ \mathrm{LT} & 0.30 \mathrm{ug} / \mathrm{L} \\ \mathrm{LT} & 10.00 \mathrm{ug} / \mathrm{L} \\ \mathrm{LT} & 10.00 \mathrm{ug} / \mathrm{L} \\ \mathrm{LT} & 45.00 \mathrm{ug} / \mathrm{L} \\ \mathrm{LT} & 10.00 \mathrm{ug} / \mathrm{L} \\ \mathrm{LT} & 0.09 \mathrm{ug} / \mathrm{L} \\ \mathrm{LT} & 10.00 \mathrm{ug} / \mathrm{L} \\ \mathrm{LT} & 10.00 \mathrm{ug} / \mathrm{L} \\ \mathrm{LT} & 10.00 \mathrm{ug} / \mathrm{L} \\ \mathrm{LT} & 10.00 \mathrm{ug} / \mathrm{L} \\ \mathrm{LT} & 10.00 \mathrm{ug} / \mathrm{L} \\ \mathrm{LT} & 10.00 \mathrm{ug} / \mathrm{L} \\ \mathrm{LT} & 10.00 \mathrm{ug} / \mathrm{L} \\ \mathrm{LT} & 20.00 \mathrm{ug} / \mathrm{L} \\ \mathrm{LT} & 10.00 \mathrm{ug} / \mathrm{L} \\ \mathrm{LT} & 10.00 \mathrm{ug} / \mathrm{L} \\ \mathrm{LT} & 10.00 \mathrm{ug} / \mathrm{L} \\ \mathrm{LT} & 10.00 \mathrm{ug} / \mathrm{L} \\ \mathrm{LT} & 10.00 \mathrm{ug} / \mathrm{L} \\ \mathrm{LT} & 10.00 \mathrm{ug} / \mathrm{L} \\ \mathrm{LT} & 10.00 \mathrm{ug} / \mathrm{L} \\ \mathrm{LT} & 10.00 \mathrm{ug} / \mathrm{L} \\ \mathrm{LT} & 10.00 \mathrm{ug} / \mathrm{L} \\ \mathrm{LT} & 50.00 \mathrm{ug} / \mathrm{L} \\ \mathrm{LT} & 10.00 \mathrm{ug} / \mathrm{L} \\ & 5.36 \mathrm{ug} / \mathrm{L} \\ \mathrm{LT} & 2.00 \mathrm{pCi} / \mathrm{L} \\ \mathrm{L} & 10+- & 1.90 \mathrm{pC} \mathrm{i} / \mathrm{L} \\ \mathrm{L} & 10+- & 2.50 \mathrm{pCi} / \mathrm{L} \\ \mathrm{L} .26+- & 0.20 \mathrm{pC} / \mathrm{i} / \mathrm{mL}\end{array}$

Gen. Eng.

Gen. Eng.

Gen. Eng.

Gen. Eng.

Gen. Eng.

Gen. Eng.

Gen. Eng.

Gen. Eng.

Gen. Eng.

Gen. Eng.

Gen. Eng.

Gen. Eng.

Gen: Eng.

Gen. Eng .

Gen. Eng.

Gen. Eng.

Gen. Eng .

Gen. Eng.

Gen. Eng.

Gen. Eng.

Gen. Eng.

Gen. Eng.

Gen. Eng.

Gen. Eng.

Gen. Eng.

Gen. Eng.

Gen. Eng.

Gen. Eng -

Gen. Eng.

Gen. Eng .

Gen. Eng.

Gen. Eng. 
WELL LFW 31

SRS Grid N 86262.2 Latitude 33.289758 N

Coordinates E 44869.0 Long itude 81.716217 W

Screen Zone Elevation 166.0-145.0 ft msl

Top of Casing Elevation $227.00 \mathrm{ft} \mathrm{msl}$

MEASUREMENTS CONDUCTED IN THE FIELD

Sample date 08/15/91 Time 1145

Depth to water - $63.03 \mathrm{ft}(19.21 \mathrm{~m})$ below the TOC

Water elevation - $163.97 \mathrm{ft}(49.98 \mathrm{~m}) \mathrm{msl}$

$\mathrm{pH}-5.1 \quad$ Alkalinity - $1 \mathrm{mg} / \mathrm{L}$

Specific conductance - 21 umhos/cm

Water temperature $=18.4$ degrees Celsius

Water evacuated from the well prior to sampling - $58 \mathrm{gal}$

LABORATORY ANALYSES

Specific conductance

pH

Silver

Aluminum

Aldrin

Arsenic

Barium

beta-Benzene hexachloride

Bromodichloromethane

Calcium

Trich lorof l uoromethane

Carbon tetrachloride

Cadmi um

Bromoform

Chloroform

Dichloromethane (Methylene chloride)

Bromomethane (Methyl bromide)

Chloromethane (Methyl chloride)

Chloride

Chlorobenzene

Chromi um

Copper

Chloroethene (Vinyl chloride)

Chloroethane

Benzene

Dibromoch loromethane

Endrin

Endosulfan sulfate

Ethylbenzene

Fluoride

Fluoride

Iron

Mercury

Potassium

Lindane

Toluene

Methosychlor

CONTINUED

\begin{tabular}{|c|c|c|c|c|}
\hline LT & $\begin{array}{r}20.00 \\
5.40 \\
2.00\end{array}$ & $\begin{array}{l}\text { umh } / \mathrm{cm} \\
\mathrm{pH} \\
\text { ug/L }\end{array}$ & $\begin{array}{l}\text { Gen. } \\
\text { Gen. } \\
\text { Gen. }\end{array}$ & $\begin{array}{l}\text { Eng. } \\
\text { Eng. } \\
\text { Eng. }\end{array}$ \\
\hline LT & 20.00 & $u_{g} / L$ & Gen. & Eng \\
\hline LT & 0.05 & $u g / L$ & Gen. & Eng. \\
\hline LT & $\begin{array}{l}2.00 \\
4.48\end{array}$ & $\begin{array}{l}\text { ug/L } \\
\text { ug/L }\end{array}$ & $\begin{array}{l}\text { Gen. } \\
\text { Gen. }\end{array}$ & $\begin{array}{l}\text { Eng } \\
\text { Eng }\end{array}$ \\
\hline LT & 0.05 & ug/L & Gen. & Eng \\
\hline LT & $\begin{array}{r}1.00 \\
674.00\end{array}$ & $\begin{array}{l}u g / L \\
\text { ug/L }\end{array}$ & $\begin{array}{l}\text { Gen. } \\
\text { Gen. }\end{array}$ & Eng \\
\hline LT & 1.00 & ug $/ L$ & Gen. & ng \\
\hline LT & 1.00 & ug/L & Gen. & 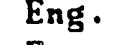 \\
\hline LT & 2.00 & ug $/ \mathrm{L}$ & Gen. & Eng \\
\hline LT & 1.00 & ug/L & Gen. & Eng \\
\hline LT & 1.00 & ug/L & Gen. & \\
\hline & 13.80 & ug $/ L$ & Gen. & \\
\hline LI & 1.00 & $u g / L$ & Gen. & \\
\hline LT & 1.00 & ug $/ L$ & en. & \\
\hline & 2440.00 & $\mathrm{ug} / \mathrm{L}$ & en. & \\
\hline LT & 1.00 & ug $/ L$ & en. & \\
\hline LT & 4.00 & $\mathrm{ug} / \mathrm{L}$ & en. & $\pi$ \\
\hline & 10.40 & ug/L & en. & \\
\hline LT & 1.00 & $\mathbf{u g} / \mathrm{L}$ & en. & \\
\hline LT & 1.00 & ug /L & en. & \\
\hline LT & 1.00 & ug/L & Gen. & $\mathrm{ng}$ \\
\hline LT & 1.00 & ug/L & Gen. & - Eng \\
\hline LT & 0.01 & $u g / L$ & Gen. & \\
\hline LT & 0.10 & $\operatorname{ug} / \mathrm{L}$ & Gen. & \\
\hline LT & 1.00 & ug/L & Gen. & \\
\hline LT & 100.00 & ug/L & Gen. & \\
\hline LT & 100.00 & $u g / L$ & Gen. & \\
\hline & 22.60 & $\mathrm{ug} / \mathrm{L}$ & Gen. & - En \\
\hline LT & 0.20 & ug $/ L$ & Gen. & En \\
\hline & 633.00 & ug/L & Geñ. & - En \\
\hline LT & 0.00 & $u g / L$ & Gen. & - $\mathbf{E}_{1}$ \\
\hline LT & 1.00 & ug/L & Gen. & • \\
\hline LT & 0.50 & $u g / L$ & Gen. & \\
\hline
\end{tabular}


WELL LFW 31 COLLECTED ON OS/15/91 LABORATORY ANALYSES CONTINUED

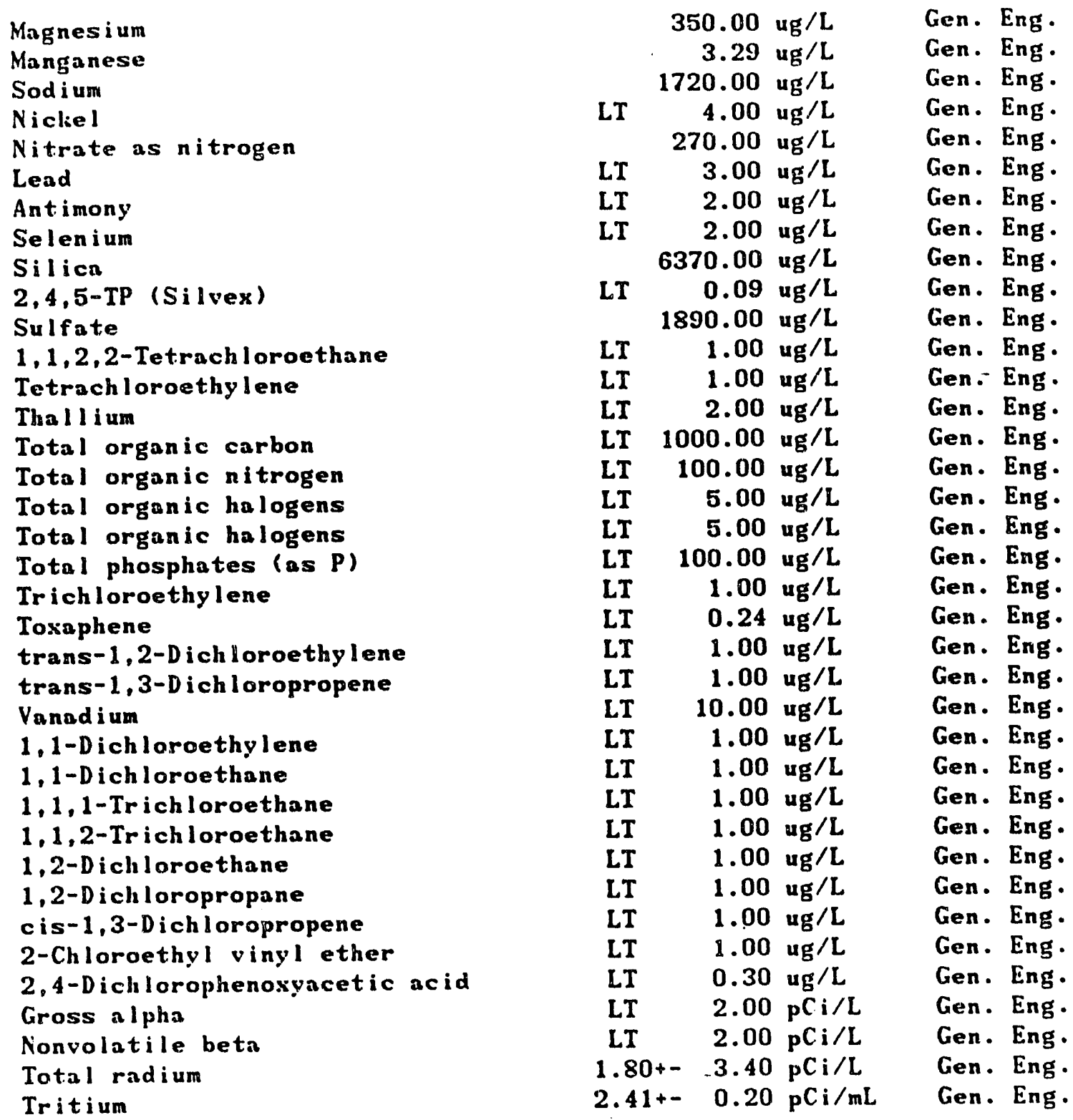


WELL LFW 32

$\begin{array}{llllll}\text { SRS Grid } & N & 85836.8 & \text { Latitude } & 33.288927 & N \\ \text { Coordinates } & E & 44935.9 & \text { Longitude } & 81.715214 & W\end{array}$

Screen Zone Elevation 165.3-144.3 ft ms

Top of Casing Elevation $221.30 \mathrm{ft} \mathrm{msl}$

MEASUREMENTS CONDUCTED IN THE FIELD

Sample date 08/15/91 Time 1120

Depth to water - $59.49 \mathrm{ft}(18.13 \mathrm{~m})$ below the TOC.

Water elevation - $161.81 \mathrm{ft}(49.32 \mathrm{~m}) \mathrm{msl}$

$\mathrm{pH}-4.9$ Alkalinity $=0 \mathrm{mg} / \mathrm{L}$

Specific conductance - 18 umhos/cm

Water temperature - 18.6 degrees Celsius

Water evacuated from the well prior to sampling - $54 \mathrm{gal}$

\section{LABORATORY ANALYSES}

Specific conductance

pH

Silver

A luminum

Aldrin

Arsenic

Barium

beta-Benzene hexachloride

Bromodichlorome thane

Calcium

Trich lorof luoromethane

Carbon tetrachloride

Cadmi um

Bromoform

Chloroform

Dichloromethane (Methylene chloride)

Bromomethane (Methyl bromide)

Chloromethane (Methyl cilloride)

Chloride

Chloride

Ch lorobenzene

Chromium

Copper

Chloroethene (Vinyl chloride)

Chloroethane

Benzene

Dibromochloromethane

Endrin

Endosulfan sulfate

Ethylbenzene

Fluoride

Iron

Mercury

Potassium

Lindane

Toluene

Methoxychlor

CONTINUED
$20.00 \mathrm{umh} / \mathrm{cm}$

$5.30 \mathrm{pll}$

LT

LT

LT

LT

LT

LT

LT

LT

LI

LT

LT

LT

LT$$
2360.00 \mathrm{ug} / \mathrm{L}
$$

$2.00 \mathrm{ug} / \mathrm{L}$

$20.00 \mathrm{ug} / \mathrm{L}$

$0.05 \mathrm{ug} / \mathrm{L}$

$2.00 \mathrm{ug} / \mathrm{L}$

$4.14 \mathrm{ug} / \mathrm{L}$

$0.05 \mathrm{ug} / \mathrm{L}$

$295.00 \mathrm{ug} / \mathrm{L}$

$1.00 \mathrm{ug} / \mathrm{L}$

$1.00 \mathrm{ug} / \mathrm{L}$

$2.00 \mathrm{ug} / \mathrm{L}$

$1.00 \mathrm{ug} / \mathrm{L}$

$1.00 \mathrm{ug} / \mathrm{L}$

$11.20 \mathrm{ug} / \mathrm{L}$

$1.00 \mathrm{ug} / \mathrm{L}$

$1.00 \mathrm{ug} / \mathrm{L}$

$2360.00 \mathrm{ug} / \mathrm{L}$

$1.00 \mathrm{ug} / \mathrm{L}$

$4.00 \mathrm{ug} / \mathrm{L}$

$4.00 \mathrm{ug} / \mathrm{L}$

$1.00 \mathrm{ug} / \mathrm{L}$

$1.00 \mathrm{ug} / \mathrm{L}$

$1.00 \mathrm{ug} / \mathrm{L}$

$1.00 \mathrm{ug} / \mathrm{L}$

$0.01 \mathrm{ug} / \mathrm{L}$

$0.10 \mathrm{ug} / \mathrm{L}$

$1.00 \mathrm{ug} / \mathrm{L}$

$100.00 \mathrm{ug} / \mathrm{L}$

$39.50 \mathrm{ug} / \mathrm{L}$

LT

$0.20 \mathrm{ug} / \mathrm{L}$

$512.00 \mathrm{ug} / \mathrm{L}$

$0.00 \mathrm{ug} / \mathrm{L}$

$1.00 \mathrm{ug} / \mathrm{L}$

$0.50 \mathrm{ug} / \mathrm{L}$
Gen. Eng -

Gen. Eng .

Gen. Eng.

Gen. Eng.

Gen. Eng -

Gen. Eng.

Gen. Eng.

Gen. Eng.

Gen. Eng.

Gen. Eng.

Gen. Eng.

Gen. Eng.

Gen. Eng.

Gen. Eng.

Gen. Eng.

Gen. Eng.

Gen. Eng.

Gen. Eng -

Gen. Eng .

Gen. Eng .

Gen. Eng.

Gen. Eng.

Gen. Eng.

Gen. Eng.

Gen. Eng.

Gen. Eng.

Gen. Eng.

Gen. Eng.

Gen. Eng.

Gen. Eng.

Gen. Eng.

Gen: Eng.

Gen. Eng.

Geñ. Eng.

Gen. Eng.

Gen. Eng .

Gen. Eng. 
WELL LFW 32 COLLECTED ON 08/15/91 LABORATORY ANALYSES CONTINUED

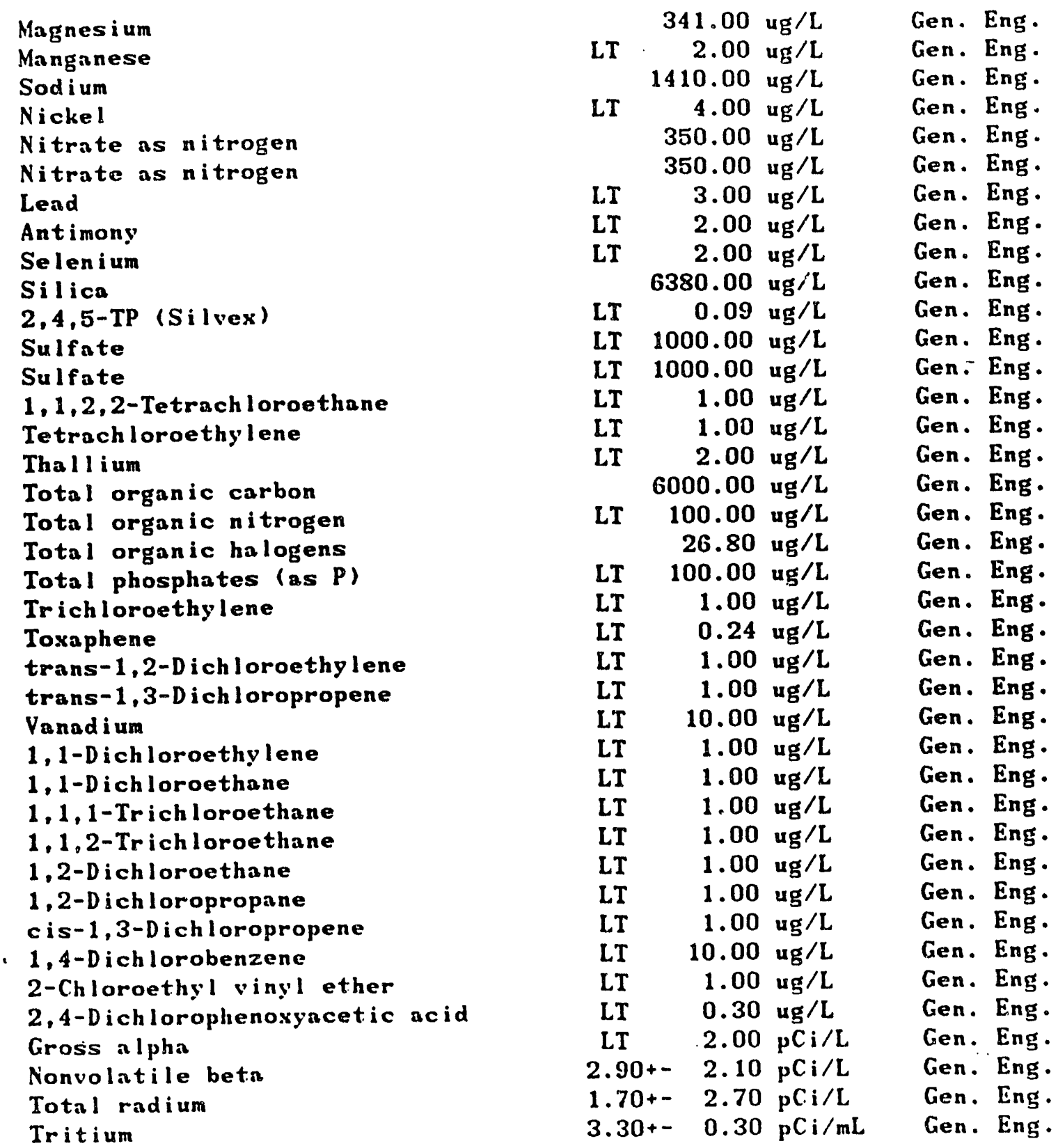


WELL LFW 33

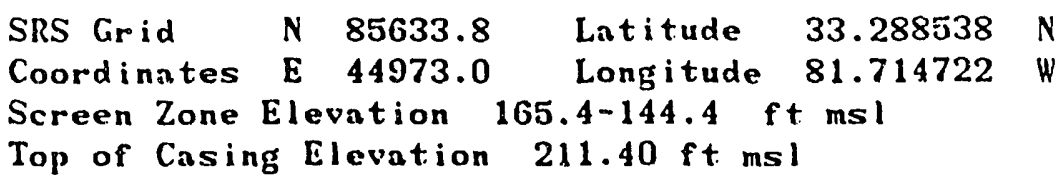

\section{MEASUREMENTS CONDUCTED IN THE FIELD}

Sample date 08/15/91 Time 1055

Depth to water - $50.59 \mathrm{ft}(15.42 \mathrm{~m})$ below the TOC

Water elevation - $160.81 \mathrm{ft}(49.02 \mathrm{~m}) \mathrm{msl}$

$\mathrm{pH}=4.8$ Alkalinity $=0 \mathrm{mg} / \mathrm{L}$

Specif ic conductance - 17 umhos/cm

Water temperature - 18.7 degrees Celsius

Water evacuated from the well prior to sampling - $56 \mathrm{gal}$

\section{LABORATORY ANALYSES}

Specific conductance
pH
Silver
Aluminum
Aldrin
Arsenic
Barium
beta-Benzene hexachloride
Bromodichloromethane
Calcium
Trichlorofluoromethane
Carbon tetrachloride
Cadmium
Bromoform
Chloroform
Dichloromethane (Methylene chloride)
Bromomethane (Methyl bromide)
Chloromethane (Methyl chloride)
Chloride
Chlorobenzene
Chromium
Copper
Chloroethene (Vinyl chloride)
Chlorocthane
Benzene
Dibromochloromethane
Endrin
Endosulfan sulfate
Ethylbenzene
Fluoride
Iron
Mercury
Potassium
Lindane
Tolucne
Methosychlor
Magnesium
NTINeD
Ch

\begin{tabular}{|c|c|c|c|c|}
\hline & $\begin{array}{r}15.00 \\
5.21 \\
2.00\end{array}$ & $\begin{array}{l}\text { umh } / \mathrm{cm} \\
\mathrm{pH}\end{array}$ & Gen. & $\begin{array}{l}\text { Eng } \\
\text { Eng } \\
\text { Eng }\end{array}$ \\
\hline $\begin{array}{l}L T \\
L T\end{array}$ & $\begin{array}{r}2.00 \\
20.00\end{array}$ & $\begin{array}{l}u g / L \\
u g / L\end{array}$ & $\begin{array}{l}\text { Gen. } \\
\text { Gen. }\end{array}$ & $\begin{array}{l}\text { Eng } \\
\text { Eng }\end{array}$ \\
\hline LT & 0.05 & $u g / L$ & Gen. & Eng \\
\hline LT & 2.00 & ug/L & Gen. & Eng \\
\hline LT & 3.00 & $u_{g} / L$ & Gen. & Eng \\
\hline LT & 0.05 & $u g / L$ & Gen. & Eng \\
\hline LT & $\begin{array}{r}1.00 \\
279.00\end{array}$ & $\begin{array}{l}u g / L \\
u g / L\end{array}$ & $\begin{array}{l}\text { Gen. } \\
\text { Gen. }\end{array}$ & $\begin{array}{l}\text { Eng } \\
\text { Eng. }\end{array}$ \\
\hline LI & 1.00 & $u_{g} / L$ & Gen. & Eng \\
\hline LT & 1.00 & $\mu g / L$ & Gen. & Eng \\
\hline LT & 2.00 & ug $/ L$ & Gen. & Eng \\
\hline LT & 1.00 & $\mathbf{u g} / \mathrm{L}$ & Gen. & En \\
\hline LT & 1.00 & ug/L & Gen. & Eng \\
\hline & 11.60 & $u_{g} / L$ & Gen. & Eng \\
\hline LT & 1.00 & ug $/ L$ & Gen. & En \\
\hline$L T$ & 1.00 & ug $/ L$ & Gen. & Eng \\
\hline & 2130.00 & ug $/ L$ & Gen. & Eng \\
\hline LT & 1.00 & $u_{g} / L$ & Gen. & Eng \\
\hline LT & 4.00 & $u g / L$ & Gen. & Eng \\
\hline LT & 4.00 & ug $/ L$ & Gen. & Eng \\
\hline LT & 1.00 & $u g / L$ & Gen. & Eng \\
\hline LT & 1.00 & $\mathbf{u g} / \mathrm{L}$ & Gen. & Eng \\
\hline LT & 1.00 & $u_{g} / L$ & Gen. & Eng \\
\hline LT & 1.00 & $\mathbf{u g} / \mathrm{L}$ & Gen. & En \\
\hline LT & 0.01 & $u g / L$ & Gen. & Eng \\
\hline LT & 0.10 & $u g / L$ & Gen. & En \\
\hline LT & 1.00 & ug $/ L$ & Gen. & EnE \\
\hline LT & 100.00 & $u g / L$ & Gen. & $E_{n}$ \\
\hline & 8.66 & $u_{g} / L$ & Gen. & Eng \\
\hline LT & 0.20 & $\mathbf{u g} / \mathrm{L}$ & Gen. & Eng \\
\hline LT & 500.00 & $\mathbf{u g} / \mathrm{L}$ & Gen. & Eng \\
\hline LT & 0.00 & $u g / L$ & Geñ. & Eng \\
\hline LT & 1.00 & $u_{g} / L$ & Gen. & En \\
\hline LT & 0.50 & ug $/ L$ & Gen. & $E_{n}$ \\
\hline & 355.00 & ug/L & & \\
\hline
\end{tabular}


WELL LFW 33 COLLECTED ON 08/15/91 LABORATORY ANALYSES CONTINUED

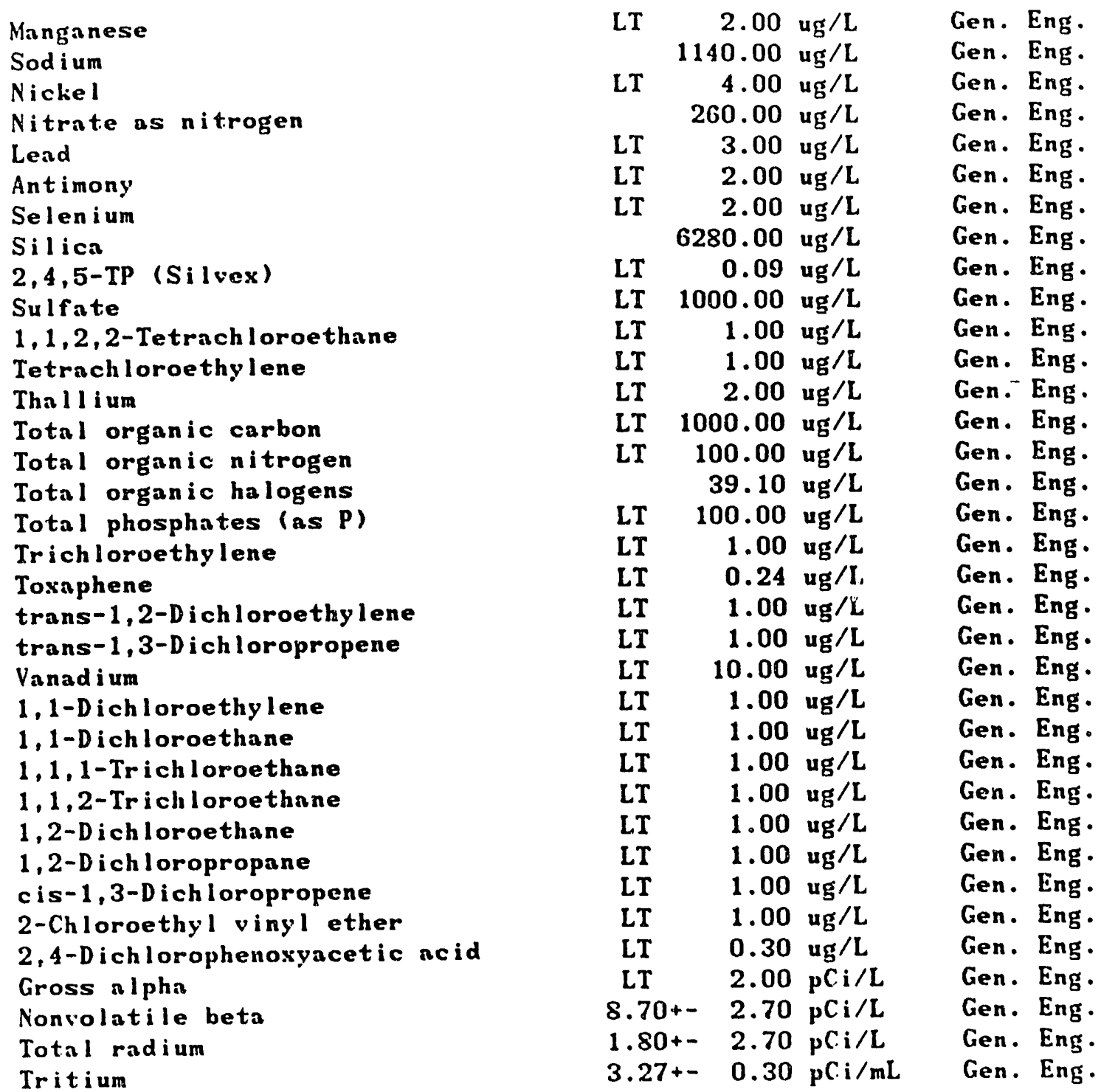


WELL LFW 34

$\begin{array}{llllll}\text { SRS Grid } & N & 85409.5 & \text { Latitude } & 33.288114 & N \\ \text { Coordinates } & E & \mathbf{4 5 0 1 6 . 9} & \text { Longitude } & 81.714171 & W\end{array}$

Screen Zone Elevation 164.7-143.7 ft ms I

Top of Casing Elevation $198.70 \mathrm{ft} \mathrm{msl}$

\section{MEASUREMENTS CONDUCTED IN THE FIELD}

Sample date 08/15/91 Time 1025

Depth to water $-39.08 \mathrm{ft}(11.91 \mathrm{~m})$ below the TOC

Water elevation $-159.62 \mathrm{ft}(48.65 \mathrm{~m}) \mathrm{msl}$

$\mathrm{pH}=4.8 \quad$ Alkalinity $-0 \mathrm{mg} / \mathrm{L}$

Specific conductance - 20 umhos $/ \mathrm{cm}$

Water temperature -18.8 degrees Celsius

Water evacunted from the well prior to sampling - $50 \mathrm{gal}$

\section{LABORATORY ANALYSES}

Specific conductance

pH

Silver

Aluminum

Aldrin

Arsenic

Barium

beta-Benzene hexachloride

Bromodichloromethane

Calcium

Trich lor of luorome thane

Carbon tetrachloride

Cadmi um

Bromoform

Chloroform

Dichloromethane (Methylene chloride)

Bromomethane (Methyl bromide)

Chloromethane (Methyl chloride)

Chloride

Chlorobenzene

Chromium

Copper

Chloroethene (Vinyl chloride)

Chloroethane

Benzene

Dibromoch lorome thane

Endrin

Endosulfan sulfate

Ethylbenzene

Fluoride

Iron

Mercury

Potassium

Lindane

Toluene

Methosychlor

Magnesium

CONTINUED
$20.00 \mathrm{umh} / \mathrm{cm}$

$5.27 \mathrm{pH}$

LT

LT

LI

LT

LT

LT

LT

LT

LT

LT

LT

LT

LT

LT

$2.00 \mathrm{ug} / \mathrm{L}$

$20.00 \mathrm{ug} / \mathrm{L}$

$0.05 \mathrm{ug} / \mathrm{L}$

$2.00 \mathrm{ug} / \mathrm{L}$

$4.59 \mathrm{ug} / \mathrm{L}$

$0.05 \mathrm{ug} / \mathrm{L}$

$1.00 \mathrm{ug} / \mathrm{L}$

$411.00 \mathrm{ug} / \mathrm{L}$

$1.00 \mathrm{ug} / \mathrm{L}$

$1.00 \mathrm{ug} / \mathrm{L}$

$2.00 \mathrm{ug} / \mathrm{L}$

$1.00 \mathrm{ug} / \mathrm{L}$

$1.00 \mathrm{ug} / \mathrm{L}$

$12.00 \mathrm{ug} / \mathrm{L}$

$1.00 \mathrm{ug} / \mathrm{L}$

$1.00 \mathrm{ug} / \mathrm{L}$

$2600.00 \mathrm{ug} / \mathrm{L}$

$1.00 \mathrm{ug} / \mathrm{L}$

LT $4.00 \mathrm{ug} / \mathrm{L}$

LT $4.00 \mathrm{ug} / \mathrm{L}$

LI $1.00 \mathrm{ug} / \mathrm{L}$

LT $1.00 \mathrm{ug} / \mathrm{L}$

LT $1.00 \mathrm{ug} / \mathrm{L}$

LT $1.00 \mathrm{ug} / \mathrm{L}$

LT $0.01 \mathrm{ug} / \mathrm{L}$

LT $0.10 \mathrm{ug} / \mathrm{L}$

LT $1.00 \mathrm{ug} / \mathrm{L}$

LT $100.00 \mathrm{ug} / \mathrm{L}$

$17.10 \mathrm{ug} / \mathrm{L}$

LT

$0.20 \mathrm{ug} / \mathrm{L}$

$517.00 \mathrm{ug} / \mathrm{L}$

$0.00 \mathrm{ug} / \mathrm{L}$

LT

LT

$1.00 \mathrm{ug} / \mathrm{L}$

LT
$0.50 \mathrm{ug} / \mathrm{L}$

$447.00 \mathrm{ug} / \mathrm{L}$
Gen. Eng -

Gen. Eng.

Gen. Eng.

Gen. Eng.

Gen. Eng.

Gen. Eng.

Gen. Eng.

Gen. Eng.

Gen. Eng.

Gen. Eng.

Gen. Eng.

Gen. Eng.

Gen. Eng.

Gen. Eng.

Gen. Eng.

Gen. Eng.

Gen. Eng.

Gen. Eng.

Gen. Eng.

Gen. Eng.

Gen. Eng.

Gen. Eng.

Gen. Eng.

Gen. Eng.

Gen. Eng.

Gen. Eng.

Gen. Eng.

Gen. Eng.

Gen. Eng.

Gen. Eng.

Gen. Eng.

Gen. Eng.

Gen. Eng.

Geñ. Eng .

Gen. Eng.

Gen. Eng.

Gen. Eng. 
WELL LFW 34 COLLECTED ON 08/15/91 LABORATORY ANALYSES CONTINUED

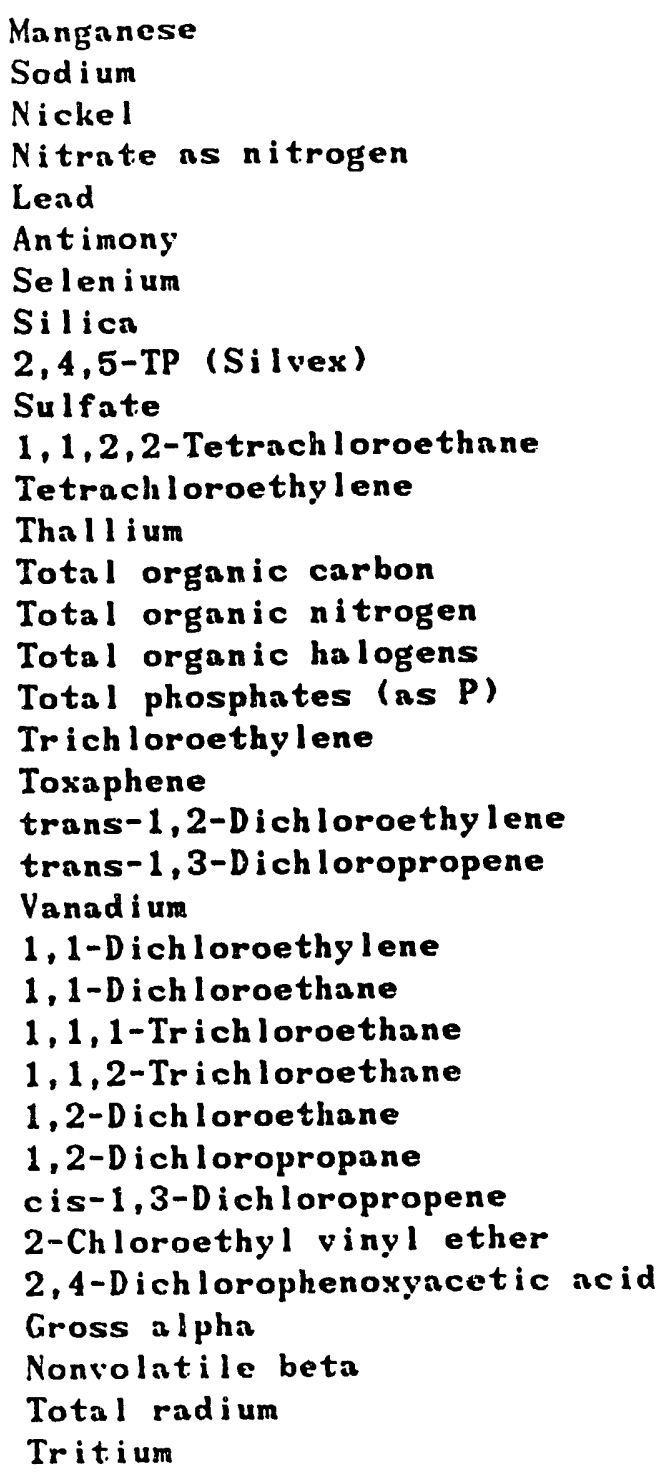

\begin{tabular}{|c|c|c|c|c|}
\hline & 2.15 & $u g / L$ & Gen. & Eng. \\
\hline & 240.00 & ug $/ \mathrm{L}$ & Gen. & Eng. \\
\hline LT & 4.00 & ug $/ \mathrm{L}$ & Gen. & Eng. \\
\hline & 420.00 & ug $/ L$ & Gen. & 1 \\
\hline LT & 3.00 & ug $/ \mathrm{L}$ & Gen. & $\mathrm{nE}_{\mathrm{E}}$ \\
\hline LT & 2.00 & ug $/ \mathrm{L}$ & Gen. & ng \\
\hline LT & 2.00 & ug/L & Gen. & g \\
\hline & 490.00 & ug/L & Gen. & \\
\hline LT & 0.09 & ug/L & Gen. & \\
\hline LT & 000.00 & $\operatorname{ug} / \mathrm{L}$ & ien. & \\
\hline LT & 1.00 & ug $/ \mathrm{L}$ & Gen. & \\
\hline LT & 1.00 & ug $/ L$ & ien. & \\
\hline LT & 2.00 & $u_{g} / L$ & en. & \\
\hline LT & 000.00 & ug/L & Gen. & \\
\hline LT & 100.00 & $\mathbf{u g} / \mathrm{L}$ & en. & \\
\hline & 14.50 & ug $/ L$ & Gen. & \\
\hline LT & 100.00 & ug $/ \mathrm{L}$ & en. & 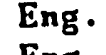 \\
\hline LT & 1.00 & ug/L & en. & \\
\hline LT & 0.24 & ug $/ L$ & en. & \\
\hline LT & 1.00 & ug $/ L$ & en. & \\
\hline LI & 1.00 & ug/L & en. & \\
\hline LT & 10.00 & ug/L & en. & n \\
\hline LT & 1.00 & ug/L & en. & n \\
\hline LT & 1.00 & ug $/ \mathrm{L}$ & en. & $\mathbf{n}$ \\
\hline LT & 1.00 & $\mathrm{ug} / \mathrm{L}$ & en. & $n_{\mathbf{g}}$ \\
\hline LT & 1.00 & ug/L & ien. & E \\
\hline LI & 1.00 & ug/L & en. & E \\
\hline L.T & 1.00 & ug $/ \mathrm{L}$ & ien. & E \\
\hline LT & 1.00 & ug/L & Gen. & \\
\hline LT & 1.00 & ug/L & Gen. & \\
\hline LT & 0.30 & ug/L & Gen. & \\
\hline LT & 2.00 & $\mathrm{pC} i / \mathrm{L}$ & ien. & \\
\hline $.00+-$ & 2.10 & $p \mathrm{Ci} / \mathrm{L}$ & ian & \\
\hline 1.8 & 3.70 & $\mathrm{p} \mathrm{C}_{\mathrm{i}} / \mathrm{L}$ & & \\
\hline $3.29+-$ & 0.30 & $p C i / m L$ & Gen. & \\
\hline
\end{tabular}


WELL LFW 35

$\begin{array}{llllll}\text { SRS Grid } & N & 85237.4 & \text { Latitude } & 33.288325 & N \\ \text { Coordinates } & E & 45378.8 & \text { Longitude } & 81.712883 & W\end{array}$

Screen Zone Elevation 164.4-143.4 ft msl

Top of Casing Elevation $181.40 \mathrm{ft} \mathrm{msl}$

\section{MEASUREMENTS CONDUCTED IN THE FIELD}

Sample date 08/15/91 Time 1000

Depth to water $=22.42 \mathrm{ft}(6.83 \mathrm{~m})$ below the TOC

Water elevation - $158.98 \mathrm{ft}(48.46 \mathrm{~m}) \mathrm{ms}$ l

pll $=4.8$ Alkalinity $=0 \mathrm{mg} / \mathrm{L}$

Specific conductance - 22 umhos/cm

Water temperature - 20.0 degrees Celsius

Water evacuated from the well prior to sampling - $49 \mathrm{gal}$

\section{LABORATORY ANALYSES}

Specific conductance

pH

Silver

Aluminum

Aldrin

Arsenic

Barium

beta-Benzene hexachloride

Bromodichloromethane

Calcium

Trichlorof luoromethane

Carbon tetrachloride

Cadmi um

Bromoform

Chloroform

Dichloromethane (Methylene chloride)

Bromomethane (Methyl bromide)

Chloromethane (Methyl chloride)

Chloride

Chlorobenzene

Chromium

Copper

Chloroethene (Vinyl chloride)

Chloroethane

Benzene

Dibromochlorome thane

Endrin

Endosulfan sulfate

Ethylbenzene

Fluoride

Iron

Mercury

Potassium

Lindane

Toluene

Methoxychlor

Magnesium

CONTINUED
$25.00 \mathrm{umh} / \mathrm{cm}$

$5.15 \mathrm{pH}$

LT

LT

LT

LT

LT

LT

LT

LT

LI

LT

LT

LT

LT

LT

LT

LT

LT

LT

LT

LT

LT

LT

LT

LT

LT

LT

LT

LT

LT

$2.00 \mathrm{ug} / \mathrm{L}$

20.00 ug/L

$0.05 \mathrm{ug} / \mathrm{L}$

$2.00 \mathrm{ug} / \mathrm{L}$

$5.26 \mathrm{ug} / \mathrm{L}$

$0.05 \mathrm{ug} / \mathrm{L}$

$1.00 \mathrm{ug} / \mathrm{L}$

$332.00 \mathrm{ug} / \mathrm{L}$

$1.00 \mathrm{ug} / \mathrm{L}$

$1.00 \mathrm{ug} / \mathrm{L}$

$2.00 \mathrm{ug} / \mathrm{L}$

$-1.00 \mathrm{ug} / \mathrm{L}$

$1.00 \mathrm{ug} / \mathrm{L}$

$12.00 \mathrm{ug} / \mathrm{L}$

$1.00 \mathrm{ug} / \mathrm{L}$

$1.00 \mathrm{ug} / \mathrm{L}$

$2830.00 \mathrm{ug} / \mathrm{L}$

$1.00 \mathrm{ug} / \mathrm{L}$

$4.00 \mathrm{ug} / \mathrm{L}$

$4.00 \mathrm{ug} / \mathrm{L}$

$1.00 \mathrm{ug} / \mathrm{L}$

$1.00 \mathrm{ug} / \mathrm{L}$

$1.00 \mathrm{ug} / \mathrm{L}$

$1.00 \mathrm{ug} / \mathrm{L}$

$0.01 \mathrm{ug} / \mathrm{L}$

$0.10 \mathrm{ug} / \mathrm{L}^{\circ}$

$1.00 \mathrm{ug} / \mathrm{L}$

$100.00 \mathrm{ug} / \mathrm{L}$

$11.20 \mathrm{ug} / \mathrm{L}$

$0.20 \mathrm{ug} / \mathrm{L}$

$500.00 \mathrm{ug} / \mathrm{L}$

$0.00 \mathrm{ug} / \mathrm{L}$

$1.00 \mathrm{ug} / \mathrm{L}$

$0.50 \mathrm{ug} / \mathrm{L}$

$576.00 \mathrm{ug} / \mathrm{L}$
Gen. Eng.

Gen. Eng.

Gen. Eng.

Gen. Eng.

Gen. Eng.

Gen. Eng.

Gen. Eng.

Gen. Eng.

Gen. Eng .

Gen. Eng.

Gen. Eng.

Gen. Eng.

Gen. Eng.

Gen. Eng.

Gen. Eng.

Gen. Eng.

Gen. Eng.

Gen. Eng.

Gen. Eng.

Gen. Eng.

Gen. Eng.

Gen. Eng.

Gen. Eng.

Gen. Eng.

Gen. Eng.

Gen. Eng.

Gen. Eng.

Gen. Eng.

Gen. Eng.

Gen. Eng .

Gen. Eng.

Gen. Eng.

Gen. Eng.

Geñ. Eng.

Gen. Eng.

Gen. Eng.

Gen. Eng. 
WELL LFW 35 COLLECTED ON 08/15/91 LABORATORY ANALYSES CONTINUED

\author{
Manganese \\ Sod i um \\ Nickel \\ Nitrate as nitrogen \\ Lead \\ Antimouy \\ Selen ium \\ Silica \\ 2,4,5-TP (Silvex) \\ Sulfate \\ 1, 1,2,2-Tetrachloroethane \\ Tetrachloroethylene \\ Thallium \\ Total organic carbon \\ Total organic nitrogen \\ Total organic halogens \\ Total phosphates (as P) \\ Trichloroethylene \\ Toxaphere \\ trans-1,2-D ich loroethyl ene \\ trans-1,3-D ich loropropene \\ Vanadi um \\ 1,1-Dichloroethylene \\ 1,1-Dichloroethane \\ 1,1,1-Trichloroethane \\ 1,1,2-Trichloroethane \\ 1,2-Dichloroethane \\ 1,2-D ich loropropane \\ cis-1,3-D ichloropropene \\ 2-Chloroethyl vinyl ether \\ 2,4-Dichlorophenoxyacetic acid \\ Gross alpha \\ Nonvolatile beta \\ Total radium \\ Tritium \\ Tritium
}

$\begin{aligned} 2.78 \mathrm{ug} / \mathrm{L} & \text { Gen. Eng. } \\ 2550.00 \mathrm{ug} / \mathrm{L} & \text { Gen. Eng. } \\ 4.00 \mathrm{ug} / \mathrm{L} & \text { Gen. Eng. } \\ 1320.00 \mathrm{ug} / \mathrm{L} & \text { Gen. Eng. } \\ 3.00 \mathrm{ug} / \mathrm{L} & \text { Gen. Eng. } \\ 2.00 \mathrm{ug} / \mathrm{L} & \text { Gen. Eng. } \\ 2.00 \mathrm{ug} / \mathrm{L} & \text { Gen. Eng. } \\ 6760.00 \mathrm{ug} / \mathrm{L} & \text { Gen. Eng. }\end{aligned}$

$\begin{array}{lrl}\text { LT } & 0.09 \mathrm{ug} / \mathrm{L} & \text { Gen. Eng. } \\ \text { LT } & 1000.00 \mathrm{ug} / \mathrm{L} & \text { Gen. Eng. }\end{array}$

LT $1.00 \mathrm{ug} / \mathrm{L}$ Gen. Eng.

LI $1.00 \mathrm{ug} / \mathrm{L}$ Gen. Eng.

LT $2.00 \mathrm{ug} / \mathrm{L}$ Gen.-Eng.

LT $1000.00 \mathrm{ug} / \mathrm{L}$ Gen. Eng.

LT $100.00 \mathrm{ug} / \mathrm{L}$ Gen. Eng.

6.70 ug/L Gen. Eng.

LT $100.00 \mathrm{ug} / \mathrm{L}$ Gen. Eng.

LT $1.00 \mathrm{ug} / \mathrm{L}$ Gen. Eng.

LT $0.24 \mathrm{ug} / \mathrm{L}$ Gen. Eng.

LT $1.00 \mathrm{ug} / \mathrm{L}$ Gen. Eng.

LT $1.00 \mathrm{ug} / \mathrm{L}$ Gen. Eng.

LT $10.00 \mathrm{ug} / \mathrm{L}$ Gen. Eng.

LT $1.00 \mathrm{ug} / \mathrm{L}$ Gen. Eng.

LT $1.00 \mathrm{ug} / \mathrm{L}$ Gen. Eng.

LT $1.00 \mathrm{ug} / \mathrm{L}$ Gen. Eng.

LT $1.00 \mathrm{ug} / \mathrm{L}$ Gen. Eng.

LT $1.00 \mathrm{ug} / \mathrm{L}$ Gen. Eng.

LT $1.00 \mathrm{ug} / \mathrm{L}$ Gen. Eng.

LT $1.00 \mathrm{ug} / \mathrm{L}$ Gen. Eng.

LT $1.00 \mathrm{ug} / \mathrm{L}$ Gen. Eng.

LT $0.30 \mathrm{ug} / \mathrm{L}$ Gen. Eng.

LT $2.00 \mathrm{pCi} / \mathrm{L}$ Gen. Eng.

$2.50+-2.10 \mathrm{pCi} / \mathrm{L}$ - Gen. Eng.

2.30+- $2.90 \mathrm{pCi} / \mathrm{L}$ Gen. Eng.

$2.41+-0.20 \mathrm{pCi} / \mathrm{mL}$ Gen. Eng.

$1.97+-0.20 \mathrm{pCi} / \mathrm{mL}$ Gen. Eng. 
WELL LFH' 36

$\begin{array}{llllll}\text { SRS Grid } & N & 83535.3 & \text { Latitude } & 33.284894 & N \\ \text { Coordinates } & E & 45582.3 & \text { Longitude } & 81.709040 & W\end{array}$

Screen Zone Elevation 151.3-130.3 ft msl

Top of Casing Elevation $168.30 \mathrm{ft} \mathrm{msl}$

MEASUREMENTS CONDUCTED IN THE FIELD

Sample date 08/15/91 Time 1605

Depth to water $=22.44 \mathrm{ft}(6.84 \mathrm{~m})$ below the TOC

Water elevation - $145.86 \mathrm{ft}(44.46 \mathrm{~m}) \mathrm{msl}$

$\mathrm{pH}=5.8 \quad$ Alkalinity $-43 \mathrm{mg} / \mathrm{L}$

Specific conductance - 158 umhos/cm

Water temperature - 20.9 degrees Celsius

Woter evacuated from the well prior to sampling - $61 \mathrm{gal}$

\section{LABORATORY ANALYSES}

Specif ic conductance

Carbon 12-labeled 2,3,7,8-TCDD

Carbon 12-labeled TCDF

Heptachlorodibenzo-p-dioxin isomers

Heptachlorodibenzo-p-furan isomers

Hexachlorodibenzo-p-dioxin isomers

Hexachlorodibenzo-p-furan isomers

Octachlorodibenzo-p-dioxin isomers

Octachlorodibenzo-p-furan isomers

Pentachlorodibenzo-p-dioxin isomers

Pentachlorodibenzo-p-furan isomers

pH

Tetrachlorodibenzo-p-dioxin isomers

Tetrachlorodibenzo-p-furan isomers

$1,2,3,4,6,7,8-$ HIPCDD

$1,2,3,4,6,7,8$-HIIPCDF

$1,2,3,4,7,8$-Hesachlorodibenzo-p-dios in LT

$1,2,3,4,7,8$-llexachlorodibenzo-p-furan LT

$1,2,3,7,8$-Pentachlorodibenzo-p-dioxin LT

$1,2,3,7,8$-Pentachlorodibenzo-p-ruran LT

$a, a-D$ imetlyy lphenethylamine

alpha-Benzene hexachloride

Acetone

Acetophenone

Acrolein

Acrylonitrile

Silver

Aluminum

Aldrin

Acenaphthene

Acenaphthylene

Aniline

Anthracene

Aramite

Arsenic

Allyl chloride

Barium

CONTINUED
LI

$180.00 \mathrm{umh} / \mathrm{cm}$

$0.45 \mathrm{ng} / \mathrm{L}$

$0.40 \mathrm{ng} / \mathrm{L}$

$0.65 \mathrm{ng} / \mathrm{L}$

$0.45 \mathrm{ng} / \mathrm{L}$

$0.45 \mathrm{ng} / \mathrm{L}$

$0.40 \mathrm{ng} / \mathrm{L}$

$1.00 \mathrm{ng} / \mathrm{L}$

$1.00 \mathrm{ng} / \mathrm{L}$

$0.55 \mathrm{ng} / \mathrm{L}$

$0.55 \mathrm{ng} / \mathrm{L}$

$6.25 \mathrm{pH}$

LT $0.45 \mathrm{ng} / \mathrm{L}$

LT $0.40 \mathrm{ng} / \mathrm{L}$

$0.65 \mathrm{ng} / \mathrm{L}$

$0.45 \mathrm{ng} / \mathrm{L}$

$0.45 \mathrm{ng} / \mathrm{L}$

$0.40 \mathrm{ng} / \mathrm{L}$

$0.55 \mathrm{ng} / \mathrm{L}$

$0.55 \mathrm{ng} / \mathrm{L}$

$10.00 \mathrm{ug} / \mathrm{L}$

$0.05 \mathrm{ug} / \mathrm{L}$

$10.00 \mathrm{ug} / \mathrm{L}$

LT

LI

LT

LT

LT

LT

LT

LT

LT

LT

LT

LT

LT
$10.00 \mathrm{ug} / \mathrm{L}$

$20.00 \mathrm{ug} / \mathrm{L}$

$20.00 \mathrm{ug} / \mathrm{L}$

$2.00 \mathrm{ug} / \mathrm{L}$

$20.00 \mathrm{ug} / \mathrm{L}$

$0.05 \mathrm{ug} / \mathrm{L}$

$10.00 \mathrm{ug} / \mathrm{L}$

$10.00 \mathrm{ug} / \mathrm{L}$

$10.00 \mathrm{ug} / \mathrm{L}$

$10.00 \mathrm{ug} / \mathrm{L}$

$10.00 \mathrm{ug} / \mathrm{L}$

$2.00 \mathrm{ug} / \mathrm{L}$

$50.00 \mathrm{ug} / \mathrm{L}$

$16.40 \mathrm{ug} / \mathrm{L}$
Gen. Eng -

Gen. Eng.

Gen. Eng.

Gen. Eng.

Gen. Eng.

Gen. Eng.

Gen. Eng.

Gen. Eng.

Gen. Eng.

Gen. Eng.

Gen. Eng.

Gen. Eng.

Gen. Eng.

Gen. Eng.

Gen. Eng.

Gen. Eng.

Gen. Eng.

Gen. Eng.

Gen. Eng.

Gen. Eng.

Gen. Eng.

Gen. Eng.

Gen. Eng.

Gen. Eng.

Gen. Eng .

Gen. Eng.

Gen. Eng.

Gen. Eng.

Gen. Eng.

Gen. Eng.

Gen. Eng.

Gen. Eng.

Gen. Eng.

Geñ. Eng.

Gen. Eng.

Gen. Eng.

Gen. Eng . 


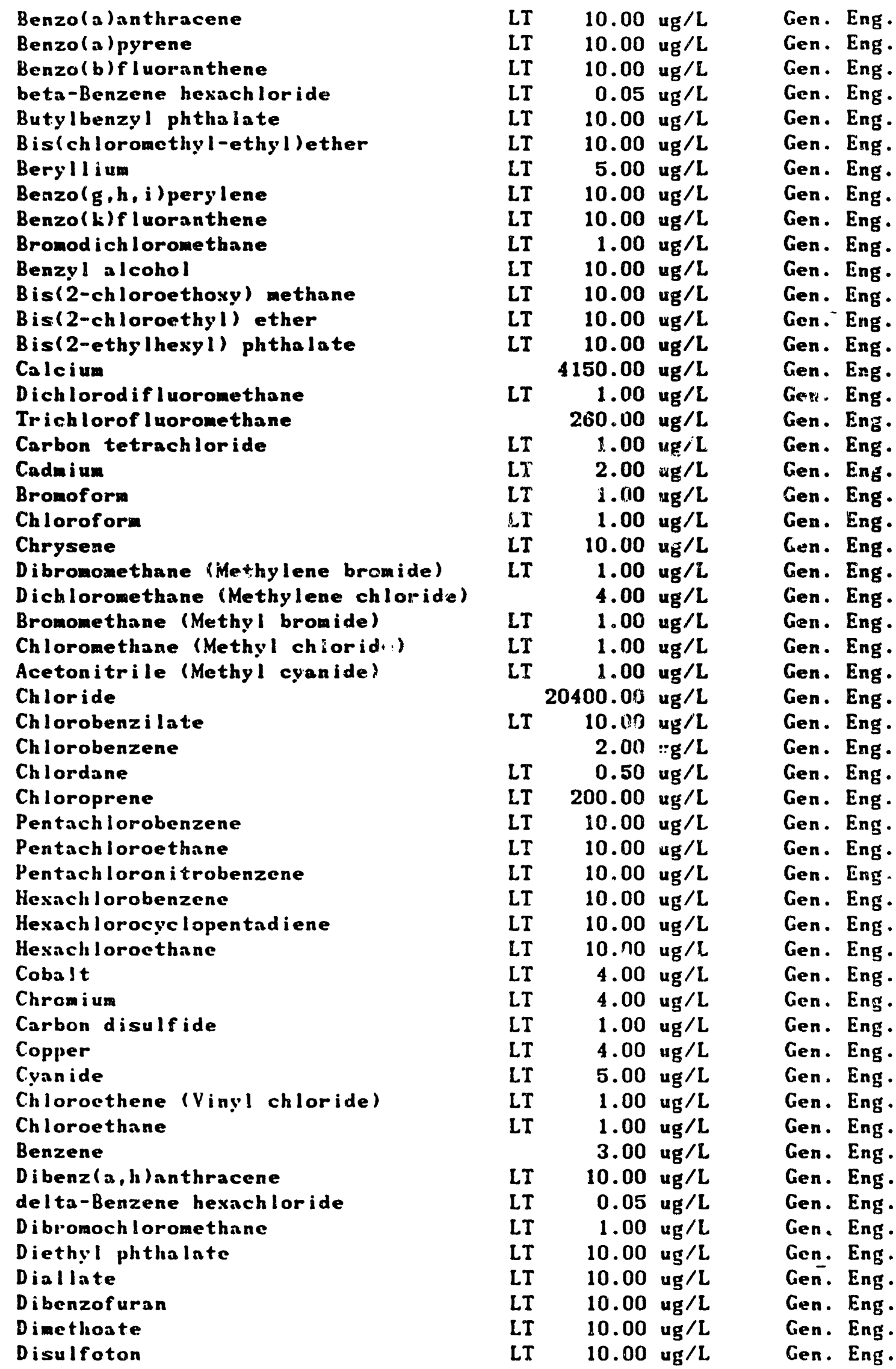


Dieldrin

Dimethyl phthalate

Di-n-butyl phthalate

Di-n-octyl phthalate

Diphenylamine

Ethyl methanesulfonate

Endrin aldehyde

Endrin

Endosulfan I

Endosulfan II

Endosulfan sulfate

Ethylbenzene

Ethyl methacrylate

Fluoride

Fluoride

Famphur

Fluoranthene

Iron

Fluorene

Hexach lorobutadiene

Hexachlorophene

Hexach loropropene

Mercury

Heptachlor

Heptachlor epoxide

Indeno(1,2,3-c,d)pyrene

Iodomethane (Methyl iodide)

Isosafrole

Isobutyl alcohol

Isadrin

Isophorone

Potassium

Kepone

Lindane

Methacrylonitrile

Toluene

Methyl ethyl ketone

Methyl methacrylate

Methyl methanesulfonate

Methoxychlor

Magnes ium

Methyl isobutyl ketone

Manganese

Methapyri lene

Sod i um

Naphthalene

Nitrobenzene

$N$-Nitrosodimethylamine

$N-N i$ trosod $i-n-b u t y l a m i n e$

$N-N$ i trosodi-propylamine

$N-N i$ trosodiethylamine

$\mathrm{N}-\mathrm{Nitrosomethylethylamine}$

$\mathrm{Nickel}$

N-N:t-osomorpho! I:
LI

LT

LT

LT

LI

LT

LT

LT

LT

LI

LT

LI

LT

LI

LT

LT

LT

LT

LT

\section{LT}

LT

LT

LT

LT

LT

LT

LT

LT

LT

LI

LI

LT

LT

LI

LT

LT

LT

LT

LT

LT

LT
LT

LT

LI

LI

LT

LI

LI

I.
$0.50 \mathrm{ug} / \mathrm{L}$

$10.00 \mathrm{ug} / \mathrm{L}$

$10.00 \mathrm{ug} / \mathrm{L}$

$10.00 \mathrm{ug} / \mathrm{L}$

$10.00 \mathrm{ug} / \mathrm{L}$

$10.00 \mathrm{ug} / \mathrm{L}$

$0.10 \mathrm{\mu g} / \mathrm{L}$

$0.01 \mathrm{ug} / \mathrm{L}$

$0.10 \mathrm{ug} / \mathrm{L}$

$0.10 \mathrm{ug} / \mathrm{L}$

$0.10 \mathrm{ug} / \mathrm{L}$

$1.00 \mathrm{ug} / \mathrm{L}$

$10.00 \mathrm{ug} / \mathrm{L}$

$100.00 \mathrm{ug} / \mathrm{L}$

$100.00 \mathrm{ug} / \mathrm{L}$

$10.00 \mathrm{ug} / \mathrm{L}$

$10.00 \mathrm{ug} / \mathrm{L}$

$1100.00 \mathrm{ug} / \mathrm{L}$

$10.00 \mathrm{ug} / \mathrm{L}$

$10.00 \mathrm{ug} / \mathrm{L}$

$10.00 \mathrm{ug} / \mathrm{L}$

$10.00 \mathrm{ug} / \mathrm{L}$

$0.20 \mathrm{ug} / \mathrm{L}$

$0.05 \mathrm{ug} / \mathrm{L}$

$0.05 \mathrm{ug} / \mathrm{L}$

$10.00 \mathrm{ug} / \mathrm{L}$

$15.00 \mathrm{ug} / \mathrm{L}$

$10.00 \mathrm{ug} / \mathrm{L}$

$100.00 \mathrm{ug} / \mathrm{L}$

$10.00 \mathrm{ug} / \mathrm{L}$

$10.00 \mathrm{ug} / \mathrm{L}$ $692.00 \mathrm{ug} / \mathrm{L}$

$10.00 \mathrm{ug} / \mathrm{L}$

$0.00 \mathrm{ug} / \mathrm{L}$

$50.00 \mathrm{ug} / \mathrm{L}$

$.1 .00 \mathrm{ug} / \mathrm{L}$

$1.00 \mathrm{ug} / \mathrm{L}$

$10.00 \mathrm{ug} / \mathrm{L}$

$10.00 \mathrm{ug} / \mathrm{L}$

$0.50 \mathrm{ug} / \mathrm{L}$

$8310.00 \mathrm{ug} / \mathrm{L}$

$1.00 \mathrm{ug} / \mathrm{L}$

$12.10 \mathrm{ug} / \mathrm{L}$

$10.00 \mathrm{ug} / \mathrm{L}$

$17100.00 \mathrm{ug} / \mathrm{L}$

$10.00 \mathrm{ug} / \mathrm{L}$

$10.00 \mathrm{ug} / \mathrm{L}$

$10.00 \mathrm{ug} / \mathrm{L}$

$10.00 \mathrm{ug} / \mathrm{L}$

$10.00 \mathrm{ug} / \mathrm{L}$

$10.00 \mathrm{ug} / \mathrm{L}$

$10.00 \mathrm{ug} / \mathrm{L}$

$4.00 \mathrm{\mu g} / \mathrm{L}$

$10.00 \mathrm{ug} / \mathrm{I}$.
Gen. Eng.

Ger. Eng.

Gen. Eng.

Gen. Eng.

Gen. Eng.

Gen. Eng.

Gen. Eng.

Gen. Eng.

Gen. Eng.

Gen. Eng.

Gen. Eng.

Gen. Eng -

Gen.- Eng.

Gen. Eng.

Gen. Eng.

Gen. Eng.

Gen. Eng.

Gen. Eng.

Gen. Eng.

Gen. Eng.

Gen. Eng.

Gen. Eng.

Gen. Eng.

Gen. Eng.

Gen. Eng.

Gen. Eng.

Gen. Eng.

Gen. Eng.

Gen. Eng -

Gen. Eng.

Gen. Eng.

Gen. Eng .

Gen. Eng.

Gen. Eng.

Gen. Eng.

Gen. Eng.

Gen. Eng.

Gen. Eng.

Gen. Eng -

Gen. Eng.

Gen. Eng.

Gen. Eng.

Gen. Eng.

Gen. Eng.

Gen. Eng.

Gen. Eng.

Gen. Eng .

Gen. Eng.

Gen. Eng.

Gen. Eng.

Geñ. Eng.

Gen. Eng.

Gen. Eng.

Gen. Eng. 


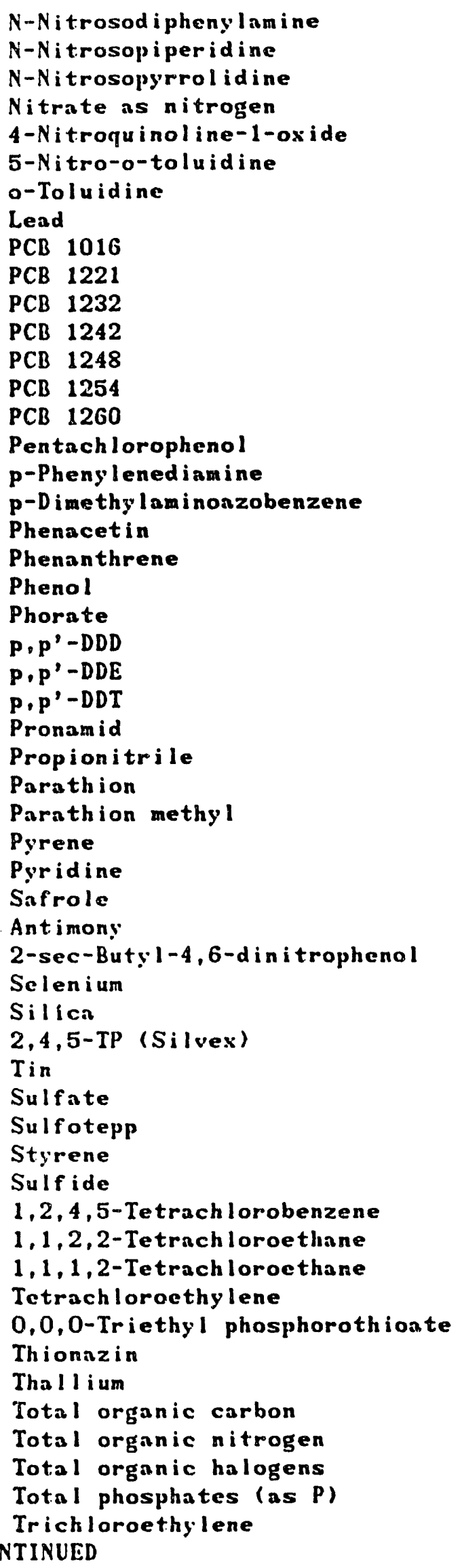

\begin{tabular}{|c|c|c|c|c|}
\hline LT & 10.00 & $\mathrm{ug} / \mathrm{L}$ & Gen. & Eng. \\
\hline LT & 10.00 & $u_{g} / L$ & Gen. & Eng. \\
\hline LI & 10.00 & $\mathrm{ug} / \mathrm{L}$ & Gen. & Eng. \\
\hline$L T$ & 50.00 & $u_{g} / L$ & Gen. & Eng \\
\hline $\mathbf{L T}$ & 10.00 & $\operatorname{ug} / \mathrm{L}$ & Gen. & Eng. \\
\hline $\mathbf{L T}$ & 10.00 & ug $/ \mathrm{L}$ & Gen. & Eng \\
\hline LT & 10.00 & $u g / L$ & Gen. & Eng. \\
\hline LT & 3.00 & $u_{g} / L$ & Gen. & Eng. \\
\hline$L x$ & 0.50 & $u_{g} / L$ & Gen. & Eng. \\
\hline LT & 0.50 & $u_{g} / L$ & Gen. & Eng . \\
\hline L.T & 0.50 & ug/L & Gen. & Eng. \\
\hline LT & 0.50 & ug/L & Gen. & Eng. \\
\hline LT & 0.50 & ug $/ L$ & Gen. & Eng. \\
\hline LT & 1.00 & $\mathrm{ug} / \mathrm{L}$ & Gen. & Eng. \\
\hline LI & 1.00 & ug $/ L$ & Gen. & Eng . \\
\hline LT & 10.00 & $u g / L$ & Gen. & Eng • \\
\hline LT & 10.00 & $u g / L$ & Gen. & Eng. \\
\hline$L T$ & 10.00 & ug $/ L$ & Gea. & Eng. \\
\hline $\mathbf{L T}$ & 10.00 & $u_{g} / L$ & Gen. & Eng. \\
\hline $\mathbf{L T}$ & 10.00 & $u_{g} / L$ & Gen. & Eng. \\
\hline $\mathbf{L T}$ & 10.00 & $u g / L$ & Gen. & Eng. \\
\hline LT & 0.10 & ug $/ L$ & Gen. & Eng. \\
\hline LT & 0.10 & $u_{g} / L$ & Gen. & Eng. \\
\hline LT & 0.10 & $u_{g} / L$ & Gen. & Eng. \\
\hline $\mathrm{LT}$ & 0.10 & $u_{g} / L$ & Gen. & Eng. \\
\hline LI & 10.00 & $\operatorname{ug} / \mathrm{L}$ & Gen. & Eng - \\
\hline$L T$ & 200.00 & ug/L & Gen. & Eng. \\
\hline$L T$ & 0.05 & ug/L & Gen. & Eng . \\
\hline LT & 0.05 & $u_{g} / L$ & Gen. & Eng. \\
\hline LT & 10.00 & ug/L & Gen. & Eng. \\
\hline LT & 10.00 & ug $/ L$ & Gen. & Eng. \\
\hline L.T & 10.00 & ug $/ L$ & Gen. & Eng. \\
\hline L.T & 2.00 & $u_{g} / L$ & Gen. & Eng . \\
\hline LT & 10.00 & ug/L & Gen. & Eng. \\
\hline LT & 2.00 & ug $/ L$ & Gen. & Eng. \\
\hline & 8130.00 & $u_{g} / L$ & Gen. & Eng. \\
\hline LT & 0.09 & $\mathbf{u g} / \mathrm{L}$ & Gen. & Eng. \\
\hline LT & 2.00 & $u g / L$ & Gen. & Eng. \\
\hline & 2670.00 & $u g / L$ & Gen. & Eng. \\
\hline LI & 10.00 & $u g / L$ & Gen. & Eng. \\
\hline LT & 1.00 & $u g / L$ & Gen. & Eng. \\
\hline LT & 1000.00 & $u_{g} / L$ & Gen. & Eng. \\
\hline LT & 10.00 & ug $/ L$ & Gen. & Eng. \\
\hline LT & 1.00 & ug/L & & Eng. \\
\hline LT & 1.00 & $u_{g} / L$ & Gen. & Eng. \\
\hline & 1.40 & $\mathrm{ug} / \mathrm{L}$ & Ger & Eng. \\
\hline$L T$ & 10.00 & $u g / L$ & Gen. & Eng. \\
\hline $\mathbf{L T}$ & 10.00 & $u g / L$ & Gen. & Eng. \\
\hline LI & 2.00 & $\mathrm{ug} / \mathrm{L}$ & & Eng. \\
\hline & 16000.00 & $u g / L$ & Gen. & Eng. \\
\hline LT & 100.00 & $u g / L$ & Geñ. & Eng. \\
\hline & 343.00 & $u g / L$ & & Eng. \\
\hline LT & $\begin{array}{r}100.00 \\
1.30\end{array}$ & $\begin{array}{l}u g / L \\
u g / L\end{array}$ & $\begin{array}{l}\text { Ge } \\
\text { Ge }\end{array}$ & Eng. \\
\hline
\end{tabular}


Toxaphene

trans-1,2-D ichloroethylene

trans-1,3-D ich loropropene

trans-1,4-D ichloro-2-butene

Vanadium

$V$ inyl acetate

Xylenes

1-Naphthylamine

1,1-Dichloroethylene

1,1-Dichloroethane

1,1,1-Trich loroethane

1,1,2-Trich loroethane

1,2-Dibromoethane

1, 2-D ibromo-3-ch loropropane

1,2-Dich lorobenzene

1,2-Dichloroethane

1,2-Dich loropropane

1,2,3-Tr ich loropropane

1,2,4-Trich lorobenzene

1,3-Dichlorobenzene

cis-1,3-Dich loropropene

1,3-Dinitrobenzene

$1,3,5-$ Tr in i trobenzene

1,4-D ich lorobenzene

1,4-Dioxane

1,4-Naphthoquinone

2-Acety lam i nof l uorene

2-Chloroethyl vinyl ether

2-Ch lorophenol

2-Chloronaphthalene

2-Hexanone

2-Methylnaphthalene

o-Cresol (.2-Methylphenol)

2-Nitroaniline

2-Naphthylamine

2-Nitrophenol

2-Picoline

2,3,4,6-Tetrachlorophenol

2,4-Dichlorophenosyacetic acid

2,4-Dich lorophenol

2,4-Dimethyl plienol

2, 4-Dinitrophenol

2,4-Din i trotoluene

2,4,5-Trichlorophenoxyacetic ncid

2,4,5-Trichlorophenol

2,4,6-Trich lorophenol

2,6-Dichlorophenol

2,6-Dinitrotoluene

3-Methylcholanthrene

m-Cresol (3-Methylphenol)

3-Nitroaniline

3, 3'-D ichlorobenzidine

3, 3'-Dimethy l benz id ine

4-Ami nobipheny!

cồTIVive
LT

LT

LT

LT

LT

LT

LT

LT

LT

LT

LI

LT

LT

LT

LT

LT

LT

LT

LT

LT

LT

LT

LT

LI

LI

LT

LT

LT

LT

LT

LT

LT

LT

LT

LT

LT

LT

LT

LI

LT

LT

LT

LT

LT

LT

LT

LT

LT

LT

LT

LT
$0.24 \mathrm{ug} / \mathrm{L}$

$1.00 \mathrm{ug} / \mathrm{L}$

$1.00 \mathrm{ug} / \mathrm{L}$

$30.00 \mathrm{ug} / \mathrm{L}$

$10.00 \mathrm{ug} / \mathrm{L}$

$1.00 \mathrm{ug} / \mathrm{L}$

$1.00 \mathrm{ug} / \mathrm{L}$

$10.00 \mathrm{ug} / \mathrm{L}$

$1.00 \mathrm{ug} / \mathrm{L}$

$46.00 \mathrm{ug} / \mathrm{L}$

$11.00 \mathrm{ug} / \mathrm{L}$

$1.00 \mathrm{ug} / \mathrm{L}$

$20.00 \mathrm{ug} / \mathrm{L}$

$1.00 \mathrm{ug} / \mathrm{L}$

$10.00 \mathrm{ug} / \mathrm{L}$

$3.00 \mathrm{ug} / \mathrm{L}$

$1.00 \mathrm{ug} / \mathrm{L}$

$20.00 \mathrm{ug} / \mathrm{L}$

$10.00 \mathrm{ug} / \mathrm{L}$

$10.00 \mathrm{ug} / \mathrm{L}$

$1.00 \mathrm{ug} / \mathrm{L}$

$10.00 \mathrm{ug} / \mathrm{L}$

$10.00 \mathrm{ug} / \mathrm{L}$

$10.00 \mathrm{ug} / \mathrm{L}$

$10.00 \mathrm{ug} / \mathrm{L}$

$10.00 \mathrm{ug} / \mathrm{L}$

$10.00 \mathrm{ug} / \mathrm{L}$

$10.00 \mathrm{ug} / \mathrm{L}$

$10.00 \mathrm{ug} / \mathrm{L}$

$10.00 \mathrm{ug} / \mathrm{L}$

$1.00 \mathrm{ug} / \mathrm{L}$

$10.00 \mathrm{ug} / \mathrm{L}$

$10.00 \mathrm{ug} / \mathrm{L}$

$10.00 \mathrm{ug} / \mathrm{L}$

$10.00 \mathrm{ug} / \mathrm{L}$

$10.00 \mathrm{ug} / \mathrm{L}$

$10.00 \mathrm{ug} / \mathrm{L}$

$10.00 \mathrm{ug} / \mathrm{L}$

0.30 ug/L

10.00 ug/L

$10.00 \mathrm{ug} / \mathrm{L}$

$45.00 \mathrm{ug} / \mathrm{L}$

$10.00 \mathrm{ug} / \mathrm{L}$

$0.09 \mathrm{ug} / \mathrm{L}$

$10.00 \mathrm{ug} / \mathrm{L}$

$10.00 \mathrm{ug} / \mathrm{L}$

$10.00 \mathrm{ug} / \mathrm{L}$

$10.00 \mathrm{ug} / \mathrm{L}$

$10.00 \mathrm{ug} / \mathrm{L}$

$10.00 \mathrm{ug} / \mathrm{L}$

$10.00 \mathrm{ug} / \mathrm{L}$

$20.00 \mathrm{ug} / \mathrm{L}$

$10.00 \mathrm{ug} / \mathrm{L}$

$10.00 \mathrm{ug} / \mathrm{L}$
Gen. Eng.

Gen. Eng.

Gen. Eng.

Gen. Eng.

Gen. Eng.

Gen. Eng.

Gen. Eng.

Gen. Eng.

Gen. Eng.

Gen. Eng.

Gen. Eng.

Gen. Eng.

Gen:- Eng.

Gen. Eng.

Gen. Eng.

Gen. Eng.

Gen. Eng.

Gen. Eng.

Gen. Eng.

Gen. Eng.

Gen. Eng.

Gen. Eng.

Gen. Eng.

Gen. Eng.

Gen. Eng.

Gen. Eng.

Gen. Eng.

Gen. Eng.

Gen. Eng.

Gen. Eng.

Gen. Eng.

Gen. Eng.

Gen. Eng.

Gen. Eng.

Gen. Eng.

Gen. Eng.

Gen. Eng.

Gen. Eng.

Gen. Eng.

Gen. Eng.

Gen. Eng.

Gen. Eng.

Gen. Eng.

Gen. Eng.

Gen. Eng.

Gen. Eng .

Gen. Eng.

Gen. Eng.

Gen. Eng.

Gen. Eng .

Geñ. Eng.

Gen. Eng.

Gen. Eng.

Gen. Eng. 
WELL LFW 36 COLLECTED ON 08/15/91 LABORATORY. ANALYSES CONTINUED

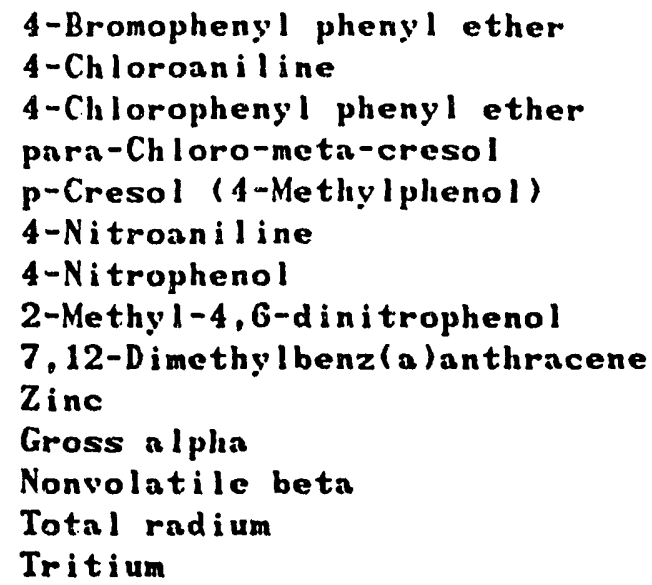

$\begin{array}{rr}\mathrm{LT} & 10.00 \mathrm{ug} / \mathrm{L} \\ \mathrm{LT} & 10.00 \mathrm{ug} / \mathrm{L} \\ \mathrm{LT} & 10.00 \mathrm{ug} / \mathrm{L} \\ \mathrm{LT} & 10.00 \mathrm{ug} / \mathrm{L} \\ \mathrm{LT} & 10.00 \mathrm{ug} / \mathrm{L} \\ \mathrm{LT} & 10.00 \mathrm{ug} / \mathrm{L} \\ \mathrm{LT} & 10.00 \mathrm{ug} / \mathrm{L} \\ \mathrm{LT} & 50.00 \mathrm{ug} / \mathrm{L} \\ \mathrm{LT} & 10.00 \mathrm{ug} / \mathrm{L} \\ & 3.69 \mathrm{ug} / \mathrm{L} \\ \mathrm{LT} & 2.00 \mathrm{pCi} / \mathrm{L} \\ 2.40+- & 2.00 \mathrm{pCi} / \mathrm{L} \\ 3.40+- & 3.40 \mathrm{pCi} / \mathrm{L} \\ 10.30+- & 0.50 \mathrm{pCi} / \mathrm{mL}\end{array}$

Gen. Eng.

Gen. Eng.

Gen. Eng.

Gen. Eng.

Gen. Eng.

Gen. Eng .

Gen. Eng.

Gen. Eng.

Gen. Eng.

Gen. Eng.

Gen. Eng.

Gen. Eng.

Gen.- Eng.

Gen. Eng.

WELL LFW 37

$\begin{array}{llllll}\text { SRS Grid } & N & 83113.2 & \text { Latitude } & 33.284100 & N \\ \text { Coordinates } & E & 45667.7 & \text { Longitude } & 81.707994 & W\end{array}$

Screen Zone Elevation 150.8-129.8 ft msl

Top of Casing Elevation $167.80 \mathrm{ft} \mathrm{ms} 1$

\section{MEASUREMENTS CONDUCTED IN THE FIELD}

Sample date 08/15/91 Tisne 1530

Depth to water $=25.15 \mathrm{ft}(7.67 \mathrm{~m})$ below the TOC

Water elevation $=142.65 \mathrm{ft}(43.48 \mathrm{~m}) \mathrm{msl}$

$\mathrm{pH}-5.2 \quad$ Alkalinity $-0 \mathrm{mg} / \mathrm{L}$

Specific conductance - 98 umhos $/ \mathrm{cm}$

Water temperature - 19.3 degrees Celsius

Water evacuated from the well prior to sampling - 62 gal

\section{LABORATORY ANALYSES}

Specific conductance

Carbon 12-labeled 2,3,7,8-TCDD

Carbon 12-labeled TCDF

Heptachlorodibenzo-p-dioxin isomers

Heptachlorodibenzo-p-furan isomers

Hexachlorodibenzo-p-dioxin isomers

Hexachlorodibenzo-p-furan isomers

Octachlorodibenzo-p-dioxin isomers

Octachlorodibenzo-p-furan isomers

Pentachlorodibenzo-p-dioxin isomers

Pentachlorodibenzo-p-furan isomers

pll

Tetrachlorodibenzo-p-dioxin isomers

Tetrachlorodibenzo-p-furan isomers

$1,2,3,4,6,7,8-11 P C D D$

$1,2,3,4,6,7,8$-HIIPCDF

$2,3,4,7,8$-Hexnchlorodibenzo-p-diox in LT

$1,2,3,4,7,8$-Hexach lorod ibenzo-p-furan $T$ CONTINUED
LT

LT

LT

LT

LT

LT

LT

LT

LT

LT

LT

LT

LT

LT

LT

$95.00 \mathrm{umh} / \mathrm{cm}$

$0.45 \mathrm{ng} / \mathrm{L}$

$0.40 \mathrm{ng} / \mathrm{L}$

$0.65 \mathrm{ng} / \mathrm{L}$

$0.45 \mathrm{ng} / \mathrm{L}$

$0.45 \mathrm{ng} / \mathrm{L}$

$0.40 \mathrm{ng} / \mathrm{L}$

$1.00 \mathrm{ng} / \mathrm{L}$

$1.00 \mathrm{ng} / \mathrm{L}$

$0.55 \mathrm{ng} / \mathrm{L}$

$0.55 \mathrm{ng} / \mathrm{L}$

$5.47 \mathrm{pH}$

$0.45 \mathrm{ng} / \mathrm{L}$.

$0.40 \mathrm{ng} / \mathrm{L}$

$0.65 \mathrm{ng} / \mathrm{L}$

$0.45 \mathrm{ng} / \mathrm{L}$

$0.45 \mathrm{ng} / \mathrm{L}$

$0.40 \mathrm{ng} / \mathrm{L}$
Gen. Eng.

Gen. Eng.

Gen. Eng.

Gen. Eng.

Gen. Eng.

Gen. Eng.

Gen. Eng.

Gen. Eng.

Gen. Eng .

Gen. Eng.

Gen. Eng.

Gen. Eng.

Gen. Eng.

Gen. Eng .

Gen. Eng.

Geñ. Eng.

Gen. Eng.

Gen. Eng. 


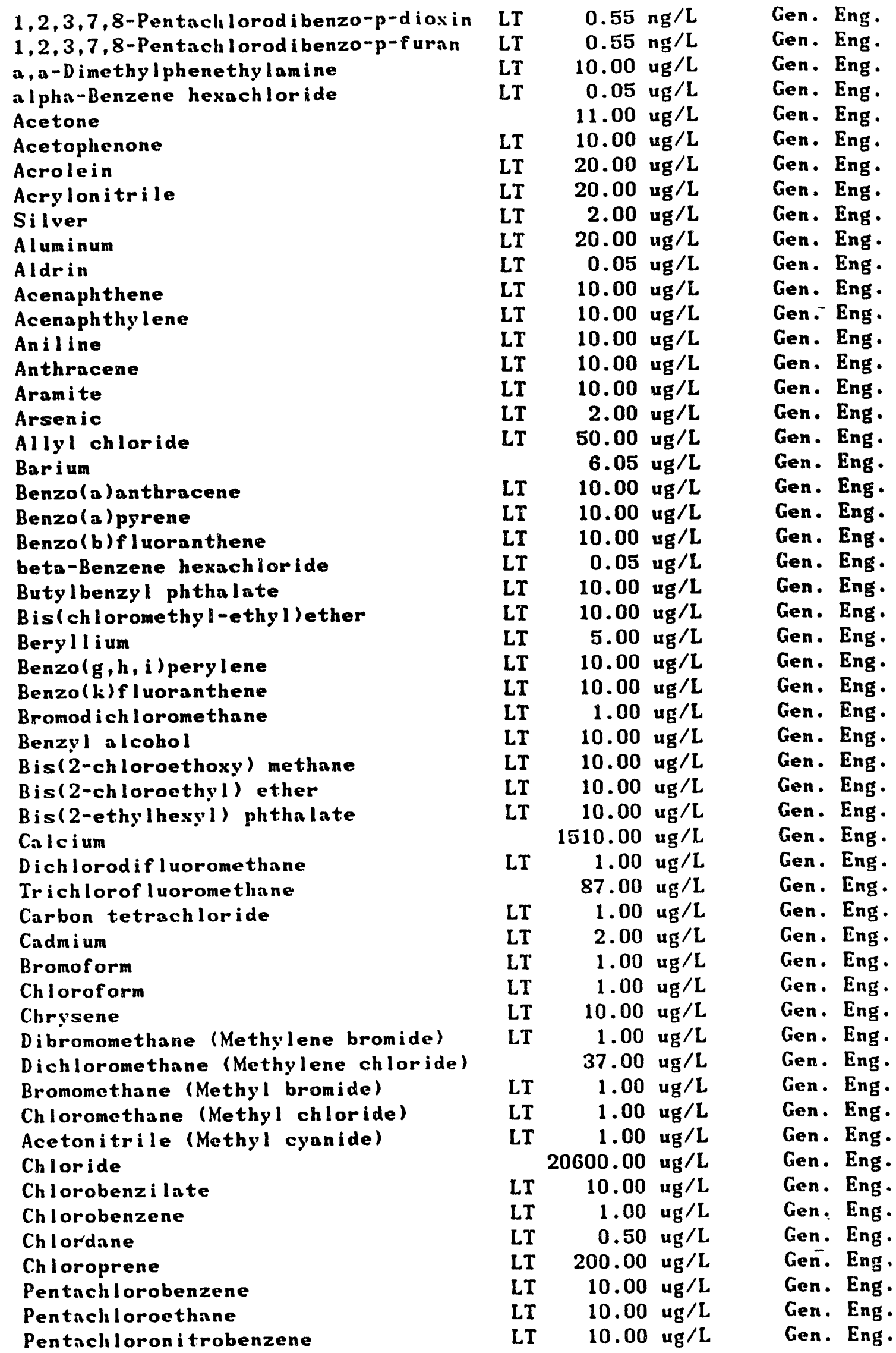


Hexachlorobenzene

Hexach lorocyclopen tad iene

Hexachloroethane

Cobalt

Chromium

Carbon disulfide

Copper

Cyanide

Chloroethene (Vingl chloride)

Chloroethane

Benzene

Dibenz (a, h)anthracene

delta-Benzene hexachloride

Dibromochloromethane

Diethyl phthalate

Diallate

Dibenzofuran

Dimethoate

Disulfoton

Dieldrin

Dimethyl phthalate

Di-n-butyl phthalate

Di-n-octyl phthalate

Diphenylamine

Ethyl methanesulfonate

Endrin aldehyde

Endrin

Endosulfan I

Endosulfan II

Endosulfan sulfate

Ethyl benzene

Ethyl methacrylate

Fluoride

Fluoride

Famphur

Fluoranthene

Iron

Fluorene

Hexachlorobutadiene

Hexachlorophene

Hexachloropropene

Mercury

Heptachlor

Heptachlor epoxide

Indeno $(1,2,3-c, d)$ pyrene

Iodomethane (Methyl iodide)

Isosaf role

Isobutyl alcohol

Isodrin

Isophorone

Potassium

Kepone

Lindane

Methacrylonitrile

CONTINUED
LT

LT

LT

LT

LT

LT

LT

LT

LT

LT

I.T

LI

LT

LT

LT

LT

LI

LT

LT

LI

LT

LT

LT

LT

LT

LT

LT

LT

LI

LT

LT

LT

LI

LT

LT

LT

LT

LT

LI

LT

LT

LT

LT

LT

LT

LT

LT

LT

LT

LI

LI
$10.00 \mathrm{ug} / \mathrm{L}$

$10.00 \mathrm{ug} / \mathrm{L}$ $10.00 \mathrm{ug} / \mathrm{L}$

$4.00 \mathrm{ug} / \mathrm{L}$

$4.00 \mathrm{ug} / \mathrm{L}$

$1.00 \mathrm{ug} / \mathrm{L}$

$4.00 \mathrm{ug} / \mathrm{L}$

$5.00 \mathrm{ug} / \mathrm{L}$

$1.00 \mathrm{ug} / \mathrm{L}$

$1.00 \mathrm{ug} / \mathrm{L}$

$2.00 \mathrm{ug} / \mathrm{L}$

$10.00 \mathrm{ug} / \mathrm{L}$.

$0.05 \mathrm{ug} / \mathrm{L}$

$1.00 \mathrm{ug} / \mathrm{L}$

$10.00 \mathrm{ug} / \mathrm{L}$

$10.00 \mathrm{ug} / \mathrm{L}$

$10.00 \mathrm{ug} / \mathrm{L}$

$10.00 \mathrm{ug} / \mathrm{L}$

$10.00 \mathrm{ug} / \mathrm{L}$

$0.50 \mathrm{ug} / \mathrm{L}$

$10.00 \mathrm{ug} / \mathrm{L}$

$10.00 \mathrm{ug} / \mathrm{L}$

$10.00 \mathrm{ug} / \mathrm{L}$

$10.00 \mathrm{ug} / \mathrm{L}$

$10.00 \mathrm{ug} / \mathrm{L}$

$0.10 \mathrm{ug} / \mathrm{L}$

$0.01 \mathrm{ug} / \mathrm{L}$

$0.10 \mathrm{ug} / \mathrm{L}$

$0.10 \mathrm{ug} / \mathrm{L}$

$0.10 \mathrm{ug} / \mathrm{L}$

$1.00 \mathrm{ug} / \mathrm{L}$

$10.00 \mathrm{ug} / \mathrm{L}$

$100.00 \mathrm{ug} / \mathrm{L}$

$100.00 \mathrm{ug} / \mathrm{L}$

$10.00 \mathrm{ug} / \mathrm{L}$

$10.00 \mathrm{ug} / \mathrm{L}$

$18.00 \mathrm{ug} / \mathrm{L}$

$10.00 \mathrm{ug} / \mathrm{L}$

$10.00 \mathrm{ug} / \mathrm{L}$

$10.00 \mathrm{ug} / \mathrm{L}$

$10.00 \mathrm{ug} / \mathrm{L}$

$0.20 \mathrm{ug} / \mathrm{L}$

$0.05 \mathrm{ug} / \mathrm{L}$

$0.05 \mathrm{ug} / \mathrm{L}$

$10.00 \mathrm{ug} / \mathrm{L}$

$15.00 \mathrm{ug} / \mathrm{L}$

$10.00 \mathrm{ug} / \mathrm{L}$

$100.00 \mathrm{ug} / \mathrm{L}$

$10.00 \mathrm{ug} / \mathrm{L}$

$10.00 \mathrm{ug} / \mathrm{L}$

$500.00 \mathrm{ug} / \mathrm{L}$

$10.00 \mathrm{ug} / \mathrm{L}$

$0.00 \mathrm{ug} / \mathrm{L}$

$50.00 \mathrm{ug} / \mathrm{L}$
Gen. Eng.

Gen. Eng.

Gen. Eng.

Gen. Eng.

Gen. Eng.

Gen. Eng.

Gen. Eng.

Gen. Eng.

Gen. Eng.

Gen. Eng.

Gen. Eng.

Gen. Eng.

Gen.-Eng -

Gen. Eng.

Gen. Eng.

Gen. Eng.

Gen. Eng.

Gen. Eng.

Gen. Eng.

Gen. Eng.

Gen. Eng.

Gen. Eng -

Gen. Eng.

Gen. Eng.

Gen. Eng.

Gen. Eng.

Gen. Eng.

Gen. Eng .

Gen. Eng.

Gen. Eng.

Gen. Eng.

Gen. Eng.

Gen. Eng.

Gen. Eng.

Gen. Eng.

Gen. Eng.

Gen. Eng.

Gen. Eng.

Gen. Eng.

Gen. Eng.

Gen. Eng.

Gen. Eng.

Gen. Eng.

Gen. Eng.

Gen. Eng.

Gen. Eng.

Gen. Eng.

Gen. Eng.

Gen. Eng.

Gen. Eng.

Geñ. Eng.

Gen. Eng.

Gen. Eng.

Gen. Eng . 
Toluene

Methyl ethyl ketone

Methyl methacrylate

Methyl methanesulfonate

Methoxychlor

Magnesium

Methyl isobutyl ketone

Manganese

Methapyrilene

Sod i um

Naphthalene

Nitrobenzene

$\mathrm{N}-\mathrm{N}$ itrosod imethylamine

$N-N i$ trosodi-n-butylamine

$N-N i t r o s o d i-p r o p y l a m i n e$

$\mathrm{N}-\mathrm{Nitrosod}$ iethylamine

$N-N i$ trosomethylethylamine

Nickel

$\mathrm{N}-\mathrm{N}$ i trosomorphol ine

$N-N i$ trosodipheny lamine

$N-N i$ trosopiperidine

$N-N i t r o s o p y r r o l i d i n e$

Nitrate as nitrogen

4-Nitroquinol ine-1-oxide

5-Nitro-o-toluidine

o-Toluidine

Lead

PCB 1016

PCB 1221

PCB 1232

PCB 1242

PCB 1248

PCB 1254

PCB 1260

Pentachlorophenol

p-Phenylenediamine

$p-D$ imethy lami noazobenzene

Phenacetin

Phenanthrene

Phenol

Phorate

p. p'-DDD

$p, p^{\prime}-D D E$

p, p'-DDT

Pronamid

Propionitrile

Parathion

Parathion methyl

Pyrene

Pyridine

Safrole

Antimony

2-sec-Butyl-4,6-din itrophenol

Selenium CONTINUED
LT

LI

LI

LT

LT

L.T

LT

LT

LT

LT

LT

LT

LI

LT

LT

LT

LT

LI

LT

LT

LT

LI

LT

LT

LT

LT

LT

LT

LT

LT

LT

LT

LT

LT

LT

LI

LT

LT

LT

LT

LT

LT

LT

LI

LT

LI

LT

LI

LT

LT
$1.00 \mathrm{ug} / \mathrm{L}$

$1.00 \mathrm{ug} / \mathrm{L}$

$10.00 \mathrm{ug} / \mathrm{L}$

$10.00 \mathrm{ug} / \mathrm{L}$

$0.50 \mathrm{ug} / \mathrm{L}$

$2410.00 \mathrm{ug} / \mathrm{L}$

$1.00 \mathrm{ug} / \mathrm{L}$

$2.85 \mathrm{ug} / \mathrm{L}$

$10.00 \mathrm{ug} / \mathrm{L}$

$11800.00 \mathrm{ug} / \mathrm{L}$

$10.00 \mathrm{ug} / \mathrm{L}$

$10.00 \mathrm{ug} / \mathrm{L}$

$10.00 \mathrm{ug} / \mathrm{s}$.

$10.00 \mathrm{ug} / \mathrm{s}$

$10.00 \mathrm{ug} / \mathrm{L}$

$10.00 \mathrm{ug} / \mathrm{L}$

$10.00 \mathrm{ug} / \mathrm{L}$

$4.00 \mathrm{ug} / \mathrm{L}$

$10.00 \mathrm{ug} / \mathrm{L}$

$10.00 \mathrm{ug} / \mathrm{L}$

$10.00 \mathrm{ug} / \mathrm{L}$

$10.00 \mathrm{ug} / \mathrm{L}$

$66.00 \mathrm{ug} / \mathrm{L}$

$10.00 \mathrm{ug} / \mathrm{L}$

$10.00 \mathrm{ug} / \mathrm{L}$

$10.00 \mathrm{ug} / \mathrm{L}$

$3.00 \mathrm{ug} / \mathrm{L}$

$0.50 \mathrm{ug} / \mathrm{L}$

$0.50 \mathrm{ug} / \mathrm{L}$

0.50 ug $/ L$

$0.50 \mathrm{ug} / \mathrm{L}$

0.50 ug $/ \mathrm{L}$

$1.00 \mathrm{ug} / \mathrm{L}$

$1.00 \mathrm{ug} / \mathrm{L}$

$10.00 \mathrm{ug} / \mathrm{L}$

$10.00 \mathrm{ug} / \mathrm{L}$

$10.00 \mathrm{ug} / \mathrm{L}$

$10.00 \mathrm{ug} / \mathrm{L}$

$10.00 \mathrm{ug} / \mathrm{L}$

$10.00 \mathrm{ug} / \mathrm{L}$

$0.10 \mathrm{ug} / \mathrm{L}$

$0.10 \mathrm{ug} / \mathrm{L}$

$0.10 \mathrm{ug} / \mathrm{L}$

$0.10 \mathrm{ug} / \mathrm{L}$

$10.00 \mathrm{ug} / \mathrm{L}$

$200.00 \mathrm{ug} / \mathrm{L}$

$0.05 \mathrm{ug} / \mathrm{L}$

$0.05 \mathrm{ug} / \mathrm{L}$

$10.00 \mathrm{ug} / \mathrm{L}$

$10.00 \mathrm{ug} / \mathrm{L}$

$10.00 \mathrm{ug} / \mathrm{L}$

$2.00 \mathrm{ug} / \mathrm{L}$

$10.00 \mathrm{ug} / \mathrm{L}$

$2.00 \mathrm{~kg} / \mathrm{L}$.
Gen. Eng.

Gen. Eng.

Gen. Eng.

Gen. Eng.

Gen. Eng.

Gen. Eng.

Gen. Eng.

Gen. Eng.

Gen. Eng.

Gen. Eng.

Gen. Eng.

Gen. Eng.

Gen.-Eng.

Gen. Eng.

Gen. Eng.

Gen. Eng.

Gen. Eng.

Gen. Eng.

Gen. Eng -

Gen. Eng.

Gen. Eng.

Gen. Eng.

Gen. Eng.

Gen. Eng.

Gen. Eng.

Gen. Eng.

Gen. Eng.

Gen. Eng.

Gen. Eng.

Gen. Eng.

Gen. Eng.

Gen. Eng.

Gen. Eng.

Gen. Eng.

Gen. Eng.

Gen. Eng.

Gen. Eng.

Gen. Eng.

Gen. Eng.

Gen. Eng.

Gen. Eng.

Gen. Eng.

Gen. Eng.

Gen. Eng.

Gen. Eng.

Gen. Eng.

Gen. Eng.

Gen. Eng.

Gen. Eng.

Gen. Eng.

Geñ. Eng.

Gen. Eng.

Gen. Eng.

Gen. Eng. 
Silica

$2,4,5-T P$ (Si lvex)

Tin

Sulfate

Sulf otepp

Styrene

Sulf ide

1,2,4,5-Tetrach lorobenzene

$1,1,2,2$-Tetrachloroethane

$1,1,1,2$-Tetrach loroethane

Tetrachloroethylene

0,0,0-Triethyl phosphorothioate

Thionazin

Thall i um

Total organic carbon

Total organic nitrogen

Total organic halogens

Total phosphates (as P)

* Trichloroethylene

Toxaphene

trans-1,2-Dichloroethylene

trans-1, 3-D ich loropropene

trans-1, 4-D ich Ioro-2-butene

Vanadium

Vinyl acetate

$\lambda y l e n e s$

1-Naphthylamine

1,1-Dichloroethylene

1,1-Dichloroethane

1,1,1-Trichloroethane

1,1,2-Trichloroethane

1,2-Dibromoethane

1, 2-D i bromo-3-chloropropare

1,2-Dichlorobenzene

1,2-Dichloroethane

1,2-Dichloropropane

1,2,3-Trich loropropane

1,2,4-Trich lorobenzene

1,3-Dichlorobenzene

c is-1,3-D ichloropropene

$1,3-D$ in itrobenzene

1,3,5-Trinitrobenzene

1,4-Dichlorobenzene

1, 4-Diosane

1, 4-Naph thoquinone

2-Acety laminof I uorene

2-Chloroethyl vinyl ether

2-Chlorophenol

2-Chloronaphthalene

2-Hesanone

2-Methylnaphthalene

o-Cresol (2-Methylpheno I)

2-Nitroaniline

2-Naphthylamine

CONTINUED

\begin{tabular}{|c|c|c|c|c|}
\hline & 10600.00 & $\mathrm{ug} / \mathrm{L}$ & Gen. & En \\
\hline LT & 0.09 & ug/L & Gen. & . Eng \\
\hline $\mathbf{T}$ & 2.00 & ugg $/ \mathrm{L}$ & Gen. & \\
\hline & 1470.00 & $\mathrm{ug} / \mathrm{L}$ & Gen. & \\
\hline LT & 10.00 & ug $/ \mathrm{L}$ & Gen. & \\
\hline LT & 1.00 & $u g / L$ & Gen. & \\
\hline LT & 1000.00 & ug $/ \mathrm{L}$ & Gen. & \\
\hline LT & 10.00 & ug $/ \mathrm{L}$ & Gen. & \\
\hline LT & 1.00 & $u_{g} / L$ & ien. & \\
\hline LI & 1.00 & ug/L & Gen. & \\
\hline & 1.00 & ug/L & Gen. & \\
\hline$T$ & 10.00 & $\mathrm{ug} / \mathrm{L}$ & Gen. & \\
\hline$T$ & 10.00 & ug $/ \mathrm{L}$ & Gen :- & $\cdots_{2}$ \\
\hline$T$ & 2.00 & ug $/ \mathrm{L}$ & Gen. & 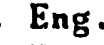 \\
\hline & 3000.00 & ug/L & Gen. & \\
\hline $\mathrm{LT}$ & 100.00 & ug/L & en. & \\
\hline & 406.00 & ug/L & - & \\
\hline LT & 100.00 & ug $/ \mathrm{L}$ & en. & \\
\hline LT & 6.00 & ug $/ \mathrm{L}$ & Ger & \\
\hline LT & 0.24 & ug/L & Gen. & \\
\hline LT & 1.00 & ug $/ \mathrm{L}$ & Gen. & \\
\hline LT & 30.00 & ug $/ \mathrm{L}$ & Gen. & \\
\hline LT & 10.00 & $u g / L$ & Gen. & \\
\hline LT & 1.00 & ug $; L$ & Gen. & \\
\hline LT & 1.00 & ug $/ \mathrm{L}$ & Gen. & \\
\hline LT & 10.00 & ug $/ \mathrm{L}$ & Gen. & \\
\hline LT & 1.00 & ug/L & Gen. & \\
\hline & 67.00 & ug /L & Gen. & \\
\hline & 8.00 & ug/L & Gen. & \\
\hline LT & 1.00 & ug $/ \mathrm{L}$ & Gen. & 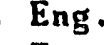 \\
\hline LT & 20.00 & $4 \mathrm{~g} / \mathrm{L}$ & Gen. & En \\
\hline LT & 1.00 & ug $/ \mathrm{L}$ & Gen. & \\
\hline LI & 10.00 & $\mathrm{ug} / \mathrm{L}$ & Gen. & \\
\hline & 4.00 & ug $/ \mathrm{L}$ & Ger & \\
\hline LT & 1.00 & ug/L & Gen. & En \\
\hline LT & 20.00 & $u g / L$ & Gen. & \\
\hline L.T & 10.00 & $u g / L$ & Gen. & - Eng \\
\hline LT & 10.00 & $u g / L$ & Gen. & $E_{1}$ \\
\hline LT & 1.00 & ug $/ \mathrm{L}$ & Gen. & \\
\hline LT & 10.00 & $u g / L$ & Cen. & \\
\hline LT & 10.00 & ug $/ \mathrm{L}$ & Gen. & \\
\hline & 25.00 & ug $/ \mathrm{L}$ & Gen & \\
\hline LT & 10.00 & ug/L & Gen. & \\
\hline LT & 10.00 & $u g / L$ & Gen. & \\
\hline LT & 10.00 & ug/L & Gen. & E \\
\hline LT & 10.00 & ug $/ \mathrm{L}$ & Gen. & $\mathbf{E}$ \\
\hline LT & 10.00 & ug $/ \mathrm{L}$ & Gen. & $\mathbf{E}$ \\
\hline LT & 10.00 & ug $/ \mathrm{L}$ & Gen. & Lit \\
\hline LT & 1.00 & $\operatorname{ug} / \mathrm{L}$ & Gen. & \\
\hline LT & 10.00 & $u g / L$ & Geñ. & \\
\hline $\mathrm{LT}$ & 10.00 & $u_{g} / L$ & & \\
\hline LT & 10.00 & $u g / L$ & G & \\
\hline LT & 10.00 & $u g / L$ & Gen. & \\
\hline
\end{tabular}




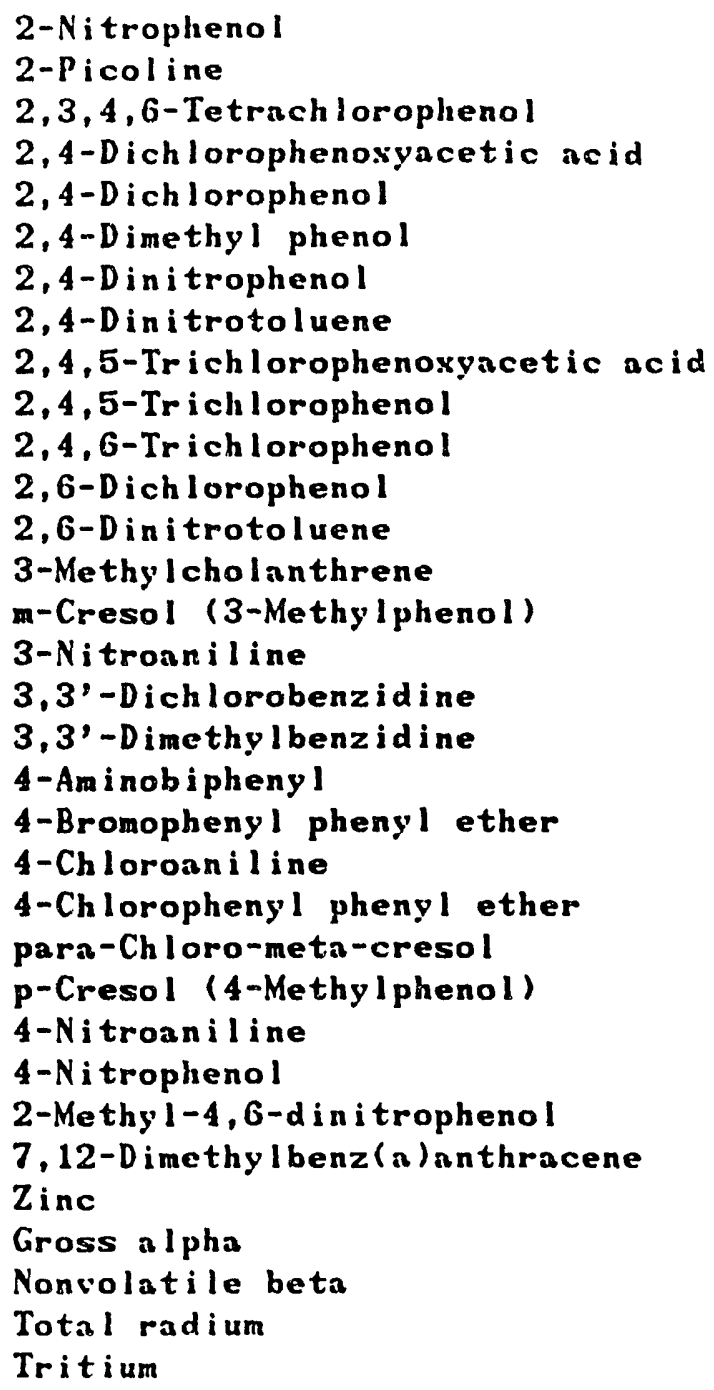

\begin{tabular}{|c|c|c|c|}
\hline LT & 10.00 & ug $/ \mathrm{L}$ & Gen. \\
\hline LT & 10.00 & ug $/ \mathrm{L}$ & Gen. \\
\hline LT & 10.00 & ug $/ \mathrm{L}$ & Gen. \\
\hline LT & 0.30 & ug $/ \mathrm{L}$ & Gen. \\
\hline LT & 10.00 & ug/L $/ \mathrm{L}$ & Gen. \\
\hline LT & 10.00 & ug $/ \mathrm{L}$ & Gen. \\
\hline LT & 45.00 & ug /L & Gen. \\
\hline LT & 10.00 & ug $/ \mathrm{L}$ & Gen. \\
\hline LT & 0.09 & ug $/ \mathrm{L}$ & Gen. \\
\hline LT & 10.00 & ug $/ \mathrm{L}$ & Gen. \\
\hline LT & 10.00 & ug $/ \mathrm{L}$ & Gen. \\
\hline LT & 10.00 & ug/L & Gen. \\
\hline LT & 10.00 & $u g / L$ & Gen. \\
\hline LT & 10.00 & ug $/ L$ & Gen. \\
\hline LT & 10.00 & ug $/ L$ & Gen. \\
\hline LT & 10.00 & ug/L & Gen. \\
\hline LT & 20.00 & ug/L & Gen. \\
\hline LT & 10.00 & ug/L & Gen. \\
\hline LT & 10.00 & ug $/ \mathrm{L}$ & Gen. \\
\hline LT & 10.00 & ug/L & Gen. \\
\hline LT & 10.00 & ug $/ L$ & Gen. \\
\hline LT & 10.00 & ug $/ \mathrm{L}$ & Gen. \\
\hline LT & 10.00 & $u g / L$ & Gen. \\
\hline LT & 10.00 & ug $/ \mathrm{L}$ & Gen. \\
\hline LT & 10.00 & ug $/ L$ & Gen. \\
\hline LT & 10.00 & ug $/ L$ & Gen. \\
\hline LT & 50.00 & $4 \mathrm{~g} / \mathrm{L}$ & Gen. \\
\hline LT & 10.00 & ug $/ \mathrm{L}$ & Gen. \\
\hline LT & 2.00 & $u g / L$ & Gen. \\
\hline LT & 2.00 & $p C i / L$ & Gen. \\
\hline $6.70+-$ & 2.40 & $\mathrm{pCi} / \mathrm{L}$ & Gen. \\
\hline $2.00+-$ & 2.90 & $p C i / L$ & Gen. \\
\hline $11.30+-$ & 0.50 & $\mathrm{pCi} / \mathrm{mL}$ & Gen. \\
\hline
\end{tabular}


WELL LFW 38

$\begin{array}{llllll}\text { SRS Grid } & N & 83172.3 & \text { Latitude } & 33.284803 & N \\ \text { Coordinates } & \text { E } 46018.5 & \text { Longitude } 81.707185 & W \\ \text { Screen Zone Elevation } & 151.5-130.5 & \text { ft ms }\end{array}$

Top of Casing Elevation $168.50 \mathrm{ft} \mathrm{msl}$

MEASUREMENTS CONDUCTED IN THE FIELD

Sample date 08/26/91 Time 1200

cepth to water $=24.81 \mathrm{ft}(7.56 \mathrm{~m})$ below the TOC

water elevation - $143.69 \mathrm{ft}(43.80 \mathrm{~m}) \mathrm{msl}$

$\mathrm{pH}=4.7$ Alkalinity - $0 \mathrm{mg} / \mathrm{L}$

Specific conductance - 54 umhos/cm

Water temperature - 19.9 degrees Celsius

Water evacunted from the well prior to snmpling - $42 \mathrm{gal}$

\section{LABORATORY ANALYSES}

Specific conductance

Carbon 12-labeled 2,3,7,8-TCDD

Heptachlorodibenzo-p-dioxin isomers

Heptachlorodibenzo-p-furan isomers

Hexachlorodibenzo-p-dioxin isomers

Hexachlorodibenzo-p-furan isomers

Octachlorodibenzo-p-dioxin isomers

Octachlorodibenzo-p-furan isomers

Pentachlorodiberzo-p-dioxin isomers

Pentachlorodibenzo-p-furan isomers

pH

Tetrachlorodibenzo-p-dioxin isomers

Tetrachlorodibenzo-p-furan isomers

$1,2,3,4,6,7,8-H P C D D$

1, 2, 3, 4, 7, 8-Hexnchlorodibenzo-p-dioxin LI

$1,2,3,7,8$-Pentachlorodibenzo-p-diosin LT

a, a-Dimethy' phenethylamine

alpha-Benzene hexachloride

Acetone

Acetophenone

Acrolein

Acrylonitrile

Silver

Aluminum

Aldrin

Acenaphthene

Acenaphthylene

Ani line

Anthracene

Aramite

Arsenic

Allyl chloride

Barium

Benzo(a)antliracene

Benzo(a) pyrene

Benzo(b)fluoranthene

beta-Benzene hexachloride

CONTINUED
$50.00 \mathrm{umh} / \mathrm{cm}$

LI

LT

LI

LT

LT

LT

LT

LT

LT

LT

LT

LT

LT

LT

LT

LT

LT

LT

LT

LT

LT

LT

LT

LT

LT

LI

LT

LT

LT

LT

LT
$0.45 \mathrm{ng} / \mathrm{L}$

$0.65 \mathrm{ng} / \mathrm{L}$

$0.65 \mathrm{ng} / \mathrm{L}$

$0.45 \mathrm{ng} / \mathrm{L}$

$0.45 \mathrm{ng} / \mathrm{L}$

$1.00 \mathrm{ng} / \mathrm{L}$

$1.00 \mathrm{ng} / \mathrm{L}$

$0.55 \mathrm{ng} / \mathrm{L}$

$0.55 \mathrm{ng} / \mathrm{L}$

$5.00 \mathrm{pll}$

$0.45 \mathrm{ng} / \mathrm{L}$

$0.45 \mathrm{ng} / \mathrm{L}$

$0.65 \mathrm{ng} / \mathrm{L}$

$0.45 \mathrm{ng} / \mathrm{L}$

$0.55 \mathrm{ng} / \mathrm{L}$

$10.00 \mathrm{ug} / \mathrm{L}$

$0.05 \mathrm{ug} / \mathrm{L}$

$1.00 \mathrm{ug} / \mathrm{L}$

$10.00 \mathrm{ug} / \mathrm{L}$

$20.00 \mathrm{ug} / \mathrm{L}$

$20.00 \mathrm{ug} / \mathrm{L}$

$2.00 \mathrm{ug} / \mathrm{L}$

$25.60 \mathrm{ug} / \mathrm{L}$

$0.05 \mathrm{ug} / \mathrm{L}$

$10.00 \mathrm{ug} / \mathrm{L}$

$10.00 \mathrm{ug} / \mathrm{L}$

$10.00 \mathrm{ug} / \mathrm{L}$

$10.00 \mathrm{ug} / \mathrm{L}$

$10.00 \mathrm{ug} / \mathrm{L}$

$2.00 \mathrm{ug} / \mathrm{L}$

$50.00 \mathrm{ug} / \mathrm{L}$

$5.81 \mathrm{ug} / \mathrm{L}$

$10.00 \mathrm{ug} / \mathrm{L}$

$10.00 \mathrm{ug} / \mathrm{L}$

$10.00 \mathrm{ug} / \mathrm{L}$

$0.05 \mathrm{ug} / \mathrm{L}$
Gen. Eng.

Gen. Eng .

Gen. Eng.

Gen. Eng.

Gen. Eng.

Gen. Eng.

Gen. Eng.

Gen. Eng.

Gen. Eng.

Gen. Eng .

Gen. Eng .

Gen. Eng -

Gen. Eng .

Gen. Eng.

Gen. Eng.

Gen. Eng.

Gen. Eng.

Gen. Eng.

Gen. Eng.

Gen. Eng.

Gen. Eng.

Gen. Eng.

Gen. Eng.

Gen. Eng.

Gen. Eng .

Gen. Eng.

Gen. Eng.

Gen. Eng.

Gen. Eng.

Gen. Eng.

Gen. Eng.

Gen. Eng.

Gen. Eng.

Gē̄. Eng.

Gen. Eng.

Gen. Eng.

Gen. Eng. 
Butylbenzyl phthalate

Bis(chloromethyl-ethyl)ether

Beryllium

Benzo( $g, h, i)$ perylene

Benzo(li)f luoranthene

Bromodichloromethane

Benzyl alcohol

Bis(2-chloroethoxy) methane

Bis(2-chloroethyl) ether

Bis(2-ethylhexyl) phthalate

Calcium

Dichlorod if I uoromethane

Trichlor of luoromethane

Carbon tetrachloride

Cadmi um

Bromoform

Chloroform

Chrysene

Dibromonethane (Methylene bromide)

Dichloromethane (Methylene chloride)

Bromomethane (Methyl bromide)

Chloromethane (Methyl chloride)

Acetonitrile (Methyl cyanide)

Chloride

Chloride

Chloride

Chlorobenzilate

Chlorobenzene

Chlordane

Ch loroprene

Pentachlorobenzene

Pentachloroethane

Pentachloronitrobenzene

Hexach lorobenzene

Hexach lorocyclopentadiene

Hesach loroe thane

Cobalt

Chromi um

Carbon disulfide

Copper

Cyanide

Chloroethene (Vinyl chloride)

Chloroethane

Benzene

Dibenz $(a, h)$ anthracene

delta-Benzene hesachloride

Dibromochlorome thane

Diethyl phthalate

Dinllate

Dibenzofuran

Dimethonte

Disulfoton

Dieldrin

Dimethyl phthalate

CONTINUED

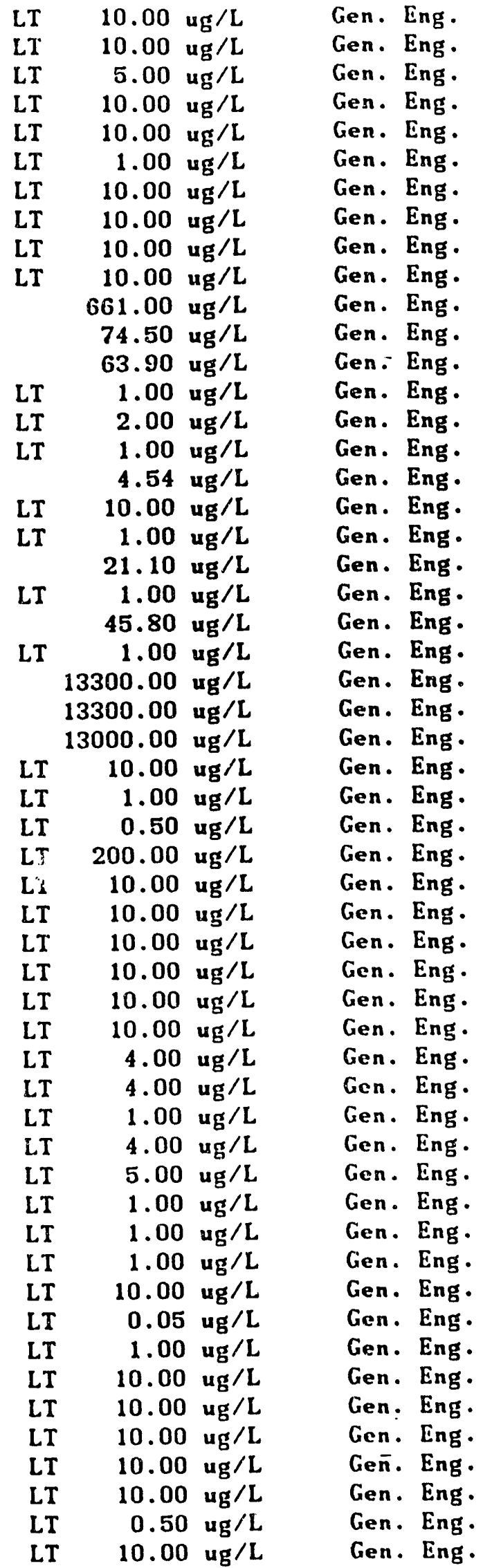




\begin{tabular}{|c|c|c|c|c|c|}
\hline $\begin{array}{l}\text { trans-1,2-D ich loroethylene } \\
\text { trans-1,3-D ich loropropene }\end{array}$ & $\begin{array}{l}\text { LT } \\
\text { LT }\end{array}$ & $\begin{array}{l}1.00 \\
1.00\end{array}$ & $\begin{array}{l}u g / L \\
u g / L\end{array}$ & $\begin{array}{l}\text { Gen. } \\
\text { Gen. }\end{array}$ & $\begin{array}{l}\text { Eng. } \\
\text { Eng. }\end{array}$ \\
\hline & LT & $\begin{array}{r}1.00 \\
30.00\end{array}$ & $\begin{array}{l}\text { ug/ } / \mathrm{L} \\
\mathrm{ug} / \mathrm{L}\end{array}$ & Gen. & $\begin{array}{l}\text { Eng. } \\
\text { Eng. }\end{array}$ \\
\hline Vanadium & LT & 10.00 & ug/L & Gen. & Eng. \\
\hline Vinyl acetate & LT & 1.00 & ug/L & Gen. & Eng. \\
\hline Xylenes & LT & 1.00 & $u_{g} / L$ & Gen. & Eng. \\
\hline 1-Naphthylamine & LT & 10.00 & ug $/ \mathrm{L}$ & Gen. & Eng. \\
\hline 1,1-Dichloroethylene & LT & 1.00 & ug/L & Gen. & Eng. \\
\hline 1,1-Dichloroethane & & 44.70 & ug/L & Gen. & Eng. \\
\hline 1,1,1-Trichloroe thane & & 13.60 & $\mathrm{ug} / \mathrm{L}$ & Gen. & Eng. \\
\hline $1,1,2-$ Trichloroethane & LT & 1.00 & ug/L & Gen. & Eng. \\
\hline 1,2-D ibromoethane & LT & 20.00 & ug $/ L$ & Gen. & Eng. \\
\hline 1,2-D i bromo-3-chl loropropane & LI & 1.00 & $\mathrm{ug} / \mathrm{L}$ & Gen.- & Eng. \\
\hline & $\mathbf{L T}$ & $\begin{array}{r}10.00 \\
2.13\end{array}$ & $\begin{array}{l}u g / L \\
u g / L\end{array}$ & $\begin{array}{l}\text { Gen. } \\
\text { Gen. }\end{array}$ & $\begin{array}{l}\text { Eng. } \\
\text { Eng. }\end{array}$ \\
\hline & LT & 1.00 & ug/L & Gen. & $\begin{array}{l}\text { Eng. } \\
\text { Eng. }\end{array}$ \\
\hline 1,2,3-Trich loropropane & LT & 20.00 & ug/L & Gen. & Eng. \\
\hline 1,2,4-Trichlorobenzene & LT & 10.00 & $\mathbf{u g} / \mathrm{L}$ & Gen. & Eng. \\
\hline 1,3-D ichlorobenzene & LI & 10.00 & ug/L & Gen. & Eng. \\
\hline cis-1,3-D ich loropropene & LT & 1.00 & $u g / L$ & Gen. & Eng. \\
\hline $1,3-D$ initrobenzene & LI & 10.00 & $\mathrm{ug} / \mathrm{L}$ & Gen. & Eng. \\
\hline $1,3,5-\mathrm{Tr}$ in itrobenzene & LT & 10.00 & ug/L & Gen. & Eng. \\
\hline 1,4-Dich lorobenzene & LT & 10.00 & $u g / L$ & Gen. & Eng. \\
\hline 1,4-Dioxane & LT & 10.00 & ug/L & Gen. & Eng. \\
\hline 1,4-Naphthoquinone & LT & 10.00 & ug/L & Gen. & Eng. \\
\hline 2-Acetylaminof luorene & LT & 10.00 & ug $/ \mathrm{L}$ & Gen. & Eng. \\
\hline 2-Chloroethyl vinyl ether & LT & 10.00 & $\operatorname{ug} / \mathrm{L}$ & Gen. & Eng. \\
\hline 2-Chlorophenol & LT & 10.00 & ug/L & Gen. & Eng. \\
\hline 2-Chloronaph tha lene & LT & 10.00 & ug/L & Gen. & Eng. \\
\hline 2-llesanone & LT & 1.00 & ug/L & Gen. & Eng. \\
\hline 2-Methylnaphthalene & LT & 10.00 & ug/L & Gen. & Eng. \\
\hline o-Cresol (2-Methylphenol) & LT & 10.00 & ug $/ \mathrm{L}$ & Gen. & Eng. \\
\hline 2-Nitroaniline & LT & 10.00 & ug /L & Gen. & Eng. \\
\hline 2-Naphthylamine & LT & 10.00 & ug $/ \mathrm{L}$ & Gen. & Eng. \\
\hline 2-Nitrophenol & LT & 10.00 & ug /L & Gen. & Eng. \\
\hline 2-Picoline & LT & 10.00 & ug/L & Gen. & Eng. \\
\hline $2,3,4,6$-Tetrachlorophenol & LT & 10.00 & ug /L & Gen. & - Eng. \\
\hline 2,4-Dichlorophenosycetic acid & LT & 0.30 & ug/L & Gen. & - Eng. \\
\hline 2,4-Dichlorophenol & LT & 10.00 & ug/L & Gen. & Eng. \\
\hline 2,4-Dimethyl phenol & LT & 10.00 & ug/L & Gen. & - Eng. \\
\hline $2,4-D$ initrophenol & LT & 45.00 & ug/L & Gen. & - Eng. \\
\hline $2,4-D$ in itrotoluene & L.T & 10.00 & $\mathrm{ug} / \mathrm{L}$ & Gen. & - Eng. \\
\hline $2,4,5-$ Trichlorophenosyacetic acid & $\mathrm{LT}$ & 0.09 & $\mathrm{ug} / \mathrm{L}$ & Gen. & - Eng. \\
\hline $2,4,5$-Trichlorophenol & LT & 10.00 & $u g / L$ & Gen. & - Eng. \\
\hline $2,4,6-\mathrm{Tr}$ ich lorophenol & LT & 10.00 & $\mathrm{ug} / \mathrm{L}$ & Gen. & - Eng. \\
\hline 2,6-Dichlorophenol & LT & 10.00 & $\mathrm{ug} / \mathrm{L}$ & Gen. & - Eng. \\
\hline $2,6-D$ in itrotoluene & LT & 10.00 & $u_{g} / L$ & Gen. & Eng. \\
\hline 3-Methylcholanthrene & LT & 10.00 & $\mathrm{ug} / \mathrm{L}$ & Gen. & Eng. \\
\hline m-Cresol (3-Methylphenol) & LT & 10.00 & $\mathbf{u g} / \mathrm{L}$ & Gen. & Eng. \\
\hline 3-Nitronniline & LT & 10.00 & $\mathrm{ug} / \mathrm{L}$ & Gen. & Eng. \\
\hline $3,3^{\prime}-D$ ichlorobenzidine & LT & 20.00 & $\mathbf{u g} / \mathrm{L}$ & Geñ. & Eng. \\
\hline 3, 3'-D imethylbenzidine & LT & 10.00 & $\mathrm{ug} / \mathrm{L}$ & Gen. & Eng. \\
\hline 4-Am inobiphenyl & LT & 10.00 & ug/L & Gen. & Eng \\
\hline 4-Bromophenyl phenyl ether & LT & 10.00 & $\mathbf{u g} / \mathrm{L}$ & Gen. & \\
\hline
\end{tabular}




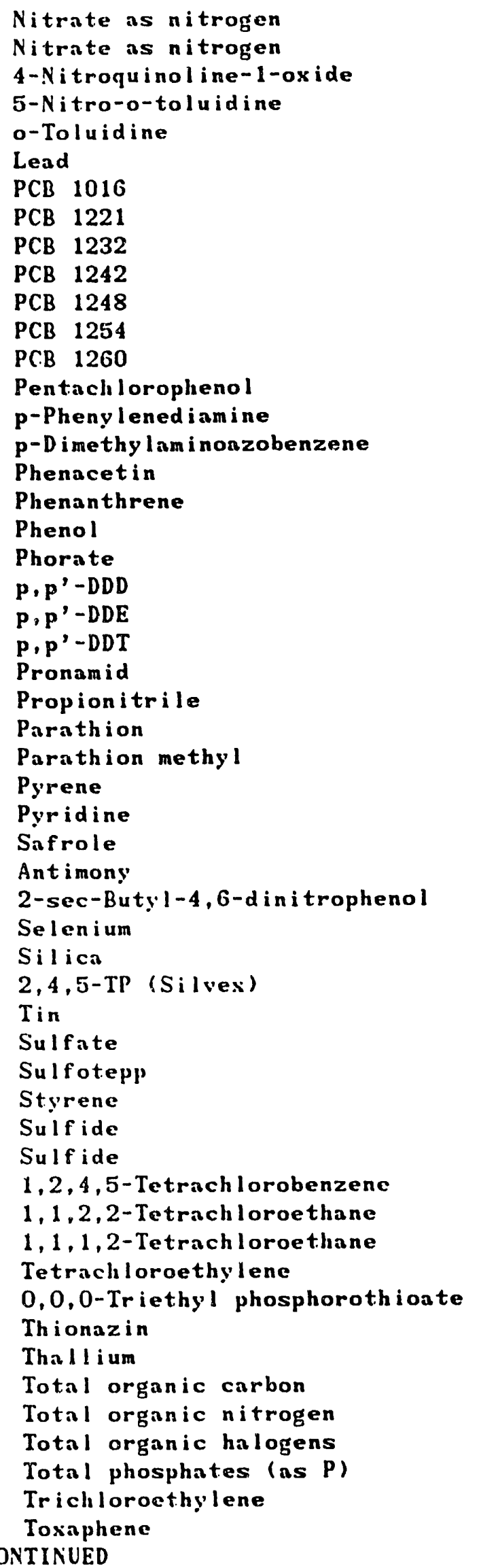

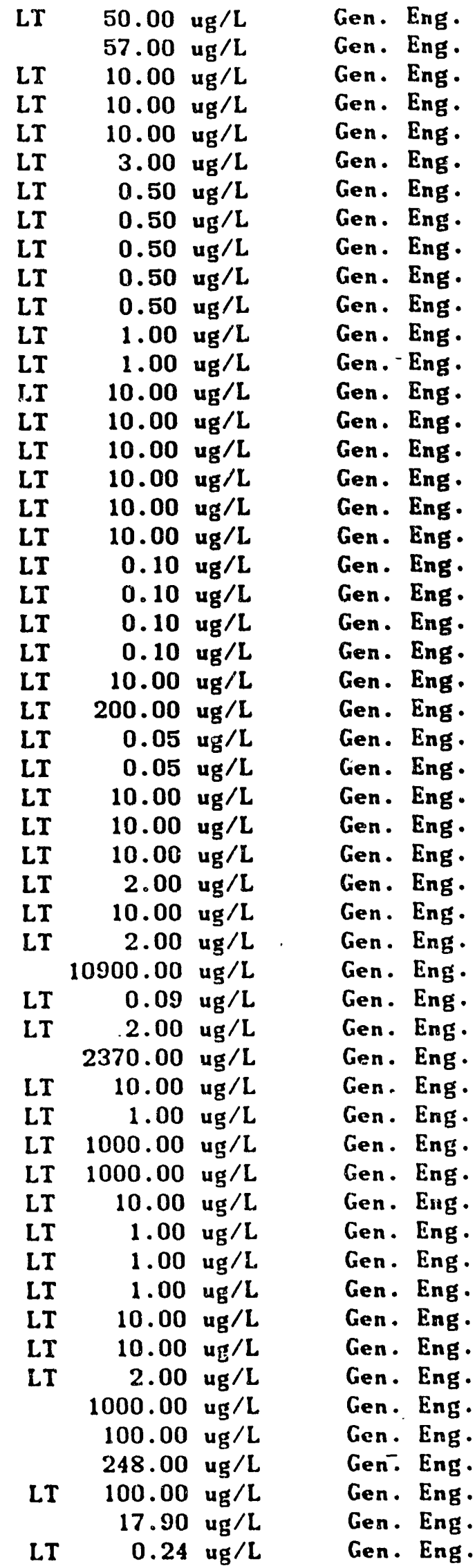




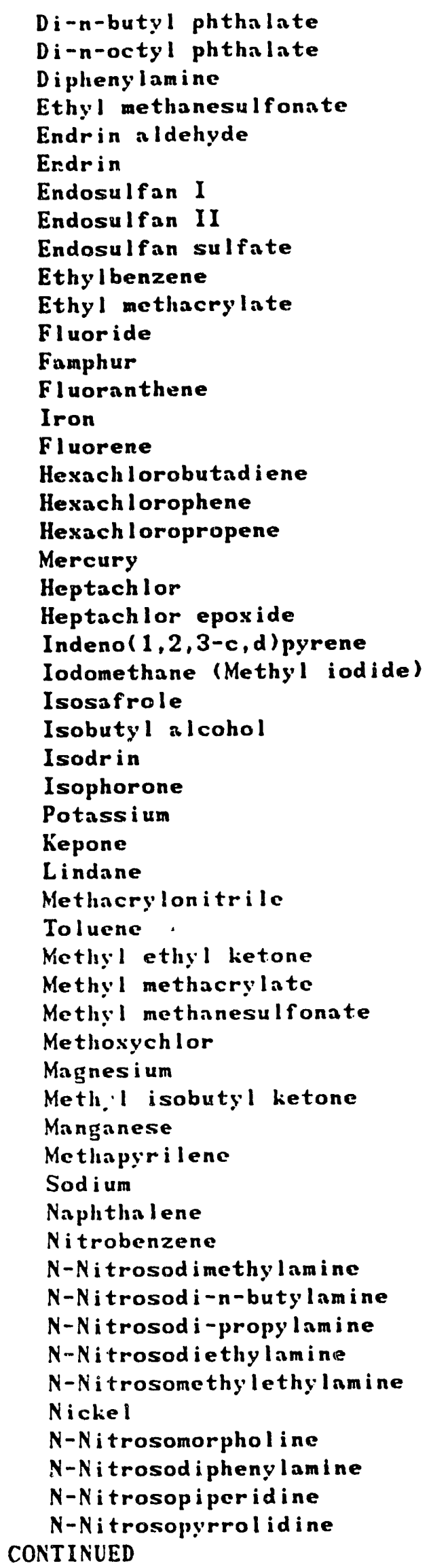

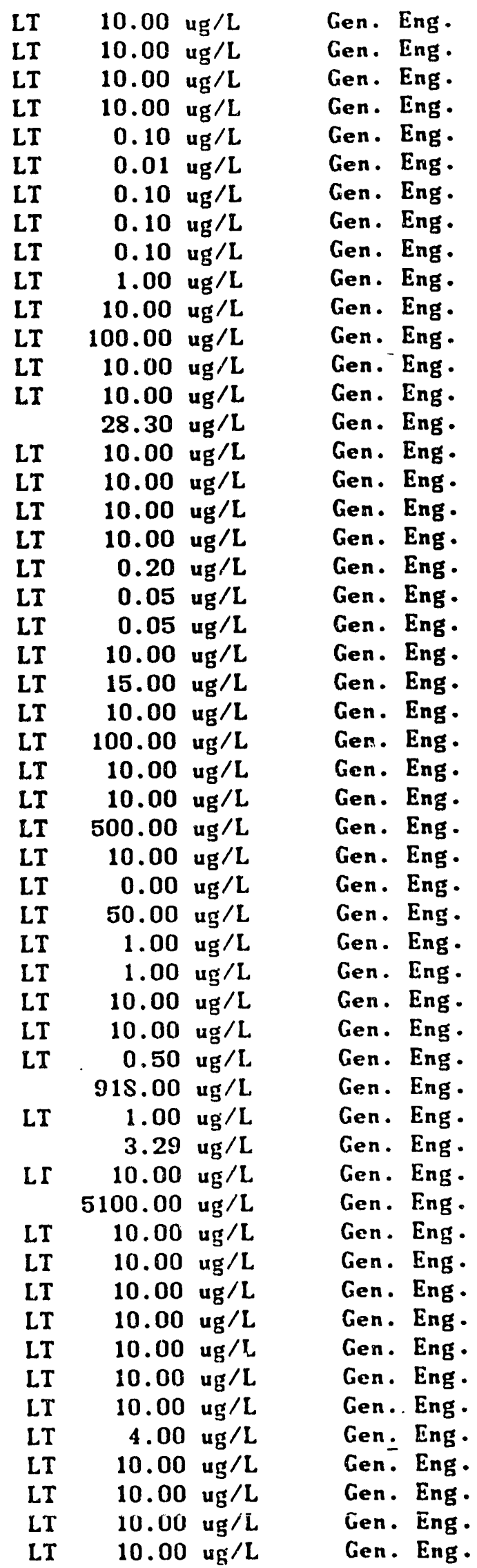


WELL LFW 38 COLEECTED ON 08/26/91 LABORATORY ANALYSES CONTINUED

4-Chlorouniline
4-Chlorophenyl phenyl ether
para-Cliloro-meta-cresol
-Cresol (4-Methylphenol)
4-Nitroaniline
4-Nitrophenol
2-Methyl-4,6-dinitrophenol
7,12-Dimethylbenz(a)anthracene
Zinc
Gross alpha
Nonvolatile beta
Total radium
Tritium

$\begin{array}{lrl}\mathrm{LT} & 10.00 \mathrm{ug} / \mathrm{L} \\ \mathrm{LT} & 10.00 \mathrm{ug} / \mathrm{L} \\ \mathrm{LT} & 10.00 \mathrm{ug} / \mathrm{L} \\ \mathrm{LT} & 10.00 \mathrm{ug} / \mathrm{L} \\ \mathrm{LT} & 10.00 \mathrm{ug} / \mathrm{L} \\ \mathrm{LT} & 10.00 \mathrm{ug} / \mathrm{L} \\ \mathrm{LT} & 50.00 \mathrm{ug} / \mathrm{L} \\ \mathrm{LT} & 10.00 \mathrm{ug} / \mathrm{L} \\ \mathrm{LT} & 2.00 \mathrm{ug} / \mathrm{L} \\ \mathrm{LT} & 2.00 \mathrm{pCi} / \mathrm{L} \\ 2.80+- & 2.00 \mathrm{pCi} / \mathrm{L} \\ 2.20+- & 2.90 \mathrm{pCi} / \mathrm{L} \\ 7.40+- & 0.40 \mathrm{pCi} / \mathrm{mL}\end{array}$

Gen. Eng .

Gen. Eng.

Gen. Eng.

Gen. Eng.

Gen. Eng.

Gen. Eng.

Gen. Eng.

Gen. Eng.

Gen. Eng.

Gen. Eng.

Gen. Eng.

Gen. Eng.

Gen. Eng.

WELL LFW 39

SRS Grid N 83213.1 Latitude $33.285220 N$

Coordinates E 46218.5 Longitude 81.706738 W

Screen Zone Elevation 152.2-131.2 ft ms l

Top of Casing Elevation $169.20 \mathrm{ft} \mathrm{msl}$

MEASUREMENTS CONDUCTED IN THE FIELD

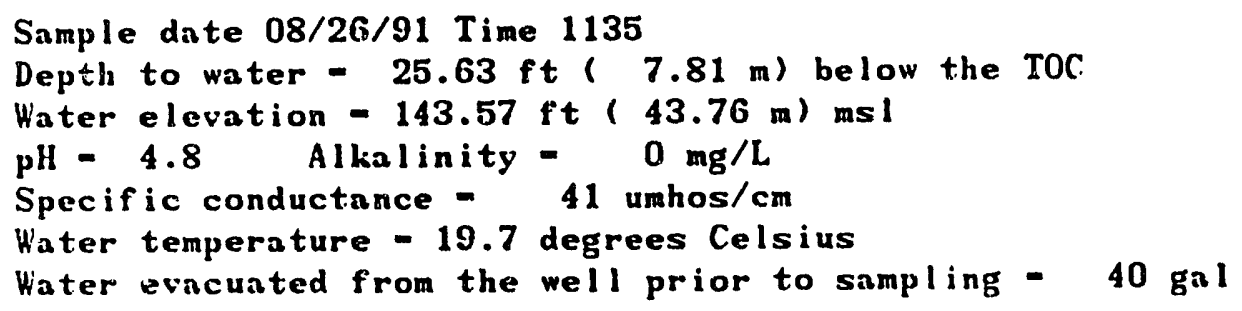

LABORATORY ANALYSES

Specific conductance

pH

Silver

Aluminum

Aldrin

Arsenic

Barium

beta-Benzene hexachloride

Bromodichloromethane

Calcium

Trichlorof luoromethane

Carbon tetrachloride

Cadmi um

Bromoform

Chloroform

Dichloromethane (Methylene chloride)

Bromomethane (Methyl bromide)

Chloromethane (Methyl chloride)

Chlorido

C.ONTINUED

$\begin{array}{lrll} & 35.00 \mathrm{umh} / \mathrm{cm} & \text { Gen. Eng. } \\ & 5.00 \mathrm{ull} & \text { Gen. Eng. } \\ \text { LT } & 2.00 \mathrm{ug} / \mathrm{L} & \text { Gen. Eng. } \\ & 23.90 \mathrm{ug} / \mathrm{L} & \text { Gen. Eng. } \\ \text { LT } & 0.05 \mathrm{ug} / \mathrm{L} & \text { Gen. Eng. } \\ \text { LT } & 2.00 \mathrm{ug} / \mathrm{L} & \text { Gen. Eng. } \\ & 5.98 \mathrm{ug} / \mathrm{L} & \text { Gen. Eng. } \\ \text { LT } & 0.05 \mathrm{ug} / \mathrm{L} & \text { Gen. Eng. } \\ \text { LT } & 1.00 \mathrm{ug} / \mathrm{L} & \text { Gen. Eng. } \\ & 402.00 \mathrm{ug} / \mathrm{L} & \text { Gen. Eng. } \\ & 400.00 \mathrm{ug} / \mathrm{L} & \text { Gen. Eng. } \\ \text { LT } & 1.00 \mathrm{ug} / \mathrm{L} & \text { Gen. Eng. } \\ \text { LT } & 2.00 \mathrm{ug} / \mathrm{L} & \text { Gen. Eng. } \\ \text { LT } & 1.00 \mathrm{ug} / \mathrm{L} & \text { Gen. Eng. } \\ \text { LT } & 1.00 \mathrm{ug} / \mathrm{L} & \text { Gen. Eng. } \\ & 51.00 \mathrm{ug} / \mathrm{L} & \text { Gen. Eng. } \\ \text { LT } & 1.00 \mathrm{ug} / \mathrm{L} & \text { Gen. Eng. } \\ \text { LT } & 1.00 \mathrm{ug} / \mathrm{L} & \text { Gen. Eng. } \\ & 5600.00 \mathrm{ug} / \mathrm{L} & \text { Gen. Eng. }\end{array}$


WELL LFW 40

Coordinates E 46395.1 Longitude 81.706343 W

Screen Zone Elevation 152.2-131.2 ft msl

Top of Casing Elevation $169.20 \mathrm{ft} \mathrm{msl}$

MEASUREMENTS CONDUCTED IN TIIE FIELD

Sample date 08/26/91 Time 1105

Depth to water - $25.69 \mathrm{ft}(7.83 \mathrm{~m})$ below the TOC

Water elevation - $143.51 \mathrm{ft}(43.74 \mathrm{~m}) \mathrm{ms}$ l

$\mathrm{pH}=4.9$ Alkalinity - $0 \mathrm{mg} / \mathrm{L}$

Specific conductance - 26 umhos/cm

Water temperature $=19.6$ degrees Celsius

Water evacuated from the well prior to sampling $=40 \mathrm{~g}$

\section{LABORATORY ANALYSES}

Specific conductance
pH
Silver
Aluminum
Aldrin
Arsenic
Barium
beta-Benzene hexachloride
Bromodichloromethane
Calcium
Trichlorofluoromethane
Carbon tetrachloride
Cadmium
Bromoform
Chloroform
Dichloromethane (Methylene chloride)
Bromomethane (Methyl bromide)
Chloromethane (Methyl chloride)
Chloride
Chlorobenzene
Chromium
Copper
Chlorocthene (Vingl chloride)
Chloroethane
Benzene
Dibromochloromethane
Endrin
Endosulfan sulfate
Ethylbenzene
Fluoride
Iron
Mercury
Potassium
Lindane
Toluene
Mothosychlor
Magnesium
ONTINUED
(a)

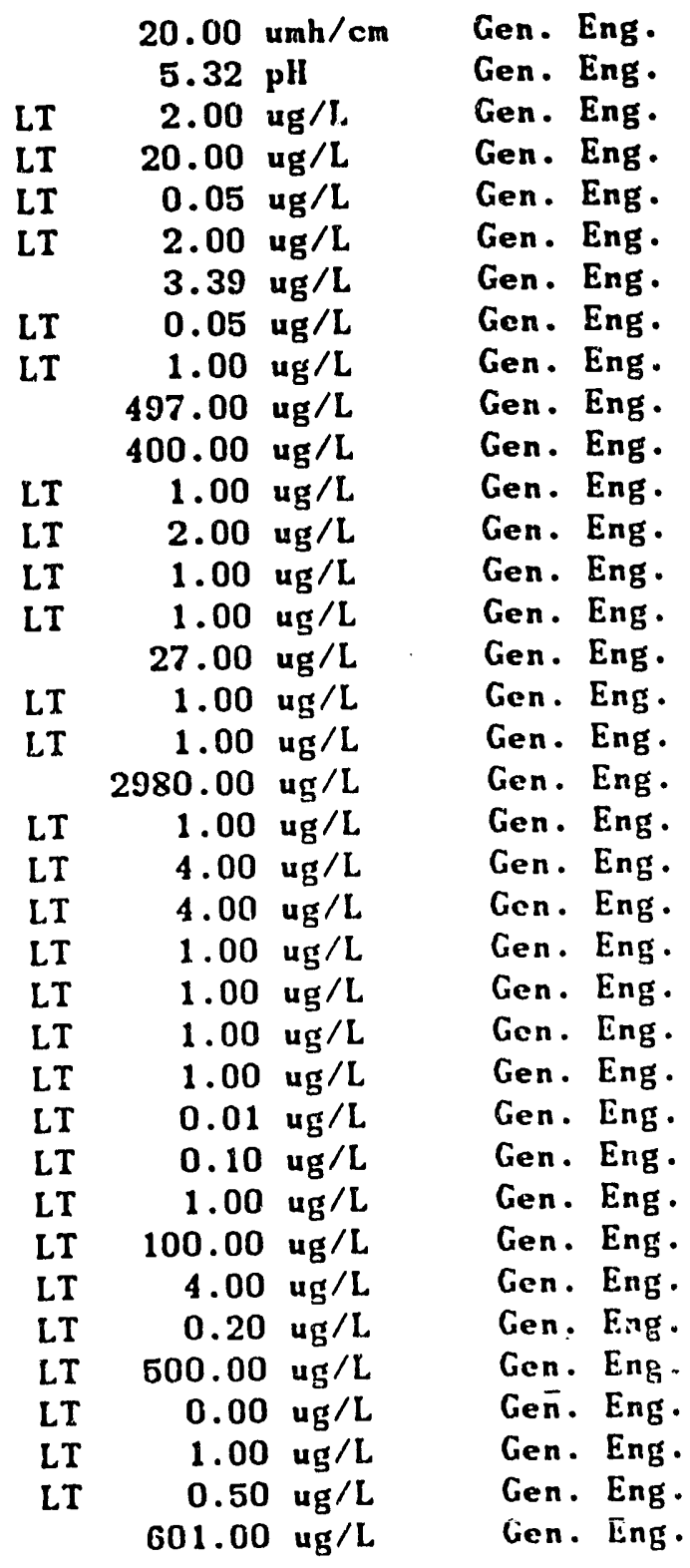


WELL LFW 40 COLLECTED ON 08/26/91 LABORATORY ANALYSES CONTINUED

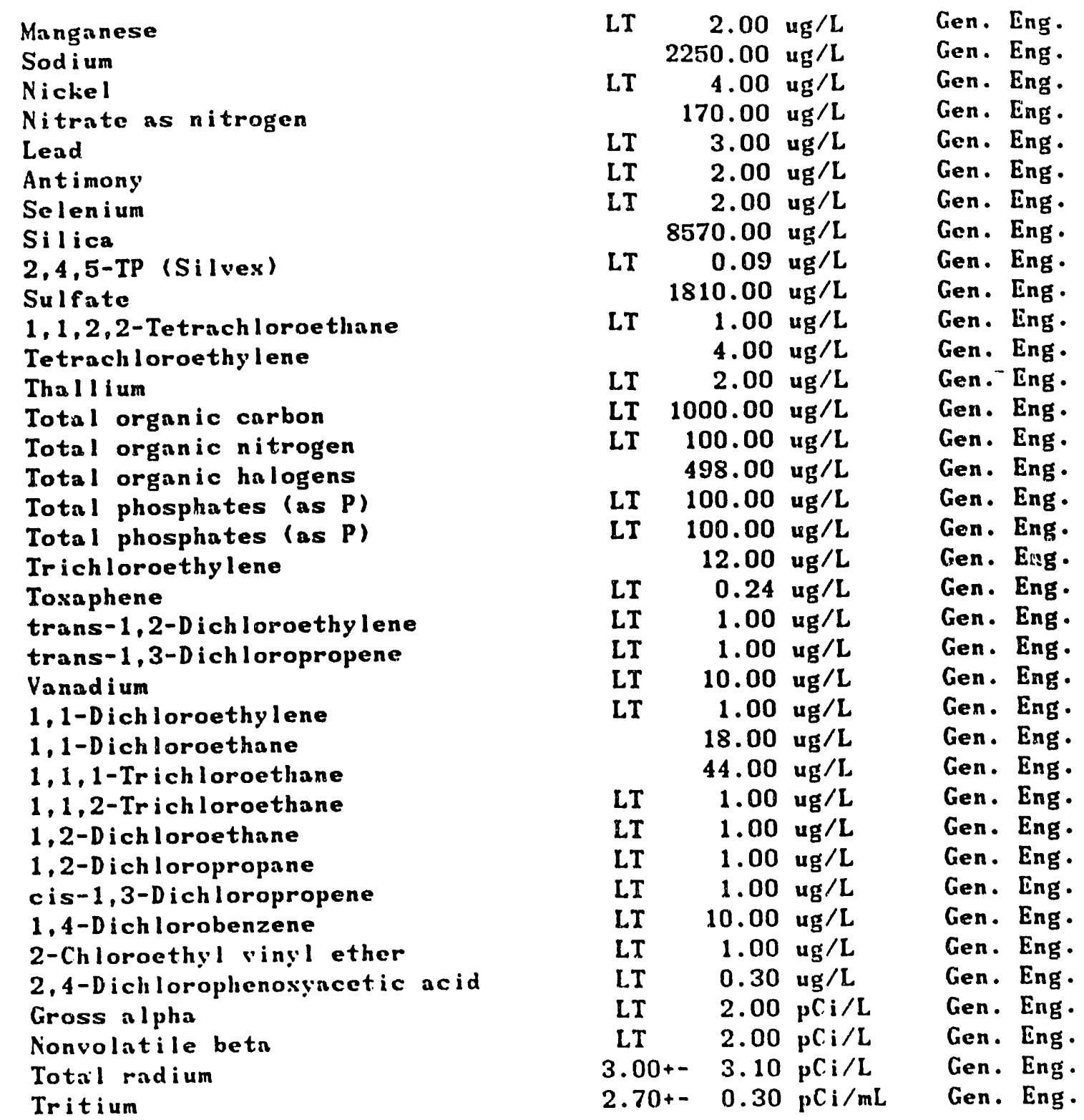


WELL LFW 40

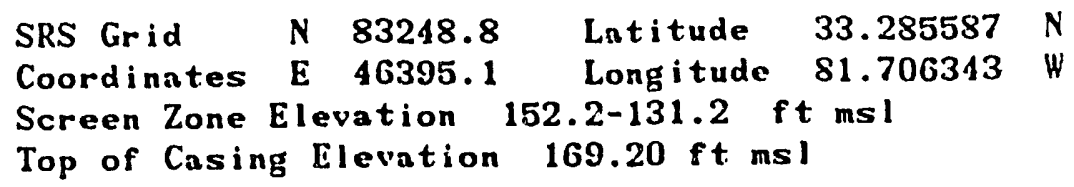

\section{MEASUREMENTS CONDUCTED IN THE FIELD}

Sample date 08/26/91 Time 1105

Depth to water - $25.69 \mathrm{ft}(7.83 \mathrm{~m})$ below the TOC

Water elevation $-143.51 \mathrm{ft}(43.74 \mathrm{~m}) \mathrm{msl}$

$\mathrm{pH}=4.9$ Alkalinity $=0 \mathrm{mg} / \mathrm{L}$

Specific conductance = 26 umhos/cm

Water temperature - 19.6 degrees Celsius

Water evacuated from the well prior to sampling - $40 \mathrm{gal}$

\section{LABORATORY ANALYSES}

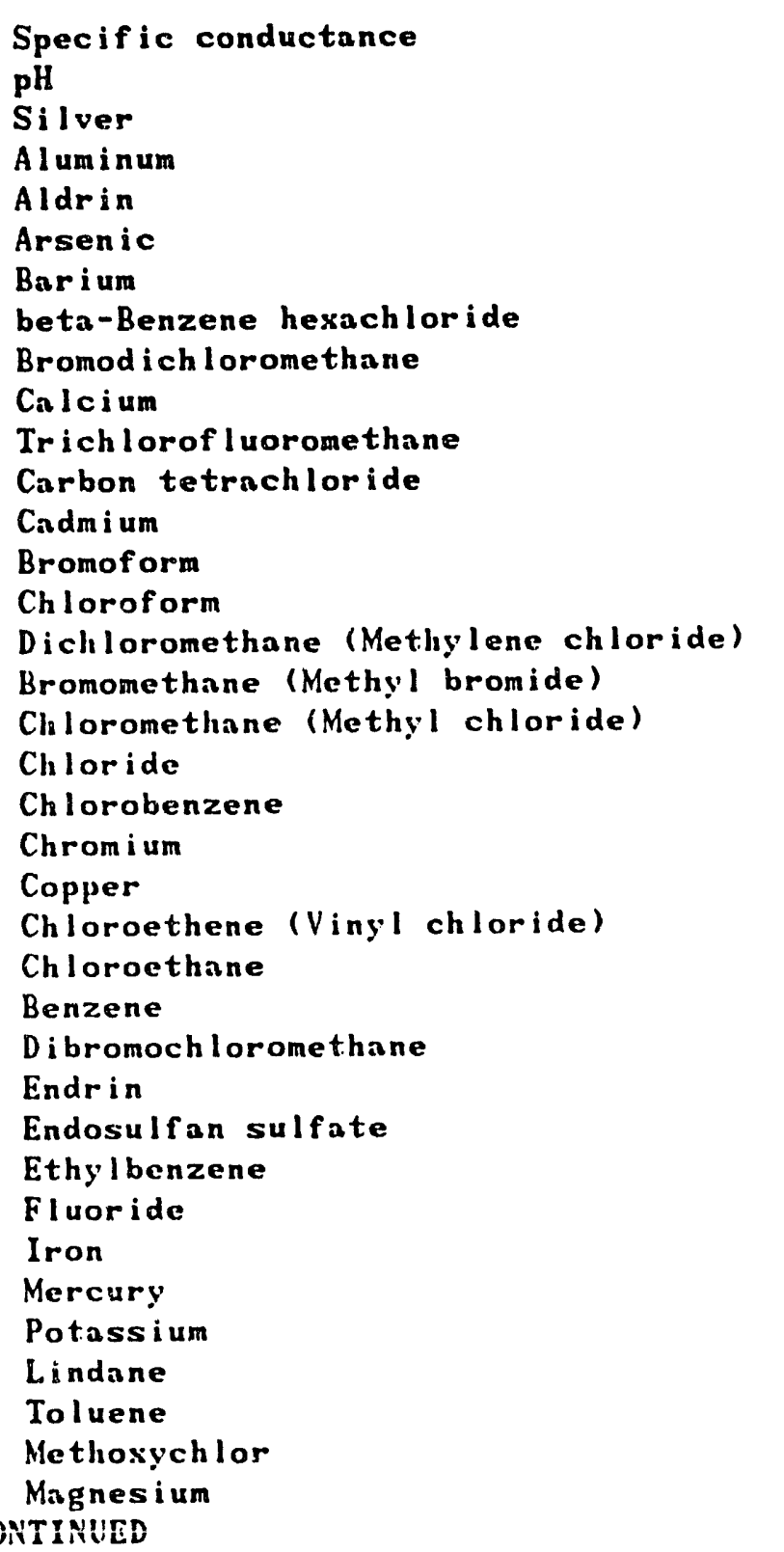

$\begin{array}{lrll} & 20.00 \mathrm{umh} / \mathrm{cm} & \text { Gen. Eng. } \\ & 5.15 \mathrm{uIl} & \text { Gen. Eng. } \\ \text { LT } & 2.00 \mathrm{ug} / \mathrm{L} & \text { Gen. Eng. } \\ \text { LT } & 20.00 \mathrm{ug} / \mathrm{L} & \text { Gen. Eng. } \\ \text { LT } & 0.05 \mathrm{ug} / \mathrm{L} & \text { Gen. Eng. } \\ \text { LT } & 2.00 \mathrm{ug} / \mathrm{L} & \text { Gen. Eng. } \\ & 3.15 \mathrm{ug} / \mathrm{L} & \text { Gen. Eng. } \\ \text { LT } & 0.05 \mathrm{ug} / \mathrm{L} & \text { Gen. Eng. } \\ \text { LT } & 1.00 \mathrm{ug} / \mathrm{L} & \text { Gen. Eng. } \\ & 494.00 \mathrm{ug} / \mathrm{L} & \text { Gen. Eng. } \\ & 400.00 \mathrm{ug} / \mathrm{L} & \text { Gen. Eng. } \\ \text { LT } & 1.00 \mathrm{ug} / \mathrm{L} & \text { Gen. Eng. } \\ \text { LT } & 2.00 \mathrm{ug} / \mathrm{L} & \text { Gen. Eng. } \\ \text { LT } & 1.00 \mathrm{ug} / \mathrm{L} & \text { Gen. Eng. } \\ \text { LT } & 1.00 \mathrm{ug} / \mathrm{L} & \text { Gen. Eng. } \\ & 28.00 \mathrm{ug} / \mathrm{L} & \text { Gen. Eng. } \\ \text { LT } & 1.00 \mathrm{ug} / \mathrm{L} & \text { Gen. Eng. } \\ \text { LT } & 1.00 \mathrm{ug} / \mathrm{L} & \text { Gen. Eng. } \\ & 2910.00 \mathrm{ug} / \mathrm{L} & \text { Gen. Eng. } \\ \text { LT } & 1.00 \mathrm{ug} / \mathrm{L} & \text { Gen. Eng. } \\ \text { LT } & 4.00 \mathrm{ug} / \mathrm{L} & \text { Gen. Eng. } \\ \text { LT } & 4.00 \mathrm{ug} / \mathrm{L} & \text { Gen. Eng. } \\ \text { LT } & 1.00 \mathrm{ug} / \mathrm{L} & \text { Gen. Eng. } \\ \text { LT } & 1.00 \mathrm{ug} / \mathrm{L} & \text { Gen. Eng. } \\ \text { LT } & 1.00 \mathrm{ug} / \mathrm{L} & \text { Gen. Eng. } \\ \text { LT } & 1.00 \mathrm{ug} / \mathrm{L} & \text { Gen. Eng. } \\ \text { LT } & 0.01 \mathrm{ug} / \mathrm{L} & \text { Gen. Eng. } \\ \text { LT } & 0.10 \mathrm{ug} / \mathrm{L} & \text { Gen. Eng. } \\ \text { LT } & 1.00 \mathrm{ug} / \mathrm{L} & \text { Gen. Eng. } \\ \text { LT } & 100.00 \mathrm{ug} / \mathrm{L} & \text { Gen. Eng. } \\ \text { LT } & 4.00 \mathrm{ug} / \mathrm{L} & \text { Gen. Eng. } \\ \text { LT } & 0.20 \mathrm{ug} / \mathrm{L} & \text { Gen. Eng. } \\ \text { LT } & 500.00 \mathrm{ug} / \mathrm{L} & \text { Gen. Eng. } \\ \text { LT } & 0.00 \mathrm{ug} / \mathrm{L} & \text { Gen. Eng. } \\ \text { LT } & 1.00 \mathrm{ug} / \mathrm{L} & \text { Gen. Eng. } \\ & 0.50 \mathrm{ug} / \mathrm{L} & \text { Gen. Eng. } \\ & 585.00 \mathrm{ug} / \mathrm{L} & \text { Gen. Eng. }\end{array}$


WELL LFW 40 COLLECTED ON 08/26/91 LABORATORY ANALYSES CONTINUFD

Manganese

Sod i um

Nickel

Nitrate as nitrogen

Lead

Antimony

Selen i um

Silica

2,4,5-TP (Silvex)

Sulfate

1,1,2,2-Tetrachloroethane

Tetrachloroethylene

Thalli um

Total organic carbon

Total organic nitrogen

Total organic halogens

Total phosphates (as P)

* Trichloroethylene

Toxaphene

trans-1,2-Dichloroethylene trans-1,3-Dich loropropene

Vanadi um

1,1-Dichloroethylene

1,1-Dichloroethane

1,1,1-Trichloroethane

$1,1,2-$ Trichloroethane

1,2-Dichloroethane

1,2-Dich loropropane

cis-1,3-Dich loropropene

1,4-Dich lorobenzene

2-Chloroethyl vinyl ether

2,4-Dichlorophenoxyacetic acid

Gross alpha

Nonvolatile beta

Total radium

Trit i um
LT

LT

T

$2.00 \mathrm{ug} / \mathrm{L}$

$2170.00 \mathrm{ug} / \mathrm{L}$

$4.00 \mathrm{ug} / \mathrm{L}$

$190.00 \mathrm{ug} / \mathrm{L}$

LT

LT

LT

LT

LT

$3.00 \mathrm{ug} / \mathrm{L}$

$2.00 \mathrm{ug} / \mathrm{L}$

$2.00 \mathrm{ug} / \mathrm{L}$

$8150.00 \mathrm{ug} / \mathrm{L}$

$0.09 \mathrm{ug} / \mathrm{L}$

$2360.00 \mathrm{ug} / \mathrm{L}$

$1.00 \mathrm{ug} / \mathrm{L}$

$3.00 \mathrm{ug} / \mathrm{L}$

LT

$2.00 \mathrm{ug} / \mathrm{L}$

$2000.00 \mathrm{ug} / \mathrm{L}$

$370.00 \mathrm{ug} / \mathrm{L}$

$461.00 \mathrm{ug} / \mathrm{L}$

LT

$100.00 \mathrm{ug} / \mathrm{L}$

$11.00 \mathrm{ug} / \mathrm{L}$

LI $0.24 \mathrm{ug} / \mathrm{L}$

LT $1.00 \mathrm{ug} / \mathrm{L}$

LT $1.00 \mathrm{ug} / \mathrm{L}$

LT

LT

$10.00 \mathrm{ug} / \mathrm{L}$

$1.00 \mathrm{ug} / \mathrm{L}$

$17.00 \mathrm{ug} / \mathrm{L}$

$46.00 \mathrm{ug} / \mathrm{L}$

LT $\quad 1.00 \mathrm{ug} / \mathrm{L}$

LT

LT

LT

LT

LT

LT

LT

$1.00 \mathrm{ug} / \mathrm{L}$

$1.00 \mathrm{ug} / \mathrm{L}$

$1.00 \mathrm{ug} / \mathrm{L}$

$10.00 \mathrm{ug} / \mathrm{L}$

$1.00 \mathrm{ug} / \mathrm{L}$

$0.30 \mathrm{ug} / \mathrm{L}$

$2.00 \mathrm{pC} i / \mathrm{L}$

LT

$2.00 \mathrm{pC} i / \mathrm{L}$

$3.50+-3.30 \mathrm{pCi} / \mathrm{L}$

$2.65+-0.30 \mathrm{pCi} / \mathrm{mL}$
Gen. Eng.

Gen. Eng.

Gen. Eng.

Gen. Eng.

Gen. Eng.

Gen. Eng .

Gen. Eng.

Gen. Eng.

Gen. Eng.

Gen. Eng.

Gen. Eng.

Gen. Eng -

Gen - Eng .

Gen. Eng.

Gen. Eng -

Gen. Eng .

Gen. Eng.

Gen. Eng.

Gen. Eng.

Gen. Eng.

Gen. Eng .

Gen. Eng.

Gen. Eng.

Gen. Eng.

Gen. Eng.

Gen. Eng.

Gen. Eng.

Gen. Eng -

Gen. Eng.

Gen. Eng.

Gen, Eng.

Gen. Eng.

Gen. Eng.

Gen. Eng.

Gen. Eng.

Gen. Eng. 
WELL LFW 41

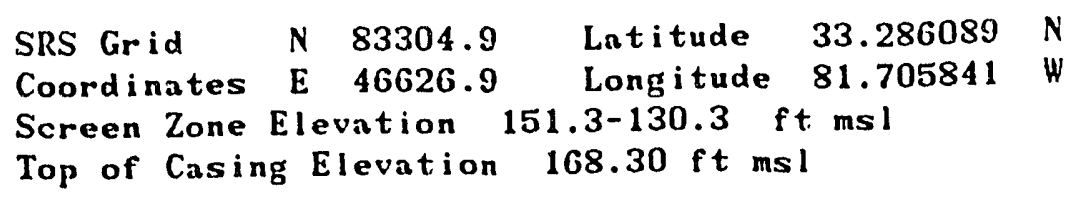

\section{MEASUREMENTS CONDUCTED IN THE FIELD}

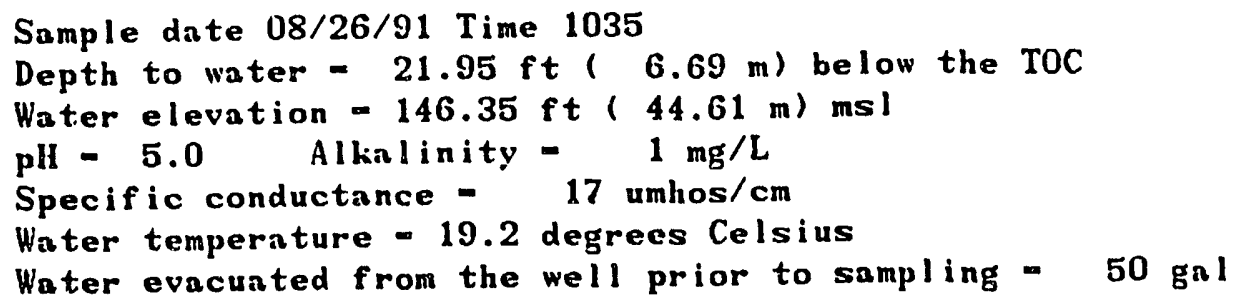

\section{LABORATORY ANALYSES}

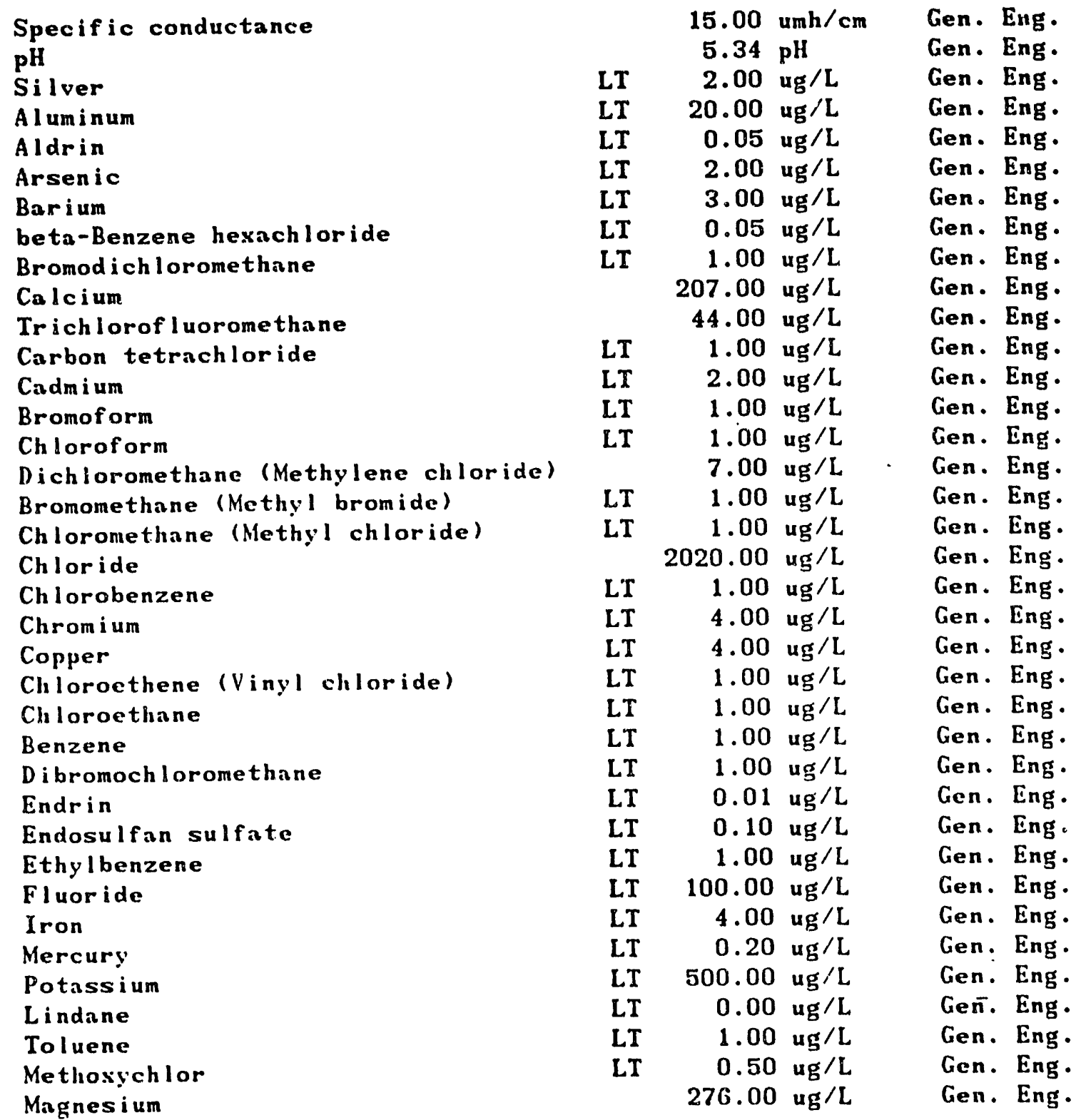


WELL LFW 41 COLLECTED ON 08/26/9: LABORATORY ANALYSES CONTINUED

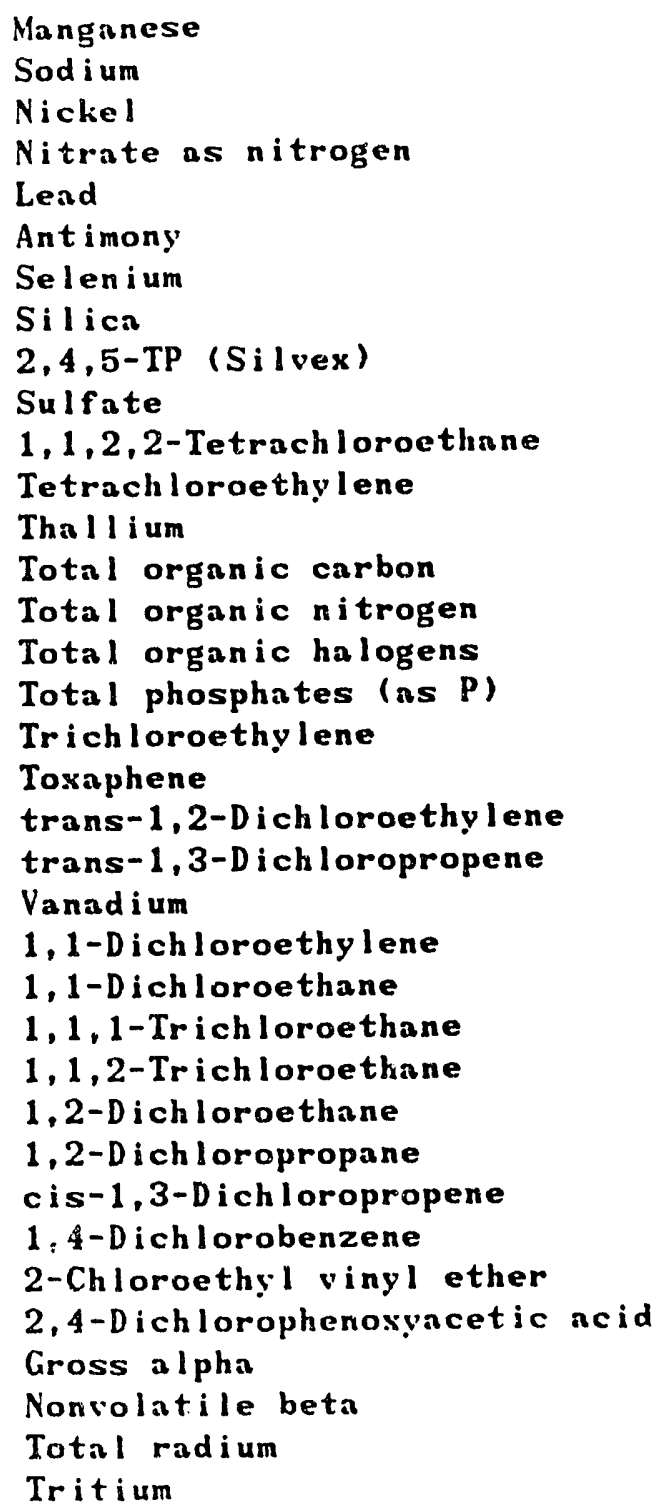

\begin{tabular}{|c|c|c|c|}
\hline LT & $\begin{array}{r}2.00 \\
1430.00\end{array}$ & $\begin{array}{l}\text { ug } / L \\
\text { ug } / L\end{array}$ & $\begin{array}{l}\text { Gen. } \\
\text { Gen. }\end{array}$ \\
\hline LT & 4.00 & ug $/ \mathrm{L}$ & Gen. \\
\hline LT & 50.00 & ug $/ \mathrm{L}$ & Gen. \\
\hline LT & 3.00 & $u_{g} / L$ & Gen. \\
\hline LT & 2.00 & $\mathrm{ug} / \mathrm{L}$ & Gen. \\
\hline LT & 2.00 & $u_{g} / L$ & Gen. \\
\hline & 7350.00 & $\mathbf{u g} / \mathrm{L}$ & Gen. \\
\hline L.T & $\begin{array}{r}0.09 \\
1100.00\end{array}$ & $\begin{array}{l}\text { ug } / L \\
\text { ug } / L\end{array}$ & $\begin{array}{l}\text { Gen. } \\
\text { Gen. }\end{array}$ \\
\hline LT & 1.00 & ug/L & Gen. \\
\hline LT & 1.00 & $u g / L$ & Gen. \\
\hline LT & 2.00 & ug $/ L$ & en - \\
\hline LT & 1000.00 & $u g / L$ & en. \\
\hline LT & $\begin{array}{r}100.00 \\
6.00\end{array}$ & $\begin{array}{l}u_{g} / L \\
u g / L\end{array}$ & $\begin{array}{l}\text { Gen. } \\
\text { Gen. }\end{array}$ \\
\hline LT & 100.00 & ug/L & Gen. \\
\hline LT & 1.00 & ug/L & en. \\
\hline LT & 0.24 & $\mathrm{ug} / \mathrm{L}$ & Gen. \\
\hline LT & 1.00 & $\mathrm{ug} / \mathrm{L}$ & en. \\
\hline LT & 1.00 & ug/L & Gen. \\
\hline LT & 10.00 & ug/L & en. \\
\hline LT & 1.00 & ug/L & en. \\
\hline LT & 1.00 & ug/L & en. \\
\hline & 4.00 & ug $/ \mathrm{L}$ & en. \\
\hline LT & 1.00 & $\mathrm{ug} / \mathrm{L}$ & Gen. \\
\hline LI & 1.00 & ug $/ \mathrm{L}$ & Gen. \\
\hline LT & 1.00 & $\mathrm{ug} / \mathrm{L}$ & Gen. \\
\hline LT & 1.00 & ug $/ \mathrm{L}$ & Gen. \\
\hline LT & 10.00 & $u_{g} / L$ & Gen. \\
\hline LT & 1.00 & ug $/ \mathrm{L}$ & Ger \\
\hline LT & 0.30 & ug/L & Gen \\
\hline LT & 2.00 & $\mathrm{pCi} / \mathrm{L}$ & Gen \\
\hline LT & 2.00 & $\mathrm{pCi} / \mathrm{L}$ & Gen \\
\hline 5 & 3.40 & $\mathrm{pC} \mathrm{i} / \mathrm{L}$ & \\
\hline 08 & 0.30 & $\mathrm{pCi} / \mathrm{mL}$ & \\
\hline
\end{tabular}


HELL LFW 42

SRS Grid $\quad N \quad 83776.2 \quad$ Latitude $33.286978 \mathrm{~N}$
Coordinates E $46532.9 \quad$ Longitude 81.707005
Screen Zone Elevation $151.2-130.2 \mathrm{ft} \mathrm{ms}$
Top of Casing Elevation $168.20 \mathrm{ft}$ ms l

\section{MEASUREMENTS CONDUCTED IN TIE FIELD}

Sample date 08/26/91 Time 1005

Depth to water $=20.03 \mathrm{ft}(6.11 \mathrm{~m})$ below the TOC

Water elevation - $148.17 \mathrm{ft}(45.16 \mathrm{~m}) \mathrm{ms}$ )

$\mathrm{pH}=5.0 \quad$ Alkalinity - $0 \mathrm{mg} / \mathrm{L}$

Specific conductance - 15 umhos/cm

Water temperature - 19.5 degrees Celsius

Water evacuated from the well prior to sampling - $55 \mathrm{gal}$

\section{LABORATORY ANALYSES}

Specific conductance

pH

Silver

Aluminum

Aldrin

Arsenic

Barium

beta-Benzene hexachloride

Bromodich loromethane

Calcium

Trichlorof l uoromethane

Carbon tetrnchloride

Cadmi um

Bromoform

Chlorof orm

Dichloromethane (Methylene chloride)

Bromomethane (Mcthyl bromide)

Chloronethane (Methyl chloride)

Chlor ide

Chlorobenzene

Chromium

Copper

Chloroethene (Vingl chloride)

Chloroethane

Benzene

Dibromochloromethane

Endrin

Endosulfan sulfate

Ethylbenzene

Fluoride

Fluoride

Iron

Mercury

Potassium

Lindane

Toluene

Methoxychlor

CONTINUED
$10.00 \mathrm{umh} / \mathrm{cm}$

$5.33 \mathrm{pH}$

LT

LT

$2.00 \mathrm{ug} / \mathrm{L}$

LT

LT

LT

LT

$0.05 \mathrm{ug} / \mathrm{L}$

$2.00 \mathrm{ug} / \mathrm{L}$

$3.00 \mathrm{ug} / \mathrm{L}$

$0.05 \mathrm{ug} / \mathrm{L}$

$1.00 \mathrm{ug} / \mathrm{L}$

$231.00 \mathrm{ug} / \mathrm{L}$

$69.20 \mathrm{ug} / \mathrm{L}$

LT $\quad 1.00 \mathrm{ug} / \mathrm{L}$

LI $2.00 \mathrm{ug} / \mathrm{L}$

LT

LT

LT

$1.00 \mathrm{ug} / \mathrm{L}$

$1.00 \mathrm{ug} / \mathrm{L}$

$1.58 \mathrm{ug} / \mathrm{L}$

$1.00 \mathrm{ug} / \mathrm{L}$

$1.00 \mathrm{ug} / \mathrm{L}$

$2070.00 \mathrm{ug} / \mathrm{L}$

LT $\quad 1.00 \mathrm{ug} / \mathrm{L}$

LI $4.00 \mathrm{ug} / \mathrm{L}$

LI

LT

LT

LT

LT

LT

LT

LT

LT

LT

LT

LT

LT

LT

LT

LT
$4.00 \mathrm{ug} / \mathrm{L}$

$1.00 \mathrm{ug} / \mathrm{L}$

$1.00 \mathrm{ug} / \mathrm{L}$

$1.00 \mathrm{ug} / \mathrm{L}$

$1.00 \mathrm{ug} / \mathrm{L}$

$0.01 \mathrm{ug} / \mathrm{L}$

$0.10 \mathrm{ug} / \mathrm{L}$

$1.00 \mathrm{ug} / \mathrm{L}$

$100.00 \mathrm{ug} / \mathrm{L}$

$100.00 \mathrm{ug} / \mathrm{L}$

$4.00 \mathrm{ug} / \mathrm{L}$

$0.20 \mathrm{ug} / \mathrm{L}$

$500.00 \mathrm{ug} / \mathrm{L}$

$0.00 \mathrm{ug} / \mathrm{L}$

$1.00 \mathrm{ug} / \mathrm{L}$

$0.50 \mathrm{ug} / \mathrm{L}$
Gen. Eng.

Gen. Eng.

Gen. Eng.

Gen. Eng.

Gen. Eng.

Gen. Eng.

Gen. Eng.

Gen. Eng.

Gen. Eng.

Gen. Eng.

Gen. Eng.

Gen. Eng.

Gen. Eng.

Gen. Eng.

Gen. Eng.

Gen. Eng.

Gen. Eng.

Gen. Eng.

Gen. Eng .

Gen. Eng.

Gen. Eng.

Gen. Eng.

Gen. Eng.

Gen. Eng.

Gen. Eng.

Gen. Eng.

Gen. Eng.

Gen. Eng.

Gen. Eng.

Gen. Eng.

Gen. Eng.

Gen. Eng.

Gen. Eng.

Ger. Eng.

Gen. Eng.

Gen. Eng.

Gen. Eng - 


\author{
Magnes i um \\ Manganese \\ Sod i um \\ Nickel \\ Nitrate as nitrogen \\ Lead \\ Antimony \\ Selenium \\ Silica \\ 2,4,5-TP (Silvex) \\ Sulfate \\ Sulfate \\ 1,1,2,2-Tetrachloroethane \\ Tetrachloroe thylene \\ Tha Il i um \\ Total organic carbon \\ Total organic carbon \\ Total organic nitrogen \\ Total organic halogens \\ Total phosphates (as P) \\ Trichloroethylene \\ Toxnphene \\ trans-1,2-Dichloroethylene \\ trans-1,3-Dich loropropene \\ Vanadium \\ 1,1-Dichloroethylene \\ 1,1-Dichloroethane \\ 1,1,1-Trichloroethane \\ 1,1,2-Trichloroethane \\ 1,2-Dichloroethane \\ 1,2-Dichloropropane \\ c is -1,3-D ichloropropene \\ $1,4-D i c h$ lorobenzene \\ 2-Chloroethyl vinyl ether \\ 2,4-Dichlorophenosyacetic acid \\ Gross alpha \\ Nonvolatile beta \\ * Total radium \\ Tritium
}

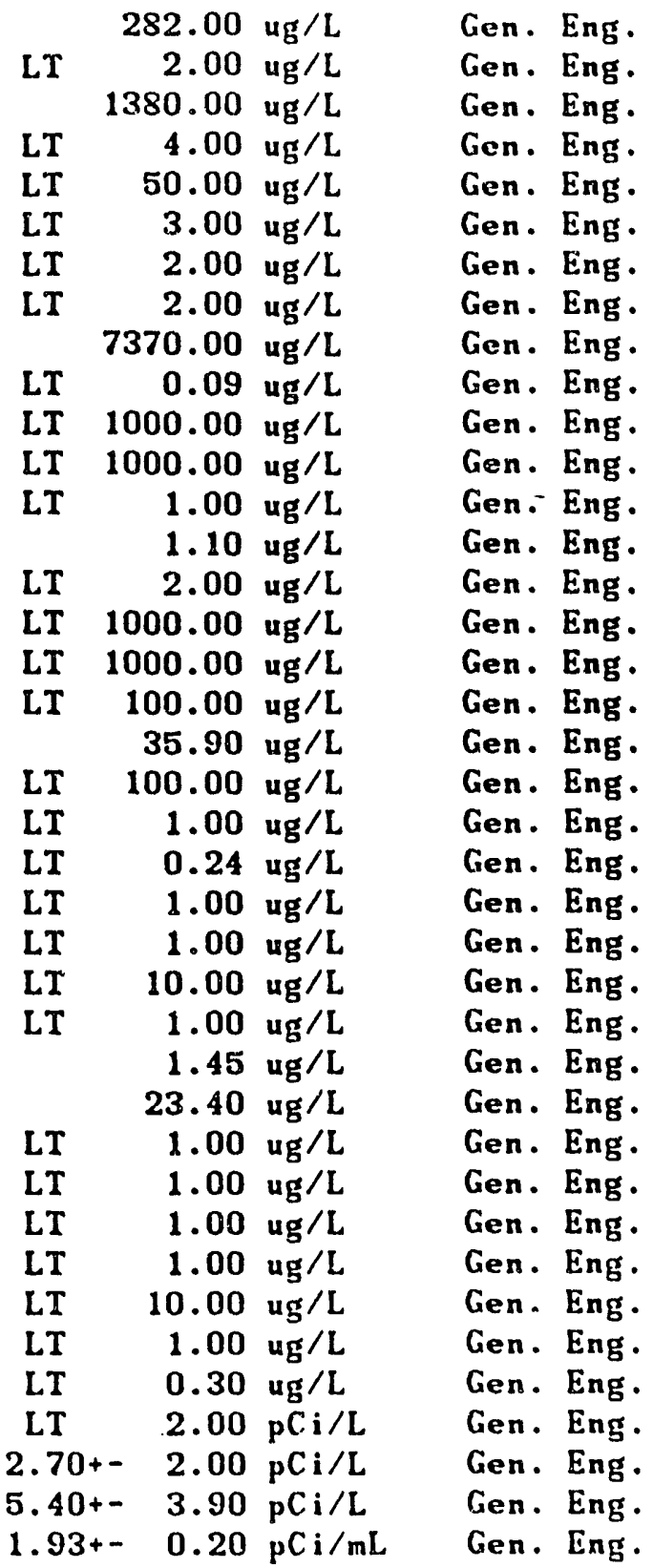


WELL LFW 43B

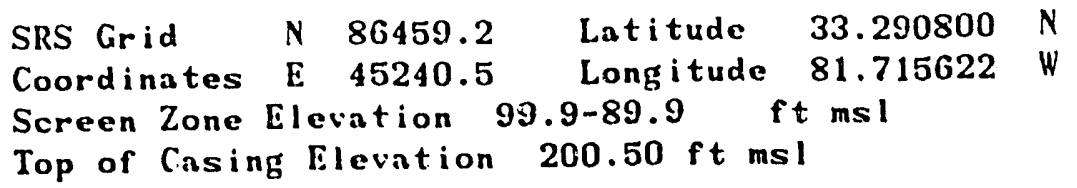

\section{MEASUREMENTS CONDUCTED IN THE FIELD}

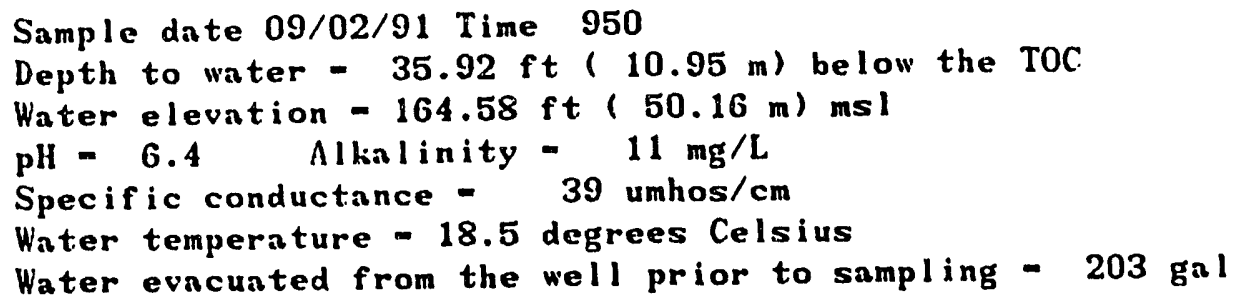

\section{LABORATORY ANALYSES}

Specific conductance
pH
Silver
Arsenic
Barium
Bromodichloromethane
Calcium
Trichlorofluoromethane
Carbon tetrachloride
Cadmium
Bromoform
Chloroform
Dichloromethane (Methylene chloride)
Bromomethane (Methyl bromide)
Chloromethane (Methyl chloride)
Chloride
Chlorobenzene
Chromium
Chloroethene (Vingl chloride)
Chlorocthane
Benzene
Dibromochloromethane
Endrin
Ethylbenzene
Fluoride
Iron
Mercury
Potassium
Lindane
Toluene
Metlosychlor
Magnesium
Manganese
Sodium
Nitrnte as nitrogen
Lend
Selenium
NTINugD

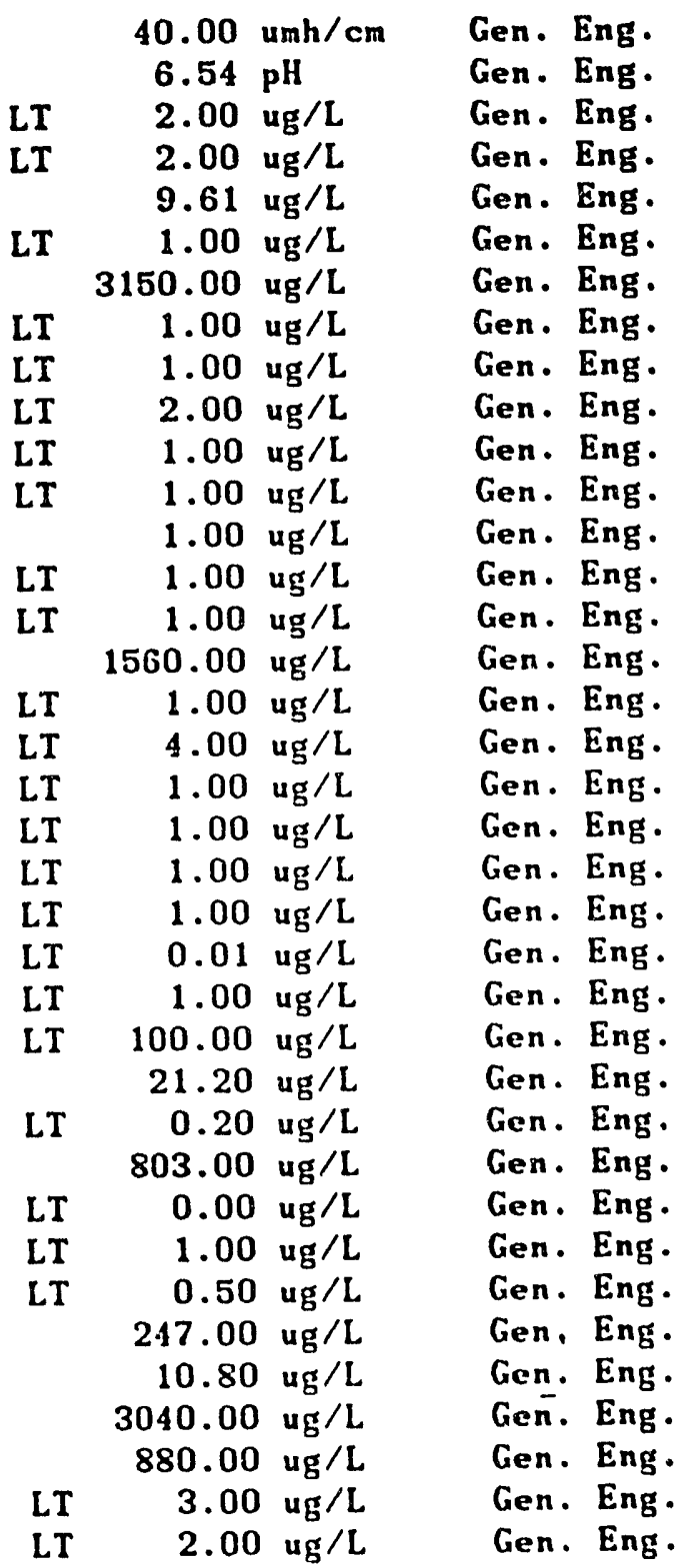


WELL LFW 43B C.OLLECTED ON 09/02/91 LABORATORY ANALYSES CONTINUED

Silica
$2,4,5-T P$ (Silvex)
Sulfate
1,1,2,2-Tetrachloroethane
Tetrachloroethylene
Total dissolved solids
Total organic carbon
Total organic halogens
Phenols
Total phosphates (as P)
Trichloroethylene
Toxaphene
trans-1,2-Dichloroethylene
trans-1,3-Dichloropropene
1,1-Dichloroethylene
1,1-Dichloroethane
1,1,1-Trichloroethane
1,1,2-Trichloroethane
1,2-Dichloroethane
1,2-Dichloropropane
cis-1,3-Dichloropropene
2-Chloroethyl vinyl ether
2,4-Dichlorophenoxyacetic acid
Gross alpha
Nonvolatile beta
Total radium
Tritium

\begin{tabular}{|c|c|c|c|c|}
\hline & 8760.00 & ug $/ \mathrm{L}$ & Gen. & Eng \\
\hline LT & 0.09 & ug $/ \mathrm{L}$ & Gen. & Eng \\
\hline LT & 1000.00 & ug $/ \mathrm{L}$ & Gen. & Eng \\
\hline $\mathrm{LT}$ & 1.00 & ug $/ \mathrm{L}$ & Gen. & Eng \\
\hline LT & 1.00 & ug/L & Gen. & Eng \\
\hline & $56000 . G J$ & ug $/ \mathrm{L}$. & Gen. & Eng \\
\hline LT & 1000.00 & $u g / L$ & Gen. & $E$ \\
\hline & 49.90 & $u g / L$ & Gen. & \\
\hline LT & 5.00 & $u g / L$ & Gen. & $n_{8}$ \\
\hline LT & 100.00 & $\mathbf{u g} / \mathrm{L}$ & Gen. & Eng \\
\hline LT & 1.00 & $u g / L$ & Gen. & Eng \\
\hline L.T & 0.24 & $u_{g} / L$ & Gen. & En \\
\hline L.T & 1.00 & ug $/ \mathrm{L}$ & Gen.- & En \\
\hline LT & 1.00 & ug/L & Gen. & En \\
\hline LT & 1.00 & ug/L & Gen. & \\
\hline LT & 1.00 & ug $/ L$ & Gen. & \\
\hline LT & 1.00 & $u_{g} / L$ & Gen. & \\
\hline LT & 1.00 & $u g / L$ & Gen. & En \\
\hline LT & 1.00 & $\mathbf{u g} / \mathrm{L}$ & Gen. & En \\
\hline LT & 1.00 & ug/L & Gen. & En \\
\hline LT & 1.00 & ug/L & Gen. & En \\
\hline LT & 1.00 & $u g / L$ & Gen. & En \\
\hline LT & 0.30 & ug/L & Gen. & \\
\hline LT & 2.00 & $\mathrm{pC} i / L$ & ien. & \\
\hline LT & 2.00 & $\mathrm{pCi} / \mathrm{L}$ & Gen. & \\
\hline LT & 1.00 & $\mathrm{pCi} / \mathrm{L}$ & Gen. & \\
\hline 80 & 0.20 & $\mathrm{i} / \mathrm{mL}$ & Ge & \\
\hline
\end{tabular}

WELL LFW $43 \mathrm{C}$

\section{SRS Grid N 86480.6 Latitude 33.290838 N \\ Coordinates E 45234.9 Longitude 81.715679 W \\ Screen Zone Elevation 138.1-128.1 ft ms I \\ Top of Casing Elevation $200.10 \mathrm{ft} \mathrm{msl}$}

\section{MEASUREMENTS CONDUCTED IN THE FIELD}

Sample date 09/02/91 Time 905

Depth to water - $35.02 \mathrm{ft}(10.67 \mathrm{~m})$ below the TOC

Water elevation $-165.08 \mathrm{ft}(50.32 \mathrm{~m}) \mathrm{msl}$

pll $=5.8 \quad$ Alkalinity $-8 \mathrm{mg} / \mathrm{L}$

Specific conductance - $\quad 34$ umhos/cm

Water temperature $=18.5$ degrees Celsius

Water evacuated from the well prior to sampling - $104 \mathrm{gal}$

\section{LABORATORY ANALYSES}

Specif ic conductance

pII

Silver

Arsenic

Bar ium

CONTINUED
$35.00 \mathrm{umh} / \mathrm{cm}$

6.13 pll

LT

2.00 ug $/ \mathrm{L}$

$2.00 \mathrm{ug} / \mathrm{L}$

$12.30 \mathrm{ug} / \mathrm{L}$
Gen. Eng .

Gen. Eng.

Geñ. Eng.

Gen. Eng.

Gen. Eng. 
WELL LFW 43C COLLECTED ON 09/02/91 LABORATORY ANALYSES CONTINUED

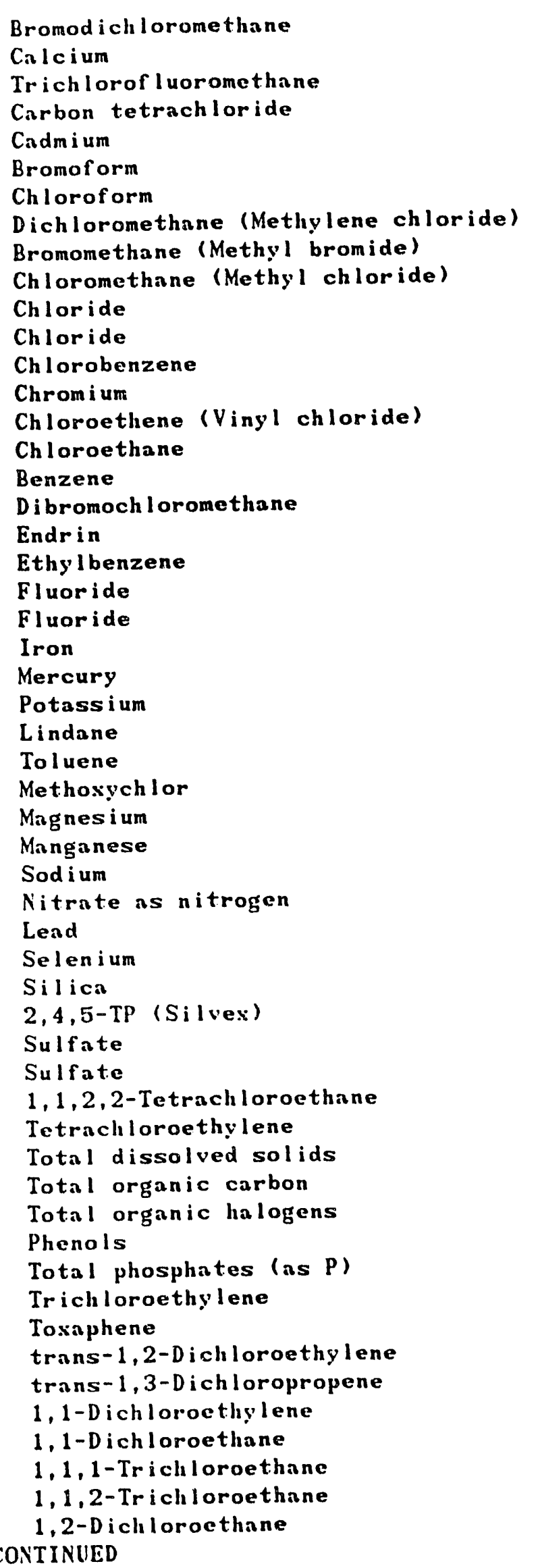

LT

\section{LT}

LT

LT

LT

LT

LI

LT

\section{LT}

LT

LT

\section{LT}

LT

LT

LT

LT

LT

LT

LT

LT

LT

LT

LT

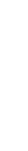

LI

LT

LT

LI

LT

LT

LT

\section{LT}

LT

LT

LT

LT

LT

LT

LT

LT

LT

LT

LT
$1.00 \mathrm{ug} / \mathrm{L}$ $3350.00 \mathrm{ug} / \mathrm{L}$

$1.00 \mathrm{ug} / \mathrm{L}$

$1.00 \mathrm{ug} / \mathrm{L}$

$2.00 \mathrm{ug} / \mathrm{L}$

$1.00 \mathrm{ug} / \mathrm{L}$

$1.00 \mathrm{ug} / \mathrm{L}$

$1.00 \mathrm{ug} / \mathrm{L}$

$1.00 \mathrm{ug} / \mathrm{L}$

$1.00 \mathrm{ug} / \mathrm{L}$

$1600.00 \mathrm{ug} / \mathrm{L}$

$1600.00 \mathrm{ug} / \mathrm{L}$

$1.00 \mathrm{ug} / \mathrm{L}$

$4.00 \mathrm{ug} / \mathrm{L}$

$1.00 \mathrm{ug} / \mathrm{L}$

$1.00 \mathrm{ug} / \mathrm{L}$

$1.00 \mathrm{ug} / \mathrm{L}$

$1.00 \mathrm{ug} / \mathrm{L}$

$0.01 \mathrm{ug} / \mathrm{L}$

$1.00 \mathrm{ug} / \mathrm{L}$

$100.00 \mathrm{ug} / \mathrm{L}$

$100.00 \mathrm{ug} / \mathrm{L}$

$4.10 \mathrm{ug} / \mathrm{L}$

$0.20 \mathrm{ug} / \mathrm{L}$

$500.00 \mathrm{ug} / \mathrm{L}$

$0.00 \mathrm{ug} / \mathrm{L}$

$1.00 \mathrm{ug} / \mathrm{L}$

$0.50 \mathrm{ug} / \mathrm{L}$

$442.00 \mathrm{ug} / \mathrm{L}$

$17.50 \mathrm{ug} / \mathrm{L}$

$1520.00 \mathrm{ug} / \mathrm{L}$

$530.00 \mathrm{ug} / \mathrm{L}$

$3.00 \mathrm{ug} / \mathrm{L}$

$2.00 \mathrm{ug} / \mathrm{L}$

$7750.00 \mathrm{ug} / \mathrm{L}$

$.0 .09 \mathrm{ug} / \mathrm{L}$

$1000.00 \mathrm{ug} / \mathrm{L}$

$1000.00 \mathrm{ug} / \mathrm{L}$

$1.00 \mathrm{ug} / \mathrm{L}$

$1.00 \mathrm{ug} / \mathrm{L}$

$47000.00 \mathrm{ug} / \mathrm{L}$

$1000.00 \mathrm{ug} / \mathrm{L}$

$119.00 \mathrm{ug} / \mathrm{L}$

$5.00 \mathrm{ug} / \mathrm{L}$

$100.00 \mathrm{ug} / \mathrm{L}$

$1.00 \mathrm{ug} / \mathrm{L}$

$0.24 \mathrm{ug} / \mathrm{L}$

$1.00 \mathrm{ug} / \mathrm{L}$

$1.00 \mathrm{ug} / \mathrm{L}$

$1.00 \mathrm{ug} / \mathrm{L}$

$1.00 \mathrm{ug} / \mathrm{L}$

$1.00 \mathrm{ug} / \mathrm{L}$

$1.00 \mathrm{ug} / \mathrm{L}$

$1.00 \mathrm{ug} / \mathrm{L}$
Gen. Eng.

Gen. Eng.

Gen. Eng.

Gen. Eng.

Gen. Eng.

Gen. Eng.

Gen. Eng -

Gen. Eng.

Gen. Eng.

Gen. Eng.

Gen. Eng -

Gen. Eng -

Gen.- Eng.

Gen. Eng.

Gen. Eng.

Gen. Eng.

Gen. Eng.

Gen. Eng.

Gen. Eng -

Gen. Eng .

Gen. Eng .

Gen. Eng.

Gen. Eng.

Gen. Eng.

Gen. Eng.

Gen. Eng.

Gen. Eng .

Gen. Eng.

Gen. Eng.

Gen. Eng.

Gen. Eng.

Gen. Eng.

Gen. Eng.

Gen. Eng.

Gen. Eng.

Gen. Eng.

Gen. Eng.

Gen. Eng.

Gen. Eng.

Gen. Eng.

Gen. Eng.

Gen. Eng.

Gen. Eng.

Gen. Eng.

Gen. Eng.

Gen. Eng.

Gen. Eng.

Gen. Eng .

Gen. Eng.

Gen. Eng.

Geñ. Eng.

Gen. Eng.

Gen. Eng.

Gen. Eng. 
WELL LFW 43C COLLECTED ON 09/02/91 LABORATORY ANALYSES CONTINUED

$\begin{array}{llll}\text { 1,2-Dichloropropane } & \mathrm{LT} & 1.00 \mathrm{ug} / \mathrm{L} & \text { Gen. Eng. } \\ \text { cis-1,3-Dichloropropene } & \mathrm{LT} & 1.00 \mathrm{ug} / \mathrm{L} & \text { Gen. Eng. } \\ \text { 2-Chloroethyl vinylether } & \mathrm{LT} & 1.00 \mathrm{ug} / \mathrm{L} & \text { Gen. Eng. } \\ \text { 2,4-Dichlorophenoxyacetic acid } & \mathrm{LT} & 0.30 \mathrm{ug} / \mathrm{L} & \text { Gen. Eng. } \\ \text { Gross alpha } & \mathrm{LT} & 2.00 \mathrm{pCi} / \mathrm{L} & \text { Gen. Eng. } \\ \text { Nonvolatile beta } & \mathrm{LT} & 2.00 \mathrm{pCi} / \mathrm{L} & \text { Gen. Eng. } \\ \text { Total radium } & \mathrm{LT} & 1.00 \mathrm{pCi} / \mathrm{L} & \text { Gen. Eng. } \\ \text { Tritium } & 2.03+- & 0.20 \mathrm{pCi} / \mathrm{mL} & \text { Gen. Eng. }\end{array}$

WELL LFW 43D

$\begin{array}{llllll}\text { SRS Grid } & \text { N } & 86443.2 & \text { Latitude } & 33.290771 & N \\ \text { Coordinates } & \text { E } & 45244.5 & \text { Long itude } & 81.715581 & W\end{array}$

Screen Zone Elevation 170.4-150.4 ft ms l

Top of Casing Elevation $200.40 \mathrm{ft} \mathrm{ms}$ !

MEASUREMENTS CONDUCTED IN THE FIELD

Sample date 09/02/91 Time 1015

Depth to water - $34.23 \mathrm{ft}(10.43 \mathrm{~m})$ below the TOC

Water elevation - $166.17 \mathrm{ft}(50.65 \mathrm{~m}) \mathrm{msl}$

$\mathrm{pH}=5.3 \quad$ Alkalinity $=11 \mathrm{mg} / \mathrm{L}$

Specific conductance - 29 umhos/cm

Water temperature - 19.1 degrees Celsius

Water evacunted from the well prior to sampling - $72 \mathrm{gal}$

\section{LABORATORY ANALYSES}

Specific conductance

pH

Silver

Arsenic

Barium

Bromodichloromethane

Calcium

Trichlor of I uoromethane

Carbon tetrachloride

Cadmi um

Bromoform

Chloroform

Dichloromethane (Methylene chloride)

Bromomethane (Methyl bromide)

Chloromethane (Methyl chloride)

Chloride

Chlorobenzene

Chrom i um

Chlorocthene (Vinyl chloride)

Chloroethane

Benzene

Dibromoch loromethane

Endrin

Ethylbenzene

CONTINUED

$\begin{array}{lrll} & 30.00 \mathrm{umh} / \mathrm{cm} & \text { Gen. Eng. } \\ & 5.56 \mathrm{uH} & \text { Gen. Eng. } \\ \text { LT } & 2.00 \mathrm{ug} / \mathrm{L} & \text { Gen. Eng. } \\ \text { LT } & 2.00 \mathrm{ug} / \mathrm{L} & \text { Gen. Eng. } \\ & 10.50 \mathrm{ug} / \mathrm{L} & \text { Gen. Eng. } \\ \text { LT } & 1.00 \mathrm{ug} / \mathrm{L} & \text { Gen. Eng. } \\ & 1410.00 \mathrm{ug} / \mathrm{L} & \text { Gen. Eng. } \\ & 40.00 \mathrm{ug} / \mathrm{L} & \text { Gen. Eng. } \\ \text { LT } & 1.00 \mathrm{ug} / \mathrm{L} & \text { Gen. Eng. } \\ \text { LT } & 2.00 \mathrm{ug} / \mathrm{L} & \text { Gen. Eng. } \\ \text { LT } & 1.00 \mathrm{ug} / \mathrm{L} & \text { Gen. Eng. } \\ \text { LT } & 1.00 \mathrm{ug} / \mathrm{L} & \text { Gen. ing. } \\ & 1.00 \mathrm{ug} / \mathrm{L} & \text { Gen. Eng. } \\ \text { LT } & 1.00 \mathrm{ug} / \mathrm{L} & \text { Gen. Eng. } \\ \text { LT } & 1.00 \mathrm{ug} / \mathrm{L} & \text { Gen. Eng. } \\ & 2520.00 \mathrm{ug} / \mathrm{L} & \text { Gen. Eng. } \\ \text { LT } & 1.00 \mathrm{ug} / \mathrm{L} & \text { Gen. Eng. } \\ \text { LT } & 4.00 \mathrm{ug} / \mathrm{L} & \text { Gen. Eng. } \\ \text { LT } & 1.00 \mathrm{ug} / \mathrm{L} & \text { Gen. Eng. } \\ \text { LT } & 1.00 \mathrm{ug} / \mathrm{L} & \text { Gen. Eng. } \\ \text { LT } & 1.00 \mathrm{ug} / \mathrm{L} & \text { Gen. Eng. } \\ \text { LT } & 1.00 \mathrm{ug} / \mathrm{L} & \text { Gen. Eng. } \\ \text { LT } & 0.01 \mathrm{ug} / \mathrm{L} & \text { Gen. Eng. } \\ \text { LT } & 1.00 \mathrm{ug} / \mathrm{L} & \text { Gen. Eng. } \\ & & & \end{array}$


WELL LFW 43D COLLECTED ON 09/02/91 LABORATORY ANALYSES CONTINUED

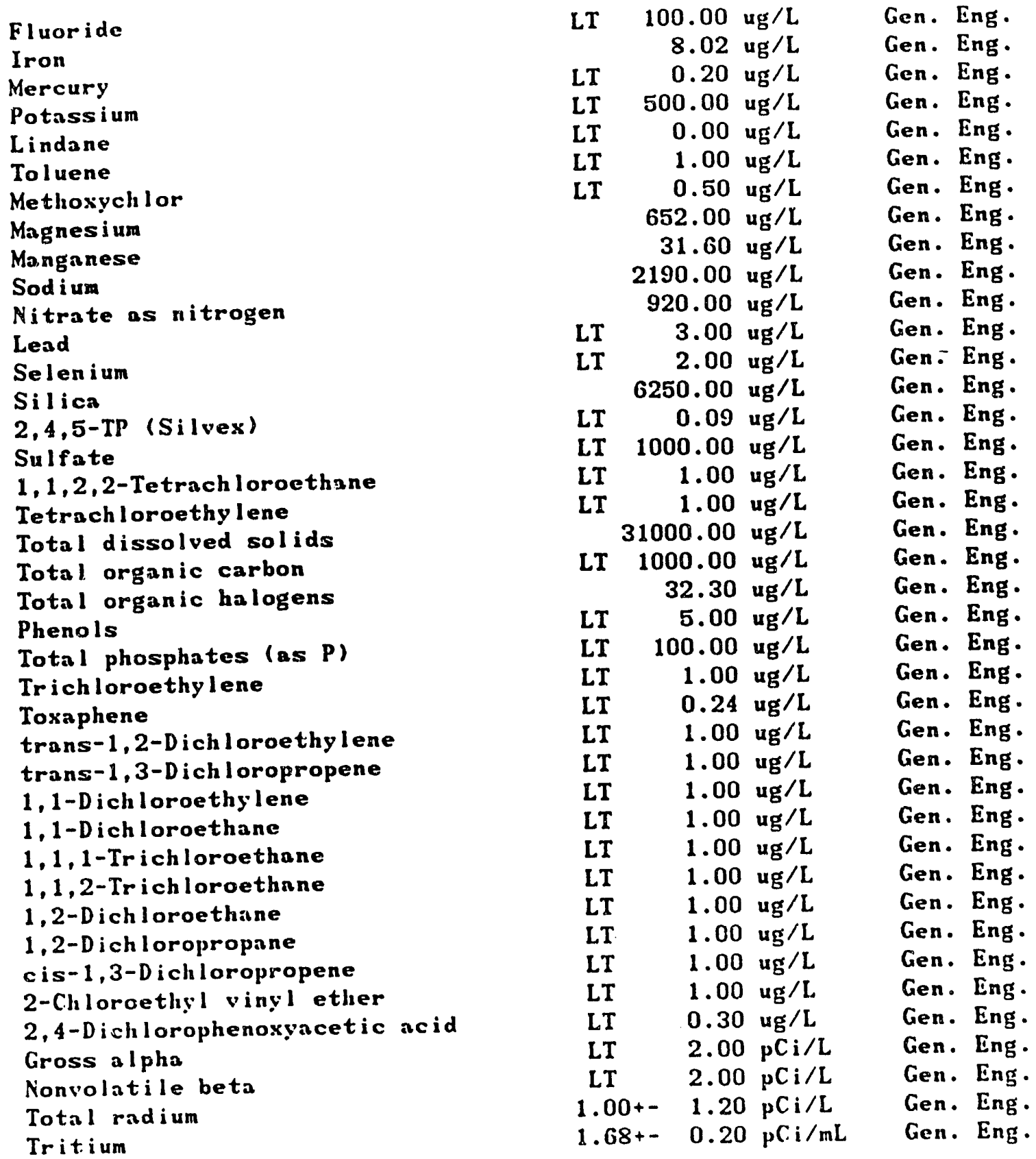


KELL LFW 44D

$\begin{array}{llllll}\text { SRS Grid } & N & 84524.4 & \text { Latitude } & 33.286167 & N \\ \text { Coordinates } & E & 45022.6 & \text { Longitude } & 81.712435 & W\end{array}$

Screen Zone Elevation 159.3-139.5 ft msl

Top of Casing Elevation $168.30 \mathrm{ft} \mathrm{msl}$

MEASUREMENTS CONDUCTED IN THE FIELD

Sample date 09/02/91 Time 1120

Depth to water - $14.18 \mathrm{ft}(4.32 \mathrm{~m})$ below the rOC

Water elevation - $154.12 \mathrm{ft}(46.98 \mathrm{~m}) \mathrm{msl}$

pll $=4.9$ Alkalinity $-0 \mathrm{mg} / \mathrm{L}$

Specif ic conductance - $19 \mathrm{umhos} / \mathrm{cm}$

Water temperature -19.7 degrees Celsius

Water evacuated from the well prior to sampling - $45 \mathrm{gal}$

\section{LABORATORY ANALYSES}

Specific conductance

pH

Silver

Arsenic

Bari um

Bromodichloromethane

Calcium

Trichlor of luoromethane

Carbon tetruchloride

Cadmi um

Bromoform

Chlorof orm

Dichloromethane (Methylene chloride)

Bromomethane (Methyl bromide)

Chloromethane (Methyl chloride)

Chloride

Chlorobenzene

Chromium

Chlorocthene (Vinyl chloride)

Chloroethane

Benzene

Dibromochloromethane

Endrin

Ethylbenzene

Fluoride

Iron

Mercury

Potassium

Lindane

Toluene

Methoxychlor

Magnesi um

Manganese

Sod i um

Nitrate ns nitrogen

Lend

Selenium

CONTINUED
$20.00 \mathrm{umh} / \mathrm{cm}$

$5.15 \mathrm{pH}$

LT

LT

LT

LT

LT

LT

LT

LT

LT

LT

LT

LT

LT

L.T

LT

LT

LT

LI

LT

LT

LT

LT

LT

$2.00 \mathrm{ug} / \mathrm{L}$

$2.00 \mathrm{ug} / \mathrm{L}$

$3.38 \mathrm{ug} / \mathrm{L}$

$1.00 \mathrm{ug} / \mathrm{L}$

$369.00 \mathrm{ug} / \mathrm{L}$

$1.00 \mathrm{ug} / \mathrm{L}$

$1.00 \mathrm{ug} / \mathrm{L}$

$2.00 \mathrm{ug} / \mathrm{L}$

$1.00 \mathrm{ug} / \mathrm{L}$

$1.00 \mathrm{ug} / \mathrm{L}$

$1.00 \mathrm{ug} / \mathrm{L}$

$1.00 \mathrm{ug} / \mathrm{L}$

$1.00 \mathrm{ug} / \mathrm{L}$

$2910.00 \mathrm{ug} / \mathrm{L}$

$1.00 \mathrm{ug} / \mathrm{L}$

$4.00 \mathrm{ug} / \mathrm{L}$

$1.00 \mathrm{ug} / \mathrm{L}$

$1.00 \mathrm{ug} / \mathrm{L}$

$1.00 \mathrm{ug} / \mathrm{L}$

$1.00 \mathrm{ug} / \mathrm{L}$

$0.01 \mathrm{ug} / \mathrm{L}$

$1.00 \mathrm{ug} / \mathrm{L}$

$100.00 \mathrm{ug} / \mathrm{L}$

$10.40 \mathrm{ug} / \mathrm{L}$

$0.20 \mathrm{~kg} / \mathrm{L}$

$624.00 \mathrm{ug} / \mathrm{L}$

$0.00 \mathrm{ug} / \mathrm{L}$

$1.00 \mathrm{ug} / \mathrm{L}$

$0.50 \mathrm{ug} / \mathrm{L}$

$344.00 \mathrm{ug} / \mathrm{L}$

i. $15 \mathrm{ug} / \mathrm{L}$

$1400.00 \mathrm{ug} / \mathrm{L}$

$400.00 \mathrm{ug} / \mathrm{L}$

LT

$3.00 \mathrm{ug} / \mathrm{L}$

LT
Gen. Eng.

Gen. Eng.

Gen. Eng.

Gen. Eng.

Gen. Eng.

Gen. Eng.

Gen. Eng.

Gen. Eng.

Gen. Eng -

Gen. Eng.

Gen. Eng.

Gen. Eng.

Gen. Eng.

Gen. Eng.

Gen. Eng.

Gen. Eng.

Gen. Eng.

Gen. Eng.

Gen. Eng.

Gen. Eng.

Gen. Eng.

Gen. Eng.

Gen. Eng.

Gen. Eng.

Gen. Eng.

Gen. Eng.

Gen. Eng.

Gen. Eng.

Gen. Eng.

Gen. Eng.

Gen. Eng.

Gen. Eng.

Gen. Eng.

Geñ. Eng.

Gen. Eng.

Gen. Eng.

Gen. Eng. 
WELL LFW 44D COLLECTED ON 09/02/91 LABORATORY ANALYSES CONTINUED

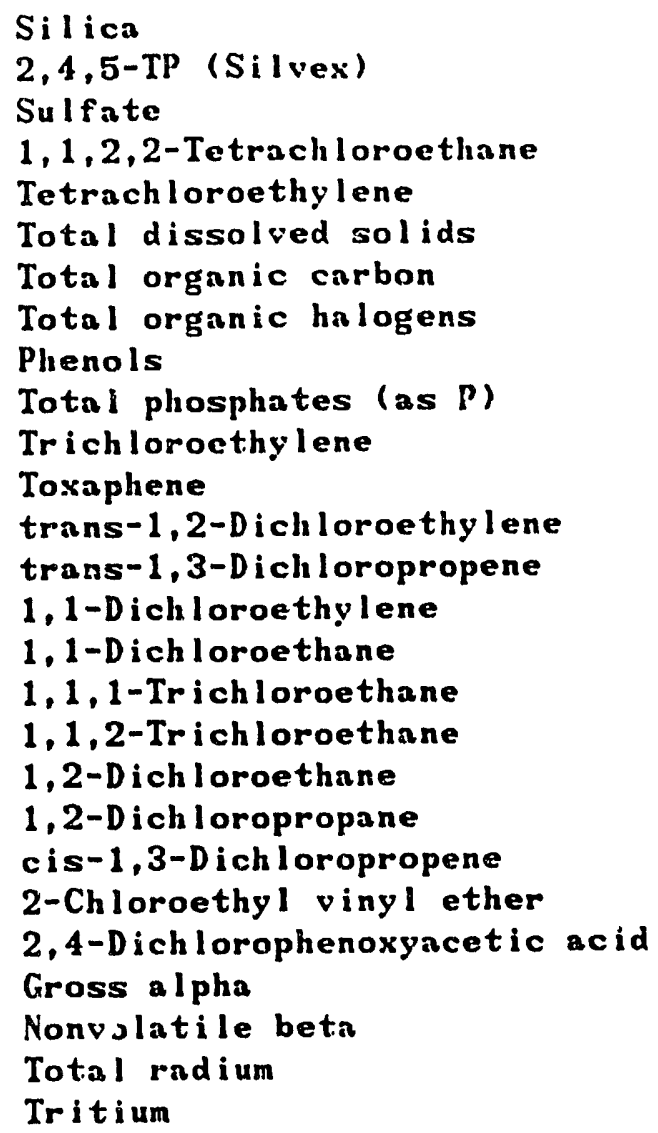

$\begin{array}{lr} & 6470.00 \mathrm{ug} / \mathrm{L} \\ \mathrm{LT} & 0.09 \mathrm{ug} / \mathrm{L} \\ \mathrm{LT} & 1000.00 \mathrm{ug} / \mathrm{L} \\ \mathrm{LT} & 1.00 \mathrm{ug} / \mathrm{L} \\ \mathrm{LT} & 1.00 \mathrm{ug} / \mathrm{L} \\ 23000.00 \mathrm{ug} / \mathrm{L} \\ \mathrm{LT} & 1000.00 \mathrm{ug} / \mathrm{L} \\ & 90.50 \mathrm{ug} / \mathrm{L} \\ \mathrm{LT} & 5.00 \mathrm{ug} / \mathrm{L} \\ \mathrm{LT} & 100.00 \mathrm{ug} / \mathrm{L} \\ \mathrm{LT} & 1.00 \mathrm{ug} / \mathrm{L} \\ \mathrm{LT} & 0.24 \mathrm{ug} / \mathrm{L} \\ \mathrm{LT} & 1.00 \mathrm{ug} / \mathrm{L} \\ \mathrm{LT} & 1.00 \mathrm{ug} / \mathrm{L} \\ \mathrm{LT} & 1.00 \mathrm{ug} / \mathrm{L} \\ \mathrm{LT} & 1.00 \mathrm{ug} / \mathrm{L} \\ \mathrm{LT} & 1.00 \mathrm{ug} / \mathrm{L} \\ \mathrm{LT} & 1.00 \mathrm{ug} / \mathrm{L} \\ \mathrm{LT} & 1.00 \mathrm{ug} / \mathrm{L} \\ \mathrm{LT} & 1.00 \mathrm{ug} / \mathrm{L} \\ \mathrm{LT} & 1.00 \mathrm{ug} / \mathrm{L} \\ \mathrm{LT} & 1.00 \mathrm{ug} / \mathrm{L} \\ \mathrm{LT} & 0.30 \mathrm{ug} / \mathrm{L} \\ \mathrm{LT} & 2.00 \mathrm{pC} / \mathrm{L} \\ \mathrm{LT} & 2.00 \mathrm{pCi} / \mathrm{L} \\ 1.40+- & 1.20 \mathrm{pC} \mathrm{i} / \mathrm{L} \\ 3.20+- & 0.30 \mathrm{pC} \mathrm{i} / \mathrm{mL}\end{array}$

Gen. Eng.

Gen. Eng.

Gen. Eng.

Gen. Eng.

Gen. Eng.

Gen. Eng.

Gen. Eng.

Gen. Eng.

Gen. Eng.

Gen. Eng.

Gen. Eng.

Gen. Eng.

Gen.- Eng.

Gen. Eng.

Gen. Eng.

Gen. Eng.

Gen. Eng.

Gen. Eng.

Gen. Eng.

Gen. Eng.

Gen. Eng.

Gen. Eng.

Gen. Eng.

Gen. Eng.

Gen. Eng .

Gen. Eng.

Gen. Eng.

HELL LFW 45D

$\begin{array}{llllll}\text { SRS Grid } & N & 84217.8 & \text { Latitude } & 33.285684 & N \\ \text { Coordinates } & E & 45142.0 & \text { Longitude } 81.711525 & W\end{array}$

Screen Zone Elevation 154.7-134.7 ft msl

Top of Casing Elevation $164.40 \mathrm{ft} \mathrm{msl}$

\section{MEASUREMENTS CONDUCTED IN TIEE FIELD}

Sample date 09/02/91 Time 1200

Depth to water - $13.01 \mathrm{ft}(3.97 \mathrm{~m})$ below the TOC

Water elevation $=151.39 \mathrm{ft}(46.14 \mathrm{~m}) \mathrm{msl}$

$\mathrm{pH}=5.0 \quad$ Alkalinity - $0 \mathrm{mg} / \mathrm{L}$

Specific conductance - 20 umhos/cm

Water temperature -19.7 degrees Celsius

Water evacuated from the well prior to sampling - $50 \mathrm{gal}$

\section{LABORATORY ANALYSES}

Specific conductance

pll

Silver

Arsenic

Barium

CONTINUED

$\begin{array}{lcl} & 20.00 \mathrm{unh} / \mathrm{cm} & \text { Gen. Eng. } \\ & 5.31 \mathrm{pll} & \text { Gen. Eng. } \\ \text { LT } & 2.00 \mathrm{ug} / \mathrm{L} & \text { Gen. Eng. } \\ \text { LT } & 2.00 \mathrm{ug} / \mathrm{L} & \text { Gen. Eng. } \\ & 5.19 \mathrm{ug} / \mathrm{I} & \text { Gen. Eng. }\end{array}$


WELL LFW 45D COLLECTED ON 09/02/91 LABORATORY ANALYSES CONTINUED

Bromodichloronethane
Calcium
Trichlorofluoromethane
Carbon tetrachloride
Cadmium
Bromoform
Chloroform
Dichloromethane (Methylene chloride)
Bromomethne (Methyl bromide)
Chloromethane (Methyl chloride)
Chloride
Chlorobenzene
Chromium
Chloroethene (Vingl chloride)
Chloroethane
Benzene
Dibromochloromethane
Endrin
Ethylbenzene
Fluoride
Iron
Mercury
Potassium
Lindane
Toluene
Metloxychlor
Magnesium
Manganese
Sodium
Nitrate as nitrogen

Nitrate as nitrogen

Selenium

Silica

2,4,5-TP (Silvex)

Sulf a te

1,1,2,2-Tetrachloroethane

Tetrachloroethylene

Total dissolved solids

Total dissolved solids

Total organic carbon

Total organic halogens

Phenols

Total phosphates (as P)

Tr.ichloroethylene

Toxaphene

trans-1,2-Dichloroethylene

trans-1,3-Dich loropropene

1,1-Dichloroethylene

1,1-Dichloroethane

1,1,1-Trichlorocthane

1,1,2-Trichloroethane

1,2-Dichloroethane

1, 2-Dichioropropane

cis-1,3-Dichloropropene
LT

LT

LT

LT

LT

LT

LT

LT

LT

LT

LT

LT

LI

LT

LT

LT

LT

LT

LT

LT

LT$$
33000.00 \text { ug } / \mathrm{L}
$$$$
\begin{aligned}
& 33000.00 \mathrm{ug} / \mathrm{L} \\
& 39000.00 \mathrm{ug} / \mathrm{L}
\end{aligned}
$$$$
\begin{array}{r}
39000.00 \mathrm{ug} / \mathrm{L} \\
1000.00 \mathrm{ug} / \mathrm{L}
\end{array}
$$$$
\text { LT } \quad 5.00 \mathrm{ug} / \mathrm{l} \text {. }
$$

$$
5.00 \mathrm{ug} / \mathrm{L}
$$
$100.00 \mathrm{ug} / \mathrm{L}$ $1.00 \mathrm{ug} / \mathrm{L}$ $0.24 \mathrm{ug} / \mathrm{L}$ $1.00 \mathrm{ug} / \mathrm{L}$ $1.00 \mathrm{ug} / \mathrm{L}$ $1.00 \mathrm{ug} / \mathrm{L}$ $1.00 \mathrm{ug} / \mathrm{L}$ $1.00 \mathrm{ug} / \mathrm{L}$ $1.00 \mathrm{ug} / \mathrm{L}$ $1.00 \mathrm{ug} / \mathrm{L}$ $1.00 \mathrm{ug} / \mathrm{L}$ $1.00 \mathrm{ug} / \mathrm{L}$
Gen. Eng.

Gen. Eng.

Gen. Eng.

Gen. Eng.

Gen. Eng.

Gen. Eng.

Gen. Eng.

Gen. Eng.

Gen. Eng.

Gen. Eng.

Gen. Eng.

Gen: Eng.

Gen. Eng.

Gen. Eng.

Gen. Eng.

Gen. Eng.

Gen. Eng.

Gen. Eng .

Gen. Eng.

Gen. Eng.

Gen. Eng.

Gen. Eng.

Gen. Eng.

Gen. Eng.

Gen. Eng.

Gen. Eng.

Gen. Eng.

Gen. Eng.

Gen. Eng.

Gen. Eng.

Gen. Eng.

Gen. Eng.

Gen. Eng.

Gen. Eng.

Gen. Eng.

Gen. Eng.

Gen. Eng.

Gen. Eng.

Gen. Eng.

Gen. Eng.

Gen. Eng.

Gen. Eng.

Gen. Eng.

Gen. Eng.

Gen. Eng.

Gen. Eng.

Gen. Eng.

Gen. Eng.

Geñ. Eng.

Gen. Eng .

Gen. Eng.

Gen. Eng.
Gen. Eng.

Gen. Eng.

$0.09 \mathrm{ug} / \mathrm{L}$
$00.00 \mathrm{ug} / \mathrm{L}$
$1.00 \mathrm{ug} / \mathrm{L}$
$1.00 \mathrm{ug} / \mathrm{L}$
$000.00 \mathrm{ug} / \mathrm{L}$
$000.00 \mathrm{ug} / \mathrm{L}$
$000.00 \mathrm{ug} / \mathrm{L}$
$5.00 \mathrm{ug} / \mathrm{L}$
$5.00 \mathrm{ug} / \mathrm{L}$
$100.00 \mathrm{ug} / \mathrm{L}$
$1.00 \mathrm{ug} / \mathrm{L}$
$0.24 \mathrm{ug} / \mathrm{L}$
$1.00 \mathrm{ug} / \mathrm{L}$
$1.00 \mathrm{ug} / \mathrm{L}$
$1.00 \mathrm{ug} / \mathrm{L}$
$1.00 \mathrm{ug} / \mathrm{L}$
$1.00 \mathrm{ug} / \mathrm{L}$
$1.00 \mathrm{ug} / \mathrm{L}$
$1.00 \mathrm{ug} / \mathrm{L}$
$1.00 \mathrm{ug} / \mathrm{L}$
$1.00 \mathrm{ug} / \mathrm{L}$

$5.38 \mathrm{ug} / \mathrm{L}$
$80.00 \mathrm{ug} / \mathrm{L}$ 
WELL LFW 45D COLLECTED ON 09/02/91 LABORATORY ANALYSES CONTINUED

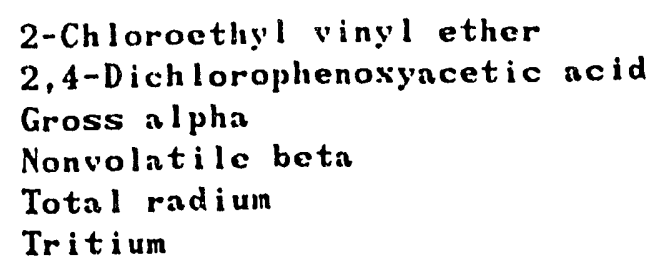

$\begin{array}{ll}\mathrm{LT} & 1.00 \mathrm{ug} / \mathrm{L} \\ \mathrm{LT} & 0.30 \mathrm{ug} / \mathrm{L} \\ \mathrm{LT} & 2.00 \mathrm{pCi} / \mathrm{L} \\ \mathrm{LT} & 2.00 \mathrm{pCi} / \mathrm{L} \\ \mathrm{LT} & 1.00 \mathrm{pCi} / \mathrm{L} \\ 2.18+- & 0.20 \mathrm{pCi} / \mathrm{mL}\end{array}$

Gen. Eng.

Gen. Eng.

Gen. Eng.

Gen. Eng.

Gen. Eng.

Gen. Eng.

WELL LFW 46D

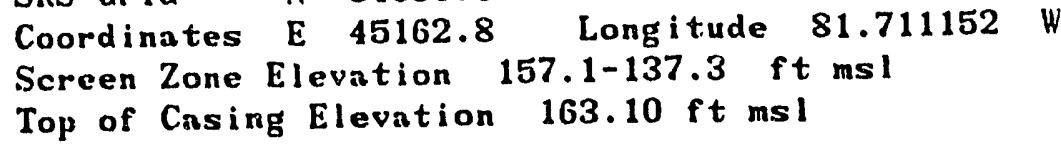

\section{MEASUREMENTS CONDUCTED IN THE FIELD}

Sample date 09/02/91 Time 1235

Depth to water - $13.25 \mathrm{ft}(4.04 \mathrm{~m})$ below the TOC

Water elevation - $149.85 \mathrm{ft}(45.67 \mathrm{~m}) \mathrm{msl}$

$\mathrm{pH}=5.5$ Alkalinity - $4 \mathrm{mg} / \mathrm{L}$

Specific conductance - 92 umhos/cm

Water temperature -21.2 degrees Celsius

Water evacuated from the well prior to sampling - $39 \mathrm{gal}$

\section{LABORATORY ANALYSES}

Specific conductance
pH
Silver
Arsenic
Barium
Bromodichloromethane
Calcium
Trichlorofluoromethne
Carbon tetrachloride
Cadmium
Bromoform
Chloroform
Dichloromethane (Methylene chloride)
Bromomethane (Methyl bromide)
Chloromethane (Methyl chloride)
Chloride
Chlorobenzene
Chromium
Chloroethene (Vingl chloride)
Chloroethane
Benzene
Dibromochloromethane
Endrin
Ethylbenzene
Fluoride
Iron

\begin{tabular}{|c|c|c|c|c|}
\hline & 100.00 & uinh/ & Gen. & Eng . \\
\hline & 5.75 & pH & Gen. & Eng. \\
\hline$T$ & 2.00 & $u_{g} / L$ & Gen. & Eng. \\
\hline LT & 2.00 & ug/L & Gen. & Eng. \\
\hline & 13.20 & $\mathrm{ug} / \mathrm{L}$ & Gen. & Eng. \\
\hline LT & 1.00 & ug $/ \mathrm{L}$ & Gen. & Eng. \\
\hline & 6960.00 & $\mathrm{~kg} / \mathrm{L}$ & Gen. & Eng. \\
\hline LT & 1.00 & ug $/ L$ & Gen. & Eng. \\
\hline LT & 1.00 & ug $/$ L & Gen. & Eng \\
\hline LT & 2.00 & $u g / L$ & Gen. & Eng \\
\hline$T$ & 1.00 & $\mathrm{ug} / \mathrm{L}$ & Gen. & Eng \\
\hline$T$ & 1.00 & $\mathrm{ug} / \mathrm{L}$ & Gen. & Eng \\
\hline & 2.00 & $\mathrm{ug} / \mathrm{L}$ & Gen. & ng \\
\hline$T$ & 1.00 & ug /L & Gen & $\exists$ \\
\hline $\mathrm{T}$ & 1.00 & $u g / L$ & Ger: & $n g$ \\
\hline & 2370.00 & ug/L & Gen. & ing \\
\hline LT & 1.00 & $\mathrm{ug} / \mathrm{L}$ & Gen. & Eng \\
\hline LT & 4.00 & $u g / L$ & Gen. & Eng: \\
\hline LT & 1.00 & ug/L & Gen. & Eng \\
\hline LT & 1.00 & $\mathrm{ug} / \mathrm{L}$ & Gen. & En \\
\hline LT & 1.00 & $\mathrm{ug} / \mathrm{L}$ & Gen. & \\
\hline LT & 1.00 & $\mathrm{ug} / \mathrm{L}$ & Gen: & $r$ \\
\hline LT & 0.01 & $\mathrm{ug} / \mathrm{L}$ & Gen. & \\
\hline LT & 1.00 & ug/L & Geñ. & En \\
\hline LT & 100.00 & $\mathrm{ug} / \mathrm{L}$ & Gen. & En \\
\hline LT & 4.00 & $\mathrm{ug} / \mathrm{L}$ & Gen. & \\
\hline
\end{tabular}


Mercury

Potassium

Lindane

Toluene

Methoxychlor

Magnesi um

Manganese

Sodium

Nitrate as nitrogen

Lead

Selen ium

Silica

$2,4,5-T P$ (Silvex)

Sulfate

1, 1,2,2-Tetrachloroethane

Tetrach loroethylene

Total dissolved solids

Total organic carbon

Total organic halogens

Phenols

Total phosphates (as P)

Trichloroethylene

Toxaphene

trans-1,2-Dichloroethylene

trans-1,3-Dichloropropene

1,1-Dichloroethylene

1.1-Dichloroethane

1,1,1-Trichloroethane

$1,1,2-$ Trich loroethane

1,2-Dichloroethane

1,2-D ichloropropane

cis-1,3-Dichloropropene

2-Chloroethyl vinyl ether

2,4-Dichlorophenoxyacetic acid

Gross a I pha

Nonvolatile beta

* Total radium

Tritium
LT

LT

LT

LT

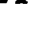

$0.20 \mathrm{ug} / \mathrm{L}$ $946.00 \mathrm{ug} / \mathrm{L}$

$0.00 \mathrm{ug} / \mathrm{L}$

$1.00 \mathrm{ug} / \mathrm{L}$

$0.50 \mathrm{ug} / \mathrm{L}$

$3740.00 \mathrm{ug} / \mathrm{L}$

$4.74 \mathrm{ug} / \mathrm{L}$

$1180.00 \mathrm{ug} / \mathrm{L}$

$300.00 \mathrm{ug} / \mathrm{L}$

LT $3.00 \mathrm{ug} / \mathrm{L}$

LT

\section{LT}

$2.00 \mathrm{ug} / \mathrm{L}$

$6580.00 \mathrm{ug} / \mathrm{L}$

$0.09 \mathrm{ug} / \mathrm{L}$

$28400.00 \mathrm{ug} / \mathrm{L}$

LT

LT

$88000.00 \mathrm{ug} / \mathrm{L}$

LT $1000,00 \mathrm{ug} / \mathrm{L}$

$84.10 \mathrm{ug} / \mathrm{L}$

LT $5.00 \mathrm{ug} / \mathrm{L}$

LT $100.00 \mathrm{ug} / \mathrm{L}$

LT

LT

LT

LT

LT

LT

LT

LT

LT

LT

LT

LT

LT

LT

$$
1.00 \mathrm{ug} / \mathrm{L}
$$

$0.24 \mathrm{\mu g} / \mathrm{L}$

$1.00 \mathrm{ug} / \mathrm{L}$

$1.00 \mathrm{ug} / \mathrm{L}$

$1.00 \mathrm{ug} / \mathrm{L}$

$1.00 \mathrm{ug} / \mathrm{L}$

$1.00 \mathrm{ug} / \mathrm{L}$

$1.00 \mathrm{ug} / \mathrm{L}$

$1.00 \mathrm{ug} / \mathrm{L}$

$1.00 \mathrm{ug} / \mathrm{L}$

$1.00 \mathrm{ug} / \mathrm{L}$

$1.00 \mathrm{ug} / \mathrm{L}$

$0.30 \mathrm{ug} / \mathrm{L}$

$2.00 \mathrm{pCi} / \mathrm{L}$

$3.90+-2.30 \mathrm{pCi} / \mathrm{L}$

$5.00+-2.10 \mathrm{pCi} / \mathrm{L}$

$1.74+-0.20 \mathrm{pCi} / \mathrm{mL}$
Gen. Eng.

Gen. Eng.

Gen. Eng.

Gen. Eng.

Gen. Eng.

Gen. Eng.

Gen. Eng.

Gen. Eng.

Gen. Eng.

Gen. Eng.

Gen. Eng.

Gen. Eng.

Gen.- Eng.

Gen. Eng.

Gen. Eng.

Gen. Eng.

Gen. Eng.

Gen. Eng.

Gen. Eng.

Gen. Eng.

Gen. Eng.

Gen. Eng.

Gen. Eng.

Gen. Eng.

Gen. Eng.

Gen. Eng.

Gen. Eng.

Gen. Eng.

Gen. Eng.

Gen. Eng.

Gen. Eng.

Gen. Eng.

Gen. Eng.

Gen. Eng.

Gen. Eng.

Gen. Eng.

Gen. Eng.

Gen. Eng. 
WELL LFW $47 \mathrm{C}$

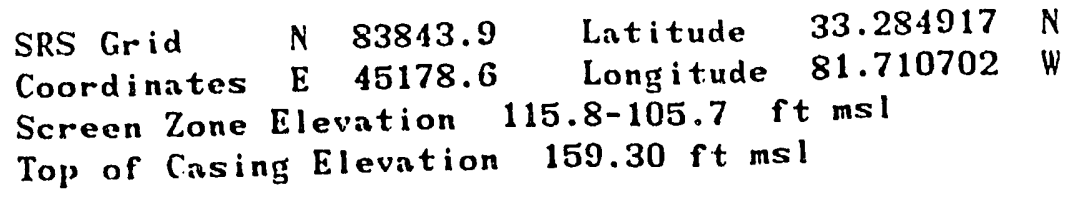

MEASUREMENTS CONDUCTED IN TIE FIELD

Sample date 09/02/91 Time 1420

Depth to water $=12.04 \mathrm{ft}(3.67 \mathrm{~m})$ below the TOC

Water elevation - $147.26 \mathrm{ft}(44.89 \mathrm{~m}) \mathrm{msl}$

pll $=5.2$ Alkalinity $=1 \mathrm{mg} / \mathrm{L}$

Specif ic conductance - 30 umhos/cm

Water temperature $=18.5$ degrees Celsius

Water evacuated from the well prior to sampling - $115 \mathrm{gal}$

\section{LABORATORY ANALYSES}

Specific conductance
pH
Silver
Arsenic
Barium
Bromodichloromethane
Calcium
Trichlorofluoromethane
Carbon tetrachloride
Cadmium
Bromoform
Chloroform
Dichloromethane (Methylene chloride)
Bromomethane (Methyl bromide)
Chloromethane (Methyl chloride)
Chloride
Chlorobenzene
Chromium
Chloroethene (Vinyl chloride)
Chloroethane
Benzene
Dibromochloromethane
Endrin
Ethylbenzene
Fluoride
Iron
Mercury
Potassium
Lindane
Toluene
Methosychlor
Magnesium
Manganese
Sodium
Nitrate as nitrogen
Lead
Selenium
CONTINUED
(a)

\begin{tabular}{|c|c|c|c|c|}
\hline & 30.00 & $u m h / c m$ & Gen. & Eng. \\
\hline & 5.41 & $\mathrm{pH}$ & Gen. & Eng. \\
\hline LT & 2.00 & ug $/ \mathrm{L}$ & Gen. & Eng. \\
\hline LT & 2.00 & ug/L & Gen. & Eng. \\
\hline & 4.89 & ug $/ \mathrm{L}$ & Gen. & Eng. \\
\hline$T$ & $\begin{array}{r}1.00 \\
729.00\end{array}$ & $u g / L$ & $\begin{array}{l}\text { Gen. } \\
\text { Gen. }\end{array}$ & $\begin{array}{l}\text { Eng. } \\
\text { Eng. }\end{array}$ \\
\hline LT & 1.00 & ug/L & Gen. & Eng. \\
\hline LT & 1.00 & ug $/ \mathrm{L}$ & Cen. & Eng. \\
\hline LT & 2.00 & $\mathrm{ug} / \mathrm{L}$ & Gen. & Eng. \\
\hline LT & 1.00 & $u_{g} / L$ & Gen. & Eng. \\
\hline LT & 1.00 & $u g / L$ & Gen. & Eng. \\
\hline & 1.00 & ug/L & Gen. & Eng. \\
\hline LT & 1.00 & $u_{g} / L$ & Gen. & Eng. \\
\hline LT & 1.00 & $u g / L$ & Gen. & Eng. \\
\hline & 3680.00 & $u g / L$ & Gen. & Eng. \\
\hline LT & 1.00 & $u_{g} / \mathrm{L}$ & Gen. & Eng. \\
\hline LT & 4.00 & ug/L & Gen. & Eng. \\
\hline & 3.00 & ug $/ \mathrm{L}$ & Gen. & Eng. \\
\hline LT & 1.00 & ug $/ \mathrm{L}$ & Gen. & Eng. \\
\hline LT & 1.00 & ug $/ \mathrm{L}$ & Gen. & Eng. \\
\hline LT & 1.00 & $\mathrm{ug} / \mathrm{L}$ & Gen. & Eng. \\
\hline LT & 0.01 & $u_{g} / L$ & Gen. & Eng. \\
\hline$L T$ & 1.00 & ug/L & Gen. & Eng. \\
\hline LT & 100.00 & ug/L & Gen. & Eng. \\
\hline & 11.50 & $u g / L$ & Gen. & Eng. \\
\hline LT & 0.20 & $\mathrm{ug} / \mathrm{L}$ & Gen. & Eng. \\
\hline & 553.00 & $\mathrm{ug} / \mathrm{L}$ & Gen. & Eng. \\
\hline LT & 0.00 & ug/L & Gen. & Eng. \\
\hline LT & 1.00 & ug/L & Gen. & Eng. \\
\hline LT & 0.50 & ug/L & Gen. & Eng. \\
\hline & 1000.00 & ug $/ \mathrm{L}$ & Gen. & Eng \\
\hline & 10.30 & $u g / L$ & Gen. & Eng. \\
\hline & 2100.00 & ug/L & Geñ. & Eng. \\
\hline & 420.00 & $u g / L$ & Gen. & Eng \\
\hline LT & 3.00 & ug/L & Gen. & - Eng \\
\hline LT & 2.00 & ug/L & Gen. & - Eng \\
\hline
\end{tabular}


WELL LFW 47C COLLECTED ON 09/02/91 LABORATORY ANALYSES CONTINUED

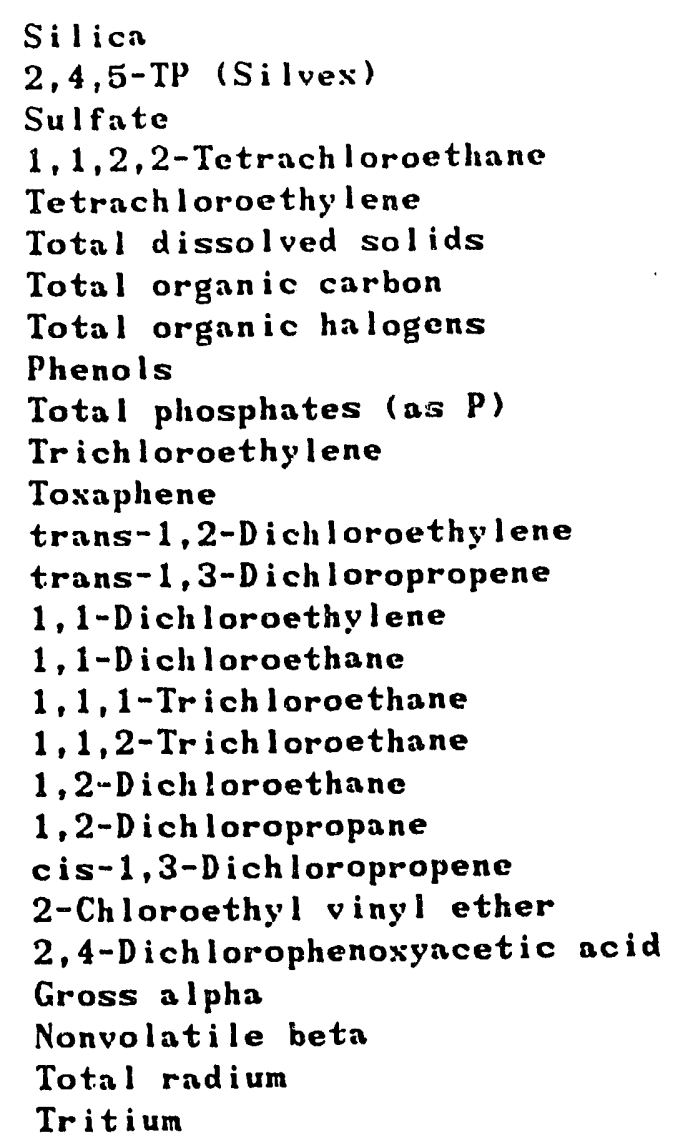

\begin{tabular}{|c|c|c|c|c|}
\hline & 7640.00 & $\mathrm{ug} / \mathrm{L}$ & Gen. & Eng. \\
\hline LT & 0.09 & ug $/ L$ & Gen. & Eng. \\
\hline LT & 1000.00 & ug $/ \mathrm{L}$ & Gen. & Eng. \\
\hline L.T & 1.00 & $u g / L$ & Gen. & Eng. \\
\hline LT & 1.00 & ug/L & Gen. & Eng. \\
\hline & 9000.00 & ug/L & Gen. & Eng. \\
\hline LT & 1000.00 & ug/L & Gen. & Eng. \\
\hline & 63.90 & ug/L & Gen. & Eng. \\
\hline LT & 5.00 & ug $/ \mathrm{L}$ & Gen. & Eng. \\
\hline LT & 100.00 & $u g / L$ & Gen. & Eng. \\
\hline LT & 1.00 & $\mathrm{ug} / \mathrm{L}$ & Gen. & Eng. \\
\hline LT & 0.24 & ug/L & Gen. & Eng. \\
\hline LT & 1.00 & ug/L & Gen. & Eng. \\
\hline LT & 1.00 & ug $/ \mathrm{L}$ & en. & ng • \\
\hline LT & 1.00 & ug/L & en. & Eng. \\
\hline & 2.00 & ug/L & Gen. & Eng. \\
\hline $\mathbf{L T}$ & 1.00 & ug/L & Gen. & Eng. \\
\hline LI & 1.00 & $u_{g} / L$ & Gen. & Eng \\
\hline LT & 1.00 & ug $/ L$ & Gen. & Eng \\
\hline LT & 1.00 & ug $/ \mathrm{L}$ & Gen. & Eng \\
\hline LT & 1.00 & ug $/ L$ & Gen. & Eng \\
\hline LT & 1.00 & ug $/ L$ & Gen. & n \\
\hline LT & 0.30 & ug $/ L$ & Gen. & \\
\hline LT & 2.00 & $\mathrm{pCi} / \mathrm{L}$ & Gen. & \\
\hline LT & 2.00 & $\mathrm{pCi} / \mathrm{L}$ & Gen. & \\
\hline .00 & 1.40 & $\mathrm{pli}$ i & & \\
\hline 4.9 & 0.30 & $\mathrm{Ci} / \mathrm{ml}$ & & 0 \\
\hline
\end{tabular}

\section{WELL LFW 47D}

$\begin{array}{llllll}\text { SRS Grid } & N & 83859.3 & \text { Latitude } & 33.284933 & N \\ \text { Coordinates } & E & 45167.9 & \text { Longitude } & 81.710760 & \mathrm{~W}\end{array}$

Screen Zone Elevation 154.7-134.9 ft msl

Top of Casing Elevation $159.70 \mathrm{ft} \mathrm{msl}$

MEASUREMENTS CONDUCTED IN THE FIELD

Sample date $09 / 02 / 91$ Time 1440

Depth to water - $11.89 \mathrm{ft}(3.62 \mathrm{~m})$ below the TOC

Water elevation - $147.81 \mathrm{ft}(45.05 \mathrm{~m}) \mathrm{msl}$

$\mathrm{pH}=5.2$ Alkalinity $=1 \mathrm{mg} / \mathrm{L}$

Specific conductance = 24 umhos/cm

Water temperature - 20.7 degrees Celsius

Water evacuated from the well prior to sampling - $40 \mathrm{gal}$

\section{LABORATORY ANALYSES}

Specific conductance

pll

Silver

Arsenic

Barium

CONTINUED
$25.00 \mathrm{umh} / \mathrm{cm}$

5.46 pll

LT

LT
$2.00 \mathrm{ug} / \mathrm{L}$

$2.00 \mathrm{ug} / \mathrm{L}$

$14.80 \mathrm{ug} / \mathrm{L}$
Gen. Eng.

Gen. Eng.

Geñ. Eng.

Gen. Eng .

Gen. Eng. 
WELL LFW 47D COLLECTED ON 09/02/91 LABORATORY ANALYSES CONTINUED

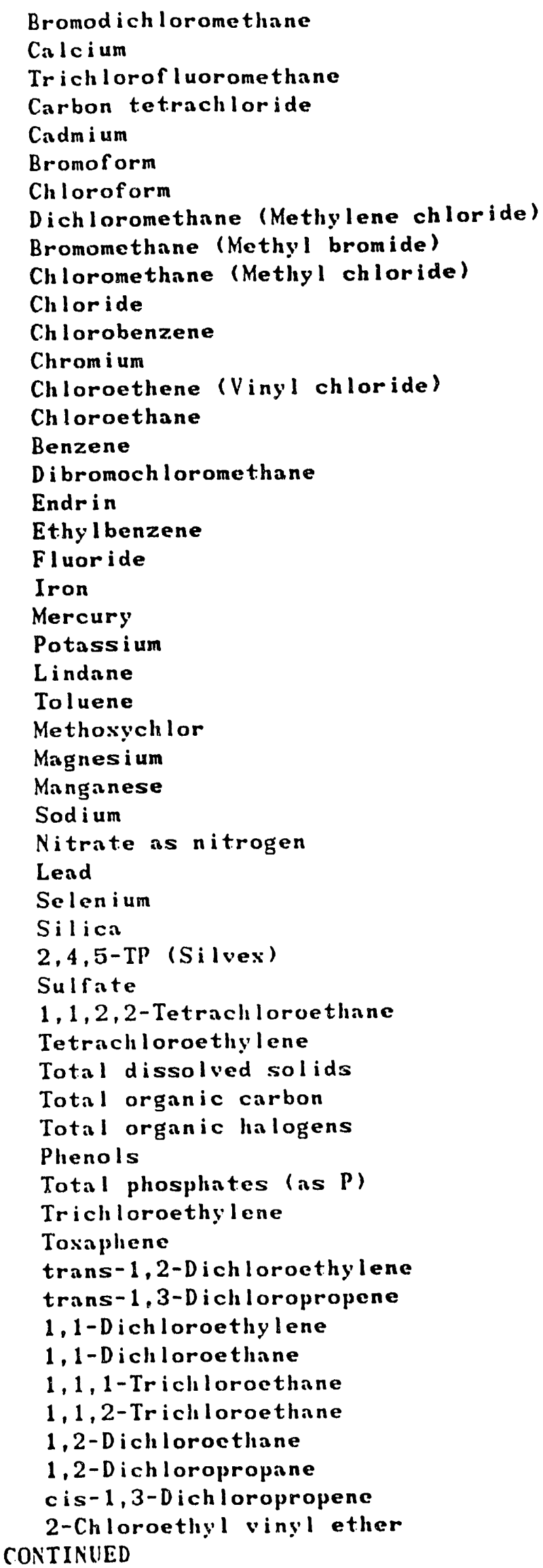

$5.00 \mathrm{ug} / \mathrm{L}$ $100.00 \mathrm{ug} / \mathrm{L}$ $1.00 \mathrm{ug} / \mathrm{L}$ $0.24 \mathrm{ug} / \mathrm{L}$ $1.00 \mathrm{ug} / \mathrm{L}$ $1.00 \mathrm{ug} / \mathrm{L}$ $1.00 \mathrm{ug} / \mathrm{L}$ $1.00 \mathrm{ug} / \mathrm{L}$ $1.00 \mathrm{ug} / \mathrm{L}$ $1.00 \mathrm{ug} / \mathrm{L}$ $1.00 \mathrm{ug} / \mathrm{L}$ $1.00 \mathrm{ug} / \mathrm{L}$ $1.00 \mathrm{ug} / \mathrm{L}$ $1.00 \mathrm{ug} / \mathrm{L}$
Gen. Eng.

Gen. Eng.

Gen. Eng.

Gen. Eng.

Gen. Eng.

Gen. Eng.

Gen. Eng.

Gen. Eng.

Gen. Eng.

Gen. Eng.

Gen. Eng.

Gen. Eng.

Gen- Eng.

Gen. Eng.

Gen. Eng.

Gen. Eng .

Gen. Eng.

Gen. Eng.

Gen. Eng.

Gen. Eng.

Gen. Eng .

Gen. Eng.

Gen. Eng.

Gen. Eng .

Gen. Eng .

Gen. Eng.

Gen. Eng.

Gen. Eng.

Gen. Eng.

Gen. Eng.

Gen. Eng.

Gen. Eng.

Gen. Eng.

Gen. Eng.

Gen. Eng.

Gen. Eng.

Gen. Eng.

Gen. Eng.

Gen. Eng.

Gen. Eng.

Gen. Eng.

Gen. Eng .

Gen. Eng.

Gen. Eng.

Gen. Eng.

Gen. Eng.

Gen. Eng.

Gen. Eng.

Gen. Eng.

Gen. Eng.

Geñ. Eng.

Gen. Eng.

Gen. Eng.

Gen. Eng . 
WELL LFW 47D COLLECTED ON 09/02/91 LABORATORY ANALYSES CONTINUTD

2,4-Dichlorophenoxyacetic acid
Gross alpha
Nonvolatile beta
Total radium
Tritium

$\begin{array}{cl}\mathrm{LT} & 0.30 \mathrm{ug} / \mathrm{L} \\ \mathrm{LT} & 2.00 \mathrm{pCi} / \mathrm{L} \\ \mathrm{LT} & 2.00 \mathrm{pCi} / \mathrm{L} \\ \mathrm{LT} & 1.00 \mathrm{pCi} / \mathrm{L} \\ 2.21+- & 0.20 \mathrm{pC} \cdot \mathrm{i} / \mathrm{mL}\end{array}$

Gen. Eng .

Gen. Eng.

Gen. Eng.

Gen. Eng.

Gen. Eng.

\section{WELL LFW $48 \mathrm{C}$}

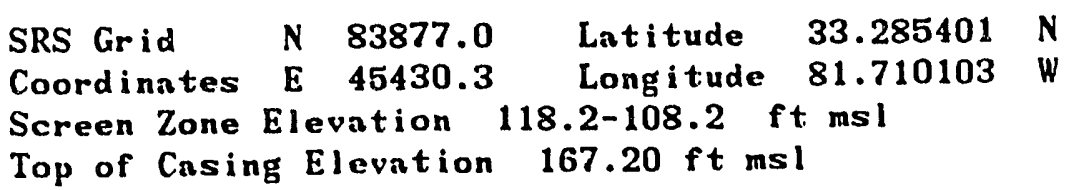

\section{MEASUREMENTS CONDUCTED IN THE FIELD}

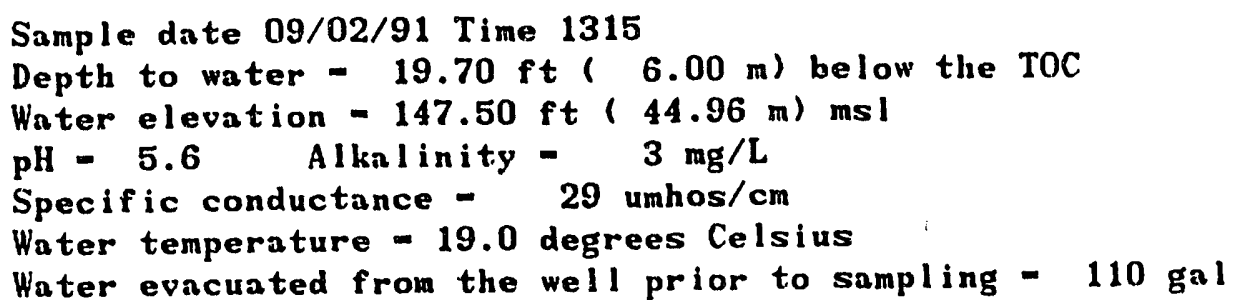

\section{LABORATORY ANALYSES}

Specific conductance

$\mathrm{pH}$

Silver

Arsenic

Barium

Bromodichloromethane

Calcium

Trichlor of luoromethane

Carbon tetrachloride

Cadin i um

Bromoform

Chloroform

Dichloromethane (Methylene chloride)

Bromomethane (Methyl bromide)

Chloromethane (Methyl chloride)

Chloride

Chlorobenzene

Chromium

Chloroethene (Vinyl chloride)

Chloroethane

Benzene

Dibromochloromethane

Endrin

Ethylbenzene

Fluoride

Iron

Mercury

CONTINUED
$28.00 \mathrm{umh} / \mathrm{cm}$

$5.81 \mathrm{pH}$

LT

LT

$2.00 \mathrm{ug} / \mathrm{L}$

$2.00 \mathrm{ug} / \mathrm{L}$

$7.42 \mathrm{ug} / \mathrm{L}$

LT

$1.00 \mathrm{ug} / \mathrm{L}$

$946.00 \mathrm{ug} / \mathrm{L}$

LT

LT

LT

LT

LT

LT

LT

LI

LT

LT

LT

LT

LT

LT

LT

LT

LT
$1.00 \mathrm{ug} / \mathrm{L}$

$1.00 \mathrm{ug} / \mathrm{L}$

$2.00 \mathrm{ug} / \mathrm{L}$

$1.00 \mathrm{ug} / \mathrm{L}$

$1.00 \mathrm{ug} / \mathrm{L}$

$2.00 \mathrm{ug} / \mathrm{L}$

$1.00 \mathrm{ug} / \mathrm{L}$

$1.00 \mathrm{ug} / \mathrm{L}$

$1850.00 \mathrm{ug} / \mathrm{L}$

$1.00 \mathrm{ug} / \mathrm{L}$

$4.00 \mathrm{ug} / \mathrm{L}$

$1.00 \mathrm{ug} / \mathrm{L}$

$1.00 \mathrm{ug} / \mathrm{L}$

$1.00 \mathrm{ug} / \mathrm{L}$

$1.00 \mathrm{ug} / \mathrm{L}$

$0.01 \mathrm{ug} / \mathrm{L}$

$1.00 \mathrm{ug} / \mathrm{L}$

$100.00 \mathrm{ug} / \mathrm{L}$

$6.17 \mathrm{ug} / \mathrm{L}$

$0.20 \mathrm{ug} / \mathrm{L}$
Gen. Eng -

Gen. Eng.

Gen. Eng.

Gen. Eng.

Gen. Eng.

Gen. Eng.

Gen. Eng.

Gen. Eng.

Gen. Eng.

Gen. Eng.

Gen. Eng.

Gen. Eng.

Gen. Eng.

Gen. Eng.

Gen. Eng.

Gen. Eng.

Gen. Eng.

Gen. Eng.

Gen. Eng.

Gen. Eng.

Gen. Eng.

Gen. Eng.

Gen. Eng.

Gen. Eng.

Geñ. Eng.

Gen. Eng.

Gen. Eng. 
WELL LFW 48C COLLECTED ON 09/02/91 LABORATORY ANALYSES CONTINUED

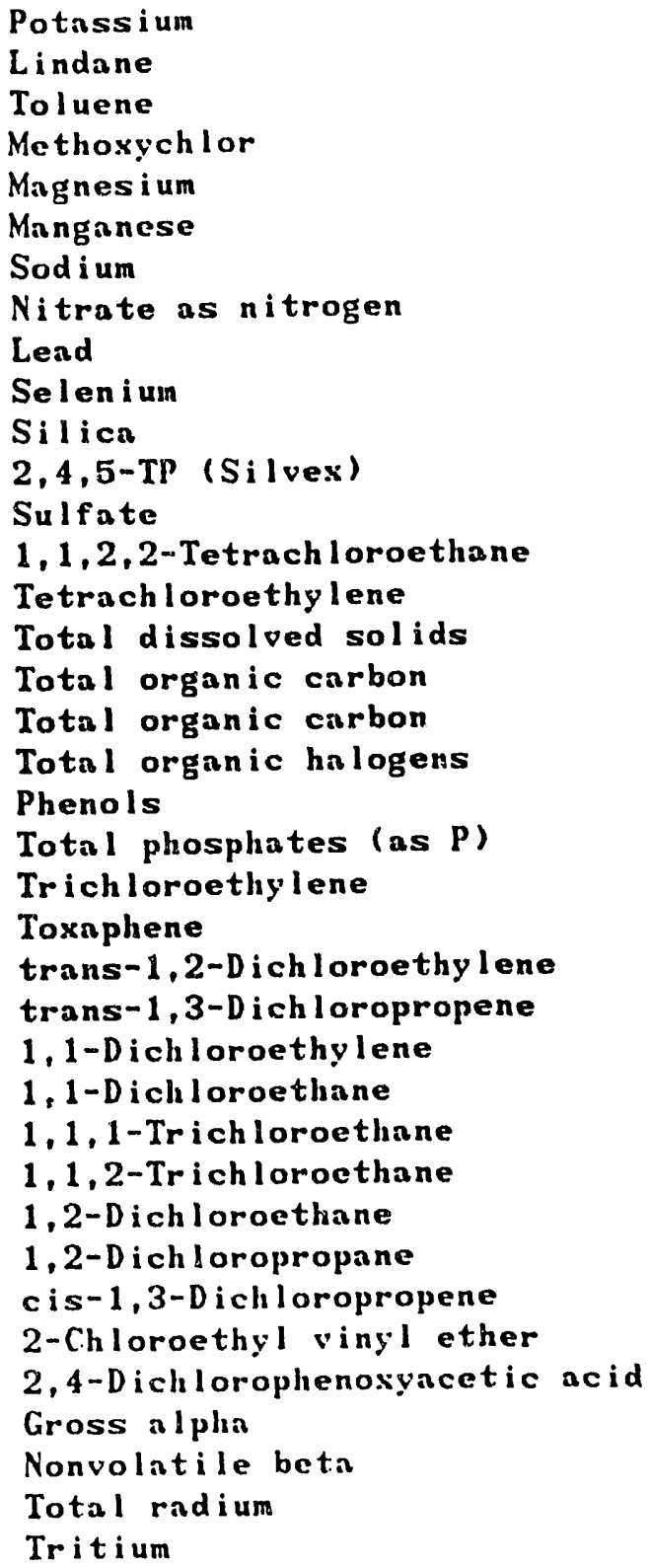

\begin{tabular}{|c|c|c|c|c|}
\hline & 806.00 & ug $/ \mathrm{L}$ & Gen. & Eng. \\
\hline $\mathrm{T}$ & 0.00 & ug $/ \mathrm{L}$ & Gen. & Eng \\
\hline$T$ & 1.00 & ug $/ \mathrm{L}$ & Gen. & Eng \\
\hline$T$ & 0.50 & ug $/ \mathrm{L}$ & Gen. & Eng \\
\hline & $\begin{array}{r}372.00 \\
17.50\end{array}$ & $\begin{array}{l}\mathrm{ug} / \mathrm{L} \\
\mathrm{ug} / \mathrm{L}\end{array}$ & $\begin{array}{l}\text { Gen. } \\
\text { Gen. }\end{array}$ & $\begin{array}{l}\text { Eng. } \\
\text { Eng. }\end{array}$ \\
\hline & 2570.00 & ug $/ \mathrm{L}$ & Gen. & Eng. \\
\hline & 580.00 & ug $/ L$ & Gen. & Eng \\
\hline $\mathbf{T}$ & 3.00 & ug $/ L$ & Gen. & Eng \\
\hline & 2.00 & ug $/ L$ & ien. & Eng \\
\hline & 7140.00 & ug $/ L$ & en. & Eng \\
\hline$T$ & 0.09 & ug $/ \mathrm{L}$ & ien. & En \\
\hline $\mathrm{T}$ & 1000.00 & ug $/ L$ & ien.- & En \\
\hline LT & 1.00 & ug/L & Gen. & Eng \\
\hline LT & 1.00 & ug $/ \mathrm{L}$ & Gen. & Eng \\
\hline & 26000.00 & $u g / L$ & en. & Eng \\
\hline LT & 1000.00 & ug $/ \mathrm{L}$ & ien. & Eng \\
\hline $\mathbf{L T}$ & 1000.00 & ug $/ L$ & en. & En \\
\hline & 94.90 & ug/L & en. & \\
\hline $\mathbf{L T}$ & 5.00 & ug $/ \mathrm{L}$ & en. & \\
\hline LT & 100.00 & $\mathrm{ug} / \mathrm{L}$ & en. & \\
\hline LT & 1.00 & ug $/ L$ & en. & \\
\hline LT & 0.24 & ug $/ \mathrm{L}$ & en. & \\
\hline LT & 1.00 & ug/L & en. & \\
\hline LT & 1.00 & ug/L & en. & \\
\hline LT & 1.00 & ug /L & & \\
\hline LI & 1.00 & $u g / L$ & en. & \\
\hline LT & 1.00 & $u g / L$ & en. & \\
\hline LT & 1.00 & $\mathrm{ug} / \mathrm{L}$ & a. & $\cdot 1$ \\
\hline LT & 1.00 & ug $/ \mathrm{L}$ & n. & \\
\hline LT & 1.00 & ug $/ L$ & & \\
\hline LT & 1.00 & $u g / L$ & & \\
\hline LT & 1.00 & $u g / L$ & & \\
\hline LT & 0.30 & $u g / L$ & & \\
\hline LT & 2.00 & $\mathrm{pCi} / \mathrm{L}$ & & \\
\hline LT & 2.00 & $\mathrm{pC} i / \mathrm{L}$ & ien. & \\
\hline LT & 1.00 & $\mathrm{pC} i / L$ & 1. & - \\
\hline .83 & 0.30 & $\mathrm{pCi} / \mathrm{m}$ & wen. & \\
\hline
\end{tabular}


WELL LFW 48D

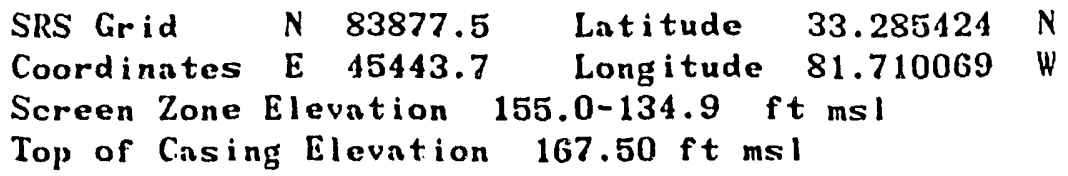

MEASUREMENTS CONDUCTED IN THE FIELD

Sample date 09/02/91 Time 1335

Depth to water - $19.30 \mathrm{ft}(5.88 \mathrm{~m})$ below the TOC.

Water elevation $-148.20 \mathrm{ft}(45.17 \mathrm{~m}) \mathrm{msl}$

$\mathrm{pH}-6.6$ Alkalinity - $101 \mathrm{mg} / \mathrm{L}$

Specif ic conductance - 342 umhos/cm

Water temperature - 19.2 degrees Celsius

Water evacuated from the well prior to sampling $=42 \mathrm{gal}$

\section{LABORATORY ANALYSES}

Specific conductance

pH

Si I ver

Arsenic

Barium

Bromodichloromethane

Calcium

Trichlor of I uoromethane

Carbon tetrachloride

Cadmi um

Bronof orm

Chlorof orm

Dichloromethane (Methylene chloride)

Bromomethane (Methyl bromide)

Chloromethane (Methyl chloride)

Chloride

Chlorobenzene

Chromium

* Chloroethene (Vinyl chloride)

Chloroethane

Benzene

Dibromochloromethane

Endrin

Ethylbenzene

Fluoride

Iron

Mercury

Potassium

Lindane

Toluene

Methosychlor

Magnesium

Manganese

Sodi um

Nitrate as nitrogen

Lend

Selenium

C.ONTINUED

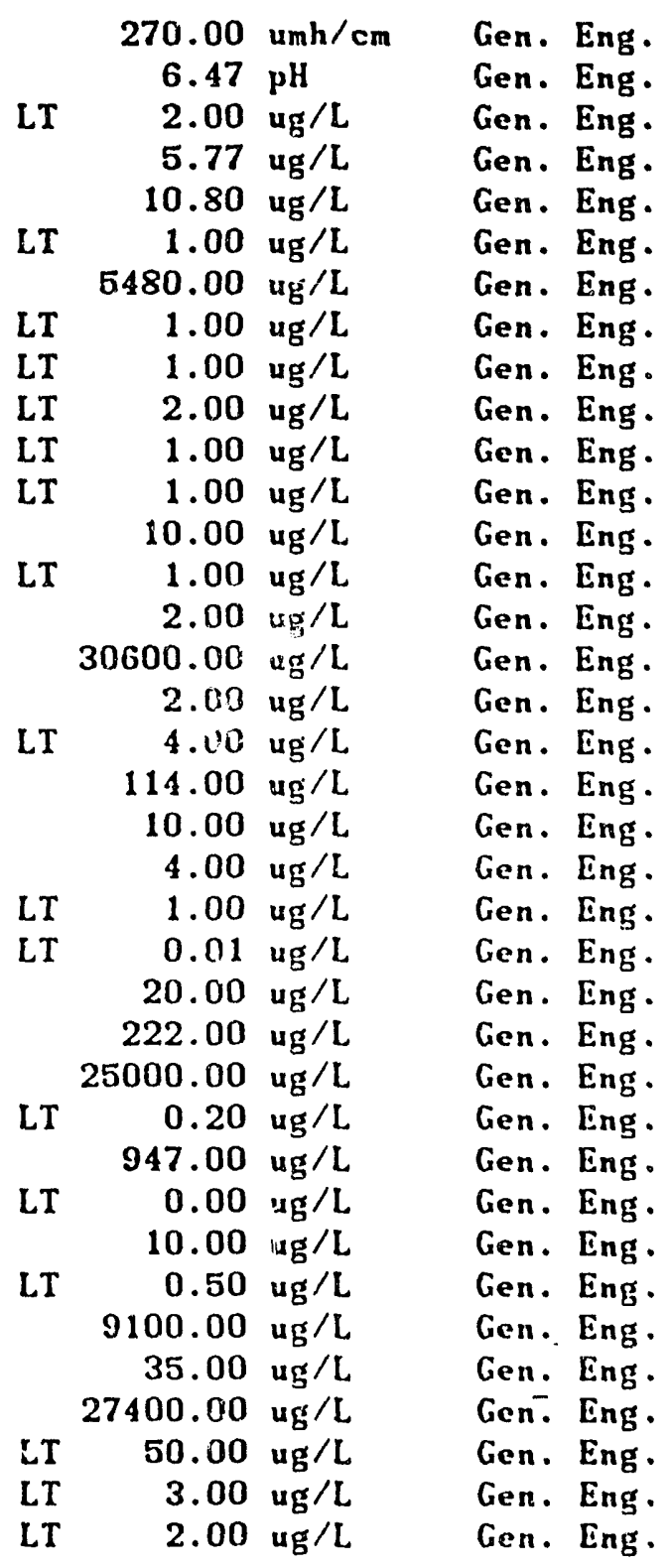


WELL LFW 48D COLLECTED ON 09/02/91 LABORATORY ANALYSES CONTINUED

\author{
Silica \\ $2,4,5-T P$ (Silvex) \\ Sulfate \\ 1,1,2,2-Tetrachloroethane \\ Tetrachloroethylene \\ Total dissolved solids \\ Total organic carbon \\ Total organic carbon \\ Total organic halogens \\ Total organic halogens \\ Phenols \\ Total phosphates (as P) \\ Trichloroethylene \\ Toxaphene \\ trans-1,2-Dichloroethylene \\ trans-1,3-Dich Joropropene \\ 1,1-Dichloroethylene \\ 1,1-Dichloroethane \\ 1,1,1-Trichloroethane \\ 1,1,2-Trichloroethane \\ 1,2-Dichloroethane \\ 1,2-Dich loropropane \\ cis-1,3-D ich loropropene \\ 2-Chloroethyl vinyl ether \\ 2,4-Dichlorophenoxyacetic acid \\ Gross a lpha \\ Nonvolatile beta \\ Total radium \\ Tritium
}

$\begin{array}{rrrr} & 6320.00 \mathrm{ug} / \mathrm{L} & \text { Gen. Eng. } \\ \mathrm{LT} & 0.09 \mathrm{ug} / \mathrm{L} & \text { Gen. Eng. } \\ \mathrm{LT} & 1000.00 \mathrm{ug} / \mathrm{L} & \text { Gen. Eng. } \\ \mathrm{LT} & 1.00 \mathrm{ug} / \mathrm{L} & \text { Gen. Eng. } \\ \mathrm{LT} & 1.00 \mathrm{ug} / \mathrm{L} & \text { Gen. Eng. } \\ 170000.00 \mathrm{ug} / \mathrm{L} & \text { Gen. Eng. } \\ 7000.00 \mathrm{ug} / \mathrm{L} & \text { Gen. Eng. } \\ 7000.00 \mathrm{ug} / \mathrm{L} & \text { Gen. Eng. } \\ & 339.00 \mathrm{ug} / \mathrm{L} & \text { Gen. Eng. } \\ & 331.00 \mathrm{ug} / \mathrm{L} & \text { Gen. Eng. } \\ \mathrm{LT} & 5.00 \mathrm{ug} / \mathrm{L} & \text { Gen. Eng. } \\ \mathrm{LT} & 100.00 \mathrm{ug} / \mathrm{L} & \text { Gen. Eng. } \\ \mathrm{LT} & 1.00 \mathrm{ug} / \mathrm{L} & \text { Gen. Eng. } \\ \mathrm{LT} & 0.24 \mathrm{ug} / \mathrm{L} & \text { Gen. Eng. } \\ \mathrm{LT} & 1.00 \mathrm{ug} / \mathrm{L} & \text { Gen. Eng. } \\ \mathrm{LT} & 1.00 \mathrm{ug} / \mathrm{L} & \text { Gen. Eng. } \\ \mathrm{LT} & 1.00 \mathrm{ug} / \mathrm{L} & \text { Gen. Eng. } \\ & 86.00 \mathrm{ug} / \mathrm{L} & \text { Gen. Eng. } \\ \mathrm{LT} & 1.00 \mathrm{ug} / \mathrm{L} & \text { Gen. Eng. } \\ \mathrm{LT} & 1.00 \mathrm{ug} / \mathrm{L} & \text { Gen. Eng. } \\ & 4.00 \mathrm{ug} / \mathrm{L} & \text { Gen. Eng. } \\ \mathrm{LT} & 1.00 \mathrm{ug} / \mathrm{L} & \text { Gen. Eng. } \\ \mathrm{LT} & 1.00 \mathrm{ug} / \mathrm{L} & \text { Gen. Eng. } \\ \mathrm{LT} & 1.00 \mathrm{ug} / \mathrm{L} & \text { Gen. Eng. } \\ \mathrm{LT} & 0.30 \mathrm{ug} / \mathrm{L} & \text { Gen. Eng. } \\ \mathrm{LT} & 2.00 \mathrm{pCi} / \mathrm{L} & \text { Gen. Eng. } \\ 3.50+- & 2.10 \mathrm{pCi} / \mathrm{L} & \text { Gen. Eng. } \\ \mathrm{LT} & 1.00 \mathrm{pCi} / \mathrm{L} & \text { Gen. Eng. } \\ 15.90+- & 0.60 \mathrm{pCi} / \mathrm{mL} & \text { Gen. Eng. } \\ & & & \end{array}$

HELL LFW 55C

$\begin{array}{llllll}\text { SRS Grid } & \text { N } & 83613.2 & \text { Latitude } & 33.284451 & \text { N } \\ \text { Coordinates } & \text { E } & \mathbf{4 5 2 0 5 . 9} & \text { Longitude } & 81.710181 & \text { W }\end{array}$

Screen Zone Elevation 103.9-93.9 ft msl

Top of Casing Elevation $153.90 \mathrm{ft} \mathrm{ms}$

\section{MEASUREMENTS CONDUCTED IN THE FIELD}

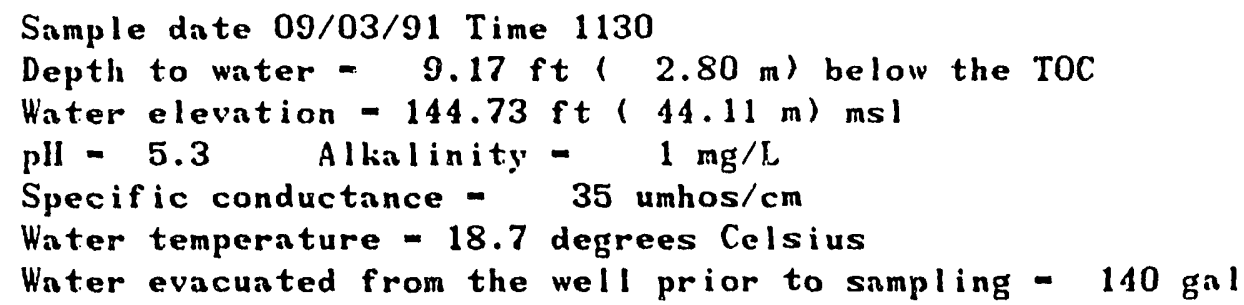

\section{LABORATORY ANALYSES}

\footnotetext{
Specific conductance

pll

Silver

CONTINUED
}

$\begin{array}{lcl} & 36.00 \mathrm{umh} / \mathrm{cm} & \text { Geñ. Eng. } \\ & 5.47 \mathrm{pH} & \text { Gen. Eng. } \\ \text { LT } & 2.00 \mathrm{ug} / \mathrm{L} & \text { Gen. Eng. }\end{array}$




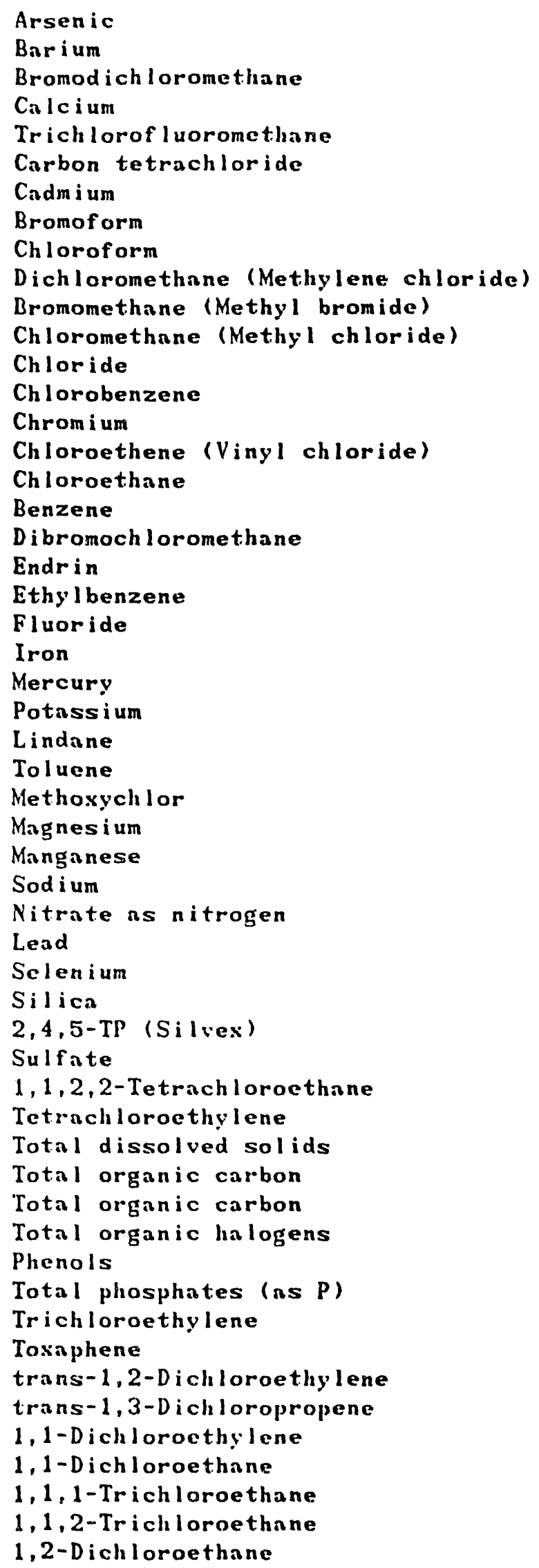

LT

LT

LT

LT

LT

LT

LT

LT

LT

LT

LT

LT

LT

LT

LT

LT

LT

LT

LT

LT

LT

LT

LT

LT

LT

LT

LT

LT

LT

$$
\text { (1) }
$$

LT

LT

LT

LT

LT

LT

LT

LT

LT

LT

LT

LT

LT

$$
\begin{array}{r}
2.00 \mathrm{ug} / \mathrm{L} \\
3.56 \mathrm{ug} / \mathrm{L} \\
1.00 \mathrm{ug} / \mathrm{L} \\
867.00 \mathrm{ug} / \mathrm{L} \\
1.00 \mathrm{ug} / \mathrm{L} \\
1.00 \mathrm{ug} / \mathrm{L} \\
2.00 \mathrm{ug} / \mathrm{L} \\
1.00 \mathrm{ug} / \mathrm{L} \\
1.00 \mathrm{ug} / \mathrm{L} \\
1.00 \mathrm{ug} / \mathrm{L} \\
1.00 \mathrm{ug} / \mathrm{L} \\
1.00 \mathrm{ug} / \mathrm{L}
\end{array}
$$$$
3560.00 \mathrm{ug} / \mathrm{L}
$$$$
1.00 \mathrm{ug} / \mathrm{L}
$$$$
4.00 \mathrm{ug} / \mathrm{L}
$$$$
1.00 \mathrm{ug} / \mathrm{L}
$$$$
1.00 \mathrm{ug} / \mathrm{L}
$$$$
1.00 \mathrm{ug} / \mathrm{L}
$$$$
1.00 \mathrm{ug} / \mathrm{L}
$$$$
0.01 \mathrm{ug} / \mathrm{L}
$$$$
1.00 \mathrm{ug} / \mathrm{L}
$$$$
100.00 \mathrm{ug} / \mathrm{L}
$$$$
9.25 \mathrm{ug} / \mathrm{L}
$$$$
0.20 \mathrm{ug} / \mathrm{L}
$$$$
500.00 \mathrm{ug} / \mathrm{L}
$$$$
0.00 \mathrm{ug} / \mathrm{L}
$$$$
1.00 \mathrm{ug} / \mathrm{L}
$$$$
0.50 \mathrm{ug} / \mathrm{L}
$$

$1160.00 \mathrm{ug} / \mathrm{L}$

$10.30 \mathrm{ug} / \mathrm{L}$

$2760.00 \mathrm{ug} / \mathrm{L}$

$970.00 \mathrm{ug} / \mathrm{L}$

$3.00 \mathrm{ug} / \mathrm{L}$

$2.00 \mathrm{ug} / \mathrm{L}$

$7690.00 \mathrm{ug} / \mathrm{L}$

0.09 ug $/ \mathrm{L}$

$1000.00 \mathrm{ug} / \mathrm{L}$

$1.00 \mathrm{ug} / \mathrm{L}$

$1.00 \mathrm{ug} / \mathrm{L}$ $27000.00 \mathrm{ug} / \mathrm{L}$

$1000.00 \mathrm{ug} / \mathrm{L}$

$1000.00 \mathrm{ug} / \mathrm{L}$

$8.20 \mathrm{ug} / \mathrm{L}$

$5.00 \mathrm{ug} / \mathrm{L}$ $100.00 \mathrm{ug} / \mathrm{L}$

$1.00 \mathrm{ug} / \mathrm{L}$

$0.24 \mathrm{ug} / \mathrm{L}$

$1.00 \mathrm{ug} / \mathrm{L}$

$1.00 \mathrm{ug} / \mathrm{L}$

$1.00 \mathrm{ug} / \mathrm{L}$

$1.00 \mathrm{ug} / \mathrm{L}$

$1.00 \mathrm{ug} / \mathrm{L}$

$1.00 \mathrm{ug} / \mathrm{L}$

$1.00 \mathrm{ug} / \mathrm{L}$
Gen. Eng.

Gen. Eng.

Gen. Eng.

Gen. Eng.

Gen. Eng.

Gen. Eng.

Gen. Eng.

Gen. Eng.

Gen. Eng.

Gen. Eng.

Gen. Eng.

Gen. Eng.

Gen: Eng.

Gen. Eng.

Gen. Eng.

Gen. Eng.

Gen. Eng.

Gen. Eng.

Gen. Eng.

Gen. Eng.

Gen. Eng .

Gen. Eng.

Gen. Eng.

Gen. Eng.

Gen. Eng.

Gen. Eng.

Gen. Eng.

Gen. Eng.

Gen. Eng.

Gen. Eng.

Gen. Eng.

Gen. Eng.

Gen. Eng.

Gen. Eng.

Gen. Eng.

Gen. Eng.

Gen. Eng.

Gen. Eng.

Gen. Eng.

Gen. Eng.

Gen. Eng.

Gen. Eng.

Gen. Eng.

Gen. Eng.

Gen. Eng.

Gen. Eng.

Gen. Eng.

Gen. Eng.

Gen. Eng.

Gen. Eng.

Geñ. Eng .

Gen. Eng.

Gen. Eng.

Gen. Eng. 
WELL LFW 55C COLLECTED ON 09/03'91 LABORATORY. ANALYSES CONTINUED

$\begin{array}{lccc}\text { 1,2-Dichloropropane } & \text { LT } & 1.00 \mathrm{ug} / \mathrm{L} & \text { Gen. Eng. } \\ \text { cis-1,3-Dichloropropene } & \text { LT } & 1.00 \mathrm{ug} / \mathrm{L} & \text { Gen. Eng. } \\ \text { 2-Chlorocthyl vinylether } & \text { LT } & 1.00 \mathrm{ug} / \mathrm{L} & \text { Gen. Eng. } \\ \text { 2,4-Dichlorophenc gacetic acid } & \text { LT } & 0.30 \mathrm{ug} / \mathrm{L} & \text { Gen. Eng. } \\ \text { Gross alpha } & \text { LT } & 2.00 \mathrm{pCi} / \mathrm{L} & \text { Gen. Eng. } \\ \text { Nonvolatile beta } & \mathrm{LT} & 2.00 \mathrm{pCi} / \mathrm{L} & \text { Gen. Eng. } \\ \text { Total radium } & 1.50+- & 2.40 \mathrm{pCi} / \mathrm{L} & \text { Gen. Eng. } \\ \text { Tritium } & 2.03+- & 0.20 \mathrm{pCi} / \mathrm{mL} & \text { Gen. Eng. }\end{array}$

WELL LFW 55D

SRS Grid N 83601.3 Latitude 33.284398 N

Coordinates E 45189.3 Longitude 81.710202 W

Screen Zone Elevation 141.4-121.2 ft msl

Top of Casing Elevation $153.40 \mathrm{ft} \mathrm{msl}$

MEASUREMENTS CONDUCTED IN THE FIELD

Sample date 09/03/91 Time 1105

Depth to water - $8.08 \mathrm{ft}(2.46 \mathrm{~m})$ below the TOC

Water elevation - $145.32 \mathrm{ft}(44.29 \mathrm{~m}) \mathrm{msl}$

$\mathrm{pH}-5.2$ Alkalinity $=1 \mathrm{mg} / \mathrm{L}$

Specific conductance - 22 umhos/cm

Water temperature -19.3 degrees Celsius

Water evacuated from the well prior to sampling - $70 \mathrm{gal}$

\section{LABORATORY ANALYSES}

Specific conductance

pH

Silver

Arsenic

Barium

Bromodichloromethane

Calcium

Trichlor of luoromethane

Carbon tetrachloride

Cadmi um

Bromof orm

Chloroform

Dichloromethane (Methylene chloride)

Bromomethane (Methyi bromicle)

Chloromethane (Methyl chloride)

Chloride

Chlorobenzene

Chromium

Chloroethene (Vinyl chloride)

Chloroethane

Benzene

Dibromoch loromethane

Endrin

Ethylbenzene

CONTINUED
$23.00 \mathrm{umh} / \mathrm{cm}$

$5.33 \mathrm{pHI}$

LT

LT

ug/L

$2.00 \mathrm{ug} / \mathrm{L}$

$9.80 \mathrm{ug} / \mathrm{L}$

LT

$1.00 \mathrm{ug} / \mathrm{L}$

$862.00 \mathrm{ug} / \mathrm{L}$

$1.00 \mathrm{ug} / \mathrm{L}$

$1.00 \mathrm{ug} / \mathrm{L}$

$2.00 \mathrm{ug} / \mathrm{L}$

$1.00 \mathrm{ug} / \mathrm{L}$

$1.00 \mathrm{ug} / \mathrm{L}$

$1.00 \mathrm{ug} / \mathrm{L}$

$1.00 \mathrm{ug} / \mathrm{L}$

$1.00 \mathrm{ug} / \mathrm{L}$

$2530.00 \mathrm{ug} / \mathrm{L}$

$1.00 \mathrm{ug} / \mathrm{L}$

$4.00 \mathrm{ug} / \mathrm{L}$

$1.00 \mathrm{ug} / \mathrm{L}$

$1.00 \mathrm{ug} / \mathrm{L}$

$1.00 \mathrm{ug} / \mathrm{L}$

$1.00 \mathrm{ug} / \mathrm{L}$

$0.01 \mathrm{ug} / \mathrm{L}$

$1.00 \mathrm{ug} / \mathrm{L}$
Gen. Eng.

Gen. Eng.

Gen. Eng.

Gen. Eng.

Gen. Eng.

Gen. Eng.

Gen. Eng.

Gen. Eng.

Gen. Eng.

Gen. Eng.

Gen. Eng.

Gen. Eng.

Gen. Eng.

Gen. Eng.

Gen. Eng.

Gen. Eng.

Gen. Eng.

Gen. Eng.

Gen. Eng.

Gen. Eng.

Gen. Eng.

Gen- Eng.

Gen. Eng.

Geil. Eng. 
WELL LFW 55D COLLECTED ON 09/03/91 LABORATORY ANALYSES CONTINUED

Fluoride
Iron
Mercury
Potassium
Lindane
Toluene
Methosychlor
Magnesium
Manganese
Sodium
Nitrate as nitrogen
Lead
Selenium
Silica
2,4,5-TP (Silvex)
Sulfate
1,1,2,2-Tetrachloroethane
Tetrachloroethylene
Total dissolved soids
Total organic carbon
Total organic halogens
Total organic halogens
Phenols
Total phosphates (as P)
Trichloroethylene
Tosaphene
trans-1,2-Dichloroethylene
trans-1,3-Dichloropropene
1,1-Dichloroethylene
1, 1-Dichloroethane
1,1,1-Trichloroethane
1,1,2-Trichloroetlane
1,2-Dichloroethare
1,2-Dichloropropane
cis-1,3-Dichloropropene
2-Chloroethyl vinyl ether
2, -Dichlorophenoxyacetic ac:d
Gross alpha
Nonvolatile beta
Total radium
Tritium

\begin{tabular}{|c|c|c|}
\hline LT & 100.00 & $u_{g} / L$ \\
\hline & 6.79 & $\mathrm{ug} / \mathrm{L}$ \\
\hline LT & 0.20 & $u g / L$ \\
\hline & 644.00 & ug/L \\
\hline LT & 0.00 & ug $/ L$ \\
\hline LT & 1.00 & ug/L \\
\hline LIT & 0.50 & $u g / L$ \\
\hline & 471.00 & $u_{g} / L$ \\
\hline & 20.80 & $u_{g} / L$ \\
\hline & 1220.00 & $u g / L$ \\
\hline & 280.00 & $u g / L$ \\
\hline LT & 3.00 & $\mathbf{u g} / \mathrm{L}$ \\
\hline LT & 2.00 & $u_{g} / L$ \\
\hline & 6350.00 & ug/L \\
\hline L.T & 0.09 & $\mathrm{ug} / \mathrm{L}$ \\
\hline LT & 1000.00 & ug/L \\
\hline LT & 1.00 & $u g / L$ \\
\hline LT & 1.00 & $\mathbf{u g} / \mathrm{L}$ \\
\hline & 24000.00 & ug $/ \mathrm{L}$ \\
\hline LT & 1000.00 & ug/L \\
\hline LT & 5.00 & $\mathrm{ug} / \mathrm{L}$ \\
\hline LT & 5.00 & $u_{g} / L$ \\
\hline LT & 5.00 & $u_{g} / L$ \\
\hline L.T & 100.00 & ug/L \\
\hline LT & 1.00 & ug/L \\
\hline LT & 0.24 & $u g / L$ \\
\hline LT & 1.00 & $u g / L$ \\
\hline LT & 1.00 & $u g / L$ \\
\hline LT & 1.00 & ug/L \\
\hline LT & 1.00 & ug/L \\
\hline LT & 1.00 & ug/L \\
\hline LT & 1.00 & ug/L \\
\hline LT & 1.00 & ug/L \\
\hline $\mathrm{LT}$ & 1.00 & ug/L \\
\hline LT & 1.00 & $u g / L$ \\
\hline LT & 1.00 & $u g / L$ \\
\hline LT & 0.30 & $u g / L$ \\
\hline LT & 2.00 & $\mathrm{p} C \mathrm{i} / \mathrm{L}$ \\
\hline 2.10 & 1.96 & $\mathrm{pCi} / \mathrm{L}$ \\
\hline LT & 1.00 & $\mathrm{pCi} / \mathrm{L}$ \\
\hline 2.99 & 0.30 & $\mathrm{pCi} / \mathrm{mL}$ \\
\hline
\end{tabular}

Gen. Eng.

Gen. Eng.

Gen. Eng.

Gen. Eng.

Gen. Eng.

Gen. Eng .

Gen. Eng.

Gen. Eng.

Gen. Eng .

Gen. Eng .

Gen. Eng .

Gen. Eng .

Gen.- Eng.

Gen. Eng.

Gen. Eng.

Gen. Eng.

Gen. Eng .

Gen. Eng .

Gen. Eng.

Gen. Eng.

Gen. Eng -

Gen. Eng.

Gen. Eng -

Gen. Eng.

Gen. Eng.

Gen. Eng.

Gen. Eng .

Gen. Eng.

Gen. Eng.

Gen. Eng.

Gen. Eng.

Gen. Eng.

Gen. Eng.

Gen. Eng.

Gen. Eng.

Gen. Eng.

Gen. Eng.

Gen. Eng.

Gen. Eng.

Gen. Eng.

Gen. Eng. 
WELL LFW 56D

$\begin{array}{llccc}\text { SRS Grid } & N & 83398.0 & \text { Latitude } 33.284140 & \mathrm{~N} \\ \text { Coordinates } & E & 45306.6 & \text { Longitude } 81.709498 & \mathrm{~W} \\ \text { Screen Zone Elevation } 151.4-131.3 \mathrm{ft} \mathrm{msl} & \\ \text { Top of Cosing Elevation } 155.90 \mathrm{ft} \mathrm{msl}\end{array}$

MEASUREMENTS CONDUCTED IN TIIE FIELD

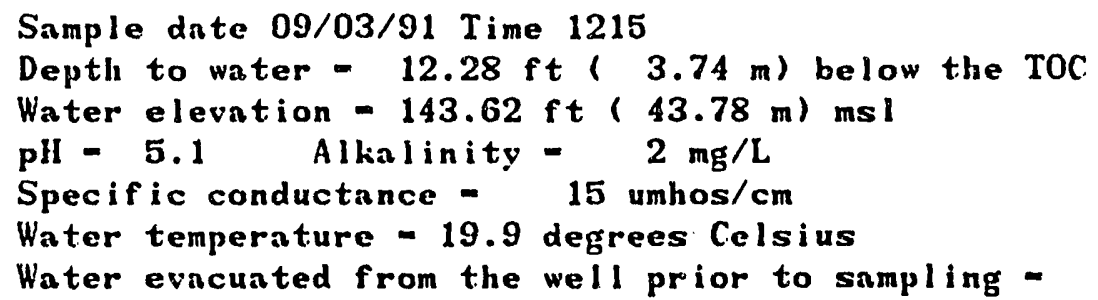

\section{LABORATORY ANALYSES}

Specif ic conductance

pll

Silver

Arsenic

Barium

Bromodichloromethane

Calcium

Trichl or of I uoromethane

Carbon tetrachloride

Cadmi um

Bromoform

Chlorof orm

Dichloromethane (Methylene chloride)

Bromomethane (Methyl bromide)

Chloromethane (Methyl chloride)

Chlor ide

Chlorobenzene

Chromium

Chlorocthene (Vinyl chloride)

Chloroctiane

Benzene

Dibromoch loromethane

Endrin

Ethylbenzene

Fluoride

Iron

Mercury

Potassium

Lindane

Toluerne

Methoxychior

Magnes $i$ um

Manganese

Sodium

Nitrate as nitrogen

Lead

Selen i um

CONTINUED
$18.00 \mathrm{umh} / \mathrm{cm}$

$5.38 \mathrm{pll}$

LI

LT

LT

$T$

LI

LT

LT

LT

LT

LT

LT

LI

LT

LT

L.T

LT

LT

LT

LT

LT

LT

LT

LT

LT

LI

LT
$2.00 \mathrm{ug} / \mathrm{L}$

$2.00 \mathrm{ug} / \mathrm{L}$

$3.80 \mathrm{ug} / \mathrm{L}$

$1.00 \mathrm{ug} / \mathrm{L}$

$399.00 \mathrm{ug} / \mathrm{L}$

$57.00 \mathrm{ug} / \mathrm{L}$

$1.00 \mathrm{ug} / \mathrm{L}$

$2.00 \mathrm{ug} / \mathrm{L}$

$1.00 \mathrm{ug} / \mathrm{L}$

$1.00 \mathrm{ug} / \mathrm{L}$

$1.00 \mathrm{ug} / \mathrm{L}$

$1.00 \mathrm{ug} / \mathrm{L}$

$1.00 \mathrm{ug} / \mathrm{L}$

$1650.00 \mathrm{ug} / \mathrm{L}$

$1.00 \mathrm{ug} / \mathrm{L}$

$4.00 \mathrm{ug} / \mathrm{L}$

$1.00 \mathrm{ug} / \mathrm{L}$

$1.00 \mathrm{ug} / \mathrm{L}$

$1.00 \mathrm{ug} / \mathrm{L}$

$1.00 \mathrm{ug} / \mathrm{L}$

0.01 ug $/ \mathrm{L}$

$1.00 \mathrm{ug} / \mathrm{L}$

$100.00 \mathrm{ug} / \mathrm{L}$

$4.00 \mathrm{ug} / \mathrm{L}$

$0.20 \mathrm{ug} / \mathrm{L}$

$500.00 \mathrm{ug} / \mathrm{L}$

$0.00 \mathrm{ug} / \mathrm{L}$

$1.00 \mathrm{ug} / \mathrm{L}$

$0.50 \mathrm{ug} / \mathrm{L}$

$327.00 \mathrm{ug} / \mathrm{L}$

$4.36 \mathrm{ug} / \mathrm{L}$

$1070.00 \mathrm{ug} / \mathrm{L}$

$82.00 \mathrm{~kg} / \mathrm{I}$.

$3.00 \mathrm{ug} / \mathrm{L}$

$2.00 \mathrm{ug} / \mathrm{L}$
Gen. Eng -

Gen. Eng.

Gen. Eng.

Gen. Eng.

Gen. Eng.

Gen. Eng.

Gen. Eng.

Gen. Eng.

Gen. Eng.

Gen. Eng.

Gen. Eng.

Gen. Eng.

Gen. Eng.

Gen. Eng.

Gen. Eng.

Gen. Eng.

Gen. Eng.

Gen. Eng .

Gen. Eng.

Gen. Eng.

Gen. Eng.

Gen. Eng.

Gen. Eng.

Gen. Eng.

Gen. Eng.

Gen. Eng.

Gen. Eng.

Gen. Eng.

Gen. Eng.

Gen. Eng.

Gen. Eng.

Gen. Eng.

Gen. Eng.

Geñ. Eng.

Gen. Eng.

Gen. Eng.

Gen. Eng. 
WELL LEW 56D COLLECTED ON 09/03/91. LABORATORY ANALYSES CONTINUED

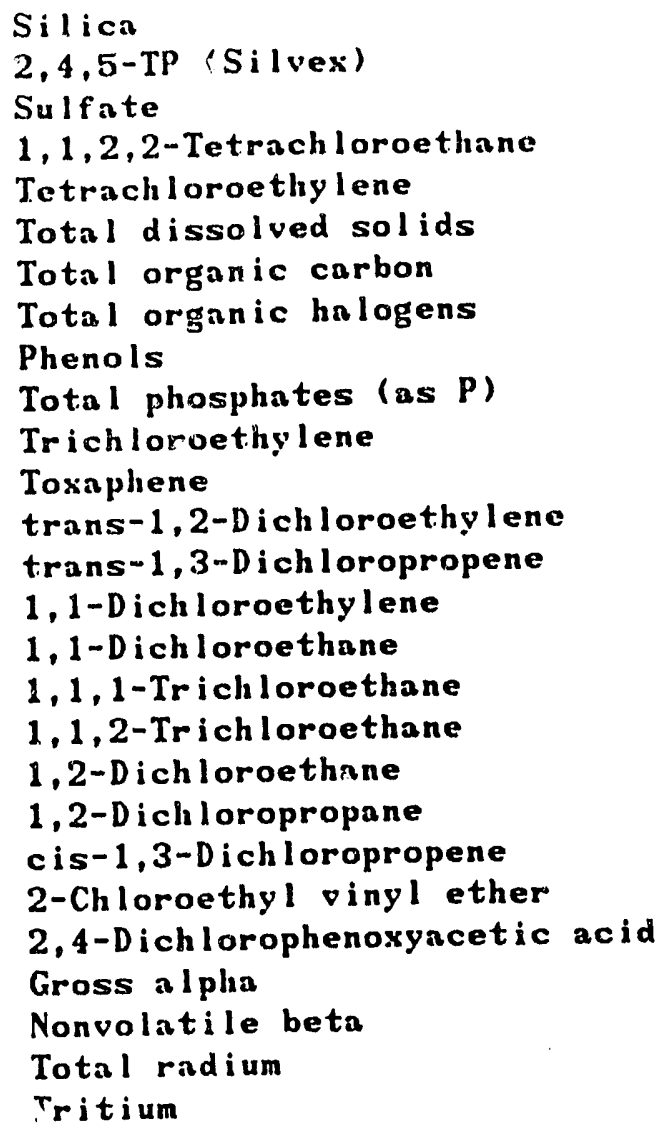

$\begin{array}{lrl} & 6130.00 \mathrm{ug} / \mathrm{L} \\ \mathrm{LT} & 0.09 \mathrm{ug} / \mathrm{L} \\ \mathrm{LT} & 1000.00 \mathrm{ug} / \mathrm{L} \\ \mathrm{LT} & 1.00 \mathrm{ug} / \mathrm{L} \\ \mathrm{LT} & 1.00 \mathrm{ug} / \mathrm{L} \\ & 18000.00 \mathrm{ug} / \mathrm{L} \\ \mathrm{LT} & 1000.00 \mathrm{ug} / \mathrm{L} \\ & 13.20 \mathrm{ug} / \mathrm{L} \\ \mathrm{LT} & 5.00 \mathrm{ug} / \mathrm{L} \\ \mathrm{LT} & 100.00 \mathrm{ug} / \mathrm{L} \\ \mathrm{LT} & 1.00 \mathrm{ug} / \mathrm{L} \\ \mathrm{LT} & 0.24 \mathrm{ug} / \mathrm{L} \\ \mathrm{LT} & 1.00 \mathrm{ug} / \mathrm{L} \\ \mathrm{LT} & 1.00 \mathrm{ug} / \mathrm{L} \\ \mathrm{LT} & 1.00 \mathrm{ug} / \mathrm{L} \\ \mathrm{LT} & 1.00 \mathrm{ug} / \mathrm{L} \\ \mathrm{LT} & 1.00 \mathrm{ug} / \mathrm{L} \\ \mathrm{LT} & 1.00 \mathrm{ug} / \mathrm{L} \\ \mathrm{LT} & 1.00 \mathrm{ug} / \mathrm{L} \\ \mathrm{LT} & 1.00 \mathrm{ug} / \mathrm{L} \\ \mathrm{LT} & 1.00 \mathrm{ug} / \mathrm{L} \\ \mathrm{LT} & 1.00 \mathrm{ug} / \mathrm{L} \\ \mathrm{LT} & 0.30 \mathrm{ug} / \mathrm{L} \\ \mathrm{LT} & 2.00 \mathrm{pCi} / \mathrm{L} \\ \mathrm{LT} & 2.00 \mathrm{pCi} / \mathrm{L} \\ 1.50+- & 2.40 \mathrm{pCi} / \mathrm{L} \\ 3.92+- & 0.30 \mathrm{pCi} / \mathrm{mL}\end{array}$

Gen. Eng.

Gen. Eng.

Gen. Eng.

Gen. Eng.

Gen. Eng.

Gen. Eng.

Gen. Eng.

Gen. Eng.

Gen. Eng.

Gen. Ing.

Gen. Eng.

Gen. Eng.

Gen.- Eng.

Gen. Eng.

Gen. Eng.

Gen. Eng.

Gen. Eng .

Gen. Eng -

Gen. Eng.

Gen. Eng.

Gen. Eng.

Gen. Eng.

Gen. Eng.

Gen. Eng.

Gen. Eng.

Gen. Eng.

Gen. Eng.

\section{HELL LFW 57B}

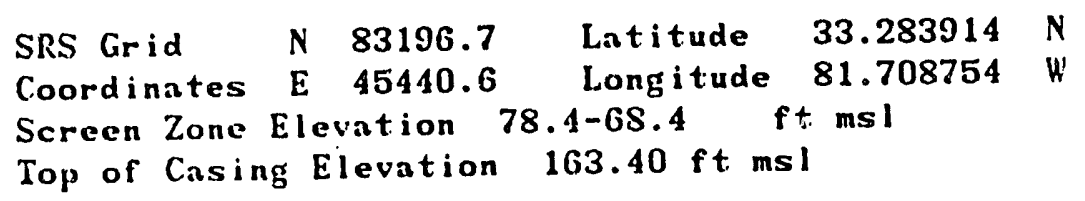

\section{MEASUREMENTS CONDUCTED IN THE FIELD}

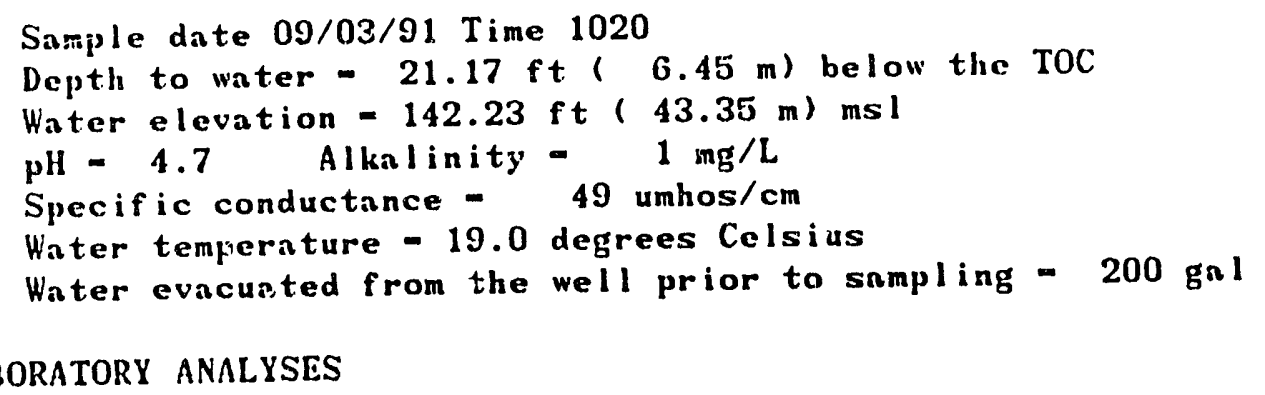

Specif ic conductance

$$
\text { pH }
$$

Silver

Arsenic

Barium

CONTINUED

$\begin{array}{lcl} & 49.00 \mathrm{umh} / \mathrm{cm} & \text { Gen. Eng. } \\ & 4.89 \mathrm{pll} & \text { Gen. Eng. } \\ \text { LT } & 2.00 \mathrm{ug} / \mathrm{L} & \text { Feñ. Eng. } \\ \text { LT } & 2.00 \mathrm{ug} / \mathrm{L} & \text { Gen. Eng. } \\ & 1 \mathrm{i} .10 \mathrm{ug} / \mathrm{L} & \text { Gen. Eng. }\end{array}$


WELL LFW 57B COLLECTED ON 09/03/91 LABORATORY ANALYSES CONTINUED

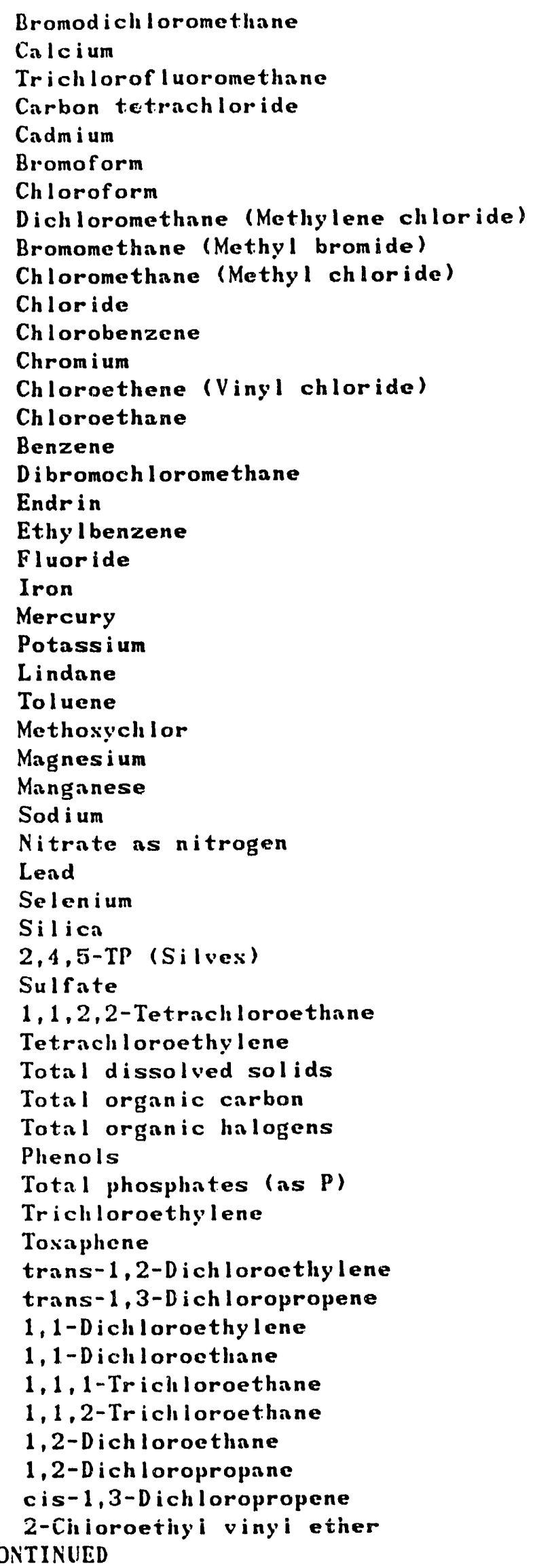

LI

$1.00 \mathrm{ug} / \mathrm{L}$
$1640.00 \mathrm{ug} / \mathrm{L}$
$4.00 \mathrm{ug} / \mathrm{L}$
$1.00 \mathrm{ug} / \mathrm{L}$
$2.00 \mathrm{ug} / \mathrm{L}$
$1.00 \mathrm{ug} / \mathrm{L}$
$1.00 \mathrm{ug} / \mathrm{L}$
$1.00 \mathrm{ug} / \mathrm{L}$
$1.00 \mathrm{ug} / \mathrm{L}$
$1.00 \mathrm{ug} / \mathrm{L}$
$2370.00 \mathrm{ug} / \mathrm{L}$

$1.00 \mathrm{ug} / \mathrm{L}$

$4.00 \mathrm{ug} / \mathrm{L}$

$1.00 \mathrm{ug} / \mathrm{L}$

$1.00 \mathrm{ug} / \mathrm{L}$

$1.00 \mathrm{ug} / \mathrm{L}$

$1.00 \mathrm{ug} / \mathrm{L}$

$0.01 \mathrm{ug} / \mathrm{L}$

$1.00 \mathrm{ug} / \mathrm{L}$

$100.00 \mathrm{ug} / \mathrm{L}$

$147.00 \mathrm{ug} / \mathrm{L}$

LT

LT

LT

LT

LT

$0.20 \mathrm{ug} / \mathrm{L}$

$500.00 \mathrm{ug} / \mathrm{L}$

$0.00 \mathrm{ug} / \mathrm{L}$

$1.00 \mathrm{ug} / \mathrm{L}$

$0.50 \mathrm{ug} / \mathrm{L}$

$560.00 \mathrm{ug} / \mathrm{L}$

$39.00 \mathrm{ug} / \mathrm{L}$

$3060.00 \mathrm{ug} / \mathrm{L}$

$940.00 \mathrm{ug} / \mathrm{L}$

LT

LT

LT

LT

LT

\section{LT}

LT

LT

LT

LT

LT

LT

LT

LT

LT

LT

LT

LT

LT

LT

LI
Gen. Eng.

Gen. Eng.

Gen. Eng.

Gen. Eng.

Gen. Eng.

Gen. Eng.

Gen. Eng.

Gen. Eng.

Gen. Eng.

Gen. Eng.

Gen. Eng.

Gen. Eng.

Gen.-Eng.

Gen. Eng.

Gen. Eng.

Gen. Eng.

Gen. Eng.

Gen. Eng.

Gen. Eng.

Gen. Eng.

Gen. Eng.

Gen. Eng.

Gen. Eng.

Gen. Eng.

Gen. Eng.

Gen. Eng.

Gen. Eng.

Gen. Eng.

Gen. Eng.

Gen. Eng.

Gen. Eng.

Gen. Eng.

Gen. Eng.

Gen. Eng.

Gen. Eng.

Gen. Eng

Gen. Eng.

Gen. Eng.

Gen. Eng.

Gen. Eng.

Gen. Eng.

Gen. Eng.

Gen. Eng.

Gen. Eng.

Gen. Eng.

Gen. Eng.

Gen. Eng.

Gen. Eng.

Gen. Eng.

Gen. Eng.

Geñ. Eng.

Gen. Eng.

Gen. Eng.

Gen. Eng. 
WELL LFW 57B COLLECTED ON 09/03/91 LABORATORY ANALYSES CONTINUED

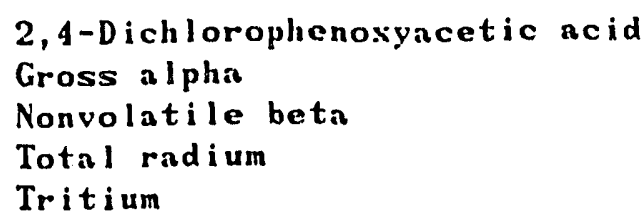

$\begin{array}{cll}\text { LT } & 0.30 \mathrm{ug} / \mathrm{L} & \text { Gen. Eng. } \\ \mathrm{LT} & 2.00 \mathrm{pCi} / \mathrm{L} & \text { Gen. Eng. } \\ 5.10+- & 2.10 \mathrm{pC} \mathrm{i} / \mathrm{L} & \text { Gen. Eng. } \\ \mathrm{LT} & 1.00 \mathrm{pCi} / \mathrm{L} & \text { Gen. Eng. } \\ \mathrm{LT} & 0.70 \mathrm{pCi} / \mathrm{mL} & \text { Gen. Eng. }\end{array}$

WELL LFW 57C

SRS Grid N 83200.1 Latitude $33.283873 \mathrm{~N}$

Coordinates E 45411.1 Longitude $81.708838 \mathrm{~W}$

Screen Zone Elevation 117.9-107.8 ft ms

Top of Casing Elevation $162.90 \mathrm{ft} \mathrm{msl}$

MEASUREMENTS CONDUCTED IN THE FIELD

Sample date 09/03/91 Time 925

Depth to water $=20.63 \mathrm{ft}(6.29 \mathrm{~m})$ below the TOC

Water elevation $=142.27 \mathrm{ft}(43.36 \mathrm{~m}) \mathrm{msl}$

pll $=5.7 \quad$ Alkalinity $=5 \mathrm{mg} / \mathrm{L}$

Specific conductance - 39 umhos/cm

Water temperature - 18.5 degrees Celsius

Water evacuated from the well prior to sampling - 97 gal

\section{LABORATORY ANALYSES}

Specific conductance

pH

Silver

Arsenic

Barium

Bromide

Bromodich loromethane

Calcium

Trichlorof l uoromethane

Carbon tetrachloride

Cadmi um

Bromoform

$\mathrm{Ch}_{1}$ loroform

Dichloromethane (Methylene chloride)

Bromumethane (Methyl bronide)

Chloromethane (Methyl chloride)

Chloride

Chlorobenzene

Chrom i um

Chloroethene (Vinyl chloride)

Chloroethane

Benzene

Dibromoch loromethane

Endrin

Ethylbenzene

Fluoride

Fluoride

CONTINUED
$37.00 \mathrm{umh} / \mathrm{cm}$

$5.75 \mathrm{pll}$

LT

$2.00 \mathrm{ug} / \mathrm{L}$

LT

$2.00 \mathrm{ug} / \mathrm{L}$

$6.45 \mathrm{ug} / \mathrm{L}$

LT $1000.00 \mathrm{ug} / \mathrm{L}$

LT $\quad 1.00 \mathrm{ug} / \mathrm{L}$

$1180.00 \mathrm{ug} / \mathrm{L}$

LT $1.00 \mathrm{ug} / \mathrm{L}$

LT

LT

LT

LT

LT

LT

LT

LT

LT

LT

LT

LT

LT

LT

LT

LT
$1.00 \mathrm{ug} / \mathrm{L}$

$2.00 \mathrm{ug} / \mathrm{L}$

$1.00 \mathrm{ug} / \mathrm{L}$

$1.00 \mathrm{ug} / \mathrm{L}$

$1.70 \mathrm{ug} / \mathrm{L}$

$1.00 \mathrm{ug} / \mathrm{L}$

$1.00 \mathrm{ug} / \mathrm{L}$

$4040.00 \mathrm{ug} / \mathrm{L}$

$1.00 \mathrm{ug} / \mathrm{L}$

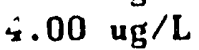

$1.00 \mathrm{ug} / \mathrm{L}$

$1.00 \mathrm{ug} / \mathrm{L}$

$1.00 \mathrm{ug} / \mathrm{L}$

$1.00 \mathrm{ug} / \mathrm{L}$

$0.01 \mathrm{ug} / \mathrm{L}$

$1.00 \mathrm{ug} / \mathrm{L}$

$100.00 \mathrm{ug} / \mathrm{L}$

$100.00 \mathrm{ug} / \mathrm{L}$
Gen. Eng

Gen. Eng.

Gen. Enğ.

Gen. Eng.

Gen. Eng.

Gen. Eng.

Gen. Eng.

Gen. Eng .

Gen. Eng .

Gen. Eng.

Gen. Eng.

Gen. Eng.

Gen. Eng.

Gen. Eng.

Gen. Eng.

Gen. Eng.

Gen. Eng.

Gen. Eng.

Gen. Eng.

Gen. Eng.

Gen. Eng.

Gen. Eng.

Gen : Eng.

Gen. Eng.

Geñ. Eng.

Gen. Eng .

Gen. Eng. 
WELL LFW 57C COLLECTED ON 09/03/91 LABORATORY ANALYSES CONTINUED

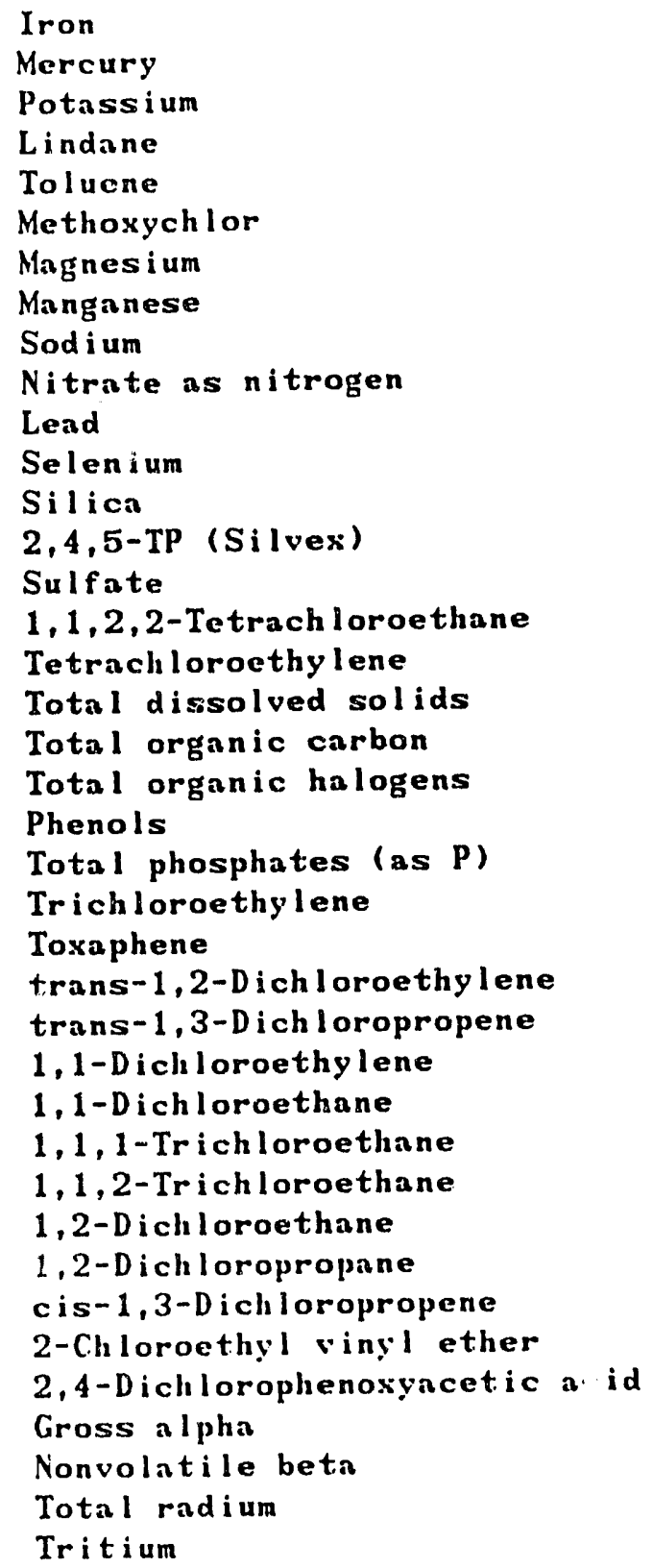

\begin{tabular}{|c|c|c|c|c|}
\hline & 38.80 & ug $/ \mathrm{L}$ & Gen. & Eng. \\
\hline$x$ & 0.20 & ug/L & Gen. & Eng \\
\hline & 500.00 & $u_{g} / L$ & Gen. & Eng \\
\hline LT & 0.00 & $\mathrm{ug}_{\mathrm{g}} / \mathrm{L}$ & en. & Eng \\
\hline $\mathrm{T}$ & 1.00 & ug $/ \mathrm{L}$ & ien. & Eng \\
\hline $\mathrm{I}$ & 0.50 & $u_{g} / L$ & Gen. & Eng \\
\hline & 180.00 & ug/L & Gen. & Eng \\
\hline & 17.50 & ug/L & Gen. & Eng \\
\hline & 680.00 & ug $/ \mathrm{L}$ & Gen. & Eng \\
\hline & 660.00 & ug/L & ien. & Eng \\
\hline$T$ & 3.00 & $\mathrm{ug} / \mathrm{L}$ & Gen. & Eng \\
\hline$T$ & 2.00 & ug $/ \mathrm{L}$ & Gen. & Eng \\
\hline & 350.00 & $\mathbf{u g} / \mathrm{L}$ & Gen. & Eng \\
\hline $\mathbf{T}$ & 0.09 & ug $/ L$ & Gen. & Eng \\
\hline$T$ & 000.00 & ug $/ \mathrm{L}$ & Gen. & Eng \\
\hline$T$ & 1.00 & $u_{g} / L$ & Gen. & EnE \\
\hline $\mathrm{T}$ & 1.00 & ug/L & Gen. & \\
\hline & 2000.00 & ug $/ \mathrm{L}$ & Gen. & \\
\hline $\mathrm{T}$ & $\begin{array}{r}000.00 \\
37.20\end{array}$ & $\begin{array}{l}u g / L \\
\mu \sigma / L\end{array}$ & Gen. & Eng \\
\hline 1 & $\begin{array}{r}37.20 \\
5.00\end{array}$ & $\begin{array}{l}\text { ug } / L \\
\text { ug } / L\end{array}$ & en. & \\
\hline 1 & 100.00 & ug $/ L$ & Gen. & Eng \\
\hline$L T$ & 1.00 & ug/L & en. & Eng \\
\hline LT & 0.24 & $u_{g} / L$ & en. & En \\
\hline LI & 1.00 & ug $/ L$ & en. & Er \\
\hline LT & 1.00 & ug/L & a. & - Er \\
\hline LT & 1.00 & ug/L & en. & \\
\hline & 6.00 & ug / L & $\bullet$ & \\
\hline LT & 1.00 & $u g / L$ & en. & - Er \\
\hline LT & 1.00 & ug $/ L$ & n. & \\
\hline LT & 1.00 & ug/L & n. & $\mathbf{E}$ \\
\hline LT & 1.00 & $\mathrm{ug} / \mathrm{L}$ & Gen. & $\mathbf{E}$ \\
\hline$L T$ & 1.00 & $u g / L$ & Gen. & En \\
\hline LT & 1.00 & ug/L & & En \\
\hline LT & 0.30 & $u g / L$ & n. & En \\
\hline LT & $\therefore .00$ & $\mathrm{pCi} / \mathrm{L}$ & n. & • \\
\hline 10 & 1.60 & $\mathrm{pCi} / \mathrm{L}$ & $n$. & • \\
\hline & 2.50 & $\mathrm{pCi} / \mathrm{L}$ & n. & 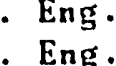 \\
\hline ר & 0.30 & $\mathrm{pCi} / \mathrm{mL}$ & & \\
\hline
\end{tabular}


HELL LFW 57D

$\begin{array}{lllll}\text { SRS Grid } & N & 83190.2 & \text { Latitude } 33.283861 & N \\ \text { Coordinates } & \text { E } 45417.4 & \text { Longitude } 81.708802 & W \\ \text { Screen Zone Elevation } 150.4-130.6 \quad \mathrm{ft} \mathrm{msl} & \\ \text { Top of Casing Elevation } 162.90 \mathrm{ft} \mathrm{msl} & \end{array}$

\section{MEASUREMENTS CONDUCTED IN THE FIELD}

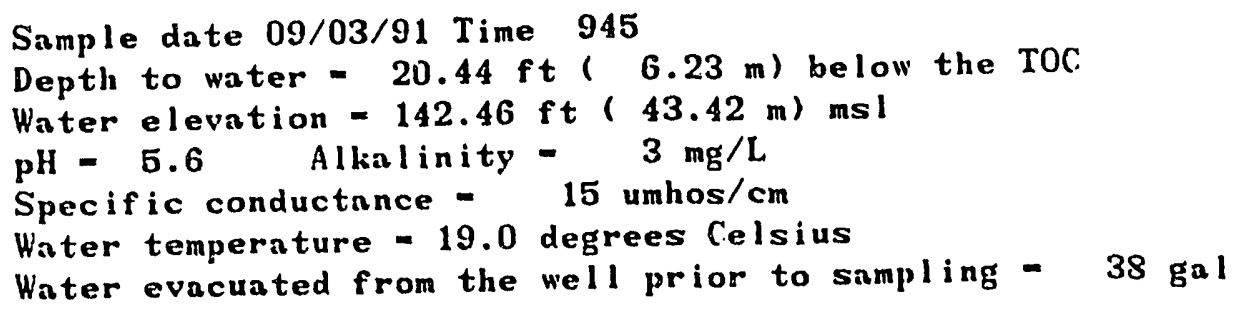

\section{LABORATORY ANALYSES}

Specific conductance
pll
Silver
Arsenic
Barium
Bromide
Bromodichloromethane
Calcium
Trichlorofluoromethane
Carbon tetrachloride
Cadmium
Bromoform
Chloroform
Dichloromethane (Methylene chloride)
Bromomethane (Methyl bromide)
Chloromethane (Methyl chloride)
Chloride
Chlorobenzene
Chromium
Chloroethene (Vinyl chloride)
Chloroethane
Benzene
Dibromochloromethane
Endrin
Ethylbenzene
Fluoride
Iron
Mercury
Potassium
Lindane
Toluene
Methosychlor
Magnesium
Manganese
Sodium
Nitrate as nitrogen
Lead
ONinded
Ming

\begin{tabular}{|c|c|c|c|c|}
\hline & 21.00 & $\mathrm{umh} / \mathrm{cm}$ & Gen. & ng. \\
\hline & 5.80 & pH & Gen. & Eng \\
\hline LT & 2.00 & ug $/ \mathrm{L}$ & Gen. & Eng \\
\hline$L T$ & 2.00 & $u g / L$ & Gen. & Eng \\
\hline & 6.38 & ug $/ \mathrm{L}$ & Gen. & Eng \\
\hline $\mathrm{T}$ & 1000.00 & ug $/ \mathrm{L}$ & Gen. & Eng \\
\hline$\Gamma$ & 1.00 & ug $/ \mathrm{L}$ & Gen. & $\mathbf{n g}_{\mathrm{g}}$ \\
\hline & 1010.00 & ug $/ L$ & Gen. & 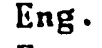 \\
\hline$T$ & 1.00 & ug $/ \mathrm{L}$ & ien. & Eng \\
\hline $\mathrm{T}$ & 1.00 & ug/L & ien. & ng \\
\hline$T$ & 2.00 & ug/L & en. & ing \\
\hline$T$ & 1.00 & ug $/ \mathrm{L}$ & Gen. & Eng \\
\hline $\mathrm{T}$ & 1.00 & ug $/ L$ & Gen. & $\pi$ \\
\hline & 1.00 & $\mathrm{ug} / \mathrm{L}$ & Gen. & 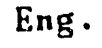 \\
\hline$T$ & 1.00 & $\mathbf{u g} / \mathrm{L}$ & Gen. & En \\
\hline T & 1.00 & $\mathrm{ug} / \mathrm{L}$ & Gen. & $\mathbf{E}$ \\
\hline & 2010.00 & $\mathbf{u g} / \mathrm{L}$ & Gen. & En \\
\hline LT & 1.00 & ug $/ \mathrm{L}$ & Gen. & Er \\
\hline LT & 4.00 & $u g / L$ & Gen. & Er \\
\hline LT & 1.00 & $u g / L$ & en. & \\
\hline LT & 1.00 & $\mathrm{ug} / \mathrm{L}$ & en. & \\
\hline LT & 1.00 & $\mathrm{ug} / \mathrm{L}$ & en. & \\
\hline LT & 1.00 & $u_{g} / L$ & Gen. & Eng \\
\hline LT & 0.01 & $\mathrm{ug} / \mathrm{L}$ & Gen. & \\
\hline LT & 1.00 & $\mathrm{ug} / \mathrm{L}$ & Gen. & \\
\hline LT & 100.00 & ug/L & Gen. & \\
\hline LT & 4.00 & $\mathrm{ug} / \mathrm{L}$ & Gen. & \\
\hline LT & 0.20 & $\mathrm{ug} / \mathrm{L}$ & Gen. & \\
\hline LT & 500.00 & ug/L & Gen. & \\
\hline LT & 0.00 & $\mathbf{u g}_{\mathrm{g}} / \mathrm{L}$ & Gen. & \\
\hline LT & 1.00 & ug $/ L$ & Gen. & \\
\hline LT & 0.50 & $u g / L$ & Gen: & \\
\hline & 734.00 & $\mathrm{ug} / \mathrm{L}$ & Gen. & \\
\hline & 21.20 & ug/L & $\operatorname{Gen}$ & \\
\hline & 2360.00 & $\mathrm{ug} / \mathrm{L}$ & Gen & \\
\hline$\omega T$ & 50.00 & ug $/ \mathrm{L}$ & Gen & \\
\hline & 3,00 & ug/L & Gen & \\
\hline
\end{tabular}


WELL LFW 57D COLLECTED ON 09/03/91 LABORATORY ANALYSES CONTINUED

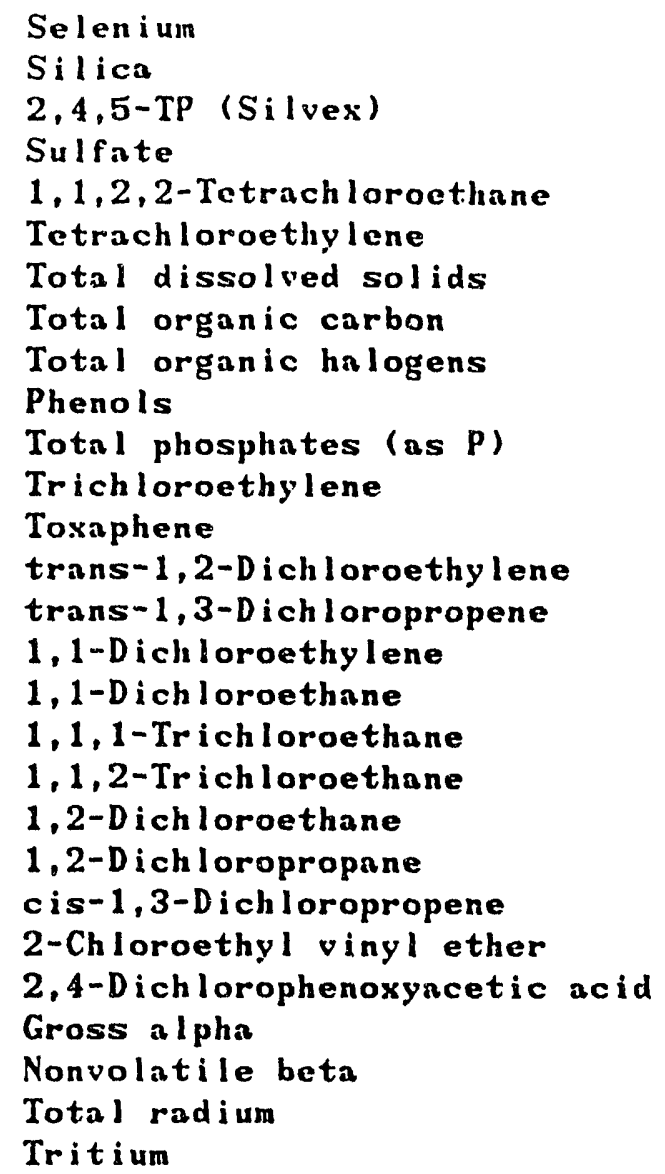

\begin{tabular}{|c|c|c|c|}
\hline$L T$ & $\begin{array}{r}2.00 \\
5530.00\end{array}$ & $\begin{array}{l}u g / L \\
u g / L\end{array}$ & $\begin{array}{l}\text { Gen. } \\
\text { Gen. }\end{array}$ \\
\hline LT & 0.09 & ug $/ \mathrm{L}$ & Gen. \\
\hline LT & 1000.00 & $\mathrm{ug} / \mathrm{L}$ & Gen. \\
\hline LT & 1.00 & ug $/ \mathrm{L}$ & Gen. \\
\hline LT & 1.00 & ug $/ L$ & Gen. \\
\hline$L^{3}$ & $\begin{array}{r}38000.00 \\
1000.00\end{array}$ & $\begin{array}{l}u g / L \\
u g / L\end{array}$ & $\begin{array}{l}\text { Gen. } \\
\text { Gen. }\end{array}$ \\
\hline & 24.80 & ug/L & Gen. \\
\hline LT & 5.00 & ug/L & Gen. \\
\hline LT & 100.00 & $\mathrm{ug} / \mathrm{L}$ & Gen. \\
\hline LT & 1.00 & ug/L & Gen. \\
\hline LI & 0.24 & ug/L & en. \\
\hline LT & 1.00 & ug $/ \mathrm{L}$ & Gen. \\
\hline LT & 1.00 & ug /L & Gen. \\
\hline LT & $\begin{array}{l}1.00 \\
4.00\end{array}$ & $\begin{array}{l}u g / L \\
u g / L\end{array}$ & $\begin{array}{l}\text { Gen. } \\
\text { Gen. }\end{array}$ \\
\hline LT & 1.00 & ug/L & Gen. \\
\hline LT & 1.00 & ug $/ \mathrm{L}$ & Gen. \\
\hline LT & 1.00 & ug $/ \mathrm{L}$ & Gen. \\
\hline LT & 1.00 & ug $/ L$ & en. \\
\hline LT & 1.00 & ug $/ \mathrm{L}$ & Gen. \\
\hline LT & 1.00 & ug $/ \mathrm{L}$ & Gen. \\
\hline LT & 0.30 & $u g / L$ & Gen \\
\hline LT & 2.00 & $i / L$ & Ger \\
\hline 90 & 1.80 & $O \mathrm{C}_{i} / \mathrm{L}$ & \\
\hline & 2.40 & $i / L$ & \\
\hline & 0.20 & $/ \mathrm{mL}$ & \\
\hline
\end{tabular}

WELL LFW 58D

Coordinates E 45700.2 Latitude 33.283771 N

Screen Zone Elevation 147.6-127.5 ft ms

Top of Casing Elevation $165.60 \mathrm{ft}$ misl

\section{MEASUREMENTS CONDUCTED IN THE FIELD}

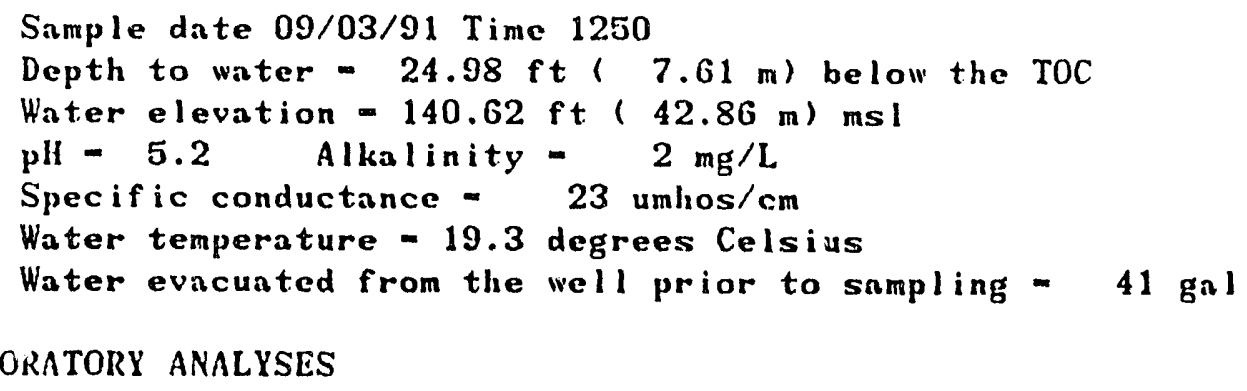

$\begin{array}{lccc}\text { Specific conductance } & 25.00 \mathrm{umh} / \mathrm{cm} & \text { Gen. Eng. } \\ \text { pll } & & 5.38 \mathrm{pH} & \text { Gen- Eng. } \\ \text { Silver } & \text { LT } & 2.00 \mathrm{ug} / \mathrm{L} & \text { Gen. Eng. } \\ \text { Arsenic } & \text { LT } & 2.00 \mathrm{ug} / \mathrm{L} & \text { Gen. Eng. } \\ \text { CONIINUED } & & & \end{array}$


WELL LFW 58D COLLECTED ON 09/03/91 LABORATORY ANALYSES CONTINUED

Barium

Bromide

Bromodichlorome thane

Calcium

Trichlorof luoromethane

Carbon tetrachloride

Cadmi um

Bromoform

Chloroform

Dichloromethane (Methylene chloride)

Bromomethane (Methyl bromide)

Chloromethane (Methyl chloride)

Chloride

Chlorobenzene

Chromi um

Chloroethene (Vinyl chloride)

Chloroethane

Benzene

Dibromoch loromethane

Endrin

Ethylbenzene

Fluoride

Iron

Mercury

Potassium

Lindane

To ene

Methosychlor

Magnes i um

Manganese

Sod i um

Nitrate as nitrogen

Lead

Selen i um

Silica

$2,4,5-T P$ (Silvex)

Sulfate

1,1,2,2-Tetrachloroethane

Tetrachloroethylene

Total dissolved solids

Total organic carbon

Total organic halogens

Phenols

Phenols

Total phosphates (as P)

* Trichloroethylene

Toxaphene

trans-1,2-Dichloroethylene

trans-1, 3-D i chloropropene

1,1-Dichloroethylene

1,1-Dichloroethane

$1,1,1-\mathrm{Tr}$ ichloroethane

$1,1,2-$ Trichloroethane

1,2-D ichloroethane
LT

LT

$T$

LT

LT

LT

LT

LT

LI

LT

LT

LT

LT

LT

LT

LT

LT

LT

LT

LT

LT

LT

LI

LT

LT

LT

LT

LT

LT

LT

LT

LT

LT

LT

LT

LT

LT
$6.88 \mathrm{ug} / \mathrm{L}$

$1000.00 \mathrm{ug} / \mathrm{L}$

$5.00 \mathrm{ug} / \mathrm{L}$

$1050.00 \mathrm{ug} / \mathrm{L}$

$145.00 \mathrm{ug} / \mathrm{L}$

$5.00 \mathrm{ug} / \mathrm{L}$

$2.00 \mathrm{ug} / \mathrm{L}$

$5.00 \mathrm{ug} / \mathrm{L}$

$5.00 \mathrm{ug} / \mathrm{L}$

$15.00 \mathrm{ug} / \mathrm{L}$

$5.00 \mathrm{ug} / \mathrm{L}$

$5.00 \mathrm{ug} / \mathrm{L}$

$3260.00 \mathrm{ug} / \mathrm{L}$

$5.00 \mathrm{ug} / \mathrm{L}$

$4.00 \mathrm{ug} / \mathrm{L}$

$5.00 \mathrm{ug} / \mathrm{L}$

$5.00 \mathrm{ug} / \mathrm{L}$

$5.00 \mathrm{ug} / \mathrm{L}$

$5.00 \mathrm{ug} / \mathrm{L}$

$0.01 \mathrm{ug} / \mathrm{L}$

$5.00 \mathrm{ug} / \mathrm{L}$

$100.00 \mathrm{ug} / \mathrm{L}$

$7.76 \mathrm{ug} / \mathrm{L}$

$0.90 \mathrm{ug} / \mathrm{L}$

$500.00 \mathrm{ug} / \mathrm{L}$

$0.00 \mathrm{ug} / \mathrm{L}$

$5.00 \mathrm{ug} / \mathrm{L}$

$0.50 \mathrm{ug} / \mathrm{L}$

$419.00 \mathrm{ug} / \mathrm{L}$

$27.90 \mathrm{ug} / \mathrm{L}$

$2060.00 \mathrm{ug} / \mathrm{L}$

$80.00 \mathrm{ug} / \mathrm{L}$

$3.00 \mathrm{ug} / \mathrm{L}$

$2.00 \mathrm{ug} / \mathrm{L}$

$9020.00 \mathrm{ug} / \mathrm{L}$

$0.09 \mathrm{ug} / \mathrm{L}$

$1000.00 \mathrm{ug} / \mathrm{L}$

$5.00 \mathrm{ug} / \mathrm{L}$

$5.00 \mathrm{ug} / \mathrm{L}$

$69000.00 \mathrm{ug} / \mathrm{L}$

$1000.00 \mathrm{ug} / \mathrm{L}$

$58.50 \mathrm{ug} / \mathrm{L}$

$5.00 \mathrm{ug} / \mathrm{L}$

$5.00 \mathrm{ug} / \mathrm{L}$

$100.00 u_{E} / L$

$5.00 \mathrm{ug} / \mathrm{L}$

$0.24 \mathrm{ug} / \mathrm{L}$

$5.00 \mathrm{ug} / \mathrm{L}$

$5.00 \mathrm{ug} / \mathrm{L}$

$5.00 \mathrm{ug} / \mathrm{L}$

$11.50 \mathrm{ug} / \mathrm{L}$

$14.00 \mathrm{ug} / \mathrm{L}$

$5.00 \mathrm{ug} / \mathrm{L}$

$5.00 \mathrm{ug} / \mathrm{L}$
Gen. Eng.

Gen. Eng.

Gen. Eng.

Gen. Eng.

Gen. Eng.

Gen. Eng.

Gen. Eng.

Gen. Eng.

Gen. Eng.

Gen. Eng.

Gen. Eng.

Gen. Eng •

Gen. - Eng.

Gen. Eng.

Gen. Eng.

Gen. Eng.

Gen. Eng.

Gen. Eng .

Gen. Eng.

Gen. Eng.

Gen. Eng.

Gen. Eng.

Gen. Eng.

Gen. Enğ.

Gen. Eng .

Gen. Eng.

Gen. Eng.

Gen. Eng.

Gen. Eng.

Gen. Eng.

Gen. Eng.

Gen. Eng.

Gen. Eng.

Gen. Eng.

Gen. Eng.

Gen. Eng.

Gen. Eng.

Gen. Eng.

Gen. Eng.

Gen. Eng.

Gen. Eng.

Gen. Eng .

Gen. Eng.

Gen. Eng .

Gen. Eng.

Gen. Eng.

Gen. Eng.

Gen. Eng.

Gen. Eng.

Gen. Eng.

Geñ. Eng.

Gen. Eng.

Gen. Eng.

Gen. Eng. 
WELL LFW 58D COLLECTED ON 09/03/91 LABORATORY ANALYSES CONTINUED

$\begin{array}{llll}\text { 1,2-Dichloropropane } & \mathrm{LT} & 5.00 \mathrm{ug} / \mathrm{L} & \text { Gen. Eng. } \\ \text { cis-1,3-Dichloropropene } & \mathrm{LT} & 5.00 \mathrm{ug} / \mathrm{L} & \text { Gen. Eng. } \\ \text { 2-Chloroethyl vinyl ether } & \mathrm{LT} & 5.00 \mathrm{ug} / \mathrm{L} & \text { Gen. Eng. } \\ \text { 2,4-Dichlorophenoxyacetic acid } & \mathrm{IT} & 0.30 \mathrm{ug} / \mathrm{L} & \text { Gen. Eng. } \\ \text { Gross alpha } & \mathrm{LT} & 2.00 \mathrm{pCi} / \mathrm{L} & \text { Gen. Eng. } \\ \text { Nonvolatile beta } & 2.50+- & 1.70 \mathrm{pCi} / \mathrm{L} & \text { Gen. Eng. } \\ \text { Total radium } & 1.60+- & 2.50 \mathrm{pCi} / \mathrm{L} & \text { Gen. Eng. } \\ \text { Tritium } & 2.57+- & 0.30 \mathrm{pCi} / \mathrm{mL} & \text { Gen. Eng. }\end{array}$

WELL LFW 59B

$\begin{array}{llllll}\text { SRS Grid } & N & 83027.1 & \text { Latitude } & 33.284529 & N \\ \text { Coordinates } & \text { E } & 46047.4 & \text { Longitude } & 81.706827 & W\end{array}$

Coordinates E 46047.4 Longitude 81.7
Screen Zone Elevation $75.6-65.6 \mathrm{ft} \mathrm{msl}$

Top of Casing Elevation $165.60 \mathrm{ft} \mathrm{ms}$ l

\section{MEASUREMENTS CONDUCTED IN THE FIELD}

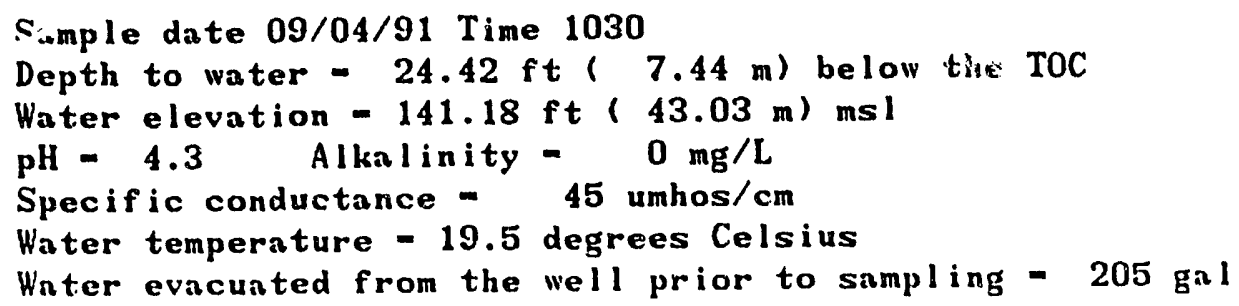

\section{LABORATORY ANALYSES}

Specific conductance
pll
Silver
Arsenic
Barium
Bromide
Bromodichloromethane
Calcium
Trichlorofluoromethane
Carbon tetrachloride
Cadmium
Bromoform
Chloroform
Dichloromethane (Methylene chloride)
Bromomethane (Methyl bromide)
Chloromethane (Methyl chloride)
Chloride
Chlorobenzene
Chromium
Chloroethene (Vinyl chloride)
Chloroethane
Benzene
Dibromochloromethane
Endrin

$\begin{array}{lrl} & 41.00 \mathrm{umh} / \mathrm{cm} & \text { Gen. Eng. } \\ & 4.53 \mathrm{pll} & \text { Gen. Eng. } \\ \text { LT } & 2.00 \mathrm{ug} / \mathrm{L} & \text { Gen. Eng. } \\ \text { LT } & 2.00 \mathrm{ug} / \mathrm{L} & \text { Gen. Eng. } \\ & 9.25 \mathrm{ug} / \mathrm{L} & \text { Gen. Eng. } \\ \text { LT } & 1000.00 \mathrm{ug} / \mathrm{L} & \text { Gen. Eng. } \\ \text { LT } & 1.00 \mathrm{ug} / \mathrm{L} & \text { Gen. Eng. } \\ & 1550.00 \mathrm{ug} / \mathrm{L} & \text { Gen. Eng. } \\ \text { LT } & 1.00 \mathrm{ug} / \mathrm{L} & \text { Gen. Eng. } \\ \text { LT } & 1.00 \mathrm{ug} / \mathrm{L} & \text { Gen. Eng. } \\ \text { LT } & 2.00 \mathrm{ug} / \mathrm{L} & \text { Gen. Eng. } \\ \text { LT } & 1.00 \mathrm{ug} / \mathrm{L} & \text { Gen. Eng. } \\ \text { LT } & 1.00 \mathrm{ug} / \mathrm{L} & \text { Gen. Eng. } \\ \text { LT } & 1.00 \mathrm{ug} / \mathrm{L} & \text { Gen. Eng. } \\ \text { LT } & 1.00 \mathrm{ug} / \mathrm{L} & \text { Gen. Eng. } \\ \text { LT } & 1.00 \mathrm{ug} / \mathrm{L} & \text { Gen. Eng. } \\ & 2130.00 \mathrm{ug} / \mathrm{L} & \text { Gen. Eng. } \\ \text { LT } & 1.00 \mathrm{ug} / \mathrm{L} & \text { Gen. Eng. } \\ \text { LT } & 4.00 \mathrm{ug} / \mathrm{L} & \text { Gen. Eng. } \\ \text { LT } & 1.00 \mathrm{ug} / \mathrm{L} & \text { Gen. Eng. } \\ \text { LT } & 1.00 \mathrm{ug} / \mathrm{L} & \text { Gen. Eng. } \\ \text { LT } & 1.00 \mathrm{ug} / \mathrm{L} & \text { Gen. Eng. } \\ \text { LT } & 1.00 \mathrm{ug} / \mathrm{L} & \text { Gen. Eng. } \\ \text { LT } & 0.01 \mathrm{ug} / \mathrm{L} & \text { Gen. Eng. }\end{array}$


WELL LFW 59B COLLECTED ON 09/04/91 LABORATORY ANALYSES CONTINUED

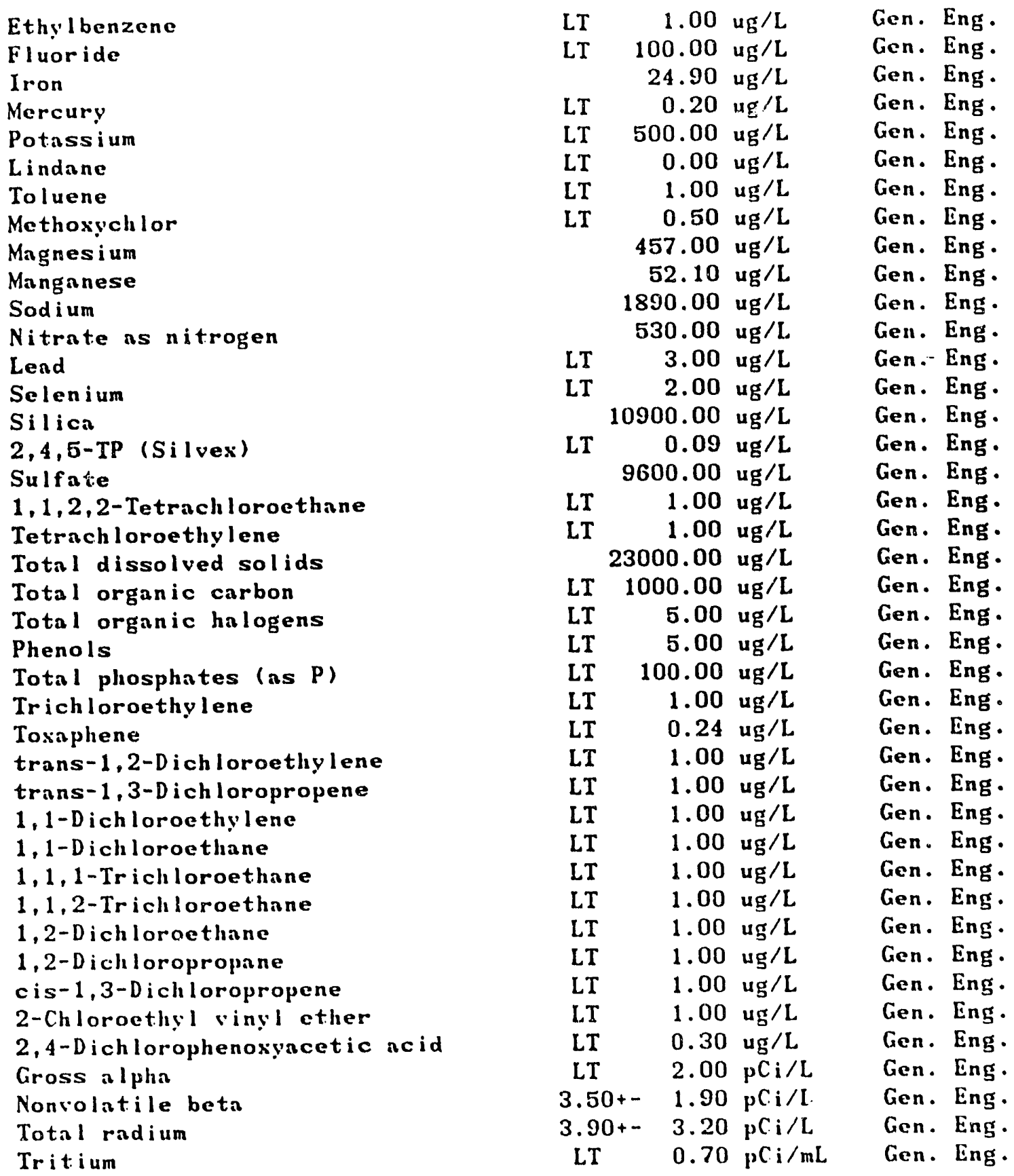


WELL LFW $59 \mathrm{C}$

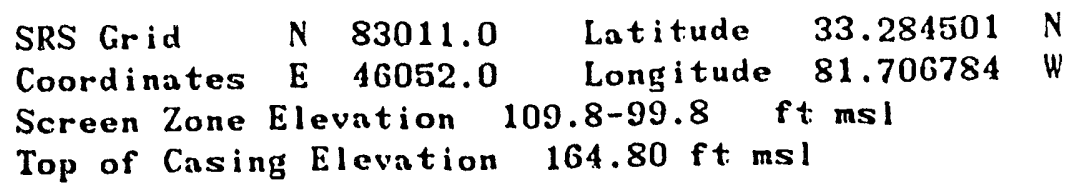

\section{MEASUREMENTS CONDUCTED IN THE FIELD}

Sample date 09/04/91 Time 1050

Depth to water - $24.02 \mathrm{ft}(7.32 \mathrm{~m})$ below the TOC

Water elevation $-140.78 \mathrm{ft}(42.91 \mathrm{~m}) \mathrm{ms}$

pll -4.9 Alkalinity - $0 \mathrm{mg} / \mathrm{L}$

Specific conductance - 44 umhos/cm

Water temperature - 19.3 degrees Celsius

Water evacuated from the well prior to sampling - $143 \mathrm{gal}$

\section{LABORATORY ANALYSES}

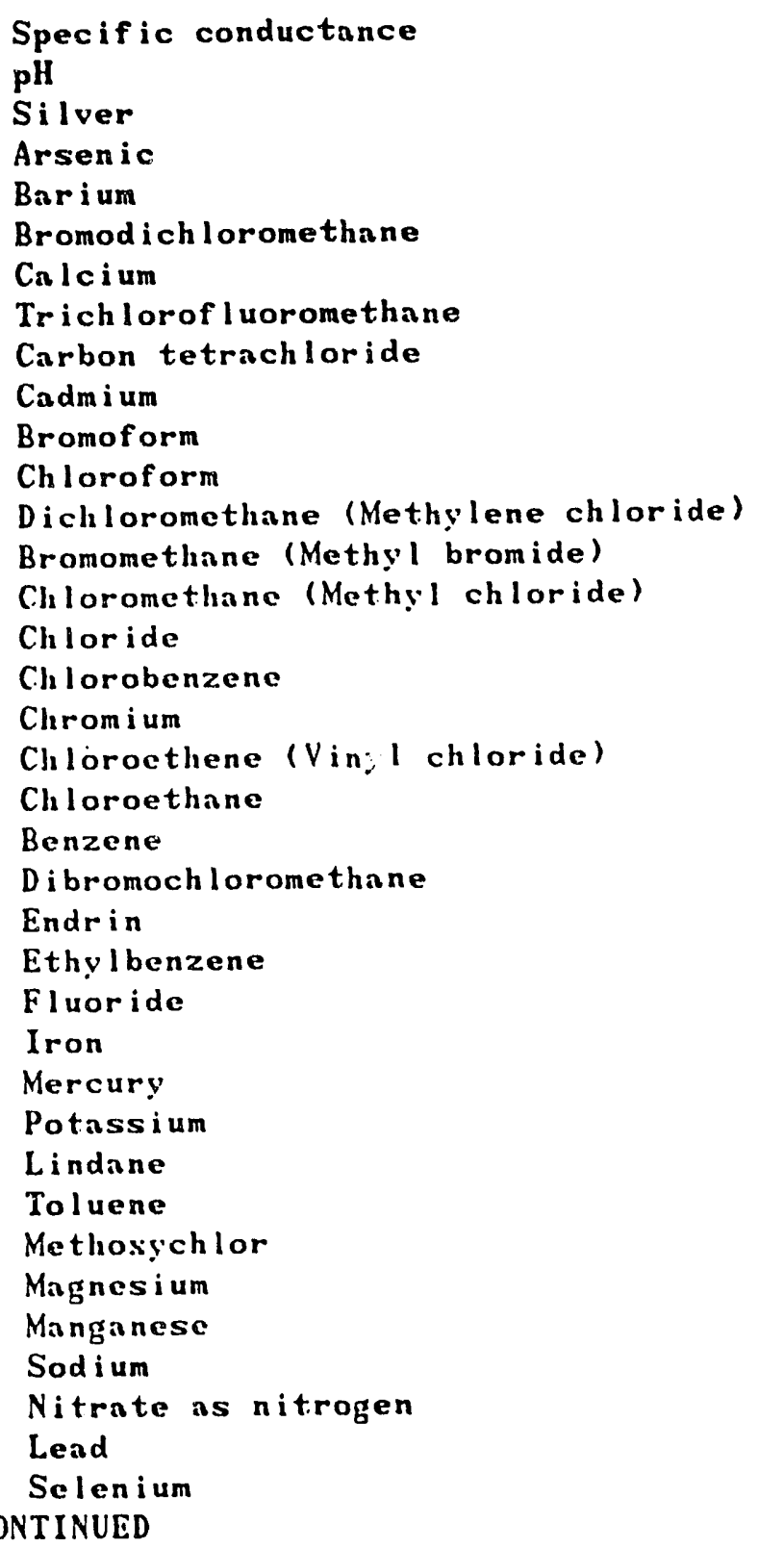

$\begin{array}{lrll} & 42.00 \mathrm{umh} / \mathrm{cm} & \text { Gen. Eng. } \\ & 5.55 \mathrm{ull} & \text { Gen. Eng. } \\ \text { LT } & 2.00 \mathrm{ug} / \mathrm{L} & \text { Gen. Eng. } \\ \text { LT } & 2.00 \mathrm{ug} / \mathrm{L} & \text { Gen. Eng. } \\ & 9.89 \mathrm{ug} / \mathrm{L} & \text { Gen. Eng. } \\ \text { LT } & 1.00 \mathrm{ug} / \mathrm{L} & \text { Gen. Eng. } \\ & 1170.00 \mathrm{ug} / \mathrm{L} & \text { Gen. Eng. } \\ & 8.00 \mathrm{ug} / \mathrm{L} & \text { Gen. Eng. } \\ \text { LT } & 1.00 \mathrm{ug} / \mathrm{L} & \text { Gen. Eng. } \\ \text { LT } & 2.00 \mathrm{ug} / \mathrm{L} & \text { Gen. Eng. } \\ \text { LT } & 1.00 \mathrm{ug} / \mathrm{L} & \text { Gen. Eng. } \\ \text { LT } & 1.00 \mathrm{ug} / \mathrm{L} & \text { Gen. Eng. } \\ & 13.00 \mathrm{ug} / \mathrm{L} & \text { Gen. Eng. } \\ \text { LT } & 1.00 \mathrm{ug} / \mathrm{L} & \text { Gen. Eng. } \\ \text { LT } & 1.00 \mathrm{ug} / \mathrm{L} & \text { Gen. Eng. } \\ & 5450.00 \mathrm{ug} / \mathrm{L} & \text { Gen. Eng. } \\ \text { LT } & 1.00 \mathrm{ug} / \mathrm{L} & \text { Gen. Eng. } \\ \text { LT } & 4.00 \mathrm{ug} / \mathrm{L} & \text { Gen. Eng. } \\ \text { LT } & 1.00 \mathrm{ug} / \mathrm{L} & \text { Gen. Eng. } \\ \text { LT } & 1.00 \mathrm{ug} / \mathrm{L} & \text { Gen. Eng. } \\ \text { LT } & 1.00 \mathrm{ug} / \mathrm{L} & \text { Gen. Eng. } \\ \text { LT } & 1.00 \mathrm{ug} / \mathrm{L} & \text { Gen. Eng. } \\ \text { LT } & 0.01 \mathrm{ug} / \mathrm{L} & \text { Gen. Eng. } \\ \text { LT } & 1.00 \mathrm{ug} / \mathrm{L} & \text { Gen. Eng. } \\ \text { LT } & 100.00 \mathrm{ug} / \mathrm{L} & \text { Gen. Eng. } \\ & 63.80 \mathrm{ug} / \mathrm{L} & \text { Gen. Eng. } \\ \text { LT } & 0.20 \mathrm{ug} / \mathrm{L} & \text { Gen. Eng. } \\ \text { LT } & 500.00 \mathrm{ug} / \mathrm{L} & \text { Gen. Eng. } \\ \text { LT } & 0.00 \mathrm{ug} / \mathrm{L} & \text { Gen. Eng. } \\ \text { LT } & 1.00 \mathrm{ug} / \mathrm{L} & \text { Gen. Eng. } \\ \text { LT } & 0.50 \mathrm{ug} / \mathrm{L} & \text { Gen. Eng } \\ & 991.00 \mathrm{ug} / \mathrm{L} & \text { Gen. Eng. } \\ & 17.60 \mathrm{ug} / \mathrm{L} & \text { Gen. Eng. } \\ & 3650.00 \mathrm{ug} / \mathrm{L} & \text { Gen. Eng. } \\ \text { LT } & 1080.00 \mathrm{ug} / \mathrm{L} & \text { Gen. Eng. } \\ \text { LT } & 3.00 \mathrm{ug} / \mathrm{L} & \text { Gen. Eng. } \\ & 2.00 \mathrm{ug} / \mathrm{L} & \text { Gen. Eng. }\end{array}$


WELL LFW 59C COLLECTED ON 09/04/91 LABORATGRY ANALYSES CONTINUED

Silica
2,4,5-TP (Silvex)
Sulfate
1,1,2,2-Tetrachloroethane
Tetrachlorocthylene
Total dissolved solids
Total dissolved solids
Total organic carbon
Total organic halogens
Phenols
Total phosphates (as P)
Total phosphates (as P)
Trichloroethylene
Tosaphene
trans-1,2-Dichloroethylene
trans-1,3-Dichloropropene
1,1-Dichloroethylene
1,1-Dichloroethane
1,1,1-Trichloroethane
1,1,2-Trichloroethane
1,2-Dichloroethane
1,2-Dichloropropane
cis-1,3-Dichloropropene
2-Chloroethyl vingl ether
2,4-Dichlorophenosyacetic acid
Gross alpha
Nonvolatile beta
Total radium
Tritium

\begin{tabular}{|c|c|c|c|c|}
\hline & 9180.00 & $u g / L$ & Gen. & Eng \\
\hline LT & 0.09 & $\mathrm{ug} / \mathrm{L}$ & Gen. & Eng \\
\hline LT & 1000.00 & ug $/ L$ & Gen. & Eng \\
\hline LT & 1.00 & $\mathrm{ug} / \mathrm{L}$ & Gen. & Eng \\
\hline LT & 1.00 & ug $/ \mathrm{L}$ & Gen. & Eng \\
\hline & 8000.00 & ug $/ \mathrm{L}$ & Gen, & Eng \\
\hline & 2000.00 & ug $/ \mathrm{L}$ & Gen. & Eng \\
\hline LT & 1000.00 & ug $/ \mathrm{L}$ & Gen. & Eng \\
\hline & 65.60 & ug $/ \mathrm{L}$ & Gen. & Eng \\
\hline LT & 5.00 & ug $/ \mathrm{L}$ & Gen. & Eng \\
\hline LT & 100.00 & $u g / L$ & Gen. & Eng \\
\hline LT & 100.00 & $u g / L$ & Gen. & Eng \\
\hline & 7.00 & ug/L & Gen: & Eng \\
\hline LT & 0.24 & ug $/ \mathrm{L}$ & Gen. & Eng \\
\hline LT & 1.00 & ug $/ \mathrm{L}$ & Gen. & Eng \\
\hline LT & 1.00 & ug $/ \mathrm{L}$ & Gen. & Eng \\
\hline & 1.00 & ug/L & Gen. & En \\
\hline & 11.00 & ug/L & Gen. & Eng \\
\hline & 1.00 & $u g / c$ & Gen. & Eng \\
\hline LT & 1.00 & ug/L & Gen. & Eng \\
\hline LT & 1.00 & ug/L & Gen. & Eng \\
\hline LT & 1.00 & ug $/ \mathrm{L}$ & Gen. & Eng \\
\hline LT & 1.00 & ug $/ L$ & Gen. & Eng \\
\hline LT & 1.00 & ug/L & Gen. & Eng \\
\hline LT & 0.30 & ug/L & Gen. & Eng \\
\hline LT & 2.00 & $\mathrm{pCi} / \mathrm{L}$ & Gen. & Eng \\
\hline $2.30+-$ & 2.00 & $\mathrm{pC} \mathrm{i} / \mathrm{L}$ & Gen. & \\
\hline $2.20+-$ & 2.60 & $\mathrm{pCi} / \mathrm{L}$ & Gen. & En \\
\hline $40+-$ & 0.50 & $\mathrm{pCi} / \mathrm{mL}$ & Gen. & \\
\hline
\end{tabular}

HELL LFH 59D

$\begin{array}{llllll}\text { SRS Grid } & N & 83000.1 & \text { Latitude } & 33.284484 & N \\ \text { Coordinates } & E & 46056.1 & \text { Longitude } & 81.706752 & W\end{array}$

Screen Zone Elevation 149.1-129.1 ft ms I

Top of Casing Elevation $165.10 \mathrm{ft} \mathrm{msl}$

\section{MEASUREMENTS CONDUCTED IN TIIE FIELD}

Sample date $09 / 04 / 91$ Time 1120

Depth to water $-23.10 \mathrm{ft}(7.04 \mathrm{~m})$ below the TOC

Water elevation $-142.00 \mathrm{ft}(43.28 \mathrm{~m}) \mathrm{msl}$

pll $=5.0 \quad$ Allialinity $=0 \mathrm{mg} / \mathrm{L}$

Specific conductance - $30 \mathrm{umhos} / \mathrm{cm}$

Water temperature - 19.4 degrees Celsius

Water evacuated from the well prior to sampling - 41 gal

\section{LABORATORY ANALYSES}

Specific conductance

pll

Silver

CONTINUED
$33.00 \mathrm{umh} / \mathrm{cm}$

$5.21 \mathrm{pll}$

$\mathrm{LT}$
Geñ. Eng.

Gen. Eng.

Gen. Eng . 


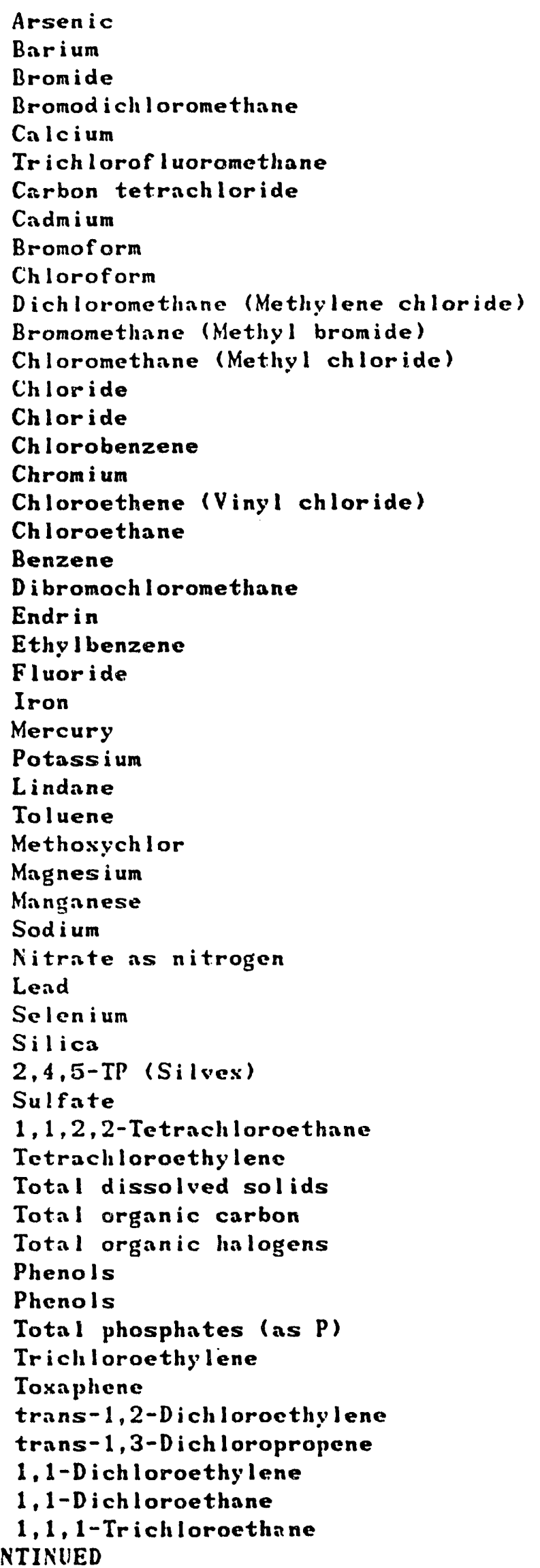

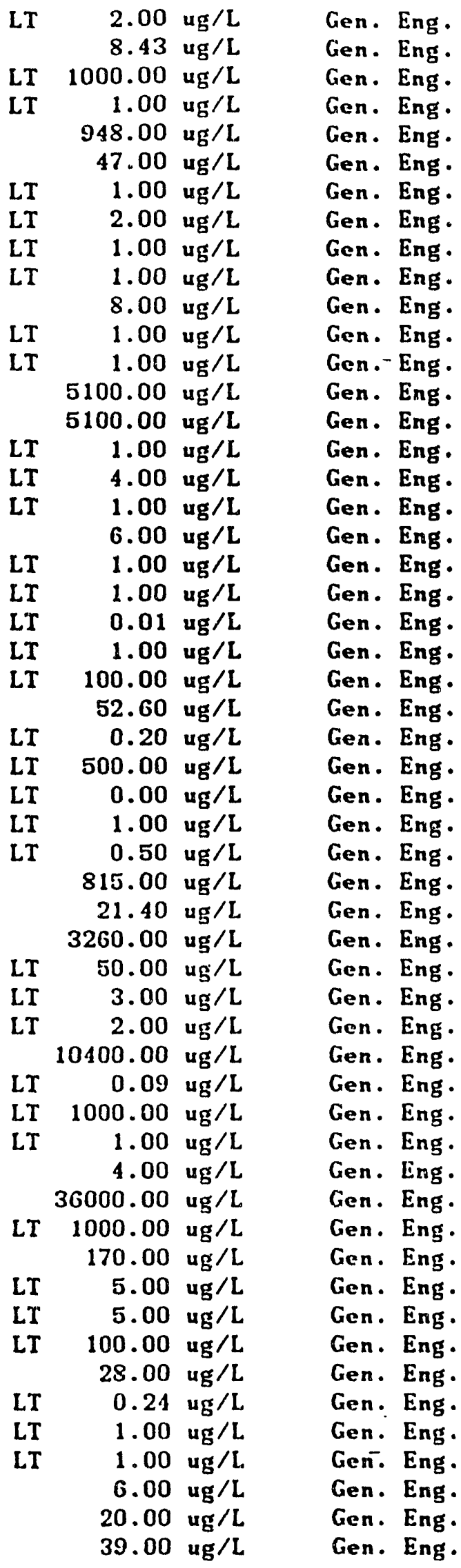


HELL LFW 59D COLLECTED ON 09/04/91 LABORATORY ANALYSES CONTINUED

1,1,2-Trichloroethane
1,2-Dichloroethane
1,2-Dichloropropane
cis-1,3-Dichloropropene
2-Chloroethyl vinyl ether
2,4-Dichlorophenoxyacetic ncid
Gross al pha
Nonvolatile beta
Total radium
Tritium

WELL LFW GOD

$\begin{array}{ll}\mathrm{LT} & 1.00 \mathrm{ug} / \mathrm{L} \\ \mathrm{LT} & 1.00 \mathrm{ug} / \mathrm{L} \\ \mathrm{LT} & 1.00 \mathrm{ug} / \mathrm{L} \\ \mathrm{LT} & 1.00 \mathrm{ug} / \mathrm{L} \\ \mathrm{LT} & 1.00 \mathrm{ug} / \mathrm{L} \\ \mathrm{LT} & 0.30 \mathrm{ug} / \mathrm{L} \\ \mathrm{LT} & 2.00 \mathrm{pCi} / \mathrm{L} \\ 3.30+- & 1.80 \mathrm{pCi} / \mathrm{L} \\ 1.70+- & 2.60 \mathrm{pCi} / \mathrm{L} \\ 3.59+- & 0.30 \mathrm{pCi} / \mathrm{mL}\end{array}$

Gen. Eng. Gen. Eng. Gen. Eng. Gen. Eng. Gen. Eng. Gen. Eng. Gen. Eng. Gen. Eng. Gen. Eng. Gen. Eng.

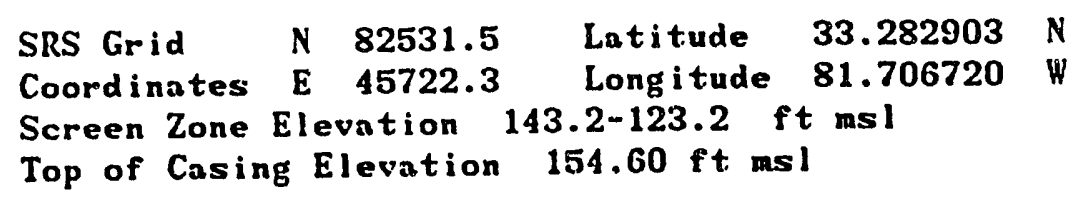

\section{LABORATORY ANALYSES}

Specific conductance

pll

Silver

Arsenic

Barium

Bromodichlorome thane

Calcium

Trichlorof l uorome thane

Carbon tetrachloride

Cadmi ium

Bromoform

Chloroform

Dichloromethane (Methylene chloride)

Bromomethane (Methyl bromide)

Chloromethane (Methyl chloride)

Chloride

Chloride

Chlorobenzene

Chromium

Chloroethene (Vinyl chloride)

Chloroethane

Benzene

CONTINUED

$\begin{array}{lrll} & 60.00 \mathrm{umh} / \mathrm{cm} & \text { Gen. Eng. } \\ & 6.70 \mathrm{pIl} & \text { Gen. Eng. } \\ \text { LT } & 2.00 \mathrm{ug} / \mathrm{L} & \text { Gen. Eng. } \\ \text { LT } & 2.00 \mathrm{ug} / \mathrm{L} & \text { Gen. Eng. } \\ & 7.51 \mathrm{ug} / \mathrm{L} & \text { Gen. Eng. } \\ \text { LT } & 1.00 \mathrm{ug} / \mathrm{L} & \text { Gen. Eng. } \\ & 2530.00 \mathrm{ug} / \mathrm{L} & \text { Gen. Eng. } \\ & 90.00 \mathrm{ug} / \mathrm{L} & \text { Gen. Eng. } \\ & 1.00 \mathrm{ug} / \mathrm{L} & \text { Gen. Eng. } \\ \text { LT } & 2.00 \mathrm{ug} / \mathrm{L} & \text { Gen. Eng. } \\ \text { LT } & 1.00 \mathrm{ug} / \mathrm{L} & \text { Gen. Eng. } \\ \text { LT } & 1.00 \mathrm{ug} / \mathrm{L} & \text { Gen. Eng. } \\ & 2.00 \mathrm{ug} / \mathrm{L} & \text { Gen. Eng. } \\ \text { LT } & 1.00 \mathrm{ug} / \mathrm{L} & \text { Gen. Eng. } \\ & 22.00 \mathrm{ug} / \mathrm{L} & \text { Gen. Eng. } \\ & 3040.00 \mathrm{ug} / \mathrm{L} & \text { Gen. Eng } \\ & 3040.00 \mathrm{ug} / \mathrm{L} & \text { Gen. Eng. } \\ \text { LT } & 1.00 \mathrm{ug} / \mathrm{L} & \text { Gen. Eng. } \\ \text { LT } & 4.00 \mathrm{ug} / \mathrm{L} & \text { Gen. Eng. } \\ \text { LT } & 1.00 \mathrm{ug} / \mathrm{L} & \text { Gen. Eng. } \\ \text { LT } & 1.00 \mathrm{ug} / \mathrm{L} & \text { Gen. Eng. } \\ \text { LT } & 1.00 \mathrm{ug} / \mathrm{L} & \text { Gen. Eng. } & \end{array}$


WELL LFW GOD COLLECTED ON 09/05/91 LABORATORY ANALYSES CONTINUED

Dibromochloromethane

Endrin

Ethylbenzene

Fluoride

Iron

Mercury

Potassium

Lindane

Toluene

Methoxychlor

Magnesium

Manganese

Sod i um

Nitrate as nitrogen

Lead

Selenium

Silica

$2,4,5-\operatorname{TP}(\mathrm{Si}$ lvex)

Sulfate

$1,1,2,2$-Tetrachloroethane

Tetrachloroethylene

Total dissolved solids

Total organic carbon

Total organic halogens

Phenols

Total phosphates (as P)

Trichloroethylene

Tosaphene

trans-1,2-Dich loroethylene

trans-1,3-Dich loropropene

1,1-D ich loroethylene

1,1-D ichloroethane

1,1,1-Trichloroethane

$1,1,2-\mathrm{Tr}$ ichloroethane

1,2-Dichloroethane

1,2-D ich loropropane

c is-1,3-D ich loropropene

2-Chloroethyl vinyl ether

2,4-Dichlorophenoxyacetic acid

Gross a lpha

Nonvolatile beta

Total radium

Tritium

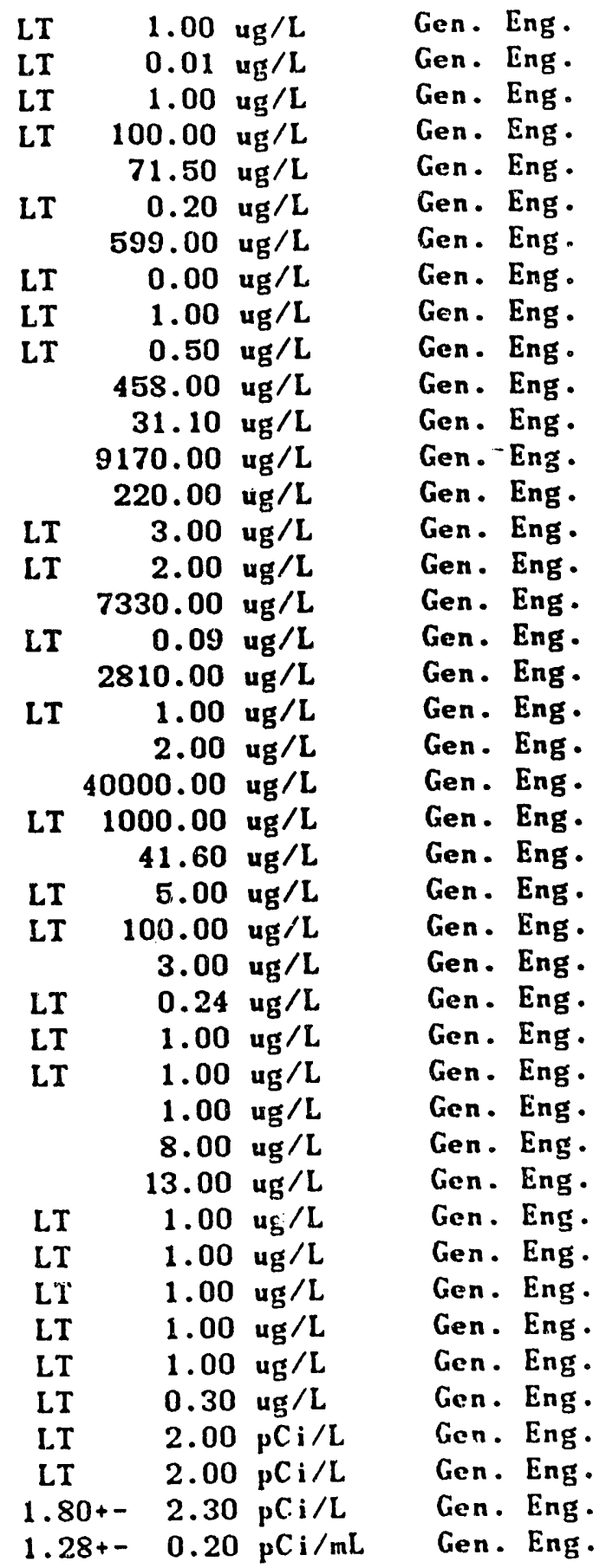


WELL LFW' G1C

$\begin{array}{llllll}\text { SRS Grid } & N & 83084.4 & \text { Latitude } & 33.285378 & N \\ \text { Coordinates } & E & 46489.6 & \text { Longitude } & 81.705774 & W\end{array}$

Screen Zone Elevation 120.8-110.7 ft msl

Top of Casing Elevation $165.80 \mathrm{ft} \mathrm{ms}$ !

MEASUREMENTS CONDUCTED IN THE FIELD

Sample date 09/04/91 Time 915

Depth to water - $25.40 \mathrm{ft}(7.74 \mathrm{~m})$ below the TOC

Water elevation - $140.40 \mathrm{ft}(42.79 \mathrm{~m}) \mathrm{msl}$

pll - 5.5 Alkalinity $=3 \mathrm{mg} / \mathrm{L}$

Specif ic conductance - $\quad 32$ umhos/cm

Water temperature - 18.7 degrees Celsius

Water evacuated from the well prior to sampling - $84 \mathrm{gal}$

\section{LABORATORY ANALYSES}

Specific conductance

pll

Silver

Arsenic

Barium

Bromodich loromethane

Calcium

Trichlorof luoromethane

Carbon tetrachloride

Cadmi um

Bromoform

Chloroform

Dichloromethane (Methylene chloride)

Bromomethane (Methyl bromide)

Chloromethane (Methyl chloride)

Chloride

Chlorobenzene

Chromi um

Chloroethene (Vinyl chloride)

Chloroethane

Benzene

Dibromochloromethane

Endrin

Ethylbenzene

Fluoride

Iron

Mercury

Potassium

Lindane

Toluene

Methosychlor

Magnesium

Manganese

Sod $i$ um

Nitrate as nitrogen

Lead

Selenium

CONTINUED
$31.00 \mathrm{umh} / \mathrm{cm}$

$5.46 \mathrm{pll}$

LT

LT

$2.00 \mathrm{ug} / \mathrm{L}$

$2.00 \mathrm{ug} / \mathrm{L}$

$8.57 \mathrm{ug} / \mathrm{L}$

LT

$1.00 \mathrm{ug} / \mathrm{L}$

$1410.00 \mathrm{ug} / \mathrm{L}$

$250.00 \mathrm{ug} / \mathrm{L}$

LT

LT

LT

LT

LT

LT

LT

$1.00 \mathrm{ug} / \mathrm{L}$

$2.00 \mathrm{ug} / \mathrm{L}$

$1.00 \mathrm{ug} / \mathrm{L}$

$1.00 \mathrm{ug} / \mathrm{L}$

$25.00 \mathrm{ug} / \mathrm{L}$

$1.00 \mathrm{ug} / \mathrm{L}$

$1.00 \mathrm{ug} / \mathrm{L}$

$2780.00 \mathrm{ug} / \mathrm{L}$

$1.00 \mathrm{ug} / \mathrm{L}$

LT

LT

LT

LT

LT

LT

L.T

LT

LT

$4.00 \mathrm{ug} / \mathrm{L}$

$1.00 \mathrm{ug} / \mathrm{L}$

$11.00 \mathrm{ug} / \mathrm{L}$

$1.00 \mathrm{ug} / \mathrm{L}$

$1.00 \mathrm{ug} / \mathrm{L}$

$0.01 \mathrm{ug} / \mathrm{L}$

$1.00 \mathrm{ug} / \mathrm{L}$

$100.00 \mathrm{ug} / \mathrm{L}$

$4.34 \mathrm{ug} / \mathrm{L}$

$0.20 \mathrm{ug} / \mathrm{L}$

$922.00 \mathrm{ug} / \mathrm{L}$

$0.00 \mathrm{ug} / \mathrm{L}$

LT

LI

$1.00 \mathrm{ug} / \mathrm{L}$

$0.50 \mathrm{ug} / \mathrm{L}$

$660.00 \mathrm{ug} / \mathrm{L}$

$23.00 \mathrm{ug} / \mathrm{L}$

$2790.00 \mathrm{ug} / \mathrm{L}$

$740.00 \mathrm{ug} / \mathrm{L}$

LT

$3.00 \mathrm{ug} / \mathrm{L}$

LT
Gen. Eng -

Gen. Eng.

Gen. Eng.

Gen. Eng.

Gen. Eng.

Gen. Eng.

Gen. Eng.

Gen. Eng.

Gen. Eng.

Gen. Eng.

Gen. Eng.

Gen. Eng.

Gen. Eng.

Gen. Eng.

Gen. Eng.

Gen. Eng.

Gen. Eng.

Gen. Eng.

Gen. Eng.

Gen. Eng.

Gen. Eng.

Gen. Eng.

Gen. Eng.

Gen. Eng.

Gen. Eng.

Gen. Eng .

Gen. Eng.

Gen. Eng.

Gen. Eng.

Gen. Eng .

Gen. Eng.

Gen. Eng.

Gen. Eng.

Geñ. Eng.

Gen. Eng.

Gen. Eng.

Gen. Eng. 
WELL LFW 61C COLLECTED ON 09/04/91 LABORATORY ANALYSES CONTINUED

Silica
2,4,5-TP (Silves)
Sulfate
1,1,2,2-Tetrachloroethane
Tetrachloroethylene
Total dissolved solids
Total organic carbon
Total organic halogens
Phenols
Total phosphates (as P)
Trichloroethylene
Toxaphene
trans-1,2-Dichloroethylene
trans-1,3-Dichloropropene
1,1-Dichloroethylene
1,1-Dichloroethane
1,1,1-Trichloroethane
1,1,2-Trichloroethane
1,2-Dichloroethane
1,2-Dichloropropane
cis-1,3-Dichloropropene
2-Chloroethyl vinyl ether
2,4-Dichlorophenosyacetic acid
Gross alpha
Nonvolatile beta
Total radium
Tritium

\begin{tabular}{|c|c|c|c|c|}
\hline & 7890.00 & $\mathbf{u g} / \mathrm{L}$ & Gen. & Eng \\
\hline LT & 0.09 & $\mathrm{ug} / \mathrm{L}$ & Gen. & Eng \\
\hline$L T$ & 1000.00 & $\mathrm{ug} / \mathrm{L}$ & Gen. & Eng \\
\hline$L T$ & 1.00 & $u g / L$ & Gen. & Eng \\
\hline & 2.00 & $u g / L$ & Gen. & Eng \\
\hline & 32000.00 & $u g / L$ & Gen. & 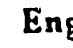 \\
\hline LT & 1000.00 & $u_{g} / L$ & Gen. & 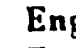 \\
\hline & 191.00 & ug $/ \mathrm{L}$ & Gen. & \\
\hline LT & 5.00 & $\mathrm{ug} / \mathrm{L}$ & Gen. & \\
\hline LT & 100.00 & ug $/ L$ & Gen. & \\
\hline & 6.00 & $\mathrm{ug} / \mathrm{L}$ & Yen. & \\
\hline LT & 0.24 & ug/L & Gen. & \\
\hline$L T$ & 1.00 & $\mathbf{u g} / \mathrm{L}$ & en: & \\
\hline LT & 1.00 & ug $/ L$ & Gen. & \\
\hline & $\begin{array}{l}4.00 \\
9.00\end{array}$ & $\begin{array}{l}u g / L \\
u g / L\end{array}$ & $\begin{array}{l}\text { Gen. } \\
\text { Gen. }\end{array}$ & \\
\hline & 22.00 & ug $/ L$ & Gen. & \\
\hline LI & 1.00 & ug/L & Gen. & \\
\hline 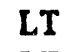 & 1.00 & $u g / L$ & Gen. & \\
\hline LT & 1.00 & ug $/ L$ & Gen. & \\
\hline LT & 1.00 & ug $/ \mathrm{L}$ & Gen. & \\
\hline LT & 1.00 & $u g / L$ & Gen. & \\
\hline L] & 0.30 & $\mathrm{ug} / \mathrm{L}$ & Gen. & \\
\hline LI & 2.00 & $\mathrm{pC} i / L$ & Gen. & \\
\hline 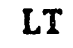 & 2.00 & $\mathrm{pCi} / \mathrm{L}$ & Gen. & \\
\hline LT & 1.00 & $\mathrm{pCi} / \mathrm{L}$ & Gen. & \\
\hline & 0.30 & $\mathrm{pCi} / \mathrm{n}$ & Gen. & \\
\hline
\end{tabular}

\section{HELL LFW 61D}

$\begin{array}{llllll}\text { SRS Grid } & N & \$ 3089.1 & \text { Latitude } & 33.285358 & N \\ \text { Coordinates } & \text { E } & 46471.1 & \text { Longitude } & 81.705832 & \text { H' }\end{array}$

Screen Zonc Elevation $150.4-130.3 \mathrm{ft} \mathrm{msl}$

Top of Casing Elevation $166.40 \mathrm{ft} \mathrm{ms}$

\section{MEASUREMENTS CONDUCTED IN THE FIELD}

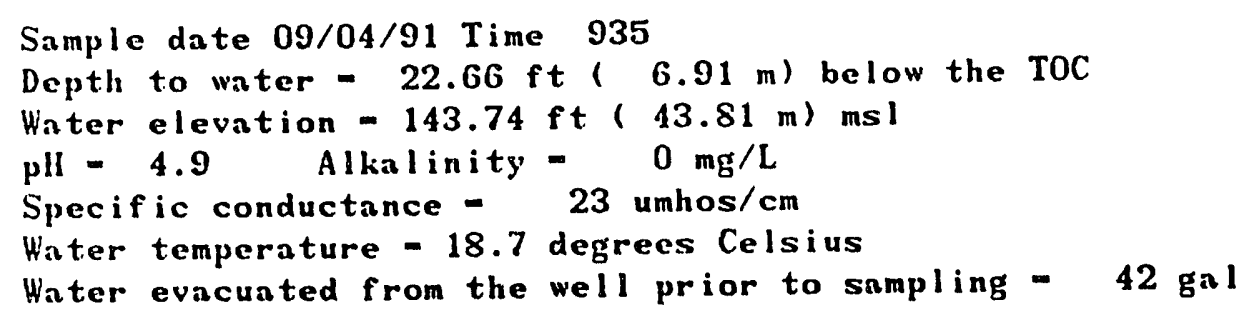

\section{LABORATORY ANALYSES}

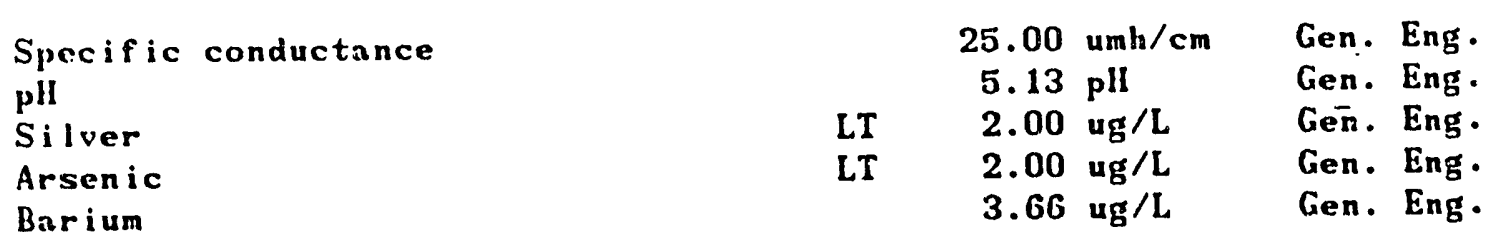




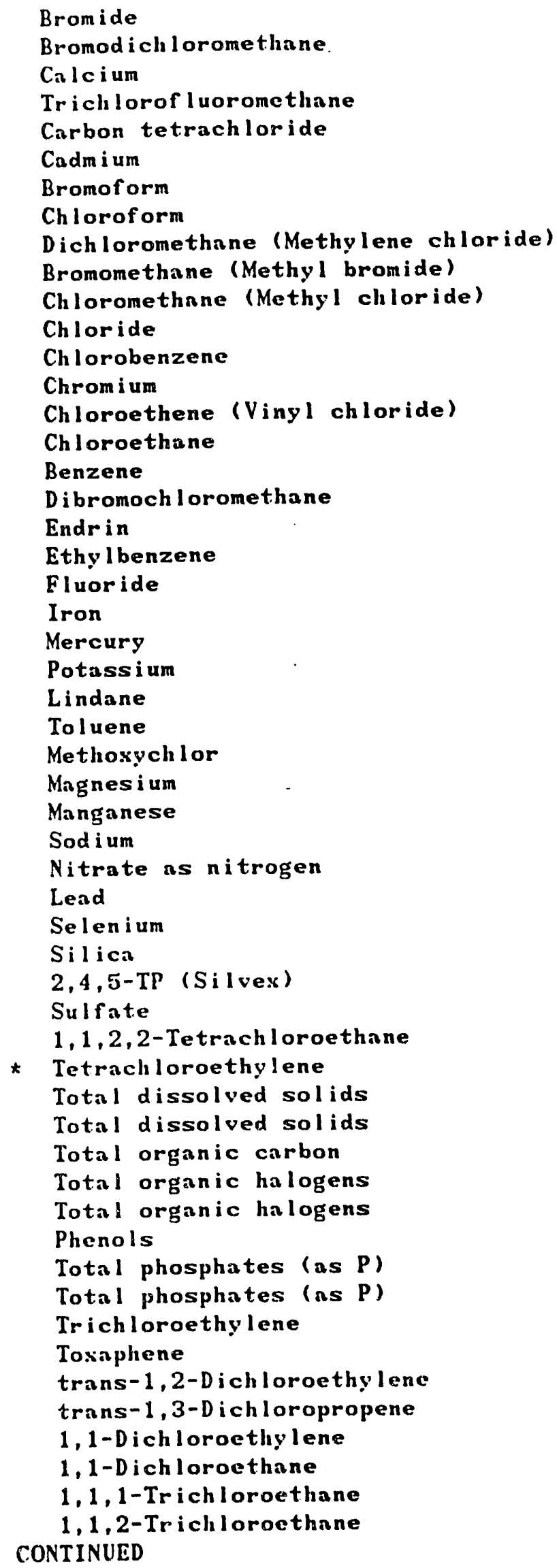

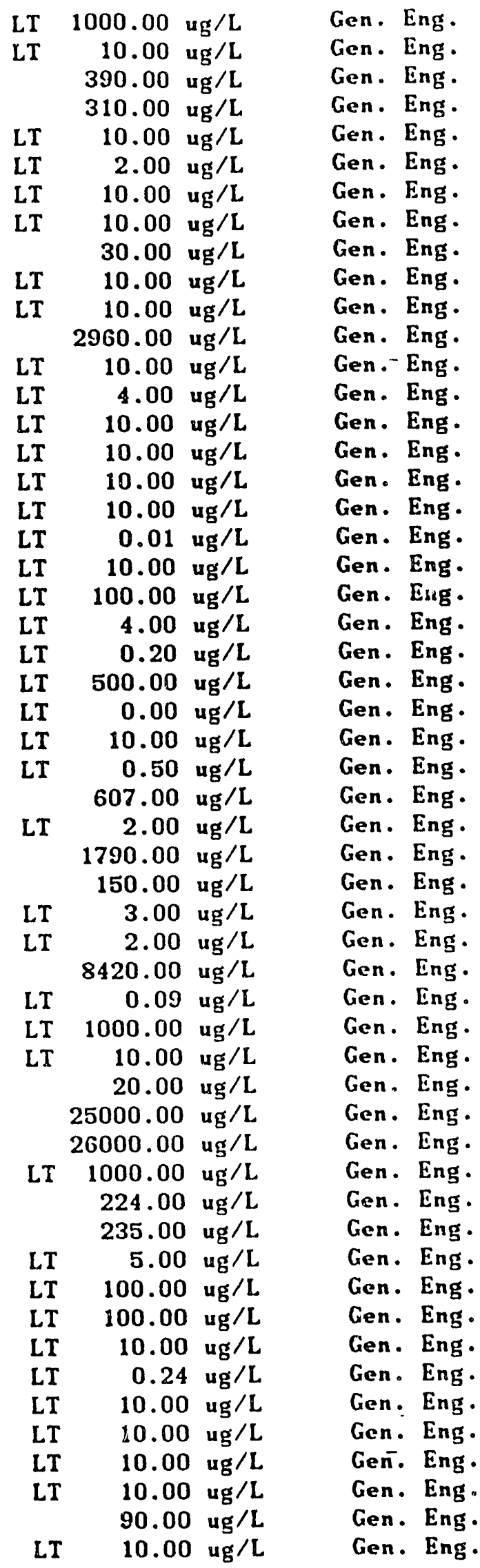


WELL LFW G1D COLLECTED ON 09/04/91 LABORATORY ANALYSES CONTINUED

$\begin{array}{llrl}\text { 1,2-Dichloroethane } & \mathrm{LT} & 10.00 \mathrm{ug} / \mathrm{L} & \text { Gen. Eng. } \\ \text { 1,2-Dichloropropane } & \mathrm{LT} & 10.00 \mathrm{ug} / \mathrm{L} & \text { Gen. Eng. } \\ \text { cis-1,3-Dichloropropene } & \mathrm{LT} & 10.00 \mathrm{ug} / \mathrm{L} & \text { Gen. Eng. } \\ \text { 2-Chlorocthyl vinylether } & \mathrm{LT} & 10.00 \mathrm{ug} / \mathrm{L} & \text { Gen. Eng. } \\ \text { 2,4-Dichlorophenosyacetic acid } & \mathrm{LT} & 0.30 \mathrm{ug} / \mathrm{L} & \text { Gen. Eng. } \\ \text { Gross alpha } & \mathrm{LT} & 2.00 \mathrm{pCi} / \mathrm{L} & \text { Gen. Eng. } \\ \text { Nonvolatile beta } & 3.60+- & 1.90 \mathrm{pCi} / \mathrm{L} & \text { Gen. Eng. } \\ \text { Total radium } & 1.10+- & 2.30 \mathrm{pCi} / \mathrm{L} & \text { Gen. Eng. } \\ \text { Tritium } & 2.83+- & 0.30 \mathrm{pCi} / \mathrm{mL} & \text { Gen. Eng. }\end{array}$

WELL LFW 62B

$\begin{array}{llllll}\text { SRS Grid } & \text { N } & 83001.2 & \text { Latitude } & 33.284257 & N \\ \text { Coordinates } & \text { E } & 45915.5 & \text { Longitude } & 81.707124 & W\end{array}$

Screen Zone Elevation 72.4-62.4 ft msl

Top of Casing Elevation $162.40 \mathrm{ft} \mathrm{msl}$

MEASUREMENTS CONDUCTED IN THE FIELD

Sample date 09/04/91 Time 1210

Depth to water - $22.09 \mathrm{ft}(6.73 \mathrm{~m})$ below the TOC

Water elevation $-140.31 \mathrm{ft}(42.77 \mathrm{~m}) \mathrm{msl}$

$\mathrm{pH}=5.4 \quad$ Alkalinity $=0 \mathrm{mg} / \mathrm{L}$

Specific conductance - 39 umhos/cm

Water temperature - 19.5 degrees Celsius

Water evacuated from the well prior to sampling - $211 \mathrm{gal}$

\section{LABORATORY ANALYSES}

Specif ic conductance

pH

Silver

Arsenic

Barium

Bromodichloromethane

Calcium

Trichlorof luoromethane

Carbon tetrachloride

Cirdm i um

Bromoform

Chloroform

Dichloromethane (Methylene chloride)

Bromomethane (Methyl bromide)

Chloromethane (Methyl chloride)

Chloride

Chlorobenzene

Chrom i um

Chloroethene (Vinyl chloride)

Chloroethane

Benzene

Dibromoch loromethane

Endrin

C.ONTINUED
$40.00 \mathrm{umh} / \mathrm{cm}$

$5.59 \mathrm{pH}$

LT

LT

$2.00 \mathrm{ug} / \mathrm{L}$

$2.00 \mathrm{ug} / \mathrm{L}$

$4.37 \mathrm{ug} / \mathrm{L}$

LT $\quad 1.00 \mathrm{ug} / \mathrm{L}$

$682.00 \mathrm{ug} / \mathrm{L}$

$\begin{array}{ll}\text { LT } & 1.00 \mathrm{ug} / \mathrm{L} \\ \text { LT } & 1.00 \mathrm{ug} / \mathrm{L}\end{array}$

LT $2.00 \mathrm{ug} / \mathrm{L}$

LT $1.00 \mathrm{ug} / \mathrm{L}$

LT $1.00 \mathrm{ug} / \mathrm{L}$

LT $1.00 \mathrm{ug} / \mathrm{L}$

LI

LT

LT

LT

LT

LT

LT

LT

LT
$1.00 \mathrm{ug} / \mathrm{L}$

$1.00 \mathrm{ug} / \mathrm{L}$

$1940.00 \mathrm{ug} / \mathrm{L}$

$1.00 \mathrm{ug} / \mathrm{L}$

$4.00 \mathrm{ug} / \mathrm{L}$

$1.00 \mathrm{ug} / \mathrm{L}$

$1.00 \mathrm{ug} / \mathrm{L}$

$1.00 \mathrm{ug} / \mathrm{L}$

$1.00 \mathrm{ug} / \mathrm{L}$

$0.01 \mathrm{ug} / \mathrm{L}$
Gen. Eng.

Gen. Eng.

Gen. Eng.

Gen. Eng .

Gen. Eng.

Gen. Eng.

Gen. Eng.

Gen. Eng.

Gen. Eng.

Gen. Eng.

Gen. Eng.

Gen. Eng.

Gen. Eng.

Gen. Eng.

Gen. Eng.

Gen. Eng.

Gen. Eng.

Gen. Eng.

Gent. Eng.

Gen. Eng.

Geñ. Eng.

Gen. Eng.

Gen. Eng. 
WELL LFW 62B COLLECTED ON 09/04/91 LABORATORY ANALYSES CONTINUED

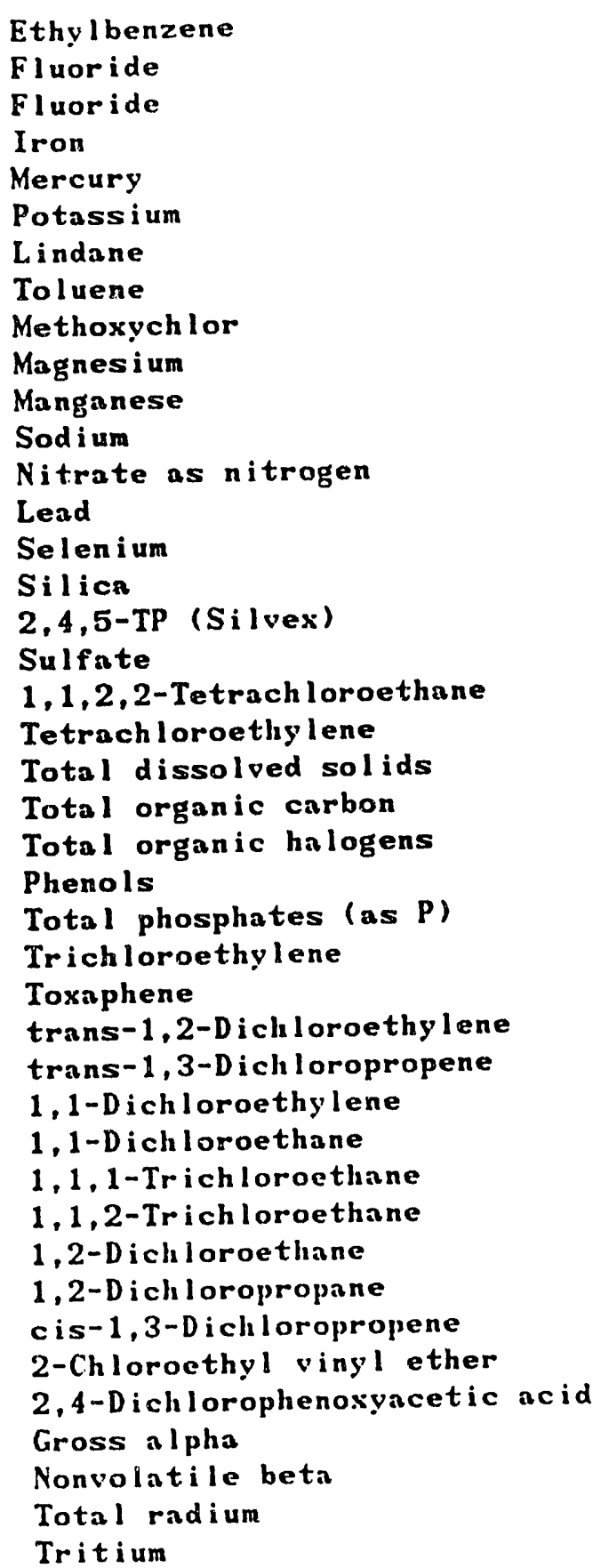

\begin{tabular}{|c|c|c|c|c|c|}
\hline LT & & 1.00 & ug/L & Gen. & Eng. \\
\hline $\mathrm{LT}$ & & 0.00 & $u_{g} / L$ & Gen. & Eng. \\
\hline$T$ & & 0.00 & ug/L & Gen. & Eng. \\
\hline & & 16.10 & ug /L & Gen. & Eng. \\
\hline$T$ & & 0.20 & ug/L & Gen. & Eng. \\
\hline & & 15.00 & ug $/ L$ & Gen. & Eng. \\
\hline$T$ & & 0.00 & $u g / \mathbb{L}$ & Gen. & Eng • \\
\hline$T$ & & 1.00 & ug/L & Gen. & Eng. \\
\hline$T$ & & 0.50 & $\mathbf{u g} / \mathrm{L}$ & en. & Eng. \\
\hline & & 19.00 & ug/L & & Eng. \\
\hline & & 15.00 & ug $/ \mathrm{L}$ & en. & Eng. \\
\hline & $54:$ & 30.00 & ug/L & en . & Eng. \\
\hline & & 80.00 & ug /L & en. & Eng. \\
\hline & & 3.00 & ug $/ L$ & en. & Eng • \\
\hline & & 2.00 & ug $/ L$ & ien. & Eng. \\
\hline & 107 & 00.00 & ug/L & en. & Eng . \\
\hline $\mathbf{T}$ & & 0.09 & ug/L & en. & Eng. \\
\hline & 63 & 40.00 & ug/L & en. & Eng. \\
\hline LI & & 1.00 & ug/L & en. & Eng • \\
\hline $\mathbf{I}$ & & 1.00 & ug $/ L$ & en. & Eng • \\
\hline & 650 & 00.00 & ug $/ L$ & en. & Eng. \\
\hline & 20 & 00.00 & ug/L & en. & Eng. \\
\hline & & 10.10 & $\mathbf{u g}_{\mathrm{g}} / \mathbf{L}$ & en. & Eng \\
\hline LI & & 5.00 & ug /L & n. & Eng • \\
\hline LT & & 00.00 & $u_{\mathrm{g}} / \mathrm{L}$ & n. & Eng \\
\hline & & 1.00 & ug $/ L$ & ien. & Eng \\
\hline LI & & 0.24 & ug /L & n. & Eng. \\
\hline LT & & 1.00 & ug/L & n. & Eng \\
\hline LT & & 1.00 & ug/L & en. & Eng. \\
\hline LT & & 1.00 & ug/L & en. & Eng \\
\hline LT & & 1.00 & ug/L & n & Eng . \\
\hline LT & & 1.00 & $\mathbf{u g} / \mathrm{L}$ & & Eng. \\
\hline $\mathrm{LT}$ & & 1.00 & $\mathbf{u} \mathfrak{g} / \mathbf{L}$ & ел. & Eng \\
\hline LT & & 1.00 & ug/L & n. & Eng. \\
\hline LT & & 1.00 & ug/L & en. & Eng \\
\hline $\mathrm{LT}$ & & 1.00 & ug/L & en. & - Eng \\
\hline LT & & 1.00 & ug/L & & - Eng \\
\hline LT & & 0.30 & $\mathbf{u g} / \mathbf{L}$ & ien & - Eng \\
\hline LT & & 2.00 & $p C i / L$ & en & Eng \\
\hline LT & & 2.00 & $\mathrm{pCi} / \mathrm{L}$ & & En \\
\hline . & & 3.00 & $p C i / L$ & & ng \\
\hline$L T$ & & 0.70 & $\mathrm{p} \mathrm{C} i / \mathrm{mL}$ & & \\
\hline
\end{tabular}


WELL LFW 62C

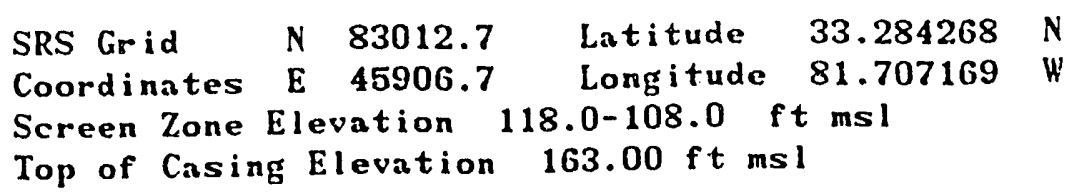

\section{MEASUREMENTS CONDUCTED IN THE FIELD}

Sample date 09/04/91 Time 1235

Depth to water - $22.02 \mathrm{ft}(6.71 \mathrm{~m})$ below the TOC

Water elevation $=140.98 \mathrm{ft}(42.97 \mathrm{~m}) \mathrm{msl}$

$\mathrm{pH}=5.6$ Alkalinity - $1 \mathrm{mg} / \mathrm{L}$

Specific conductance - 27 umhos/cm

Water temperature - 19.4 degrees Celsius

Water evacuated from the well prior to sampling - $93 \mathrm{gal}$

\section{LABORATORY ANALYSES}

\begin{tabular}{|c|}
\hline $\begin{array}{l}\text { Specific conductance } \\
\text { pH } \\
\text { Silver } \\
\text { Arsenic } \\
\text { Barium } \\
\text { Bromide } \\
\text { Bromodichloromethane } \\
\text { Calcium } \\
\text { Trichlorofluoromethane } \\
\text { Carbon tetrachloride } \\
\text { Cadmium } \\
\text { Bromoform } \\
\text { Chloroform } \\
\text { Dichloromethane (Methylene chloride) } \\
\text { Bromomethane (Methyl bromide) } \\
\text { Chloromethane (Methyl chloride) } \\
\text { Chloride } \\
\text { Chlorobenzene } \\
\text { Chromium } \\
\text { Chloroethene (Vingl chloride) } \\
\text { Chloroethane } \\
\text { Benzede } \\
\text { Dibromochloromethane } \\
\text { Endrin } \\
\text { Ethylbenzene } \\
\text { Fluoride } \\
\text { Iron } \\
\text { Mercury } \\
\text { Potassium } \\
\text { Lindane } \\
\text { Toluene } \\
\text { Methosychlor } \\
\text { Magnesium } \\
\text { Manganese } \\
\text { Sodium } \\
\text { Nitrate as nitrogen } \\
\text { Lead } \\
\text { NTINUED }\end{array}$ \\
\hline
\end{tabular}

\begin{tabular}{|c|c|c|c|c|}
\hline & 30.00 & $\mathrm{umh} / \mathrm{cm}$ & Gen. & Eng. \\
\hline & 5.62 & $\mathrm{pH}$ & Gen. & Eng. \\
\hline & 2.00 & ug $/ \mathrm{L}$ & Gen. & Eng. \\
\hline & 2.00 & $\mathrm{ug} / \mathrm{L}$ & Gen. & Eng. \\
\hline & 7.07 & ug/L & Gen. & Eng. \\
\hline LT & 1000.00 & ug/L & Gen. & Eng. \\
\hline$L T$ & 1.00 & ug/L & Gen. & g. \\
\hline & 1240.00 & ug $/ \mathrm{L}$ & Gen. & ng. \\
\hline & 4.00 & $u_{g} / L$ & Gen. & Eng. \\
\hline$T$ & 1.00 & ug $/ L$ & Gen. & ing. \\
\hline T & 2.00 & ug/L & Gen. & ing. \\
\hline$T$ & 1.00 & ug $/ \mathrm{L}$ & Gen. & Eng. \\
\hline $\mathrm{T}$ & 1.00 & ug/L & Gen. & ing. \\
\hline & 2.00 & ug $/ L$ & Gen. & ng. \\
\hline LT & 1.00 & ug $/ L$ & Gen. & Eng. \\
\hline$T$ & 1.00 & $u g / L$ & Gen. & Eng. \\
\hline & 1740.00 & ug/L & Gen. & Eng. \\
\hline LT & 1.00 & ug $/ \mathrm{L}$ & Gen. & Eng. \\
\hline LT & 4.00 & ug $/ \mathrm{L}$ & Gen. & Eng. \\
\hline LT & 1.00 & $u g / L$ & Gen. & Eng. \\
\hline LT & 1.00 & $u g / L$ & Gen. & Eng \\
\hline $\mathrm{LT}$ & 1.00 & $u_{g} / \mathrm{L}$ & Gen. & Eng \\
\hline LT & 1.00 & $u g / L$ & Gen. & Eng \\
\hline LT & 0.01 & $u_{\mathfrak{g}} / \mathrm{L}$ & Gen. & Eng \\
\hline LT & 1.00 & $\mathrm{ug} / \mathrm{L}$ & Gen. & Eng \\
\hline LT & 100.00 & $\mathrm{ug} / \mathrm{L}$ & Gen. & Eng \\
\hline LT & 4.00 & ug/L & Gen. & Eng \\
\hline LT & 0.20 & ug/L & Gen. & 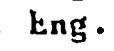 \\
\hline & 510.00 & $u_{g} / L$ & Gen. & \\
\hline LT & 0.00 & $u g / L$ & Gen. & Eng \\
\hline LT & 1.00 & ug $/ L$ & Gen. & • \\
\hline $\mathrm{LT}$ & 0.50 & $u g / L$ & Gen. & Eng. \\
\hline & 731.00 & ug $/ \mathrm{L}$ & Gen. & \\
\hline & 21.20 & $4 g / L$ & Geñ. & \\
\hline & 1450.00 & $\omega g / L$ & Gen. & \\
\hline & 1600.00 & ug $/ \mathrm{L}$ & Gen. & \\
\hline LI & 3.00 & ug/L & Gen. & \\
\hline
\end{tabular}


WELL LFW 62C COLLECTED ON 09/04/91 LABORATORY ANALYSES CONTINUED

Selenium
Silica
2,4,5-TP (Silvex)
Sulfatc
1,1,2,2-Tetrachloroethane
Tetrachloroethylene
Total dissolved solids
Total organic carbon
Total organic halogens
Phenols
Total phosphates (as P)
Trichloroethylene
Toxaphene
trans-1,2-Dichloroethylene
trans-1,3-Dichloropropene
1,1-Dichloroethylene
1,1-Dichloroethane
1,1,1-Trichloroethane
1,1,2-Trichloroethane
1,2-Dichloroethane
1,2-Dichloropropane
cis-1,3-Dichloropropene
2-Chlorethyl vinyl ether
$2,4-D i c h$ irophenoxyacetic acid
Gross a bha
Nonvolati be beta
Total radium
Tritium

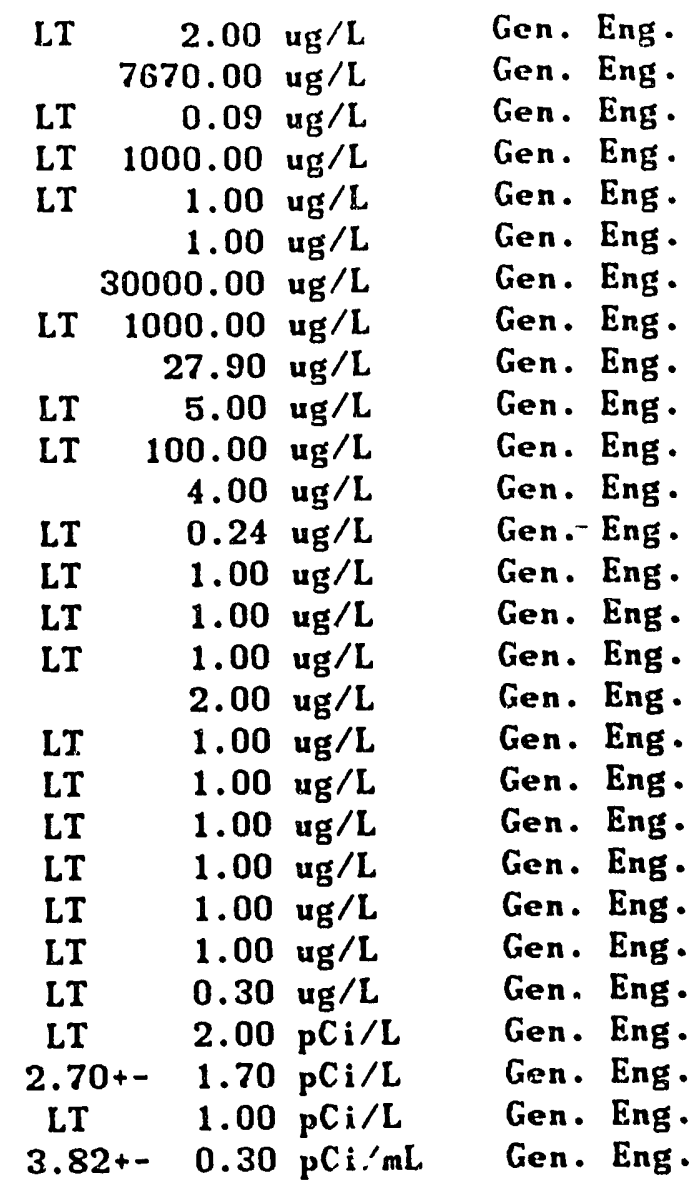

WELL LFW 62D

$\begin{array}{llllll}\text { SRS Grid } & N & 82991.6 & \text { Latitude } & 33.284247 & N \\ \text { Coordinates } & E & 45922.9 & \text { Longitude } & 81.707086 & \text { W }\end{array}$

Screen Zone Elevation 147.6-127.6 ft msl

Top of Casing Elevation $162.60 \mathrm{ft} \mathrm{msl}$

\section{MEASUREMENTS CONDUCTED IN THE FIELD}

Sample date 09/05/91 Time 930

Depth to water - $20.18 \mathrm{ft}(6.15 \mathrm{~m})$ below the TOC.

Water elevation $=142.42 \mathrm{ft}(43.41 \mathrm{~m}) \mathrm{msl}$

pll $=5.1$ Alkalinity $=10 \mathrm{mg} / \mathrm{L}$

Specific conductance - 26 umhos/cm

Water temperature - 19.1 degrees Celsius

Water evncuated from the well prior to sampling - 12 gal

The well went dry during purging.

\section{LABORATORY ANALYSES}

Specific conductance

pll

Silver

Arsenic

CONTINUED

$\begin{array}{lrll} & 20.00 \mathrm{umbi} i \mathrm{~cm} & \text { Geñ. Eng. } \\ & 5.54 \mathrm{pH} & \text { Gen. Eng. } \\ \text { LT } & 2.00 \mathrm{ug} / \mathrm{L} & \text { Gen. Eng. } \\ \text { LT } & 2.00 \mathrm{ug} / \mathrm{L} & \text { Gen. Eng. }\end{array}$

Geñ. Eng.

Gen. Eng.

Gen. Eng. 
WELL LFW 62D COLLECTED ON 09/05/91 LABORATORY ANALYSES CONTINUED

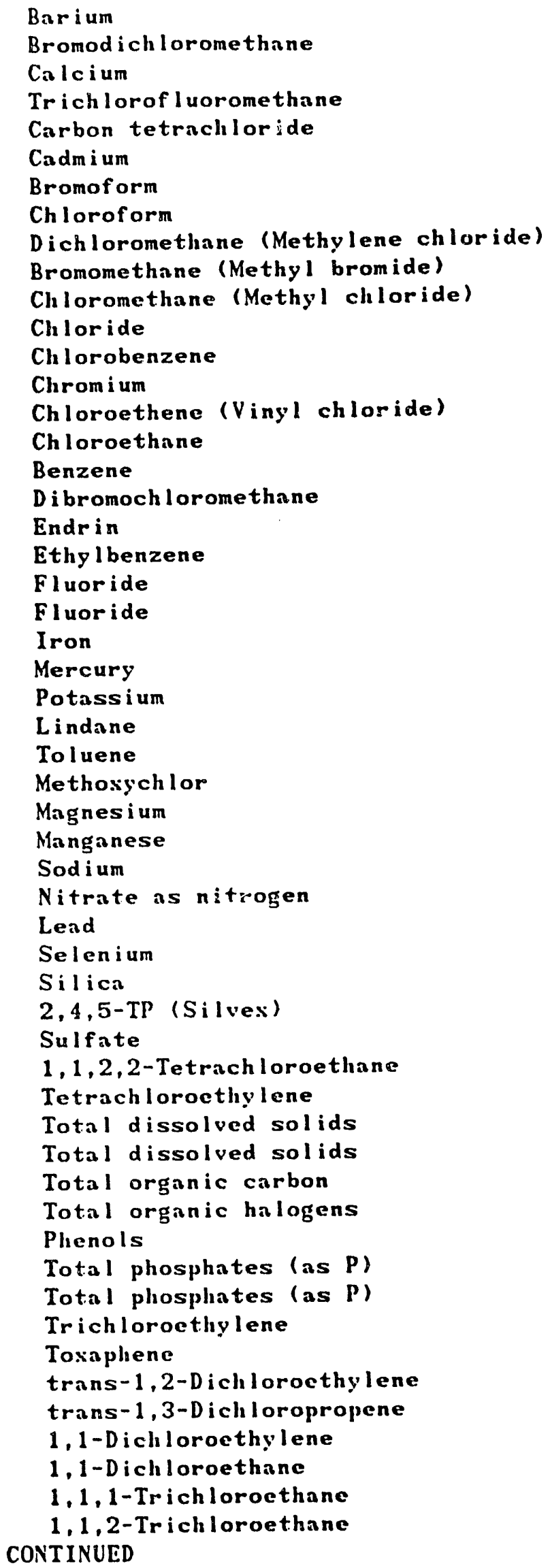

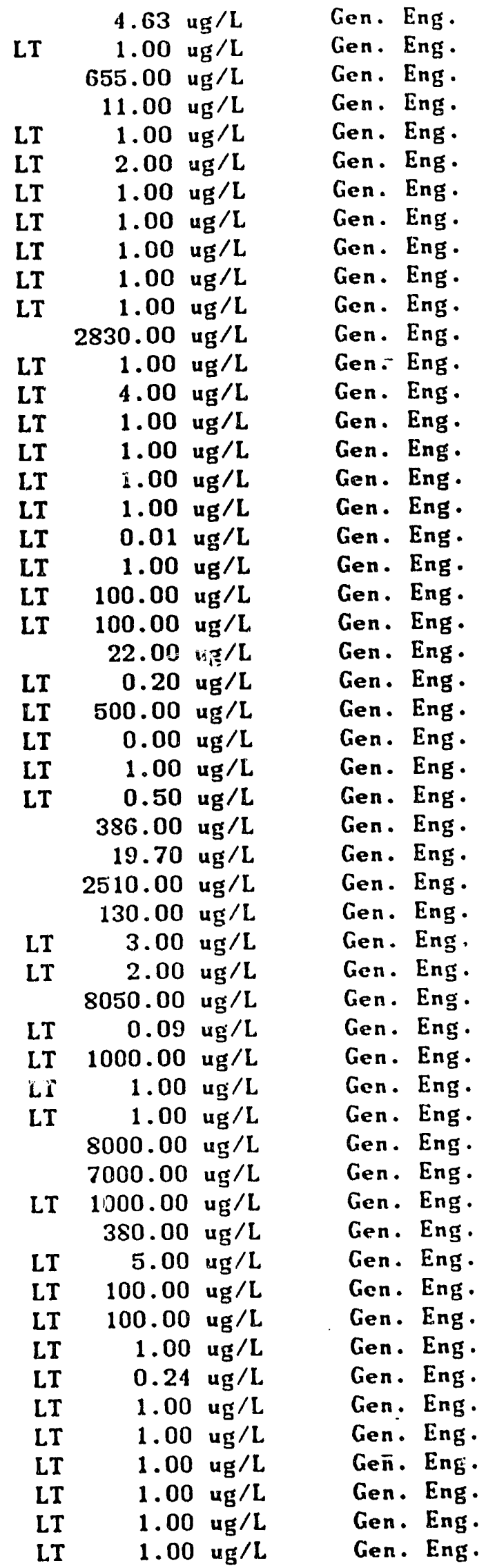


WELL LFW 62D COLLECTED ON 09/05/91 LABORATORY ANALYSES CONTINUED

1,2-Dichloroethane

1,2-Dichloropropane

cis-1,3-D ich loropropene

2-Chloroethyl vinyl ether

2, 4-Dichlorophenoxyacetic acid

Gross a lpha

Nonvolatile beta

Total radium

Tritium

$\begin{array}{lll}\text { LT } & 1.00 \mathrm{ug} / \mathrm{L} & \text { Gen. Eng. } \\ \mathrm{LT} & 1.00 \mathrm{ug} / \mathrm{L} & \text { Gen. Eng. } \\ \mathrm{LT} & 1.00 \mathrm{ug} / \mathrm{L} & \text { Gen. Eng. } \\ \mathrm{LT} & 1.00 \mathrm{ug} / \mathrm{L} & \text { Gen. Eng. } \\ \mathrm{LT} & 0.30 \mathrm{ug} / \mathrm{L} & \text { Gen. Eng. } \\ \mathrm{LT} & 2.00 \mathrm{pC} \mathrm{i} / \mathrm{L} & \text { Gen. Eng. } \\ \mathrm{LT} & 2.00 \mathrm{pCi} / \mathrm{L} & \text { Gen. Eng. } \\ 1.20+- & 2.20 \mathrm{pCi} / \mathrm{L} & \text { Gen. Eng. } \\ 1.17+- & 0.20 \mathrm{pCi} / \mathrm{mL} & \text { Gen. Eng. }\end{array}$




\section{APPENDIX C}

\section{FOURTH QUARTER 1991 ANALYTICAL DATA}




\section{WELL LFW 6}

$\begin{array}{llll}\text { SRS Coord. } & \text { Screen Zone Elevation } & \text { Top of Casing } & \text { Casing } \\ \text { N84537.8 } & 160.4-141.1 \mathrm{ft} \mathrm{msl} & 171.7 \mathrm{ft} \mathrm{msl} & 4^{\text {" Steel }} \\ \text { E45241.2 } & & \end{array}$

MEASUREMENTS CONDUCTED IN THE FIEI $?$

Sample date: $10 / 24 / 91$

Depth to water: $16.29 \mathrm{ft}(4.97 \mathrm{~m})$ below TOC

Time: 15:05

Water elevation: $155.41 \mathrm{ft}(47.37 \mathrm{~m}) \mathrm{msl}$

$\mathrm{pH}: 6.3$

So. conductance: $429 \mu \mathrm{S} / \mathrm{cm}$

Alkalinity: $96 \mathrm{mg} / \mathrm{L}$

Water evacuated before sampling: $50 \mathrm{gal}$

Water temperature: $20.5^{\circ} \mathrm{C}$

\section{LABORATORY ANALYSES}

H D Analyte

- $\quad \mathrm{pH}$

Specific conductance

Aluminum

Antimony

Arsenic

Barium

Benzene

Bromodichloromethane

Bromoform

Bromomethane (Methyl bromide)

Cadmium

Calcium

Carbon tetrachloride

Chloride

Chlorobenzene

Chloroethane

- Chloroethene (Vinyl chloride)

2-Chloroethyl vinyl ether

Chloroform

Chloromethane (Methyl chloride)

Chromium

Copper

Dibromochloromethane

1, 1-Dishloroethane

1,2.j)ictiloroethane

1,1-bichloroethylene

trans-1,2-Dichloroethylene

Dichloromethane (Methylene chloride)

2,4-Dichlorophenoxyacetic acid

1,2-Dichloropropane

trans-1,3-Dichloropropene

cis-1,3-Dichloropropene

Endrin

Ethylbenzene

Fluoride
Result

6.2

300

$<20$

$<2.0$

5.1

11

2.2

$<1.0$

$<1.0$

$<1.0$

$<2.0$

5,090

$<1.0$

11,200

1.1

4.1

4.7

$<1.0$

$<1.0$

$<1.0$

$<4.0$

$<4.0$

$<1.0$

7.7

$<1.0$

$<1.0$

$<1.0$

5.1

$<0.30$

$<1.0$

$<1.0$

$<1.0$

$<0.0060$

$<1.0$

120

\begin{tabular}{|c|c|c|}
\hline Units & Flag & $\underline{\text { Lab }}$ \\
\hline $\mathrm{pH}$ & 0 & $\mathrm{GE}$ \\
\hline$\mu \mathrm{S} / \mathrm{cm}$ & 1 & GE \\
\hline$\mu g / L$ & 0 & GE \\
\hline$\mu g / L$ & 0 & GE \\
\hline$\mu g / L$ & 0 & GE \\
\hline$\mu g / L$ & 0 & GE \\
\hline$\mu g / L$ & 0 & GE \\
\hline$\mu g / L$ & 0 & $\mathrm{GE}$ \\
\hline$\mu g / L$ & 0 & GE \\
\hline$\mu g / L$ & 0 & GE \\
\hline$\mu \mathrm{g} / \mathrm{L}$ & 0 & GE \\
\hline$\mu g / L$ & 0 & GE \\
\hline$\mu g / L$ & 0 & GE \\
\hline$\mu g / L$ & 0 & GE \\
\hline$\mu g / L$ & 0 & GE \\
\hline$\mu g / L$ & 0 & GE \\
\hline$\mu g / L$ & 2 & GE \\
\hline$\mu g / L$ & 0 & GE \\
\hline$\mu g / L$ & 0 & GE \\
\hline$\mu g / L$ & 0 & GE \\
\hline$\mu g / L$ & 0 & GE \\
\hline$\mu g / L$ & 0 & GE \\
\hline$\mu g / L$ & 0 & GE \\
\hline$\mu g / L$ & 1 & GE \\
\hline$\mu g / L$ & 0 & GE \\
\hline$\mu \mathrm{g} / \mathrm{L}$ & 0 & GE \\
\hline$\mu \mathrm{g} / \mathrm{L}$ & 0 & GE \\
\hline$\mu g / L$ & 0 & $\mathrm{GE}$ \\
\hline$\mu g / L$ & 0 & GE \\
\hline$\mu g / L$ & 0 & GE \\
\hline$\mu g / L$ & 0 & GE \\
\hline$\mu g / L$ & 0 & $\mathrm{GE}$ \\
\hline$\mu g / L$ & 0 & $\mathrm{GE}$ \\
\hline$\mu g / L$ & 0 & $\mathrm{GE}$ \\
\hline$\mu g / L$ & 0 & GE \\
\hline
\end{tabular}

$\overline{-}$ = exceeded holding time.

= exceeded primary drinking water standard 
WELL LFW 6, laboratory analyses (cont.)

\begin{tabular}{|c|c|c|c|c|c|}
\hline \multirow[t]{30}{*}{$\underline{H} \underline{D}$} & Analvte & Result & Unit & Flag & Lab \\
\hline & Lead & $<3.0$ & $\mu g / L$ & 0 & GE \\
\hline & Lindane & $<0.0050$ & $\mu g / L$ & 0 & GE \\
\hline & Magnesium & 11,000 & $\mu g / L$ & 0 & GE \\
\hline & Manganese & 36 & $\mu \mathrm{g} / \mathrm{L}$ & 1 & GE \\
\hline & Mercury & $<0.20$ & $\mu g / L$ & 0 & GE \\
\hline & Methoxychlor & $<0.50$ & $\mu g / L$ & 0 & GE \\
\hline & Nickel & 5.8 & $\mu g / L$ & 0 & $G$ \\
\hline & Nitrate as nitrogen & 62 & $\mu g / L$ & 0 & GE \\
\hline & Potassium & 2,470 & $\mu g / L$ & 0 & L \\
\hline & Selenium & $<2.0$ & $\mu g / L$ & 0 & GE \\
\hline & Silica & 9,460 & $\mu g / L$ & 0 & G \\
\hline & Silver & $<2.0$ & $\mu g / L$ & 0 & . \\
\hline & Sodium & 10,400 & $\mu g /$ & 0 & Gt \\
\hline & Sulfate & 3,130 & $\mu g / L$ & 0 & - \\
\hline & $1,1,2,2$-Tetrachloroethane & $<1.0$ & $\mu g / L$ & 0 & E \\
\hline & Tetrachloroethylene & $<1.0$ & $\mu g / L$ & 0 & 0 \\
\hline & Toluene & $<1.0$ & $\mu g / L$ & 0 & 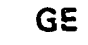 \\
\hline & Total organic carbon & 5,000 & $\mu g / L$ & 1 & \\
\hline & Total organic halogens & 153 & $\mu g / L$ & 2 & $\mathbf{E}$ \\
\hline & Toxaphene & $<0.24$ & $\mu g / L$ & 0 & \\
\hline & 2,4,5-TP (Silvex) & $<0.090$ & $\mu g / L$ & 0 & \\
\hline & 1,1,1-Trichloroethane & $<1.0$ & $\mu g / L$ & 0 & \\
\hline & 1.1,2-Trichloroethane & $<1.0$ & $\mu_{\theta} / L$ & 0 & Jᄃ \\
\hline & Trichloroethylene & $<1.0$ & $\mu g / L$ & 0 & \\
\hline & Trichlorofluoromethane & $<1.0$ & $\mu g / L$ & 0 & \\
\hline & Vanadium & $<8.0$ & $\mu g / L$ & 0 & \\
\hline & Gross alpha & $<2.0 E+00$ & $\mathrm{pCi} / \mathrm{L}$ & 0 & \\
\hline & Nonvolatile beta & $<2.0 E+00$ & $\mathrm{pCi} / \mathrm{L}$ & 0 & \\
\hline & Total radium & $<1.0 E+00$ & pCin. & 0 & \\
\hline & Tritium & $<7.0 E-01$ & $\mathrm{pCi} / \mathrm{mi}$ & 0 & \\
\hline
\end{tabular}

\section{WELL LFW 8}

$\begin{array}{llll}\text { SRS Coord. } & \text { Screen Zone Elev?ion } & \text { Top of Casing } & \text { Casing } \\ \text { N84032.6 } & 159.2-139.9 \mathrm{ft} \mathrm{msl} & 170.5 \mathrm{ft} \mathrm{msl} & 4^{\text {" Steel }} \\ \text { E45415.3 } & & \end{array}$

MEASUREMENTS CONDUCTED IN THE FIELD

Sample date: $10 / 28 / 91$

Depth to water: $19.64 \mathrm{ft}(5.99 \mathrm{~m})$ below TOC

Time: $12: 30$

Water elevation: $150.86 \mathrm{ft}(45.98 \mathrm{~m}) \mathrm{ms}$

pH: 6.6

Sp. conductance: $389 \mu \mathrm{S} / \mathrm{cm}$

Alkalinity: $173 \mathrm{mg} / \mathrm{L}$

Water evacuated before sampling: $32 \mathrm{gal}$

Water temperature: $21.0^{\circ} \mathrm{C}$

$\overline{-=\text { exceeded holding time. }}=$ exceeded primary drinking water standard. 


\begin{tabular}{|c|c|c|c|c|c|}
\hline $1 \underline{D}$ & Analyte & Result & Unit & Flag & $\underline{\text { Lab }}$ \\
\hline & $\mathrm{pH}$ & 6.4 & $\mathrm{pH}$ & 0 & GE \\
\hline & Specific conductance & 270 & $\mu \mathrm{S} / \mathrm{cm}$ & 1 & GE \\
\hline & Aluminum & $<20$ & $\mu g / L$ & 0 & GE \\
\hline & Antimony & $<2.0$ & $\mu g / L$ & 0 & GE \\
\hline & Arsenic & 8.4 & $\mu \mathrm{g} / \mathrm{L}$ & 0 & GE \\
\hline & Barium & 6.5 & $\mu g / L$ & 0 & GE \\
\hline - & Benzene & 5.1 & $\mu g / L$ & 2 & GE \\
\hline & Bromodichloromethane & $<1.0$ & $\mu g / L$ & 0 & GE \\
\hline & Bromoform & $<1.0$ & $\mu g / L$ & 0 & GE \\
\hline & Bromomethane (Methyl bromide) & $<1.0$ & $\mu g / L$ & 0 & GE \\
\hline & Cadmium & $<2.0$ & $\mu g / L$ & 0 & GE \\
\hline & Calcium & 6,960 & $\mu g / L$ & 0 & . GE \\
\hline & Carbon tetrachloride & $<1.0$ & $\mu g / L$ & 0 & GE \\
\hline & Chloride & 25,400 & $\mu g / L$ & 0 & GE \\
\hline & Chlorobenzene & 13 & $\mu g / L$ & 2 & GE \\
\hline & Chloroethane & 8.6 & $\mu g / L$ & 1 & GE \\
\hline a & Chloroethene (Vinyl chloride) & 167 & $\mu g / L$ & 2 & GE \\
\hline & 2-Chloroethyl vinyl ether & $<1 . r$ & $\mu g / L$ & 0 & GE \\
\hline & Chlor :orm & $<1.0$ & $\mu g / L$ & 0 & GE \\
\hline & Chloromethane (Methyl chloride) & $<1.0$ & $\mu g / L$ & 0 & GE \\
\hline & Chromium & $<4.0$ & $\mu g / L$ & 0 & GE \\
\hline & Copper & $<4.0$ & $\mu g / L$ & 0 & GE \\
\hline & Dibromochloromethane & $<1.0$ & $\mu g / L$ & 0 & GE \\
\hline & 1,1-Dichloroethane & 53 & $\mu g / L$ & 2 & GE \\
\hline & 1,2-Dichloroethane & 2.3 & $\mu g / L$ & 0 & GE \\
\hline & 1,1-Dichloroethylene & $<1.0$ & $\mu g / L$ & 0 & GE \\
\hline & trans-1,2-Dichloroethylene & $<1.0$ & $\mu g / L$ & 0 & GE \\
\hline & Dichloromethane (Methylene chloride) & 5.4 & $\mu g / L$ & 0 & GE \\
\hline & 2,4-Dichlorophenoxyacetic acid & $<0.30$ & $\mu g / L$ & 0 & GE \\
\hline & 1,2-Dichloropropane & $<1.0$ & $\mu g / L$ & 0 & GE \\
\hline & trans-1,3-Dichloropropene & $<1.0$ & $\mu g / L$ & 0 & GE \\
\hline & cis-1,3-Dichloropropene & $<1.0$ & $\mu g / L$ & 0 & GE \\
\hline & Endrin & $<0.0060$ & $\mu g / L$ & 0 & GE \\
\hline & Endrin & $<0.0060$ & $\mu g / L$ & 0 & GE \\
\hline & Ethylbenzene & 22 & $\mu g / L$ & 0 & GE \\
\hline & Fluoride & 216 & $\mu g / L$ & 0 & GE \\
\hline - & Lead & 16 & $\mu g / L$ & 2 & GE \\
\hline & Lindane & $<0.0050$ & $\mu g / L$ & 0 & GE \\
\hline & Lindane & $<0.0050$ & $\mu g / L$ & 0 & GE \\
\hline & Magnesium & 14,100 & $\mu g / L$ & 0 & GE \\
\hline & Manganese & 22 & $\mu g / L$ & 0 & GE \\
\hline & Mercury & $<0.20$ & $\mu g / L$ & 0 & GE \\
\hline & Methoxychlor & $<0.50$ & $\mu g / L$ & 0 & GE \\
\hline & Methoxychlor & $<0.50$ & $\mu g / L$ & 0 & GE \\
\hline & Nickel & $<4.0$ & $\mu g / L$ & 0 & GE \\
\hline & Nitrate as ritrogen & $<50$ & $\mu g / L$ & 0 & $\mathrm{GE}$ \\
\hline & Potassium & 1,700 & $\mu g / L$ & 0 & GE \\
\hline & Selenium & $<2.0$ & $\mu g / L$ & 0 & GE \\
\hline & Silica & 6,060 & $\mu g / L$ & 0 & GE \\
\hline & Silver & $<2.0$ & $\mu g / L$ & 0 & GE \\
\hline & Sodium & 21,300 & $\mu g / L$ & 0 & GE \\
\hline
\end{tabular}

- = exceeded holding time. - = exceeded primary drinking water standard 
WELL LFW 8, lat:oratory analyses (cont.)

\begin{tabular}{|c|c|c|c|c|c|}
\hline \multirow[t]{19}{*}{$\underline{H} \underline{D}$} & Analyte & Result & $\underline{\text { Unit }}$ & Flag & $\underline{\text { Lab }}$ \\
\hline & Sulfate & $<1,000$ & $\mu g / L$ & 0 & GE \\
\hline & $1,1,2,2$-Tetrachloroethane & $<1.0$ & $\mu g / L$ & 0 & GE \\
\hline & Tetrachloroethylene & $<1.0$ & $\mu g / L$ & 0 & GE \\
\hline & Toluene & 8.9 & $\mu g / L$ & 0 & GE \\
\hline & Total organic carbon & 8,000 & $\mu g / L$ & 1 & $E$ \\
\hline & Total organic halogens & 210 & $\mu g / L$ & 2 & GE \\
\hline & Toxaphene & $<0.24$ & $\mu g / L$ & 0 & $\mathrm{GE}$ \\
\hline & Toxaphene & $<0.24$ & $\mu g / L$ & 0 & 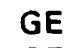 \\
\hline & $2,4,5-T P$ (Silvex) & $<0.090$ & $\mu g / L$ & 0 & $\mathbf{E}$ \\
\hline & $1,1,1$-Trichloroethane & $<1.0$ & $\mu g / L$ & 0 & GE \\
\hline & 1,1,2-Trichloroethane & $<1.0$ & $\mu g / L$ & 0 & GE \\
\hline & Trichloroethylene & $<1.0$ & $\mu g / L$ & 0 & $\mathbf{E}$ \\
\hline & Trichlorofluoromethane & $<1.0$ & $\mu g / L$ & 0 & $\mathbf{E}$ \\
\hline & Vanadium & $<8.0$ & $\mu_{g} / L$ & 0 & $\mathrm{jE}$ \\
\hline & Gross alpha & $4.5 E+00 \pm 1.8 E+00$ & $\mathrm{pCi} / \mathrm{L}$ & 0 & \\
\hline & Nonvolatile beta & $9.5 E+00 \pm 1.9 E+00$ & $\mathrm{pCi} / \mathrm{L}$ & 0 & GE \\
\hline & Total radium & $1.6 E+00 \pm 1.5 E+00$ & $\mathrm{pCi} / \mathrm{L}$ & 0 & $\mathbf{E}$ \\
\hline & Tritium & $.9 E+00 \pm 7.0 E-01$ & $\mathrm{pCi} / \mathrm{mL}$ & 0 & $\mathbf{E}$ \\
\hline
\end{tabular}

\section{WELL LFW 10A}

$\begin{array}{lllll}\text { SRS Coord. } & \text { Screen Zone Elevation } & \text { Too of Casing } & \text { Casing } \\ \text { N84369.6 } & 159.2-129.2 \mathrm{ft} \mathrm{msl} & 175.5 \mathrm{ft} \mathrm{msl} & 4^{\text {" PVC }}\end{array}$

$E 45935.6$

MEASUREMENTS CONDUCTED M THE FIELD

Sample date: $10 / 24 / 91$

Depth to water: $21.61 \mathrm{ft}(6.59 \mathrm{~m})$ below TOC Water elevation: $153.89 \mathrm{ft}(46.91 \mathrm{~m}) \mathrm{msl}$ Sp. conductance: $668 \mu \mathrm{S} / \mathrm{cm}$

Water evacuated before sampling: $67 \mathrm{gal}$

\section{LABORATORY ANALYSES}

H $D \quad$ Analyte
pH
Specific conductance
Aluminum
Antimony
Arsenic
Barium
Benzene
Brornodichloromethare
Bromoform
Bromomethane (Methyl bromide)
Cadmium
Calcium

$\begin{aligned} & \text { Result } \\ & \\ & 6.1 \\ & 500 \\ &<20 \\ &<2.0 \\ & 5.5 \\ & 12 \\ & 9.9 \\ &<1.0 \\ &<1.0 \\ &<1.0 \\ &<2.0 \\ & 25.800\end{aligned}$

Time: 9:15

pH: 6.4

Alkalinity: $131 \mathrm{mg} / \mathrm{L}$

Water temperature: $20.5^{\circ} \mathrm{C}$ 
WELL LFW 10A, laboratory analyses (cont.)

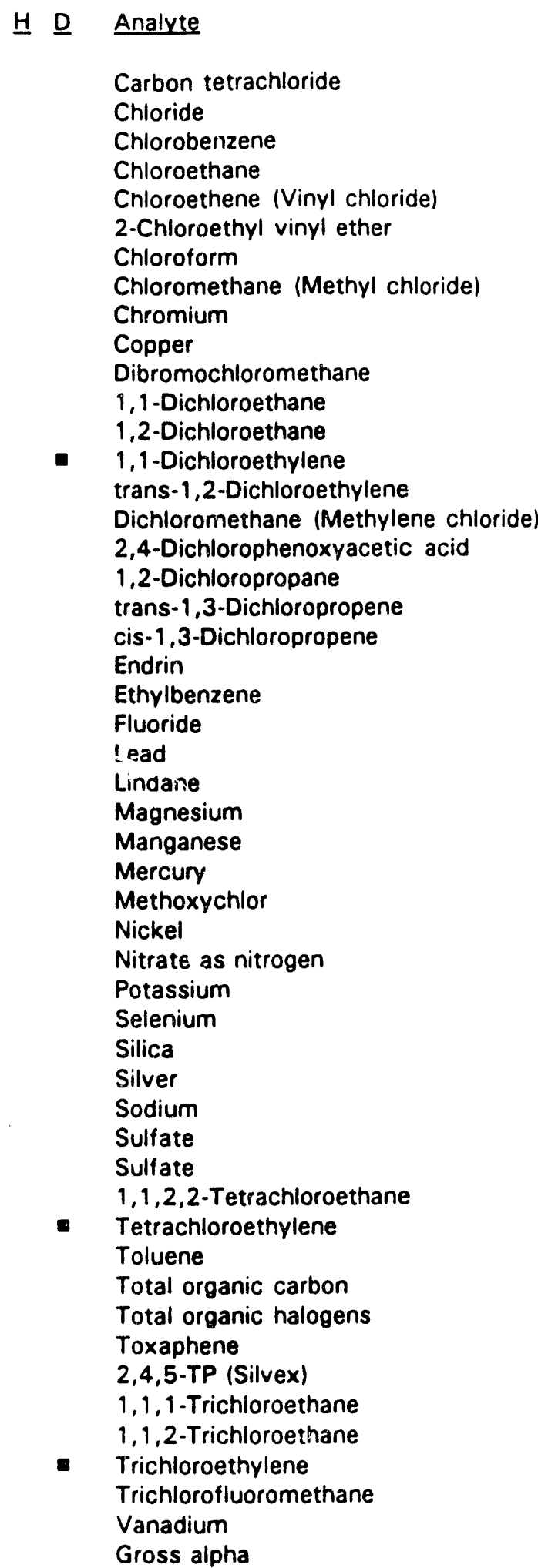

\begin{tabular}{|c|c|c|c|}
\hline Result & Unit & Flag & $\underline{\text { Lab }}$ \\
\hline$<1.0$ & $\mu g / L$ & 0 & GE \\
\hline 36,400 & $\mu g / L$ & 0 & GE \\
\hline$<1.0$ & $\mu \mathrm{g} / \mathrm{L}$ & 0 & GE \\
\hline$<1.0$ & $\mu g / L$ & 0 & GE \\
\hline$<1.0$ & $\mu g / L$ & 0 & GE \\
\hline$<1.0$ & $\mu g / L$ & 0 & GE \\
\hline$<1.0$ & $\mu \mathrm{g} / \mathrm{L}$ & 0 & GE \\
\hline$<1.0$ & $\mu g / L$ & 0 & GE \\
\hline$<4.0$ & $\mu g / L$ & 0 & GE \\
\hline$<4.0$ & $\mu g / L$ & 0 & GE \\
\hline$<1.0$ & $\mu g / L$ & 0 & GE \\
\hline 6.6 & $\mu g / L$ & 1 & GE \\
\hline$<20$ & $\mu g / L$ & 0 & GE \\
\hline 7.6 & $\mu g / L$ & 2 & GE \\
\hline$<1.0$ & $\mu g / L$ & 0 & GE \\
\hline 757 & $\mu \mathrm{g} / \mathrm{L}$ & 0 & GE \\
\hline$<0.30$ & $\mu \mathrm{g} / \mathrm{L}$ & 0 & GE \\
\hline$<1.0$ & $\mu \mathrm{g} / \mathrm{L}$ & 0 & GE \\
\hline$<1.0$ & $\mu g / L$ & 0 & GE \\
\hline$<1.0$ & $\mu g / L$ & 0 & GE \\
\hline$<0.0060$ & $\mu g / L$ & 0 & GE \\
\hline 36 & $\mu g / L$ & 0 & GE \\
\hline 188 & $\mu g / L$ & 0 & GE \\
\hline$<3.0$ & $\mu g / L$ & 0 & GE \\
\hline$<0.0050$ & $\mu g / L$ & 0 & GE \\
\hline 19.700 & $\mu g / L$ & 0 & GE \\
\hline 91 & $\mu g / L$ & 2 & GE \\
\hline$<0.20$ & $\mu g / L$ & 0 & GE \\
\hline$<0.50$ & $\mu g / L$ & 0 & GE \\
\hline 6.5 & $\mu g / L$ & 0 & GE \\
\hline 63 & $\mu g / L$ & 0 & GE \\
\hline$<500$ & $\mu g / L$ & 0 & GE \\
\hline$<2.0$ & $\mu g / L$ & 0 & GE \\
\hline 10,300 & $\mu g / L$ & 0 & GE \\
\hline$<2.0$ & $\mu g / L$ & 0 & GE \\
\hline 45,300 & $\mu g / L$ & 0 & GE \\
\hline 3,080 & $\mu g / L$ & 0 & GE \\
\hline 3,100 & $\mu \mathrm{g} / \mathrm{L}$ & 0 & GE \\
\hline$<1.0$ & $\mu \mathrm{g} / \mathrm{L}$ & 0 & GE \\
\hline 23 & $\mu g / L$ & 2 & GE \\
\hline 87 & $\mu g / L$ & 0 & GE \\
\hline 47,000 & $\mu g / L$ & 2 & GE \\
\hline 982 & $\mu g / L$ & 2 & GE \\
\hline$<0.24$ & $\mu \mathrm{g} / \mathrm{L}$ & 0 & GE \\
\hline 0.11 & $\mu g / L$ & 0 & GE \\
\hline 21 & $\mu \mathrm{g} / \mathrm{L}$ & 0 & GE \\
\hline$<1.0$ & $\mu g / L$ & 0 & GE \\
\hline 50 & $\mu g / L$ & 2 & GE \\
\hline$<1.0$ & $\mu g / L$ & 0 & GE \\
\hline$<8.0$ & $\mu g / L$ & 0 & GE \\
\hline$<2.0 E+00$ & $\mathrm{pCi} / \mathrm{L}$ & 0 & GE \\
\hline
\end{tabular}

- = exceeded holding time. = exceeded primary drinking water standard 
WELL LFW 10A, laboratory analyses (cont.)

H $\underline{\text { Analyte }}$

Nonvolatile beta

Total radium

- Tritium
Result

$4.6 E+00 \pm 8.4 E+00$

$1.0 E+00 \pm 8.0 E-01$

$1.2 E+02 \pm 1.9 E+00$
Unit

Flag

$\underline{L a b}$

$\mathrm{pCi} / \mathrm{L} \quad 0$

GE

$\mathrm{pCi} / \mathrm{L} \quad \mathrm{G} \quad \mathrm{GE}$

$\mathrm{pCi} / \mathrm{mL} \quad 2$

\section{WELL LFW 16}

SRS Coord.

N84748.9

E45852.6

MEASUREMENTS CONDUCTED IN THE FIELD

Sample date: $10 / 24 / 91$

Depth to water: $21.46 \mathrm{ft}(6.54 \mathrm{~m})$ below TOC Water elevation: $157.34 \mathrm{ft}(47.96 \mathrm{~m}) \mathrm{msl}$

Sp. conductance: $79 \mu \mathrm{S} / \mathrm{cm}$

Water evacuated before sampling: $69 \mathrm{gal}$

\section{LABORATORY ANALYSES}

H D Analyte

- $\quad \mathrm{pH}$

Specific conductance

Screen Zone Elevation

$161.2-131.2 \mathrm{ft} \mathrm{msl}$

Top of Casing

Casing

$178.8 \mathrm{ft} \mathrm{msl}$

4" PVC

Result

Time: 8:45

$\mathrm{pH}: 5.2$

Alkalinity: $9 \mathrm{mg} / \mathrm{L}$

Water temperature: $21.3^{\circ} \mathrm{C}$

5.5

75

Aluminum

Antimony

Arsenic

Barium

Benzene

Bromodichloromethane

Bromoform

Bromomethane (Methyl bromide)

Cadmium

$\therefore$ ciurr

Carbon tetrachloride

Chloride

Chloride

Chlorobenzene

Chloroethane

Chloroethene (Vinyl chloride)

2-Chloroethyl vinyl ether

Chloroform

Chloromethane (Methyl chloride)

Chromium

Copper

Dibromochloromethane

$<20$

$<2.0$

$<2.0$

10

1.5

$<1.0$

$<1.0$

$<1.0$

$<2.0$

3,730

$<1.0$

5,400

5,250

$<1.0$

$<1.0$

$<1.0$

$<1.0$

$<1.0$

$<1.0$

$<4.0$

$<4.0$

$<1.0$

1,1-Dichloroethane

42

1,2-Dichloroethane

$<1.0$

1,1-Dichloroethylene

Unit Flag Lab

$\mathrm{pH} \quad \mathrm{O} \quad \mathrm{GE}$

$\mu \mathrm{S} / \mathrm{cm} \quad 0 \quad \mathrm{GE}$

$\mu g / L \quad 0 \quad$ GE

$\mu g / L \quad 0 \quad$ GE

$\mu g / L \quad 0 \quad$ GE

$\mu g / L \quad 0 \quad$ GE

$\mu g / L \quad 0 \quad$ GE

$\mu g / L \quad 0 \quad$ GE

$\mu g / L \quad 0 \quad$ GE

$\mu g / L \quad 0 \quad G E$

$\mu g / L \quad 0 \quad$ GE

$\mu \mathrm{g} / \mathrm{L} \quad \mathrm{O} \quad \mathrm{GE}$

$\mu \mathrm{g} / \mathrm{L} \quad \mathrm{O} \quad \mathrm{GE}$

$\mu g / \mathrm{L} \quad 0 \quad \mathrm{GE}$

$\mu g / L \quad 0 \quad$ GE

$\mu g / L \quad 0 \quad$ GE

$\mu g / L \quad 0 \quad$ GE

$\mu g / L \quad 0 \quad$ GE

$\mu \mathrm{g} / \mathrm{L} \quad 0 \quad \mathrm{GE}$

$\mu g / L \quad 0 \quad$ GE

$\mu g / L \quad 0 \quad G E$

$\mu g / L \quad 0 \quad$ GE

$\mu g / L \quad 0 \quad$ GE

$\mu g / L \quad 0 \quad G E$

$\mu g / L \quad 2 \quad G E$

$\mu g / L \quad 0 \quad$ GE

$\mu \mathrm{g} / \mathrm{L} \quad 0 \quad \mathrm{GE}$

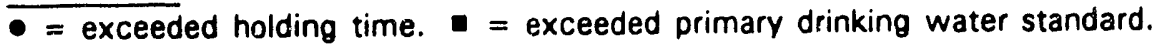


WELL LFW 16, laboratory analyses (cont.)

H

Analyte
trans-1,2-Dichloroethylene
Dichloromethane (Methylene chloride)
2,4-Dichlorophenoxyacetic acid
1,2-Dichloropropane
trans-1,3-Dichloropropene
cis-1,3-Dichloropropene
Endrin
Ethylbenzene
Fluoride
Lead
Lindane
Magnesium
Manganese
Mercury
Methoxychlor
Nickel
Nitrate as nitrogen
Potassium
Selenium
Silica
Silver
Sodium
Sulfate
1,1,2,2-Tetrachloroethane
Tetrachloroethylene
Toluene
Total organic carbon
Total organic halogeris
Toxaphene
2,4,5-TP (Silvex)
1,1,1-Trichloroethane
1,1,2-Trichloroethane
Trichloroethylene
Trichlorofluoromethane
Vanadium
Gross alpha
Nonvolatile beta
Total radium
Tritium

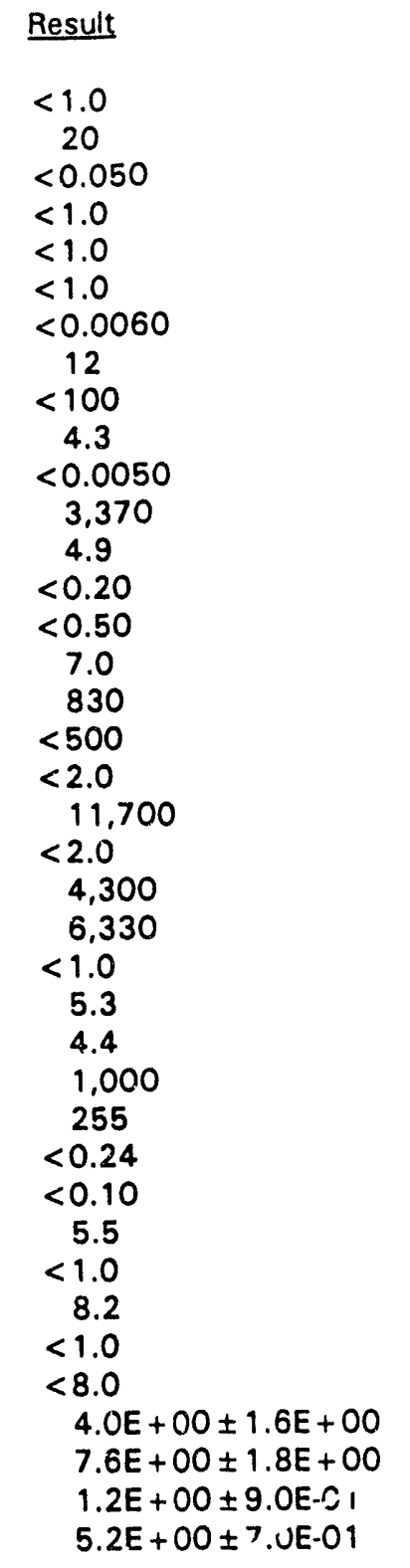

$\begin{array}{lll}\text { Unit } & \text { Flag } & \text { Lab } \\ \mu \mathrm{g} / \mathrm{L} & 0 & \mathrm{GE} \\ \mu \mathrm{g} / \mathrm{L} & 0 & \mathrm{GE} \\ \mu \mathrm{g} / \mathrm{L} & 0 & \mathrm{GE} \\ \mu \mathrm{g} / \mathrm{L} & 0 & \mathrm{GE} \\ \mu \mathrm{g} / \mathrm{L} & 0 & \mathrm{GE} \\ \mu \mathrm{g} / \mathrm{L} & 0 & \mathrm{GE} \\ \mu \mathrm{g} / \mathrm{L} & 0 & \mathrm{GE} \\ \mu \mathrm{g} / \mathrm{L} & 0 & \mathrm{GE} \\ \mu \mathrm{g} / \mathrm{L} & 0 & \mathrm{GE} \\ \mu \mathrm{g} / \mathrm{L} & 0 & \mathrm{GE} \\ \mu \mathrm{g} / \mathrm{L} & 0 & \mathrm{GE} \\ \mu \mathrm{g} / \mathrm{L} & 0 & \mathrm{GE} \\ \mu \mathrm{g} / \mathrm{L} & 0 & \mathrm{GE} \\ \mu \mathrm{g} / \mathrm{L} & 0 & \mathrm{GE} \\ \mu \mathrm{g} / \mathrm{L} & 0 & \mathrm{GE} \\ \mu \mathrm{G} / \mathrm{L} & 0 & \mathrm{GE} \\ \mu \mathrm{g} / \mathrm{L} & 0 & \mathrm{GE} \\ \mu \mathrm{g} / \mathrm{L} & 0 & \mathrm{GE} \\ \mu \mathrm{g} / \mathrm{L} & 0 & \mathrm{GE} \\ \mu \mathrm{g} / \mathrm{L} & 0 & \mathrm{GE} \\ \mu \mathrm{g} / \mathrm{L} & 0 & \mathrm{GE} \\ \mu \mathrm{g} / \mathrm{L} & 0 & \mathrm{GE} \\ \mu \mathrm{g} / \mathrm{L} & 0 & \mathrm{GE} \\ \mu \mathrm{g} / \mathrm{L} & 0 & \mathrm{GE} \\ \mu \mathrm{g} / \mathrm{L} & 2 & \mathrm{GE} \\ \mu \mathrm{g} / \mathrm{L} & 0 & \mathrm{GE} \\ \mu \mathrm{g} / \mathrm{L} & 0 & \mathrm{GE} \\ \mu \mathrm{g} / \mathrm{L} & 2 & \mathrm{GE} \\ \mu \mathrm{g} / \mathrm{L} & 0 & \mathrm{GE} \\ \mu \mathrm{g} / \mathrm{L} & 0 & \mathrm{GE} \\ \mu \mathrm{g} / \mathrm{L} & 0 & \mathrm{GE} \\ \mu \mathrm{g} / \mathrm{L} & 0 & \mathrm{GE} \\ \mu \mathrm{g} / \mathrm{L} & 2 & \mathrm{GE} \\ \mu \mathrm{g} / \mathrm{L} & 0 & \mathrm{GE} \\ \mu \mathrm{g} / \mathrm{L} & 0 & \mathrm{GE} \\ \mathrm{pCi} / \mathrm{L} & 0 & \mathrm{GE} \\ \mathrm{pCC} / \mathrm{L} & 0 & \mathrm{GE} \\ \mathrm{pC} \mathrm{C} / \mathrm{L} & 0 & \mathrm{GE} \\ \mathrm{pCI} / \mathrm{mL} & 0 & \mathrm{GE} \\ & & \end{array}$

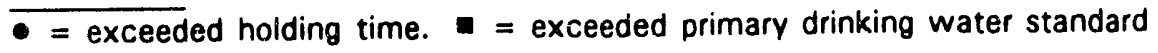


WELL LFW 17

SRS Coord.

N84602.8

$E 45607.3$

MEASUREMENTS CONDUCTED IN THE FIELD

Sample date: $10 / 24 / 91$

Depth to water: $21.38 \mathrm{ft}(6.52 \mathrm{~m})$ below TOC Water elevation: $156.42 \mathrm{ft}(47.68 \mathrm{~m}) \mathrm{msl}$

Sp. conductance: $301 \mu \mathrm{S} / \mathrm{cm}$

Water evacuated before sampling: $93 \mathrm{gal}$

\section{LABORATORY ANALYSES}

므 Analyte.

pH
Specific conductance
Aluminum
Antimony
Arsenic
Barium
Benzene
Bromodichloromethane
Bromoform
Bromomethane (Methyl bromide)
Cadmium
Calcium
Carbon tetrachloride
Chloride
Chlorobenzene
Chloroethane
Chloroethene (Vinyl chloride)
2-Chloroethyl vinyl ether
Chloroform
Chloromethane (Methyl chloride)
Chromium
Copper
Dibromochloromethane
1,1 -Dichloroethane
$1,2-$ Dichloroethane
1,1 -Dichloroethylene
trans-1,2-Dichloroethylene
Dichloromethane (Methylene chloride)
$2,4-$ Dichlorophenoxyacetic acid
$1,2-$ Dichloropropane
trans-1,3-Dichloropropene
cis-1,3-Dichloropropene
Endrin
Ethylbenzene
Fluoride
Lead

- $\mathrm{pH}$

Antimony

Arsenic

Benzene

Bromodichloromethane

Bromomethane (Methyl bromide)

Cadmium

Carbon tetrachloride

Chloride

Chloroethene (Vinyl chloride)

chloroethyl vinyl ether

Chloromethane (Methyl chloride)

Dibromochloromethane
Screen Zone Elevation

$158.5-128.5 \mathrm{ft} \mathrm{ms}$

\section{Top of Casing}

$177.8 \mathrm{ft} \mathrm{msl}$
Casing

4" PVC
Time: $14: 40$

pH: 6.4

Alkalinity: $106 \mathrm{mg} / \mathrm{L}$

Water temperature: $20.0^{\circ} \mathrm{C}$
Result

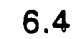

160

$<20$

$<2.0$

18

4.1

3.9

$<1.0$

$<1.0$

$\because 1.0$

$<2.0$

4,740

$<1.0$

14,700

79

3.9

$<1.0$

$<1.0$

$<1.0$

$<1.0$

$<4.0$

$<4.0$

$<1.0$

28

4.4

$<1.0$

$<1.0$

3.0

$<0.050$

$<1.0$

$<1.0$

$<1.0$

$<0.0060$

25

$<100$

$<3.0$

\begin{tabular}{|c|c|c|}
\hline Unit & Flag & Lab \\
\hline $\mathrm{pH}$ & 0 & GE \\
\hline$\mu \mathrm{S} / \mathrm{cm}$ & 0 & GE \\
\hline$\mu g / L$ & 0 & GE \\
\hline$\mu g / L$ & 0 & GE \\
\hline$\mu g / L$ & 0 & GE \\
\hline$\mu g / L$ & 0 & GE \\
\hline$\mu g / L$ & 1 & GE \\
\hline$\mu g / L$ & 0 & GE \\
\hline$\mu g / L$ & 0 & GE \\
\hline$\mu g / L$ & 0 & GE \\
\hline$\mu g / L$ & 0 & GE \\
\hline$\mu \mathrm{g} / \mathrm{L}$ & 0 & GE \\
\hline$\mu \mathrm{g} / \mathrm{L}$ & 0 & GE \\
\hline$\mu g / L$ & 0 & GE \\
\hline$\mu g / L$ & 2 & GE \\
\hline$\mu g / L$ & 0 & GE \\
\hline$\mu g / L$ & 0 & GE \\
\hline$\mu \mathrm{g} / \mathrm{L}$ & 0 & GE \\
\hline$\mu g / L$ & 0 & GE \\
\hline$\mu g / L$ & 0 & GE \\
\hline$\mu g / L$ & 0 & GE \\
\hline$\mu g / L$ & 0 & GE \\
\hline$\mu \mathrm{g} / \mathrm{L}$ & 0 & GE \\
\hline$\mu g / L$ & 2 & GE \\
\hline$\mu g / L$ & 1 & GE \\
\hline$\mu g / h$ & 0 & GE \\
\hline$\mu g / L$ & 0 & GE \\
\hline$\mu g / L$ & 0 & GE \\
\hline$\mu g / L$ & 0 & GE \\
\hline$\mu g / L$ & 0 & GE \\
\hline$\mu g / L$ & 0 & \\
\hline$\mu g / L$ & 0 & GI \\
\hline$\mu g / L$ & 0 & \\
\hline$\mu g / L$ & 0 & \\
\hline$\mu g / L$ & 0 & 38 \\
\hline$\mu \mathrm{g} / \mathrm{L}$ & 0 & \\
\hline
\end{tabular}

$\overline{- \text { = exceeded holding time. }} \mathbf{a}=$ exceeded primary drinking water standard. 
WELL LFW 17, laboratory analyses (cont.)

D. Analyte
Lindane
Magnesium
Manganese
Mercury
Methoxychlor
Nickel
Nitrate as nitrogen
Potassium
Selenium
Silica
Silver
Sodium
Sulfate
1,1,2,2-Tetrachloroethane
Tetrachloroethylene
Toluene
Total organic carbon
Total organic halogens
Toxaphene
$2,4,5-$ TP (Silvex)
$1,1,1-$ Trichloroethane
$1,1,2-$ Trichloroethane
Trichloroethylene
Trichlorofluoromethane
Vanadium
Gross alpha
Nonvolatile beta
Total radium
Tritium

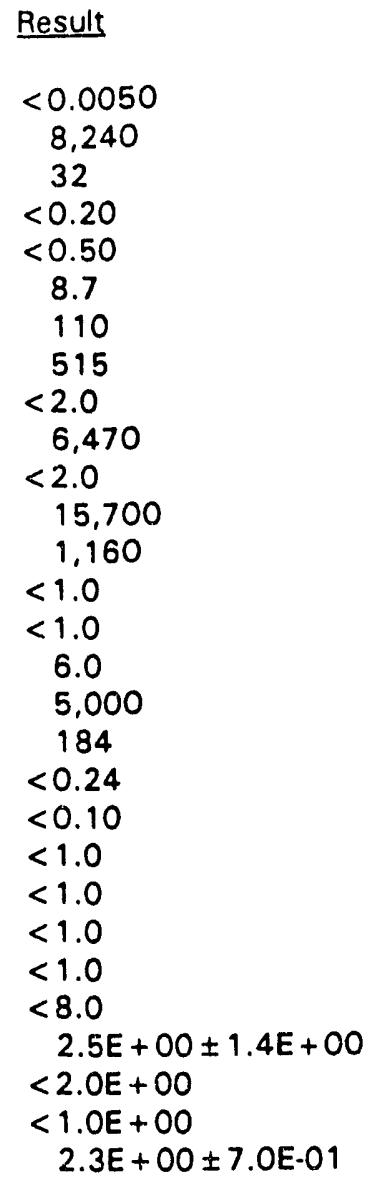

\begin{tabular}{|c|c|c|}
\hline Unit & Flag & $\underline{L a b}$ \\
\hline$\mu g / L$ & 0 & GE \\
\hline$\mu g / L$ & 0 & GE \\
\hline$\mu g / L$ & 1 & GE \\
\hline$\mu g / L$ & 0 & GE \\
\hline$\mu g / L$ & 0 & GE \\
\hline$\mu g / L$ & 0 & GE \\
\hline$\mu g / L$ & 0 & GE \\
\hline$\mu \mathrm{g} / \mathrm{L}$ & 0 & GE \\
\hline$\mu g / L$ & 0 & GE \\
\hline$\mu g / L$ & 0 & GE \\
\hline$\mu g / L$ & 0 & GE \\
\hline$\mu g / L$ & 0 & GE \\
\hline$\mu \mathrm{g} / \mathrm{L}$ & 0 & GE \\
\hline$\mu \mathrm{g} / \mathrm{L}$ & 0 & GE \\
\hline$\mu g / L$ & 0 & GE \\
\hline$\mu g / L$ & 0 & GE \\
\hline$\mu \mathrm{g} / \mathrm{L}$ & 1 & GE \\
\hline$\mu g / L$ & 2 & GE \\
\hline$\mu g / L$ & 0 & GE \\
\hline$\mu g / L$ & 0 & GE \\
\hline$\mu g / L$ & 0 & GE \\
\hline$\mu g / L$ & 0 & GE \\
\hline$\mu g / L$ & 0 & GE \\
\hline$\mu g / L$ & 0 & GE \\
\hline$\mu g / L$ & 0 & $\mathrm{GE}$ \\
\hline $\mathrm{pCi} / \mathrm{L}$ & 0 & $\mathrm{GE}$ \\
\hline $\mathrm{pCi} / \mathrm{L}$ & 0 & GE \\
\hline $\mathrm{pCi} / \mathrm{L}$ & 0 & $\mathbf{G}$ \\
\hline $\mathrm{pCi} / \mathrm{mL}$ & 0 & \\
\hline
\end{tabular}

\section{WELL LFW 18}

\begin{tabular}{|c|c|c|c|}
\hline SRS Coord. & Screen Zone Elevation & Top of Casing & Casing \\
\hline $\begin{array}{l}\text { N84577.3 } \\
\text { E45459.4 }\end{array}$ & $160.1-130.1 \mathrm{ft} \mathrm{msl}$ & $175.0 \mathrm{ft} \mathrm{msi}$ & 4" PVC \\
\hline
\end{tabular}

\section{MEASUREMENTS CONDUCTED IN THE FIELD}

Sample date: $10 / 24 / 91$

Depth to water: $18.98 \mathrm{ft}(5.79 \mathrm{~m})$ below TOC Water elevation: $156.02 \mathrm{ft}(47.56 \mathrm{~m}) \mathrm{msl}$

Sp. conductance: $258 \mu \mathrm{S} / \mathrm{cm}$

Water evacuated before sampling: $70 \mathrm{gal}$
Time: $14: 10$

pH: 6.4

Alkalinity: $117 \mathrm{mg} / \mathrm{L}$

Water temperature: $20.9^{\circ} \mathrm{C}$

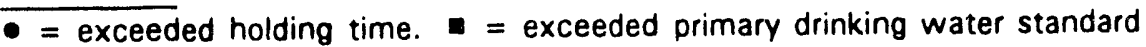




\begin{tabular}{|c|c|c|c|c|c|c|}
\hline$\underline{H} \underline{D}$ & Analyte & Result & $\underline{\text { Unit }}$ & Flag & & $\underline{\text { Lab }}$ \\
\hline$\bullet$ & $\mathrm{pH}$ & 6.2 & $\mathrm{pH}$ & 0 & & GE \\
\hline & Specific conductance & 165 & $\mu \mathrm{S} / \mathrm{cm}$ & 0 & & GE \\
\hline & Aluminum & $<20$ & $\mu_{g} / L$ & 0 & & GE \\
\hline & Antimony & $<2.0$ & $\mu g / L$ & 0 & & GE \\
\hline & Arsenic & 19 & $\mu g / L$ & 0 & & GE \\
\hline & Barium & 4.9 & $\mu g / L$ & 0 & & GE \\
\hline & Benzene & 1.5 & $\mu g / L$ & 0 & & GE \\
\hline & Bromodichloromethane & $<1.0$ & $\mu \mathrm{g} / \mathrm{L}$ & 0 & & GE \\
\hline & Bromoform & $<1.0$ & $\mu g / L$ & 0 & & GE \\
\hline & Bromomethane (Methyl bromide) & $<1.0$ & $\mu g / L$ & 0 & & GE \\
\hline & Cadmium & $<2.0$ & $\mu g / L$ & 0 & & GE \\
\hline & Calcium & 3,130 & $\mu g / L$ & 0 & & GE \\
\hline & Carbon tetrachloride & $<1.0$ & $\mu g / L$ & 0 & - & GE \\
\hline & Chloride & 10,120 & $\mu g / L$ & 0 & & GE \\
\hline & Chlorobenzene & 7.6 & $\mu g / L$ & 1 & & GE \\
\hline & Chloroethane & $<1.0$ & $\mu g / L$ & 0 & & GE \\
\hline & Cliloroethene (Vinyl chloride) & $<1.0$ & $\mu g / L$ & 0 & & GE \\
\hline & 2-Chloroethyl vinyl ether & $<1.0$ & $\mu g / L$ & 0 & & GE \\
\hline & Chloroform & $<1.0$ & $\mu \theta / L$ & 0 & & GE \\
\hline & Chloromethane (Methyl chloride) & $<1.0$ & $\mu g / L$ & 0 & & GE \\
\hline & Chromium & $<4.0$ & $\mu g / L$ & 0 & & GE \\
\hline & Copper & $<4.0$ & $\mu g / L$ & 0 & & GE \\
\hline & Dibromochloromethane & $<1.0$ & $\mu g / L$ & 0 & & GE \\
\hline & 1,1-Dichloroethane & 9.2 & $\mu g / L$ & 1 & & GE \\
\hline & 1,2-Dichloroethane & 2.0 & $\mu g / L$ & 0 & & GE \\
\hline & 1,1-Dichloroethylene & $<1.0$ & $\mu g / L$ & 0 & & GE \\
\hline & trans-1,2-Dichloroethylene & $<1.0$ & $\mu \mathrm{g} / \mathrm{L}$ & 0 & & GE \\
\hline & Dichloromethane (Methylene chloride) & 2.1 & $\mu g / L$ & 0 & & GE \\
\hline 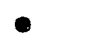 & 2,4-Dichlorophenoxyacetic acid & $<0.30$ & $\mu g / L$ & 0 & & GE \\
\hline & 1,2-Dichloropropane & $<1.0$ & $\mu g / L$ & 0 & & GE \\
\hline & trans-1,3-Dichloropropene & $<1.0$ & $\mu g / L$ & 0 & & GE \\
\hline & cis-1,3-Dichloropropene & $<1.0$ & $\mu g / L$ & 0 & & GE \\
\hline & Endrin & $<0.0060$ & $\mu g / l$. & 0 & & GE \\
\hline & Ethylbenzene & 9.6 & $\mu \phi, L$ & 0 & & GE \\
\hline & Fluoride & $<100$ & $\mu_{\mathrm{d}} / \mathrm{L}$ & 0 & & GE \\
\hline & Lead & $<3.0$ & $.19 / \mathrm{L}$ & 0 & & GE \\
\hline & Lindane & $<0.0050$ & $\mu \Omega / L$ & 0 & & GE \\
\hline & Magnesium & 5.760 & $\mu g / L$ & 0 & & GE \\
\hline & Manganese & 13 & $\mu g / L$ & 0 & & GE \\
\hline & Mercury & $<0.20$ & $\mu g / L$ & 0 & & GE \\
\hline & Methoxychlor & $<0.50$ & $\mu g / L$ & 0 & & GE \\
\hline & Nickel & $<4.0$ & $\mu g / L$ & 0 & & GE \\
\hline & Nitrate as nitrogen & $<50$ & $\mu g / L$ & 0 & & GE \\
\hline & Potassium & 1,100 & $\mu g / L$ & 0 & & GE \\
\hline & Selenium & $<2.0$ & $\mu g / t$. & 0 & & GE \\
\hline & Silica & 7,680 & $\mu g / L$ & 0 & & GE \\
\hline & Silver & $<2.0$ & $\mu g / L$ & 0 & & GE \\
\hline & Sodium & 8,010 & $\mu g / L$ & 0 & & GE \\
\hline & Sulfate & $<1,000$ & $\mu g / L$ & 0 & & GE \\
\hline & 1,1,2,2-Tetrachloroethane & $<1.0$ & $\mu g / L$ & 0 & & GE \\
\hline & Tetrachloroethylene & $<1.0$ & $\mu g / L$ & 0 & & GE \\
\hline
\end{tabular}

$\overline{- \text { = exceeded holding time. }}$ = exceeded primary drinking water standard. 
WELL LFW 18, laboratory analyses (cont.)

H $\underline{\text { Analyte }}$

Toluene

Total organic carbon

Total organic halogens

Toxaphene

- 2,4,5-TP (Silvex)

1,1,1-Trichloroethane

1,1,2-Trichloroethane

Trichloroethylene

Trichlorofluorometnane

Vanadium

Gross alpha

Nonvolatile beta

Total radium

Tritium
Result

$\begin{aligned} & 1.9 \\ & 2.000 \\ & 155 \\ < & 0.24 \\ < & 0.090 \\ < & 1.0 \\ < & 1.0 \\ < & 1.0 \\ < & 1.0 \\ < & 8.0 \\ < & 2.0 E+00 \\ & 4.6 E+00 \pm 1.6 E+00 \\ & 1.9 E+00 \pm 1.0 E+00 \\ < & 7.0 E-01\end{aligned}$

Unit $\quad \underline{\text { Flag }} \quad \underline{\text { Lab }}$

$\mu g / L \quad$ GE

$\mu g / L \quad O \quad$ GE

$\mu g / L \quad 2 \quad G E$

$\mu g / L \quad 0 \quad G E$

$\mu g / L \quad 0 \quad$ GE

$\mu g / L \quad 0 \quad$ GE

$\mu g / L \quad 0 \quad$ GE

$\mu g / L \quad 0 \quad$ GE

$\mu g / L \quad 0 \quad$ GE

$\mu \mathrm{g} / \mathrm{L} \quad 0 \quad \mathrm{GE}$

$\mathrm{pCi} / \mathrm{L} \quad \mathrm{C}$ GE

$\mathrm{pCi} / \mathrm{L} \quad \mathrm{O} \quad \mathrm{GE}$

$\mathrm{pCi} / \mathrm{L} \quad 0 \quad-\mathrm{GE}$

$\mathrm{pCi} / \mathrm{mL} \quad 0 \quad \mathrm{GE}$

\section{WELL LFW 19}

\section{SRS Coord.}

N84817.2

E45135.4

\section{Screen Zone Elevation}

$160.0-130.0 \mathrm{ft} \mathrm{mst}$
Too of Casing

$176.7 \mathrm{ft} \mathrm{msl}$

Casing

4" PVC

\section{MEASUREMENTS CONDUCTED IN THE FIELD}

Sample date: $10 / 24 / 91$

Time: $13: 50$

Inaccessibility or pump failure prevented sample collection.

\section{WELL LFW 19}

SRS Coord.

Screen Zone Elevation

160.0-130.0 ft msl

N84817.2

E45135.4

\section{MEASUREMENTS CONDUCTED IN THE FIELD}

Sample date: $12 / 16 / 9$ i

Depth to water: $20.14 \mathrm{ft}(6.14 \mathrm{~m})$ below TOC

Water elevation: $156.56 \mathrm{ft}(47.72 \mathrm{~m}) \mathrm{msl}$

Sp. conductance: $18 \mu \mathrm{S} / \mathrm{cm}$

Water evacuated before sampling: 70 gal
Top of Casing Casing

$176.7 \mathrm{ft} \mathrm{msl} \quad$ 4" PVC
Time: 15:40

pH: 5.1

Alkalinity: $0 \mathrm{mgil}$

Water temperature: $17.4^{\circ} \mathrm{C}$

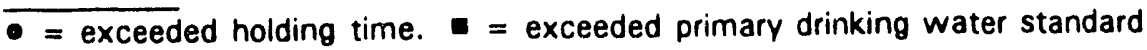


Specific conductance $\quad 20$

Aluminum $<20$

Antimony $\quad<2.0$

Arsenic $\quad<2.0$

Barium $<3.0$

Benzene

$<1.0$

Bromodichloromethane $\quad<1.0$

Bromoform $<1.0$

Bromomethane (Methyl bromide) $\quad<1.0$

Cadmium $<2.0$

Calcium $\quad 374$

Carbon tetrachloride $\quad<1.0$

Chloride

2,230

Chlorobenzene

$<1.0$

Chloroethane

$<1.0$

Chloroethene (Vinyl chloride)

$<1.0$

2-Chloroethyl vinyl ether

Chloroform

$<1.0$

$<1.0$

Chloromethane (Methyl chloride)

$<1.0$

Chromium

$<4.0$

Copper

Dibromochloromethane $<1.0$

1,1-Dichloroethane $\quad<1.0$

1,2-Dichloroethane

$<1.0$

1,1-Dichloroethylene

$<1.0$

trans-1,2-Dichloroethylene

Dichloromethane (Methylene chloride)

$<1.0$

2,4-Dichlorophenoxyacetic acid

1.6

$<0.30$

1,2-Dichloropropane

$<1.0$

trans-1,3-Dichloropropene

$<1.0$

cis-1,3-Dichloropropene

$<1.0$

Endrin

$<0.0060$

$\mathrm{pH} \quad \mathrm{O} \quad \mathrm{GE}$

Ethylbenzene

$<1.0$

Fluoride

Lead

$<100$

7.4

Lindane

Magnesium

$<0.0050$

Manganese

441

Mercury

3.6

$<0.20$

Methoxychlor

$<0.50$

Nickel

4.0

Nitrate as nitrogen $\quad 380$

Potassium

$<500$

Selenium

$<2.0$

Silica

Silver

$<2.0$

Sodium

1,150

Sulfate

$<1,000$

1,1,2,2-Tetrachloroethane

$<1.0$

Tetrachloroethylene

$<1.0$

$\begin{array}{lll}\mu \mathrm{S} / \mathrm{cm} & 0 & \mathrm{GE} \\ \mu \mathrm{g} / \mathrm{L} & 0 & \mathrm{GE}\end{array}$

$\begin{array}{lll}\mu g / L & 0 & \text { GE } \\ \mu g / L & 0\end{array}$

$\mu g / L \quad 0 \quad$ GE

$\mu g / L \quad 0 \quad$ GE

$u g / L \quad 0 \quad$ GE

$\mu g / L \quad 0 \quad$ GE

$\mu g / L \quad O \quad$ GE

$\mu g / L \quad 0 \quad$ GE

$\mu g / L \quad O \quad$ GE

$\mu g / L \quad 0 \quad$ GE

$\mu g / L \quad 0 \quad-$ GE

$\mu g / L \quad 0 \quad$ GE

$\mu g / L \quad 0 \quad G E$

$\mu g / L \quad 0 \quad$ GE

$\mu \mathrm{g} / \mathrm{L} \quad 0 \quad \mathrm{GE}$

$\mu g / L \quad 0 \quad$ GE

$\mu g / L \quad 0 \quad$ GE

$\mu g / L \quad 0 \quad$ GE

$\mu g / L \quad 0 \quad$ GE

$\mu g / L \quad 0 \quad$ GE

$\mu g / L \quad O \quad$ GE

$\mu g / L \quad O \quad$ GE

$\mu g / L \quad O \quad$ GE

$\mu g / L \quad 0 \quad$ GE

$\mu g / L \quad 0 \quad$ GE

$\mu g / L \quad O \quad G E$

$\mu g / L \quad 0 \quad$ GE

$\mu g / L \quad 0 \quad$ GE

$\mu g / L \quad 0 \quad$ GE

$\mu g / L \quad 0 \quad$ GE

$\mu g / L \quad 0 \quad$ GE

$\mu g / L \quad 0 \quad$ GE

$\mu g / L \quad 0 \quad$ GE

$\mu g / L \quad 0 \quad$ GE

$\mu g / L \quad 0 \quad$ GE

$\mu g / L \quad 0 \quad$ GE

$\mu g / L \quad 0 \quad$ GE

$\mu \mathrm{g} / \mathrm{L} \quad 0 \quad \mathrm{GE}$

$\mu \mathrm{g} / \mathrm{L} \quad \mathrm{O} \quad \mathrm{GE}$

$\mu g / L \quad 0 \quad$ GE

$\mu g / L \quad 0 \quad$ GE

$\mu g / L \quad 0 \quad$ GE

$\mu g / L \quad 0 \quad$ GE

$\mu g / L \quad 0 \quad G E$

$\mu g / L \quad 0 \quad$ GE

$\mu^{\prime} \mathrm{g} / \mathrm{L} \quad 0 \quad \mathrm{GE}$

$\mu g / L \quad 0 \quad$ GE

$\mu g / L \quad 0 \quad$ GE

$\mu \mathrm{g} / \mathrm{L} \quad \mathrm{O}$ GE

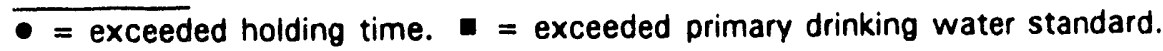


WELL LFW 19, laboratory analyses (cont.)

H $D \quad$ Analyte
Toluene
Total organic carbon
Total organic halogens
Toxaphene
$2,4,5-T P$ (Silvex)
$1,1,1$-Trichloroethane
$1,1,2-$ Trichloroethane
Trichloroethylene
Trichlorofluoromethane
Vanadium
Gross alpha
Nonvolatile beta
Total radium
Tritium

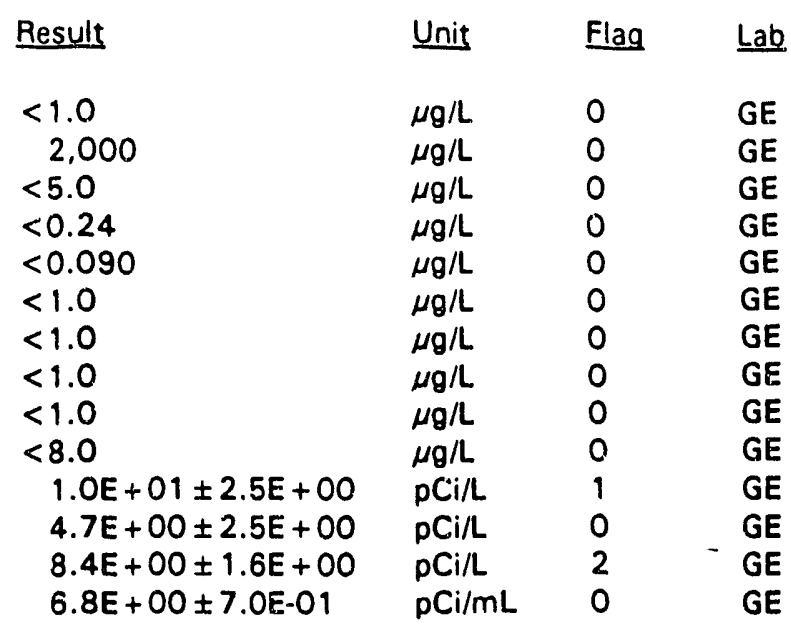

GE

GE

GE

GE

GE

GE

GE

GE

GE

GE

WELL LFW 20

SRS Coord.

N85262.6

E45582.9

\section{Screen Zone Elevation}

$165.0-135.0 \mathrm{ft} \mathrm{ms}$
Top of Casing

$180.5 \mathrm{ft} \mathrm{msl}$

\section{Casing}

4" PVC

MEASUREMENTS CONDUCTED IN THE FIELD

Sample date: $10 / 24 / 91$

Depth to water: $19.70 \mathrm{ft}(6.00 \mathrm{~m})$ below TOC Water elevation: $160.80 \mathrm{ft}(49.01 \mathrm{~m}) \mathrm{msl}$

Sp. conductance: $16 \mu \mathrm{S} / \mathrm{cm}$

Water evacuated before sampling: $70 \mathrm{gal}$

\section{LABORATORY ANALYSES}

H D Analyte

- $\mathrm{pH}$

Specific conductance

Aluminum

Antimony

Arsenic

Barium

Benzene

Bromodichloromethane

Bromoform

Bromomethane (Methyl bromide)

Cadmium

Calcium

Carbon tetrachloride

Chloride

Chlorobenzene

Chloroethane
Result

$\begin{aligned} & 5.2 \\ & 20 \\ < & 20 \\ < & 2.0 \\ < & 2.0 \\ & 3.9 \\ < & 1.0 \\ < & 1.0 \\ < & 1.0 \\ < & 1.0 \\ < & 2.0 \\ & 190 \\ < & 1.0 \\ & 3.060 \\ < & 1.0 \\ < & 1.0\end{aligned}$

Time: $13: 30$

pH: 4.8

Alkalinity: $0 \mathrm{mg} / \mathrm{L}$

Water temperature: $21.1^{\circ} \mathrm{C}$ 
WELL LFW 20, laboratory analyses (cont.)

H D Analyte

Chloroethene (Vinyl chloride)

2-Chloroethyl vinyl ether

Chloroform

Chloromethane (Methyl chloride)

Chromium

Copper

Dibromochloromethane

1.1-Dichloroethane

1,2-Dicinloroethane

1.1-Dichloroethylene

trans-1,2-Dichloroethylene

Dichloromethane (Methylene chloride)

2,4-Dichlorophenoxyacetic acid

1.2-Dichloropropane

trans-1,3-Dichloropropene

cis-1,3-Dichloropropene

Endrin

Ethylbenzene

Fluoride

Lead

Lindane

Magnesium

Manganese

Mercury

Methoxychlor

Nickel

Nitrate as nitrogen

Potassium

Selenium

Silica

Silver

Sodium

Sulfate

1,1,2,2-Tetrachloroethane

Tetrachloroethylene

Toluene

Total organic carbon

Total organic halogens

Toxaphene

2,4,5-TP (Silvex)

1,1,1-Trichloroethane

1,1,2-Trichloroethane

Trichloroethylene

Trichlorofluorornethane

Vanadium

Gross alpha

Nonvolatile beta

- Total radium

Tritium
Results

$<1.0$

$<1.0$

$<1.0$

$<1.0$

$<4.0$

11

$<1.0$

$<1.0$

$<1.0$

$<1.0$

$<1.0$

$<1.0$

$<0.30$

$<1.0$

$<1.0$

$<1.0$

$<0.0060$

$<1.0$

$<100$

5.7

$<0.0050$

293

2.6

$<0.20$

$<0.50$

11

300

$<500$

$<2.0$

5.670

$<2.0$

1,250

$<1,000$

$<1.0$

$<1.0$

$<1.0$

$<1,000$

66

$<0.24$

$<0.090$

$<1.0$

$<1.0$

$<1.0$

$<1.0$

$<8.0$

$<2.0 E+00$

$2.9 E+00 \pm 1.5 E+00$

$1.4 E+01 \pm 3.9 E+00$

$<7.0 E-01$

\begin{tabular}{|c|c|c|}
\hline Unit & Flag & Lab \\
\hline$\mu g / L$ & 0 & GE \\
\hline$\mu g / L$ & 0 & GE \\
\hline$\mu g / L$ & 0 & GE \\
\hline$\mu g / L$ & 0 & GE \\
\hline$\mu \mathrm{g} / \mathrm{L}$ & 0 & GE \\
\hline$\mu g / L$ & 0 & GE \\
\hline$\mu g / L$ & 0 & GE \\
\hline$\mu g / L$ & 0 & GE \\
\hline$\mu g / L$ & 0 & GE \\
\hline$\mu g / L$ & 0 & GE \\
\hline$\mu g / L$ & 0 & GE \\
\hline$\mu g / L$ & 0 & GE \\
\hline$\mu g / L$ & 0 & GE \\
\hline$\mu g / L$ & 0 & GE \\
\hline$\mu g / L$ & 0 & GE \\
\hline$\mu g / L$ & 0 & GE \\
\hline$\mu \mathrm{g} / \mathrm{L}$ & 0 & GE \\
\hline$\mu g / L$ & 0 & GE \\
\hline$\mu g / L$ & 0 & GE \\
\hline$\mu g / L$ & 0 & GE \\
\hline$\mu g / L$ & 0 & GE \\
\hline$\mu g / L$ & 0 & GE \\
\hline$\mu g / L$ & 0 & GE \\
\hline$\mu g / L$ & 0 & GE \\
\hline$\mu g / L$ & 0 & GE \\
\hline$\mu g / L$ & 0 & GE \\
\hline$\mu g / L$ & 0 & GE \\
\hline$\mu g / L$ & 0 & GE \\
\hline$\mu g / L$ & 0 & GE \\
\hline$\mu g \Omega$ & 0 & GE \\
\hline$\mu g / L$ & 0 & GE \\
\hline$\mu g / L$ & 0 & GE \\
\hline$\mu g / L$ & 0 & GE \\
\hline$\mu g / L$ & 0 & GE \\
\hline$\mu g / L$ & 0 & GE \\
\hline$\mu g / L$ & 0 & GE \\
\hline$\mu g / L$ & 0 & GE \\
\hline$\mu g / L$ & 2 & GE \\
\hline$\mu g / L$ & 0 & GE \\
\hline$\mu g / L$ & 0 & GE \\
\hline$\mu g / L$ & 0 & GE \\
\hline$\mu g / L$ & 0 & GE \\
\hline$\mu g / L$ & 0 & GE \\
\hline$\mu g / L$ & 0 & GE \\
\hline$\mu g / L$ & 0 & GE \\
\hline pCi/L & 0 & GE \\
\hline pCi/L & 0 & $\mathrm{GE}$ \\
\hline $\mathrm{pCi} / \mathrm{L}$ & 2 & GE \\
\hline & & \\
\hline
\end{tabular}

$\overline{- \text { = exceeded holding time. }}-$ = exceeded primary drinking water standard. 
WELL LFW 21

SAS Coord.

N84178.3

E46149.4

MEASUREMENTS CONDUCTED IN THE FIELD

Sample date: 10/25/91

Depth to water: $23.78 \mathrm{ft}(7.25 \mathrm{~m})$ below TOC

Water elevation: $151.82 \mathrm{ft}(46.28 \mathrm{~m}) \mathrm{msl}$

Sp. conductance: $522 \mu \mathrm{S} / \mathrm{cm}$

Water evacuated before sampling: $74 \mathrm{gal}$

\section{LABORATOAY ANALYSES}

H D Analyte

- $\quad \mathrm{pH}$

Specific conductance

Aluminum

Antimony

Arsenic

Barium

Benzene

Bromodichloromethane

Bromoform

Bromomethane (Methyl bromide)

Cadmium

Calcium

Carbon tetrachloride

Chloride

Chlorobenzene

Chloroethane

Chloroethene (Vinyl chloride)

2-Chloroethyl vinyl ether

Chloroform

Chloromethane (Methyl chloride)

Chromium

Copper

Dibromochloromethane

1,1-Dichloroethane

1,2-Dichloroethane

1,1-Dichloroethylene

trans-1,2-Dichloroethylene

Dichloromethane (Methylene chloride)

2,4-Dichlorophenoxyacetic acid

1,2-Dichloropropane

trans-1,3-Dichloropropene

cis-1,3-Dichloropropene

Endrin

Endrin

Ethylbenzene

Fluoride
Result

6.1

360

$<20$

$<2.0$

3.4

8.6

5.6

$<1.0$

$<1.0$

$<1.0$

$<2.0$

10,100

$<1.0$

15,400

1.2

$<1.0$

$<1.0$

$<1.0$

$<1.0$

$<1.0$

$<4.0$

$<4.0$

$<1.0$

82

$<1.0$

3.9

$<1.0$

633

$<0.30$

$<1.0$

$<1.0$

$<1.0$

$<0.0060$

$<0.0060$

23

230
Top of Casing Casing

$175.6 \mathrm{ft} \mathrm{msl} \quad 4^{n}$ PVC
Time: $12: 30$

$\mathrm{pH}: 6.3$

Alkalinity: $216 \mathrm{mg} / \mathrm{L}$

Water temperature: $21.3^{\circ} \mathrm{C}$
Unit Flag Lab

pH 0 GE

$\mu S / c m \quad$ GE

$\mu \mathrm{g} / \mathrm{L} \quad 0 \quad \mathrm{GE}$

$\mu g /$ L $\quad 0 \quad$ GE

$\mu g / L \quad 0 \quad$ GE

$\mu g / L \quad 0 \quad G E$

$\mu g / L \quad 2 \quad$ GE

$\mu g / L \quad 0 \quad$ GE

$\mu g /$ $\quad 0 \quad$ GE

$\mu g / L \quad 0 \quad$ GE

$\mu g / L \quad 0 \quad$ GE

$\mu g / L \quad 0 \quad$ GE

$\mu g / L \quad 0 \quad$ GE

$\mu g \Omega \quad 0 \quad$ GE

$\mu g /$ L $\quad 0 \quad$ GE

$\mu g / \quad 0 \quad$ GE

$\mu g / L \quad 0 \quad$ GE

$\mu g / L \quad 0 \quad$ GE

$\mu g / L \quad 0 \quad$ GE

$\mu g / L \quad 0 \quad$ GE

$\mu g / 2 \quad 0 \quad$ GE

$\mu g / L \quad 0 \quad$ GE

$\mu g / \mathrm{L} \quad 0 \quad \mathrm{GE}$

$\mu g / L \quad 2 \quad$ GE

$\mu g / L \quad 0 \quad$ GE

$\mu g / L \quad 1 \quad$ GE

$\mu g / L \quad 0 \quad$ GE

$\mu g / L \quad 0 \quad$ GE

$\mu g / L \quad 0 \quad$ GE

$\mu g / L \quad 0 \quad$ GE

$\mu g / \mathrm{L} \quad 0 \quad \mathrm{GE}$

$\mu g / L \quad O \quad$ GE

$\mu g / L \quad 0 \quad$ GE

$\mu g / L \quad 0 \quad$ GE

$\mu g / L \quad 0 \quad$ GE

$\mu g /$ GE

- = exceeded holding time. = exceeded primary drinking water standard 
WELL LFW 21, laboratory analyses (cont.)

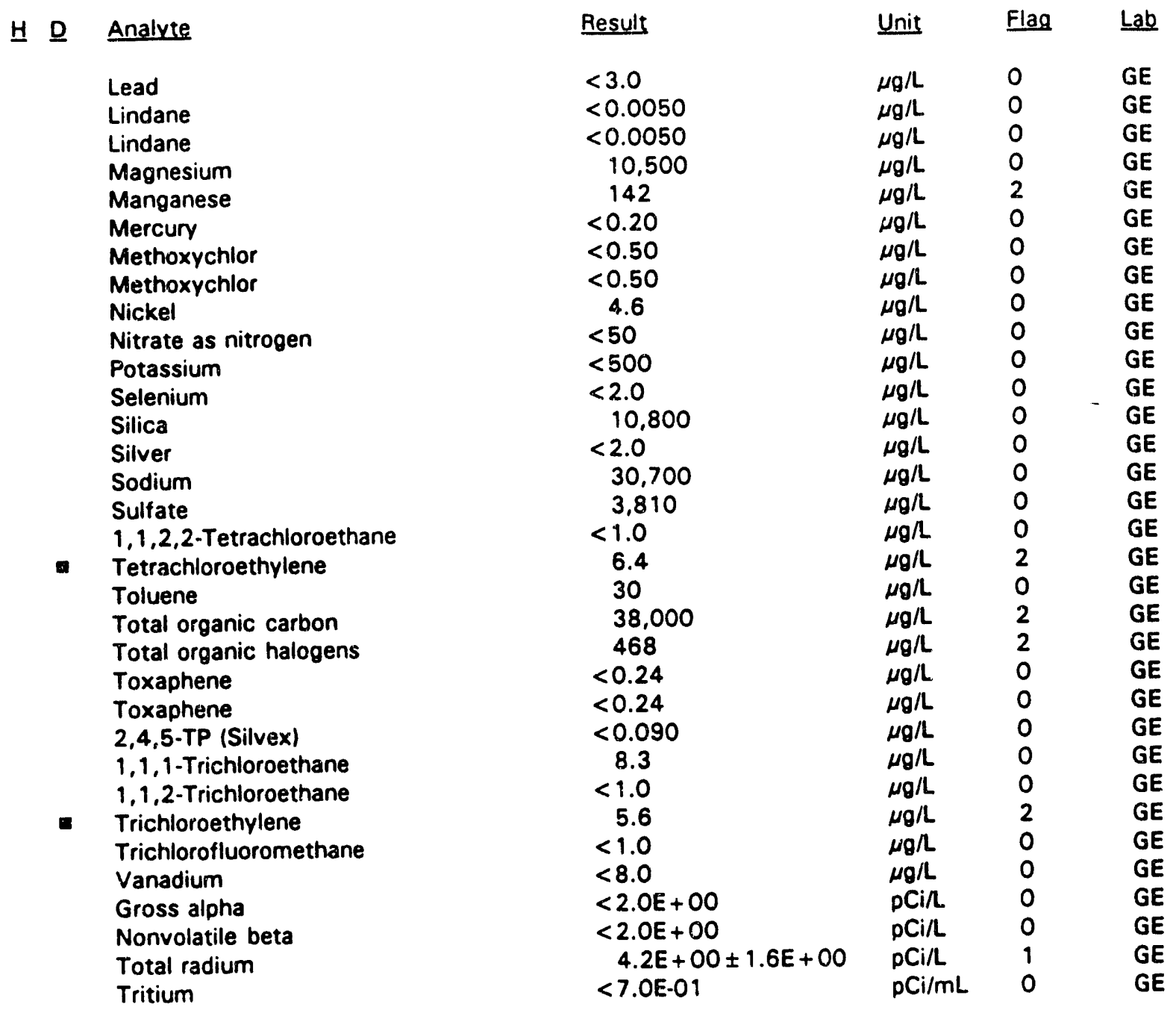

\section{WELL LFW 22}

SRS Coord.

N84223.6

E46325.2

\section{Screen Zone Elevation}

$152.4-122.4 \mathrm{ft} \mathrm{msl}$
Too of Casing

$174.2 \mathrm{ft} \mathrm{msl}$
Casing

4" PVC

\section{MEASUREMENTS CONDUCTED IN THE FIELD}

Sample date: 10/25/91

Depth to water: $21.80 \mathrm{ft}(6.64 \mathrm{~m})$ below TOC

Water elevation: $152.40 \mathrm{ft}(46.45 \mathrm{~m}) \mathrm{msl}$

Sp. conductance: $434 \mu \mathrm{S} / \mathrm{cm}$

Water evacuated before sampling: $80 \mathrm{gal}$
Time: 11:55

$\mathrm{pH}: 6.3$

Alkalinity: $153 \mathrm{mg} / \mathrm{L}$

Water temperature: $20.9^{\circ} \mathrm{C}$

- = exceeded holding time. $=$ exceeded primary drinking water standard. 


\begin{tabular}{|c|c|c|c|c|c|}
\hline$\underline{H} \underline{\mathrm{D}}$ & Analyte & Result & Unit & Flag & $\underline{\text { Lab }}$ \\
\hline - & $\mathrm{pH}$ & 5.7 & $\mathrm{pH}$ & 0 & GE \\
\hline & Specific conductance & 230 & $\mu \mathrm{S} / \mathrm{cm}$ & 0 & GE \\
\hline & Aluminum & $<20$ & $\mu g / L$ & 0 & GE \\
\hline & $\begin{array}{l}\text { Antimony } \\
\text { Arsenic }\end{array}$ & $<2.0$ & $\mu g / L$ & 0 & GE \\
\hline & $\begin{array}{l}\text { Arsenic } \\
\text { Barium }\end{array}$ & $\begin{array}{l}4.3 \\
5.5\end{array}$ & $\mu g / L$ & 0 & $\begin{array}{l}\text { GE } \\
\text { GE }\end{array}$ \\
\hline & $\begin{array}{l}\text { Barium } \\
\text { Benzene }\end{array}$ & $\begin{array}{l}5.5 \\
2.3\end{array}$ & $\mu g / L$ & 0 & $\begin{array}{l}\text { GE } \\
\text { GE }\end{array}$ \\
\hline & Bromodichloromethane & $\begin{array}{r}2.3 \\
<1.0\end{array}$ & $\begin{array}{l}\mu g / L \\
\mu g / L\end{array}$ & 0 & $\begin{array}{l}\text { GE } \\
\text { GE }\end{array}$ \\
\hline & Bromoform & $<1.0$ & $\mu g / L$ & 0 & $\begin{array}{l}\text { GE } \\
\text { GE }\end{array}$ \\
\hline & Bromomethane (Methyl bromide) & $<1.0$ & $\mu g / L$ & 0 & $\begin{array}{l}\text { GE } \\
\text { GE }\end{array}$ \\
\hline & Cadmium & $<2.0$ & $\mu g / L$ & 0 & $\begin{array}{l}\text { GE } \\
\text { GE }\end{array}$ \\
\hline & Calcium & 3,040 & $\mu g / L$ & 0 & GE \\
\hline & Carbon tetrachloride & $<1.0$ & $\mu g / L$ & 0 & $-\mathrm{GE}$ \\
\hline & Chloride & 17,700 & $\mu g / L$ & 0 & GE \\
\hline & Chlorobenzene & $<1.0$ & $\mu g / L$ & 0 & GE \\
\hline & Chloroethane & $<1.0$ & $\mu g / L$ & 0 & GE \\
\hline & Chloroethene (Vinyl chloride) & $<1.0$ & $\mu g / L$ & 0 & GE \\
\hline & 2-Chloroethyl vinyl ether & $<1.0$ & $\mu g / L$ & 0 & GE \\
\hline & $\begin{array}{l}\text { Chloroform } \\
\text { Chloromethane (Methyl chloride) }\end{array}$ & $\begin{array}{l}<1.0 \\
<1.0\end{array}$ & $\mu g / L$ & $\begin{array}{l}0 \\
0\end{array}$ & GE \\
\hline & & $\begin{array}{l}<1.0 \\
<4.0\end{array}$ & $\begin{array}{l}\mu g / L \\
\mu \rho / L\end{array}$ & $\begin{array}{l}0 \\
0\end{array}$ & $\begin{array}{l}\text { GE } \\
\text { GE }\end{array}$ \\
\hline & Copper & $<4.0$ & $\mu g / L$ & 0 & $\begin{array}{l}\text { GE } \\
\text { GE }\end{array}$ \\
\hline & Dibromochloromethane & $<1.0$ & $\mu g / L$ & 0 & GE \\
\hline & 1,1-Dichloroethane & 64 & $\mu g / L$ & 2 & GE \\
\hline & 1,2-Dichloroethane & $<1.0$ & $\mu g / L$ & 0 & GE \\
\hline & 1.1-Dichloroethylene & 4.6 & $\mu g / \mathrm{L}$ & 1 & GE \\
\hline & & $<1.0$ & $\mu g / L$ & $\begin{array}{l}0 \\
0\end{array}$ & GE \\
\hline & $\begin{array}{l}\text { Dichloromethane (Methylene chloride) } \\
\text { 2,4-Dichlorophenoxyacetic acid }\end{array}$ & $\begin{aligned} & 103 \\
< & 0.30\end{aligned}$ & $\mu g / L$ & $\begin{array}{l}0 \\
0\end{array}$ & $\begin{array}{l}\text { GE } \\
\text { GE }\end{array}$ \\
\hline & & & $\mu g /$ & 0 & $\begin{array}{l}\text { GE } \\
\text { GE }\end{array}$ \\
\hline & trans-1,3-Dichloropropene & $<1.0$ & $\mu g / L$ & 0 & GE \\
\hline & cis-1,3-Dichloropropene & $<1.0$ & $\mu g / L$ & 0 & GE \\
\hline & Endrin & $<0.0060$ & $\mu g / L$ & 0 & GE \\
\hline & Endrin & $<0.0060$ & $\mu g / L$ & 0 & GE \\
\hline & Ethylbenzene & 39 & $\mu g / L$ & 0 & GE \\
\hline & Fluoride & 149 & $\mu g / L$ & 0 & GE \\
\hline & Fluoride & 148 & $\mu g / L$ & 0 & GE \\
\hline & Lead & $<3.0$ & $\mu g / L$ & 0 & GE \\
\hline & Lindane & $<0.0050$ & $\mu g /$ & 0 & GE \\
\hline & Lindane & $<0.0050$ & $\mu g / L$ & 0 & GE \\
\hline & Magnesium & 5,760 & $\mu g / L$ & 0 & GE \\
\hline & Manganese & 31 & $\mu g /$ & 1 & GE \\
\hline & Mercury & 0.22 & $\mu g / L$ & 0 & GE \\
\hline & Methoxychlor & $<0.50$ & $\mu g / L$ & 0 & GE \\
\hline & Methoxychlor & $<0.50$ & $\mu g / L$ & 0 & GE \\
\hline & Nickel & $<4.0$ & $\mu g / L$ & 0 & GE \\
\hline & Nitrate as nitrogen & 100 & $\mu g / L$ & 0 & GE \\
\hline & Potassium & $<500$ & $\mu g / L$ & 0 & GE \\
\hline & Selenium & $<2.0$ & $\mu g / L$ & 0 & GE \\
\hline & Silica & 7,530 & $\mu g /$ & 0 & GE \\
\hline & Silver & $<2.0$ & $\mu g \Omega$ & 0 & GE \\
\hline
\end{tabular}

- = exceeded holding time. - = exceeded primary drinking water standard 
WELL LFW 22, laboratory analyses (cont.)

H $\mathrm{D} \quad$ Analute
Sodium
Sulfate
1,1,2,2-Tetrachloroethane
Tetrachloroethylene
Toluene
Total organic carbon
Total organic halogens
Toxaphene
Toxaphene
2,4,5-TP (Silvex)
1,1,1-Trichloroethane
1,1,2-Trichloroethane
Trichloroethylene
Trichlorofluoromethane
Vanadium
Gross alpha
Nonvolatile beta
Total radium
Tritium

Result

9,950
3,260
$<1.0$
2.1
74
50,000
461
$<0.24$
$<0.24$
$<0.090$
11
$<1.0$
12
102
$<8.0$
$4.4 E+00 \pm 1.9 E+00$
$<2.0 E+00$
$<1.0 E+00$
$<7.0 E-01$

\begin{tabular}{|c|c|c|}
\hline Unit & Flag & Lab \\
\hline$\mu g / L$ & 0 & GE \\
\hline$\mu g / L$ & 0 & GE \\
\hline$\mu \mathrm{g} / \mathrm{L}$ & 0 & GE \\
\hline$\mu g / L$ & 0 & GE \\
\hline$\mu g / L$ & 0 & GE \\
\hline$\mu g / L$ & 2 & SE \\
\hline$\mu g / L$ & 2 & GE \\
\hline$\mu g / L$ & 0 & GE \\
\hline$\mu g / L$ & 0 & GE \\
\hline$\mu g / L$ & 0 & GE \\
\hline$\mu g / L$ & 0 & GE \\
\hline$\mu g / L$ & 0 & GE \\
\hline$\mu g / L$ & 2 & GE \\
\hline$\mu g / L$ & 2 & GE \\
\hline$\mu g / L$ & 0 & GE \\
\hline $\mathrm{pCi} / \mathrm{L}$ & 0 & G \\
\hline $\mathrm{pCi} / \mathrm{L}$ & 0 & G \\
\hline $\mathrm{pCi} / \mathrm{L}$ & 0 & \\
\hline $\mathrm{pCi} / \mathrm{mL}$ & 0 & \\
\hline
\end{tabular}

\section{WELL LFW 23}

\section{SRS Coord.}

N84251.3

E46456.1

\section{MEASUREMENTS CONDUCTED IN THE FIELD}

Sample date: 10/25/91

Depth to water: $19.16 \mathrm{ft}(5.84 \mathrm{~m})$ below TOC Water elevation: $152.64 \mathrm{ft}(46.53 \mathrm{~m}) \mathrm{msl}$ Sp. conductance: $21 \mu \mathrm{S} / \mathrm{cm}$ Water evacuated before sampling: $73 \mathrm{gal}$

\section{LABORATORY ANALYSES}

H D Analyte

- $\quad \mathrm{pH}$

Specific conductance

Aluminum

Antimony

Arsenic

Barium

Benzene

Bromodichloromethane

Bromoform

Bromomethane (Methyl bramide)

Cadmium

\section{Screen Zone Elevation}

$155.1-125.1 \mathrm{ft} \mathrm{msl}$
Top of Casing

$171.8 \mathrm{ft} \mathrm{msl}$
Casing

4" PVC
Time: $11: 30$

pH: 4.6

Alkalinity: $0 \mathrm{mg} / \mathrm{L}$

Water temperature: $20.8^{\circ} \mathrm{C}$

\begin{tabular}{|c|c|c|}
\hline Reșult & Unit & Flag \\
\hline 5.0 & $\mathrm{pH}$ & 0 \\
\hline 20 & $\mu \mathrm{S} / \mathrm{cm}$ & 0 \\
\hline$<20$ & $\mu g / L$ & 0 \\
\hline$<2.0$ & $\mu g / L$ & 0 \\
\hline$<2.0$ & $\mu g / L$ & 0 \\
\hline 4.1 & $\mu g / L$ & 0 \\
\hline$<1.0$ & $\mu g / L$ & 0 \\
\hline$<1.0$ & $\mu g / L$ & 0 \\
\hline$<1.0$ & $\mu g / L$ & 0 \\
\hline$<1.0$ & $\mu g /$ & 0 \\
\hline$<2.0$ & $\mu g / L$ & 0 \\
\hline
\end{tabular}

$\overline{0}=$ exceeded holding time.

w = exceeded primary drinking water standard. 
WELL LFW $2 \Im$ i. laboratory analyses (cont.)

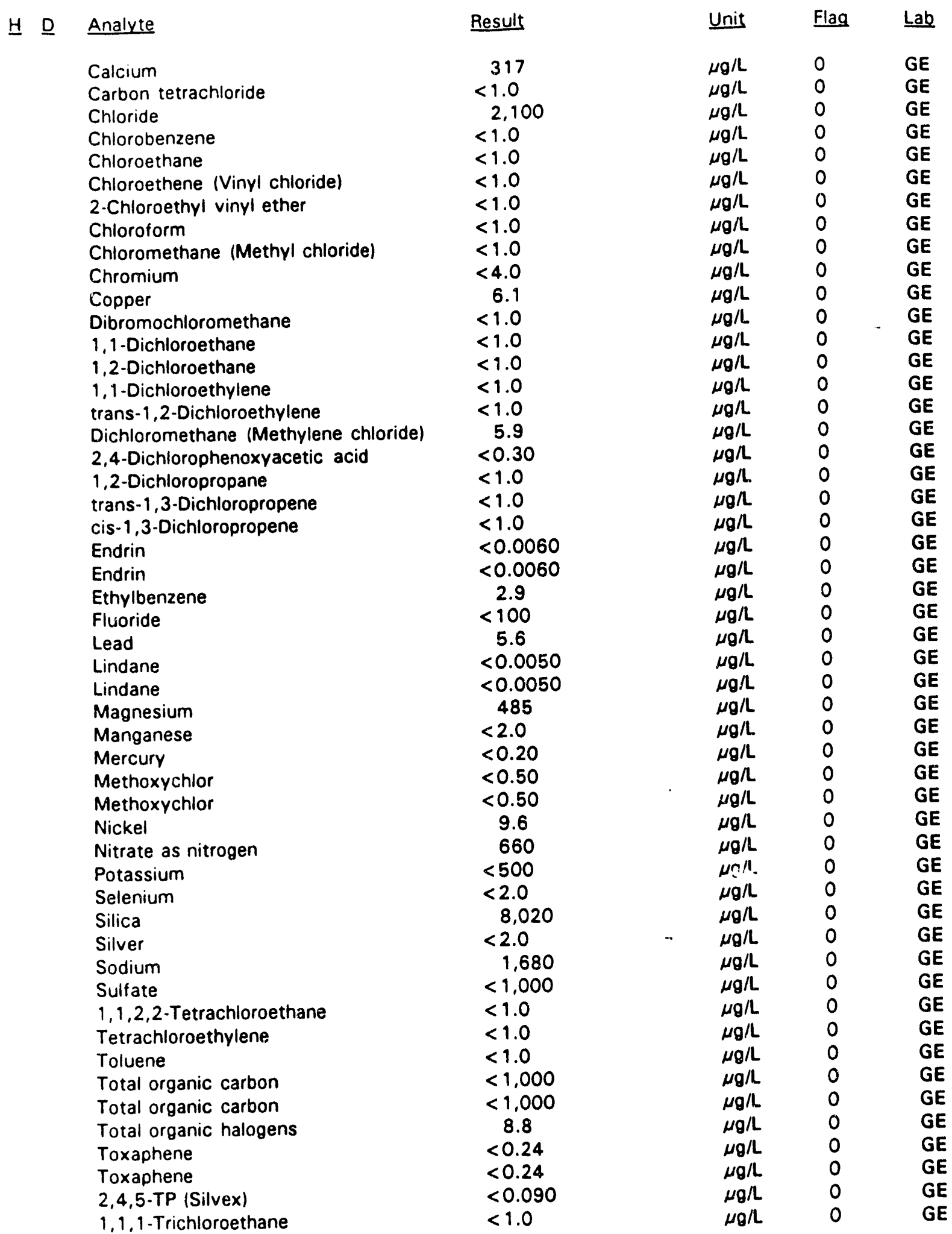

$\overline{- \text { = exceeded holding time. }}$ = exceeded primary drinking water standard 
WELL LFW 23, laboratory analyses (cont.)

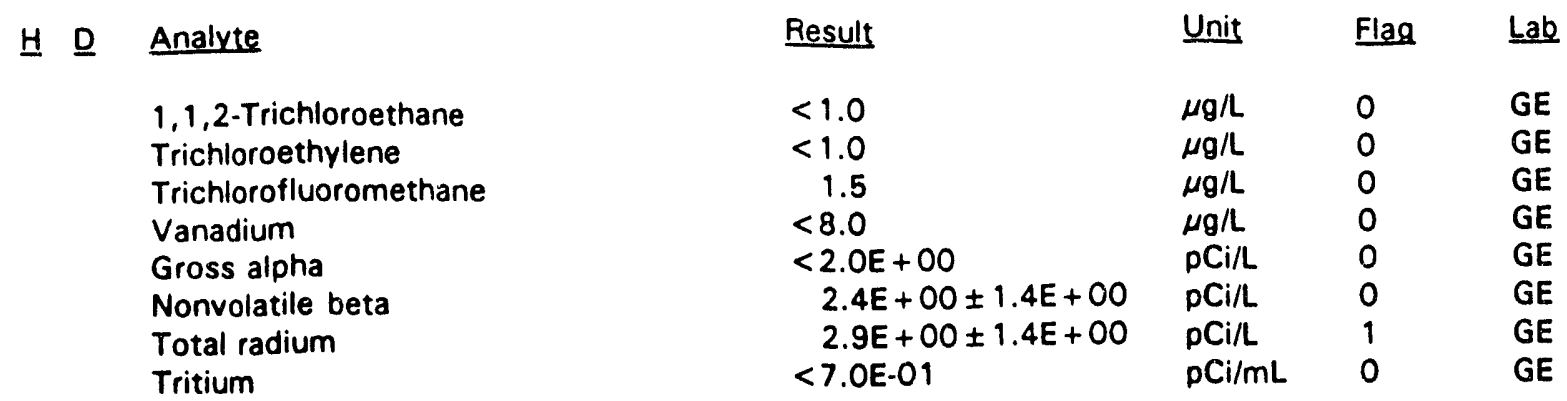

\section{WELL. LFW 24}

SRS Coord.

N84544.2

E46520.8

\section{MEASUREMENTS CONDUCTED IN THE FIELD}

Sample date: $10 / 25 / 91$

Depth to water: $15.99 \mathrm{ft}(4.87 \mathrm{~m})$ below TOC

Water elevation: $155.31 \mathrm{ft}(47.34 \mathrm{~m}) \mathrm{msl}$

Sp. conductance: $17 \mu \mathrm{S} / \mathrm{cm}$

Water evacuated before sampling: $82 \mathrm{gal}$

\section{LABORATORY ANALYSES}

\section{H D Analyte}

๑

pH
Specific conductance
Aluminum
Antimony
Arsenic
Barium
Benzene
Bromodichloromethane
Bromoform
Bromomethane (Methyl bromide)
Cadmium
Calcium
Carbon tetrachloride
Chloride
Chlorobenzene
Chloroethane
Chloroethene (Vinyl chloride)
2 Chloroethyl vinyl ether
Chloroform
Chloromethane (Methyl chloride)
Chromium
Copper

Screen Zone Elevation

$154.5-124.5 \mathrm{ft} \mathrm{msl}$
Toe of Casing

$171.3 \mathrm{ft} \mathrm{msl}$
Casing

4" PVC
Time: 11:00

pH: 4.6

Alkalinity: $0 \mathrm{mg} / \mathrm{L}$

Water temperature: $19.7^{\circ} \mathrm{C}$

$\begin{array}{clll}\text { Result } & \text { Unit } & \text { Flag } & \text { Lab } \\ & & & \\ 4.9 & \mathrm{pH} & 0 & \mathrm{GE} \\ 20 & \mu \mathrm{S} / \mathrm{cm} & 0 & \mathrm{GE} \\ <20 & \mu \mathrm{g} / \mathrm{L} & 0 & \mathrm{GE} \\ <2.0 & \mu \mathrm{g} / \mathrm{L} & 0 & \mathrm{GE} \\ <2.0 & \mu \mathrm{g} / \mathrm{L} & 0 & \mathrm{GE} \\ 5.5 & \mu \mathrm{g} / \mathrm{L} & 0 & \mathrm{GE} \\ <1.0 & \mu \mathrm{g} / \mathrm{L} & 0 & \mathrm{GE} \\ <1.0 & \mu \mathrm{g} / \mathrm{L} & 0 & \mathrm{GE} \\ <1.0 & \mu \mathrm{g} / \mathrm{L} & 0 & \mathrm{GE} \\ <1.0 & \mu \mathrm{g} / \mathrm{L} & 0 & \mathrm{GE} \\ <2.0 & \mu \mathrm{g} / \mathrm{L} & 0 & \mathrm{GE} \\ 317 & \mu \mathrm{g} / \mathrm{L} & 0 & \mathrm{GE} \\ <1.0 & \mu \mathrm{g} / \mathrm{L} & 0 & \mathrm{GE} \\ 2.440 & \mu \mathrm{g} / \mathrm{L} & 0 & \mathrm{GE} \\ <1.0 & \mu \mathrm{g} / \mathrm{L} & 0 & \mathrm{GE} \\ <1.0 & \mu \mathrm{g} / \mathrm{L} & 0 & \mathrm{GE} \\ <1.0 & \mu \mathrm{g} / \mathrm{L} & 0 & \mathrm{GE} \\ <1.0 & \mu \mathrm{g} / \mathrm{L} & 0 & \mathrm{GE} \\ <1.0 & \mu \mathrm{g} / \mathrm{L} & 0 & \mathrm{GE} \\ <1.0 & \mu \mathrm{g} / \mathrm{L} & 0 & \mathrm{GE} \\ <4.0 & \mu \mathrm{g} / \mathrm{L} & 0 & \mathrm{GE} \\ 7.6 & \mu \mathrm{g} / \mathrm{L} & 0 & \mathrm{GE} \\ & & & \end{array}$

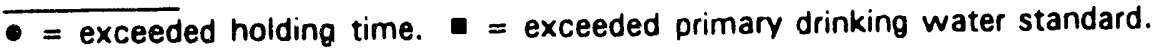


WELL LFW 24, laboratory analyses (cont.)

\begin{tabular}{|c|c|c|c|c|c|c|}
\hline$\underline{H} \underline{D}$ & Analvte & Result & Unit & Flag & & $\underline{L a b}$ \\
\hline & Dibromochioromethane & $<1.0$ & $\mu g / L$ & 0 & & GE \\
\hline & 1,1-Dichloroethane & $<1.0$ & $\mu g / L$ & 0 & & GE \\
\hline & 1,2-Dichloroethane & $<1.0$ & $\mu \mathrm{g} / \mathrm{L}$ & 0 & & GE \\
\hline & 1,1 -Dichloroethylene & $<1.0$ & $\mu g / L$ & 0 & & GE \\
\hline & trans-1,2-Dichloroethylene & $<1.0$ & $\mu g / L$ & 0 & & GE \\
\hline & Dichloromethane (Methylene chloride) & 3.3 & $\mu g / L$ & 0 & & GE \\
\hline & 2,4-Dichlorophenoxyacetic acid & $<0.30$ & $\mu g / L$ & 0 & & GE \\
\hline & 1,2-Dichloropropane & $<1.0$ & $\mu g / L$ & 0 & & GE \\
\hline & trans-1,3-Dichloropropene & $<1.0$ & $\mu g / L$ & 0 & & jE \\
\hline & cis-1,3-Dichloropropene & $<1.0$ & $\mu g / L$ & 0 & & GE \\
\hline & Endrin & $<0.0060$ & $\mu \mathrm{g} / \mathrm{L}$ & 0 & & GE \\
\hline & Endrin & $<0.0060$ & $\mu g / L$ & 0 & - & GE \\
\hline & Ethylbenzene & $<1.0$ & $\mu g / L$ & 0 & & GE \\
\hline & Fluoride & $<100$ & $\mu g / L$ & 0 & & GE \\
\hline & Lead & 4.4 & $\mu g / L$ & 0 & & GE \\
\hline & Lindane & $<0.0050$ & $\mu g / L$ & 0 & & GE \\
\hline & Lindane & $<0.0050$ & $\mu g / L$ & 0 & & GE \\
\hline & Magnesium & 622 & $\mu_{g} / L$ & 0 & & GE \\
\hline & Manganese & $<2.0$ & $\mu g / L$ & 0 & & GE \\
\hline & Mercury & $<0.20$ & $\mu g / L$ & 0 & & GE \\
\hline & Methoxychlor & $<0.50$ & $\mu g / L$ & 0 & & GE \\
\hline & Methoxychlor & $<0.50$ & $\mu g / L$ & 0 & & GE \\
\hline & Nickel & 7.1 & $\mu g / L$ & 0 & & GE \\
\hline & Nitrate as nitrogen & 790 & $\mu g / L$ & 0 & & GE \\
\hline & Potassium & $<500$ & $\mu_{g} / L$ & 0 & & GE \\
\hline & Selenium & $<2.0$ & $\mu g / L$ & 0 & & GE \\
\hline & Silica & 6,500 & $\mu g / L$ & 0 & & GE \\
\hline & Silver & $<2.0$ & $\mu g / \mathrm{L}$ & 0 & & GE \\
\hline & Sodium & 1,080 & $\mu g / L$ & 0 & & GE \\
\hline & Sulfate & 4,660 & $\mu g / L$ & 0 & & GE \\
\hline & $1,1,2,2$-Tetrachloroethane & $<1.0$ & $\mu g / L$ & 0 & & GE \\
\hline & Tetrachloroethylene & $<1.0$ & $\mu g / L$ & 0 & & GE \\
\hline & Toluene & $<1.0$ & $\mu g / L$ & 0 & & GE \\
\hline & Total organic carbon & $<1,000$ & $\mu g / L$ & 0 & & GE \\
\hline & Total organic halogens & $<5.0$ & $\mu g / L$ & 0 & & GE \\
\hline & Total organic halogens & $<5.0$ & $\mu \mathrm{g} / \mathrm{L}$ & 0 & & GE \\
\hline & Toxaphene & $<0.24$ & $\mu g / L$ & 0 & & GE \\
\hline & Toxaphene & $<0.24$ & $\mu g / L$ & 0 & & $\overrightarrow{G E}$ \\
\hline & 2,4,5-TP (Silvex) & $<0.090$ & $\mu g / L$ & 0 & & GE \\
\hline & $1,1,1$-Trichloroethane & $<1.0$ & $\mu g / L$ & 0 & & $\overline{\mathrm{GE}}$ \\
\hline & $1,1,2$-Trichloroethane & $<1.0$ & $\mu g / L$ & 0 & & GE \\
\hline & Trichloroethylene & $<1.0$ & $\mu g / L$ & 0 & & GE \\
\hline & Trichlorofluoromethane & $<1.0$ & $\mu g / L$ & 0 & & GE \\
\hline & Vanadium & $<8.0$ & $\mu g / L$ & 0 & & GE \\
\hline & Gross alpha & $3.3 E+00 \pm 1.4 E+00$ & $\mathrm{pCi} / \mathrm{L}$ & 0 & & GE \\
\hline & Nonvolatile beta & $6.7 E+00 \pm 1.7 E+00$ & $\mathrm{pCi} / \mathrm{L}$ & 0 & & GE \\
\hline & Total radium & $1.8 E+00 \pm 1.1 E+00$ & $\mathrm{pCi} / \mathrm{L}$ & 0 & & GE \\
\hline & Tritium & $<7.0 E-01$ & $\mathrm{pCi} / \mathrm{mL}$ & 0 & & GE \\
\hline
\end{tabular}

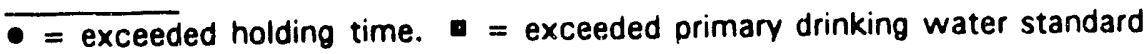




\section{WELL LFW 25}

SRS Coord.

N84967.2

E46425.7

MEASUREMENTS CONDUCTED IN THE FIELD

Sample date: $10 / 25 / 91$

Depth to water: $16.41 \mathrm{ft}(5.00 \mathrm{~m})$ below TOC

Water elevation: $158.29 \mathrm{ft}(48.25 \mathrm{~m}) \mathrm{msl}$

Sp. conductance: $16 \mu \mathrm{S} / \mathrm{cm}$

Water evacuated before sampling: $93 \mathrm{gal}$

\section{LABORATORY ANALYSES}

H D Analyte

- $\quad$ pH

Specific conductance

Aluminum

Antimony

Arsenic

Barium

Benzene

Bromodichloromethane

Bromoform

Bromomethane (Methyl bromide)

Cadmium

Calcium

Carbon tetrachloride

Chloride

Chlorobenzene

Chloroethane

Chloroethene (Vinyl chloride)

2-Chloroethyl vinyl ether

Chloroform

Chloromethane (Methyl chloride)

Chromium

Copper

Dibromochloromethane

1,1-Dichloroethane

1.2-Dichloroethane

1,1-Dichloroethylene

trans-1,2-Dichloroethylene

Dichloromethane (Methylene chloride)

2,4-Dichlorophenoxyacetic acid

1.2-Dichloropropane

trans-1,3-Dichloropropene

cis-1,3-Dichloropropene

Endrin

Endrin

Ethylbenzene

Fluoride
Resuit

5.0

15

$<20$

$<2.0$

$<2.0$

4.6

$<1.0$

$<1.0$

$<1.0$

$<1.0$

$<2.0$

212

$<1.0$

1,720

$<1.0$

$<1.0$

$<1.0$

$<1.0$

$<1.0$

$<1.0$

$<4.0$

5.8

$<1.0$

$<1.0$

$<1.0$

$<1.0$

$<1.0$

5.4

$<0.30$

$<1.0$

$<1.0$

$<1.0$

$<0.0060$

$<0.0060$

$<1.0$

$<100$
Top of Casing Casing

$174.7 \mathrm{ft} \mathrm{msl} \quad$ 4" PVC
Time: 9:10

$\mathrm{pH}: 4.7$

Alkalinity: $0 \mathrm{mg} / \mathrm{L}$

Water temperature: $19.3^{\circ} \mathrm{C}$
Unit Flag L Lab

pH $\quad 0 \quad$ GE

$\mu \mathrm{S} / \mathrm{cm} \quad 0 \quad$ GE

$\mu g / L \quad 0 \quad$ GE

$\mu g / L \quad 0 \quad$ GE

$\mu g /$ L $\quad 0 \quad$ GE

$\mu g / L \quad 0 \quad$ GE

$\mu g / L \quad 0 \quad G E$

$\mu g / 2 \quad 0 \quad$ GE

$\mu g / L \quad O \quad G E$

$\mu g / L \quad 0 \quad$ GE

$\mu g / L \quad 0 \quad$ GE

$\mu g / L \quad O \quad$ GE

$\mu \mathrm{g} / \mathrm{L} \quad \mathrm{O} \quad \mathrm{GE}$

$\mu g / L \quad O \quad$ GE

$\mu g / L \quad 0 \quad$ GE

$\mu g / L \quad 0 \quad$ GE

$\mu g / L \quad O \quad$ GE

$\mu g / L \quad 0 \quad$ GE

$\mu g / L \quad 0 \quad G E$

$\mu \mathrm{g} / \mathrm{L} \quad 0 \quad \mathrm{GE}$

$\mu g / L \quad O \quad$ GE

$\mu g / L \quad 0 \quad$ GE

$\mu g / L \quad 0 \quad$ GE

$\mu g / L \quad 0 \quad$ GE

$\mu g / L \quad 0 \quad$ GE

$\mu g / L \quad 0 \quad G E$

$\mu g / L \quad 0 \quad$ GE

$\mu g / L \quad O \quad$ GE

$\mu g / L \quad 0 \quad$ GE

$\mu \mathrm{g} / \mathrm{L} \quad \mathrm{O} \quad \mathrm{GE}$

$\mu g / L \quad$ GE

$\mu g / L \quad 0 \quad$ GE

$\mu g / L \quad 0 \quad G E$

$\mu g / L \quad O \quad G E$

$\mu \mathrm{g} / \mathrm{L} \quad \mathrm{O} \quad \mathrm{GE}$

$\mu g / L \quad$ GE

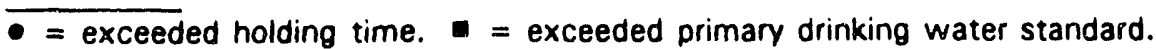


WELL LFW 25, laboratory analyses (cont.)

․ㅡ $\quad$ Analyte
Lead
Lindane
Lindane
Magnesium
Manganese
Mercury
Methoxychlor
Methoxychlor
Nickel
Nitrate as nitrogen
Nitrate as nitrogen
Potassium
Selenium
Silica
Silver
Sodium
Sulfate
1,1,2,2-Tetrachloroethane
Tetrachloroethylene
Toluene
Total organic carbon
Total organic halogens
Toxaphene
Toxaphene
$2,4,5-T P$ (Silvex)
1,1,1-Trichloroethane
1,1,2-Trichloroethane
Trichloroethylene
Trichlorofluoromethane
Vanadium
Gross alpha
Nonvolatile beta
Total radium
Tritium

\begin{tabular}{|c|c|c|c|}
\hline Result & Unit & Flag & Lab \\
\hline$<3.0$ & $\mu g / L$ & 0 & GE \\
\hline$<0.0050$ & $\mu g / L$ & 0 & GE \\
\hline$<0.0050$ & $\mu g / L$ & 0 & GE \\
\hline 464 & $\mu g / L$ & 0 & GE \\
\hline$<2.0$ & $\mu g / L$ & 0 & GE \\
\hline$<0.20$ & $\mu g / L$ & 0 & GE \\
\hline$<0.50$ & $\mu g / L$ & 0 & GE \\
\hline$<0.50$ & $\mu g / L$ & 0 & GE \\
\hline 5.7 & $\mu \mathrm{g} / \mathrm{L}$ & 0 & GE \\
\hline 650 & $\mu g / L$ & 0 & GE \\
\hline 650 & $\mu g / L$ & 0 & GE \\
\hline$<500$ & $\mu \mathrm{g} / \mathrm{L}$ & 0 & GE \\
\hline$<2.0$ & $\mu g / L$ & 0 & GE \\
\hline 6,320 & $\mu g / L$ & 0 & GE \\
\hline$<2.0$ & $\mu g / L$ & 0 & GE \\
\hline 967 & $\mu g / L$ & 0 & GE \\
\hline 2,090 & $\mu g / L$ & 0 & GE \\
\hline$<1.0$ & $\mu g / L$ & 0 & GE \\
\hline$<1.0$ & $\mu g / L$ & 0 & GE \\
\hline$<1.0$ & $\mu g / L$ & 0 & GE \\
\hline$<1,000$ & $\mu g / L$ & 0 & GE \\
\hline 12 & $\mu g / L$ & 0 & GE \\
\hline$<0.24$ & $\mu \mathrm{g} / \mathrm{L}$ & 0 & GE \\
\hline$<0.24$ & $\mu g / L$ & 0 & GE \\
\hline$<0.090$ & $\mu g / L$ & 0 & GE \\
\hline$<1.0$ & $\mu g / L$ & 0 & GE \\
\hline$<1.0$ & $\mu \mathrm{g} / \mathrm{L}$ & 0 & GE \\
\hline$<1.0$ & $\mu \mathrm{g} / \mathrm{L}$ & 0 & GE \\
\hline$<1.0$ & $\mu g / L$ & 0 & GE \\
\hline$<8.0$ & $\mu g / L$ & 0 & GE \\
\hline $5.9 E+00 \pm 1.7 E+00$ & $\mathrm{pCi} / \mathrm{L}$ & 0 & GE \\
\hline $1.1 E+01 \pm 1.9 E+00$ & $\mathrm{pCi} / \mathrm{L}$ & 0 & GE \\
\hline $2.7 E+00 \pm 1.4 E+00$ & $\mathrm{pCi} / \mathrm{L}$ & 1 & GE \\
\hline$<7.0 E-01$ & $\mathrm{pCi} / \mathrm{mL}$ & 0 & GE \\
\hline
\end{tabular}

\section{WELL LFW 26}

\begin{tabular}{|c|c|c|}
\hline SRS Coord. & Screen Zone Elevation & Too of Casing \\
\hline $\begin{array}{l}\text { N85654.6 } \\
\text { E45633.8 }\end{array}$ & $164.2-143.2 \mathrm{ft} \mathrm{msl}$ & $186.5 \mathrm{ft} \mathrm{msl}$ \\
\hline
\end{tabular}

\section{MEASUREMENTS CONDUCTED IN THE FIELD}

Sample date: 10/25/91

Depth to water: $23.35 \mathrm{ft}(7.12 \mathrm{~m})$ below TOC

Water elevation: $163.15 \mathrm{ft}(49.73 \mathrm{~m}) \mathrm{msl}$

Sp. conductance: $13 \mu \mathrm{S} / \mathrm{cm}$

Water evacuated before sampling: $52 \mathrm{gal}$
Time: 10:30

pH: 4.7

Alkalinity: $0 \mathrm{mg} / \mathrm{L}$

Water temperature: $19.8^{\circ} \mathrm{C}$

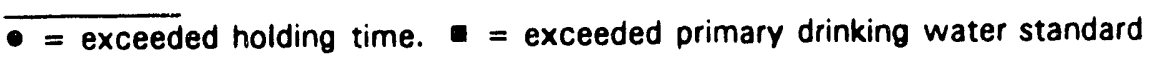


H D Analyte

- $\mathrm{pH}$

$$
\text { Specific conductance }
$$

Aluminum

Antimony

Arsenic

Barium

Benzene

Bromodichloromethane

Bromoform

Bromomethane (Methyl bromide)

Cadmium

Calcium

Carbon tetrachloride

Chloride

Chlorobenzene

Chloroethane

Chloroethene (Vinyl chloride)

2-Chloroethyl vinyl ether

Chloroform

Chloromethane (Methyl chloride)

Chromium

Copper

Dibromochloromethane

1,1-Dichloroethane

1,2-Dichloroethane

1,1-Dichloroethylene

trans-1,2-Dichloroethylene

Dichloromethane (Methylene chloride)

2,4-Dichlorophenoxyacetic acid

1,2-Dichloropropane

trans-1,3-Dichloropropene

cis-1,3-Dichloropropene

Endrin

Endrin

Ethylbenzene

Fluoride

Lead

Lindane

Lindane

Magnesium

Manganese

Mercury

Methoxychlor

Methoxychlor

Nickel

Nitrate as nitrogen

Potassium

Selenium

Silica

Silver

Sodium

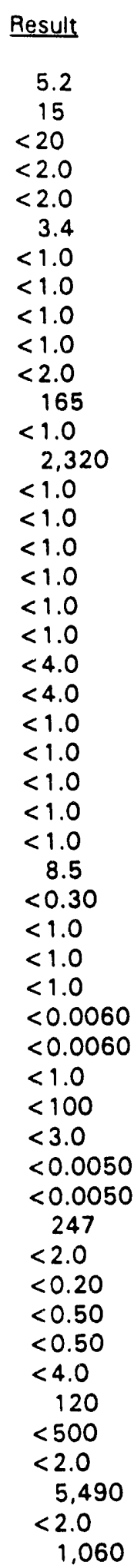

\begin{tabular}{|c|c|c|}
\hline Unit & Flag & $\underline{\text { Lab }}$ \\
\hline $\mathrm{pH}$ & 0 & GE \\
\hline$\mu \mathrm{S} / \mathrm{cm}$ & 0 & GE \\
\hline$\mu g / L$ & 0 & GE \\
\hline$\mu g / L$ & 0 & GE \\
\hline$\mu g / L$ & 0 & GE \\
\hline$\mu g / L$ & 0 & GE \\
\hline$\mu g / L$ & 0 & GE \\
\hline$\mu \mathrm{g} / \mathrm{L}$ & 0 & GE \\
\hline$\mu g / L$ & 0 & GE \\
\hline$\mu \mathrm{g} / \mathrm{L}$ & 0 & GE \\
\hline$\mu g / L$ & 0 & GE \\
\hline$\mu g / L$ & 0 & GE \\
\hline$\mu g / L$ & 0 & GE \\
\hline$\mu g / L$ & 0 & GE \\
\hline$\mu g / L$ & 0 & GE \\
\hline$\mu g / L$ & 0 & $\overline{G E}$ \\
\hline$\mu g / L$ & 0 & GE \\
\hline$\mu g / L$ & 0 & GE \\
\hline$\mu g / L$ & 0 & GE \\
\hline$\mu g / L$ & 0 & GE \\
\hline$\mu g / L$ & 0 & GE \\
\hline$\mu g / L$ & 0 & GE \\
\hline$\mu g / L$ & 0 & GE \\
\hline$\mu g / L$ & 0 & GE \\
\hline$\mu g / L$ & 0 & GE \\
\hline$\mu g / L$ & 0 & GE \\
\hline$\mu g / L$ & 0 & GE \\
\hline$\mu g / L$ & 0 & GE \\
\hline$\mu g / L$ & 0 & GE \\
\hline$\mu g / L$ & 0 & GE \\
\hline$\mu g / L$ & 0 & GE \\
\hline$\mu g / L$ & 0 & GE \\
\hline$\mu g / L$ & 0 & GE \\
\hline$\mu g / L$ & 0 & GE \\
\hline$\mu g / L$ & 0 & GE \\
\hline$\mu g / L$ & 0 & GE \\
\hline$\mu g / L$ & 0 & GE \\
\hline$\mu g / L$ & 0 & GE \\
\hline$\mu g / L$ & 0 & GE \\
\hline$\mu g / L$ & 0 & GE \\
\hline$\mu g / L$ & 0 & GE \\
\hline$\mu g / L$ & 0 & GE \\
\hline$\mu g / L$ & 0 & GE \\
\hline$\mu g / L$ & 0 & GE \\
\hline$\mu g / L$ & 0 & GE \\
\hline$\mu g / L$ & 0 & GE \\
\hline$\mu g / L$ & 0 & GE \\
\hline$\mu g / L$ & 0 & GE \\
\hline$\mu g / L$ & 0 & $\mathrm{GE}$ \\
\hline$\mu g / L$ & 0 & GE \\
\hline$\mu g / L$ & 0 & GE \\
\hline
\end{tabular}

- = exceeded holding time. $=$ exceeded primary drinking water standard. 
WELL LFW 26, laboratory analyses (cont.)

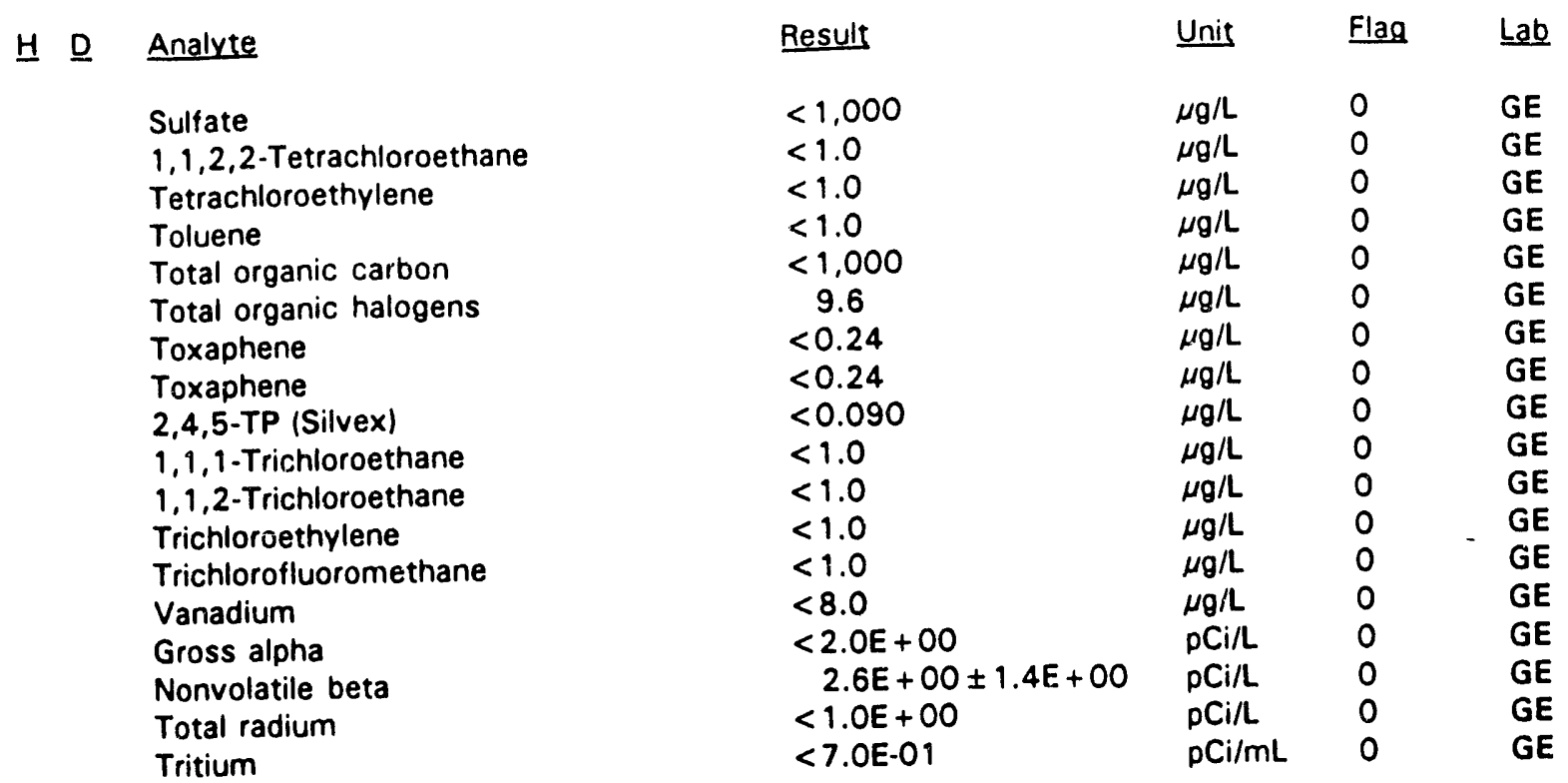

\section{WELL LFW 27}

\section{SRS Coord.}

N85839.1

E45596.1

\section{MEASUREMENTS CONDUCTED IN THE FIELD}

Sample date: $10 / 25 / 91$

Depth to water: $25.18 \mathrm{ft}(7.67 \mathrm{~m})$ below TOC

Water elevation: $164.02 \mathrm{ft}(49.99 \mathrm{~m} / \mathrm{msl}$

Sp. conductance: $14 \mu \mathrm{S} / \mathrm{cm}$

Water evacuated before sampling: $\mathbf{5 7}$ gal

\section{LABORATORY ANALYSES}

H D Analyte

- $\mathrm{pH}$

- $\quad \mathrm{pH}$

Specific conductance

Specific conductance

Aluminum

Aluminum

Antimony

Antimony

Arsenic

Arsenic

Barium

Barium

\section{Screen Zone Elevation}

$163.9-142.9 \mathrm{ft} \mathrm{msl}$
Too of Casing

$189.2 \mathrm{ft} \mathrm{msl}$
Casing

4" PVC
Time: 10:00

pH: 4.9

Alkalinity: $0 \mathrm{mg} / \mathrm{L}$

Water temperature: $19.5^{\circ} \mathrm{C}$

\begin{tabular}{c} 
Result \\
\hline \\
5.2 \\
5.3 \\
15 \\
15 \\
$<20$ \\
$<20$ \\
$<2.0$ \\
$<2.0$ \\
$<2.0$ \\
$<2.0$ \\
3.7 \\
3.8
\end{tabular}

Unit Flag Lab

pH $\quad 0 \quad$ GE

$\mathrm{pH} \quad 0 \quad \mathrm{GE}$

$\mu \mathrm{S} / \mathrm{cm} \quad 0 \quad \mathrm{GE}$

$\mu \mathrm{S} / \mathrm{cm} \quad 0 \quad \mathrm{GE}$

$\mu g / L \quad 0 \quad$ GE

$\mu g / L \quad 0 \quad$ GE

$\mu \mathrm{g} / \mathrm{L} \quad \mathrm{O} \quad \mathrm{GE}$

$\mu \mathrm{g} / \mathrm{L} \quad \mathrm{O} \quad \mathrm{GE}$

$\mu g / \mathrm{L} \quad 0 \quad \mathrm{GE}$

$\mu g / L \quad 0 \quad$ GE

$\mu g / \mathrm{L} \quad 0 \quad \mathrm{GE}$

$\mu g / L \quad 0 \quad$ GE

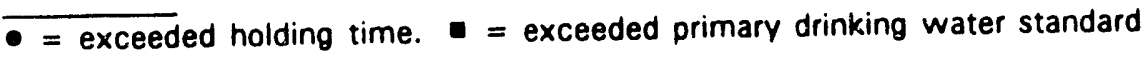


WELL LFW 27, laboratory analyses (cont.)

H $\underline{\text { Analyte }}$

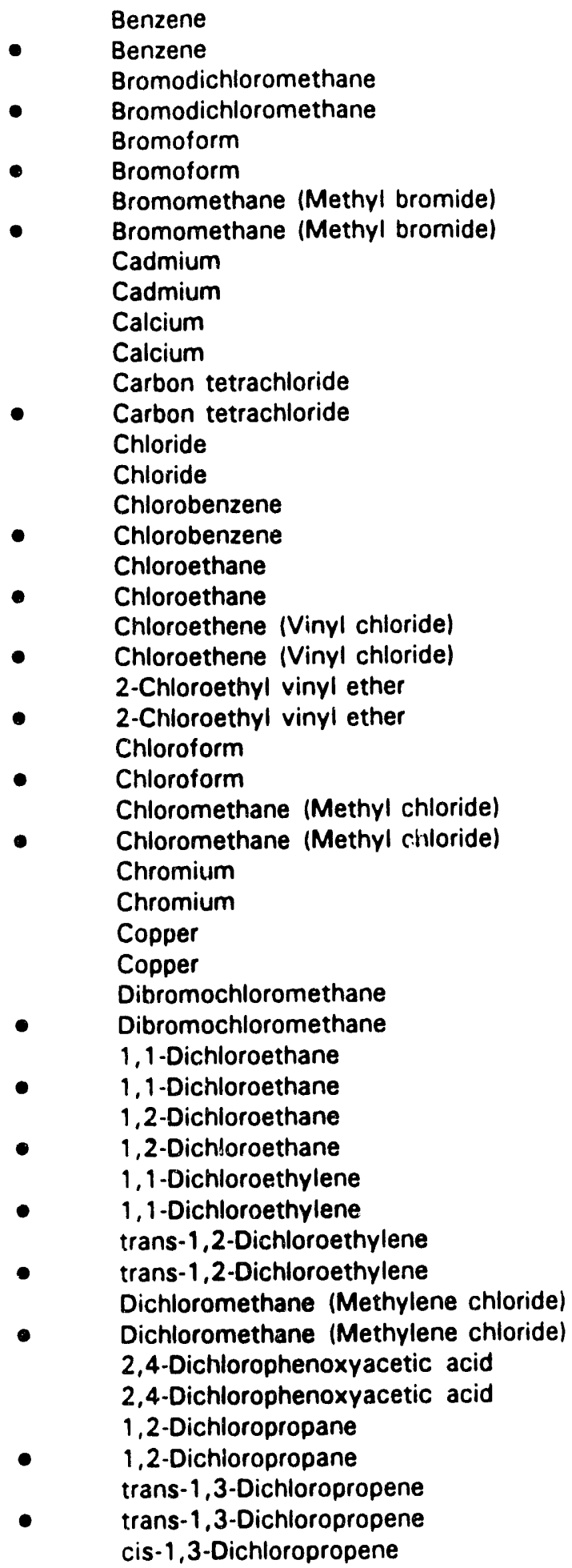

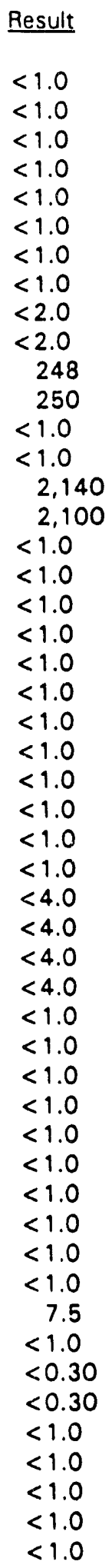

\begin{tabular}{|c|c|c|}
\hline Unit & Flag & Lab \\
\hline$\mu g / L$ & 0 & GE \\
\hline$\mu g / L$ & 0 & GE \\
\hline$\mu g / L$ & 0 & GE \\
\hline$\mu g / L$ & 0 & GE \\
\hline$\mu g / L$ & 0 & GE \\
\hline$\mu g / L$ & 0 & GE \\
\hline$\mu \mathrm{g} / \mathrm{L}$ & 0 & GE \\
\hline$\mu g / L$ & 0 & $\mathrm{GE}$ \\
\hline$\mu g / L$ & 0 & GE \\
\hline$\mu g / L$ & 0 & GE \\
\hline$\mu g / L$ & 0 & $\overline{G E}$ \\
\hline$\mu \mathrm{g} / \mathrm{L}$ & 0 & GE \\
\hline$\mu g / L$ & 0 & $-\quad \mathrm{GE}$ \\
\hline$\mu \mathrm{g} / \mathrm{L}$ & 0 & GE \\
\hline$\mu g / L$ & 0 & GE \\
\hline$\mu \mathrm{g} / \mathrm{L}$ & 0 & GE \\
\hline$\mu g / L$ & 0 & GE \\
\hline$\mu g / L$ & 0 & GE \\
\hline$\mu g / L$ & 0 & GE \\
\hline$\mu g / L$ & 0 & GE \\
\hline$\mu g / L$ & 0 & GE \\
\hline$\mu g / L$ & 0 & GE \\
\hline$\mu g / L$ & 0 & GE \\
\hline$\mu g / L$. & 0 & GE \\
\hline$\mu g / L$ & 0 & GE \\
\hline$\mu g / L$ & 0 & GE \\
\hline$\mu g / L$ & 0 & GE \\
\hline$\mu g / L$ & 0 & GE \\
\hline$\mu g / L$ & 0 & GE \\
\hline$\mu g / L$ & 0 & GE \\
\hline$\mu g / L$ & 0 & GE \\
\hline$\mu g / L$ & 0 & GE \\
\hline$\mu g / L$ & 0 & GE \\
\hline$\mu g / L$ & 0 & GE \\
\hline$\mu g / L$ & 0 & GE \\
\hline$\mu g / L$ & 0 & GE \\
\hline$\mu g / L$ & 0 & GE \\
\hline$\mu g / L$ & 0 & GE \\
\hline$\mu g / L$ & 0 & GE \\
\hline$\mu g / L$ & 0 & GE \\
\hline$\mu \mathrm{g} / \mathrm{L}$ & 0 & GE \\
\hline$\mu g / L$ & 0 & GE \\
\hline$\mu g / L$ & 0 & GE \\
\hline$\mu g / L$ & 0 & GE \\
\hline$\mu \mathrm{g} / \mathrm{L}$ & 0 & GE \\
\hline$\mu g / L$ & 0 & GE \\
\hline$\mu g / L$ & 0 & GE \\
\hline$\mu g / L$ & 0 & GE \\
\hline$\mu g / L$ & 0 & GE \\
\hline$\mu \mathrm{g} / \mathrm{L}$ & 0 & GE \\
\hline$\mu g / L$ & 0 & GE \\
\hline
\end{tabular}

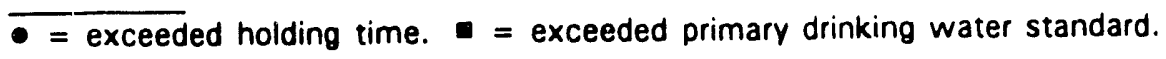


WELL LFW 27, laboratory analyses (cont.)

\begin{tabular}{|c|c|c|}
\hline$\underline{H} \quad \underline{D}$ & Analyte & Result \\
\hline$\bullet$ & cis-1,3-Dichloropropene & $<1.0$ \\
\hline & Endrin & $<0.0060$ \\
\hline & Endrin & $<0.0060$ \\
\hline & Endrin & $<0.0060$ \\
\hline & Ethylbenzene & $<1.0$ \\
\hline • & Ethylbenzene & $<1.0$ \\
\hline & Fluoride & $<100$ \\
\hline & Fluoride & $<100$ \\
\hline & Lead & $<3.0$ \\
\hline & Lead & $<3.0$ \\
\hline & Lindane & $<0.0050$ \\
\hline & Lindane & $<0.0050$ \\
\hline & Lindane & $<0.0050$ \\
\hline & Magnesium & 255 \\
\hline & Magnesium & 255 \\
\hline & Manganese & 2.1 \\
\hline & Manganese & 2.1 \\
\hline & Mercury & 1.1 \\
\hline & Mercury & $<0.20$ \\
\hline & Methoxychlor & $<0.50$ \\
\hline & Methoxychlor & $<0.50$ \\
\hline & Methoxychlor & $<0.50$ \\
\hline & Nickel & $<4.0$ \\
\hline & Nickel & $<4.0$ \\
\hline$\bullet$ & Nitrate as nitrogen & 230 \\
\hline & Nitrate as nitrogen & 130 \\
\hline & Potassium & $<500$ \\
\hline & Potassium & $<500$ \\
\hline & Selenium & $<2.0$ \\
\hline & Selenium & $<2.0$ \\
\hline & Silica & 6,290 \\
\hline & Silica & $6,31 C$ \\
\hline & Silver & $<20$ \\
\hline & Silver & $<2.0$ \\
\hline & Sodium & 1,400 \\
\hline & Sodium & 1,420 \\
\hline & Sulfate & $<1,000$ \\
\hline & Sulfate & $<1,000$ \\
\hline & 1,1,2,2-Tetrachloroethane & $<1.0$ \\
\hline • & $1,1,2,2$-Tetrachloroethane & $<1.0$ \\
\hline & Tetrachloroethylene & $<1.0$ \\
\hline 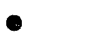 & Tetrachloroethylene & $<1.0$ \\
\hline & Toluene & $<1.0$ \\
\hline 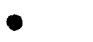 & Toluene & $<1.0$ \\
\hline & Total organic carbon & $<1,000$ \\
\hline & Total organic carbon & $<1,000$ \\
\hline & Total organic halogens & $<5.0$ \\
\hline & Total organic halogens & $<5.0$ \\
\hline & Total organic halogens & $<5.0$ \\
\hline & Toxaphene & $<0.24$ \\
\hline & Toxaphene & $<0.24$ \\
\hline
\end{tabular}

\begin{tabular}{|c|c|c|}
\hline Unit & Flag & \\
\hline$\mu g / L$ & 0 & \\
\hline$\mu g / L$ & 0 & \\
\hline$\mu g / L$ & 0 & \\
\hline$\mu \mathrm{g} / \mathrm{L}$ & 0 & \\
\hline$\mu g / L$ & 0 & \\
\hline$\mu g / L$ & 0 & \\
\hline$\mu g / L$ & 0 & \\
\hline$\mu g / L$ & 0 & \\
\hline$\mu g / L$ & 0 & \\
\hline$\mu g / L$ & 0 & \\
\hline$\mu g / L$ & 0 & \\
\hline$\mu g / L$ & 0 & - \\
\hline$\mu g / L$ & 0 & - \\
\hline$\mu g / L$ & 0 & \\
\hline$\mu g / L$ & 0 & \\
\hline$\mu g / L$ & 0 & \\
\hline$\mu g / L$ & 0 & \\
\hline$\mu g / L$ & 1 & \\
\hline$\mu g / L$ & 0 & \\
\hline$\mu g / L$ & 0 & \\
\hline$\mu g / L$ & 0 & \\
\hline$\mu g / L$ & 0 & \\
\hline$\mu g / L$ & 0 & \\
\hline$\mu g / L$ & 0 & \\
\hline$\mu g / L$ & 0 & \\
\hline$\mu g / L$ & 0 & \\
\hline$\mu g / L$ & 0 & \\
\hline$\mu_{g} / L$ & 0 & \\
\hline$\mu g / L$ & 0 & \\
\hline$\mu g / L$ & 0 & \\
\hline$\mu g / L$ & 0 & \\
\hline$\mu g / L$ & 0 & \\
\hline$\mu g / L$ & 0 & \\
\hline$\mu g / L$ & 0 & \\
\hline$\mu g / L$ & 0 & \\
\hline$\mu g / L$ & 0 & \\
\hline$\mu \mathrm{g} / \mathrm{L}$ & 0 & \\
\hline$\mu \mathrm{g} / \mathrm{L}$ & 0 & \\
\hline$\mu \mathrm{g} / \mathrm{L}$ & 0 & \\
\hline$\mu g / L$ & 0 & \\
\hline$\mu g / L$ & 0 & \\
\hline$\mu g / L$ & 0 & \\
\hline$\mu g / L$ & 0 & \\
\hline$\mu \mathrm{g} / \mathrm{L}$ & 0 & \\
\hline$\mu \mathrm{g} / \mathrm{L}$ & 0 & \\
\hline$\mu g / L$ & 0 & \\
\hline$\mu g / L$ & 0 & \\
\hline$\mu g / L$ & 0 & \\
\hline$\mu \sigma / L$ & 0 & \\
\hline$\mu g / L$ & 0 & \\
\hline$\mu \mathrm{g} / \mathrm{L}$ & 0 & \\
\hline
\end{tabular}

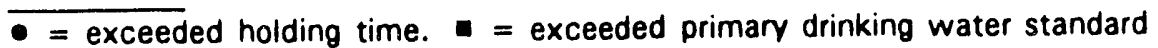


WELL LFW 27, laboratory arialyses (cont.)

$\begin{array}{ll}\text { H. } & \text { Result } \\ \text { Analyte } & <0.24 \\ \text { Toxaphene } & <0.090 \\ 2,4,5-T P \text { (Silvex) } & <0.090 \\ 2,4,5-T P \text { (Silvex) } & <1.0 \\ 1,1,1-\text { Trichloroethane } & <1.0 \\ 1,1,1 \text {-Trichloroethane } & <1.0 \\ 1,1,2-\text { Trichloroethane } & <1.0 \\ 1,1,2-\text { Trichloroethane } & <1.0 \\ \text { Trichloroethylene } & <1.0 \\ \text { Trichloroethylene } & <1.0 \\ \text { Trichlorofluoromethane } & <1.0 \\ \text { Trichlorofluoromethane } & <8.0 \\ \text { Vanadium } & <8.0 \\ \text { Vanadium } & <2.0 E+00 \\ \text { Gross alpha } & <2.0 E+00 \\ \text { Gross alpha } & 3.1 E+00 \pm 1.5 E+00 \\ \text { Nonvolatile beta } & <2.0 E+00 \\ \text { Nonvolatile beta } & 1.1 E+00 \pm 1.2 E+00 \\ \text { Total radium } & 1.3 E+00 \pm 1.2 E+00 \\ \text { Total radium } & <7.0 E-01 \\ \text { Tritium } & <7.0 E-01 \\ \text { Tritium } & \end{array}$

\begin{tabular}{|c|c|c|}
\hline Unit & Flag & \\
\hline$\mu g / L$ & 0 & \\
\hline$\mu g / L$ & 0 & \\
\hline$\mu g / L$ & 0 & \\
\hline$\mu g / \mathrm{l}$. & 0 & \\
\hline$\mu g / L$ & 0 & \\
\hline$\mu g / L$ & 0 & \\
\hline$\mu g / L$ & 0 & \\
\hline$\mu g /$ & 0 & \\
\hline$\mu g /$ & 0 & \\
\hline$\mu g / L$ & 0 & \\
\hline$\mu g / L$ & 0 & \\
\hline$\mu g / L$ & 0 & - \\
\hline$\mu g / L$ & 0 & \\
\hline pCi/L & 0 & \\
\hline $\mathrm{pCi} / \mathrm{L}$ & 0 & \\
\hline $\mathrm{pCi} / \mathrm{L}$ & 0 & \\
\hline $\mathrm{CCi} / \mathrm{L}$ & 0 & \\
\hline pCill & 0 & \\
\hline pC.n & 0 & \\
\hline $\mathrm{pC}: / \mathrm{mL}$ & 0 & \\
\hline $\mathrm{pCi} / \mathrm{mL}$ & 0 & \\
\hline
\end{tabular}

\section{WELL LFW 27}

\section{SRSS Coord.}

N85839.1

E45596.1

\section{MEASUREMENTS COIIDUCTED IN THE FIELD}

Sample da.e: 10/25/91

Depth to water: $25.18 \mathrm{ft}(7.67 \mathrm{~m})$ below TOC

Water elevation: $164.02 \mathrm{ft}(49.99 \mathrm{~m}) \mathrm{msl}$

Sp. conductance: $14 \mu \mathrm{S} / \mathrm{cm}$

Water evacuated before sampling: $57 \mathrm{gal}$
Time: 10:00

$\mathrm{pH}: 4.9$

Alkalinity: $0 \mathrm{mg} / \mathrm{L}$

Water temperature: $19.5^{\circ} \mathrm{C}$

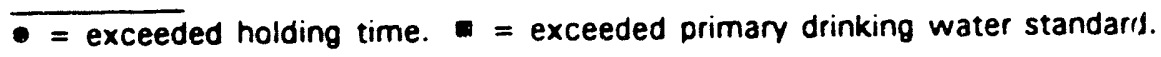


WELL LFW 28

\section{SRS Coord.}

N86079.6

E45555.3

MEASUREMENTS CONDUCTED IN THE FIELD

Sample date: 10/29/91

Depth to water: $27.06 \mathrm{ft}(8.25 \mathrm{~m})$ below TOC Water elevation: $165.34 \mathrm{ft}(50.40 \mathrm{~m} / \mathrm{ms})$

Sp. conductance: $31 \mu \mathrm{S} / \mathrm{cm}$

Water evacuated before sampling: $16 \mathrm{gal}$

The well went dry during purging.

\section{LABORF TORY ANALYSES}

\section{H D Analyte}

pH

Specific conductance

Aluminum

Antimony

Antimony

Arsenic

Arsenic

Barium

Benzene

Bromodichloromethane

Bromoform

Bromomethane (Methyl bromide)

Cadmium

Calcium

Cárbin tetrachloride

C. lloride

Chlorobenzene

Chloroethane

Chloroethene (Vinyl chloride)

2-Chioroethyl vinyl ether

Chloroform

Chloromethane (Methyl chloride)

Chromium

Copper

Dibromochloromethane

1,1-Dichloroetriane

1,2-Dichloroethane

1,1-Dichloroethylene

trans-1,2-Dichloroethylene

Dichloromethane (Methylene chloride)

2,4-Dichlorophenoxyacetic acid

1,2-Dichloropropane

trans-1,3-Dichloropropene

cis-1,3-Dichloropropene

Endrin
Screen Zone Elevation

$162.1-141.1 \mathrm{ft} \mathrm{msl}$

Top of Casing

Casing

$192.4 \mathrm{ft} \mathrm{msl}$

4" PVC
Time: 9:00

pH: 5.5

Alkalinity: $7 \mathrm{mg} / \mathrm{L}$

Water temperature: $18.5^{\circ} \mathrm{C}$
Result

5.7

30

$<20$

$<2.0$

$<2.0$

$<2.0$

$<2.0$

8.9

$<1.0$

$<1.0$

$<1.0$

$<1.0$

$<2.0$

2,810

$<1.0$

1.580

$<1.0$

$<1.0$

$<1.0$

$<1.0$

$<1.0$

$<1.0$

$<4.0$

4.6

$<1.0$

$<1.0$

$<1.0$

$<1.0$

$<1.0$

8.0

$<0.30$

$<1.0$

$<1.0$

$<1.0$

$<0.0060$
Unit Flag Lab

pH $\quad 0 \quad$ GE $\mu S / \mathrm{cm} \quad 0 \quad \mathrm{GE}$ $\mu g / L \quad 0 \quad$ GE $\mu g / L \quad 0 \quad$ GE $\mu \mathrm{g} / \mathrm{L} \quad 0 \quad \mathrm{GE}$ $\mu g / L \quad 0 \quad$ GE $\mu g / L \quad 0 \quad$ GE $\mu g / L \quad 0 \quad$ GE $\mu g / L \quad 0 \quad$ GE $\mu g / L \quad 0 \quad G E$ $\mu g / L \quad 0 \quad$ GE $\mu g / L \quad O \quad$ GE $\mu g / L \quad 0 \quad G E$ $\mu g / L \quad 0 \quad$ GE $\mu g / L \quad 0 \quad$ GE $\mu g / L \quad 0 \quad$ GE $\mu g / L \quad 0 \quad$ GE $\mu g / L \quad 0 \quad$ GE $\mu g / L \quad 0 \quad G E$ $\mu g / L \quad 0 \quad$ GE $\mu g / L \quad 0 \quad G E$ $\mu g / L \quad 0 \quad G E$ $\mu g / L \quad 0 \quad$ GE $\mu g / L \quad 0 \quad$ GE $\mu g / L \quad 0 \quad$ GE $\mu g / L \quad 0 \quad$ GE $\mu g / L \quad 0 \quad$ GE $\mu g / L \quad 0 \quad$ GE $\mu g / L \quad O \quad$ GE $\mu g / L \quad O \quad G E$ $\mu g / L \quad 0 \quad$ GE $\mu g / L . \quad 0 \quad$ GE $\mu g / L . \quad O \quad G E$ $\mu g / L \quad O \quad$ GE $\mu g / L \quad$ GE

\footnotetext{
- exceeded holding time. = exceeded primary drinking water standard
} 
WELL LFW 28, laboratory analyses (cont.)

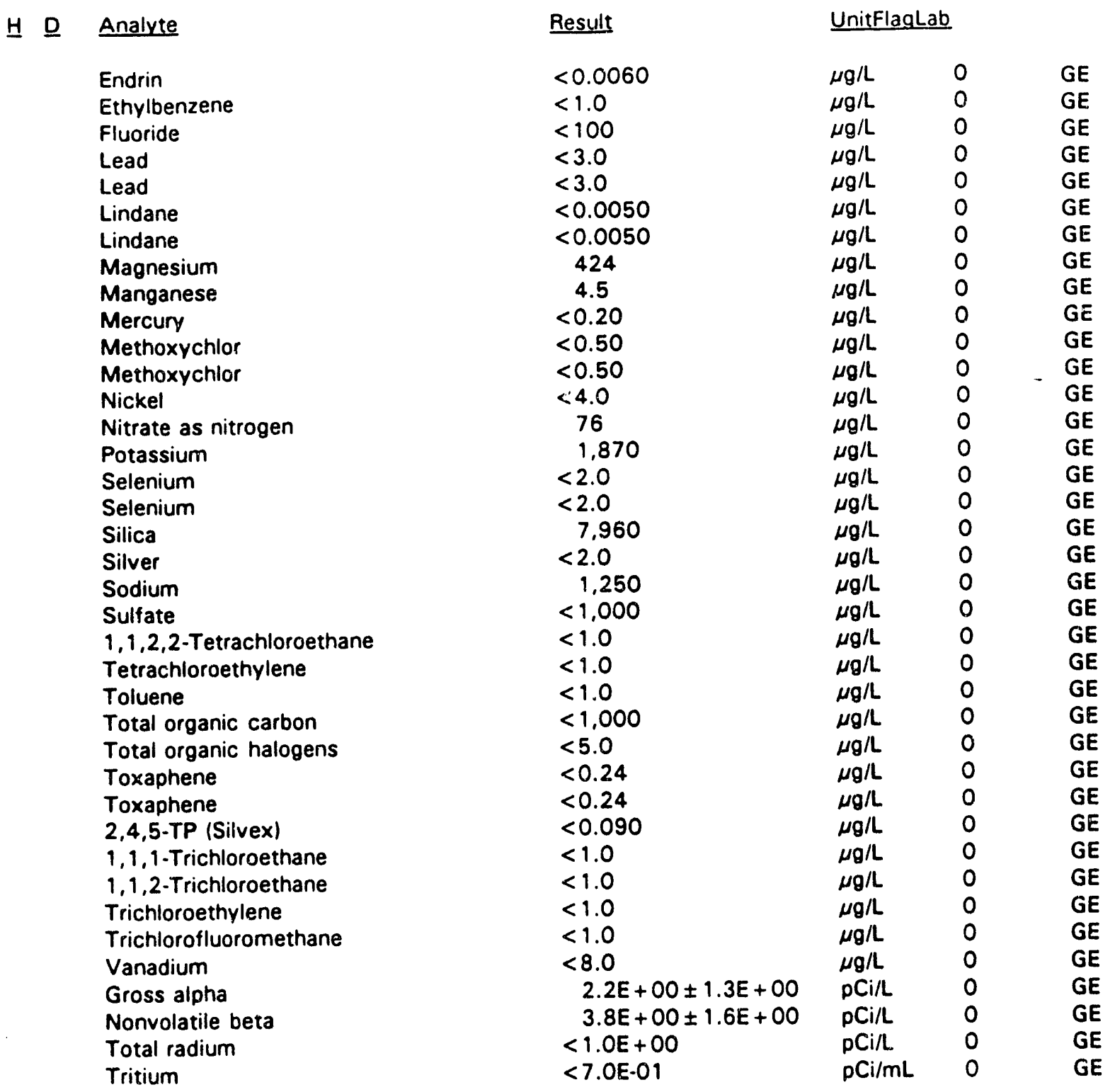

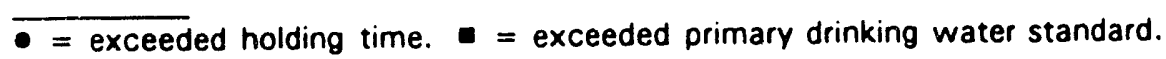


WELL LFW 29

SRS Coord.

Screen Zone Elevation

Top of Casing

Casing

N86372.7

$164.9-143.9 \mathrm{ft} \mathrm{msl}$

$195.3 \mathrm{ft} \mathrm{msl}$

4" PVC

E45503.3

MEASUREMENTS CONDUCTED IN THE FIELD

Sample date: $10 / 28 / 91$

Depth to water: $28.40 \mathrm{ft}(8.66 \mathrm{~m})$ below TOC

Water elevation: $166.90 \mathrm{ft}(50.87 \mathrm{~m}) \mathrm{msl}$

Sp. conductance: $21 \mu \mathrm{S} / \mathrm{cm}$

Water evacuated before sampling: 62 gal

Time: 9:45

pH: 4.5

Alkalinity: $0 \mathrm{mg} / \mathrm{L}$

Water temperature: $18.3^{\circ} \mathrm{C}$

\section{LABORATORY ANALYSES}

H D Analyte

- $\quad \mathrm{pH}$

Specific conductance
Aluminum
Antimony
Arsenic
Barium
Benzene
Bromodichloromethane
Bromoform
Bromomethane (Methyl bromide)
Cadmium
Calcium
Carbon tetrachloride
Chloride
Chlorobenzene
Chloroethane
Chloroethene (Vinyl chloride)
2-Chloroethyl vinyl ether
Chloroform
Chloromethane (Methyl chloride)
Chromium
Copper
Dibromochloromethane
1,1-Dichloroethane
1,2-Dichloroethane
1,1-Dichloroethylene
trans-1,2-Dichloroethylene
Dichloromethane (Methylene chloride)
2,4-Dichlorophenoxyacetic acid
1,2-Dichloropropane
trans-1,3-Dichloropropene
cis-1,3-Dichloropropene
Endrin
Ethylbenzene
Fluoride
Lead

Result

$$
5.1
$$$$
20
$$$$
<20
$$$$
<2.0
$$$$
<2.0
$$

8.2

$<1.0$

$<1.0$

$<1.0$

$<1.0$

$<2.0$

605

$<1.0$

1.810

$<1.0$

$<1.0$

$<1.0$

$<1.0$

$<1.0$

$<1.0$

$<4.0$

$<4.0$

$<1.0$

$<1.0$

$<1.0$

$<1.0$

$<1.0$

$<1.0$

$<0.30$

$<1.0$

$<1.0$

$<1.0$

$<0.0060$

$<1.0$

$<100$

$<3.0$
Unit Flag Lab

$\mathrm{pH} \quad \mathrm{O} \quad \mathrm{GE}$

$\mu S / \mathrm{cm} \quad 0 \quad$ GE

$\mu g / L \quad O \quad$ GE

$\mu g / L \quad 0 \quad$ GE

$\mu g / L \quad 0 \quad$ GE

$\mu g / L \quad 0 \quad$ GE

$\mu g / L \quad 0 \quad$ GE

$\mu g / L \quad 0 \quad$ GE

$\mu g / L \quad O \quad$ GE

$\mu g / L \quad 0 \quad$ GE

$\mu g / L \quad 0$. GE

$\mu g / L \quad O \quad G E$

$\mu g / L \quad 0 \quad$ GE

$\mu g / L \quad O \quad$ GE

$\mu g / L \quad 0 \quad G E$

$\mu g / L \quad 0 \quad$ GE

$\mu g / L \quad 0 \quad$ GE

$\mu g / L \quad 0 \quad G E$

$\mu g / L \quad 0 \quad$ GE

$\mu g / L \quad 0 \quad$ GE

$\mu g / L \quad 0 \quad G E$

$\mu g / L \quad 0 \quad$ GE

$\mu g / L \quad 0 \quad$ GE

$\mu g / L \quad 0 \quad$ GE

$\mu g / L \quad 0 \quad$ GE

$\mu g / L \quad 0 \quad$ GE

$\mu g / L \quad 0 \quad$ GE

$\mu g / L \quad 0 \quad G E$

$\mu g / L \quad 0 \quad$ GE

$\mu g / L \quad O \quad$ GE

$\mu g / L \quad O \quad G E$

$\mu g / L \quad 0 \quad$ GE

$\mu g / L \quad O \quad$ GE

$\mu g / L \quad O \quad G E$

$\mu g / L \quad O \quad$ GE

$\mu g / L \quad 0 \quad$ GE

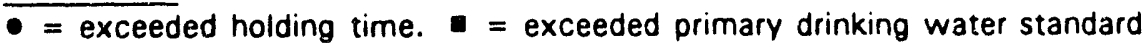


WELL LFW 29, laboratory analyses (cont.)

H $\underline{D}$

Analyte
Lindane
Magnesium
Manganese
Mercury
Methoxychlor
Nickel
Nitrate as nitrogen
Potassium
Selenium
Silica
Silver
Sodium
Sulfate
1,1,2,2-Tetrachloroethane
Tetrachloroethylene
Toluene
Total organic carbon
Total organic carbon
Total organic halogens
Toxaphene
$2,4,5-$ TP (Silvex)
1,1,1-Trichloroethane
1,1,2-Trichloroethane
Trichloroethylene
Trichlorofluoromethane
Vanadium
Gross alpha
Nonvolatile beta
Total radium
Tritium

$\begin{aligned} & \text { Pesult } \\ & \\ &<0.0050 \\ & \quad 587 \\ & 4.0 \\ &<0.20 \\ &<0.50 \\ &<4.0 \\ & \quad 860 \\ &<500 \\ &<2.0 \\ & \quad 6.200 \\ &<2.0 \\ & 1.010 \\ &<1.000 \\ &<1.0 \\ &<1.0 \\ &<1.0 \\ &<1.000 \\ &<1.000 \\ & 12 \\ &<0.24 \\ &<0.090 \\ &<1.0 \\ &<1.0 \\ &<1.0 \\ &<1.0 \\ &<8.0 \\ &<2.0 E+00 \\ & 2.0 E+00 \pm 1.5 E+00 \\ & 3.5 E+00 \pm 1.5 E+00 \\ &<7.0 E-01 \\ &\end{aligned}$

\begin{tabular}{|c|c|c|}
\hline Unit & Flag & $\underline{\text { Lab }}$ \\
\hline$\mu g / L$ & 0 & GE \\
\hline$\mu g, L$ & 0 & GE \\
\hline$\mu g / L$ & 0 & GE \\
\hline$\mu \mathrm{g} / \mathrm{L}$ & 0 & GE \\
\hline$\mu \mathrm{g} / \mathrm{L}$ & 0 & GE \\
\hline$\mu g / L$ & 0 & GE \\
\hline$\mu g / L$ & 0 & $\mathrm{GE}$ \\
\hline$\mu g / L$ & 0 & GE \\
\hline$\mu g / L$ & 0 & GE \\
\hline$\mu g / L$ & 0 & GE \\
\hline$\mu g / L$ & 0 & GE \\
\hline$\mu g / L$ & 0 & GE \\
\hline$\mu g / L$ & 0 & $\mathrm{GE}$ \\
\hline$\mu g / L$ & 0 & GE \\
\hline$\mu g / L$ & 0 & GE \\
\hline$\mu g / L$ & 0 & GE \\
\hline$\mu g / L$ & 0 & GE \\
\hline$\mu g / L$ & 0 & GE \\
\hline$\mu g / L$ & 0 & $\mathrm{GE}$ \\
\hline$\mu g / L$ & 0 & GE \\
\hline$\mu g / L$ & 0 & GE \\
\hline$\mu g / L$ & 0 & $\mathrm{GE}$ \\
\hline$\mu g / L$ & 0 & GE \\
\hline$\mu g / L$ & 0 & GE \\
\hline$\mu g / L$ & 0 & GE \\
\hline$\mu g / L$ & 0 & $\mathrm{GE}$ \\
\hline $\mathrm{pCi} / \mathrm{L}$ & 0 & GE \\
\hline $\mathrm{pCi} / \mathrm{L}$ & 0 & $\mathrm{GE}$ \\
\hline $\mathrm{pCi} / \mathrm{L}$ & 1 & GE \\
\hline $\mathrm{pCi} / \mathrm{mL}$ & 0 & $\mathrm{GE}$ \\
\hline
\end{tabular}

\section{WELL LFW 30}

$\begin{array}{llll}\text { SRS Coord. } & \text { Screen Zone Elevation } & \text { Too of Casing } & \text { Casing } \\ \text { N86318.4 } & 162.7-141.7 \mathrm{ft} \mathrm{msl} & 210.0 \mathrm{ft} \mathrm{msl} & 4^{\text {" PVC }}\end{array}$

E45170.9

MEASUREMENTS CONDUCTED IN THE FIELD

Sample date: $10 / 28 / 91$

Depth to water: $42.76 \mathrm{ft}(13.03 \mathrm{~m})$ below TOC Water elevation: $167.24 \mathrm{ft}(50.98 \mathrm{~m}) \mathrm{msl}$

Sp. conductance: $22 \mu \mathrm{S} / \mathrm{cm}$

Water evacuated before sampling: $45 \mathrm{gal}$
Time: 9:15

pH: 5.1

Alkalinity: $0 \mathrm{mg} / \mathrm{L}$

Water temperature: $18.1^{\circ} \mathrm{C}$

$\overline{0=\text { exceeded holding time. }}=$ exceeded primary drinking water standard. 


\section{LABORATORY ANALYSES}

H D Analyte

- $\quad \mathrm{pH}$

Specific conductance

Aluminum

Antimony

Arsenic

Barium

Benzene

Bromodichloromethane

Bromoform

Bromomethane (Methyl bromide)

Cadmium

Calcium

Carbon tetrachloride

Chloride

Chlorobenzene

Chloroethane

Chloroethene (Vinyl chloride)

2-Chloroethyl vinyl ether

Chloroform

Chloromethane (Methyl chloride)

Chromium

Copper

Dibromochloromethane

1,1-Dichloroethane

1,2-Dichloroethane

1,1-Dichloroethylene

trans-1,2-Dichloroethylene

Dichloromethane (Methylene chloride)

2,4-Dichlorophenoxyacetic acid

1,2-Dichloropropane

trans-1,3-Dichloropropene

cis-1,3-Dichloropropene

Endrin

Ethylbenzene

Fluoride

Lead

Lindane

Magnesium

Manganese

Mercury

Methoxychlor

Nickel

Nitrate as nitrogen

Potassium

Selenium

Silica

Silver

Sodium

Sulfate

1,1,2,2-Tetrachloroethane

Tetrachloroethylene
Result

5.4

20

20

$<2.0$

$<2.0$

5.3

$<1.0$

$<1.0$

$<1.0$

$<1.0$

$<2.0$

927

$<1.0$

1,580

$<1.0$

$<1.0$

$<1.0$

$<1.0$

$<1.0$

$<1.0$

$<4.0$

$<4.0$

$<1.0$

$<1.0$

$<1.0$

$<1.0$

$<1.0$

$<1.0$

$<0.30$

$<1.0$

$<1.0$

$<1.0$

$<0.0060$

$<1.0$

$<100$

$<3.0$

$<0.0050$

505

3.8

0.32

$<0.50$

$<4.0$

560

$<500$

$<2.0$

6.010

$<2.0$

1.100

$<1,000$

$<1.0$

$<1.0$
Unit Flag Lab

$\mathrm{pH} \quad \mathrm{O} \quad \mathrm{GE}$

$\mu \mathrm{S} / \mathrm{cm} \quad 0 \quad \mathrm{GE}$

$\mu \mathrm{g} / \mathrm{L} \quad 0 \quad \mathrm{GE}$

$\mu g / L \quad 0 \quad G E$

$\mu g / L \quad 0 \quad$ GE

$\mu g / L \quad 0 \quad$ GE

$\mu g / L \quad 0 \quad$ GE

$\mu \mathrm{g} / \mathrm{L} \quad \mathrm{O} \quad \mathrm{GE}$

$\mu g / L \quad 0 \quad$ GE

$\mu g / L \quad 0 \quad$ GE

$\mu g / L \quad 0 \quad G E$

$\mu g / L \quad 0 \quad$ GE

$\mu g / L-0 \rightarrow G E$

$\mu g / L \quad 0 \quad$ GE

$\mu g / L \quad 0 \quad$ GE

$\mu g / L \quad 0 \quad$ GE

$\mu g / L \quad 0 \quad$ GE

$\mu g / L \quad 0 \quad G E$

$\mu \mathrm{g} / \mathrm{L} \quad 0 \quad \mathrm{GE}$

$\mu g / \mathrm{L} \quad 0 \quad$ GE

$\mu g / L \quad 0 \quad$ GE

$\mu g / L \quad 0 \quad$ GE

$\mu g / L \quad 0 \quad$ GE

$\mu g / L \quad 0 \quad$ GE

$\mu g / L \quad 0 \quad G E$

$\mu g / L \quad 0 \quad$ GE

$\mu g / L \quad 0 \quad$ GE

$\mu g / L \quad 0 \quad G E$

$\mu g / L \quad 0 \quad G E$

$\mu g / L \quad 0 \quad$ GE

$\mu g / L \quad 0 \quad$ GE

$\mu g / L \quad 0 \quad$ GE

$\mu g / L \quad 0 \quad$ GE

$\mu g / L \quad 0 \quad$ GE

$\mu g / L \quad 0 \quad G E$

$\mu g / L \quad 0 \quad G E$

$\mu g / L \quad 0 \quad$ GE

$\mu g / L \quad 0 \quad G E$

$\mu g / L \quad 0 \quad$ GE

$\mu g / L \quad 0 \quad$ GE

$\mu g / L \quad 0 \quad$ GE

$\mu g / L \quad 0 \quad$ GE

$\mu g / L \quad 0 \quad$ GE

$\mu g / L \quad 0 \quad$ GE

$\mu g / L \quad 0 \quad$ GE

$\mu g / L \quad 0 \quad$ GE

$\mu g / L \quad 0 \quad$ GE

$\mu g / L \quad 0 \quad$ GE

$\mu g / L \quad 0 \quad$ GE

$\mu g / L \quad 0 \quad$ GE

$\mu \mathrm{g} / \mathrm{L}$

GE

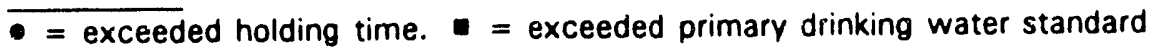


WELL LFW 30, laboratory analyses (cont.)

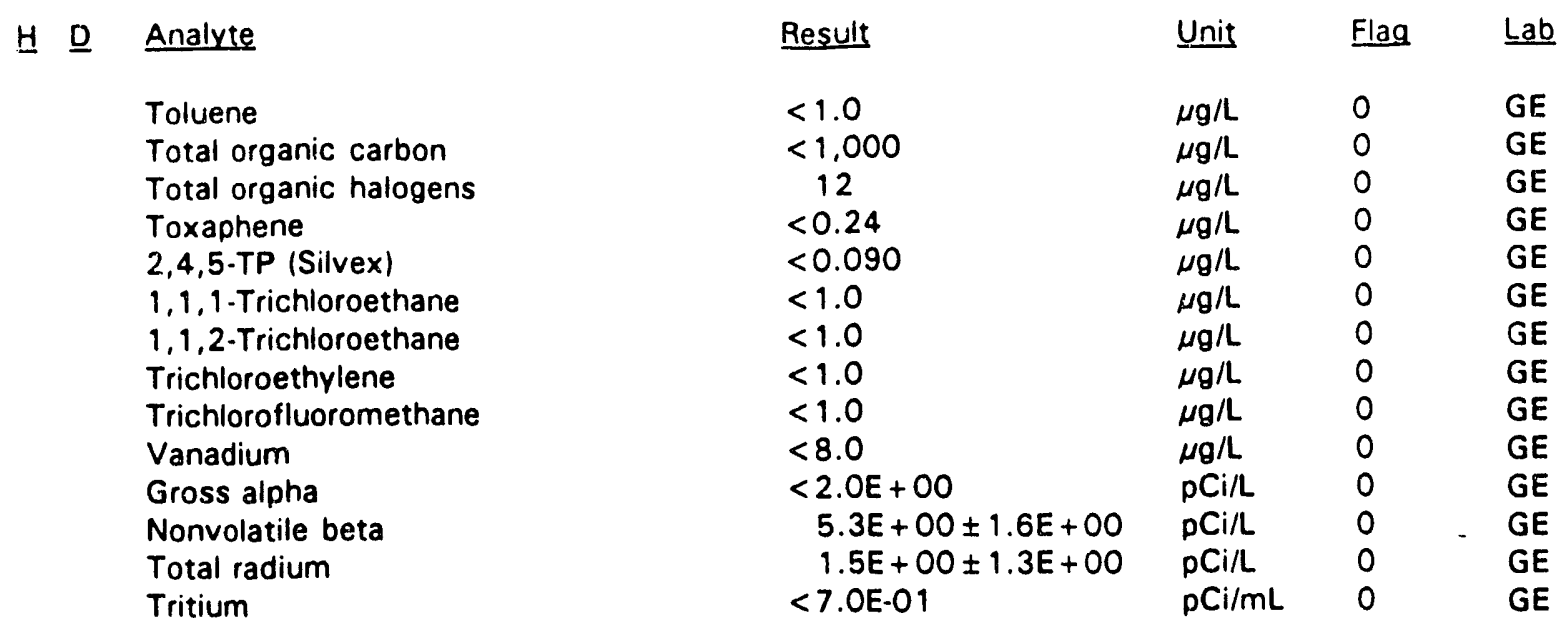

\section{WELL LFW 31}

SRS Coord.

N86262.2

E44869.0

MEASUREMENTS CONDUCTED IN THE FIELD

Sample date: $10 / 25 / 91$

Depth to water: $62.50 \mathrm{ft}(19.05 \mathrm{~m})$ below TOC

Water elevation: $166.80 \mathrm{ft}(50.84 \mathrm{~m}) \mathrm{msl}$

Sp. conductance: $24 \mu \mathrm{S} / \mathrm{cm}$

Water evacuated before sampling: $59 \mathrm{gal}$

\section{LABORATORY ANALYSES}

H D Analyte

$\mathrm{pH}$

Specific conductance

Aluminum

Antimony

Arsenic

Barium

Benzene

Bromodichloromethane

Bromoform

Bromomethane (Methyl bromide)

Cadmium

Calcium

Carbon tetrachloride

Chloride

Chlorobenzene

Chloroethane
Screen Zone Elevation

$166.0-145.0 \mathrm{ft} \mathrm{msl}$
Toe of Casing

$229.3 \mathrm{ft} \mathrm{msl}$
Casing

4" PVC
Time: $14: 20$

pH: 5.0

Alkalinity: $0 \mathrm{mg} / \mathrm{L}$

Water temperature: $18.5^{\circ} \mathrm{C}$

\begin{tabular}{c} 
Result \\
\hline \\
5.5 \\
25 \\
$<20$ \\
$<2.0$ \\
$<2.0$ \\
4.5 \\
$<1.0$ \\
$<1.0$ \\
$<1.0$ \\
$<1.0$ \\
$<2.0$ \\
676 \\
$<1.0$ \\
3.520 \\
$<1.0$ \\
$<1.0$
\end{tabular}

Unit Flag Lab

pH $\quad 0 \quad$ GE

$\mu S / c m$ GE

$\mu g / L \quad 0 \quad$ GE

$\mu g / L \quad 0 \quad G E$

$\mu g / L \quad 0 \quad$ GE

$\mu g / L \quad 0 \quad$ GE

$\mu g / L \quad 0 \quad G E$

$\mu g / L \quad 0 \quad$ GE

$\mu g / \mathrm{L} \quad 0 \quad \mathrm{GE}$

$\mu \mathrm{g} / \mathrm{L} \quad 0 \quad \mathrm{GE}$

$\mu g / L \quad 0 \quad G E$

$\mu g / L \quad 0 \quad$ GE

$\mu g / L \quad 0 \quad G E$

$\mu g / L \quad 0 \quad$ GE

$\mu g / L \quad 0 \quad G E$

$\mu g / L \quad 0 \quad$ GE

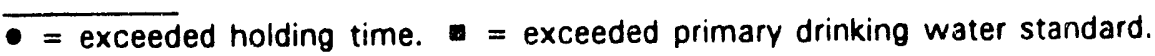


WELL LFW 31, laboratory analyses (cont.)

H D Analyte

Chloroethene (Vinyl chloride)

2-Chloroethyl vinyl ether

Chloroform

Chloromethane (Methyl chloride)

Chromium

Copper

Dibromochloromethane

1,1-Dichloroethane

1,2-Dichloroethane

1,1-Dichloroethylene

trans-1,2-Dichloroethylene

Dichloromethane (Methylene chloride)

2,4-Dichlorophenoxyacetic acid

1,2-Dichloropropane

trans-1,3-Dichloropropene

cis-1,3-Dichloropropene

Endrin

Ethylbenzene

Fluoride

Lead

Lindane

Magnesium

Manganese

Mercury

Methoxychlor

Nickel

Nitrate as nitrogen

Potassium

Selenium

Silica

Silver

Sodium

Sulfate

1,1,2,2-Tetrachloroethane

Tetrachloroethylene

Toluene

Total organic carbon

Total organic halogens

Toxaphene

2,4,5-TP (Silvex)

$1,1,1$-rrichloroethane

1,1,2-Trichloroethane

Trichloroethylene

Trichlorofluoromethane

Vanadium

Gross alpha

Nonvolatile beta

Total radium

Tritium
Result

$<1.0$

$<1.0$

$<1.0$

$<1.0$

$<4.0$

5.4

$<1.0$

$<1.0$

$<1.0$

$<1.0$

$<1.0$

11

$<0.30$

$<1.0$

$<1.0$

$<1.0$

$<0.0060$

$<1.0$

$<100$

$<3.0$

$<0.0050$

423

2.3

$<0.20$

$<0.50$

$<4.0$

530

811

$<2.0$

5.920

$<2.0$

2,230

1,440

$<1.0$

$<1.0$

$<1.0$

$<1,000$

10

$<0.24$

$<0.090$

$<1.0$

$<1.0$

$<1.0$

$<1.0$

$<8.0$

$<2.0 E+00$

$<2.0 E+00$

$<1 . \mathrm{OE}+\mathrm{OO}$

$<7.0$-01 $\underline{\text { Unit }} \quad \underline{\text { Flag }} \quad \underline{\text { Lab }}$

$\mu g / L \quad 0 \quad$ GE

$\mu g / L \quad 0 \quad$ GE

$\mu g / L \quad O \quad$ GE

$\mu g / L \quad O \quad G E$

$\mu g / L \quad O \quad$ GE

$\mu g / L \quad 0 \quad$ GE

$\mu g / L \quad 0 \quad$ GE

$\mu g / L \quad 0 \quad$ GE

$\mu g / L \quad 0 \quad$ GE

$\mu \mathrm{g} / \mathrm{L} \quad 0 \quad \mathrm{GE}$

$\mu g / L \quad O \quad$ GE

$\mu g / L \quad 0 \quad$ GE

$\mu g / L \quad 0 \quad G E$

$\mu g / L \quad 0 \quad$ GE

$\mu g / L \quad 0 \quad$ GE

$\mu \mathrm{g} / \mathrm{L} \quad 0 \quad \mathrm{GE}$

$\mu g / L \quad O \quad G E$

$\mu \mathrm{g} / \mathrm{L} \quad \mathrm{O} \quad \mathrm{GE}$

$\mu g / L \quad 0 \quad$ GE

$\mu g / L \quad 0 \quad G E$

$\mu g / L \quad 0 \quad$ GE

$\mu g / L \quad 0 \quad$ GE

$\mu g / L \quad 0 \quad$ GE

$\mu g / L \quad 0 \quad$ GE

$\mu \mathrm{g} / \mathrm{L} \quad \mathrm{O} \quad \mathrm{GE}$

$\mu g / L \quad 0 \quad$ GE

$\mu g / L \quad 0 \quad G E$

$\mu g / L \quad 0 \quad G E$

$\mu g / L \quad 0 \quad$ GE

$\mu g / L \quad 0 \quad G E$

$\mu g / L \quad 0 \quad$ GE

$\mu g / L \quad 0 \quad$ GE

$\mu g / L \quad 0 \quad$ GE

$\mu g / L \quad 0 \quad$ GE

$\mu \mathrm{g} / \mathrm{L} \quad \mathrm{O} \quad \mathrm{GE}$

$\mu g / L \quad 0 \quad$ GE

$\mu g / L \quad 0 \quad$ GE

$\mu \mathrm{g} / \mathrm{L} \quad 0 \quad \mathrm{GE}$

$\mu g / L \quad 0 \quad$ GE

$\mu g / L \quad 0 \quad$ GE

$\mu g / L \quad 0 \quad$ GE

$\mu g / L \quad 0 \quad$ GE

$\mu g / L \quad 0 \quad$ GE

$\mu g / L \quad 0 \quad$ GE

$\mu g / L \quad 0 \quad G E$

pCill $\quad 0 \quad$ GE

$\mathrm{pCi} / \mathrm{L} \quad 0 \quad \mathrm{GE}$

$\mathrm{pCi} / \mathrm{L} \quad 0 \quad \mathrm{GE}$

$\mathrm{pCi} / \mathrm{mL} \quad 0 \quad \mathrm{GE}$

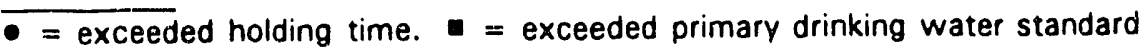




\section{WELL LFW 32}

SRS Coord.

N85836.8

E44935.9

MEASUREMENTS CONDUCTED IN THE FIELD

Sample date: $10 / 25 / 91$

Depth to water: $59.14 \mathrm{ft}(18.03 \mathrm{~m})$ below TOC

Water elevation: $164.56 \mathrm{ft}(50.16 \mathrm{~m}) \mathrm{msl}$

Sp. conductance: $21 \mu \mathrm{S} / \mathrm{cm}$

Water evacuated before sampling: $55 \mathrm{gal}$

\section{LABORATORY ANALYSES}

H $\underline{\text { Analyte }}$

pH
Specific conductance
Aluminum
Antimony
Arsenic
Barium
Benzene
Bromodichloromethane
Bromoform
Bromomethane (Methyl bromide)
Cadmium
Calcium
Carbon tetrachloride
Chloride
Chlorobenzene
Chloroethane
Chloroethene (Vinyl chloride)
2-Chloroethyl vinyl ether
Chloroform
Chloromethane (Methyl chloride)
Chromium
Copper
Dibromochloromethane
1,1-Dichloroethane
1,2-Dichloroethane
1,1-Dichloroethylene
trans-1,2-Dichloroethylene
Dichloromethane (Methylene chloride)
$2,4-$ Dichlorophenoxyacetic acid
1,2-Dichloropropane
trans-1,3-Dichloropropene
cis-1,3-Dichloropropene
Endrin
Ethylbenzene
Fluoride
Lead

- $\quad \mathrm{pH}$
Top of Casing

Casing

$223.7 \mathrm{ft} \mathrm{msl}$

4" PVC
Time: $13: 55$

pH: 4.9

Alkalinity: $0 \mathrm{mg} / \mathrm{L}$

Water temperature: $18.8^{\circ} \mathrm{C}$
Result

5.2

20

$<20$

$<2.0$

$<2.0$

3.3

$<1.0$

$<1.0$

$<1.0$

$<1.0$

$<2.0$

292

$<1.0$

2,700

$<1.0$

$<1.0$

$<1.0$

$<1.0$

$<1.0$

$<1.0$

$<4.0$

$<4.0$

$<1.0$

$<1.0$

$<1.0$

$<1.0$

$<1.0$

7.3

$<0.30$

$<1.0$

$<1.0$

$<1.0$

$<0.0060$

$<1.0$

$<100$

$<3.0$
Unit Flag Lab

$\mathrm{pH} \quad \mathrm{O} \quad \mathrm{GE}$

$\mu \mathrm{S} / \mathrm{cm} \quad 0 \quad \mathrm{GE}$

$\mu g / L \quad 0 \quad$ GE

$\mu g / L \quad 0 \quad$ GE

$\mu g / L \quad 0 \quad$ GE

$\mu g / L \quad 0 \quad$ GE

$\mu \mathrm{g} / \mathrm{L} \quad \mathrm{O} \quad \mathrm{GE}$

$\mu g / L \quad 0 \quad$ GE

$\mu g / L \quad 0 \quad$ GE

$\mu g / L \quad 0 \quad$ GE

$\mu g / L \quad O \quad G E$

$\mu g / L \quad 0 \quad$ GE

$\mu g / L \quad 0 \quad$ GE

$\mu g / L \quad 0 \quad$ GE

$\mu g / L \quad 0 \quad$ GE

$\mu g / L \quad O \quad G E$

$\mu \mathrm{g} / \mathrm{L} \quad 0 \quad \mathrm{GE}$

$\mu \mathrm{g} / \mathrm{L} \quad 0 \quad \mathrm{GE}$

$\mu g / L \quad 0 \quad$ GE

$\mu \mathrm{g} / \mathrm{L} \quad \mathrm{O} \quad \mathrm{GE}$

$\mu g / L \quad 0 \quad$ GE

$\mu g / L \quad 0 \quad$ GE

$\mu g / L \quad 0 \quad G E$

$\mu \mathrm{g} / \mathrm{L} \quad \mathrm{O} \quad \mathrm{GE}$

$\mu g / L \quad O \quad$ GE

$\mu g / L \quad 0 \quad$ GE

$\mu g / L \quad 0 \quad G E$

$\mu g / L \quad 0 \quad$ GE

$\mu g / L \quad O \quad$ GE

$\mu g / L \quad O \quad$ GE

$\mu g / L \quad 0 \quad$ GE

$\mu \mathrm{g} / \mathrm{L} \quad \mathrm{O} \quad \mathrm{GE}$

$\mu \mathrm{g} / \mathrm{L} \quad \mathrm{G} \quad \mathrm{GE}$

$\mu g / L \quad 0 \quad G E$

$\mu g / L \quad$ GE

$\mu g / L \quad$ GE

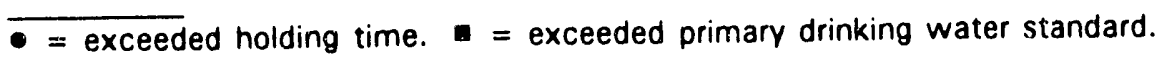


WELL LFW 32, laboratory analyses (cont.)

\section{H}

Analyte
Lindane
Magnesium
Manganese
Mercury
Methoxychlor
Nickel
Nitrate as nitrogen
Potassium
Selenium
Silica
Silver
Sodium
Sulfate
1,1,2,2-Tetrachloroethane
Tetrachloroethylene
Toluene
Total organic carbon
Total organic halogens
Toxaphene
$2,4,5-T P$ (Silvex)
$1,1,1$-Trichloroethane
1,1,2-Trichloroethane
Trichloroethylene
Trichlorofluoromethane
Vanadium
Gross alpha
Nonvolatile beta
Total radium
Tritium

$\begin{aligned} & \text { Result } \\ & \\ &<0.0050 \\ & \quad 436 \\ &<2.0 \\ &<0.20 \\ &<0.50 \\ &<4.0 \\ & \quad 450 \\ & 563 \\ &<2.0 \\ & \quad 6.100 \\ &<2.0 \\ & 1.730 \\ &< 1.000 \\ &<1.0 \\ &<1.0 \\ &<1.0 \\ &<1.000 \\ & 8.1 \\ &<0.24 \\ &<0.090 \\ &<1.0 \\ &<1.0 \\ &<1.0 \\ &<1.0 \\ &<8.0 \\ & 2.5 E+00 \pm 1.3 E+00 \\ & 3.5 E+00 \pm 1.5 E+00 \\ &< 1.0 E+00 \\ &<7.0 E-01 \\ &\end{aligned}$

\begin{tabular}{|c|c|c|}
\hline Unit & Flag & $\underline{\text { Lab }}$ \\
\hline$\mu \mathrm{g} / \mathrm{L}$ & 0 & GE \\
\hline$\mu \mathrm{g} / \mathrm{L}$ & 0 & GE \\
\hline$\mu g / L$ & 0 & GE \\
\hline$\mu g / L$ & 0 & GE \\
\hline$\mu \mathrm{g} / \mathrm{L}$ & 0 & $\mathrm{GE}$ \\
\hline$\mu g / L$ & 0 & GE \\
\hline$\mu \mathrm{g} / \mathrm{L}$ & 0 & $G$ \\
\hline$\mu g / L$ & 0 & $\mathrm{GE}$ \\
\hline$\mu g / L$ & 0 & 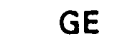 \\
\hline$\mu g / L$ & 0 & $\mathrm{GE}$ \\
\hline$\mu g / L$ & 0 & \\
\hline$\mu g / L$ & 0 & 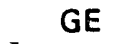 \\
\hline$\mu g / L$ & 0 & G \\
\hline$\mu g / L$ & 0 & GE \\
\hline$\mu_{\mathrm{g}} / \mathrm{L}$ & 0 & \\
\hline$\mu g / L$ & 0 & $\mathbf{G}$ \\
\hline$\mu g / L$ & 0 & 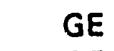 \\
\hline$\mu_{g} / L$ & 0 & \\
\hline$\mu_{g} / L$ & 0 & $\mathrm{GE}$ \\
\hline$\mu g / L$ & 0 & \\
\hline$\mu g / L$ & 0 & $\mathbf{G}$ \\
\hline$\mu g / L$ & 0 & \\
\hline$\mu g / L$ & 0 & \\
\hline$\mu g / L$ & 0 & \\
\hline$\mu g / L$ & 0 & \\
\hline $\mathrm{pCi} / \mathrm{L}$ & 0 & \\
\hline $\mathrm{pCi} / \mathrm{L}$ & 0 & \\
\hline $\mathrm{pCi} / \mathrm{L}$ & 0 & \\
\hline $\mathrm{pCi} / \mathrm{mL}$ & 0 & \\
\hline
\end{tabular}

\section{WELL LFW 33}

SRS Coord.

N85633.8

E44973.0

\section{Screen Zone Elevation}

$165.4-144.4 \mathrm{ft} \mathrm{msl}$
Top of Casing

$213.7 \mathrm{ft} \mathrm{msl}$
Casing

4" PVC

\section{MEASUREMENTS CONDUCTED IN THE FIELD}

Sample date: $10 / 25 / 91$

Depth to water: $50.52 \mathrm{ft}(15.40 \mathrm{~m})$ below TOC

Water elevation: $163.18 \mathrm{ft}(49.74 \mathrm{~m}) \mathrm{msl}$

Sp. conductance: $18 \mu \mathrm{S} / \mathrm{cm}$

Water evacuated before sampling: 51 gal
Time: $13: 30$

pH: 4.9

Alkalinity: $0 \mathrm{mg} / \mathrm{L}$

Water temperature: $18.8^{\circ} \mathrm{C}$

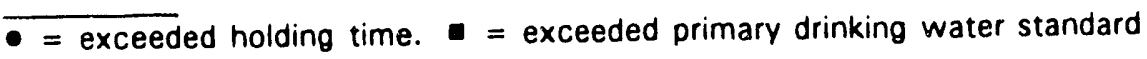


H D Analyte

- $\quad \mathrm{pH}$

Specific conductance

Aluminum

Antimony

Arsenic

Barium

Benzene

Bromodic:oromethane

Bromoforri:

Bromomethane (Methyl bromide)

Cadmium

Calcium

Carbon tetrachloride

Chloride

Chlorobenzene

Chloroethane

Chloroethene (Vinyl chloride)

2-Chloroethyl vinyl ether

Chloroform

Chloromethane (Methyl chloride)

Chromium

Copper

Dibromochloromethane

1,1-Dichloroethane

1,2-Dichloroethane

1,1-Dichloroethylene

trans-1,2-Dichloroethylene

Dichloromethane (Methylene chloride)

2,4-Dichlorophenoxyacetic acid

1,2-Dichloropropane

trans-1,3-Dichloropropene

cis-1,3-Dichloropropene

Endrin

Ethylbenzene

Fluoride

Lead

Lindane

Magnesium

Manganese

Mercury

Methoxychlor

Nickel

Nitrate as nitrogen

Potassium

Selenium

Silica

Silver

Sodium

Sulfate

1,1,2,2-Tetrachloroethane

Tetrachloroethylene
Result

5.2

20

$<20$

$<2.0$

$<2.0$

3.5

$<1.0$

$<1.0$

$<1.0$

$<1.0$

$<2.0$

368

$<1.0$

2,800

$<1.0$

$<1.0$

$<1.0$

$<1.0$

$<1.0$

$<1.0$

$<4.0$

$<4.0$

$<1.0$

$<1.0$

$<1.0$

$<1.0$

$<1.0$

$<1.0$

$<0.30$

$<1.0$

$<1.0$

$<1.0$

$<0.0060$

$<1.0$

$<100$

$<3.0$

$<0.0050$

460

$<2.0$

$<0.20$

$<0.50$

$<4.0$

380

538

$<2.0$

6.120

$<2.0$

1,260

$<1,000$

$<1.0$

$<1.0$ $\underline{\text { Unit }} \quad \underline{\text { Flag }} \quad \underline{\text { Lab }}$

$\mathrm{pH} \quad \mathrm{O} \quad \mathrm{GE}$

$\mu \mathrm{S} / \mathrm{cm} \quad \mathrm{G}$ GE

$\mu g / L \quad O \quad$ GE

$\mu g / L \quad O \quad$ GE

$\mu g / L \quad 0 \quad$ GE

$\mu g / L \quad O \quad$ GE

$\mu g / L \quad O \quad$ GE

$\mu g / L \quad O \quad$ GE

$\mu g / L \quad 0 \quad$ GE

$\mu g / L \quad 0 \quad$ GE

$\mu g / L \quad O \quad$ GE

$\mu g / L \quad O \quad G E$

$\mu g / L \quad 0 \quad-$ GE

$\mu \mathrm{g} / \mathrm{L} \quad \mathrm{O} \quad \mathrm{GE}$

$\mu g / L \quad O \quad G E$

$\mu g / L \quad O \quad$ GE

$\mu g / L \quad O \quad$ GE

$\mu g / L \quad O \quad$ GE

$\mu g / L \quad 0 \quad$ GE

$\mu g / L \quad O \quad$ GE

$\mu g / L \quad O \quad$ GE

$\mu g / L \quad O \quad G E$

$\mu g / L \quad 0 \quad$ GE

$\mu g / L \quad O \quad$ GE

$\mu g / L \quad O \quad$ GE

$\mu g / L \quad O \quad G E$

$\mu \mathrm{g} / \mathrm{L} \quad \mathrm{O} \quad \mathrm{GE}$

$\mu g / L \quad O \quad$ GE

$\mu g / L \quad O \quad G E$

$\mu g / L \quad 0 \quad$ GE

$\mu g / L \quad O \quad$ GE

$\mu g / L \quad 0 \quad$ GE

$\mu g / L \quad 0 \quad$ GE

$\mu g / L \quad O \quad$ GE

$\mu g / L \quad O \quad$ GE

$\mu g / L \quad O \quad$ GE

$\mu \mathrm{g} / \mathrm{L} \quad \mathrm{O} \quad \mathrm{GE}$

$\mu g / L \quad 0 \quad$ GE

$\mu g / L \quad O \quad$ GE

$\mu g / L \quad 0 \quad$ GE

$\mu g / L \quad 0 \quad$ GE

$\mu g / L \quad 0 \quad$ GE

$\mu g / L \quad 0 \quad$ GE

$\mu \mathrm{g} / \mathrm{L} \quad \mathrm{O} \quad \mathrm{GE}$

$\mu g / L \quad 0 \quad$ GE

$\mu g / L \quad 0 \quad$ GE

$\mu g / L \quad 0 \quad$ GE

$\mu g / L \quad O \quad$ GE

$\mu g / L \quad 0 \quad$ GE

$\mu g / L \quad O \quad G E$

$\mu g / L \quad O \quad$ GE

- = exceeded holding time. = exceeded primary drinking water standard. 
WELL LFW 33, laboratory analyses (cont.)

\begin{tabular}{|c|c|c|c|c|c|c|}
\hline$\underline{H} \underline{D}$ & Analvte & Result & Unit & Flag & & $\underline{\mathrm{Lab}}$ \\
\hline & Tolvene & $<1.0$ & $\mu g / L$ & 0 & & GE \\
\hline & Total organic carbon & $<1,000$ & $\mu_{g} / L$ & 0 & & GE \\
\hline & Total organic halogens & 11 & $\mu g / L$ & 0 & & GE \\
\hline & Toxaphene & $<0.24$ & $\mu g / L$ & 0 & & GE \\
\hline & 2,4,5-TP (Silvex) & $<0.090$ & $\mu g / L$ & 0 & & GE \\
\hline & 1,1,1-Trichloroethane & $<1.0$ & $\mu g / L$ & 0 & & GE \\
\hline & 1,1,2-Trichloroethane & $<1.0$ & $\mu g / L$ & 0 & & GE \\
\hline & Trichloroethylene & $<1.0$ & $\mu g / L$ & 0 & & GE \\
\hline & Trichlorofluoromethane & $<1.0$ & $\mu g / L$ & 0 & & GE \\
\hline & Vanadium & $<8.0$ & $\mu g / L$ & 0 & & GE \\
\hline & Gross alpha & $<2.0 E+O O$ & $\mathrm{pCi}$ & 0 & & 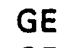 \\
\hline & Nonvolatile beta & $9.4 E+00 \pm 1.9 E+00$ & $\mathrm{pCi} / \mathrm{L}$ & 0 & & GE \\
\hline - & Total radium & $5.0 E+00 \pm 5.6 E+00$ & $\mathrm{pCi} / \mathrm{L}$ & 2 & & \\
\hline & Tritium & $<7.0 \mathrm{E}-01$ & $\mathrm{pCi} / \mathrm{mL}$ & 0 & & \\
\hline
\end{tabular}

\section{WELL LFW 34}

$\begin{array}{llll}\text { SRS Coord. } & \text { Screen Zone Elevation } & \text { Top of Casing } & \text { Casing } \\ \begin{array}{l}\text { N85409.5 } \\ \text { E45016.9 }\end{array} & 164.7-143.7 \mathrm{ft} \mathrm{msl} & 201.0 \mathrm{ft} \mathrm{msl} & 4^{\text {" PVC }}\end{array}$

\section{MEASUREMENTS CONDUCTED IN THE FIELD}

Sample date: 10/25/91

Depth to water: $39.23 \mathrm{ft}(11.96 \mathrm{~m})$ below TOC

Water elevation: $161.77 \mathrm{ft}(49.31 \mathrm{~m}) \mathrm{msl}$

Sp. conductance: $19 \mu \mathrm{S} / \mathrm{cm}$

Water evacuated before sampling: $49 \mathrm{gal}$

\section{LABORATORY ANALYSES}

Time: 13:05

pH: 5.1

Alkalinity: $0 \mathrm{mg} / \mathrm{L}$

Water temperature: $19.0^{\circ} \mathrm{C}$

H D $\quad$ Analyte
- $\quad$ SH
Specific conductance
Aluminum
Antimony
Arsenic
Barium
Benzene
Bromodichloromethane
Bromoform
Bromomethane (Methyl bromide)
- Cadmium
Calcium
Carbon tetrachloride
- Chloride
Chlorobenzene
Chloroethane

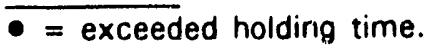

- = exceeded primary drinking water standard

$\begin{aligned} & \text { Result } \\ & \\ & \\ & 5.3 \\ & 20 \\ &<20 \\ &<2.0 \\ &<2.0 \\ & \quad 6.8 \\ &<1.0 \\ &<1.0 \\ &<1.0 \\ &<1.0 \\ &<2.0 \\ & 746 \\ &< 1.0 \\ & 2.650 \\ &< 1.0 \\ &< 1.0\end{aligned}$

\begin{tabular}{llll} 
Unit & & Flag & Lab \\
\cline { 3 - 4 } $\mathrm{pH}$ & 0 & GE \\
$\mu \mathrm{S} / \mathrm{cm}$ & 0 & $\mathrm{GE}$ \\
$\mu \mathrm{g} / \mathrm{L}$ & 0 & $\mathrm{GE}$ \\
$\mu \mathrm{g} / \mathrm{L}$ & 0 & $\mathrm{GE}$ \\
$\mu \mathrm{g} / \mathrm{L}$ & 0 & $\mathrm{GE}$ \\
$\mu \mathrm{g} / \mathrm{L}$ & 0 & $\mathrm{GE}$ \\
$\mu \mathrm{g} / \mathrm{L}$ & 0 & $\mathrm{GE}$ \\
$\mu \mathrm{g} / \mathrm{L}$ & 0 & $\mathrm{GE}$ \\
$\mu \mathrm{g} / \mathrm{L}$ & 0 & $\mathrm{GE}$ \\
$\mu \mathrm{g} / \mathrm{L}$ & 0 & $\mathrm{GE}$ \\
$\mu \mathrm{g} / \mathrm{L}$ & 0 & $\mathrm{GE}$ \\
$\mu \mathrm{g} / \mathrm{L}$ & 0 & $\mathrm{GE}$ \\
$\mu \mathrm{g} / \mathrm{L}$ & 0 & $\mathrm{GE}$ \\
$\mu \mathrm{g} / \mathrm{L}$ & 0 & $\mathrm{GE}$ \\
$\mu \mathrm{g} / \mathrm{L}$ & 0 & $\mathrm{GE}$ \\
$\mu \mathrm{g} / \mathrm{L}$ & 0 & $\mathrm{GE}$
\end{tabular}

Fourth Quarter 1991 
WELL LFW 34, laboratory analyses (cont.)

\begin{tabular}{|c|c|c|c|c|c|}
\hline$\underline{H} \underline{D}$ & Analyte & Result & $\underline{\text { Unit }}$ & Flag & $\underline{\text { Lab }}$ \\
\hline & Chloroethene (Vinyl chloride) & $<1.0$ & $\mu g / L$ & 0 & GE \\
\hline • & 2-Chloroethyl vinyl ether & $<1.0$ & $\mu g / L$ & 0 & GE \\
\hline & Chloroform & $<1.0$ & $\mu g / L$ & 0 & GE \\
\hline 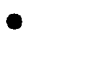 & Chloromethane (Methyl chloride) & $<1.0$ & $\mu g / L$ & 0 & GE \\
\hline & Chromium & $<4.0$ & $\mu g / L$ & 0 & GE \\
\hline & Copper & $<4.0$ & $\mu g / L$ & 0 & GE \\
\hline & Dibromochloromethane & $<1.0$ & $\mu g / L$ & 0 & GE \\
\hline & 1,1-Dichloroethane & $<1.0$ & $\mu g / L$ & 0 & GE \\
\hline & 1.2-Dichloroethane & $<1.0$ & $\mu g / L$ & 0 & GE \\
\hline 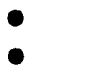 & 1,1-Dichloroethylene & $<1.0$ & $\mu g / L$ & 0 & GE \\
\hline & trans-1,2-Dichloroethylene & $<1.0$ & $\mu g / L$ & 0 & GE \\
\hline & Dichloromethane (Methylene chloride) & $<1.0$ & $\mu g / L$ & 0 & GE \\
\hline & 2,4-Dichlorophenoxyacetic acid & $<0.30$ & $\mu g / L$ & 0 & GE \\
\hline & 1,2-Dichloropropane & $\begin{array}{l}<1.0 \\
<1.0\end{array}$ & $\mu g / L$ & 0 & GE \\
\hline & trans-1,3-Dichloropropene & & $\mu g / L$ & 0 & GE \\
\hline & $\begin{array}{l}\text { cis-1,3-Dichloropropene } \\
\text { Endrin }\end{array}$ & $\begin{array}{l}<1.0 \\
<0.0060\end{array}$ & $\mu g / L$ & 0 & GE \\
\hline & & $<1.0$ & $\begin{array}{l}\mu g / L \\
\mu g / L\end{array}$ & $\begin{array}{l}0 \\
0\end{array}$ & $\begin{array}{l}\text { GE } \\
G E\end{array}$ \\
\hline & Fluoride & $<100$ & $\mu g / L$ & 0 & $\begin{array}{l}\text { GE } \\
\text { GE }\end{array}$ \\
\hline & Lead & $<3.0$ & $\mu g / L$ & 0 & GE \\
\hline & Lindane & $<0.0050$ & $\mu g / L$ & 0 & GE \\
\hline & Magnesium & 460 & $\mu g / L$ & 0 & GE \\
\hline & Manganese & 2.3 & $\mu g / L$ & 0 & GE \\
\hline & Mercury & $<0.20$ & $\mu g / L$ & 0 & GE \\
\hline & Methoxychlor & $<0.50$ & $\mu g / L$ & 0 & GE \\
\hline & Nickel & $<4.0$ & $\mu g / L$ & 0 & GE \\
\hline - & Nitrate as nitrogen & 450 & $\mu g / L$ & 0 & GE \\
\hline & Potassium & $<500$ & $\mu g / L$ & 0 & GE \\
\hline & Selenium & $<2.0$ & $\mu g / L$ & 0 & GE \\
\hline & Silica & 6,260 & $\mu g / L$ & 0 & GE \\
\hline & Silver & $<2.0$ & $\mu g / L$ & 0 & GE \\
\hline & Sodium & 1,320 & $\mu g / L$ & 0 & GE \\
\hline & Sulfate & $<1,000$ & $\mu g / L$ & 0 & GE \\
\hline - & 1,1,2,2-Tetrachloroethane & $<1.0$ & $\mu g / L$ & 0 & GE \\
\hline & Tetrachloroethylene & $<1.0$ & $\mu g / L$ & 0 & GE \\
\hline 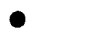 & Toluene & $<1.0$ & $\mu g / L$ & 0 & GE \\
\hline & Total organic carbon & $<1,000$ & $\mu g / L$ & 0 & GE \\
\hline & Total organic halogens & $<5.0$ & $\mu g / L$ & 0 & GE. \\
\hline & Total organic halogens & $<5.0$ & $\mu g / L$ & 0 & GE \\
\hline & Toxaphene & $<0.24$ & $\mu g / L$ & 0 & GE \\
\hline & 2,4,5-TP (Silvex) & $<0.090$ & $\mu g / L$ & 0 & GE \\
\hline & $1,1,1$-Trichloroethane & $<1.0$ & $\mu g / L$ & 0 & GE \\
\hline & 1,1,2-Trichloroethane & $<1.0$ & $\mu g / L$ & 0 & GE \\
\hline & Trichloroethylene & $<1.0$ & $\mu \mathrm{g} / \mathrm{L}$ & 0 & GE \\
\hline & Trichlorofluoromethane & $<1.0$ & $\mu \mathrm{g} / \mathrm{L}$ & 0 & GE \\
\hline & Vanadium & $<8.0$ & $\mu g / L$ & 0 & GE \\
\hline & Gross alpha & $<2.0 \mathrm{E}+00$ & $\mathrm{pCi} / \mathrm{L}$ & 0 & GE \\
\hline & Nonvolatile beta & $4.0 E+00 \pm 1.6 E+00$ & $\mathrm{pCi} / \mathrm{L}$ & 0 & GE \\
\hline & Total radium & $4.2 E+00 \pm 1.5 E+00$ & $\mathrm{pCi} / \mathrm{L}$ & 1 & GE \\
\hline & Tritium & $<7.0 E-01$ & $\mathrm{pCi} / \mathrm{mL}$ & 0 & GE \\
\hline
\end{tabular}

$\overline{- \text { = exceeded holding time. }}-$ = exceeded primary drinking water standard. 
WELL LFW 35

\section{SRS Coord.}

N85237.4

E45378.8

MEASUREMENTS CONDUCTED IN THE FIELD

Sample date: $10 / 24 / 91$

Depth to water: $23.18 \mathrm{ft}(7.07 \mathrm{~m})$ below TOC

Water elevation: $160.52 \mathrm{ft}(48.93 \mathrm{~m}) \mathrm{msl}$

Sp. conductance: $23 \mu \mathrm{S} / \mathrm{cm}$

Water evacuated before sampling: $47 \mathrm{gal}$

\section{LABORATORY ANALYSES}

\section{H $\underline{\text { Analyte }}$}

- $\mathrm{pH}$

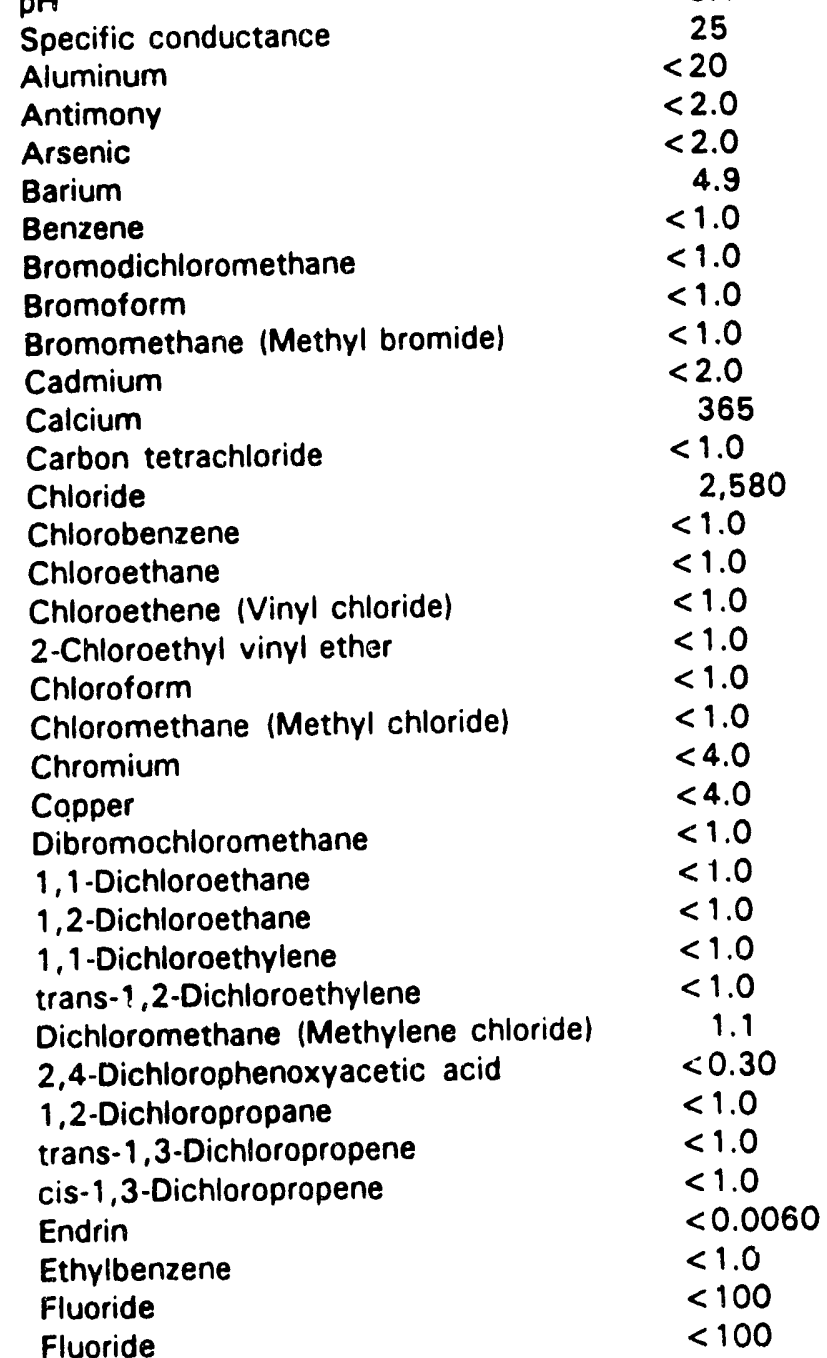

Time: 13:05

$\mathrm{pH}: 4.8$

Alkalinity: $0 \mathrm{mg} / \mathrm{L}$

Water temperature: $20.7^{\circ} \mathrm{C}$

\begin{tabular}{|c|c|c|}
\hline Result & Unit & Flag \\
\hline 5.1 & $\mathrm{pH}$ & 0 \\
\hline 25 & $\mu \mathrm{S} / \mathrm{cm}$ & 0 \\
\hline$<20$ & $\mu g / L$ & 0 \\
\hline$<2.0$ & $\mu g / L$ & 0 \\
\hline$<2.0$ & $\mu g / L$ & 0 \\
\hline 4.9 & $\mu g / L$ & 0 \\
\hline$<1.0$ & $\mu g / L$ & 0 \\
\hline$<1.0$ & $\mu g / L$ & 0 \\
\hline$<1.0$ & $\mu g / L$ & 0 \\
\hline$<1.0$ & $\mu g / L$ & 0 \\
\hline$<2.0$ & $\mu g / L$ & 0 \\
\hline 365 & $\mu g / L$ & 0 \\
\hline$<1.0$ & $\mu g / L$ & 0 \\
\hline 2,580 & $\mu g / L$ & 0 \\
\hline$<1.0$ & $\mu g / L$ & 0 \\
\hline$<1.0$ & $\mu g / L$ & 0 \\
\hline$<1.0$ & $\mu \mathrm{g} / \mathrm{L}$ & 0 \\
\hline$<1.0$ & $\mu g / L$ & 0 \\
\hline$<1.0$ & $\mu g / L$ & 0 \\
\hline$<1.0$ & $\mu g / L$ & 0 \\
\hline$<4.0$ & $\mu g / L$ & 0 \\
\hline$<4.0$ & $\mu g / L$ & 0 \\
\hline$<1.0$ & $\mu g / L$ & 0 \\
\hline$<1.0$ & $\mu \mathrm{g} / \mathrm{L}$ & 0 \\
\hline$<1.0$ & $\mu g / L$ & 0 \\
\hline$<1.0$ & $\mu g / L$ & 0 \\
\hline$<1.0$ & $\mu g / L$ & 0 \\
\hline 1.1 & $\mu g / L$ & 0 \\
\hline$<0.30$ & $\mu g / L$ & 0 \\
\hline$<1.0$ & $\mu g / L$ & 0 \\
\hline$<1.0$ & $\mu g / L$ & 0 \\
\hline$<1.0$ & $\mu g / L$ & 0 \\
\hline$<0.0060$ & $\mu \mathrm{g} / \mathrm{L}$ & 0 \\
\hline$<1.0$ & $\mu g / L$ & 0 \\
\hline$<100$ & $\mu g / L$ & 0 \\
\hline$<100$ & $\mu g / L$ & 0 \\
\hline
\end{tabular}

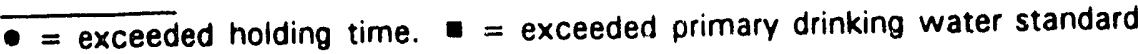


WELL LFW 35, laboratory analyses (cont.)

\begin{tabular}{|c|c|c|c|c|c|}
\hline$\underline{\mathrm{H}} \underline{\mathrm{D}}$ & Analyte & Result & Unit & Flag. & $\underline{\text { Lab }}$ \\
\hline & Lead & $<3.0$ & $\mu g / L$ & 0 & GE \\
\hline & Lindane & $<0.0050$ & $\mu g / L$ & 0 & GE \\
\hline & Magnesium & 618 & $\mu g / L$ & 0 & GE \\
\hline & Manganese & 3.2 & $\mu g / L$ & 0 & GE \\
\hline & Mercury & $<0.20$ & $\mu g / L$ & 0 & GE \\
\hline & Methoxychlor & $<0.50$ & $\mu g / L$ & 0 & GE \\
\hline & Nickel & $<4.0$ & $\mu g / L$ & 0 & GE \\
\hline & Nitrate as nitrogen & 1,240 & $\mu g / L$ & 0 & GE \\
\hline & Potassium & $<500$ & $\mu g / L$ & 0 & GE \\
\hline & Selenium & $<2.0$ & $\mu g / L$ & 0 & GE \\
\hline & Silica & 6,420 & $\mu g / L$ & 0 & GE \\
\hline & Silver & $<2.0$ & $\mu g / L$ & 0 & GE \\
\hline & Sodium & 2,710 & $\mu g / L$ & 0 & GE \\
\hline & Sulfate & $<1,000$ & $\mu g / L$ & 0 & GE \\
\hline & 1,1,2,2-Tetrachloroethane & $<1.0$ & $\mu g / L$ & 0 & GE \\
\hline & Tetrachloroethylene & $<1.0$ & $\mu g / L$ & 0 & GE \\
\hline & Toluene & $<1.0$ & $\mu g / L$ & 0 & GE \\
\hline & Total organic carbon & $<1,000$ & $\mu g / L$ & 0 & GE \\
\hline & Total organic halogens & 44 & $\mu g / L$ & q & GE \\
\hline & Total organic halogens & 45 & $\mu g / L$ & 1 & GE \\
\hline & Toxaphene & $<0.24$ & $\mu g / L$ & 0 & GE \\
\hline & 2,4,5-TP (Silvex) & $<0.090$ & $\mu g / L$ & 0 & GE \\
\hline & 1,1,1-Trichloroethane & $<1.0$ & $\mu g / L$ & 0 & GE \\
\hline & 1,1,2-Trichloroethane & $<1.0$ & $\mu g / L$ & 0 & GE \\
\hline & Trichloroethylene & $<1.0$ & $\mu g / L$ & 0 & GE \\
\hline & Trichiorofluoromethane & $<1.0$ & $\mu g / L$ & 0 & GE \\
\hline & Vanadium & $<8.0$ & $\mu g / L$ & 0 & GE \\
\hline & Gross alpha & $<2.0 E+00$ & $\mathrm{pCi} / \mathrm{L}$ & 0 & GE \\
\hline & Nonvolatile beta & $2.9 E+00 \pm 1.5 E+00$ & $\mathrm{pCi} / \mathrm{L}$ & 0 & GE \\
\hline & Total radium & $<1.0 E+00$ & $\mathrm{pCi} / \mathrm{L}$ & 0 & GE \\
\hline & Tritium & $<7.0 \mathrm{E}-01$ & $\mathrm{pCi} / \mathrm{mL}$ & 0 & GE \\
\hline
\end{tabular}

\section{WELL LFW 36}

SRS Coord.

N83535.5

E45582.3

\section{Screen Zone Elevation}

151.3-130.3 ft msl
Too of Casing

$170.4 \mathrm{ft} \mathrm{msl}$
Casing

4" PVC

\section{MEASUREMENTS CONDUCTED IN THE FIELD}

Sample date: 10/24/91

Depth to water: $23.96 \mathrm{ft}(7.30 \mathrm{~m})$ below TOC

Water elevation: $146.44 \mathrm{ft}(44.64 \mathrm{~m}) \mathrm{msl}$

Sp. conductance: $323 \mu \mathrm{S} / \mathrm{cm}$

Water evacuated before sampling: $45 \mathrm{gal}$
Time: $12: 35$

pH: 6.4

Alkalinity: $96 \mathrm{mg} / \mathrm{L}$

Water temperature: $20.2^{\circ} \mathrm{C}$

- = exceeded holding time. = exceeded primary drinking water standard. 
H $\underline{\text { Analyte }}$

- $\mathrm{pH}$

Specific conductance

Aluminum

Antimiony

Arsenic

Barium

Benzene

Bromodichloromethane

Bromoform

Bromomethane (Methyl bromide)

Cadmium

Calcium

Carbon tetrachloride

Chloride

Chlorobenzene

Chloroethane

Chloroethene (Vinyl chloride)

2-Chloroethyl vinyl ether

Chioroform

Chloromethane (Methyl chloride)

Chromium

Copper

Dibromochloromethane

1,1-Dichloroethane

1,2-Dichloroethane

1.9-Dichloroethylene

trans-1,2-Dichloroethylene

Dichloromethane (Methylene chloride)

- 2,4-Dichlorophenoxyacetic acid

1,2-Dichloropropane

trans-1,3-Dichloropropene

cis-1,3-Dichloropropene

Endrin

Ethylbenzene

Fluoride

Lead

Lindane

Magnesium

Manganese

Mercury

Methoxychlor

Nickel

Nitrate as nitrogen

Potassium

Selenium

Silica

Silver

Sodium

Sulfate

1,1,2,2-Tetrachloroethane

Tetrachloroethylene
Result

6.4

240

$<20$

$<2.0$

$<2.0$

4.0

4.9

$<1.0$

$<1.0$

$<1.0$

$<2.0$

5,610

$<1.0$

25,300

6.0

4.1

$<1.0$

$<1.0$

$<1.0$

$<1.0$

$<4.0$

$<4.0$

$<1.0$

72

2.9

$<1.0$

$<1.0$

5.2

$<0.30$

$<1.0$

$<1.0$

$<1.0$

$<0.0060$

1.7

127

$<3.0$

$<0.0050$

15,800

21

$<0.20$

$<0.50$

$<4.0$

$<50$

$<500$

$<2.0$

7,530

$<2.0$

26,700

2,640

$<1.0$

$<1.0$
Unit $\quad \underline{F l a g} \quad \underline{\text { Lab }}$

$\mathrm{pH} \quad 0 \quad \mathrm{GE}$

$\mu \mathrm{S} / \mathrm{cm} \quad 0 \quad \mathrm{GE}$

$\mu g / L \quad 0 \quad$ GE

$\mu g / L \quad 0 \quad$ GE

$\mu g / L \quad 0 \quad G E$

$\mu g / L \quad 0 \quad$ GE

$\mu g / L \quad 1 \quad$ GE

$\mu g / L \quad O \quad$ GE

$\mu g / L \quad 0 \quad G E$

$\mu g / L \quad 0 \quad G E$

$\mu g / L \quad 0 \quad$ GE

$\mu g / L \quad O \quad G E$

$\mu g / \mathrm{h} \quad 0 \quad-\mathrm{GE}$

$\mu g / L \quad 0 \quad$ GE

$\mu g / L \quad 1 \quad$ GE

$\mu g / L \quad 0 \quad$ GE

$\mu g / L \quad O \quad$ GE

$\mu g / L \quad 0 \quad$ GE

$\mu g / L \quad 0 \quad$ GE

$\mu g / L \quad 0 \quad$ GE

$\mu g / L \quad O \quad G E$

$\mu \mathrm{g} / \mathrm{L} \quad 0 \quad \mathrm{GE}$

$\mu g / L \quad 0 \quad$ GE

$\mu g / L \quad 2 \quad$ GE

$\mu g / L \quad 1 \quad$ GE

$\mu g / L \quad 0 \quad$ GE

$\mu g / L \quad 0 \quad$ GE

$\mu g / L \quad 0 \quad$ GE

$\mu g / L \quad 0 \quad$ GE

$\mu g / L \quad 0 \quad$ GE

$\mu g / L \quad 0 \quad$ GE

$\mu g / L \quad 0 \quad$ GE

$\mu g / L \quad 0 \quad$ GE

$\mu \mathrm{g} / \mathrm{L} \quad \mathrm{O} \quad \mathrm{GE}$

$\mu g / L \quad 0 \quad$ GE

$\mu g / L \quad 0 \quad$ GE

$\mu g / L \quad 0 \quad$ GE

$\mu g / L \quad 0 \quad$ GE

$\mu g / L \quad 0 \quad G E$

$\mu g / L \quad 0 \quad$ GE

$\mu g / L \quad 0 \quad$ GE

$\mu g / L \quad 0 \quad$ GE

$\mu g / L \quad 0 \quad$ GE

$\mu g / L \quad O \quad$ GE

$\mu g / L \quad 0 \quad G E$

$\mu g / L \quad 0 \quad G E$

$\mu g / L \quad 0 \quad$ GE

$\mu g /$ L $\quad 0 \quad$ GE

$\mu g / L \quad 0 \quad$ GE

$\mu g / L \quad 0 \quad$ GE

$\mu g / L \quad 0 \quad$ GE

$\overline{-=\text { exceeded holding time. }}-$ = exceeded primary drinking water standard 
WELL LFW 36, laboratory analyses (cont.)

H D Analute

Toluene

Total organic carbon

Total organic halogens

Toxaphene

- 2,4,5-TP (Silvex)

1,1,1-Trichloroethane

1,1,2-Trichloroethane

Trichloroethylene

Trichlorofluoromethane

Vanadium

Gross alpha

Nonvolatile beta

Total radium

Tritium

Result
1.0
5.000
309
$<0.24$
0.58
4.1
$<1.0$
1.3
16
$<8.0$
$5.9 E+00 \pm 1.9 E+00$
$4.3 E+00 \pm 1.6 E+00$
$1.9 E+00 \pm 1.5 E+00$
$1.6 E+00 \pm 7.0 E-01$

$\begin{array}{lll}\text { Unit } & \text { Flag } & \text { Lab } \\ \mu g / L & 0 & \mathrm{GE} \\ \mu g / \mathrm{L} & 1 & \mathrm{GE} \\ \mu \mathrm{g} / \mathrm{L} & 2 & \mathrm{GE} \\ \mu g / \mathrm{L} & 0 & \mathrm{GE} \\ \mu g / \mathrm{L} & 0 & \mathrm{GE} \\ \mu g / \mathrm{L} & 0 & \mathrm{GE} \\ \mu g / \mathrm{L} & 0 & \mathrm{GE} \\ \mu \mathrm{g} / \mathrm{L} & 0 & \mathrm{GE} \\ \mu \mathrm{g} / \mathrm{L} & 2 & \mathrm{GE} \\ \mu \mathrm{g} / \mathrm{L} & 0 & \mathrm{GE} \\ \mathrm{pCi} / \mathrm{L} & 0 & \mathrm{GE} \\ \mathrm{pCi} / \mathrm{L} & 0 & \mathrm{GE} \\ \mathrm{pCi} / \mathrm{L} & 0 & -\mathrm{GE} \\ \mathrm{pCi} / \mathrm{mL} & 0 & \mathrm{GE}\end{array}$

\section{WELL LFW 37}

\section{SRS Coord.}

N83113.2

E45667.7

\section{Screen Zone Elevation}

$150.8-129.8 \mathrm{ft} \mathrm{msl}$
Top of Casing

$169.9 \mathrm{ft} \mathrm{msl}$
Casing

4" PVC

\section{MEASUREMENTS CONDUCTED IN THE FIELD}

Sample date: 10/24/91

Depth to water: $26.84 \mathrm{ft}(8.18 \mathrm{~m})$ below TOC

Water elevation: $143.06 \mathrm{ft}(43.61 \mathrm{mi} \mathrm{msl}$

Sp. conductance: $115 \mu \mathrm{S} / \mathrm{cm}$

Water evacuated before sampling: $37 \mathrm{gal}$

\section{LABORATORY ANALYSES}

H D Analyte

$\mathrm{pH}$

Specific conductance

Aluminum

Antimony

Arsenic

Barium

Benzene

Bromodichloromethane

Bromoform

Bromomethane (Methyl bromide)

Cadmium

Calcium

Carbon tetrachloride

Chloride

Chlorobenzene

Chloroethane
Result

$\begin{aligned} & 5.3 \\ & 110 \\ &<20 \\ & 2.3 \\ &<2.0 \\ & 6.5 \\ & 4.9 \\ &<1.0 \\ &<1.0 \\ &<1.0 \\ &<2.0 \\ & 1.700 \\ &<1.0 \\ & 23.100 \\ &<1.0 \\ &<1.0\end{aligned}$

Time: 12:05

$\mathrm{pH}: 5.1$

Alkalinity: $0 \mathrm{mg} / \mathrm{L}$

Water temperature: $19.7^{\circ} \mathrm{C}$ 
WELL LFW 37, laboratory analyses (cont.)

H D Analyte

Chloroethene (Vinyl chloride)

2-Chloroethyl vinyl ether

Chloroform

Chloromethane (Methyl chloride)

Chromium

Copper

Dibromochloromethane

1,1-Dichloroethane

1,2-Dichloroethane

1,1-Dichloroethylene

trans-1,2-Dichloroethylene

Dichloromethane (Methylene chloride)

- 2,4-Dichlorciphenoxyacetic acid

1,2-Dichloropropane

trans-1,3-Dichloropropene

cis-1,3-Dichloropropene

Endrin

Ethylbenzene

Fluoride

Lead

Lindane

Magnesium

Manganese

Mercury

Methoxychlor

Nickel

Nitrate as nitrogen

Potassium

Selenium

Silica

Silver

Sodium

Sulfate

1,1,2,2-Tetrachloroethane

Tetrachloroethylene

Toluene

Total organic carbon

Total organic halogens

Toxaphene

- $\quad 2,4,5-$ TP (Silvex)

$1,1,1$-Trichloroethane

1,1,2-Trichloroethane

- Trichloroethylene

Trichlorofluoromethane

Vanadium

Gross alpha

Nonvolatile beta

Total radium

Tritium

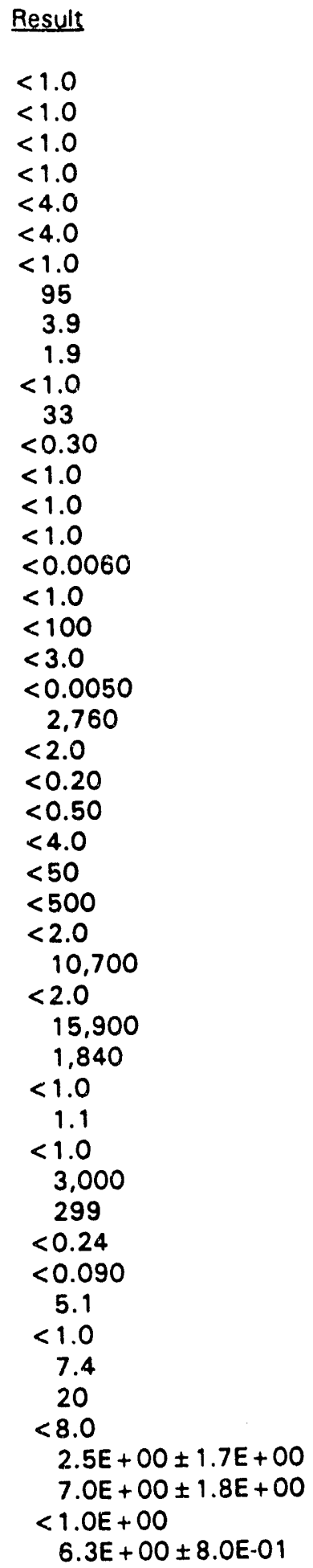

$\mu g / L \quad O \quad G E$

$\mu g / L \quad 0 \quad$ GE

$\mu g / L \quad O \quad G E$

$\mu g / L \quad 0 \quad$ GE

$\mu g / L \quad 0 \quad G E$

$\mu g / L \quad 0 \quad G E$

$\mu g / L \quad 0 \quad G E$

$\mu g / L \quad 2 \quad G E$

$\mu g / L \quad 1 \quad G E$

$\mu g / L \quad 0 \quad$ GE

$\mu g / L \quad 0 \quad$ GE

$\mu g / L \quad 0 \quad$ GE

$\mu g / L \quad 0 \quad-\quad G E$

$\mu g / L \quad 0 \quad$ GE

$\mu g / L \quad O \quad G E$

$\mu g / L \quad 0 \quad G E$

$\mu g / L \quad 0 \quad G E$

$\mu g / L \quad 0 \quad$ GE

$\mu g / L \quad 0 \quad G E$

$\mu g / L \quad 0 \quad$ GE

$\mu g / L \quad 0 \quad$ GE

$\mu g / L \quad 0 \quad G E$

$\mu g / L \quad 0 \quad$ GE

$\mu g / L \quad 0 \quad$ GE

$\mu g / L \quad 0 \quad$ GE

$\mu g / L \quad 0 \quad$ GE

$\mu g / L \quad 0 \quad$ GE

$\mu g / L \quad 0 \quad$ GE

$\mu g / L \quad 0 \quad$ GE

$\mu g$ h $\quad 0$ GE

$\mu g / L \quad 0 \quad$ GE

$\mu g / L \quad 0 \quad$ GE

$\mu g / \mathrm{L} \quad 0 \quad \mathrm{GE}$

$\mu g / L \quad 0 \quad$ GE

$\mu g / L \quad 0 \quad$ GE

$\mu g / L \quad 0 \quad$ GE

$\mu g / L \quad 0 \quad$ GE

$\mu g / L \quad 2 \quad$ GE

$\mu g / L \quad 0 \quad G E$

$\mu g / L \quad 0 \quad$ GE

$\mu g / L \quad 0 \quad$ GE

$\mu g / L \quad 0 \quad G E$

$\mu g / L \quad 2 \quad G E$

$\mu g / L \quad 2 \quad \mathrm{GE}$

$\mu g / L \quad 0 \quad$ GE

pCi/L $0 \quad$ GE

PCi/L $\quad 0 \quad$ GE

$\mathrm{pCi} / \mathrm{L} \quad 0 \quad \mathrm{GE}$

$\mathrm{pCi} / \mathrm{mL} \quad 0 \quad \mathrm{GE}$

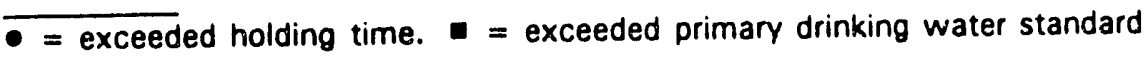




\section{WELL LFW 38}

SRS Coord.

N83172.3

E46018.5

\section{MEASUREMENTS CONDUCTED IN THE FIELD}

Sample date: 10/24/91

Depth to water: $26.45 \mathrm{ft}(8.06 \mathrm{~m})$ below TOC

Water elevation: $143.85 \mathrm{ft}(43.85 \mathrm{~m}) \mathrm{msl}$

Sp. conductance: $54 \mu \mathrm{S} / \mathrm{cm}$

Water evacuated before sampling: $38 \mathrm{gal}$

\section{LABORATORY ANALYSES}

.

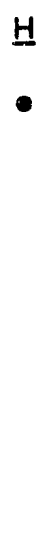

\section{Screen Zone Elevation}

$151.5-130.5 \mathrm{ft} \mathrm{msl}$
Casing

$170.3 \mathrm{ft} \mathrm{msl} \quad$ 4" PVC
Time: 11:40

pH: 4.6

Alkalinity: $0 \mathrm{mg} / \mathrm{L}$

Water temperature: $20.1^{\circ} \mathrm{C}$

\begin{tabular}{|c|c|c|c|}
\hline Result & Unit & Flag & $\underline{L a b}$ \\
\hline 4.9 & $\mathrm{pH}$ & 0 & GE \\
\hline 50 & $\mu \mathrm{S} / \mathrm{cm}$ & 0 & GE \\
\hline 30 & $\mu g / L$ & 0 & GE \\
\hline$<2.0$ & $\mu g / L$ & 0 & GE \\
\hline$<2.0$ & $\mu g / L$ & 0 & GE \\
\hline 6.3 & $\mu g /$ & 0 & GE \\
\hline 2.6 & $\mu g / L$ & 1 & GE \\
\hline$<1.0$ & $\mu g / L$ & 0 & GE \\
\hline$<1.0$ & $\mu g / L$ & 0 & GE \\
\hline$<1.0$ & $\mu g / L$ & 0 & GE \\
\hline$<2.0$ & $\mu g / L$ & 0 & GE \\
\hline 849 & $\mu g / L$ & 0 & GE \\
\hline$<1.0$ & $\mu g / L$ & 0 & GE \\
\hline 11,200 & $\mu g / L$ & 0 & GE \\
\hline 10,900 & $\mu g / L$ & 0 & GE \\
\hline$<1.0$ & $\mu g / L$ & 0 & GE \\
\hline$<1.0$ & $\mu g / L$ & 0 & GE \\
\hline$<1.0$ & $\mu g / L$ & 0 & GE \\
\hline$<1.0$ & $\mu g / L$ & 0 & GE \\
\hline 2.1 & $\mu g / L$ & 0 & GE \\
\hline$<1.0$ & $\mu g / L$ & 0 & GE \\
\hline$<4.0$ & $\mu g / L$ & 0 & GE \\
\hline$<4.0$ & $\mu_{g} / L$ & 0 & GE \\
\hline$<1.0$ & $\mu g / L$ & 0 & GE \\
\hline 42 & $\mu g / L$ & 2 & GE \\
\hline 1.5 & $\mu g / L$ & 0 & GE \\
\hline 2.7 & $\mu g / L$ & 0 & GE \\
\hline$<1.0$ & $\mu g / L$ & 0 & GE \\
\hline 37 & $\mu g / L$ & 0 & GE \\
\hline$<0.30$ & $\mu g \Omega$ & 0 & GE \\
\hline$<1.0$ & $\mu g /$ & 0 & GE \\
\hline$<1.0$ & $\mu g / L$ & 0 & GE \\
\hline$<1.0$ & $\mu g / L$ & 0 & GE \\
\hline$<0.0060$ & $\mu g / L$ & 0 & GE \\
\hline$<1.0$ & $\mu g / L$ & 0 & GE \\
\hline$<100$ & $\mu_{g} / L$ & 0 & GE \\
\hline
\end{tabular}

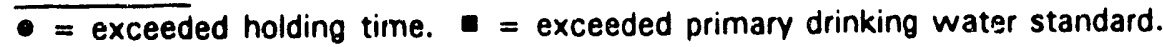


WELL LFW 38, laboratory analyses (cont.)

\begin{tabular}{|c|c|c|c|c|c|}
\hline \multirow[t]{25}{*}{$\underline{H} \underline{D}$} & Analyte & Result & Unit & Flag & $\underline{L a b}$ \\
\hline & Lead & $<3.0$ & $\mu g / L$ & 0 & GE \\
\hline & Lindane & $<0.0050$ & $\mu g / L$ & 0 & GE \\
\hline & Magnesium & 1,070 & $\mu g / L$ & 0 & GE \\
\hline & Manganese & 8.6 & $\mu g / L$ & 0 & GE \\
\hline & Mercury & $<0.20$ & $\mu g / L$ & 0 & GE \\
\hline & Methoxychlor & $<0.50$ & $\mu g / L$ & 0 & GE \\
\hline & Nickel & $<4.0$ & $\mu g / L$ & 0 & GE \\
\hline & Nitrate as nitrogen & $<50$ & $\mu g / L$ & 0 & GE \\
\hline & Nitrate as nitrogen & $<50$ & $\mu g / L$ & 0 & GE \\
\hline & Potassium & $<500$ & $\mu \mathrm{g} / \mathrm{L}$ & 0 & GE \\
\hline & Selenium & $<2.0$ & $\mu g / L$ & 0 & GE \\
\hline & Silica & 10,500 & $\mu g / L$ & 0 & GE \\
\hline & Silver & $<2.0$ & $\mu g / L$ & 0 & GE \\
\hline & Sodium & 6,460 & $\mu g / L$ & 0 & GE \\
\hline & Sulfate & $<1,000$ & $\mu g / L$ & 0 & GE \\
\hline & 1,1,2,2-Tetrachloroethane & $<1.0$ & $\mu g / L$ & 0 & GE \\
\hline & Tetrachloroethylene & 2.7 & $\mu g / L$ & 1 & GE \\
\hline & Toluene & $<1.0$ & $\mu g / L$ & 0 & GE \\
\hline & Total organic carbon & 1.000 & $\mu g / L$ & 0 & GE \\
\hline & Total organic halogens & 236 & $\mu g / L$ & 2 & GE \\
\hline & Toxaphene & $<0.24$ & $\mu g / L$ & 0 & GE \\
\hline & 2,4,5-TP (Silvex) & $<0.090$ & $\mu g / L$ & 0 & GE \\
\hline & 1,1,1-Trichloroethane & 10 & $\mu g / L$ & 0 & GE \\
\hline & 1,1,2-Trichloroethane & $<1.0$ & $\mu g / L$ & 0 & GE \\
\hline \multirow[t]{7}{*}{ - } & Trichloroethylene & 15 & $\mu g / L$ & 2 & GE \\
\hline & Trichlorofluoromethane & 19 & $\mu \mathrm{g} / \mathrm{L}$ & 2 & GE \\
\hline & Vanadium & $<8.0$ & $\mu g / L$ & 0 & GE \\
\hline & Gross alpha & $<2.0 E+00$ & $\mathrm{pCi} / \mathrm{L}$ & 0 & GE \\
\hline & Nonvolatile beta & $3.3 E+00 \pm 1.5 E+00$ & $\mathrm{pCi} / \mathrm{L}$ & 0 & \\
\hline & Total radium & $<1.0 E+00$ & $\mathrm{pCi} / \mathrm{L}$ & 0 & GE \\
\hline & Tritium & $<7.0 \mathrm{E}-01$ & $\mathrm{pCi} / \mathrm{mL}$ & 0 & GE \\
\hline
\end{tabular}

\section{WELL LFW 39}

\section{SRS Coord.}

N83213.1

E46218.5

\section{Screen Zone Elevation}

$152.2-131.2 \mathrm{ft} \mathrm{msl}$
Top of Casing

$171.4 \mathrm{ft} \mathrm{msl}$
Casing

4" PVC

\section{MEASUREMENTS CONDUCTED IN THE FIELD}

Sample date: $10 / 24 / 91$

Depth to water: $27.31 \mathrm{ft}(8.32 \mathrm{~m})$ below TOC

Water elevation: $144.09 \mathrm{ft}(43.92 \mathrm{~m}) \mathrm{msl}$

Sp. conductance: $34 \mu \mathrm{S} / \mathrm{cm}$

Water evacuated before sampling: $36 \mathrm{gal}$
Time: $11: 15$

pH: 4.5

Alkalinity: $0 \mathrm{mg} / \mathrm{L}$

Water temperature: $20.0^{\circ} \mathrm{C}$

= exceeded holding time. $\quad=$ exceeded primary drinking water standard 


\section{LABORATORY ANALYSES}

\begin{tabular}{|c|c|c|c|c|c|}
\hline$\underline{H} \underline{D}$ & Analyte & Result & Unit & Flag & $\underline{L a b}$ \\
\hline & $\mathrm{pH}$ & 4.9 & $\mathrm{pH}$ & 0 & GE \\
\hline & Specific conductance & 30 & $\mu \mathrm{S} / \mathrm{cm}$ & 0 & GE \\
\hline & Aluminum & 26 & $\mu g / L$ & 0 & GE \\
\hline & Antimony & $<2.0$ & $\mu_{g} / L$ & 0 & GE \\
\hline & Arsenic & $<2.0$ & $\mu g / L$ & 0 & GE \\
\hline & Barium & 5.5 & $\mu g / L$ & 0 & GE \\
\hline & Benzene & $<1.0$ & $\mu g / L$ & 0 & GE \\
\hline & Bromodichloromethane & $<1.0$ & $\mu g / L$ & 0 & GE \\
\hline & Bromoform & $<1.0$ & $\mu g / L$ & 0 & GE \\
\hline & Bromomethane (Methyl bromide) & $<1.0$ & $\mu g / L$ & 0 & GE \\
\hline & Cadmium & $<2.0$ & $\mu g / L$ & 0 & GE \\
\hline & Calcium & 477 & $\mu g / L$ & 0 & GE \\
\hline & Carbon tetrachloride & $<1.0$ & $\mu g / L$ & 0 & - $\mathbf{G E}$ \\
\hline & Chloride & 4,120 & $\mu g / L$ & 0 & GE \\
\hline & Chlorobenzene & $<1.0$ & $\mu g / L$ & 0 & GE \\
\hline & Chloroethane & $<1.0$ & $\mu g / L$ & 0 & GE \\
\hline & Chloroethene (Vinyl chloride) & $<1.0$ & $\mu g / L$ & 0 & GE \\
\hline & 2-Chloroethyl vinyl ether & $<1.0$ & $\mu g / L$ & 0 & GE \\
\hline & Chloroform & $<1.0$ & $\mu g / L$ & 0 & GE \\
\hline & Chloromethane (Methyl chloride) & $<1.0$ & $\mu g / L$ & 0 & GE \\
\hline & Chromium & $<4.0$ & $\mu g / L$ & 0 & GE \\
\hline & Copper & $<4.0$ & $\mu g / L$ & 0 & GE \\
\hline & Dibromochloromethane & $<1.0$ & $\mu g / L$. & 0 & GE \\
\hline & 1,1-Dichloroethane & 42 & $\mu g / L$ & 2 & GE \\
\hline & 1,2-Dichloroethane & $<1.0$ & $\mu g / L$ & 0 & GE \\
\hline $\mathbf{0}$ & 1,1-Dichloroethylene & 34 & $\mu g / L$ & 2 & GE \\
\hline & trans-1,2-Dichloroethylene & $<1.0$ & $\mu g / L$ & 0 & GE \\
\hline & Dichloromethane (Methylene chloride) & 14 & $\mu g / L$ & 0 & GE \\
\hline & 2,4-Dichlorophenoxyacetic acid & $<0.30$ & $\mu g / L$ & 0 & GE \\
\hline & 1,2-Dichloropropane & $<1.0$ & $\mu g / L$ & 0 & GE \\
\hline & trans-1,3-Dichloropropene & $<1.0$ & $\mu g / L$ & 0 & GE \\
\hline & cis-1,3-Dichloropropene & $<1.0$ & $\mu g / L$ & 0 & GE \\
\hline & Endrin & $<0.0060$ & $\mu g / L$ & 0 & GE \\
\hline & Ethylbenzene & $<1.0$ & $\mu g / L$ & 0 & GE \\
\hline & Fluoride & $<100$ & $\mu g / L$ & 0 & GE \\
\hline & Lead & $<3.0$ & $\mu g / L$ & 0 & GE \\
\hline & Lindane & $<0.0050$ & $\mu g / L$ & 0 & GE \\
\hline & Magnesium & 879 & $\mu g / L$ & 0 & GE \\
\hline & Manganese & 2.6 & $\mu \mathrm{g} / \mathrm{L}$ & 0 & GE \\
\hline & Mercury & 1.2 & $\mu g / L$ & 1 & GE \\
\hline & Methoxychlor & $<0.50$ & $\mu g / L$ & 0 & GE \\
\hline & Nickel & $<4.0$ & $\mu g / L$ & 0 & GE \\
\hline & Nitrate as nitrogen & 990 & $\mu g / L$ & 0 & GE \\
\hline & Potassium & $<500$ & $\mu g / L$ & 0 & GE \\
\hline & Selenium & $<2.0$ & $\mu g / L$ & 0 & GE \\
\hline & Silica & 9,440 & $\mu g / L$ & 0 & GE \\
\hline & Silver & $<2.0$ & $\mu g / L$ & 0 & GE \\
\hline & Sodium & 3,310 & $\mu g / L$ & 0 & $\mathrm{Gt}$ \\
\hline & Sulfate & $<1,000$ & $\mu g / L$ & 0 & GE \\
\hline & $1,1,2,2$-Tetrachloroethane & $<1.0$ & $\mu g / L$ & 0 & jt \\
\hline $\boldsymbol{v}$ & Tetrachloroethylene & 9.0 & $\mu g / L$ & 2 & GE \\
\hline
\end{tabular}

\footnotetext{
$\overline{- \text { = exceeded holding time. }}=$ exceeded primary drinking water standard.
} 
WELL LFW 39, laboratory analyses (cont.)

\begin{tabular}{|c|c|c|c|c|c|}
\hline$\underline{\mathrm{D}}$ & Analyte & Result & Unit & Flag & $\underline{\text { Lab }}$ \\
\hline & Toluene & $<1.0$ & $\mu g / L$ & 0 & GE \\
\hline & Total organic carbon & $<1,000$ & $\mu g / L$ & 0 & GE \\
\hline & Total organic halogens & 716 & $\mu g / L$ & 2 & GE \\
\hline & Toxaphene & $<0.24$ & $\mu g / L$ & 0 & GE \\
\hline & 2,4,5-TP (Silvex) & $<0.090$ & $\mu g / L$ & 0 & GE \\
\hline - & 1,1,1-Trichloroethane & 249 & $\mu g / L$ & 2 & GE \\
\hline & $1,1,2$-Trichloroethane & $<1.0$ & $\mu g / L$ & 0 & E \\
\hline $\mathbf{a}$ & Trichloroethylene & 9.7 & $\mu g / L$ & 2 & GE \\
\hline & Trichlorofluoromethane & 206 & $\mu g / L$ & 2 & $\mathbf{E}$ \\
\hline & Vanadium & $<8.0$ & $\mu g / L$ & 0 & \\
\hline & Gross alpha & $3.3 E+00 \pm 1.4 E+00$ & $\mathrm{pCi} / \mathrm{L}$ & 0 & SE \\
\hline & Nonvolatile beta & $3.1 E+00 \pm 1.5 E+00$ & $\mathrm{pCi} / \mathrm{L}$ & 0 & GE \\
\hline & Total radium & $1.1 E+00 \pm 1.3 E+00$ & $p C i / L$ & 0 & jE \\
\hline & Tritium & $<7.0 \mathrm{E}-01$ & $\mathrm{pCi} / \mathrm{mL}$ & 0 & $F$ \\
\hline
\end{tabular}

\section{WELL LFW 40}

SRS Coord.

N83248.8

E46395.1

MEASUREMENTS CONDUCTED IN THE FIELD

Sample date: $10 / 24 / 91$

Depth to water: $27.36 \mathrm{ft}(8.34 \mathrm{~m})$ below TOC

Water elevation: $143.64 \mathrm{ft}(43.78 \mathrm{~m}) \mathrm{msl}$

Sp. conductance: $14 \mu \mathrm{S} / \mathrm{cm}$

Water evacuated before sampling: $36 \mathrm{gal}$

\section{LABORATORY ANALYSES}

H D Ainalute

- $\quad \mathrm{pH}$

Specific conductance
Aluminum
Antimony
Arsenic
Barium
Benzene
Bromodichloromethane
Bromoform
Bromomethane (Methyl bromide)
Cadmium
Calcium
Carbon tetrachloride
Chloride
Chlorobenzene
Chloroethane

Screen Zone Elevation

$152.2-131.2 \mathrm{ft} \mathrm{msl}$
Top of Casing

$171.0 \mathrm{ft} \mathrm{msl}$
Casing

4" PVC
Time: $10: 45$

$\mathrm{pH}: 4.7$

Alkalinity: $0 \mathrm{mg} / \mathrm{L}$

Water temperature: $19.9^{\circ} \mathrm{C}$

Result
$\quad 5.2$
15
$<20$
$<2.0$
$<2.0$
$<3.0$
$<1.0$
$<1.0$
$<1.0$
$<1.0$
$<2.0$
265
$<1.0$
1.780
$<1.0$
3.4

Unit Flag Lab

pH $\quad 0 \quad$ GE

$\mu \mathrm{S} / \mathrm{cm} \quad 0 \quad \mathrm{GE}$

$\mu g / L \quad 0 \quad$ GE

$\mu g / L \quad 0 \quad$ GE

$\mu g / L \quad 0 \quad$ GE

$\mu g / L \quad 0 \quad$ GE

$\mu g / L \quad 0 \quad$ GE

$\mu g / L \quad 0 \quad$ GE

$\mu g / L \quad 0 \quad G E$

$\mu g / L \quad 0 \quad G E$

$\mu g / L \quad 0 \quad$ GE

$\mu g / L \quad 0 \quad$ GE

$\mu g / L \quad 0 \quad$ GE

$\mu g / L \quad 0 \quad$ GE

$\mu g / L \quad 0 \quad G E$

$\mu g / L \quad O \quad$ GE

- exceeded holding time. = exceeded primary drinking water standard 
WELL LFW 40, laboratory analyses (cont.)

H D Analyte

Chloroethene (Vinyl chloride)

2-Chloroethyl vinyl ether

Chloroform

Chloromethane (Methyl chloride)

Chromium

Copper

Dibromochloromethane

1,1-Dichloroethane

1,2-Dichloroethane

1,1-Dichloroethylene

trans-1,2-Dichloroethylene

Dichloromethane (Methylene chloride)

2,4-Dichiorophenoxyacetic acid

1,2-nichloropropane

trans-1,3-Dichloropropene

cis-1,3-Dichloropropene

Endrin

Ethylbenzene

Fluoride

Lead

Lindane

Magnesium

Manganese

Mercury

Methoxychlor

Nickel

Nitrate as nitrogen

Potassium

Selenium

Silica

Silver

Sodium

Sulfate

1,1,2,2-Tetrachloroethane

Tetrachloroethylene

Toluene

Total organic carbon

Total organic halogens

Toxaphene

2,4,5-TP (Silvex)

1,1,1-Trichloroethane

1,1,2-Trichloroethane

- Trichloroethylene

Trichlorofluoromethane

Vanadium

Gross alpha

Nonvolatile beta

Total radium

Tritium
Result

$<1.0$

$<1.0$

$<1.0$

$<1.0$

$<4.0$

$<4.0$

$<1.0$

11

$<1.0$

3.6

$<1.0$

26

$<0.30$

$<1.0$

$<1.0$

$<1.0$

$<0.0060$

$<1.0$

$<100$

$<3.0$

$<0.0050$

320

$<2.0$

$<0.20$

$<0.50$

$<4.0$

66

$<500$

$<2.0$

6,660

$<2.0$

1,360

$<1,000$

$<1.0$

2.0

$<1.0$

$<1,000$

144

$<0.24$

$<0.090$

14

$<1.0$

5.4

63

$<8.0$

$2.5 E+00 \pm 1.3 E+00$

$<2.0 E+00$

$<1.0 E+00$

$<7.0 E-01$
Unit $\quad \underline{\text { Flag }} \quad \underline{\text { Lab }}$

$\mu g / L \quad 0 \quad G E$

$\mu g / L \quad O \quad G E$

$\mu \mathrm{g} / \mathrm{L} \quad 0 \quad \mathrm{GE}$

$\mu g / L \quad 0 \quad G E$

$\mu g / L \quad 0 \quad$ GE

$\mu g / L \quad O \quad G E$

$\mu g / L \quad 0 \quad G E$

$\mu g / L \quad 2 \quad$ GE

$\mu g / L \quad 0 \quad$ GE

$\mu g / L \quad 1 \quad$ GE

$\mu g / L \quad O \quad$ GE

$\mu g / L \quad 0 \quad G E$

$\mu g / L \quad 0 \quad-\quad G E$

$\mu g / L \quad 0 \quad G E$

$\mu g / L \quad 0 \quad$ GE

$\mu g / L \quad 0 \quad G E$

$\mu g / L \quad 0 \quad$ GE

$\mu g / L \quad 0 \quad$ GE

$\mu g / L \quad 0 \quad$ GE

$\mu g / L \quad 0 \quad G E$

$\mu g / L \quad 0 \quad$ GE

$\mu g / L \quad 0 \quad$ GE

$\mu g / L \quad 0 \quad$ GE

$\mu g / L \quad 0 \quad$ GE

$\mu g / L \quad 0 \quad$ GE

$\mu g / L \quad 0 \quad G E$

$\mu g / L \quad 0 \quad$ GE

$\mu g / L \quad 0 \quad$ GE

$\mu g / L \quad 0 \quad G E$

$\mu g / \mathrm{L} \quad 0 \quad \mathrm{GE}$

$\mu g / L \quad 0 \quad G E$

$\mu g / L \quad 0 \quad G E$

$\mu g / L \quad 0 \quad G E$

$\mu g / L \quad 0 \quad$ GE

$\mu g / L \quad 0 \quad$ GE

$\mu g / L \quad 0 \quad$ GE

$\mu g / L \quad 0 \quad$ GE

$\mu g / L \quad 2 \quad G E$

$\mu \mathrm{g} / \mathrm{L} \quad 0 \quad \mathrm{GE}$

$\mu g / L \quad 0 \quad$ GE

$\mu g / L \quad 0 \quad$ GE

$\mu g / L \quad 0 \quad$ GE

$\mu g / L \quad 2 \quad G E$

$\mu g / L \quad 2 \quad G E$

$\mu g / L \quad 0 \quad$ GE

$\mathrm{pCi} / \mathrm{L} \quad 0 \quad \mathrm{GE}$

$\mathrm{pCi} / \mathrm{L} \quad 0 \quad \mathrm{GE}$

$\mathrm{pCi} / \mathrm{L} \quad 0 \quad \mathrm{GE}$

$\mathrm{pCi} / \mathrm{mL} \quad 0 \quad \mathrm{GE}$

$\overline{-=\text { exceeded holding time. }}=$ exceeded primary drinking water standard. 


\section{WELL LFW 41}

SRS Coord.

N83304.9

E46626.9

\section{MEASUREMENTS CONDUCTED IN THE FIELD}

Sample date: 10/24/91

Depth to water: $24.95 \mathrm{ft}(7.60 \mathrm{~m})$ below TOC Water elevation: $145.55 \mathrm{ft}(44.36 \mathrm{~m}) \mathrm{msl}$

Sp. conductance: $14 \mu \mathrm{S} / \mathrm{cm}$

Water evacuated before sampling: 42 gal

\section{LABORATORY ANALYSES}

H D Analyte

pH

Specific conductance

Aluminum

Antimony

Arsenic

Barium

Benzene

Bromodichloromethane

Bromoform

Bromomethane (Methyl bromide)

Cadmium

Calcium

Carbon tetrachloride

Chloride

Chlorobenzene

Chloroethane

Chloroethene (Vinyl chloride)

2-Chloroethyl vinyl ether

Chloroform

Chloromethane (Methyl chloride)

Chromium

Copper

Dibromochloromethane

1,1-Dichloroethane

1,2-Dichloroethane

1,1-Dichloroethylene

trans-1,2-Dichloroethylene

Dichloromethane (Methylene chloride)

2,4-Dichlorophenoxyacetic acid

1,2-Dichloropropane

trans-1,3-Dichloropropene

cis-1,3-Dichloropropene

Endrin

Ethylbenzene

Fluoride

Lead $\underline{\text { Result }}$

5.3

10

$<20$

$<2.0$

$<2.0$

$<3.0$

$<1.0$

$<1.0$

$<1.0$

$<1.0$

$<2.0$

196

$<1.0$

1,900

$<1.0$

$<1.0$

$<1.0$

$<1.0$

$<1.0$

$<1.0$

$<4.0$

$<4.0$

$<1.0$

$<1.0$

$<1.0$

$<1.0$

$<1.0$

1.4

$<0.30$

$<1.0$

$<1.0$

$<1.0$

$<0.0060$

$<1.0$

$<100$

$<3.0$
Top of Casing Casing

$170.5 \mathrm{ft} \mathrm{msl} \quad 4$ " PVC
Time: 10:15

$\mathrm{pH}: 4.8$

Alkalinity: $0 \mathrm{mg} / \mathrm{L}$

Water temperature: $19.3^{\circ} \mathrm{C}$
Unit Flag Lab

$\mathrm{pH} \quad \mathrm{O} \quad \mathrm{GE}$

$\mu \mathrm{S} / \mathrm{cm} \quad 0 \quad \mathrm{GE}$

$\mu g / L \quad 0 \quad$ GE

$\mu \mathrm{g} / \mathrm{L} \quad 0 \quad$ GE

$\mu g / L \quad 0 \quad$ GE

$\mu g / L \quad 0 \quad$ GE

$\mu g / L \quad 0 \quad$ GE

$\mu g / L \quad 0 \quad$ GE

$\mu g / L \quad 0 \quad$ GE

$\mu g / L \quad 0 \quad$ GE

$\mu g / L \quad 0 \quad$ GE

$\mu g / L \quad 0 \quad$ GE

$\mu g / L \quad 0 \quad$ GE

$\mu g / L \quad 0 \quad$ GE

$\mu g / L \quad 0 \quad$ GE

$\mu \mathrm{g} / \mathrm{L} \quad 0 \quad \mathrm{GE}$

$\mu g / L \quad O \quad$ GE

$\mu g / L \quad O \quad$ GE

$\mu g / L \quad O \quad G E$

$\mu \mathrm{g} / \mathrm{L} \quad 0 \quad$ GE

$\mu g / L \quad 0 \quad$ GE

$\mu g / L \quad 0 \quad$ GE

$\mu g / L \quad 0 \quad$ GE

$\mu g / L \quad 0 \quad$ GE

$\mu g / L \quad 0 \quad$ GE

$\mu g / L \quad 0 \quad$ GE

$\mu g / L \quad 0 \quad$ GE

$\mu g / L \quad 0 \quad$ GE

$\mu g / L \quad 0 \quad$ GE

$\mu g / L \quad 0 \quad$ GE

$\mu g / L \quad 0 \quad$ GE

$\mu g / L \quad 0 \quad$ GE

$\mu \mathrm{g} / \mathrm{L} \quad 0 \quad \mathrm{GE}$

$\mu g / L \quad 0 \quad$ GE

$\mu g / L \quad 0 \quad$ GE

$\mu g / L \quad 0 \quad$ GE

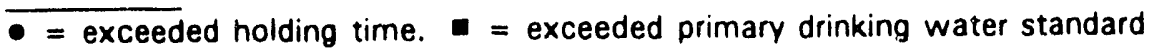


WELL LFW 41, laboratory analyses (cont.)

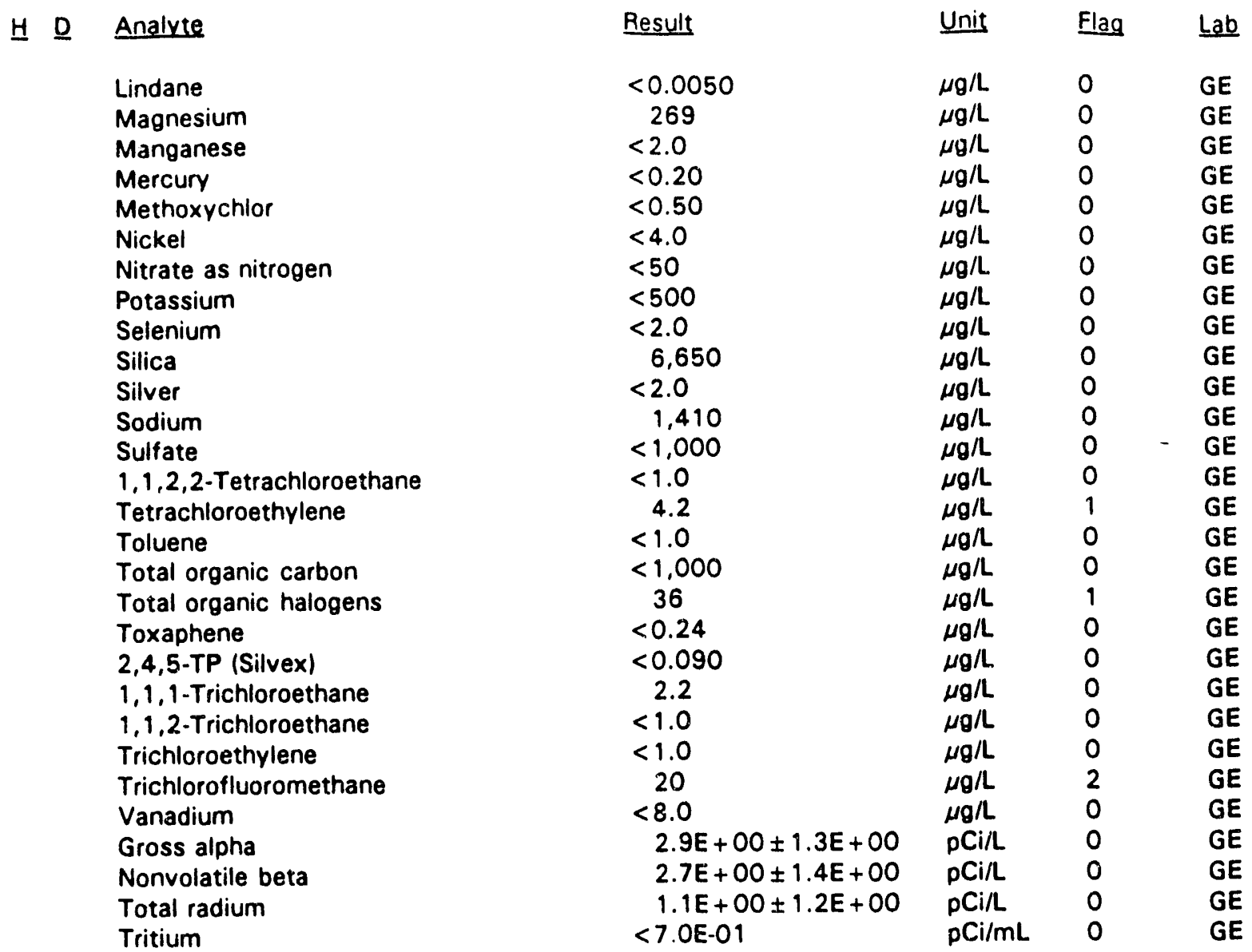

\section{WELL LFW 42}

SRS Coord.

N83776.2

E46532.9

\section{Screen Zone Elevation}

$151.2-130.2 \mathrm{ft} \mathrm{msl}$
Top of Casing

$170.1 \mathrm{ft} \mathrm{msl}$
Casing

4" PVC

MEASUREMENTS CONDUCTED IN THE FIELD

Sample date: $10 / 24 / 91$

Depth to water: $22.37 \mathrm{ft}(6.82 \mathrm{~m})$ below TOC Water elevation: $147.73 \mathrm{ft}(45.03 \mathrm{~m}) \mathrm{msl}$

Sp. conductance: $14 \mu \mathrm{S} / \mathrm{cm}$

Water evacuated before sampling: $49 \mathrm{gal}$
Time: $9: 50$

$\mathrm{pH}: 4.8$

Alkalinity: $0 \mathrm{mg} / \mathrm{L}$

Water temperature: $20.0^{\circ} \mathrm{C}$

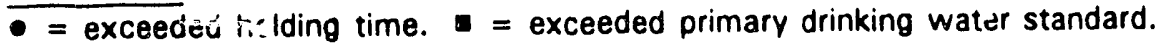


H D Analyte

$\mathrm{pH}$

Specific conductance

Aluminum

Antimony

Arsenic

Barium

Benzene

Bromodichloromethane

Bromoforrn

Bromomethane (Methyl bromide)

Cadmium

Calcium

Carbon tetrachloride

Chloride

Chloride

Chlorobenzene

Chloroethane

Chloroethene (Vinyl chloride)

2-Chloroethyl vinyl ether

Chloroform

Chloromethane (Methyl chloride)

Chromium

Copper

Dibromochloromethane

1.1-Dichloroethane

1,2-Dichloroethane

1,1-Dichloroethylene

trans-1,2-Dichloroethylene

Dichloromethane (Methylene chloride)

2,4-Dichlorophenoxyacetic acid

1,2-Dichloropropane

trans-1,3-Dichloropropene

cis-1,3-Dichloropropene

Endrin

Ethylbenzene

Fluoride

Fluoride

Lead

Lindane

Magnesium

Manganese

Mercury

Methoxychlor

Nickel

Nitrate as nitrogen

Nitrate as nitrogen

Potassium

Selenium

Silica

Silver

Sodium
Result

5.4

15
$<20$

3.0

$<2.0$

$<3.0$

$<1.0$

$<1.0$

$<1.0$

$<1.0$

$<2.0$

217

$<1.0$

1,930

1,890

$<1.0$

$<1.0$

$<1.0$

$<1.0$

$<1.0$

$<1.0$

$<4.0$

$<4.0$

$<1.0$

$<1.0$

$<1.0$

$<1.0$

$<1.0$

$<1.0$

$<0.30$

$<1.0$

$<1.0$

$<1.0$

$<0.0060$

$<1.0$

$<100$

$<100$

$<3.0$

$<0.0050$

281

$<2.0$

$<0.20$

$<0.50$

$<4.0$

$<50$

$<50$

$<500$

$<2.0$

6,840

$<2.0$

1,360
Unit Flag $\underline{\text { Lab }}$

$\mathrm{pH} \quad 0 \quad \mathrm{GE}$

$\mu \mathrm{S} / \mathrm{cm} \quad 0 \quad \mathrm{GE}$

$\mu g / L \quad 0 \quad G E$

$\mu g / L \quad 1 \quad$ GE

$\mu g / L \quad 0 \quad$ GE

$\mu g / L \quad 0 \quad$ GE

$\mu g / L \quad 0 \quad$ GE

$\mu g / L \quad 0 \quad$ GE

$\mu g / L \quad 0 \quad$ GE

$\mu g / L \quad 0 \quad$ GE

$\mu g / L \quad 0 \quad$ GE

$\mu g / L \quad 0 \quad$ GE

$\mu g / L \quad 0 \quad-$ GE

$\mu g / L \quad 0 \quad$ GE

$\mu g / L \quad 0 \quad$ GE

$\mu g / L \quad 0 \quad$ GE

$\mu g / L \quad 0 \quad$ GE

$\mu g \Omega \quad 0 \quad$ GE

$\mu g / L \quad 0 \quad$ GE

$\mu g / L \quad 0 \quad$ GE

$\mu g / L \quad 0 \quad$ GE

$\mu g / L \quad 0 \quad$ GE

$\mu g / \mathrm{L} \quad 0 \quad \mathrm{GE}$

$\mu g /$ L $\quad 0 \quad$ GE

$\mu g / L \quad 0 \quad$ GE

$\mu g /$ L $\quad 0 \quad$ GE

$\mu g / L \quad 0 \quad$ GE

$\mu g / L \quad O \quad$ GE

$\mu g / L \quad 0 \quad$ GE

$\mu g / \mathrm{L} \quad 0 \quad \mathrm{GE}$

$\mu g / L \quad O \quad$ GE

$\mu g / L \quad 0 \quad$ GE

$\mu g / L \quad 0 \quad$ GE

$\mu g / L \quad 0 \quad$ GE

$\mu g / L \quad 0 \quad$ GE

$\mu g / L \quad 0 \quad$ GE

$\mu g / L \quad 0 \quad$ GE

$\mu g / L \quad 0 \quad$ GE

$\mu g / L \quad 0 \quad$ GE

$\mu g / L \quad 0 \quad$ GE

$\mu \mathrm{g} / \mathrm{L} \quad 0 \quad \mathrm{GE}$

$\mu g / L \quad 0 \quad$ GE

$\mu g / L \quad 0 \quad$ GE

$\mu g / L \quad 0 \quad$ GE

$\mu \mathrm{g} / \mathrm{L} \quad \mathrm{O} \quad \mathrm{GE}$

$\mu g / L \quad 0 \quad$ GE

$\mu g / L \quad 0 \quad G E$

$\mu g / L \quad 0 \quad$ GE

$\mu g / L \quad O \quad$ GE

$\mu g / L \quad 0 \quad$ GE

$\mu g / L \quad O \quad$ GE

$\overline{-=\text { exceeded holding time. }}=$ exceeded primary drinking water standard 
WELL LFW 42, laboratory analyses (cont.)

\begin{tabular}{|c|c|c|c|c|}
\hline Analyte & Result & Unit & Flag & \\
\hline Sulfate & $<1,000$ & $\mu g / L$ & 0 & \\
\hline Sulfate & $<1,000$ & $\mu g / L$ & 0 & \\
\hline 1,1,2,2-Tetrachloroethane & $<1.0$ & $\mu g / L$ & 0 & \\
\hline Tetrachloroethylene & $<1.0$ & $\mu_{g} / \mathrm{L}$ & 0 & \\
\hline Tolvene & $<1.0$ & $\mu g / L$ & 0 & \\
\hline Total organic carbon & $<1,000$ & $\mu g / L$ & 0 & \\
\hline Total organic carbon & $<1,000$ & $\mu g / L$ & 0 & \\
\hline Total organic halogens & 19 & $\mu g / L$ & 0 & \\
\hline Toxaphene & $<0.24$ & $\mu g / L$ & 0 & \\
\hline 2,4,5-TP (Silvex) & $<0.090$ & $\mu g / L$ & 0 & \\
\hline 1,1,1-Trichloroethane & 6.6 & $\mu g / L$ & 0 & \\
\hline 1,1,2-Trichloroethane & $<1.0$ & $\mu g / L$ & 0 & \\
\hline Trichloroethylene & $<1.0$ & $\mu_{g} / \mathrm{L}$ & 0 & - \\
\hline Trichlorofluoromethane & 7.8 & $\mu g / L$ & 1 & \\
\hline Vanadium & $<8.0$ & $\mu g / L$ & 0 & \\
\hline Gross alpha & $4.0 E+00 \pm 1.5 E+00$ & pCill & 0 & \\
\hline Nonvolatile beta & $3.6 E+00 \pm 1.5 E+00$ & $\mathrm{pCi} / \mathrm{L}$ & 0 & \\
\hline Total radium & $<1.0 E+00$ & $\mathrm{pCi} / \mathrm{L}$ & 0 & \\
\hline Tritium & $<7.0$ E-01 & $\mathrm{pCi} / \mathrm{mL}$ & 0 & \\
\hline
\end{tabular}

\section{WELL LFW 43B}

\section{SRS Coord.}

N86459.2

$E 45240.5$

\section{MEASUREMENTS CONDUCTED IN THE FIELD}

Sample date: $10 / 28 / 91$

Depth to water: $36.08 \mathrm{ft}(11.00 \mathrm{~m})$ below TOC Water elevation: $166.92 \mathrm{ft}(50.88 \mathrm{~m}) \mathrm{ms}$ )

Sp. conductance: $30 \mu \mathrm{S} / \mathrm{cm}$

Water evacuated before sampling: $253 \mathrm{gal}$

\section{LABORATORY ANALYSES}

\section{Screen Zone Elevation}

100.4-90.4 ft msl
Top of Casing

$203.0 \mathrm{ft} \mathrm{msl}$
Casing

4" PVC
Time: 11:05

pH: 6.0

Alkalinity: $9 \mathrm{mg} / \mathrm{L}$

Water temperature: $18.5^{\circ} \mathrm{C}$

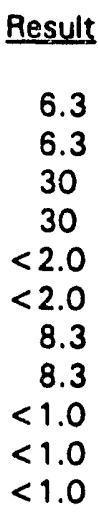

$\underline{\text { Unit }} \quad \underline{\text { Flag }} \underline{\text { Lab }}$

$\mathrm{pH} \quad \mathrm{O} \quad \mathrm{GE}$

$\mathrm{pH} \quad \mathrm{O} \quad \mathrm{GE}$

$\mu \mathrm{S} / \mathrm{cm} \quad 0 \quad \mathrm{GE}$

$\mu \mathrm{S} / \mathrm{cm} \quad 0 \quad \mathrm{GE}$

$\mu g / L \quad 0 \quad$ GE

$\mu g / L \quad 0 \quad$ GE

$\mu g / L \quad O \quad$ GE

$\mu g / L \quad 0 \quad$ GE

$\mu \mathrm{g} / \mathrm{L} \quad \mathrm{O} \quad \mathrm{GE}$

$\mu g / L \quad 0 \quad$ GE

$\mu g / L \quad$ GE

$\overline{-=\text { exceeded holding time. }}=$ = exceeded primary drinking water standard. 
WELL LFW 438, laboratory analyses (cont.)

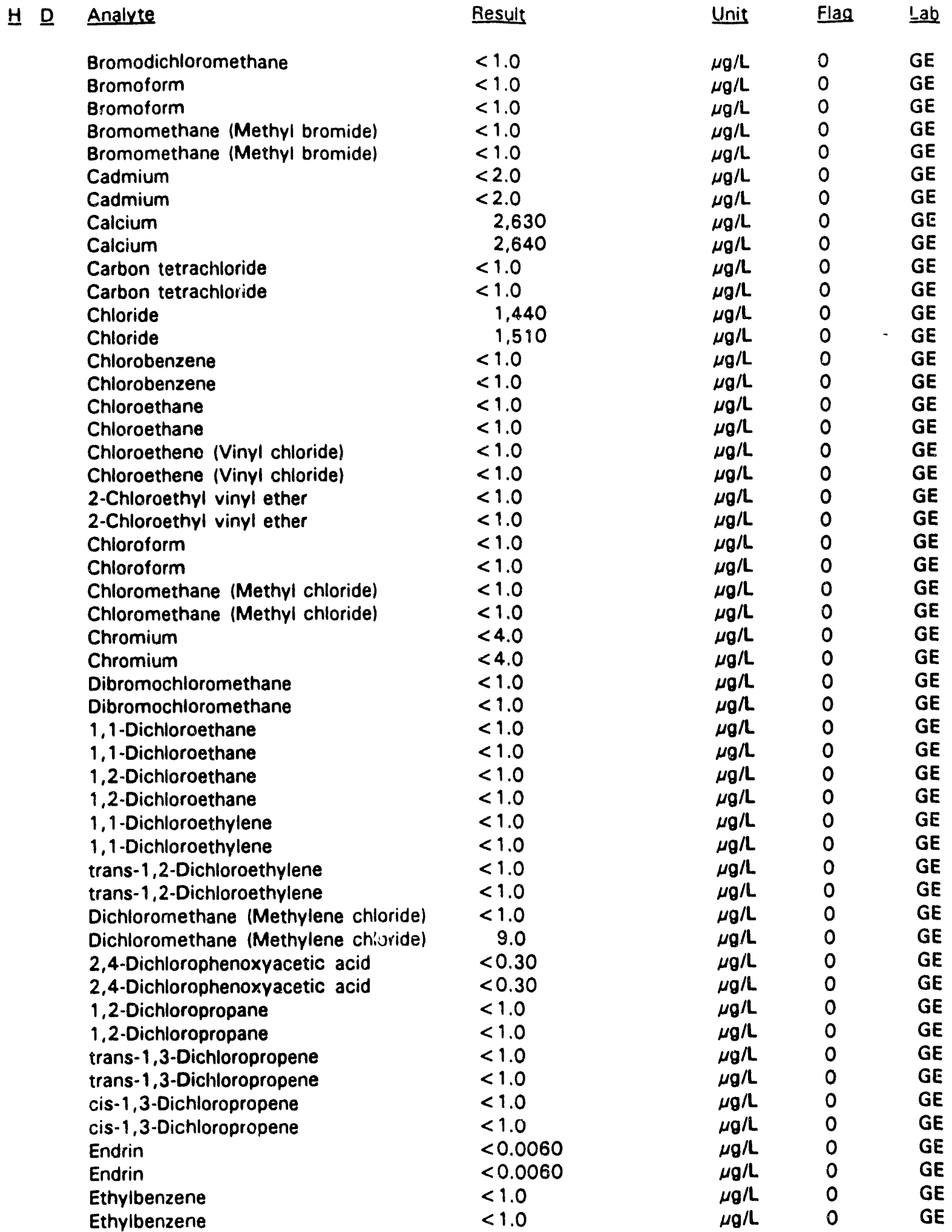

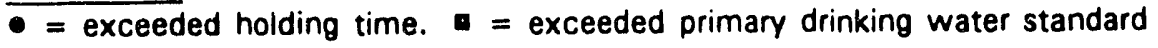


WELL LFW 43B, laboratory analyses (cont.)

\begin{tabular}{|c|c|c|}
\hline 브 $\underline{D}$ & Analyte & Result \\
\hline & Fluoride & $<100$ \\
\hline & Fluoride & $<100$ \\
\hline & Iron & 15 \\
\hline & Iron & 24 \\
\hline & Lead & $<3.0$ \\
\hline & Lead & $<3.0$ \\
\hline & Lindane & $<0.0050$ \\
\hline & Lindane & $<0.0050$ \\
\hline & Magnesium & $? 55$ \\
\hline & Magnesium & 258 \\
\hline & Manganese & 12 \\
\hline & Manganese & 12 \\
\hline & Mercury & 0.25 \\
\hline & Mercury & $<0.20$ \\
\hline & Methoxychlor & $<0.50$ \\
\hline & Methoxychlor & $<0.50$ \\
\hline & Nitrate as nitrogen & 810 \\
\hline & Nitrate as nitrogen & 810 \\
\hline & Phenols & $<5.0$ \\
\hline & Phenols & $<5.0$ \\
\hline & Phenols & $<5.0$ \\
\hline & Potassium & 534 \\
\hline & Potassium & 518 \\
\hline & Selınium & $<2.0$ \\
\hline & Selenium & $<2.0$ \\
\hline & Silica & 8,120 \\
\hline & Silica & 8,290 \\
\hline & Silver & $<2.0$ \\
\hline & Silver & $<2.0$ \\
\hline & Sodium & 2,110 \\
\hline & Súdium & 2,100 \\
\hline & Sulfate & $<1,000$ \\
\hline & Sulfate & $<1,000$ \\
\hline & $1,1,2,2$-Tetrachloroethane & $<1.0$ \\
\hline & 1,1,2,2-Tetrachloroethane & $<1.0$ \\
\hline & Tetrachloroethylene & $<1.0$ \\
\hline & Tetrachloroethylene & $<1.0$ \\
\hline & Toluene & $<1.0$ \\
\hline & Tolvene & $<1.0$ \\
\hline$\bullet$ & Total dissolved solids & 13,000 \\
\hline & Total dissolved solids & 17,000 \\
\hline & Total organic carbon & $<1,000$ \\
\hline & Total organic carbon & $<1,000$ \\
\hline & Tctal organic halogens & $<5.0$ \\
\hline & Total organic halogens & $<5.0$ \\
\hline & Total phnsphates (as P) & $<50$ \\
\hline & Total phosphates (as P) & $<50$ \\
\hline & Total phosphates (as P) & $<50$ \\
\hline & Toxaphene & $<0.24$ \\
\hline & Toxaphene & $<0.24$ \\
\hline & 2,4,5-TP (Silvex) & $<0.090$ \\
\hline
\end{tabular}

Unit Flag Lab

$\bullet$

$<100$

$<3.0$

$<0.0050$

258

0.25

$<0.20$

$<0.50$

810

$<5.0$

5.0

518

8,120

8,290

2.0

2,110

$<1,000$

1.0

17,000

1,000

$<1,000$

$<5.0$

$<50$

$<50$

$<0.24$

$<0.090$ $\mu g / L \quad 0 \quad$ GE

$\mu g / L \quad 0 \quad$ GE

$\mu g / L \quad 0 \quad G E$

$\mu g / L \quad 0 \quad$ GE

$\mu g / L \quad 0 \quad$ GE

$\mu g / L \quad 0 \quad G E$

$\mu g / L \quad 0 \quad$ GE

$\mu g / L \quad 0 \quad G E$

$\mu g / L \quad 0 \quad$ GE

$\mu g / L \quad D \quad G E$

$\mu g / L \quad 0 \quad$ GE

$\mu g / L \quad 0 \quad$ GE

$\mu_{y} \mathrm{~L} \quad 0 \quad-\mathrm{GE}$

$\mu g / L \quad 0 \quad$ GE

$\mu \mathrm{g} / \mathrm{L} \quad 0 \quad \mathrm{GE}$

$\mu g / L \quad 0 \quad$ GE

$\mu g / L \quad 0 \quad$ GE

$\mu g / L \quad 0 \quad$ GE

$\mu g / L \quad 0 \quad G E$

$\mu g / \mathrm{L} \quad 0 \quad \mathrm{GE}$

$\mu g / L \quad 0 \quad$ GE

$\mu g / L \quad O \quad$ GE

$\mu g / L \quad 0 \quad$ GE

$\mu g / L \quad 0 \quad$ GE

$\mu g / L \quad 0 \quad$ GE

$\mu g / L \quad 0 \quad G E$

$\mu g / L \quad 0 \quad$ GE

$\mu g / L \quad 0 \quad G E$

$\mu g / L \quad 0 \quad G E$

$\mu g / L \quad 0 \quad$ GE

$\mu g / L \quad 0 \quad G E$

$\mu \mathrm{g} / \mathrm{L} \quad 0 \quad \mathrm{GE}$

$\mu g / L \quad 0 \quad$ GE

$\mu g / L \quad 0 \quad$ GE

$\mu g / L \quad 0 \quad$ GE

$\mu g / L \quad 0 \quad$ GE

$\mu g / L \quad 0 \quad$ GE

$\mu g / L \quad 0 \quad$ GE

$\mu g / L \quad 0 \quad$ GE

$\mu g / L \quad 0 \quad G E$

$\mu g / L \quad 0 \quad$ GE

$\mu g / L \quad O \quad G E$

$\mu g / L \quad 0 \quad G E$

$\mu g / L \quad 0 \quad$ GE

$\mu g / L \quad 0 \quad$ GE

$\mu g / L \quad 0 \quad G E$

$\mu g / 2 \quad 0 \quad$ GE

$\mu g / L \quad 0 \quad$ GE

$\mu g / L \quad 0 \quad$ GE

$\mu g / L \quad 0 \quad G E$

$\mu g / L \quad 0 \quad$ GE

- = exceeded holding time. $=$ exceeded primary drinking water standard. 
WELL LFW 43B, laboratory analyses (cont.)

므 Analyte

2,4,5-TP (Silvex)
1,1,1-Trichloroethane
1,1,1-Trichloroethane
1,1,2-Trichloroethane
1,1,2-Trichloroethane
Trichloroethylene
Trichloroethylene
Trichlorofluoromethane
Trichlorofluoromethane
Gross alpha
Gross alpha
Nonvolatile beta
Nonvolatile beta
Total radium
Total radium
Tritium
Tritium

$\begin{aligned} & \text { Result } \\ &<0.090 \\ &<1.0 \\ &<1.0 \\ &<1.0 \\ &<1.0 \\ &<1.0 \\ &<1.0 \\ &<1.0 \\ &<1.0 \\ &<2.0 E+00 \\ &<2.0 E+00 \\ &<2.0 E+00 \\ &<2.0 E+00 \\ & 1.0 E+00 \pm 1.4 E+00 \\ &<1.0 E+00 \\ &<7.0 E-01 \\ &<7.0 E-01\end{aligned}$

Unit Flag Lab

$\mu g / L \quad O \quad$ GE

$\mu g / L \quad O \quad$ GE

$\mu g / L \quad$ GE

$\mu g / L \quad O \quad G E$

$\mu g / L \quad 0 \quad$ GE

$\mu g / L \quad O \quad$ GE

$\mu g / L \quad 0 \quad G E$

$\mu g / L \quad$ GE

$\mu g / L \quad O \quad G E$

$\mathrm{pCi} / \mathrm{L} \quad \mathrm{O} \quad \mathrm{GE}$

$\mathrm{pCi} / \mathrm{L} \quad \mathrm{O}$ GE

$\mathrm{pCi} / \mathrm{L} \quad \mathrm{O}$ GE

$\mathrm{pCi} / \mathrm{L} \quad \mathrm{O}-\mathrm{GE}$

$\mathrm{pCi} / \mathrm{L} \quad \mathrm{O}$ GE

pCi/L $\quad 0 \quad$ GE

$\mathrm{pCi} / \mathrm{mL} \quad 0 \quad \mathrm{GE}$

$\mathrm{pCi} / \mathrm{mL} \quad 0 \quad$ GE

\section{WELL LFW 43B}

\section{SRS Coord.}

N86459.2

E45240.5

\section{Screen Zone Elevation}

100.4-90.4 ft msl
Top of Casing Casing

$203.0 \mathrm{ft} \mathrm{msl} \quad$ 4" PVC $^{\circ}$

MEASUREMENTS CONDUCTED IN THE FIELD

Sample date: 10/28/91

Depth to water: $36.08 \mathrm{ft}(11.00 \mathrm{~m})$ below TOC

Water elevation: $166.92 \mathrm{ft}(50.88 \mathrm{~m}) \mathrm{msl}$

Sp. conductance: $30 \mu \mathrm{S} / \mathrm{cm}$

Water evacuated before sampling: $253 \mathrm{gal}$

WELL LFW 43C

$\underline{\text { SRS Coord. }}$

Screen Zone Elevation

N86480.6

E45234.9
Time: 11:05

pH: 6.0

Alkalinity: $9 \mathrm{mg} / \mathrm{L}$

Water temperature: $18.5^{\circ} \mathrm{C}$

\section{MEASUREMENTS CONDUCTED IN THE FIELD}

Sample date: 10/28/91

Depth to water: $35.36 \mathrm{ft}(10.78 \mathrm{~m})$ below TOC

Water elevation: $167.24 \mathrm{ft}(50.98 \mathrm{~m}) \mathrm{msl}$

Sp. conductance: $21 \mu \mathrm{S} / \mathrm{cm}$

Water evacuated before sampling: $103 \mathrm{gal}$
Time: 10:30

$\mathrm{pH}: 5.3$

Alkalinity: $3 \mathrm{mg} / \mathrm{L}$

Water temperature: $18.3^{\circ} \mathrm{C}$

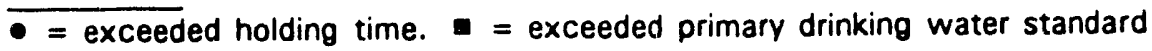


H D Analyte $\quad$ Result

$\mathrm{pH}$

Specific conductance 20

Arsenic.

Barium

Benzene

Bromodichloromethane

Bromoform

Bromomethane (Methyl bromide)

Cadmium

Calcium

Carbon tetrachloride

Chloride

Chlorobenzene

Chloroethane

Chloroethene (Vinyl chloride)

2-Chloroethyl vinyl ether

Chloroform

Chloromethane (Methyl chloride)

Chromium

Dibromochloromethane

1,1-Dichloroethane

1.2-Dichloroethane

1,1-Dichloroethylene

trans-1,2-Dichloroethylene

Dichloromethane (Methylene chloride)

2,4-Dichlorophenoxyacetic acid

1,2-Dichloropropane

trans-1,3-Dichloropropene

cis-1,3-Dichloropropene

Endrin

Ethylbenzene

Fluoride

Iron

Lead

Lindane

Magnesium

Manganese

Mercury

Methoxychlor

Nitrate as nitrogen

Phenols

Potassium

Selenium

Silica

Silver

Sodium

Sulfate
5.8
20

$<2.0$

7.6

$<1.0$

$<1.0$

$<1.0$

$<1.0$

$<2.0$

1,850

$<1.0$

1,610

$<1.0$

$<1.0$

$<1.0$

$<1.0$

$<1.0$

$<1.0$

$<4.0$

$<1.0$

$<1.0$

$<1.0$

$<1.0$

$<1.0$

8.0

$<0.30$

$<1.0$

$<1.0$

$<1.0$

$<0.0060$

$<1.0$

$<100$

5.7

$<3.0$

$<0.0050$

361

13

$<0.20$

$<0.50$

420

$<5.0$

$<500$

$<2.0$

7,050

$<2.0$

1,140

$<1,000$
Unit $\quad \underline{\text { lag }} \quad \underline{\text { Lab }}$

pH $\quad 0 \quad$ GE

$\mu \mathrm{S} / \mathrm{cm} \quad 0 \quad \mathrm{GE}$

$\mu g / L \quad 0 \quad$ GE

$\mu \mathrm{g} / \mathrm{L} \quad \mathrm{O} \quad \mathrm{GE}$

$\mu g / L \quad 0 \quad$ GE

$\mu g / L \quad 0 \quad$ GE

$\mu g / L \quad 0 \quad$ GE

$\mu g / L \quad 0 \quad$ GE

$\mu g / L \quad 0 \quad$ GE

$\mu g / L \quad 0 \quad$ GE

$\mu g / L \quad 0 \quad G E$

$\mu g / L \quad 0 \quad$ GE

$\mu g / \mathrm{L} \quad 0 \quad-\mathrm{GE}$

$\mu g / L \quad 0 \quad$ GE

$\mu g / L \quad 0 \quad$ GE

$\mu g / L \quad 0 \quad$ GE

$\mu g / L \quad 0 \quad$ GE

$\mu g / L \quad 0 \quad$ GE

$\mu g / L \quad 0 \quad$ GE

$\mu g / L \quad 0 \quad G E$

$\mu g / L \quad 0 \quad$ GE

$\mu g / L \quad 0 \quad$ GE

$\mu g / L \quad 0 \quad$ GE

$\mu g / L \quad 0 \quad G E$

$\mu g / L \quad 0 \quad$ GE

$\mu g / L \quad 0 \quad$ GE

$\mu g / L \quad 0 \quad$ GE

$\mu g / L \quad 0 \quad$ GE

$\mu g / L \quad O \quad$ GE

$\mu g / L \quad 0 \quad$ GE

$\mu g / L \quad 0 \quad$ GE

$\mu g / L \quad 0 \quad$ GE

$\mu g / L \quad 0 \quad$ GE

$\mu g / L \quad 0 \quad$ GE

$\mu g / L \quad 0 \quad$ GE

$\mu \mathrm{g} / \mathrm{L} \quad \mathrm{O} \quad \mathrm{GE}$

$\mu g / L \quad 0 \quad$ GE

$\mu g / L \quad 0 \quad$ GE

$\mu g / L \quad 0 \quad$ GE

$\mu g / L \quad 0 \quad$ GE

$\mu g / L \quad 0 \quad G E$

$\mu g / L \quad 0 \quad$ GE

$\mu g / L \quad 0 \quad$ GE

$\mu g / L \quad 0 \quad G E$

$\mu g / L \quad 0 \quad G E$

$\mu g / L \quad 0 \quad$ GE

$\mu g / L \quad O \quad$ GE

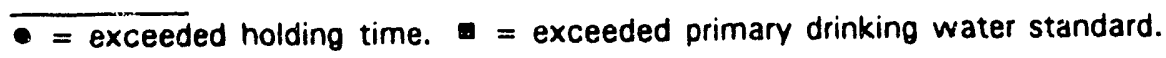


WELL LFW 43C, laboratory analyses (cont.)

H. $\quad$ Analyte
1,1,2,2-Tetrachloroethane
Tetrachloroethylene
Toluene
Total dissolved solids
Total organic carbon
Total organic halogens
Total phosphates las P)
Toxaphene
2,4,5-TP (Silvex)
1,1,1-Trichloroethane
1,1,2-Trichloroethane
Trichloroethylene
Trichlorofluoromethane
Gross alpha
Nonvolatile beta
Total radium
Tritium

$\begin{aligned} & \text { Result } \\ & \\ &<1.0 \\ &<1.0 \\ &<1.0 \\ & \quad 9.000 \\ &<1.000 \\ &<5.0 \\ &<50 \\ &<0.24 \\ &<0.090 \\ &<1.0 \\ &<1.0 \\ &<1.0 \\ &<1.0 \\ &<2.0 E+00 \\ & 2.6 E+00 \pm 1.5 E+00 \\ &<1.0 E+00 \\ &<7.0 E-01\end{aligned}$

UnitFlagLab

$\mu g / L \quad O \quad G E$

$\mu g / L \quad O \quad G E$

$\mu g / L \quad O \quad G E$

$\mu g / L \quad O \quad$ GE

$\mu g / L \quad$ GE

$\mu g / L \quad$ GE

$\mu g / L \quad O \quad$ GE

$\mu g / L \quad$ GE

$\mu g / L \quad 0 \quad$ GE

$\mu g / L \quad 0 \quad$ GE

$\mu g / L \quad 0 \quad G E$

$\mu g / L \quad 0 \quad$ GE

$\mu g / L \quad 0 \quad-$ GE

pCi/L $\quad 0 \quad$ GE

pCi/L $\quad 0 \quad$ GE

PCi/L $\quad 0 \quad$ GE

$\mathrm{pCi} / \mathrm{mL} \quad 0 \quad$ GE

\section{WELL LFW 43D}

SRS Coord.

N86443.2

E45244.5

\section{MEASUREMENTS CONDUCTED IN THE FIELD}

Sample date: 10/28/91

Depth to water: $34.96 \mathrm{ft}(10.66 \mathrm{~m})$ below TOC

Water elevation: $167.94 \mathrm{ft}(51.19 \mathrm{~m}) \mathrm{msl}$

Sp. conductance: $24 \mu \mathrm{S} / \mathrm{cm}$

Water evacuated before sampling: $46 \mathrm{gal}$

\section{LABORATORY ANALYSES}

\section{H D Analyte}

- $\mathrm{pH}$

Specitic conductance

Arsenic

Barium

Benzene

Bromodichloromethane

Bromoform

Bromomethane (Methyl bromide)

Cadmium

Calcium

Carbon tetrachloride

Chloride

Chlorobenzene
Screen Zone Elevation

$170.9-150.9 \mathrm{ft} \mathrm{msl}$
Tor of Cassing

$202.9 \mathrm{ft} \mathrm{msl}$
Casing

4" PVC
Time: $10: 45$

pH: 5.1

Alkalinity: $1 \mathrm{mg} / \mathrm{L}$

Water temperature: $18.6^{\circ} \mathrm{C}$ 
WELL LFW 43D, laboratory analyses (cont.)

H $\underline{\text { Analyte }}$

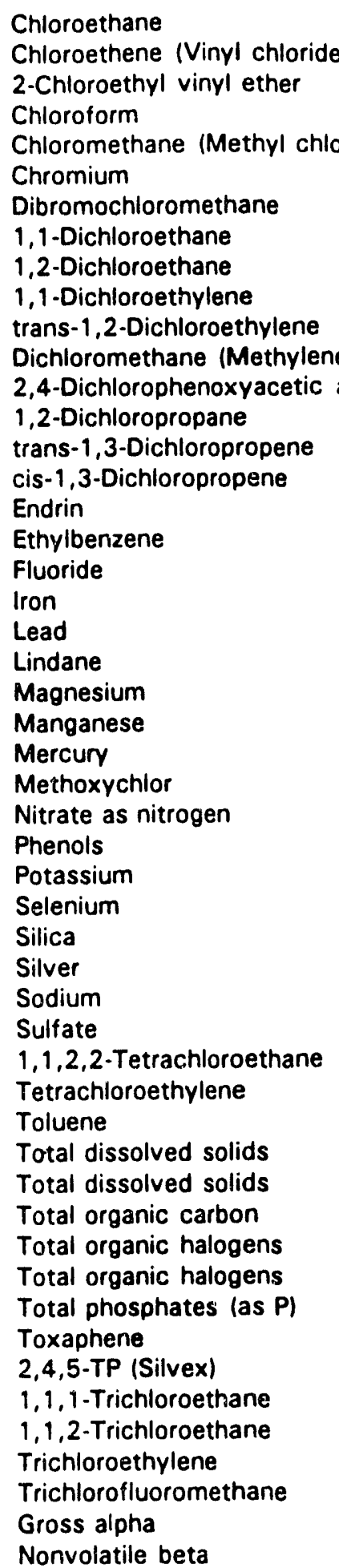

\begin{tabular}{|c|c|c|c|}
\hline Result & Unit & Flag & Lab \\
\hline$<1.0$ & $\mu g / L$ & 0 & GE \\
\hline$<1.0$ & $\mu g / L$ & 0 & GE \\
\hline$<1.0$ & $\mu g / L$ & 0 & GE \\
\hline$<1.0$ & $\mu g / L$ & 0 & GE \\
\hline$<1.0$ & $\mu g / L$ & 0 & GE \\
\hline$<4.0$ & $\mu g / L$ & 0 & GE \\
\hline$<1.0$ & $\mu g / L$ & 0 & GE \\
\hline$<1.0$ & $\mu g / L$ & 0 & GE \\
\hline$<1.0$ & $\mu g / L$ & 0 & GE \\
\hline$<1.0$ & $\mu g / L$ & 0 & GE \\
\hline$<1.0$ & $\mu g / L$ & 0 & GE \\
\hline 3.0 & $\mu g / L$ & 0 & GE \\
\hline$<0.30$ & $\mu g / L$ & 0 & - $\mathrm{GE}$ \\
\hline$<1.0$ & $\mu g / L$ & 0 & GE \\
\hline$<1.0$ & $\mu g / L$ & 0 & GE \\
\hline$<1.0$ & $\mu g / L$ & 0 & GE \\
\hline$<0.0060$ & $\mu g / L$ & 0 & GE \\
\hline$<1.0$ & $\mu g / L$ & 0 & GE \\
\hline$<100$ & $\mu g / L$ & 0 & GE \\
\hline 6.6 & $\mu g / L$ & 0 & GE \\
\hline$<3.0$ & $\mu g / L$ & 0 & GE \\
\hline$<0.0050$ & $\mu g / L$ & 0 & GE \\
\hline 697 & $\mu g / L$ & 0 & GE \\
\hline 22 & $\mu g / L$ & 0 & GE \\
\hline$<0.20$ & $\mu O / L$ & 0 & GE \\
\hline$<0.50$ & $\mu g / L$ & 0 & GE \\
\hline 840 & $\mu g / L$ & 0 & GE \\
\hline$<5.0$ & $\mu g / L$ & 0 & GE \\
\hline$<500$ & $\mu g / L$ & 0 & GE \\
\hline$<2.0$ & $\mu g / L$ & 0 & GE \\
\hline 5,800 & $\mu g / L$ & 0 & GE \\
\hline$<2.0$ & $\mu g / L$ & 0 & GE \\
\hline 1,440 & $\mu g / L$ & 0 & GE \\
\hline$<1,000$ & $\mu \mathrm{g} / \mathrm{L}$ & 0 & GE \\
\hline$<1.0$ & $\mu g / L$ & 0 & GE \\
\hline$<1.0$ & $\mu g / L$ & 0 & GE \\
\hline$<1.0$ & $\mu g / L$ & 0 & GE \\
\hline 8,000 & $\mu g / L$ & 0 & GE \\
\hline 9,000 & $\mu g / L$ & 0 & GE \\
\hline$<1,000$ & $\mu g / L$ & 0 & GE \\
\hline$<5.0$ & $\mu g / L$ & 0 & GE \\
\hline$<5.0$ & $\mu g / L$ & 0 & GE \\
\hline$<50$ & $\mu g / L$ & 0 & GE \\
\hline$<0.24$ & $\mu g / L$ & 0 & GE \\
\hline$<0.090$ & $\mu g / L$ & 0 & GE \\
\hline$<1.0$ & $\mu g / L$ & 0 & GE \\
\hline$<1.0$ & $\mu g / L$ & 0 & GE \\
\hline$<1.0$ & $\mu_{g} / L$ & 0 & GE \\
\hline$<1.0$ & $\mu g / L$ & 0 & GE \\
\hline$<2.0 E+00$ & $\mathrm{pCi} / \mathrm{L}$ & 0 & GE. \\
\hline$<2.0 E+00$ & $\mathrm{pCi} / \mathrm{L}$ & 0 & GE \\
\hline
\end{tabular}

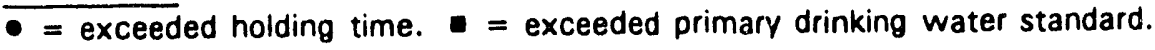


WELL LFW 430, laboratory analyses (cont.)

H D Analyte

Total radium
Tritium
Result

$<1.0 E+00$

$<7.0 \mathrm{E}-01$ $\underline{\text { Unit }} \quad \underline{\text { Flag }} \quad \underline{\text { Lab }}$

$\mathrm{pCi} / \mathrm{L} \quad \mathrm{O} \quad \mathrm{GE}$

\section{WELL LFW 44D}

SRS Coord.

N84524.4

E45022.6

MEASUREMENTS CONDUCTED IN THE FIELD

Sample date: $10 / 28 / 91$

Depth to water: $14.81 \mathrm{ft}(4.51 \mathrm{~m})$ below TOC

Water elevation: $155.49 \mathrm{ft}(47.39 \mathrm{~m}) \mathrm{msl}$

Sp. conductance: $20 \mu \mathrm{S} / \mathrm{cm}$

Water evacuated before sampling: $\mathbf{4 3}$ gal

\section{LABORATORY ANALYSES}

\section{H $\underline{\text { Analyte }}$}

- $\quad \mathrm{pH}$

Specific conductance

Arsenic

Barium

Benzene

Bromodichloromethane

Bromoform

Bromomethane (Methyl bromide)

Cadmium

Calcium

Carbon tetrachloride

Chloride

Chlorobenzene

Chloroethane

Chloroethene (Vinyl chloride)

2-Chloroethyl vinyl ether

Chloroform

Chloromethane (Methyl chloride)

Chromium

Dibromochloromethane

1,1-Dichloroethane

1,2-Dichloroethane

1,1-Dichloroethylene

trans-1,2-Dichloroethylene

Dichloromethane (Methylene chloride)

2,4-Dichlorophenoxyacetic acid 1,2-Dichloropropane

trans-1,3-Dichloropropene
Result

$$
5.2
$$

20

$<2.0$

3.2

$<1.0$

$<1.0$

$<1.0$

$<1.0$

$<2.0$

432

$<1.0$

3,180

$<1.0$

$<1.0$

$<1.0$

$<1.0$

$<1.0$

$<1.0$

$<4.0$

$<1.0$

$<1.0$

$<1.0$

$<1.0$

$<1.0$

16

$<0.30$

$<1.0$

$<1.0$
Top of Casing Casing

$170.3 \mathrm{ft} \mathrm{msl} \quad 4^{n}$ PVC
Time: 12:05

$\mathrm{pH}: 4.8$

Alkalinity: $0 \mathrm{mg} / \mathrm{L}$

Water temperature: $19.4^{\circ} \mathrm{C}$

- = exceeded holding time. $=$ exceeded primary drinking water standard 
WELL LFW 44D, laboratory analyses (cont.)

H $\underline{\text { Analyte }}$

cis-1,3-Dichloropropene

Endrin

Ethylbenzene

Fluoride

Iron

Lead

Lindane

Magnesium

Manganese

Mercury

Methoxychlor

Nitrate as nitrogen

Phenols

Potassium

Selenium

Silica

Silver

Sodium

Sulfate

1,1,2,2-Tetrachloroethane

Tetrachloroethylene

Toluene

- Total dissolved solids

Total organic carbon

Total organic halogens

Total phosphates (as P)

Toxaphene

2,4,5-TP (Silvex)

1,1,1-Trichloroethane

1,1,2-Trichloroethane

Trichloroethylene

Trichlorofluoromethane

Gross alpha

Nonvolatile beta

- Total radium

Tritium

Result
$<1.0$
$<0.0060$
$<1.0$
$<100$
14
$<3.0$
$<0.0050$
368
2.9
$<0.20$
$<0.50$
390
$<5.0$
600
$<2.0$
6,360
$<2.0$
1.390
1.600
$<1.0$
$<1.0$
$<1.0$
8.000
$<1.000$
$<5.0$
$<50$
$<0.24$
$<0.090$
$<1.0$
$<1.0$
$<1.0$
$<1.0$
$<2.0 E+00$
$2.9 E+00 \pm 1.4 E+00$
$5.0 E+00 \pm 1.7 E+00$
$<7.0 E-01$

\begin{tabular}{|c|c|c|}
\hline Unit & Flag & $\underline{\operatorname{Lab}}$ \\
\hline$\mu \mathrm{g} / \mathrm{L}$ & 0 & GE \\
\hline$\mu g / L$ & 0 & $\mathrm{GE}$ \\
\hline$\mu g / L$ & 0 & GE \\
\hline$\mu g / L$ & 0 & GE \\
\hline$\mu \mathrm{g} / \mathrm{L}$ & 0 & $\mathrm{GE}$ \\
\hline$\mu g / L$ & 0 & GE \\
\hline$\mu_{g / L}$ & 0 & GE \\
\hline$\mu g / L$ & 0 & GE \\
\hline$\mu g / L$ & 0 & GE \\
\hline$\mu g / L$ & 0 & GE \\
\hline$\mu g / L$ & 0 & GE \\
\hline$\mu \mathrm{g} / \mathrm{L}$ & 0 & GE \\
\hline$\mu g / L$ & 0 & GE \\
\hline$\mu \mathrm{g} / \mathrm{L}$ & 0 & GE \\
\hline$\mu g / L$ & 0 & GE \\
\hline$\mu g / L$ & 0 & GE \\
\hline$\mu g / L$ & 0 & GE \\
\hline$\mu g / L$ & 0 & GE \\
\hline$\mu g / L$ & 0 & GE \\
\hline$\mu \mathrm{g} / \mathrm{L}$ & 0 & GE \\
\hline$\mu g / L$ & 0 & GE \\
\hline$\mu g / L$ & 0 & GE \\
\hline$\mu \mathrm{g} / \mathrm{L}$ & 0 & GE \\
\hline$\mu g / L$ & 0 & GE \\
\hline$\mu g / L$ & 0 & GE \\
\hline$\mu \mathrm{g} / \mathrm{L}$ & 0 & $\mathrm{GE}$ \\
\hline$\mu \mathrm{g} / \mathrm{L}$ & 0 & GE \\
\hline$\mu g / L$ & 0 & GE \\
\hline$\mu \mathrm{g} / \mathrm{L}$ & 0 & GE \\
\hline$\mu g / L$ & 0 & GE \\
\hline$\mu g / L$ & 0 & GE \\
\hline$\mu \mathrm{g} / \mathrm{L}$ & 0 & GE \\
\hline pCi/L & 0 & GE \\
\hline $\mathrm{pCi} / \mathrm{L}$ & 0 & GE \\
\hline $\mathrm{pCi} / \mathrm{L}$ & 2 & GE \\
\hline $\mathrm{pCi} / \mathrm{mL}$ & 0 & GE \\
\hline
\end{tabular}

$\overline{-=\text { exceeded holding time. }}=$ exceeded primary drinking water standard. 


\section{WELL LFW 45D}

SRS Coord.

N84217.8

E451420

MEASUREMENTS CONDUCTED IN THE FIELD

Sample date: 10/28/91

Depth to water: $13.55 \mathrm{ft}(4.13 \mathrm{~m})$ below TOC

Water elevation: $152.75 \mathrm{ft}(46.56 \mathrm{~m}) \mathrm{msl}$

Sp. conductance: $15 \mu \mathrm{S} / \mathrm{cm}$

Water evacuated before sampling: $49 \mathrm{gal}$

\section{LABORATORY ANALYSES}

H D Analyte

-

$\mathrm{pH}$

Arsenic

Arsenic

Barium

- Benzene

Benzene
Specific conductance

Bromodichloromethane

Bromodichloromethane

Bromoform

Bromoform

Bromomethane (Methyl bromide)

Bromomethane (Methyl bromide)

Cadmium

Calcium

- Carbon tetrachloride

- Carbon tetrachloride

Chloride

- Chlorobenzene

- Chlorobenzene

- Chloroethane

- Chloroethane

- Chloroethene (Vinyl chloride)

- Chloroethene (Vinyl chloride)

- 2-Chloroethyl vinyl ether

- 2-Chloroethyl vinyl ether

- Chloroform

- Chloroform

- Chloromethane (Methyl chloride)

- Chloromethane (Methyl chloride) Chromium

- Dibromochloromethane

- Dibromochloromethane

- 1,1-Dichloroethane

- 1,1-Dichloroethane

- 1,2-Dichloroethane
Result

5.2

15

$<2.0$

$<2.0$

3.8

$<1.0$

$<1.0$

$<1.0$

$<1.0$

$<1.0$

$<1.0$

$<1.0$

$<1.0$

$<2.0$

337

$<1.0$

$<1.0$

2,100

$<1.0$

$<1.0$

$<1.0$

$<1.0$

$<1.0$

$<1.0$

$<1.0$

$<1.0$

$<1.0$

$<1.0$

$<1.0$

$<1.0$

$<4.0$

$<1.0$

$<1.0$

$<1.0$

$<1.0$

$<1.0$
Top of Casing

Casing

$166.3 \mathrm{ft} \mathrm{msl}$

4" PVC
Time: $14: 45$

pH: 4.9

Alkalinity: $0 \mathrm{mg} / \mathrm{L}$

Water temperature: $20.0^{\circ} \mathrm{C}$
Unit Flag Lab

pH $O \quad$ GE

$\mu \mathrm{S} / \mathrm{cm} \quad 0 \quad$ GE

$\mu g / L \quad O \quad$ GE

$\mu g / L \quad 0 \quad$ GE

$\mu g / L \quad 0 \quad$ GE

$\mu g / L \quad O \quad$ GE

$\mu g / L \quad 0 \quad$ GE

$\mu \mathrm{g} / \mathrm{L} \quad \mathrm{O} \quad \mathrm{GE}$

$\mu \mathrm{g} / \mathrm{L} \quad \mathrm{O} \quad \mathrm{GE}$

$\mu g / L \quad 0 \quad$ GE

$\mu g / L \quad 0 \quad$ GE

$\mu g / L \quad O \quad$ GE

$\mu g / L \quad O \quad$ GE

$\mu g / L \quad 0 \quad$ GE

$\mu g / L \quad O \quad G E$

$\mu g / L \quad 0 \quad$ GE

$\mu g / L \quad 0 \quad$ GE

$\mu \mathrm{g} / \mathrm{L} \quad \mathrm{O} \quad \mathrm{GE}$

$\mu g / L \quad 0 \quad$ GE

$\mu g / L \quad 0 \quad G E$

$\mu g / L \quad 0 \quad$ GE

$\mu g / L \quad 0 \quad$ GE

$\mu g / L \quad 0 \quad$ GE

$\mu g / L \quad O \quad$ GE

$\mu g / \mathrm{L} \quad 0 \quad \mathrm{GE}$

$\mu g / L \quad 0 \quad$ GE

$\mu g / L \quad 0 \quad$ GE

$\mu g / L \quad 0 \quad$ GE

$\mu g / L \quad 0 \quad$ GE

$\mu g / L \quad 0 \quad$ GE

$\mu g / L \quad 0 \quad$ GE

$\mu \mathrm{g} / \mathrm{L} \quad \mathrm{O} \quad \mathrm{GE}$

$\mu g / L \quad 0 \quad$ GE

$\mu g / L \quad 0 \quad$ GE

$\mu g / L \quad 0 \quad G E$

$\mu g / L \quad O \quad$ GE

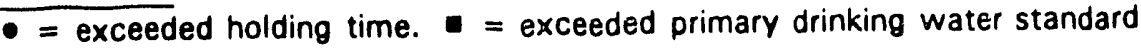


WELL LFW 45D, laboratory analyses (cont.)

\begin{tabular}{|c|c|c|c|c|c|}
\hline$\underline{H} \underline{D}$ & Analyte & Result & Unit & Flag & Lab \\
\hline$\bullet$ & 1,2-Dichloroethane & $<1.0$ & $\mu g / L$ & 0 & GE \\
\hline$\bullet$ & 1,1-Dichloroethylene & $<1.0$ & $\mu g / L$ & 0 & GE \\
\hline$\bullet$ & 1.1-Dichloroethylene & $<1.0$ & $\mu g / L$ & 0 & GE \\
\hline$\bullet$ & trans-1,2-Dichloroethylene & $<1.0$ & $\mu g / L$ & 0 & GE \\
\hline$\bullet$ & trans-1,2-Dichloroethylene & $<1.0$ & $\mu g / L$ & 0 & GE \\
\hline - & Dichloromethane (Methylene chloride) & $<1.0$ & $\mu g / L$ & 0 & GE \\
\hline - & Dichloromethane (Methylene chloride) & $<1.0$ & $\mu g / L$ & 0 & GE \\
\hline & 2,4-Dichlorophenoxyacetic acid & $<0.30$ & $\mu g / L$ & 0 & GE \\
\hline - & 1,2-Dichloropropane & $<1.0$ & $\mu g / L$ & 0 & GE \\
\hline - & 1,2-Dichloropropane & $<1.0$ & $\mu g / L$ & 0 & GE \\
\hline - & trans-1,3-Dichloropropene & $<1.0$ & $\mu g / L$ & 0 & GE \\
\hline$\bullet$ & trans-1,3-Dichloropropene & $<1.0$ & $\mu \mathrm{g} / \mathrm{L}$ & 0 & GE \\
\hline$\bullet$ & cis-1,3-Dichloropropene & $<1.0$ & $\mu g / L$ & 0 & GE \\
\hline - & cis-1,3-Dichloropropene & $<1.0$ & $\mu g / L$ & 0 & GE \\
\hline & Endrin & $<0.0060$ & $\mu g / L$ & 0 & GE \\
\hline & Endrin & $<0.0060$ & $\mu g / L$ & 0 & GE \\
\hline - & Ethylbenzene & $<1.0$ & $\mu g / L$ & 0 & GE \\
\hline • & Ethylbenzene & $<1.0$ & $\mu g / L$ & 0 & GE \\
\hline & Fluoride & $<100$ & $\mu g / L$ & 0 & GE \\
\hline & Lead & $<3.0$ & $\mu g / L$ & 0 & GE \\
\hline & Lead & $<3.0$ & $\mu g / L$ & 0 & GE \\
\hline & Lindane & $<0.0050$ & $\mu g / L$ & 0 & GE \\
\hline & Lindane & $<0.0050$ & $\mu g / L$ & 0 & GE \\
\hline & Magnesium & 286 & $\mu g / L$ & 0 & E \\
\hline & Manganese & 3.9 & $\mu g / \Omega$ & 0 & GE \\
\hline & Mercury & $<0.20$ & $\mu g / L$ & 0 & GE \\
\hline & Methoxychlor & $<0.50$ & $\mu g / L$ & 0 & GE \\
\hline & Methoxychlor & $<0.50$ & $\mu g / L$ & 0 & GE \\
\hline & Nitrate as nitrogen & 170 & $\mu g / L$ & 0 & GE \\
\hline & Phenols & $<5.0$ & $\mu g / L$ & 0 & GE \\
\hline & Phenols & $<5.0$ & $\mu g / L$ & 0 & GE \\
\hline & Potassium & 699 & $\mu g / L$ & 0 & GE \\
\hline & Selenium & $<2.0$ & $\mu g / L$ & 0 & GE \\
\hline & Selenium & $<2.0$ & $\mu g / L$ & 0 & GE \\
\hline & Silica & 6,580 & $\mu g / L$ & 0 & GE \\
\hline & Silver & $<2.0$ & $\mu g / L$ & 0 & GE \\
\hline & Sodium & 934 & $\mu g / L$ & 0 & GE \\
\hline & Sulfate & $<1,000$ & $\mu g / L$ & 0 & GE \\
\hline 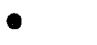 & 1,1,2,2-Tetrachloroethane & $<1.0$ & $\mu g / L$ & 0 & JE \\
\hline . & $1,1,2,2$-Tetrachloroethane & $<1.0$ & $\mu g / L$ & 0 & GE \\
\hline - & Tetrachloroethylene & $<1.0$ & $\mu g / L$ & 0 & GE \\
\hline 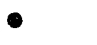 & Tetrachloroethylene & $<1.0$ & $\mu g / L$ & 0 & GE \\
\hline - & Toluene & $<1.0$ & $\mu g / L$ & 0 & GE \\
\hline$\bullet$ & Toluene & $<1.0$ & $\mu g / L$ & 0 & GE \\
\hline - & Total dissolved solids & 7,000 & $\mu g / L$ & 0 & GE \\
\hline & Total organic carbon & $<1,000$ & $\mu g / L$ & 0 & UE \\
\hline & Total organic halogens & $<5.0$ & $\mu \mathrm{g} / \mathrm{L}$ & 0 & GE \\
\hline & Total organic halogens & $<5.0$ & $\mu g / L$ & 0 & $\mathrm{G}$ \\
\hline & Total phosphates (as P) & $<50$ & $\mu g / L$ & 0 & UE \\
\hline & Toxaphene & $<0.24$ & $\mu g / L$ & 0 & GE \\
\hline & Toxaphene & $<0.24$ & $\mu g / L$ & 0 & إ \\
\hline
\end{tabular}

$\overline{-=\text { exceeded holding time. }}$ = exceeded primary drinking water standard. 
WELL LFW 45D, laboratory analyses (cont.)

$\begin{array}{ll}\text { H } & \text { Analyte } \\ & \\ \text { 2,4,5-TP (Silvex) } \\ \text { - } \quad 1,1,1 \text {-Trichloroethane } \\ \text { 1,1,1-Trichloroethane } \\ \text { 1,1,2-Trichloroethane } \\ \text { 1,1,2-Trichloroethane } \\ \text { Trichloroethylene } \\ \text { Trichloroethylene } \\ \text { Trichlorofluoromethane } \\ \text { Trichlorofluoromethane } \\ \text { Gross alpha } \\ \text { Nonvolatile beta } \\ \text { Total radium } \\ \text { Tritium }\end{array}$

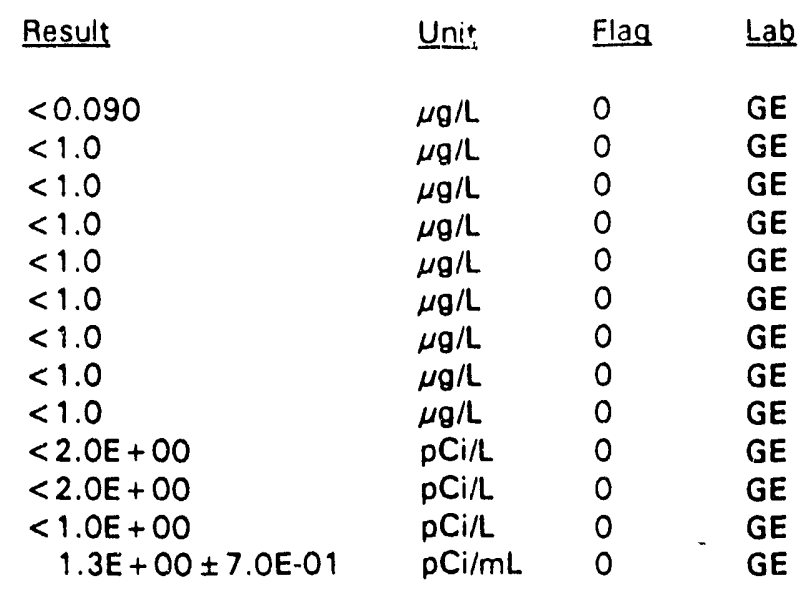

\section{WELL LFW 46D}

\begin{tabular}{|c|c|c|c|}
\hline SRS Coord. & Screen Zone Elevation & Top of Casing & Casing \\
\hline $\begin{array}{l}N 84054.0 \\
E 45162.8\end{array}$ & $157.1 \cdot 137.3 \mathrm{ft} \mathrm{msl}$ & $165.1 \mathrm{ft} \mathrm{msl}$ & 4" PVC \\
\hline
\end{tabular}

\section{MEASUREMENTS CONDUCTED IN THE FIELD}

Sample date: $10 / 28 / 91$

Depth to water: $13.75 \mathrm{ft}(4.19 \mathrm{~m})$ below TOC

Water elevation: $151.35 \mathrm{ft}(46.13 \mathrm{~m}) \mathrm{msl}$

Sp. conductance: $97 \mu \mathrm{S} / \mathrm{cm}$

Water evacuated before sampling: $38 \mathrm{gal}$

\section{LABORATORY ANALYSES}

H $\underline{\text { snalyte }}$

- $\mathrm{pH}$

Specific conductance

Arsenic

Barium

Benzene

Bromodichloromethane

Bromoform

Bromomethane (Methyl bromide)

Cadmium

Calcium

Carbon tetrachloride

Chloride

Chlorobenzene

Chloroethane

Chloroethene (Vinyl chloride)

2-Chloroethyl vinyl ether

Chloroform
Result

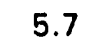

90

$<2.0$

13

$<1.0$

$<1.0$

$<1.0$

$<1.0$

$<2.0$

8,150

$<1.0$

2,730

$<1.0$

$<1.0$

$<1.0$

$<1.0$

$<1.0$
Time: 14:05

$\mathrm{pH}: 5.5$

Alkalinity: $64 \mathrm{mg} / \mathrm{L}$

Water temperature: $20.6^{\circ} \mathrm{C}$

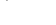

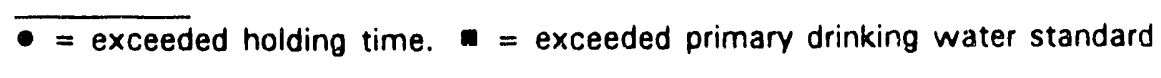

$\underline{\text { Unit }} \quad \underline{\text { Flag }} \underline{\underline{L a b}}$

$\mathrm{pH} \quad \mathrm{O}$ GE

$\mu \mathrm{S} / \mathrm{cm} \quad \mathrm{GE}$

$\mu g / L \quad$ GE

$\mu g / L \quad 0 \quad$ GE

$\mu g / L \quad 0 \quad$ GE

$\mu \mathrm{g} / \mathrm{L} \quad 0 \quad$ GE

$\mu g / L \quad 0 \quad$ GE

$\mu g / L \quad O \quad$ GE

$\mu g / L \quad 0 \quad$ GE

$\mu \mathrm{g} / \mathrm{L} \quad 0 \quad$ GE

$\mu g / L \quad O \quad$ GE

$\mu g / L \quad O \quad$ GE

$\mu g / L \quad O \quad$ GE

$\mu g / L \quad 0 \quad$ GE

$\mu g / L \quad 0 \quad$ GE

$\mu g / L \quad 0 \quad$ GE

$\mu \mathrm{g} / \mathrm{L} \quad \mathrm{O} \quad \mathrm{GE}$ 
WELL LFW 46D, laboratory analyses (cont.)

H D Analyte

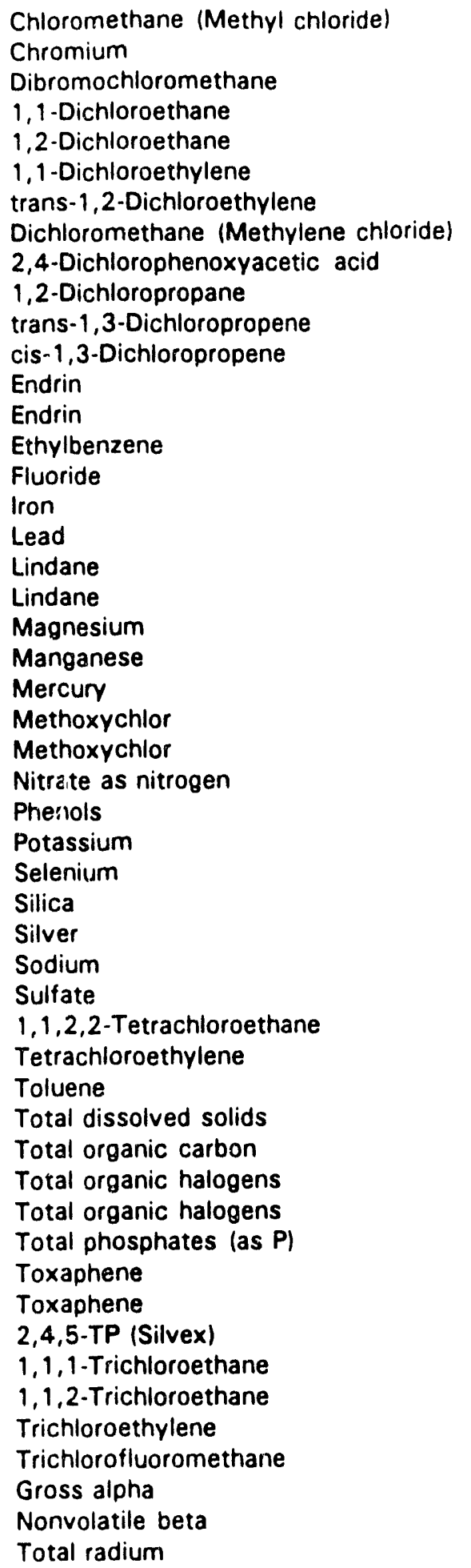

\begin{tabular}{|c|c|c|c|}
\hline Result & Unit & Flag & \\
\hline$<1.0$ & $\mu g / L$ & 0 & \\
\hline$<4.0$ & $\mu g / L$ & 0 & \\
\hline$<1.0$ & $\mu g / L$ & 0 & \\
\hline$<1.0$ & $\mu \mathrm{g} / \mathrm{L}$ & 0 & \\
\hline$<1.0$ & $\mu g / L$ & 0 & \\
\hline$<1.0$ & $\mu g / L$ & 0 & \\
\hline$<1.0$ & $\mu g / L$ & 0 & \\
\hline 1.0 & $\mu \theta / L$ & 0 & \\
\hline$<0.30$ & $\mu g / L$ & 0 & \\
\hline$<1.0$ & $\mu g / L$ & 0 & \\
\hline$<1.0$ & $\mu g / L$ & 0 & \\
\hline$<1.0$ & $\mu \mathrm{g} / \mathrm{L}$ & 0 & \\
\hline$<0.0060$ & $\mu g / L$ & 0 & - \\
\hline$<0.0060$ & $\mu g / L$ & 0 & \\
\hline$<1.0$ & $\mu g / L$ & 0 & \\
\hline$<100$ & $\mu g / L$ & 0 & \\
\hline 12 & $\mu g / L$ & 0 & \\
\hline$<3.0$ & $\mu g / L$ & 0 & \\
\hline$<0.0050$ & $\mu \mathrm{g} / \mathrm{L}$ & 0 & \\
\hline$<0.0050$ & $\mu g / L$ & 0 & \\
\hline 3,970 & $\mu g / L$ & 0 & \\
\hline 7.0 & $\mu g / L$ & 0 & \\
\hline$<0.20$ & $\mu g / L$ & 0 & \\
\hline$<0.50$ & $\mu g / L$ & 0 & \\
\hline$<0.50$ & $\mu g / L$ & 0 & \\
\hline 87 & $\mu g / L$ & 0 & \\
\hline$<5.0$ & $\mu g / L$ & 0 & \\
\hline 867 & $\mu g / L$ & 0 & \\
\hline$<2.0$ & $\mu \mathrm{g} / \mathrm{L}$ & 0 & \\
\hline 6.180 & $\mu g / L$ & 0 & \\
\hline$<2.0$ & $\mu \theta \Omega$ & 0 & \\
\hline 1,220 & $\mu g / L$ & 0 & \\
\hline 26,400 & $\mu g / L$ & 0 & \\
\hline$<1.0$ & $\mu \theta / L$ & 0 & \\
\hline$<1.0$ & $\mu g / L$ & 0 & \\
\hline$<1.0$ & $\mu g / L$ & 0 & \\
\hline 46,000 & $\mu g / L$ & 0 & \\
\hline$<1,000$ & $\mu g / L$ & 0 & \\
\hline$<5.0$ & $\mu g / L$ & 0 & \\
\hline 5.8 & $\mu g / L$ & 0 & \\
\hline 410 & $\mu g / L$ & 0 & \\
\hline$<0.24$ & $\mu g / L$ & 0 & \\
\hline$<0.24$ & $\mu \theta / L$ & 0 & \\
\hline$<0.090$ & $\mu g / L$ & 0 & \\
\hline$<1.0$ & $\mu g / L$ & 0 & \\
\hline$<1.0$ & $\mu g / L$ & 0 & \\
\hline$<1.0$ & $\mu g / L$ & 0 & \\
\hline$<1.0$ & $\mu g / L$ & 0 & \\
\hline $3.6 E+00 \pm 1.5 E+00$ & $\mathrm{pCi} / \mathrm{L}$ & 0 & \\
\hline $5.2 E+00 \pm 1.7 E+00$ & $\mathrm{pCi} / \mathrm{L}$ & 0 & \\
\hline $3.1 E+00 \pm 1.7 E+00$ & $\mathrm{pCi} / \mathrm{L}$ & 1 & \\
\hline
\end{tabular}

$\overline{- \text { exceeded holding time. }}-$ = exceeded primary drinking water standard. 
WELL LFW 46D, laboratory analyses (cont.)

$\begin{array}{lllll}\underline{H} \underline{\mathrm{D}} \text { Analyte } & \underline{\text { Result }} & \text { Unit } & \underline{\mathrm{Flag}} & \underline{\text { Lab }} \\ \text { Tritium } & <7.0 \mathrm{E}-01 & \mathrm{pCi} / \mathrm{mL} & 0 & \mathrm{GE}\end{array}$

WELL LFW 47C

$\begin{array}{llll}\text { SRS Coord. } & \text { Screen Zone Elevation } & \text { Top of Casing } & \text { Casing } \\ \text { N83843.9 } & 115.8-105.7 \mathrm{ft} \mathrm{msl} & 161.4 \mathrm{ft} \mathrm{msl} & 4^{\text {" PVC }}\end{array}$

E45178.6

MEASUREMENTS CONDUCTED IN THE FIELD

Sample date: $10 / 28 / 91$

Depth to water: $12.46 \mathrm{ft}(3.80 \mathrm{~m})$ below TOC Water elevation: $148.94 \mathrm{ft}(45.40 \mathrm{~m}) \mathrm{msl}$

Sp. conductance: $42 \mu \mathrm{S} / \mathrm{cm}$

Water evacuated before sampling: $114 \mathrm{gal}$

\section{LABORATORY ANALYSES}

H D Analyte

- $\quad \mathrm{pH}$

Specific conductance

Arsenic

Barium

Benzene

Bromodichloromethane

Bromoform

Bromomethane (Methyl bromide)

Cadmium

Calcium

Carbon tetrachloride

Chloride

Chlorobenzene

Chloroethane

- Chloroethene (Vinyl chloride)

2-Chloroethyl vinyl ether

Chloroform

Chloromethane (Methyl chloride)

Chromium

Dibromochloromethane

1.1-Dichloroethane

1,2-Dichloroethane

1,1-Dichloroethylene

trans-1,2-Dichloroethylene

Dichloromethane (Methylene chloride)

2,4-Dichlorophenoxyacetic acid

1,2-Dichloropropane

trans-1,3-Dichloropropene

cis-1,3-Dichloropropene
Result

5.5

40

$<2.0$

4.9

$<1.0$

$<1.0$

$<1.0$

$<1.0$

$<2.0$

1,060

$<1.0$

5,120

$<1.0$

$<1.0$

10

$<1.0$

$<1.0$

$<1.0$

$<4.0$

$<1.0$

3.8

$<1.0$

$<1.0$

$<1.0$

1.6

$<0.30$

$<1.0$

$<1.0$

$<1.0$
Time: 13:35

$\mathrm{pH}: 5.2$

Alkalinity: $2 \mathrm{mg} / \mathrm{L}$

Water temperature: $18.3^{\circ} \mathrm{C}$
Unit Flag $\underline{\text { Lab }}$

pH 0 GE

$\mu S / \mathrm{cm} \quad 0 \quad$ GE

$\mu g / L \quad 0 \quad$ GE

$\mu g / L \quad 0 \quad$ GE

$\mu g / L \quad O \quad$ GE

$\mu g /$ $\quad 0 \quad$ GE

$\mu g / L \quad 0 \quad$ GE

$\mu g / L \quad 0 \quad$ GE

$\mu g / L \quad 0 \quad$ GE

$\mu g / L \quad O \quad$ GE

$\mu g / L \quad O \quad$ GE

$\mu g / L \quad 0 \quad$ GE

$\mu g / L \quad O \quad$ GE

$\mu g / L \quad 0 \quad$ GE

$\mu g / L \quad 2 \quad$ GE

$\mu g / L \quad 0 \quad$ GE

$\mu g / L \quad 0 \quad$ GE

$\mu \mathrm{g} / \mathrm{L} \quad 0 \quad$ GE

$\mu g / L \quad O \quad$ GE

$\mu g / L \quad O \quad$ GE

$\mu g / L \quad 0 \quad$ GE

$\mu g / L \quad 0 \quad$ GE

$\mu g / L \quad 0 \quad$ GE

$\mu g / L \quad 0 \quad G E$

$\mu g / L \quad 0 \quad$ GE

$\mu g / L \quad 0 \quad$ GE

$\mu \mathrm{g} / \mathrm{L} \quad \mathrm{O} \quad \mathrm{GE}$

$\mu g / L \quad O \quad G E$

$\mu \mathrm{g} / \mathrm{L} \quad \mathrm{O} \quad \mathrm{GE}$

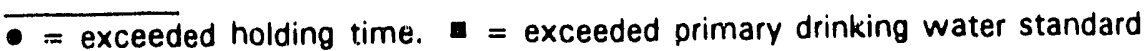


WELL LFW 47C, laboratory analyses (cont.)

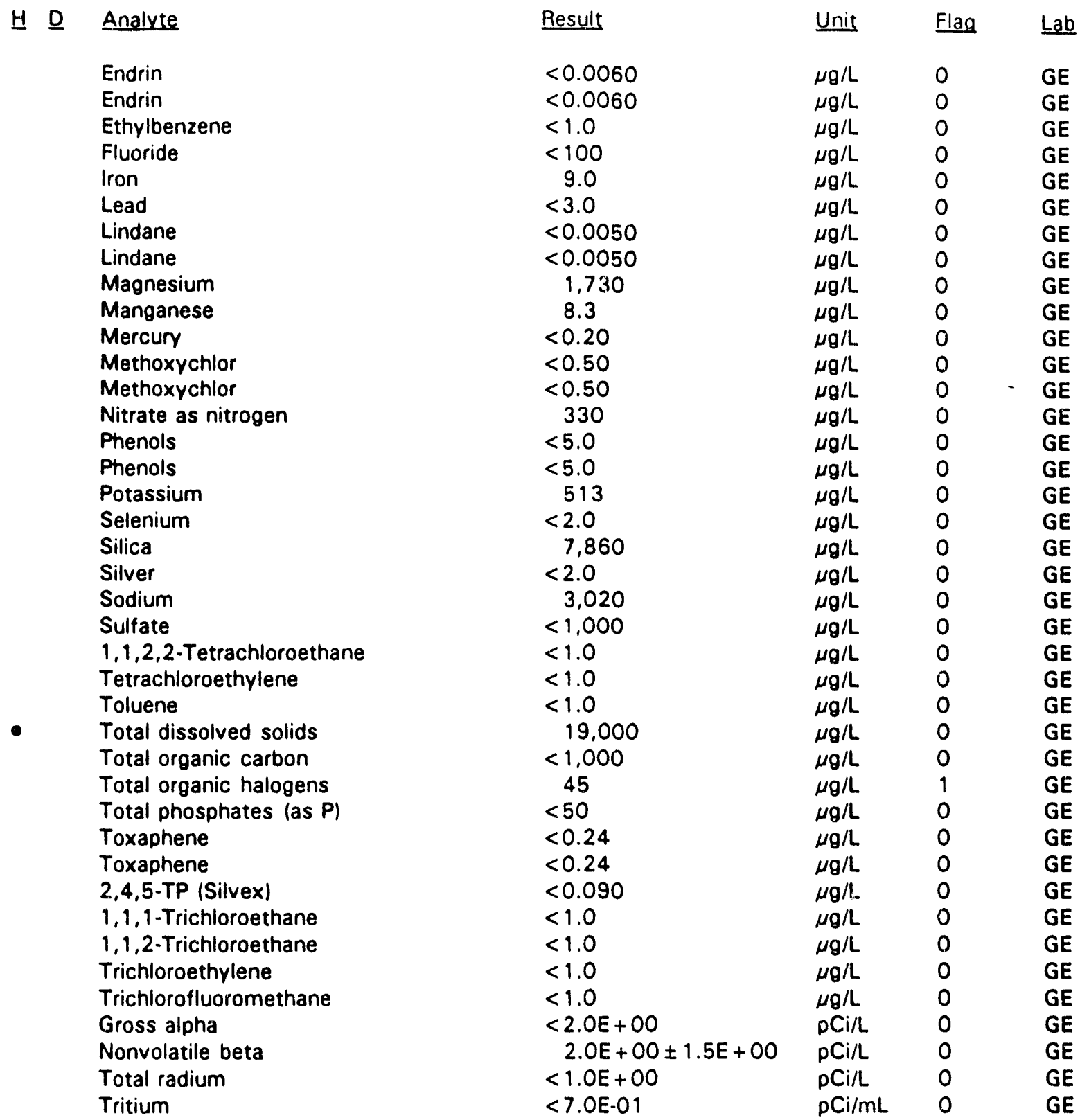

$\overline{-=\text { exceeded holding time. }} \mathbf{0}=$ exceeded primary drinking water standard. 
WELL LFW 47D

SRS Coord.

N83859.3

E45167.9

MEASUREMENTS CONDUCTED IN THE FIELD

Sample date: $10 / 28 / 91$

Depth to water: $12.22 \mathrm{ft}(3.72 \mathrm{~m})$ below TOC

Water elevation: $149.48 \mathrm{ft}(45.56 \mathrm{~m}) \mathrm{msl}$

Sp. conductance: $24 \mu \mathrm{S} / \mathrm{cm}$

Water evacuated before sampling: $40 \mathrm{gal}$

\section{LABORATORY ANALYSES}

\section{H}

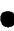

Screen Zone Elevation

$154.7-134.9 \mathrm{ft} \mathrm{msl}$
Top of Casing

$161.7 \mathrm{ft} \mathrm{msl}$
Casing

4" PVC
Time: 13:10

$\mathrm{pH}: 5.2$

Alkalinity: $1 \mathrm{mg} / \mathrm{L}$

Water temperature: $20.1^{\circ} \mathrm{C}$

Analyte
pH
Specific conductance
Arsenic
Barium
Benzene
Bromodichloromethane
Bromoform
Bromomethane (Methyl bromide)
Cadmium
Calcium
Carbon tetrachloride
Chloride
Chlorobenzene
Chloroethane
Chloroethene (Vinyl chloride)
2-Chloroethyl vinyl ether
Chloroform
Chloromethane (Methyl chloride)
Chromium
Dibromochloromethane
1,1-Dichloroethane
1,2-Dichloroethane
1,1-Dichloroethylene
trans-1,2-Dichloroethylene
Dichloromethane (Methylene chloride)
2,4-Dichlorophenoxyacetic acid
1,2-Dichloropropane
trans-1,3-Dichloropropene
cis-1,3-Dichloropropene
Endrin
Endrin
Ethylbenzene
Fluoride
Fluoride
Iron
Lead

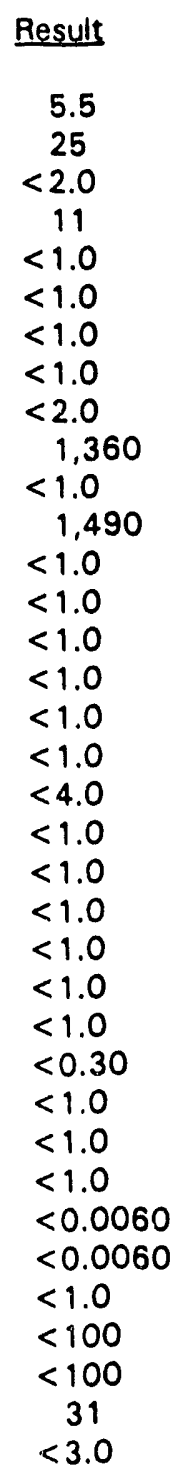

\begin{tabular}{lll} 
Unit & Flag & Lab \\
\cline { 2 - 3 } & & \\
$\mu \mathrm{H}$ & 0 & $\mathrm{GE}$ \\
$\mu \mathrm{C} / \mathrm{cm}$ & 0 & $\mathrm{GE}$ \\
$\mu \mathrm{g} / \mathrm{L}$ & 0 & $\mathrm{GE}$ \\
$\mu \mathrm{g} / \mathrm{L}$ & 0 & $\mathrm{GE}$ \\
$\mu \mathrm{g} / \mathrm{L}$ & 0 & $\mathrm{GE}$ \\
$\mu \mathrm{g} / \mathrm{L}$ & 0 & $\mathrm{GE}$ \\
$\mu \mathrm{g} / \mathrm{L}$ & 0 & $\mathrm{GE}$ \\
$\mu \mathrm{g} / \mathrm{L}$ & 0 & $\mathrm{GE}$ \\
$\mu \mathrm{g} / \mathrm{L}$ & 0 & $\mathrm{GE}$ \\
$\mu \mathrm{g} / \mathrm{L}$ & 0 & $\mathrm{GE}$ \\
$\mu \mathrm{g} / \mathrm{L}$ & 0 & $\mathrm{GE}$ \\
$\mu \mathrm{g} / \mathrm{L}$ & 0 & $\mathrm{GE}$ \\
$\mu \mathrm{g} / \mathrm{L}$ & 0 & $\mathrm{GE}$ \\
$\mu \mathrm{g} / \mathrm{L}$ & 0 & $\mathrm{GE}$ \\
$\mu \mathrm{g} / \mathrm{L}$ & 0 & $\mathrm{GE}$ \\
$\mu \mathrm{g} / \mathrm{L}$ & 0 & $\mathrm{GE}$ \\
$\mu \mathrm{g} / \mathrm{L}$ & 0 & $\mathrm{GE}$ \\
$\mu \mathrm{g} / \mathrm{L}$ & 0 & $\mathrm{GE}$ \\
$\mu \mathrm{g} / \mathrm{L}$ & 0 & $\mathrm{GE}$ \\
$\mu \mathrm{g} / \mathrm{L}$ & 0 & $\mathrm{GE}$ \\
$\mu \mathrm{g} / \mathrm{L}$ & 0 & $\mathrm{GE}$ \\
$\mu \mathrm{g} / \mathrm{L}$ & 0 & $\mathrm{GE}$ \\
$\mu \mathrm{g} / \mathrm{L}$ & 0 & $\mathrm{GE}$ \\
$\mu \mathrm{g} / \mathrm{L}$ & 0 & $\mathrm{GE}$ \\
$\mu \mathrm{g} / \mathrm{L}$ & 0 & $\mathrm{GE}$ \\
$\mu \mathrm{g} / \mathrm{L}$ & 0 & $\mathrm{GE}$ \\
$\mu \mathrm{g} / \mathrm{L}$ & 0 & $\mathrm{GE}$ \\
$\mu \mathrm{g} / \mathrm{L}$ & 0 & $\mathrm{GE}$ \\
$\mu \mathrm{g} / \mathrm{L}$ & 0 & $\mathrm{GE}$ \\
$\mu \mathrm{g} / \mathrm{L}$ & 0 & $\mathrm{GE}$ \\
$\mu \mathrm{g} / \mathrm{L}$ & 0 & $\mathrm{GE}$ \\
$\mu \mathrm{g} / \mathrm{L}$ & 0 & $\mathrm{GE}$ \\
$\mu \mathrm{g} / \mathrm{L}$ & 0 & $\mathrm{GE}$ \\
$\mu \mathrm{g} / \mathrm{L}$ & 0 & $\mathrm{GE}$ \\
$\mu \mathrm{g} / \mathrm{L}$ & 0 & $\mathrm{GE}$ \\
& 0 &
\end{tabular}

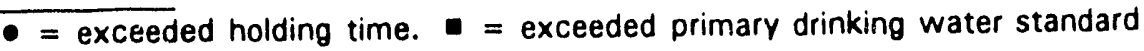


WELL LFW 47D, laboratory analyses (cont.)

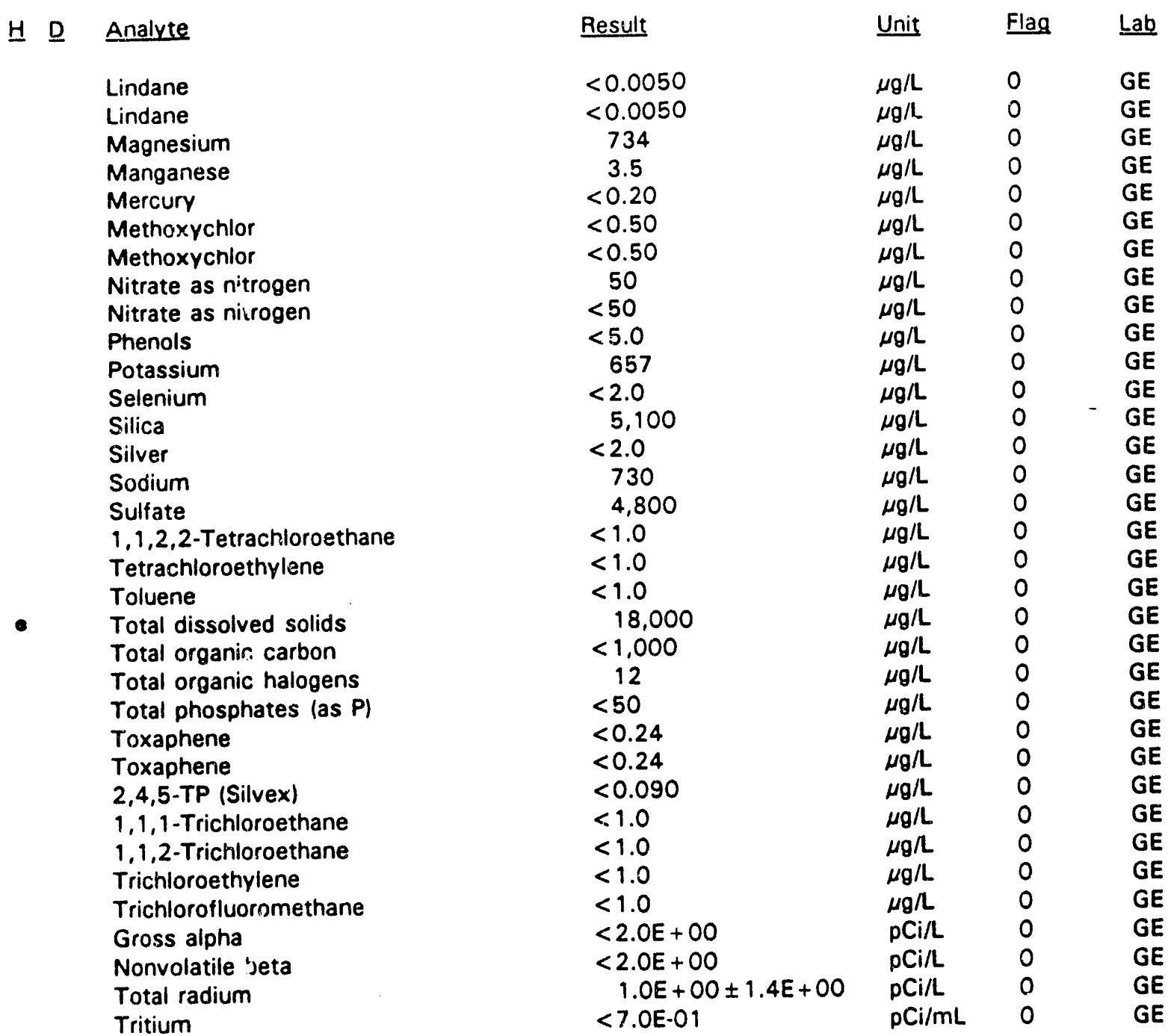

WELL LFW 48C

SRS Coord.

N83877.0

E45430.3

\section{Screen Zone Elevation}

$118.2-108.2 \mathrm{ft} \mathrm{msl}$
Top of Casing

$169.3 \mathrm{ft} \mathrm{msl}$
Casing

4" PVC

MEASUREMENTS CONDUCTED IN THE FIELD

Sample date: $10 / 29 / 91$

Depth to water: $20.29 \mathrm{ft}(6.18 \mathrm{~m})$ below TOC

Water elevation: $149.01 \mathrm{ft}(45.42 \mathrm{~m}) \mathrm{msl}$

Sp. conductance: $25 \mu \mathrm{S} / \mathrm{cm}$

Water evacuated before sampling: $13 E \mathrm{gal}$
Time: 9:50

$\mathrm{pH}: 5.4$

Alkalinity: $3 \mathrm{mg} / \mathrm{L}$

Water temperature: $18.1^{\circ} \mathrm{C}$

= exceeded holding time. $=$ exceeded primary drinking water standard. 


\section{LABORATORY ANALYSES}

H D Analvte

- $\quad \mathrm{pH}$

\begin{tabular}{|c|c|}
\hline $\begin{array}{l}\text { Specific conductance } \\
\text { Arsenic }\end{array}$ & $\begin{array}{r}20 \\
<2.0\end{array}$ \\
\hline $\begin{array}{l}\text { Arsenic } \\
\text { Arsenic }\end{array}$ & $<2.0$ \\
\hline Barium & 6.3 \\
\hline Benzene & $<1.0$ \\
\hline Bromodichloromethane & $<1.0$ \\
\hline Bromoform & $<1.0$ \\
\hline Bromomethane (Methyl bromide) & $<1.0$ \\
\hline Cadmium & $<2.0$ \\
\hline Calcium & 998 \\
\hline Carbon tetrachloride & $<1.0$ \\
\hline Chloride & 1.780 \\
\hline Chlorobenzene & $<1.0$ \\
\hline Chloroethane & $<1.0$ \\
\hline Chloroethene (Vinyl chloride) & $<1.0$ \\
\hline 2-Chloroethyl vinyl ether & $<1.0$ \\
\hline Chloroform & $<1.0$ \\
\hline Chloromethane (Methyl chloride) & $<1.0$ \\
\hline Chromium & $<4.0$ \\
\hline Dibromochloromethane & $<1.0$ \\
\hline 1,1-Dichloroethane & $<1.0$ \\
\hline 1,2-Dichloroethane & $<1.0$ \\
\hline 1,1-Dichloroethylene & $<1.0$ \\
\hline trans-1,2-Dichloroethylene & $<1.0$ \\
\hline Dichloromethane (Methylene chloride) & 4.1 \\
\hline 2,4-Dichlorophenoxyacetic acid & $<0.30$ \\
\hline 1,2-Dichloropropane & $<1.0$ \\
\hline trans-1,3-Dichloropropene & $<1.0$ \\
\hline cis-1,3-Dichloropropene & $<1.0$ \\
\hline Endrin & $<0.0060$ \\
\hline Endrin & $<0.0060$ \\
\hline Ethylbenzene & $<1.0$ \\
\hline Fluoride & $<100$ \\
\hline Lead & $<3.0$ \\
\hline Lead & $<3.0$ \\
\hline Lindane & $<0.0050$ \\
\hline Lindane & $<0.0050$ \\
\hline Magnesium & 363 \\
\hline Manganese & 18 \\
\hline Mercury & $<0.20$ \\
\hline Methoxychlor & $<0.50$ \\
\hline Methoxychlor & $<0.50$ \\
\hline Nitrate as nitrogen & 430 \\
\hline Phenols & $<5.0$ \\
\hline Potassium & 508 \\
\hline Selenium & $<2.0$ \\
\hline Selenium & $<2.0$ \\
\hline Silica & 7,050 \\
\hline Silver & $<2.0$ \\
\hline Sodium & 1,440 \\
\hline
\end{tabular}

\begin{tabular}{|c|c|c|}
\hline Unit & Flag & $\underline{\text { Lab }}$ \\
\hline $\mathrm{pH}$ & 0 & $\mathrm{GE}$ \\
\hline$\mu \mathrm{S} / \mathrm{cm}$ & 0 & GE \\
\hline$\mu g / L$ & 0 & GE \\
\hline$\mu \mathrm{g} / \mathrm{L}$ & 0 & GE \\
\hline$\mu g / L$ & 0 & GE \\
\hline$\mu g / L$ & 0 & GE \\
\hline$\mu \mathrm{g} / \mathrm{L}$ & 0 & GE \\
\hline$\mu g / L$ & 0 & GE \\
\hline$\mu \mathrm{g} / \mathrm{L}$ & 0 & GE \\
\hline$\mu g / L$ & 0 & GE \\
\hline$\mu \mathrm{g} / \mathrm{L}$ & 0 & GE \\
\hline$\mu g / L$ & 0 & GE \\
\hline$\mu g / L$ & 0 & $G E$ \\
\hline$\mu g / L$ & 0 & GE \\
\hline$\mu g / L$ & 0 & GE \\
\hline$\mu g / L$ & 0 & GE \\
\hline$\mu g / L$ & 0 & GE \\
\hline$\mu g / L$ & 0 & GE \\
\hline$\mu g / L$ & 0 & GE \\
\hline$\mu g / L$ & 0 & GE \\
\hline$\mu g / L$ & 0 & GE \\
\hline$\mu g / L$ & 0 & GE \\
\hline$\mu g / L$ & 0 & GE \\
\hline$\mu g / L$ & 0 & GE \\
\hline$\mu g / L$ & 0 & GE \\
\hline$\mu g / L$ & 0 & GE \\
\hline$\mu g / L$ & 0 & GE \\
\hline$\mu g / L$ & 0 & GE \\
\hline$\mu g / L$ & 0 & GE \\
\hline$\mu g / L$ & 0 & GE \\
\hline$\mu g / L$ & 0 & GE \\
\hline$\mu g / L$ & 0 & GE \\
\hline$\mu g / L$ & 0 & GE \\
\hline$\mu g / L$ & 0 & GE \\
\hline$\mu g / L$ & 0 & GE \\
\hline$\mu g / L$ & 0 & GE \\
\hline$\mu g / L$ & 0 & $\mathrm{GE}$ \\
\hline$\mu \mathrm{g} / \mathrm{L}$ & 0 & GE \\
\hline$\mu g / L$ & 0 & GE \\
\hline$\mu \mathrm{g} / \mathrm{L}$ & 0 & GE \\
\hline$\mu \mathrm{g} / \mathrm{L}$ & 0 & GE \\
\hline$\mu \mathrm{g} / \mathrm{L}$ & 0 & GE \\
\hline$\mu \mathrm{g} / \mathrm{L}$ & 0 & GE \\
\hline$\mu \mathrm{g} / \mathrm{L}$ & 0 & $\mathrm{GE}$ \\
\hline$\mu \mathrm{g} / \mathrm{L}$ & 0 & $\mathrm{GE}$ \\
\hline$\mu g / L$ & 0 & $\mathrm{GE}$ \\
\hline$\mu g / L$ & 0 & $\mathrm{GE}$ \\
\hline$\mu g / L$ & 0 & GE \\
\hline$\mu g / L$ & 0 & $\mathrm{GE}$ \\
\hline$\mu g / L$ & 0 & $\mathrm{GE}$ \\
\hline$\mu g / L$ & 0 & GE \\
\hline
\end{tabular}

\footnotetext{
- = exceeded holding time. $=$ exceeded primary drinking water standard
} 
WELL LFW 48C, laboratory analyses (cont.)

H $\underline{\text { Analyte }}$

Sulfate
1,1,2,2-Tetrachloroethane
Tetrachloroethylene
Toluene
Total dissolved solids
Tota! organic carbon
Total organic carbon
Total organic halogens
Total phosphates las P)
Toxaphene
Toxaphene
2,4,5-TP (Silvex)
1,1,1-Trichloroethane
1,1,2-Trichloroethane
Trichloroethylene
Trichlorofluoromethane
Gross alpha
Nonvolatile beta
Total radium
Tritium

Result

$<1.000$
$<1.0$
$<1.0$
$<1.0$
$\quad 14,000$
$<1.000$
$<1.000$
$\quad 7.7$
$<50$
$<0.24$
$<0.24$
$<0.090$
$<1.0$
$<1.0$
$<1.0$
$<1.0$
$<2.0 E+00$
$<2.0 E+00$
$<1.0 E+00$
$<7.0 E-01$

$\begin{array}{lll}\text { Unit } & \text { Flag } & \text { Lab } \\ \mu g / \mathrm{L} & 0 & \text { GE } \\ \mu \mathrm{g} / \mathrm{L} & 0 & \mathrm{GE} \\ \mu \mathrm{g} / \mathrm{L} & 0 & \mathrm{GE} \\ \mu \mathrm{g} / \mathrm{L} & 0 & \mathrm{GE} \\ \mu \mathrm{g} / \mathrm{L} & 0 & \mathrm{GE} \\ \mu \mathrm{g} / \mathrm{L} & 0 & \mathrm{GE} \\ \mu \mathrm{g} / \mathrm{L} & 0 & \mathrm{GE} \\ \mu \mathrm{g} / \mathrm{L} & 0 & \mathrm{GE} \\ \mu \mathrm{g} / \mathrm{L} & 0 & \mathrm{GE} \\ \mu \mathrm{g} / \mathrm{L} & 0 & \mathrm{GE} \\ \mu \mathrm{g} / \mathrm{L} & 0 & \mathrm{GE} \\ \mu \mathrm{g} / \mathrm{L} & 0 & \mathrm{GE} \\ \mu \mathrm{g} / \mathrm{L} & 0 & \mathrm{GE} \\ \mu \mathrm{g} / \mathrm{L} & 0 & \mathrm{GE} \\ \mu \mathrm{g} / \mathrm{L} & 0 & \mathrm{GE} \\ \mu \mathrm{g} / \mathrm{L} & 0 & \mathrm{GE} \\ \mathrm{pCi} / \mathrm{L} & 0 & \mathrm{GE} \\ \mathrm{pCi} / \mathrm{L} & 0 & \mathrm{GE} \\ \mathrm{pCi} / \mathrm{L} & 0 & \mathrm{GE} \\ \mathrm{pCi} / \mathrm{mL} & 0 & \mathrm{GE}\end{array}$

\section{WELL LFW 48D}

\section{SRS Coord.}

N83877.5

E45443.7

\section{MEASUREMENTS CONDUCTED IN THE FIELD}

Sample date: $10 / 29 / 91$

Depth to water: $20.16 \mathrm{ft}(6.14 \mathrm{~m})$ below TOC Water elevation: $149.34 \mathrm{ft}(45.52 \mathrm{~m}) \mathrm{msl}$

Sp. conductance: $405 \mu \mathrm{S} / \mathrm{cm}$

Water evacuated before sampling: $39 \mathrm{gal}$

\section{LABORATORY ANALYSES}

H $\underline{\text { Analvte }}$

- $\mathrm{pH}$

Specific conductance

Arsenic

Arsenic

Barium

Benzene

Bromodichloromethane

Bromotorm

Biomomethane (Nethyl bromide)

Cadmium
Result

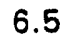

275

9.8

9.8

8.2

$<1.0$

$<1.0$

$<1.0$

$<1.0$

$<2.0$
Toe of Casing

$169.5 \mathrm{ft} \mathrm{msl}$
Casing

4" PVC
Time: 9:30

pH: 6.7

Alkalinity: $168 \mathrm{mg} / \mathrm{L}$

Water temperature: $18.9^{\circ} \mathrm{C}$

- exceeded holding time. $=$ exceeded primary drinking water standard. 
WELL LFW 48D, laboratory analyses (cont.)

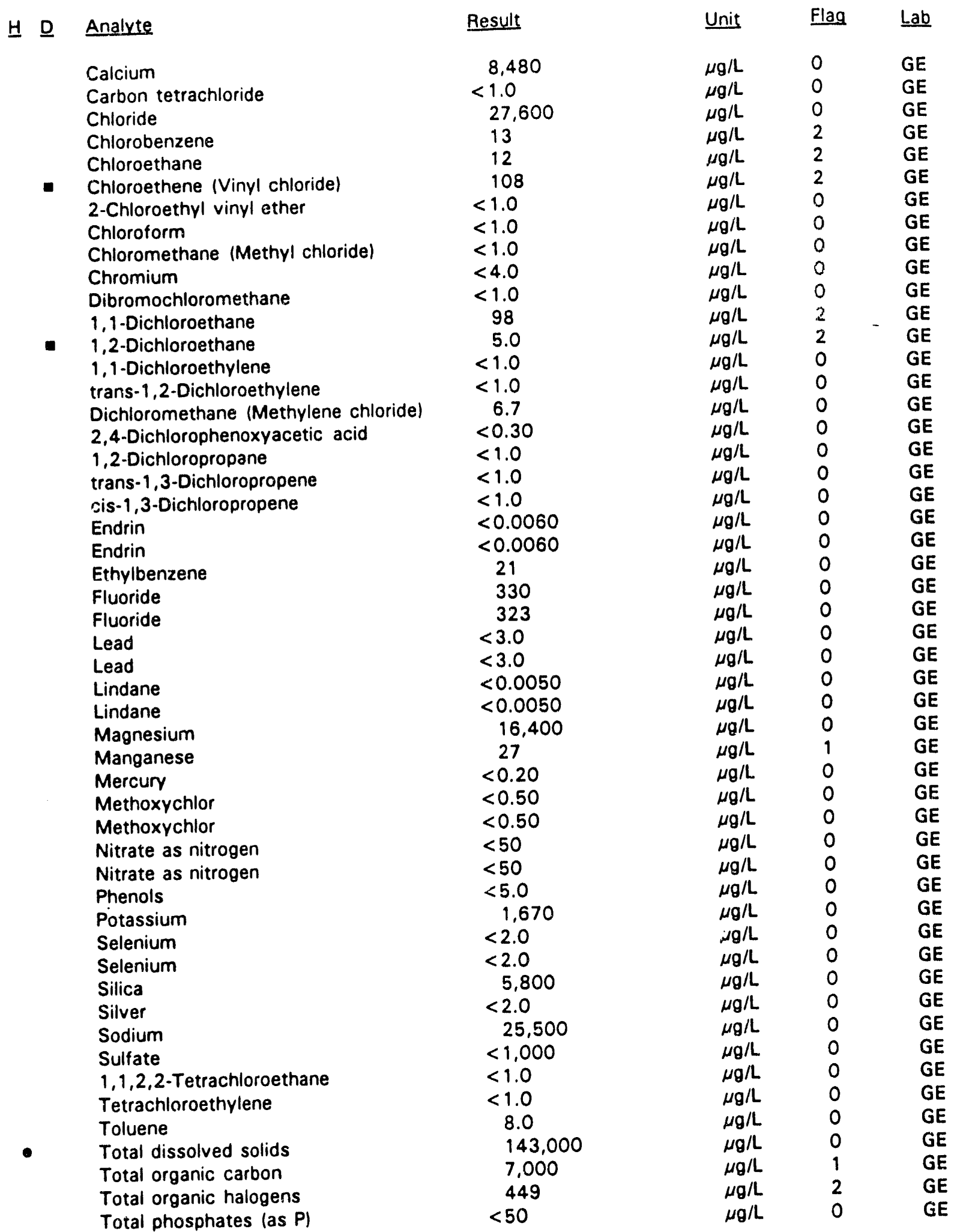

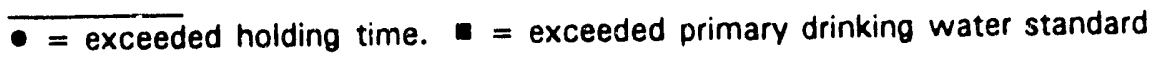


WELL LFW 48D, laboratory analyses (cont.)

$\underline{H} \underline{\mathrm{D}}$ Analyte

Toxaphene

Toxaphene

2,4,5-TP (Silvex)

$1,1,1$-Trichloroethane

1,1,2-Trichloroethane

Trichloroethylene

Trichlorofluoromethane

Gross alpha

Nonvolatile beta

Total radium

Tritium

$\begin{aligned} & \text { Result } \\ &<0.24 \\ &<0.24 \\ &<0.090 \\ &<1.0 \\ &<1.0 \\ &<1.0 \\ &<1.0 \\ & 2.1 E+00 \pm 1.4 E+00 \\ & 4.2 E+00 \pm 1.6 E+00 \\ &< 1.0 E+00 \\ & 5.3 E+00 \pm 7.0 E-01\end{aligned}$

$\underline{\text { Unit }}$

Flag

$\underline{L a b}$

$\mu g / L \quad O \quad$ GE

$\mu g / L \quad 0 \quad G E$

$\mu g / L \quad O \quad G E$

$\mu g / L \quad O \quad G E$

$\mu g / L \quad O \quad G E$

$\mu g / L \quad O \quad G E$

$\mu g / L \quad O \quad G E$

pCi/L $\quad 0 \quad$ GE

$\mathrm{pCi} / \mathrm{L} \quad \mathrm{GE}$

$\mathrm{pCi} / \mathrm{L} \quad \mathrm{O} \quad \mathrm{GE}$

$\mathrm{pCi} / \mathrm{mL} \quad \mathrm{O}$ GE

\section{WELL LFW 55C}

\section{SRS Coord.}

N83613.2

E45205.9

\section{MEASUREMENTS CONDUCTED IN THE FIELD}

Sample date: 10/29/91

Depth to water: $9.38 \mathrm{ft}(2.86 \mathrm{~m})$ below TOC

Water elevation: $147.02 \mathrm{ft}(44.81 \mathrm{~m}) \mathrm{msl}$

Sp. conductance: $40 \mu \mathrm{S} / \mathrm{cm}$

Water evacuated before sampling: $139 \mathrm{gal}$

\section{LABORATORY ANALYSES}

H D Analyte

- $\mathrm{pH}$

Specific conductance

Arsenic

Arsenic

Barium

Benzene

Bromodichloromethane

Bromoform

Bromomethane (Methyl bromide)

Cadmium

Calcium

Carbon tetrachloride

Chloride

Chlorobenzene

Chloroethane

- Chloroethene (Vinyl chloride)

2.Chloroethyl vinyl ether

Chloromethane (Methyl chloride)
Chloroform
Result

5.5

35

$<2.0$

$<2.0$

3.4

$<1.0$

$<1.0$

$<1.0$

$<1.0$

$<2.0$

1,060

$<1.0$

4,540

$<1.0$

$<1.0$

5.6

$<1.0$

$<1.0$

$<1.0$
Time: $10: 50$

$\mathrm{pH}: 5.2$

Alkalinity: $1 \mathrm{mg} / \mathrm{L}$

Water temperature: $18.0^{\circ} \mathrm{C}$
Casing

4" PVC 
WELL LFW 55D, laboratory analyses (cont.)

H D Analyte

Endrin
Ethylbenzene
Fluoride
Lead
Lead
Lindane
Lindane
Magnesium
Manganese
Mercury
Methoxychlor
Methoxychlor
Nitrate as nitrogen
Phenols
Potassium
Selenium
Selenium
Silica
Silver
Sodium
Sulfate
1,1,2,2-Tetrachloroethane
Tetrachloroethylene
Toluene
Total dissolved solids
Total organic carbon
Total organic halogens
Total phosphates (as P)
Total phosphates (as P)
Total phosphates las P)
Toxaphene
Toxaphene
2,4,5-TP (Silvex)
$1,1,1$-Trichloroethane
1,1,2-Trichloroethane
Trichloroethylene
Trichlorofluoromethane
Gross alpha
Nonvolatile beta
Total radium
Tritium

Result

$<0.0060$

$<1.0$

$<100$

$<3.0$

$<3.0$

$<0.0050$

$<0.0050$

643

19

$<0.20$

$<0.50$

$<0.50$

220

$<5.0$

827

$<2.0$

$<2.0$

6,570

$<2.0$

1,150

2,350

$<1.0$

$<1.0$

$<1.0$

10,000

$<1,000$

19

$<50$

$<50$

$<50$

$<0.24$

$<0.24$

$<0.090$

$<1.0$

$<1.0$

$<1.0$

$<1.0$

$<2.0 E+00$

$<2.0 E+00$

$<1.0 E+00$

$<7.0 \mathrm{E}-01$

\begin{tabular}{|c|c|c|}
\hline Unit & Flag & Lat \\
\hline$\mu \mathrm{g} / \mathrm{L}$ & 0 & $\mathrm{GE}$ \\
\hline$\mu \mathrm{g} / \mathrm{L}$ & 0 & $\mathrm{GE}$ \\
\hline$\mu g / L$ & 0 & $\mathrm{GE}$ \\
\hline$\mu g / L$ & 0 & GE \\
\hline$\mu g / L$ & 0 & $\mathrm{GE}$ \\
\hline$\mu g / L$ & 0 & $\mathrm{GE}$ \\
\hline$\mu g / L$ & 0 & GE \\
\hline$\mu g / L$ & 0 & GE \\
\hline$\mu g / L$ & 0 & GE \\
\hline$\mu g / L$ & 0 & GE \\
\hline$\mu g / L$ & 0 & GE \\
\hline$\mu \mathrm{g} / \mathrm{L}$ & 0 & $\mathrm{GE}$ \\
\hline$\mu g / L$ & 0 & $\mathrm{GE}$ \\
\hline$\mu g / L$ & 0 & $\mathrm{GE}$ \\
\hline$\mu g / L$ & 0 & $\mathrm{GE}$ \\
\hline$\mu g / L$ & 0 & $\mathrm{GE}$ \\
\hline$\mu g / L$ & 0 & $\mathrm{GE}$ \\
\hline$\mu g / L$ & 0 & $\mathrm{GE}$ \\
\hline$\mu g / L$ & 0 & $G E$ \\
\hline$\mu g / L$ & 0 & $\mathrm{GE}$ \\
\hline$\mu g / L$ & 0 & GE \\
\hline$\mu g / L$ & 0 & $\mathrm{GE}$ \\
\hline$\mu g / L$ & 0 & $\mathrm{GE}$ \\
\hline$\mu g / L$ & 0 & GE \\
\hline$\mu g / L$ & 0 & GE \\
\hline$\mu g / L$ & 0 & $\mathrm{GI}$ \\
\hline$\mu g / L$ & 0 & GI \\
\hline$\mu g / L$ & 0 & $\mathrm{Gl}$ \\
\hline$\mu \mathrm{g} / \mathrm{L}$ & 0 & $\mathrm{G}$ \\
\hline$\mu g / L$ & 0 & G \\
\hline$\mu g / L$ & 0 & $\mathrm{Gl}$ \\
\hline$\mu g / L$ & 0 & $\mathrm{Gt}$ \\
\hline$\mu g / L$ & 0 & $\mathrm{G}$ \\
\hline$\mu g / L$ & 0 & GI \\
\hline$\mu g / L$ & 0 & (s) \\
\hline$\mu g / L$ & 0 & $G$ \\
\hline$\mu g / L$ & 0 & $\mathbf{G}$ \\
\hline $\mathrm{pCi} / \mathrm{L}$ & 0 & $\mathbf{G}$ \\
\hline $\mathrm{pCi} / \mathrm{L}$ & 0 & G \\
\hline $\mathrm{pCi} / \mathrm{L}$ & 0 & G \\
\hline $\mathrm{pCi} / \mathrm{mL}$ & 0 & G \\
\hline
\end{tabular}

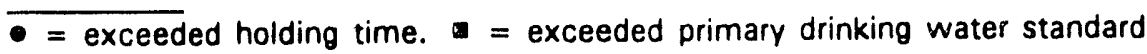


WELL LFW 56D

SRS Coord.

N83398.0

E45306.6

MEASUREMENTS CONDUCTED IN THE FIELD

Sample date: 10/29/91

Depth to water: $12.61 \mathrm{ft}(3.84 \mathrm{~m})$ below TOC Water elevation: $145.49 \mathrm{ft}(44.35 \mathrm{~m}) \mathrm{msl}$

Sp. conductance: $16 \mu \mathrm{S} / \mathrm{cm}$

Water evacuated before sampling: $38 \mathrm{gal}$

LABORATORY ANALYSES
Screen Zone Elevation

$151.4-131.3 \mathrm{ft} \mathrm{msl}$
Top of Casing

$158.1 \mathrm{ft} \mathrm{msl}$
Casing

4" PVC
Time: $11: 30$

pH: 4.9

Alkalinity: $0 \mathrm{mg} / \mathrm{L}$

Water temperature: $19.5^{\circ} \mathrm{C}$

\section{브 Analyte}

-

pH

Specific conductance

Arsenic

Arsenic

Barium

Benzene

Bromodichloromethane

Bromoform

Bromomethane (Methyl bromide)

Cadmium

Calcium

Carbon tetrachloride

Chloride

Chloroberizene

Chloroethane

Chloroethene (Vinyl chloride)

2-Chloroethyl vinyl ether

Chloroform

Chloromethane (Methyl chloride)

Chromium

Dibromochloromethane

1.1-Dichloroethane

1,2-Dichloroethane

1,1-Dichloroethylene

trans-1,2-Dichloroethylene

Dichloromethane (Methylene chloride)

2,4-Dichlorophenoxyacetic acid

1,2-Dichloropropane

trans-1,3-Dichioropropene

cis-1,3-Dichloropropene

Endrin

Endrin

Ethylbenzene

Fluoride

Lead

Lead
Result

5.3

15

$<2.0$

$<2.0$

$<3.0$

$<1.0$

$<1.0$

$<1.0$

$<1.0$

$<2.0$

368

$<1.0$

2,220

$<1.0$

$<1.0$

$<1.0$

$<1.0$

$<1.0$

$<1.0$

$<4.0$

$<1.0$

1.8

$<1.0$

$<1.0$

$<1.0$

3.4

$<0.30$

$<1.0$

$<1.0$

$<1.0$

$<0.0060$

$<0.0060$

$<1.0$

$<100$

$<3.0$

$<3.0$
Unit Flag Lab

$\mathrm{pH} \quad \mathrm{O} \quad \mathrm{GE}$

$\mu \mathrm{S} / \mathrm{cm} \quad 0 \quad \mathrm{GE}$

$\mu g / L \quad 0 \quad$ GE

$\mu g / L \quad 0 \quad$ GE

$\mu g / L \quad 0 \quad$ GE

$\mu g / L \quad 0 \quad$ GE

$\mu g / L \quad 0 \quad$ GE

$\mu g / L \quad 0 \quad$ GE

$\mu g / L \quad 0 \quad$ GE

$\mu g / L \quad 0 \quad$ CE

$\mu g / L \quad 0 \quad G E$

$\mu g / L \quad 0 \quad$ GE

$\mu g / L \quad 0 \quad$ GE

$\mu g / L \quad 0 \quad G E$

$\mu g / L \quad 0 \quad$ GE

$\mu g / L \quad 0 \quad$ GE

$\mu g / L \quad 0 \quad$ GE

$\mu g / L \quad 0 \quad$ GE

$\mu g / L \quad O \quad$ GE

$W / L \quad 0 \quad$ GE

He: 0 GE

$\mu$ H $\quad 0$ GE

$\mu_{\mathrm{G}} / . \quad 0 \quad \mathrm{GE}$

$\mu$ (n). $\quad 0 \quad$ GE

$\mu g / s_{\infty} \quad 0 \quad$ GE

$\mu g / h . \quad 0 \quad$ GE

$\mu g /$ i. $\quad 0 \quad$ GE

$\mu g /$ h. $\quad 0 \quad$ GE

Wo/ $\quad 0$ GE

Wh $\quad 0 \quad$ GE

WGL $\quad 0 \quad$ GE

$\mathrm{kig} / \mathrm{L} \quad 0 \quad \mathrm{GE}$

$\mu g / L . \quad 0 \quad$ GE

$\mu g / L \quad 0 \quad$ GE

$\mu g / L \quad 0 \quad$ GE

$\mu g / L \quad 0 \quad$ GE

- exceeded holding time. = exceeded primary drinking water standard. 
WELL LFW 57B

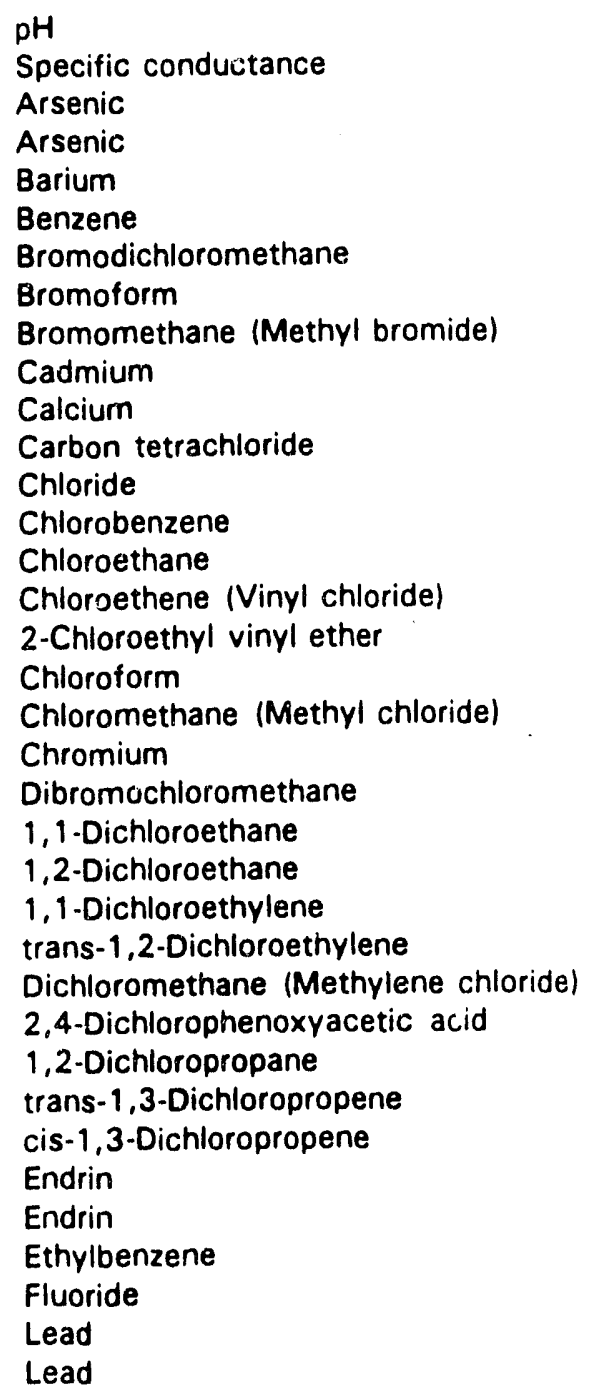

SRS Coord.

N83196.7

E45440.6

\section{MEASUREMENTS CONDUCTED IN THE FIELD}

\section{Sample date: 10/29/91}

Depth to water: $21.60 \mathrm{ft}(6.58 \mathrm{~m})$ below TOC Water elevation: $143.80 \mathrm{ft}(43.83 \mathrm{~m}) \mathrm{msl}$

Sp. conductance: $55 \mu \mathrm{S} / \mathrm{cm}$

Water evacuated before sampling: $249 \mathrm{gal}$

\section{LABORATORY ANALYSES}

H D Analyte

Screen Zone Elevation

78.4-68.4 $\mathrm{ft} \mathrm{msl}$

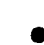

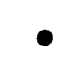

$\bullet$
Result

$\begin{aligned} & 4.4 \\ & 50 \\ &< 2.0 \\ &< 2.0 \\ & 8.1 \\ &< 1.0 \\ &< 1.0 \\ &< 1.0 \\ &< 1.0 \\ &< 2.0 \\ & 1.480 \\ &< 1.0 \\ & 2.550 \\ &< 1.0 \\ &< 1.0 \\ &< 1.0 \\ &< 1.0 \\ &< 1.0 \\ &< 1.0 \\ &< 4.0 \\ &<1.0 \\ &<1.0 \\ &<1.0 \\ &<1.0 \\ &<1.0 \\ & 3.1 \\ &<<0.30 \\ &<1.0 \\ &<1.0 \\ &<1.0 \\ &<0.0060 \\ &<0.0060 \\ &<1.0 \\ &<100 \\ &<3.0 \\ &<3.0 \\ &\end{aligned}$

Time: $12: 40$

$\mathrm{pH}: 4.2$

Alkalinity: $0 \mathrm{mg} / \mathrm{L}$

Water temperature: $18.5^{\circ} \mathrm{C}$
Top of Casing Casing

$165.4 \mathrm{ft} \mathrm{msl} \quad 4^{n}$ PVC 
WELL LFW 578, laboratory analyses (cont.)

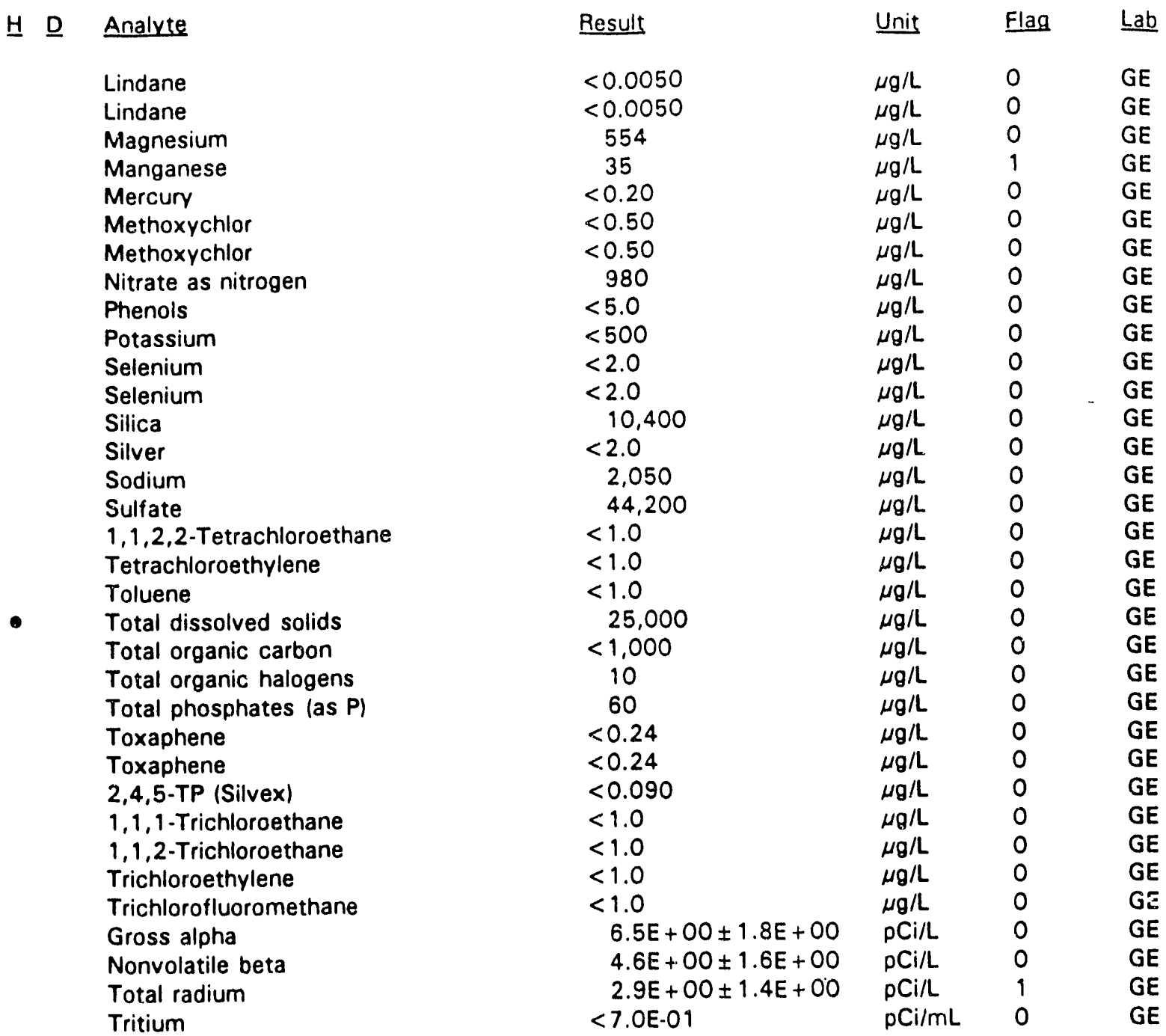

\section{WELL LFW 57C}

\section{SRS Coord.}

N83200.1

E45411.1

\section{Screen Zone Elevation}

$117.9-107.8 \mathrm{ft} \mathrm{msl}$
Top of Casing

$165.0 \mathrm{ft} \mathrm{msl}$
Casing

4" PVC

\section{MEASUREMENTS CONDUCTED IN THE FIELD}

Sample date: 10/29/91

Depth to water: $21.08 \mathrm{ft}(6.43 \mathrm{~m})$ below TOC

Water elevation: $143.92 \mathrm{ft}(43.87 \mathrm{~m}) \mathrm{ms}$ |

Sp. conductance: $31 \mu \mathrm{S} / \mathrm{cm}$

Water evacuated before sainpling: $96 \mathrm{gal}$
Time: 12:15

pH: 5.3

Alkalinity: $2 \mathrm{mg} / \mathrm{L}$

Water temperature: $18.1^{\circ} \mathrm{C}$

$\overline{- \text { = exceeded holding time. }}=$ exceeded primary drinking water standard 


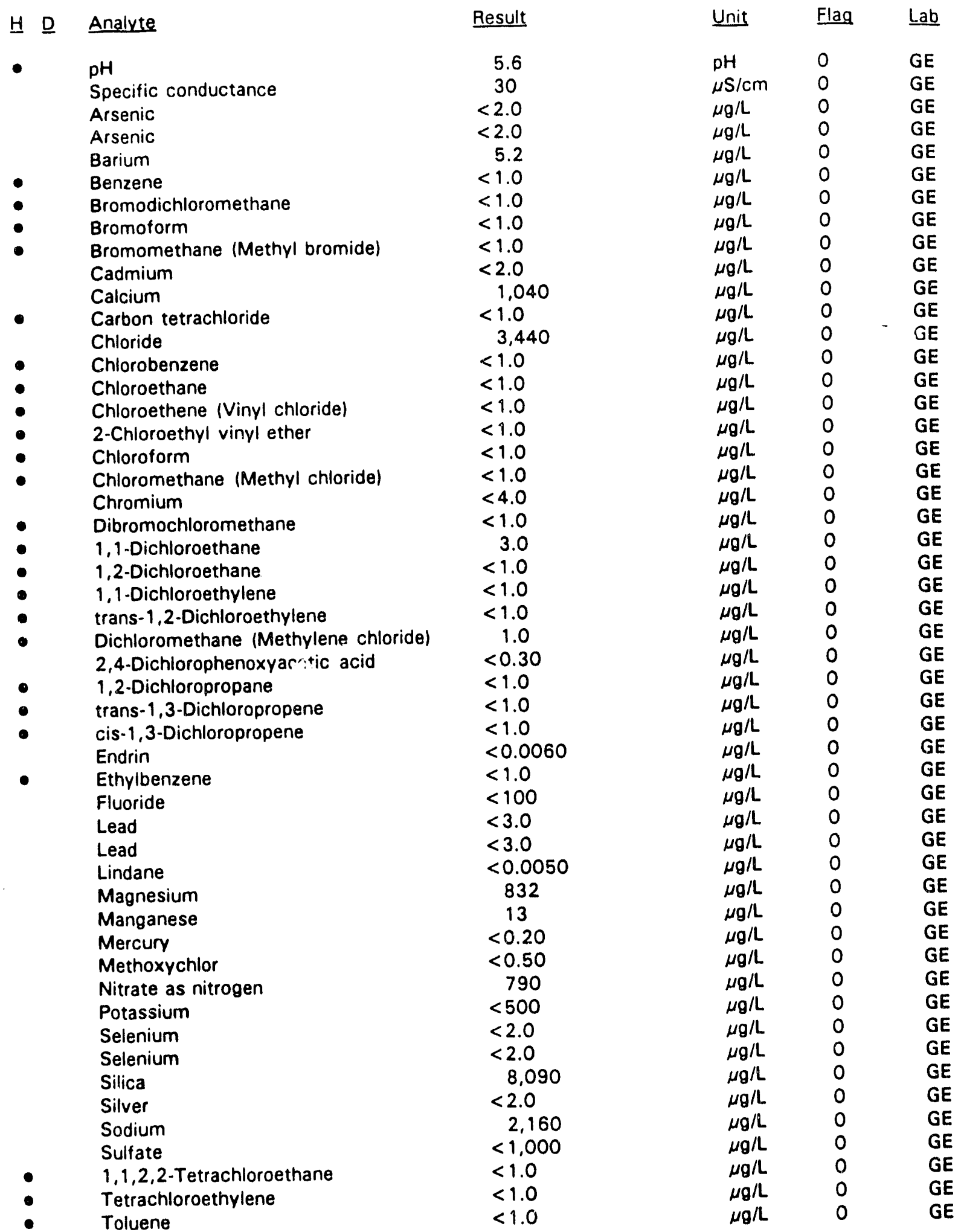

$\overline{0}=$ exceeded holding time. $=$ exceeded primary dinking water standard. 
WELL LFW 57C, laboratory analyses (cont.)

$\begin{array}{ll}\text { H. } & \text { Analyte } \\ & \text { Total organic carbon } \\ \text { Total organic carbon } \\ \text { Total organic halogens } \\ \text { Total organic halogens } \\ \text { Toxaphene } \\ \text { 2,4,5-TP (Silvex) } \\ \text { 1,1,1-Trichloroethane } \\ \text { 1,1,2-Trichloroethane } \\ \text { Trichloroethylene } \\ \text { Trichlorofluoromethane } \\ \text { Gross alpha } \\ \text { Nonvolatile beta } \\ \text { Total radium } \\ \text { Tritium }\end{array}$

Result

$<1,000$
$<1,000$
$\quad 12$
$\quad 13$
$<0.24$
$<0.090$
$<1.0$
$<1.0$
$<1.0$
$<1.0$
$<2.0 E+00$
$<2.0 E+00$
$<1.0 E+00$
$<7.0 E-01$

\begin{tabular}{llll} 
Unit & & Flag & Lab \\
\cline { 3 - 4 }$\mu \mathrm{g} / \mathrm{L}$ & 0 & $\mathrm{GE}$ \\
$\mu \mathrm{g} / \mathrm{L}$ & 0 & $\mathrm{GE}$ \\
$\mu \mathrm{g} / \mathrm{L}$ & 0 & $\mathrm{GE}$ \\
$\mu \mathrm{g} / \mathrm{L}$ & 0 & $\mathrm{GE}$ \\
$\mu \mathrm{g} / \mathrm{L}$ & 0 & $\mathrm{GE}$ \\
$\mu \mathrm{g} / \mathrm{L}$ & 0 & $\mathrm{GE}$ \\
$\mu \mathrm{g} / \mathrm{L}$ & 0 & $\mathrm{GE}$ \\
$\mu \mathrm{g} / \mathrm{L}$ & 0 & $\mathrm{GE}$ \\
$\mu \mathrm{g} / \mathrm{L}$ & 0 & $\mathrm{GE}$ \\
$\mu \mathrm{g} / \mathrm{L}$ & 0 & $\mathrm{GE}$ \\
$\mathrm{pCi} / \mathrm{L}$ & 0 & $\mathrm{GE}$ \\
$\mathrm{pCi} / \mathrm{L}$ & 0 & $\mathrm{GE}$ \\
$\mathrm{pCi} / \mathrm{L}$ & 0 & GE \\
$\mathrm{pCi} / \mathrm{mL}$ & 0 & GE
\end{tabular}

\section{WELL LFW 57D}

\begin{tabular}{|c|c|c|c|}
\hline SRS Coord. & Scrten Zone Elevation & Top of Casing & Casing \\
\hline $\begin{array}{l}N 83190.2 \\
E 45417.4\end{array}$ & $150.4-130.6 \mathrm{ft} \mathrm{msl}$ & $164.8 \mathrm{ft} \mathrm{msl}$ & 4" PVC \\
\hline
\end{tabular}

MEASUREMENTS CONDUCTED IN THE FIELD

Sample date: 10/29/91

Depth to water: $20.91 \mathrm{ft}(6.37 \mathrm{~m})$ below TOC

Water elevation: $143.89 \mathrm{ft}(43.86 \mathrm{~m}) \mathrm{msl}$

Sp. conductance: $19 \mu \mathrm{S} / \mathrm{cm}$

Water evacuated before sampling: $36 \mathrm{gal}$

\section{LABORATORY ANALYSES}

H D Analyte

- $\quad \mathrm{pH}$

Specific conductance
Arsenic
Arsenic
Barium
Benzene
Bromodichloromethane
Bromoform
Bromomethane (Methyl bromide)
Cadmium
Calcium
Carbon tetrachloride
Chloride
Chlorobenzene
Chloroethane
Chloroethene (Vinyl chloride)

Result

$\begin{aligned} & 5.9 \\ & 30 \\ < & 2.0 \\ < & 2.0 \\ & 5.0 \\ < & 1.0 \\ < & 1.0 \\ < & 1.0 \\ < & 1.0 \\ < & 2.0 \\ & 1.210 \\ < & 1.0 \\ & 3.280 \\ < & 1.0 \\ < & 1.0 \\ < & 1.0\end{aligned}$

Time: $12: 00$

$\mathrm{pH}: 5.4$

Alkalinity: $7 \mathrm{mg} / \mathrm{L}$

Water temperature: $19.3^{\circ} \mathrm{C}$

$\overline{- \text { = exceeded holding time. }} \mathbf{-}=$ exceeded primary drinking water standard

\begin{tabular}{|c|c|}
\hline Unit & Flag \\
\hline $\mathrm{pH}$ & 0 \\
\hline$\mu \mathrm{S} / \mathrm{cm}$ & 0 \\
\hline$\mu g / L$ & 0 \\
\hline$\mu g / L$ & 0 \\
\hline$\mu g / L$ & 0 \\
\hline$\mu g / L$ & 0 \\
\hline$\mu \mathrm{g} / \mathrm{L}$ & 0 \\
\hline$\mu \mathrm{g} / \mathrm{L}$ & 0 \\
\hline$\mu_{g} / L$ & 0 \\
\hline$\mu g / L$ & 0 \\
\hline$\mu g / L$ & 0 \\
\hline$\mu g / L$ & 0 \\
\hline$\mu \mathrm{g} / \mathrm{L}$ & 0 \\
\hline$\mu g / L$ & 0 \\
\hline$\mu g / L$ & 0 \\
\hline$\mu \mathrm{g} / \mathrm{L}$ & 0 \\
\hline
\end{tabular}


WELL LFW 570, laboratory analyses (cont.)

\section{H D Analyte}

2.Chloroethyl vinyl ether

Chioroform

Chloromethane (Methyl chloride)

Chromium

Dibromochloromethane

1,1-Dichloroethane

1,2-Dichloroethane

1,1-Dichloroethylene

trans-1,2-Dichloroethylene

Dichloromethane (Methylene chloride)

- 2,4-Dichlorophenoxyacetic acid

1,2-Dichloropropane

trans-1,3-Dichloropropene

cis-1,3-Dichloropropene

Endrin

Endrin

Ethylbenzene

Fluoride

Lead

Lead

Lindane

Lindane

Magnesium

Manganese

Mercury

Methoxychlor

Methoxychlor

Nitrate as nitrogen

Phenols

Potassium

Selenium

Selenium

Silica

Silver

Sodium

Sulfate

1,1,2,2-Tetrachloroethane

Tetrachloroethylene

Toluene

- Total dissolved solids

Total organic carbon

Total organic halogens

Total phosphates (as P)

Toxaphene

Toxaphene

2,4,5-TP (Silvex)

1,1,1-Trichloroethane

1,1,2-Trichloroethane

Trichloroethylene

Trichlorofluoromethane

Gross alpha
Result

$<1.0$

$<1.0$

$<1.0$

$<4.0$

$<1.0$

12

$<1.0$

$<1.0$

$<1.0$

4.4

$<0.30$

$<1.0$

$<1.0$

$<1.0$

$<0.0060$

$<0.0060$

$<1.0$

$<100$

$<3.0$

$<3.0$

$<0.0050$

$<0.0050$

1,370

20

$<0.20$

$<0.50$

$<0.50$

$<50$

$<5.0$

$<500$

$<2.0$

$<2.0$

5.890

$<2.0$

2,950

1,220

$<1.0$

$<1.0$

$<1.0$

14,000

$<1,000$

19

$<50$

$<0.24$

$<0.24$

$<0.090$

$<1.0$

$<1.0$

$<1.0$

$<1.0$

$<2.0 \mathrm{E}+00$ $\underline{\text { Unit }} \quad \underline{F l a g} \quad \underline{L a b}$

$\mu g / L \quad O \quad$ GE

$\mu g / L \quad O \quad G E$

$\mu \mathrm{g} / \mathrm{L} \quad \mathrm{O} \quad \mathrm{GE}$

$\mu \mathrm{g} / \mathrm{L} \quad \mathrm{O} \quad \mathrm{GE}$

$\mu g / L \quad 0 \quad$ GE

$\mu g / L \quad 2 \quad G E$

$\mu g / L \quad 0 \quad$ GE

$\mu g / L \quad O \quad G E$

$\mu \mathrm{g} / \mathrm{L} \quad 0 \quad$ GE

$\mu g / L \quad 0 \quad$ GE

$\mu g / L \quad O \quad$ GE

$\mu g / L \quad 0 \quad$ GE

$\mu g / L . \quad 0 \quad-$ GE

$\mu \mathrm{g} / \mathrm{L} \quad 0 \quad \mathrm{GE}$

$\mu g / L \quad 0 \quad$ GE

$\mu \mathrm{g} / \mathrm{L} \quad 0 \quad$ GE

$\mu g / L \quad 0 \quad$ GE

$\mu g / L \quad 0 \quad$ GE

$\mu g / L \quad 0 \quad$ GE

$\mu g / L \quad 0 \quad$ GE

$\mu g / L \quad 0 \quad$ GE

$\mu g / L \quad 0 \quad$ GE

$\mu g / L \quad 0 \quad$ GE

$\mu \mathrm{g} / \mathrm{L} \quad 0 \quad$ GE

$\mu g / L \quad 0 \quad$ GE

$\mu g / L \quad 0 \quad$ GE

$\mu \mathrm{g} / \mathrm{L} \quad 0 \quad$ GE

$\mu g / L \quad 0 \quad$ GE

$\mu \mathrm{g} / \mathrm{L} \quad \mathrm{O} \quad \mathrm{GE}$

$\mu g / L \quad 0 \quad$ GE

$\mu g / L \quad O \quad$ GE

$\mu g / L \quad 0 \quad$ GE

$\mu g / L \quad 0 \quad$ GE

$\mu g / L \quad 0 \quad$ GE

$\mu g / L \quad 0 \quad$ GE

$\mu \mathrm{g} / \mathrm{L} \quad 0 \quad \mathrm{GE}$

$\mu g / L \quad 0 \quad$ GE

$\mu \mathrm{g} / \mathrm{L} \quad 0 \quad \mathrm{GE}$

$\mu \mathrm{g} / \mathrm{L} \quad 0 \quad$ GE

$\mu g / L \quad 0 \quad$ GE

$\mu g / L \quad 0 \quad$ GE

$\mu g / h \quad 0 \quad$ GE

$\mu g / L \quad 0 \quad$ GE

$\mu g / L \quad 0 \quad$ GE

$\mu g / L \quad 0 \quad$ GE

$\mu g / L \quad 0 \quad$ GE

$\mu g / L \quad O \quad$ GE

$\mu g / L \quad O \quad G E$

$\mu \mathrm{g} / \mathrm{L} \quad \mathrm{O} \quad \mathrm{GE}$

$\mu g / L \quad 0 \quad$ GE

$\mathrm{pCi} / \mathrm{L} \quad 0 \quad \mathrm{GE}$

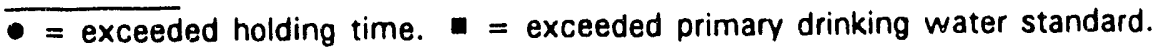


WELL LFW 570, laboratory analyses (cont.)

H $\underline{D}$

Analyte
Nonvolatile beta
Total radium
Tritium
Result
$<2.0 E+00$
$1.2 E+00 \pm 1.3 E+00$
$<7.0 \mathrm{E}-01$

Unit Flag Lab

Total radium

pCi/L 0

GE

$\begin{array}{ll}\mathrm{pCi} / \mathrm{L} & 0 \\ \mathrm{pCi} / \mathrm{mL} & 0\end{array}$

GE

GE

\section{WELL LFW 58D}

\section{SRS Coord.}

N82940.6

E45700.2

\section{MEASUREMENTS CONDUCTED IN THE FIELD}

Sample date: $10 / 29 / 91$

Depth to water: $25.67 \mathrm{ft}(7.82 \mathrm{~m})$ below TOC

Water elevation: $141.93 \mathrm{ft}(43.26 \mathrm{~m}) \mathrm{msl}$

Sp. conductance: $32 \mu \mathrm{S} / \mathrm{cm}$

Water evacuated before sampling: $49 \mathrm{gal}$

\section{LABORATORY ANALYSES}

H D Analyte

- $\mathrm{pH}$

Specific conductance
Specific conductance
Arsenic
Arsenic
Arsenic
Arsenic
Barium
Barium
Benzene
Benzene
Bromodichloromethane
Bromodichloromethane
Bromoform
Bromoform
Bromomethane (Methyl bromide)
Bromomethane (Methyl bromide)
Cadmium
Cadmium
Calcium
Calcium
Carbon tetrachloride
Carbon tetrachloride
Chloride
Chloride
Chlorobenzene

Result

5.3

5.3

30

30

$<2.0$

$<2.0$

$<2.0$

$<2.0$

6.7

6.5

$<1.0$

$<1.0$

$<i .0$

$<1.0$

$<1.0$

$<1.0$

$<1.0$

$<1.0$

$<2.0$

$<2.0$

1,140

1,130

$<1.0$

$<1.0$

$4,62 \mathrm{C}$

4,360

$<1.0$
Time: 13:20

$\mathrm{pH}: 5.1$

Alkalinity: $3 \mathrm{mg} / \mathrm{L}$

Water temperature: $19.4^{\circ} \mathrm{C}$
Lab.

Flag

pH $\quad 0 \quad$ GE

pH $O \quad$ GE

$\mu \mathrm{S} / \mathrm{cm} \quad \mathrm{G}$ GE

$\mu \mathrm{S} / \mathrm{cm} \quad 0 \quad \mathrm{GE}$

$\mu g / L \quad 0 \quad$ GE

$\mu g / L \quad O \quad$ GE

$\mu g / L \quad O \quad$ GE

$\mu g / L \quad O \quad$ GE

$\mu g / L \quad 0 \quad$ GE

$\mu g / L \quad 0 \quad$ GE

$\mu g / L \quad O \quad G E$

$\mu g / L \quad 0 \quad$ GE

$\mu g / L \quad O \quad$ GE

$\mu \mathrm{g} / \mathrm{L} \quad \mathrm{O} \quad \mathrm{GE}$

$\mu \mathrm{g} / \mathrm{L} \quad \mathrm{O} \quad \mathrm{GE}$

$\mu \mathrm{g} / \mathrm{L} \quad \mathrm{O} \quad \mathrm{GE}$

$\mu g / L \quad O \quad$ GE

$\mu g / L \quad O \quad$ GE

$\mu g / L \quad O \quad G E$

$\mu g / L \quad 0 \quad$ GE

$\mu g / L \quad 0 \quad$ GE

$\mu g / L \quad 0 \quad$ GE

$\mu g / L \quad O \quad$ GE

$\mu g / L \quad O \quad$ GE

$\mu \mathrm{g} / \mathrm{L} \quad 0 \quad \mathrm{GE}$

$\mu g / L \quad O \quad$ GE

$\mu g / L \quad O \quad$ GE

$\overline{0 \text { = exceeded holding time. }}$ = exceeded primary drinking water standard 
WELL LFW 58D, laboratory analyses (cont.)

\section{H D Analyte}

Chlorobenzene
Chloroethane
Chloroethane
Chloroethene (Vinyl chloride)
Chloroethene (Vinyl chloride)
2-Chloroethyl vinyl ether
2-Chloroethyl vinyl ether
Chloroform
Chloroform
Chloromethane (Methyl chloride)
Chloromethane (Methyl chloride)
Chromium
Chromium
Dibromochloromethane
Dibromochloromethane
1,1-Dichloroethane
1,1-Dichloroethane
1,2-Dichloroethane
1,2-Dichloroethane
1,1-Dichloroethylene
1,1-Dichloroethylene
trans-1,2-Dichloroethylene
trans-1,2-Dichloroethylene
Dichloromethane (Methylene chl:)ride)
Dichloromethane (Methylene chiuride)
2,4-Dichlorophenoxyacetic acid
2,4-Dichlorophenoxyacetic acid
1,2-Dichloropropane
1,2-Dichloropropane
irans-1,3-Dichloropropene
trans-1,3-Dichloropropene
cis-1,3-Dichloropropene
cis-1,3-Dichloropropene
Endrin
Endrin
Endrin
Endrin
Ethylbenzene
Ethylbenzene
Fluoride
Fluoride
Lead
Lead
Lead
Lead
Lindane
Lindane
Lindane
Lindane
Magnesiumesium

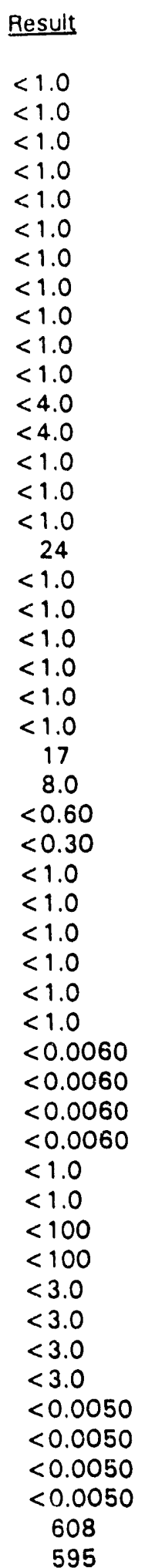

\begin{tabular}{|c|c|c|}
\hline Unit & Flag & $\underline{L a b}$ \\
\hline$\mu g / L$ & 0 & GE \\
\hline$\mu g / L$ & 0 & $\mathrm{GE}$ \\
\hline$\mu g / L$ & 0 & GE \\
\hline$\mu g / L$ & 0 & $\mathrm{GE}$ \\
\hline$\mu g / L$ & 0 & GE \\
\hline$\mu g / L$ & 0 & GE \\
\hline$\mu g / L$ & 0 & GE \\
\hline$\mu \mathrm{g} / \mathrm{L}$ & 0 & GE \\
\hline$\mu g / L$ & 0 & GE \\
\hline$\mu \mathrm{g} / \mathrm{L}$ & 0 & GE \\
\hline$\mu g / L$ & 0 & $\mathrm{GE}$ \\
\hline$\mu g / L$ & 0 & GE \\
\hline$\mu \mathrm{g} / \mathrm{L}$ & 0 & GE \\
\hline$\mu g / L$ & 0 & GE \\
\hline$\mu g / L$ & 0 & GE \\
\hline$\mu g / L$ & 0 & $\mathrm{GE}$ \\
\hline$\mu g / L$ & 2 & GE \\
\hline$\mu \mathrm{g} / \mathrm{L}$ & 0 & GE \\
\hline$\mu g / L$ & 0 & GE \\
\hline$\mu g / L$ & 0 & GE \\
\hline$\mu g / L$ & 0 & GE \\
\hline$\mu g / L$ & 0 & $\mathrm{GE}$ \\
\hline$\mu g / L$ & 0 & GE \\
\hline & 0 & GE \\
\hline$\mu g / L$ & 0 & GE \\
\hline$\mu \mathrm{g} / \mathrm{L}$ & 0 & GE \\
\hline$\mu g / L$ & 0 & GE \\
\hline$\mu \mathrm{g} / \mathrm{L}$. & 0 & GE \\
\hline$\mu g / L$ & 0 & GE \\
\hline$\mu g / L$ & 0 & GE \\
\hline$\mu g / L$ & 0 & GE \\
\hline$\mu \mathrm{g} / \mathrm{L}$ & 0 & GE \\
\hline$\mu \mathrm{g} / \mathrm{L}$ & 0 & GE \\
\hline$\mu g / L$ & 0 & GE \\
\hline$\mu g / L$ & 0 & $\mathrm{GE}$ \\
\hline$\mu \mathrm{g} / \mathrm{L}$ & 0 & GE \\
\hline$\mu g / L$ & 0 & GE \\
\hline$\mu g / L$ & 0 & GE \\
\hline$\mu \mathrm{g} / \mathrm{L}$ & 0 & $\mathrm{GE}$ \\
\hline$\mu \mathrm{g} / \mathrm{L}$ & 0 & $\mathrm{GE}$ \\
\hline$\mu g / L$ & 0 & $\mathrm{GE}$ \\
\hline$\mu \mathrm{g} / \mathrm{L}$ & 0 & $\mathrm{GE}$ \\
\hline$\mu g / L$ & 0 & $\mathrm{GE}$ \\
\hline$\mu \mathrm{g} / \mathrm{L}$ & 0 & $\mathrm{GE}$ \\
\hline$\mu \mathrm{g} / \mathrm{L}$ & 0 & $\mathrm{GE}$ \\
\hline$\mu g / L$ & 0 & $\mathrm{GE}$ \\
\hline$\mu \mathrm{g} / \mathrm{L}$ & 0 & $\mathrm{GE}$ \\
\hline$\mu \mathrm{g} / \mathrm{L}$ & 0 & $G E$ \\
\hline$\mu g / L$ & 0 & GE \\
\hline$\mu g / L$ & 0 & GE \\
\hline $\mathrm{g} / \mathrm{h}$ & 0 & GE \\
\hline
\end{tabular}

- =xceeded holding time. = exceeded primary drinking water standard. 
WELL LFW 58D, laboratory analyses (cont.)

\section{H D Analyte}

Manganese
Manganese
Mercury
Mercury
Methoxychlor
Methoxychlor
Methoxychlor
Methoxychlor
Nitrate as nitrogen
Nitrate as nitrogen
Phenols
Phenols
Potassium
Potassium
Selenium
Selenium
Selenium
Selenium
Silica
Silica
Silver
Silver
Sodium
Sodium
Sulfate
Sulfate
1,1,2,2-Tetrachloroethane
1,1,2,2-Tetrachloroethane
Tetrachloroethylene
Tetrachloroethylene
Toluene
Toluene
Total dissolved solids
Total dissolved solids
Total organic carbon
Total organic carbon
Total organic halogens
Total organic halogens
Total phosphates (as P)
Total phosphates (as P)
Toxaphene
Toxaphene
Toxaphene
Toxaphene
2,4,5-TP (Silvex)
$2,4,5-$-TP (Silvex)
1,1,1-Trichloroethane
1,1,1-Trichloroethane
1,1,2-Trichloroethane
1,1,2-Trichloroethane
Trichloroethylene

Results

Unit Flag Lab

30

30

1.7

1.6

$<0.50$

$<0.50$

$<0.50$

$<0.50$

190

170

$<5.0$

$<5.0$

$<500$

$<500$

$<2.0$

$<2.0$

$<2.0$

$<2.0$

10,700

10,600

$<2.0$

$<2.0$

3,010

2,990

$<1,000$

$<1,000$

$<1.0$

$<1.0$

$<1.0$

2.0

1.0

$<1.0$

16,000

19,000

$<1,000$

$<1,000$

103

129

$<50$

$<50$

$<0.24$

$<0.24$

$<0.24$

$<0.24$

$<0.10$

$<0.090$

3.0

19

$<1.0$

$<1.0$

$<1.0$ $\mu g / L \quad 1 \quad$ GE

$\begin{array}{lll}\mu g / L & 1 & \text { GE } \\ \mu g / L & 1 & \text { GE }\end{array}$

$\begin{array}{lll}\mu g / L & 1 & \text { GE } \\ \mu g / L & 1 & \text { GE }\end{array}$

$\mu g / L \quad 0 \quad G E$

$\mu g / \mathrm{L} \quad 0 \quad \mathrm{GE}$

$\mu g / L \quad 0 \quad$ GE

$\mu g / L \quad 0 \quad G E$

$\mu g / L \quad 0 \quad G E$

$\mu g / L \quad O \quad$ GE

$\mu g / L \quad 0 \quad$ GE

$\mu g / L \quad 0 \quad$ GE

$\mu g / L \quad 0 \quad-$ GE

$\mu g / L \quad 0 \quad$ GE

$\mu g / L \quad 0 \quad$ GE

$\mu g / L \quad O \quad$ GE

$\mu g / L$ GE

$\mu g / L \quad 0 \quad$ GE

$\mu g / L \quad O \quad G E$

$\mu g \Omega$ GE

$\mu g /$ L $\quad 0 \quad$ GE

$\mu g / L \quad 0 \quad$ GE

$\mu g / L \quad 0 \quad$ GE

$\mu g / L \quad 0 \quad$ GE

$\mu g /$ $\quad 0 \quad$ GE

$\mu g / L \quad 0 \quad$ GE

$\mu g / L \quad O \quad G E$

$\mu g /$ $\quad 0 \quad$ GE

$\mu g / L \quad 0 \quad$ GE

$\mu g / L \quad 0 \quad$ GE

$\mu g / L \quad 0 \quad$ GE

$\mu g / L \quad 0 \quad$ GE

$\mu g / L \quad 0 \quad$ GE

$\mu g / L \quad 0 \quad$ GE

$\mu g / L \quad O \quad G E$

$\mu g / L \quad 2 \quad G E$

$\mu g / L \quad 2 \quad G E$

$\mu g /$ L $\quad 0 \quad$ GE

$\mu g / L \quad 0 \quad$ GE

$\mu g / L \quad O \quad$ GE

$\mu g / L \quad 0 \quad G E$

$\mu g / L \quad 0 \quad$ GE

$\mu g /$ L $\quad 0$ GE

$\mu g / L \quad O \quad$ GE

$\mu g / L \quad 0 \quad$ GE

$\mu g / L \quad 0 \quad$ GE

$\mu g / L \quad 0 \quad$ GE

$\mu g / L \quad 0 \quad G E$

$\mu g / L \quad 0 \quad G E$

$\mu g / L \quad O \quad$ GE $\mu g / L \quad 0 \quad$ GE

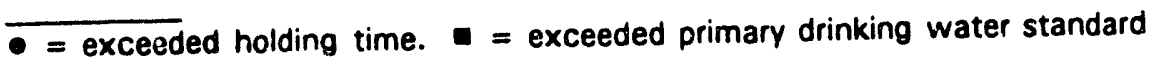


WELL LFW 58D, laboratory analyses (cont.)

$\begin{array}{ll}\text { H. } & \text { Analyte } \\ & \text { Trichloroethylene } \\ \text { Trichlorofluoromethane } \\ \text { Trichlorofluoromethane } \\ \text { Gross alpha } \\ \text { Gross alpha } \\ \text { Nonvolatile beta } \\ \text { Nonvolatile beta } \\ \text { Total radium } \\ \text { Total radium } \\ \text { Tritium } \\ \text { Tritium }\end{array}$

\begin{tabular}{|c|c|c|}
\hline Result & Unit & Flag \\
\hline 8.3 & $\mu g / L$ & 2 \\
\hline$<1.0$ & $\mu g / L$ & 0 \\
\hline 123 & $\mu g / L$ & 2 \\
\hline$<2.0 E+00$ & $\mathrm{pCi} / \mathrm{L}$ & 0 \\
\hline$<2.0 E+00$ & $\mathrm{pCi} / \mathrm{L}$ & 0 \\
\hline$<2.0 E+00$ & $\mathrm{pCi} / \mathrm{L}$ & 0 \\
\hline$<2.0 E+00$ & $\mathrm{pCi} / \mathrm{L}$ & 0 \\
\hline$<1.0 E+00$ & $\mathrm{pCi} / \mathrm{L}$ & 0 \\
\hline$<1.0 E+00$ & $\mathrm{pCi} / \mathrm{L}$ & 0 \\
\hline$<7.0 E-01$ & $\mathrm{pCi} / \mathrm{mL}$ & 0 \\
\hline $3.0 E+00 \pm 7.0 E-01$ & pCi/mL & 0 \\
\hline
\end{tabular}

\section{WELL LFW 58D}

SRS Coord.

N82940.6

E45700.2

\section{Screen Zone Elevation}

$147.6-127.5 \mathrm{ft} \mathrm{msl}$
Top of Casing

$167.6 \mathrm{ft} \mathrm{msl}$
Casing

4" PVC

\section{MEASUREMENTS CONDUCTED IN THE FIELD}

Sample date: 10/29/91

Depth to water: $25.67 \mathrm{ft}(7.82 \mathrm{~m})$ below TOC

Water elevation: $141.93 \mathrm{ft}(43.26 \mathrm{~m}) \mathrm{msl}$

Sp. conductance: $32 \mu \mathrm{S} / \mathrm{cm}$

Water evacuated before sampling: $49 \mathrm{gal}$

\section{WELL LFW 59B}

\section{SRS Coord.}

N83027.1

E46047.4

MEASUREMENTS CONDUCTED IN THE FIELD

Sample date: 10/31/91

Depth to water: $25.07 \mathrm{ft}(7.64 \mathrm{~m})$ below TOC Water elevation: $143.03 \mathrm{ft}(43.60 \mathrm{~m}) \mathrm{msl}$

Sp. conductance: $46 \mu \mathrm{S} / \mathrm{cm}$

Water evacuated before sampling: $203 \mathrm{gal}$
Time: 13:20

pH: 5.1

Alkalinity: $3 \mathrm{mg} / \mathrm{L}$

Water temperature: $19.4^{\circ} \mathrm{C}$
Time: 12:45

pH: 3.9

Alkalinity: $0 \mathrm{mg} / \mathrm{L}$

Water temperature: $19.0^{\circ} \mathrm{C}$

- = exceeded holding time. - = exceeded primary drinking water standard. 
H D Analyte

pH

Specific conductance

Arsenic

Barium

Benzene

Bromodichloromethane

Bromoform

Bromomethane (Methyl bromide)

Cadmium

Calcium

Carbon testachloride

Chloride

Chlorobenzene

Chloroethane

Chloroethene (Vinyl chloride)

2-Chloroethyl vinyl ether

Chloroform

Chloromethane (Methyl chloride)

Chromium

Dibromochloromethane

1,1-Dichloroethane

1,2-Dichloroethane

1,1-Dichloroethylene

trans-1,2-Dichloroethylene

Dichloromethane (Methylene chloride)

2,4-Dichlorophenoxyacetic acid

1,2-Dichloropropane

trans-1,3-Dichloropropene

cis-1,3-Dichloropropene

Endrin

Ethylbenzene

Fluoride

Iron

Lead

Lindane

Magnesium

Manganese

Mercury

Methoxychior

Nitrate as nitrogen

Phenols

Potassium

Selenium

Silica

Silver

Sodium
Result

4.4

40

$<2.0$

6.3

$<1.0$

$<1.0$

$<1.0$

$<1.0$

$<2.0$

1,110

$<1.0$

2,430

$<1.0$

$<1.0$

$<1.0$

$<1.0$

$<1.0$

$<1.0$

$<4.0$

$<1.0$

$<1.0$

$<1.0$

$<1.0$

$<1.0$

$<1.0$

$<0.30$

$<1.0$

$<1.0$

$<1.0$

$<0.0060$

$<1.0$

$<100$

19

$<3.0$

$<0.0050$

449

31

$<0.20$

$<0.50$

480

$<5.0$

$<500$

$<2.0$

10,600

$<2.0$

1.530
Unit Flag Lab

pH 0 GE

$\mu \mathrm{S} / \mathrm{cm} \quad 0 \quad \mathrm{GE}$

$\mu g / L \quad O \quad$ GE

$\mu g / L \quad 0 \quad$ GE

$\mu g / L \quad O \quad$ GE

$\mu g / L \quad$ GE

$\mu g / L \quad O \quad$ GE

$\mu g / L \quad O \quad$ GE

$\mu g / L \quad O \quad$ GE

$\mu g / L \quad 0 \quad$ GE

$\mu g / L \quad 0 \quad$ GE

$\mu g / L \quad O \quad$ GE

$\mu g / L \quad 0 \quad-G E$

$\mu g / L \quad 0 \quad$ GE

$\mu g / L \quad O \quad$ GE

$\mu g / L \quad O \quad$ GE

$\mu g / L \quad O \quad$ GE

$\mu g \Omega \quad 0 \quad$ GE

$\mu g / 2 \quad O \quad G E$

$\mu g / L \quad O \quad$ GE

$\mu \mathrm{g} / \mathrm{L} \quad 0 \quad$ GE

$\mu g / L \quad$ GE

$\mu g / L \quad O \quad$ GE

$\mu g / L \quad 0 \quad$ GE

$\mu g /$ G $O$ GE

$\mu g /$ $\quad 0$ GE

$\mu g / L \quad O \quad$ GE

$\mu g / \mathrm{L} \quad 0 \quad$ GE

$\mu g / L \quad 0 \quad$ GE

$\mu g / L \quad 0 \quad$ GE

$\mu g / L \quad 0 \quad$ GE

$\mu g /$ L $\quad$ GE

$\mu g / L \quad 0 \quad$ GE

$\mu g \Omega \quad$ GE

$\mu g / L \quad$ GE

$\mu g / L \quad 0 \quad$ GE

$\mu g / L \quad 1 \quad$ GE

$\mu g / L \quad 0 \quad$ GE

$\mu g / L \quad 0 \quad$ GE

$\mu g / L \quad 0 \quad$ GE

$\mu g / L \quad O \quad$ GE

$\mu g / L \quad 0 \quad$ GE

$\mu g / L \quad 0 \quad$ GE

$\mu g / L \quad O \quad$ GE

$\mu g / L \quad$ GE

$\mu g / L \quad$ GE

- = exceeded holding time. $\quad=$ exceeded primary drinking water stanifard 
WELL LFW 598, laboratory analyses (cont.)

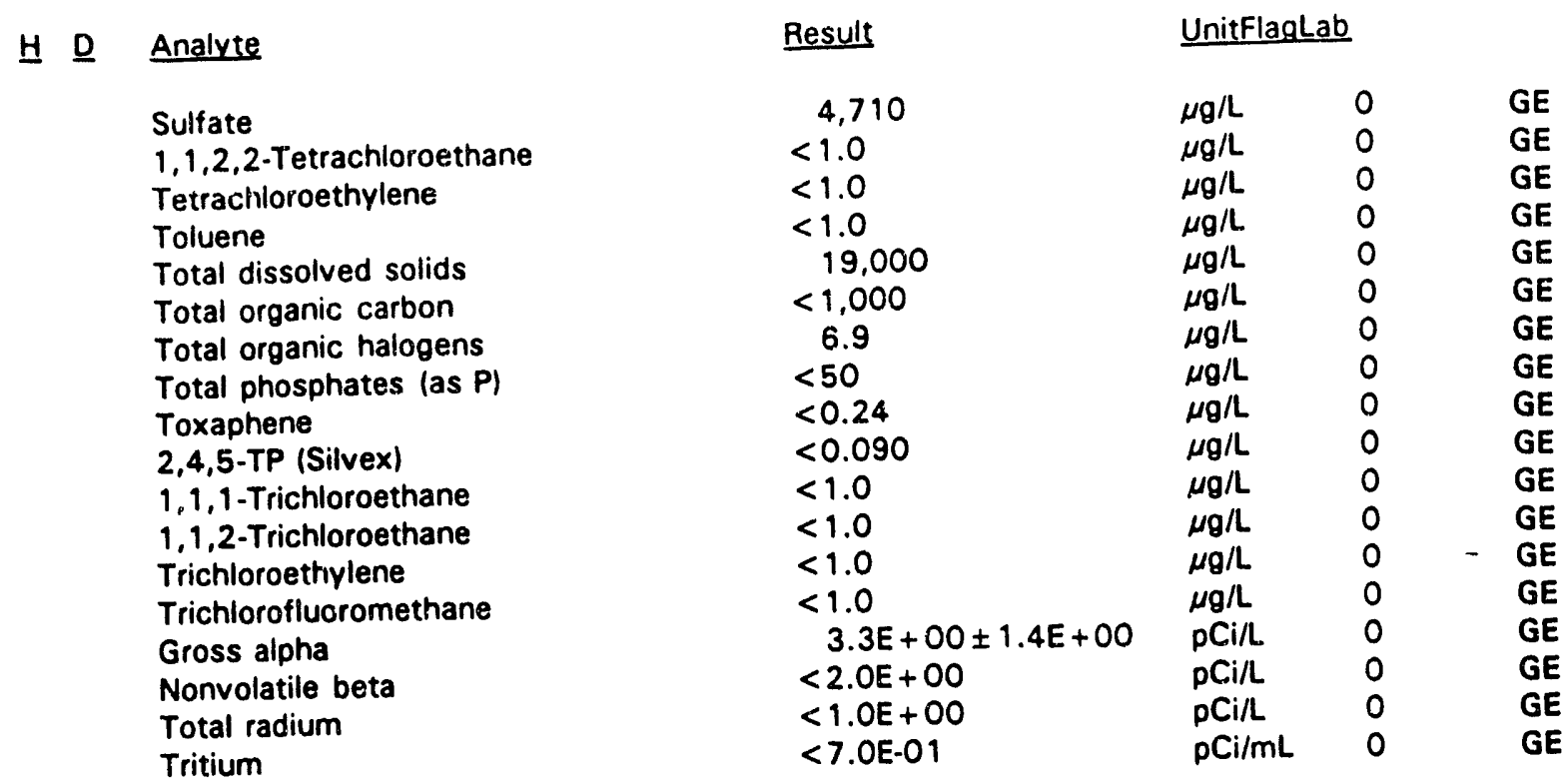

\section{WELL LFW 59C}

\section{SRS Coord.}

N83011.0

E46052.0

\section{MEASUREMENTS CONDUCTED IN THE FIELD}

Sample date: $10 / 31 / 91$

Depth to water: $24.92 \mathrm{ft}(7.60 \mathrm{~m})$ below TOC

Water elevation: $142.38 \mathrm{ft}(43.40 \mathrm{~m}) \mathrm{msl}$

Sp. conductance: $45 \mu \mathrm{S} / \mathrm{cm}$

Water evacuated before sampling: $112 \mathrm{gal}$

\section{LABORATORY ANALYSES}

\section{H D Analyte}

pH
Specific conductance
Arsenic
Barium
Benzene
Bromodichloromethane
Bromoform
Bromomethane (Methyl bromide)
Cadmium
Calcium
Carbon tetrachloride
Chloride

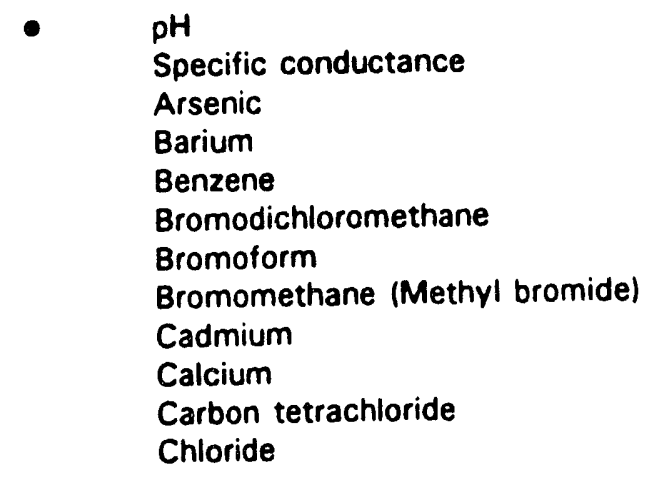

\section{Screen Zone Elevation}

$110.3-100.3 \mathrm{ft} \mathrm{msl}$

\section{Ton of Casing}

$167.3 \mathrm{ft} \mathrm{msl}$
Casing

4" PVC
Time: $12: 25$

$\mathrm{pH}: 4.5$

Alkalinity: $0 \mathrm{mg} / \mathrm{L}$

Water temperature: $19.0^{\circ} \mathrm{C}$

\begin{tabular}{c} 
Result \\
\hline \\
5.0 \\
40 \\
$<2.0$ \\
9.5 \\
$<1.0$ \\
$<1.0$ \\
$<1.0$ \\
$<1.0$ \\
$<2.0$ \\
\\
$<1.150$ \\
$<1.0$ \\
5.950
\end{tabular}

Unit Flag Lab

$\begin{array}{lll}\mathrm{pH} & 0 & \mathrm{GE} \\ \mu \mathrm{S} / \mathrm{cm} & 0 & \mathrm{GE} \\ \mu \mathrm{g} / \mathrm{L} & 0 & \mathrm{GE} \\ \mu \mathrm{g} / \mathrm{L} & 0 & \mathrm{GE} \\ \mu \mathrm{g} / \mathrm{L} & 0 & \mathrm{GE} \\ \mu \mathrm{g} / \mathrm{L} & 0 & \mathrm{GE} \\ \mu \mathrm{g} / \mathrm{L} & 0 & \mathrm{GE} \\ \mu \mathrm{g} / \mathrm{L} & 0 & \mathrm{GE} \\ \mu \mathrm{g} / \mathrm{L} & 0 & \mathrm{GE} \\ \mu \mathrm{g} / \mathrm{L} & 0 & \mathrm{GE} \\ \mu \mathrm{g} / \mathrm{L} & 0 & \mathrm{GE} \\ \mu \mathrm{g} / \mathrm{L} & 0 & \mathrm{GE}\end{array}$

$\overline{-=\text { exceeded holding time. }}-$ = exceeded primary drinking water standard. 
WELL LFW 59C, laboratory analyses (cont.)

H

D Alalyte
Chloride
Chlorobenzene
Chloroethane
Chloroethene (Vinyl chloride)
2-Chloroethyl vinyl ether
Chloroform
Chloromethane (Methyl chloride)
Chromium
Dibromochloromethane
1,1-Dichloroethane
1,2-Dichloroethane
1,1-Dichloroethylene
trans-1,2-Dichloroethylene
Dichloromethane (Methylene chloride
2,4-Dichlorophenoxyacetic acid
1,2-Dichloropropane
trans-1,3-Dichloropropene
cis-1,3-Dichloropropene
Endrin
Ethylbenzene
Fluoride
Iron
Lead
Lindane
Magnesium
Manganese
Mercury
Methoxychlor
Nitrate as nitrogen
Phenols
Potassium
Selenium
Silica
Silver
Sodium
Sulfate
1,1,2,2-Tetrachloroethane
Tetrachloroethylene
Toluene
Total dissolved solids
Total organic carbon
Total organic halogens
Total phosphates (as P)
Toxaphene
2,4,5-TP (Silvex)
1,1,1-Trichlorothothyluoromethane
Nonvolatile beta

Result

$$
5.950
$$$$
<1.0
$$$$
<1.0
$$$$
<1.0
$$$$
<1.0
$$$$
1.0
$$$$
<1.0
$$$$
<4.0
$$$$
<1.0
$$$$
17
$$$$
1.0
$$$$
2.0
$$$$
<1.0
$$$$
10
$$$$
<0.30
$$$$
<1.0
$$$$
<1.0
$$$$
<1.0
$$$$
<0.0060
$$$$
<1.0
$$$$
<100
$$$$
44
$$$$
<3.0
$$$$
<0.0050
$$$$
1,150
$$$$
12
$$$$
0.31
$$$$
<0.50
$$$$
1,030
$$$$
<5.0
$$$$
578
$$$$
<2.0
$$$$
9,400
$$$$
<2.0
$$$$
3,880
$$$$
1,830
$$$$
<1.0
$$$$
1.0
$$$$
<1.0
$$$$
22,000
$$$$
<1,000
$$$$
266
$$$$
<50
$$$$
<0.24
$$$$
<0.090
$$$$
1.0
$$$$
<1.0
$$$$
8.0
$$$$
<1.0
$$$$
<2.0 E+00
$$$$
<2.0 E+00
$$

\begin{tabular}{|c|c|c|}
\hline Unit & Flag & $\underline{\mathrm{Lab}}$ \\
\hline$\mu g / \mathrm{L}$ & 0 & GE \\
\hline$\mu g / L$ & 0 & GE \\
\hline$\mu g / 2$ & 0 & GE \\
\hline$\mu g / L$ & 0 & GE \\
\hline$\mu \mathrm{g} / \mathrm{L}$ & 0 & GE \\
\hline$\mu g / L$ & 0 & GE \\
\hline$\mu \mathrm{g} / \mathrm{L}$ & 0 & GE \\
\hline$\mu \mathrm{g} / \mathrm{L}$ & 0 & GE \\
\hline$\mu \mathrm{g} / \mathrm{L}$ & 0 & GE \\
\hline$\mu g / L$ & 2 & GE \\
\hline$\mu \mathrm{g} / \mathrm{L}$ & 0 & GE \\
\hline$\mu \mathrm{g} / \mathrm{L}$ & 0 & GE \\
\hline$\mu \mathrm{g} / \mathrm{L}$ & 0 & $-\quad G E$ \\
\hline$\mu g / L$ & 0 & GE \\
\hline$\mu g / L$ & 0 & GE \\
\hline$\mu g / L$ & 0 & GE \\
\hline$\mu g / L$ & 0 & GE \\
\hline$\mu g / L$ & 0 & GE \\
\hline$\mu \mathrm{g} / \mathrm{L}$ & 0 & GE \\
\hline$\mu \mathrm{g} / \mathrm{L}$ & 0 & GE \\
\hline$\mu g / L$ & 0 & GE \\
\hline$\mu g / L$ & 0 & GE \\
\hline$\mu g / L$ & 0 & GE \\
\hline$\mu g / L$ & 0 & GE \\
\hline$\mu g / L$ & 0 & GE \\
\hline$\mu g / L$ & 0 & GE \\
\hline$\mu g / L$ & 0 & GE \\
\hline$\mu g / L$ & 0 & GE \\
\hline$\mu g / L$ & 0 & GE \\
\hline$\mu g / L$ & 0 & GE \\
\hline$\mu g / L$ & 0 & GE \\
\hline$\mu g / L$ & 0 & GE \\
\hline$\mu g / L$ & 0 & GE \\
\hline$\mu g / L$ & 0 & GE \\
\hline$\mu g / L$ & 0 & $\mathrm{GE}$ \\
\hline$\mu g / L$ & 0 & GE \\
\hline$\mu g / L$ & 0 & GE \\
\hline$\mu g / L$ & 0 & GE \\
\hline$\mu g / L$ & 0 & GE \\
\hline$\mu g / L$ & 0 & GE \\
\hline$\mu g / L$ & 0 & GE \\
\hline$\mu g / L$ & 2 & GE \\
\hline$\mu g / L$ & 0 & $\mathrm{GE}$ \\
\hline$\mu g / L$ & 0 & $\mathrm{GE}$ \\
\hline$\mu g / L$ & 0 & GE \\
\hline$\mu g / L$ & 0 & GE \\
\hline$\mu g / L$ & 0 & GE \\
\hline$\mu g / L$ & 2 & GE \\
\hline$\mu g / L$ & 0 & GE \\
\hline $\mathrm{pCi} / \mathrm{L}$ & 0 & GE \\
\hline $\mathrm{pCi} / \mathrm{L}$ & 0 & GE \\
\hline
\end{tabular}

$\overline{-}=$ exceeded holding time.

- =xceeded primary drinking water standard 
WELL LFW 59C, laboratory analyses (cont.)

H D Analyte

Total radium

Tritium
Result

$1.1 E+00 \pm 1.2 E+00$

$2.3 E+00 \pm 7.0 E-01$
Unit Flag

Lab

pCill 0

$\mathrm{pCi} / \mathrm{mL} \quad 0$
GE GE

\section{WELL LFW 59D}

\section{SRS Coord.}

N83000.1

E46056.1

\section{MEASUREMENTS CONDUCTED IN THE FIELD}

Sample date: $10 / 31 / 91$

Depth to water: $24.66 \mathrm{ft}(7.52 \mathrm{~m})$ below TOC Water elevation: $142.94 \mathrm{ft}(43.57 \mathrm{~m}) \mathrm{msl}$

Sp. conductance: $26 \mu \mathrm{S} / \mathrm{cm}$

Water evacuated before sampling: $36 \mathrm{gal}$

\section{LABORATORY ANALYSES}

H D Analyte

pH
Specific conductance
Arsenic
Barium
Benzene
Bromodichloromethane
Bromoform
Bromomethane (Methyl bromide)
Cadmium
Calcium
Carbon tetrachloride
Chloride
Chlorobenzene
Chloroethane
Chloroethene (Vinyl chloride)
2-Chloroethyl vinyl ether
Chloroform
Chloromethane (Methyl chloride)
Chromium
Dibromochloromethane
1,1-Dichloroethane
1,2-Dichloroethane
1,1-Dichloroethylene
trans-1,2-Dichloroethylene
Dichloromethane (Methylene chloride)
2,4-Dichlorophenoxyacetic acid
1,2-Dichloropropane
trans-1,3-Dichloropropene

- $\quad$ PH

\begin{tabular}{c} 
Result \\
\hline 5.3 \\
25 \\
$<2.0$ \\
7.1 \\
$<1.0$ \\
$<1.0$ \\
$<1.0$ \\
$<1.0$ \\
$<2.0$ \\
835 \\
$<1.0$ \\
3.880 \\
$<1.0$ \\
$<1.0$ \\
$<1.0$ \\
$<1.0$ \\
$<1.0$ \\
$<1.0$ \\
$<4.0$ \\
$<1.0$ \\
19 \\
$<1.0$ \\
31.8 \\
$<1.0$ \\
3.8 \\
$<0.30$ \\
$<1.0$ \\
$<1.0$ \\
\end{tabular}

Time: 13:05

pH: 4.8

Alkalinity: $0 \mathrm{mg} / \mathrm{L}$

Water temperature: $19.7^{\circ} \mathrm{C}$ 
WELL LFW 59D, laboratory analyses (cont.)

H D Analyte

cis-1, 3-Dichloropropene

Endrin

Ethylbenzene

Fluoride:

Iron

Lead

Lindane

Magnesium

Manganese

Mercury

Methoxychlor

Nitrate as nitrogen

Nitrate as nitrogen

Phenols

Potassium

Selenium

Silica

Silver

Sodium

Sulfate

1,1,2,2-Tetrachloroethane

Tetrachloroethyiene

Toluene

Total dissolved solids.

Total organic carbon

Total organic halogens

Total phosphates (as P)

Toxaphene

2,4,5-TP (Silvex)

1,1,1-Trichloroethane

1,1,2-Trichloroethane

- Trichloroethylene

Trichlorofluoromethane

Gross alpha

Nonvolatile beta

- Total radium

Tritium
Result

$<1.0$

$<0.0060$

$<i .0$

$<100$

6.6 ,

$<3.0$

$<0.0050$

738

19

$<0.20$

$<0.50$

$<50$

$<50$

$<5.0$

$<500$

$<2.0$

10,600

$<2.0$

2,140

$<1,000$

$<1.0$

2.5

$<1.0$

15,000

$<1,000$

271

$<50$

$<0.24$

$<0.090$

40

$<1.0$

27

109

$<2.0 E+00$

$<2.0 E+00$

$9.3 E+00 \pm 2.2 E+00$

$<7.0 \mathrm{E}-01$
Unit $\quad \underline{\text { Flag }} \quad \underline{\text { Lab }}$

$\mu g / L \quad 0 \quad$ GE

$\mu g / L \quad 0 \quad$ GE

$\mu g / L \quad 0 \quad G E$

$\mu g / L \quad 0 \quad G E$

$\mu g / L \quad 0 \quad G E$

$\mu g / L \quad 0 \quad G E$

$\mu \mathrm{g} / \mathrm{L} \quad \mathrm{O} \quad \mathrm{GE}$

$\mu g / L \quad 0 \quad G E$

$\mu g / L \quad 0 \quad$ GE

$\mu \mathrm{g} / \mathrm{L} \quad \mathrm{O} \quad \mathrm{GE}$

$\mu g / L \quad 0 \quad$ GE

$\mu g / L \quad 0 \quad$ GE

$\mu g / L \quad 0 \quad-G E$

$\mu g / L \quad 0 \quad G E$

$\mu g / L \quad 0 \quad$ GE

$\mu g / L \quad 0 \quad$ GE

$\mu g / L \quad 0 \quad$ GE

$\mu g / L \quad 0 \quad$ GE

$\mu g / L \quad 0 \quad$ GE

$\mu g / L \quad 0 \quad$ GE

$\mu g / L \quad 0 \quad$ GE

$\mu g / L \quad 1 \quad$ GE

$\mu g / L \quad 0 \quad$ GE

$\mu g / L \quad 0 \quad$ GE

$\mu g / L \quad 0 \quad G E$

$\mu g / L \quad 2 \quad G E$

$\mu g / L \quad 0 \quad$ GE

$\mu g / L \quad 0 \quad$ GE

$\mu g / L \quad 0 \quad$ GE

$\mu g / L \quad 0 \quad$ GE

$\mu g / L \quad 0 \quad$ GE

$\mu g / L \quad 2 \quad G E$

$\mu g / L \quad 2 \quad G E$

pCi/L 0 GE

pCi/L 0 GE

pCi/L 2 GE

$\mathrm{pCi} / \mathrm{mL} \quad 0 \quad \mathrm{GE}$

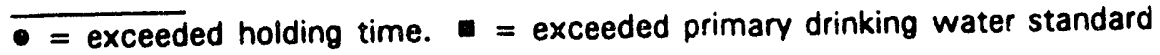


WELL LFW 60D

SR'S Coord.

N82531.5

E45722.3

MEASUREMENTS CONDUCTED IN THE FIELD

Sample date: $10 / 29 / 91$

Depth to water: $18.79 \mathrm{ft}(5.73 \mathrm{~m})$ below TOC

Water elevation: $138.31 \mathrm{ft}(42.16 \mathrm{~m}) \mathrm{msl}$

Sp. conductance: $40 \mu \mathrm{S} / \mathrm{cm}$

Water evacuated before sampling: $10 \mathrm{gal}$

The well went dry during purging.

\section{LABORATORY ANALYSES}

\section{H D Analyte}

pH
Specific conductance
Arsenic
Barium
Benzene
Bromodichloromethane
Bromoform
Bromomethane (Methyl bromide)
Cadmium
Calcium
Carbon tetrachloride
Chloride
Chlorobenzene
Chloroethane
Chloroethene (Vinyl chloride)
2-Chloroethyl vinyl ether
Chloroform
Chloromethane (Methyl chloride)
Chromium
Dibromochloromethane
1,1-Dichloroethane
1,2-Dichloroethane
1,1-Dichloroethylene
trans-1,2-Dichloroethylene
Dichloromethane (Methylene chloride)
2,4-Dichlorophenoxyacetic acid
1,2-Dichloropropane
trans-1,3-Dichloropropene
cis-1,3-Dichloropropene
Endrin
Ethylbenzene
Fluoride
Lead
Lindane
Magnesium

Screen Zone Elevation

143.8-123.8 ft msl
Ton of Casing Casing

$157.1 \mathrm{ft} \mathrm{msl} \quad$ 4" PVC
Time: 13:55

pH: 6.0

Alkalinity: $12 \mathrm{mg} / \mathrm{L}$

Water temperature: $19.1^{\circ} \mathrm{C}$

\begin{tabular}{|c|c|c|c|}
\hline Result & Unit & Flag & Lab \\
\hline 6.5 & $\mathrm{pH}$ & 0 & GE \\
\hline 30 & $\mu \mathrm{S} / \mathrm{cm}$ & 0 & GE \\
\hline$<2.0$ & $\mu \mathrm{g} / \mathrm{L}$ & 0 & $\mathbf{G E}$ \\
\hline 5.5 & $\mu g / L$ & 0 & GE \\
\hline$<1.0$ & $\mu g / L$ & 0 & GE \\
\hline$<1.0$ & $\mu g / L$ & 0 & GE \\
\hline$<1.0$ & $\mu g / L$ & 0 & GE \\
\hline$<1.0$ & $\mu g / L$ & 0 & GE \\
\hline$<2.0$ & $\mu g / L$ & 0 & GE \\
\hline 1,930 & $\mu \mathrm{g} / \mathrm{L}$ & 0 & GE \\
\hline$<1.0$ & $\mu g / L$ & 0 & GE \\
\hline 2,210 & $\mu g / L$ & 0 & GE \\
\hline$<1.0$ & $\mu g / L$ & 0 & GE \\
\hline$<1.0$ & $\mu g / L$ & 0 & GE \\
\hline$<1.0$ & $\mu g / L$ & 0 & GE \\
\hline$<1.0$ & $\mu g / L$ & 0 & GE \\
\hline 1.0 & $\mu g / L$ & 0 & GE \\
\hline$<1.0$ & $\mu g / L$ & 0 & GE \\
\hline$<4.0$ & $\mu g / L$ & 0 & GE \\
\hline$<1.0$ & $\mu g / L$ & 0 & GE \\
\hline$<1.0$ & $\mu g / L$ & 0 & GE \\
\hline$<1.0$ & $\mu g / L$ & 0 & GE \\
\hline$<1.0$ & $\mu_{g} / L$ & 0 & GE \\
\hline$<1.0$ & $\mu g / L$ & 0 & GE \\
\hline 12 & $\mu g / L$ & 0 & GE \\
\hline$<0.30$ & $\mu g / L$ & 0 & GE \\
\hline$<1.0$ & $\mu g / L$ & 0 & GE \\
\hline$<1.0$ & $\mu g / L$ & 0 & GE \\
\hline$<1.0$ & $\mu g / L$ & 0 & GE \\
\hline$<0.0060$ & $\mu g / L$ & 0 & GE \\
\hline$<1.0$ & $\mu g / L$ & 0 & GE \\
\hline$<100$ & $\mu g / L$ & 0 & GE \\
\hline$<3.0$ & $\mu g / L$ & 0 & GE \\
\hline$<0.0050$ & $\mu g / L$ & 0 & GE \\
\hline 374 & $\mu g / L$ & 0 & $E$ \\
\hline
\end{tabular}

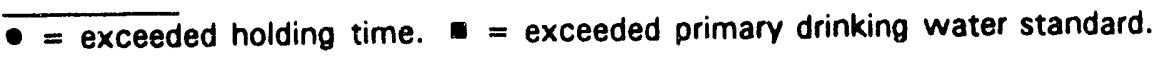


WELL LFW 60D, laboratory analyses (cont.)

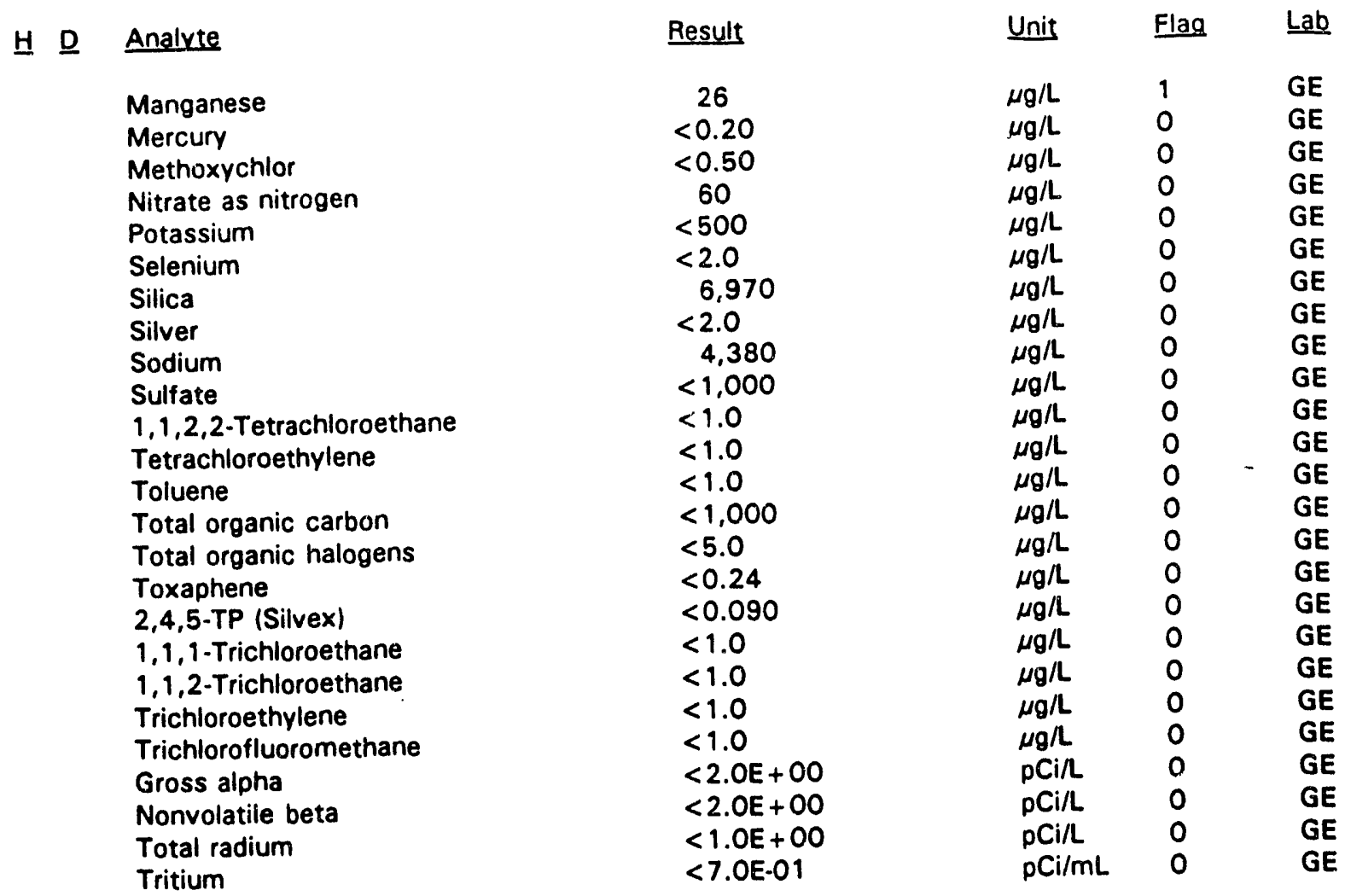

\section{WELL LFW 61C}

\section{SRS Coord.}

N83084.4

E46489.6

\section{MEASUREMENTS CONDUCTED IN THE FIELD}

Sample date: $10 / 31 / 91$

Depth to water: $26.60 \mathrm{ft}(8.11 \mathrm{~m})$ below TOC

Water elevation: $141.70 \mathrm{ft}(43.19 \mathrm{~m}) \mathrm{msl}$

Sp. conductance: $25 \mu \mathrm{S} / \mathrm{cm}$

Water evacuated before sampling: $81 \mathrm{gal}$

\section{LABORATORY ANALYSES}

H D Analyte.

- $\quad \mathrm{pH}$

Specific conductance

Arsenic

Barium

Benzene
Screen Zone Elevation

$121.1-111.0 \mathrm{ft} \mathrm{msl}$
Top of Casing Casing

$108.3 \mathrm{ft} \mathrm{msl} \quad 4^{\prime \prime} \mathrm{PVC}$
Time: 13:50

$\mathrm{pH}: 5.0$

Alkalinity: $1 \mathrm{mg} / \mathrm{L}$

Water temperature: $19.0^{\circ} \mathrm{C}$

- =xceeded holding time. = exceeded primary drinking water standard

Unit Flag Lab

pH $\quad$ GE

$\mu \mathrm{S} / \mathrm{cm} \quad \mathrm{G} \quad \mathrm{GE}$

$\mu g / \mathrm{L} \quad \mathrm{GE}$

$\mu g / L \quad O \quad$ GE

$\mu g / L \quad O \quad G E$ 
WELL LFW 61C, laboratory analyses (cont.)

\section{H D Analyte}

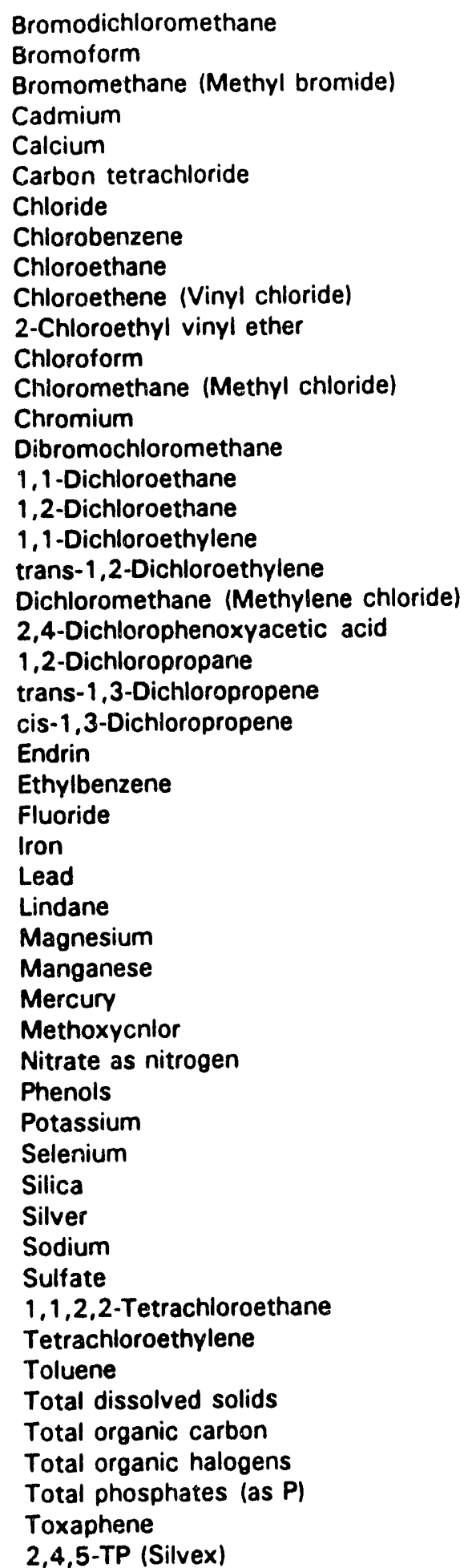

Result

$<1.0$

$<1.0$

$<1.0$

$<2.0$

926

$<1.0$

2,460

$<1.0$

$<1.0$

$<1.0$

$<1.0$

$<1.0$

$<1.0$

$<4.0$

$<1.0$

6.0

$<1.0$

2.0

$<1.0$

10

$<0.30$

$<1.0$

$<1.0$

$<1.0$

$<0.0060$

$<1.0$

$<100$

7.2

$<3.0$

$<0.0050$

594

17

$<0.20$

$<0.50$

400

$<5.0$

900

$<2.0$

7.600

$<2.0$

2,120

$<1,000$

$<1.0$

1.0

$<1.0$

17,000

$<1,000$

196

$<50$

$<0.24$

$<0.090$
Unit Flag Lab

$\mu g / L \quad 0 \quad$ GE

$\mu g / L \quad 0 \quad$ GE

$\mu \mathrm{g} / \mathrm{L} \quad \mathrm{O} \quad \mathrm{GE}$

$\mu g / L \quad 0 \quad$ GE

$\mu \mathrm{g} / \mathrm{L} \quad \mathrm{O} \quad \mathrm{GE}$

$\mu g / L \quad 0 \quad G E$

$\mu g / L \quad 0 \quad$ GE

$\mu g / L \quad 0 \quad$ GE

$\mu g / L \quad 0 \quad$ GE

$\mu g / L \quad 0 \quad$ GE

$\mu g / L \quad 0 \quad$ GE

$\mu g / L \quad 0 \quad$ GE

$\mu g / L \quad 0 \quad-$ GE

$\mu g / L \quad 0 \quad$ GE

$\mu g / L \quad 0 \quad$ GE

$\mu g / L \quad 1 \quad$ GE

$\mu g / L \quad 0 \quad$ GE

$\mu g / L \quad 0 \quad$ GE

$\mu \mathrm{g} / \mathrm{L} \quad 0 \quad \mathrm{GE}$

$\mu g / L \quad O \quad$ GE

$\mu g / L \quad 0 \quad$ GE

$\mu g / L \quad$ GE

$\mu g / L \quad 0 \quad$ GE

$\mu \mathrm{g} / \mathrm{L} \quad \mathrm{O} \quad \mathrm{GE}$

$\mu g / L \quad 0 \quad$ GE

$\mu g / L \quad O \quad$ GE

$\mu g / L \quad 0 \quad$ GE

$\mu g / L \quad 0 \quad$ GE

$\mu g / L \quad 0 \quad$ GE

$\mu g / L \quad 0 \quad$ GE

$\mu g / L \quad 0 \quad$ GE

$\mu g / L \quad O \quad$ EE

$\mu g / L \quad 0 \quad$ GE

$\mu g / L \quad 0 \quad$ GE

$\mu g / L \quad 0 \quad$ GE

$\mu g / L \quad 0 \quad$ GE

$\mu g / L \quad 0 \quad G E$

$\mu g / L \quad 0 \quad$ GE

$\mu g / L \quad 0 \quad$ GE

$\mu g / L \quad 0 \quad$ GE

$\mu g / L \quad 0 \quad$ GE

$\mu g / L \quad 0 \quad$ GE

$\mu g / L \quad 0 \quad$ GE

$\mu g / L \quad 0 \quad$ GE

$\mu g / L \quad 0 \quad$ GE

$\mu g / L \quad 0 \quad$ GE

$\mu g / L \quad 0 \quad$ GE

$\mu \mathrm{g} / \mathrm{L} \quad 2 \quad \mathrm{GE}$

$\mu g / \mathrm{L} \quad \mathrm{G} \quad \mathrm{GE}$

$\mu g / L \quad 0 \quad$ GE

$\mu g / L \quad$ GE

$\overline{- \text { = exceeded holding time. }}=$ =xceeded primary drinking water standard. 
WELL LFW 61C, laboratory analyses (cont.)

\begin{tabular}{|c|c|c|c|c|c|}
\hline \multirow[t]{9}{*}{$\underline{H} \underline{D}$} & Analyte & Result & Unit & Flag & $\underline{\text { Lab }}$ \\
\hline & 1,1,1-Trichloroethane & 13 & $\mu \mathrm{g} / \mathrm{L}$ & 0 & $\mathrm{GE}$ \\
\hline & 1,1,2-Trichloroethane & $<1.0$ & $\mu g / L$ & 0 & GE \\
\hline & Trichloroethylene & 4.0 & $\mu g / L$ & 1 & GE \\
\hline & Trichlorofluoromethane & 54 & $\mu g / L$ & 2 & GE \\
\hline & Gross alpha & $<2.0 \mathrm{E}+00$ & $\mathrm{pCi} / \mathrm{L}$ & 0 & $E$ \\
\hline & Nonvolatile beta & $<2.0 E+00$ & $\mathrm{pCi} / \mathrm{L}$ & 0 & GE \\
\hline & $\begin{array}{l}\text { Total radium } \\
\text { Tritium }\end{array}$ & $\begin{array}{l}<1.0 E+00 \\
<7.0 E-01\end{array}$ & $\begin{array}{l}\mathrm{pCi} / \mathrm{L} \\
\mathrm{pCi} / \mathrm{mL}\end{array}$ & 0 & G \\
\hline & & & & & \\
\hline
\end{tabular}

\section{WELL LFW 61D}

\section{SRS Coord.}

N83089.1

E46471.1

\section{MEASUREMENTS CONDUCTED IN THE FIELD}

Sample date: $10 / 31 / 91$

Depth to water: $24.88 \mathrm{ft}(7.58 \mathrm{~m})$ below TOC Water elevation: $143.42 \mathrm{ft}(43.71 \mathrm{~m}) \mathrm{msl}$ Sp. conductance: $25 \mu \mathrm{S} / \mathrm{cm}$ Water evacuated before sampling: $36 \mathrm{gal}$

\section{LABORATORY ANALYSES}

H D Analyte

pH
Specific conductance
Arsenic
Barium
Benzene
Bromodichloromethane
Bromoform
Bromomethane (Methyl bromide)
Cadmium
Calcium
Carbon tetrachloride
Chloride
Chlorobenzene
Chloroethane
Chloroethene (Vinyl chloride)
2-Chloroethyl vinyl ether
Chloroform
Chloromethane (Methyl chloride)
Chromium
Dibromochloromethane
1,1-Dichloroethane
1,2-Dichloroethane

Time: 13:35

pH: 4.5

Alkalinity: $0 \mathrm{mg} / \mathrm{L}$

Water temperature: $19.0^{\circ} \mathrm{C}$

\section{Result}

$\begin{aligned} & 5.1 \\ & 20 \\ &< 2.0 \\ & 3.8 \\ &< 1.0 \\ &< 1.0 \\ &< 1.0 \\ &< 1.0 \\ &< 2.0 \\ & 458 \\ &< 1.0 \\ & 2.900 \\ &< 1.0 \\ &< 1.0 \\ &< 1.0 \\ &< 1.0 \\ &< 1.0 \\ &<1.0 \\ &<4.0 \\ &<1.0 \\ & 17 \\ &<<1.0\end{aligned}$

\begin{tabular}{lll} 
Unit & Flag & Lab \\
\cline { 2 - 3 }$p H$ & 0 & GE \\
$\mu S / c m$ & 0 & GE \\
$\mu g / L$ & 0 & GE \\
$\mu g / L$ & 0 & GE \\
$\mu g / L$ & 0 & GE \\
$\mu g / L$ & 0 & GE \\
$\mu g / L$ & 0 & GE \\
$\mu g / L$ & 0 & GE \\
$\mu g / L$ & 0 & GE \\
$\mu g / L$ & 0 & GE \\
$\mu g / L$ & 0 & GE \\
$\mu g / L$ & 0 & GE \\
$\mu g / L$ & 0 & GE \\
$\mu g / L$ & 0 & GE \\
$\mu g / L$ & 0 & GE \\
$\mu G / L$ & 0 & GE \\
$\mu g / L$ & 0 & GE \\
$\mu g / L$ & 0 & GE \\
$\mu g / L$ & 0 & GE \\
$\mu g / L$ & 0 & GE \\
$\mu g / L$ & 2 & GE \\
$\mu g / L$ & 0 & GE
\end{tabular}

Fourth Quarter 1991 
WELL LFW 61D, laboratory analyses (cont.)

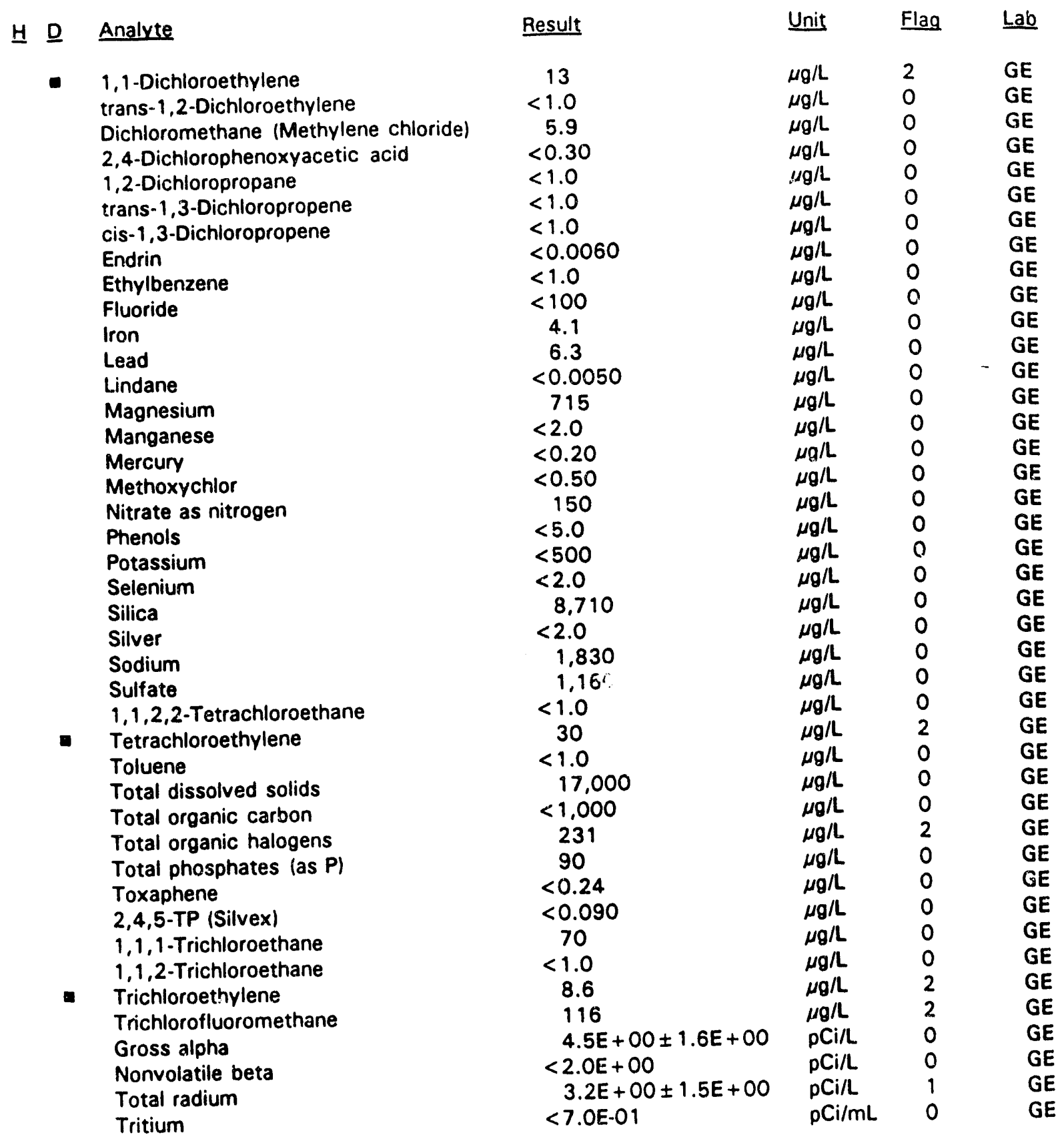

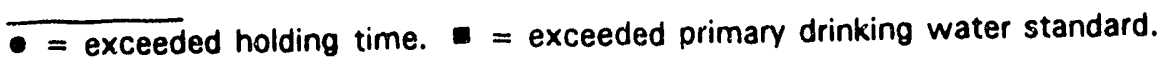


WELL LFW 62B

SPS Coord.

N83001.2

E45915.5

MEASUREMENTS CONDUCTED IN THE FIELD

Sample date: 10/31/91

Depth to water: $22.59 \mathrm{ft}(6.89 \mathrm{~m})$ below TOC

Water elevation: $142.31 \mathrm{ft}(43.38 \mathrm{~m}) \mathrm{ms}$ |

Sp. conductance: $32 \mu \mathrm{S} / \mathrm{cm}$

Water evacuated before sampling: $210 \mathrm{gal}$

\section{LABORATORY ANALYSES}

H D Analyte

Result

$\mathrm{pH}$

Specific conductance

Arsenic

Barium

Benzene

Bromodichloromethane

Bromoform

Bromomethane (Methyl bromide)

Cadmium

Calcium

Carbon tetrachloride

Chloride

Chlorobenzene

Chloroethane

Chloroethene (Vinyl chloride)

2-Chloroethyl vinyl ether

Chloroform

Chloromethane (Methyl chloride)

Chromium

Dibromochloromethane

1,1-Dichloroethane

1,2-Dichlor jethane

1,1-Dichloroethylene

trans-1,2-Dichloroethylene

Dichloromethane (Methylene chloride)

2,4-Dichlorophenoxyacetic acid

1,2-Dichloropropane

trans-1,3-Dichloropropene

cis-1,3-Dichloropropene

Endrin

Ethylbenzene

Fluoride

Iron

Lead

Lindane

Magnesium
5.3

30

$<2.0$

5.2

$<1.0$

$<1.0$

$<1.0$

$<1.0$

$<2.0$

785

$<1.0$

1,830

$<1.0$

$<1.0$

$<1.0$

$<1.0$

$<1.0$

$<1.0$

$<4.0$

$<1.0$

$<1.0$

$<1.0$

$<1.0$

$<1.0$

$<1.0$

$<0.30$

$<1.0$

$<1.0$

$<1.0$

$<0.0060$

$<1.0$

$<100$

20

14

$<0.0050$

259
Top of Casing Casing

$164.9 \mathrm{ft} \mathrm{msl} \quad 4^{\mathrm{n}} \mathrm{PVC}$
Time: $11: 40$

pH: 5.0

Alkalinity: $0 \mathrm{mg} / \mathrm{L}$

Water temperature: $19.1^{\circ} \mathrm{C}$
Unit Flag Lab

pH $\quad 0 \quad$ GE

$\mu S / c m$ GE

$\mu g / L \quad 0 \quad$ GE

$\mu g / L \quad 0 \quad$ GE

$\mu g / 2 \quad 0 \quad$ GE

$\mu g / L \quad 0 \quad$ GE

$\mu g / L \quad 0 \quad$ GE

$\mu g / L \quad 0 \quad$ GE

$\mu g / L \quad 0 \quad$ GE

$\mu g / L \quad 0 \quad$ GE

$\mu g / L \quad 0 \quad \mathrm{GE}$

$\mu g / L \quad 0 \quad G E$

$\mu g / L \quad 0 \quad G E$

$\mu g / 2 \quad 0 \quad G E$

$\mu Q . L \quad O \quad G E$

$\mu g / L \quad 0 \quad$ GE

$\mu g / L . \quad 0 \quad$ GE

$\mu g / L \quad 0 \quad \mathrm{GE}$

$\mu g / L \quad 0 \quad$ GE

$\mu g / L \quad 0 \quad$ GE

$\mu g / L \quad O \quad G E$

$\mu g / L \quad 0 \quad$ GE

$\mu g / L \quad 0 \quad$ GE

$\mu g / L \quad 0 \quad$ GE

$\mu g / L \quad O \quad G E$

$\mu g /$ $\quad 0 \quad G E$

$\mu g / L \quad 0 \quad$ GE

$\mu g /$ O $\quad$ GE

$\mu g / \quad 0 \quad$ GE

$\mu g / L \quad 0 \quad$ GE

$\mu g \Lambda$ GE

$\mu g / L \quad O \quad$ GE

$\mu g / L \quad 0 \quad G E$

$\mu g / L \quad 1 \quad$ GE

$\mu g /$ L $\quad 0 \quad$ GE

$\mu g / L \quad O \quad G E$

$\overline{0}$ = exceeded holding time. = exceeded primary drinixing water standard 
WELL LFW 62B, laboratory analyses (cont.)

H D Analyte

Manganese
Mercury
Methoxychlor
Nitrate as nitrogen
Phenols
Potassium
Selenium
Silica
Silver
Sodium
Sulfate
$1,1,2,2-$ Tetrachloroethane
Tetrachloroethylene
Toluene
Total dissolved solids
Total dissolved solids
Total organic carbon
Total organic halogens
Total phosphates (as P)
Toxaphene
$2,4,5-$ TP iSilvex)
$1,1, i-$ Trichloroethane
$1,1,2-$ Trichloroethane
Trichloroethylene
Trichlorofluoromethane
Gross alpha
Nonvolatile beta
Total radium
Tritium

$\begin{aligned} & \text { Result } \\ & \\ & 19 \\ &<0.20 \\ &<0.50 \\ & 530 \\ &<5.0 \\ &<500 \\ &<2.0 \\ & 10,400 \\ &<2.0 \\ & 3,960 \\ & 5,660 \\ &<1.0 \\ &<1.0 \\ &<1.0 \\ & 45,000 \\ & 47.000 \\ &<1,000 \\ & 10 \\ & 200 \\ &<0.24 \\ &<0.090 \\ &<1.0 \\ &<1.0 \\ &<1.0 \\ &<1.0 \\ & 1.4 E+01 \pm 4.8 E+00 \\ & 7.7 E+00 \pm 4.3 E+00 \\ & 2.2 E+00 \pm 1.7 E+00 \\ &<7.0 E-01 \\ &\end{aligned}$

\section{WELL LFW 62C}

\begin{tabular}{|c|c|c|}
\hline Unit & Flag & $\underline{\text { Lab }}$ \\
\hline$\mu g / L$ & 0 & $\mathrm{GE}$ \\
\hline$\mu g / L$ & 0 & GE \\
\hline$\mu g / L$ & 0 & GE \\
\hline$\mu g / L$ & 0 & GE \\
\hline$\mu g / L$ & 0 & GE \\
\hline$\mu g / L$ & 0 & GE \\
\hline$\mu g / L$ & 0 & GE \\
\hline$\mu g / L$ & 0 & GE \\
\hline$\mu g / L$ & 0 & GE \\
\hline$\mu g / L$ & 0 & GE \\
\hline$\mu g / L$ & 0 & GE \\
\hline$\mu g / L$ & 0 & GE \\
\hline$\mu \mathrm{g} / \mathrm{L}$ & 0 & GE \\
\hline$\mu g / L$ & 0 & GE \\
\hline$\mu g / L$ & 0 & o \\
\hline$\mu \mathrm{g} / \mathrm{L}$ & 0 & 2 \\
\hline$\mu \mathrm{g} / \mathrm{L}$ & 0 & GE \\
\hline$\mu g / L$ & 0 & GE \\
\hline$\mu g /$ & 0 & GE \\
\hline$\mu g / L$ & 0 & GE \\
\hline$\mu g / L$ & 0 & GE \\
\hline$\mu g / L$ & 0 & GE \\
\hline$\mu g / L$ & 0 & GE \\
\hline$\mu g / L$ & 0 & GE \\
\hline$\mu g /$ & 0 & GE \\
\hline pCin & 1 & GE \\
\hline $\mathrm{pCi} / \mathrm{L}$ & 0 & $C E$ \\
\hline $\mathrm{Ci} / \mathrm{L}$ & 0 & GE \\
\hline $\mathrm{pCi} / \mathrm{mL}$ & 0 & GE \\
\hline
\end{tabular}

\section{SRS Coord.}

N83012.7

E45906.7

\section{MEASUREMENTS CONDUCTED IN THE FIELD}

Sample date: 10/29/91

Depth to water: $22.87 \mathrm{ft}(6.97 \mathrm{~m})$ below TOC Water elevation: $142.63 \mathrm{ft}(43.47 \mathrm{~m}) \mathrm{ms}$ Sp. conductance: $26 \mu \mathrm{S} / \mathrm{cm}$ Screen Zone Elevation 118.4-108.4 ft msl Top of Casing Casing $165.5 \mathrm{ft} \mathrm{msl}$ 4" PVC
Time: $14: 45$

pH: 5.2

Alkalinity: $1 \mathrm{mg} / \mathrm{L}$

Water temperature: $19.1^{\circ} \mathrm{C}$

Water evacuated before sampling: 91 gal

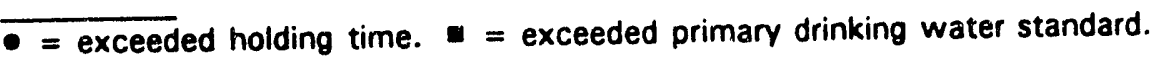




\section{H D Analyte}

- pH

Specific conductance

Arsenic

Arsenic

Barium

Benzene

Bromodichloromethane

Bromoform

Bromomethane (Methyl bromide)

Cadmium

Calcium

Carbon tetrachloride

Chloride

Chlorobenzene

Chloroethane

Chloroethene (Vinyl chloride)

2-Chloroethyl vinyl ether

Chloroform

Chloromethane (Methyl chloride)

Chromium

Dibromochlornmethane

1,1-Dichloroethann

1,2-Dichloroethane

1,1-Dichloroethylene

trans-1,2-Dichloroethylene

Dichloromethane (Methylene chloride)

- 2,4-Dichlorophenoxyacetic acid

1,2-Dichloropropane
trans-1,3-Dichloropropene
cis-1,3-Dichloropropene
Endrin
Endrin
Ethylbenzene
Fluoride
Lead
Lead
Lindane
Lindane
Magnesium
Manganese
Mercury
Methoxychlor
Methoxychlor
Nitrate as nitrogen
Phenols
Phenols
Potassium
Selenium
Selenium
Silica
Silver

Result

5.4

25

$<2.0$

$<2.0$

6.3

$<1.0$

$<1.0$

$<1.0$

$<1.0$

$<2.0$

1,250

$<1.0$

2,100

$<1.0$

$<1.0$

$<1.0$

$<1.0$

$<1.0$

$<1.0$

$<4.0$

$<1.0$

5.0

$<1.0$

2.0

$<1.0$

5.0

$<0.30$

$<1.0$

$<1.0$

$<1.0$

$<0.0060$

$<0.0060$

$<1.0$

$<100$

$<3.0$

$<3.0$

$<0.0050$

$<0.0050$

812

18

0.34

$<0.50$

$<0.50$

1.470

$<5.0$

$<5.0$

540

$<2.0$

$<2.0$

7,940

$<2.0$
Unit Flag Lab

pH $O$ GE

$\mu S / \mathrm{cm} \quad 0 \quad \mathrm{GE}$

$\mu g / L \quad O \quad$ GE

$\mu g / L \quad 0 \quad$ GE

$\mu g / L \quad 0 \quad$ GE

$\mu g / L \quad 0 \quad$ GE

$\mu g / L \quad O \quad$ GE

$\mu g / L \quad 0 \quad$ GE

$\mu g / L \quad O \quad$ GE

$\mu g / L \quad 0 \quad$ GE

$\mu g /$ G $\quad 0$ GE

$\mu g / \mathrm{L} \quad 0 \quad$ GE

$\mu g / L \quad 0 \quad-$ GE

$\mu g / L \quad 0 \quad$ GE

$\mu g / L \quad 0 \quad$ GE

$\mu g / L \quad O \quad$ GE

$\mu g / L \quad 0 \quad$ GE

$\mu g / 2 \quad 0 \quad$ GE

$\mu g / L \quad$ GE

$\mu g / L \quad 0 \quad$ GE

$\mu g \Lambda \quad$ GE

$\mu g /$ L 1 GE

$\mu g / L \quad$ GE

$\mu g / L \quad 0 \quad$ GE

$\mu g \Omega \quad$ GE

$\mu g \Lambda \quad$ GE

$\mu g \Lambda \quad \quad 0 \quad$ GE

$\mu g / L . \quad$ GE

$\mu g / L \quad$ GE

$\mu g / L \quad O \quad$ GE

$\mu g / L \quad$ GE

$\mu g / 2 \quad 0 \quad$ GE

$\mu g / L \quad 0 \quad$ GE

$\mu g / \quad 0 \quad$ GE

$\mu g \Omega \quad$ GE

$\mu g / 2 \quad 0 \quad$ GE

$\mu g /$ $\quad 0 \quad$ GE

$\mu g / L \quad$ GE

$\mu g /$ GE

$\mu g /$ $\quad 0 \quad$ GE

$\mu g / L \quad 0 \quad$ GE

$\mu g / L \quad 0 \quad$ GE

$\mu g / L \quad 0 \quad$ GE

$\mu g / L \quad 0 \quad$ GE

$\mu g / L \quad O \quad$ GE

$\mu g \Omega \quad$ GE

$\mu g / L \quad 0$ GE

$\mu g h \quad 0$ GE

$\mu g /$. 0 GE

$\mu g /$ $\quad 0 \quad$ GE

$\mu g h$ GE

= exceeded holding time. = exceeded primary drinking water standard 
WELL LFW 62C, laboratory analyses (cont.)

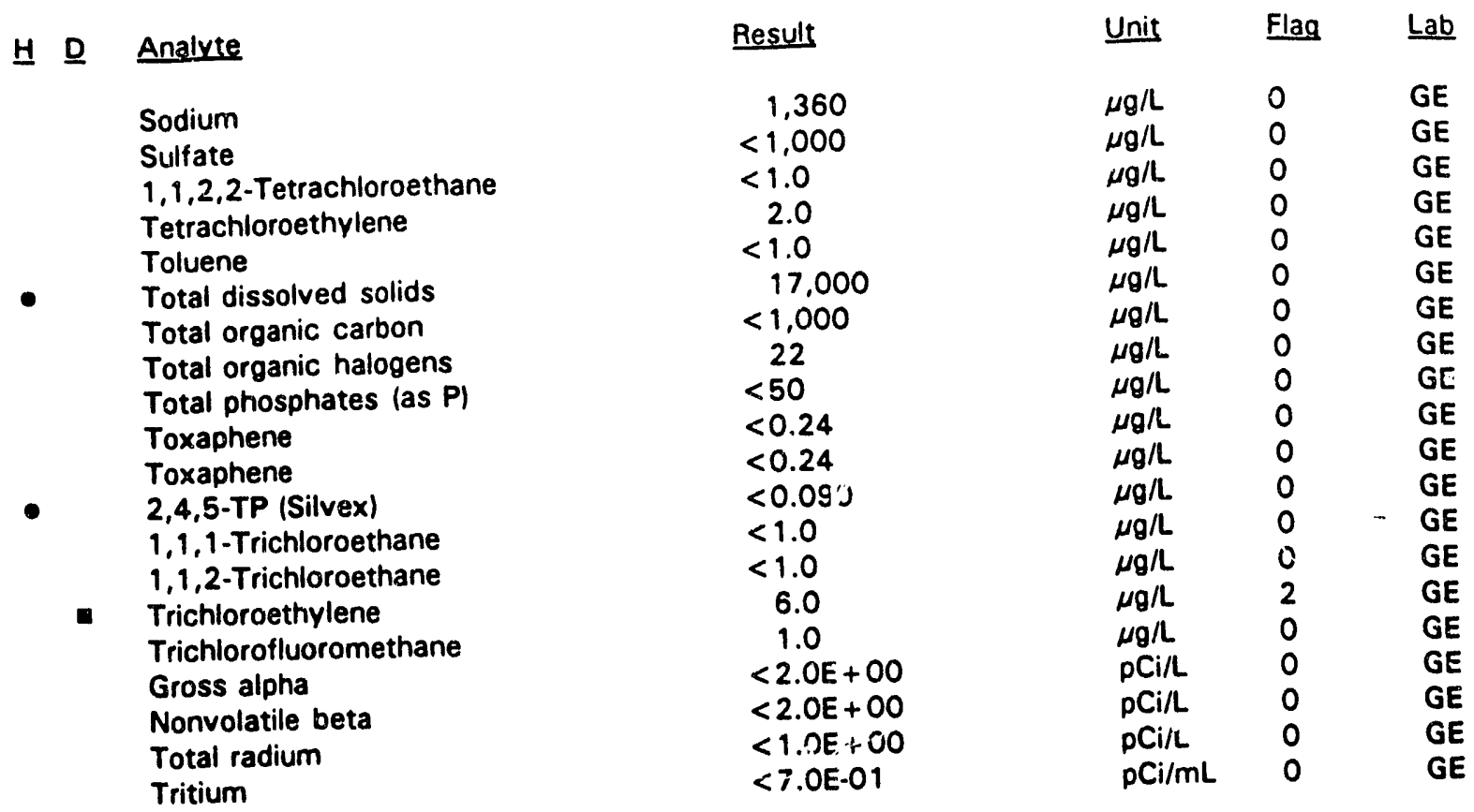

\section{WELL LFW 62D}

SRS coord.

N82991.6

E45922.9

MEASUREMENTS CONDUCTED IN THE FIELD

Sample date: 10/29/91

Depth to water: $21.69 \mathrm{ft}(6.61 \mathrm{~m})$ below TOC Water elevation: $143.11 \mathrm{ft}(43.62 \mathrm{~m}) \mathrm{msl}$

Sp. conductance: $34 \mu \mathrm{S} / \mathrm{cm}$

Screen Zone Elevation

$147.6-127.6 \mathrm{ft} \mathrm{msl}$
Top of Casing

$164.8 \mathrm{ft} \mathrm{msl}$
Casing

4" PVC

Water evacuated before sampling: $10 \mathrm{gal}$

The well went dry during purging.

\section{LABORATORY ANALYSES}

브 Analyte

- $\quad$ pH

Specific conductance

Arsenic

Arsenic

Barium

Benzene

Bromodichloromethane

Bromoform

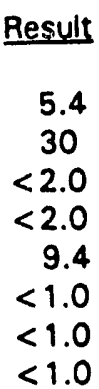

Time: $14: 25$

pH: 5.0

Alkalinity: $5 \mathrm{mg} / \mathrm{L}$

Water temperature: $19.1^{\circ} \mathrm{C}$

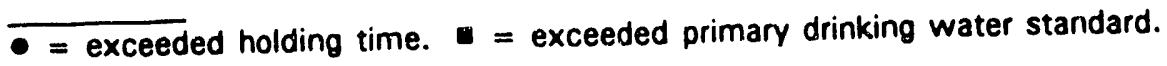

\begin{tabular}{lll} 
Unit & Flag & Lab \\
\cline { 2 - 3 } & & \\
$\mu \mathrm{SH} / \mathrm{cm}$ & 0 & GE \\
$\mu \mathrm{g} / \mathrm{L}$ & 0 & GE \\
$\mu \mathrm{g} / \mathrm{L}$ & 0 & GE \\
$\mu \mathrm{g} / \mathrm{L}$ & 0 & GE \\
$\mu \mathrm{g} / \mathrm{L}$ & 0 & GE \\
$\mu \mathrm{g} / \mathrm{L}$ & 0 & GE \\
$\mu \mathrm{g} / \mathrm{L}$ & 0 & GE
\end{tabular}


WELL LFW 62D, laboratory analyses (cont.)

H D Analyte

Bromomethane (Methyl bromide)

Cadmium

Calcium

Carbon tetrachloride

Chloride

Chlorobenzene

Chloroethane

Chloroethene (Vinyl chloride)

2-Chloroethyl vinyl ether

Chloroform

Chloromethane (Methyl chloride)

Chromium

Dibromochloromethane

1,1-Dichloroethane

1,2-Dichloroethane

1,1-Dichloroethylene

trans-1,2-Dichloroethylene

Dichloromethane (Methylene chloride)

2,4-Dichlorophenoxyacetic aciu

1,2-Dichloropropane

trans-1,3-Dichloropropene

cis-1,3-Dichloropropene

Endrin

Endrin

Ethylbenzene

Fluoride

Lead

Lead

Lindane

Lindane

Magnesium

Manganese

Mercury

Methoxychlor

Methoxychlor

Nitrate as nitrogen

Phenols

Patassium

Selenium

Selenium

Silica

Silver

Sodium

Sulfate

1,1,2,2-Tetrachloroethane

Tetrachloroethylene

Tolvene

- Total dissolved solids

Total organic carbon

Total organic halogens

Total phosphates (as P)
Result

$<1.0$

$<2.0$

1,450

$<1.0$

4,380

$<1.0$

$<1.0$

$<1.0$

$<1.0$

$<1.0$

$<1.0$

$<4.0$

$<1.0$

27

$<1.0$

4.0

$<1.0$

10

$<0.30$

$<1.0$

$<1.0$

$<1.0$

$<0.0060$

$<0.0060$

$<i .0$

$<100$

$<3.0$

$<3.0$

$<0.0050$

$<0.0050$

729

46

$<0.20$

$<0.50$

$<0.50$

2.70

$<5.0$

$<500$

$<2.0$

$<2.0$

11,600

$<2.0$

3,020

$<1,000$

$<1.0$

4.0

$<1.0$

26,000

$<1,000$

247

$<50$
Unit Flag Lab

$\mu g / \mathrm{L} \quad \mathrm{O} \quad \mathrm{GE}$

$\mu g / L \quad 0 \quad$ GE

$\mu g / L \quad 0 \quad$ GE

$\mu g / L \quad 0 \quad$ GE

$\mu g / L \quad 0 \quad$ GE

$\mu g / L \quad 0 \quad$ GE

$\mu g / L \quad 0 \quad$ GE

$\mu g / L \quad 0 \quad$ GE

$\mu g / L \quad 0 \quad$ GE

$\mu g / L \quad 0 \quad$ GE

$\mu g / L \quad 0 \quad$ GE

$\mu g / L \quad 0 \quad$ GE

$\mu g / L \quad 0 \quad-G E$

$\mu g / L \quad 2 \quad$ GE

$\mu g / L \quad 0 \quad$ GE

$\mu g / L \quad 1 \quad$ GE

$\mu g / L \quad 0 \quad$ GE

$\mu g / L \quad 0 \quad$ GE

$\mu g / L \quad 0 \quad$ GE

$\mu g / \quad 0 \quad G E$

$\mu g / L \quad 0 \quad$ GE

$\mu g / L \quad 0 \quad$ GE

$\mu g / L \quad 0 \quad$ GE

$\mu g / L \quad 0 \quad$ GE

$\mu G, L \quad 0 \quad$ GE

$\mu g / \quad 0 \quad$ GE

$\mu g / L \quad 0 \quad G E$

$\mu g / L \quad 0 \quad$ GE

$\mu g / L \quad 0 \quad$ GE

$\mu g / L \quad 0 \quad$ GE

$\mu \mathrm{g} / \mathrm{L} \quad 0 \quad \mathrm{GE}$

$\mu g / L \quad 1 \quad$ GE

$\mu g / L \quad 0 \quad$ GE

$\mu g / L \quad O \quad G E$

$\mu g / L \quad 0 \quad$ GE

$\mu g / L \quad 0 \quad$ GE

$\mu g / L \quad 0 \quad$ GE

$\mu g / L \quad 0 \quad G E$

$\mu g / L \quad 0 \quad$ GE

$\mu g / L \quad 0 \quad$ GE

$\mu g / L \quad 0 \quad$ GE

$\mu g / L \quad 0 \quad$ GE

$\mu g / L \quad 0 \quad G E$

$\mu g / L \quad 0 \quad$ GE

$\mu g / L \quad 0 \quad$ GE

$\mu g / L \quad 1 \quad$ GE

$\mu g / L \quad 0 \quad$ GE

$\mu g / L \quad 0 \quad$ GE

$\mu g / L \quad O \quad$ GE

$\mu g / L \quad 2 \quad G E$

$\mu g / L \quad 0 \quad$ GE

- = exceeded holding time.

. exceeded rimary drinking water standard 
WELL LFW 620, laboratory analyses (cont.)

\section{H D Analyte}

Toxaphene

Toxaphene

- 2,4,5-TP (Silvex)

1,1,1-Trichloroethane

1,1,2-Trichloroethane

- Trichloroethylene

Trichlorofluoromethane

Gross aipha

Nonvolatile beta

Total radium

Tritium

Result

$<0.24$
$<0.24$
$<0.090$
36
$<1.0$
$\quad 8.0$
26
$<2.0 E+00$
$<2.0 E+00$
$<1.0 E+00$
$<7.0 E-01$

Unit

$\mu g / L \quad 0$

$\mu g / L \quad 0$

$\mu g / L \quad 0$

$\mu g / L \quad 0$

$\mu g / L \quad 0$

$\mu g / L \quad 2$

$\mu g / L \quad 2$

$\mathrm{pCi} / \mathrm{L} \quad 0$

pCill $\quad 0$

$\mathrm{pCi} / \mathrm{L} \quad 0$

$\mathrm{pCi} / \mathrm{mL} \quad 0$
$\underline{L a b}$

GE

GE

GE

GE

GE

GE

GE

GE

GE

GE

GE

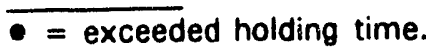

- exceeded primary drinking water standard. 


\section{APPENDIX D}

\section{FIELD MEASUREMENTS}


Water Level in the LFW-Series Monitoring Wells

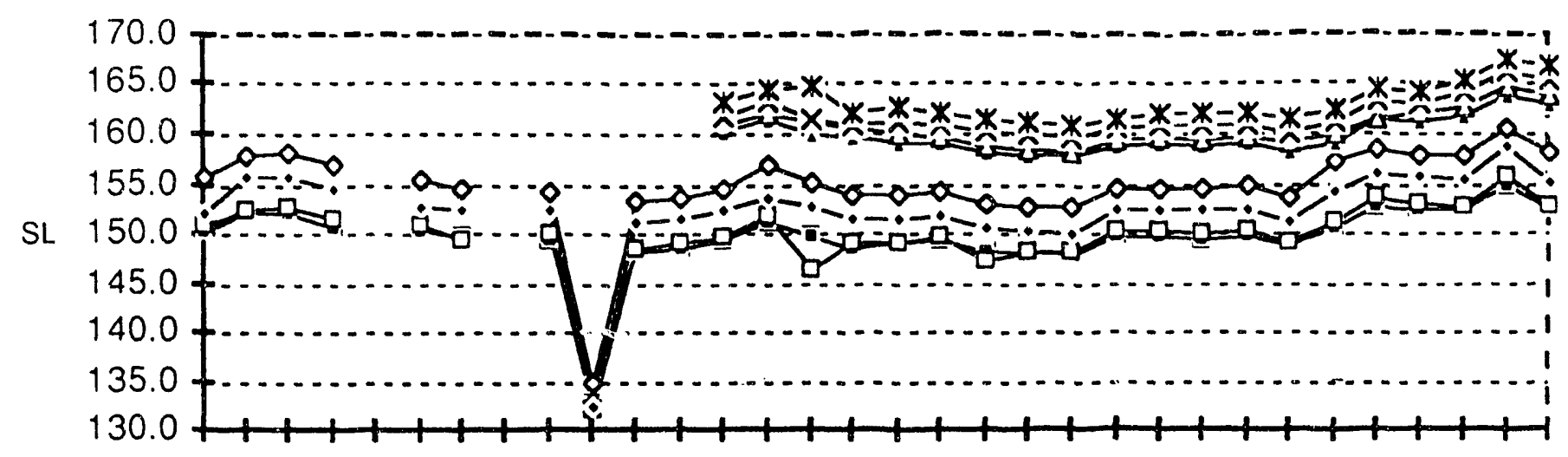

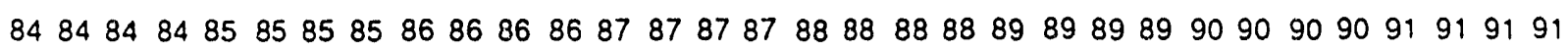

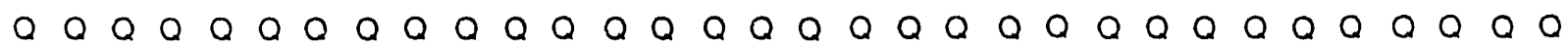

$\begin{array}{lllllllllllllllllllllllllllllllll}1 & 2 & 3 & 4 & 1 & 2 & 3 & 4 & 1 & 2 & 3 & 4 & 1 & 2 & 3 & 4 & 1 & 2 & 3 & 4 & 1 & 2 & 3 & 4 & 1 & 2 & 3 & 4 & 1 & 2 & 3 & 4\end{array}$

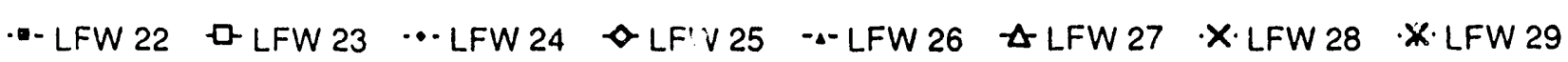

\section{Water Level in the LFW-Series Monitoring Wells}

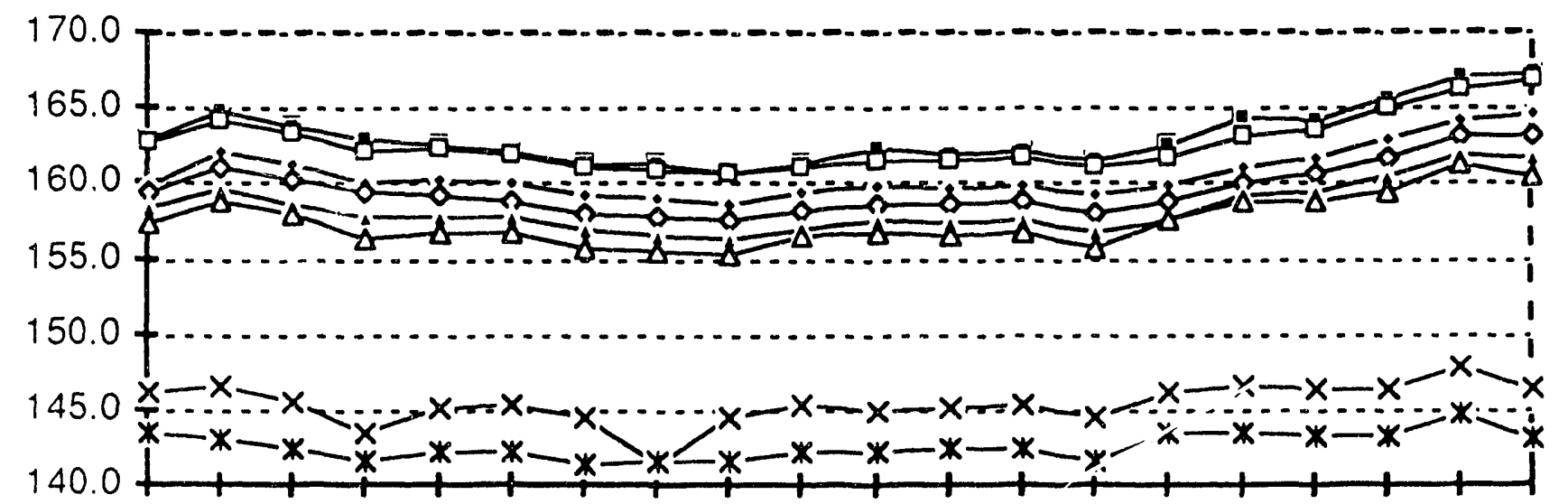

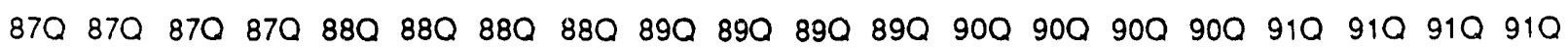
$\begin{array}{lllllllllllllllllllll}1 & 2 & 3 & 4 & 1 & 2 & 3 & 4 & 1 & 2 & 3 & 4 & 1 & 2 & 3 & 4 & 1 & 2 & 3 & 4\end{array}$

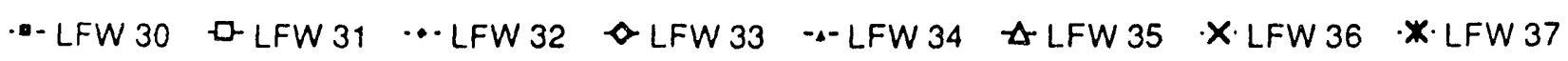


Water Level in the LFW-Series Monitoring Wells

ISL 145.0

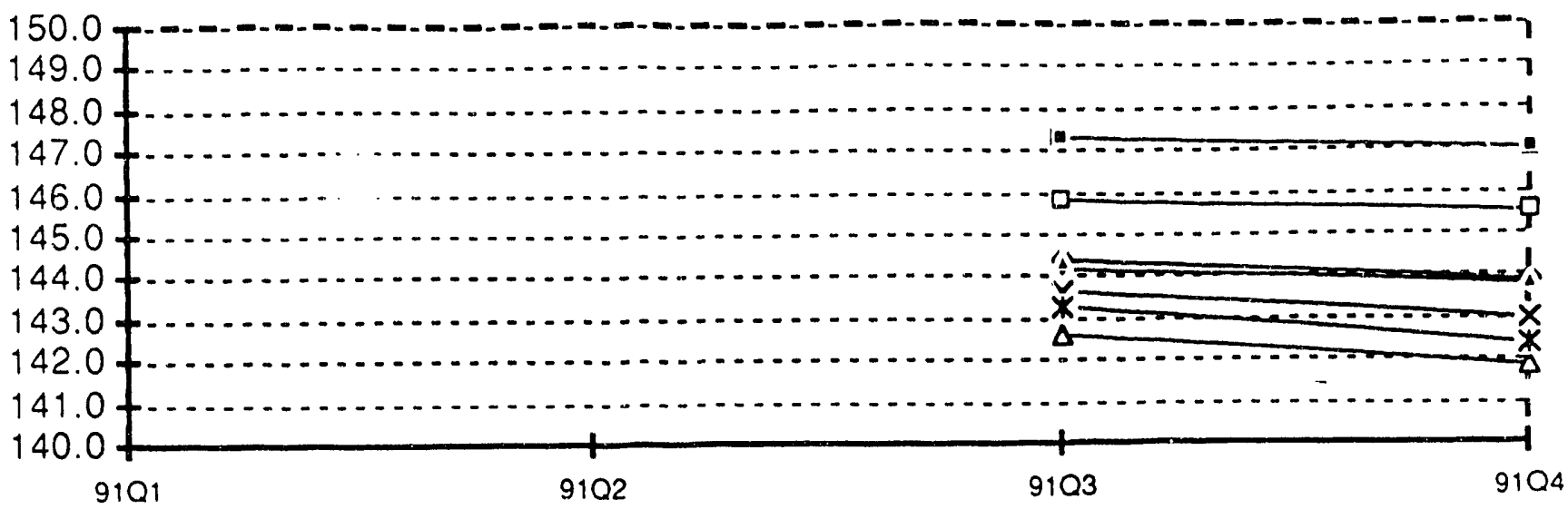

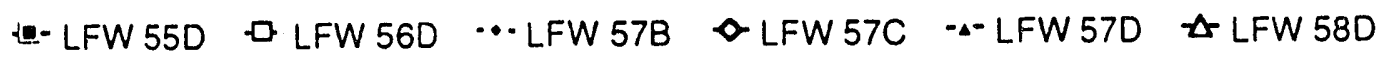

X. LFW 59B $\quad$ X. LFW 59C

Water Level in the LFW-Series Monitoring Wells

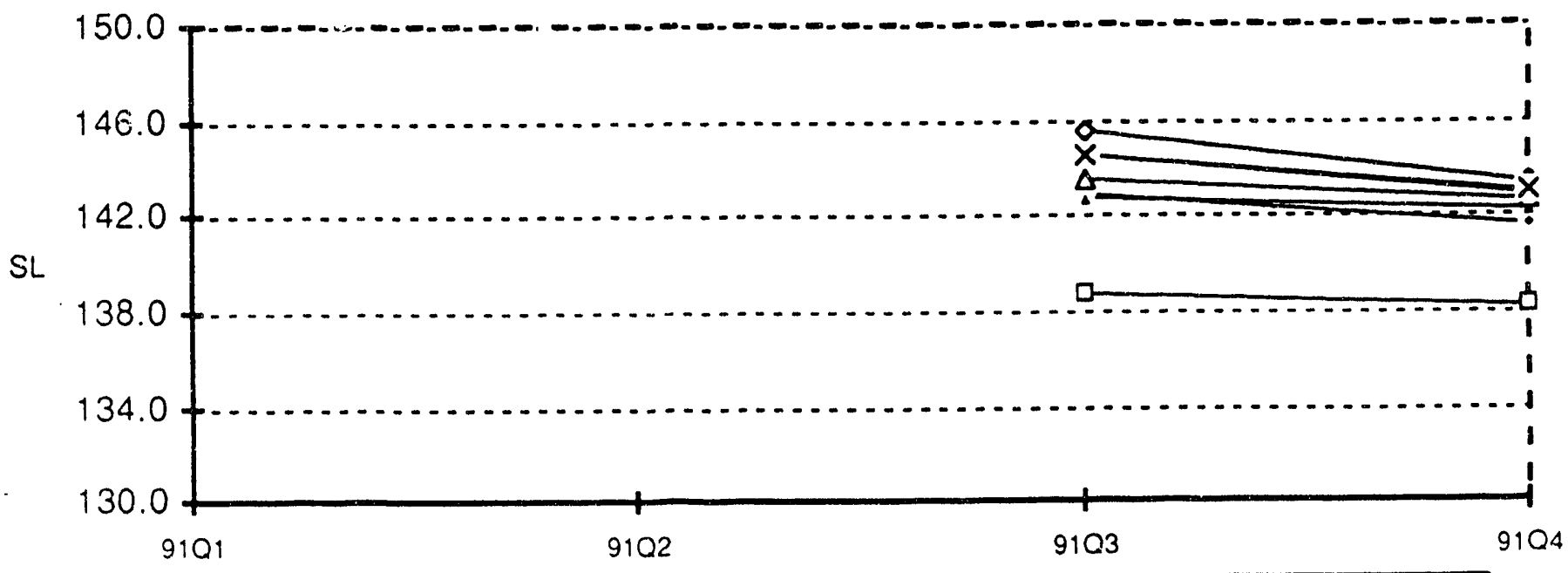

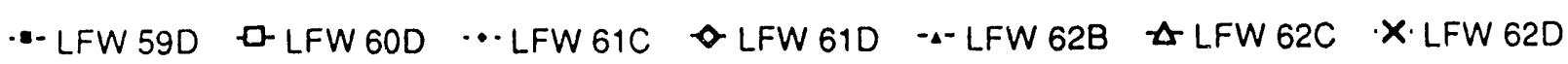


Field $\mathrm{pH}$ in the LFW-Series Monitoring Wells

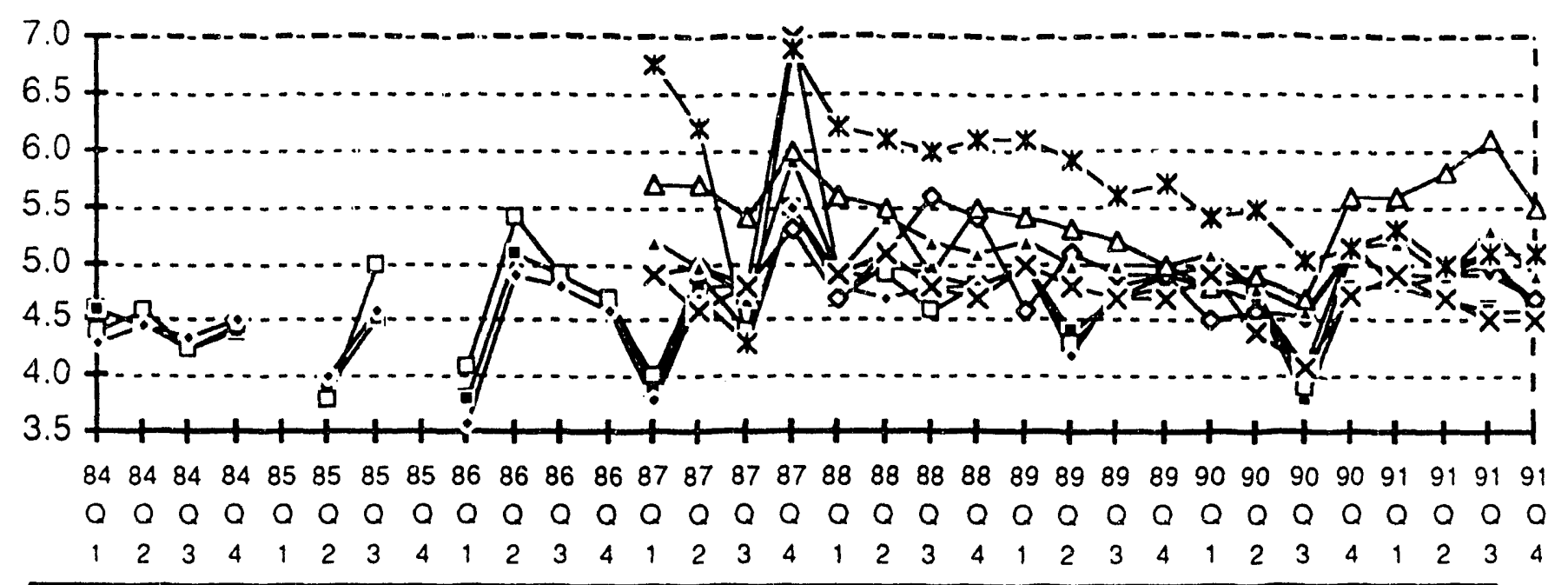

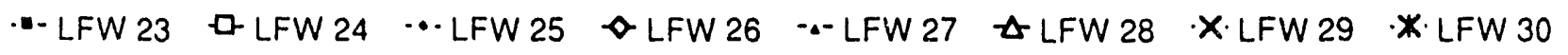

Field $\mathrm{pH}$ in the LFW-Series Monitoring Wells

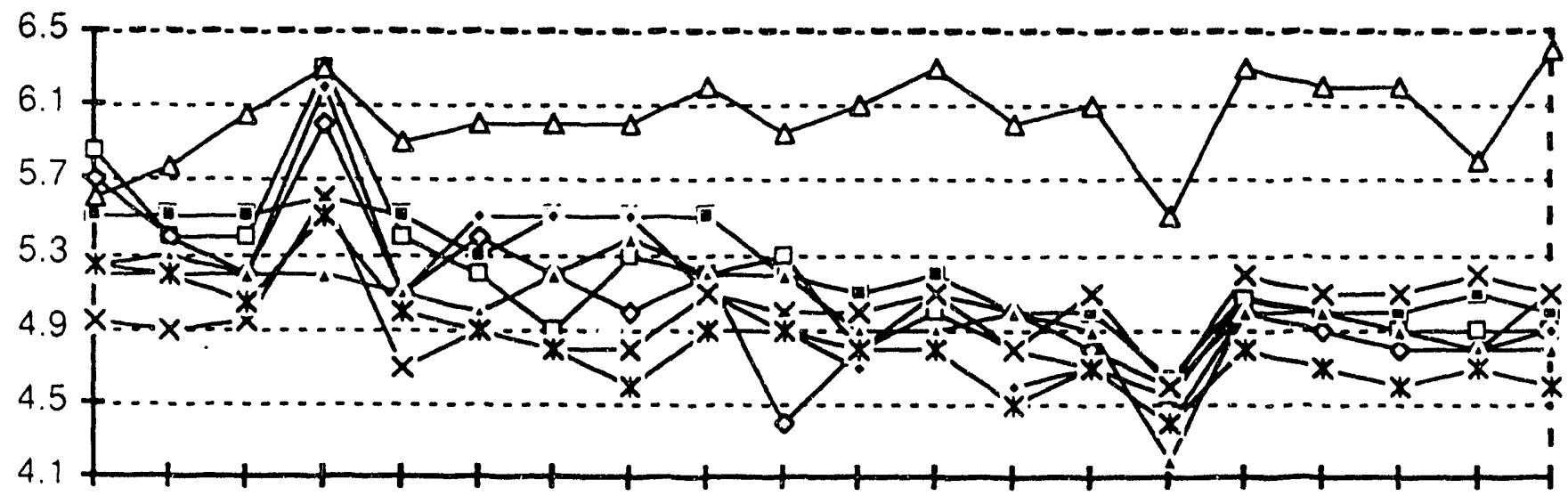

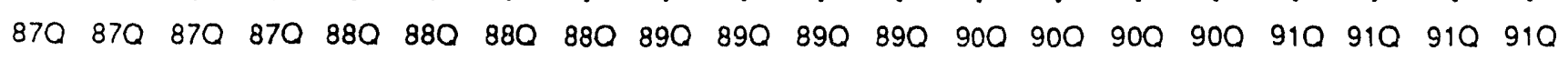

$\begin{array}{lllllllllllllllllllll}1 & 2 & 3 & 4 & 1 & 2 & 3 & 4 & 1 & 2 & 3 & 4 & 1 & 2 & 3 & 4 & 1 & 2 & 3 & 4\end{array}$

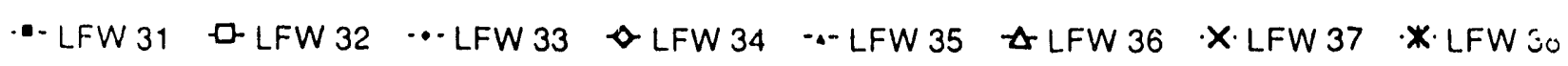


Field $\mathrm{pH}$ in the LFW-Series Monitoring Wells

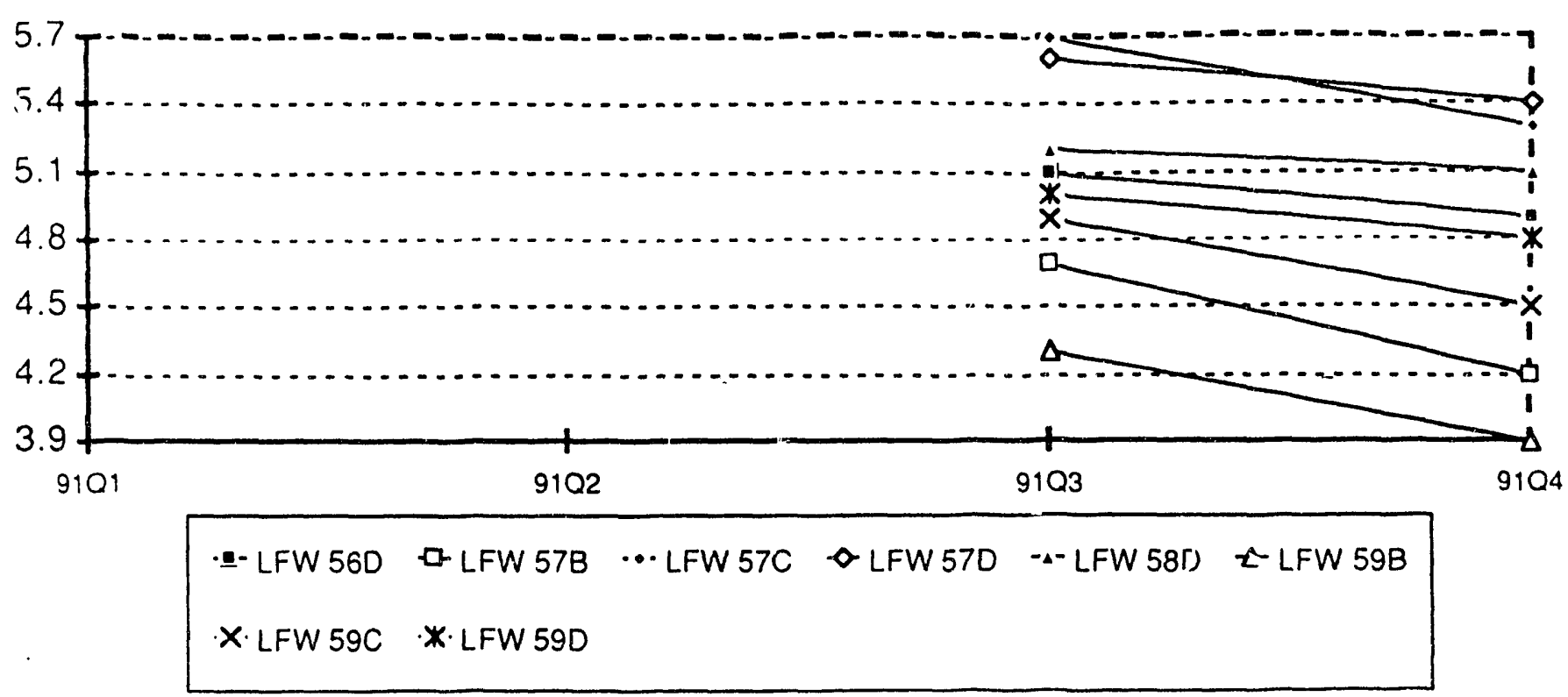

Field $\mathrm{pH}$ in the LFW-Series Monitoring Wells

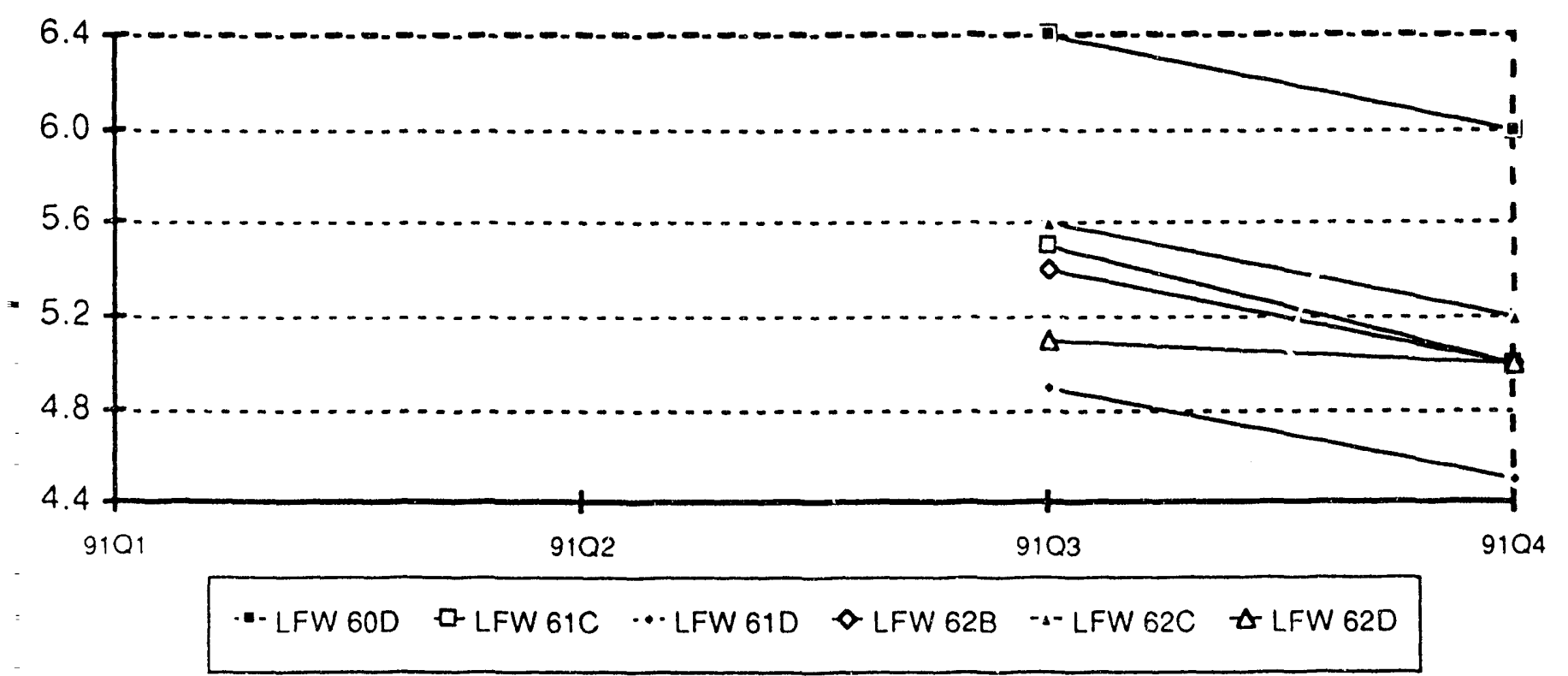


Field Conductivity in the LFW-Series Monitoring Wells

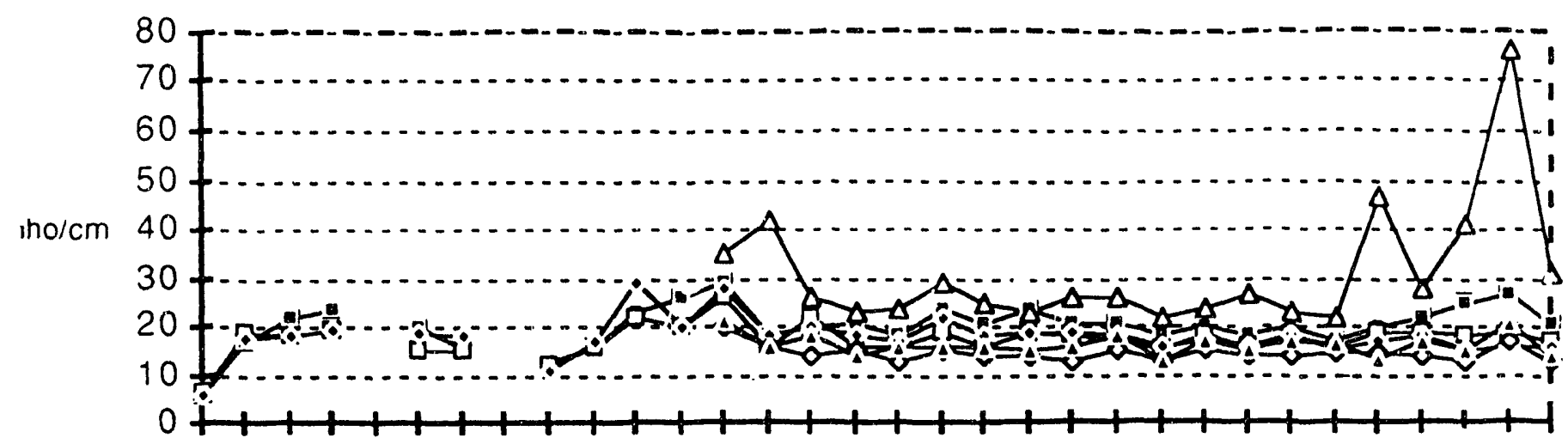

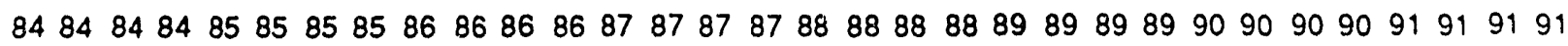

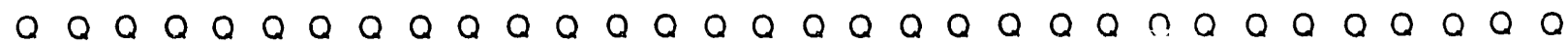

$\begin{array}{lllllllllllllllllllllllllllllllll}1 & 2 & 3 & 4 & 1 & 2 & 3 & 4 & 1 & 2 & 3 & 4 & 1 & 2 & 3 & 4 & 1 & 2 & 3 & 4 & 1 & 2 & 3 & 4 & 1 & 2 & 3 & 4 & 1 & 2 & 3 & 4\end{array}$

-LFW $23 \quad$ - LFW $24 \cdots$ LFW $25 \diamond$ LFW 26 --LFW $27 \quad \triangle$ LFW 28

Field Conductivity in the LFW-Series Monitoring Wells

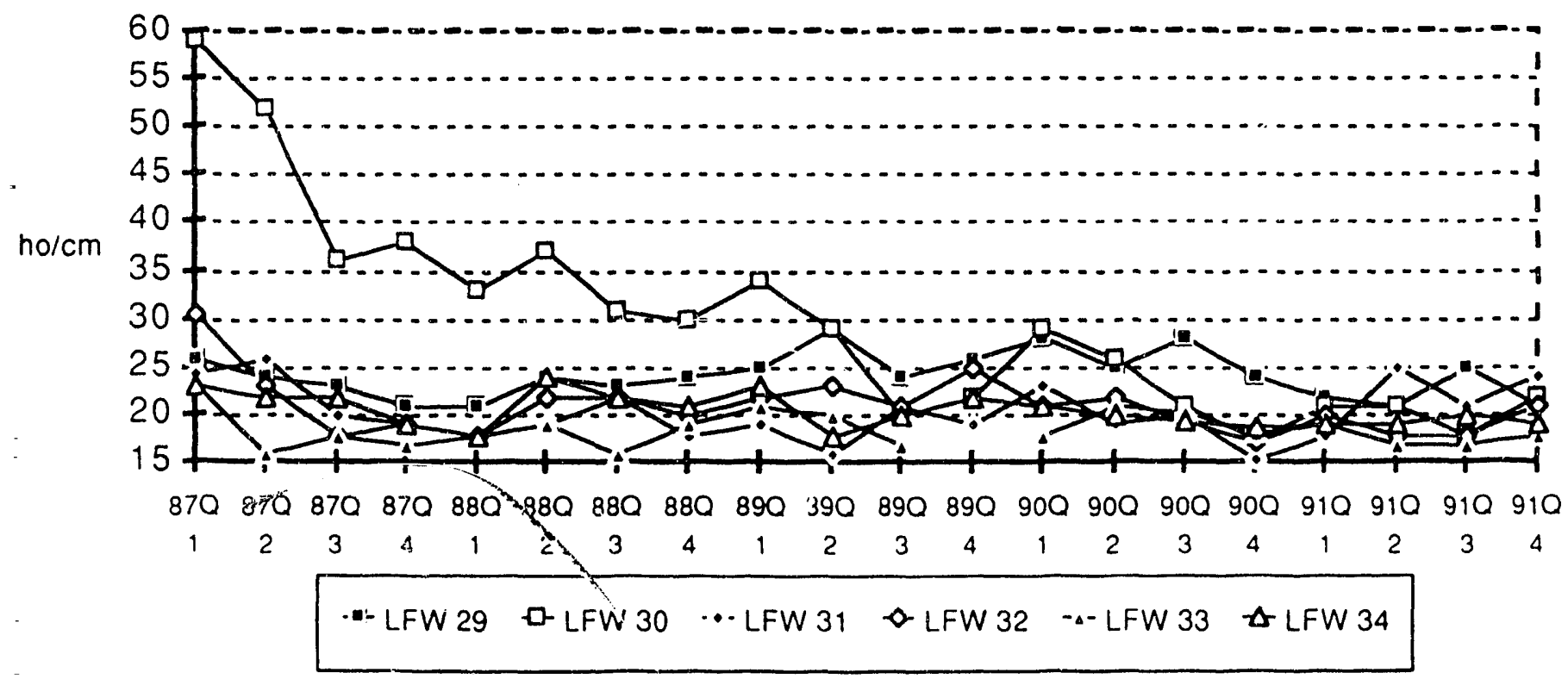


Field Conductivity in the LFW-Series Monitoring Wells

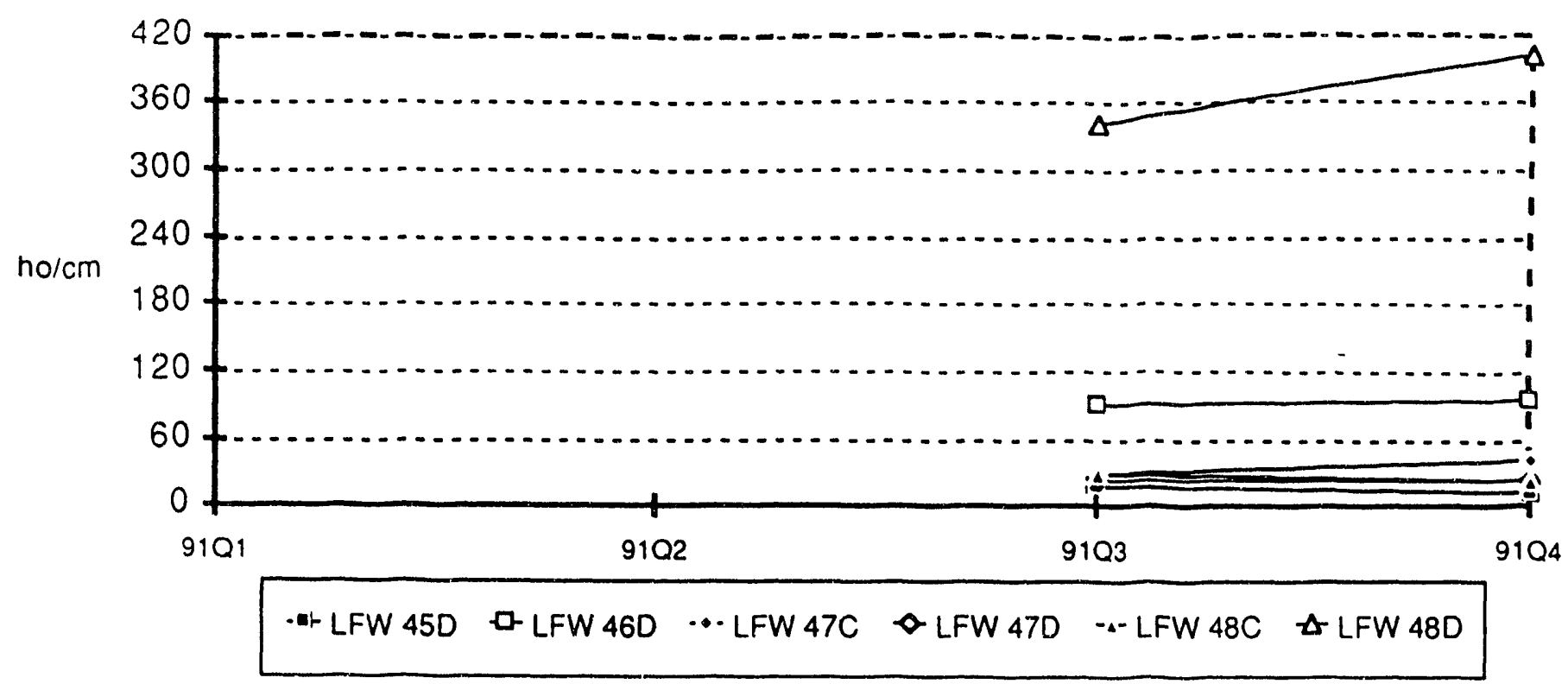

Field Conductivity in the LFW-Series Monitoring Wells

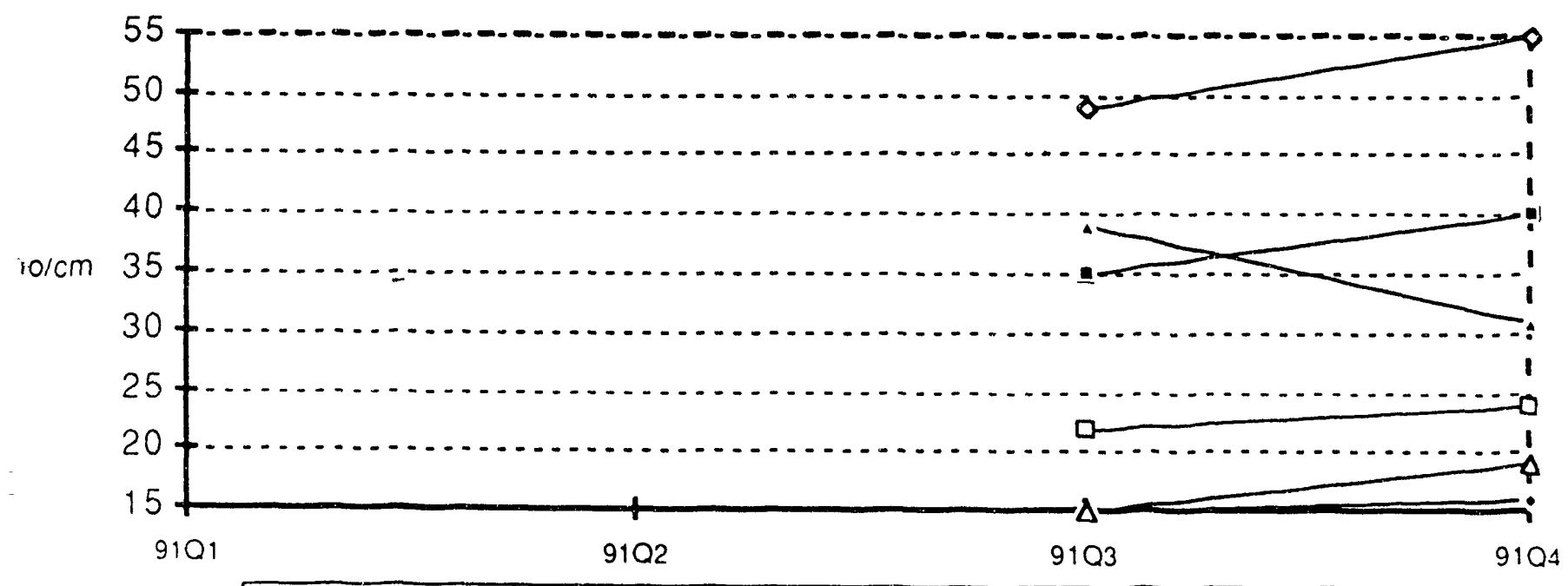

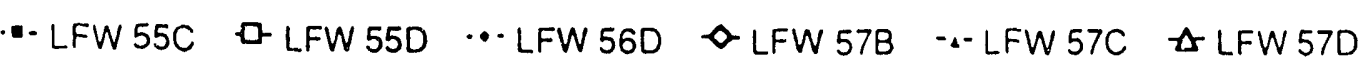




\section{APPENDIX E}

\section{TIME-SERIES OF WATER LEVELS}




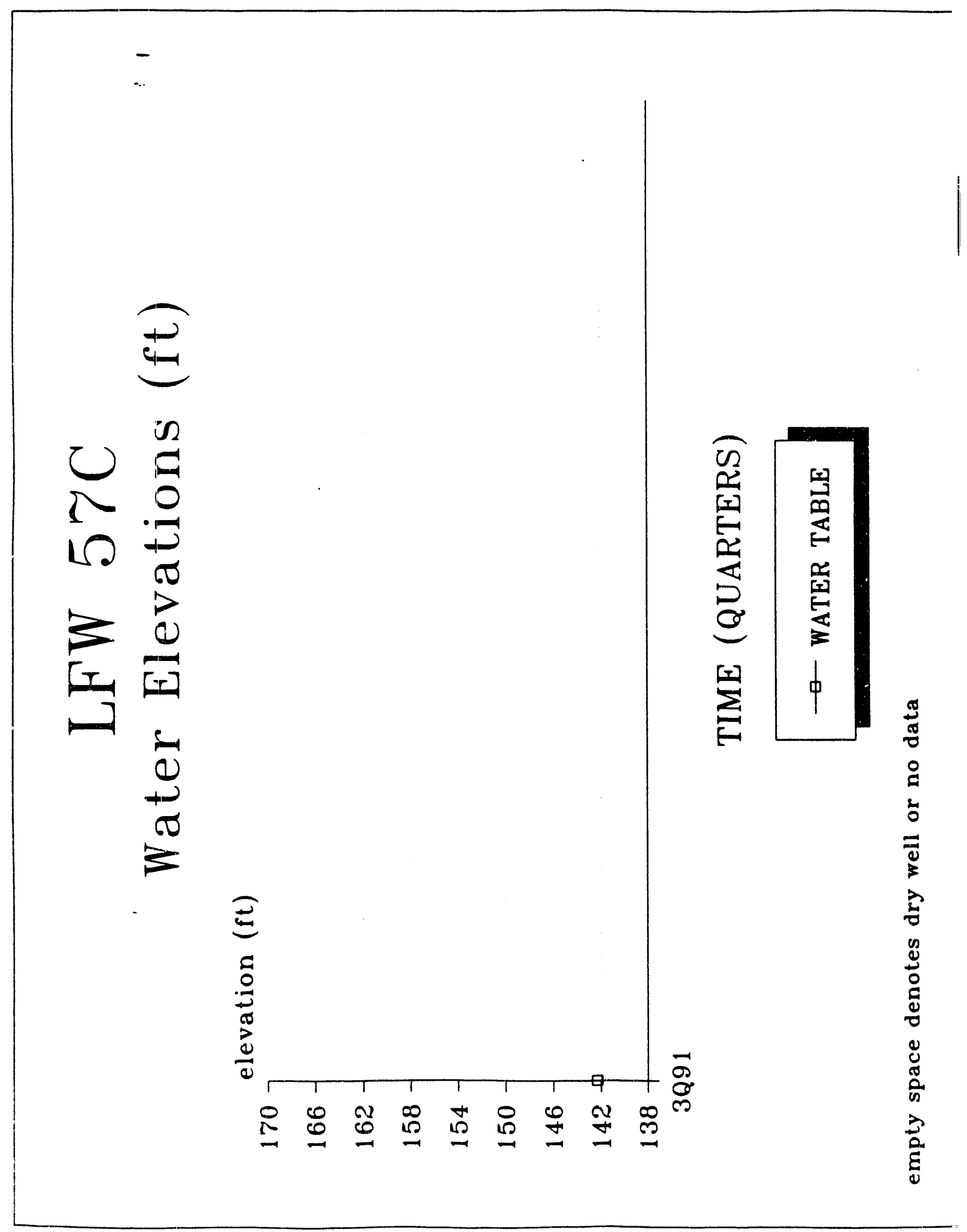




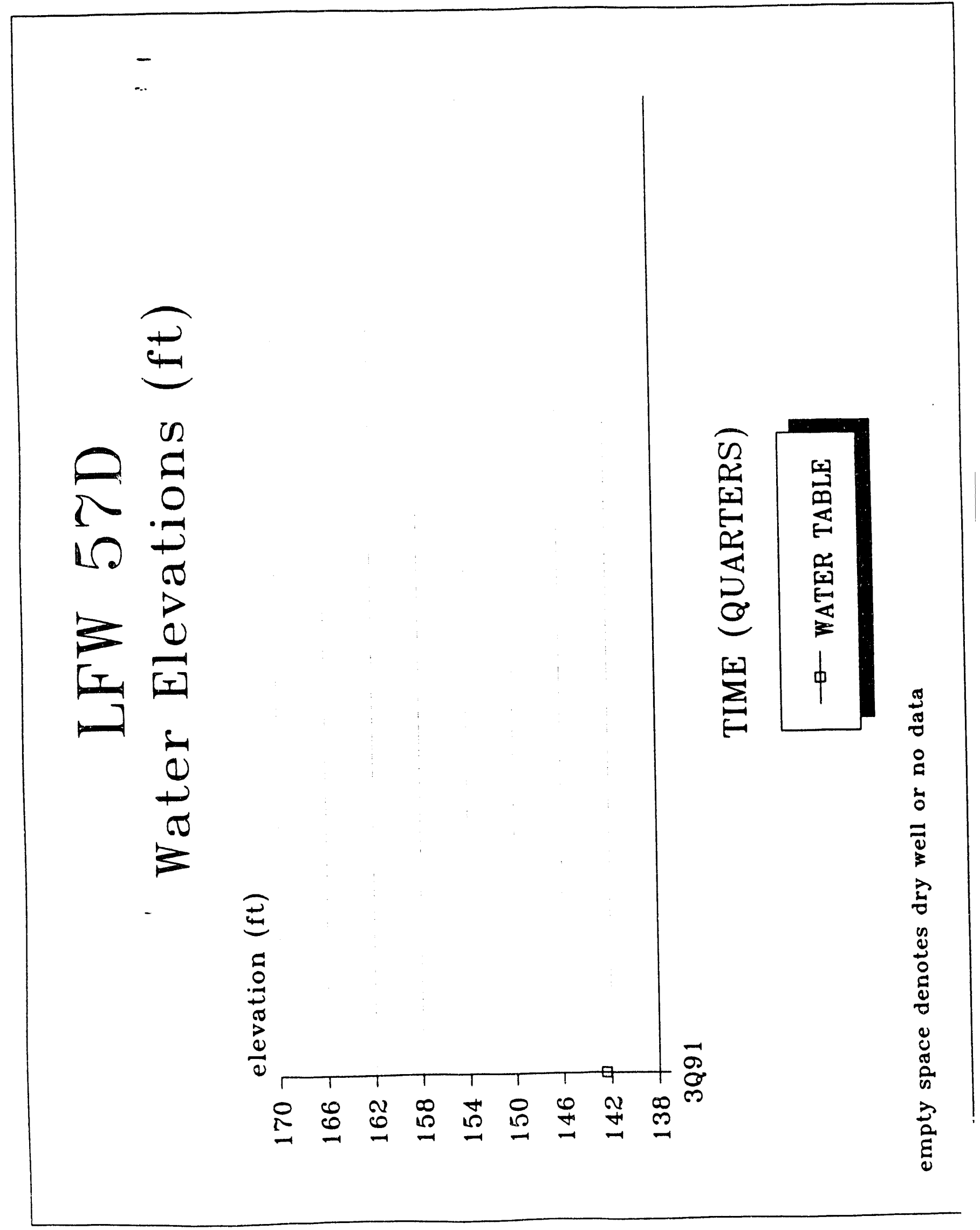




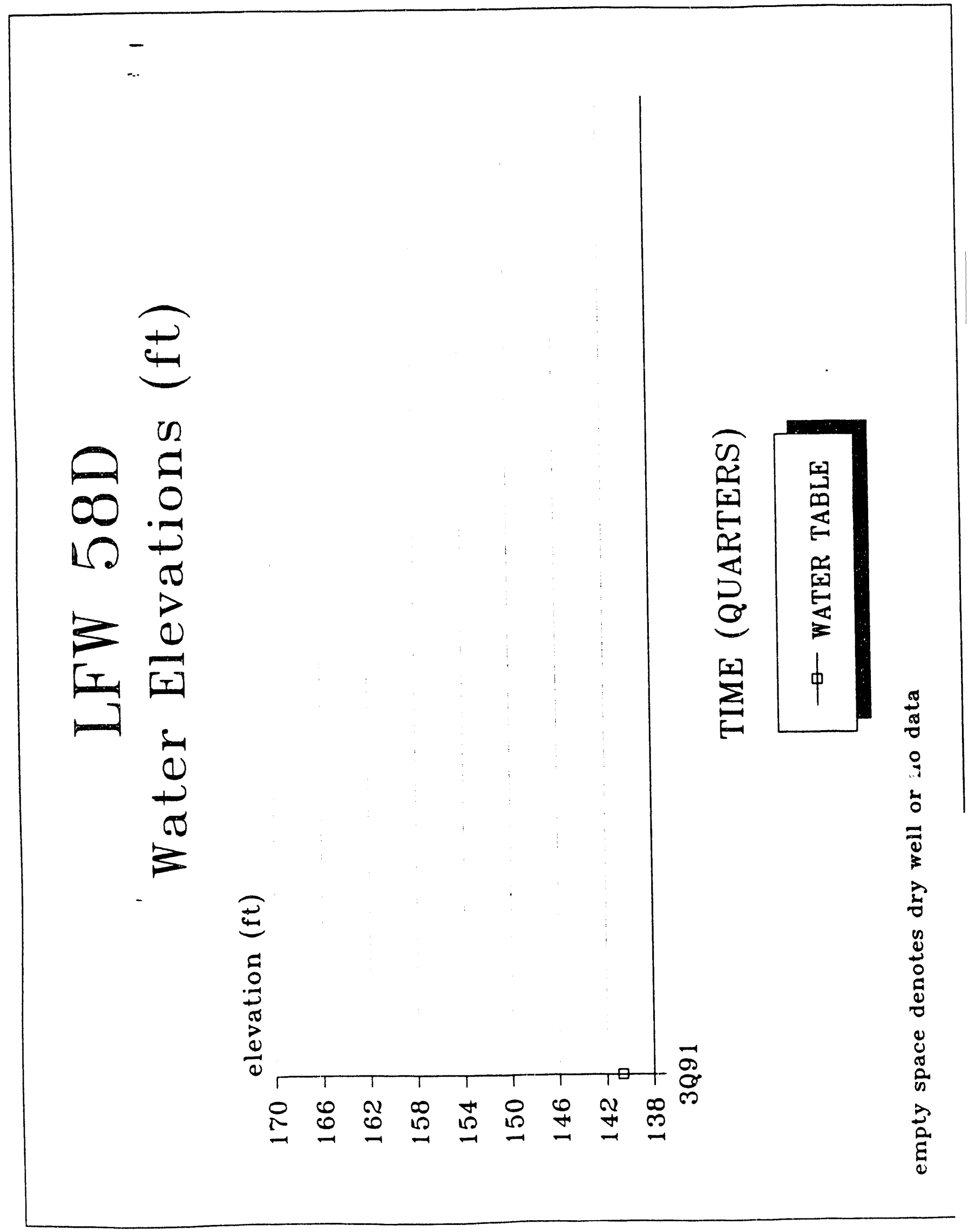




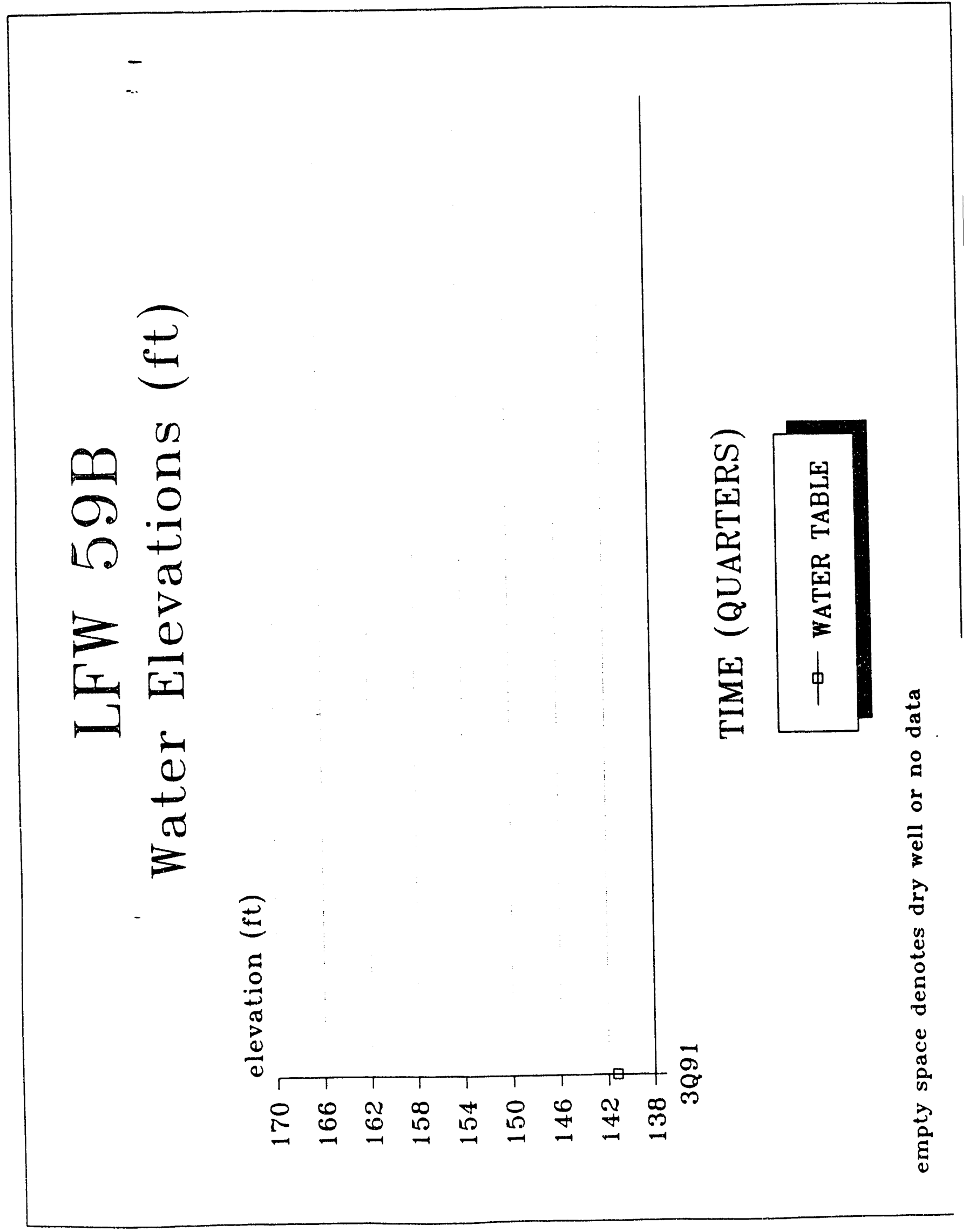




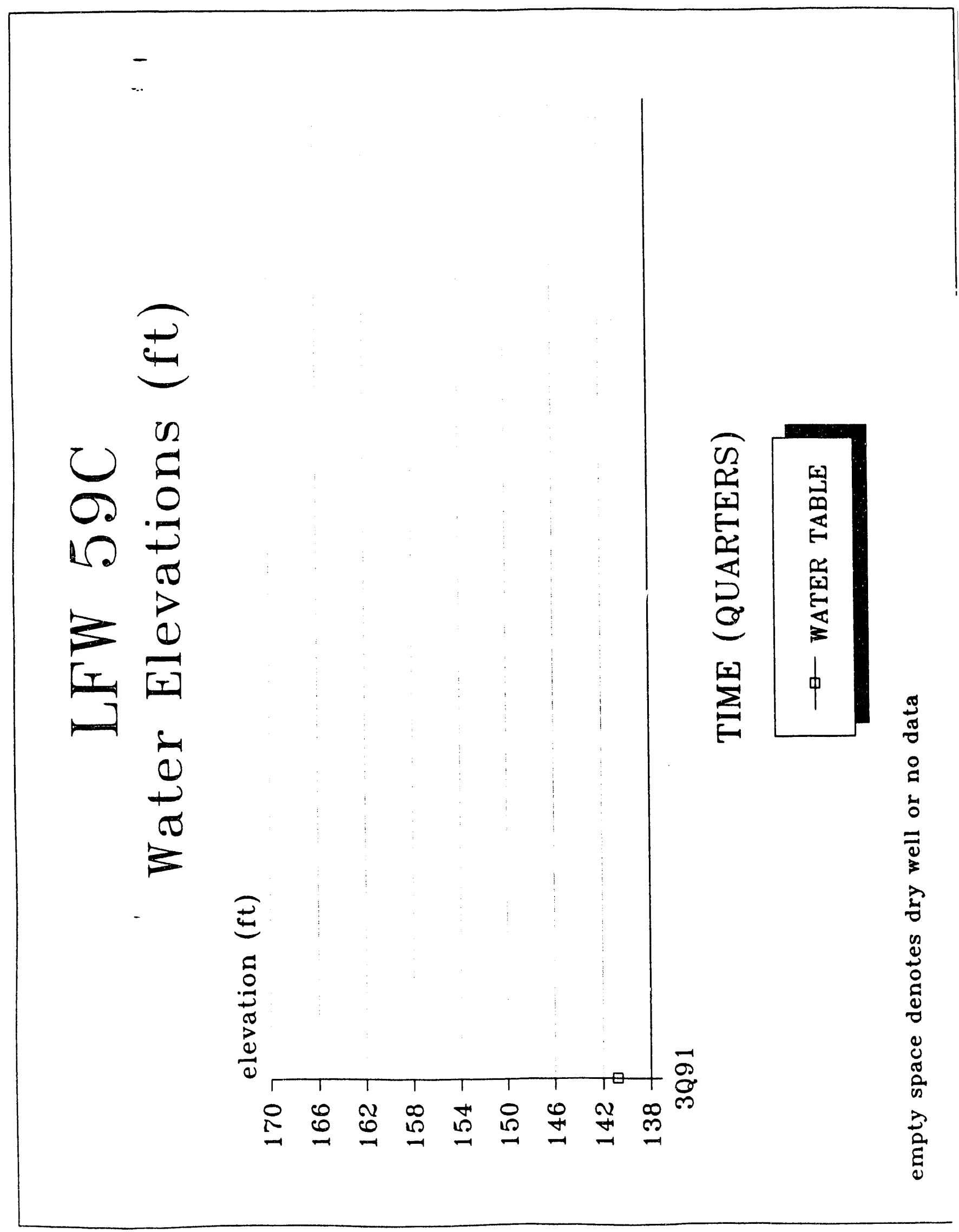




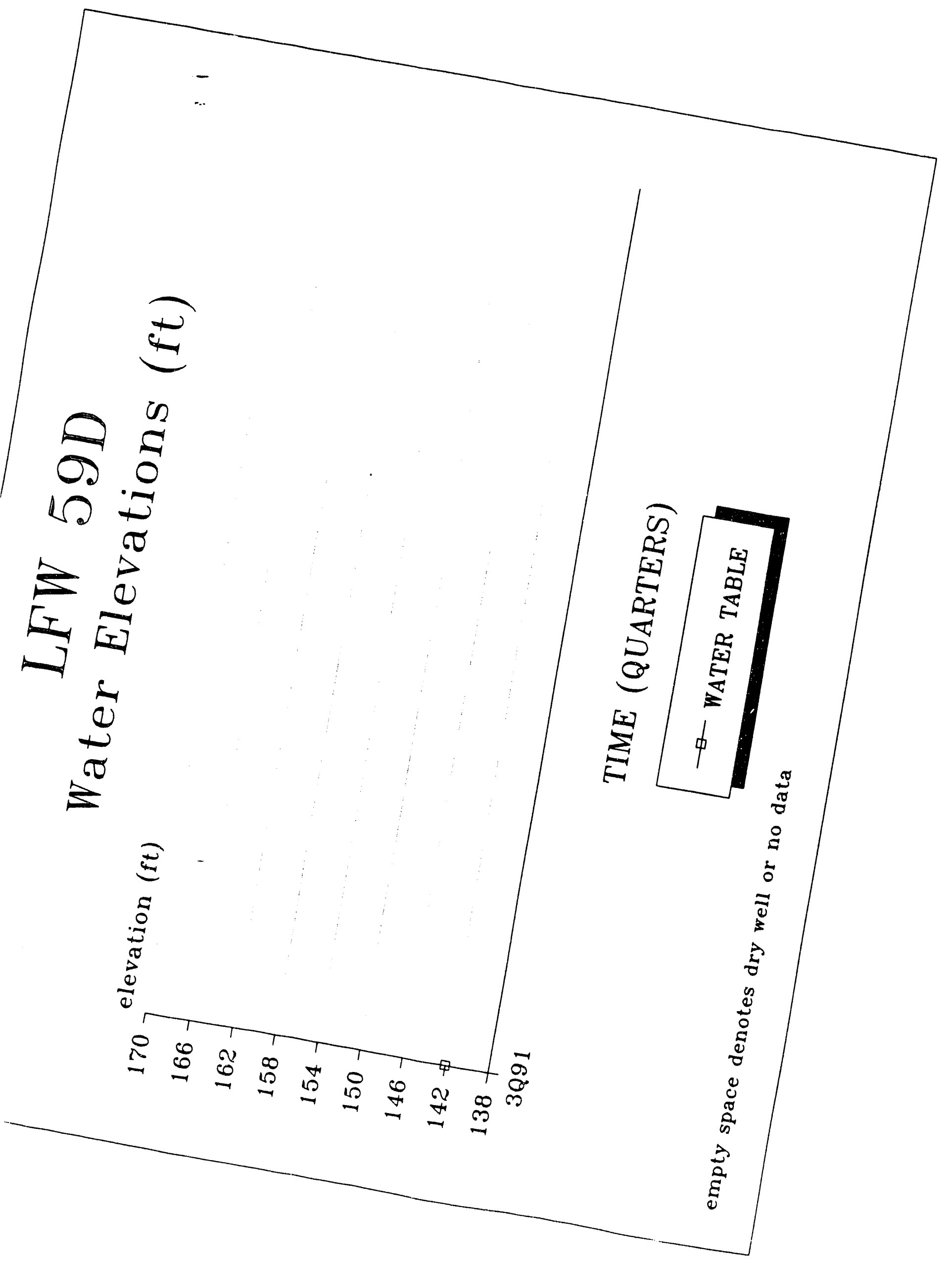




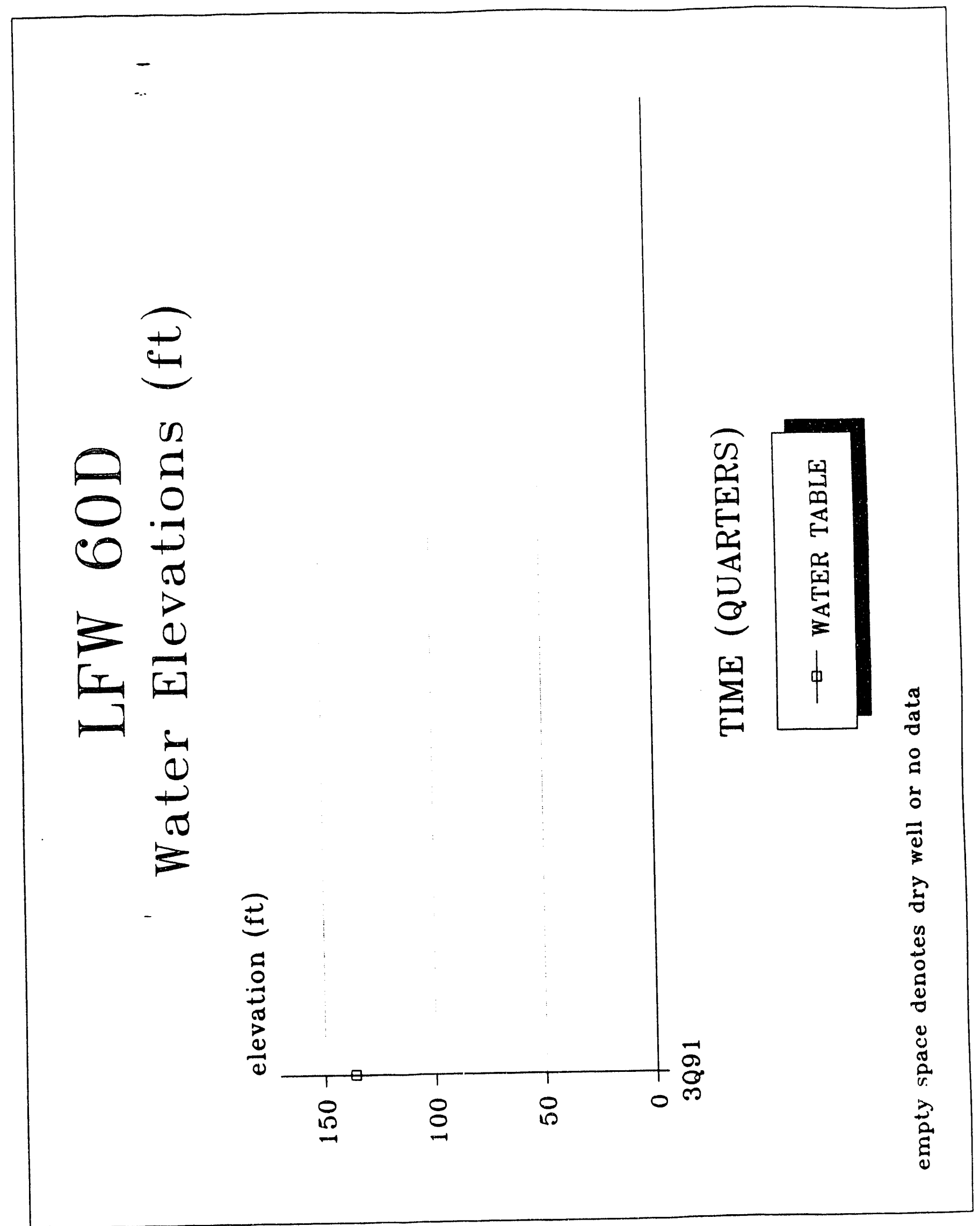




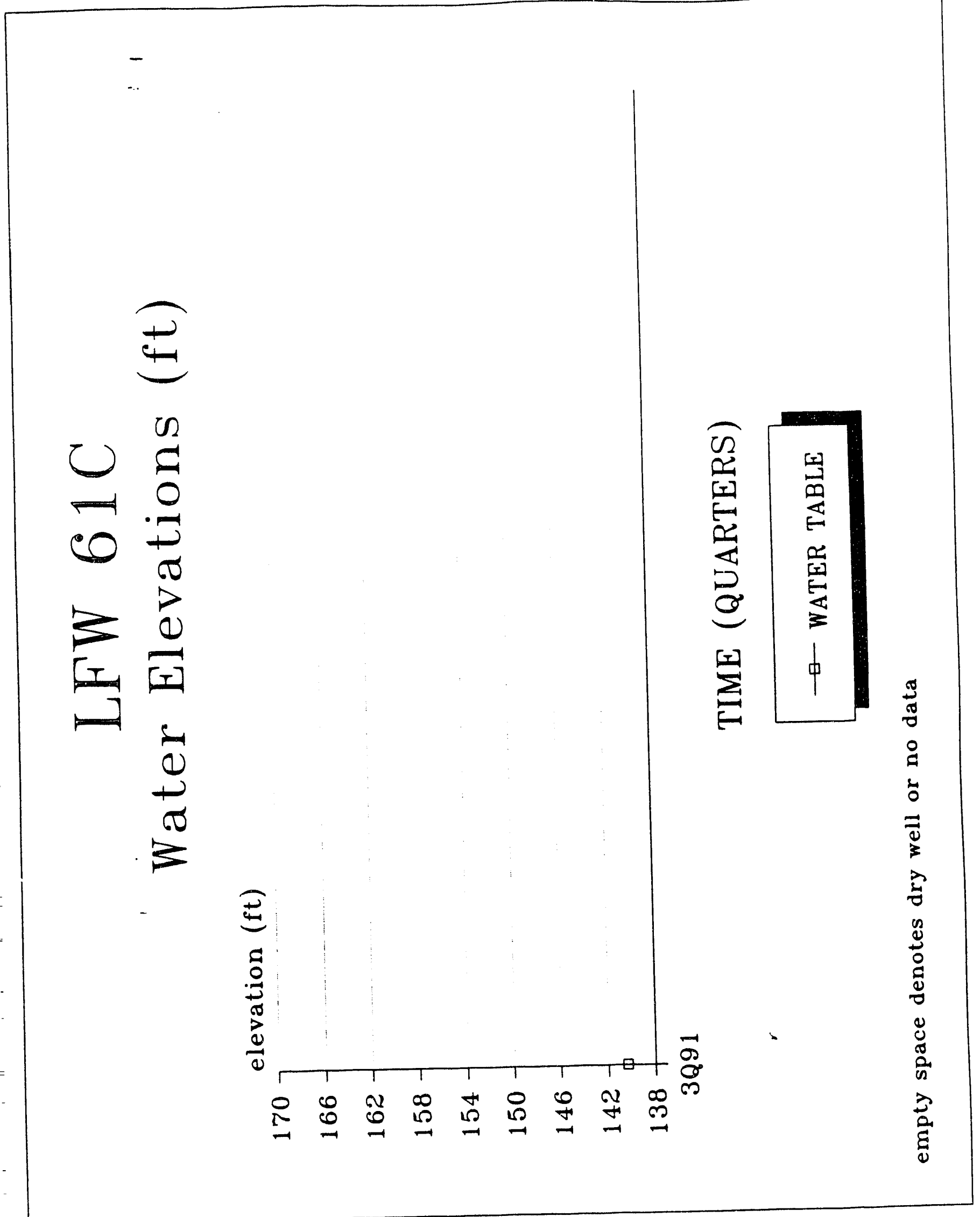




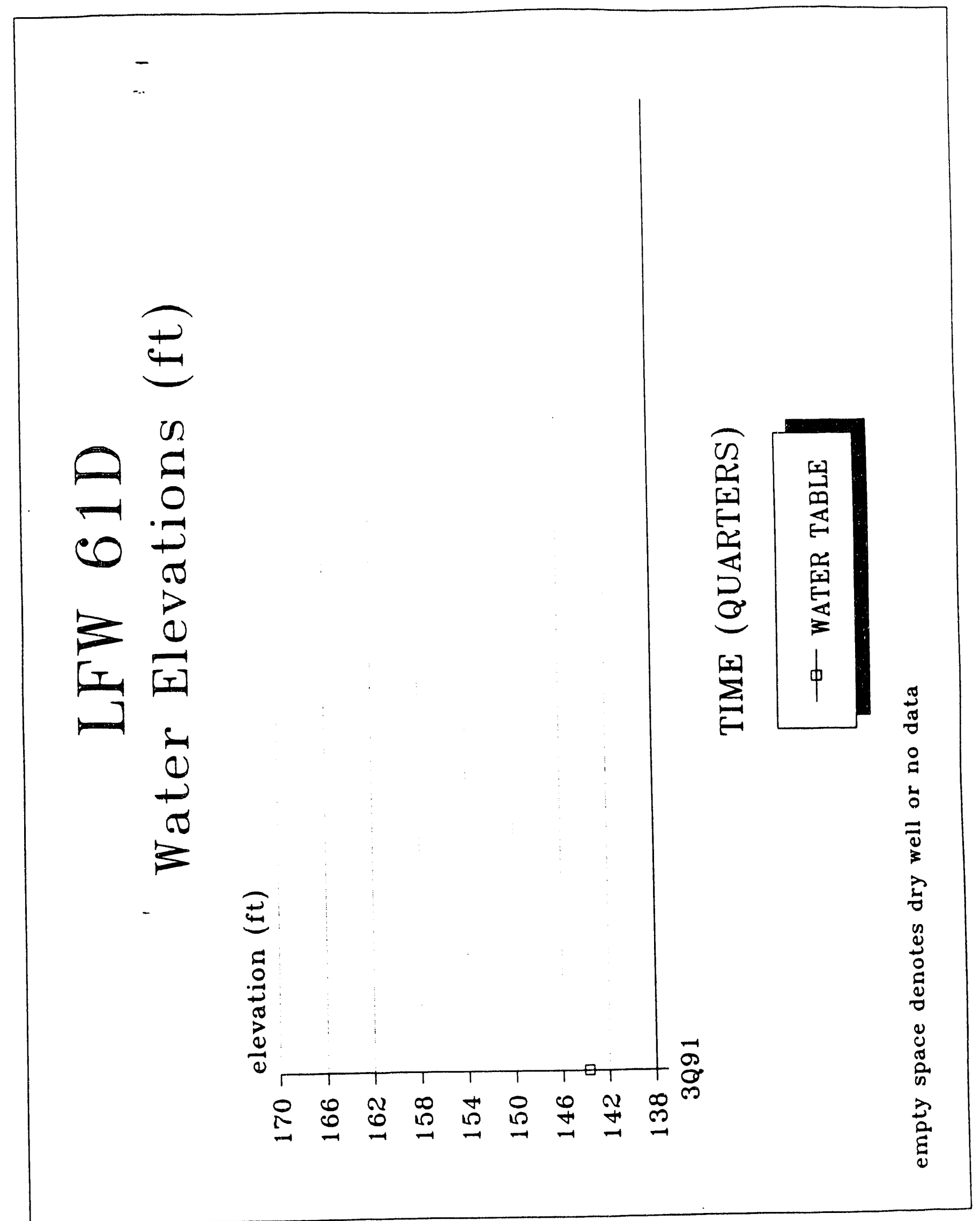




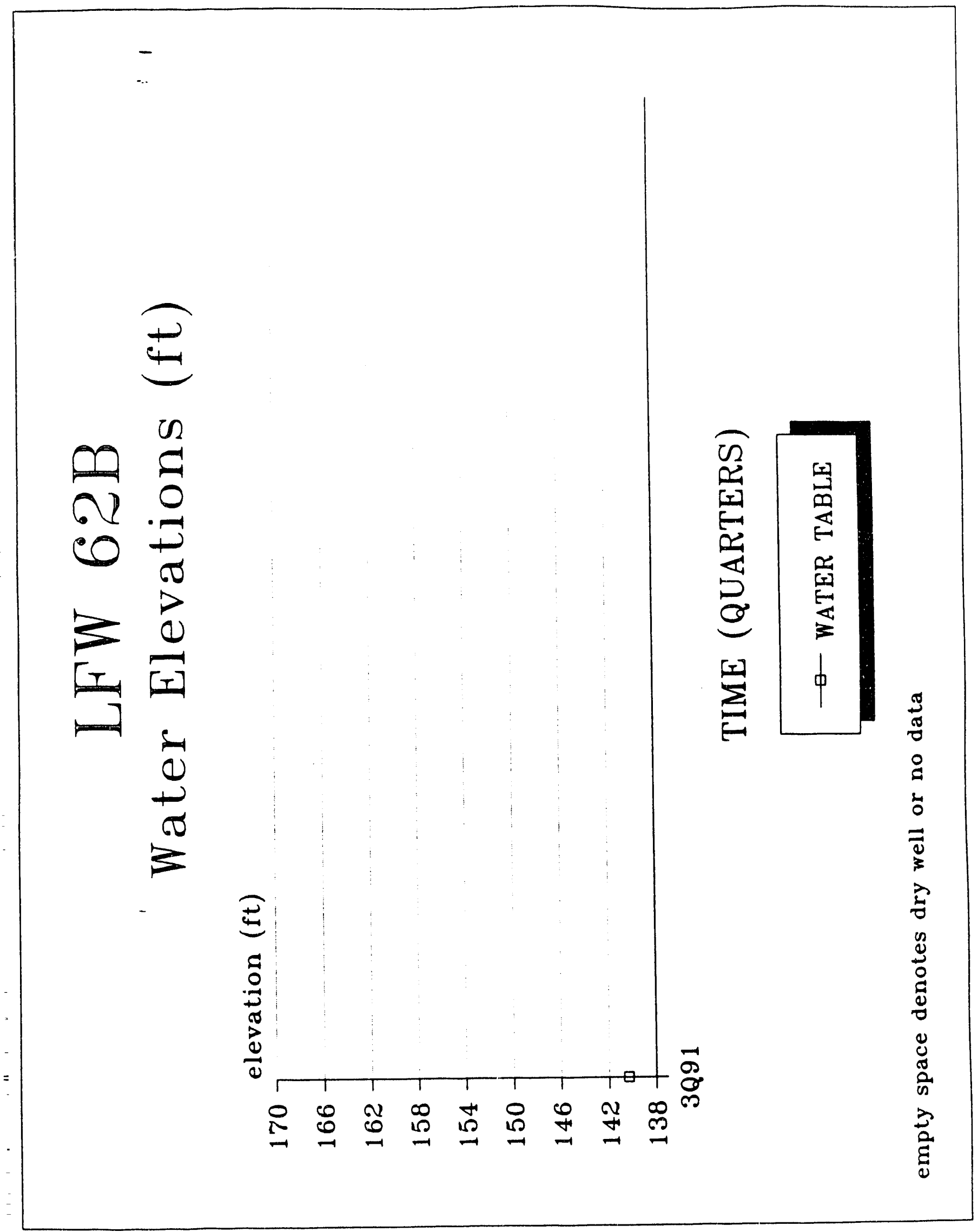




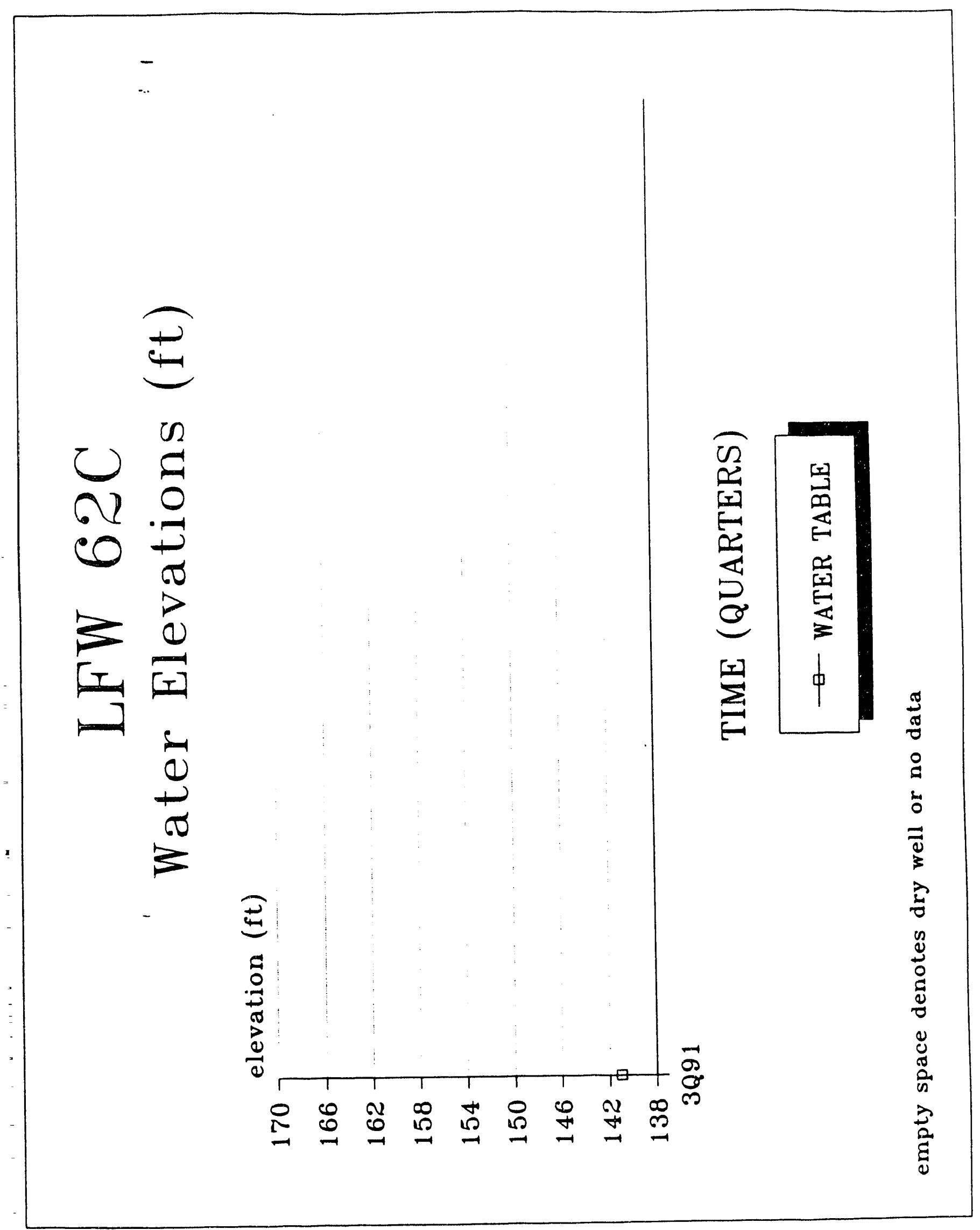




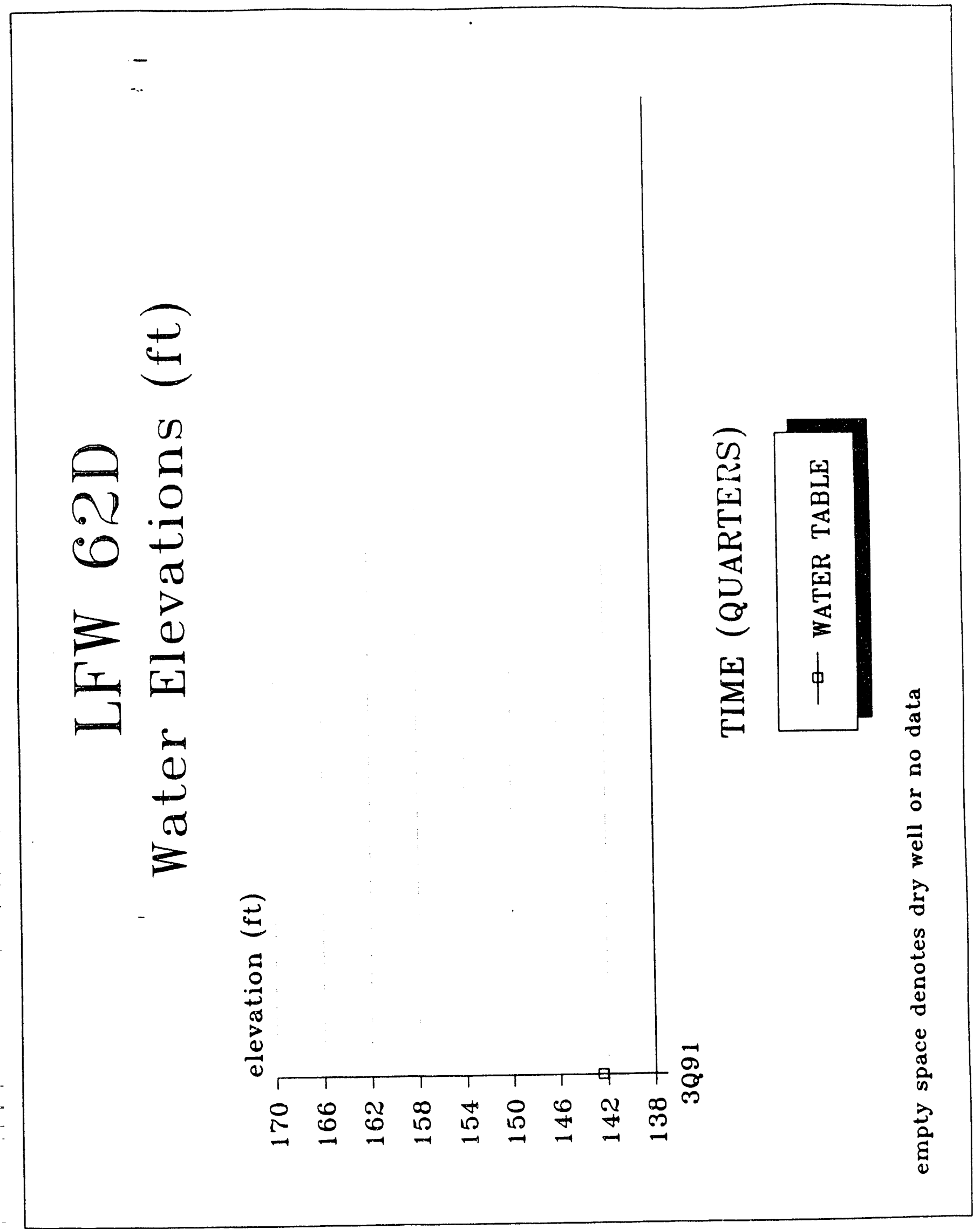




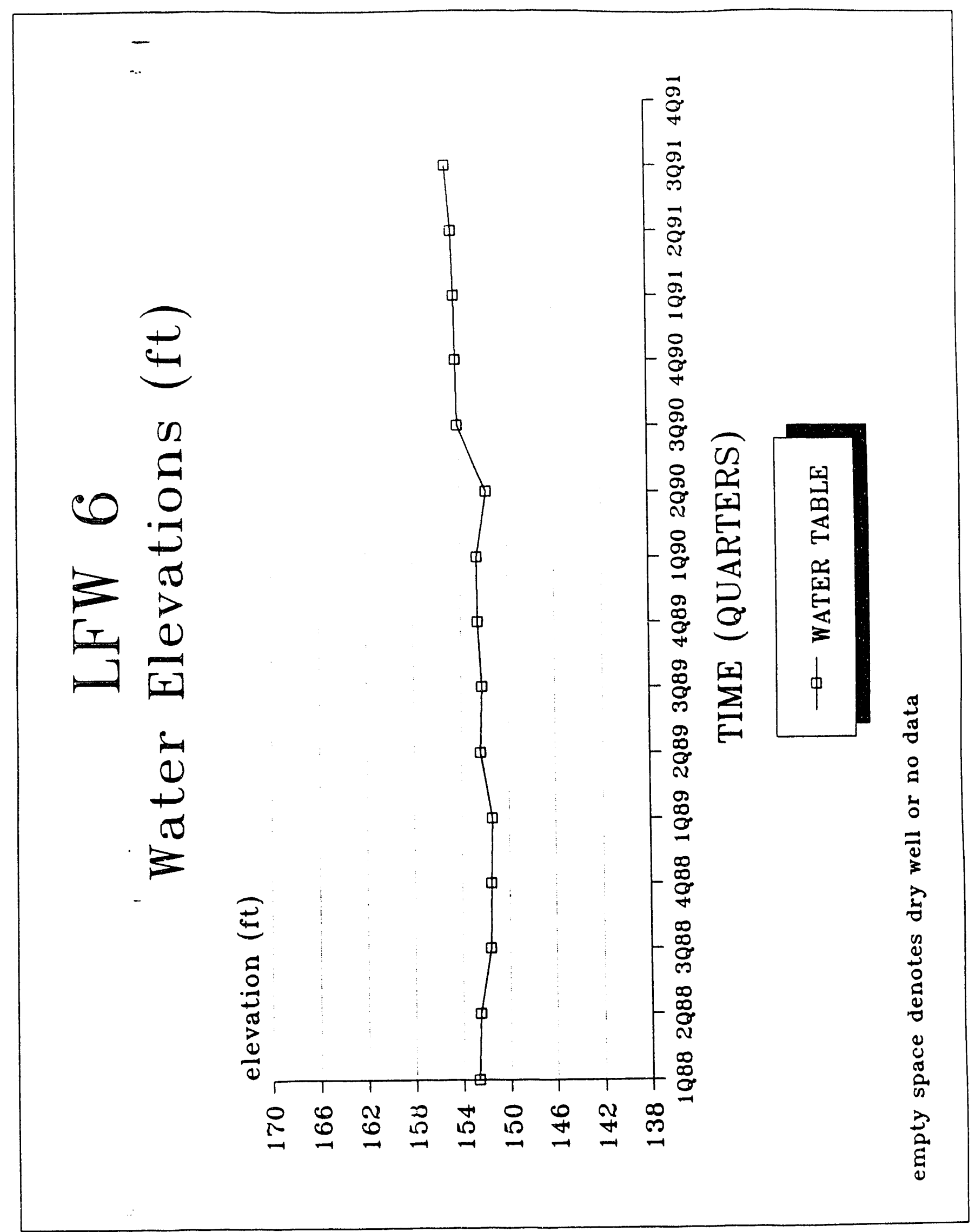




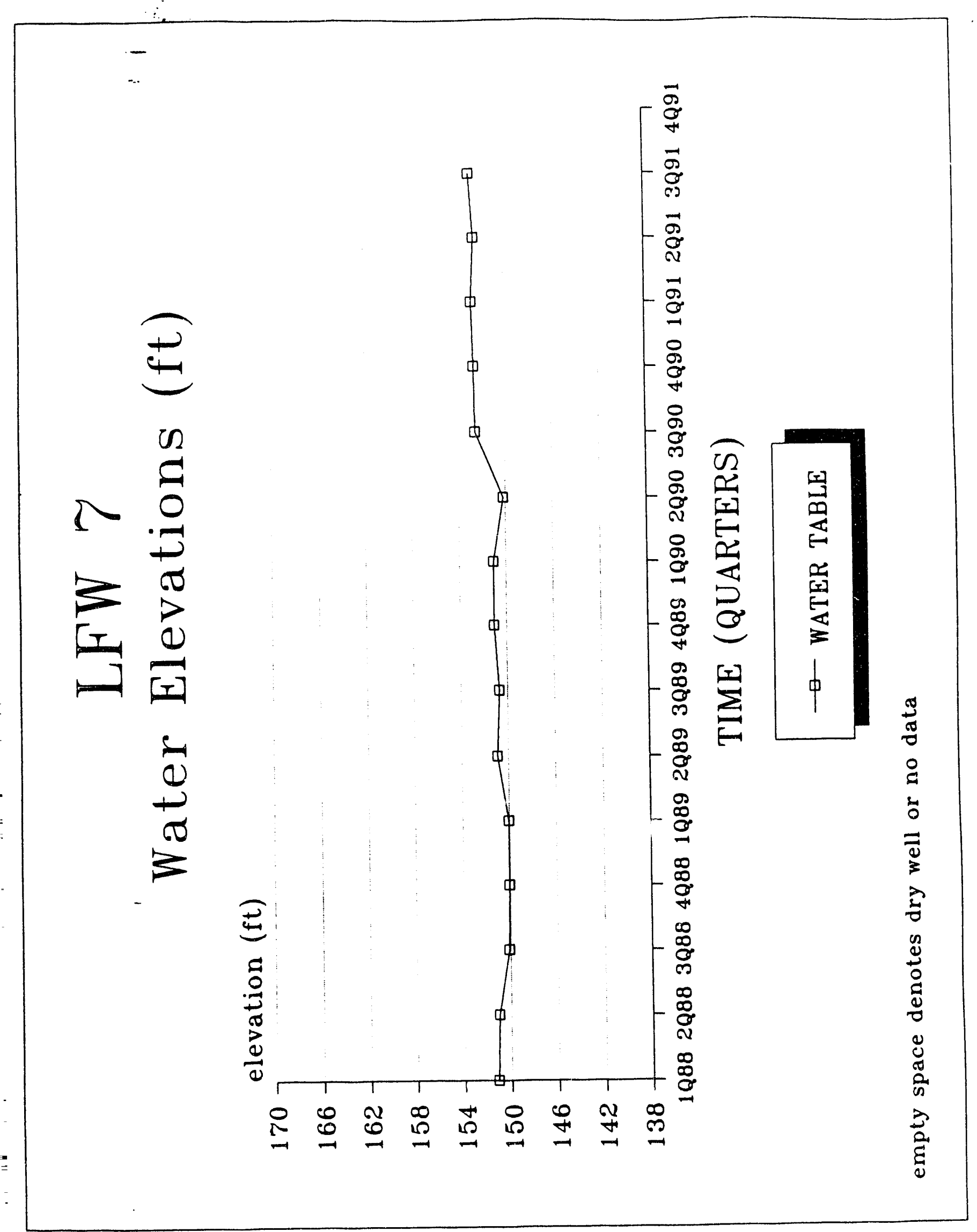




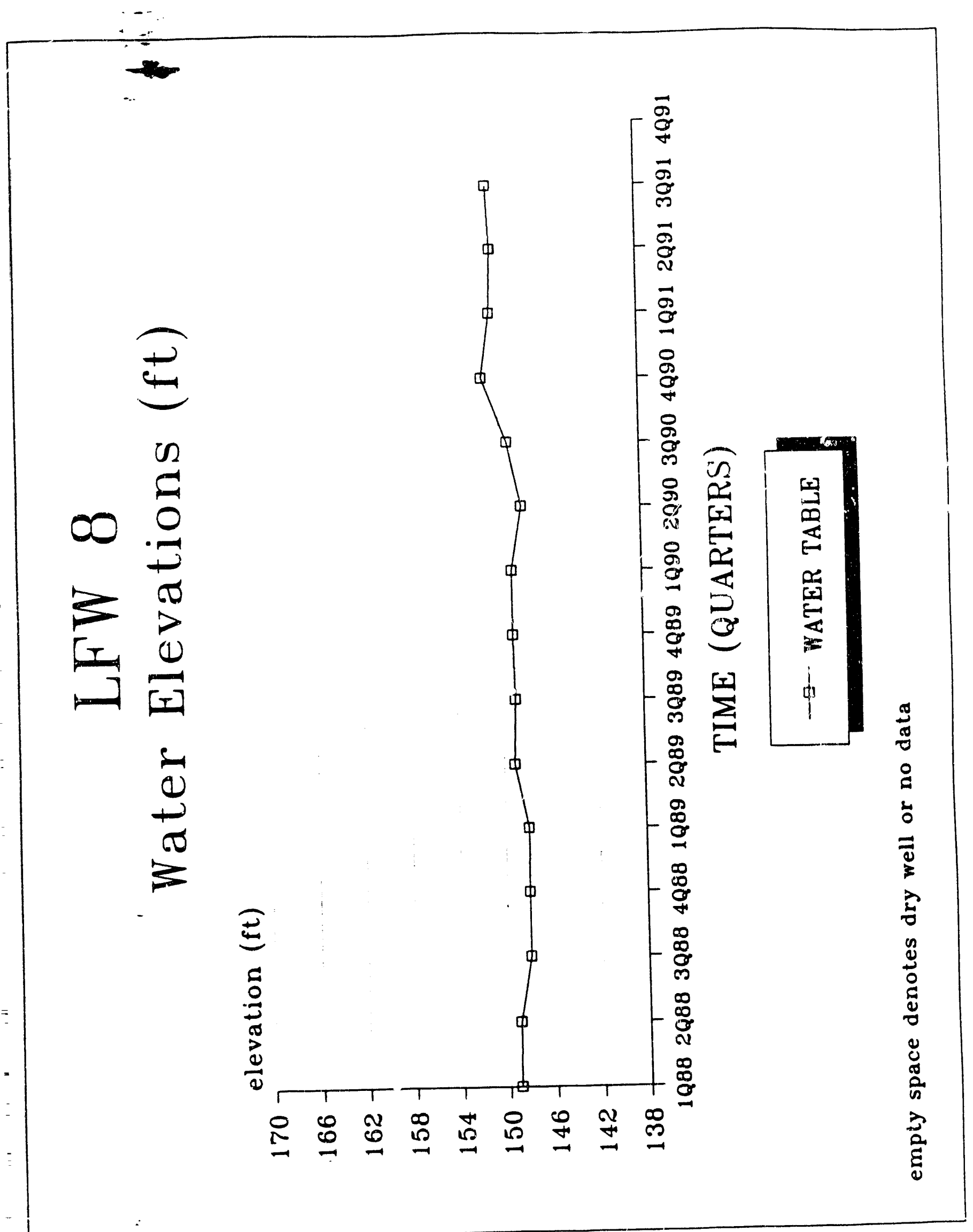




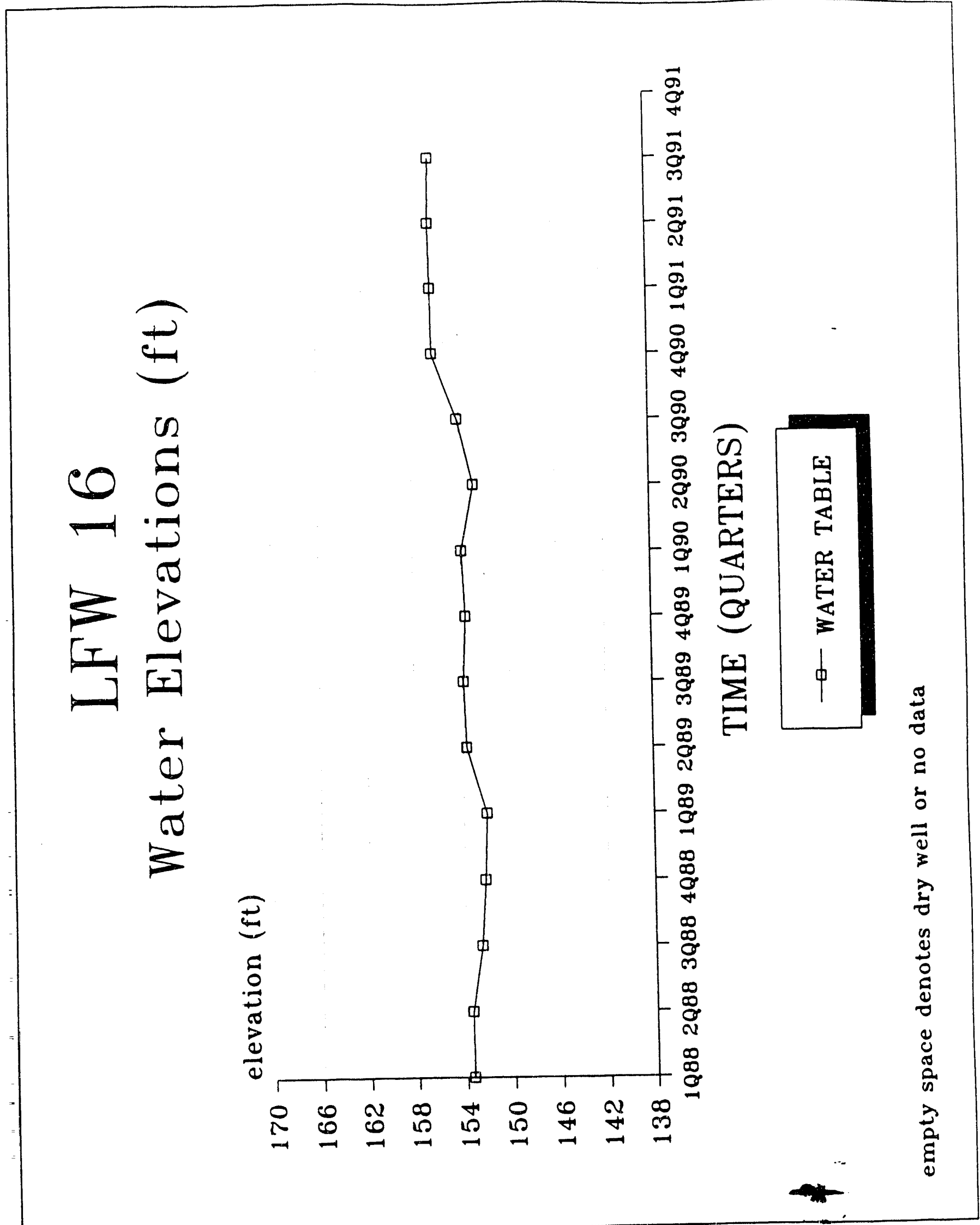




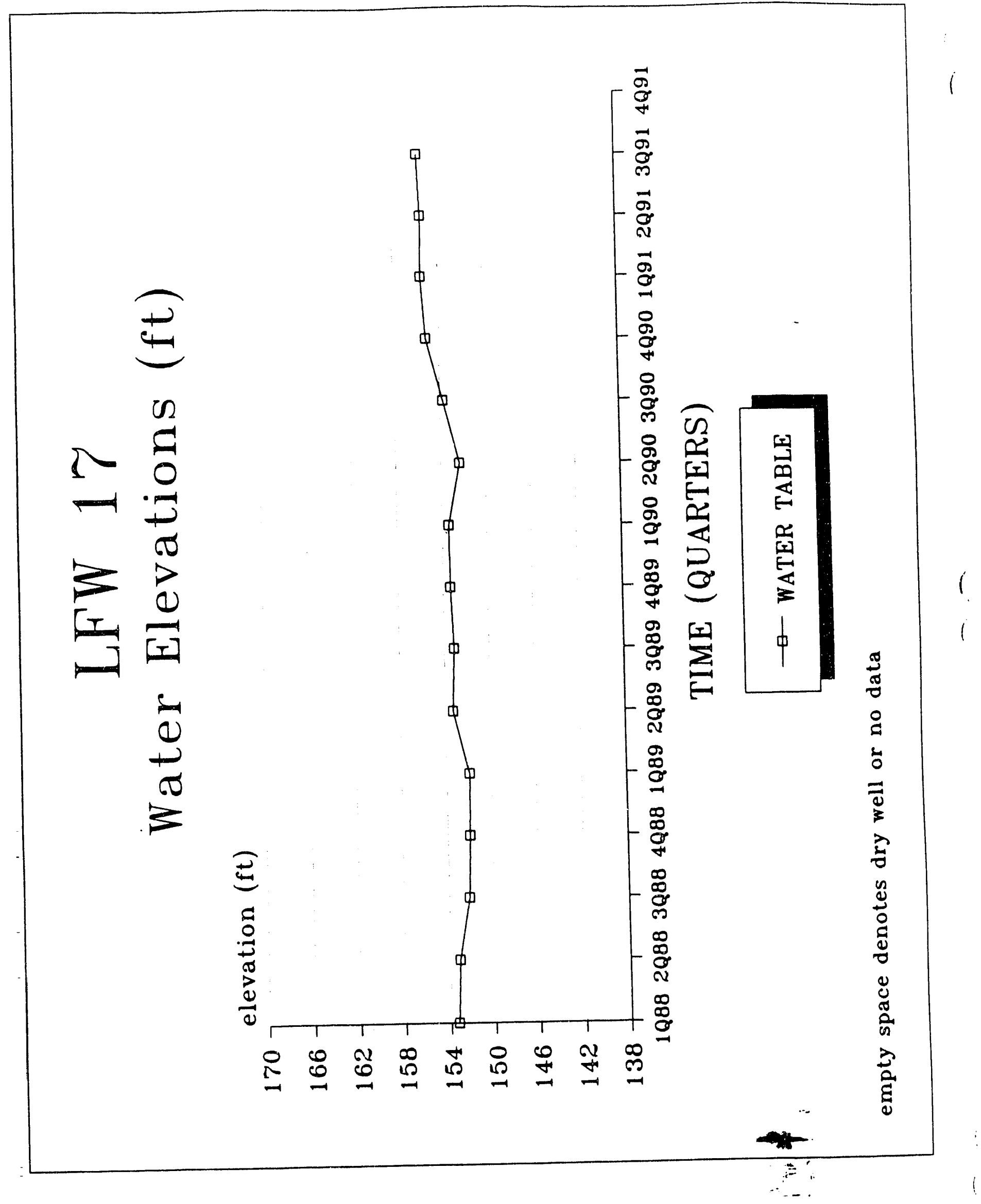




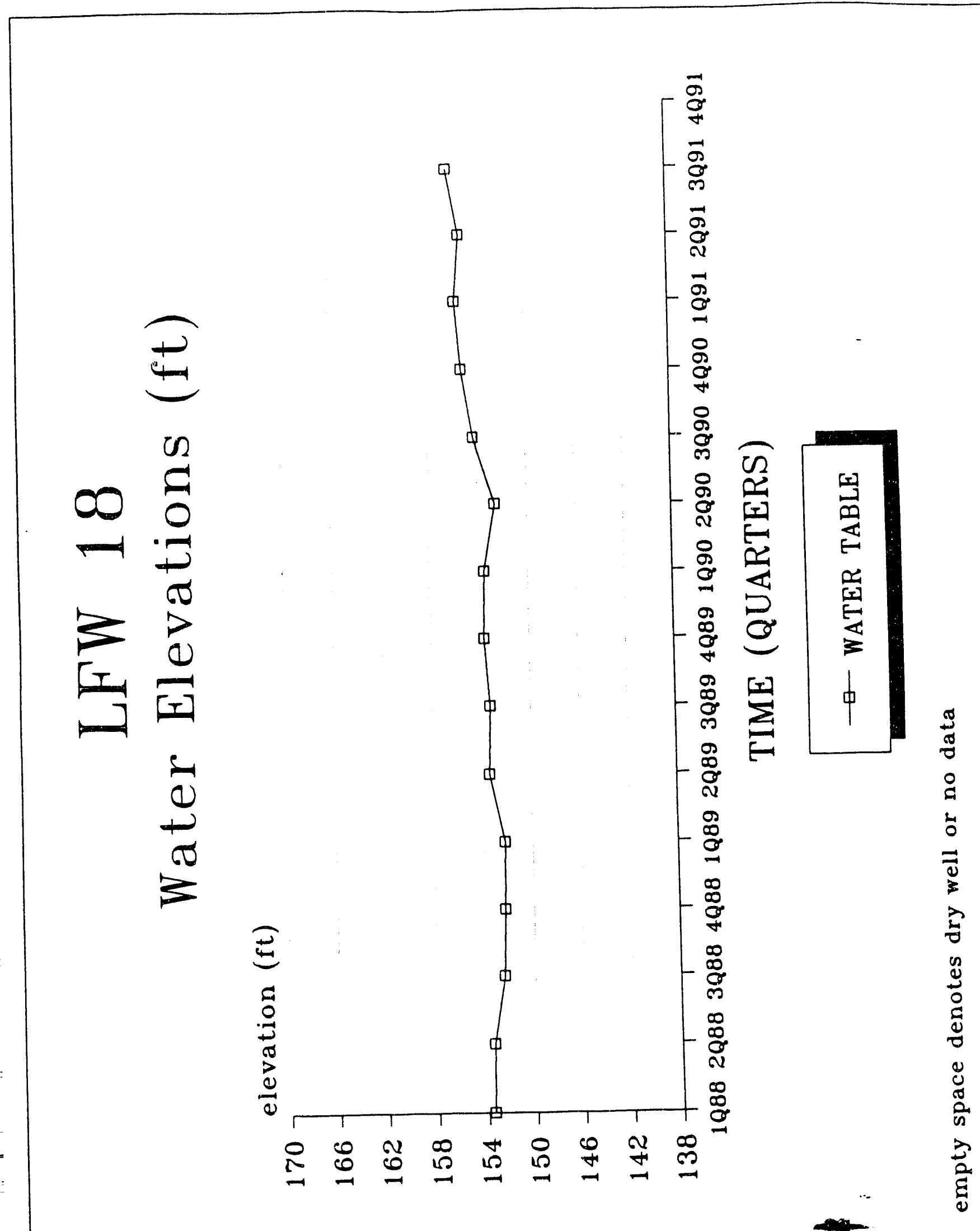




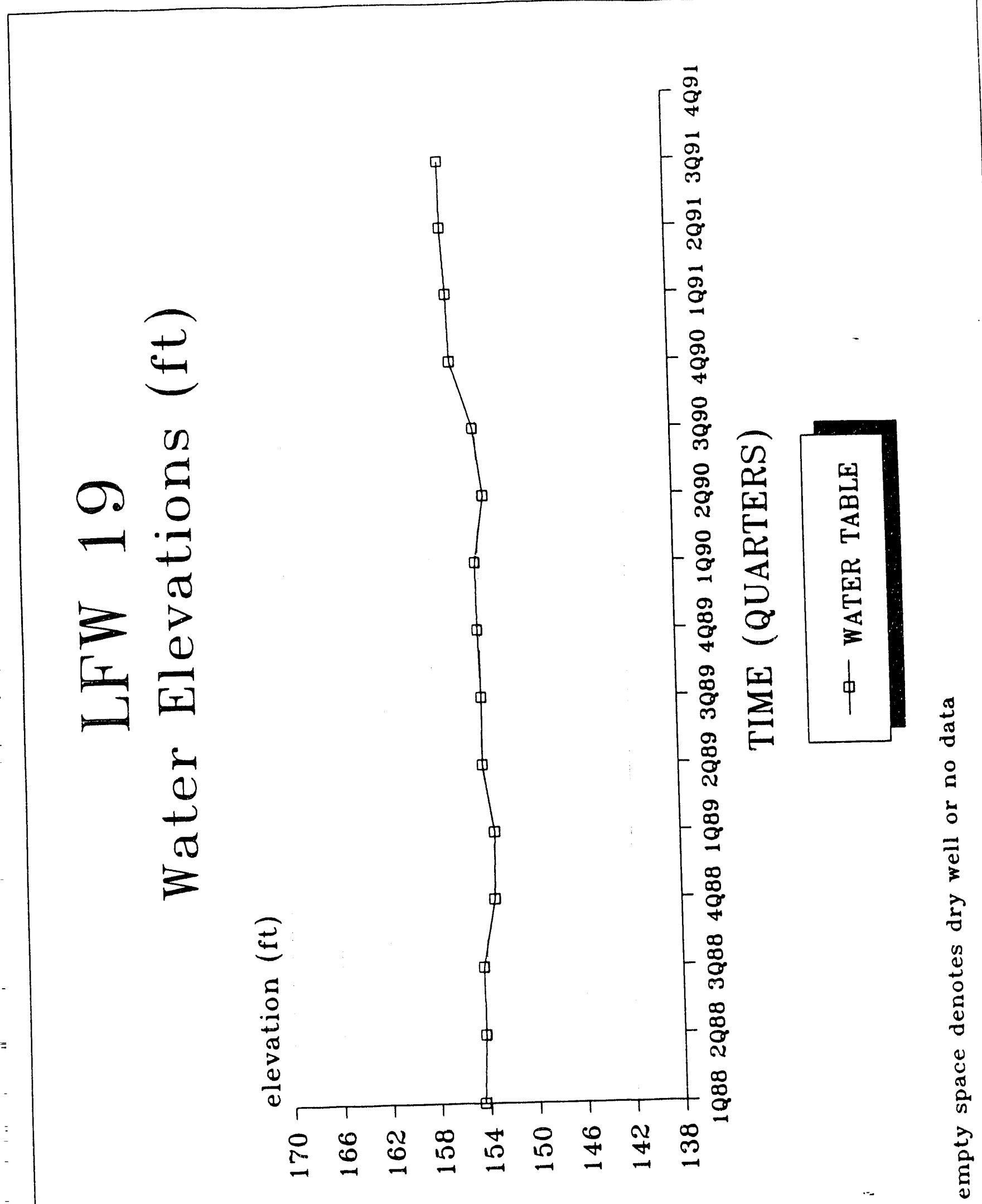




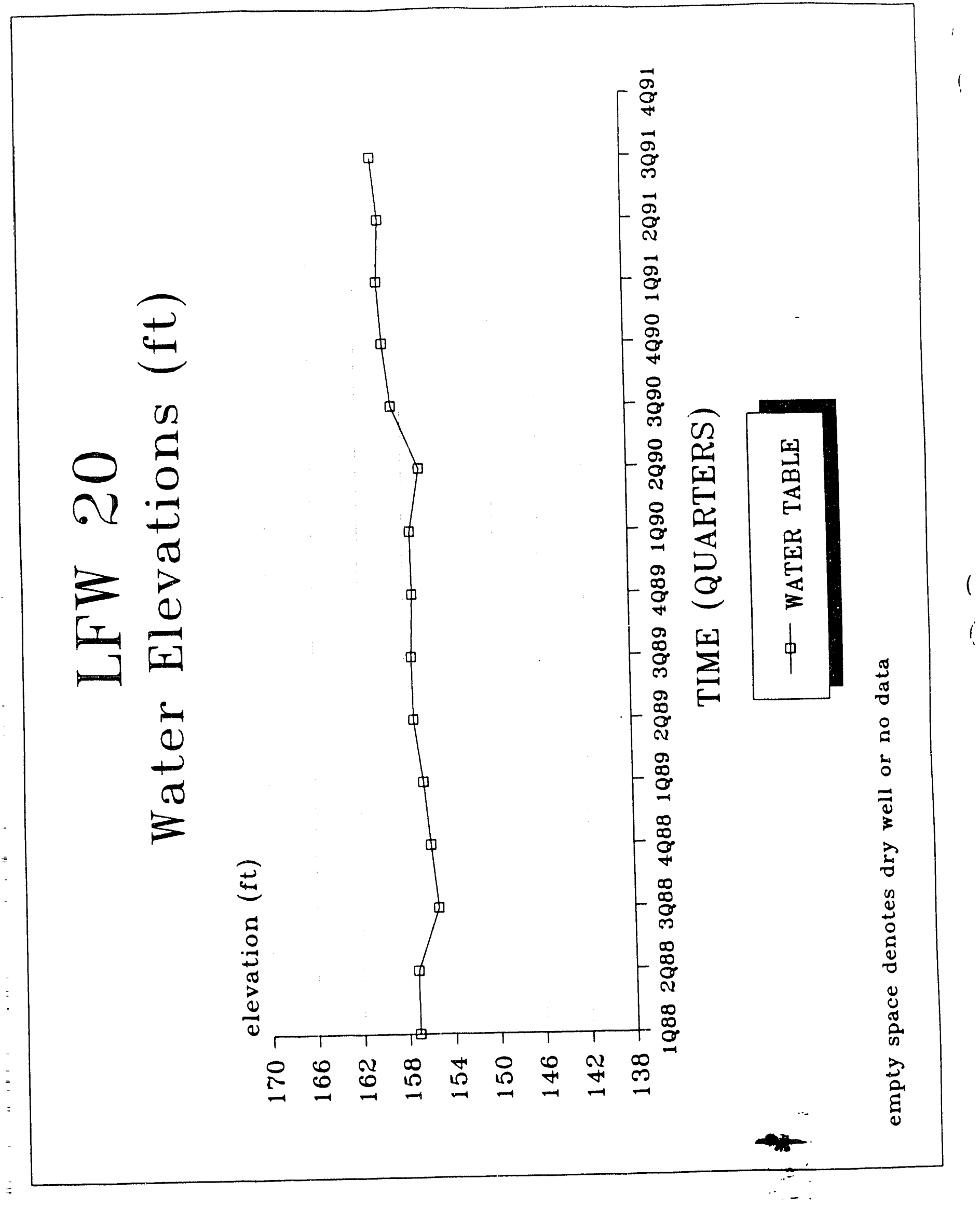




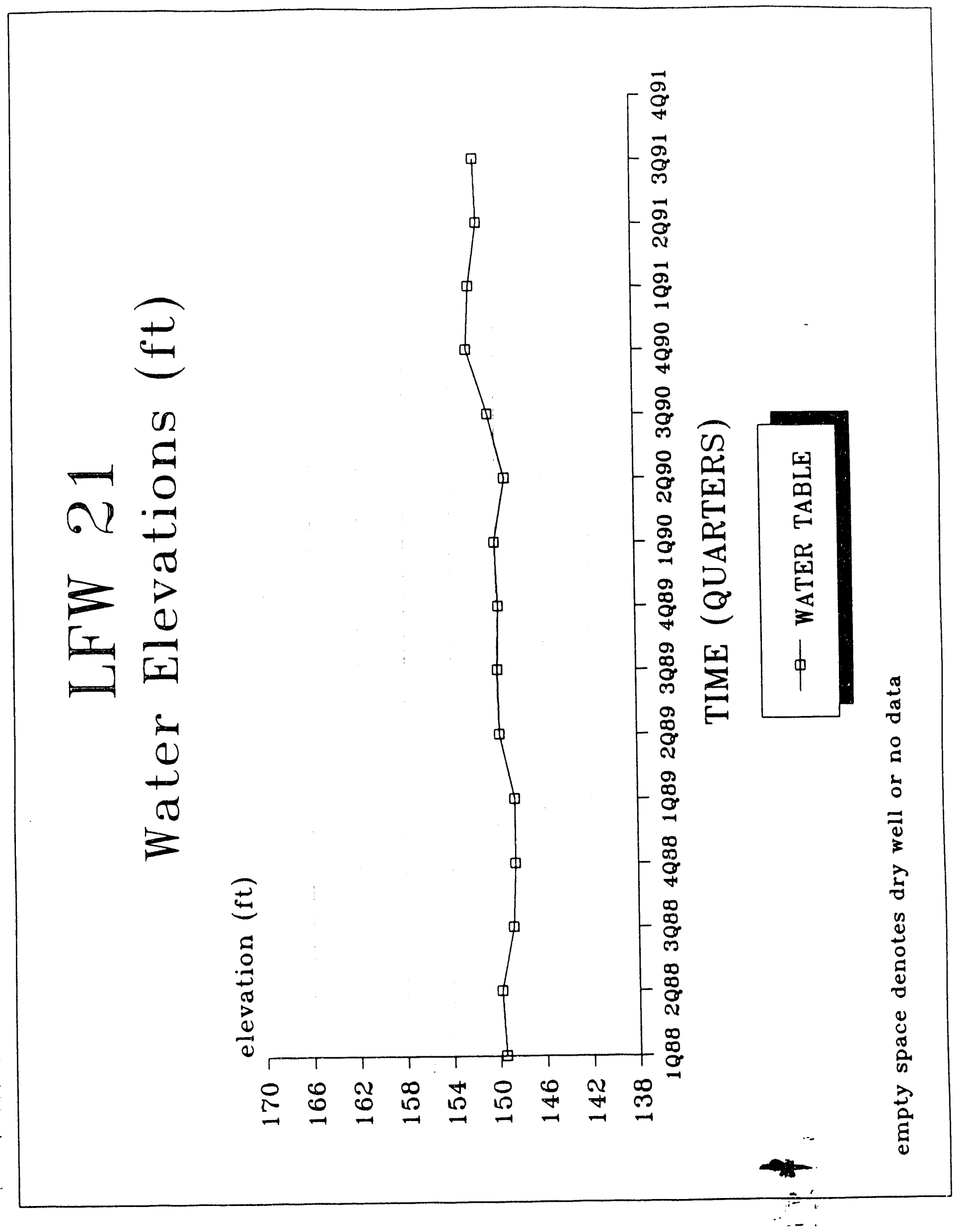




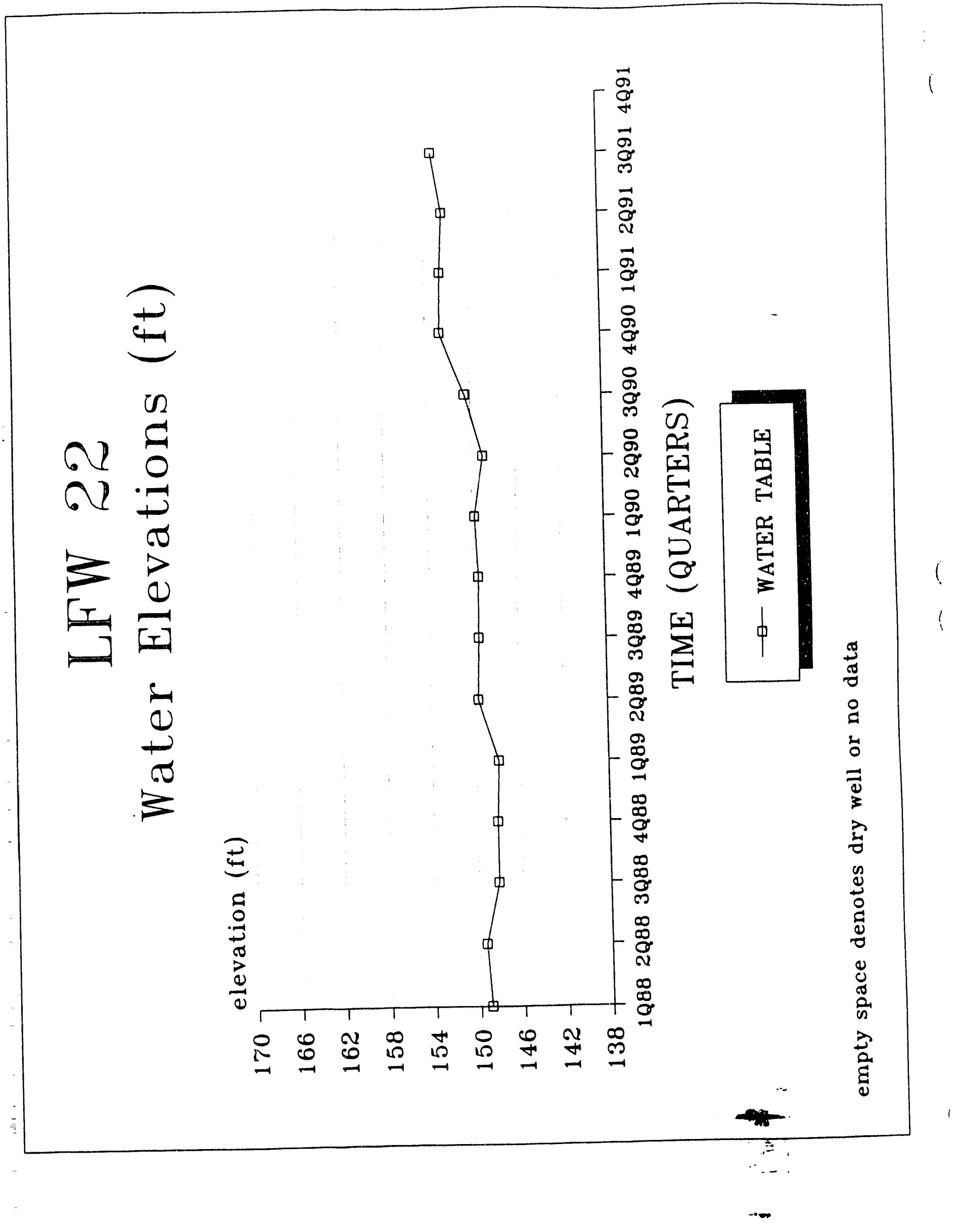




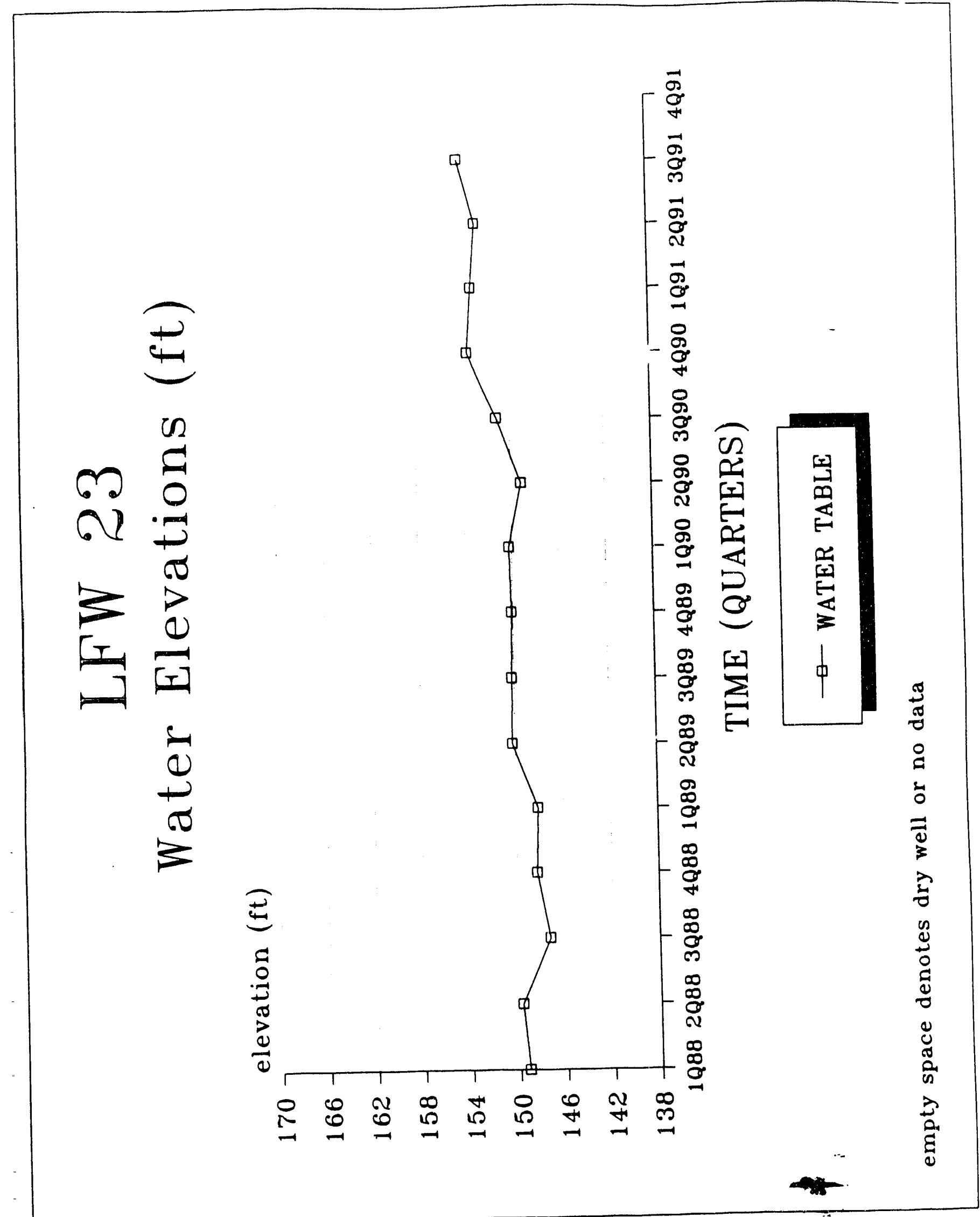




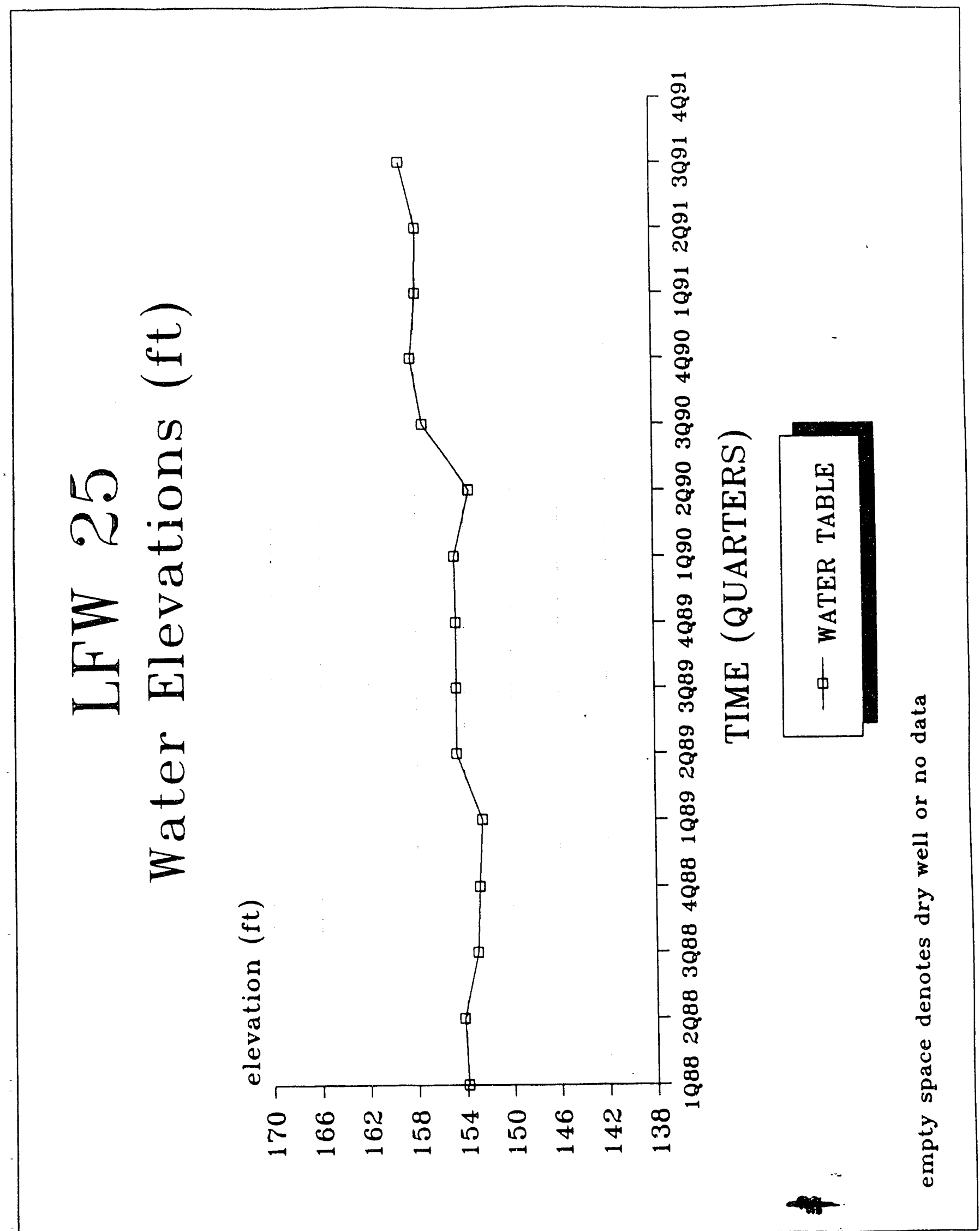




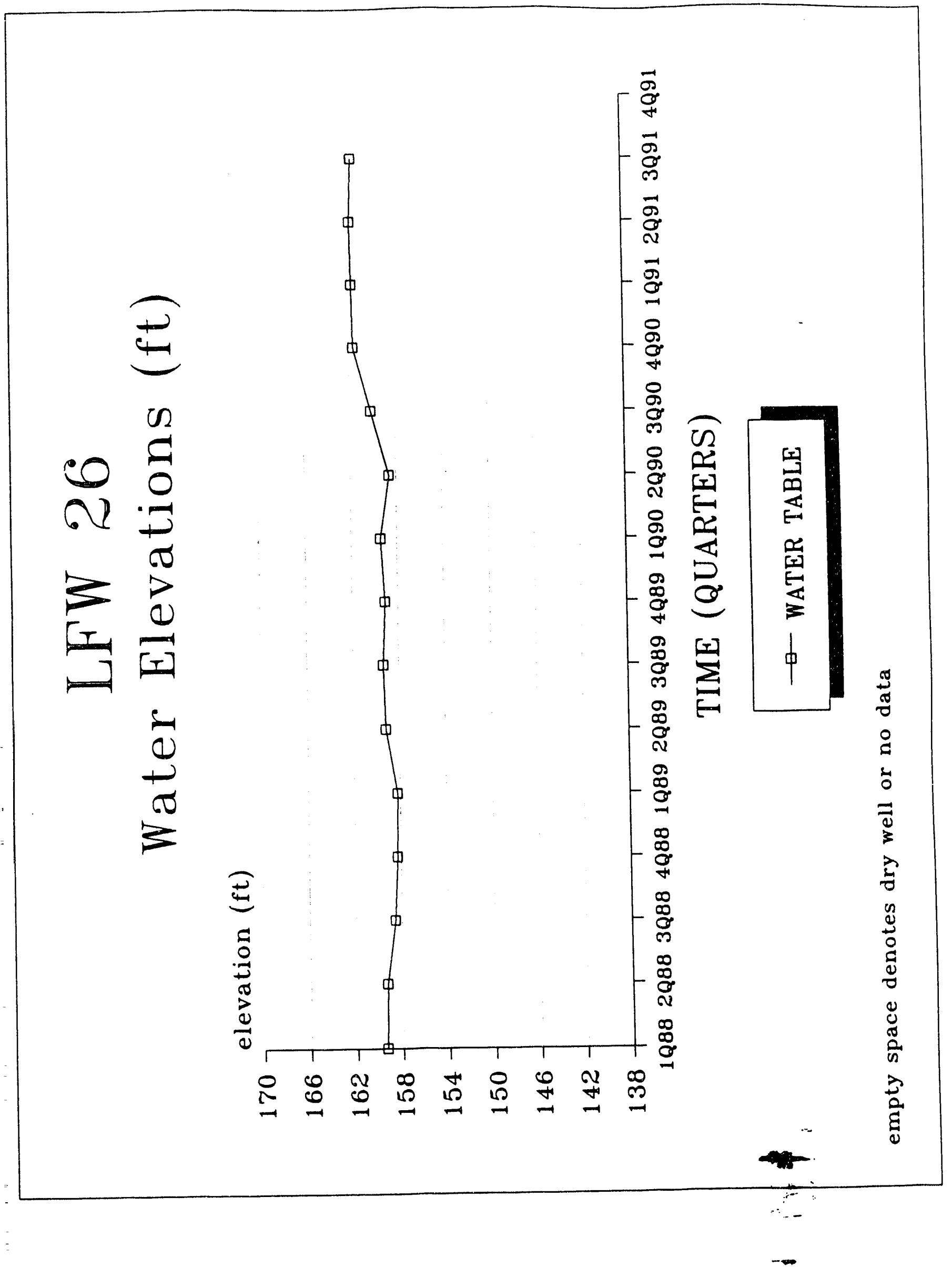




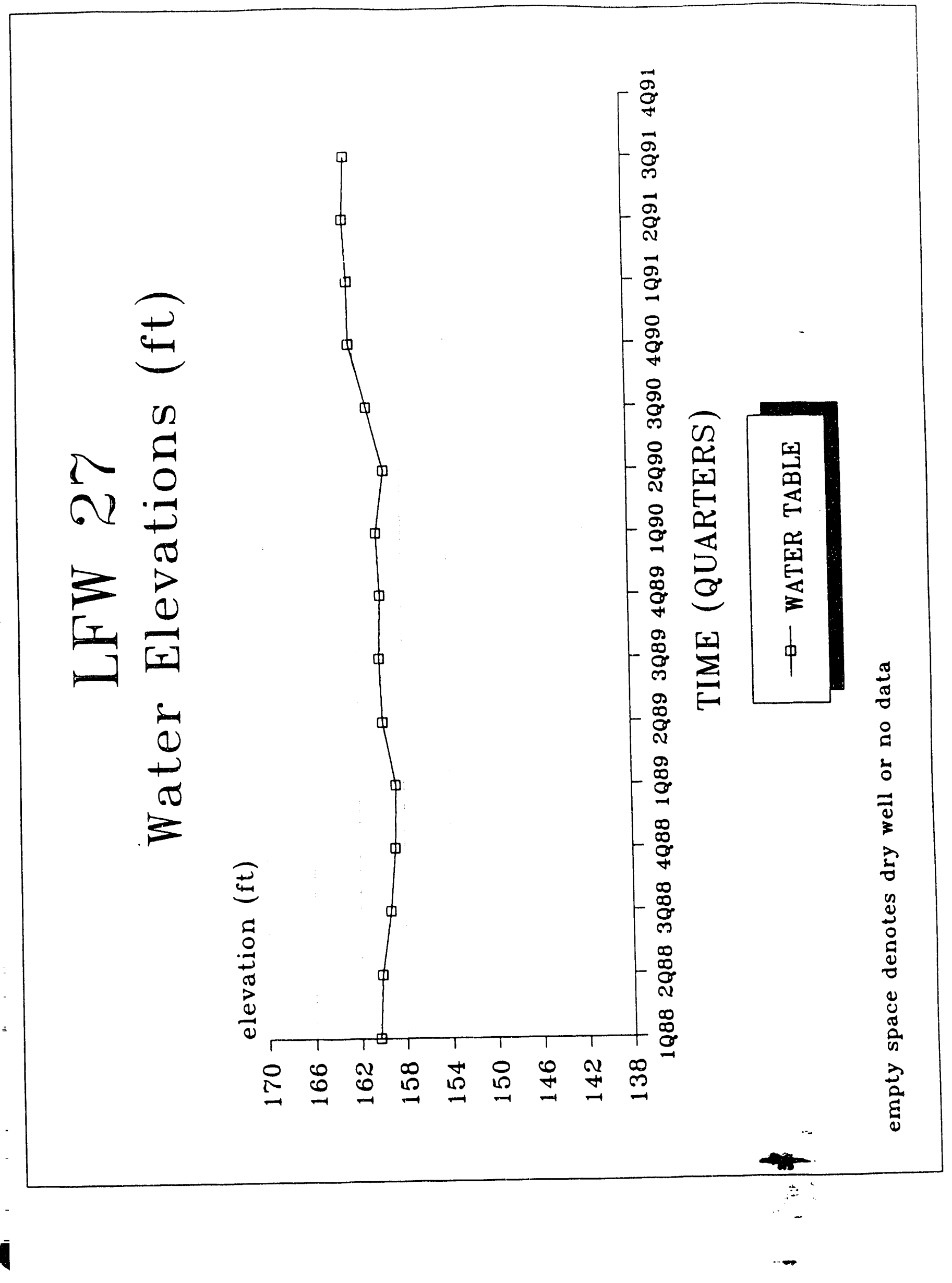




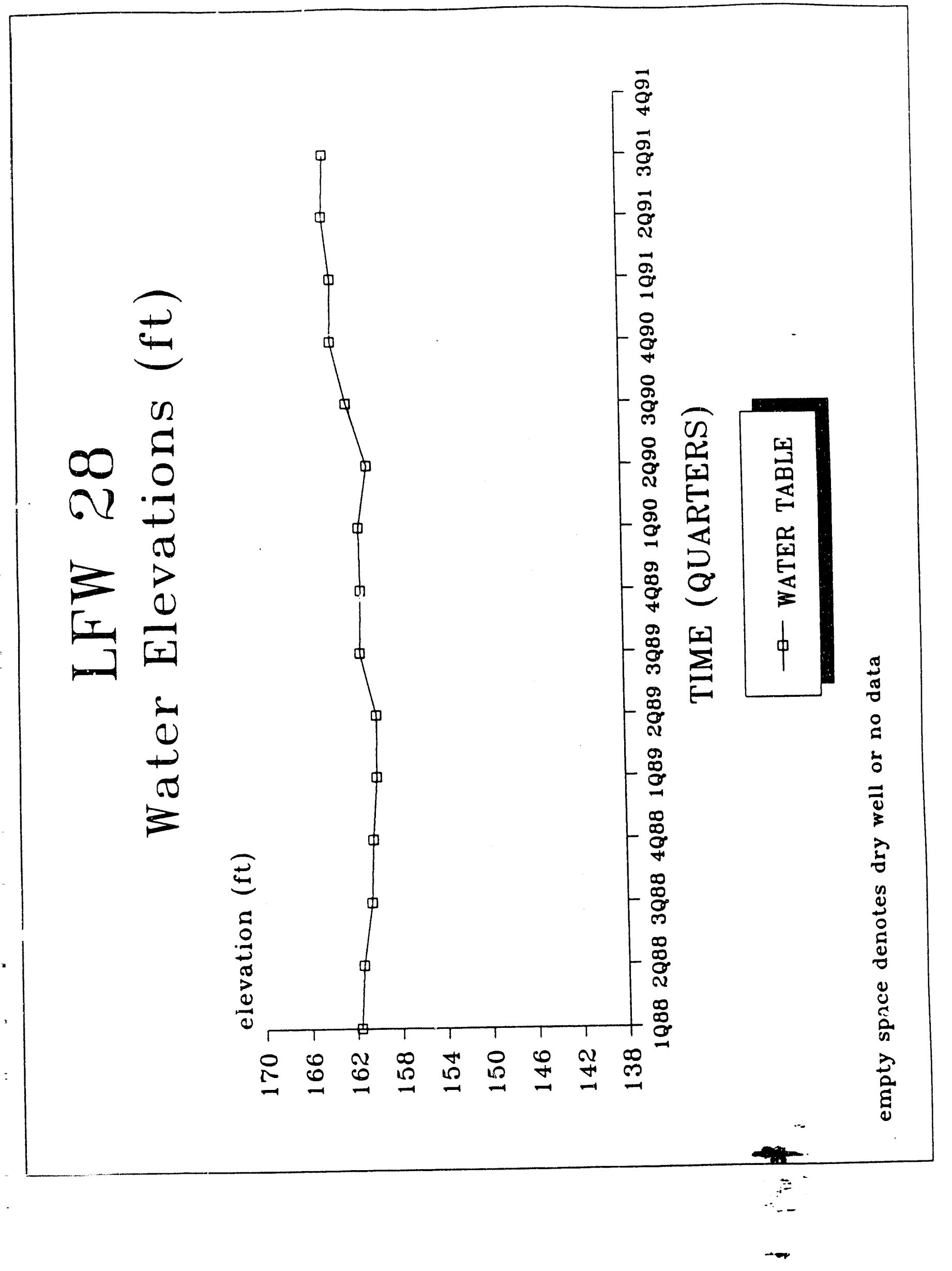




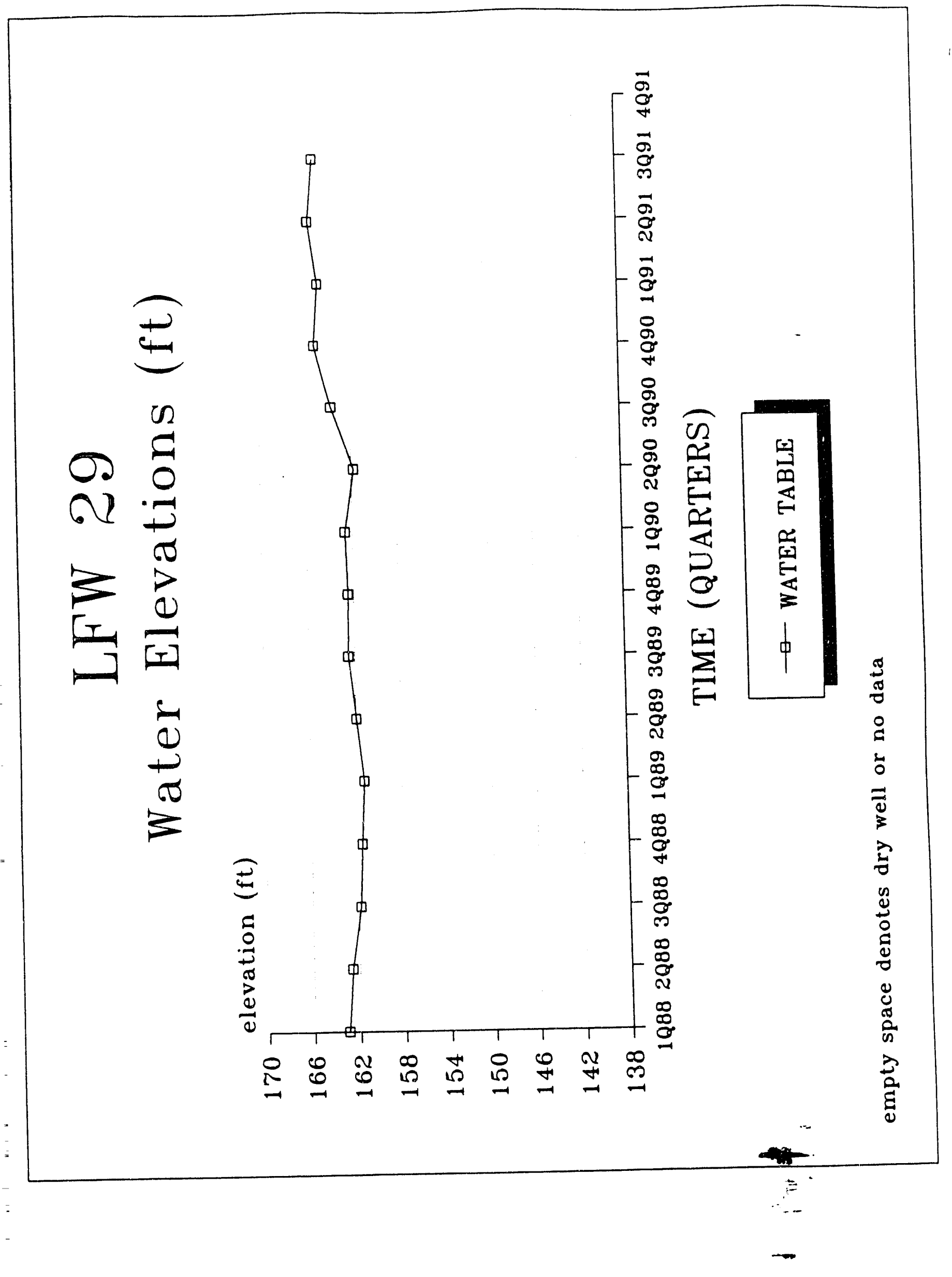




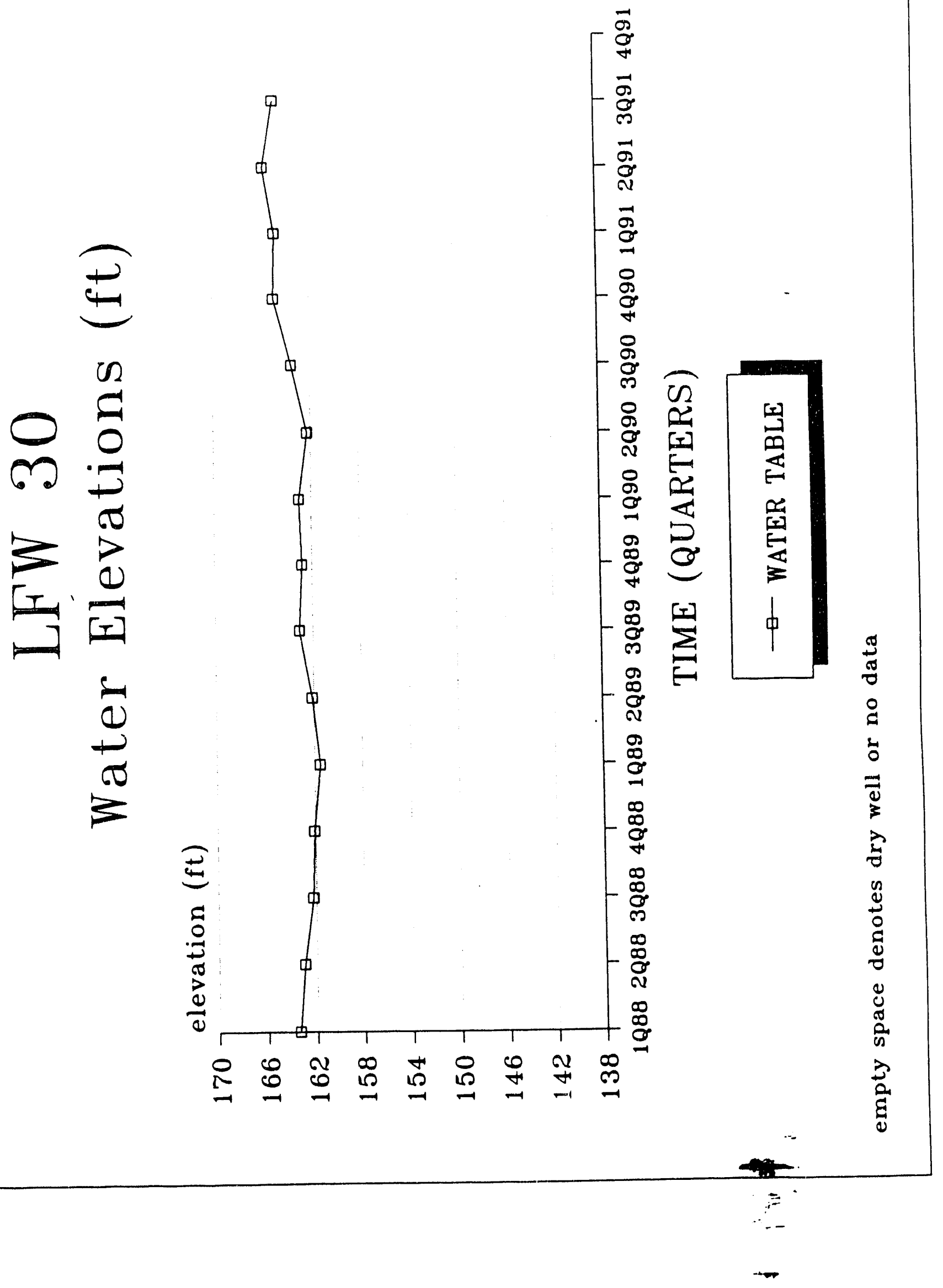




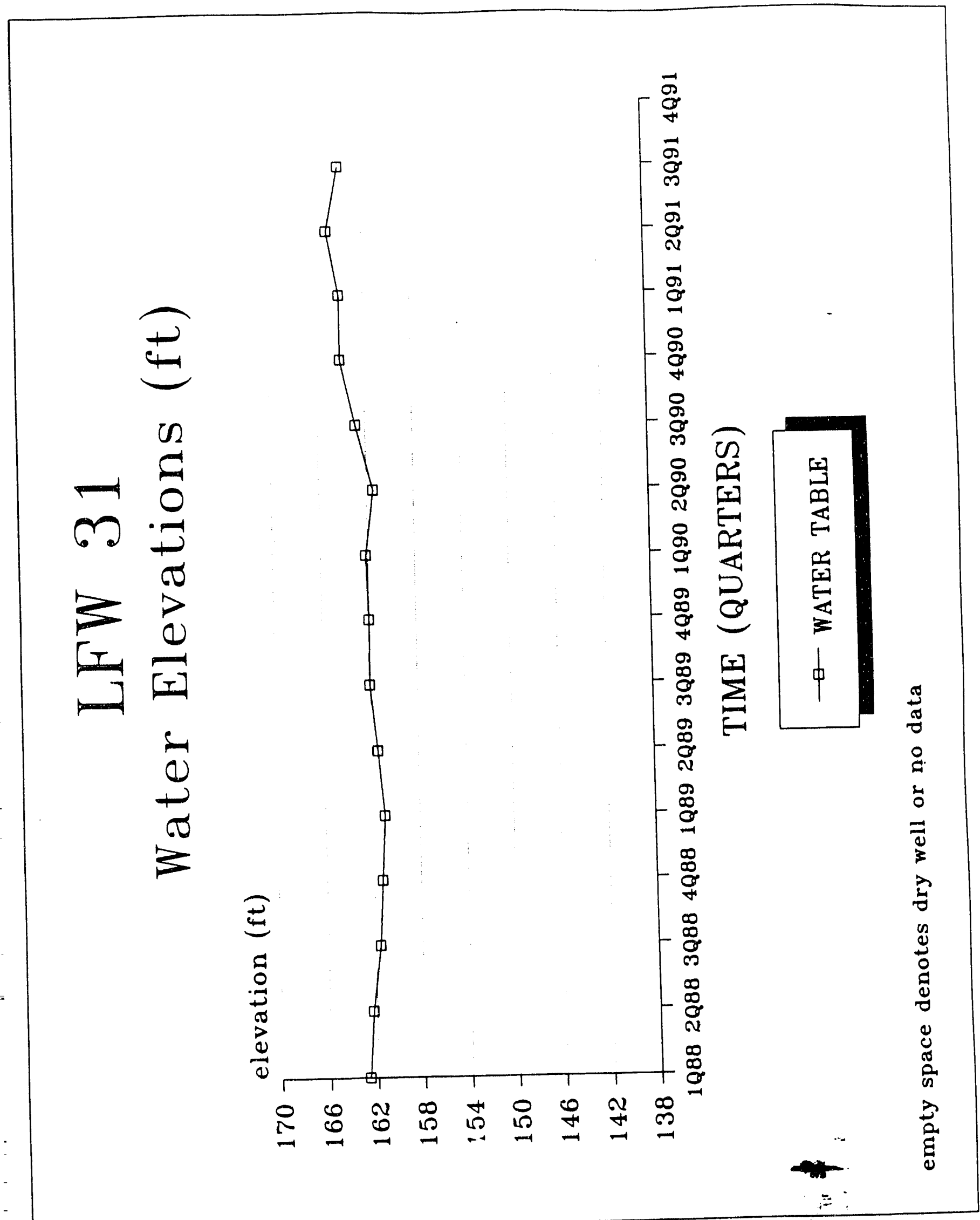




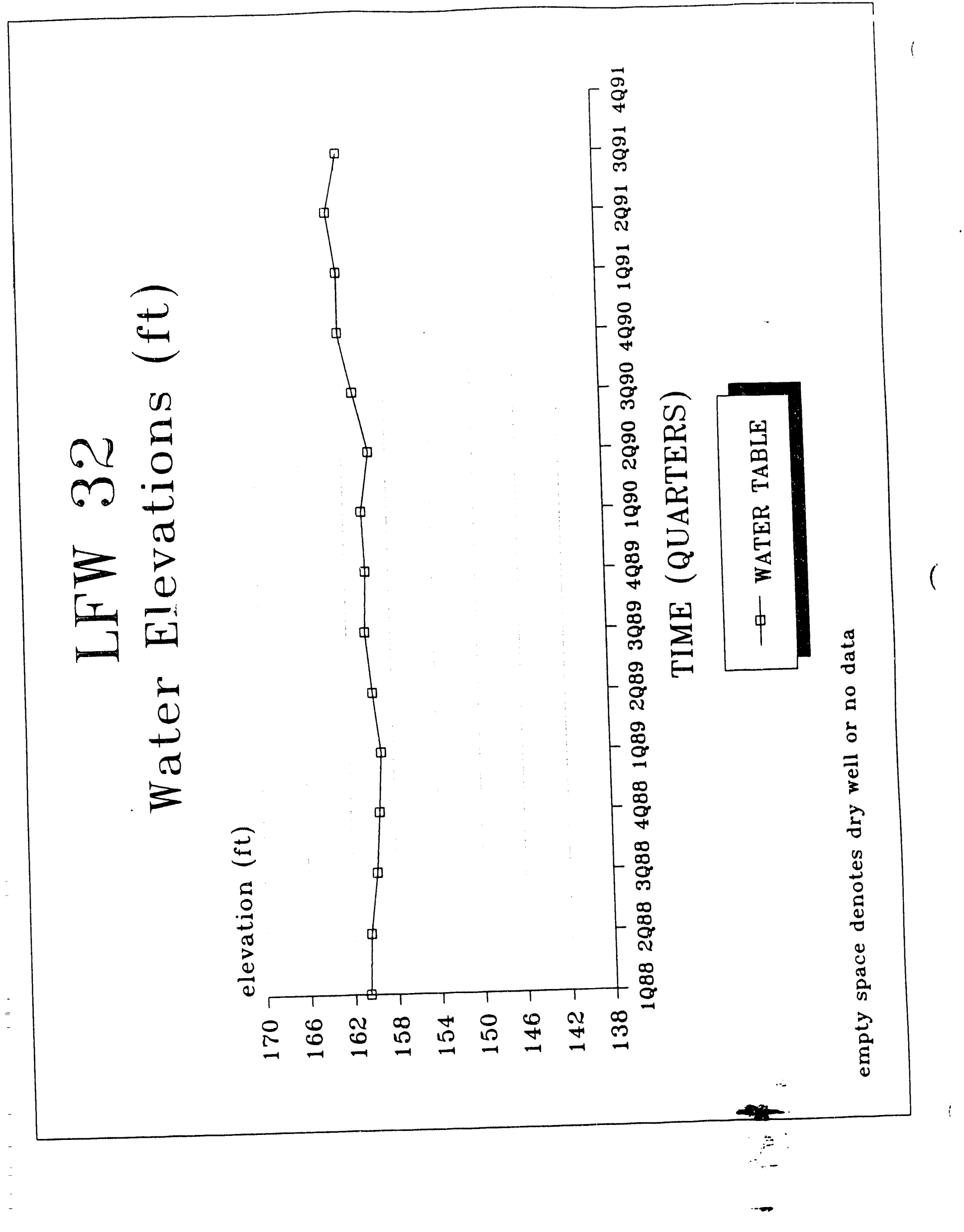




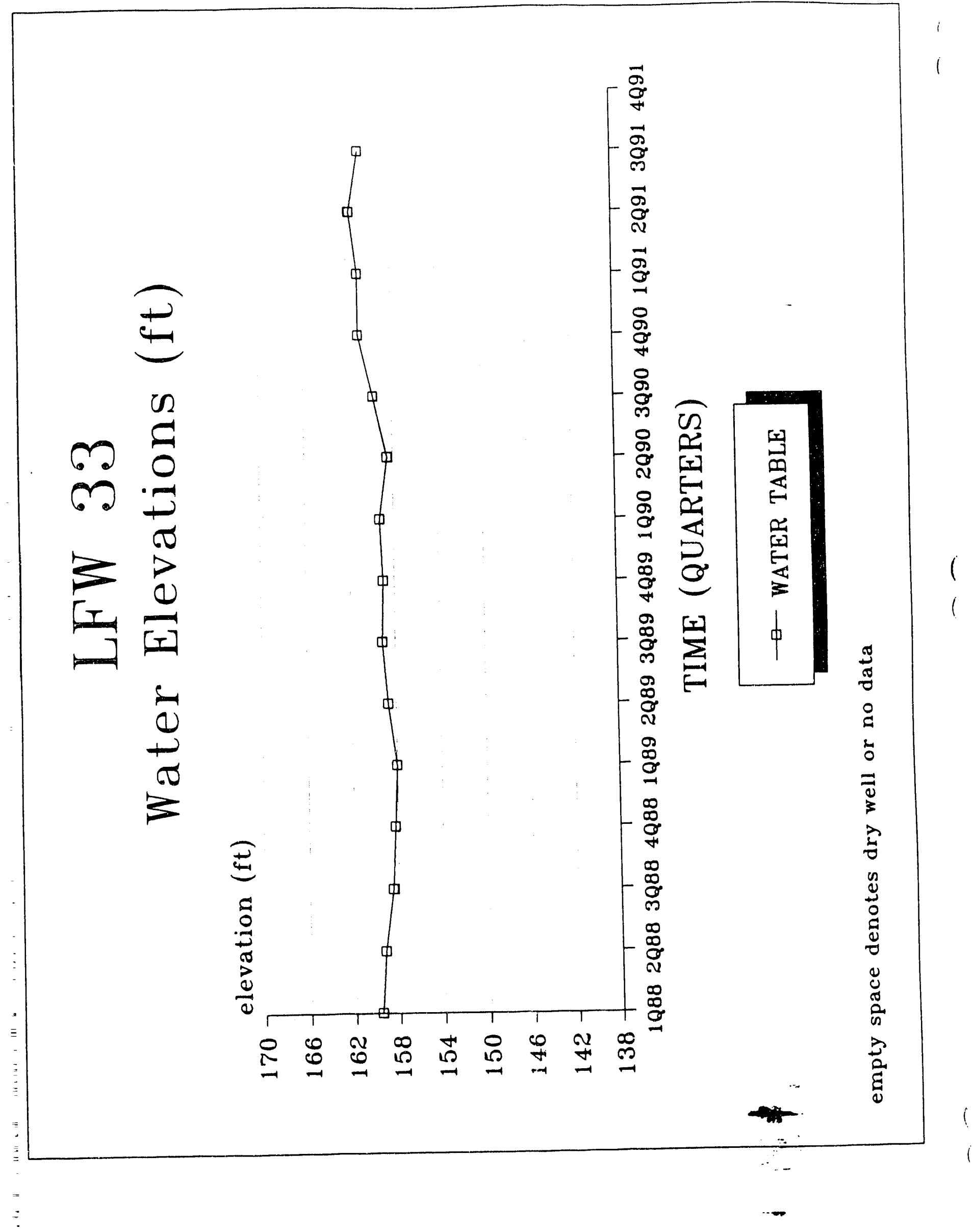




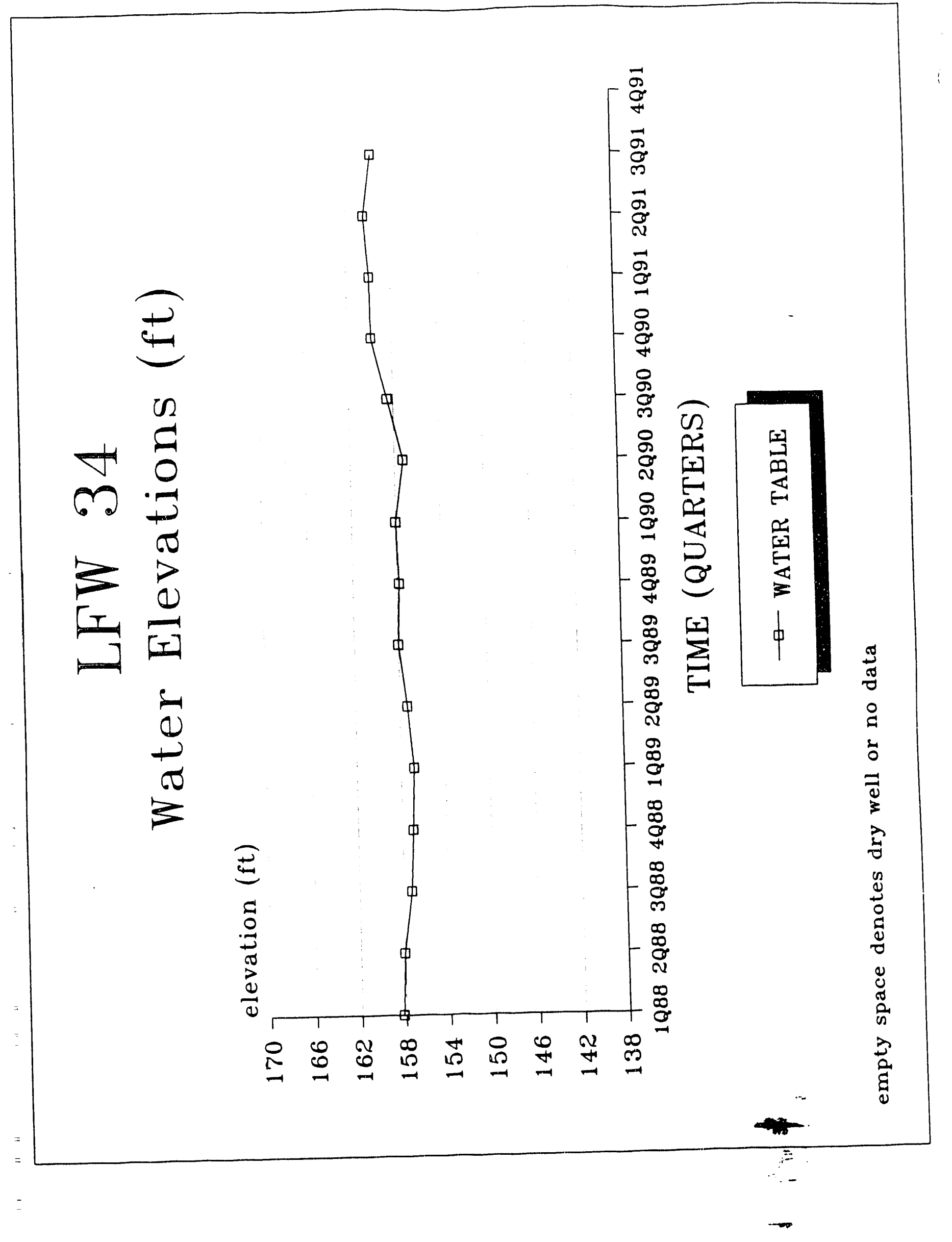



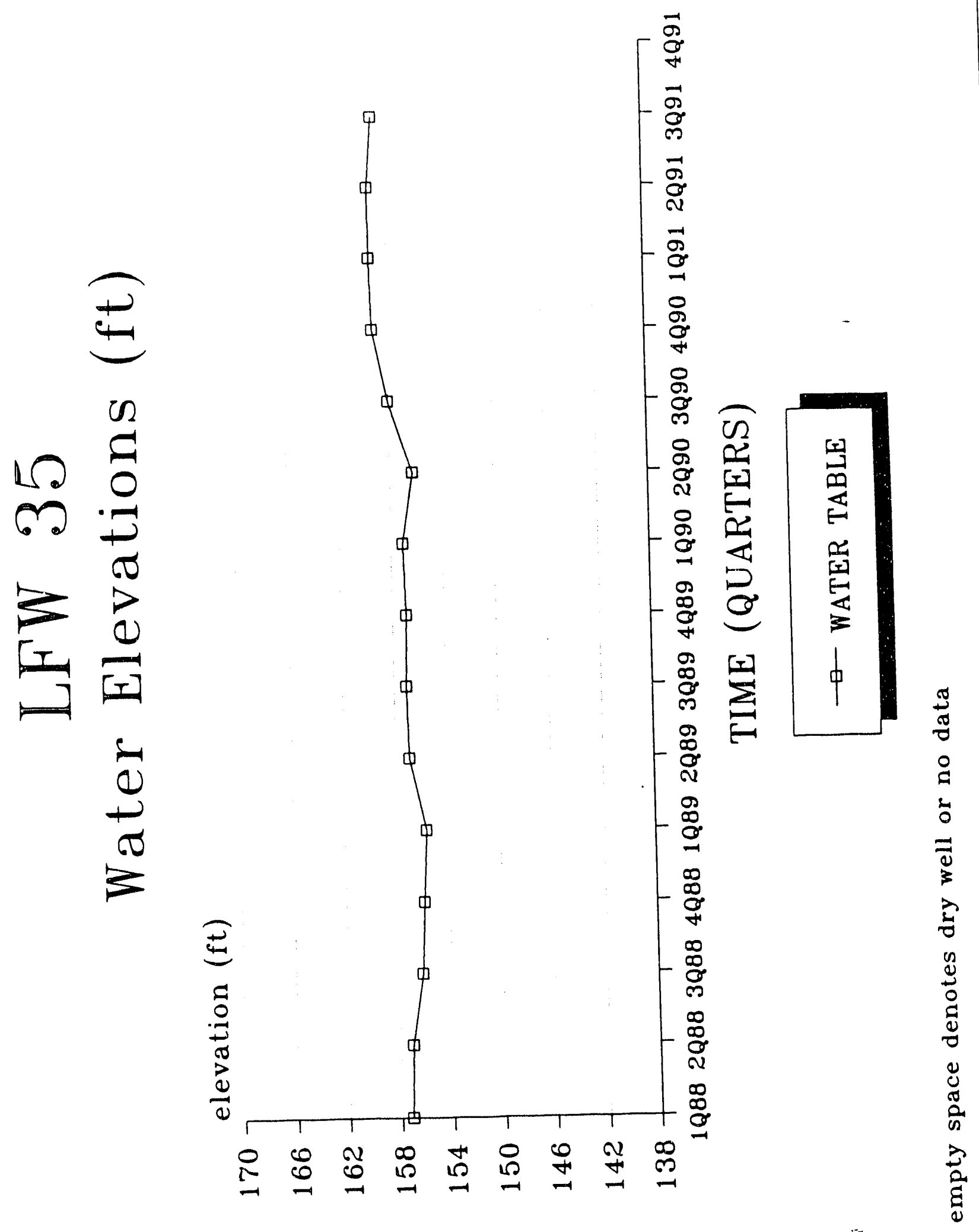


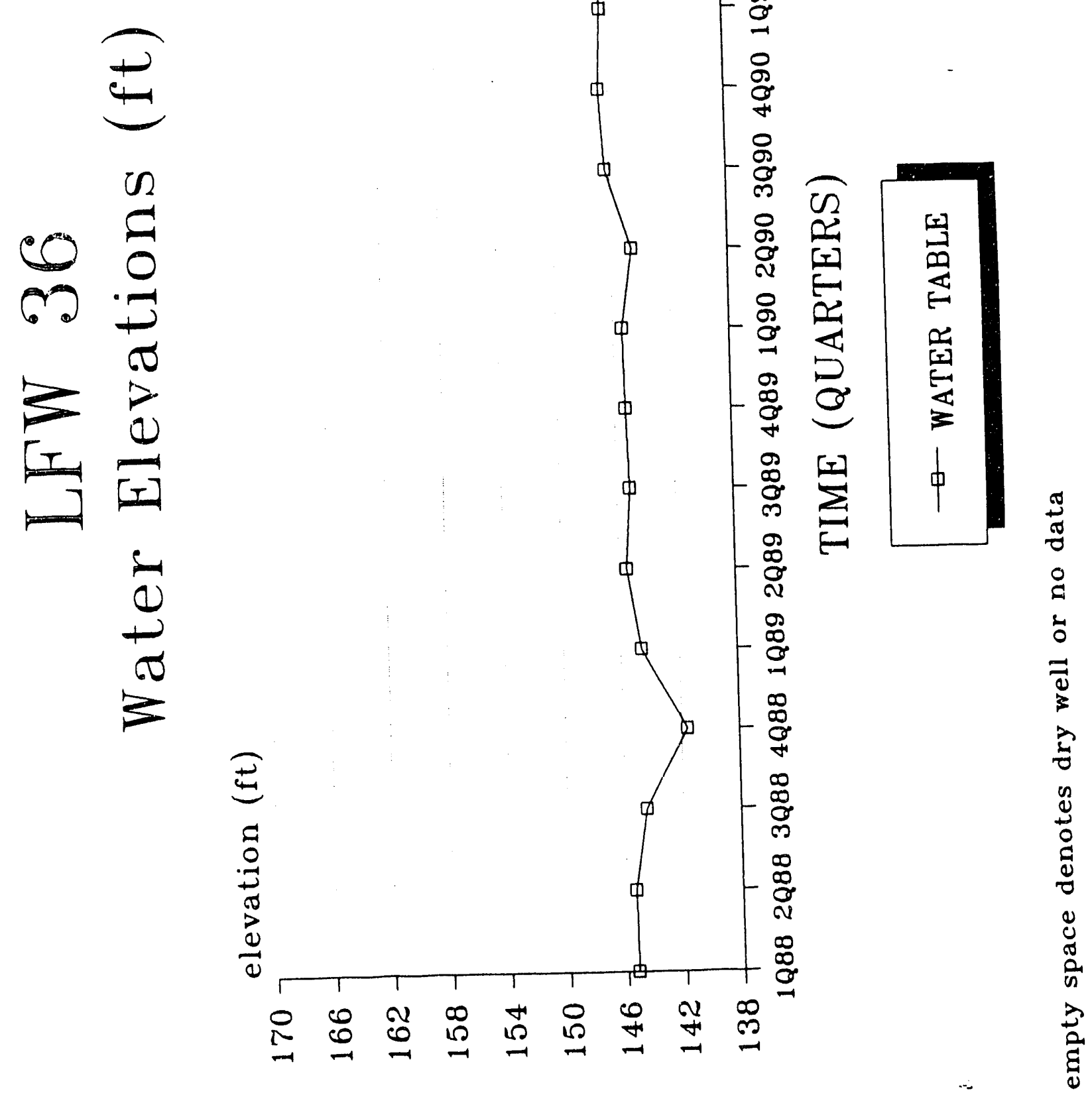




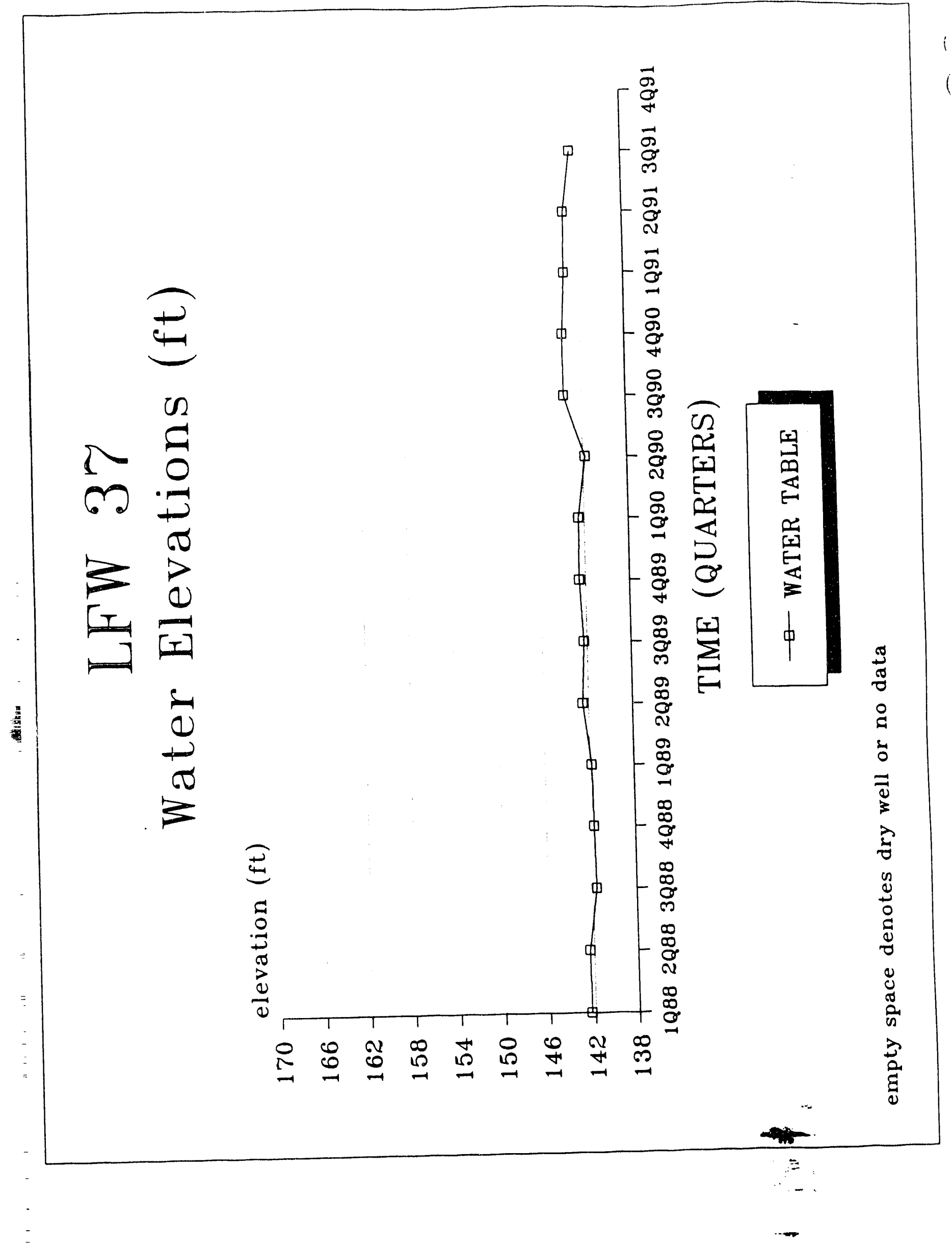




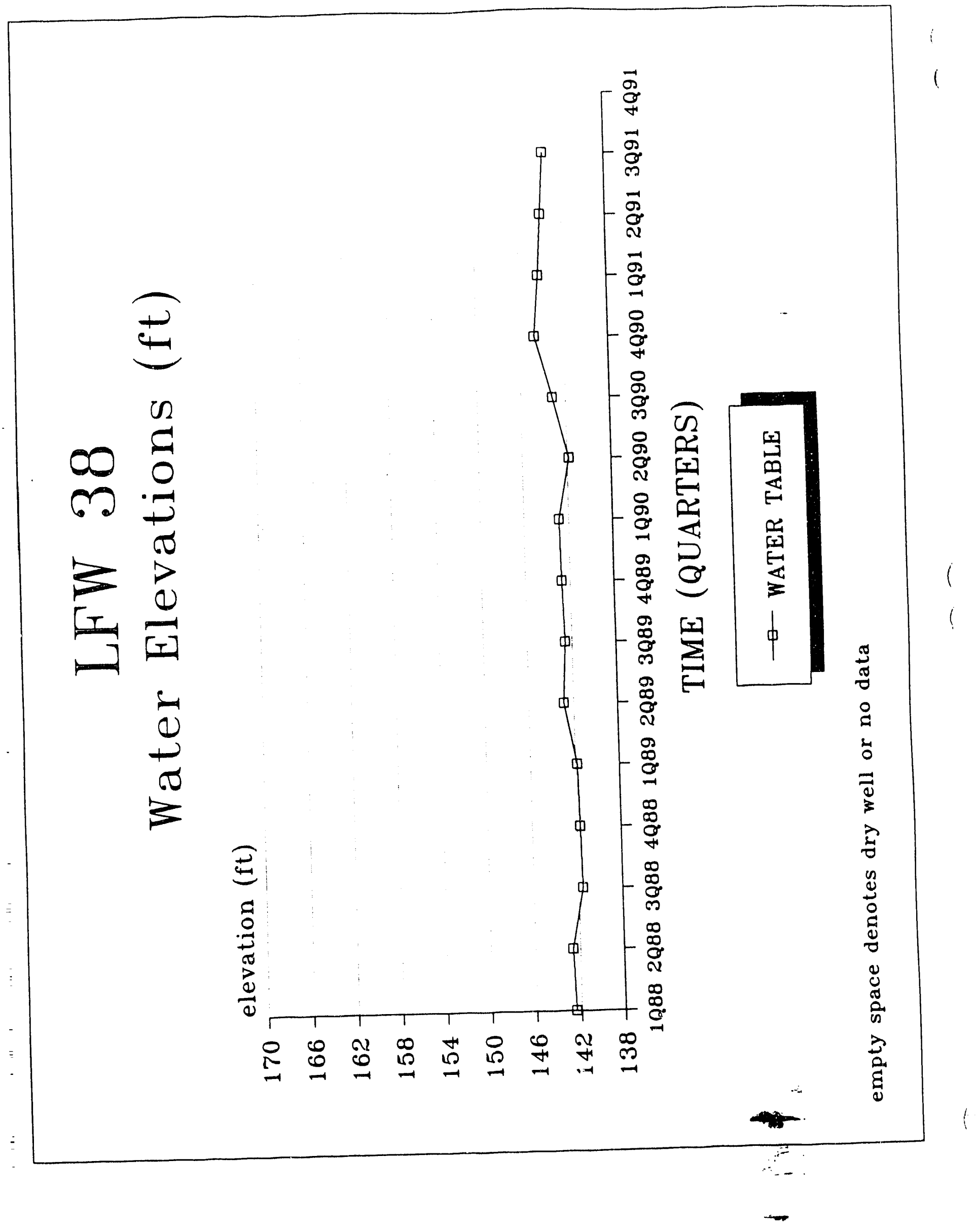




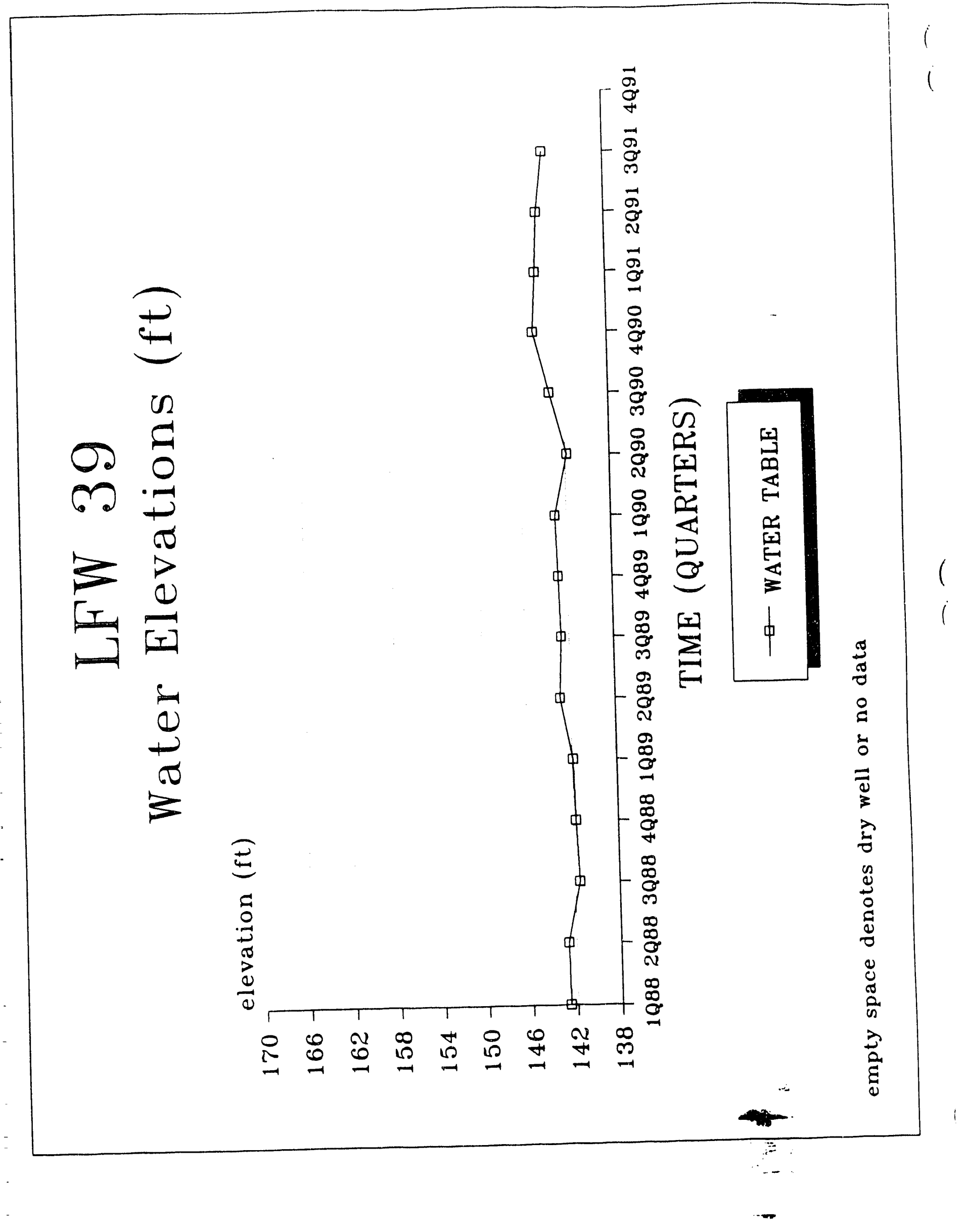




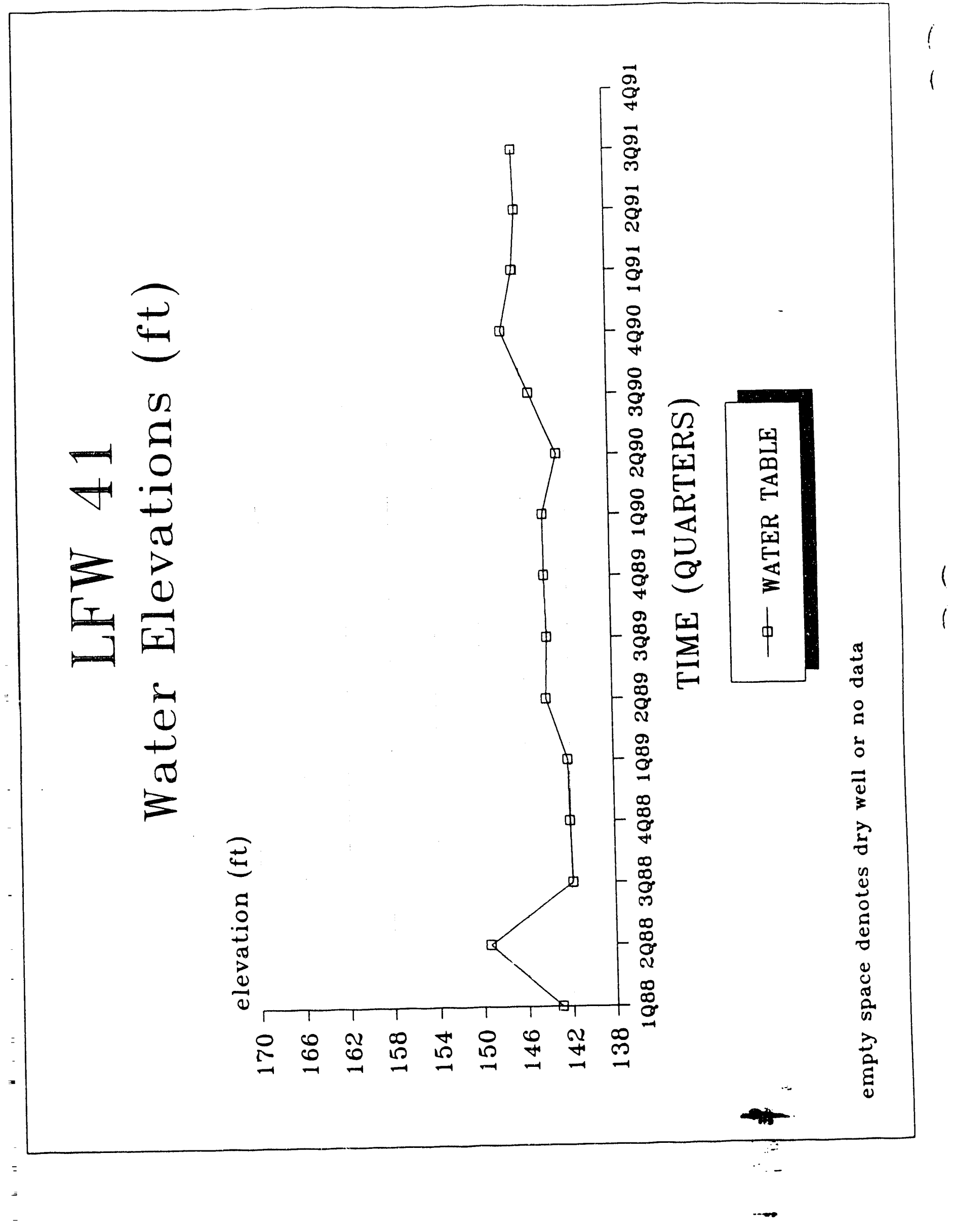




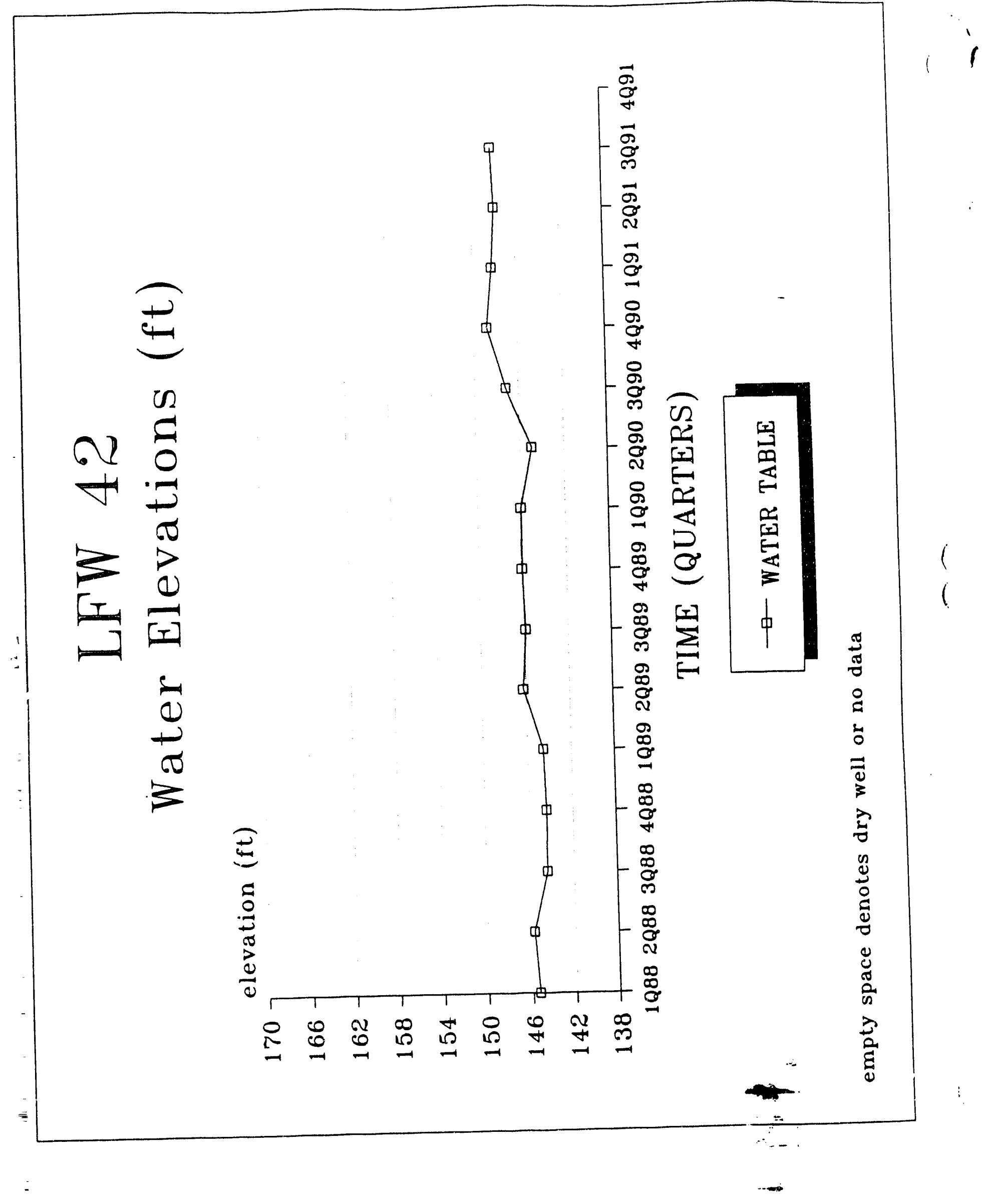




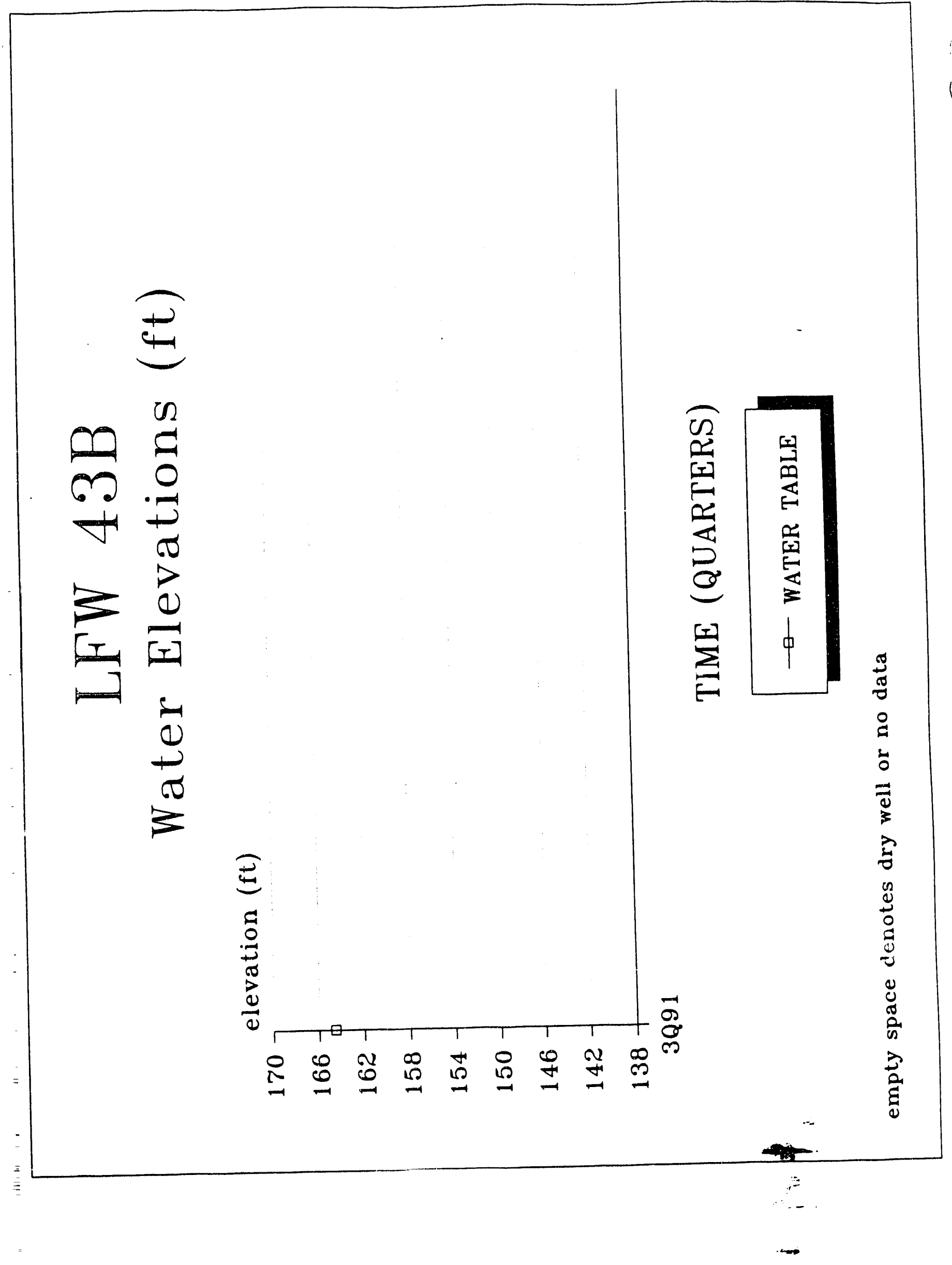




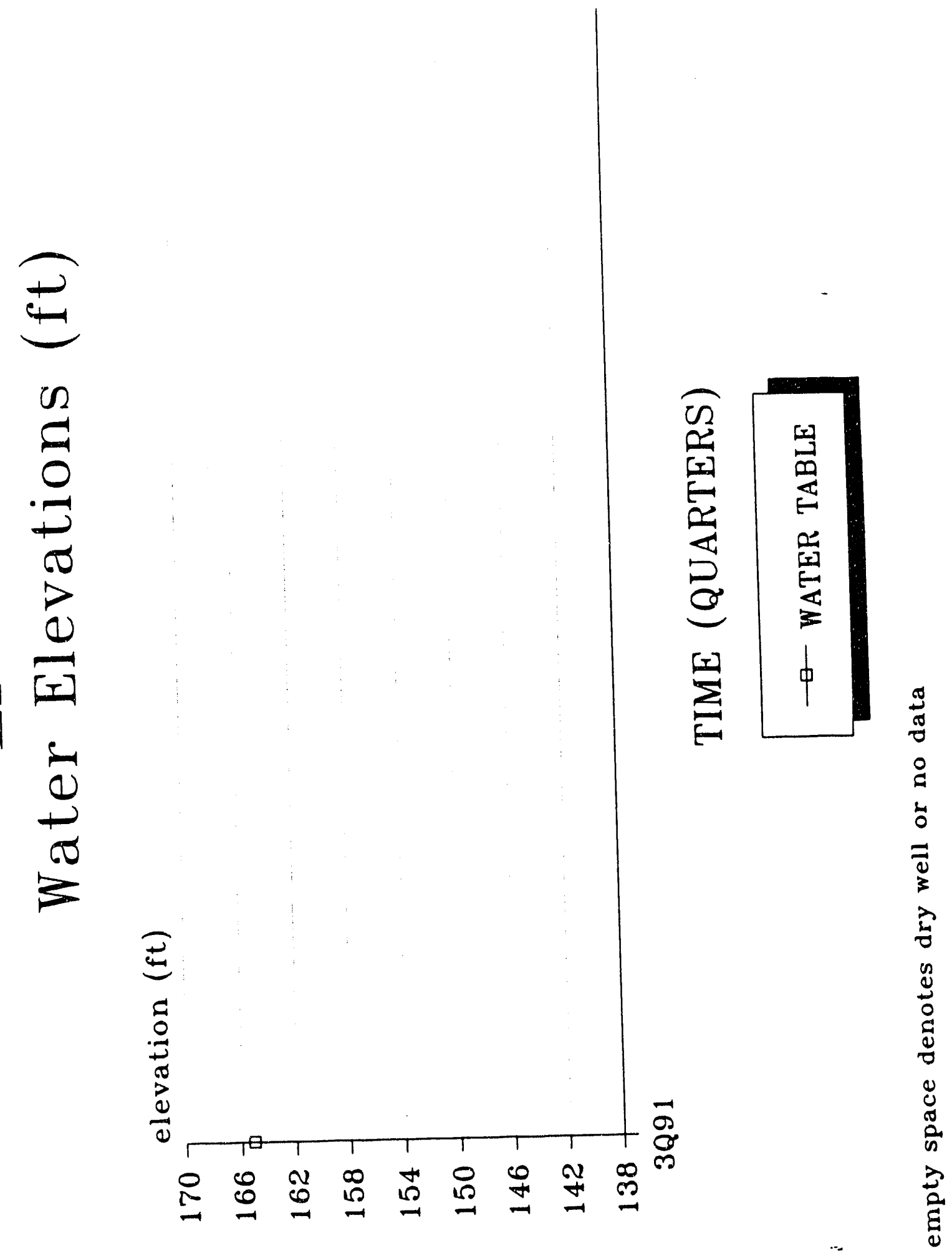




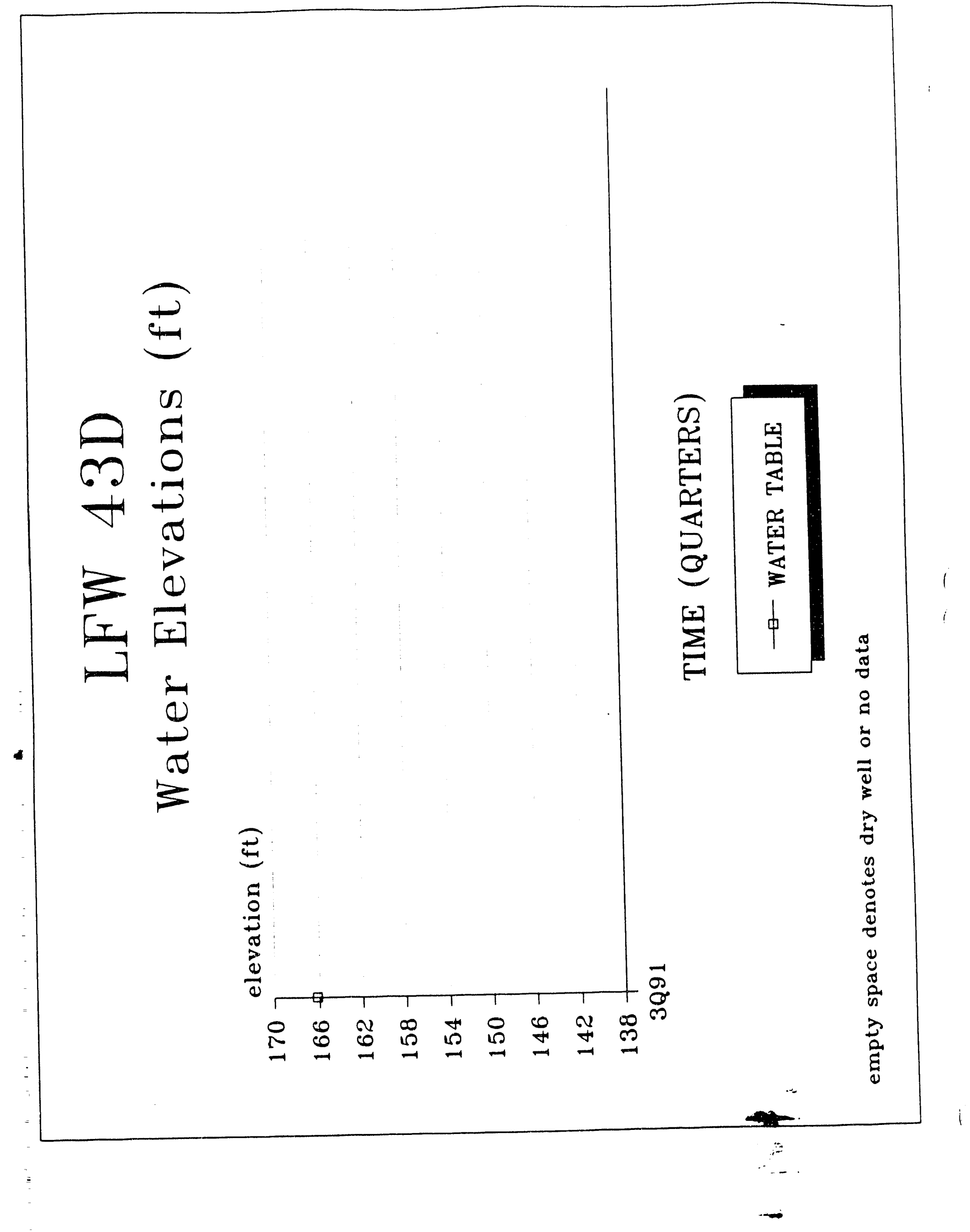




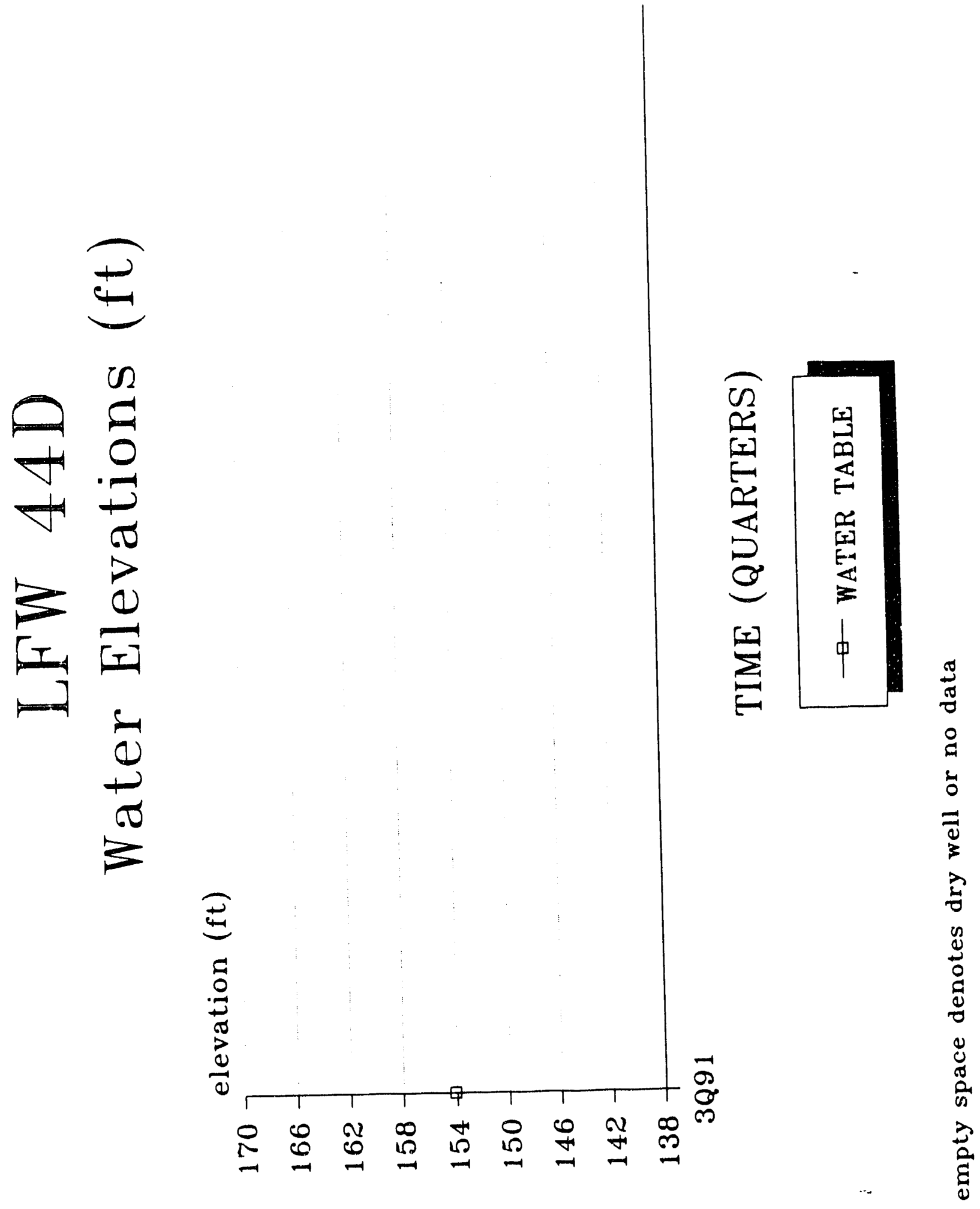




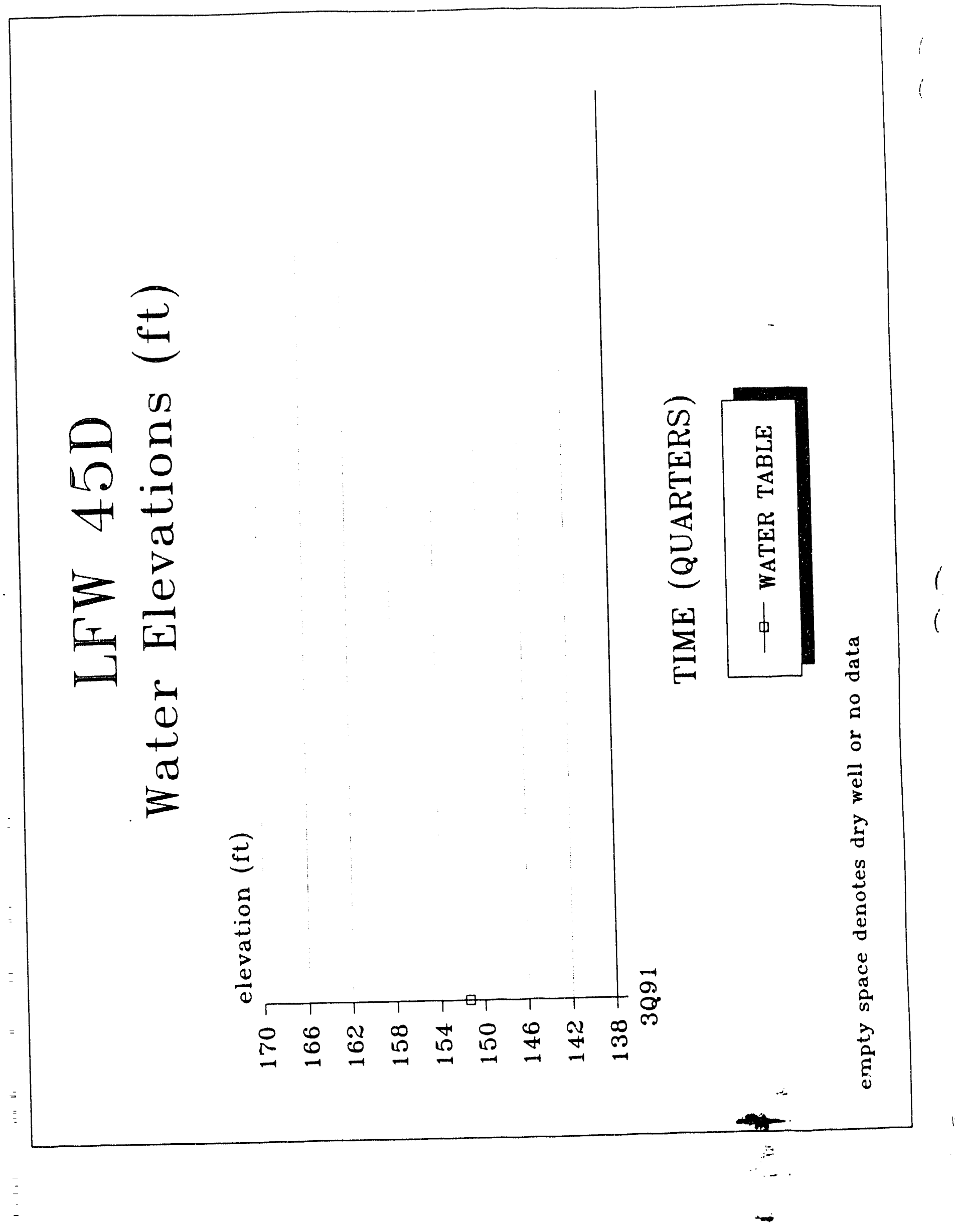




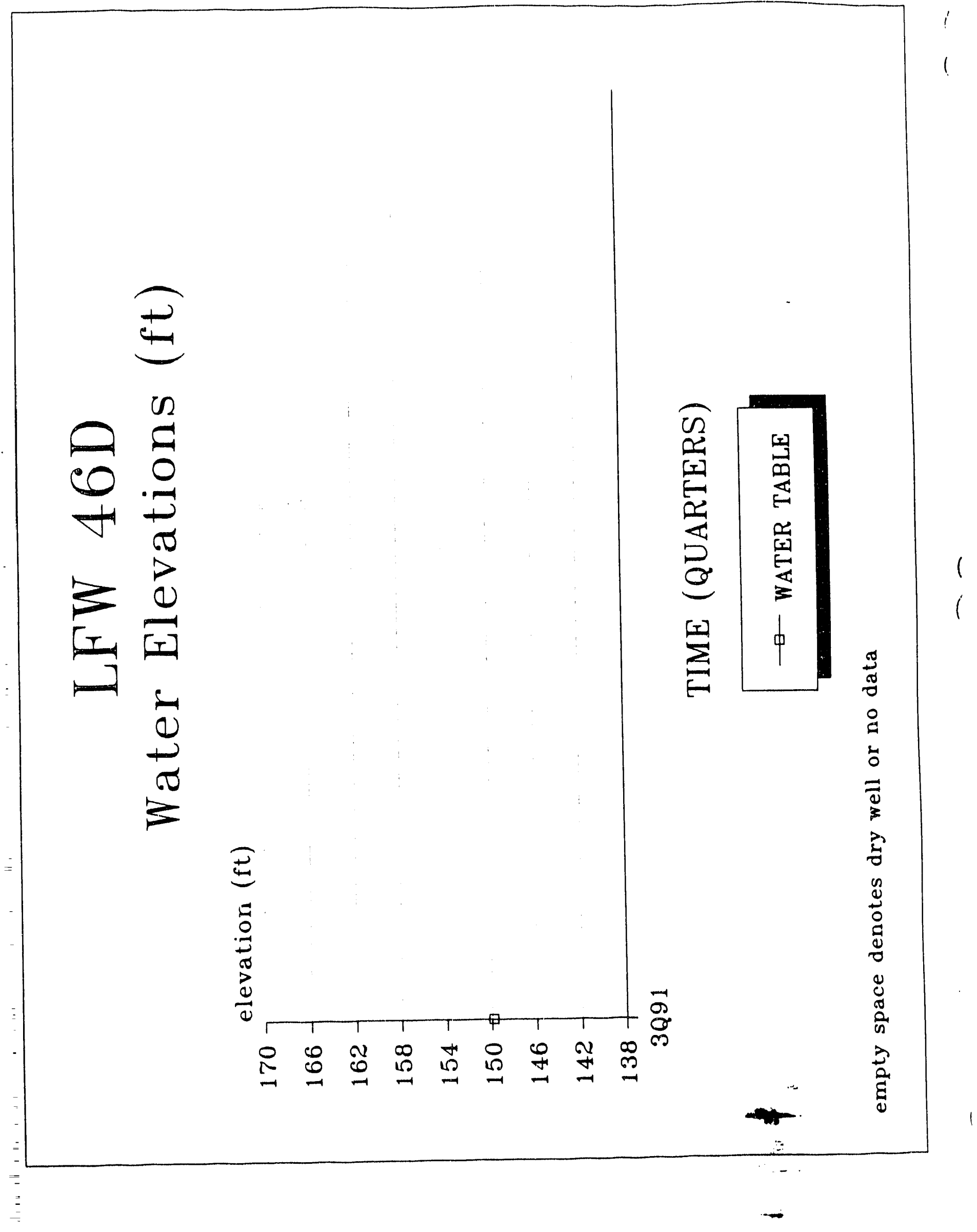




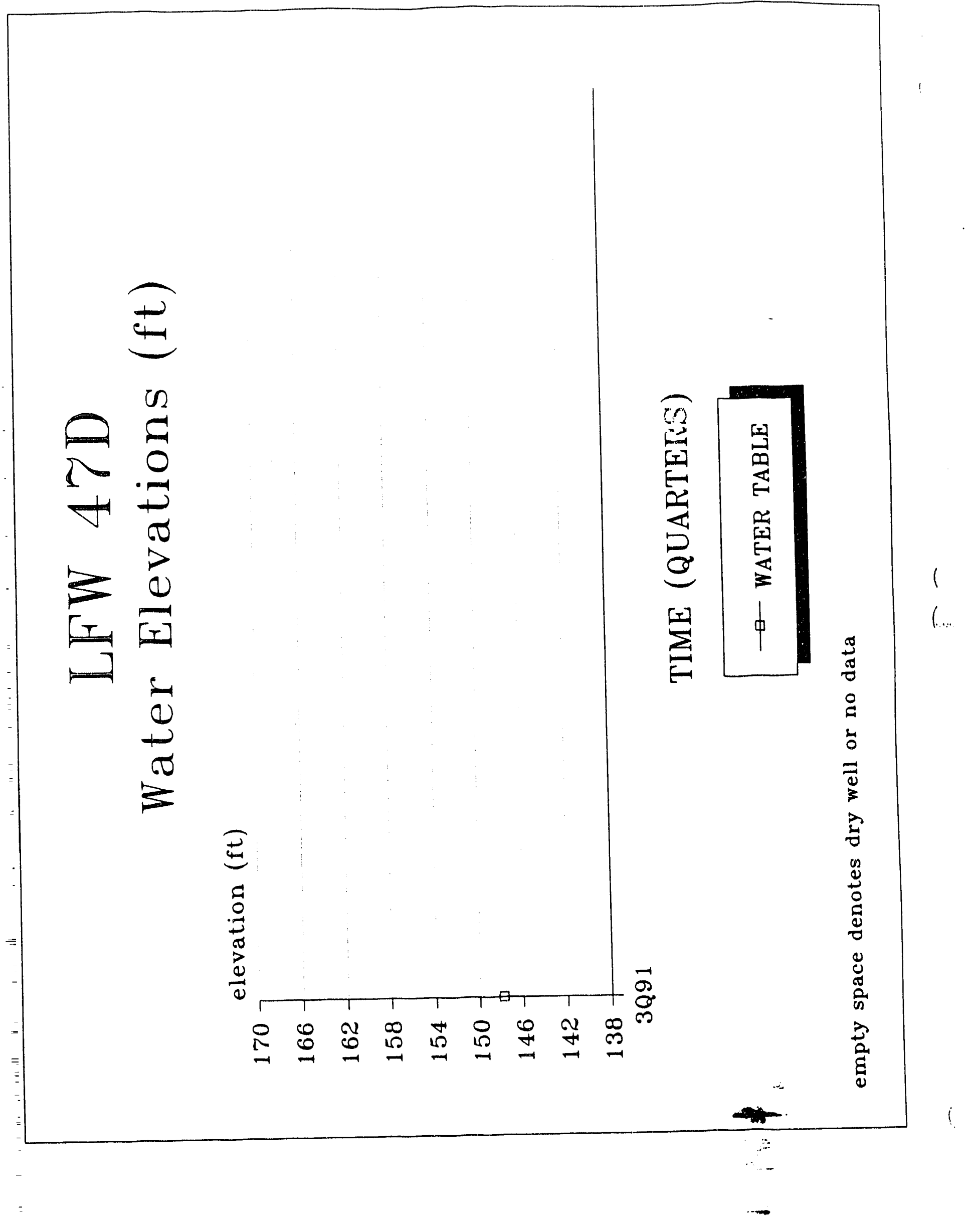




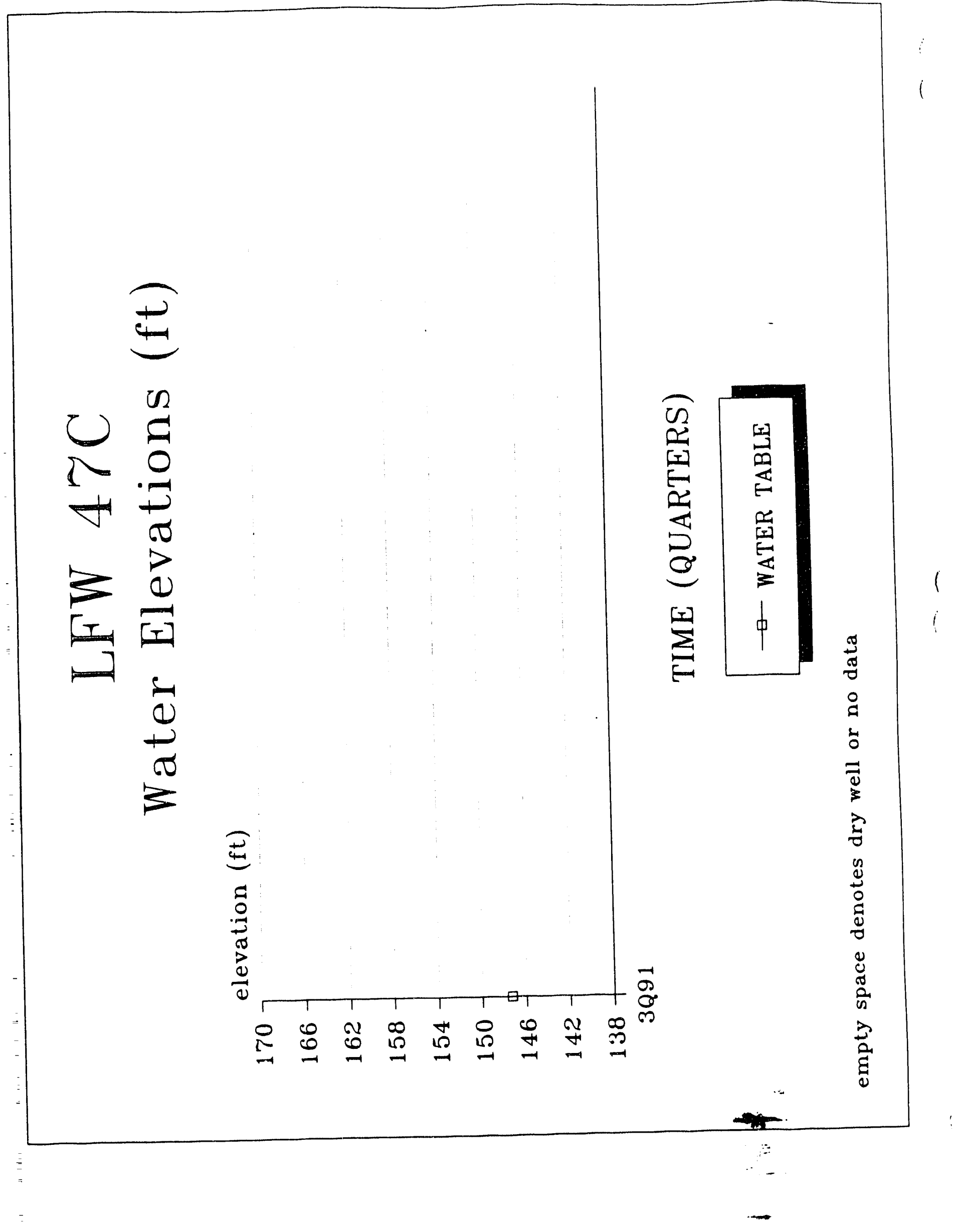




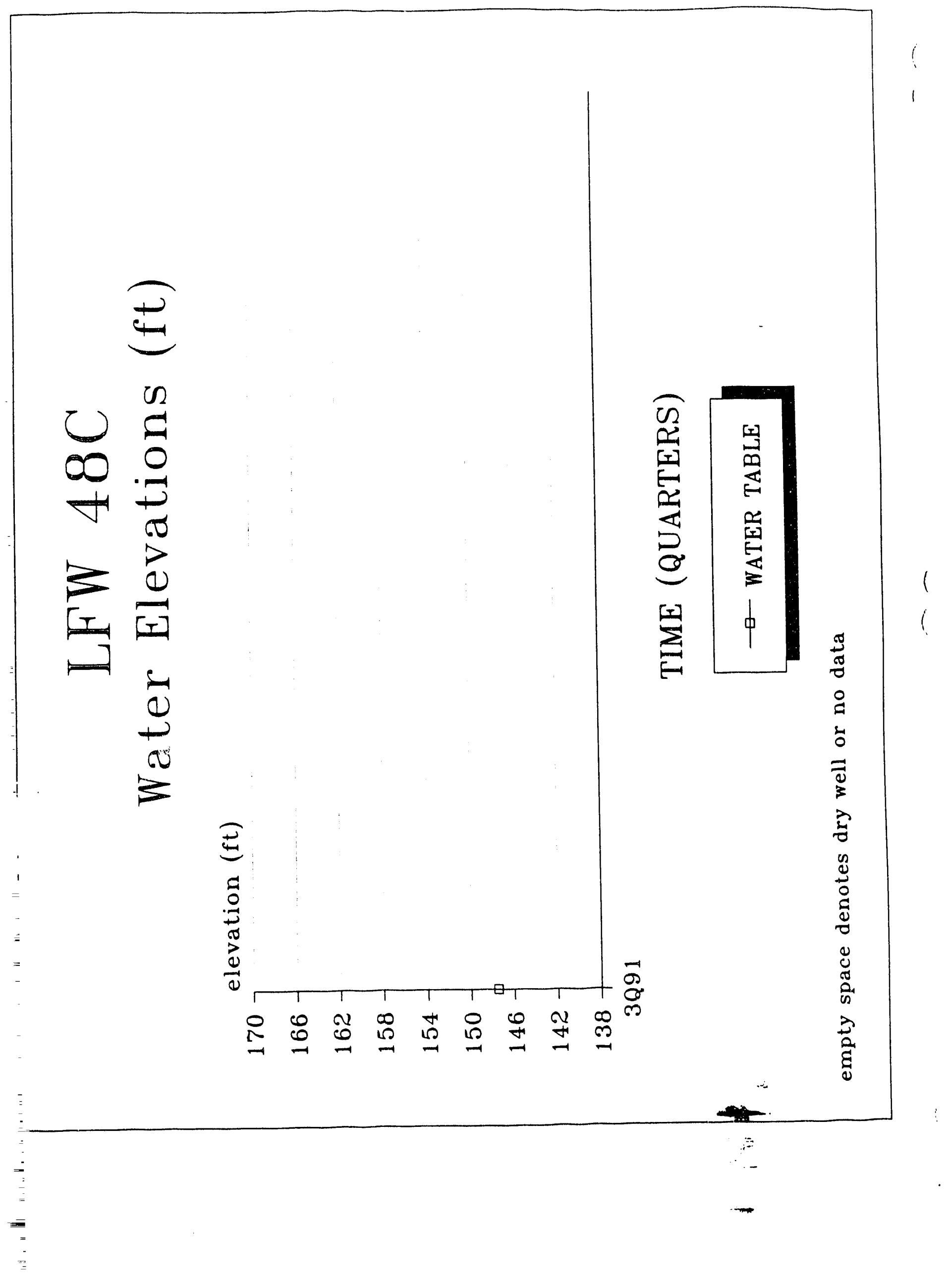




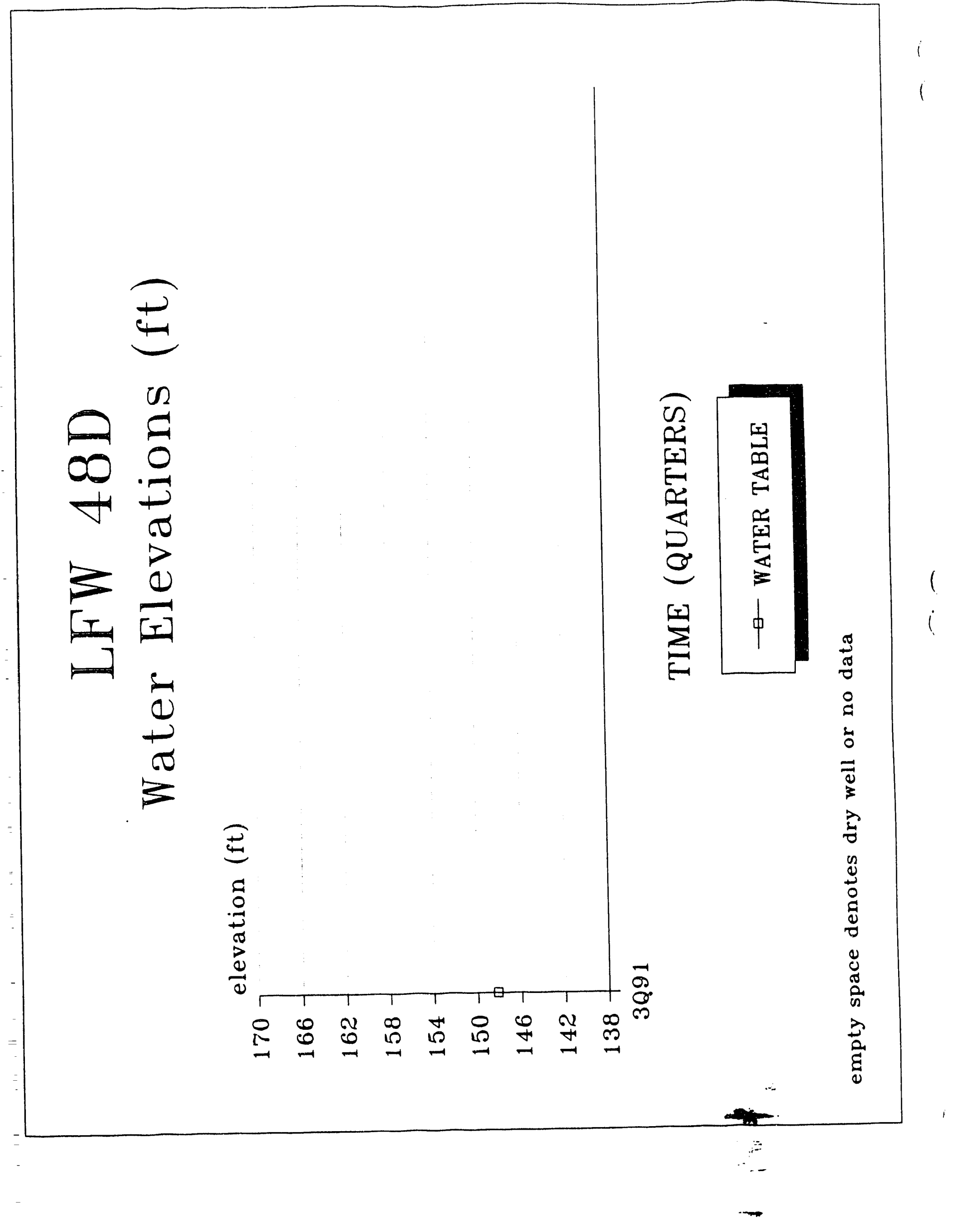




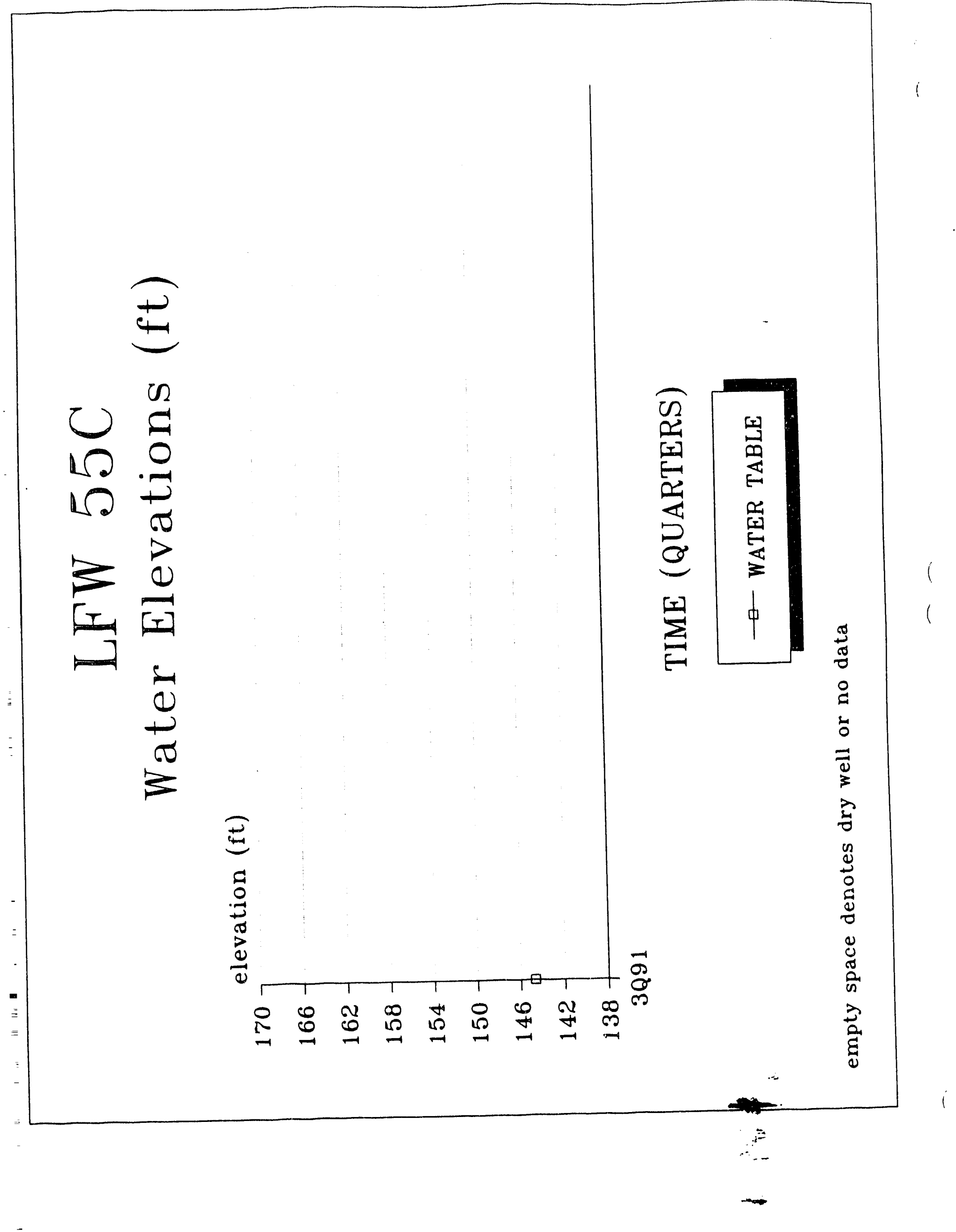




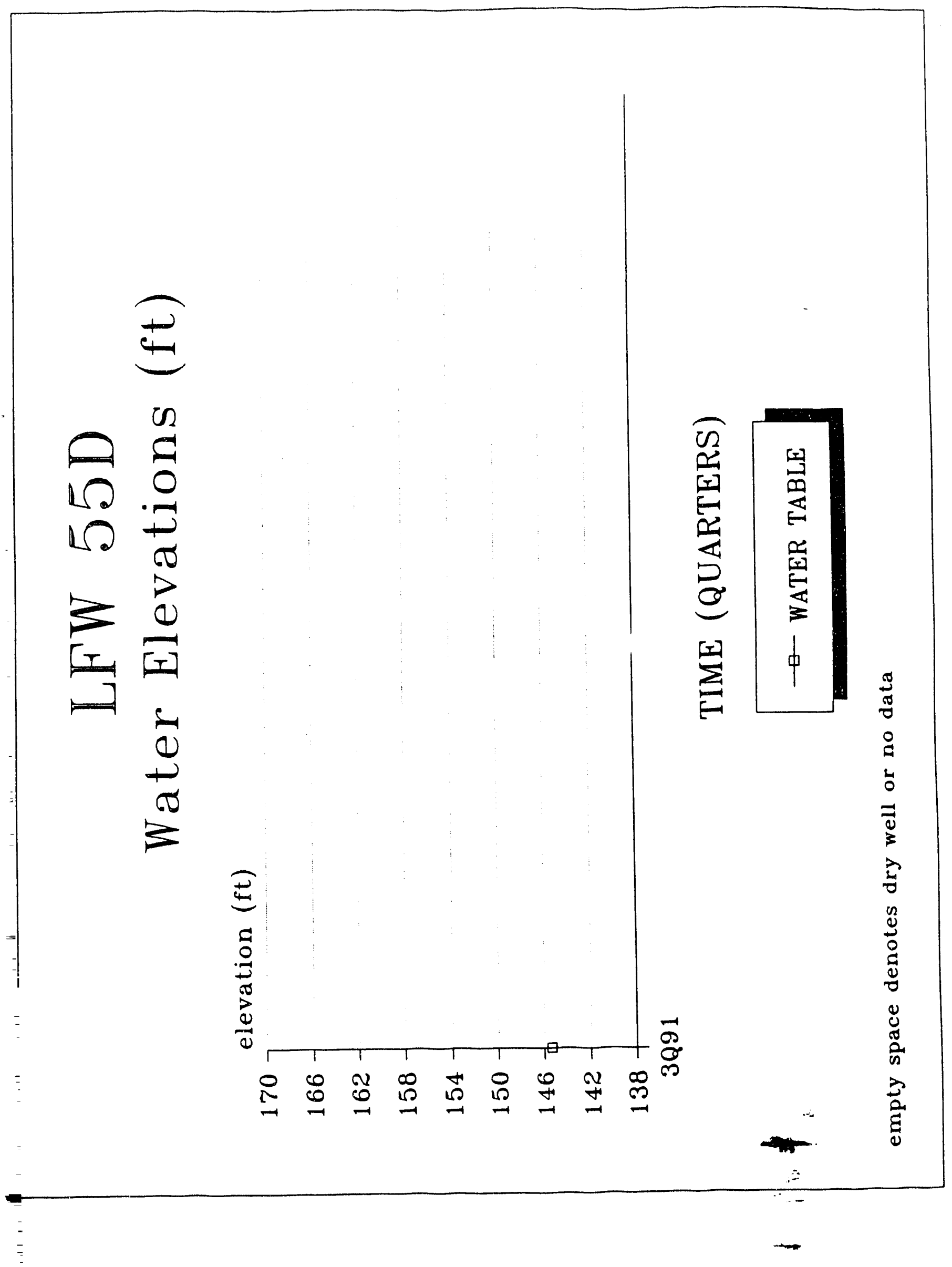




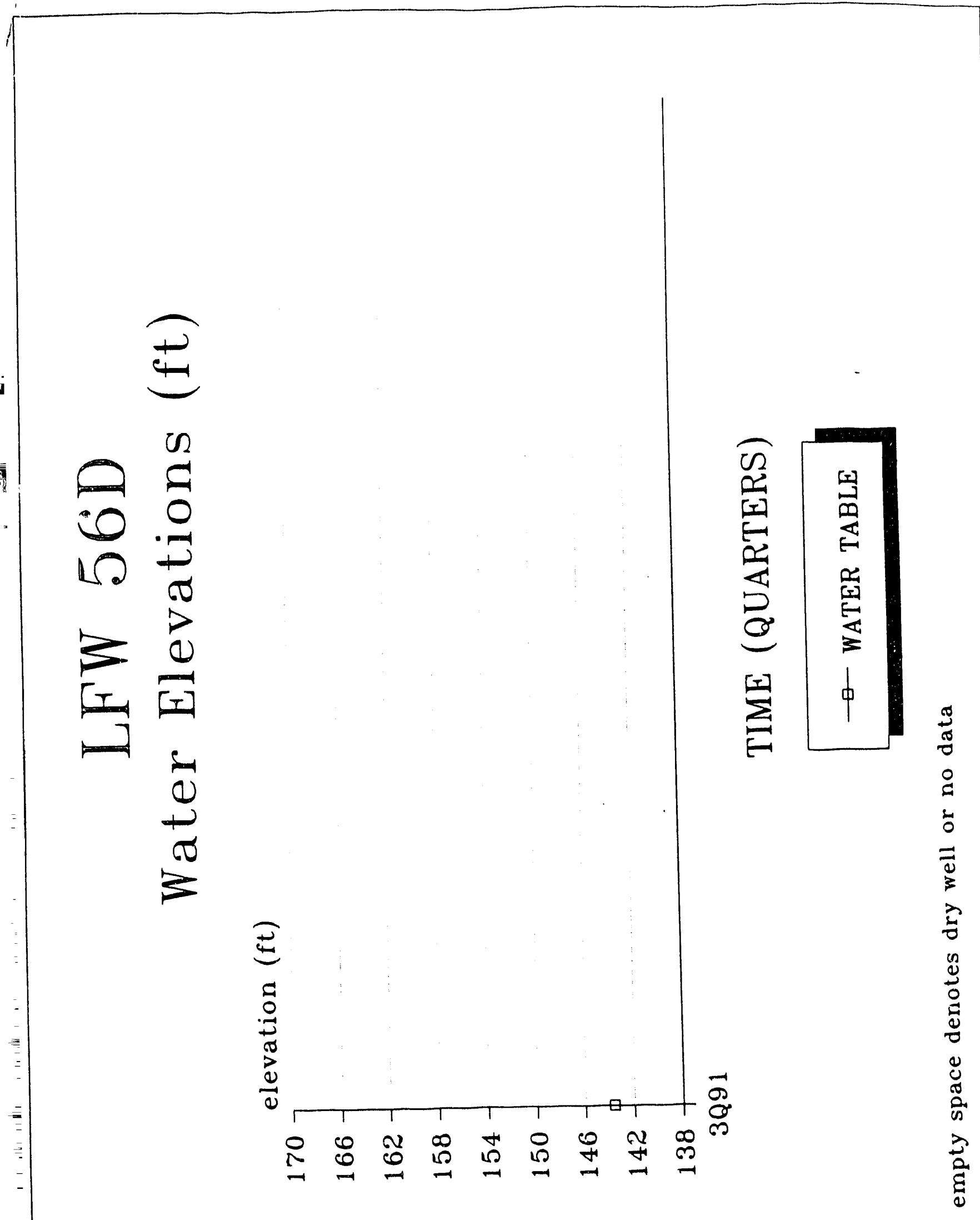




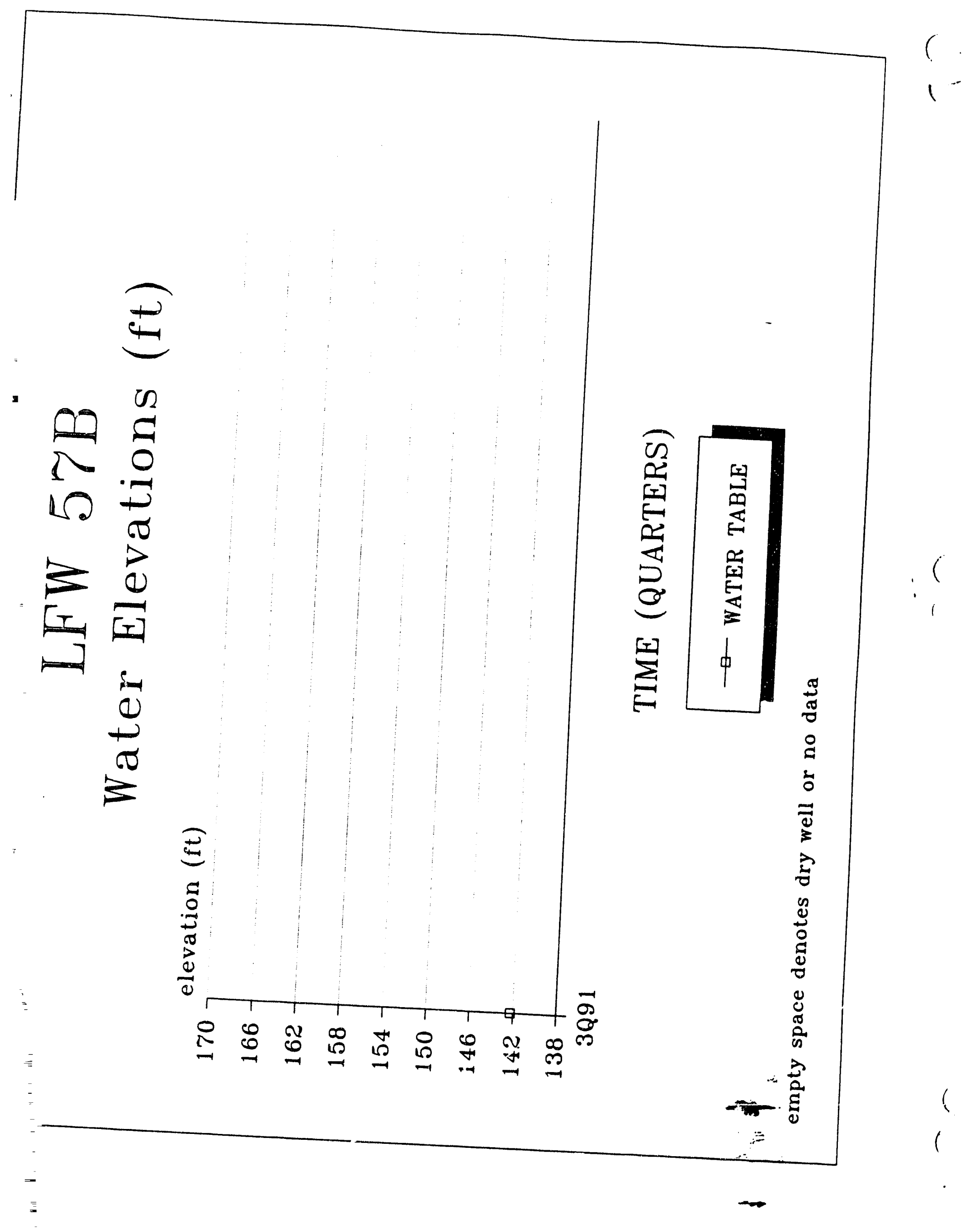



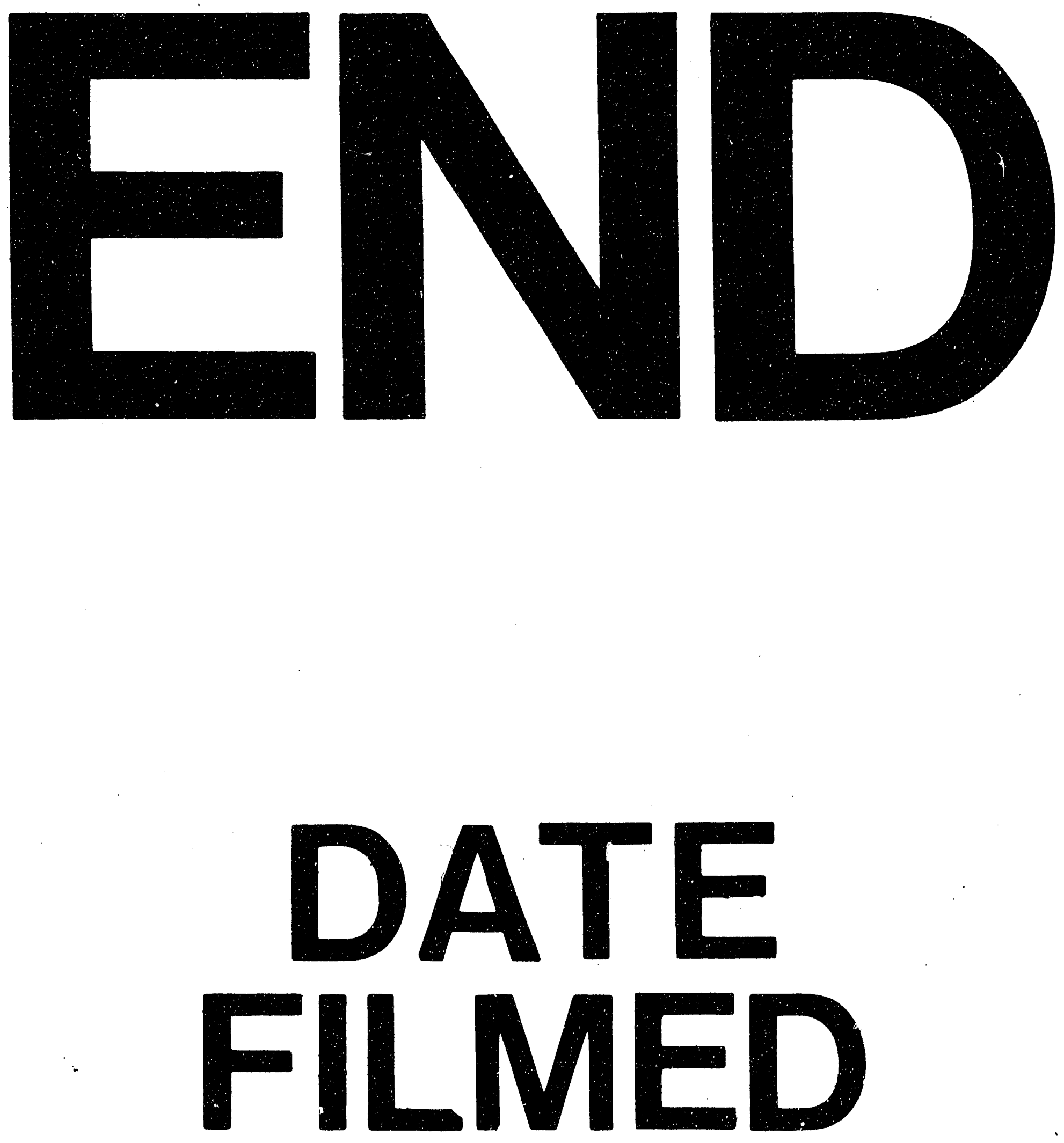

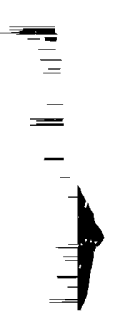

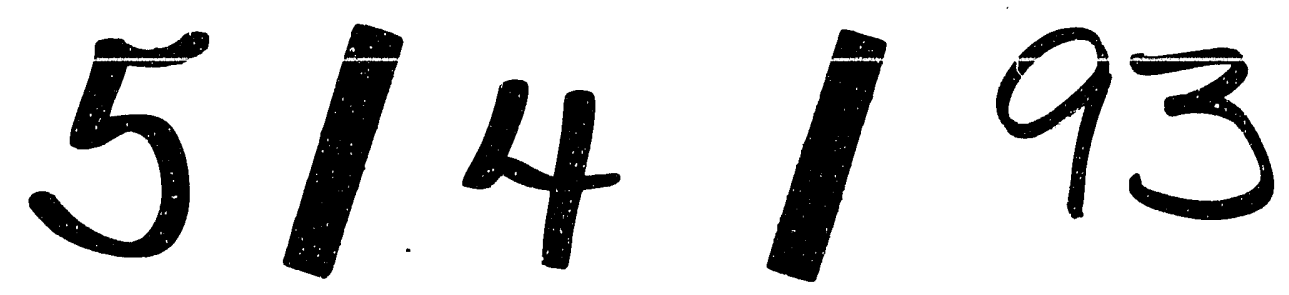




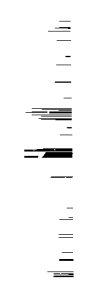

